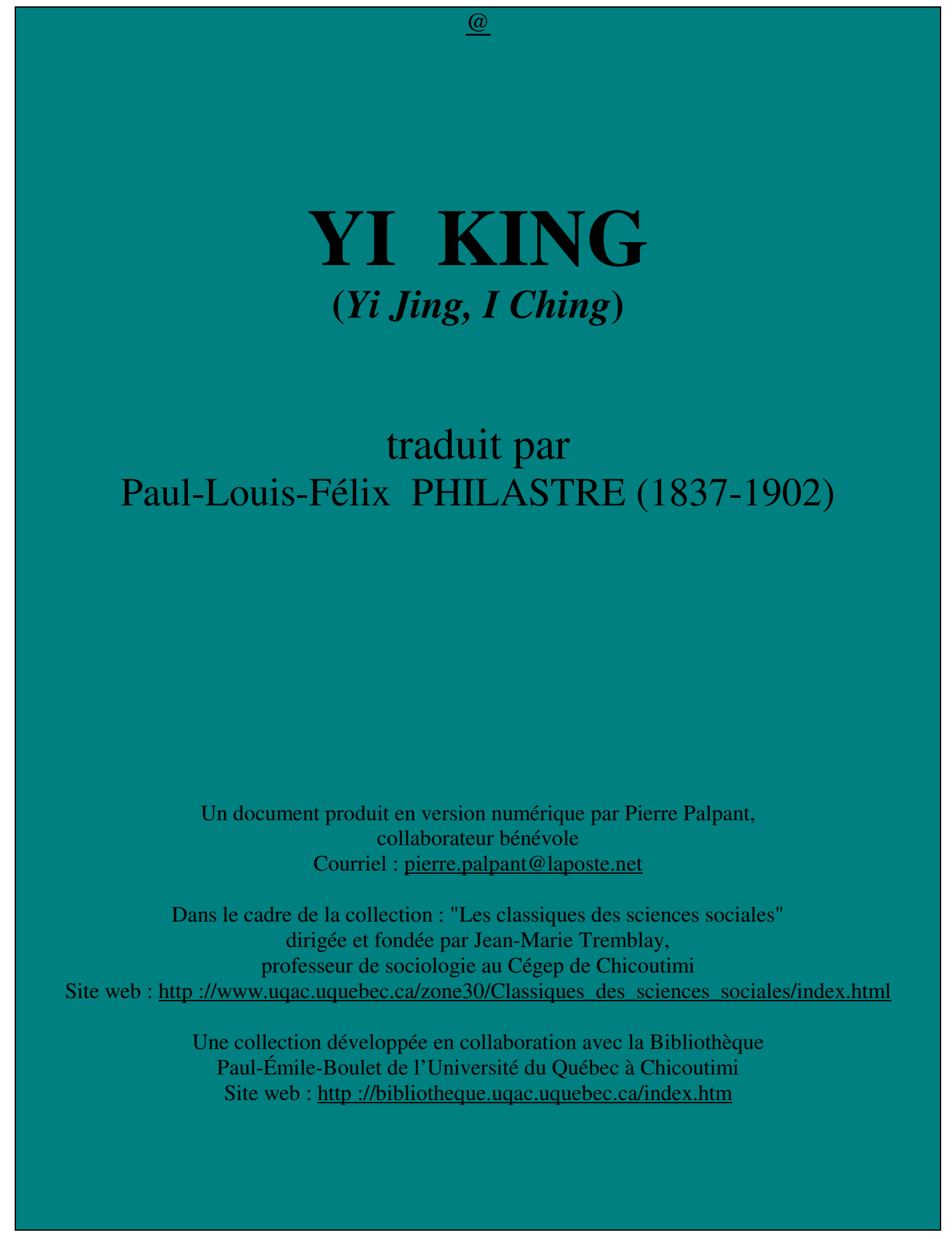


Un document produit en version numérique par Pierre Palpant, collaborateur bénévole, Courriel : pierre.palpant@laposte.net

à partir de :

\section{YI KING}

\section{Traduit par Paul-Louis-Félix PHILASTRE (1837-1902)}

Editions Zulma, 1992, 890 pages.

Première édition, Ernest LEROUX, Paris, 1885

Polices de caractères utilisée : Times, 10 et 12 points.

Mise en page sur papier format LETTRE (US letter), 8.5' $\mathrm{x} 11$ '”.

Édition complétée le 30 novembre 2004 à Chicoutimi, Québec. 


\section{T A B LE DES M A T I ÈRES}

\section{$\underline{\text { Note css }}$}

$\underline{\text { Introduction }}-\underline{\text { Conclusion }}-\underline{\text { Formules annexées }}(\underline{1}-\underline{2})$ - $\underline{\text { Postface }}$

Koua : $\underline{\text { Définitions }}-\underline{\text { Ordre }}-\underline{\text { Oppositions }}-\underline{\text { Table }}-\underline{\text { Table analytique }}$

\section{PREMIÈRE PARTIE}

1. - Khien, activité

2. - Khouen, passivité

3. - Tshouen, naissance des êtres

4. - Mong, développ. de l'intelligence

5. - $\underline{\text { Su, l'attente }}$

6. - Song, le doute

7. - $\underline{\text { Shi, }}$ le groupement de la foule

8. - Pi, l'association

9. - Siao tshou, petit arrêt

10. - $\underline{\mathbf{L i}}$, les règles rituelles

11. - Thae, prospérité

12. - P'i, décadence

13. - Thong jen, identité des hommes

14. - Tae yeou, grand avoir

15. - Khien, modestie

16. - Yu, satisfaction

17. - $\underline{\text { Souei, }}$, entraînement et action de suivre

18. - Kou, les causes

19. - $\underline{\text { Lin }}$, troubles et surveillance

20. - Kouan, l'objet de l'observation

21. - $\underline{\text { She ho }}$, couper court à l'erreur

22. - $\underline{\mathbf{P i}}$, orner et régulariser

23. - Po, user peu à peu, déclin

24. - Fou, retour en sens opposé

25. - Wou wang, absence dirrégularité

26. - Tae tsou, grand arrêt

27. - Yi , la nourriture

28. - Tae kuo, ce qui est grand traverse

29. - Khan, chute dans l'a bîme

30. - $\underline{\mathbf{L i}}$, jonction et séparation

\section{DEUXIÈME PARTIE}

31. - Hien, l'influence

32. - Heng, perpétuité

33. - Thouen, se retirer en arrière

34. - Ta tshang, parfait épanouissement

35. - Tsin, progression en avant

36. - Ming yi, blessure

37. - Kia jen, hommes de la famille

38. - Khouei, opposition

39. - Kien, difficulté

40. - Kiae, délivrance

41. - Souen, décroissance

42. - $\underline{\mathbf{Y} \mathbf{i}}$, croissance

43. - Kouae, détermination

44. - Keou, rencontre (conjonction)

45. - Tsouei, rassemblement, réunion

46. - Sheng, naissance, élévation

47. - Khouen, misère

48. - Tsing, le puits

49. - Ko, modifier, changer

50. - Ting, trépied

51. - Tshen, l'objet, l'ustensile

52. - Ken, l'arrêt

53. - Tsien, progression en avant

54. - Kouei mei, mariage

55. - Fong, grandeur

56. - Lou, voyageur, étranger

57. - Souen, entrer

58. - Touei, plaire

59. - Hoan, séparation

60. - Tsie, définir, régler

61. - Tshong fou, confiance, certitude

62. - Siao kuo, petit excès

63. - Ki tsi, déjà établi

64. - Vi tsi, pas encore établi 


\section{NOTE C S S}

\section{Quelques modalités de circulation spécifiques au Yi king.}

Dans chacune des 64 sections du livre, à la présentation très similaire, on trouvera en première page, $1+6$ courtes sentences.

Un clic sur le symbole [] après la première sentence permet le retour à la table des matières.

Un clic sur l'une des sentence s conduit à la sous-section où, après son rappel, la sentence est commentée. Un clic sur l'hexagramme repris en tête de chaque sous-section permet de revenir à la section.

Au bas des 1+6 sentences, deux symboles rouges : < et $>$.

Un clic sur le < permet d'accéder à l'hexagramme correspondant étudié sur le site d'expression anglaise : http://www.sacred-texts.com/ich/index.htm, traduction de James Legge. Ce très riche site reprend par ailleurs bon nombre des traductions du sinologue anglais.

Un clic sur le > permet d'accéder à l'hexagramme correspondant étudié sur le remarqueble site de l'association française des professeurs de Chinois (traduction allemande du Père Richard Wilhelm, adaptation française d'Étienne Perrot. Par rapport à CSS : autre traducteur, autres commentateurs du texte) : http://afpc.asso.fr/wengu/wg/wengu.php?l=Yijing

\section{Présentations}

Les références au Yi king sont très nombreuses dans les éditions CSS, notamment chez Henri Cordier, Henri Maspero, Marcel Granet, Léon Wieger.

Il est également instructif d'explorer les classiques, par exemple le Tso tchouan, avec les mots 'hexagramme', 'devin', 'achillée', ...: quelques exemples, concernant $\underline{\mathrm{Pi} \text { Ouan, }} \underline{\mathrm{Ki} \text { Iou, }}$ Mou Kiang ou $\underline{\text { Mou tseu. }}$ Mais aussi le Chou king ou le $\underline{\text { I-li}}$.

Les deux sites précités sacred-texts et afpc fournissent également une présentation détaillée. 


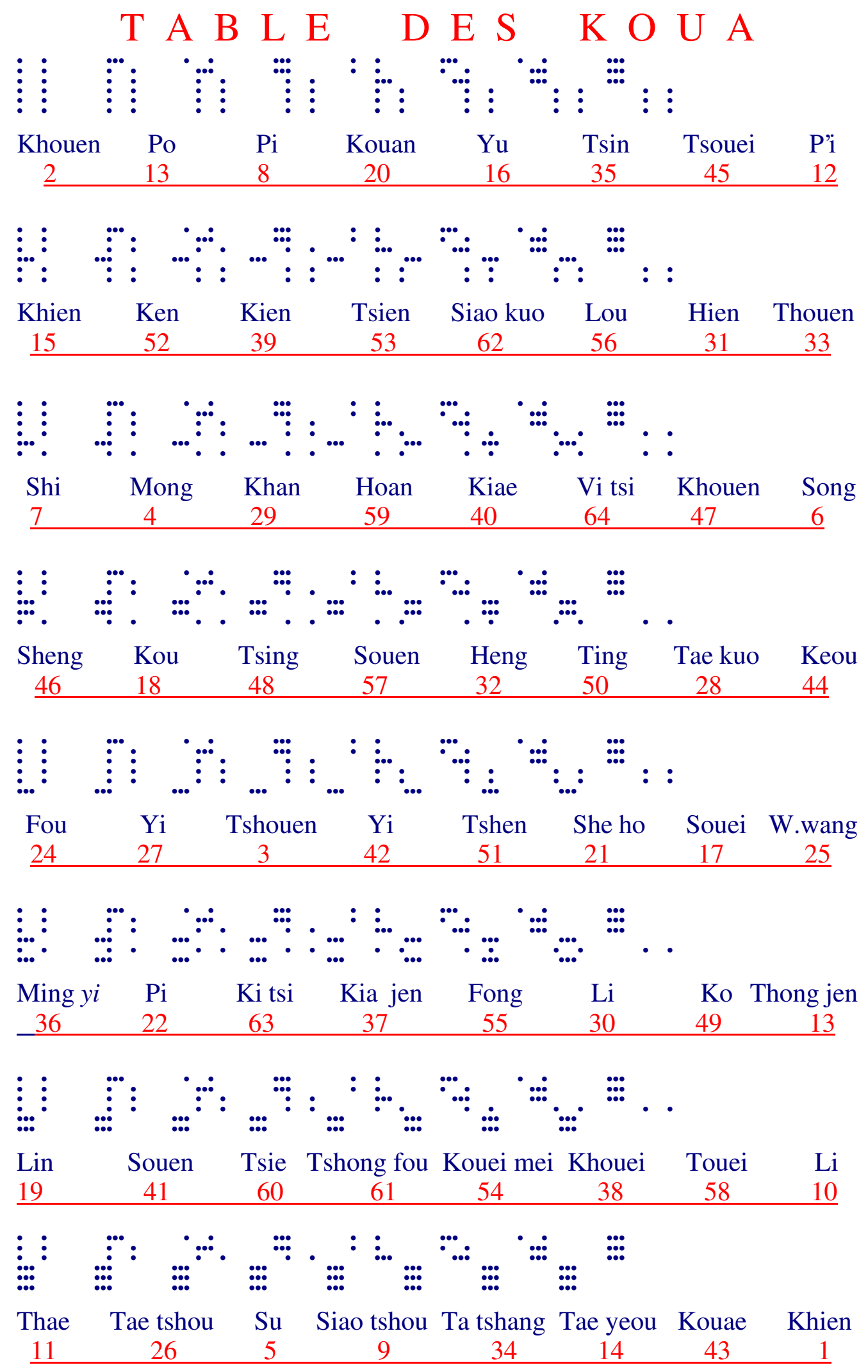




\section{N T R O D U C T I O N}

Le Yi king est considéré par les Chinois comme le plus antique monument de leur littérature ; toutes les écoles sont d'accord sur ce point.

D'après un passage des Rites de Tsheou, le magistrat chargé de la surintendance de la divination avait dans ses attributions la surveillance des règles posées par les trois livres appelés $Y i$, ou des Changements. Le premier de ces trois livres était intitulé Lien shan, Chaîne des montagnes, c'est-à-dire succession ininterrompue de montagnes. Ce titre provenait de la classification adoptée des hexagrammes, dont le premier figurait «la montagne sur la montagne »; le symbole adopté était les nuages émanant des montagnes. Le second était intitulé Kouei mang, Retour et Concèlement, parce qu'il n'était aucune question qui ne pût y être ramenée et que toutes s'y trouvaient cachées et contenues. Le dernier avait pour titre Tsheou $^{1} y i$, Changements dans la révolution circulaire, ce qui exprimait que la doctrine du livre des changements s'étend à tout et embrasse toutes choses dans son orbe. Cette explication des titres de ces trois ouvrages est personnelle à son auteur et n'est appuyée sur aucun texte faisant autorité ; elle n'est plus admise par personne ; je la crois cependant plus près de la vérité que les autres, qui vont suivre.

On remarque, d'autre part, que Shen Nong, héros anté-historique, est quelquefois appelé Lien Shan Shi, ou Li Shan Shi ; de même aussi, Hoang Ti, autre héros, est aussi appelé Kouei Tsang Shi ; ces deux expressions Lien shan et Kouei tsang étant donc également des vocables de règnes, on en déduit que ce titre de Tsheou yi vient aussi du vocable de la maison de Tsheou. Cette dernière supposition est officiellement et universellement admise aujourd'hui.

Mais quelques critiques font à ce sujet des objections très plausibles; il résulte du texte de quelques formules, ou sentences du Yi king, attribuées à Wen Wang, fondateur de la dynastie de Tsheou, que ces sentences auraient forcément dû être écrites postérieurement à la mort de Wen Wang. On en a conclu qu'une partie des formules seulement devait être attribuée à Wen Wang et le reste à Tsheou Kong, son fils.

Du passage des Rites de Tsheou, cité plus haut, on conclut encore que le Lien shan était le livre des Changements, ou Yi king, de la première dynastie (Hia) ; que le Kouei tsang était celui de la seconde dynastie (Sheang), et que le Tsheou yi fut celui de la troisième dynastie (Tsheou). On admet que le fond du livre était le même et que la forme seule différait quelque peu. Les deux premiers ont disparu sans laisser d'autre trace que celle de leurs titres mentionnés dans les Rites de Tsheou.

Le Yi king, tel qu'il nous est parvenu, est l'œuvre de plusieurs personnes.

\footnotetext{
${ }^{1}$ Tsheou, mouvement circulaire, révolution embrassant tout l'univers. Et aussi, nom d'un fief dont le prince feudataire fonda la troisième dynastie impériale de la Chine.
} 
La substance primitive est une série de soixante-quatre hexagrammes ; ces hexagrammes sont formés avec deux sortes de traits : un trait plein —_ et un trait brisé — — . La tradition rapporte que Fou Hi contemplant le ciel, puis baissant les yeux vers la terre et en observant les particularités, considérant l'apparence des oiseaux et les productions de la terre, les caractères du corps humain et ceux des êtres et des choses extérieures, commença par tracer huit koua, ou trigrammes, avec les deux lignes en question; ensuite, combinant ces huit premiers koua simples deux à deux, il en forma soixante-quatre hexagrammes; c'est là son œuvre et la trame du Yi king.

Wen Wang, prince feudataire, sujet du dernier empereur de la dynastie des Sheang, exilé et interné comme suspect, rédigea, pendant son bannissement, pour chacun de ces soixante-quatre signes, une formule de quelques mots, en exprimant la valeur générale. Son fils Tsheou Kong composa à son tour une formule pour chaque trait de chaque hexagramme. Plus tard, Khong Tse, reprenant leur œuvre, composa plusieurs commentaires particuliers qu'on désigne ensemble, et assez arbitrairement, sous la rubrique de Dix coups d'aile ; ce sont :

1. - Les «formules déterminatives », commentaires ou gloses des formules attribuées à Wen Wang;

2. — Les «formules symboliques », commentaires des formules attribuées à Tsheou Kong ;

3. - L'« expression parlée de la représentation graphique de la forme, ou expressions des représentations », commentaire spécial aux deux premiers koua ;

4. - Les «formules annexées », qu'on désigne généralement sous la rubrique de Grand Commentaire et qui embrassent tout l'ouvrage à un point de vue général en résumant la doctrine de Khong Tse sur cette question ;

5. — La « définition des koua »;

6. - L'« ordre des koua» expliquant l'ordre de classification des hexagrammes ;

7. - Les « oppositions des koua » autre vue sur leur ordre de classification.

Les Chinois attachent une haute importance à établir que la tradition orale de l'ensei gnement de la doctrine contenue dans le Yi king n'a jamais été interrompue ; ils citent les maîtres et leurs disciples et continuateurs depuis Khong Tse jusqu'aux philosophes de la renaissance des lettres, sous la dynastie des Song.

Le Yi king ne fut point condamné par l'Empereur Shi Hoang Ti ; ce prince n'y vit qu'un livre de divination dont la destruction semblait inutile au plan qu'il poursuivait. 
Ce résumé très succinct est tiré des premières lignes de lintroduction de l'édition officielle de la dynastie régnante ${ }^{1}$. Nous allons la compléter de quelques renseignements moins orthodoxes.

Fou $\mathrm{Hi}$ est un mythe; la tradition le représente avec de légères protubérances en forme de cornes sur le front. Pour être moins gracieux, le symbole n'en est pas moins le même que le croissant lunaire que Diane porte sur le front. Selon moi, Fou Hi symbolise les phases de la lune, résultant du mouvement apparent du soleil et de la lune autour de la terre considérée comme centre.

Fou Hi assistant à la séparation du Chaos d'où naissaient le Ciel et la Terre en comprit la genèse ; plus tard il vit un cheval-dragon sortant d'un fleuve et prit pour règle les figures apparentes sur son dos. Ces figures, qu'on appelle le «Tableau du fleuve », sont formées de points ronds, noirs ou blancs, groupés dans un certain ordre, et ce fut, dit la tradition, d'après ces signes que Fou Hi traça les huit premiers koua simples (trigrammes). J'avance enc ore que le dragon symbolise le lever du soleil et le cheval, très probablement, son coucher; que les figures qui forment ce tableau représentent des astres et des constellations ; qu'enfin les deux traits —_ et — _ premiers éléments des koua, représentent ou symbolisent deux grands moments dans la marche combinée et apparente du soleil et de la lune et que la base fondamentale du $\mathrm{Yi}$ king est essentiellement une observation astronomique. Les koua, ou diagrammes, représentent tous également la série des phases de la lune.

Après Fou Hi, le premier commentateur est Wen Wang. Pour les Chinois ce personnage est indiscutablement historique. Je suis certainement seul contre tous en avançant qu'il est permis de douter. Le brevet d'authenticité historique est délivré par le Shou king ; or, je considère cette autorité comme suspecte et essentiellement sujette à discussion. Très certainement, il a dû exister un personnage appelé Wen Wang; je n'en doute pas ; mais entre le rôle que le Shou king lui attribue et la réalité il peut y avoir une distance considérable. Tous les peuples qui ont une antiquité attribuent les grandes inventions du génie humain, les grands faits mémorables, à tel ou tel de leurs grands hommes ; le fait rappelé est souvent défiguré mais il a toujours un fond de vérité ; le personnage a presque toujours existé, mais il peut souvent n'y avoir rien de commun entre le héros et l'œuvre qu'on lui attribue. Il est même très probable que souvent, après un certain laps de temps, la tradition transpose le mérite de l'acte sur la tête d'un autre personnage dont la gloire plus récente fait oublier le héros précédent. Je soupçonne que tel est le cas pour Wen Wang; mais qu'il soit ou non l'auteur de la première glose, je ne crois pas que le titre Tsheou Yi vienne du vocable de la dynastie de Tsheou.

Après Wen Wang et son fils Tsheou Kong, le premier commentateur réellement historique est Khong Tse. À première vue, son auvre n'est guère moins obscure que celle de ses devanciers.

\footnotetext{
${ }^{1}$ Un magnifique exemplaire de cette édition est conservé à la bibliothèque de Lyon.
} 
La persécution des lettrés par Shi Hoang Ti plongea dans un désarroi complet et pour plusieurs siècles toutes les traditions littéraires; sous les Han il s'agit plus de reconstituer les textes et de les collationner que de les éclaircir. Bien que le Yi king n'eût pas été pros crit, son étude resta stationnaire et il faut franchir d'un bond une période de quinze siècles pour passer de Khong Tse à une école nouvelle qui, sous la dynastie des Song, releva un moment la gloire des lettres chinoises. Tsheou Tse, le premier dans les temps modernes, reprit d'une façon originale l'étude du Yi king et en déduisit un système cosmogonique qui, sans être neuf, résume sous une forme brève et nette les conceptions et la doctrine de tous ses devanciers. Tsheng Tse, son disciple, écrivit un commentaire traditionnel complet du Yi king; selon moi, c'est le plus remarquable, bien que l'école chinoi se moderne donne la préférence à celui de Tshou Hi, un peu postérieur, et intitulé Sens Primitif.

Depuis Tshou $\mathrm{Hi}$, on a encore délayé beaucoup d'encre et noirci énormément de papier, mais on n'a plus rien écrit d'original sur le Yi king; les taoïstes ont, il est vrai, composé un pastiche intitulé Thai huien king qui n’a aucune valeur réelle. La mine semble épuisée, mais, en réalité, c'est le génie d'un pe uple qui est engourdi. Les Chinois ne cherchent plus, ils conservent ; ifs se cramponnent à la tradition admise et leur unique souci est de se maintenir toujours d'accord avec elle.

Cependant, la vérité ne perd jamais complètement ses droits, même en Chine. On trouvera dans un ouvrage du P. de Prémare, publié en 1878 par MM. A. Bonnetty et P. Perny, de nombreuses citations d'auteurs chinois d'où résulte que bon nombre des meilleurs esprits qu'ait produit la Chine considèrent que la véritable interprétation du Yi king s'est perdue à la mort de Khong Tse et qu'on n'en connaît plus le vrai sens. Le livre que je cite ici, Vestiges des principaux dogmes chrétiens tirés des anciens livres de la Chine, est une auvre très remarquable et très digne d'étude, non pour y suivre la pensée et les vues exclusives de l'auteur, dont la grande érudition et le haut sens critique étaient enchaînés par la foi, mais pour y trouver, réunis et groupés, un nombre très considérable d'indices précieux sur la véritable valeur des livres classiques de la Chine. De tels travaux ont certainement dû ne pas être étrangers aux persécutions dirigées un peu plus tard contre les jésuites, en Chine, par les catholiques plus orthodoxes ; si la face de la médaille considérée par le $\mathrm{P}$. de Prémare était séduisante pour des hommes d'une foi inébranlée, le revers pouvait à bon droit alarmer des esprits plus froids et plus clairvoyants. Pour ce qui nous importe en ce moment, il suffit de citer le « Ve point » discuté p. 30 et suivantes ; la thèse du P. de Prémare, la Connaissance de la véritable doctrine des King est entièrement perdue chez les Chinois, s'y trouve surabondamment prouvée, exclusivement par des témoignages chinois. Considéré par les Chinois, le Yi king est avant tout un livre de divination; telle est sa forme, tel est son usage, et c'est dans ce sens qu'il est commenté et expliqué. Ce n'est ni le lieu ni le moment de rechercher pourquoi cette forme a été choisie par les auteurs du livre; il suffit de justifier en quelques mots l'utilité de cette tradition et son intérêt. Or : 
1. - En chinois, le mot n'a presque jamais de sens absolument défini et limité ; le sens résulte très généralement de la position dans la phrase, mais avant tout de son emploi dans tel ou tel livre plus ancien et de l'interprétation admise dans ce cas. Ici, point de « racines » au-delà desquelles on n'atteint plus et qui justifient le sens des dérivés dans les divers idiomes ou dialectes d'une même famille; le mot n'a de valeur que par ses acceptions traditionnelles. On n'a pas, à ma connaissance, tiré tout le parti possible de cette particularité de la langue chinoise, au point de vue de l'étude et de la recherche de la nature réelle du langage humain. Le mot chinois nous apparaît «comme si », expression naturelle et spontanée d'une pensée abstraite étrangère aux circonstances et aux conditions de la vie animale de l'homme, celui-ci, saisissant dans cette pensée un rapport avec les circonstances et les conditions de sa vie, avait emprunté le son de cette expression pour créer sa parole raisonnée.

De là, nécessité absolue, pour l'étude de la langue chinoise, de connaître les sources originales de la littérature, et, entre ces sources, la plus antique et la plus importante est incontestablement le $Y i$ king.

2. - Si on ne considérait que les diagrammes, il serait absolument impossible d'y décou vrir aucune idée intelligible ; les formules de Wen Wang, de Tsheou Kong, et même celles de Khong Tse ne seraient guère plus compréhensibles sans la Tradition, c'est-à-dire sans les commentaires de Tsheng Tse et de Tshou Tse, qui la résument. Il est donc indispensable de traduire en entier ces deux commentaires qui contiennent, d'une façon complète, toutes les notions des Chinois en fait de naturalisme, de morale et de philosophie. De plus, ces deux commentaires sont des modèles excellents du meilleur style chinois, clair et simple, encore préservé du goût amphigourique des modernes ${ }^{1}$.

Donc, le Yi king, considéré dans son ensemble, est encore indispensable à tous ceux qui, dans un but quelconque, veulent connaître les idées chinoises sur toutes ces questions.

3. - Je ne mentionne provisoirement que pour mémoire le côté le plus sérieux et le plus intéressant, à mon point de vue, de l'étude de ce livre ; j'en ai parlé ailleurs, et je laisse à ceux qui auront la patience d'en lire la traduction, à juger du bien fondé des hypothèses et des opinions que j'ai émises en commençant sur la vraie origine du Yi king.

Enfin, j'ajouterai encore que cette traduction française aura au moins le mérite de la nouveauté. Je n'ai trouvé dans les renseignements bibliographiques dont je dispose, que la mention d'une seule traduction de ce livre: y King antiquissimus sinarum liber quem ex latina interpretatione $\mathrm{P}$.

\footnotetext{
${ }^{1}$ Cette appréciation du style ne peut naturellement pas s'étendre à la méthode d'exposition et aux longueurs qui en résultent.
} 
Regis aliorumque ex Soc. Jesu PP., edidit julius Mohl, 1834. Stuttgartiæ et Tubingæ, 2 vol. ${ }^{1}$.

Beaujeu, le 21 mars 1881.

P.- L.- F. PHILASTRE

${ }^{1}$ A. Wylie, Notes on chinese literature, p. XIV. 


\section{AVERTISSEMENT AU LECTEUR}

Cette édition présente le texte canonique du Yi king (en italique), suivi des deux commentaires traditionnels de Tsheng Tse et de Tshou Hi (en romain).

Le Yi king est constitué de 64 koua : un koua est un hexagramme, formé de deux trigrammes, ou koua simples. Il y a huit koua simples, qui assemblés deux à deux forment les 64 koua tracés par Fou HI.

Les textes en italique qui suivent les caractères chinois sont les formules initiales attribuées à Wen Wang sur chaque koua, et celles attribuées à Tsheou Kong sur les six traits qui composent le koua. Les autres textes en italique, notamment les commentaires des formules déterminative et symbolique, sont attribués à Khong Tse (Confucius).

D'autres commentaires attribués à Khong Tse sont souvent cités par Tsheng Tse et Tshou Hi ; ils sont rappelés dans leur intégralité à la fin du volume : les Formules annexées, les Définitions des koua, l'Ordre des koua, et les Oppositions des koua.

Philastre a repris quelques commentaires supplémentaires qui apparaissent sous les titres Définitions diverses (extraits d'un recueil de citations de divers commentateurs) et jugements (gloses impériales de l'édition officielle de la dynastie des Ming).

Le Shi king est le Classique de la poésie, le Shou king est le Classique des documents, et le Li king, le Classique des rites.

Khong Tse (Khong Fou Tse) est aussi appelé Fou Tse, ou Tse, le Maître, ou encore l'homme saint ; il s'agit bien sûr de Confucius (550 ? - 479 avant Jésus-Christ).

Paul-Louis-Félix Philastre (1837-1902) est le premier traducteur du Yi king en français.

Il a également traduit un code du droit annamite et chinois. 


\section{P R E M I È R E P A R T I E}

TSHOU HI. - Tsheou, nom d'une dynastie ; $y i^{1}$, titre du livre. Les koua, ou diagrammes, qui constituent la substance première de ce livre, furent primitivement tracés par Fou Hi ; ils expriment le changement par jonction et le changement par modifications progressives, d'où le titre de livre des changements. Les formules y ont été adjointes par Wen Wang et par Tsheou Kong ${ }^{2}$, d'où le nom de Tsheou joint au titre. Comme le volume des lames de bambou, sur lesquelles il était écrit, était gros et lourd, on le divisa en deux livres : première et seconde parties ${ }^{3}$. Le King, ou texte, comprend les traits tracés par Fou Hi, les formules de Wen Wang et Tsheou Kong, et également les dix livres de Commentaires, ouvre de Khong Tse, en tout douze livres. Le

${ }^{1} \mathrm{Yi}$, les Chinois disent que le caractère qui représente ce mot est composé de deux autres caractères représentant l'un le soleil et l'autre la lune. Ce mot symbolise la loi fondamentale d'évolution qui est le fond de la doctrine admise du livre. M'appuyant sur le Shuo wen (le plus ancien dictionnaire chinois), je nie cette origine : le caractère $y i$, dans sa forme antique est composé du caractère [], soleil, et du caractère wou, qui exprime la négation impérative. $\mathrm{Si}$ Shan Tshen Shi dit: Le soleil disparaît, la lune surgit; le froid cesse et est remplacé par la chaleur ; le jour et la nuit, l'obscurité et la clarté se succèdent sans fin ; tel est le cours ordinaire de la marche (tao) des phénomènes célestes. Les hommes saints, en l'observant pour faire le livre des changements, ne firent que mettre en lumière la loi immuable (li) de croissance et de décroissance de la négativité et de la positivité. Si la positivité croît, la négativité diminue ; si la négativité grandit, la positivité se dissipe ; une période de croissance et une période de décroissance, telle est la loi (tao) du ciel. Lhomme qui étudie le livre des changements connaîtra la raison d’être (li) du bonheur et du malheur, de la décadence et de l'élévation, et la voie rationnelle (tao) selon laquelle il convient d'avancer ou de reculer, de laquelle enfin résulte le salut ou la perte. Lin Tshouen Wou Shi dit que de ce temps (époque où vivait Fou $\mathrm{Hi}$ ) le caractère yi n'existait pas encore ; il y avait des figures et pas de texte. Plus tard, lorsqu'on inventa les caractères, on commença à réunir les deux caractères jie (soleil) et yue (lune) pour former le caractère $y i$.

2 Wen Wang, père du fondateur de la dynastie des Tsheou, composa ses formules pendant son internement, vers 1150 A. C .; Tsheou Kong aurait rédigé les siennes vers 1100 ( ?).

${ }^{3}$ Cette opinion est combattue par Shouang Hou Hou Shi avec ses raisons assez probantes tirées de l'ordre suivant lequel les koua, ou dia grammes, sont rangés et de linnégalité des deux parties. Cette inégalité semble plutôt être une conséquence d'un plan adopté par Wen Wang. Il fait remarquer que la première partie débute par les koua khien et khouen qui expriment le commencement de la transformation de l'éther et que la seconde partie débute par les koua hieng et heng qui expriment le commencement de la forme. 
désordre fut, d'ailleurs, mis dans ces textes par les divers philosophes confucéens ${ }^{1}$. Dans les temps modernes, Tshiao Shi, le premier commença à corriger ces erreurs, mais il ne put cependant pas encore reconstituer complètement l'ancienne forme du texte. Lu Shi, à son tour, rétablit la distinction entre les deux volumes de texte et les dix volumes de commentaires, en revenant ainsi à l'ancienne distribution de Khong Ts e.

\footnotetext{
${ }^{1}$ J'emploie ce terme doublement inexact faute d'en trouver un autre suffisamment approprié. Le terme chinois pour désigner la doctrine en question est Jou; les adhérents sont appelés Jou shi ou «docteurs de la représentation graphique de l'image » (Wen sha). C'est donc d' une doctrine plus antique qu'il s'agit et non pas d'une doctrine particu lière à Khong Tse.
} 


\section{Khien}

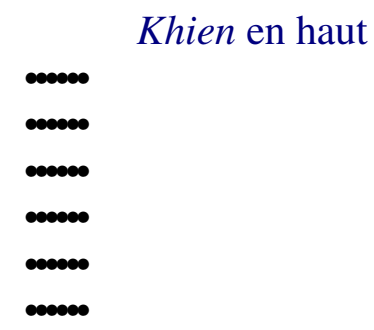

Khien en bas

1. Khien : Cause initiale, liberté, bien, perfection

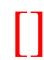

8. Premier trait nonaire : dragon caché ; ne pas agir.

$*$

10. Deuxième trait nonaire : dragon visible dans la rizière; avantage à voir un grand homme.

$*$

12.Troisième trait nonaire : l'activité de l'homme doué dure tout le jour; le soir, il est encore comme préoccupé. Danger ; pas de culpabilité.

$*$

14. Quatrième trait nonaire : parfois il saute dans les abîmes ; pas de culpabilité.

$*$

16. Cinquième trait nonaire : dragon volant dans le ciel ; avantage à voir un grand homme.

$*$

18. Trait supérieur nonaire : dragon élevé; il y a regret.

$*$

20. Emploi des traits nonaires : dans la troupe des dragons visibles, aucun ne précède les autres ; bonheur.

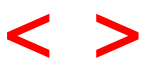




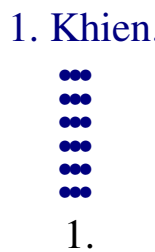

Khien : Cause initiale, liberté, bien, perfection ${ }^{1}$.

TSHENG TSE. - Dans la plus haute antiquité, l'homme saint commença par tracer les huit koua ${ }^{2}$ : la voie rationnelle (tao) des trois causes actives ${ }^{3}$ était dès lors complète. Il se servit ensuite de cette base en combinant ces huit koua simples deux à deux par superposition, pour embrasser la totalité des modifications dans l'univers, et c'est ainsi qu'il obtint les koua parfaits de six traits. Le koua simple khien, répété sur lui-même constitue le koua parfait khien. Khien exprime le ciel, Ti-thien. Thien, le ciel, c'est la substance et la forme du ciel ; khien, c'est la nature et le sentiment du ciel ${ }^{4}$. Khien équivaut à «force d'action» ou «activité » kien ${ }^{5}$; l'activité s ans repos est appelée khien.

Or, lorsqu'il s'agit du ciel ( thien), si on en parle d'une façon absolue, c'est la voie rationnelle ou morale (tao) ; c'est précisément : « le ciel qui, d'ailleurs, ne s'oppose point $\gg{ }^{6}$. Si on en parle en distinguant, alors, au point de vue de la forme et de la substance, on dit «le ciel» (thien); au point de vue de la puissance qui régit, on dit «le pouvoir suprême» $t i$; au point de vue de l'action et des effets ${ }^{7}$, on dit « esprits et génies »; au point de vue des effets transcendantaux, on dit 《l'esprit ${ }^{8}$; au point de vue de la nature et du sentiment, on dit khien, ou «activité ».

\footnotetext{
${ }^{1}$ Les feuilles de l'arbre commençant à pousser, c'est l'action de la cause initiale ; les fleurs s'ouvrant, c'est la liberté d'expansion ; les fruits qui se nouent, c'est le bien ; ces fruits se développent et mûrissent, c'est la pure té ou perfection (Tshou Hi).

${ }^{2}$ Les huit koua primitifs de trois traits.

${ }^{3}$ San tsae. Essentiellement, le caractère tsae représente un germe végétal perçant la terre. De là, est-il dit, par extension, le sens de force, capacité productive et causative, et enfin l'emploi de ce mot pour désigner les qualités et les talents. Dans le cas actuel, ce mot sert à former une expression technique qui désigne le ciel, la terre et l'homme, considérés comme les trois causes des lois physiques, intellectuelles et morales.

${ }^{4}$ C'est seulement par figure que ces termes sont employés en parlant du ciel. La nature, c'est le naturel particulier de l'être considéré ; le sentiment est l'effet manifesté du naturel. Tsheng Tse dit : La substance ou essence de l'activité, c'est sa nature ; les effets de cette activité sont ce qu'on appelle le sentiment.

${ }^{5}$ Le véritable sens du mot tsin est « haut, élevé ».

${ }^{6}$ Citation d'un passage ulté rieur.

${ }^{7}$ Les phénomènes naturels tels que la pluie, la foudre, etc.

${ }^{8}$ Les esprits et génies ne sont rien que l'action et la réaction, la contraction et la dilatation (de l'éther) ; l'action et les effets en sont les manifestations visibles. Quant à l'esprit, c'est une expression pour désigner la cause mystérieuse de toutes choses. Cette cause mystérieuse et transcendante réside précisément dans l'esprit ; lorsqu'elle se manifeste et qu'on la voit dans
} 
Cette activité, exprimée par le mot khien, est l'origine et le commencement de tous les êtres et de toutes choses ${ }^{1}$; c'est pour cela que le koua représente le ciel, la positivité, le père, le prince.

Cause initiale, liberté, bien, perfection, sont ce qu'on appelle les quatre vertus ${ }^{2}$. La première exprimée par le mot yuan, c'est le commencement de tous les êtres et de toutes choses. La seconde, exprimée par le caractère heng, c'est la croissance de toutes choses ; la troisième, exprimée par le caractère [] , c'est la faculté de satisfaction des besoins, tels qu'ils résultent de la condition de chaque être ; la quatrième, exprimée par le caractère tsheng, c'est le développement normal et parfait de toutes choses.

Les koua khien et khouen, seuls ${ }^{3}$ ont ces quatre vertus; dans les autres koua, elles se modifient selon le sujet, de sorte que la première exprime spécialement et exclusivement le bien et la grandeur ; la troisième consiste surtout dans la droiture et la fermeté, et les substances de la seconde et de la quatrième correspondent dans chaque cas à la nature spéciale du sujet. Le sens de ces quatre vertus est large et étendu.

TSHOU HI. - Ces six traits sont le koua tracé par Fou Hi.

Le trait plein — est appelé $k i$, ou unité ; c'est le nombre de la positivité. Khien, c'est la force d'action, ou activité, exprimée par le mot kien; c'est la nature de la positivité. Dans les annotations ${ }^{4}$, le caractère khien est le nom du koua de trois traits ; celui d'en bas est le koua intérieur ; celui d'en haut est le koua extérieur ${ }^{5}$. Dans le texte, le caractère khien est le nom du koua de six traits.

Fou Hi leva les yeux et regarda; il les baissa et examina. Il vit que la négativité et la positivité ont la dualité et l'unité pour nombres. Il traça donc un trait simple pour symboliser la positivité et un trait double pour symboliser la négativité. Il vit qu'une négativité et une positivité ont chacun e l'apparence d'engendrer une négativité ou une positivité ; aussi, à partir d'en haut et en montant, il traça encore un second, puis un troisième trait, pour achever les huit koua simples primitifs. Il vit que la nature de la positivité est l'activité et que la plus grande de ses formes réalisées est le ciel ; c'est pour cela que le koua composé de trois traits simples fut nommé khien et qu'il l'attribua à la représentation du ciel. Les trois traits complétés, les huit koua achevés, il

son action et ses effets on désigne cette action et ces effets par les termes esprits et génies. Mais quant à ce qui ne peut être atteint, on l'appelle l'esprit (Tsheng Tse).

${ }^{1}$ Tsheng Tse n'est pas fort éloigné du taoïsme ; ici, ses paroles se rapportent au moins autant au livre de Lao Tse qu'aux paroles de Wen Wang.

2 Vertus attributives.

${ }^{3}$ Selon Tsheng Tse les deux caractères khien et khouen n'existaient point antérieurement et ils ont été imaginés pour exprimer des «idées difficiles à rendre clairement ».

${ }^{4}$ Le terme que je traduis par «Commentaire traditionnel» exprime plus spécialement la transmission d'une doctrine. Celui qui est traduit par « annotations » signifierait aussi bien commentaire, mais il a le sens d'expliquer, préciser le sens des mots.

${ }^{5}$ Parce que lorsque les koua sont rangés sur une circonférence l'un se trouve en dedan s et l'autre en dehors par rapport au centre. 
augmenta encore trois fois d'un trait, pour en porter le nombre à six, c'est-à-dire qu'au-dessus de chacun des huit koua simples, il ajouta successivement chacun de ces mêmes signes, pour former les soixante-quatre koua parfaits.

Dans ce koua, les six traits sont tous des traits simples; le koua simple inférieur et le koua simple supérieur sont tous les deux le koua simple khien de sorte qu'il exprime la pureté de substance de la positivité et l'extrême activité, et c'est pour cela que le nom, khien, et la représentation symbolique, le ciel, ne changent ni l'un ni l'autre.

Les mots yuan, heng, li, tsheng, sont la formule attachée à ce diagramme par Wen Wang, pour définir les présages fastes ou néfastes du koua entier; c'est ce qu'on appelle thouan, ou formule déterminative d'un koua. Le mot yuan exprime la grandeur ${ }^{1}$; le mot heng, l'intelligence ; le mot li, l'utilité ; le mot tsheng, la droiture et la fermeté. Wen Wang considéra la voie rationnelle de l'activité comme exprimant la grandeur de l'intelligence et l'extrême droiture. Aussi lorsqu'en consultant le sort, on obtient ce koua, et qu'aucun des six traits ne se modifie ${ }^{2}$, le sens divinatoire exprimé est qu'il faut posséder une grande intelligence et qu'alors il y aura certainement avantage parla droiture et la fermeté ; ces conditions remplies, il est possible de garantir le succès final de l'entreprise. C'est ainsi que les hommes saints, en faisant le livre des changements, ont appris aux hommes à consulter le sort par le moyen de l'écaille de la tortue ou par celui d es brins de paille et qu'il est possible, par ces moyens, d'atteindre l'idée pure et exacte au sujet de l'entreprise et de l'accomplissement d'une chose ou d'une affaire. Ceci est applicable à tous les autres koua.

DÉFINITIONS DIVERSES. - Khong Shi Ying Ta dit : Koua signifie mettre à part ; cela veut dire suspendre à part, en le mettant en évidence, le symbole d'une chose, pour servir d'avis aux hommes ; c'est pour cela que ces signes sont appelés koua. Ce koua khien symbolise essentiellement le ciel. Le ciel est formé par l'accumulation de tout l'éther positif, c'est pourquoi les six traits de ce koua sont tous positifs. Une fois qu'il est tracé, on ne le nomme pas thien, ciel, mais khien. Le mot ciel est le nom de la substance déterminée ; khien est l'ex pression qui désigne l'effet de cette même substance. Aussi, les «Définitions des koua» disent: «khien, activité »; cela exprime que la substance du ciel a l'activité pour effet. Les hommes saints qui firent le livre des changements eurent essentiellement en vue l'instruction des hommes : ils voulurent que ceux-ci prissent les effets du ciel pour modèle et non pas quils se réglassent sur sa substance, aussi appelèrent-ils ce koua khien et non pas thien, ciel ${ }^{3}$. Tshou Tse dit encore: Les quatre caractères yuan, heng, li,

\footnotetext{
${ }^{1}$ Tshou Hi adopte une lecture que je n'ai pas suivie dans la traduction du texte, aussi il est indispensable de préciser en répétant le terme chinois du texte.

${ }^{2}$ Expressions techniques, termes de divination qui seront expliqués plus tard.

${ }^{3}$ La pensée du commentateur est difficile à saisir. S'agit-il de vouloir que les hommes se règlent sur les saisons, sur les lois de la nature, pour pourvoir aux soins de leur existence matérielle, comme sur les lois morales qu'on prétend déduire de l'action des phénomènes
} 
tsheng, n'étaient essentiellement, dans l'idée de Wen Wang, rien autre chose dans le cas des deux koua khien et khouen que lorsqu'il s'agit de tout autre koua. Ce ne fut qu'à l'époque où Khong Tse fit le Commentaire des Formules déterminatives et l'Expression des représentations que, lui, le premier, les considéra comme désignant les quatre vertus des deux premiers diagrammes, tandis que, dans les autres koua, il leur laissait leur ancienne valeur. Ce n'est pas que liidée de chacun de ces deux hommes saints soit différente, mais chacun met en lumière un point de vue distinct. Aujourd'hui ceux qui étudient ce livre doivent d'ailleurs se pénétrer de son esprit, sans se laisser influencer par les préventions et s'en assimiler la substance, dans chaque cas, selon l'idée contenue dans les textes primitifs ${ }^{1}$. Les passages dissemblables ne sont d'ailleurs pas mutuellement en désaccord et personne ne doit s'en rapporter à son propre jugement et se permettre mal à propos de nouvelles interprétations ${ }^{2}$. Hou Shi Ping Wen dit: Tous les commentateurs expliquent ces quatre caractères comme exprimant quatre vertus; le «Sens primitif» seul, les considère comme une simple formule divinatoire ${ }^{3}$.

2 .

LE COMMENTAIRE TRADITIONNEL DE LA FORMULE DÉTERMINATIVE dit: Qu' elle est grande la cause initiale de l'activité (khien)! Toutes choses lui doivent leur commencement ${ }^{4}$; c' est le ciel tout entier!

TSHOU HI. - La formule déterminative, ou thouan, c'est précisément la formule attachée au koua par Wen Wang (...) ${ }^{5}$. Le commentaire, c'est l'explication de la formule du texte, ou king, donnée par Khong Tse ; ceci s'applique à tout ce qui, dans la suite, est appe lé commentaire traditionnel (tshouan).

naturels, pour régler leur conduite et leurs mours, dans tous les cas, sans entrer dans aucune spéculation inutile sur la nature elle-même du principe premier dont résultent ces lois et cette action?

1 Sous cette ambiguïté, Tshou Hi laisse voir que, selon lui, on doit surtout s'en rapporter à l’idée qu'il attribue à Wen Wang.

${ }^{2}$ C'est là tout l'esprit de l'ensei gnement chinois depuis Khong Tse. L'axiome : Magister dixit n'a jamais eu nulle part autant de vale ur qu'en Chine.

3 «Sens primitif », titre du commentaire de Tshou Hi. Les «Jugements », titre des gloses impériales dans l'édition de la dynastie des Ming intitulée « grande édition complète », reviennent sur l'opinion de Tshou Hi et se donnent une certa ine peine pour prouver que, tout en disant le contraire, Khong Tse était justement de l'avis de Wen Wang.

4 Devoir le commencement, littéralement : être doué de commencement, c'est être redevable de la création de l'éther constitutif. Être doué de la vie, c'est acquérir et réaliser la forme. (Tshou Hi).

5 Le passage sauté explique les mots «première partie ». Dans certaines éditions, au lieu de suivre la formule du diagramme, le commentaire forme un livre séparé, divisé comme le texte en première et seconde partie. 
JUGEMENTS. - Le commentaire traditionnel de la formule déterminative, c'est l'expli cation de l'idée de Wen Wang donnée par Khong Tse. D’abord il explique le nom ; ensuite il explique la formule. Dans l'explication du nom, il se sert indifféremment du symbole, des propriétés des deux koua simples et de la substance du koua parfait lui-même. Quelquefois il tient compte de tous ces éléments simultanément; d'autres fois il n'en emploie qu'un ou deux ; c'est toujours l'idée exposée dans la première phrase du commentaire qu'on doit considérer comme la plus importante.

TSHOU HI. - Ce commentaire éclaire le sens du mot khien, simplement au moyen de la voie immuable (tao) du ciel. De plus il énonce séparément les quatre caractères de la formule, qu'il considère comme exprimant quatre vertus ou propriétés, afin d'en faire ressortir clairement la valeur. Ce premier paragraphe explique d'abord le sens du pre mier, yuan. "Qu' elle est grande », exclamation admirative; yuan, grandeur, commencement. Le mot yuan, ici appliqué au caractère khien, c'est le grand principe d'où pr ocède la vertu du ciel ; la naissance de tous les êtres est due à cette grandeur et c'est cette gran deur qui constitue leur commencement. De plus il est encore considéré comme exprimant la première entre les quatre vertus attributives et comme s'étendant du principe au résultat de la vertu du ciel, c'est pourquoi le texte dit : C'est le ciel tout entier ${ }^{1}$.

DÉFINITIONS DIVERSES. - Les neuf commentaires du livre des changements ${ }^{2}$ disent : Khien, pureté de la substance de la positivité ; symbole du ciel, (...) yuan, commencement de l'éther. — Les Sujets de dissertations ${ }^{3}$ de Tshou Hi disent encore : Yuan, c'est le point de départ de l'effet, et la raison d'être des trois termes suivants : liberté, bien et perfection, y est virtuellement contenue, au point que ce qui est l'intelligence (heng), ce qui est le bien (li), ce qui est la perfection (tsheng), c'est toujours l'action de ce qui est exprimé par le mot yuan ; c'est ainsi que ce mot yuan implique en luimême les quatre vertus. Si on en parle en distinguant, alors, la grandeur et la liberté (yuan et heng) sont la libre expansion du vrai ; le bien et la perfection (li et tsheng) sont la conséquence du vrai ; la substance et l'effet s'y trouvent certainement. En en parlant au point de vue de l'effet, c'est la grandeur, yuan, qui prédomine ; en en parlant au point de vue de la substance, c'est la pureté qui prédomine. - Ils disent encore : Le mot yuan exprime le principe en vertu duquel le ciel et la terre engendrent les êtres et les choses ; c'est l'idée de la vie. Dans le mot heng, liberté, c'est la continuité de l'idée de la vie qui se trouve exprimée. Dans le mot $l i$, bien, c'est la possibilité de satisfaction aux conditions de la vie. Dans le mot tsheng, perfection, c'est la réalisation de

\footnotetext{
${ }^{1}$ C'est le ciel tout entier ! en effet le mot thien, ciel, s'emploie seulement pour exprimer la forme et la substance ; khien yuan, la grandeur de l'activité, c'est ce qui fait que le ciel est le ciel. (Tshou Hi).

2 Titre d'un recueil de comment aires.

3 Titre d'un ouvrage contenant un recueil des opinions émises sur toutes sortes de sujets par Tshou Hi ; ne l'ayant point lu, je ne puis répondre de l'exactitude de la traduction du titre.
} 
l'idée de la vie. Lorsqu'on nomme l'humanité 1, c'est précisément de cette pensée qu'il s'agit ; l'humanité, c'est essentiellement l'idée de la vie. C'est par l'idée de la vie que la pitié germe dans le cour ; si on provoque cette idée, le germe de la pitié se manifeste immédiatement; s'il s'agit de la répulsion qu'inspire le vice, c'est que l'humanité s'efface et que le devoir s'élève et se montre; s'il s'agit de la modestie et de l'humilité, c'est l'humanité qui disparaît et fait place à la bienséance ; s'il s'agit du vr ai et du faux, du pour et du contre, l'humanité fait place à la raison. Quant à l'homme dépour vu d'humanité, comment donc pourrait-il encore avoir le sentiment du devoir et des convenances ou de la raison?

3.

Les nuages marchent, la pluie étend son effet; les genres des êtres se perpétuent dans la forme ${ }^{2}$.

TSHOU HI. - C'est l'explication du mot heng, ou liberté, dans l'activité (khien).

DÉFINITIONS DIVERSES. - Yu Shi Yen dit: Plus haut le Commentaire traditionnel emploie l'expression « tous les êtres »; ici il est question des « genres des êtres ». « Tous », ou totalité, et « genres » ont des valeurs communes et d'autres qui sont différentes. Le mot yuan, cause initiale, exprime l'origine du don de l'éther ${ }^{3}$; il n'est pas encore possible de faire de distinction, aussi on dit, en généralisant, tous, totalité. Mais quand il s'agit de la liberté, heng, alors le courant ébranle la molécule du fluide ${ }^{4}$; la forme se manifeste, et dans chaque cas il y a lieu de distinguer d'après l'ampleur ou l'exiguitté, entre ce qui est élevé ou bas, aussi le commentaire spécialise en employant le mot «genres ».

4.

\footnotetext{
${ }^{1}$ Le commentateur prend les « cinq vertus » comme objet d'ap plication de l'explication qui précède ; l'humanité, jen, est le pivot de ces cinq vertus.

${ }^{2}$ Littéralement : s'écoulent dans le courant de la forme.

${ }^{3}$ C'est par cette cause créatrice que la chose et douée de l'éther dont elle est formée.

${ }^{4}$ Courant de l'eau au moment où le liquide s'ébranle et commence à couler.
} 
La fin et le commencement sont éclairés d'une grande lumière ; les six situations se présentent avec le temps. Suivant le temps aussi, monter les six dragons pour parcourir le ciel ${ }^{1}$.

TSHOU HI. - Le commencement, c'est la grandeur, yuan ${ }^{2}$; la fin désigne la perfection. Ce qui est sans fin n'a pas de commencement ; sans perfection rien ne constituerait la grandeur (yuan). Ceci exprime que, l'homme saint projetant une grande clarté sur la fin et le commencement de la voie rationnelle de l'activité (khien), on voit les six situations du koua ${ }^{3}$ se présenter chacune avec le temps, et qu'employer le moyen de ces six positivi tés pour parcourir la voie rationnelle tracée par le ciel, c'est véritablement là la grandeur (yuan) et l'intelligence ( heng) de l'homme saint.

DÉFINITIONS DIVERSES. - Tshou Hi dit: se présentent avec le temps : c'est-à-dire que chacune survient en son temps; ainsi, selon qu'il (le dragon) est plongé ou visible, qu'il vole ou qu'il bondit, c'est toujours dans le temps opportun.

5.

La voie de l'activité ${ }^{4}$, c'est la modification et la transformation. Chaque chose se conformant exactement à sa nature et à sa destinée, maintenant, en s'y accordant, l' extrême harmonie ; c'est là le bien et la perfection.

TSHOU HI. - La modification, c'est la transformation progressive ; la transformation c'est l'accomplissement parfait de la modification. Ce que reçoivent les êtres, c'est leur nature ; ce que le ciel leur confère, c'est la destinée ${ }^{5}$. L'extrême harmonie, c'est l'assem blage et la réunion de la négativité et de la positivité, l'éther harmoniquement coagulé. Chaque chose se conformant exactement, c'est-à-dire se conformant à son état originel au moment où elle vient à la vie. Maintenir en s'y accordant, c'est -à-dire conserver l'intégrali té de cet état après la naissance. Ceci exprime que les

\footnotetext{
${ }^{1}$ Ce n'est pas sans hésitation et sans crainte de subir l’influence du commentaire étroit de Tshou Hi, que ce sens est adopté.

${ }^{2}$ Le sens donné au même mot variant avec le commentateur, il en résulte pour le traducteur l'obligation d'intercaler souvent le terme chinois dans le texte français.

3 Situation, terme technique pour désigner certaines situations symbolisées par chacun des six traits.

${ }^{4}$ Khien, ciel, activité. Voir la formule $\mathrm{n}^{\circ} 1$; tao, voie logique, rationnelle, morale ; la morale conséquence naturelle de la loi céleste, ou raison d'être, li.

${ }^{5}$ Même idée envisagée successivement au point de vue de celui qui reçoit et de la puissance qui impose ou confère.
} 
modifications et les transformations de la voie rationnelle de l'activité ne sont jamais autre chose que le bien, et que tous les êtres ont chacun leur nature et leur destinée, afin de se perpétuer sans dégénérescence. Ce passage a pour objet l'explication du sens des termes bien (la) et perfection ( $t$ sheng) ${ }^{1}$.

DÉFINITIONS DIVERSES. - Tshou Tse dans les Sujets de dissertations dit: Maintenir en s'y conformant l'extrême harmonie, c'est précisément entretenir cette raison d'être de la vie et s'y conformer. La réunion ${ }^{2}$ des deux éthers du ciel et de la terre, c'est le ciel et la terre maintenant en s'y conformant cette raison d'être de la création des êtres ; l'œavre de création et de transformation est incessante, et après que les êtres ont été transformés et sont venus à la vie, chacun de ces êtres maintient dans sa sphère cette raison d'être de la vie, et il s'y conforme. S'il ne la maintenait pas en s'y conformant, alors il n'y aurait plus d'êtres. Hou Shi Ping Wen dit : En en parlant d'après la distinction entre les deux éthers, alors, la modification est le mécanisme qui produit tous les êtres ; c'est la cause initiale et la liberté (yuan, heng); la transformation est le mécanisme dans lequel s'absorbent les êtres : c'est le bien et la perfection (li, tsheng). Si on en parle au point de vue de la circulation d'un seul éther, la modification est le progrès, la transformation est l'achèvement.

6.

Elle précède tous les êtres ${ }^{3}$; tous les états sont également en paix.

TSHOU HI. - L'homme saint est au-dessus de tout; il dépasse tous les êtres en élévation et il agit lui-même comme la modification et la transformation de la voie rationnelle de l'activité. Chaque état possède ce quil lui faut, et tous sont en paix. C'est encore chaque chose se conformant exactement à sa nature et à sa destinée et maintenant, en s'y accordant, l'extrême harmonie. Il s'agit ici d u bien et de la perfection (li et tsheng) chez l'homme saint. En effet, quand il en a été parlé d'une façon générale, il a été dit que yuan, la grandeur, exprime le commencement de la naissance des êtres; que heng, liberté, exprime leur multiplication florissante ; que $l i$, le bien, est leur tendance à la reproduction de l'espèce ${ }^{4}$ et que tsheng, la

\footnotetext{
${ }^{1}$ Dans les «sujets de dissertations », Tshou Hi donne comme exemple de cette puissance de conservation du genre, qui réside dans chaque être, le grain qui devient germe, puis fleur, puis grain, et qui se reproduit sans varier dans ses transformations.

${ }^{2}$ Les deux mots yin yun, qu'on expliqu e par l'expression « réunion des éthers du ciel et de la terre » sont peu ou point compris. Ils paraissent signifier réellement cause et effet.

3 Selon l'explication donnée par Tshou Hi, il faudrait lire : Il précède, au lieu de : Elle précède. Je rapporte l'action de précéder à la voie de l'activité, voir la formule $\mathrm{n}^{\circ} 2$.

${ }^{4}$ Littéralement : tendre à la semence et achèvement de la semence.
} 
perfection, consiste dans l'achèvement régulier de cette reproduction. Du moment où la semence est parfaite, la radicule s'en échappe, elle peut être plantée et vivre. C'est ainsi que les quatre vertus se suivent en cercle, sans point de départ. Mais toutefois, dans le cycle de ces quatre conditions, le souffle de la vie circule et agit sans qu'à l'origine il y ait intermittence ou point de départ; c'est par là que la grandeur, yuan, contient implicitement l'essence des quatre vertus et résume le ciel tout entier ${ }^{1}$. Ce qui fait que j'en parle au point de vue de l'homme saint, c'est que telle est l'idée de Khong Tse. Effectivement, il considère ce koua comme représentant l'homme saint ${ }^{2}$, occupant la situation du ciel ${ }^{3}$, pratiquant la loi morale du ciel, de sorte quil en résulte un sens divinatoire exprimant l'extrême pacification. Bien que les expressions qu'il emploie et le sens qu'il donne aux caractères aient quelque chose d'étranger à l'ancien texte de Wen Wang, cependant, le lecteur doit chercher dans chaque texte l’idée qui y est contenue, de telle sorte que ces idées diverses soient également admissibles et sans contradiction entre elles. Ceci est encore applicable dans le cas du koua khouen.

TSHENG TSE ${ }^{4}$. - La formule placée au-dessous du koua, ou diagramme, est ce qu'on appelle thouan, ou formule déterminative. Fou Tse 5 la suit et l'explique, et son propre commentaire est couramment appelé du même nom de « formule déterminative ». La formule appelée thouan traite du sens d'un koua pris dans son ensemble et sans avoir égard au sens particulier de chaque trait; aussi, ceux qui le savent, en examinant la formule déterminative, embrassent dans leur pensée plus de la moitié de ce qu'il y a à en dire. Qu'elle est grande la cause initiale de l'activité ! exclamation admirative sur la grandeur de la voie suivant laquelle la cause initiale de l'activité est l'origine de toutes choses. Entre les quatre vertus att ributives, yuan, la cause initiale, ou faculté causative, est comme l'humanité entre les cinq vertus ordinaires ${ }^{6}$. À un point de vue particulier c'est une chose distincte; en parlant au point de vue absolu, elle implique toutes les quatre

\footnotetext{
${ }^{1}$ Tshou Hi ne voit dans la formule du koua qu'une simple formule divinatoire que Khong Tse aurait, plus ou moins à propos, interprétée au point de vue de la loi immuable qui régit le monde. Quant à ce qu'il avance plus bas que Khong Tse, en écrivant ces quelques phrases, avait en vue de peindre la voie morale et rationnelle de "l'homme saint », c'est peut-être exact, mais il faudrait être bien sûr de s'entendre sur ce que Khong Tse entendait alors par cette expression. Exotériquement il pouvait être question de l'homme supérieur, du prince modèle qui gouverne les hommes comme le ciel gouverne le monde, mais il me semble qu'en réalité il s'agit du soleil, générateur et cause physique de la vie sur la terre et qu'enfin les « royaumes » ne sont que les divisions du zodiaque, déterminées par les douze conjonctions annuelles.

${ }^{2}$ Cette expression, qui souvent dans ces commentaires désigne Fou Hi, Wen Wang, Tsheou Kong ou Khong Tse est employée ici d'une façon générale, l'homme parfait.

${ }^{3}$ Le rang suprême; le rang souverain de l'empereur, pontife et représentant la providence et la justice divine sur la terre.

${ }^{4}$ Lorsque, comme dans le cas actuel, le commentaire de Tsheng s'applique en un seul paragraphe à plusieurs propositions commentées séparément par Tshou $\mathrm{Hi}$, il a fallu intervertir l'ordre chronologique et placer le commentaire de Tshou le premier.

${ }^{5}$ Une des appellations qui désignent Khong Tse.

${ }^{6}$ Humanité, devoir, bienséance, raison et foi.
} 
vertus. Toutes choses lui doivent leur commencement, c'est le ciel tout entier ; il s'agit de la cause initia le ; la cause initiale de l'activité exprime d'une façon générale et complète la voie rationnelle du ciel. La voie de l'action du ciel commence ${ }^{1}$ tous les êtres ; tous doivent leur origine au ciel.

Les nuages marchent; la pluie étend son effet; les genres des êtres se perpétuent dans la forme. Il s'agit de la liberté (heng). La voie immuable du ciel agit par un mouvement sans fin ; elle engendre tous les êtres.

La fin et le commencement de la voie du ciel sont éclairés d'une grande lumière, de sorte qu'on voit les six situations du koua se présenter chacune avec le temps. Le premier et le dernier traits du koua sont le commencement et la fin de la voie rationnelle du ciel ; profiter $^{2}$ de linstant de chacun de ces traits, tel est le mouvement du ciel. Pour parcourir, c'est-à-dire pour correspondre au mouvement du ciel.

La voie rationnelle de l'activité, c'est la modification et la transformation ; elle engendre tous les êtres ; immenses ou minimes, élevés ou bas, chacun selon sa propre espèce se conforme exactement à sa nature et à sa destinée. Ce que le ciel confère, c'est la destinée ; ce que les êtres reçoivent, c'est leur nature. Maintenir en s'y accordant l' extrême harmonie, c'est le bien et la perfection. Maintenir, veut dire faire subsister d'une façon perma nente; s'accorder, veut dire que l'harmonie est continue. Maintenir, en s'y accordant, l'extrême harmonie, c'est suivre le bien et la perfection ; la voie rationnelle du ciel et de la terre est permanente et éternelle ; elle maintient, en s'y accordant, l'extrême harmonie.

Le ciel est l'ancêtre de tous les êtres ; le roi est la souche à laquelle se rattachent tous les états. La voie de l'activité précè de la foule des êtres et les mille variétés se développent librement. La voie rationnelle du prince consiste à surveiller, du haut de son rang prééminent, le plan de la providence, tandis que tout entre les quatre mers ${ }^{3}$ suit son gouvernement et s'y soumet; si le prince réalise et met en action la voie rationnelle du ciel, alors tous les états jouissent également de la paix ${ }^{4}$.

7.

\footnotetext{
${ }^{1}$ Elle les crée.

${ }^{2}$ C'est le même mot traduit par monter dans la formule $n^{\circ} 4$.

${ }^{3}$ L'univers.

${ }^{4}$ Le prince, c'est l'empereur ; les (États) sont les États feudataires relevant du pontife suprême.
} 
LE COMMENTAIRE TRADITIONNEL DE LA FORMULE SYMBOLIQUE dit: L'action du ciel, c'est l'activité ; l'homme doué ${ }^{1}$, limite, en s'efforçant sans cesse.

TSHENG TSE. - La formule symbolique placée à la suite d'un koua explique l'image symbolique de ce koua ; la formule symbolique placée à la suite de la formule d'un trait explique l'image symbolique de ce trait particulier. Chaque koua, ou diagramme, prend une valeur symbolique qui sert à constituer une règle. Le sens symbolique de recouvrir en abritant et d'engendrer, attribué à la voie rationnelle du koua khien est le plus étendu et le plus important; tout autre qu'un homme saint est incapable de le réaliser en le mettant en pratique. Aussi, dans le dessein que tous les hommes indistinctement puissent $\mathrm{y}$ trouver une règle à la portée de chacun, le commentaire ne relève rien que la pratique de l'activité. L'extrême activité suffit certainement pour illustrer la voie immuable du ciel ; l'homme doué l'imite en s'efforçant sans cesse, il se règle sur l'activité de l'action du ciel.

TSHOU HI. - Les symboles, ce sont les deux symboles des koua simples inférieur et supérieur, ainsi que ceux des six traits de ces deux koua simples; ce sont les formules annexées par Tsheou Kong. Le ciel est limage symbolique du koua khien. Toutes les fois qu'un hexagramme est formé par la répétition du même trigramme, le sens attribué à ce koua simple est toujours répété ; dans le cas actuel seulement, il n'en est point ainsi ; le ciel est un et unique. Mais du moment où il est question de l'action du ciel, on voit quil s'agit de sa révolution diurne, suivie de nouvelles révolutions identiques. C'est encore l'image symbolique d'une répétition continue ; sans une extrême activité, cette action serait impossible. L'homme doué en fait sa règle pour ne pas laisser altérer l'énergie de la vertu du ciel ${ }^{2}$ par les désirs humains, de sorte qu'il ne cesse pas ses pro pres efforts.

\footnotetext{
${ }^{1}$ Terme scolastique qui désigne un état de perfectionnement inférieur à la sainteté et supérieur à la simple sagesse.

${ }^{2}$ L'homme à sa naissance est doué du principe de toutes les vertus, lois du ciel.
} 


\section{Khien.}

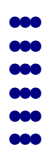

8.

Premier trait nonaire : dragon caché ${ }^{1}$; ne pas agir.

TSHENG TSE. — Le trait inférieur est considéré comme le premier; le chiffre neuf est la perfection dans les nombres positifs, aussi on l'emploie pour désigner les traits positifs.

La raison d'être ${ }^{2}$ n'a pas de forme visible, aussi on emploie une image symbolique pour éclairer le sens. Dans le koua khien, c'est le dragon qui est pris comme image symbolique ${ }^{3}$. Le dragon, considéré comme être, c'est une intelligence ${ }^{4}$ dont les modifications sont illimitées, aussi on le choisit comme symbole des modifications et transformations de la voie rationnelle de l'activité exprimée par le mot khien. L'éther de la positivité diminue ou croît, l'homme saint avance ou recule ; le premier trait nonaire est en bas du koua, il est considéré comme constituant le point de départ du commencement des êtres. Au moment où l'éther positif vient à germer, l'homme saint est enc ore au berceau ${ }^{5}$; il est comme le dragon encore caché, et il ne peut pas encore agir de lui-même ; il convient qu'il se développe dans l'ombre en attendant le moment opportun.

TSHOU HI. - Premier trait nonaire, c'est la désignation du trait positif, placé as du koua. Toutes les fois qu'on trace un koua, c'est en commençant à partir d'en bas et en montant, aussi c'est le trait inférieur qui est considéré comme le premier. Dans les nombres positifs, le chiffre neuf exprime la vieillesse et le chiffre sept, la jeunesse ${ }^{6}$; ce qui est vieux se modifie, ce qui est jeune ne se modifie pas, et c'est pour cela que les traits positifs sont appelés traits nonaires.

Dragon caché ; ne pas agir : c'est la formule attachée par Tsheou Kong pour déterminer la valeur faste ou néfaste d'un trait, et ce qu'on appelle aussi formule d'un trait. Caché, invisible ; dragon, être positif. Le premier trait

\footnotetext{
${ }^{1}$ Caché : plongé, immergé, invisible.

${ }^{2}$ La raison d'être d'une chose, li, c'est la cause ; tao, la voie rationnelle, est l'effet de cette raison d'être et la loi qui en découle.

${ }^{3}$ Cette formule est la première de celles qu'on nomme formules symboliques ; c'est l'œuvre de Tsheou Kong.

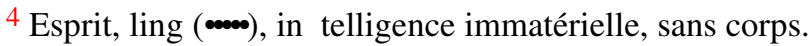

${ }^{5}$ Littéralement: incliné et minime; ces deux termes du texte sont ceux qu'on emploie en parlant des astres à leur lever et à leur coucher.

${ }^{6}$ La positivité déjà vieille est sur le point de se transformer en négativité jeune, et inversement. Ces termes et plusieurs qui suivent sont techniques et employés dans la divination; on en trouvera plus tard l'explication.
} 
positif est en bas du koua, il ne peut pas encore étendre son effet et le développer, aussi l'image symbolique est celle du dra gon caché et le sens divinatoire dit : Ne pas agir. Toutes les fois qu'on rencontre le koua khien ${ }^{1}$ et que ce trait se transforme ${ }^{2}$, il convient de considérer cette image symbolique et d'en méditer le sens divinatoire. Ceci est applicable au sujet de tous les autres traits.

DÉFINITIONS DIVERSES. - Tshen Shi Lin Shi dit: Employer l'appellation dragon, c'est emprunter une image symbolique ; les éthers du ciel et de la terre montent et descendent; la voie logique (tao) de l'homme doué comporte l'action et la retraite. Le dragon, considéré comme être, peut voler comme il peut plonger dans les eaux ; c'est pour cela qu'on emprunte son image pour servir de terme de comparaison avec les facultés de l'homme doué. Le premier trait nonaire se rapportant à l'état $\mathrm{d} u$ dragon encore caché et invisible, la formule dit : Ne pas agir ${ }^{3}$.

Dragon caché ; ne pas agir : la positivité est en dessous ${ }^{4}$.

TSHENG TSE. — L'éther positif est en dessous ; l'homme doué est placé dans une position infime, il ne doit pas encore agir.

TSHOU HI. - Positivité, désigne le trait nonaire ; en dessous, c'est -à-dire caché.

\footnotetext{
${ }^{1}$ Terme de divination; on recherche d'abord le koua, puis le trait particulier correspondant à la question posée.

2 Autre terme technique pour la divination.

${ }^{3}$ Tshou Hi dit que la raison ou pensée morale contenue dans les formules est infinie dans ses applications, et que, pour ce motif, Tsheng Tse peut en faire des déductions sans fin. Mais, ajoute-t-il, «ce n'est pas là le sens primitif du Yi king ». Selon lui, ce sens primitif se réduit à une simple formule divinatoire : la positivité étant cachée ne peut produire son effet ; c'est le dragon caché, donc on ne doit pas agir. Il faut s'en tenir là, méditer le sens et ensuite on en déduit naturellement les attributions et les applications à toutes les circonstances. $\mathrm{Si}$, au contraire, on prend comme Tsheng Tse, les applications possibles pour des définitions, on perd de vue ce que montre essentiellement le Yi king (c'est-à-dire la matière agissant par une loi ou force qui en est inséparable). Tsheng Tse a répondu d'avance à cette critique en s'adressant à un précurseur de Tshou, qui avait soutenu que chaque trait ne com portait que la seule idée exprimée dans la formule correspondante, en disant que ce serait réduire la morale et la philosophie à trois cent quatre-vingt quatre axiomes, c'est-à-dire les circonstances dans lesquelles l'homme peut se trouver, à trois cent quatre -vingt-quatre cas.

${ }^{4}$ Shouang Hou Hou Shi avance que c'est ici qu'on rencontre pour la première fois le terme, positivité ; le terme opposé, négativité, se rencontre pour la première fois dans la formule correspondante du second diagramme. Cette remarque a son importance.
} 


\section{Khien.}

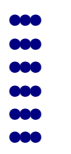

10.

\section{Deuxième trait nonaire : dragon visible dans la rizière ; avantage à voir un grand homme.}

TSHENG TSE. - La rizière, c'est la surface de la terre. Il est sorti et visible sur la terre ; sa vertu est déjà manifestée. Si on en parle au point de vue de l'homme saint, c'est le temps où Shouen cultivait la terre et se livrait à la pêche ${ }^{1}$. Avantage à voir un prince doué d'une grande vertu ${ }^{2}$ poursuivre et pratiquer sa morale ; de même, le prince a avantage à voir le sujet doué d'une grande vertu ${ }^{3}$ pour s'en faire aider dans l'achèvement de son ouvre ${ }^{4}$. L'univers a avantage à voir l'homme doué d'une grande vertu, afin d'être imprégné de son influence bienfaisante. Le prince doué d'une grande vertu, c'est le cinquième trait nonaire. La substance de chacun des deux koua khien et khouen est immélangée ; elle ne se partage pas en dureté énergique et malléabilité ; aussi, dans ces deux koua, c'est l'iden tité de qualités qui est considérée comme constituant la correspondance sympathique ${ }^{5}$.

TSHOU HI. - Deuxième, c'est-à-dire le second en montant à partir du trait inférieur; dans la suite, observer cette manière de compter. Le second trait nonaire possède la dureté énergique, l'activité, la justice et la droiture ${ }^{6}$; il émerge et cesse d'être caché ; son influence bienfaisante atteint les êtres : c'est ce que ceux -ci ont avantage à voir, aussi son image symbolique est un dragon visible dans la rizière, et le sens divinatoire est : avantage à voir un grand homme.

\footnotetext{
${ }^{1}$ Détail de la vie de Shouen, alors quill n’avait pas encore été appelé au pouvoir par Yao et qu'il menait la vie agricole, dans l'obscurité.

2 Yao.

${ }^{3}$ Shouen.

${ }^{4}$ En l'associant à son règne.

5 Termes techniques. Les traits qui occupent le même rang dans chacun des deux koua simples superposés passent pour se correspondre sympathiquement. En général, il n'y a correspondance qu'autant qu'un trait représente l'unité et l'autre la dua lité. Ici, le cinquième et le second trait font exception et s'ils se correspondent sympathiquement, c'est à cause de leurs vertus communes.

${ }^{6}$ Positivité, dureté énergique et activité ; négativité, douceur et malléabilité. Le deuxième trait et le cinquième sont, chacun, au rang intermédiaire d'un des koua simples, de là la condition de «milieu » et de «justice». En général la droiture consiste à ce que le trait soit positif s’il occupe un rang impair et négatif sil occupe un rang pair ; cependant, suivant le cas, les commentateurs ne sont jamais embarrassés pour trouver une raison concluante lorsque ces conditions ne sont pas remplies et que le sens admis, qu'il faut tou jours justifier, réclame lidée de droiture.
} 
Bien que le second trait nonaire n'occupe pas encore une situation définie ${ }^{1}$, cependant sa vertu, qui est celle d'un grand homme, est déjà évidente ; un homme ordinaire serait incapable de répondre à ces indications, aussi lorsqu'on rencontre ce trait ${ }^{2}$ se modifiant, il exprime uniquement qu'il y a avantage à voir cet homme et non pas un autre. En effet, ce terme désigne encore le grand homme placé dans une situation inférieure et représenté par le second trait; de cette façon, ce trait et la personne qui consulte le sort sont considérés respectivement comme un hôte et son hôte ${ }^{3}$ et la règle à laquelle chacun est soumis est la même; si celui-ci possède les vertus du dragon visible, il aura avantage à voir le grand homme qui occupe un rang supérieur et qui est représenté par le cinquième trait nonaire.

11.

Dragon visible dans la rizière: l'effet de sa vertu se répand universellement.

TSHENG TSE. - Visible sur la terre, sa vertu et son action transformatrice atteignent les êtres par une extension universelle ${ }^{4}$.

\footnotetext{
${ }^{1}$ C'est à partir du troisième rang que le trait indique une situation ; le troisième rang est la situation du sujet; le quatrième indique la situation du grand dignitaire, du ministre ou conseiller du prince ; le cinquième rang indique la situation du prince.

${ }^{2}$ En consultant le sort.

${ }^{3}$ Expression très usitée pour exprimer que deux personnes, ou même deux choses, occupent des positions respectivement analogues l'une par rapport à l'autre. C'est aussi une allusion aux règles rituelles de la bienséance.

${ }^{4}$ Comme le soleil commençant à s'élever ; bien quill ne soit pas encore au méridien, cependant sa clarté s'étend déjà sur toutes choses ( Pan kien tong sha). Lou Shi Hi Sheng dit: L'éther positif visible dans la rizière engendre les végétaux pour l'usage des hommes ; l'homme saint visible au monde améliore peu à peu les êtres par son enseignement.
} 


\section{Khien.}

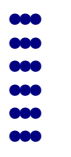

12.

\section{Troisième trait nonaire : l'activité de l'homme doué dure tout le jour ; le soir, il est encore comme préoccupé. Danger ; pas de culpabilité.}

TSHENG TSE. - Bien que le troisième rang représente la situation de l'homme, ce trait est en même temps le trait supérieur dans la substance du koua simple inférieur; il représente celui qui n'a pas encore quitté les rangs inférieurs et qui est déjà distingué par son illustration; c'est le moment où la renommée de la vertu transcendante de Shouen ${ }^{1}$ se répandait. Jour et nuit, sans se laisser aller à la négligence, il est essentiellement préoccupé par la crainte, de sorte que, bien que placé sur un terrain périlleux, il ne commet cependant point d'erreurs ${ }^{2}$. Lorsqu'il s'agit d'un homme placé dans une condition inférieure et chez qui les vertus du prince sont déjà manifestées ${ }^{3}$, l'univers tend à venir à lui ; le péril et les motifs de crainte sont évidents. Bien qu'il soit question de ce qui est relatif à l'homme saint, si la formule ne comportait pas un avertissement, comment pourrait-elle être considérée comme constituant un enseignement pour les hommes ? C'est là l'esprit dans lequel le Yi king a été rédigé.

TSHOU HI. - Nonaire, trait positif ; troisième rang, situation positive : répétition de dureté énergique sans justice ${ }^{4}$. Il occupe le rang supérieur du koua simple inférieur, c'est là un terrain périlleux. Mais sa nature et sa substance $^{5}$ sont la dureté énergique et l'activité, c'est donc l'image symbolique de l'activité incessante, de la crainte et du danger, ce qui fait que

\footnotetext{
${ }^{1}$ Qui devint le second empereur des temps historiques, selon Khong Tse

${ }^{2}$ Le même mot a le sens de faute, erreur, culpabilité ; le choix du sens de ce mot dépend de celui de la phrase.

3 Allusion à l'histoire de Wen Wang, alors que simple feudataire, sa vertu lui attirait les sympathies de l'empire et, en même temps, les persécutions jalouses du dernier empereur de la dynastie des Sheang.

${ }^{4}$ Les rangs impairs sont positifs ; un trait positif placé à un rang positif indique la répétition de la qualité de dureté énergique. Il est sans justice parce quill n’o ccupe pas le milieu d'un koua simple.

${ }^{5}$ Sa nature, c'est sa qualité de trait positif ; sa substance, c'est-à-dire le koua simple dont il fait partie, et, dans ce cas, c'est le koua simple khien, activité. Toutes ces expressions sont des termes techniques qui se présentent continuellement au sujet de chaque trait ; pénibles à saisir, d'abord, on s'y habitue facilement ensuite et leur nombre est en somme très limité.
} 
tel est le sens divinatoire ${ }^{1}$. L'expression (l'homme doué) désigne celui qui consulte le sort; la formule exprime que sil peut avoir cette sollicitude tranquille et cette circonspection, bien que placé sur un terrain périlleux, il ne commet point de fautes ${ }^{2}$.

13.

Activité qui dure tout le jour; retourner et revenir dans la même voie
(tao).

TSHENG TSE. - Qu'il avance ou se recule, qu'il se meuve ou demeure en repos, il faut toujours que ce soit d'après la voie morale (tao).

TSHOU HI. - Retourner et revenir, idée qui exprime la répétition de l'aller et du retour en marchant dans la même voie.

1 Dans les formules symboliques annexées à chaque trait par Tsheou Kong, le premier membre de phrase indique l'image symbolique attribuée à ce trait et le second est une formule divinatoire qui donne la clef de la réponse à la question posée par celui qui consulte le sort.

2 Tshou Hi dit: Ce trait est nonaire, c'est-à-dire positif, il occupe un rang positif (impair) : donc c'est un excès de positivité ou énergie. N'étant pas au milieu, il manque de justice. Il occupe le rang supérieur dans sa sphère, ce qui constitue un péril. Il doit donc sans cesse développer son activité tout le jour et être encore sur ses gardes le soir, bien que ce soit là le temps ordinairement consacré au repos. Alors, malgré le péril, il ne commettra point de fautes. Telle est exactement l'idée de l'homme saint qui a écrit la for mule. 


\section{Khien.}

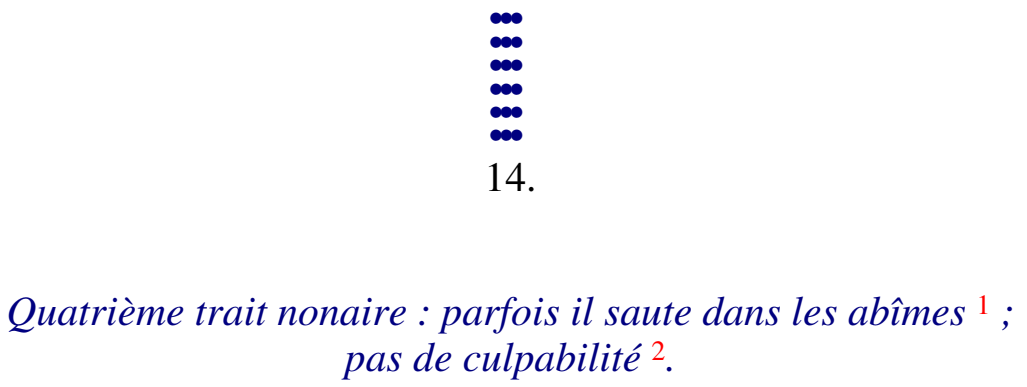

TSHENG TSE. - L'abîme est le lieu de repos du dragon ; parfois, expression qui marque l'incertitude, elle marque que ce n'est point absolu. Il saute ou ne saute pas ; s'il saute, c'est seulement au moment opportun et pour chercher le repos. Le mouvement chez l'homme saint n'est jamais sans rapport avec le moment où il a lieu. C'est le temps où Shouen faisait ses expériences pour établir le calendrier ${ }^{3}$.

TSHOU HI..- Parfois, expression de doute et d’indétermination. Sauter, parvenir brusquement dans un milieu sans avoir aucun accès pour y atteindre ${ }^{4}$, spécialement sans voler. Abîme, le vide des espaces supérieurs où les profondeurs des gouffres marins, lieux profondément obscurs et insondables, séjour des dragons. Comme lorsque, étant descendu dans la rizière, il arrive qu'il saute et s'élève, par conséquent en se dirigeant vers le ciel. Il est nonaire, c'est-à-dire positif ; le quatrième rang est une position négative ; il occupe le rang inférieur du koua simple supérieur : donc c'est une circonstance qui implique le changement et la réforme, un moment où il y a indétermination sur l'opportunité d'avancer ou de reculer, et c'est pourquoi telle est l'image symbolique. Le sens divinatoi re est que, si on peut avancer ou reculer suivant l'opportunité et la convenance du moment, il n'y aura point d'erreur ni de culpabilité.

15.

Parfois il saute dans les abîmes ; en avançant, pas de culpabilité.

\footnotetext{
${ }^{1}$ Il passe du troisième rang au quatrième, c'est-à-dire d'un koua simple dans l'autre ; il franchit la distance et saute pour aller occuper le rang inférieur dans le koua simple supérieur, le fond.

${ }^{2}$ Expression qui indique un danger, un inconvénient inhérent à la voie suivie, mais qui sera compensé par les avantages qui résultent moralement de cette voie.

${ }^{3}$ Allusion à l'histoire de Shouen, dans le Shou king.

${ }^{4}$ Comme le poisson qui bondit hors de l'eau.
} 
TSHENG TSE. - Apprécier qu'il y a possibilité et avancer, précisément dans le moment opportun, de sorte qu'il n'y a pas de culpabilité.

TSHOU HI. - Il est possible d'avancer, sans quil y ait obligation d'avancer. 


\section{Khien.}

\section{Cinquième trait nonaire : dragon volant dans le ciel ; avantage à voir un grand homme.}

TSHENG TSE. - Il avance siéger dans la situation du ciel ${ }^{1}$. Du moment où l'homme saint possède la situation du ciel, l'avantage, pour lui, consiste à voir l'homme de grande vertu qui est en bas, au-dessous de lui ${ }^{2}$, pour achever ensemble de régler les affaires de l'univers ${ }^{3}$. D'un autre côté, l'univers a évidemment avantage à voir le prince doué de grandes vertus.

TSHOU HI. - Dureté énergique, activité, justice et droiture ; avec cela il occupe la situation prééminente, comme celui qui avec la vertu de l'homme saint occupe la situation qui revient à un homme saint. C'est pour cela que telle qu'elle est l'image symbo lique et la règle d’interprétation divinatoire est la même que dans le cas du second trait nonaire. Seulement, celui qui a avantage à voir, c'est le grand homme placé dans la situa tion supérieure. Si celui qui consulte le sort possède cette situation, cela exprime qu'il aura avantage à voir le grand homme placé dans un rang inférieur et représenté par le second trait nonaire.

17.

Dragon volant dans le ciel : le grand homme agit.

TSHENG TSE. — L'action du grand homme, le fait de l'homme saint.

TSHOU HI. - Agir, faire.

\footnotetext{
${ }^{1}$ Le rang suprême ; le prince réalise sur la terre le plan de la providence ; il représente le ciel et maintient sa loi parmi les hommes.

${ }^{2}$ Le second trait.

${ }^{3}$ Nouvelle allusion à l'association de Shouen à l'empire.
} 


\section{Khien.}

18.

Trait supérieur nonaire : dragon élevé ; il y a regret.

TSHENG TSE. - Le cinquième trait nonaire, la plus haute de toutes les situations; justice et droiture, indication de la limite extrême du moment opportun ; ce niveau dépassé, c'est l'excès d'élévation ${ }^{1}$.

Aussi il y a des regrets. Lorsqu'il y a excès ${ }^{2}$, il y a regret. L'homme saint, seul, sait avancer et reculer, demeurer ou disparaître, sans jamais commettre d'excès dans son action, de sorte qu'il n'en arrive point aux regrets.

TSHOU HI. - Supérieur est la désignation particulière du trait le plus élevé dans un koua. Le caractère kang exprime l’idée que la hauteur convenable est dépassée et qu'il n'y a plus possibilité de descendre. La positivité étant parvenue à l'extrême limite de l'élévation, si elle se meut, elle doit nécessairement avoir des regrets, c'est pourquoi tels sont l'image symbolique et le sens divinatoire.

19.

Le dragon élevé a des regrets; ce qui est complètement achevé ne peut durer longtemps ${ }^{3}$.

TSHENG TSE. - Ce qui est complètement achevé doit se modifier ; il y a regret.

\footnotetext{
${ }^{1}$ Le texte chinois du commentaire emploie le même mot traduit dans la formule parle mot «élevé ». Pour justifier un sens admis, les commentateurs lui attribuent la valeur de « excès d'élévation », quill n'a réellement point.

${ }^{2}$ Le même mot signifie : trop, excès, faute et erreur.

${ }^{3}$ Le terme chinois traduit par «complètement achevé », signifie «plein et s'applique, par exemple, à la pleine lune.
} 
1. Khien.

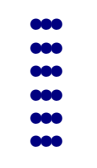

20.

\section{Emploi des traits nonaires : dans la troupe des dragons visibles, aucun ne précède les autres; bonheur.}

TSHENG TSE. - Employer les traits nonaires ${ }^{1}$, c'est se placer dans la voie de la dureté énergique et de l'activité. Pui sque la positivité considérée ${ }^{2}$ fait partie de la substance du koua khien, c'est donc la pureté immélangée dans la dureté énergique. La dureté ${ }^{3}$ et la malléabilité ${ }^{4}$ se corrigeant mutuellement constituent la justice, tandis qu'ici, à cause de l'unité de substance de la dureté énergique, il y a excès dans cette énergie. Voir ${ }^{5}$ la troupe des dragons, c'est-à-dire regarder le sens des divers traits positifs de ce koua; aucun ne prenant le pas sur les autres, c'est un présage heureux. Considérer la dureté énergique, comme étant la première condition à réaliser dans l'univers, c'est une voie logique de malheur.

TSHOU HI. - Employer les traits nonaires ${ }^{6}$, signifie que toutes les fois qu'on consulte le sort et qu'on rencontre des traits positifs, on les considère toujours comme ayant les qualités du nombre neuf et on ne leur suppose jamais celles du nombre sept ; c'est, en effet, la règle commune pour les cent quatre-vingt-douze traits positifs des divers koua. Cette règle est posée ici, parce que, dans ce koua, la positivité est immélangée, et parce qu'il est présenté le premier. De plus, la formule donnée par l'homme saint enseigne que, lorsqu'on rencontre ce koua et que tous les traits se modifient, c'est précisément ce sens divinatoire qu'il faut appliquer. Effe ctivement, si les six positivités se modifient toutes, il s'agit de la dureté énergique susceptible de douceur ; c'est une voie logique de bonheur, c'est pour cela que cette formule exprime l'image symbolique d'une troupe de dragons dont aucun ne prend le pas sur les autres, et que, si celui qui consulte le sort est dans ces mêmes conditions, ce sera un présage heureux. Le commentaire traditionnel du Tshouen tsieou ${ }^{7}$ dit : La transformation du koua khien en koua khouen dit $^{1}$ :

\footnotetext{
${ }^{1}$ Le génie de la langue chinoise permet de lire : emploi de la «nonarité » en forgeant un substantif qui exprime l'état ou la qualité de ce qui est nonaire ou plus correctement de ce qui tient à la nature du nombre neuf.

${ }^{2}$ Les traits nonaires considérés.

${ }^{3}$ Exprimée par les traits positifs.

${ }^{4}$ Exprimée par les traits négatifs.

${ }^{5}$ C'est le mot traduit par « visible », dans la formule. Selon qu'on le lit kien ou hien, le même caractère a l'un ou l'autre de ces deux sens. Tsheng Tse en adopte un, Tsh ou Hi l'autre.

${ }^{6}$ C'est encore une autre lecture des mêmes mots de la formule.

${ }^{7}$ Commentaire d'un des cinq King
} 
Dans la troupe des dragons visibles, aucun ne précède les autres; présage heureux. En effet, c'est préci sément l'idée contenue dans la formule du koua khouen, dont la substance est également immélangée : «perfection de la jument », etc ${ }^{2}$.

21.

Emploi des traits nonaires: La vertu du ciel ne doit pas être estimée au-dessus des autres.

TSHENG TSE. - L'emploi des traits nonaires, c'est la vertu du ciel. La vertu du ciel est la positivité et la dureté énergique; employer la dureté énergique d'une façon répétée, et l'estimer comme primant les autres, serait commettre un excès.

TSHOU HI. - Exprime que la dureté énergique et la positivité ne doivent pas être les qualités prédominantes des êtres; c'est pour cela que les six positivités se modifient toutes. Le présage est heureux. Depuis les mots: L'action du ciel... jusqu'à la fin de la formule $\left(n^{\circ} 7\right)$, c'est ce que les anciens philosophes ${ }^{3}$ appelaient la grande formule symbolique; depuis les mots: Dragon caché... et en continuant $\left(n^{\circ} 9\right)$, c'est ce qu’ils appelaient les petites formules symboliques. Dans les autres koua, cette même remarque est encore applicable.

\section{COMMENTAIRE SUR L'EXPRESSION DES REPRÉSENTATIONS 4}

TSHOU HI. - Ce livre revient sur les idées déjà émises dans le commentaire de la formule déterminative $\left(\mathrm{du} \mathrm{n}^{\circ} 2\right.$ au $\left.\mathrm{n}^{\circ} 6\right)$ et les commentaires des formules symboliques $\left(\mathrm{n}^{\circ} \mathrm{g} 7,9,11,13,15,17\right.$ et 19), afin d'épuiser le sens abstrait du sujet dans les deux koua khien et khouen. Quant aux définitions relatives aux autres diagrammes, on peut prendre ce commentaire pour règle et procéder par analogie et déduction.

\footnotetext{
${ }^{1}$ Titre d'un livre. Si tous les traits de l'un de ces deux diagrammes se transforment à la fois, le résultat de cette transformation est évidemment l'autre dia gramme.

${ }^{2}$ Voir la formule $\mathrm{n}^{\circ} 58$.

${ }^{3}$ Les disciples de la doctrine de la représentation graphique de l'image.

${ }^{4}$ L'un des commentaires de Khong Tse ; représentation graphique de l'image dans les caractères. Sens des mots représentés par les caractères.
} 
La cause initiale, c'est ce qu'il y a de plus primordial dans le bien ${ }^{1}$; la liberté, c'est la réunion de toutes les beautés; le bien ${ }^{2}$, c'est l'harmonie des devoirs ; la perfection, c'est la tige qui supporte toutes choses.

TSHENG TSE. - Les autres koua n'ont que la formule déterminative et les formules symboliques ; pour les deux koua khien et khouen, seuls, a été fait le commentaire sur l'expression des représentations, afin d'en éclaircir le sens. Ce commentaire analyse la voie rationnelle de l'activité (khien), et il en déduit l'application aux choses humaines. La cause initiale, la liberté, le bien, la perfection, sont les quatre vertus de koua khien; chez l'homme, la cause initiale sera la cause de toute bonté ; la liberté sera la réunion de toutes les beautés; le bien sera l'accord harmonique aux devoirs ; la perfection sera l'effet de cette cause considérée comme tuteur ou base de toutes choses.

TSHOU HI. - Le mot yuan exprime la puissance capable de commencer (créer), la vie des êtres; c'est la vertu du ciel et de la terre et aucune ne précède celle-ci. Aussi, dans les saisons, c'est le printemps ; dans l'homme, ce sera l'humanité et ce quil y a de plus élevé dans la bonté sous tous les rapports. La liberté, c'est la libre expansion des êtres vivants. Parvenus à ce point, les êtres ne sont jamais sans beauté, aussi, dans les saisons, c'est l'été ; dans l'homme ce sera la bienséance et la réunion de toutes les beautés. Le bien, c'est le développement des êtres vivants selon leurs aptitudes et les conditions particulières de leur nature ; ils ont chacun ce qui leur convient et ne se nuisent pas entre eux. Aussi, dans les saisons, c'est l'automne ; dans l'homme, ce sera le devoir et la possession de l'harmo nie des lois de sa condition. La perfection, c'est le développement parfait des êtres vivants ; la raison d'être de leur reproduction est complète et entière, suffisante dans chaque cas selon le lieu de leur habitation. Aussi, dans les saisons, c'est l'hi ver; dans l'homme ce sera l'intelligence et cela constituera le point d'appui (tronc, tuteur) de toutes choses. Le tronc ${ }^{3}$, c'est le corps de l'arbre et ce qui supporte les rameaux et les feuilles, en leur donnant un point d'appui.

DÉFINITIONS DIVERSES. - Heang Shi Ngan She dit : La bonté, la beauté, le devoir sont autant de noms différents du bien. Dans le début de toutes choses, c'est le bien ; toutes les perfections du bien constituent le beau ; la possession par chacun de ce qui lui est dû constitue le devoir; ce que le devoir établit constitue le fait. Il ne s'agit que d'une seule et même raison d'être sous quatre noms différents..., le mot tronc désigne ce qui peut sou tenir et maintenir.

\footnotetext{
${ }^{1}$ Shien, bonté, bien ; ce n'est plus le mot li du texte.

${ }^{2} \mathrm{Li}$, le caractère de la formule $\mathrm{n}^{\circ} 1$.

${ }^{3}$ C'est le mot du texte traduit, suivant le sens de la proposition, par tronc, souche, tuteur, tige, point d'appui, etc.
} 
Tshou Hi dit encore : le mot droiture ne peut pas exprimer complètement le sens du mot perfection; il faut employer simultanément, dans la définition, les deux termes droiture et fermeté et alors seulement le sens commence à être complet.

23.

L'homme doué réalise l'humanité suffisamment pour s'élever au-dessus des hommes; il honore la société suffisamment pour se conformer à la bienséance; il fait le bien aux êtres suffisamment pour s'accorder au devoir ; sa perfection et sa fermeté sont suffisantes pour soutenir toutes choses.

TSHENG TSE. - Constituer sa propre règle selon la loi d'humanité symbolisée dans le koua khien, telle est la voie morale du prince et de l'homme placé dans une position émi nente, et cette voie est suffisante pour élever au-dessus du niveau ordinaire des hommes. Réaliser l'humanité, c'es t réaliser les vertus exprimées par le mot yuan du texte ; apprécier et s'efforcer d'imiter, c'est ce qu'on appelle réaliser. Pouvoir comprendre et apprécier les beautés de la liberté dans les relations, c'est se conformer à la bienséance ; ce qui n'est pas la bienséance n'est pas la conséquence de la raison d'être, comment cela pourrait-il être considéré comme constituant la beauté ! Sans la raison d'être, comment y aurait-il liberté ${ }^{1}$ ? S'accorder au devoir ${ }^{2}$, c'est pouvoir être bienfaisant envers les êtres. Comment serait-il possible de ne pas se conformer à ce qui est juste et de ne point faire le bien aux êtres ? La perfection et la fermeté sont ce qui permet d'être le soutien et le tuteur de toutes choses.

TSHOU HI. - Puisqu'il considère l'humanité 3 comme constituant ce qu'il doit tendre à réaliser, il en résulte qu'il n'y a pas un seul être qui ne soit compris dans son amour, et c'est pour cela que c'est suffisant pour l'élever

${ }^{1}$ C'est la liberté d'expansion et de développement dans les limites tracées par les lois de la nature.

${ }^{2}$ Yi TshaeTshen Shi dit: Le devoir et le bien, si on en parle au point de vue du cour de l'homme, sont le premier, la conséquence de la raison d'être posée par le ciel et, le second, ce qui engendre le désir chez l'homme. Si on en parle au point de vue de la raison d'être céleste, alors le bien c'est la conséquence convenable (le droit) qui résulte du devoir, et le devoir est la raison d'être du bien. Assurer équitablement le bien dans tout l'univers, c'est conduire tous les êtres de telle façon que chacun se conforme exactement à sa nature et à sa destinée. Cette double définition du devoir et du bien, considérés au point de vue absolu, et relativement à la nature humaine, est bien près d'être irréprochable.

${ }^{3}$ Le mot jeu, traduit par le mot français humanité, n'exprime cependant pas exactement la même idée. Pour les Chinois, jeu exprime la possession complète de toutes les vertus qui sont l'apanage de l'homme ; c'est la conformité aux lois de la nature humaine, telles qu'elles résultent de son cour et de sa raison. 
au-dessus des hommes. Témoignant sa bonté à ce quil rencontre ${ }^{1}$, il en résulte qu'il n'est jamais sans se conformer à la bien séance ${ }^{2}$. Dirigeant les êtres de façon que chacun ait ce qui lui est avantageux, il n'est aucun devoir auquel il ne soit satisfait. La perfection et la fermeté consistent à savoir reconnaître en quoi consiste la droiture et à s'y maintenir avec fermeté ; c'est ce qu'on appelle : «connaître et ne pas abandonner » ${ }^{3}$, et c'est suffisant pour qu'il soit le tuteur et la base de toutes choses ${ }^{4}$.

24.

L'homme doué pratique ces quatre vertus, c'est pourquoi le texte dit: « activité, cause initiale, liberté, bien, perfection. »

TSHENG TSE. - Pratiquer ces quatre vertus, c'est se conformer à l'activité exprimée par le koua khien.

TSHOU HI. - Sans l'extrême activité de l'homme doué, il est impossible de les pratiquer, aussi la formule dit: khien, cause initiale, liberté, bien, perfection.

-Ici finit le premier paragraphe ; il revient sur l’idée exprimée dans la formule déterminative et ne diffère en rien des paroles de Mou Keang rapportées dans le commentaire traditionnel du Tshouen tsieou. Quelqu'un doutait si l'antiquité avait déjà connu ces définitions ; Mou Keang les énonça et Fou Tse les recueillit également ; aussi, dans la suite du texte, on distingue en faisant précéder le commentaire des mots «Tse dit» (le Maître dit), afin de montrer clairement que lorsqu'il s'agit d'opinions exprimées par Khong Tse, les commentateurs ont voulu spécifier que les propositions qui précèdent $\left(\mathrm{n}^{\circ} \mathrm{s}\right.$ 22, 23 et 24) sont des définitions qui viennent de l'antiquité.

25.

\footnotetext{
${ }^{1}$ Tshou Hi dénature quelque peu le sens de la formule $\mathrm{n}^{\circ} 23$.

${ }^{2}$ Ce terme chinois n'a pas d'équivalent en français ; il exprime l'ensemble des règles et lois rituelles qui régissent les actions des hommes dans leurs relations sociales, depuis la politesse et la convenance, et aussi les prescriptions et les devoirs religieux.

${ }^{3}$ Citation de Mang Tse.

${ }^{4}$ Selon Tshou Hi, les quatre membres de phrase de la formule $\mathrm{n}^{\circ} 25$ contiennent les définitions des quatre vertus du ciel, tandis que les quatre membres de phrase de la formule $\mathrm{n}^{\circ}$ 23 expriment les conséquences qui en résultent dans les affaires humaines.
} 
La formule du premier trait nonaire dit: Dragon caché; ne pas agir, qu' est-ce que cela exprime? Tse dit ${ }^{1}$ : Vertu du dragon qui, de plus, est cachée. Ne pas changer selon le siècle; ne pas s'attacher à la renommée; fuir le monde sans chagrin; ne pas être apprécié et ne pas s'en chagriner. Faire ce qui plaît, résister aux chagrins, être opiniâtre dans ce qui ne doit pas être abandonné : tel est le dragon caché 2.

TSHENG TSE. - À partir d'ici et en suivant, il s'agit des effets de l'activité désignée par le mot khien, c'est la voie rationnelle de l'emploi des traits nonaires. Le premier trait nonaire exprime le point où la positivité commence à exister. L'état de concèlement de la vertu du dragon, c'est la condition de l'homme saint laissé dans l'oubli e t tenu à l'écart. Il se maintient dans la voie de la morale et ne modifie pas ses principes suivant le temps ; il cache ses actions sans chercher à être compris de son siècle ; il a confiance en soi-même et se suffit. Lorsqu'il voit une opportunité, il agit; s'il reconnaît le danger, il l'évite. Dans l'observation de ses principes, il met une telle fermeté que rien ne peut altérer en lui cette vertu du dragon caché.

TSHOU HI. - Vertu du dragon, vertu de l'homme saint. Ce trait est en bas du koua, donc la vertu est cachée. Changer veut dire modifier les principes observés. En général, au sujet des six traits du koua khien, le commentaire de l'expression des représentations éclaire toujours le sens par l'exemple de l'homme saint ; caché ou en évidence, sa profondeur ne change point.

26.

La formule du second trait nonaire dit: Dragon visible dans la rizière; avantage à voir un grand homme. Qu'est-ce que cela exprime? Tse dit: Vertu du dragon avec justice et droiture; bonne foi dans les moindres paroles; circonspection dans les actions les plus ordinaires. Etre en garde contre le mensonge, sauvegarder la vérité. Améliorer son siècle sans s'en vanter; vertu universelle et transformatrice. Le Yi king dit: dragon visible dans la rizière ; avantage à voir un grand homme : vertu du prince.

TSHENG TSE. - C'est celui qui, possédant la vertu du dragon, se place avec justice et droiture. Être placé juste au milieu d'un koua simple est considéré comme exprimant le sens de possession de la justice et de la droiture. Circonspection et bonne foi dans les choses les plus ordinaires: C'est à cela qu'il faut s'en tenir dans la suite des actions. Du moment où il se place sur un terrain qui ne comporte ni erreur, ni excès, c'est qu'il est

${ }^{1}$ C'est-à-dire Khong Tse ; application de ce qui vient d'être dit dans le commentaire de TSHOU HI.

${ }^{2}$ Il est permis de supposer que Khong Tse s'est peint lui-même dans ce passage. 
spécialement en garde contre le mensonge; étant en garde contre le mensonge, la vérité se trouve préservée. Améliorer son siècle sans s'en vanter, sans s'en attribuer le mérite. Vertu universelle et transformatrice, c'est la réforme de soi-même dont résulte la réforme des êtres. Il s'a git toujours de choses relatives à un grand homme; bien que ce ne soit pas la situation du prince, c'est nécessairement la vertu du prince.

TSHOU HI. - Juste au milieu ${ }^{1}$, c'est le moment où il n'est pas caché et où il ne saute pas encore. Alors même qu'il ne s'agit que de paroles sans importance et d'actions jour nalières, conserver néanmoins la bonne foi et la circonspection, c'est le comble de la vertu la plus parfaite. Être en garde contre le mensonge et préserver la vérité, c'est l'idée de ne pas la bl esser et même de la garantir. L'expression « vertu du prince » explique que le grand homme est représenté par le second trait nonaire.

27.

La formule du troisième trait nonaire dit: L'activité de l'homme doué dure tout le jour; le soir, il est encore comme préoccupé : danger ; pas de culpabilité. Qu'est-ce que cela exprime? Tse dit: L'homme doué développe ses vertus et perfectionne ses aptitudes. C'est par la bonne foi qu'il développe ses vertus; c'est en corrigeant ses expressions et en maintenant la vérité qu'il se tient au niveau de ses aptitudes, connaissant la limite, il y tend ${ }^{2}$ et peut en approcher de près; connaissant la fin, il la prend pour but et peut ainsi maintenir le devoir. C'est pour cela qu'il peut occuper une situation élevée sans être enorgueilli, comme il peut occuper une situation inférieure sans s'en chagriner. Il déploie donc son activité suivant les moments et il ne laisse pas endormir sa vigilance; bien qu'il y ait péril, il ne commet pas de fautes ${ }^{3}$.

TSHENG TSE. — Le troisième trait occupe le rang supérieur dans le koua simple inférieur, et, chez lui, la vertu du prince est déjà manifeste ; comment cela peut-il être interprété ? Uniquement comme exprimant l'action de développer ses vertus et de perfectionner ses aptitudes. En lui-même il

\footnotetext{
${ }^{1}$ C'est l'expression traduite par droiture et justice, dans la formule. On peut prendre le premier mot comme adjectif et lire exact, juste, mais ce n'est plus le sens du texte de Khong Tse.

${ }^{2}$ Ceux qui voulaient assurer la droiture dans leur coaur commençaient par assurer la sincérité dans leurs intentions; ceux qui voulaient assurer la sincérité dans leurs intentions commençaient par étendre leurs connaissances. L'extension des connaissances consiste dans l'extrême analyse des choses ; lorsque les choses sont analysées, on sait ensuite vers quoi il faut tendre (Khong Tse, grande étude).

3 Ce passage présente sous une forme un peu différente presque toute la doctrine du perfectionnement exposée dans la «grande étude».
} 
concentre la fidélité et la bonne foi, ce qui développe sa vertu ; il choisit ses expressions et domine ses tendances, ce qui fait qu'il se tient au niveau de ses aptitudes et les conserve. Connaître la limite et y tendre, c'est étendre le savoir ; chercher à savoir où il faut tendre et ensuite y tendre, c'est ce qui précède le savoir et c'est à cause de cela qu'il peut s'en approcher de très près. C'est ce qu'on appelle : «commencer à suivre les veines du bois » ${ }^{1}$, et c'est quelque chose qui est relatif au savoir ${ }^{2}$. Connaître la fin et la prendre pour but, c'est l'action de l'énergie ; du moment où il connaît la fin vers laquelle il doit tendre, il avance avec énergie et y aboutit. C'est ce qui vient après le soin de la conservation et du maintien des facultés et c'est pour cela qu'il peut maintenir le devoir et l'observer. C'est là ce qu'on appelle « aller jusqu'à la fin des veines du bois », et c'est quelque chose qui est inhérent à la sainteté 3 . Tels sont le commencement et la fin de l'étude ; c'est ainsi qu'étudie l'homme doué, aussi il sait se placer suivant la voie rationnelle, quelle que soit la hauteur ou l'infériorité de sa position, sans orgueil, comme sans tristesse. Il ne se laisse pas aller à la négligence et sait être circonspect, de sorte que bien que sur un terrain périlleux il ne commet pas de fautes.

TSHOU HI. - La fidélité et la bonne foi dirigeant son cour, il n'est aucune de ses pensées qui soit sans franchise et sincérité. La correction des expressions, c'est la conséquence qui se manifeste dans le fait ; aucune de ses paroles n'est autrement que vraie. Bien que le cour soit plein de fidélité et de bonne foi, cependant, sans le soin de corriger les expressions et de maintenir la vérité, il n'y aurait aucun moyen de se maintenir à ce niveau. Connaître la limite et y tendre, c'est quelque chose qui est relatif au développe ment de la vertu. Connaître la fin et la prendre pour but, c'est quelque chose qui est rela tif au soin de se tenir au niveau des aptitudes, c'e st ce qui fait que jusqu'à la fin du jour son activité ne cesse pas, et que, le soir, il est encore comme inquiet et préoccupé. Il peut occuper une position élevée ou inférieure, il n'est ni orgueilleux, ni chagrin : c'est ce qu'on appelle ne commettre auc une faute.

28.

La formule du quatrième trait nonaire dit: Parfois il saute dans les abîmes, pas de culpabilité. Qu'est-ce que cela exprime? Tse dit : Élevée ou basse, sa position n'est pas toujours la même; jamais elle ne constitue une irrégularité ; il avance ou recule, son mouvement n'est pas constamment le même. ,jamais il ne s'écarte de ses semblables. L'homme doué développe ses

${ }^{1}$ L'expression figurée : suivre les veines des fibres du bois, qui indiquent la voie du développement des rameaux.

${ }^{2}$ Expression scolastique qui reporte encore à la « grande étude » de Khong Tse.

${ }^{3}$ Terme scolastique très usité dans l'analyse du sujet d'une proposition. 
vertus et il perfectionne ses aptitudes; il sait profiter du moment opportun, c'est pourquoi il ne commet pas de fautes.

TSHENG TSE. - Parfois il saute, parfois il reste en place ; élevée ou basse, sa position n'est pas toujours la même. Parfois il avance, parfois il recule; qu’il s'éloigne ou se rap proche, il suit la voie convenable et juste. Jamais sa position ne constitue une irrégularité ou la violation d'un principe ; jamais il ne se sépare de ses semblables et de ceux de son genre. Il développe ses vertus et perfectionne ses aptitudes ; il veut profiter du moment opportun. À certains moments, il agit, dans d'autres, il s'arrête : son action ne peut pas être constamment la même, aussi la formule dit: " Parfois. » Les abîmes profonds sont les lieux de repos du dragon. «Dans les abîmes » exprime quil saute pour gagner son lieu de repos. Les abîmes sont des lieux profonds, et cependant la formule emploie le terme sauter ${ }^{1}$; ce mot est pris uniquement dans l'acception de se porter vers le lieu de son repos. Parfois, exprime l'incertitude 2 ; c'est suivant le moment et sans quil soit possible de l'affirmer. L'homme doué se conforme aux nécessités du moment comme l'ombre suit la forme ; s'il pouvait s'écarter de cette règle, c'est qu'elle ne constituerait plus la voie rationnelle.

TSHOU HI. - Au sujet du koua simple intérieur, il est question de l'étude de la vertu; au sujet du koua extérieur, il est question du moment et de la situation; avec le développement de la vertu et le perfectionnement des aptitudes, l'explication du troisième trait nonaire est complète ; ici l'explication montre qu'il veut profiter du mome nt opportun et avancer.

29.

La formule du cinquième trait nonaire dit: Dragon volant dans le ciel; avantage à voir un grand homme. Qu'est-ce que cela exprime? Tse dit : Les tons (musicaux) équivalents se correspondent mutuellement; les éthers analogues s'attirent entre eux; l'eau coule dans les déclivités, le feu se porte vers ce qui est $\mathrm{sec}^{3}$. Le nuage suit le dragon, le vent suit le tigre. L'homme saint agit et tous les êtres le suivent des yeux. Ce qui a son origine dans le ciel monte; ce qui a son origine sur la terre descend, de sorte que chaque chose suit son propre genre.

\footnotetext{
${ }^{1}$ Le mot employé signifie sauter en s'élevant, sauter en l'air, surgir hors des abîmes.

${ }^{2}$ En général, le rapprochement du supérieur et de linnférieur, le point intermédiaire entre ce qui est haut et ce qui est bas est toujours une occasion ou un lieu de péril. C'est pour cela que le troisième trait est menacé d'un danger et que dans le cas du quatrième il y a encore doute et incertitude (Heang Shi Ngan She).

${ }^{3}$ L'eau et le feu sont de ux des cinq agents produits par les deux éthers qui, eux-mêmes, ne sont que deux états de l'extrême origine ou principe primordial.
} 
TSHENG TSE. — Le lien commun entre l'homme et l'homme saint est le genre ; le cinquième trait, avec la vertu du dragon, monte occuper la situation prééminente ; rien de ce qui appartient au genre humain ne reste sans accourir vers lui et sans lever les yeux dans sa direction; à plus forte raison doit-il en être de même de ceux qui possèdent les mêmes vertus. Celui qui occupe une position élevée correspond sympathiquement à celui qui occupe un rang inférieur et celui-ci suit le premier. Les mêmes tons se correspondent, les mêmes éthers s'attirent; couler vers les déclivités, se porter vers ce qui est sec, suivre le dragon, suivre le tigre : c'est toujours la conséquence du genre de l'éther. Aussi l'homme saint agit et tous les êtres le regardent. Le supérieur regardant l'inférieur, celui-ci, à son tour, regarde vers le supérieur. Le mot «être désigne les hommes. Les «anciennes expressions ${ }^{1}$ disent : «L'homme est "être " un être ; l'être qui discute ${ }^{2}$ s'appel le homme. » Dans le Yi king, la formule : avantage à voir un grand homme, ne change pas, mais sa valeur est variable. Par exemple, dans le cas du koua song, cette même formule veut dire qu'il convient de voir un homme de grande vertu, juste et droit, et qu'alors la décision sera intelligente et claire ; il s'agit d'un conseil à donner et il en est question avant que l'homme dont il s'agit n'ait été vu. Dans le cas des formules des second et cinquième traits du koua khien, du moment où l'homme saint est déjà révélé, le supérieur et l'inférieur se voient mutuellement ; ils achèvent l'œuvre de concert. Ce qui est avanta geux, c'est de voir le grand homme, et il s'agit de ce qui a lieu après l'action de voir. « $\mathrm{Ce}$ qui a son origine dans le ciel », par exemple : le soleil, la lune, les étoiles, les astres ; « ce qui a son origine sur la terre », par exemple : les reptiles, insectes et quadrupèdes, les plantes et les arbres. La négativité et la positivité suivent chacune leur propre genre; l'homme et les êtres ne font jamais exception à ce principe.

TSHOU HI. — Agir, littéralement, s'élever. « Être » homme ; suivre des yeux, ou regarder, explique liidée contenue dans les mots « avantage à voir »; l'expression «ce qui a son origine dans le ciel» désigne les choses qui se meuvent ; l'expression « ce qui a son origine sur la terre » désigne les choses immobiles. Les êtres suivent chacun leur propre genre; lhomme saint est le premier à la tête du genre humain, aussi il s'élève et monte, de sorte que tous les hommes le voient.

30.

\footnotetext{
${ }^{1}$ Probablement le titre d'un livre.

2 Il faudrait pouvoir lire le passage cité, dans son entier, pour être sûr de la traduction. On pourrait presque lire : l'être qui raisonne.
} 
La formule du trait supérieur nonaire dit: Dragon élevé; il y a regret. Qu'est-ce que cela exprime Tse dit: Noble et sans situation ${ }^{1}$, élevé et sans peuple ${ }^{2}$; l'homme sage est dans une position inférieure et ne l'aide pas, c'est ce qui fait que, s'il se meut, il aura des regrets ${ }^{3}$.

TSHENG TSE. - Le trait nonaire occupe le rang supérieur sans mériter ou atteindre la situation prééminente ; c'est pour cela qu'il ne possède pas de peuple et qu'on ne l'aide pas ; sil se meut il aura des regrets.

TSHOU HI. - L'expression « les sages dans les situations inférieures » désigne le cinquième trait nonaire et ceux qui sont au-dessous ${ }^{4}$. " Ils ne l'aident pas », puisque le trait supérieur nonaire est monté trop haut, son esprit est infatué de lui-même et on ne vient pas à lui pour l'aider et l'assister. - Ici finit le second paragraphe, qui revient sur les idées exprimées dans le commentaire de la formule symbolique.

31.

Dragon caché ; ne pas agir : infériorité 5 .

TSHENG TSE. - À partir d'ici et en continuant, il est question du moment indiqué par le koua khien. Ne pas agir ${ }^{6}$; puisqu'il est en bas il ne peut pas encore agir.

32.

Dragon visible dans la rizière : le moment cesse 7 .

\footnotetext{
${ }^{1}$ Noble, c'est-à-dire positif, le trait supérieur ne marque aucune situation.

2 Tous les traits au-dessous de lui sont positifs, ils n'indiquent pas le peuple.

${ }^{3}$ Bien quill y ait en lui excès d'une quali té (énergie), sỉl peut rester en repos sans agir, il réussira à éviter les regrets.

${ }^{4}$ La lecture de Tshou Hi est incorrecte au point de vue chinois. Il ne s'agit que du second trait.

${ }^{5}$ Yun Fong Hou Shi dit : «La positivité est en dessous » $\left(\mathrm{n}^{\circ} 9\right)$ est dit par rapport à l'éther. Ici le commentaire dit : «infériorité », cela se rapporte à l'homme.

${ }^{6}$ Tsheng Tse lit : il n'agit pas.

${ }^{7}$ Je crois que la vraie lecture serait: le temps change.
} 
TSHENG TSE. - Il s'arrête suivant le moment. TSHOU HI. - Cela exprime qu'il n'est pas encore temps d'agir.

33.

Activité qui dure tout le jour : faire quelque chose ${ }^{1}$.

TSHENG TSE. - Développement de la vertu, perfectionnement des aptitudes.

34.

Parfois il saute dans les abîmes, s'essayer soi-même.

TSHENG TSE. - S'employer suivant le moment ${ }^{2}$.

TSHOU HI. - Ne pas se hâter dans ce quỉl y a à faire et en essayer la possibilité.

35.

Dragon volant dans le ciel : il monte pour régir.

TSHENG TSE. - Il possède la situation et régit; gouvernement du supérieur.

TSHOU HI. - Il occupe le rang supérieur pour gouverner les inférieurs.

36.

${ }^{1}$ Quelque chose, ce qu'il convient de faire. (Lin shi hi yuan.)

${ }^{2}$ S'e ssayer, comme le jeune oiseau essaie ses ailes sur le bord du nid. (L'action de sauter est comparée à l'essai des ailes.) 
Dragon élevé, il y a regret : calamité inhérente à la fin.

TSHENG TSE. - À la limite extrême, les calamités surgissent.

37.

Khien, cause initiale ; emploi des traits nonaires ; l'univers en ordre.

TSHENG TSE. — La voie rationnelle de l'emploi des traits nonaires est la même, qu'il s'agisse du ciel ou de l'homme saint se conformant à son effet ; l'univers est en ordre.

TSHOU HI. - Il s'agit de la grandeur ( yuan) du koua khien et de l'emploi des traits nonaires. Cette qualité est considérée autrement que dans les autres koua. Si la voie suivie par le prince est la dureté énergique, et sỉl peut aussi y allier la douceur, il n'est r ien qui ne soit en ordre dans l'univers. - Ici finit le troisième paragraphe, qui revient de nouveau sur les idées précédemment émises.

38.

Dragon caché; ne pas agir : l'éther de la positivité est caché et invisible.

TSHENG TSE. - Cette formule et les suivantes traitent du sens du koua khien. $A u$ moment où la positivité est encore extrêmement minime, cachée et invisible, l'homme doué doit de même se tenir dans l'ombre ; il ne doit pas encore agir. 
Dragon visible dans la rizière : l' univers est embelli et éclairé ${ }^{1 .}$

TSHENG TSE. - La vertu du dragon se manifeste sur la terre ; l'univers contemple sa beauté et son intelligence et se transforme en s'améliorant.

TSHOU HI. - Bien qu'il ne soit pas dans une position supérieure, l'univers en ressent c ependant déjà l'effet transformateur ${ }^{2}$.

40.

Activité qui dure tout le jour : agir avec énergie selon le moment.

TSHENG TSE. - Se conformant au moment pour avancer.

TSHOU HI. - Conséquence naturelle du moment.

41.

Parfois il saute dans les abîmes : la voie rationnelle du koua khien, c'est le renouvellement ${ }^{3}$.

TSHENG TSE. - Il quitte une situation inférieure et monte dans une position supérieure ; en haut et en bas, il y a renouvellement.

TSHOU HI. - Il quitte le koua simple inférieur et monte; c'est le moment du changement et de la modification.

\footnotetext{
${ }^{1}$ Expression dont le sens est très mal défini ; très souvent employée d'après le sens qu'on lui attribue traditionnellement dans ce commentaire. Généralement, d'après l'interprétation admise dans le Shou king, on considère que wen exprime une suite d'actions visibles, la conduite suivie ; tandis que ming, exprime la clarté et le discernement dans l'esprit.

${ }^{2}$ Dans ce cas et avec cet emploi, ce terme correspond très exactement à progrès, civilisation.

${ }^{3}$ Ce terme signifie aussi changement, mais avec le sens de renouvellement de ce qui a fait son temps et est démodé ou hors d'usage. Yu Shi Yen dit : renouveler, c'est modifier (transformer graduellement).
} 
42.

Dragon volant dans le ciel : c'est s'établir dans la situation qui comporte la vertu du ciel.

TSHENG TSE. - Position correcte dans l'élévation ; cette situation demande la vertu du ciel.

TSHOU HI. - La « vertu du ciel », c'est précisément la « situation du ciel »; en effet, ce n'est qu'à la condition d'avoir cette vertu qu'il convient d'occuper cette situation, et c'est pour cela qu'elle est ainsi appelée ${ }^{1}$.

43.

Dragon élevé; il y a regret: avec le temps, tout arrive à son extrême limite.

TSHENG TSE. - Le moment étant arrivé à sa limite ${ }^{2}$, il en résulte que la position et le moment sont également extrêmes.

44.

Cause initiale de l'activité, emploi des traits nonaires : alors on voit les lois du ciel.

TSHENG TSE. — La voie rationnelle de l'emploi des traits nonaires, c'est la loi du ciel. «Loi du ciel» désigne la voie rationnelle du ciel. Aussi, si on demandait si les six traits du koua khien se rapportent tous à l'homme saint, il faudrait répondre que s'il s'agit d'épui ser la voie rationnelle en la parcourant jusqu'au bout il est question de l'homme saint ; selon qu'on réussit à s'y conformer ou qu'on y manque, c'est le bonheur ou le malheur qui en résultent.

\footnotetext{
${ }^{1}$ Situation du ciel : c'est -à-dire pouvoir suprême sur la terre entière.

2 Tsheng Tse prend le mot «moment» avec son sens technique dans l'application du Yi king à la divination, c'est-à-dire le sens de moment, époque, indiquée par le koua. Le moment du koua est écoulé et le rang occupé est le dernier. Ce n'est pas là le sens naturel de la phrase de Khong Tse.
} 
Comment cela serait-il vrai dans le seul cas du koua khien? Il en est de même lorsqu'il s'agit de tout autre koua.

TSHOU HI. - Posséder la dureté énergique et pouvoir employer la douceur, telle est la règle du ciel. - Ici finit le quatrième paragraphe, qui revient encore sur les idées déjà émises.

45.

La cause initiale dans le koua khien, c'est le commencement et la liberté d'expansion.

TSHENG TSE. - Nouvelle répétition expliquant les définitions afin d'épuiser le sens. Du moment où il y a commencement, il faut nécessairement qu'il y ait liberté; sans cette liberté d'expansion, il y aurait arrêt et interruption.

TSHOU HI. - S’il y a commencement, il y a nécessairement liberté ; la raison d'être et la force inhérente des choses l'exigent ainsi ${ }^{1}$.

46.

Le bien et la perfection sont le naturel et le sentiment ${ }^{2}$.

${ }^{1}$ Ce qui a été créé doit se reproduire librement ; c'est la loi universelle.

2 On demandait l'explication de ces mots : Le bien et la perfection sont la nature et le sentiment. Tshou Hi répondit : Ici, dans l'emploi de ces deux mots « nature et sentiment », c'est comme si on disait « substance essentielle». La cause initiale et la liberté en sont la manifestation et l'effet : le bien et la perfection sont le point où cette substance essentielle se recueille, se réunit et revient sur elle-même. Par exemple, la saison du printemps fait éclore la vie ; l'été arrivé, les bourgeons des rameaux s'épanouissent ; à l'automne le fruit se no ue. Il y a déjà tendance au recueillement, au rassemblement, et au résultat qui est d'amasser (il y a déjà tendance vers le repos de l'hiver qui arrête la circulation de la sève) ; mais le résultat n'est pas encore certain et la semence n'est pas encore ap te à se reproduire. Enfin vient l'hiver et l'œuvre s'achève. Bien qu'à l'automne le fruit soit déjà formé et la semence pleine, que peu à peu elle tende à s'échapper de son enveloppe en attendant le moment propice, cependant la concentration de l'éther n'e st pas complète. Si on la semait en cet état, elle ne germerait pas et ce n'est qu'à l'hiver que l'ouvre est accomplie. L'homme seul arrivé à l'hiver et à l'automne doute encore comme s'il ne voyait plus l'idée de la vie (intention providentielle de préser ver la vie); il ignore que, réellement, elle est recueillie et rassemblée à l'intérieur. Sujets de dissertation. 
TSHENG TSE. — Le naturel et le sentiment de l'activité exprimée par le koua khien. Selon qu'il y a déjà commencement et liberté, s'il n'y avait en outre le bien et la perfection, serait-il possible qu'il n'y eût ni arrêt, n i interruption?

TSHOU HI. - Recueillir, c'est récolter, rentrer et serrer. Dans ces actions on voit la réalité du naturel et du sentiment.

47.

Le commencement dî̀ à l'activité peut faire agir le bien et le beau pour l'avantage de l'univers. Ce qui constitue l'avantage (li) n'est pas défini ; c'est immense!

TSHENG TSE. — La voie rationnelle du commencement par l'activité du koua khien peut produire la vie et l'achèvement de tous les genres, l'univers contemple sa beauté et le bien qu'elle représente, $m$ ais il n'est pas dit en quoi consistent ces avantages. C'est que, effectivement, elle n'est jamais sans avantages et il est impossible de les énumérer tous. Aussi la formule comporte une exclamation admirative sur l'immensité des avantages qui en résultent et dit : c'est immense !

TSHOU HI. - Le commencement ${ }^{1}$, c'est la grandeur ( yuan) et la liberté (heng); ce qui produit l'avantage de l'univers, c'est le bien (li). Ce qui est avantageux n'est pas exprimé, c'est la perfection (tsheng). On a dit: Dans le koua khien, le texte dit: «avantage de la jument», et dans ce cas ce qui est avantageux est nommé.

48.

Qu'elle est grande l'activité! énergie, force, justice, droiture ; pureté de substance, de qualités, d'essence.

TSHOU HI. - L'énergie (ou dureté é nergique) se dit au sujet de la substance ; force (ou activité) s'applique indifféremment à l'effet. La justice, c'est dans l'action, ne pas dépasser le point qu'il faut atteindre et ne point

1 Pour désigner d'un seul mot les quatre vertus attributives, on dit « cause initiale de l'activité », ou encore : "commencement de l'activi té », ce qui comporte les quatre vertus. Définitions diverses (création). 
rester en deçà. La droiture, c'est la quali té qui consiste à se maintenir sans partialité. Ces quatre conditions sont les vertus de l'acti vité représentée par le koua khien. Pureté immélangée de la substance, non mélangée de malléabilité négative ; pureté de qualités, qualités qui ne sont pas entachées de fausseté et de vice ; c'est, en effet, l'extrême degré de l'énergie de la force, de la justice et de la droiture. Enfin, pureté d'essence, c'est -à-dire encore pureté immélangée de substance et de qualité. On a douté si, l'énergie du koua khien n'étant pas tempérée par la malléabilité (douceur) ${ }^{1}$, il était néanmoins possible d'employer les qualifications de justice et droitu re. Cela est inexact ; entre le ciel et la terre, il n'y a essentiellement qu'un seul courant d'action d'un éther unique et cet éther affecte de ux états, le mouvement et le repos. Si on en parle d'après la totalité absolue de la substance qui produit le courant d'action, on l'appelle simplement activité khien et il n'est rien qui ne s'y trouve compris. Si on distingue d'après les états de mouveme nt et de repos, il y a, en outre de cette distinction, les différences qualifiées par les mots positivité et négativité, énergie et malléabilité ${ }^{2}$.

49.

Les six formules des traits mettent en relief et montrent les sentiments sous tous les aspects.

TSHOU HI. - Les mots traduits par « sous tous les aspects » ont encore le sens de suivre, en les épuisant, tous les méandres des conséquences d'une proposition quelconque.

50.

Suivant le temps, monter les six dragons pour parcourir le ciel $\left(n^{\circ} 4\right)$; les nuages marchent, la pluie étend son effet $\left(n^{\circ} 3\right)$; l'univers est en paix.

\footnotetext{
${ }^{1}$ C'est-à-dire puisquill n'y a pas de traits négatifs dans le koua.

${ }^{2} \mathrm{Ce}$ commentaire résume très nettement l'exposition cosmogonique de Tsheou Tse, dans le Thai ki.
} 
TSHENG TSE 1. - Qu'elle est grande ! exclamation admirative arrachée par la grandeur de la voie rationnelle. Par ces six termes : dureté énergique, force ou activité, justice, droiture, pureté immélangée de substance et de qualité, la voie logique de l'activité exprimée par le koua khien est peinte et figurée. L'expression pureté d'essence exprime l'extrême et absolue pureté dans ces six traits. Les formules des six traits épuisent, en les généralisant dans toutes les applications possibles, le sentiment et le sens $\left(\mathrm{n}^{\circ} 49\right)$. Profiter 2 de l'instant indiqué par chacun des six traits $\left(\mathrm{n}^{\circ} 50\right)$, afin de correspondre au mouvement du ciel ${ }^{3}$, alors l'action et l'effet du ciel sont manifestes. C'est pourquoi on voit que les nuages en marchant, la pluie en étendant ses effets, la négativité et la positivité dans leur choc et leur libre expansion, posent la voie logique de l'harmonie et de la paix dans l'univers.

TSHOU HI. - Ceci exprime que l'ho mme saint profitant du moment pour monter chacun des six dragons afin de parcourir le ciel, il en résulte que l'effet produit est comme le cours des nuages dans le ciel et comme leur résolution en pluie, et que l'uni vers jouit de la paix. - Ceci est le cinquième paragraphe. Il revient de nouveau sur l’idée exprimée dans les premières propositions.

51.

L'homme doué considère le perfectionnement de la vertu comme son action naturelle. $C$ est une action qui peut être journellement observée. Le sens exprimé par le mot caché est qu'elle est cachée et pas encore manifestée, qu'il la pratique, mais qu'elle n'est pas encore entièrement perfectionnée. $C$ est à cause de cela que l'homme doué n'agit pas.

TSHENG TSE. - Dans le perfectionnement de la vertu, ce qui peut être vu, c'est son action, ou pratique ; ce n'est que quand la vertu est perfectionnée que son effet peut s'étendre. Dans le cas du premier trait, elle commence, mais elle est encore cachée, pas encore manifestée ; son action n'est pas encore produite par la pratique. Ne pas encore être mise en pratique, c'est ne pas encore être manifestée. C'est à cause de cela que l'homme doué n'agit pas.

TSHOU HI. - Vertu perfectionnée ${ }^{4}$, vertu déjà perfectionnée ; dans le premier trait nonaire, la vertu est certainement déjà achevée, mais seulement son action, ou mise en pratique, n'est pas encore visible.

\footnotetext{
${ }^{1}$ Ce commentaire se rapporte aux formules $n^{\circ} 48,49$ et 50 .

${ }^{2}$ Le même mot signifie profiter de..., ou bien employer comme moyen de locomotion, monter en barque, à cheval, etc.

${ }^{3}$ Mouvement astronomique.

${ }^{4} \mathrm{Si}$ on adopte la lecture de Tshou Hi le texte devient : « Il considère la vertu achevée comme son action naturelle » et le sens n'est point correct.
} 
52.

L'homme doué étudie pour l'accumuler; il interroge pour la déterminer ; il est bienfaisant pour ne point s'en départir ; il est humain pour la pratiquer. La formule du Yi king dit : Dragon visible dans la rizière ; avantage à voir un grand homme; c'est la vertu du prince.

TSHENG TSE. — L'homme saint est dans une position inférieure ${ }^{1}$; bien qu’il soit déjà illustre, il ne possède encore aucune situation offi cielle, de sorte qu'il se borne à dévelop per ses vertus et à perfectionner ses aptitudes. Étudier et accumuler, interroger et déterminer, c'est le développement de la vertu. Être bienfaisant pour ne point s'en départir, être humain et la pratiquer, c'est le perfectionnement des aptitudes. La vertu du prince est déjà manifeste en lui, il a avantage à voir un grand homme et à avancer pour mettre cette vertu en pratique. Avancer pour occuper une situation, comme Shouen et Vu ; avancer en pratiquant la morale comme Hi Tshouan.

TSHOU HI. - C'est, en effet, par ces quatre conditions, qu'il achève de réaliser la vertu du grand homme. Le commentaire répète l'expression « vertu du prince », afin d'éclairer parfaitement que c'est bien le second trait nonaire qui représente le grand homme.

53.

Troisième trait nonaire: répétition de dureté énergique et absence de justice; il est en haut sans atteindre le ciel; il est en bas sans être encore dans la rizière, aussi son activité ne cesse point, et, suivant le moment, il est inquiet et préoccupé. Bien qu'il y ait péril, il ne commet point de fautes.

TSHENG TSE. - Dans le troisième trait, il y a répétition de dureté énergique ; c'est la perfection complète de la dureté énergique. Il a dépassé le juste milieu et il occupe le rang supérieur dans le koua simple inférieur; la position est élevée, sans qu'il ait déjà atteint le ciel, tandis qu'en bas, il s'est déjà écarté de la rizière. C'est un terrain périlleux et à craindre. Suivant le moment il se plie aux nécessités de sa position ; son activité dure sans cesse, il est craintif et circonspect pour prévenir le péril. Aussi, malgré le péril, il n'en

\footnotetext{
${ }^{1}$ Le second trait.
} 
arrive point à commettre des fautes. L’homme doué se plie aux nécessités du moment ; il est prudent et circonspect, ce qui fait qu'il peut prospérer.

TSHOU HI. - Répétition de dureté énergique, c'est-à-dire trait positif occupant un rang positif.

54.

Quatrième trait nonaire: répétition de dureté énergique sans justice; élevé sans atteindre le ciel ; bas sans être encore dans la rizière ; au milieu sans être parmi les hommes, c'est pour cela que c'est un objet de doute. Parfois, exprime ce doute ; c'est pour cela qu'il n'y a point de culpabilité ${ }^{1}$.

TSHENG TSE. - Le quatrième trait n'est pas à la hauteur du ciel ; il n'est pas dans la rizière, et il est déjà sorti d'entre les hommes en s'élevant au-dessus d'eux; le terrain qu’il foule est périlleux. «Doute» est une expression qui marque l'absence de décision défi nie ; la position n'est pas assurée. Parfois il avance, parfois il recule, et seulement pour son repos ; c'est à cause de cela qu'il ne commet pas de fautes.

TSHOU HI. - Dans le cas du quatrième trait nonaire, il n'y a pas répétition de dureté énergique ${ }^{2}$, le caractère qui exprime le sens de répétition est peut-être en trop dans le texte. Être parmi les hommes, c'est occuper le troisième rang. Parfois, c'est-à-dire selon le moment, et sans que rien soit fixé d'avance.

55.

Or, le grand homme accorde sa vertu à celle du ciel et de la terre; il égale par son intelligence la clarté du soleil et celle de la lune; il conforme l'ordre de son action à la régularité des quatre saisons; il déduit des esprits et des génies (des phénomènes naturels) les pronostics heureux ou

\footnotetext{
${ }^{1}$ Khong Shi Ying Ta dit: Le troisième trait avec le quatrième expriment toujours la voie rationnelle de l'homme. Au-dessous de l'homme la terre est proche ; au-dessus de lui le ciel est éloigné. Le troisième trait nonaire est près du second : c'est exactement la voie logique de l'homme. Le quatrième trait nonaire monte et se rapproche du ciel ; en bas, il s'éloigne de la terre; ce n'est pas un lieu où l'homme soit apte à se placer et c'est pour cela que la formule spécifie qu'il n'est plus entre les hommes.

${ }^{2}$ Puisque le quatrième rang est pair et, par suite, négatif.
} 
malheureux ${ }^{1}$. Il devance l'action du ciel, et le ciel ne le dément pas; il suit l'action du ciel et se conforme aux saisons telles qu'elles sont tracées par le ciel, qui ne le désavoue point. Combien, à plus forte raison, est-il impossible qu'il en soit autrement de la part des hommes, et, à plus forte raison encore, de la part des esprits et des génies ${ }^{2}$.

TSHENG TSE. - L'accord du grand homme avec le ciel et la terre, avec le soleil et la lune, les quatre saisons, les esprits et les génies, c'est son accord avec la voie morale. Le ciel et la terre constituent la voie rationnelle; les esprits et les génies sont les traces visibles de l'action et de la transformation. L'homme saint devance l'action du ciel et le ciel le confirme ; il suit le ciel et est capable de se conformer passivement à sa loi, c'est là s'accor der uniquement à la voie rationnelle. Du moment où il s'accorde avec la voie morale et logique, comment les hommes, les esprits et les génies pourraient-ils se mettre en opposition avec lui ?

TSHOU HI. - Les mots « grand homme » désignent précisément le grand homme quil y a avantage à voir et dont il s'agit dans la formule du trait. Ayant cette vertu, et digne de la situation qu'il occupe, il peut répondre à cette désignation. L'homme d'une part, et le ciel, la terre, les esprits et génies, de l'autre, ne sont essentiellement pas soumis à deux rai sons d'être différentes l'une de l'autre. C'est uniquement parce que cette raison d'être lui est cachée par l'égoïsme qui coexiste avec la personnalité, parce qu'il est entravé par sa forme et sa substance, qu'ils ne peuvent communiquer librement ensemble. Le grand homme est sans égoïsme, il considère la voie morale comme constituant sa propre substance. Comment donc serait-il encore possible d'en parler en distinguant entre l'un et les autres, entre ce qui précède et ce qui suit ? «Devancer le ciel qui ne le désavoue point », c'est-à-dire que l'action qui résulte de sa propre idée coïncide sans aucun effort avec les règles et les prescriptions de la voie morale. «Suivre le ciel », « obéir au ciel » sont des expressions qui marquent que, sachant que telle est la raison d'être des choses, il y défère dans ses actions. Houei Ho parlant de Kuo Tse Yi dit: Ceux qui cultivent la divination par les koua avancent que ce passage exprime qu'en agissant on devra rencontrer et voir un grand homme, puis revenir ; le sens divinatoire, en effet, s'accorde avec cette explication. Du moment où il s'agissait de Tse Yi, bien qu'il n'atteignît point au degré exprimé par Khong Tse, cependant, à cause de son extrême désintéressement sans aucun égoïsme, on pouvait encore, dans son temps, le considérer comme un grand homme.

\footnotetext{
${ }^{1}$ Le sens littéral serait : Il se rend favorable ou nuisible en s'accordant à l'action des espr its ou des génies.

2 Accorder sa vertu à celle du ciel et de la terre, c'est-à-dire abriter et supporter ; égaler par son intelligence la clarté du soleil et de la lune, c'est -à-dire examiner et surveiller ; conformer l'ordre de son action à la régularité des quatre saisons, c'est, par exemple, récompenser, pour représenter les saisons du printemps et de l'été, et punir en imitant l'automne et l'hiver. Déduire des esprits et des génies les pronostics heureux ou malheureux, c'est récompenser le bien par le bonheur et punir le mal par le malheur (Khong Shi Ying Ta).
} 
56.

Ce qu'exprime le mot traduit par «élevé » ( $\left.n^{\circ} 18\right)$, c'est, savoir avancer sans savoir reculer; savoir conserver et ne pas savoir abandonner; savoir posséder et ne pas savoir perdre.

TSHOU HI. - C'est à cause de cela que sill se meut il a des regrets.

57.

N'est-ce pas l'homme saint seul qui sait avancer et reculer, conserver et perdre, et cela sans jamais manquer à la droiture ; n'est-ce pas l'homme saint seul!

TSHENG TSE. — L'excès extrême est exprimé par le caractère traduit par le mot «élevé ». Celui qui est arrivé à cet excès d'élévation ne sait plus reconnaître la raison d'être des actions, soit qu'il convienne d'avancer ou de reculer, de conserver ou d'aban donner, de posséder ou de perdre. L'homme saint la connaît, et il se place en conséquence. En aucun cas il ne manque de droiture, de sorte qu'il n'en arrive pas à l'excès d'éléva tion.

TSHOU HI. - Connaissant que telles sont la raison d'être et la force naturelle des choses, et se plaçant dans chaque cas d'après la voie logique du moment, on n'arrive pas aux regrets. Cela n'est certainement pas calculer dans un but d'intérêt privé pour éviter le malheur. Le commentaire répète deux fois les mots : n'est -ce pas l'homme saint seul ; d'abord, c'est comme pour poser la question, ensuite, c'est pour poser la réponse. - Ceci constitue le sixième paragraphe. Il revient de nouveau sur les idées émises dans le second, le troisième et le quatrième paragraphes. 


\section{Khouen}

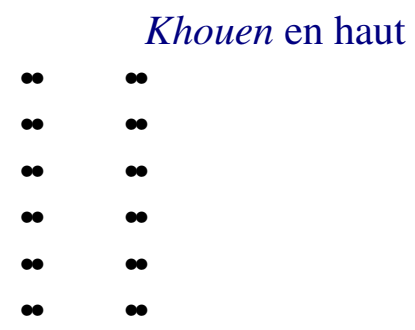

Khouen en bas

58. Khouen: Cause initiale et liberté; bien et perfection de la jument. L'homme doué a le moyen d'agir. En précédant, «aveuglement»; en suivant, «possibilité de se guider sur le bien». Dans le Sud-Ouest, "possibilité d'avoir des amis »; dans le Nord Est, « perte des amis ». Calme dans la perfection, «présage heureux».

[]

65. Premier trait hexaire : en piétinant sur le givre, la glace solide survient.

$*$

67. Deuxième trait hexaire : rectitude, régularité, grandeur. Sans exercice préparatoire, rien cependant qui ne soit le bien.

$*$

69. Troisième trait hexaire : en taisant le mérite il peut y avoir perfection; parfois suivre les affaires du roi ; sans posséder l'autorité exclusive, arriver au but

*

71. Quatrième trait hexaire : lier le sac : pas de culpabilité, pas de louanges.

$*$

73. Cinquième trait hexaire : jupe jaune ; excellence du présage heureux.

$*$

75. Trait supérieur hexaire : les dragons combattent dans le désert ; leur sang est noir et jaune.

*

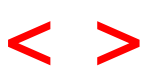




\section{Khouen.}

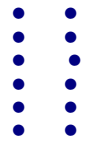

58.

Khouen : Cause initiale et liberté ${ }^{1}$; bien et perfection de la jument. L'homme doué a le moyen d'agir. En précédant, « aveuglement»; en suivant, "possibilité de se guider sur le bien ${ }^{2}$ ». Dans le Sud-Ouest, "possibilité d'avoir des amis »; dans le Nord Est, «perte des amis ». Calme dans la perfection, «présage heureux».

TSHENG TSE. - Khouen est l'antithèse de khien; les quatre vertus sont les mêmes, mais l'essence ${ }^{3}$ de la perfection est différente. Dans le koua khien, c'est la dureté énergique et la fermeté qui sont considérées comme constituant la perfection ; dans le cas du koua khouen, c'est par la douceur malléable et la passivité qu'il y a perfection. La jument est douce et obéissante, de plus elle marche avec une énergie équivalente ${ }^{4}$; c'est pour cela qu'elle est prise comme symbole, et que la formule dit : perfection de la jument. Dans son action, l'homme doué agit avec douceur et obéissance, et il se montre bienfaisant avec perfection, il se conforme aux vertus du koua khouen. La négativité est ce qui suit la positivité ; elle attend la mesure et s'y accorde ${ }^{5}$.

Si la négativité précède la positivité, c'est ce qui constitue l'aveuglement et l'erreur; si elle reste en arrière, alors elle se maintient dans la règle. Se guider sur le bien ; le bien pour tous les êtres dépend de ce qui est désigné par le mot khouen; sa naissance et son développement sont l'œuvre de la terre. Il en est encore de même de la voie morale du sujet; le prince ordonne, le sujet agit 6 ; user ses forces dans l'exécution des affaires, telle est la fonction du sujet. Le Sud-Ouest est une région négative; le Nord-Est est une région positive. La négativité doit suivre la positivité en se séparant de ce qui constitue son propre genre ${ }^{7}$ et en sacrifiant ses amis, elle peut alors accomplir l'auvre méritoire de la transformation et de la génération et jouir du présage heureux du calme dans la perfection. Se maintenant dans la règle, elle jouit du

\footnotetext{
1 Suivant la ponctuation adoptée par les Chinois, il vaudrait peut-être mieux lire : liberté initiale ; avantage de la perfection de la jument.

${ }^{2}$ Le sens est à peu près celui du mot utilité.

${ }^{3}$ C'e st le terme qui est généralement traduit par le mot substance.

${ }^{4}$ Équivalente à l'énergie ou activité du koua khien.

5 Terme technique de musique. Écouter le ton donné par celui qui dirige l'orchestre et s'y accorder.

6 Le terme du texte désigne le sujet, mais il comporte l'idée d'exercice d'une fonction publique ou d'un service confié par le prince.

7 Abandonner son propre genre; c'est-à-dire sacrifier les affections privées, abandonner famille et amis pour se dévouer tout entier au prince.
} 
calme et s'en contente ; se contentant et se maintenant dans la règle elle atteint la perfection : c'est par là qu'il y a présage heureux.

TSHOU HI. — Le trait brisé — — est appelé ngeou, ou dualité ${ }^{1}$; c'est le nombre de la négativité. Khouen, passivité ; c'est le naturel de la négativité. Dans les annotations, c'est le nom du koua simple de trois traits; dans le texte, ou king, c'est le nom du koua parfait de six traits.

Parmi les formes définies de la négativité, aucune n'est aussi grande que la terre. Dans ce koua simple, les traits représentent tous trois la dualité ; c'est pour cela qu'on le nomme khouen ${ }^{2}$ et il symbolise la terre. En le répétant sur lui-même, on obtient encore l'image de ce qui est exprimé par le mot khouen, de sorte qu'il est réellement la représen tation de la pureté de la substance de la négativité et le comble de la passivité, aussi le nom et la représentation symbolique ne changent ni l'un ni l'autre.

La jument ${ }^{3}$, c'est ce qui est passif, ou obéissant, et ce qui marche avec une énergie équivalente ${ }^{4}$. La positivité précède, la négativité suit; la positivité se guide sur le devoir, la négativité se guide sur le bien (utile). Le Sud-Ouest est une région négative; le Nord-Est une région positive. Le calme, c'est l'état de la positivité ; la perfection, c'est la conserva tion et le maintien d'une activité équivalente.

Lorsqu'on rencontre ce koua ${ }^{5}$, le sens divinatoire exprime une grande liberté ${ }^{6}$ et l'avantage (bien) est considéré comme consistant dans la droiture résultant de l'emploi de l'activité obéissante. S'il y a quelq ue chose à entreprendre, alors, en précédant, il y aura aveuglement, tandis qu'en suivant, il y aura réussite et conformité au bien. En allant dans le Sud-Ouest, on pourra posséder des amis, et en allant dans le Nord-Est, on les perdra. Dans la majorité des cas, lorsqu'on sait se contenter de la droiture, c'est un présage de bonheur.

59.

\footnotetext{
${ }^{1}$ Littéralement : labourer ensemble, deux hommes ensemble, labourant. Selon moi ce sens attribué à un caractère ne peut être qu'exotérique et je pense qu’il s'agit des deux astres décrivant ensemble l'horizon, au coucher du soleil, à l'équinoxe d'automne.

${ }^{2}$ Le mot khouen est considéré comme exprimant la passivité et la négativité ; tous les traits de ce koua étant négatifs, on lui donne ce nom.

3 Tsien Shi Pao dit: Parmi ce qui parcourt le ciel, rien n'est comparable au dragon ; parmi ce qui parcourt la terre, rien n'e st égal au cheval. C'est pour cela que le koua khouen a la jument pour symbole.

${ }^{4}$ Les commentateurs adoptent plutôt le sens d'activité, force équivalente, mais il s'agit bien d'une relation de correspondance établie ou supposée entre les deux koua.

${ }^{5}$ Dans la divination ; cette expression se rencontrera très fréquemment.

${ }^{6}$ Tshou Hi traduit le mot yuan par grandeur.
} 
Le commentaire de la formule déterminative dit ${ }^{1}$ : Qu' elle est extrême la faculté productrice ${ }^{2}$ de la passivité (khouen). Toutes choses lui doivent leur naissance ${ }^{3}$; c'est la sujétion passive au ciel.

TSHOU HI. - Ceci éclaire le sens attribué au koua par l'exemple de la loi naturelle (tao) de la terre, en commençant par parler de la vertu exprimée par le mot yuan ${ }^{4}$. Extrême, marque ce qui est poussé à la dernière limite. Entre cette expression et le mot grande, employé dans la formule $\mathrm{n}^{\circ} 2$, il y a une légère nuance. Le commencement (formule $\mathrm{n}^{\circ} 2$ ), c'est le commencement de l'éther ${ }^{5}$; la naissance, c'est le commencement de la forme. Sujétion passive à l'influence émanant du ciel ; c'est la voie rationnelle ( tao) de la terre.

DÉFINITIONS DIVERSES. — Lu Shi Ta Lin dit : La substance (essence) du koua khien, c'est la grandeur ; l'objectif du koua khouen, c'est la loi de l'activité (khien) ; il atteint jusqu'à la grandeur de l'activité et s'y arrête. C'est pour cela qu'en parlant du qualificatif yuan au sujet du koua khien, la formule dit : «Qu'elle est grande », et qu'en en parlant au sujet du koua khouen, elle dit : «Qu'elle est extrême ! " 6 -Tshou Hi dans les «Sujets de dissertation» dit. : Doués de leur commencement par l'activité, ils sont naturellement doués de la naissance par la passivité (khouen). Il n'y a pas de contestation sur le moment de l'obtention tous les êtres sont doués de leur commencement par l'acti vité et par cela même, leur être existe ; ils sont doués de la naissance par la passivité et, dès ce moment, ils ont leur forme. L'éther surgissant, ils naissent ; c'est précisément là la faculté productrice (yuan) du koua khouen (passivité). Tsae Shi Tsing dit : Si la formule disait seulement : «Qu'elle est extrême la faculté productrice de la passivité ! Toutes choses lui doivent leur naissance », on douterait alors si cette faculté est équivalente à celle qui est exprimée par la formule $n^{\circ} 2$.

«Qu'elle est grande la cause initiale de l'activité ! Toutes choses lui doivent leur commencement »; mais elle ajoute : C'est la sujétion passive au ciel. Non seulement on peut donc voir par là que la voie rationnelle de la passivité ne comporte pas par elle-même le sens d'achèvement et de fin, mais encore on peut voir que l'activité et la passivité unissent leurs vertus pour accomplir ensemble l'œure de la vie dans les êtres. S’il n'en était pas ainsi, le koua khien ayant les quatre vertus attributives de l'activité, le koua khouen ayant les quatre vertus attributives de la passivité, leurs noms exprimeraient en réalité des choses qui se confondraient.

\footnotetext{
${ }^{1}$ Voir la formule $\mathrm{n}^{\circ} 2$ et les commentaires.

${ }^{2}$ Yuan; c'est le même terme traduit par faculté créatrice dans le cas de la formule ${ }^{\circ} 2$. Cette différence de valeur dans la traduction est justifiée par ce qui suit.

${ }^{3}$ La différence entre « commencement » et «naissance » est expliquée plus loin.

${ }^{4}$ Ce passage montre bien qu'un koua ne représente pas une chose en particulier ; c'est l'expression abstra ite d'une loi.

${ }^{5}$ La création.

${ }^{6}$ Le même terme signifie aussi parvenir à... atteindre jusqu'à... On peut donc lire : Qu'elle parvient loin ! Qu'elle est près d'atteindre sa limite !
} 
60.

L'ampleur de la passivité contient les êtres; sa vertu réunit sans limites; sa faculté de contenance est immense; son éclat est grand. Les genres des êtres jouissent tous de leur liberté d'expansion.

TSHOU HI. - Il s'agit de la liberté (heng). Vertu réunissant sans limites, qui exprime qu'elle est équipollente à celle de l'activité.

61.

La jument est de la classe des choses terrestres; elle parcourt la terre sans limites; elle est douce et obéissante et avantageuse par sa perfection. L'homme doué agit ainsi.

TSHOU HI. - Il s'agit du bien et de la perfection. Le cheval est le symbole de l'activité et cependant il est considéré comme de la classe des choses terrestres. L'animal femelle est un être négatif et, de plus, le cheval est un être qui parcourt la terre ; parcourant la terre sans limites, elle est donc passive et active. La douceur malléable, l'obéissance passi ve, le bien avec la perfection, telles sont les vertus de la passivité (khouen). L'homme doué agit ainsi ; l'action de l'homme doit être comme la vertu du koua khouen. Son action étant telle, le sens divinatoire sera comme il est dit dans la suite du texte.

TSHENG TSE. — La voie à laquelle les êtres doivent leur naissance peut certainement être appelée «grande»; mais du moment où le terme « grandeur» est employé comme qualification de l'activité, la passivité est qualifiée par le terme extrême. Le sens de ce dernier terme comporte une légère gradation; il n'équivaut pas à la perfection achevée de l'idée de grandeur. Telle est la circonspection sévère de l'homme saint dans la classification de l'éminence et de l'infériorité.

Tous les êtres doivent leur commencement à l'activité, ils doivent leur naissance à la passivité, c'est la voie logique de la paternité et de la maternité. La sujétion passive à l'influence émanant du ciel, afin d'accomplir son ouvre, c'est l'ampleur de la vertu de la passivité (khouen), elle s'étend à tous les êtres, les maintient et les supporte et concorde à l'absence de limites de l'activité (khien). La voie de la passivité (khouen) est figurée et décrite par les 
quatre expressions, faculté de contenance, immensité, éclat et grandeur, et c'est encore là l'équivalent des qualifications de dureté énergique et activité, justice et droiture, pureté de substance et de qualités, employées pour l'activité (khien).

La faculté de contenance, c'est l'aptitude à renfermer e n embrassant et à supporter; l’immensité est la magnanimité et la générosité ; l'éclat est la clarté resplendissante; la grandeur, c'est l'ampleur puissante. C'est par cela même qu'elle réunit ces quatre conditions qu'elle peut achever, en s'y soumettant, l'ouvre du ciel.

Les divers genres des êtres possèdent tous la liberté d'expansion suivant les lois de leur nature particulière. La jument est prise comme symbole, parce qu'elle est douce, obéis sante et active. Les expressions «classe des choses terrestres », «parcourir la terre sans limites » expriment l'activité. Le koua khien représente l'activité, le koua khouen exprimé la passivité, le koua khouen exprime-t-il donc aussi l'activité ? Sans activité, comment serait-il l'équivalent du koua khien ${ }^{1}$ ? L'activité (khien) ne peut agir, tandis que la passivité (khouen) resterait immobile ${ }^{2}$. Son mouvement est la dureté énergique et n'altère pas ce qui constitue sa douceur malléable. La douceur malléable et la passivité, le bien et la perfection, sont les vertus du koua khouen, ou passivité ; tel doit être le mode d'action de l'homme doué. La voie de l'homme doué concorde avec la vertu de la passivité (khouen).

62.

En précédant, aveuglement, perte de la voie rationnelle; en suivant passivement, possibilité de se maintenir dans la règle immuable ${ }^{3}$. Dans le Sud-Ouest possibilité de posséder des amis ; c'est marcher avec ceux de son propre genre. Dans le Nord Est perte des amis; c'est, à la fin, avoir le bonheur.

TSHOU HI. - La positivité est la grandeur, la négativité est la petitesse. La positivité comporte en elle-même la négativité, mais celle-ci ne peut point comporter la positivité. Aussi, la vertu de la passivité est toujours inférieure de moitié à celle de la positivité (khien). Bien que, perdant les amis dans le

\footnotetext{
${ }^{1}$ Le terme chinois du texte exprime lidée de former paire avec quelque cho se, les deux objets se complétant mutuellement, par exemple, le mari et la femme, le mâle et la femelle, etc.

${ }^{2}$ La force d'activité ne peut exister sans la matière ; si celle-ci n'existait point la force serait sans application et sans effets ; telle est la traduction très exacte d'une explication de Tsheng Tse.

${ }^{3}$ Sans le sens établi par la tradition, on lirait : d'abord, aveuglement, perte de la vie ; après, atteindre passivement la régularité ordinaire.
} 
Nord-Est, cependant, en revenant dans le Sud-Ouest, finalement, il y aura le bonheur.

63.

Le présage heureux de la satisfaction dans la perfection correspond à la terre par son absence de limites.

TSHOU HI. - Calme ou satisfaction, et, en outre, perfection ; c'est la vertu de la terre.

TSHENG TSE, -L'effet de l'activité ( khien), c'est l'existence de la positivité ; l'effet de la passivité (khouen), c'est l'existence de la négativité. Avant la forme, on dit voie rationnelle du ciel et de la terre; après la forme, on dit: auvre de la négativité et de la positivité. Depuis les mots : «En précédant, aveuglément .... en suivant, possibilité... » et en continuant, il est question de la voie rationnelle de la négativité. En donnant la première la mesure, elle s'aveugle et perd la voie logique de la négativité ; au contraire, en la suivant pour s'y accorder, elle s'y conforme passivement et elle réussit à atteindre la raison naturelle constante. Le Sud-Ouest est une région négative, en suivant ce qui constitue son propre genre, elle possède des amis. Le Nord-Est est une région positive, en s'écartant de ce qui constitue son propre genre, elle perd ses amis. Si elle s'écarte de son propre genre pour suivre la positivité, elle peut alors accomplir l'œuvre de l'enfantement des êtres et, à la fin, il y a un présage heureux et bonheur.

Agir avec ce qui constitue son propre genre, telle est sa manière d'être originelle ; suivre la positivité, c'est là son usage, ou emploi. La substance de la négativité est la douceur malléable dans le mouvement; aussi, si elle suit la positivité, elle peut être calme dans la perfection et comporter un présage heureux : c'est là correspondre à l'absence de limites de la voie de la terre. La négativité ne se contentant pas du calme dans la perfection, comme pourrait-il y avoir correspondance avec la voie rationnelle de la terre ? Dans la formule déterminative, l'expression «sans limites » revient trois fois. Dans chaque cas, le sens n'est pas le même. La vertu se ré unissant sans limites, c'est l'infini du ciel ; correspondre à la terre par son absence de limites, c'est l'absence de limites de la terre ; parcourir la terre sans limites, c'est l'action de l'activité du cheval. 
Le commentaire de la formule symbolique dit: La force naturelle à la condition de la terre ${ }^{1}$, c'est la passivité. L'homme doué embrasse tous les êtres par l'ampleur de sa vertu.

TSHENG TSE. - La grandeur de la voie de la passivité est encore comme celle de l'acti vité (khien), sans être un homme saint, qui donc pourrait la réaliser ${ }^{2}$. La terre est vaste et la force naturelle inhérente à sa condition fait qu'elle remplit un rôle passif et incliné ${ }^{3}$; aussi, dans cette formule, c'est l'image symbolique de la passivité jointe à l'ampleur qu i est adoptée, et le texte dit : La force naturelle à la condition de la terre est la passivité (khouen). L'homme doué regarde l'image symbolique de l'ampleur de la passivité (khouen) et il tolère et supporte les différents êtres, en les embrassant dans les effets de sa vertu étendue et profonde.

TSHOU HI. - La terre est l'image symbolique de la passivité exprimée par le koua khouen; de même encore, elle est unique, aussi n'est-il point question de la répétition du koua simple et il ne s'agit que de la pas sivité obéissante qui constitue la force inhérente à sa condition. De cette façon, on voit l'éternité sans fin de la relation entre ce qui est haut et ce qui est bas ${ }^{4}$. Extrême passivité obéissante, ampleur superlative, telle que rien n'exis te qui n'en so it embrassé.

\footnotetext{
${ }^{1}$ La force naturelle d'une chose, qui fait que sa manière d'être et ses conséquences sont telles; force invincible qui résulte d'une loi.

${ }^{2}$ La mettre en action, en pratique. Littéralement, lui donner un corps.

${ }^{3}$ La terre est horizontale et le ciel s'étend en hauteur ; telle est l'explication admise ; sans tenir compte de la tradition, ce passage dit littéralement : la terre décline passivement.

${ }^{4}$ Le ciel et la terre, le prince et le sujet, etc.
} 


\section{Khouen.}

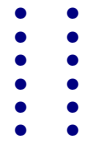

65.

Premier trait hexaire ${ }^{1}$ : en piétinant sur le givre, la glace solide survient.

TSHENG TSE. — Les traits négatifs sont qualifiés par le terme hexaire ; il exprime le parfait épanouissement de la négativité. Dans le nombre huit, la positivité est déjà née et la pureté de substance n'est plus parfaite. La négativité commence à naître par en bas, elle est encore extrêmement minime. Au moment où la négativité commence à naître, l'homme saint considérant qu'elle e st sur le point de se développer, en fait l'objet d'un avertissement. Lorsque la négativité commence à se coaguler, elle constitue le givre ; si on piétine sur le givre, on doit savoir que la négativité augmentera progressivement et arrivera à l'état de glace solide. De même, bien que les commencements des effets de l'action de l'homme inférieur soient infimes, on ne doit pas les laisser grandir; grandissant, ils en arriveraient à des effets extrêmes.

TSHOU HI. - Hexaire est le nom qu'on donne aux traits négatifs. Dans les nombres négatifs, six est la vieillesse et huit est le jeune âge, aussi, en parlant de traits négatifs, on les appelle hexaires, c'est-à-dire qui sont du genre du nombre six. Le givre est la concrétion de l'éther négatif ; quand cette concrétion est arrivée à son parfait développement, l'eau se durcit et devient glace.

Dans ce trait, la négativité commence à naître en bas du koua ; son germe est extrêmement ténu, et la force naturelle à sa condition fait qu'elle doit nécessairement arriver à son parfait développement. Aussi, l’image symbolique est que si on piétine sur le givre, on doit savoir que la glace solide est sur le point de survenir. Or, la négativité et la positivité sont l'essence fondamentale de la création et de la transformation ; l'une ne peut exister sans l'autre, et leur décroissance comme leur développement, sont soumis à des règles constantes; c'est encore quelque chose où l'homme ne peut ni diminuer ni ajouter. Mais la positivité régit la vie; la négativité régit la mort, de sorte que leur différence générique consiste dans la distinction entre ce qui est le bien et ce qui est le mal. Aussi, l'homme saint en faisant le livre des changements, après avoir fait ressortir clairement par ce qui est relatif à l'activité et à la passivité, à l'humanité et au devoir, qu'elles ne peuvent jamais exister l'une sans l'autre et qu'elles n'ont point de tendances particulières dans leur influence régulatrice, s'occupe enfin des circonstances de leur annihilation et de leur développement et de la distinction entre le bien

\footnotetext{
${ }^{1}$ Ici encore, je suis obligé de forger un mot pour rendre la valeur du terme chinois.
} 
et le mal, de sorte qu'il ne manque jamais d'arriver à l'idée de l’influence réciproque de la positivité et de la négativité, dont l'une maintient, tandis que l'autre renverse.

C'est effectivement ainsi qu'elles procè dent dans la transformation et la production et qu'elles mettent en contact actif le ciel et la terre. Le thème est profond. Il n'est pas ques tion du sens divinatoire; l'idée de nécessité de la circonspection dans les choses les plus minimes, se voit suffisamment dans la formule symbolique.

66.

En piétinant sur le givre, la glace durcit, la négativité commence à se coaguler. En suivant cette voie jusqu'à son extrême limite, il arrive un moment où la glace est solidifiée.

TSHENG TSE. - La négativité commence à se coaguler ${ }^{1}$ et produit le givre; son développement s'achève peu à peu, de sorte qu'elle parvient à l'état de glace solide. Bien que l'homme inférieur ne possède d'abord qu'une influence minime, si cette influence grandit, elle arrivera petit à petit jusquà son parfait développement, de sorte qu'il est néces saire d'avertir dès le début. Le mot traduit par «en suivant» signifie s'accoutumer par la répétition ; c'est en s'accoutumant par la répétition qu'on arrive à la perfection. On s'accou tume par la répétition en suivant une impulsion ${ }^{2}$.

TSHOU HI. - Il est à remarquer que c'est Wei Tshi qui a avancé que le premier trait hexaire a pour formule les mots «en piétinant sur le givre »; actuellement on doit suivre cette lecture. Le terme traduit par « en suivant» exprime le sens d'exercice par la répéti tion avec soumission passive.

\footnotetext{
${ }^{1}$ Condensation d'une vap eur en liquide, ou coagulation d'un liquide en solide ; l'éther passant d'un état insaisissable à celui de matière.

2 Khong Shi Ying Ta dit: Le terme qui est traduit par «en suivant» signifie s'exercer passivement par répétition ; par exemple, comme les oiseaux et les quadrupèdes se dressent par l'habitude. Cela exprime l'idée de suivre passivement la voie naturelle de la malléabilité négative. Répétition incessante qui fait qu'ensuite la glace solide survient. La glace dure est mentionnée comme avertissement, à cause de l'expression piétiner sur le givre, qui est employée au sujet du mal ; cet avertissement sert à mettre en garde contre l'habitude, et à prévenir les suites d'une cause en apparence minime ; il montre que, dès le début, il faut savoir prévoir la fin.
} 


\section{Khouen.}

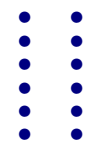

67.

Deuxième trait hexaire : rectitude, régularité, grandeur. Sans exercice préparatoire, rien cependant qui ne soit le bien.

TSHENG TSE. - Le second rang est une situation négative et inférieure, aussi ce trait est le trait principal ${ }^{1}$ du koua khouen, et il exprime d'une façon générale la voie rationnelle de la passivité. La justice, la droiture et l'infériorité constituent la voie naturelle de la terre. Par ces trois qualités de rectitude, régularité et grandeur, les effets de sa vertu se trouvent dépeints et figurés ; ces simples mots épuisent ce qui peut être dit de la voie naturelle de la terre. Procédant par la rectitude, la régularité et la grandeur, il en résulte que, même sans étude ni exercice préparatoire, rien cependant n'est autrement que bien. Sans exercice préparatoire, c'est-à-dire spontanément. Dans la voie de la passivité (khouen), c'est ce qui est sans l'action de personne ; lorsqu'il s'agit de l'homme saint, c'est le calme dans la stricte confo rmité à la morale. Rectitude, régularité, grandeur, c'est ce que Mang Tse appelle « extrême grandeur, extrême énergie par la rectitude ». Ici, il s'agit de la substance du koua khouen, c'est pour cela que le mot « énergie » est remplacé par le mot « régularité », encore pour la même raison qui fait qu'au sujet de la perfection la formule ajoute les mots : de la jument. Lorsqu'on parle de l'éther, c'est la grandeur qui est énoncée la première ; la grandeur, c'est l'essence de l'éther ; au sujet du koua khouen, ou passivité, c'est la rectitude et la régularité qui passent avant tout ; c'est par la rectitude et la régularité qu'il y a grandeur. Les mots « rectitude, régularité » et « grandeur» suffisent à épuiser la définition de la voie naturelle de la terre. C'est à l'homme de la connaître. Les deux koua khien et khouen expriment la pureté de substance. Quant à la correspondance mutuelle et sympathique entre les situations, le second trait étant le maître du koua khouen, on ne relève pas sa correspondance sympathique avec le cinquième, et ce dernier n'est pas considéré comme exprimant la voie du prince ; dans le koua khien, le second et le cinquième traits se correspondent mutuellement.

TSHOU HI. - Malléabilité, passivité, droiture et fermeté : telle est la rectitude du koua khouen. Le don de types déterminés dans les formes est l'ouvre de sa régularité ; la réunion illimitée des vertus constitue sa grandeur. Le second trait hexaire possède la douceur malléable, l'obéissance et la justice avec la droiture ; de plus, il implique la pureté immélangée de la voie morale de la passivité. Au-dedans, sa vertu est la rectitude; au-dehors, c'est la

\footnotetext{
${ }^{1}$ Littéralement, « le maître du koua ».
} 
régularité et, enfin, la perfection absolue de la grandeur. Il exprime l'inutilité de l'exercice préparatoire pour attei ndre à ce résultat que rien ne soit autrement que le bien. Le sens divinatoire est que si celui qui consulte le sort possède une telle vertu, tel aussi sera l'augure.

68.

Le mouvement du second trait hexaire, c'est la rectitude par la régularité : «Sans aucun exercice préparatoire, rien n'est cependant autrement que bien »; la voie de la terre est éclatante.

TSHENG TSE. - Sujétion à l'impulsion du ciel et, de là, mouvement ; rectitude par la régularité. La rectitude et la régularité comportent la grandeur. Le sens des mots « rectitude» et « régularité » est que la grandeur est infinie. La voie de la terre est éclatante et claire ; son œuvre s'achève et s'accomplit passivement; comment serait-il nécessaire qu'il y eût d'abord exercice préalable et que le bien fût le résultat de cet exercice ${ }^{1}$ ?

${ }^{1}$... Le mouvement du second trait hexaire constitue la vertu de ce trait ; ce n'est que par sa mise en acte qu'elle devient visible. Donner naissance aux êtres suivant leur nature particulière, c'est là sa rectitude; achever la forme des êtres sans variation, c'est là sa régularité. 


\section{Khouen.}

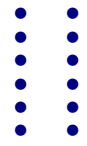

69.

Troisième trait hexaire : en taisant le mérite il peut y avoir perfection; parfois suivre les affaires du roi ; sans posséder l'autorité exclusive, arriver au but ${ }^{1}$.

TSHENG TSE. - Le troisième trait occupe le rang supérieur dans le koua simple inférieur; c'est celui qui possède la situation. La voie rationnelle du sujet comporte nécessairement le devoir de taire et de cacher son mérite et ses talents ; s’il fait quelque chose de bien, il en rapporte le mé rite au prince, et, de cette façon, il peut se maintenir avec continuité et posséder la droiture. De cette façon, le supérieur ne sera pas entraîné par la suspicion et la haine ; l'inférieur s'accordera à la voie rationnelle de la douceur et de la sou mission. Possibilité de perfection, signifie possibilité de se maintenir avec une fermeté parfaite, et, de plus, possibilité de continuité durable et permanente sans regrets ni culpabilité. Si parfois il vient à suivre, en s'en occupant, les affaires du supérieur, il n'ose point s'attribuer l'autorité exclusive et le mérite de l'auvre accomplie ; il s'occupe seule ment de l'exécution, afin de garantir la fin. Observer les devoirs de son rang pour conduire les affaires à leur terme, telle est la voie logique du sujet.

TSHOU HI. - Trait hexaire, négativité ; troisième rang, positivité. En renfermant en soi ses belles qualités, possibilité d'atteindre à la perfection par l'observation stricte des lois de sa propre condition. Mais ce trait occupe le rang supérieur dans le koua simple inférieur ; il ne renferme et ne cache pas indéfiniment ses talents, aussi, parfois il sort de la retraite, se met en avant, et s'occupe des affaires du supérieur, de sorte que bien que n'ayant pas d'autorité en commençant, à la fin il réussit et arrive au but. Le trait a cette image symbolique et la formule avertit celui qui consulte le sort que, si telle est sa vertu, ce sens divinatoire lui sera applicable.

70.

\footnotetext{
${ }^{1}$ Littéralement, il faudrait lire : pas achevé (complet) il y a fin. La valeur «d'attribuer une autorité exclusive » donnée aux deux caractères mou tsheng leur est acquise, par tradition, dans l'explication de ce passage. Le sens naturel des mots employés serait que la chose dont il s'agit finit sans être complètement achevée.
} 
Taire son mérite, possibilité de perfection; avec le temps elle éclate. Parfois s'occuper des affaires du roi : Savoir brillant et grand.

TSHENG TSE. - Fou Tse, craignant que les hommes ne s'appliquent à l'observation de la lettre sans en pénétrer le sens, continue l'explication de cette formule en exposant la voie rationnelle du sujet placé dans une position inférieure. Il ne convient pas qu'il ait le mérite du bien qu'il accomplit ; il doit absolument cacher et maintenir dans l'ombre ses plus belles qualités ; après cela, il possède la droiture et peut suivre la voie ordinaire. Mais, ce que le devoir lui prescrit de faire surgit et se présente avec le temps; ne pas s'attribuer le mérite, ne pas manquer à ce qui est convenable, c'est agir suivant le moment. Sans renfermer en soi ses qualités, et sans cacher ses talents, il n'arriverait pas au but ; en les cachant, mais sans agir, il n'accomplirait pas jusqu'au bout ce que la fidélité au prince exige du sujet. Parfois il s'occupe des affaires du roi ; la formule symbolique ne relève que le premier membre de phrase, en expliquant le sens de cette proposition, le texte qui suit se trouve également éclairci; dans les autres koua il en est toujours ainsi. S'occuper parfois des affaires du roi, pouvoir se passer de l'autorité exclusive et arriver au but, tout cela montre clairement que réellement le savoir est considérable et brillant. C'est unique ment à cause de l'éclat et de la grandeur de son savoir qu'il peut se renfermer dans l'obs curité ${ }^{1}$. L'homme superficiel et peu clairvoyant, lorsqu'il a fait le bien, ne craint qu'un e seule chose, et cette chose est que les hommes n'en aient point connaissance ; comment serait-il capable de renfermer en lui-même les plus belles qualités ?

\footnotetext{
${ }^{1}$ Ce n'est ni à cause de l'orgueil, ni à cause de la vanité déçue. ( Lu shi tsou khien.)
} 


\section{Khouen.}

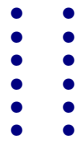

71.

Quatrième trait hexaire : lier le sac : pas de culpabilité, pas de louanges.

TSHENG TSE. - Le quatrième trait occupe une position voisine de celle du cinquième, mais il ne comporte aucun sens d'accord entre eux; c'est le moment où le supérieur et l'inférieur sont séparés et ne s'entendent point. Il se place d'après la droiture, c'est un terrain douteux et périlleux. S'il cache et dissimule son savoir, comme en liant et fermant l'ouverture d'un sac, sans le laisser manifester au-dehors, alors il pourra être sans aucune culpabilité ; autrement il lui arrivera malheur. Du moment où il cache et dissimule son savoir, il ne reçoit naturellement aucune louange.

TSHOU HI. — «Lier le sac » veut dire lier la bouche du sac sans en rien sortir. Le mot «louange » exprime que le degré réel est dépassé. Avec une telle circonspection mystérieuse, il ne commettra aucune faute, mais, de même, il ne recevra aucune louange. Le quatrième trait hexaire comporte répétition de négativité sans justice, c'est à cause de cela que tels sont la formule symbolique et le sens divinatoire. En effet, tantôt les choses doivent être faites avec circonspection et mystère, tantôt il convient qu'il se cache en se retirant à l'écart.

72.

Lier le sac, pas de culpabilité ; avec de la circonspection, pas de mal.

TSHENG TSE. - Capable d'une telle circonspection, il n'éprouvera aucun mal. 


\section{Khouen.}

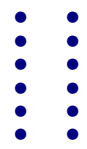

73.

Cinquième trait hexaire : jupe jaune ; excellence du présage heureux.

TSHENG TSE. - Bien que le koua khouen expose la voie rationnelle du sujet, le cinquième rang représente réellement la situation du prince, aussi ce trait est l'objet d'un avertis sement qui dit: "Jupe jaune, excellence du présage heureux. » Le jaune est la couleur de la justice ${ }^{1}$; la jupe est le vêtement inférieur ; en conservant la justice et demeurant dans l'infériorité, ce sera un présage absolument heureux. Cela veut dire se maintenir dans sa propre condition. Le mot yuan ${ }^{2}$ exprime la grandeur et le bien. La formule symbolique du trait exprime seulement qu'en conservant la justice et en demeurant dans l'infériori té, ce sera un présage absolument heureux ; elle ne développe pas complètement le sens. Du moment où la jupe jaune indique la grandeur et l'excellence du présage heureux, il en résulte évidemment que l'occupation du rang prééminent serait le présage d'un grand malheur pour l'univers. Les hommes des siècles postérieurs ${ }^{3}$ n'ont pas pénétré le sens de cette formule, de sorte qu'il s'est obscurci et il est indispensable de le préciser. Le cinquième rang représente la situation prééminente ; dans les autres koua, lorsqu'un trait hexaire occupe le cinquième rang, tantôt il représente la douceur et la passivité, tantôt il exprime l'élégance de la forme et l'intelligence, tantôt enfin il comporte le sens de faiblesse et d'obscurité. Dans le koua khouen, il exprime l'occupation d'une situation émi nente. La négativité est la voie logique du sujet, ou de la femme; le sujet occupant la situation prééminente, c'est, par exemple, le cas de Wei Mang. On peut encore dire : La femme occupant la situation prééminente, c'est, par exemple, le cas de $\mathrm{Nu}$ Kuo et de Wou Shi. Ce ne sont point là des circonstances ordinaires dans l'évolution et on ne peut en parler, de sorte que la formule comporte l'avertissement de la jupe jaune, mais n'en déduit pas toutes les conséquences. On pourrait conserver quelques doutes; puisque, au sujet des affaires de Ko Thang Wou toutes les conséquences sont encore expliquées, pourquoi dans le cas actuel, seul, n'en serait-il point parlé ? Or, l'élévation ou l'abaisse ment sont des circonstances ordinaires qui résultent de la raison d'être des choses, mais occuper la situation prééminente avec les qualités de la négativité, ce n'est point là une circonstance ordinaire de l'évolution.

\footnotetext{
${ }^{1}$ Milieu, justice.

${ }^{2}$ C'est toujours le terme yuan, cause initiale de la formule $\mathrm{n}^{\circ} 1$.

${ }^{3}$ Les siècles postérieurs au temps de Khong Tse.
} 
TSHOU HI. - Le jaune est la couleur de la justice, la jupe est l'ajustement inférieur. Le cinquième trait hexaire, avec les qualités de la négativité, occupe un rang prééminent, c'est la vertu de la justice et de l'obéissance complète dans l'individu et manifeste hors de lui, aussi telle est l'image symbolique, tandis que le sens divinatoire est le présage heureux de la grandeur et de la bonté. Chez celui qui consulte le sort, la vertu devra être équivalente et alors le sens divinatoire de la formule lui sera également applicable. D'après le commentaire traditionnel du Tshouen tsieou, Nan Khouei, sur le point de se révolter, consulta le sort et obtint ce trait quil considéra comme un signe heureux de grandeur. Tse Fou Houei Po dit: Lorsqu'il s'agit de quelque chose qui est relatif à la fidélité et à la bonne foi, l'application de cette formule est possible, mais dans tout autre cas, le résultat final sera la destruction. La force et l'énergie à l'extérieur et la douceur au-dedans, c'est là la fidélité ; la concorde guidant la pureté, c'est là la bonne foi, aussi la formule dit: «Jupe jaune, excellence du présage heureux. » Le jaune est la couleur de la justice, la jupe est l'ajustement inférieur, le terme yuan exprime le développement du bien. Sans fidélité au-dedans, cette couleur ne convient pas ; si l'inférieur n'est pas guidé par l'amour du bien public, cet ajustement ne lui convient pas ; si l'affaire en qu estion n'est pas conforme au bien, elle ne peut arriver à son développement extrême. D'ailleurs, en fait, le livre des changements ne doit pas servir à consulter le sort au sujet de choses ou d'affaires périlleuses et malsaines. Si une de ces trois conditions fait défaut, bien que la réponse obtenue par la divination semble concorder, en réalité, il n'en est rien. Par la suite, Houei fut bien effectivement détruit, et cet exemple peut servir à montrer la loi qui régit la divination.

74.

Jupe jaune, excellence du présage heureux : la représentation figurative est à l'intérieur ${ }^{1}$.

TSHENG TSE. - Les signes indicatifs de la couleur jaune et de la justice sont à l'inté rieur et ne le dépassent point ; au-dedans, accumulation des plus belles qualités, coïncidant avec l'occupation d'une position inférieure, c'est pour cela que cette formule est considérée comme exprimant l'excellence du présage heureux.

TSHOU HI. - La représentation figurative est au-dedans et elle se manifeste au-dehors.

\footnotetext{
${ }^{1}$ Wen, représentation graphique de l'image - exprime la représentation de l'image, la forme, les contours, les dessins du pelage ou de la robe des animaux; il sert encore à désigner les caractères d'écriture. Dans un autre ordre d'idées, il désigne la manifestation des vertus, visible dans les actes et dans la conduite d'un homme.
} 


\section{Khouen.}

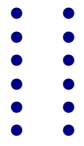

75.

Trait supérieur hexaire : les dragons combattent dans le désert ; leur sang est noir et jaune.

TSHENG TSE. - La négativité est ce qui suit la positivité, mais, cependant, quand elle est arrivée à l'extrême limite de son développement, elle lui résiste et entre en lutte avec elle. Le trait hexaire étant déjà arrivé à l'extrême limite de l'élévation et avançant encore sans cesse, il doit nécessairement y avoir combat et lutte, c'est pourquoi la formule dit : Combattent dans le désert. Le terme traduit par désert ${ }^{1}$ exprime que le mouvement en avant continue jusqu'à l'extérieur du koua. Du moment où elles s'opposent l'une à l'autre en ennemis, toutes deux sont nécessairement blessées, c'est pourquoi le sang est no ir et jaune.

TSHOU HI. - La négativité arrivée à l'extrême limite de son développement, au point qu'elle entre en lutte avec la positivité, toutes deux sont atteintes et blessées. Telle est l'image symbolique. Si celui qui consulte le sort est dans ce cas, le présage néfaste est manifeste.

76.

Les dragons combattent dans le désert ; la voie est épuisée.

TSHENG TSE. - La négativité parvenue à son parfait développement, jusqu’à sa plus extrême limite, il y a nécessairement lutte et blessures.

77.

Emploi des traits hexaires : bien, perfection éternelle.

${ }^{1}$ Yie, traduit par désert, exprime l'espace au -delà des régions les plus lointaines habitées. Le son « yie », selon son accent, exprime, le doute, le désert, ou la nuit. 
TSHENG TSE. - L'emploi des traits hexaires dans le koua khouen est analogue à l'emploi des traits nonaires dans le koua Khien; c'est la voie naturelle de l'emploi et des effets de la négativité. La voie naturelle de la négativité est la douceur malléable avec difficulté de persistance continue ; aussi dans la voie rationnelle de l'usage des traits hexaires, le bien consiste dans la perfection ferme et indéfiniment persistante.

TSHOU HI. - Emploi des traits hexaires ; ces mots expriment que toutes les fois qu'en consultant le sort on obtient un trait négatif, on emploie toujours le nombre six et jamais le nombre huit. C'est encore une règle générale et toujours applicable ; c'est parce que ce koua comporte la pureté de substance de la négativité, et qu'il est placé au début du livre, qu'elle est donnée ici. Sion rencontre ce koua et que tous les traits soient en voie de transformation, le sens divinatoire sera tel qu'il est énoncé dans la formule. En effe t, la négativité et la malléabilité ne peuvent pas subsister et se maintenir avec fermeté ; elles se transforment et deviennent positivité, et alors il peut y avoir perfection durable. C'est pour cela que la formule avertit celui qui consulte le sort par les mots : «bien, perfection éternelle », qui correspondent précisément aux mots : bien et perfection, dans le koua Khien. Comme cette nouvelle positivité provient de la transformation du koua khouen, elle est insuffisante pour comporter les deux autres vertus, « cause initiale et liberté ».

78.

Emploi des traits hexaires, perfection durable : pour une grande fin.

TSHENG TSE. - Du moment où la fermeté de la perfection de la négativité est insuffisante, il est impossible qu'elle dure indéfiniment ; aussi, dans la voie naturelle de l'emploi des traits hexaires, le bien consiste dans le parfait développement de la grandeur finale. Pouvoir finir par la grandeur, c'est là la perfection durable.

TSHOU HI. - D'abord négativité, ensuite positivité ; c'est pourquoi la formule dit « grande fin ${ }^{1}$.

\section{COMMENTAIRE TRADITIONNEL SUR L'EXPRESSION DES REPRÉSENTATIONS}

\footnotetext{
${ }^{1}$ La positivité constitue la grandeur; la négativité constitue la petitesse. La négativité se transformant en positivité c'est ce qu'o $\mathrm{n}$ appelle finir par la grandeur. (Tshou Tse).
} 
79.

Khouen, extrême douceur malléable et dureté énergique dans le mouvement ; extrême repos et régularité de la vertu ${ }^{1}$.

TSHOU HI. - Dureté énergique et régularité expliquent le sens de l'expression «perfection de la jument». Le mot «régularité » exprime la continuité dans la génération des êtres.

80.

En suivant elle ${ }^{2}$ possède une direction et une continuité permanentes.

TSHOU HI. - Le commentaire traditionnel de Tsheng dit qu'au -dessous du mot $t$ shou traduit par direction, il devrait y avoir le mot $l i$, bien ${ }^{3}$.

81.

Elle supporte et contient tous les êtres et son action transformatrice est éclatante ${ }^{4}$.

TSHOU HI. - Nouvel éclaircissement du sens du mot heng, «liberté ».

\footnotetext{
1 Kien Ngan Khieu Shi dit: La substance de la passivité est essentiellement l'extrême malléabilité ; mais, au moment où elle engendre les êtres et leur donne la vie, et où elle produit son mouvement, cette malléabilité n'est jamais dépourvue d'énergie active. Elle est essentiellement l'extrême repos et son immense vertu engendre journellement en donnant la forme suivant des types une fois déterminés. En ceci on peut voir la régularité de la vertu.

${ }^{2}$ La passivité exprimée par le koua khouen

${ }^{3}$ En ajoutant le mot $l i$ on aurait le membre de phrase de la formule $n^{\circ} 51$ traduit par : «en suivant possibilité de se guider sur le bien ».

${ }^{4}$ Littéralement le texte dit : «Et transforme l'éclat »; on s'est conformé dans la traduction au sens de convention attribué à ce passage par les Chinois. Le koua khouen représente la terre qui transforme et produit en fournissant la matière et la forme.
} 
82.

La voie de la passivité (khouen), n'est-ce pas l'obéissance? Elle est soumise au ciel et agit avec le temps.

TSHOU HI. - Nouvel éclaircissement de l'idée contenue dans l'expression « sujétion passive au ciel ». Ce qui précède est un retour sur les idées émises dans le commentaire de la formule déterminative.

TSHENG TSE. — La voie de la passivité est l'extrême malléabilité, tandis que son mouvement est énergique ; la substance de la passivité exprimée par le koua khouen est le repos ${ }^{1}$, tandis que sa vertu est la régularité. Son mouvement étant énergique, elle correspond sympathiquement à l'activité du koua Khien, sans aucune contradiction. Sa vertu est la régularité, ce qui fait qu'elle donne naissance aux êtres d'une fa çon constante et continue. La voie logique de la négativité ne consiste pas à donner la mesure, mais à s'y accor der ; aussi, en restant en arrière, elle réussit dans son action et se guide sur le bien ${ }^{2}$. Achever la production des êtres, telle est l'acti on ordinaire et continue de la passivité exprimée par le koua khouen. Contenant et supportant tous les genres et toutes les classes d'êtres et d'objets, son ouvre transformatrice est brillante et grande. Après le mot $t$ shou ${ }^{3}$, il manque le mot $l i^{4}$. La voie de la passivité n'est-elle pas l'obéissance ? Elle est soumise au ciel et agit suivant le moment ; elle est soumise à l'impulsion émanant du ciel et elle agit sans se mettre en opposition avec le temps ${ }^{5}$. C'est encore là une exclamation d'admiration sur la soumission qui constitue la voie rationnelle du koua khouen.

83.

La famille qui accumule le bien doit avoir un surcroît de félicité ; la famille qui accumule ce qui n'est pas le bien doit avoir un surcroît de malheur. Si le sujet massacre le prince, si le fils massacre le père, ce ne sont point là des résultats d'une cause née entre le matin et le soir. Ce qui fait que de telles actions surviennent a dû se produire en croissant peu à peu; c'est

\footnotetext{
${ }^{1}$ Beaucoup de ces termes chinois n'ont pas d'équivalents en français. Selon le cas, il faut traduire par «substance», ou par «essence», ou par «corps». On remarquera cette conception tout à fait particulière qui à chaque instant, fait considérer une qualité ou un état comme constituant la substance ou l'essence du sujet considéré.

${ }^{2}$ Tsheng Tse rétablit le texte tel quill se trouve dans la formule $\mathrm{n}^{\circ} 65$.

${ }^{3}$ Maître, diriger ; prendre pour guide.

${ }^{4}$ Bien, utilité.

${ }^{5} \mathrm{La}$ terre donne ses produits selon les saisons.
} 
qu'en y remédiant on n'y a point remédié de bonne heure. Le livre des changements dit: En piétinant sur le givre la glace dure survient $\left(n^{\circ} 65\right)$; il s'agit, en effet, du cours passif des choses.

TSHENG TSE. - Aucune chose dans l'univers ne s'achève sans avoir été produite par une accumulation successive d'élém ents. Si ce qu'une famille pratique et accumule est le bien, le bonheur et la félicité atteindront ses enfants et petits-enfants; si ce qu'elle accumule n'est pas le bien, les calamités et le malheur fondront sur ses descendants. Les malheurs extrêmes, tels que le meurtre et la rébellion, résultent tous d'une accumulation et d'une répétition de causes qui s'enchaînent ; ils ne peuvent pas être le résultat d'une cause subite naissant et produisant ses effets dans le même jour. Celui qui est intelligent saura que ce qui augmente peu à peu ne peut point ne pas se développer en grandissant et que les petites quantités accumulées finissent par produire la grandeur; il y portera remède de bonne heure, sans laisser passivement le germe se développer et grandir. En agissant ainsi, dans l'univers, le mal ne peut pas arriver à se produire et c'est là connaître et comprendre l'avertissement relatif au givre et à la glace. Le givre qui devient de la glace dure, un petit mal qui finit par grandir, sont toujours le résultat de la tolérance passive du développement de la force naturelle et des conséquences d'une chose.

TSHOU HI. - Dans l'antiquité, le terme shouen «obéissance passive, cours passif », et le mot shen «circonspection», étaient indifféremment employés l'u n pour l'autre. Ici on doit lire shen (et traduire) : en effet, il s'agit de la circonspection, ce qui exprime qu'il faut se préoccuper des causes pendant qu'elles sont encore minimes ${ }^{1}$.

84.

Rectitude dans la droiture, régularité dans le devoir; l'homme doué est respectueux pour se rectifier en lui-même; il respecte le devoir pour rectifier ce qui lui est extérieur. Le respect et le devoir subsistant, la vertu n'est pas isolée. Avec la rectitude, la régularité et la grandeur, même sans exercice préparatoire, rien n'est autrement que bien, de sorte qu'il n'y a aucun doute sur ce qui doit être fait.

TSHENG TSE. - Rectitude exprime la droiture ; régularité exprime le devoir. L'homme doué se guide par le respect pour maintenir la droiture en

\footnotetext{
${ }^{1}$ Ce singulier moyen de trouver un sens étranger au texte est fréquemment employé. Dans la traduction, j'ai suivi la lecture de Tsheng Tse.
} 
lui-même ${ }^{1}$; il observe le devoir afin de régulariser son action extérieure ${ }^{2}$. Lorsque le respect subsiste, la rectitude existe à l'intérieur ; lorsque le devoir se traduit en actions ${ }^{3}$, le mouvement extérieur est régulier.

Le devoir se traduit en actions extérieures, il n'est pas extérieur. Lorsque le respect et le devoir sont maintenus, la vertu est parfaite ; elle ne vise pas à la grandeur et elle est grande. La vertu n'est point unique et isolée, il n'est rien qu'elle n'embrasse quand elle est appliquée à quelque o bjet ; son impulsion n'est jamais sans avantage : Qu'est -ce qui pourrait être douteux ${ }^{4}$ ?

TSHOU HI. - Ici, il est question de l'étude. La droiture désigne la substance essentielle ; le devoir désigne ce qui précise et détermine la loi ; le respect est le maintien et la conservation de la substance essentielle. Quant à la rectitude intérieure et à la régularité extérieure, le commentaire traditionnel de Tsheng est suffisant et complet. Les mots «pas isolée » expriment la grandeur. C'est parce qu'il y a do ute, qu'il y a nécessité d'apprendre par la continuité de l'exercice et qu'ensuite l'acte constitue le bien ; s'il n'y a point de doute, qu'emprunterait -on à l'exercice 5 ?

85.

Bien que la négativité ait de belles qualités, elle les cache, afin de s'occuper du service du roi; elle n'ose point s'en attribuer la direction exclusive. Telle est la voie de la terre, de l'épouse, du sujet. La voie de la terre, c'est de n'avoir pas la puissance suprême d'action et de se substituer pour arriver au but final.

TSHENG TSE. - La voie naturelle de l'infériorité, consiste à ne point s'attribuer le méri te de l'œuvre accomplie, à renfermer, en les cachant, ses belles qualités, pour se consacrer au service du roi, enfin à remplacer le supérieur dans ce qu’il s'agit de faire, sans oser s'attribuer la direction

\footnotetext{
${ }^{1}$ Dans le cour, Définitions diverses.

2 On interprète aussi le mot « extérieur » comme désignant les êtres extérieurs.

${ }^{3}$ Se dessiner au-dehors, prendre une forme.

${ }^{4}$ Le commentateur dénature certainement le véritable sens du terme du texte traduit par le mot isolé.

${ }^{5}$ Le terme traduit par «exercice » comporte le sens d'«étude » et d'«essai » qui précède la réussite parfaite. Au sujet de cette formule et à titre de curiosité, je crois pouvoir ajouter la citation suivante. Sie Shi Bang Tso dit : Ce qui constitue la différence entre les bouddhistes et nous autres philosophes de l'école de la représentation figurative, c'est qu'ils n'ont point le dogme de l'observation du devoir par la régularité dans l'accomplissement des actions extérieures qui est précisément la dernière conséquence de la raison d'être de toutes choses. Les bouddhistes, au contraire, considèrent la raison comme un danger. On ne peut pas dire que les bouddhistes ne discernent pas le fait ; mais, après qu'ils l'ont dis cerné, ils se refusent à en arriver jusqu'à la raison qui produit son existence.
} 
exclusive et le mérite. Elle est semblable à la voie naturelle de l'évolution de la terre remplaçant le ciel pour achever les êtres, tandis que la puissance exclusive et le mérite de l'auvre appartiennent et revie nnent au ciel. Il en est encore de même de la voie morale de l'épouse ${ }^{1}$.

86.

Le ciel et la terre modifient et transforment; les plantes et les arbres croissent abondamment. Si le ciel et la terre interrompent leur relation, le sage se cache. Le livre des changements dit: Lier le sac; pas de culpabilité, pas de louanges ; il s'agit, en effet, de la circonspection.

TSHENG TSE. - Le quatrième trait se trouve dans le koua simple supérieur, près du prince, mais il ne comporte point le sens d'accord entre eux. Aussi il est l'image symbolique de la séparation et de l'interruption de relations. Lorsque le ciel et la terre échangent leurs influences, ils modifient et transforment toutes choses, les plantes et les arbres croissent et se développent, le prince et le sujet poursuivent leurs relations mutuelles et la voie morale est librement ouverte. Lorsque le ciel et la terre sont séparés et sans action réciproque, tous les êtres manquent des conditions nécessaires à leur évolution ; la voie morale entre le prince et le sujet est obstruée et fermée et le sage se cache et se retire à l'écart. Pendant le moment de l'interruption des relations mutuelles, le quatrième trait ferme le sac et cache ce quil contient. Aussi, bien quil ne puisse donner lieu à aucune lo uange, il peut cependant n'avoir aucune culpabilité, ce qui exprime l'opportunité de s'observer soi -même avec circonspection.

87.

L'homme doué porte la couleur jaune en lui-même et pénètre la raison d'être.

TSHOU HI. - Les deux mots hoang tshong du texte (traduits par « porter la couleur jaune en soi-même », littéralement « jaune en dedans »), expriment que la vertu de justice est intérieure. Cette formule explique le sens du mot jaune.

${ }^{1}$ Ce qui engendre les êtres dans la terre, c'est toujours l'éther émanant du ciel. (Tsheng Tse). 
88.

Il accomplit avec droiture les devoirs de sa situation et n'oublie pas sa propre substance.

TSHOU HI. - Bien qu'il se trouve dans une situation prééminente, cependant il est d'une essence inférieure. C'est l'explication du sens du mot « jupe ${ }^{1}$.

89.

La beauté est dans son intérieur et elle s'étend aux quatre membres; lorsqu' elle éclate dans les actes, c'est l'extrême degré de la beauté.

TSHOU HI. - La beauté est dans son intérieur: c'est une nouvelle explication des mots «jaune en dedans ». L’influence s'étendant aux quatre membres, est une nouvelle explication des mots « ne pas oublier sa propre substance $»$.

TSHENG TSE. - Jaune en dedans; les capacités (voir $\mathrm{n}^{\circ} 74$ ) sont intérieures. Chez l'homme doué, les capacités sont intérieures et pénètrent jusqu'à la raison d'être des choses. Il occupe la situation qui lui revient régulièrement, sans perdre de vue qu'il est d'une essence inférieure. Le cinquième rang indique la situation prééminente, -mais dans le cas du koua khouen, on ne relève que le sens de justice et droiture. Les belles qualités sont accumulées au-dedans et se reflètent librement dans les quatre membres (par la correction des mouvements); elles se manifestent et deviennent visibles dans les actions ; c'est l'extrême et parfait développement de la vertu et des belles qualités ${ }^{2}$.

\footnotetext{
${ }^{1}$ D'après les commentaires traditionnels, il s'agit de ne point oublier sa propre condition quelle que soit l'élévation de la position momentanément occupée. Littéralement le texte dit : Situation correcte, demeurer dans la substance.

${ }^{2}$ Les qualités sont intérieures ; elles se manifestent dans les actions extérieures et jusque dans la contenance et les mouvements. Ces qualités sont, la rectitude, ou droiture, et la régularité ou conformité au devoir dans les actes extérieurs ; dans le cas du cinquième, elles sont en acte et on considère leurs conséquences.
} 
90.

La négativité étant en antagonisme ${ }^{1}$ avec la positivité, il doit nécessairement y avoir lutte, parce qu'elle est jalouse de n'être pas elle-même positivité. C'est pour cela quelle est qualifiée dragon. Elle n'a cependant pas encore quitté son propre genre, c'est pourquoi le terme sang est employé. Or, le noir et le jaune sont le mélange des couleurs du ciel et de la terre; le ciel est noir et la terre est jaune.

TSHENG TSE. - La positivité est la grandeur, la négativité est la petitesse; la négativité doit suivre la positivité. Du moment où la négativité est arrivée à son extrême perfection, elle égale la positivité ; c'est là être en antagonisme avec elle. Ne se suivant plus mutuellement, il y a nécessairement lutte. Bien que le koua représente l'unité de substance de la négativité, il y a crainte et suspicion à cause du défaut de positivité, et c'est pour cela que la formule emploie le terme dragon, afin de montrer quil y a combat contre la positivité. Dans le désert, indique que c'est en avançant sans cesse et en parvenant à l'extérieur. La perfection étant arrivée à son extrême développement et le mouvement de progression ne cessant point, il en résulte qu'il y a combat. Bien que la perfection soit arrivée à son entier développement, aucun trait n'a encore ab andonné le genre de la négativité et, puisqu’il y a lutte avec la positivité, il est évident qu'il doit y avoir blessures ; c'est pour cela que le terme sang est employé. La négativité étant parvenue à son extrême perfection, jusqu'à lutter contre la positivité, cette dernière, elle-même, ne peut éviter les blessures, aussi le sang qui coule est noir et jaune. Le jaune et le noir sont les couleurs du ciel et de la terre et ceci indique que toutes deux sont blessées.

TSHOU HI. - Le mot «douter», du texte, veut dire se poser en adversaire sans qu'il y ait différence de grandeur entre elles deux. Bien que le koua khouen ne contienne pas de traits positifs, cependant la positivité n'en est jamais absolument exclue. Le sang est quelque chose qui appartient au genre négatif ; en effet le souffle ${ }^{2}$ est positif et le sang négatif. Le noir et le

\footnotetext{
${ }^{1}$ Le mot du texte signifie littéralement «douter» et s'emploie avec le sens de « se méfier de $»$.

${ }^{2}$ Ici je traduis le terme khi par le mot « souffle », respiration. C'est qu'en effet l'argument est emprunté à une théorie physiologique. On trouve dans les traités de médecine quantité d'axiomes tels que les suivants : «C'est pourquoi l'homme seul, par sa naissance, possède les éthers du ciel et de la terre. La tête est ronde, image symbolique du ciel ; les pieds sont rectilignes, image symbolique de la terre. Le ciel a la négativité et la positivité ; l'homme a le souffle $k h i$ et le sang. Le ciel a les cinq agents, l'homme reçoit à sa naissance l'éther du ciel et de la terre ; l'éther positif du ciel constitue le souffle ; l'éther négatif de la terre constitue le sang, aussi le souffle est toujours en excès et le sang fait toujours défaut. » (Tiré de lIntroduction à l'étude de la médecine, hygiène). Selon sa valeur dans la même phrase, le mot $k h i$ doit se traduire par éther, ou par souffle.
} 
jaune sont les couleurs respectives du ciel et de la terre ; ceci exprime que la négativité et la positivité sont toutes deux blessées. Ce qui précède revient sur des idées déjà exprimées dans le commentaire de la formule symbolique. 


\section{Tshouen}

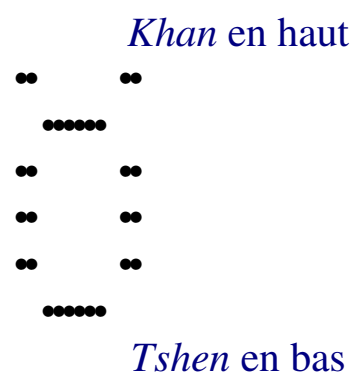

91. Grande liberté, perfection avantageuse, ne pas agir dans ce qu'il ya à entreprendre ; avantage à instituer des feudataires.

[]

96. Premier trait nonaire : immobilité de la borne; avantage à demeurer dans la perfection; avantage à instituer des feudataires.

$*$

98. Deuxième trait hexaire : comme embarrassé, comme perplexe ; montant à cheval et comme demeurant en rang ; s'il ne s'agit de brigands, c'est un mariage ; la jeune fille vierge n'est pas accordée ; après dix ans, alors elle est accordée.

100. Troisième trait hexaire : poursuivre le daim sans guide ; c'est seulement entrer dans la forêt. L'homme doué apprécie dès le début qu'il vaut mieux renoncer; en entreprenant, appréhension.

$*$

102. Quatrième trait hexaire : monter à cheval et comme demeurer en rang ; rechercher en mariage ; en entreprenant, présage heureux ; rien qui ne soit avantageux.

*

104. Cinquième trait nonaire : obstacle à l'influence; petite perfection, présage heureux; grande perfection, présage malheureux.

$*$

106. Trait supérieur hexaire : monter à cheval et comme demeurer en rang ; pleurs de sang, les larmes comme ruisselant

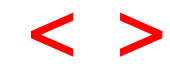


TSHENG TSE. - Au sujet du koua (diagramme), « L'Ordre des koua ${ }^{1} »$ dit : «Aussitôt que le ciel et la terre existent, tous les êtres naissent; ce qui comble l'intervalle entre le ciel et la terre n'est rien autre que tous les être s et toutes les choses; aussi le koua tshouen suit immédiatement (les koua khien et khouen qui représentent le ciel et la terre). Tshouen signifie remplir.» Tshouen exprime le commencement et la naissance des êtres. Lorsque les êtres commencent à naître, la coagulation de l'éther ne s'effectue pas encore libre ment, aussi elle constitue un obstacle qui intercepte l'intervalle entre le ciel et la terre et ce n'est qu'au moment du libre développement florissant et parfait que l'idée d’intercep tation disparaît. Le ciel et la terre donnent naissance à tous les êtres et tshouen exprime le commencement de la vie chez ces êtres ; c'est pour cela que ce koua est placé immédiate ment à la suite des koua khien et khouen. Si on en parle au point de vue des deux symboles ${ }^{2}$ c'est la formation du nuage et de la foudre. La négativité et la positivité commencent à s'unir. Si on en parle au point de vue des deux substances ${ }^{3}$, dans le koua simple tshen l'union commence par en bas, et dans le koua simple khan l'union commence par le milieu ${ }^{4}$. La négativité et la positivité s'unissent, c'est ce qui produit les nuages et la foudre ; la négativité et la positivité commençant à s'unir, les nuages et la foudre se cor respondent mutuellement, mais l'effet bienfaisant de cette union ne s'achève pas encore et c'est pourquoi le koua est appelé tshouen ${ }^{5}$. Si l'effet bienfaisant de cette union était déjà produit, cela constituerait le koua kiae. Ou bien encore : mouvement au milieu du danger ${ }^{6}$, ce qui constitue encore le sens exprimé par le mot tshouen. Lorsque la négativité et la positivité ne s'unissent pas, elles constituent le koua $p i$; lorsqu'elles commencent à s'unir sans y parvenir déjà librement, elles constituent le koua tshouen. Dans le temps, c'est le moment où l'univers ${ }^{7}$ est entravé par des difficultés et ne jouit pas encore librement de la liberté de son essor et de sa prospérité.

\footnotetext{
${ }^{1}$ Titre d'un des dix « coups d'aile » ou commentaires de Khong Tse dont la traduction suit.

${ }^{2}$ Les images symboliques attribuées aux koua simples de trois traits.

${ }^{3}$ La substance ; c'est l'ensemble des traits qui compo sent le koua simple.

${ }^{4}$ Dans le koua simple inférieur, l'unique trait positif est en bas, dans le koua simple supé rieur, il est au milieu. Chacun de ces deux koua simples représente le koua khouen $\left(\mathrm{n}^{\circ} 2\right)$ dans lequel un seul trait devient positif.

${ }^{5}$ Le mot tshouen exprimant cet embarras.

${ }^{6}$ Tshen ébranlement, mouvement ; khan, danger ; au milieu, c'est-à-dire en dedans du cercle sur lequel les koua sont supposés rangés.

${ }^{7}$ L'État, l'empire.
} 


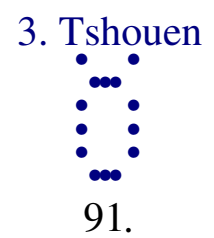

Grande liberté, perfection avantageuse ${ }^{1}$, ne pas agir dans ce qu' il y a à entreprendre ; avantage à instituer des feudataires.

TSHENG TSE. - L'état exprimé par le mot tshouen a une voie naturelle de grande liberté et pour celui qui se trouve dans les conditions quil comporte, l'avantage consiste dans la perfection et la fermeté. Sans perfection dans la fermeté, comment traverser cet état d'empêchement et d'embarras ? Pendant le moment du commencement de cet empêchement, il ne doit rien y avoir à entreprendre. Lorsqu'il s'agit d'un empêchement qui entrave l'univers, comment la force seule pourrait-elle y remédier ? Il faut absolument recourir à l'assistance et à l'aide d'autrui, aussi il est avantageux d'instituer des princes feudataires ${ }^{2}$.

TSHOU HI. - Tshen et khan sont également les noms de deux koua simples de trois traits. Dans le koua simple tshen, une seule positivité s'agite au-dessous de deux négativités ${ }^{3}$, aussi sa vertu est le mouvement et son image symbolique est la foudre. Dans le koua simple khan, une positivité est insérée dans l'intervalle entre deux négativités, aussi sa vertu ${ }^{4}$ est la chute, le péril, et son image symbolique est le nuage, la pluie, l'eau.

Tshouen est le nom du koua parfait de six traits; ce mot exprime la difficulté ; c'est l’idée des êtres commençant à naître et ne jouissant pas encore de leur liberté d'expansion. C'est pour cela que, comme caractère d'écriture, il représente un germe perçant la terre, commençant à sortir, et pas encore redressé. Comme koua, puisque le koua simple tshen rencontre le koua simple khan, il en résulte que le koua khien et le koua khouen commencent à s'unir et se trouvent en face du péril, aussi le nom du koua tshouen exprime l'embarras. Le mouvement représenté par le koua simple tshen, est au-dessous ; le péril, exprimé par le koua khan est au-dessus ; c'est donc la possibilité du mouvement au milieu du péril. Pouvant se mouvoir, bien quil soit possible que ce soit avec liberté d'action, cependant, pu isqu'il s'agit d'être dans le péril, il convient donc de conserver la droiture et il n'est pas encore possible d'avancer hâtivement. Aussi, pour celui qui, en consultant le sort, obtient ce koua, le sens divinatoire est « grande liberté et avantage par la

${ }^{1}$ Ce sont toujours les quatre mêmes termes yuan, heng, li, tsheng, mais la phrase est autrement ponctuée, du moins par les commentateurs et d'après la tradition.

2 Pour aider au gouvernement de l'empire universel.

${ }^{3}$ Un trait positif au-dessous de deux traits négatifs.

${ }^{4}$ Dans ce cas il faut donner au mot te vertu, le sens de propriété exprimée, ou valeur attribuée au koua. 
droiture ». Toutefois, il ne doit pas apporter de précipitation dans ce qu’il a à entreprendre. De plus, dans le premier trait nonaire, la positivité se trouve placée au-dessous de la négativité et représente le maître du koua parfait; c'est l'image de p ouvoir, par la sagesse, se mettre au-dessous des hommes, posséder l'affection du peuple et pouvoir le régir. Lorsqu'on consulte le sort au sujet de l'établissement d'un prince, si on rencontre ce koua, c'est un signe faste.

92.

Le commentaire de la formule déterminative dit: tshouen, la dureté énergique et la douceur malléable commencent à s'unir et la difficulté naît.

TSHOU HI. - C'est une explication du sens du nom du koua au moyen des deux substances (des koua simples). Les mots «commencement de l'union » se rapportent au koua simple tshen; les mots «difficulté naissante » se rapportent au koua simple khan.

93.

Mouvement au milieu du péril : grande liberté et perfection.

TSHOU HI. - Explication de la formule du koua au moyen des vertus des deux substances (des koua simples). Le mouvement, c'est l'action du koua simple tshen; le péril, c'est le lieu représenté par le koua simple khan. À partir de ce koua, dans l'explication des quatre mots yuan, heng, li, tsheng, on a suivi l'idée fondamental e de Wen Wang ${ }^{1}$.

94.

L'agitation de la foudre et de la pluie est pleine et complète; l'action du ciel est confuse et obscure; il convient d'instituer des feudataires et de ne point se livrer au repos.

\footnotetext{
${ }^{1}$ Tshou Hi revient sur ce point toutes les fois qu'il en trouve l'occasion.
} 
TSHOU HI. - Explication de la formule du koua au moyen de limage symbolique des deux substances. La foudre est le symbole du koua simple tshen; la pluie est le symbole du koua simple khan. L'action du ciel est une expression équivalente à l'expression «translation du ciel» ${ }^{1}$. Le terme «plantes» du texte (traduit par «confuse») exprime la confusion et le désordre ; l'obscurité est l'absence de lumière, comme pendant les ténèbres de la nuit. La négativité et la positivité s'unissent et la foudre et la pluie se forment ; c'est la confusion et le désordre, les ténèbres et l'obscurité ; c'est l'interceptation entre les deux origines. L'univers n'étant pas encore définitivement constitué, les appellations (noms) et les conditions ne sont pas encore clairement établies; il convient dinstituer un prince pour le gouvernement suprême et on ne peut pas encore dire que c'est un moment de calme et de repos. Ce commentaire ne choisit pas, pour objet de son explication, le sens de la formule du premier trait nonaire; il y a un grand nombre de moyens d'explication du sens, le commentaire en choisit un, quil adopte provisoirement.

TSHENG TSE. - En en parlant au point de vue des deux images symboliques, nuage et foudre, alors, la dureté énergique et la malléabilité commencent à s'unir. En en parlant au point de vue des deux substances des koua simples khan et tshen, c'est le mouvement au milieu du péril. Lorsque la dureté énergique et la malléabilité commencent à s'unir, elles ne peuvent pas encore se manifester librement, de sorte qu'il y a difficulté et embarras, et c'est pour cela que la formule dit : la difficulté naît ; les hommes s'agitant au milieu des périls, tel est le sens des mots « difficulté et embarras ». Ce qu'on entend par grande liberté et perfection, c'est le mouvement de la foudre et de la pluie remplissant totalement l'espace. La négativité et la positivité commençant à s'unir, par suite de la difficulté et de l'embarras ou impénétrabilité, elles ne peuvent pas encore manifester librement ; lorsqu'elles sont parvenues à se joindre harmoniquement, la foudre et la pluie s'achèvent et remplissent totalement l'espace entre le ciel et la terre ${ }^{2}$. Les êtres vivants peuvent ensuite se développer suivant les nécessités de leur condition; donc l'embarras exprimé par le mot tshouen, ou «impénétrabilité », comporte une voie de grande liberté d'expansion. Ce qui fait quill peut y avoir une grande liberté d'expansion, c'est la perfection ; sans une parfaite fermeté, comment sortir d'embarras ? L'homme placé dans l'embarras a aussi devant lui une voie naturelle de grande liberté et c'est aussi par la perfection et la ferme té. L'action du ciel est confuse et obscure ; le texte qui précède expose le sens du ciel et de la terre donnant la vie aux êtres ; ici, il s'agit des choses relatives au temps. L'action du ciel est une expression qui exprime la succession des saisons. Le texte emploie le mot « herbes »; ce mot exprime l'absence d'ordre et d'arrangement et la confusion de la végétation. L'obscurité exprime les ténèbres et l'absence de clarté. En présence de ce cours des saisons, ce qui est

\footnotetext{
${ }^{1}$ Ce terme désigne le mouvement astronomique attribué à la voûte céleste, la terre étant supposée immobile au centre.

${ }^{2}$ Cette explication des mots du texte «plein et complet » n'est pas du tout rigoureuse ; elle ne résulte nullement de la lecture du texte.
} 
convenable, c'est d'établir et d'instituer des moyens de concours et d'assistance, ce qui rend possible de traverser la période d'embarras et d'empêchements. Bien qu'il y ait institution de feudataires, afin de se faire aider par eux, il convient néanmoins encore de se préoccuper avec vigilance de tout motif de crainte et d'alarme, sans se livrer prématurément au calme du repos. Tel est l'avertissement donné par l'homme saint.

95.

Le commentaire traditionnel de la formule symbolique dit: Nuages et foudre, empêchement; l'homme doué en conclut la nécessité de préparer la trame et la chaîne.

TSHENG TSE. - Au sujet du koua simple khan, le commentaire ne dit pas «pluie », mais il dit «nuages »; les nuages sont constitués par la pluie non encore achevée. La pluie ne pouvant pas encore s'achever, c'est ce qui est considéré comme constituant l'embarras et l'empêchement. L'homme doué considère l'image symbolique de cet embarras et il prépare la trame et la chaîne du tissu de toutes les affaires de l'univers, afin de remédier à l'empêchement et aux difficultés. La trame, c'est l'assemblage des fils placés en longueur, la chaîne est celui des fils qui croisent les premiers. Cela exprime l'idée de faire et d'établir.

TSHOU HI. - Au sujet du koua simple khan, le commentaire emploie l'expression « nuages » et ne parle pas de l'eau ${ }^{1}$ : c'est à cause de l'idée que la liberté d'évolution n'existe pas encore. Trame et chaîne sont des termes techniques du tissage ; le mot trame exprime l’idée de conduire et de mener ; le mot chaîne exprime l'idée de régler pour mettre en ordre et diriger. Les temps d'empêchements et de difficultés sont les moments où l'homme doué doit agir.

\footnotetext{
${ }^{1}$ Le koua simple khan symbolise aussi l'eau.
} 


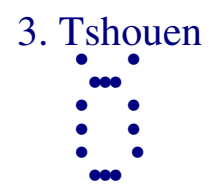

96.

Premier trait nonaire : immobilité de la borne ${ }^{1}$; avantage à demeurer dans la perfection; avantage à instituer des feudataires.

TSHENG TSE. - Puisque le premier est positif et se trouve au rang inférieur, c'est donc celui qui, avec les capacités de l'intelligence et de l'énergie, et en présence d'une période de difficultés, occupe une situation inférieure. Il n'est pas encore à propos qu'il entre prenne de remédier aux difficultés et aux embarras du moment et c'est pour cela qu'il est immob ile comme une borne de pierre ou de bois. Dès le début de la difficulté, sîl ne restait pas immobile et comme fixé en terre, sỉl avançait prématurément, il braverait la difficulté. Aussi il convient qu'il s'en tienne à la droiture et qu'il assure la fermeté de ses desseins. Toutes les fois que des hommes sont placés dans l'embarras et la difficulté, bien peu sont capables d'observer la droiture ; sans l'observation de la perfection et de la fermeté, ils sont portés à manquer au devoir; comment donc pourraient-ils traverser l'empêchement et la difficulté du moment en y apportant un remède ? Dans une période d'empêchement, et faisant face à la difficulté dans une position inférieure, ce quil importe, c'est d'avoir de l'aide et de l'assistance ; c'est là la voie pour demeurer dans la difficulté et la traverser en y remédiant et c'est pour cela que la formule adopte le sens d'établissement de feudataires, ce qui veut dire réclamer aide et assistance.

TSHOU HI. - Être immobile comme une borne ou un pieu est une expression qui indique l'apparence de l'immobilité et de l'impossibilité d'avancer. Au début de l'empê chement et de la difficulté, puisque le trait est positif et se trouve au rang inférieur, puisque de plus il fait partie de la substance du mouvement et que, enfin, au-dessus de lui, il correspond sympathiquement à un trait qui exprime la malléabilité négative dans le danger et la chute ${ }^{2}$, il a donc l'apparence symbolique d'être immobile comme une borne ou un pieu. Cependant, dans la position où il demeure, il possède la droiture $^{3}$, aussi le sens divinatoire est qu’il y a avantage à demeurer dans la perfection. D'ailleurs, essentiel lement, ce trait est en même temps le maître du koua parfait; puisque, lui-même étant positif, il se trouve au-dessous de la négativité, c'est vers lui que le peuple se porte ; c'est là l'image symbolique du feudataire, et c'est pour cela que telle est la formule symbolique. Enfin, si

\footnotetext{
${ }^{1}$ De pierre ou de bois.

2 C'est-à-dire dans le koua simple khan

${ }^{3}$ Puisqu'il est positif et qu'il occupe un rang impair.
} 
celui qui consulte le sort est dans ces mêmes conditions, alors il y aura avantage à ce quill soit élevé au rang de feudataire.

97.

Bien qu'immobile comme une borne ou un pieu, ses tendances le portent à agir avec droiture. Etre noble et se placer au-dessous de ce qui est humble, c'est une grande puissance d'attraction sur le peuple.

TSHENG TSE. - L homme sage étant dans une position inférieure, le moment n'est provisoirement pas encore avantageux. Bien qu'immobilisé comme une pierre ou un pieu et ne pouvant pas encore à son gré entreprendre de remédier aux empêchements du moment, cependant ses tendances le portent à y remédier et, dans l'action d'y remé dier, ses tendances le portent à pratiquer la droiture. Un trait nonaire se trouve en présence d'un moment d'empêchement et de difficulté ; positif, il vient se placer au-dessous de la négativité ; cela est considéré comme image symbolique de la possession de la noblesse et de l'abaissement volontaire au-dessous de ce qui est vil et humble. En présence d'un moment de difficulté et d'empêchement, la douceur malléable et la négativité ne peuvent pas se préserver par elles-mêmes ; sil existe quelqu'un qui possède les qualités de l'énergie positive, c'est vers lui que se porte la foule et c'est lui qu'elle suit. Si, en outre, celui -là peut de luimême se placer humblement et dans un rang inférieur, cela lui permettra d'exercer une grande puissance d'attraction sur le peuple. On pourrait douter que faire face aux empêchements dans une position inférieure puisse s'allier à la possession de la noblesse ; or, employer les qualités de l'énergie et de l'intelligence et s'abaisser au -dessous de la malléabilité négative, employer les aptitudes qui permettent de remédier aux empêchements et se placer au-dessous de ceux qui en sont incapables, c'est là, possédant la noblesse, s’incliner au-dessous de ce qui est humble; $a$ fortiori, lorsqu'il s'agit de la positivité par rapport à la négativité, cela constitue-t-il encore la noblesse. 


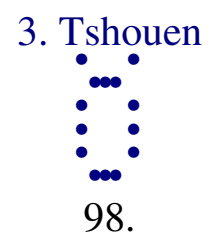

Deuxième trait hexaire : comme embarrassé, comme perplexe; montant à cheval et comme demeurant en rang ${ }^{1}$; s'il ne s'agit de brigands, c'est un mariage ; la jeune fille vierge n'est pas accordée ; après dix ans, alors elle est accordée.

TSHENG TSE. — Le second trait emploie la négativité et la douceur pour se maintenir pendant une période d'embarras et d'empêchements ; bien quil corresponde sympathiquement selon la droiture à un trait supérieur, il est cependant porté par son inclination vers la dureté énergique du premier trait, c'est pour cela qu'embarrassé et entravé par la difficulté, il est perplexe et revient sur ses pas. Le mot «comme » est un terme auxiliaire qui ne change pas la portée des termes employés. Monter à cheval, c'est vouloir aller et circuler; il veut suivre celui avec qui il sympathise selon la droiture et il demeure comme aligné en rang, sans pouvoir avancer. Le terme pan, demeurer en rang, comporte le sens de diviser et d'aligner en haie ; descendre de cheval pour former la haie, de sorte que le cheval et le cavalier sont séparés et dans des lieux différents ${ }^{2}$. Le second trait se trouve en présence d'une période de difficulté et d'empêchements ; bien quill ne puisse y remédier par lui-même, cependant il se maintient dans la justice ${ }^{3}$ et possède la droiture ${ }^{4}$; il a une correspondance sympathique au-dessus de lui ${ }^{5}$ : C'est celui qui ne manque pas au devoir. Cependant il est pressé et serré contre le premier trait ; la négativité est recherchée par la positivité, la douceur malléable est dominée par la dureté énergique. La douceur malléable étant aux prises avec une période de difficultés est évidemment incapable de les aplanir elle-même; de plus elle est pressée par la dureté énergique de la positivité, de sorte que ce trait est considéré comme exprimant la difficulté. Si on suppose qu'il ne s'agit pas de la difficulté qui consiste à être poursuivi et serré de près par des brigands, alors ce sera quelqu'un qui va faire une demande en mariage. Le mariage est la correspondance sympathique conforme à la droiture; le brigandage est le résultat du mépris de toute raison. Le second trait observe la

\footnotetext{
${ }^{1}$ En rang ; c'est la position des fonctionnaires de la cour rangés sur deux files à droite et à gauche du trône. Le mot exclut l'idée de mouvement.

2 Ce mot chinois pan devient ban dans les dialectes dérivés, tels que l'annamite. C'est exactement l'équivalent (pour ne pas dire le même mot lui-même) du mot ban des langues aryennes de l'Europe.

${ }^{3}$ Au milieu du koua simple.

${ }^{4}$ Rang pair occupé par un trait négatif.

5 Le second rang correspond au cinquième, lorsque l'un est occupé par un trait négatif et l'autre par u n trait positif, la correspondance sympathique est dite conforme à la droiture.
} 
justice et la droiture ; il ne s'unit pas inconsidérément au premier, ce qui fait qu'il n'y a pas accord et fiançailles. S'il garde fermement la pureté, sans varier, pendant une période qui atteint dix ans, l'empêchement arrivé à son extrême limite doit enfin faire place à la liberté d'action ; alors le second trait parviendra à posséder celui avec lequel il correspond sympathiquement, et l'œuvre de conception s'accomplira. Puisqu'alors qu'il s'agit de la jeune fille douée de la douceur malléable négative elle doit, en observant les convenances et en suivant ses propres tendances, parvenir après un long délai, à la liberté d'action, à plus forte raison doit-il encore en être de même lorsquill s'agit de l'homme doué qui observe la voie morale sans s'en détourner. Le premier trait est considéré comme représentant un brigand qui opprime et lèse quelqu'un. Ceci résulte naturellement de ce qu'on tient compte de la situation du second trait doué de malléabilité et proche d'un trait qui exprime la dureté énergique, et de ce qu'on en déduit un sens, sans tenir compte des qualités propres au premier trait, quelles qu'elles soient. Tel est, dans le livre des changements, la manière de choisir le sens.

TSHOU HI. - Demeurer en rang: c'est l'apparence de personnes alignées sur deux files, sans avancer. Accorder, promettre en mariage ; le livre des Rites dit: La jeune fille accordée en mariage est coiffée de l'épingle et reçoit un surnom ${ }^{1}$. Le second trait hexaire est doué de la douceur malléable de la négativité, de la justice et de la droiture; en haut il a un trait qui lui correspond sympathiquement et il est monté sur la dureté énergique du premier trait. C'est pour ces motifs qu'il est considéré comme retenu par les difficultés, perplexe, et se retournant sans avancer. Cependant le premier trait n'est pas considéré comme représentant le brigand, c'est donc qu'il sollicite le second pour conclure un mariage, mais celui-ci conserve la droiture et n'y consent point. Au bout de la période de dix années, la série des nombres est épuisée, la raison d'être du fait est arrivée à sa limite extrême, de sorte que celui qui sollicite à tort s'en va, tandis que ceux qui se correspondent sympathiquement avec droiture se réunissent et qu'il est licite d'accorder et de consentir. Le trait a cette image symbolique et c'est pourquoi la formule en profite pour avertir ceux qui consultent le sort ${ }^{2}$.

99.

Difficulté du second trait hexaire; il est monté sur la dureté énergique; au bout de dix ans accord et consentement; retour aux règles ordinaires.

\footnotetext{
${ }^{1}$ Le même caractère signifie surnommer, comme dans le livre des Rites, et il est considéré comme ayant le sens d'accorder, unir en mariage, dans ce passage du Yi king.

${ }^{2}$ Dans la «Genèse du langage » j'ai relevé l'analogie qui existe entre cette formule et un passage tronqué de l'Avesta.
} 
TSHENG TSE. - Le second trait hexaire se trouve dans un moment d'empêchement, et, de plus, il est monté sur la dureté énergique. Être serré de près et opprimé par la dureté énergique de la positivité, c'est là ce qui constitue le malheur et la difficulté. Au bout de dix ans, la difficulté ayant duré longtemps doit s'aplanir, alors il devient possible de reve nir aux règles ordinaires et il y a réunion entre ceux qui se correspondent sympathiquement avec droiture. Dix est le dernier des chiffres et exprime la fin du nombre. 


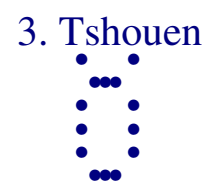

100.
Troisième trait hexaire : poursuivre le daim sans guide ; c'est seulement entrer dans la forêt.

L'homme doué apprécie dès le début qu'il vaut mieux renoncer ; en entreprenant, appréhension.

TSHENG TSE. - Le troisième trait hexaire, avec sa douceur malléable, occupe un rang qui comporte la dureté énergique. Du moment où la douceur n'est pas apte à apaiser les difficultés, occupant un rang qui comporte la dureté énergique, sans justice ni droiture, elle s'agitera mal à propos. Bien que désirant passionnément obtenir ce qu'elle poursuit, puisqu'elle n'est pas capable de remettre elle-même les choses en ordre et que, de plus, elle est sans correspondance sympathique pour l'accueillir, peut-elle donc être sur le point d'apaiser ces difficultés ? Elle est comme l'homme qui poursuit les daims sans avoir un guide. Celui qui entre dans une forêt doit avoir un guide pour le conduire; sans conducteur il ne fera qu'errer dans l'épaisseur de la forêt. Lhomme doué voit les choses dans leur premier début; il vaut mieux abandonner et cesser la poursuite; en entreprenant on ne ferait qu'aller au-devant de causes de misère et d'appréhension.

TSHOU HI. - Malléabilité négative demeurant dans un rang inférieur, sans justice ni droiture; en haut, pas de correspondance sympathique selon la droiture ; agissements irréguliers allant au-devant de misères et de souffrances, ce qui constitue l'image symbo lique de l'action de poursuivre le daim sans guide et d'errer au milieu d'une forêt. L'homme doué voit dès le premier début qu'il est préférable de renoncer et de se retirer ; s'il entreprenait la poursuite au lieu d'y renoncer, il en résulterait certainement de la confusion et l'appréhension d'un malheur. C'est un avertissement à celui qui consulte le sort, pour le prévenir qu'il lui convient d'agir ainsi.

101.

Chasser le daim sans guide, poursuivre le gibier; l'homme doué y renonce; en entreprenant, appréhension, misère. 
TSHENG TSE. - Quelque chose dont il s'agisse, il ne faut pas se mettre en train mal à propos, pour suivre ses propres désirs. Poursuivre le daim sans guide, c'est le résulta t de la convoitise du gibier. En présence d'un moment d'empêchement, agir quand on ne doit point agir, c'est encore être sans guide et poursuivre le daim, en suivant le désir qui porte à poursuivre le gibier. L’homme doué verra les effets futurs dans leur premier germe et il renoncera, sans s'obstiner à suivre sa première intention. S’il entreprenait, il devrait ensuite en éprouver de l'appréhension et en souffrir. 


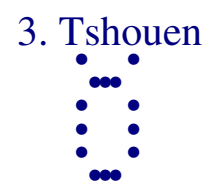

102.

Quatrième trait hexaire : monter à cheval et comme demeurer en rang ; rechercher en mariage; en entreprenant, présage heureux; rien qui ne soit avantageux.

TSHENG TSE. - Le quatrième trait hexaire, avec sa douceur et sa soumission, occupe une situation voisine du prince ; c'est celui qui s'accorde avec le supérieur et dont les capacités sont insuffisantes pour remédier aux difficultés, de sorte que, voulant avancer, il reste cependant en place. Il monte à cheval et reste comme fixé en rang. N'étant pas, par soi -même, capable de remédier aux difficultés et aux empêchements du moment, sil peut appeler le sage à son aide, il pourra dès lors y remédier. Le sage, représenté par la dureté énergique du premier trait, est précisément en correspondance sympathique selon la droiture avec le quatrième trait ; c'est celui avec qui il y a union en mariage. S'il recherche une alliance en mariage avec cette positivité énergique, et entreprend avec elle d'assister le prince doué des vertus de la positivité énergique, de la justice et de la droiture, afin de remédier aux embarras de l'époque, ce sera un présage heureux et rien ne sera sans avantages. Demeurant dans la situation de ceux qui portent les titres de Kong et de Khing ${ }^{1}$, bien que ses propres capacités ne soient pas suffisantes pour remédier aux embarras et aux difficultés du moment, s'il peut cependant rechercher le concours du sage placé au-dessous de lui, se l'attacher et l'employer, à quelles difficultés ne pourrait -il point parer ?

TSHOU HI. - Malléabilité négative placée dans l'embarras par un obstacle impénétrable. Il ne peut avancer en montant, c'est pourquoi il est considéré comme image symbolique de l'action de monter à cheval et de rester comme fixé en rang. Mais le premier trait nonaire se maintient dans la droiture et demeure dans l'infériorité pour lui corres pondre sympathiquement et c'est pour cela que le sens divinatoire est qu'en se baissant pour faire une demande en mariage, il y aura un présage heureux.

103.

Appeler à soi et entreprendre; intelligence.

\footnotetext{
${ }^{1}$ Titres de noblesse accordés au mérite.
} 
TSHENG TSE. - Reconnaître sa propre insuffisance, appeler le sage à son aide et ensuite entreprendre, c'est ce qu'on peut appeler l'intelligence. Occuper une position très importante, être par soi-même incapable, et s'en fier à sa propre manière de voir, c'est le comble de l'aveuglement. 


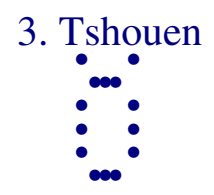

104.

Cinquième trait nonaire : obstacle à l'influence ; petite perfection, présage heureux; grande perfection, présage malheureux.

TSHENG TSE. - Le cinquième trait occupe le rang prééminent; il possède la droiture, et se trouve en face d'un moment d'empêchement. S'il a, pour l'assister, un sage doué d'intelligence et d'énergie, il pourra tr averser ces difficultés. C'est parce qu'il est sans ministres que son influence est entravée. Même dans une période d'empêchements et de difficultés, l'éminence du prince n'est pas diminuée dans ses titres et sa situation; c'est seulement l'action extérie ure de cette influence qui ne peut pas toujours se faire sentir et l'effet bienfaisant de sa vertu ne peut pas, dans certain cas, descendre sur la foule. C'est là l'obstacle à l'influence ; c'est ce qui, pour le prince, constitue l'empêchement. Du moment où l'influence bienfaisante de sa vertu éprouve certains obstacles et ne peut pas toujours atteindre au-dessous de lui, c'est que l'autorité et la puissance ne résident plus en lui. L'autorité et le pouvoir lui échappant, s'il veut hâtivement corriger cet état de choses, c'est suivre une voie qui appelle le malheur ; c'est, par exemple, le cas de Tshiao Kong, de l'état de Lu, élevé et ennobli jusqu'à être revêtu des titres de Kong et de Khing. C'est pour cela qu'une petite perfection constituera un présage heureux; avec une petite perfection, il redressera petit à petit, comme par exemple Pan Kang et Tsheou Suien ramenant la vertu et employant les sages, revenant aux institutions politiques des premiers rois et ramenant ainsi tous les princes feudataires à la Cour. C'est ce qu'on appelle atteindre lentement au résultat par l'emploi de la voie morale et en agissant sans violence. D'ailleurs, ce n'est pas là non plus l'inaction du calme impassible et imperturbable 1, comme dans les cas de $\mathrm{Hi}$ et de Tshiao de la dynastie des Theang. Par l'inaction, une diffi culté ordinaire en arrive jusqu'à causer la chute ${ }^{2}$.

TSHOU HI. - Bien que le cinquième trait nonaire, avec la dureté énergique de la positivité, la justice et la droiture, occupe la situation prééminente, cependant, en présence d'un moment d'empêchements et de difficultés, il se trouve tombé au milieu de périls. Bien qu’il possède la sympathie correcte ${ }^{3}$ du second trait hexaire, cependant, celui-ci possède la douceur malléable de la négativité et ses capacités sont faibles et insuffisantes pour traverser les dangers du moment, en y remédiant. Le premier trait nonaire, dans sa position inférieure, possède les sympathies du peuple ; toute

\footnotetext{
${ }^{1}$ Allusion à la doctrine bouddhique.

${ }^{2}$ La destruction de la dynastie.

${ }^{3}$ Correspondance sympathique conforme à la droiture.
} 
la multitude vient se grouper autour de lui. Le cinquième trait nonaire fait partie de la substance du koua simple khan $^{1}$, il possède une vertu onctueuse et bienfaisante, mais il ne peut pas en étendre et en développer l'influence ; il est considéré comme constituant le symbole de l'obstacle à l'influence. Celui qui consulte le sort, et qui s'occupe de choses de moindre importance, pourra encore, s'il conserve la droiture, en tirer un présage heureux ; s'il s'occupe de traiter les affaires graves, alors, bien que conservant la droiture, il n'évitera point le malheur ${ }^{2}$.

105.

Obstacle à l'influence ; l'étendue de son action n'est pas encore brillante.

TSHENG TSE. - L'effet bienfaisant de l'influence ne se fait pas encore sentir au-dessous de lui ; c'est parce que l'effet extérieur de la vertu ne peut pas encore être grand et brillant ; c'est l'empêchement des princes.

${ }^{1}$ Qui exprime le danger, le péril, la chute, etc.

${ }^{2}$ Affaires graves, ou grandes affaires, est presque toujours, sous la plume d'un auteur chinois, synonyme d'affaires d'État. 


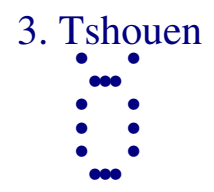

106.

Trait supérieur hexaire : monter à cheval et comme demeurer en rang ; pleurs de sang, les larmes comme ruisselant.

TSHENG TSE. - Ce trait est hexaire et, avec la malléabilité négative, il occupe le dernier rang du koua tshouen, il est au comble du péril et, sans correspondance sympathique ni accueil. Sil demeure en place il ne sera pas en paix, s'il se meut il n'a aucun lieu où il puis se aller. Il monte à cheval, veut entreprendre, et il reste comme fixé en rang sans pouvoir avancer. C'est l'excès dans le malheur et le péril, au point qu'il verse des larmes de sang et que les pleurs sont comme ruisselants ; c'est le dernier degré de la difficulté. S'il possédait l'énergie active de la positivité et sỉl avait de l'aide, l'empêchement étant déjà arrivé à son comble, il serait capable d'y remédier et de le franchir.

TSHOU HI. - Malléabilité négative sans correspondance sympathique placée à la fin de l'empêchement, en avançant, aucun lieu où aller ; rien que tristesse et crainte ; c'est pour cela que telle est l’image symbolique.

107.

Pleurs de sang, larmes comme ruisselant; comment cet état pourrait-il durer?

TSHENG TSE. - L'empêchement et la difficulté sont arrivés à leur extrême limite; il ne sait ce qu'il y a à faire et c'est pourquoi il en arrive à verser des larmes de sang. L'effet arri vé à ce degré d’intensité, est-il possible qu'il puisse durer longtemps ? Or, les koua représentent la chose ou le fait; les traits représentent le moment de cette chose ou état; on en distingue trois et, de plus, on les double ${ }^{1}$, ce qui est suffisant pour renfermer la raison d'être

\footnotetext{
${ }^{1} \mathrm{Si}$, comme je l'avance, le véritable sens du Yi king est astronomique, on peut déjà émettre une hypothèse sur la formation des koua. Le koua simple de trois traits représentant trois moments principaux de la course du soleil et de la lune sur l'horizon, la superposition des deux koua simples peut avoir pour but de représenter les trois mêmes moments au-dessus de l'horizon et les trois moments correspondants au-dessous le l'horizon. Cette hypothèse permettrait déjà de comprendre la théorie de correspondance entre les traits des koua simples
} 
de toutes choses. Présenter la chose et la développer, préciser le genre et l'éten due, c'est épuiser la série des cas possibles dans l'unive rs

inférieurs et supérieurs. Remarquons que le cinquième trait représente la «situation la plus élevée », admettons provisoirement le soleil au méridien; le second qui lui correspond représente « le sage dans une situation inférieure », par exemple, le soleil passant au méridien inférieur. 


\section{Mong}

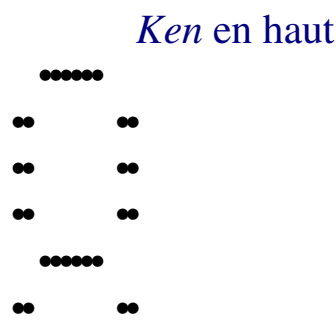

Khan en bas

108. Mong, liberté d'expansion. Ce n'est pas moi qui cherche à obtenir de l'aveuglement du jeune garçon; c'est l'aveuglement du jeune garçon qui cherche à obtenir de moi. La première fois que le sort est consulté, avertissement; une seconde ou une troisième fois entraînent la lassitude; du moment où il y a lassitude, il n'y a plus d'avertissement; avantage de la perfection.

112. Premier trait hexaire : dissiper l'aveuglement; avantage à agir en châtiant les hommes; agir pour supprimer les entraves des mains et des pieds ; s'il s'agit d'entreprendre, appréhension d'un mal futur.

$*$

114. Deuxième trait nonaire : tolérer l'aveuglement, présage heureux; s'enquérir auprès de la femme, présage heureux; l'enfant domine la famille.

$*$

116. Troisième trait hexaire : ne pas procéder au choix d'une fille; elle regarde la richesse de l'époux; ne pas avoir le corps ; aucun moyen davantage.

118. Quatrième trait hexaire : misère et aveuglement ; appréhension d'un mal. $*$

120. Cinquième trait hexaire : aveuglement du jeune garçon; présage heureux. $*$

122. Trait supérieur nonaire : frapper l'ignorant; pas davantage à agir avec violence; avantage à réprimer la violence.

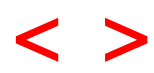


TSHENG TSE. - Mong. «L'Ordre des koua » dit : «Tshouen exprime l'idée de remplir ; tshouen c'est le comme ncement de la naissance des êtres ; lorsque les êtres naissent ils sont nécessairement incapables de discernement, c'est pourquoi ce koua est suivi du koua mong. Le koua mong exprime l'aveuglement et le manque de discernement ; c'est le jeune âge des êtres »1. Le koua tshouen exprime le commencement de la vie chez les êtres; au moment où les êtres commencent à vivre et sont dans leur premier âge, l'obscurité et l'aveuglement dans lesquels ils sont plongés ne sont pas encore dissipés ${ }^{2}$; c'est ce qui fait que le koua mong suit immédiatement le koua tshouen. Il est composé du koua simple ken, en haut, et du koua simple khan, en bas. Le koua simple ken représente la montagne, il exprime l'idée d'arrêt ; le koua simple khan représente l'eau, il exprime le dang er; au-dessous de la montagne il y a péril ; rencontrer le péril et s'arrêter ; ne point savoir où aller; telle est l'image symbolique de l'aveuglement exprimé par le mot mong. L'eau est certainement quelque chose qui change de place ; au moment où elle commence à sortir elle n'a point encore de lit pour couler, c'est pourquoi le koua est considéré comme exprimant l'idée d'aveuglement, ignorance et incertitude; lorsque le moment où l'eau coule et avance sera venu, l’idée exprimée aura le sens de liberté d'expansion.

\footnotetext{
${ }^{1}$ Il est impossible de trouver pour traduire le mot mong, un terme français qui puisse rendre toutes les acceptions dont le mot chinois est susceptible. Mong exprime le manque de lumière au figuré et dans la réalité.

${ }^{2}$ Le mot mong est expliqué par le commentaire au moyen du terme $\mathrm{H}$ dont le sens le plus archaïque est : Clarté imparfaite au moment du lever du soleil.
} 


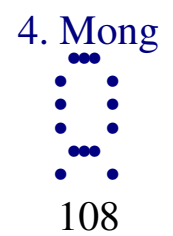

Mong, liberté d'expansion. Ce n'est pas moi qui cherche à obtenir de l'aveuglement du jeune garçon ; c'est l'aveuglement du jeune garçon qui cherche à obtenir de moi. La première fois que le sort est consulté ${ }^{1}$, avertissement ; une seconde ou une troisième fois entraînent la lassitude; du moment où il y a lassitude, il n'y a plus d'avertissement; avantage de la perfection.

TSHENG TSE. - La raison d'être des choses veut que l'aveuglement soit dissipé et éclairé ; tel est le sens du terme liberté d'expansion. Lorsque les aptitudes et les moments indiqués par le koua mong concordent, il en résulte une voie naturelle de liberté d'expansion. Le cinquième trait hexaire représente celui de qui dépend l'aveuglement, tandis que le second trait nonaire représente celui qui dissipe l'aveuglement. Le mot « moi » désigne le second trait ; ce second trait n'est pas le maître du koua mong, c'est-à-dire celui de. qui dépend l'aveuglement. Du moment où le cinquième trait cède passivement le pas au second, c'est que le second est celui qui dissipe l'aveuglement et c'est pourquoi il est parlé du second comme sill était le maître du koua. Ce n'est pas moi qui cherche à obte nir de l'aveuglement du jeune garçon, c'est l'aveuglement du jeune garçon qui cherche à obtenir de moi 2 ; le cinquième trait occupe la situation prééminente, il possède les vertus de la douceur malléable et de l'obéissance, et, au moment où il se trouve dans l'état d'aveuglement du jeune âge, il est en corresponda nce sympathique selon la droiture avec le second trait ; enfin la vertu de justice est la même chez tous deux. Il peut employer la voie morale du second trait pour dissiper son aveuglement. Le second, avec les vertus de la dureté énergique et de la justice, se trouve dans une position inférieure ; c'est celui vers qui le premier se tourne avec confiance et il convient qu'il s'observe et se préserve par la voie morale. Il doit attendre que le prince s'adresse à lui avec une complète franchise d'intention, et ensuite répondre à son appel de façon que le prince puisse profiter de sa voie morale. Ce n'est pas moi qui cherche à obtenir de l'aveuglement du jeune garçon, c'est l'aveuglement du jeune garçon qui l'amène pour chercher à obtenir de moi ${ }^{2}$. Consulter le sort, c'est étudier le sort pour prendre une décision. La première fois que le sort est consulté, avertissement; cela exprime que celui qui est consulté avec simplicité et parfaite sincérité d'intention doit répondre par un avertissement. Une seconde et une troisième question amèneront la lassitude et le dégoût, de

\footnotetext{
${ }^{1}$ Consulter le sort par la divination au moyen des brins d'herbe.

${ }^{2}$ Il paraitrait plus naturel de dire «le jeune garçon aveuglé », mais la position respective des deux mots défend cette lecture.
} 
sorte qu'il n'y aura plus d'aver tissement. La voie rationnelle de la dissipation de l'aveuglement est avantageuse par la pureté de la droiture. Enfin, bien que le second trait comporte l'énergi e et la justice, cependant, il occupe un rang négatif, c'est pour cela qu'il convient qu'il y ait avertisse ment ${ }^{1}$.

TSHOU HI. - Ken est encore le nom d'un koua simple de trois traits ; ce koua simple est composé d'une positivité arrêtée ${ }^{2}$ au-dessus de deux négativités ; aussi sa vertu, ou qualité, est l'arrêt, ou station, et son image symbolique est la montagne. Mong signifie : «Le soleil sur l'horizon sans clarté. » $\mathrm{Au}$ début de la vie, chez les êtres, l'aveuglement et le manque de discernement ne sont pas encore éclairés. Dans le koua parfait mong, puisque le koua simple khan rencontre le koua simple ken, sous la montagne il y a danger; c'est un terrain obscur et mal éclairé. Au-dedans péril, au-dehors arrêt ; c'est l'idée d'aveugle ment et de manque de discernement, et c'est pour cela qu'il est appelé mong. Après le terme «liberté d'expansion » et en continuant, le texte est une formule divinatoire. Le second trait nonaire est le maître du koua simple intérieur ; il emploie la dureté énergique et se maintient dans la justice, il peut dissiper l'aveuglement d'autrui, et comme avec le cinquième trait hexaire les qualités de négativité et de positivité se correspondent sympathiquement, il en résulte que celui qui rencontre ce koua en consultant le sort jouit de liberté d'expansion. Le mot moi désigne le second trait. L'aveuglement du jeune garçon, c'est la jeunesse dépourvue de discernement et de lumière ; cette expression désigne le cinquième trait. Si celui qui consulte le sort est une personne éclairée, c'est que quel qu'un doit s'adresser à lui et que la liberté d'expansion sera pour cette dernière person ne; si celui qui consulte le sort est encore plongé dans les ténèbres de l'ignorance, c'est que lui -même doit chercher à obtenir quelque chose d'autr ui et que la liberté sera pour lui-même. Lorsque quelqu'un nous interroge, il convient d'examiner l'opportunité ou l'inopportunité de la question et d'y répondre. Lorsque nous interrogeons quelqu'un, nous devons rechercher la simplicité et l'unité pour réduire nos interrogations. Mais qu'il s'agisse de l'intelligence s'occupant de ceux qui sont aveuglés, ou de l'aveuglement travaillant directement à sa propre réforme, il y a toujours avantage dans l'emploi de la droiture.

109.

Le commentaire de la formule déterminative dit: Mong, en bas de la montagne, il y a péril ; péril et immobilité ; aveuglement.

\footnotetext{
${ }^{1}$ Dans ce cas le terme avertissement » comporte l'idée de conseil donné à l'avance pour prévenir des suites fâcheuses, mais son emploi implique l'idée d'un défaut déjà existant.

${ }^{2}$ Elle est arrêtée puisqu'elle est au rang supérieur et n'a plus de place pour avancer.
} 
TSHOU HI. - Explication du nom du koua au moyen des images symboliques et des vertus des koua simples; il y a deux sens.

110.

Mong, liberté ; puisqu'il y a liberté, l'action et le moment concordent avec précision. Ce n'est pas moi qui cherche à obtenir de l'aveuglement du jeune garçon, c'est l'aveuglement du jeune garçon qui cherche à obtenir de moi : les deux tendances se correspondent mutuellement. La première fois que le sort est consulté, avertissement; par la dureté énergique et la justice. Une seconde et une troisième fois entraînent la lassitude; du moment où il y a lassitude, il n'y a plus d'avertissement, aveuglement de la lassitude; l'aveuglement employé à maintenir la droiture, c'est l'œuvre de la sainteté.

TSHENG TSE. - En bas de la montagne il y a péril, le péril étant à l'intérieur il est impossible de rester en place ; au-dehors, il y a arrêt et on ne peut avancer; on ne sait pas encore ce qu'il y a à faire, et c'est pourquoi le koua est considéré comme exprimant le sens d'obscurité et d'aveuglement. La liberté dans l'aveuglement, c'est l'action et le moment en concordance exacte par suite de la liberté d'expansion des influences. La p ossibilité de liberté dans l'aveuglement résulte de la pratique de la voie rationnelle de liberté des influences. Ce qu'on entend par voie rationnelle de liberté d'expansion, c'est la concordance précise avec le moment; par moment on entend l'instant où on possède la sympathie du prince ; par concordance exacte on entend qu'il s'agit de se placer en s'accordant avec la justice ; l'accord existant avec la justice, le moment sera arrivé. Ce n'est pas moi qui cherche à obtenir de l'aveuglement du jeune garçon, c'est l'aveugle ment du jeune garçon qui cherche à obtenir de moi ; les tendances se correspondent sympathiquement. Le second trait, avec la sagesse que donnent l'énergie et l'intelligence, est placé dans un rang inférieur; le cinquième, avec l'aveugle ment du jeune âge occupe le rang supérieur. Ce n'est pas le second qui cherche à obtenir du cinquième ; en effet, les tendances du cinquième le font correspondre sympathiquement au second. Le sage se trouvant dans l'infériorité, comment pourrait-il s'avancer de luimême pour chercher à obtenir du prince ? S'il sollicitait le premier, il n'y aurait aucune raison pour que le prince pût avoir confiance en lui et l'employer. C'est à cause de cela que les hommes de l'anti quité attendaient que les princes se fussent montrés absolument respectueux, qu'ils eus sent accompli tous les devoirs rituels de convenance, et qüils ne s'avançaient qu'ensuite. Ce n'était point qu'ils voulussent se grandir et s'exalter eux-mêmes, car ils n'exaltaient que la vertu et ne se plaisaient que dans la morale, et s'ils n'avaient point agi ainsi, ils eus sent été au-dessous de ce qu'ils avaient à faire. La première fois que le sort est consulté est une expression qui 
indique qu'on vient avec une sincérité absolue pour chercher à faire mettre un terme à son propre aveuglement; dans ce cas ; il convient donc d'employer la voie de la justice et de l'énergie, d'avertir, et de dissiper cet aveuglement. Une seconde et une troisième fois, c'est-à-dire un grand nombre de fois; les intentions de celui qui vient consulter sont multiples, elles ne peuvent être simples et se concentrer sur un seul sujet, de sorte qu'il en résulte de la négligence et il ne convient plus d'avertir. Si on avertissait en répondant, la réponse ne pourrait plus être reçue avec confiance, elle ne constituerait plus qu'une répétition inutile et c'est pour cela que le commentaire dit «aveuglement de la lassitude »; celui qui demande et celui qui répond sont également las et fatigués de répétitions. La formule du koua dit : Avantage de la perfection ; le commentaire de la formule déterminative expose de nouveau le sens de ces termes pour bien éclaircir quil ne s'agit pas seulement d'un avertissement adressé au sujet du second trait, mais bien réellement de la voie logique à suivre pour soigner et guérir l'aveuglement. L’ignorance pas encore dissipée est appelée mong, ou aveuglement; employer cette ignorance primordiale non encore dissipée et entretenir la droiture, c'est là accomplir l'œuvre de la sainteté. Dissiper et en suite défendre ce qui doit être interdit, pour arriver à son extinction et l'empêcher de prendre le dessus, développer la droiture dans l'aveuglement, c'est l'extrême excellence dans l'étude. Parmi les six traits du koua mong, les deux traits positifs sont ceux qui remédient à l'aveuglement et les quatre traits négatifs représentent ceux qui sont plongés dans cet aveuglement.

TSHOU HI. - Explication de la formule du koua au moyen de la substance de ce même koua parfait. Le second trait nonaire emploie la voie logique résultant de la possibilité de liberté d'expansion; il dissipe l'aveuglement des hommes, et, de plus, il se conforme à l'exacte opportunité du moment; c'est -à-dire que s'il s'agit, par exemple, des sujets traités dans le texte qui suit, il emploie toujours la liberté d'expansion et agit conformément à ce qui est possible. Les tendances se correspondent sympathiquement: le second trait possède l'intelligence et l'énergie ; le cinquième, plongé dans les ténèbres, est doux et malléable, de sorte que le second n’nvoque pas le cinquième, tandis que le cinquième invoque le second. Leurs tendances sont naturellement sympathiques. Les mots «par la dureté énergique et la justice » signifient : en employant l'énergie et la justice ; c'est à cause de cela quil peut avertir et qu’il agit suivant des principes déterminés. Le terme tra duit par « lassitude » exprime la répétition; si le sort est consulté une seconde et une troisième fois, il en résultera que celui qui interroge éprouvera certainement de la lassitude et que celui qui répond en éprouvera également. L'aveuglement de l'ignorance employé à la conservation de la droiture, c'est là l'ouvre de la sainteté et c'est ce qui explique le sens des mots « avantage de la perfection ». 
Le commentaire de la formule symbolique dit: En bas de la montagne sort une source: mong, incertitude de l'ignorance aveugle; l'homme doué emploie la décision dans ses actions pour entretenir sa vertu.

TSHENG TSE. - En bas de la montagne sort une source; elle sort et rencontre un obstacle ; elle n'a pas encore d'écoulement et de direction : c'est l'image symbolique de l'aveuglement de l'ignorance, comme l'aveuglement de l'ignorance de l'homme dans son premier âge, quand il ne sait pas encore vers quoi il doit tendre. L'homme doué considère l'image symbolique de cette ignorance et il en conclut l'emploi de la décision dans ses actions et le soin du développement de ses facultés. Considérant que cette eau sort et ne peut pas encore couler librement, il emploie la décision dans ce quill a à faire ; il la considère alors qu'elle n'a pas encore de direction pour couler vers un point quelconque, et il en conclut la nécessité d'entretenir les facultés de son intelligence ${ }^{1}$.

TSHOU HI. - La source, c'est l'eau qui comme nce à sortir; elle doit nécessairement courir et augmenter peu à peu.

${ }^{1}$ Littéralement «brillante vertu». Terme technique qui désigne l'ensemble des facultés attribuées à l'âme et à l'esprit. Voir la « Grande Étude ». 


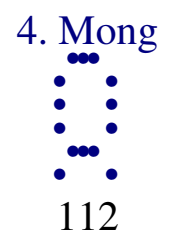

Premier trait hexaire : dissiper l'aveuglement; avantage à agir en châtiant les hommes; agir pour supprimer les entraves des mains et des pieds ; s'il s'agit d'entreprendre, appréhension d'un mal futur ${ }^{1}$.

TSHENG TSE. - Le premier trait, avec sa négativité et son manque de lumières, occupe le rang inférieur ; c'est l'ignorance aveugle du bas peuple et la formule traite de la voie rationnelle pour la dissiper. Pour dissiper l'aveuglement du bas peuple, il convient de montrer clairement les lois pénales et les défenses pour l'avertir, afin quỉl sache les craindre ; immédiatement ensuite il faut continuer en l'instruisant et en le guidant. Depuis l'antiquité, les saints rois, dans leur régime de gouvernement, instituèrent des peines et des châtiments, afin de maintenir la multitude dans l'ordre, et ils éclairèrent l'action transformatrice de l'enseignement, afin d'améliorer les moars. Les supplices et les châtiments établis, l'action transformatrice de l'enseignement pouvait ensuite se produire. Bien que l'homme saint prise la vertu et n'estime point les supplices, cependant jamais il n'adopte exclusivement un de ces deux moyens en abandonnant complètement l'autre. Aussi, au moment où commencent les institutions politiques, c'est l'établissement des règles qui précède. Au début du traitement de l'ignorance, faire sentir la puissance de l'autorité par les peines, c'est le moyen de délivrer les hommes des entraves de l'ignorance et de l'aveuglement. Les deux caractères traduits par « entraves pour les mains et les pieds $»^{2}$ expriment des liens qui retiennent. Si on ne supprime point les entraves de l'ignorance et de l'aveuglement, l'enseignement du bien n'a point de voie pour pénétrer; au contraire, quand on a employé les peines et les prohibitions pour dominer, bien que le cour des hommes n'en soit pas encore arrivé à estimer le bien pour lui-même, il est néanmoins contraint de craindre la puissance de l'autorité et à obéir, sans oser don ner libre cours aux désirs qui naissent de l'ignorance et de l'aveuglement. Ensuite, peu à peu, les hommes peuvent apprendre à connaître la voie du bien et corriger les mauvaises inclinations de leur cour. Aussi il devient possible de changer les moars et d'améliorer les coutumes. Si les supplices sont exclusivement employés comme moyen de gouvernement, alors, bien que la crainte puisse coexister avec l'ignorance, cependant, finalement l'aveuglement ne peut pas être dissipé ; si au contraire ils sont dispensés de ces peines et ne subissent aucune

\footnotetext{
${ }^{1}$ La construction du texte est obscure. Littéralement il faudrait lire «avantage à l'employer pour punir les hommes...».

${ }^{2}$ Ces deux mots sont les noms de sortes de ceps, ou cangues pour les mains ou pour les pieds.
} 
honte, l'effet transformateur du régime de gouvernement ne peut pas prévaloir et se compléter. C'est pour cela que, dans ces conditions, entreprendre quelque chose doit donner lieu à l'appréhension d'un mal futur.

TSHOU HI. - Puisque ce trait occupe le rang inférieur avec les qualités de la négativité, il exprime l'extrême aveuglement. Celui qui consulte le sort et qui rencontre ce trait doit dissiper l'aveuglement ; mais la voie rationnelle pour le dissiper comporte l'avertissement d'un châtimen t douloureux suivi de l'abandon momentané du sujet, afin de voir les résultats obtenus. Si on continue ${ }^{1}$ sans laisser le sujet à lui-même, il en résultera de la confusion et l'appréhension de conséquences fâcheuses. C'est un avertissement à celui qui consulte le sort, pour le prévenir qu'il doit en être ainsi.

113.

Avantage à agir en châtiant les hommes, afin de maintenir exactement la règle.

TSHENG TSE. - Au moment où commence le soin de remédier à l'aveuglement, on le limite et on fixe les barrières qui ne doivent pas être franchies; on indique clairement les fautes et les peines, on précise avec rectitude quelles sont les règles. On conduit les hommes dans ces limites, on les dirige par ces règles, et peu à peu on arrive à les transformer. On a émis des doutes sur la question de savoir si, au début de la dissipation de l'aveu glement, l'emploi prématuré des peines ne constitue pas ce qu'on appelle « frapper sans instruire ». C'est là ne pas savoir qu'établir des règles, instituer des pénalités, constituent précisément les moyens d'instruire. En effet, ceux qui, dans les temps modernes, ont discouru sur les institutions pénales ne se souvenaient plus que l'instruction et la transfor mation s'y trouvent inclus.

TSHOU HI. - Au début de la dissipation de l'aveuglement, il est indispensable de préciser strictement les règles. La répression par les avertissements correctifs est le moyen de maintenir strictement les règles.

\footnotetext{
1 Tsheng Tse termine son commentaire par une conclusion arbitraire ; Tshou Hi, de son côté, donne à l'avant-dernier caractère du texte, wang aller, entreprendre, le sens de « continuer l'emploi des peines ». Si son interprétation est exacte, il faudrait lire la formule : Dissiper l'aveuglement, avantage à employer les châti ments ; pour supprimer les entraves des mains et des pieds ; en continuant, appréhension.
} 


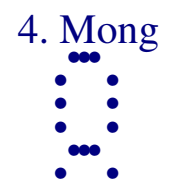

114.

Deuxième trait nonaire : tolérer l'aveuglement, présage heureux; s'enquérir auprès de la femme, présage heureux; l'enfant domine la famille.

TSHENG TSE. - Tolérer, c'est supporter, contenir. Le second trait se trouve dans une période d'aveuglement ; il a les capacités de la dureté énergique et de l'intelligence, et lui et le prince représenté par le cinquième trait hexaire se correspondent mutuellement avec sympathie. La vertu de justice est encore la même chez tous deux ; ce sont ceux qui, dans le moment en question, sont les détenteurs de l'autorité. Il doit absolument étendre et augmenter sa tolérance et avoir de la pitié et de l'indulgence pour ceux qui sont grossiers et sans instruction. Alors il pourra dissiper l'aveuglement de l'univers et accomplir l' auvre de l'extinction de l'aveuglement. La voie à suivre est vaste, ses effets sont considérables et, dans ces conditions, ce sera un présage heureux, dans le koua il n'y a que deux traits positifs. Le trait supérieur nonaire possède la dureté énergique, mais avec excès ; le second trait nonaire, seul, possède les vertus de l'énergie et de la justice et il correspond sympathiquement au cinquième ; c'est celui qui est employé dans le moment et qui seul est éclairé. S'il se fie à son intelligence et s'appuie exclusivement sur son autorité personnelle, sa vertu sera sans grandeur. Aussi alors même qu'il s'agit de l'ignorance douce et malléable de la femme, il convient encore de prendre ce qu'elle a de bon, de sorte que l'intelligence s'agrandit. De plus, c'es t parce que tous les traits sont négatifs que la formule parle de femme. Dans l'univers, personne n'atteint à la sainteté de Yao et de Shouen, cependant eux-mêmes interrogeaient journellement des gens du bas peuple, afin de prendre ce qu'ils pouvaient avo ir de bon. Le second trait peut tolérer et s'enquérir, de sorte qu'il est capable de mettre les affaires du prince en ordre, comme un fils qui dirige la famille. Du moment où le cinquième trait a les qualités de la malléabilité négative, il en résulte que le mérite de la dissipation de l'aveuglement incombe tout entier au second trait. S’il s'agit d'une famille, le cinquième trait représente le père et le deuxième trait représente l'enfant. Le second trait pouvant présider à l'œuvre de la direction des ignorants, il représente donc le fils qui est capable de diriger la famille.

TSHOU HI. - Le second trait nonaire, avec sa dureté énergique positive, représente le maître du koua simple inférieur ; il dirige d'une façon générale la troupe des négativités; c'e st celui qui est actuellement investi de l'autorité nécessaire pour dissiper l'aveugle ment. Mais ce qu’il régit étant considérable, comme le naturel des êtres n'est pas uniforme, il ne doit pas toujours employer des moyens absolument identiques, et la vertu de ce trait, qui est 
l'énergie sans excès, constitue l’image symbolique de la nécessité de tolé rance. De plus, puisque positif, il supporte un trait négatif, il constitue l'image symbolique de s'enquérir auprès de la femme. Enfin, occupant la situation inférieure, il peut supporter le poids des affaires du supérieur, ce qui constitue l'image symbolique du fils capable de diriger la famille. Aussi, si celui qui consulte le sort possède cette vertu et se trouve dans un cas analogue, il en sera de même, et c'est un présage heureux.

115.

L'enfant domine la famille ; l'énergie et la douceur se donnent la main.

TSHENG TSE. — L'enfant qui domine et dirige la famille agit en vertu de la confiance du père qui lui délègue son autorité exclusive ; ce qui fait que le second trait peut présider à l'œuvre de la dissipation de l'aveuglement, c'est que la confiance du cinquième lui délègue l'autorité absolue. Les sentiments d'énergie et de douceur du second et du cinquième trait s'attirent réciproquement ; aussi, il réussit à parcourir la voie de l'énergie et de la justice et à accomplir l'ouvre de la dissipation de l'aveuglement. Si les sentiments du supérieur et de l'inférieur ne les attiraient point l'un vers l'autre, comment le second trait, malgré sa justice et son énergie, pourrait-il prendre la direction des affaires?

TSHOU HI. - Fait ressortir la correspondance sympathique du second et du cinquième trait. 


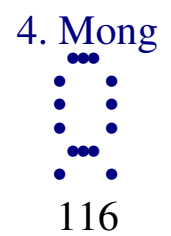

Troisième trait hexaire : ne pas procéder au choix d' une fille; elle regarde la richesse de l'époux; ne pas avoir le corps ; aucun moyen davantage.

TSHENG TSE. — Le troisième trait, avec sa malléabilité négative, est placé dans l'aveu glement et l'obscurité ; il est sans justice et sans droiture : c'est la fille agissant vicieuse ment. Celui qui lui correspond sympathiquement selon la droiture est le trait supérieur ; elle ne peut le suivre au loin. Près d'elle elle voit le second trait nonaire qui représente celui vers qui se porte la foule des ignorants aveuglés et qui possède la perfection relative au moment présent, ce qui fait qu'elle abandonne ses sympathies correctes pour le suivre. C'est la fille qui regarde la richesse de l'époux. Lorsqu'une jeune fille suit un homme, ce doit être conformément à la droiture et aux règles rituelles ${ }^{1}$; celle-ci regarde à la plus ou moins grande quantité d'or possédée par l'homme, elle en est réjouie et le suit ; on ne peut garantir qu'on possédera sa personne ${ }^{2}$. Il n'y a rien à entreprendre qui puisse être avantageux.

TSHOU HI. - Le troisième trait est hexaire, négatif, et possède la douceur malléable; il est dépourvu de justice et de droiture ; c'est l'image symbolique de la fille qui regarde à la richesse de l'époux et dont on ne peut posséder la personne. Si celui qui consulte le sort rencontre ce trait, il doit, sil choisit une fille, tomber sur une personne de ce genre ; rien ne peut lui être avantageux. Il est question de l'époux qui possède de l'or ; en effet, elle se laisse corrompre par l'or et le choisit, et c'est le cas de Tsieou Hou de l'état de Lou.

117.

Ne pas procéder au choix d'une fille; ses mours ne sont pas réservées ${ }^{3}$.

\footnotetext{
${ }^{1}$ Lorsqu'elle épouse un homme.

${ }^{2}$ Qu'elle respectera ses devoirs et sera fidèle.

${ }^{3}$ Le texte porte shouen, obéissance, soumission. Voir plus loin l'explication assez libre de Tshou Hi.
} 
TSHENG TSE. - La fille qui est dans de telles conditions a des mours basses et dépravées, elle est sans soumission, on ne doit pas la choisir.

TSHOU HI. - À la place du caractère shouen, soumission, il faut lire shen, circonspection, réserve ; en effet, dans l'antiquité les deux caractères shen et shouen s'employaient indifféremment l'un pour l'autre (?). Ceci résulte d'ailleurs d'une citation de Souen Tse lisant shen mo au lieu de shouen mo. Enfin, mours réservées se rapporte bien mieux à l'idée exprimée dans le texte. 


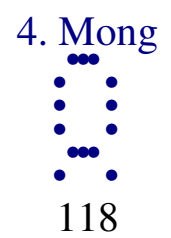

Quatrième trait hexaire : misère et aveuglement ; appréhension d'un mal ${ }^{1}$.

TSHENG TSE. — Le quatrième trait, à cause de sa douceur négative et de son ignorance aveugle, étant sans assistance ni aide de la part de l'intelligence énergique, n'ayant par lui-même aucun moyen de dissiper son propre aveuglement, est celui qui est misérable par son aveuglement ; la probabilité de l'appréhension d'un mal futur est on ne peut plus évidente. Le terme traduit par appréhension pour l'avenir exprime l'insuffisance ; cela veut dire que quelque chose doit lui manquer.

TSHOU HI. - Déjà éloigné de la positivité, il est encore sans correspondance sympathique selon la droiture; il constitue limage symbolique de la misère dans l'aveuglement. Si celui qui consulte le sort est dans un tel cas, il devra éprouver de la confusion et l'appré hension d'un mal futur; s'il peut invoquer les vertus de l'énergie et de l'intelligence et se rapprocher de celui qui les possède, il pourra se soustraire aux conséquences de ce pronostic.

119.

Appréhension causée par la misère et l'aveuglement; seul il s'éloigne du vrai (she).

TSHENG TSE. - Dans un moment d'aveuglement, l'énergie de la positivité est ce qui dissipe cet aveuglement. Le quatrième trait est malléable et négatif et extrêmement éloigné de l'énergie ; c'est donc un homme grossier et sans intelligence qui ne se rapproche pas du sage. Il n'a aucun moyen d'arriver à s'éclairer, donc c'est celui qui, misérable par son aveuglement, doit éprouver de la confusion et l'appréhension d'u n mal futur. Puisque seul il est éloigné de l'homme sage et intelligent, il ne peut pas s'approcher de la sagesse ; puisqu'il en est arrivé jusqu'à la misère, tout le porte à appréhender un mal futur. Le caractère she du texte, réalité, désigne l'énergie p ositive.

${ }^{1}$ Les commentateurs lisent : misère de l'aveuglement ; en réalité le texte porte : aveuglement de la misère, ou mieux : aveuglement misérable. J'ai choisi une lecture admissible et qui comporte les deux sens. 
TSHOU HI. - Le caractère she du texte, doit être lu $t s h i$.

DÉFINITIONS DIVERSES. - Khong Shi Ying Ta dit: C'est de la positivité que dépendent la vie et la respiration et c'est pour cela qu'on l'appelle le vrai (she); de la négativité dépendent l'évanouissement et la dissolution, elle ne peut donc pas être appelée le vrai, ou réel. Heang Shi Ngan She dit : Le premier et le troisième traits sont voisins du second trait nonaire ; le cinquième est proche du trait supérieur nonaire; le troisième et le cinquième correspondent chacun à un trait positif. Pour le quatrième trait, seul, le voisinage et la correspondance sont également des négativités et c'est pour cela que le texte dit que seul il s'éloigne du vrai. 


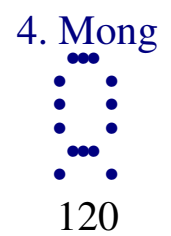

Cinquième trait hexaire : aveuglement du jeune garçon ; présage heureux.

TSHENG TSE. - Le cinquième trait, avec ses caractères de douceur malléable et de soumission, occupe la situation du prince; en bas, il correspond sympathiquement au second. À cause de ses vertus de douceur et de justice, il investit de l'autorité celui dont les qualités sont l'énergie et l'intelligence, ce qui est suffisant pour remédier à l'aveugle ment de l'univers, donc c'est un présage heureux. Le terme «jeune garçon » est choisi comme exprimant que l'aveuglement n'est pas encore dissipé et qu'il reçoit l'instruction d'autrui. Si le prince peut, avec une parfaite sincérité d'intention, conférer l'autorité au sage, afin que celui-ci accomplisse l'ouvre, quelle différence y -a-t-il entre ce cas et celui où il l'accomplit lui -même ?

TSHOU HI. - La douceur malléable et la justice occupent la situation prééminente; en bas, correspondance sympathique avec le second trait nonaire. Simplicité parfaite pas encore altérée. Pouvant écouter autrui, son image symbolique est celle de l'aveuglement du jeune garçon, et le sens divinatoire étant tel, le présage est heureux.

121.

Présage heureux de l'aveuglement du jeune garçon; soumission par humilité.

TSHENG TSE. - Faire abstraction de soi-même pour suivre les conseils d'autrui ; le terme soumission comporte l'idée de suivre, écouter. Abaisser son propre jugement et demander pour obtenir de ce qui est inférieur à soi-même ; humilité de l'inférieur. Pouvoir agir ainsi, c'est s'accorder avec harmonie aux voarx de l'univers. 


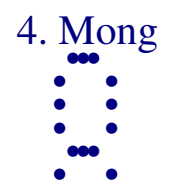

122.

Trait supérieur nonaire : frapper l'ignorant ; pas davantage à agir avec violence; avantage à réprimer la violence.

TSHENG TSE. - Un trait nonaire occupe le dernier rang du koua mong ; c'est le moment du maximum de l'aveuglement. Du moment où l'aveuglement et la grossièreté ignorante de l'homme sont arrivés à un point extrême, comme lorsque le peuple des Miao se refusait à se laisser guider, où cet homme agit avec violence, sans frein et comme un brigand, il convient de le battre et de le dompter. Mais ce trait nonaire occupe le rang supérieur; sa dureté énergique est extrême et il est sans justice, c'est pourquoi la formule avertit quill n'y a point avantage à agir par la violence ; donner un libre cours à la dureté et à la violence, c'est agir en brigand (comme dit littérairement le texte). Par exemple, l'empereur Shouen réduisant le pays des Miao, Tsheou Kong détruisant les trois rebelles, c'est toujours la répression de la violence. L'empereur Tshin et l'emper eur Yu de la dynastie des Han, portant partout la guerre et la destruction, représentent l'action par la violence.

TSHOU HI. - Puisque la dureté énergique occupe le rang supérieur, elle réprime l'aveuglement avec excès de dureté et c'est pour cela que ce trait constitue l'image symbolique de l'action de frapper l'ignorant. Toutefois l'expression comporte nécessaire ment l'idée d'excès ; l'action corrective est absolument trop sévère ; il en résulte forcément que, au contraire, le résultat sera nuisible. En se bornant à réprimer la séduction extérieure, afin de préserver intacte l'innocence pure, il en résultera que bien quill y ait excès de sévérité, l'effet obtenu sera encore convenable et c'est pour cela que la formule avertit ainsi celui qui consulte le sort; il en est de même en toute chose, il ne s'agit pas seu lement du cas où il est question d'éclairer les hommes en les instruisant.

123.

Avantage à agir pour réprimer la violence ; le supérieur et l'inférieur s'y conforment. 
TSHENG TSE. — Avantage à agir pour réprimer la violence ; le supérieur et l'inférieur s'y conforment tous deux. Le supérieur ne se montre pas trop cruel, l’inférieur est frappé et délivré de son aveuglement ; tel est le sens de l'expression « réprimer la violence ».

TSHOU HI. — Réprimer la violence en employant l'énergie ; le supérieur et l'inférieur se conforment chacun à sa voie. 


\section{Su}

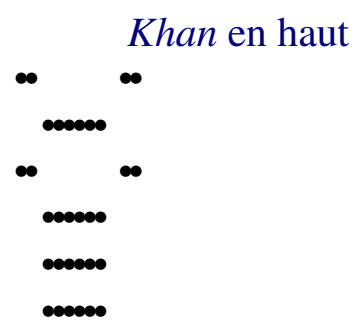

Khien en bas

124. Su : attente; avoir foi ; Liberté éclatante ; présage heureux de la perfection ; avantage à traverser un grand cours d'eau.

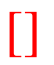

128. Premier trait nonaire : attendre dans la plaine; avantage à employer la persévérance; pas de culpabilité.

$*$

130. Second trait nonaire : attendre sur le sable ; il y a quelques légères observations verbales; à la fin bonheur.

$*$

132. Troisième trait nonaire : attendre dans la boue; il en résulte que la culpabilité survienÉ

*

134. Quatrième trait hexaire : attendre dans le sang ; sortir du gouffre.

$*$

136. Cinquième trait nonaire : attendre au milieu des vins et de la nourriture; présage heureux de la perfection.

$*$

138. Trait supérieur hexaire : entrer dans une caverne; il y a trais hommes, hôtes pas invités, qui viennent ; en les respectant, à la fin, bonheur.

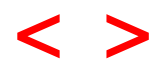


TSHENG TSE. - Su. «L'Ordre des koua » dit : «Le koua mong exprime les ténèbres de l'ignorance, c'est le jeune âge des êtres. Pendan t le jeune âge des êtres il est indispensable de les nourrir, c'est pourquoi le koua mong est suivi par le koua su. Le koua su exprime la voie naturelle de l'acte de boire et de manger. » Or, pendant le jeune âge des êtres, il faut nécessairement les soigner et les nourrir, pour quils se développent complètement. Ce qui est nécessaire pour nourrir les êtres, c'est le boire et le manger, et c'est pour cela que ce commentaire dit que le koua su exprime la voie naturelle de l'action de boire et de manger. Le nuage ${ }^{1}$ monte en haut $\mathrm{du}$ ciel $^{2}$; il y a image symbolique de la condensation de l'humidité dans l'air. Le boire et le manger sont ce qui profite aux êtres et les fait croître ${ }^{3}$, de sorte que le koua su représente la voie rationnelle de l'action de boire et de manger et c'est ce qui fait qu'il suit immédiatement le koua mong. L’idée fondamentale exprimée dans ce koua, c'est le sens de nécessité d'attendre ; l'Ordre des koua choisit comme exemple et cite la plus impérieuse de toutes les nécessités. La nature du koua simple Khien, qui est l'activité, l'oblige à avancer ; dans le cas actuel, ce koua simple est placé au-dessous du danger représenté par le koua simple khan ; le danger constitue pour lui un empêchement, de sorte quil est obligé d'attendre et qu'i 1 n'avance qu'ensuite.

\footnotetext{
${ }^{1}$ Figuré par le koua simple khan.

${ }^{2}$ Figuré par le koua simple khien.

${ }^{3}$ Dans le texte chinois c'est le même mot qui exprime, dans ce passage, les idées d'humidité, arrosement des végétaux et de substance qui profite à l'homme par ses sucs. Le nom du koua su exprime surtout la nécessité ; par extension, le sens d'attendre ce qui est nécessaire.
} 


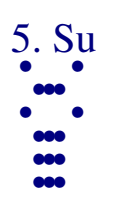

124.

Su : attente; avoir foi ; Liberté éclatante ; présage heureux de la
perfection ; avantage à traverser ${ }^{1}$ un grand cours d'eau.

TSHENG TSE. - Su : nécessité d'attendre. En en parlant au point de vue des deux substances, l'activité et l'énergie du koua simple khien avance en montant et rencontre le danger ; il n'y a pas encore possibilité d'avancer, ce qui constitue donc le sens de «nécessité d'attendre ». En en parlant d'après les propriétés des koua simples, le cinquième trait occupe la situation du prince, c'est de lui que dépend la nécessité d'attendre. Il possède les vertus d'activité, d'énergie, de justice et de droiture et il est plein de véracité et de bonne foi en lui-même ${ }^{2}$. Étant plein de véracité en lui-même, il est de bonne foi et confiant ; étant de bonne foi et confiant, son intelligence est éclatante et il peut comprendre et pénétrer librement; il possède une droiture parfaite et le présage est heureux. Dans ces conditions et attendant, que ne pourrait-il pas traverser $^{3}$ ? Bien qu'il y ait danger, rien ne lui est impossible, et c'est pourquoi il a avantage à traverser un grand cours d'eau. L'expression «présage heureux de la perfection » signifie quelquefois que du moment où il y a perfection, il y a d'ailleurs présage heureux ; d'autres fois elle signifie que si on s'accorde à la droiture, il y aura un présage heureux ; il y a lieu de distinguer.

TSHOU HI. - Su : attendre. Le koua simple khan rencontré par la koua simple khien; khien : activité ; khan : danger. La dureté énergique rencontre le danger et elle n'avance pas prématurément, ce qui la mènerait à tomber dans le danger, d'où le sens d'attente. La foi, c'est la confiance ou sincéri té intérieure. Puisque le cinquième trait nonaire du koua fait partie de la substance du koua simple khan et qu'il est plein au milieu, puisqu’il possède l'énergie de la positivité, la justice et la droiture, et qu'enfin il occupe la situation prééminente, il constitue l'image symbolique d'avoir foi et de s'accorder à la droiture. Le koua simple khan, c'est-à-dire l'eau, se trouve devant ; le koua simple khien, c'est-à-dire la force active, la contemple et la surveille, ce qui donne l'image symbolique d'êtr e sur le point de traverser

\footnotetext{
1 Traverser à pied, à gué.

${ }^{2}$ Les termes du texte ont tous plusieurs sens, de sorte quill est impossible de rendre en français les véritables jeux de mots qui constituent ces déductions, le trait est «plein au milieu » et les deux caractères tshong she, qui expriment cette condition, signifient en même temps « sincère en dedans ».

${ }^{3}$ Le sens primitif du mot est «traverser à gué ou à la nage »; pris au figuré il exprime l'idée de traverser un danger, une situation périlleuse en remédiant aux inconvénients du moment.
} 
l'eau et de ne pas avancer à la légère. Aussi, si celui qui consulte le sort a quelque chose à attendre et peut avoir confiance, il en résultera pour lui une éclatante liberté d'action ; si, de plus, il se conforme à la droiture, ce sera un présage heureux et il aura avantage à traverser un grand cours d'eau. Par la fermeté inébranlable dans la droiture, il n'est rien qui soit sans avantage et c'est surtout dans le cas où il s'agit de traverser un grand cours d'eau qu'il est extrêmement précieux de savoir attendre, parce qu'alors le désir n'entraîne pas prématurément et ne fait pas braver témérairement le danger.

DÉFINITIONS DIVERSES. -Heang Shi Ngan She dit : attendre, ce n'est pas rester indéfiniment sans avancer ; c'est se te nir à la réalité et, rencontrant un péril, attendre pour n'avancer qu'ensuite. L'attente a toujours lieu parce que, dans la circonstance en question, il y a une raison réelle qui oblige à attendre. Lorsque réellement nous avons à aller en avant, mais que nous attendons un peu, il faut absolument qu'il en existe une raison manifeste et clairement compréhensible ; si cette raison n'existait pas, pourquoi y aurait-il lieu d'attendre ? C'est pour cela que la formule dit que dans ce koua su il y a foi et liberté éclatante. Cette liberté éclatante de compréhension ne doit pas conduire à la satisfaction orgueilleuse de soi-même ; il faut du respect et de la circonspection pour arriver à bonne fin. C'est pour cela que la formule dit présage heureux de la perfection. Si on peut ainsi agir avec confiance, l'attente ne sera pas vaine et l'action d'avancer ne conduira pas à la submersion et c'est pour cela que la formule dit encore : avantage à traverser un grand cours d'eau. Avoir foi, posséder une liberté éclatante de compréhension et le présage heureux de la perfection, telle est la voie rationnelle de l'attente. L'avantage dans l'action de traverser un grand cours d'eau, c'est le symbole de l'attente.

125.

Le commentaire de la formule déterminative dit: Su, nécessité, attente; le danger est en avant; avec l'énergie et l'activité, on n'y tombe pas; le sens exprime que la situation misérable actuelle n'est pas définitive.

TSHENG TSE. - Le sens du mot su est «attendre ${ }^{1}$ puisque le danger est en avant, on ne doit pas encore avancer hâtivement, c'est pourquoi il est nécessaire d'attendre, puis d'agir. Avec l'énergie active du koua simple khien, et pouvant attendre sans se mouvoir à la légère, il n'y aura donc pas chute dans le péril, le sens ne comporte pas jusqu'à l’idée d'une mesure indéfinie. S'il s'agit de l'homme doué d'énergie et d'activité, son mouve ment comporte nécessairement la détermination et la rapidité ; c'est là savoir attendre et se

\footnotetext{
${ }^{1}$ Ici, le nom du koua $s u$ est expliqué par un caractère différent, mais dont le son est également $s u$, le sens ne varie pas.
} 
mouvoir ensuite et c'est aussi se placer au point de l'extrême excellence ${ }^{1}$. C'est pour cela que Fou Tse ${ }^{2}$ s'exclame en disant : le sens exprime que la situation misérable n'est pas définitive.

TSHOU HI. - Ceci explique le sens du nom du koua au moyen des vertus propres aux koua simples.

126.

Su: attente; avoir foi; liberté éclatante; présage heureux de la perfection. Être placé dans la situation du ciel employer la droiture et la justice. Avantage à traverser à gué un grand cours d'eau; en entreprenant, il y a du mérite.

TSHENG TSE. - Le cinquième trait avec son énergie et sa sincérité se maintient dans la justice ${ }^{3}$; c'est l'image symbolique de la bonne foi. Et comme il arrive à obtenir ce qu'il attend, il donne aussi le sens d'avoir foi. À cause de l'énergie active du koua khien et de l'extrême sincérité, s es aptitudes sont l'intelligence éclatante et la possibilité de comprendre et de pénétrer librement ; il s'accorde à la parfaite droiture et le présage est heu reux. Ce qui fait qu'il peut en être ainsi, c'est qu'il stationne dans la situation du ciel et qu'il s'accorde avec la droiture et la justice ${ }^{4}$. Occuper la situation du ciel est une expression qui désigne le cinquième trait ; l'emploi de la droiture et de la justice est commun au deuxième trait et au cinquième, aussi le texte dit droiture et justice. Du moment où il y a foi et perfection dans la droiture, bien qu'il s'agisse de traverser des dangers et des embar ras, en entreprenant il aura du mérite; c'est l'extrême excellence dans la voie rationnelle de l'attente. Puisqu'il s'agit de l'énergie de l'activité et qu'elle sait attendre, qu'est -ce qui pourrait ne pas être avantageux ?

TSHOU HI. - Explication de la formule du koua au moyen de la substance du koua parfait et des deux images symboliques des koua simples.

127.

\footnotetext{
1 Allusion à la doctrine développée dans la « Grande Étude ».

${ }^{2}$ Khong Tse, ou Khong Fou Tse, ou encore Tse, dont le nom latinisé est devenu Confucius.

3 Tshong, milieu; au figuré, justice.

${ }^{4}$ Le trône ; la situation de celui qui représente le ciel sur la terre.
} 
Le commentaire de la formule symbolique dit: Le nuage monte au ciel, attente ; l'homme doué en conclut l'opportunité de boire, de manger, de se livrer au repos et au plaisir ${ }^{1}$.

TSHENG TSE. - La vapeur du nuage se condense et monte en s'élevant au ciel; il faut attendre l'harmonie et l'union entre la négativité et la positivité, et ensuite la pluie se produira. Au moment où le nuage commence à monter au ciel, la pluie n'est pas encore for mée, ce qui comporte le sens de nécessité d'attendre. Les éthers négatifs et positifs se joignent et se confondent, mais ils n'ont pas encore achevé de produire la pluie bienfaisan te. Il en est de même de l'homme doué qui entretient ses vertus et ses capacités en les développant, mais qui n'en fait pas encore usage. L'homme doué considère l'ima ge symbolique du nuage qui monte au ciel, attend et produit la pluie; il s'attache à ses vertus morales ${ }^{2}$, demeure dans le calme en attendant le moment opportun, boit et mange afin d'entretenir sa substance matérielle ${ }^{3}$, se livre au repos et au plaisir pour maintenir lhar monie dans son cour et ses pensées, c'est ce qu'on appelle demeurer à l'aise en attendant la destinée.

TSHOU HI. - Le nuage monte au ciel ; il n'y a rien de plus à faire que d'attendre que l'harmonie soit établie entre la négativité et la positivité et que la pluie se produise spontanément. La nécessité d'attendre dans les choses ou affaires ordinaires ne comporte pas non plus la possibilité qu'il y ait quoi que ce soit à faire. Il n'y a qu'à boire, manger, se maintenir dans le calme et se distraire, en attendant que l'événement survienne de lui même; s'il y avait quoi que ce fût à faire, ce ne serait plus l'attente.

\footnotetext{
${ }^{1}$ Littéralement : emploie le boire et le manger

2 Tao te: c'est l'objet du livre de Lao Tse, auquel Tsheng Tse fait souvent allusion. Littéralement ces deux mots signifient «facultés, ou vertus de la voie ».

${ }^{3}$ Son éther et sa substance, ou corps.
} 


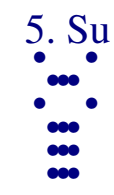

128.

Premier trait nonaire : attendre dans la plaine ; avantage à employer la persévérance; pas de culpabilité.

TSHENG TSE. - Attendre parce qu'il y a rencontre inattendue d'un danger, ce qui fait qu'il y a attente et ensuite mouvement en avant. Le premier trait est le plus éloigné du danger, c'est po ur cela qu'il exprime l'attente dans la plaine. La plaine est un lieu désert et éloigné. Placé dans un endroit désert et éloigné, l'avantage consiste dans l'observation paisible des devoirs ordinaires, de sorte qu'il n'y a point de culpabilité, ou. fautes. Si on ne sait se contenter d'une situation ordinaire, il y aura précipitation dans le mouvement et on bravera les difficultés; dans ce cas, comment serait-on capable d'attendre au loin sans commettre de fautes !

TSHOU HI. — La plaine, c'est le lieu déser t et éloigné, image symbolique du cas où on n'est pas encore près du péril. Mais comme le premier trait nonaire exprime la dureté énergique de la positivité, il donne encore l’image symbolique de pouvoir rester en permanence là où on se trouve placé. C'es $t$ pour cela que la formule avertit celui qui consulte le sort que, s’il peut remplir ces conditions, il sera sans culpabilité.

129.

Attendre dans la plaine, ne pas agir en bravant le danger. Avantage à employer la persévérance, pas de culpabilité: ne pas manquer aux règles ordinaires.

TSHENG TSE. - Celui qui est placé dans un lieu désert et éloigné ne s'expose pas en bravant le danger et les difficultés pour agir. La positivité considérée comme chose, c'est une activité énergique qui avance en montant. Le premier trait peut attendre dans un lieu désert et éloigné, sans braver le péril et le danger pour avancer. Il convient, de plus, qu'il se contente de sa position sans manquer aux règles ordinaires et alors il pourra être sans culpabilité. Bien qu'il n'avance pas, si cependant ses tendances le portent à se mouvoir, il ne pourra point se contenter de sa situation ordinaire. L'homme doué qui attend l'heure se maintient dans la paix et le repos en s'observant ; 
bien que son esprit attende et désire, c'es t encore avec calme et comme s'il devait rester ainsi jusqu'à la fin de son exis tence : c'est là pouvoir employer la constance. 


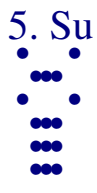

130.

Second trait nonaire : attendre sur le sable; il y a quelques légères observations verbales; à la fin bonheur.

TSHENG TSE. — Le koua simple khan représente l'eau ; près de l'eau il y a nécessairement du sable. Le second trait est un peu plus rapproché du péril, aussi représente-t-il l'attente sur le sable. Ét ant un peu plus rapproché du danger, bien qu'il ne soit pas encore arrivé jusqu'au malheur, cependant il reçoit déjà quelques observations verbales. Dans toutes les formules relatives au danger et au malheur, il y a des nuances entre le plus ou le moins de gravité des cas. Lorsqu'il s'agit de quelque chose de peu d’importance, il en résulte des observations verbales; les blessures causées par les paroles sont les moindres. Le second trait emploie les aptitudes de l'énergie et de la positivité et il demeure dans la douceur tout en conservant la justice ; il se place luimême avec grandeur et magnanimité, c'est là le bien dans l'attente. Bien qu'un peu plus rapproché du danger, il n'y est cepen dant pas encore parvenu, c'est pourquoi il souffre la blessure de quelques légères observations verbales, sans toutefois en ressentir un mal grave, et à la fin il arrivera au bonheur.

TSHOU HI. - Sur le sable, donc c'est déjà près du danger. Les blessures causées par les paroles sont encore un bien petit malheur. Se rapprochant de plus en plus du koua khan, le trait comporte cette image symbolique. Énergique et juste, pouvant attendre, il arrive finalement au bonheur. La formule avertit celui qui consulte le sort quill doit être dans ces conditions.

\section{1.}

Attendre sur le sable; grandeur dans la justice; bien qu'il y ait quelques observations verbales, tout finit par le bonheur.

TSHENG TSE. - Grandeur, magnanimité et générosité. Bien que le second trait soit rapproché du péril, cependant il emploie la grandeur et la magnanimité et se maintient dans la justice. Aussi, bien que quelques légères observations verbales l'atteignent, à la fin, il arrive au bonheur ; c'est celui qui se place suivant le bien. 
TSHOU HI. - Grandeur exprime l'idée de magnanimité ; se maintenant dans la justice au moyen de la magnanimité, il n'avance pas prématurément. 


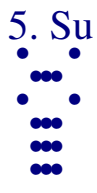

132.

Troisième trait nonaire : attendre dans la boue; il en résulte que la culpabilité survient.

TSHENG TSE. — La boue, c'est-à-dire juste contre l'eau. Du moment où il avance tout auprès du danger, il doit en résulter que la culpabilité et le malheur surviennent. Le troisième trait est énergique mais sans justice; de plus il occupe le rang supérieur dans la substance de l'activité et il représente l'image symbolique d'avancer et de se mouvoir, c'est pourquoi il en arrive jusqu’à la criminalité. S'il est dépourvu de circonspection et de res pect, il en arrivera jusqu'à sa perte et à sa destruction.

TSHOU HI. - Dans la boue, c'est être sur le point de tomber dans le péril. Criminalité, donc c'est un grand malheur. Le troisième trait nonaire est de plus en plus proche du danger, son énergie est excessive, et il est sans justice ; c'est pour cela que telle est l'image s ymbolique.

133.

Attendre dans la boue ; la calamité est au-delà ; en arriver de soi-même à la criminalité ; avec le respect et la circonspection il évite la destruction.

TSHENG TSE. - Le troisième trait est tout à fait rapproché du danger et des difficultés représentées par la substance du koua simple supérieur, c'est pour cela que le texte dit: «la calamité est au-delà ». Calamité est une appellation générale du malheur; lorsqu'elle est employée avec un autre terme qui comporte plus spécialement le sens de faute commise par malheur ou par erreur et du malheur qui en résulte, alors il y a lieu de faire la distinction ${ }^{1}$. Ce qui fait que le troisième trait en arrive à la criminalité, c'est que, de lui -même il avance et se rapproche du péril ; aussi le texte dit « en arriver de soi-même, à la criminalité », cette criminalité résulte de son propre fait. S’il peut être respectueux et circonspect $»^{2}$, apprécier l'opportunité et avancer, alors il évitera la destruction et la ruine. Le moment de l'attente, c'est celui où on

${ }^{1}$ Le terme du texte exprime plus particulièrement les calamités naturelles, considérées comme châtiments célestes.

${ }^{2}$ Respect est presque synonyme de crainte. 
attend ce qu'on désire et où on avance ensuite. Le sens consiste donc dans l'idée de ne se mouvoir qu'en tenant compte de l'opportunité du moment ; ce n'est pas un avertissement comportant qu'on ne doit pas avancer ; il prescrit strictement le respect et la circonspection, pour ne pas perdre l'opportunité.

TSHOU HI. - Au-delà, c'est-à-dire dans le koua extérieur. Avec le respect et la circonspection, pas de destruction ; c'est un éclaircissement qui met en lumière un sens divinatoire qui n'est pas directement exprimé dans la formule, preuve du soin méticuleux que l'homme saint apporte à l'idée d'avertir les hommes. 


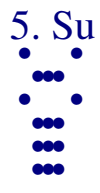

134.

Quatrième trait hexaire : attendre dans le sang ; sortir du gouffre 1.

TSHENG TSE. - Le quatrième trait avec son caractère de malléabilité négative est placé dans le péril et au-dessous de lui il est en présence du mouvement en avant de trois positivités ; c'est celui qui est atteint et blessé par le danger, et c'est pourquoi le texte dit : «attendre dans le sang ». Étant blessé par le péril et le danger, il ne peut donc se contenter de sa position, il doit absolument quitter sa situation, et c'est pourquoi le texte dit qu'il sort de la caverne. La caverne est le lieu de repos des êtres. Il se soumet passivement aux nécessités du moment, sans lutter contre le péril et la difficulté, ce qui fait qu'il n'en arri ve pas jusqu'au malheur. Puisqu’il est doux et malléable et qu’il occupe un rang négatif, c'est celui qui ne peut pas lutter ; si un trait positif occupait ce rang, ce serait nécessairement un présage de malheur. En effet, dépourvu des vertus de la justice et de la droiture, n'employant que l'énergie pour lutter contre le péril, c'est précisément suffisant pour en arriver jusqu'au malheur.

TSHOU HI. - Dans le sang, c'est-à-dire dans un lieu de meurtre et de carnage; gouffre, lieu dangereux, précipice. Le quatrième trait se joint à la substance du koua simple khan et s'y incorpore ; il est entré dans le péril, de sorte qu'il est considéré comme image sym bolique de l'attente dans le sang. Cependant il possède la douceur malléable et s'accorde avec la droiture ${ }^{2}$; il attend et n'avance pas, de sorte qu'il est encore considéré comme comportant l'image symbolique de sortir d'un gouffre. Si celui qui consulte le sort est dans ces conditions, il en résultera que, bien qu'étant dans un lieu de souffrance, à la fin il réussit à en sortir.

135.

Attendre dans le sang; obéir en écoutant.

\footnotetext{
${ }^{1}$ Le sens de ce mot est plus spécialement caverne ; il sert à désigner les cavernes habitées par les hommes des premiers âges. J'ai adopté la version de TSHOU HI.

${ }^{2}$ Rappelons que la droiture est indiquée par la condition qu'un trait négatif ou positif occupe un rang pair ou impair.
} 
TSHENG TSE. - Le quatrième trait, avec sa malléabilité négative demeure au milieu du péril et de la difficulté; il ne peut y rester définitivement, de sorte qu'il s'en retire et sort de la caverne. En effet, la douceur malléable de la négativité ne peut lutter contre le moment; ne pouvant rester en place elle se retire en amère, c'est là obéir passivement en se conformant aux nécessités et en écoutant les injonctions que comporte la nature du moment. C'est par là qu'il n'en arrive pas jusqu'au malheur. 


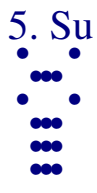

136.

Cinquième trait nonaire : attendre au milieu des vins et de la nourriture ; présage heureux de la perfection ${ }^{1}$.

TSHENG TSE. - Le cinquième trait, avec l'énergie de la positivité, demeure dans la justice et s'accorde avec la droiture ; il se tient dans la situation du ciel, et il est capable de suivre jusqu'au bout la voie rationnelle. Avec ces qualités et attendant, que pourrait-il souhaiter sans arriver à y atteindre ? C'est pour cela qu'il reste calme et en repos au milieu des vins et de la bonne chair et qu'il attend; il doit nécessairement arriver à posséder l'objet de ses désirs. Du moment où il possède la perfection de la droiture et où ce qu'il attend doit se réaliser suivant ses prévisions, c'est ce qu'on peut appeler un présage heureux.

TSHOU HI. - Le vin et la nourriture sont les éléments du repos et des plaisirs ; cela exprime qu'il est calme dans son attente. Le cinquième trait nonaire est positif, énergique, juste et droit; il attend dans la situation prééminente et c'est pour cela qu’il a cette image symbolique, si celui qui consulte le sort est dans ces conditions, et s’il possède une fermeté parfaite, il atteindra au bonheur.

137.

Vin et nourriture, présage heureux de la perfection; employer la justice et la droiture.

TSHENG TSE. - C'est celui qui attend au milieu des vins et de la nourriture, qui possède la perfection, et c'est d'ailleurs un présage heureux. Il en est ainsi parce que le cinquième trait possède la justice et la droiture et qu'il suit jusqu'au bout la voie rationnelle.

${ }^{1}$... Au sujet de la substance du koua simple khan, les expressions «boire et manger » sont souvent employées. Il semble que ce koua doit figurer les images symboliques de ces choses ; mais à présent il est impossible d'en approfondir les raisons. (TSHOU HI.) 


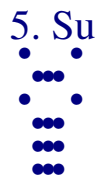

138.

Trait supérieur hexaire : entrer dans une caverne ; il y a trais hommes, hôtes pas invités, qui viennent; en les respectant, à la fin, bonheur.

TSHENG TSE. — L'attente a lieu parce que le péril est en avant ; attendre le moment et ensuite avancer. Le trait supérieur hexaire se trouve à la fin du péril ; la fin comporte nécessairement la modification. Placé à l'extrême limite de l'attente, cette attente a duré longtemps, et le moment est venu d'atteindre au résultat désiré. La négativité s'arrête au nombre six ${ }^{1}$, donc ce trait se contente de sa position, et c'est pour cela qu'il est considéré comme entrant dans la caverne ; la caverne étant le lieu de repos ${ }^{2}$. Étant en repos et déjà arrêté, ce qui suit derrière lui doit nécessairement parvenir, et l'atteindre. «Trois hommes hôtes pas invités », expression qui désigne les trois traits positifs du koua simple inférieur. Les trois traits du koua simple khien ne sauraient représenter des choses ou des êtres qui doivent rester en bas : ce sont ceux qui attendent le moment et avancent. L'attente étant arrivée à son extrême limite, il en résulte que tous avancent et montent. Pas invités, pas appelés et qui viennent d'eux -mêmes. Le trait supérieur hexaire ayant attendu, atteint une position de repos ; lorsque la troupe des duretés énergiques arrive, s'il ne se laisse point aller à ses sentiments de défiance et d'aversion, de colère et de résistance, sỉl est parfaitement sincère et respectueux en les accueillant, bien qu'extrême ment énergiques et violentes, quelles raisons auraient-elles de se montrer envahissantes et tyranniques ? C'est pour cela qu'à la fin il y a un présage heureux. On a émis des doutes sur la possibilité quill y a que la négativité placée au-dessus de trois positivités soit considérée comme exprimant le repos ? À cela on peut répondre : Les trois positivités font partie de la substance du koua simple khien; leurs tendances les portent à avancer en montant ; le trait étant hexaire et occupant une position négative, cela exprime qu'ils ne peuvent pas s'arrêter dans cette position et y demeurer avec droiture ;

\footnotetext{
${ }^{1}$ Voici un de ces passages dont il est impossible de donner un équivalent exact en français ; tout au plus pourrait-on développer tous les sens possibles, pendant plusieurs pages. Chaque mot pouvant être pris dans son sens réel ou au figuré, chacun pouvant être pris verbalement ou substantivement, il en résulte un faisceau d’idées tantôt parallèles, tantôt en opposition, qui, tout en préservant la détermination du sens lorsque le texte est appliqué à un cas spécial, conserve cependant une généralité presque indéfinie d'applications diverses.

${ }^{2}$ Puisque ce trait est négatif et qu'il occupe le premier rang, le nombre six étant la limite des nombres négatifs, cela exprime qu'il est satisfait de sa position. Étant satisfait de sa position il reste en repos et, le lieu de repos étant la caverne, c'est qu'il entre dans la caverne. Telle est l'idée exprimée par le commentateur.
} 
ils n'ont donc pas l'idée de contester et d'usurper cette position et, en les respectant, le présage sera heureux.

TSHOU HI. - La négativité est placée au comble du péril ; elle n'a plus rien à attendre, donc il y a image symbolique de tomber et d'entrer dans un précipice. En bas, ce trait correspond sympathiquement au troisième trait nonaire. Le troisième trait nonaire avec les deux traits positifs inférieurs, ont attendu jusqu'au dernier moment et avancent égale ment, ce qui constitue l'image symbolique des trois hommes hôtes pas invités. La malléabilité ne peut pas leur commander, mais elle peut leur obéir, image symbolique de les respecter. Celui qui consulte le sort doit tomber dans le danger ; cependant, s'il traite respectueusement ceux qui arrivent inopinément, il atteindra finalement au bonheur.

139.

Des hôtes pas invités arrivent; en les respectant, la fin sera heureuse; bien que la situation ne soit pas méritée, il n'y a pas encore un grand inconvénient.

TSHÉNG TSÉ. - Situation imméritée, C'est -à-dire parce qu'il est négatif et qu'il est placé au rang supérieur. Lorsque les traits sont hexaires et qu'ils occupent un rang négatif, ils sont considérés comme exprimant le repos et la satisfaction. Le commentaire de la formule symbolique revient de nouveau sur le sujet et épuise le sens qu'il comporte ; il éclaire cette idée que la négativité doit occuper un rang inférieur et que, pour elle, occuper un rang élevé constitue le fait de ne pas mériter sa situation. Cependant, si elle peut être respectueuse et attentive en se plaçant, les traits positifs ne peuvent la maltraiter, et à la fin elle obtiendra le bonheur. Bien qu'elle ne mérite pas sa situation, elle ne va pas jusqu'à commettre un manquement grave.

TSHOU HI. - Être négatif et occuper le rang supérieur, c'est précisément ce qui est considéré comme mériter sa situation, la formule dit : « situation imméritée »; le sens n'est pas encore é clairci. 


\section{Song}

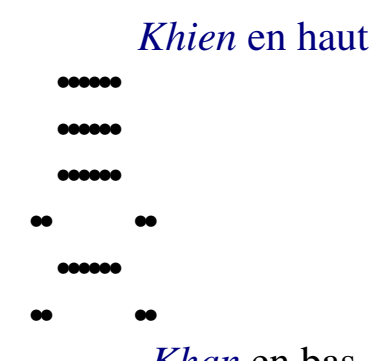

141. Song : être de bonne foi et entravé ; inquiétude, justice, présage heureux; en continuant indéfiniment malheur; avantage à voir un grand homme ; pas davantage à traverser à gué un grand cours d'eau.

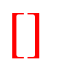

144. Premier trait hexaire : ne pas éterniser la chose en question; il y a quelques légères observations verbales; à la fin, bonheur.

$*$

146. Deuxième trait nonaire : ne pas être capable de l'emporter dans la contestation ; revenir et se mettre à l'abri ; la population du district est de trois cents familles; pas de calamités.

$*$

148. Troisième trait hexaire : nourrir sa vertu et sa perfection anciennes ; à la fin du péril, bonheur. Parfois s'occuper des affaires du roi; ne pas s'attribuer la direction.

150. Quatrième trait nonaire : ne pas être à même de l'emporter dans la contestation; revenir à la destinée; se modifier progressivement dans le calme et la perfection; bonheur.

*

152. Cinquième trait nonaire : grand présage heureux de la contestation.

*

154. Trait supérieur nonaire : parfois il reçoit le don d'une grande ceinture ; à la fin du jour le troisième la lui arrache.

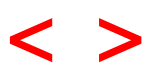


TSHENG TSE. - Song. «L'Ordre des koua » dit : «L'obligation de 'boire et de manger doit nécessairement donner lieu aux contestations, aussi le koua su est-il immédiatement suivi du koua song. »Ce que l'homme attend et désire comme indispensable ${ }^{1}$ c'est le boire et le manger. Du moment où il a à attendre et à faire face à des nécessités, c'est de là que résultent et s'élèvent les contestations ; c'est ce qui fait que le koua song suit immédiatement le koua su. Il est constitué par le koua simple khien en haut, et par le koua simple khan en bas. Si on en parle d'après les images symboliques de ces deux koua simples, la positivité du ciel agit en montant ; la nature de l'eau la porte à descendre ; les actions sont en sens opposés, ce qui produit la contestation. Si on en parle d'après les deux sub stances, en haut c'est la dureté énergique et en bas c'est le péril. L'énergie et le péril venant mutuellement en contact, est -il possible qu'il n'y ait pas de contestation ? Ou bien encore : l'homme, en lui même, est gêné et embarrassé par un danger, et dans ses actions extérieures il est dur, énergique et violent ; c'est ce qui amène la contestation.

\footnotetext{
1 «Attendre et désirer comme indispensable »; c'est lỉdée exprimée par le seul mot su.
} 


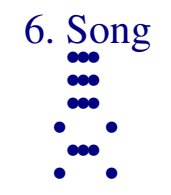

140.

Song : être de bonne foi et entravé ${ }^{1}$; inquiétude, justice, présage heureux ; en continuant indéfiniment ${ }^{2}$ malheur; avantage à voir un grand homme ; pas

davantage à traverser à gué un grand cours d'eau.

TSHENG TSE. — La voie rationnelle dans les contestations est qu'il faut absolument y apporter la bonne foi et la sincérité ; si dans la contestation il ne s'agit pas de la réalité, alors ce n'est plus que calomnie et fausseté, voie logique de malheur ${ }^{3}$. Le trait du milieu dans chaque koua simple étant plein ${ }^{4}$ constitue l'image symbolique d'être de bonne foi. La contestation 5, c'est avoir une contestation litigieuse avec quelqu'un et en attendre la solution d'un tiers. Bien que de bonne foi, il est cependant nécessaire qu’il y ait un motif de gêne et d'entraves pas encore aplani ; sans entraves, il est clair qu'il n'y aurait pas de contestation. Tant que le fait n'est pas encore élucidé, le bonheur ou le malheur qui en peuvent résulter ne peuvent pas encore être précisés avec certitude ; c'est pour cela qu'il y a crainte et inquiétude. « Présage heureux de la justice » : en possédant la justice, le présage sera heureux ${ }^{6}$. « Finalement, malheur », en poussant indéfiniment l'affaire jusqu'à son extrême limite, ce sera un présage de malheur ${ }^{7}$. Celui qui a une contestation demande qu'on détermine et qu'on élucide les torts et les droits, aussi il a avantage à voir un grand homme ; avec son énergie, son intelligence, sa justice et sa droiture, un grand homme pourra prononcer sur l'objet de la contestation. Une contestation n'est pas une chose d'harmonie et de paix, il convient de choisir un terrain sûr et de s'y placer, pour n'être pas exposé à tomber dans le péril et le danger ; c'est pour cela qu'il n'y a pas avantage à trav erser à gué un grand torrent.

TSHOU HI. - Song, contestation et élucidation; en haut, le koua simple khien, en bas le koua simple khan. Khien, dureté énergique ; khan, danger. En

\footnotetext{
${ }^{1}$ Dans son droit, dans sa liberté d'action.

2 Littéralement: à la fin, malheur; la traduction adoptée permet de suivre les deux raisonnements des commentateurs.

${ }^{3}$ En droit chinois, même en affaires civiles, toute demande non justifiée devient un acte de calomnie et est punie comme tel.

${ }^{4}$ Le terme she qu'on est obligé de traduire, selon les cas, par réalité, ou par sincérité signifie en même temps plein, non évidé ; et aussi semence des végétaux.

${ }^{5}$ Contestation, procès.

6 Tsheng Tse lit: présage heureux de la justice; Tshou $\mathrm{Hi}$ prend chaque terme indépendamment de l'autre (comme dans la traduction); c'est plus correct.

${ }^{7}$ Il y aurait autant de raisons pour lire « présage malheureux de la fin » quill y avait plus haut de lire «présage heureux de la justice». Le sens est arbitrairement étendu.
} 
haut la dureté énergique pour régir et contenir les inférieurs ; en bas, péril à cause duquel l'inférieur a recours au supérieur. Ou bien encore le koua parfait exprime le péril au-dedans et la dureté énergique au-dehors; ou enfin il exprime qu'étant soi-même en péril, la partie opposée déploie son énergie active ; c'est toujours une voie rationnelle de contestation et de procès. Le second trait nonaire (étant plein au milieu) exprime la justice et la réalité, en haut personne ne lui correspond sympathiquement, ce qui est encore considéré comme ajoutant une cause de tristesse et de préoccupations. D'ailleurs, au point de vue de la transformation des koua ${ }^{1}$, celui-ci provient du koua thouen, dans lequel la dureté énergique vient occuper le second rang et se trouve ainsi au milieu du koua simple inférieur; il y a l'image symboli que d'être de bonne foi et de voir un sujet de gêne, de savoir craindre et de s'accorder avec la justice. Le trait supérieur nonaire, c'est l'excès de la dureté énergique s'en tenant au point aigu de la contestation; il donne l'image symbolique de pousser la contestation indéfiniment et jusqu'à ses dernières limites. Le cinquième trait nonaire possède l'énergie, l'activité, la justice et la droiture et il emploie ces qualités pour se maintenir dans la situation prééminente ; c'est l'image symbolique d'un g rand homme. Puisque l'énergie est appuyée sur le péril $^{2}$, puisque la réalité est basée sur un abîme ${ }^{3}$, le koua a l'image symbolique d'absence d'avantages à traverser un grand cours d'eau. Aussi la formule avertit celui qui consulte le sort qu'il aura certa inement une question litigieuse à faire vider et que, selon la façon dont il se placera, le présage sera heureux ou malheureux.

141.

Le commentaire traditionnel de la formule déterminative dit: Song ; en haut, dureté énergique; en bas, péril ; péril et activité : contestation.

TSHENG TSE. — Le koua song est constitué par la dureté énergique, en haut, et le danger, en bas ; danger, et, en outre, activité. Ou bien encore, il est constitué par le péril et l'activité allant à la rencontre l'un de l'autr e. Au-dedans danger, au-dehors activité ; c'est toujours ce qui donne lieu à contestation. Sill s'agissait d'activité sans périls, elle ne don nerait pas naissance à un procès; s'il y avait danger, mais sans énergie active, il ne pourrait point y avoir contestation ; c'est parce qu'il y a péril et, de plus, activité énergique, qu'il y a contestation.

\footnotetext{
${ }^{1}$ Transformation qui résulte de la transposition de deux ou de plusieurs traits dans le même koua.

${ }^{2}$ Le koua simple khien sur le koua simple khan

${ }^{3}$ Second trait plein, placé au-dessus d'un trait brisé.
} 
TSHOU HI. - Explication du sens du nom du koua au moyen des vertus, ou propriétés, des deux koua simples.

Song : être de bonne foi et entravé ; inquiétude, justice, présage heureux. La dureté énergique vient et possède la justice; en continuant indéfiniment, malheur. La contestation ne doit pas s'achever. Avantage à voir un grand homme, estimer la justice et la droiture. Pas d'avantage à traverser un grand cours d'eau; entrer dans une eau profonde ${ }^{1}$.

TSHENG TSE. - Telle est certainement la voie naturelle de la contestation. De plus, si on s'attache aux aptitudes ou propriétés du koua pour l'expliquer, le second trait nonaire emploie la dureté énergique, il vient de l'extérieur ${ }^{2}$ et consomme la contestation; il en résulte donc que le second trait est le mâtre du koua song, c'est-à-dire celui de qui dépend la contestation. Employer la dureté énergique et se placer suivant la justice ${ }^{3}$, c'est l'image sy mbolique de la justice et de la réalité en soi-même, et c'est pour cela que le koua est considéré comme exprimant la bonne foi. Placé dans un moment de contestation, bien que possédant la bonne foi et la sincérité confiante, il subira nécessairement des difficultés et des embarras; il sera entravé dans sa liberté par des obstacles, et il aura de l'inquié tude et de la crainte ; s'il n'était pas entravé il ne soulèverait pas de contestation. De plus, il demeure au milieu du péril et des embûches, ce qui donne encore le sens d'être gêné et entravé, et d'éprouver un sentiment de circonspection et de crainte. Le second trait emploie la dureté énergique de la positivité, il vient de l'extérieur et possède la justice ; cela donne le sens d'employer l'énergie pou r venir soutenir une contestation, mais sans dépasser les bornes convenables. C'est à cause de cela que le présage est heureux. Dans l'explication des koua, on relève encore les transformations par lesquelles le koua s'est produit, comme constituant le sens ${ }^{4}$, ceci en est précisément un exemple. $\mathrm{Si}$, dans le sens des koua, il n'était pas tenu compte des moyens par lesquels le koua s'est produit, il ne serait plus question des traits qui se modifient. En se rapportant à la formule du koua, le second trait représente le bien et cependant, dans la formule de ce même trait, on ne voit pas quel bien et ce qui le constitue ; c'est qu'en effet, dans la formule du koua, il est tenu compte de ce qu'il y a bonne foi et accord avec la justice et c'est là-dessus que la formule raisonne ; là est le

\footnotetext{
${ }^{1}$ Un gouffre ; une eau profonde et dormante.

${ }^{2}$ Comparer ce koua avec le koua thouen.

${ }^{3}$ Au milieu.

${ }^{4}$ Ce qu'on appelle la transformation des koua.
} 
bien. Dans la formule du trait, c'est cette circonstance que, d'une posi tion inférieure, il vient soutenir une contestation contre quelqu'un qui est dans une position supérieure qui constitue le sens; les particularités relevées ne sont pas les mêmes. La contestation n'est pas une bonne chose ; c'est quelque chose qu'on ne fait que parce qu'on ne peut faire autrement ; comment serait-il possible qu'on dût la continuer indéfi niment et la pousser à ses conséquences les plus extrêmes ? En poussant à ses dernières limites une idée quelconque il en résultera un présage de malheur et c'est pour cela que le commentaire de la formule dit : «Ne doit pas s'achever »; achever veut dire pousser la chose à ses dernières conséquences. La contestation, ou procès, consiste à rechercher l'élucidation du pour et du contre ; ce qui importe dans l'élucidation, c'est la justice et la droiture, et c'est pour cela qu'il y a avantage à voir un grand homme, parce que ce qu'il estime et apprécie est la justice et la droiture. Si celui qui écoute les parties n'est pas l'homme qui convient, peut-être n'arrivera -t-il pas à décider suivant la justice et la droiture. La justice et la droiture constituent le grand homme, c'est-à-dire précisément le cinquième trait nonaire. Celui qui est en contestation avec quelqu'un doit personnellement se placer sur un terrain sûr et tranquille ; s'il s'aventure au milieu des dangers et du péril, il y tombera ; c'est là ce qui est exprimé par les termes: Entrer dans une eau profonde. Dans ce koua, il y a l'image symbolique de justice, de droiture, de danger et d'embûches.

TSHOU HI. - Explication de la formule du koua au moyen de la modification du koua, de sa substance, et des images symboliques des koua simples.

143.

Le commentaire traditionnel de la formule symbolique dit: le ciel et l'eau agissent suivant des tendances opposées : contestation; l'homme doué, en faisant quelque chose, médite sur Le début.

TSHENG TSE. - Le ciel monte, l'eau descend ; leurs tendances sont opposées dans leur action. Les deux substances sont antagoniques, d'où la contestation. Si le supérieur et l'inférieur s'accordent mutuellement, d'où pourrait s'élever la contestation ? L'homme doué regarde l'image symbolique ; il reconnaît que les sentiments humains ont une voie naturelle de contestation et de litige, de sorte que, dans chaque chose quil fait, il doit absolument apprécier et calculer au début. En coupant court à tout germe de contestation dans le début d'une affaire, il en résulter a que la contestation n'aura pas lieu de naître. Le sens des mots « méditer sur le début » est très étendu ; il comprend, par exemple, la sincérité et la circonspection dans les engagements et la clarté dans les contrats. 
TSHOU HI. - Le ciel monte, l'eau d escend, les actions sont opposées. En faisant quelque chose, méditer sur le commencement, pour couper court au germe de la contestation. 


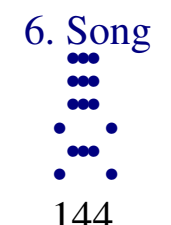

Premier trait hexaire : ne pas éterniser la chose en question; il y a quelques légères observations verbales; à la fin, bonheur.

TSHENG TSE. - Ce trait hexaire, avec sa douceur malléable et sa faiblesse, occupe le rang inférieur; c'est celui qui ne peut pas pousser indéfiniment la contestation jusqu'à ses extrêmes limites. C'est pour cela qu'au début du koua song, relatif à la contestation, les aptitudes des traits hexaires sont prises comme thème d'un avertissement qui exprime que si le sujet de la contestation n'est pas indéfiniment prolongé et éternisé, alors, bien qu'il y ait quelques légères observations verbales, à la fin on arrivera à un résultat heureux. En effet, la contestation n'est pas une chose qui doiv e durer et se prolonger éternellement; employant les aptitudes de la malléabilité négative et, étant placé dans une position inférieure, soutenant une contestation, il est impossible qu'il en résulte quelque chose d'heureux. C'est parce que, au-dessus de lui, il rencontre une assistance sympathique et parce qu'il peut ne pas éterniser le sujet de la contestation que, bien qu’il reçoi ve quelques légères observations verbales, il atteint à la fin au bonheur. Recevoir des observations verbales, c'est une des plus petites calamités; ne pas éterniser le motif et ne pas arriver jusqu'au malheur, c'est là le bonheur lorsqu'il s'agit de contestations.

TSHOU HI. - Malléable, négatif et occupant une position inférieure, il n'est pas à même de prolonger indéfiniment la contestation. C'est pour cela que telles sont l'image symbolique et la formule divinatoire.

145.

Ne pas éterniser la chose en question : les contestations ne doivent pas durer longtemps.

Bien qu'il y ait quelques observations verbales, la décision est intelligente ${ }^{1}$.

TSHENG TSE. - Le trait est hexaire; il emploie la douceur et la faiblesse et il soutient une contestation dans une position inférieure. Le sens est évidemment qu'un tel état de choses ne peut pas durer et s'éterniser. En

\footnotetext{
${ }^{1}$ Ou bien : « Le jugement est clair. »
} 
éternisant la contestation il ne réussirait pas à l'emporter sur ses adversaires, tandis que les malheurs et les difficultés l'atteindraient. De plus, dès le début du koua song, relatif aux contestations, c'est précisément un avertisse ment qui rappelle que la contestation n'est pas une chose qui doive durer longtemps. Doux et faible, occupant un rang inférieur, ses capacités ne lui permettent pas de soutenir la contestation. Bien que n'éternisant pas la contestation, du moment où cette contestation existe, il doit nécessairement en éprouver un peu de mal, et c'est à cause de cela quill reçoit quelques légères observations verbales. N'éternisant pas la question pendante et de plus, en haut, ayant la sympathie, correcte selon la droiture, de la dureté énergique de la positivité, la clarté, ou intelligence dans l'élucidation et le jugement est marquée, de sorte que finalement le présage est heureux. S’il n'en était pas ainsi, serait -il possible qu'il échappât au malheur ? Lorsqu'il s'agit de contestations, le sens est que ceux qui occupent des situations analogues ${ }^{1}$ et qui se correspondent sympathiquement sont ceux qui parviennent à obtenir une solution claire et précise. Les traits qui occupent des situations analogues sans s'accorder ensemble sont ceux qui ont une contestation entre eux; aussi le second trait et le cinquième représentent deux antagonistes.

\footnotetext{
${ }^{1}$ Le même rang dans chacun des deux koua simples.
} 


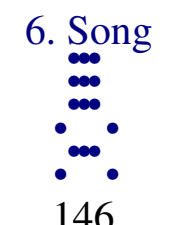

Deuxième trait nonaire : ne pas être capable de l'emporter dans la contestation ; revenir et se mettre à l'abri ; la population du district est de trois cents familles; pas de calamités.

TSHENG TSE. - Le second et le cinquième rang sont des positions qui se correspondent mutuellement, mais les deux traits étant tout deux énergiques ne s'entendent point ensemble ; ce sont ceux qui sont en contestation l'un contre l'autre. Le second trait nonaire vient de l'extérieur ${ }^{1}$; puisque avec sa dureté énergique, il se place dans le danger, c'est de lui que dépend la contestation et c'est donc lui qui est l'adversaire du cin quième. Le cinquième trait emploie la justice et la droiture et il est placé dans la situation du prince : est-il possible et licite de se poser comme son adversaire ? C'est là entreprendre une contestation que le devoir ne tolère point. S'il peut reconnaître que le devoir ne la tolère point, se retirer et revenir en arrière, se mettre à l'abri et se garer en se plaçant de façon à se contraindre et à se restreindre, alors il réussira à ne pas souffrir des calamités. Devoir se mettre à l'abri, c'est savoir quitter le rôle d'adversaire. " Trois cents familles », c'est-à-dire ce quil $\mathrm{y}$ a de plus petit en fait de district; s’il se plaçait dans une situation considérable, ce serait encore résister et lutter; pourrait-il dans ce cas éviter les calamités ? Le terme traduit par calamité indique qu'il y a faute ou erreur de la part de celui qui en souffre, comme se placer autrement qu'il ne convient. Il y a cependant une distinction à faire entre ce cas et celui où le mal est commis avec connaissance de cause.

TSHOU HI. - Le second trait nonaire, énergique et positif, est considéré comme représentant celui de qui dépend le péril ; c'est celui qui primitivement veut contester. Cependant, puisque, dur et énergique, il occupe un rang qui comporte la douceur et la malléabilité, puisqu'il possède la justice des gens de nature inférieure ${ }^{2}$ et qu’il s'élève pour correspondre sympathiquement au cinquième trait nonaire, puisque la dureté énergique occupe la situation prééminente et que la force des choses s'oppose à ce qu'il se pose en adversaire, pour toutes ces raisons, tels sont et limage symbolique et le sens divinatoire. Un district dont la population est de trois cents familles, est ce qu'il y a de plus petit en fait de district ; ceci exprime le fait de se placer soimême en se restreignant et en s'humiliant pour éviter les calamités et les

\footnotetext{
1 Voir $n^{\circ} 140$.

${ }^{2}$ Puisqu'il est placé au milieu du koua simple inférieur.
} 
malheurs. Si celui qui consulte le sort est dans ces conditions, il n'éprouvera aucune calamité ${ }^{1}$.

147.

Ne pas être capable de l'emporter dans la contestation; se retirer en se mettant à l'abri et en se réfugiant. D'un rang inférieur soutenir une contestation contre un supérieur, le malheur survient comme cueilli avec la main.

TSHENG TSE. - Du moment où le devoir défend de se poser en adversaire, il en résulte l'impossibilité de soutenir la contestation. Se retirer en se mettant à l'abri et en se réfu giant, c'est se garer en quittant la place. D'un rang inférieur élever une contestation contre un supérieur, c'est violer le devoir et déprimer la force naturelle des choses ; le malheur et les calamités en résultent comme si on les prenait en les cueillant avec la main. Cette expression indique la facilité avec laquelle on arrive à ce résultat fâcheux.

TSHOU HI. — Cueillir avec la main : prendre ou s'attirer à soi -même.

\footnotetext{
${ }^{1}$ Tsheng Tse et Tshou Tse ont adopté tous deux le sens indiqué avant eux par Wang Si. Mais on a donné bien d'autres explications de ces deux derniers membres de phrase. Heang Shi Ngan She, par exemple, dit qu'une seule famille, qui cause des troubles par les contestations qu'elle soutient, peut être la cause des souffrances de trois cents familles ; il considère quil s'agit du sacrific e de sa propre ambition au salut de la foule. Yu Shi Yen dit que celui qui sent que la force lui manque dans son entreprise fuit et l'abandonne ; du moment où il fuit, sa famille et son entourage échappent aux conséquences de sa faute.
} 


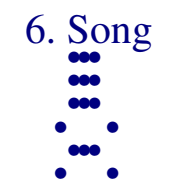

148.

Troisième trait hexaire : nourrir sa vertu et sa perfection anciennes; à la fin du péril, bonheur. Parfois s'occuper des affaires du roi ; ne pas s'attribuer la direction.

TSHENG TSE. - Bien que le troisième trait occupe une position qui comporte la dureté énergique et qu'il corresponde sympathiquement au trait supérieur, cependant le caractère de sa substance est essentiellement la malléabilité négative. Il est placé dans le péril et il sert de démarcation entre deux duretés énergiques; c'est celui qui est en danger, qui craint, et qui n'entreprend pas de contestations. Le traitement affecté à une fonction publique est proportionné au mérite de celui qui en est pourvu ${ }^{1}$; se nourrir de son ancienne vertu veut dire se maintenir dans une condition modeste et simple; perfection veut dire s'observer et se contenir avec une fermeté durable. L'expression « à la fin du péril, bonheur» veut dire que, quoique placé sur un terrain dangereux, en étant capable de reconnaître le péril et de le craindre, on arrivera certainement à la fin à posséder le bonheur. Conservant une position simple sans rien chercher à obtenir, il n'y aura aucun sujet de contestation. On dit quỉl est placé dans le péril parce qu'il est dans le koua simple qui représente le danger et que le trait quilil supporte, comme celui sur lequel il s'appuie, sont tous deux énergiques ; parce que, enfin, il se trouve, en outre, dans un moment de contestation. Ce qui est doux et malléable suit ce qui est énergique ; linfé rieur suit le supérieur et lui obéit ; le troisième trait ne fait pas de contestation, mais il suit le trait supérieur dans ce que fait celui-là. C'est pour cela que la formule dit : Parfois s'occuper des affaires du prince ${ }^{2}$. Ne pas s'attribuer la direction veut dire qu’il suit le supérieur en lui obéissant et que la direction ne lui appartient point. La contestation est une chose qui demande de l'énergie et de l'activité ; aussi le premier trait ne doit-il pas l'éterniser ; le troisième trait suit le trait supérieur, tous deux sont ceux qui ne sont pas à même de soutenir la contestation. Les deux traits, à cause de leur douceur négative, ne poussent pas les choses indéfiniment et ils atteignent au bonheur. Le quatrième trait, n'étant pas à même de l' emporter et se corrigeant, obtient aussi le bonheur. Dans les contestations, savoir s'arrêter constitue le bien.

\footnotetext{
${ }^{1}$ Le commentateur lit : « Se nourrir de son ancienne vertu, perfection. » Cette lecture, admise dans d'autres passages, est contraire à la ponctuation indiquée et admise par la tradition.

${ }^{2}$ Cette explication est incorrecte, même en se plaçant au point de vue des commentateurs chinois. Le trait supérieur ne représente pas le prince.
} 
TSHOU HI. - Le mot she a la valeur du même mot dans l'expression « manger le district ${ }^{1}$; il exprime ce dont on jouit. Le troisième trait hexaire possède la malléabilité négative ; c'est celui qui n'est pas capable de soutenir une contestation. C'est pour cela qu'il conserve son ancienne position et qu'il se maintient dans la droiture, de sorte que bien qu'il y ait péril, cependant, à la fin, arrive le bonheur. Mais parfois il sort de sa retraite et s'occupe des affaires du supérieur; alors, même dans ce cas, il ne doit pas se prévaloir de son mérite et s'attribuer la direction. Si celui qui consulte le sort conserve sa situation ordinaire et n'en sort pas, ce sera le bien.

149.

Nourrir son ancienne vertu ; en suivant le supérieur, présage heureux.

TSHENG TSE. - En conservant sa condition modeste, bien quil s'occupe de ce que fait le supérieur, ce n'est pas par lui -même qu'il agit. C'est pour cela qu'il ne s'attribue pas la direction et qu'à la fin il atteint au bonheur.

TSHOU HI. - En suivant le supérieur, présage heureux ; cela veut dire qu'en suivant quelqu'un ce sera un présage heureux. Cela montre clairement qu'en dirigeant soi-même l'affaire en question on ne réussirait pas à la terminer avec mérite.

\footnotetext{
${ }^{1}$ Se nourrir du revenu du district.
} 


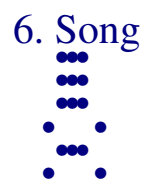

150.

Quatrième trait nonaire : ne pas être à même de l'emporter dans la contestation; revenir à la destinée; se modifier progressivement dans le calme et la perfection; bonheur.

TSHENG TSE. - Le quatrième trait emploie la dureté énergique de la positivité et fait partie de la substance de l'activité, sans posséder ni justice, ni droiture ; c'est essentiellement ce qui fait la contestation. Il supporte le cinquième, foule le troisième aux pieds, et correspond sympathiquement au premier. Le cinquième trait, c'est le prince ; le devoir ne tolère point de contestation contre lui ${ }^{1}$. Le troisième trait demeure dans un rang inférieur et il est doux et malléable; il n'y a pas de contestation de sa part. Le premier correspondant sympathiquement avec droiture et suivant avec obéissance, il n'y a pas de contes tation avec lui. Le quatrième trait, bien qu'énergique, actif et entreprenant, désireux de contestations, ne rencontre aucun adversaire qui lui résiste ; la contestation n'a pas lieu de s'élever, c'est pourquoi il ne peut pas faire de contestation ${ }^{2}$. De plus, ce trait occupe un rang qui comporte la malléabilité et il correspond à un trait qui possède cette même qualité ; cela constitue encore le sens de pouvoir s'arrêter. $\mathrm{D}$ u moment où le devoir ne permet pas la contestation, s'il peut dominer ${ }^{3}$ ses sentiments de dureté et de colère, ainsi que son désir de contester, revenir à sa destinée, purifier son cour, calmer ses sens, se modi fier et devenir calme et parfait, ce sera un présage de bonheur. Le mot destinée désigne la véritable raison d'être des choses; manquer à l'exacte raison d'être des choses constitue ce qu'on appelle «contrarier la destinée », aussi la soumission à la destinée est considérée comme constituant un retour. Contrarier, c'est ne pas se conformer avec soumission. Le Shou king dit: "Contrarier la destinée et ruiner les relations de la famille »; Mang Tse dit : «Contrarier la destinée et tyranniser le peuple. » Or, la dureté énergique sans la justice et la droiture entraîne la précipitation intempestive dans les actions, de sorte qu'il ne peut y avoir satisfaction de la position occupée. Étant sans justice ni droiture, il ne peut posséder la perfection; n'étant point satisfait et manquant de pureté, il est

\footnotetext{
${ }^{1}$ Ici, comme dans le cas de la formule du second trait, les commentateurs donnent au terme $k h o$, quatrième caractère du texte, la valeur de «possibilité ». Cette valeur ne ressort pas du texte et elle n'est justifiée que par une citation tirée du Shou king. Mais le texte du Shou king est on ne peut plus sujet à caution et même dans l'exemple cité, bien qu'on lise " pouvoir », «possibilité », le sens indique la puissance de l'agent. La vraie valeur de kho est "puissance de faire », «puissance de dominer »; exemple : puissance de dominer ses passions

${ }^{2}$ Même observation que dans le renvoi précédent au sujet du mot kho

${ }^{3}$ C'est encore le même terme kho mais cette fois il est pris dans son vrai sens.
} 
conduit à aimer les contestations. Si le devoir ne permet point la contestation et s'il ne conteste pas, s'il revient au contraire à la vraie raison d'être de toutes choses, sil modifie son manque de calme et de perfection pour devenir satisfait de son sort et pur, alors le présage sera heureux.

TSHOU HI. — « À », dans l'expression revenir à, c'est se porter vers. La destinée, ou mandat céleste, c'est la vraie et droite raison d'être de chaque chose. Se modifier, c'est se modifier progressivement et peu à peu. Le quatrième trait nonaire est énergique, mais sans justice, aussi il est l’image symbolique de la contestation. C'est parce quil occupe un rang qui comporte la douceur et la malléabilité, qu'il constitue encore l'image symbo lique de ne point pouvoir et de revenir à la vraie raison d'être des choses en modifiant peu à peu son cour, en se contentant de se placer suivant la droiture. Si celui qui consulte le sort est dans ces conditions, ce sera un présage de bonheur.

DÉFINITIONS DIVERSES. - Kung Shi Yuan dit: Le second est en contestation avec le cinquième; le quatrième est en contestation avec le premier ; ceux qui sont adversaires différent entre eux par leur force ou leur faiblesse et, dans les deux cas, la formule dit: incapacité de soutenir la contestation. En effet, puisque le second est dans une position inférieure et en contestation avec un supérieur, il ne peut l'emporter, et cela à cause de la force naturelle des choses. Puisque le quatrième occupe un rang supérieur et qu'il est en contestation avec un inférieur, ce qui l'empêche de pouvoir l'emporter, c'est seulement la raison. Le second trait considère la force naturelle des choses qui fait qu'il ne peut pas l'emporter, il revient donc et se met à l'abri en se réfugiant. Le qua trième reconnaît que la raison ne lui permet pas, c'est pourquoi il revient et se reporte vers la destinée. Le second et le quatrième traits étant tous deux durs et énergiques, et occupant d'ailleurs des situations qui comportent la douceur, c'est ce qui fait qu'ils sont capables d'agir ainsi.

151.

Revenir à la destinée et se modifier dans le calme et la perfection : pas de faute.

TSHENG TSE. - Si on peut être dans ces conditions, cela sera ne pas commettre de fautes, d'où résultera le bonheur. 


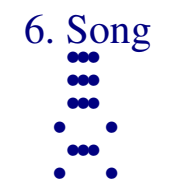

152.

Cinquième trait nonaire : grand présage heureux de la contestation.

TSHENG TSE. - Puisque, avec justice et droiture, il occupe la situation prééminente, c'est celui qui règle les contestations. Chargé du règlement des contestations, posséder la justice et la droiture est ce qui produit la grandeur du présage heureux. Grand présage heureux, c'est-à-dire parfaitement grand et atteignant les limites extrêmes du bien. Il peut y avoir un grand bonheur sans que les limites du bien soient atteintes.

TSHOU HI. - Positivité, énergie, justice et droiture dans l'occupation de la situation prééminente, c'est celui qui écoute le procès et sait atteindre à l'équité. Celui qui ren contre ce trait en consultant le sort, s'il soutient u ne contestation et a la raison de son côté, obtiendra certainement le redressement de ces griefs.

153.

Grand présage heureux de la contestation; à cause de la justice et de la droiture.

TSHENG TSE. — La voie de la justice et de la droiture ne peut pas être étendue à quoi que ce soit sans quil n'en résulte un grand bonheur.

TSHOU HI. - Avec la justice il écoutera la contestation sans partialité ; avec la droiture il prononcera d'accord avec la raison. 


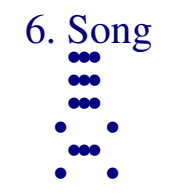

154.

Trait supérieur nonaire : parfois il reçoit le don d' une grande ceinture; à la fin du jour le troisième la lui arrache ${ }^{1 .}$

TSHENG TSE. - Ce trait étant nonaire et employant la positivité pour demeurer au rang supérieur, marque le comble de la dureté énergique et de l'activité dans l'action. De plus, il est placé à la fin de la contestation (dernier rang du koua song), donc c'est celui qui pousse la contestation à ses dernières limites. L'homme, en donnant un li bre cours à son énergie et à sa violence, en allant jusqu'aux dernières limites de la contestation, attire le malheur et se perd; la raison d'être des choses est certainement ainsi. Si on suppose que dans une juste contestation il puisse parfois réussir à l'emporter, qu'il pousse les choses à leur dernière limite sans s'arrêter, et qu'il arrive ainsi jusqu'à recevoir en récompense un vêtement de cour et une mission officielle, c'est encore par une lutte contre quelqu'un qu'il obtient ces faveurs ; est-il donc possible qu'il soit assuré d'en jouir en paix ? Aussi, à la fin de la même journée le troisième le voit et la lui arrache par force.

TSHOU HI. - La grande ceinture est un ornement du costume de cour ${ }^{2}$. Arracher, enlever par force. Il emploie la dureté énergique et se trouve au point extrême de la contestation, il prolonge indéfiniment cette contestation et peut arriver à l'emporter sur son adversaire, c'est pourquoi ce trait a l'image symbolique de celui à qui le souverain confie une mission et qu'il revêt des insignes d'une fonction publique. Toutefois c'est par la voie des contestations qu'il obtient ces avantages, aussi, comment pourrait-il en jouir longtemps en repos ? Il y a donc encore l’image symbolique qu'à la fin du jour le troisiè me trait la lui arrache. Le sens divinatoire est qu'en poussant indéfiniment une contestation, sans avoir la raison de son côté, il peut parfois arriver qu'on réussisse à l'emporter, mais que ce qu'on obtient ainsi doit finalement être reperdu. Lidée de l'avertissem ent donné par l'homme saint est profonde.

\footnotetext{
${ }^{1}$ Ceci ne peut être le vrai sens du second membre de phrase, car la construction serait absolument fautive. $1^{\circ}$ ) Le mot qui exprime l'idée de fin précède le mot traduit par jour ; donc il y a écrit dans le texte « jour de la fin » et non pas « fin du jour »; $2^{\circ}$ ) le mot traduit par jour signifie matinée et nullement « fin du jour »; il signifie aussi « réception à la cour », Il serait plus correct de lire : «en finissant d'assister à la réception à la cour », ou bien encore : «s'il reste jusquà la fin de la réception ».

${ }^{2}$ Le terme est pan, ceinture (sixième caractère du texte). Or, il faut remarquer que le même son représenté par une autre forme graphique, est le nom du premier être humain dans la mythologie chinoise.
} 
155.

À cause de la contestation un vêtement de cour lui est conféré; ce n'est pas encore assez de respect.

TSHENG TSE. - En poussant une contestation jusqu'à ses dernières limites, si on suppose qu'il reçoit la faveur du don d'un vêtement de cour et d'une mission officielle, ce n'est pas encore suffisant pour qu'il ait droit au respect et il doit être abaissé et avili. À plus forte raison le malheur et les déceptions surviennent ensuite.

Il est difficile d'ad mettre cette explication du second membre de phrase. Il faudrait ajouter le mot tshi à la fin, et alors seulement la lecture serait possible. 


\section{Shi}

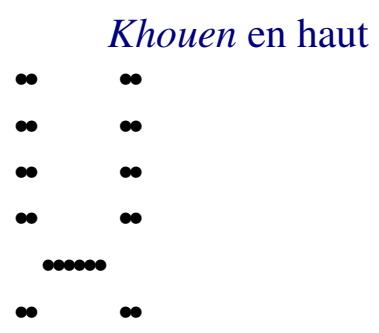

Khan en bas

156. Perfection militaire ; l'homme au bâton : présage heureux, pas de culpabilité

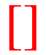

160. Premier trait hexaire : l'armée sort d'après la loi ; si ce n'est pour le bien, présage malheureux.

$*$

162. Deuxième trait nonaire : être au milieu de l'armée ; présage heureux, pas de culpabilité ; le roi donne trois fois une mission.

$*$

164. Troisième trait hexaire : l'armée a parfois plusieurs chefs ; présage malheureux.

$*$

166. Quatrième trait hexaire : l'armée retourne et renonce ; pas de culpabilité.

*

168. Cinquième trait hexaire : la rizière a du gibier ; avantage à retenir les paroles ; pas de culpabilité. Le fils aîné commande l'armée ; le fils cadet participe au commandement; perfection, présage malheureux.

*

170. Trait supérieur hexaire: un grand prince donne des ordres; fondation de royaumes, ennoblissement de familles ; l'homme inférieur ne doit pas agir.

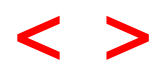


TSHENG TSE. - Shi. «L'Ordre des koua » dit : «Du moment où il y a contestation, il doit nécessairement y avoir soulèvement de la foule, aussi le koua song est suivi du koua shi. » L'agitation de la foule ${ }^{1}$ survient à cause de contestations et de disputes, c'est ce qui fait que le koua shi suit immédiatement le koua song. Il est constitué par le koua simple khouen en haut et le koua simple khan en bas. Si on en parle au point de vue des deux substances ${ }^{2}$, au milieu de la terre il y a de l'eau ${ }^{3}$, ce qui est considéré comme constituant limage symbolique de la foule se rassemblant. Si on en parle au point de vue du sens des deux koua simples, au-dedans péril et au-dehors soumission passive; une voie périlleuse dans laquelle on marche avec soumission, ce qui est le sens du mot $s h{ }^{4}$. Si on en parle d'après les traits, il n'y a qu'une seule positivité et c'est d'elle que dépend la foule des négativités, donc c'est limage symbolique de la concentration du pouvoir de commande ment sur la foule. Dans le koua pi l'unique positivité est considérée comme représentant celui de qui dépend la foule des négativités, mais elle est placée en haut et est l'image symbolique du prince; dans le koua shi l'unique positivité est aussi considérée comme celui de qui dépend la foule des négativités, mais elle est dans le koua simple inférieur et elle constitue l'image symbolique du chef d'armée.

\footnotetext{
${ }^{1}$ Yun Fong Hou Shi dit: «hing tshong », agiter les masses; c'est ce qu'il y a de plus périlleux.

2 Des koua simples.

${ }^{3}$ En supposant les koua rangés autour d'un cercle, le koua simple khouen, ou de la terre, est à l'exté rieur et le koua simple khan, eau, est à l'intérieur, c'est -à-dire entouré par la terre.

4 Shi, exprime encore une armée, troupe militaire ; le général, chef militaire qui commande une armée ; «Shi, un corps d'armée de 2.500 hommes est appelé shi » (Shuo men) — «c'est la réunion de cinq lu, ou corps de 500 hommes chacun » (Tse tien).
} 


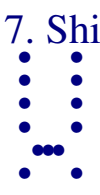

156.

\section{Perfection militaire ; l'homme au bâton : présage heureux, pas de culpabilité ${ }^{1}$.}

TSHENG TSE. — La voie rationnelle des choses militaires est basée sur la droiture. Lorsqu'il s'agit de lever des armées, de réu nir la foule pour nuire à l'univers 2 et sans que cela soit correct selon la droiture, le peuple ne suit pas l'agitateur et le repousse par la force ; aussi dans le commandement des armées (ski), c'est la pureté qui importe avant tout. Bien que le mouvemen t des armées soit commandé par la droiture, celui qui les commande doit absolument être un homme (à bâton) déjà âgé, et alors il y a présage heureux et pas de culpabilité. En effet, il y a des cas où il y a présage heureux et où cependant il y a culpabilité ; il y en a d'autres où, sans culpabilité, il n'y a cependant pas de présage heureux. Lorsqu'il y a présage heureux et que d'ailleurs il n'y a pas de culpabilité, c'est le bien absolu. « Homme à bâton » est une appellation pour les personnes éminentes et d'un caractère sévère et imposant. Le mot shi a le sens de réunir et rassembler la foule. Si celui qui commande n'est pas celui que la foule respecte et en qui elle a confiance, celui qu'elle craint et à qui elle se soumet, comment pourrait -il être écouté et suivi de la foule ? C'est pour cela que le Se ma jeang Tsu Tshuo se vit, d'une position infime et humble, appelé au commandement de la multitude, mais qu'ensuite, comme le cour de la foule n'était pas avec lui, il demanda que Tshouang Kia fût nommé général à sa place. Ce qu'on entend par l'expression «homme à bâton» ne doit pas nécessairement être un homme illustre et noble ; il suffit que, d'une façon quelconque, la foule le craigne et se soumette à lui à cause de ses capacités, de sa finesse, et de ses qualités vertueuses. C'est là tout ce qu'il faut. Aussi, lorsque jeang Tsu eut tué Tshouang Kia, le cour de la foule le craignit et se soumit à lui ; ce fut alors un « homme à bâton ». De même encore Wei Yin Heou s'éleva d'une position infime et vile et parvint au rang de général d'armée ; c'est que, en effet, son habileté et sa ruse furent suffisantes pour amener les hommes à le craindre et à l'esti mer.

TSHOU HI. - Shi : foule de soldats. En bas le koua simple khan, en haut le koua simple khouen; le koua khan représente le péril, le koua khouen l'obéissance passive. Le koua khan exprime l'eau, et le koua khouen la terre. Dans l'antiquité les soldats étaient logés chez l'agriculteur. Supporter l'extrême péril avec une grande soumission ; cacher un abîme insondable au

1 «L'homme au bâton », expression qu'on rencontrera souvent dans la suite, correspond exactement à l'exemple Dandi, fréquemment répété dans la grammaire de Kaccayana.

${ }^{2}$ On peut lire : à l'empire. 
milieu du repos le plus complet. De plus dans le koua, il n'y a qu'une seule positivité, le second trait nonaire, qui occupe le milieu du koua simple inférieur; il est considéré comme image symbolique du général. Au-dessus et au-dessous, cinq négativités obéissent avec soumission et le suivent; elles constituent l'image symbolique de la foule. Le second trait nonaire emploie la dureté énergique, occupe une position inférieure, et dirige les affaires; le cinquième trait hexaire emploie la douceur malléable, occupe la position supérieure et lui confère l'autorité ; cela constitue donc l'image symbolique du prince donnant l'ordre au général de faire avancer son armée. C'est pour cela que le koua est appelé shi. "Homme à bâton » est une appellation pour les gens âgés et les supérieurs. Dans la voie rationnelle de l'emploi des armées, l'avantage réside dans l'accord avec la droiture et dans le choix d'hommes âgés et accomplis pour leur confier le com mandement ; c'est ainsi que le présage est heureux et qu'il n'y a pas de culpabilité. C'est un avertissement à celui qui consulte le sort pour le prévenir que lui aussi doit remplir ces conditions.

\section{7.}

Le commentaire traditionnel de la formule déterminative dit: Shi, multitude ; perfection, droiture; pouvoir employer la foule avec droiture, être capable de s'en servir pour régner.

TSHENG TSE. - Pouvoir diriger la foule des hommes de façon que tous suivent la droiture; être capable de s'en servir pour régner sur l'univers. Posséder le cour de la foule qui se soumet et obéit en revenant à la droiture ; la voie de la royauté se borne à ceci.

TSHOU HI. - Ceci explique le sens des mots «perfection militaire » au moyen de la substance du koua parfait. Le mot traduit par «employer » veut dire seconder, aider quelqu'un ${ }^{1}$. L'unique positivité occupe le milieu du koua simple inférieur et les cinq négativités sont, toutes, ce qui aide et seconde. Pouvant aider la foule selon la droiture, c'est donc l'armée du roi.

\footnotetext{
${ }^{1}$ Cette explication dénature le sens du mot yi, employer; elle n'a pas été suivie dans la
} traduction. 
158.

Dureté énergique, justice et correspondance sympathique; agir dans le danger avec obéissance passive. Infliger un mal à l'univers en employant ces moyens et être suivi par le peuple ; présage heureux, de plus quelle culpabilité pourrait-il y avoir encore?

TSHENG TSE. - Il s'agi t du second trait ; il emploie la dureté énergique et il est placé au milieu (du koua simple inférieur) ; c'est la voie normale de la dureté énergique qui possède la justice. Le prince représenté par le cinquième trait hexaire est celui auquel il correspond sympathiquement et suivant la droiture, ce qui indique la confiance absolue et une délégation exclusive de l'autorité. Bien qu'agissant dans une voie périlleuse, c'est cependant par obéissance qu'il se meut ; c'est ce qu'on appelle un " soldat du devoir ${ }^{1}$; c'est le général du roi. En haut, soumission passive ; en bas, danger ; c'est agir dans le danger avec obéissance. Les armées ne sont jamais mises sur pied sans qu'il en résulte un dommage pour la fortune publique et des maux pour les individus, c'est ce qui est enten du dans l'expression "infliger un mal à l'univers ». Cependant le coar du peuple l'accompagne, c'est parce qu'il se meut par devoir. Dans l'antiquité, " l'Ouest était jaloux de la marche vers $l^{\text {'Est }} \gg^{2}$; c'est que le cour du peuple s uivait ${ }^{3}$. Dans de telles conditions, le présage est certainement heureux et il n'y a pas de culpabilité. Présage heureux veut dire qu'il y aura certainement victoire ou succès, pas de culpabilité indique que l'action est conforme au devoir. Quelle pourrait encore être la culpabilité ? Le sens est certainement qu'il n'y a point de culpabilité.

TSHOU HI. - C'est encore une explication du sens des expressions « homme à bâton », «présage heureux », «pas de culpabilité », au moyen de la substance du koua et des vertus ou propriétés des koua simples. "Dureté énergique et justice» se rapporte au second trait nonaire ; «correspondance sympathique » indique que le cinquième trait hexaire lui correspond sympathiquement ; " agir dans le danger », c'est-à-dire marcher dans une voie périlleuse; "obéissance passive» veut dire se conformer au désir de quelqu'un ; sans les vertus de l'âge mûr, tout ceci est impossible. " Infliger un mal », nuire, causer un mal ; lorsque les armées sont mises sur pied, il est impossible qu'il n'en résulte pas des maux pour l'univers, mais cependant, puisqu’il y a ces qualités et ces aptitudes, il en résulte que le peuple est satisfait et suit.

159.

\footnotetext{
1 Volontaires qui s'organisent pour défendre le prince et le pays.

2 Allusion à un passage du Shou king.

${ }^{3}$ Le héros en question.
} 
Le commentaire traditionnel de la formule symbolique dit: Au milieu de la terre, il y a de l'eau; shi, armée. L'homme doué s'en autorise pour protéger le peuple et rassembler la multitude.

TSHENG TSE. - Au milieu de la terre il y a de l'eau ; l'eau s'amasse au milieu de la terre, ce qui constitue l'image symbolique de rassembler la multitude, et c'est pourquoi ce koua est appelé shi, et représente l'armée. L'homme doué considère l'image symbolique de l'eau au milieu de la terre et il s'en autorise pour protéger et garantir le peuple et pour rassembler et réunir la foule.

TSHOU HI. - L'eau n'est pas en dehors de la terre, l'armée n'est pas en dehors de la population, de sorte que, pouvant prendre soin du peuple, il sera possible de posséder le concours de la multitude. 


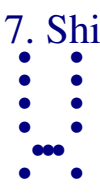

160.

\section{Premier trait hexaire : l'armée sort d'après la loi ; si ce n'est pour le bien, présage malheureux.}

TSHENG TSE. - Premier rang, commencement du koua shi, aussi il est question du sens de «faire sortir l'armée » et de la voie rationnelle de l'action des armées; il s'agit de la levée des armées dans un État ou un royaume. Si elle a lieu d'accord avec le devoir et la raison, ce sera donc d'après les lois et les règles, ce qui exprime que les armées sont mises en mouvement pour réprimer le désordre et les troubles et pour châtier la violence. Si elles sont mises en mouvement sans que ce soit d'après le devoir, alors, quoique pour le bien, ce sera une voie de malheur. Dans cette phrase le mot «bien » veut dire qu'il est licite de l'emporter dans la lutte ${ }^{1}$; le mot «malheur» veut dire : Causer du mal au peuple et blesser le devoir. Lorsqu'il s'agit de l'action des armées le mot «loi » désigne les ordres du prince, les règlements et les institutions de l'État. La voie rationnelle de l'action des armées est basée sur les ordres royaux et sur les institutions de l'État, moyens par lesquels est concentrée l'action générale du pouvoir sur la foule. Si l'armée sort sans que ce soit d'après la loi, quoiqu'elle remporte l'avantage, ce sera cependant encore un présage malheureux ; bien que conduite à la victoire, ce sera encore une voie logique de malheur. Lorsque les armées sont dirigées sans règles, il peut parfois arriver, par une chance heureuse, qu'elles ne soient pas mises en déroute et même qu'elles remportent l'avantage, c'est là ce qui fait l'objet de l'avertissement de l'homme saint.

TSHOU HI. - Loi, règles. Les deux mots feou tsang signifient « ce qui n'est pas bien ». Tshao Shi dit : La plupart des anciens philosophes prenaient indistinctement le mot feou pour le mot pou (ne pas, négation); tel est le cas. Étant au commencement du koua, ce trait représente le début de l'action des armées. La voie rationnelle de la mise d'une armée en campagne comporte nécessairement la circonspection dans les préparatifs et le début. Si c'est d'après la loi, ce sera un présage heureux ; si ce n'est pour le bien, ce sera un présage de malheur. C'est un avertissement préventif donné à celui qui consulte le sort pour lui rappeler quill doit être circonspect dans le début et observer les règles.

\footnotetext{
${ }^{1}$ Chez les commentateurs chinois, les allégations de ce genre ne sont pas rares ; mais elles ne doivent pas être acceptées sans contrôle. Feou est bien une négation, mais généralement c'est une interrogation affirmative sous forme de négation et qui se place à la fin de la phrase, où elle équivaut à l'expression : N'est -il pas vrai ?
} 
161.

L'armée sort d'après la loi ; en manquant à la loi, présage malheureux.

TSHENG TSE. - Lorsque l'armée sort, ce doit être d'après la loi ; en manquant aux lois ce serait un présage malheureux; bien que par une chance heureuse il puisse y avoir succès, c'est cependant une voie logique de malheur. 


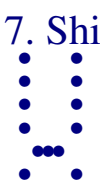

162.

Deuxième trait nonaire : être au milieu de l'armée; présage heureux, pas de culpabilité ; le roi donne trois fois une mission.

TSHENG TSE. - Dans le koua shi, le second trait nonaire est la seule positivité et c'est autour de lui que vient se grouper la foule des négativités. Le cinquième occupe la situation du prince et c'est lui qui correspond sympathiquement et selon la droiture avec le second. Le second trait est le maître du koua shi, ou de l'armée ; c'e st celui qui a la direction exclusive des affaires. Occuper une position inférieure et avoir la direction exclusive des affaires est une circonstance qui ne peut se rencontrer qu'au sujet des armées. Depuis l'antiquité, lorsque le souverain donne une miss ion à un général, sil s'agit d'affaires en dehors des frontières, celui -ci jouit d'une autorité absolue. Le second trait est à l'armée, il jouit de l'autorité absolue et il se conforme à la voie naturelle de la justice, aussi le présage est heureux et il n'y a pas de culpabilité. En effet, si le général se fie à son autorité exclusive et s'appuie sur elle, il manque à la voie rationnelle de l'inférieur ; s'il n'a point la direc tion exclusive, il n'y a pas de raison qui puisse le faire prétendre au succès, et c'est pour cela que s'accorder à la justice, en se tenant dans un juste milieu, constitue un présage heureux. Toutes les fois qu'il s'agit de la voie rationnelle des armées, si l'autorité et la douceur sont également extrêmes, c'est un présage heureux. Du moment où, placé dans cette position, le chef épuise la voie du bien, il peut assurer le succès et la paix de l'empire et c'est pour cela que le roi le favorise en lui confiant jusqu'à trois fois une mission. Toutes les fois qu'une chose est répétée jusqu'à trois fois, la limite extrême est atteinte ${ }^{1}$. Le cinquième trait hexaire se trouve dans une position supérieure ; outre quil confie et délègue une autorité exclusive, il répète encore largement le nombre de ses faveurs; en effet, si les égards ne sont pas proportionnés au mérite, l'autorité n'est pas suffisamment respectée et les inférieurs manquent de confiance dans le chef. Dans d'autres koua, il arrive aussi que le second trait nonaire représente celui que le cinquième trait hexaire investit de l'autorité mais ce n'est que dans le koua shi qu’il possède la direction exclusive des affaires et qu'il représente celui autour de qui se groupe la foule des négativités, ce qui fait que le sens est extrêmement large. Dans la voie rationnelle du sujet, il ne faut pas oser assumer la direction exclusive des affaires ; ce n'est que dans le cas où il s'agit d'affaires qui ont lieu hors des limites de l'État que cette autorité exclusive est possédée. Bien que la direction réside en lui, cependant c'est par la force des armées qui lui sont

\footnotetext{
${ }^{1}$ Règle d’interprétation du Yi king.
} 
confiées que le résultat peut être obtenu ; c'est toujours le résultat des moyens confiés par le prince et la conséquence naturelle et convenable de la dignité dont il est revêtu. Les philosophes de l'époque ont disserté sur ce fait que les sacrifices offerts en l'honneur de Tsheou Kong, avaient été accomplis avec les mêmes cérémonies et la même musique que pour l'empereur ; ils estimaient que Tsheou Kong avait agi en qualité de sujet et ne tenaient pas compte de son mérite, de sorte qu'ils appréciaient qu'on eût dû le traiter en sujet, sans employer ces cérémonies et cette musique. C'est là ne pas connaître la voie rationnelle du sujet, car dans la situation de Tsheou Kong, il s'agissait de faits qui lui étaient propres, c'étai t en raison de la possession de cette situation quil avait pu les accomplir ; c'était, en tous cas, des choses qu'il lui incombait de faire. Tsheou Kong avait atteint le faîte des honneurs. Il en est de même de la voie rationnelle des devoirs de fils; Mang Tse, seul sut apprécier et reconnaître ce sens, aussi il dit: «Servir ses parents comme Tsang Tse est bien »; jamais il ne considéra la piété filiale de Tsang Tse comme exagérée ; en effet, ce que le fils peut personnellement faire donne toujours la mesure de ce qu'il doit faire.

TSHOU HI. - Le second trait nonaire est dans une position inférieure ; il représente celui autour de qui vient se grouper la foule des négativités et il a les vertus de la dureté énergique et de la justice. En haut, il correspond sympathiquement au cinquième et il représente celui qui est favorisé de la délégation de l'autorité. C'est pour cela que tels sont l'image symbolique et le sens divinatoire.

163.

Etre au milieu de l'armée, présage heureux; recevoir les faveurs du ciel. Le roi donne trois fois une mission; il maintient en paix tous les États.

TSHENG TSE. - Ce qui fait le présage heureux de la situation au milieu de l'armée, c'est de recevoir la faveur céleste qui lui confie l'autorité. « Ciel » désigne le roi. Puisqu'il s'agit d'un sujet, s’il ne jouissait de la faveur du prince qui lui délègue l'autorité, com ment pourrait-il avoir le pouvoir exclusif de diriger les affaires militaires et le présage heureux de l'accomplissement de l'œuvre ? La formule symbolique est basée sur ce que le second trait possède la direction absolue des affaires, elle développe ce sens et est en désaccord avec les vues des philosophes de l'époque, telles qu'elles ont été rapportées plus haut. Le roi lui confère trois fois la faveur d'une mission pour le récompenser de ses succès et c'est par là qu'il maintient en paix tous les États. 
1.-C'est d'autant moins probable que, dans la formule elle -même, après qu'il a été question des « faveurs du ciel », il est spécialement question du « roi donnant des missions ». 


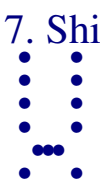

164.

\section{Troisième trait hexaire : l'armée a parfois plusieurs chefs ${ }^{1}$; présage malheureux.}

TSHENG TSE. - Le troisième trait occupe le rang supérieur dans le koua simple inférieur ; c'est celui qui occupe la situatio n et est pourvu de l'autorité. Non seulement ses aptitudes sont la douceur malléable de la négativité sans justice ni droiture, mais encore, dans les affaires militaires, l'autorité doit appartenir exclusivement à un seul homme. Du moment où le second trait emploie les aptitudes de la dureté énergique et de la justice, et où il représente celui en qui le supérieur a confiance et sur qui il s'appuie, il doit nécessai rement avoir la direction exclusive des affaires, et alors il parviendra au succès en achevant son auve. S'il arrive que plusieurs personnes sont encore envoyées pour participer à la direction des affaires, c'est une voie qui conduit certainement au malheur. L'expression $y u$ shi, du texte, signifie plusieurs chefs ; en effet, il s'agit du troisi ème trait, et c'est parce que le troisième occupe le rang supérieur dans le koua simple inférieur que ce sens est développé. Dans les affaires relatives aux corps de troupe et aux armées, si l'autorité n'est pas exclusivement concentrée dans les mains d'u ne même personne, la déroute est certaine.

TSHOU HI. - Yu shi, veut dire l'armée en désordre et dispersée, en désordre et s'en retournant. Puisque la négativité occupe un rang qui comporte la positivité, les qualités et les aptitudes sont faibles tandis que les tendances sont dures et énergiques ; la justice et la droiture font défaut, et ce trait indique l'usurpation de fonctions qui ne résultent pas de la condition. C'est pour cela que tels sont l’image symbolique et le sens divinatoire.

\section{5.}

L'armée a parfois plusieurs chefs ; grand insuccès.

\footnotetext{
${ }^{1} \mathrm{Yu}$, le fond du char portant sur l'essieu et qui permet de transporter quelque chose à l'aide des roues ; shi, corps, cadavres, etc. Rien ne justifie les lectures des commentateurs chinois, et, pour traduire, on est réduit à s'en rapporter à la tradition.
} 
TSHENG TSE. - Adjonction et concours d'un second ou d'un troisième chef ; comment serait-il possible d'arriver au succès ? Mais ce n'est pas seulement l'impossibilité du succès ; c'est encore ce qui conduit aux revers. 


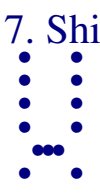

166.

Quatrième trait hexaire : l'armée retourne et renonce ; pas de culpabilité ${ }^{1}$.

TSHENG TSE. - Le mouvement en avant des armées résulte du courage et de la force ; le quatrième trait emploie la douceur et il occupe une position qui comporte la négativité : c'est celui qui n'est pas capable d'avancer et de remporter la victoire. Il reconnaît quil ne peut avancer et il recule, de sorte que les haltes de l'armée sont dans le sens de la gauche; faire les haltes vers la gauche, c'est retourner et renoncer. Apprécier l'opportu nité du mouvement en avant ou en arrière, c'est précisément ce qui convient ; c'est pour cela quil n'y a pas de culpabilité. Voir la possibilité et avancer, reconnaître l'impossibilité et se re tirer, c'est une règle permanente pour les armées. La formule relève seulement le sens d'opportunité et de convenance du mouvement de retraite; elle ne discute pas si les aptitudes du chef lui permettent ou non de se retirer. Il apprécie qu'il ne peut pas vaincre et il conserve l'armée intacte en se retirant : c'est de beaucoup préférable au cas où il l'entraînerait dans une déroute. Devoir avancer et se retirer serait un cas de culpabilité. Le Yi king développe ce sens pour avertir les générations postérieures ; le mérite de cette pensée est profond.

TSHOU HI. - L'expression tso tse veut dire se retirer en arrière et renoncer. Il s'agit de la douceur malléable et négative, sans justice, qui occupe un rang négatif et possède la droiture, et c'est pour cela que telle est l'image symbolique. Conserver l'armée intacte et se retirer est plus sage de beaucoup que ce qui a lieu dans le cas du troisième trait, aussi tel est le sens divinatoire.

167.

Retourner et renoncer, pas de culpabilité ; ce n'est pas encore manquer aux règles ordinaires.

\footnotetext{
${ }^{1}$ Tso tse, quatrième et cinquième caractères du texte. Le premier terme désigne la gauche. Malgré les explications de quelques commentateurs qui cherchent à établir que par suite de certaines considérations d'ordre tactique le terme gauche désigne l'arrière d'une armée, il est impossible de justifier l'exactitude de cette interprétation. Le second terme signifie « fois », « succession de fois ». « ordre de succession, après ». Enfin on lui donne aussi le sens de halte d'une armée.
} 
TSHENG TSE. - La voie rationnelle de l'action des armées consiste à profiter de l'opportunité du moment : c'est là la règle ordinaire et constante et c'est pourquoi se retirer en arrière en renonçant ne constitue pas encore un manquement. Reculer et renoncer, dans les mêmes conditions que le quatrième trait, c'est faire ce qui est convenable et c'est à cause de cela qu'il n'y a pas de culpabilité.

TSHOU HI. - Reconnaître l'impossibilité et la difficulté et se retirer en arrière, c'est la règle ordinaire et constante des armées. 


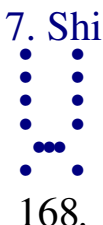

Cinquième trait hexaire : la rizière a du gibier; avantage à retenir les paroles ; pas de culpabilité. Le fils aîné commande l'armée ; le fils cadet participe au commandement; perfection, présage malheureux.

TSHENG TSE. - Le cinquième rang marque la situation du prince; celui-ci est maître de lever les armées, et c'est pour cela que la formule traite de la voie rationnelle de l'action de lever les armées et d'investir quelqu'un de l'autorité du commandement. Les armées ne doivent absolument être levées que «lorsque les peuples barbares troublent le peuple de Hia ou à cause des brigandages et rebellions criminelles $\gg{ }^{1}$, ce qui est cause du malheur des peuples; alors seulement le prince donne des instructions pour les faire détruire. C'est comme lorsque le gibier entre dans les rizières et commet des dégradations dans les récoltes et les plantations; le devoir commande de le chasser et de le prendre, et alors il est poursuivi et capturé. Mouvoir les armées dans des conditions analogues, c'est ne pas commettre de fautes ; mais si on se met légèrement en mouvement pour nuire à l'empire, la culpabilité est alors considérable. Tshi yen (retenir les paroles), c'est justement recevoir respectueusement les instructions, montrer clairement leur faute aux coupables et les châtier. S’il s'agit de Tsin Hoang et de l'empereur Wou des Han, détruisant les forêts des montagnes pour capturer tout le gibier, ce n'est plus le cas où «la rizière a du gibier». La voie rationnelle à suivre en conférant l'autorité à un géné ral et en lui confiant une armée consiste nécessairement à prendre le fils aîné pour commander l'armée. Le second trai t se trouve dans une position inférieure et il est le maître de l'armée : c'est le fils aîné. Si les fils cadets sont associés à la direction de l'armée, ce sera le cas où, bien qu'on agisse avec droiture, le présage est malheureux. Le fils cadet, c'est -à-dire tout autre que le fils aîné ; depuis l'antiquité, lorsque des généraux ont été investis du commandement sans jouir de l'autorité exclusive et absolue, il en est toujours résulté du désordre et la défaite. C'est, par exemple, le cas de la guerre de Suen Lin Fou sous la dynastie des Tsin, ou le cas de la déroute de Kuo Tse Yi, à Seang tsheou, sous la dynastie des Theang.

TSHOU HI. - Le cinquième trait hexaire est celui de qui dépend l'emploi des armées; il est doux, passif, et juste ; il n'est pas cause de la guerre. L'ennemi l'attaque et la lui impose ; il ne peut faire autrement et répond à la provocation. Ces conditions constituent l'image symbolique de la rizière qui a du gibier ${ }^{2}$, tandis que le sens divinatoire est qu'il y a avantage à capturer ce

\footnotetext{
${ }^{1}$ Citation tirée du Shou king

${ }^{2}$ La traduction est littérale et la forme aussi défectueuse en chinois.
} 
gibier, et pas de culpabilité. Yen, paroles, instructions verbales. Le fils aîné, c'est le second trait nonaire ; les fils cadets, ce sont le troisième et le quatrième traits ${ }^{1}$. De plus, la formule avertit celui qui consulte le sort au sujet de la nécessité de la délégation absolue de l'autorité. Si on charge un homme doué du soin de quelque affaire, et qu'on charge en même temps quelques hommes inférieurs de participer à la direction, ce sera multiplier le commandement et les obliger à rétrograder, de sorte que malgré la perfection ${ }^{2}$ il sera encore impossible d'éviter le malheur.

169.

Le fils aîné commande l'armée ; il emploie la justice en agissant. Les fils cadets participent au commandement : investir du commandement ceux qui n'en sont pas dignes.

TSHENG TSE. - Le fils aîné, c'est-à-dire le second trait qui, par les vertus de la justice et de la droiture, s'unit au supérieur et reçoit de celui -ci l'autorité pour agir. Si d'autres en sont investis en même temps, c'est multiplier le commandement au sujet d'une seule affaire et investir de l'autorité ceux qui ne doivent pas en être investis ; le présage est logiquement malheureux.

\footnotetext{
${ }^{1}$ Le pluriel n'est pas marqué, on peut lire le, ou les fils cadets.

${ }^{2}$ Fermeté absolue dans la droiture.
} 


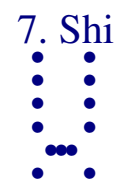

170.

Trait supérieur hexaire : un grand prince donne des ordres ; fondation de royaumes, ennoblissement de familles, l'homme inférieur ne doit pas agir ${ }^{1}$.

TSHENG TSE. - Le trait supérieur marque la fin du koua shi, la fin de l'armée, le moment où l'ouvre est accomplie. Le grand prince récompense ceux qui ont acquis du mérite par des dignités et des missions. Fonder des royaumes, c'est conférer des apanages de princes feudataires ; ennoblir des familles, c'est conférer des titres de Khinget de Tae fou ${ }^{2}$. Le mot tsheng, traduit par ennoblir, signifie conférer. Les hommes inférieurs, bien qu'ayant acquis du mérite, ne doivent pas être employés, c'est pourquoi la formule avertit qu'il ne faut pas les employer ${ }^{3}$. Lorsque les armées et les troupes sont mises sur pied, la voie pour acquérir du mérite par le succès n'est pas unique. Il n'est pas indispensable que tous soient des hommes doués, et c'est pour cela que la formule avertit que lorsque ce sont des hommes inférieurs qui ont acquis ce mérite on ne doit pas employer cette règle à leur égard. Les récompenser avec de l'or, des choses précieuses (étoffes), des pensions, et des titres est légitime, mais on ne doit pas leur conférer des royaumes, des gouvernements de régions, et les charger de fonctions politiques et civiles. En temps ordinaire, les hommes inférieurs deviennent facilement orgueilleux et suffisants ; combien à plus forte raison lorsquils ont déjà acquis du mérite ? C'est là ce qui, sous la dynastie des Han, causa la, perte de Ying Pang. Telle est la profondeur de vues et la portée des avertissements de l'homme saint. Ceci traite exclusivement du sens relatif à la fin des affaires des armées ; il n'est pas question du sens spécial relatif au trait lui-même. En effet, c'est à cause de la grandeur du sujet en général. Sill s'agit spécialement du trai t, alors, il est question d'un trait hexaire qui emploie la douceur pour occuper une position qui comporte la soumission ${ }^{4}$ et cela, au plus haut degré. Le rôle de l'armée étant déjà fini, et puisqu’il se trouve dans un rang qui n'indique aucune situation déterminée, c'est celui qui se place suivant le bien et qui est sans culpabilité.

\footnotetext{
${ }^{1}$ La traduction suit le sens naturel qui résulte du texte. - La tradition fait lire «ne pas employer l'homme inférieur », pour que la phrase eût ce sens, il faudrait que l'ordre du texte fût renversé et qu'il y eût, wou yong siao jen.

2 Titres inférieurs à celui de prince feudataire.

3 Tsheng Tse renverse l'ordre du texte pour lire le sens adopté ; c'est contre les règles de l'expression de la pensée en chinois. On pourrait tout au plus don ner au mot yong, employer, le sens de «participer à » et lire : l'homme inférieur n’y participe pas.

${ }^{4}$ Rang pair, négatif.
} 
TSHOU HI. - Fin de l'armée ; comble de la soumission passive ${ }^{1}$; c'est le moment de discuter les mérites et d'attribuer les récompenses. Le koua simple supérieur khouen représente la terre, de sorte qu'il y a l'image symbolique de fondation de royaumes et de distribution d'apanages avec dignités héréditaires dans la famille. Mais s'il s'agit d'hommes inférieurs, bien qu'ils aient acquis du mérite par leurs succès, on ne doit $\mathrm{p}$ as cependant leur conférer des gouvernements territoriaux, mais simplement leur témoigner de la bienveillance en les récompensant avec de l'or ou des étoffes précieuses. C'est un avertissement pour ceux qui récompensent; s'il s'agit de récompenser un homme inférieur on ne doit pas employer cette formule divinatoire et si, de même, des hommes inférieurs rencontrent ce trait en consultant le sort, ils ne doivent pas non plus employer les règles tracées par cette formule.

\section{1.}

Un grand prince donne des ordres, d'après la droiture et le mérite; l'homme inférieur ne doit pas être employé, il porterait certainement le trouble dans l'État.

TSHENG TSE. - Le grand prince est le dispensateur des faveurs et des récompenses; il les distribue d'après le mérite dan s les affaires de l'armée. Lors du licenciement de l'armée, bien qu'il récompense le mérite acquis, s’il s'agit d'hommes inférieurs, il ne doit cependant, à cause de leur mérite acquis, leur conférer de l'autorité et les employer ; s'il les employait, ils porteraient le trouble dans l'État. Dans l'antiquité, il y a des exemples d'hommes inférieurs qui, s'appuyant sur les mérites qu'ils avaient acquis, ont porté le trouble dans certains États.

TSHOU HI. — L'avertissement de l'homme saint est profond.

\footnotetext{
${ }^{1}$ Trait négatif, rang négatif.
} 


\section{8. $\mathrm{Pi}$}

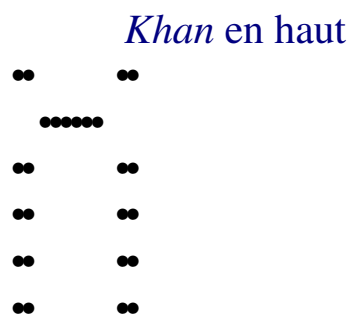

Khouen en bas

172. Pi, présage heureux; consulter le sort à plusieurs reprises pour s'assurer de la possession dune perfec tion absolue et durable; pas de culpabilité. N'étant pas tranquille, commencer à venir ; pour l'homme robuste qui vient tard, présage malheureux.

[]

177. Premier trait hexaire : avoir foi et s'associer ; pas de culpabilité ; avoir foi et remplir le vase; le dernier arrivé a d'autres présages heureux.

$*$

179. Deuxième trait hexaire : l'association se produit de dedans ; présage heureux de la perfection.

$*$

181. Troisième trait hexaire : s'associer à des hommes indignes.

183. Quatrième trait hexaire : de l'extérieur on vient s'associer ; présage heureux de la perfection.

*

185. Cinquième trait nonaire : association illustre ; le roi emploie trois lignes de rabatteurs et perd le gibier qui est devant; les gens du district ne s'en préoccupent point; présage heureux.

*

187. Trait supérieur hexaire : absence de tête dans l'association ; présage malheureux.

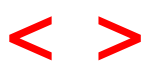


TSHENG TSE. - Pi. «L'Ordre des koua » dit: «La foule doit nécessairement avoir quelqu'un autour de qui elle se groupe; c'est pourquoi le koua shi est suivi du koua $p i$; pi, se rapprocher et s'assister. » La nature des hommes les oblige à se rapprocher et à s'entrai der mutuellement, ce n'est qu'à cette condition qu'ils peuvent jouir de la paix ; aussi, dès qu’i 1 y a foule, il faut nécessairement que cette foule ait quelqu'un autour de qui elle se serre en se groupant, et c'est là ce qui fait que le koua pi suit immédiatement le koua shi. Comme koua, il est composé du koua simple khan, en haut, et du koua simple khouen, en bas. Si on en parle d'après les deux substances, l'eau est au -dessus de la terre ; le rapprochement le plus intime des choses qui ne laisse subsister aucun interstice entre elles n'est jamais aussi parfait que dans le cas de l'eau sur la terre, et c'est pour cela que le koua est appelé pi. De plus, tous les traits sont négatifs, sauf le cinquième, qui, avec la dureté énergique de la positivité, occupe la situation du prince. C'est autour de lui que la foule se serre et se groupe, et le trait supérieur lui-même se rapproche en descendant; donc, ce koua est appelé Siao tshouet exprime l’idée de groupement. 


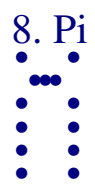

172.

$P i$, présage heureux; consulter le sort à plusieurs reprises pour s'assurer de la possession dune perfection absolue et durable; pas de culpabilité. N'étant pas tranquille, commencer à venir ; pour l'homme robuste qui vient tard, présage malheureux.

TSHENG TSE. - Le rapprochement ${ }^{1}$ est une voie naturelle de bonheur ; les hommes, en se rapprochant mutuellement et en se groupant, préparent eux-mêmes la voie de leur bonheur. Aussi le commentaire appelé «interversion des koua»dit: «Pi, rapprochement en société, félicité ; shi, rassemblement des armées, chagrin.» Les hommes en se rapprochant mutuellement pour se grouper doivent nécessairement suivre une certaine voie morale; sils n'obéissent pas à cette voie morale, ils en auront des regrets et commettront des fautes; donc, ils doivent absolument consulter le sort à plusieurs reprises, pour déterminer la légitimité de la réunion et du groupement, et ne se réunir qu'ensuite. Le terme she, consulter le sort, veut dire consulter le sens divinatoire et décider les règles d’interprétation; il ne veut pas dire : employer les moyens de divination par les brins d'herbe, ou l' « écaille de la tortue ». Si celui autour de qui il s'agit de se grouper possède une perfection absolue et durable, il n'y aura point de culpabilité. Le terme yuan du texte, traduit par le mot «absolu», veut dire «conforme à la voie morale du prince ou du supérieur»; durable veut dire permanent et indéfiniment persistant; perfection veut dire conformité à la voie rationnelle de la droiture. Lorsque le supérieur rallie et groupe les inférieurs, il doit absolument réunir ces trois conditions; les inférieurs, en se mettant à la suite d'un supérieur, doivent absolument réclamer ces trois co nditions ; alors, il n'y aura pas de culpabilité. L'homme qui ne peut pas lui -même assurer son repos et sa tranquillité, cherche à venir se joindre et s'associer en se groupant ; s'il a quelqu'un auprès de qui il puisse se serrer, il pourra assurer sa propre tranquillité. En présence d'un moment où il ne peut être en paix, il convient très certainement qu'il se hâte de chercher quelqu'un auprès de qui il puisse se serrer ; s'il reste isolé et se fie à ses propres forces, si sa tendance à rechercher un point d'appui n'est pas immédiate, s'il tarde, alors, bien que vigoureux, ce sera encore un présage de malheur. Si c'est un présage malheureux même pour l'homme vigoureux, combien à plus forte raison en sera-t-il de même pour celui qui est faible et manque d'énergie ! Le terme fou, du texte, traduit par les expressions «homme robuste», «vigoureux», «viril», est une appellation employée pour désigner ceux qui se soutiennent par l'énergie. Le

\footnotetext{
${ }^{1} \mathrm{Pi}$, dans ce passage, la traduction serait plus complète si on disait : «le rapprochement en société »; plus loin cette expression sera employée, mais il m’a paru préférable de laisser d'abord subsister le vague du terme chinois, qui peut s'appliquer à tout autre ordre d'idées.
} 
commentaire traditionnel dit : «Tse possède la virilité du méridional ${ }^{1}$; il dit encore : «c'est dire que je ne suis pas viril. » Rien de ce qui vit entre le ciel et la terre ne peut se préserver en restant isolé, sans se rapprocher en société ; malgré une force énergique, nul ne peut se maintenir dans l’isoleme nt. La voie rationnelle du rapprochement (pi) résulte de deux tendances qui s'appellent mutuellement; si les deux tendances ne s'appelaient pas mutuellement, ce serait le koua khouei ${ }^{2}$. Qu’il s'agisse du prince qui embrasse le peuple dans sa protection, des inférieurs qui se rapprochent du supérieur pour l'assister, des parents et alliés, des amis, des concitoyens, des associés, dans tous les cas, il en est toujours ainsi. Aussi, pendant que le supérieur et les inférieurs accordent leurs tendances pour se suivre mutuellement, si quelques-uns n'ont point l'idée de se rechercher mutuellement, ils se sépareront et ce sera pour eux un présage de malheur. En général, si les sentiments des hommes s'accordent, ils se réunissent; si ces sentiments sont antipathiques, ils se dispersent. «Mutuellement antipathiques », c'est-à-dire que ces hommes se traitent mutuellement sans admettre de gradation de supériorité. Le rapprochement des hommes entre eux est certainement soumis à une loi logique, et c'est préci sément ce qui fait que, lorsqu'ils veulent se grouper, ils ne doivent pas ajourner et tarder.

TSHOU HI. - Pi, se rapprocher et s'assister. Le cinquième trait nonaire emploie la dureté énergique de la positivité pour occuper le rang intermédiaire dans le koua simple supérieur et il s'accorde avec la droiture ; au-dessus et au-dessous de lui, cinq négativités se groupent et le suivent: c'est l'image symbolique d'un seul homme protégeant tous les États et l'image des peuples des quatre horizons ${ }^{3}$ levant les yeux vers le même homme. Aussi, si celui qui consulte le sort obtient ce koua, cela exprimera quil doit devenir le point d'appui autour duquel les hommes se grouperont pour s'entraider. Toutefois il devra consulter le sort de nouveau, afin d'examiner ce qui le concerne personnellement lui-même; s’il a les vertus de l'excellence absolue, de la fermeté durable et persistante dans la droiture, alors seulement il sera digne du mouvement qui amène la foule vers lui et sera sans culpabilité. Ceux qui ne se sont pas encore associés et qui ont quelque motif de crainte pour leur tranquillité sont aussi, tous, sur le point de se rallier à lui. Si quelqu'un tarde encore et vient après les autres, alors, les premiers ont déjà resserré et affermi les liens de leur union, tandis que lui n'arrive que lorsqu'il est déjà tard, et il rencontre le malheur. Quand on veut s'associer à quelqu'un, c'est encore d'après ces données qu'on doit se conduire en se considérant dans une position inverse.

\footnotetext{
${ }^{1}$ Il s'agit probablement de Lou Tse, un des disciples de Khong Tse ; dans les deux citations, c'est le mot fou qui est traduit par « viril ».

${ }^{2}$ Les deux tendances de l'eau et de la terre. Le koua khouei est formé de deux koua simples qui représentent le feu et le marais. Le feu tend à monter, l'eau du marais tend à descendre, les tendances sont opposées, et le koua khouei exprime l'idée d'opposition et de dispersion.

${ }^{3}$ Littéralement : «Des quatre mers levant les yeux vers un seul et même homme. »
} 
173.

Le commentaire traditionnel de la formule déterminative dit : Association, bonheur.

TSHOU HI. - On suppose que les trois caractères de cette formule sont une superfétation dans le texte.

174.

Pi, assistance ; l' inférieur suit et obéit passivement ${ }^{1}$.

TSHENG TSE. - «Association, bonheur. » L'association est la voie rationnelle du bonheur. Lorsque les êtres se rapprochent mutuellement et se groupent ensemble, c'est une voie logique de bonheur. Les mots «Siao tshou assistance », expliquent le sens du mot Siao tshou; Siao tshouexprime le rapprochement mutuel pour s'entraider ${ }^{2}$. Les mots : "L'inférieur suit et obéit passivement» expliquent comment le koua exprime l'association. Le cinquième trait emploie les qualités de la positivité pour occuper le rang prééminent; la troupe des inférieurs le suit passivement pour se grouper et s'entraider, c'est pour cela que le koua est appelé pi.

TSHOU HI. - Ceci explique le sens du nom du koua au moyen de la substance même de ce koua.

175.

\footnotetext{
${ }^{1}$ Dans cette formule, les deux premiers membres de phrase de la formule $\mathrm{n}^{\circ} 172$ sont lus sans temps d'arrêt, c'est -à-dire que la ponctuation est supprimée, et il en résulte une légère modification du sens.

${ }^{2}$ Le caractère qui sert de nom à ce koua est [] ; il se lit pi, pí et $p \hat{\imath}$. Ce caractère, dans son sens le plus général, se prononce $p i$ et il exprime la comparaison entre deux choses analogues qu'on assimile. C'est très évidemment le sens le plus archaïque, ainsi qu'on peut le vérifier par l'examen des formes graphiques antiques. La lecture pi, avec le sens de groupement, n'est qu'une acception dérivée. P'i a le même sens que $p$ ’̀. Cette racine, en passant dans les langues agglutinantes a formé le préfixe pe avec le sens d'assimiler, mettre au rang de.
} 
Consulter le sort à plusieurs reprises pour s'assurer de la possession dune perfection absolue et durable sans culpabilité ; à cause de la dureté énergique et de la justice. N'étant pas tranquille, commencer à venir; le supérieur et l'inférieur se correspondent sympathiquement. Pour l'homme robuste qui vient tard, présage malheureux; la voie rationnelle de l'association est épuisée.

TSHENG TSE. - Déterminer à plusieurs reprises les conditions de l'association mutuelle; ce n'est que par la possession d'une perfection absolue et durable qu'il est possible que la suite soit sans culpabilité. Ce qu'on entend par perfection absolue et durable, c'est l'ensemble des conditions que réunit le cinquième trait; demeurer dans la justice et la droiture en employant la dureté énergique de la positivité, c'est épuiser le bien dans la voie rationnelle de l'association ; se montrer digne du rang suprême par la dureté énergique de la positivité constitue la vertu du prince. Le mot yuan, traduit par « absolu », exprime la condition de se maintenir dans la justice en possédant la droiture et de pouvoir indéfiniment observer la perfection. La formule du trait parle essentiellement et d'une façon générale de la voie morale de l'association ; la formule déterminative traite. de la perfection absolue et durable; c'est le cas du cinquième trait nonaire employant son énergie à se placer selon la justice et la droiture. Pendant la vie de l'homme, lorsqu'il ne peut assurer son repos et sa tranquillité, il se décide alors à venir demander à se joindre à la société. Le peuple ne peut se protéger de lui-même, aussi il se place sous l'autorité du prince ${ }^{1}$, afin d'obtenir la tranquillité. Le prince ne peut pas exister seul, aussi il protège le peuple pour assurer son repos. Privés de repos, les hommes viennent et se groupent : le supérieur et les inférieurs se correspondent sympathiquement. $\mathrm{Si}$ on en parle au point de vue du dévouement de l'homme saint au bien public, il recherche assurément avec la plus parfaite sincérité l'association de tout l'univers, afin d'assurer la paix des peuples. Si on en parle au point de vue des mobiles privés des rois des époques postérieures, lorsqu'ils n'ont pas recherché le concours des classes inférieures de la population, il en est résulté pour eux des désastres et leur chute, aussi les tendances des supérieurs et des inférieurs doivent absolument se correspondre sympathiquement. Si on en parle au sujet du koua lui-même, la troupe des négativités placées en haut et en bas se groupe autour du cinquième trait; le cinquième trait groupe la foule ; c'est do nc la correspondance sympathique entre le supérieur et les inférieurs. La foule doit absolument se grouper en association, et ce n'est qu'à cette condition qu'elle peut jouir facilement de la vie. Entre le ciel et la terre, rien ne peut jouir de son développement normal sans rapprochement mutuel et association. Si la tendance qui porte à suivre le mouvement commun n'est pas ardente, et sil y a retard et délais, alors l'association ne peut plus s'accomplir. Pour l'homme robuste lui-même, c'est un présage de malheur. N'avoir personne de qui on puisse se rapprocher pour s'associer, c'est plier sous le poids de la misère sans

\footnotetext{
${ }^{1}$ Littéralement : «il se place un prince sur la tête. »
} 
pouvoir se redresser, et arriver ainsi jusqu'au malheur ; c'est la voie logique de la misère finale.

TSHOU HI. - C'est encore l'explica tion de la formule du koua au moyen de la substance de ce koua. «Dureté énergique et justice» désigne le cinquième trait ; « supérieur et inférieurs » désigne les cinq traits négatifs.

176.

Le commentaire traditionnel de la formule symbolique dit : Sur la terre, il y a de l'eau : rapprochement (pi); les premiers rois agirent pour instituer tous les états, pour rapprocher les princes feudataires.

TSHENG TSE. - Or, le rapprochement mutuel et l'association mutuelle des choses entre elles sans laisser aucun interstice n'est jamais tel que lorsqu'il s'agit de l'eau placée sur la terre, et c'est pourquoi ce koua est considéré comme exprimant l'association et est appelé pi. Les premiers rois, considérant cette image symbolique de l'association, se fon daient sur elle pour instituer tous les États et rapprocher entre eux les princes feudataires. Instituer les divers États, c'était le moyen de grouper le peuple en société ; rapprocher les princes feudataires en les protégeant avec une affection paternelle, c'était le moyen de grouper l'univers en une seule société.

TSHOU HI. - Sur la terre il y a de l'eau, l'eau s'associe à la terre ; elle ne souffre aucun interstice vide. Instituer des royaumes, rapprocher les princes feudataires était, de même, le moyen employé par les premiers rois pour s'associer l'univers sans laisser de lacunes. L’idée relevée dans le commentaire de la formule déterminative est que les hommes Viennent se grouper autour du sujet dont il est question; ici l'idée relevée est que le sujet va se joindre aux hommes. 


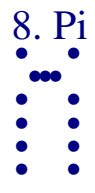

177.

Premier trait hexaire : avoir foi et s'associer ${ }^{1}$; pas de culpabilité ; avoir foi et remplir le vase ${ }^{2}$; le dernier arrivé a d'autres présages heureux ${ }^{3}$.

TSHENG TSE. - Le premier trait hexaire figure le commencement de l'association ; la voie rationnelle de l'association est basée sur la sincérité et la confiance; si quelqu'un ne porte pas la bonne foi dans son coar et se rapproche d'autrui, quel est l'homme qui vou drait s'allier à lui ? Aussi au début de l'association, il faut absolument qu'il y ait foi et sin cérité, et alors il n'y a pas de culpabilité. La foi interne, c'est la confiance et l'absolue sin cérité qu'on porte en soi-même, comme lorsque quelque chose remplit complètement l'intérieur d'un vase. Le terme du texte, traduit par le mot vase, désige un objet fait d'une matière simple et sans ornementations; cela exprime que l'intérieur d'un vase est rempli complètement, sans qu'on ajoute aucun ornement à l'extérieur. Alors, à la fin, il peut arriver qu'il y ait d'autres présages heureux ; «autres » indique quils diffèrent de ceux qui sont déjà énoncés. Au sujet du mot «extérieur», si la sincérité remplit complètement l'intérieur, aucun être ne sera sans avoir confiance, pourquoi employer encore l'orne mentation extérieure pour appeler l'association ? Si l'intérieur est plein de véracité et de sincérité, quel que soit l'extérieur, tous doivent ressentir l'influence de ces qualités et venir se mettre à la suite de celui qui les possède. La foi et la sincérité sont les bases de l'association.

TSHOU HI. - Ce qui fait la noblesse du début de l'association, c'est qu'il y a bonne foi, et alors il est possible qu'il n'y ait point de culpabilité. S i cette bonne foi remplit complètement (le cœur), alors il y aura encore d'autres présages heureux.

178.

Premier trait hexaire $d u$ koua Siao tshou; il y a d'autres présages heureux.

\footnotetext{
${ }^{1}$ S'associer à...

2 Vase en terre cuite ; bol, tasse.

3 La traduction est aussi littérale que possible et s'écarte souvent de la lecture des commentateurs chinois. Dans le cas actuel, ceux-là lisent : à la fin il arrive d'autres présages heureux. Pour adopter cette dernière lecture il faut violenter le génie du style chinois.
} 
TSHENG TSE. - Il s'agit du premier trait hexaire du koua pi. La voie rationnelle de l'association est déterminée par le commencement. Si au commencement il peut y avoir bonne foi, il en résultera qu'à la fin il y aura d'autres présages heureux. Si le début est sans sincérité, comment à la fin le présage pourrait-il être heureux ? Le présage malheureux du trait supérieur hexaire, c'est l'absence de principe ${ }^{1}$.

\footnotetext{
${ }^{1}$ Littéralement « parce qu'il n'y a point de tête »; le texte et loin d'être clair.
} 


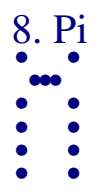

179.

Deuxième trait hexaire : l'association se produit de dedans; présage heureux de la perfection.

TSHENG TSE. - Le premier et le cinquième traits sont ceux qui se correspondent sympathiquement avec droiture ; tous deux possèdent la justice et la droiture : ce sont ceux qui s'associent et se groupent suivant la voie naturelle de la justice et de la droiture. Le second est placé dans le koua simple intérieur ; " de dedans » veut dire " de soi-même », " de son propre mouvement ». Bien que le droit de choisir les aptitudes et de les employer appartienne au supérieur, cependant, le fait de consacrer sa personne au service du pays, doit certainement résulter de la volonté propre du sujet. Posséder en soi-même le sentiment de l'accord à la voie morale du prince et se mettre en avant pour son service, c'est se conformer à la droiture, et le présage est heureux. Employer la voie rationnelle de la justice et de la droiture pour répondre sympathiquement à l'appel du supérieur, c'est là agir de soi -même et sans se manquer à soi-même. Se hâter prématurément en cherchant à s'associer, ce n'est pas la voie ra tionnelle de l'estime de soi -même, telle que la pratique l'homme doué, c'est se manquer à soi -même.

TSHOU HI. - Douceur, passivité, justice et droiture; en haut, correspondance sympathique avec le cinquième trait nonaire; de dedans, mouvement de rapprochement vers quelque chose d'extérieur et accord avec la droiture ; c'est une voie logique de bonheur. Si celui qui consulte le sort est dans ces conditions, il agira avec droiture et le présage est heureux.

180.

L'association se produit de dedans ; ne pas se manquer à soi-même.

TSHENG TSE. - Conserver sa propre voie de justice et de droiture en accueillant l'appel du supérieur, c'est là ne pas se manquer à soi -même. Les avertissements du Yi king sont graves et abstraits. Bien que le second trait possède la justice et la droiture, son caractère naturel (résultant de sa substance physique) est la douceur malléable, et son essence est la soumission passive. C'est pour cela qu'il y a l'avertissement relatif au présage heureux de 
la perfection et au fait de se manquer à soi-même ; c'est avertir de s'observer en attendant l'appel du supérieur, afin de ne pas s'engager dans une voie conduisant à des malheurs futurs. Il a été dit: la réforme de soi-même par l'homme instruit, c'est la voie morale pour appeler l'a ttention du supérieur; abaisser son jugement, s'humilier en se déconsidérant, n'est pas la voie rationnelle d'estime de soi-même. Ainsi, chez Yi Yin et Wou Heou le désir de secourir l'empire était loin de n'être pas ardent, mais ils devaient attendre que les convenances fussent observées à leur égard, avant de sortir de leur retraite.

TSHOU HI. - En possédant la droiture, on ne se manque pas à soi-même. 


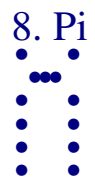

181.

Troisième trait hexaire : s'associer à des hommes indignes.

TSHENG TSE. — Le troisième trait hexaire est sans justice ni droiture, et ceux à qui il s'associe sont tous sans justice ni droiture; le quatrième trait est malléable, négatif et sans justice, tandis que le second conserve ses sympathies et s'associe avec le premier ; tous sont sans justice ni droiture ; ce sont des hommes indignes. S'associant avec des hommes indignes, les inconvénients sont évidents et il n'est pas nécessaire de parler des remords et de l'appréhension de maux futurs, c'est pourquoi on peut en souffrir. Malgré la justice et la droiture du second trait, il est qualifié comme «homme indigne ${ }^{1}$; le sens est adopté suivant l'opportunité du moment et il est différent dans chaque cas.

TSHOU HI. - Douceur malléable négative sans justice ni droiture ; ceux qui le supportent, le surmontent, ou lui correspondent sympathiquement, sont tous négatifs ; ceux à qui il s'associe ont tous l'image symbolique d'hommes indignes. Le sens divinatoire est un présage très malheureux, et cela est évident sans qu'il soit nécessaire de le mention ner.

182.

S'associer à des hommes indignes ; n'est-ce pas encore nuisible?

TSHENG TSE. - L'association des hommes entre eux a pour but la recherche de la paix et du bonheur; ici il est question d'association avec des hommes indignes, il est donc certain que, au contraire, il en résultera des regrets et l'appréhension d'un mal futur ; c'est encore quelque chose qui peut nuire. L'avertissement insiste sur les inconvénients de l'asso ciation.

\footnotetext{
${ }^{1}$ Sens douteux. Réflexion calquée sur le commentaire de la formule symbolique $\mathrm{n}^{\circ} 182$.
} 


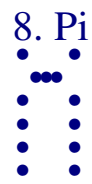

183.

Quatrième trait hexaire : de l'extérieur on vient s'associer ; présage heureux de la perfection.

TSHENG TSE. — Le quatrième ne correspond point sympathiquement au premier et le cinquième s'associe avec lui : vers l'extérieur il s'associe au cinquième; c'est là atteindre à la parfaite droiture, et c'est un présage heureux. Le prince et le sujet s'associant ensemble, c'est la droiture ; s'associer ensemble en se rapprocha nt, s'associer ensemble en se donnant l'un à l'autre, c'est ce qui convient et est opportun. Le cinquième est éner gique, positif, juste et droit ; c'est le sage ; il occupe le rang prééminent et est dans une position supérieure. Or, se rapprocher du sage, suivre le supérieur, c'est la droiture dans l'association, de sorte que cela constitue le présage heureux de la perfection. L'occupa tion du quatrième rang par un trait hexaire donne encore le sens d'accord avec la droiture. Enfin, un homme doux et négatif, sans justice, pouvant s'associer au sage doué d'éner gie, de justice, d'intelligence et de droiture, indique l'accord avec la droiture et donne un présage heureux. Ou bien encore, en s'associant au sage, en suivant le supérieur, il faut absolument agir par la voie naturelle de la droiture et le présage sera heureux. Toutes ces définitions s'enchaînent mutuellement et le sens est dès lors complet.

TSHOU HI. - Il emploie la douceur malléable et occupe un rang qui comporte la douceur; au-dehors il s'asso cie au cinquième trait nonaire, contre lequel il se presse ; cela est considéré comme exprimant l'accord à la droiture et comme une voie rationnelle de bonheur. Si celui qui consulte le sort est dans ces conditions, ce sera la droiture et un présage heureux.

184.

À l'extérieur s'associer au sage; pour suivre le supérieur.

TSHENG TSE. - «Association extérieure ${ }^{1}$ veut dire suivre le cinquième trait. Celui-ci représente le sage énergique, intelligent, juste et droit; de plus il occupe la situation du prince ; le quatrième s'associe à lui. C'est là s'associer au sage, en suivant d'ailleurs le supérieur, et de là résulte le présage heureux.

\footnotetext{
${ }^{1}$ Association avec le trait extérieur.
} 


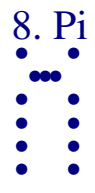

185.

Cinquième trait nonaire : association illustre ${ }^{1}$; le roi emploie trois lignes de rabatteurs et perd le gibier qui est devant; les gens du district ne s'en préoccupent point; présage heureux.

TSHENG TSE. - Le cinquième trait occupe la situation du prince ; il se place suivant la justice et possède la droiture ; c'est celui qui épuise le bien dans la voie rationnelle de l'association. La voie logique du prince groupant l'univers en société consiste seulement en ce qu'il doit illustrer et montrer clairement la voie morale de l'association, par exemple, au moyen de la sincérité de ses idées dans sa manière d'être envers les êtres, par le soin de juger sur ses propres sentiments de l'effet de ses actions sur autrui, par le développement des institutions sociales et la pratique de l'humanité, afin que tout l'univers aspire à éprouver l'influence bienfaisante de ses qualités : telle est la voie rationnelle du prince pour réunir et rapprocher l'univers en société. Dans de telles conditions, qui donc, dans l'univers, ne se rapprocherait du supérieur pour se grouper autour de lui ? Mais s’il n'agit que par la violence et si son humanité est minime; s'il méconnaît la voie morale et recherche la flatterie, sîl ne tend qu'à obtenir le concours des inférieurs, cette voie mora le est encore déprimée et étouffée : lui serait-il donc possible d'obtenir ainsi le concours de l'univers ? Aussi, l'homme saint emploie la droiture exprimée par le cinquième trait nonaire accomplissant parfaitement les prescriptions de la voie rationnelle de l'associa tion et prend comme exemple les «trois rangs de rabatteurs ». Il est dit : «Le roi emploie trois lignes de rabatteurs et perd le gibier qui est devant; les gens du district ne s'en préoccupent point ; présage heureux. » Les premiers rois considéraient les chasses des quatre saisons comme une coutume qui ne pouvait pas être délaissée, aussi consultant l'humanité de leur cour, ils établirent la règle de trois lignes de rabatteurs ${ }^{2}$. C'e st ce que le livre des Rites exprime en disant : «L'empereur (Thien Tse) ne ferme point la circonvallation. ${ }^{3}{ }^{3}$ L'expression : Tsheng theang ${ }^{4}$, faisant interrompre la continuité du filet, a le même sens.

Dans l'enceinte de chasse de l'empereur, on réuni ssait trois faces sans discontinuité, tandis que dans celle de devant on ouvrait une voie praticable, afin que le gibier puisse y passer et s'en aller, et cela par crainte de

\footnotetext{
${ }^{1}$ On peut lire aussi : «Illustration de l'idée d'association » ou « s'associer ostensiblement ».

2 Sens mal défini. Le huitième caractère du texte signifie « courir vite », « cheval qui court vite ». J'ai suivi l'interprétation traditionnelle.

${ }^{3}$ Palissade ou fossé qui entoure ; assiéger ; parc à bestiaux.

${ }^{4}$ Roi de la dynastie des Tsheou.
} 
l'extermination des animaux ${ }^{1}$; c'est là l'humani té se manifestant dans l'amour de la vie. N'était pris que ce qui ne savait pas préserver sa vie, ceux qui au lieu de sortir s'enfonçaient dans l'enceinte ; les oiseaux et autres animaux qui fuyaient par devant pouvaient se sauver, et c'est pour cela que le texte dit: «Perdre le gibier qui est devants. ${ }^{2}$ Le roi illustre et montre clairement la voie morale de l'association; l'univers vient spontanément se grouper autour de lui. Il enveloppe ceux qui Viennent de son affection et de sa protection. Certes, ce n'est pas là un empressem ent désordonné et né de la convoitise, lui faisant rechercher l'aide et la société des êtres. Ainsi, dans l'exemple des trois rangs de rabatteurs à la chasse, les animaux qui s'en vont n'en sont pas empêchés et ne sont pas poursuivis, tandis que ceux qui viennent sont pris. C'est là la grandeur dans la voie rationnelle du prince, ce qui fait que le peuple jouit du bienêtre sans savoir de qui il lui vient. «Les gens du district ne s'en préoccupent point, présa ge heureux »; ce passage exprime le point extrême auquel est poussé le désintéressement et l'absence d'égoïsme, sans distinction entre ce qui est proche ou éloigné, entre ce qui est personnel ou étranger. Le district, c'est le district de la résidence ; dans le Yi king, ce qu'on entend par ce mot di strict, c'est toujours le lieu où le roi tient sa cour, le royaume central au milieu des divers États feudataires. Le terme traduit par «se préoccuper» exprime l'idée d'assigner une limite ${ }^{3}$; unité de manière d'agir dans la façon de recevoir et de trait er les êtres, sans limiter l'affection à ceux qui résident dans le district; dans ces conditions le présage est heureux. L'homme saint gouverne le monde par la grandeur du désintéressement et par l'absence d'égoïsme ; on peut le voir par l'exemple de l'il lustration de l'idée d'association développée ci-dessus. Ce n'est pas la voie du prince réunissant l'univers en société qui est seule basée sur ces principes ; en général, dans l'association entre les hommes, il n'en est jamais autrement. Si on en parle a u point de vue du sujet envers le prince, épuiser la fidélité et la sincérité, développer jusqu'à leurs dernières limites les capacités et la force d'action, c'est illustrer la voie morale de l'association aux intérêts du prince et à sa fortune. Être employé ou ne pas l'être dépend uniquement de la volonté du prince ; on ne doit point, par une approbation servile, aller au-devant de son choix et chercher à se faire agréer dans son entourage. Entre amis il en est encore de même; il faut se réformer soi-même et assurer la sincérité de ses intentions dans les relations avec autrui. Être affectionné et recherché, ou ne

\footnotetext{
1 «Ne pouvant supporter la destruction absolue des êtres. »

${ }^{2}$ En réalité le terme kin du texte, ne désigne que les oiseaux; on l'emploie quelquefois aussi pour désigner tous les animaux, du moins on est forcé de lui donner cette valeur pour expliquer quelques textes anciens, tels que ce passage.

3 Tsheng Tse lit: «Au sujet de la population du district, pas de restriction. » Si le terme kiae, avant-dernier caractère du texte, avait le sens qu'on lui prête ici, c'est -à-dire s’il sign ifiait : fixer une limite, cette lecture des commentateurs chinois ne serait pas impossible ; mais ce caractère n'a pas ce sens. Kae signifie : «avertissement, remontrance; correction légère ; crainte. » Quelle que soit l'acception que l'on choisisse, le sens naturel, résultant de la construction de la phrase, est que, bien qu'une partie du gibier s'échappe, les gens du district ne «s'en inquiètent » pas ou « ne sont pas réprimandés ». La version de ces commentateurs n'est pas universellement admise. Voir le Tse tien.
} 
pas l'être, dépend des sentiments d'autrui et non pas de sa propre volonté à soi-même; on ne doit pas employer les discours insinuants et se parer de couleurs empruntées, suivre pas à pas en approuvant quand même, et profiter des occasions fortuites en visant à faire rechercher sa propre société par quelqu'un. Dans toute société entre concitoyens, parents ou alliés, personnes quelconques, il n'en est jamais autrement; tel est le sens de la formule relative aux trois lignes de rabatteurs et au gibier qui est en avant.

TSHOU HI. - L'unique positivité occupe le rang prééminent : énergie, activité, justice et droiture ; la foule des négativités, dans le koua, vient se grouper autour de cette positivité; c'est là manifester ostensiblement son association sans arrière-pensée d'intérêt privé. Par exemple, c'est l'empereur qui ne fait pas joindre les extrémités et qui ouvre une des faces du filet. Ceux qui viennent ne sont pas repoussés ; ceux qui s'en vont ne sont pas poursuivis. Aussi, ce koua est considéré comme exprimant l'image symbolique de l'emploi des trois rangées de rabatteurs en perdant le gibier qui est avant et sans que les hommes du district s'en préoccupent. En effet, bien qu'il s'agisse d'un cas tout person nel, c'est encore un exemple de l'idée exprimée plus haut. Ils ne s'avertissent pas mutuel lement afin d'être prêts à capturer quand même. Tout ceci exprime toujours une voie rationnelle conduisant au bonheur et si celui qui interprète le sens divinatoire est dans ces conditions, le présage est heureux.

186.

Présage heureux de l'association illustre; occuper la situation avec droiture et justice, abandon de ce qui est réfractaire, accueil de ce qui suit avec passivité; perdre le gibier qui est devant; les gens du district ne s'en préoccupent pas ; le supérieur inspire le sentiment de la justice.

TSHENG TSE. - Ce qui fait le présage heureux de l'association illustre, c'est que la situation occupée s'accorde avec la droiture et la justice ; se placer sur le terrain de la droiture et de la justice, c'est suivre la voie rationnelle de la droiture et de la justice. Dans l'association, c'est l'absence de partialité qui constitue le bien et c'est pour cela que la for mule porte «droiture et justice ». Toutes les fois que le texte porte tsheng tshong, c'est qu'en se plaçant avec droiture on se conforme à la justice ; c'est le cas dans les koua pi et sui, par exemple. Lorsque le texte porte tshong tsheng, c'est qu'il s'agit de posséder la justice et la droiture; c'est le cas dans les koua song et $s u$. La coutume de capturer tout ce qui ne sait pas profiter de la destinée (en protégeant sa propre vie) consiste précisément à laisser ce qui est obéissant et à prendre ce qui est rebelle ; ceux qui obéissent à la destinée et qui s'en vont évitent tous d'être capturés. Dans le cas du koua pi il s'agit d'attraction ou de répulsion ; quand 
on parle de ceux qui s'en vont, on considère ceux qui sont réfractaires ou opposants; quand on parle de ceux qui viennent, on considère ceux qui suivent avec obéissance. Aussi ce qui est perdu, c'est le gibier qui s'en va par devant ; cela exprime que ce qui vient est protégé et que ce qui s'enfuit n'est pas poursuivi ; aucune limite ne réduit l'affection à ce qui est proche et voisin. Le supérieur, en dirigeant l'inférieur, doit agir avec équité et justice ; il doit considérer et traiter de la même manière ce qui est proche et ce qui est éloigné.

TSHOU HI. — Être dirigé vers l'équité par la vertu du supérieur. 


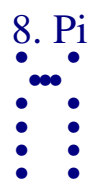

187.

Trait supérieur hexaire : absence de tête dans l'association; présage malheureux.

TSHENG TSE. - Ce trait hexaire occupe le rang supérieur ; c'est la fin de l'association. Le mot sheou, tête, avant-dernier caractère du texte, veut dire commencement, origine ; dans la voie rationnelle de toute association, si le commencement est bien, la fin sera le bien. Il arrive quelquefois que le commencement n'est pas suivi de résultat ; jamais il ne peut y avoir absence de commencement et ensuite résultat. Aussi, dans le cas où il n'y a pas de principe dans le fait d'association, lorsqu'arrive la fin, le résultat doit être malheureux. Ceci est dit par rapport à la fin de l'association. Cependant le trait supérieur est hexairé, c'est-à-dire malléable et négatif et il est sans justice ; il est placé au comble du péril ; c'est évidemment celui qui ne peut pas atteindre au résultat. Commencer une association autrement que par la voie morale et rationnelle et arriver à une impossibilité finale, c'est là un cas fréquent.

TSHOU HI. - La douceur malléable de la négativité occupe le rang supérieur; elle ne s'associe point aux traits inférieurs ; c'est une voie logique de malheur et c'est pour cela que ce trait constitue l'image sy mbolique d'absence de principe ${ }^{1}$; et que le sens divinatoire indique un présage malheureux.

188.

Absence de tête dans l'association ; résultat impossible.

TSHENG TSE. - Du moment où il n'y a pas de principe dans l'association, comment arri verait-elle à un résultat ? S'associer ensemble en ayant un principe n'est pas encore une garantie absolue d'un résultat favorable; mais si le début n'est pas conforme à la voie morale, comment garantir encore la fin? C'est pour cela que le texte dit : «résultat impossible. »

\footnotetext{
${ }^{1}$ Sheou, tête, commencement, chef.
} 
TSHOU HI. - Si on en parle au point de vue de l'image symbolique de supériorité et d'infériorité, ce sera l'absence de tête ou de chef ; si on en parle au point de vue de l'image symbolique de commencement et de fin, ce sera l'absence de rés ultat ; sans tête, ou commencement, pas de fin, ou résultat. 


\section{Siao tshou}

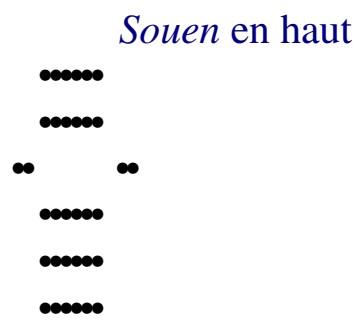

Khien en bas

189. Siao tshou, petit arrêt ; liberté ; nuages épais sans pluie; de nos plaines de l'ouest.

[]

194. Premier trait nonaire : revenir à sa propre voie ; quelle serait la faute? Présage heureux.

*

196. Deuxième trait nonaire : entraînement dans le retour, présage heureux.

198. Troisième trait nonaire : le char manque d'essieu ; l'époux et l'épouse croisent leurs regards.

$*$

200. Quatrième trait hexaire : avoir foi ; le sangs écoule, la préoccupation déborde ; pas de culpabilité.

$*$

202. Cinquième trait nonaire : avoir une foi comme inébranlable ; riche parle voisinage.

*

204. Trait supérieur nonaire : il a déjà plu; chaque chose est déjà à sa place ; estimer l'accumulation de vertu ; danger de la pureté chez la femme ; la lune près de son plein ; l'homme doué se met en mou vement, présage malheureux.

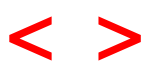


TSHENG TSE. - Siao tshou. «L'Ordre des koua » dit : « Du moment où il y a association, il doit y avoir quelque chose à réunir, aussi le koua pi est suivi du koua siao tshou. » Lorsque des êtres se groupent ensemble, ils constituent un rassemblement ; tshou signifie rassemblement. Ou bien encore, lorsqu'il y a rapprochement mutuel et association, les ten dances individuelles s'unissent ensemble, (tshou); c'est ce qui fait que le koua siao tshou suit immédiatement le koua pi. Tshou, arrêt, s'arrêter ; s'il y a arrêt, il y a rassemblement. Il est constitué par le koua simple souen, en haut, et par le koua simple khien, en bas. Le koua simple khien représente quelque chose d'élevé ; ici il se trouve sous le koua simple souen. Or, pour arrêter (tshou) l'activité de la dureté énergique, il n'y a rien de t el que l'humilité obéissante (souen) ; exprimant l'arrêt ( tshou) par l'humilité ( souen), le koua est considéré comme exprimant le rassemblement résultant de l'arrêt et de la station ( tshou). Mais l'humilité (souen), est une qualité négative ; sa substance est la douceur malléable et obéissante; il est simplement possible d'adoucir l'activité violente de la force active en employant l'humilité obéissante, mais on ne peut pas l'arrêter de force ; c'est donc la peti te voie de l'arrêt par opposition (tshou). Ou encore : le quatrième trait étant le seul trait négatif, correspond à la situation et la mérite; il est l'objet de la satisfaction des cinq traits positifs ; correspondre à sa situation, c'est se conformer à la voie rationnelle de la douceur et de l'hu milité. Il peut arrêter (tshou) les tendances de la troupe des positivités : c'est par là que le koua exprime l'arrêt ( $t$ shou). Siao tshou veut dire arrêt de ce qui est grand par ce qui est petit ; ce qui arrête et rassemble est petit, la chose qui arrête est petite, et cela à cause de sa négativité. Le commentaire de la formule déterminative précise le sens du koua par le cas du quatrième trait hexaire arrêtant les divers traits positifs; il ne parle pas des substances des deux koua simples, il ne relève que ce quill y a de plus important.

Trait négatif occupant un rang pair.

Enlisant : « La petitesse arrête. » 


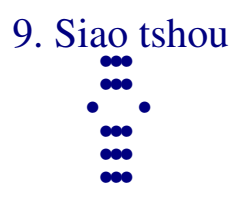

189.

Siao tshou, petit arrêt ; liberté ; nuages épais sans pluie ; de nos plaines de l'ouest.

TSHENG TSE. - Les nuages sont formés par les éthers négatifs et positifs; si les deux éthers s'unissent harmoniquement, ils s'arrêtent en se fixant mutuellement et produisent la pluie. Lorsque la positivité donne le ton et que la négativité s'y accorde en s'y confor mant, c'est ce qui produit l'harmonie ; si la négativité précède le ton que doit donner la positivité, elle ne s'y conforme plus, de sorte que l'harmonie fait défaut. Du moment où l'union n'est pas harmonique la pluie ne peut plus se produire. Lorsque les nuages arrêtés et amoncelés, bien qu'épais, ne produisent cependant point de pluie, c'est qu'ils Vien nent des plaines de l'ouest. Le Nord-Est est une région positive ; le Sud-Ouest est une région négative ; l'accord, ou ton, est donné par la négativité, donc il n'y a point d'harmo nie et il est impossible que la pluie se produise. Au point de vue de l'observateur, le mouvement ascensionnel des vapeurs nuageuses se produit toujours d'un des quatre points de l'horizon, c'est $\mathrm{p}$ our cela que la formule emploie le terme «plaines » 4 ; c'est parce qu'il s'agit de l'ouest qu'elle dit : « de nos ». Ce qui arrête la positivité, c'est le quatrième trait ; c'est donc lui le maître de l'arrêt.

TSHOU HI. - Souen est aussi le nom d'un koua simple de trois traits; il est composé d'une négativité courbée au -dessous de deux positivités, aussi ses vertus, ou aptitudes, sont l'humilité et l'introduction, ses images symboliques sont le vent et le bois. La petitesse est une qualité négative, tshou a le sens « d'arrêter quelque chose ». En haut humilité, en bas activité ; emploi de la négativité pour arrêter la positivité. De plus, dans le koua, le quatrième trait hexaire est le seul trait négatif ; les cinq traits positifs placés au-dessus et au-dessous de lui représentent tout ce qui est arrêté, et c'est pourquoi le koua lui-même est considéré comme exprimant le petit arrêt, ou la petitesse arrêtant. Ou bien encore, arrêt de la positivité par la négativité ; c'est là une chose possible, mais qui ne peut pas être durable, et cela constitue encore l'image symbolique de la petitesse dans ce qui arrête. Au-dedans activité, au-dehors humilité ; le second et le cinquième trait sont tous deux positifs ; chacun d'eux occupe le milieu d'un des koua simples et dirige l'action ou pratique la vertu indiquée; possédant la dureté énergique et étant capable de justice, c'est l'image symbolique des tendances susceptibles de prévaloir, aussi le sens divinatoire exprime quil doit y avoir liberté d'expansion et d'action. Mais toutefois, l'arrêt n'est pas absolu et l'effet n'est pas encore produit, de sorte qu'il y à l'image symbolique des nuages épais, sans pluie, 
venant de nos plaines de l'ouest. En effet, les nuages épais ont quelque chose de négatif ; les plaines de l'ouest sont une région négative. Le pronom « nos » est employé par Wen Wang pour marquer quelque chose qui lui est personnel ; Wen Wang commenta le Yi king pendant qu'il était à Yeou $l i$, de sorte qu'il considérait Khi tsheou comme une région occidentale. C'était précisément là un moment de petit arrêt. Si on obtient ce koua en consultant le sort, l’interprétation du sens divinatoire sera telle qu'elle résulte de l'image symbolique.

C'est principalement sur ce passage qu'on s'appuie pour démontrer que les formules déterminatives du Yi kingsont l'œuvre personnelle de Wen Wang, prince de Tsheou.

Le mot $k h i$ traduit par éther, désigne aussi l'air, les vapeurs, effluves, etc.

Expression empruntée à la musique et souvent employée en chinois.

Le terme du texte est vague; il désigne un espace éloigné, hors des limites de l'empire. (Probablement au-dessous de l'horizon.)

Le maître du koua.

Dans le texte chinois, le sens du mot vertu, ou propriété essentielle, qualité, peut s'allier au terme «introduction », (entrer); dans la traduction, il en résulte forcément un barbarisme choquant.

Allusion à l'histoire de Wen Wang ; un petit arrêt dans le développement de sa fortune, pendant qu'il était en prison.

Qui sera adoptée en raison de la position de celui qui consulte le sort et des circonstancés dans lesquelles il se trouvera.

190.

Le commentaire de la formule déterminative dit: Petit arrêt; la douceur répond à la situation et le supérieur, comme les inférieurs, lui correspondent sympathiquement. On l'appelle siao tshou, petit arrêt.

TSHENG TSE. - Il s'agit du sens du koua parfait. Puisqu'un trait négatif occupe le quatrième rang, que de plus il se trouve placé dans une situation supérieure, la douceur répond à la situation qu'elle occupe. Au-dessus et au-dessous les cinq traits positifs lui correspondent tous sympathiquement et représentent ce qui est arrêté. Arrêter cinq positivités au moyen d'une seule négativité est quelque chose faisable, mais qui ne peut durer, et c'est à cause de cela que le koua représente le petit arrêt. Le commentaire de la formule déterminative explique le sens du koua parfait et lorsqu'il emploie le terme yue «on l'appelle », c'est toujours pour appuyer sur les conséquences naturelles des termes qui forment le nom du koua. Lorsque ce commentaire 
donne le nom du koua et que le caractère yue est supprimé, la force de ce terme est encore la même.

TSHOU HI. - Explication du sens du nom du koua au moyen de la substance de ce koua. La douceur correspond à la situation : c'est-à-dire qu'un trait hexaire occupe le quatrième rang. Les mots «supérieurs et inférieurs » désignent les cinq traits positifs.

191.

Activité et humilité ; dureté énergique, justice et tendances qui prévalent: c'est là la liberté.

TSHENG TSE. - Il est question des aptitudes des koua simples; au-dedans activité et au-dehors humilité ; activité susceptible d'humilité. Le second et le cinquième traits occupent chacun le milieu d'un koua simple : dureté énergique et justice. La nature de la positivité est d'avancer en montant; les traits inférieurs font en outre partie de la substance du koua simple khien : leurs tendances les portent à agir. L'énergie active occupant le rang du milieu dans un koua simple est considérée comme exprimant l'énergie concordant avec la justice; elle est aussi considérée comme exprimant l'énergie active de la justice. Ces diverses conditions des traits du koua expriment que l'arrêt de la positivité a lieu par la douceur et l'humilité, et que la liberté possible résulte de l'énergie active de la justice. Si on en parle au point de vue du koua parfait, ce sera la négativité arrêtant la positivité ; si on en parle d'après les propriétés du koua, la positivité constitue la dureté énergique et la justice. Telles sont ces propriétés, aussi, quoique l'arrêt soit petit, la liberté est cependant possible.

TSHOU HI. - En en parlant au point de vue des vertus et de la substance du koua, la positivité peut encore se développer librement.

192.

Nuages épais et sans pluie; ils continuent à marcher de nos plaines de l'ouest; leur influence ne se fait pas encore sentir.

TSHENG TSE. - La voie logique de l'arrêt est qu'il ne peut devenir considérable, comme lorsque les nuages sont épais et quils ne produisent 
néanmoins pas de pluie. Lorsque la négativité et la positivité s'unissent et sont dans des conditions harmoniques, elles se joignent intimement et produisent la pluie. Si les deux éthers ne s'harmonisent pas, la positivité continue à marcher en montant, de sorte que la pluie ne se produit pas. En effet, les vapeurs de nos plaines de l'ouest donnant l'impulsion initiale, il en résulte qu'il n'y a point harmonie et que la pluie ne peut se produire; l'effet de l'action ne se produit pas encore. L'impossibilité où se trouve le petit arrêt de devenir grand est encore comme l'impossibilité où se trouvent les nuages de nos plaines de l'ouest de produire de la pluie.

TSHOU HI. - Continuant à marcher, exprime que l'arrêt n'est pas encore définitif ; l'éther avance encore en montant.

193.

Le commentaire traditionnel de la formule symbolique dit: Le vent court en haut du ciel : petit arrêt; l'homme doué en déduit la nécessité du bien absolu dans ses actions et ses vertus.

TSHENG TSE. — Ce sont l'activité et la dureté énergique de l'esse nce de la positivité qui sont arrêtées par l'humilité ; or, la nature de l'activité et de la dureté énergique est telle qu'elles ne peuvent être arrêtées que par la douceur et la soumission. Bien que ces qualités soient capables de les arrêter, cependant elles ne peuvent pas contenir définitivement et avec fermeté cette activité et cette dureté énergique ; la douceur et la soumission ne peuvent être employées que pour les dominer en les impressionnant momentanément, et c'est pour cela que le koua est considéré comme exprimant le petit arrêt. L'homme doué considère le sens exprimé par ce petit arrêt, et il l'emploie pour arriver au bien absolu dans ses actions et ses vertus. Arrêter en rassemblant donne le sens d'amasser en arrêtant; ce que l'homme doué amasse et retient de plus important, c'est l'aptitu de aux pratiques fondamentales de la voie morale de la vertu2; dans l'ordre des choses inférieures, c'est l'art et le talent dans les belles lettres. L'homme doué considère l'image symbolique du koua siao tshou et il en conclut au bien absolu par l'amélioration de ses actions et de ses aptitudes. Dans les préceptes relatifs à la conduite et aux vertus, la voie logique et le sens sont l'action par les petites causes.

TSHOU HI. - Le vent est constitué par un éther mais il est sans caractère physique ; on peut l'arrêter, mais cet arrêt ne peut durer longtemps, ce qui constitue l'image symbo lique du petit arrêt. «Améliorer jusqu'au bien absolu les actions et les vertus », exprime que le sujet n'a pas encore pu amasser une somme considérable de ces vertus et en étendre au loin l'effet bienfaisant. 
Khien, activité, ou essence de l'activité ; mais comme le terme kien, du texte, exprime spécialement l'action active ou force active, il a fallu, dans la traduction, remplacer la valeur ordinaire du mot khien (activité) par une expression équivalente (essence de la positivité).

Cette phrase est remarquable; on peut lire: «c'est l'art, (ou capacité) du principe fondamental du Tao te king » (livre de Lao Tse). 


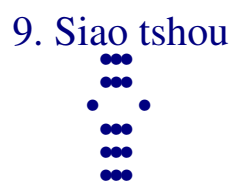

194.

Premier trait nonaire : revenir à sa propre voie ; quelle serait la faute? Présage heureux.

TSHENG TSE. - Le premier trait nonaire est positif et il fait partie de la substance de l'ac tivité ; la positivité est quelque chose qui tend à s'élever. De plus, les aptitudes de l'activité dure et énergique sont suffisantes pour avancer en montant et pour ramener à ce qui, en haut, participe aux mêmes tendances. Ce mouvement en avant, qui ramène en haut, constitue précisément sa voie, et c'est pour cela que le texte dit « revenir à sa propre voie ». Le retour ayant lieu vers sa propre voie, quelle faute, ou culpabilité, pourrait exister ? Il n'y a pas culpabilité et, de plus, le présage est heureux. Dans les diverses formules des traits, lorsqu'il est dit : «pas de culpabilité », c'est que, de la façon indiquée, il n'y aura point faute et culpabilité ; aussi, lorsque cette expression «pas de culpabilité » est employée, le bien compense le mal. Bien qu'on suppose le sens de la formule essentiellement bon, cela n'empêche pas quil ne puisse en être autrement que ce sens, et alors il y a aussi le sens de culpabilité, ou erreur. Dans le cas du premier trait nonaire, c'est par la voie rationnelle qu'il agit ; il n'y a ni faute, ni erreur, et c'est pour cela que la formule dit

«Quelle serait la faute ? »Il est on ne peut plus évident que cela exprime qu'il n’y a ni cul pabilité ni faute.

TslloU HI. - Le koua simple inférieur a pour substance l'acti vité ; c'est toujours et essentiellement une chose qui tend vers l'élévation. Les tendances portent à avancer en montant, mais cette volonté est arrêtée par la négativité. Cependant, le premier trait nonaire fait partie de la substance de l'activité ; il occupe une position inférieure et possède la droiture ; il précède de loin la négativité. Bien qu’il corresponde sympathiquement avec le quatrième trait, il peut cependant se contenir et s'observer par la droiture. Ce n'est pas lui qui est arrêté, aussi il a l’image symbolique d'avancer en revenant à sa propre voie. Si celui qui interprète le sens divinatoire est dans de telles conditions, il sera sans culpabilité et le présage sera heureux.

Koua simple inférieur khien.

195. 
Revenir à sa propre voie; le sens est un présage heureux.

TSHENG TSE. - Aptitudes de dureté énergique positive; c'est par sa propre voie qu'il revient, et le sens est un présage heureux. Le premier et le quatrième traits sont considérés comme en correspondance sympathique conforme à la droiture ; dans le moment de l'arrêt, ce sont ceux qui s'arrêtent et se rassemblent ensemble. 


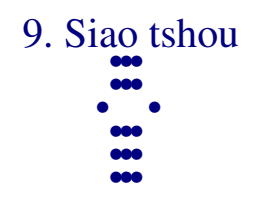

196.

Deuxième trait nonaire : entraînement dans le retour, présage heureux.

TSHENG TSE. - Le second trait emploie les qualités de la positivité pour se maintenir au milieu de la substance du koua simple inférieur; le cinquième, avec les mêmes qualités, occupe le milieu du koua simple supérieur. Tous deux avec l'énergie de l'activité, se maintiennent dans la justice, sont arrêtés par la négativité, et veulent également revenir vers les régions élevées. Bien que le cinquième trait soit au-dessus du quatrième, cependant, si on considère ce qui les arrête, c'est toujours la même chose ; ce sont donc ceux dont les tendances sont identiques. Or ceux qui souffrent des mêmes maux se plaignent mutuellement; le second et le cinquième traits ont les mêmes tendances, aussi ils s'entraînent mutuellement et reviennent. Les deux positivités avancent en même temps, de sorte que la négativité ne peut l'emporter sur elles et que leur mouvement de retour peut, par suite, s'effectuer librement, de sorte que le présage est heureux. On a dit : pouvant réussir dans leur mouvement de retour, s'écartent-ils de ce qui arrête, c'est-à-dire du rassemblement ? Réponse : dans toutes les formules des traits, il est toujours dit que si telle chose est dans telles conditions, il pourra en résulter telle autre condition; si la chose en question est déjà terminée, c'est que le moment a déjà changé; quel avertissement et quel enseignement pourrait-on encore en tirer ? Le cinquième trait fait partie de la substance de l'humilité ; l'humilité oppose un arrêt à l'activité et, au contraire, ce trait et le second s'entraînent mutuellement ; comment peut-il en être ainsi ? Réponse : si on se reporte aux deux substances, alors l'immobilité met un arrêt à l'activité ; si on en parle au point de vue de l'ensemble général du koua parfait, alors une unique négativité arrête cinq positivités. Dans le Yi king, le sens est choisi selon l'occasion qui se présente et c'est là une règle constante.

TSHOU HI. - Les tendances des trois traits positifs sont identiques, mais le second trait nonaire est un peu plus près de la négativité ; c'est à cause de son énergie et de sa justice qu'il peut s'entraîner mutuellement avec le second et revenir, ce qui est encore une voie logique de bonheur. Si celui qui interprète le sens divinatoire est dans ces conditions, ce sera un présage heureux.

Le koua souen, placé à l'extérieur entrave le mouvement naturel du koua khien.

197. 

En
même.

Entraînement au retour dans la justice; de même sans se manquer à soi.

TSHENG TSE. - Le second trait est celui qui demeure dans la justice et qui se conforme à la droiture; quîl agisse avec énergie ou douceur, quiil avance ou se recule, il ne perd jamais la voie de la justice. Lorsqu'il s'agit du mouvement de retour de la positivité, sa force naturelle est telle que ce retour doit être violent. Le second trait, puisqu'il est placé dans la justice, ne peut pas, cependant, quelle que soit la force de son mouvement en avant, en arriver à commettre un excès d'énergie; commettre un excès d'énergie, c'est se manquer à soi-même. La formule du trait parle seulement du sens de l'entraînement dans le retour et du présage heureux ; la formule syinbolique met encore en lumière et fait éclater aux yeux la beauté de l'obser vation de la justice.

TSHOU HI. — Le terme $y i$, « encore » ou « de même que », se rapporte à la formule du second trait. 


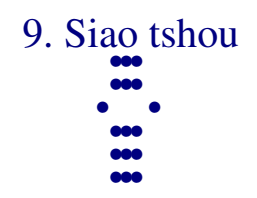

198.

\section{Troisième trait nonaire : le char manque d'essieu ; l'époux et l'épouse croisent leurs regards.}

TSHENG TSE. - Puisque, au troisième rang, un trait positif demeure sans se conformer à la justice et qu'il se rapproche secrètement du quatrième, les sentiments de la négativité et de la positivité s'appellent mutuellemen t. De plus, le rapprochement intime sans la justice indique que c'est la négativité qui arrête et qui domine, de sorte que le troisième trait ne peut avancer ; il est comme un char auquel on a enlevé les fusées d'essieu, c'est -à-dire qui ne peut plus rouler. L'époux et l'épouse croisant leurs regards ; la négativité, c'est ce qui est dominé par la positivité ; ici, au contraire, elle domine la positivité, et c'est comme l'époux et l'épouse qui croisent leurs regards. Croiser les regards veut dire se regarder mutuellement avec des yeux courroucés. Elle n'obéit pas à l'époux et, au contraire, elle le domine. Lorsque la femme mariée est l'objet de la passion aveugle de son époux, quand elle est parvenue, contre la règle logique, à dominer celui-là, il est impossible que cet époux n'ait pas déjà perdu la voie rationnelle de sa position. C'est pour cela que le troi sième trait est naturellement considéré comme un char privé d'essieu, ou comme l'image des regards qui se choquent en s'entrecroisant.

TSHOU HI. - Le troisième trait nonaire veut aussi avancer en montant ; mais s’il est énergique, il est sans justice ; il se presse contre la négativité et, de plus, il est sans correspondance sympathique suivant la droiture. Toutefois, puisque la négativité et la positivité se plaisent mutuellement et constituent le bien qui l'arrête et le retient, il ne peut avancer à son gré, de sorte quil a l'apparence symbolique d'un char dépourvu de fusées d'essieu. Cependant, comme ses tendances sont énergiques, il ne peut d'aille urs pas être en paix et il lutte contre ce qui l'arrête, de sorte qu'il donne encore l'image de l'époux et de l'épou se croisant des regards hostiles. C'est un avertissement donné à celui qui interprète le sens divinatoire que, s’il est dans ces conditions, il ne pourra pas avancer et il aura des sujets de contestation.

Peut-être plutôt de «fusées d'essieu »; cela correspondrait à la fabrication du char cambodgien où l'essieu est remplacé par deux fusées attachées sous la voiture.

«Tournent leurs regards en sens contraire. »

Cela ne résulte pas naturellement du sens de ces deux caractères. 
199.

L'époux et l'épouse croisent leurs regards; impossibilité de redresser la maison.

TSHENG TSE. - L'époux et l'épouse croisent leurs regards ; c'est en effet parce qu'il est incapable de redresser sa famille. Le troisième trait, en se plaçant, ne tient pas compte de la voie morale, de sorte que le quatrième réussit à le dominer et ne lui permet point d'avancer. Il en est de lui comme de l'époux incapable de redresser les défauts de sa famille, situation d'où résulte l'échange de regards courroucés.

TSHOU HI. - Tsheng Tse dit : c'est pour cela que le troisième trait est naturellement considéré comme un char privé d'essieu, ou comme l'image des regards qui se choquent en s'entrecroisant.

Tsheng Tse ajoute le mot kia, pour donner au terme du texte, le sens de «famille». 


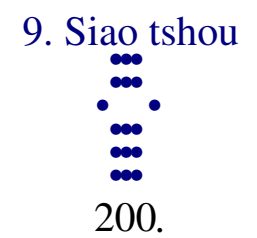

Quatrième trait hexaire : avoir foi ; le sangs écoule, la préoccupation déborde ; pas de culpabilité.

TSHENG TSE. - Le quatrième trait, pendant le moment de l'arrêt, est placé près de la situation du prince : c'est celui qui retient le prince. Si en lui même il a la bonne foi et la sincérité, les tendances du cinquième porteront celui-ci à avoir confiance et à se laisser imposer cette retenue. Dans le koua il n'y a qu'une seule négativité qui arrête la foule des positivités. Les tendances des diverses positivités sont dirigées vers le quatrième; si celui-ci veut inconsidérément employer la force pour les arrêter et les retenir, négativité isolée se posant en adversaire de toute énergie, il en résultera nécessairement pour elle du mal et des accidents. Ce n'est qu'en épuisant la bonne foi et la sincérité pour sympathiser avec ces positivités qu'il lui sera possible de leur faire subir son influence; de cette façon le mal sera écarté et les accidents éloignés, le péril et la crainte seront évités. C'est ainsi qu’il sera possible qu'il n'y ait pas de culpabilité ; de toute façon le mal sera inévitable, et telle est la voie rationnelle pour arrêter la dureté énergique par la douceur. Étant donné la majesté redoutable du prince et la petitesse infime du sujet, s'il arrive que le premier peut suspendre et contenir le cours des désirs du premier, c'est parce qu'il aura employé la bonne foi et la sincérité pour l'influencer.

TSHOU HI. - Une seule négativité arrêtant la foule des positivités il en résulte essentiellement que celle-là doit en éprouver du mal et de l'appréhension. Puis que ce trait emploie sa douceur malléable à se conformer à la droiture, qưil est sans prévention et fait partie de la substance de l'humilité, puisque deux positivités lui prêtent leur concours, il représente l'image symbolique d'avoir foi tandis que le $\mathrm{s}$ ang coule et que la préoccupation déborde; l'absence de culpabilité en est la conséquence logique. C'est pour cela que la formule avertit celui qui interprète le sens divinatoire que, si lui aussi possède cette vertu, il sera sans culpabilité.

Littéralement : «vide au milieu », évidé en dedans, pris au figuré ce terme exprime que le cour est libre de toute prévention.

C'est -à-dire de la substance du koua simple sauen. 
Avoir foi, la préoccupation déborde; le supérieur conforme ses tendances.

TSHENG TSE. - Du moment où le quatrième trait est de bonne foi, il en résulte que le cinquième a confiance en lui et l'investit de l'autorité ; il conforme ses tendances aux siennes. C'est ainsi que la préoccupation s'envole et qu'il n'y a point de culpabil ité. La préoccupation disparaissant, il est évident que le sang disparaît ; c'est le détail le moins important qui est cité. Du moment où le cinquième trait conforme et accorde ses tendances, toutes les positivités suivent également.

Le texte de la formule $\mathrm{n}^{\circ} 200$ dit littéralement: «Le sang part, la préoccupation sort.» Chaque commentaire donne à ces deux verbes « partir » et « sortir » une valeur différente qui en aucun cas ne résulte de la lecture seule du texte. 


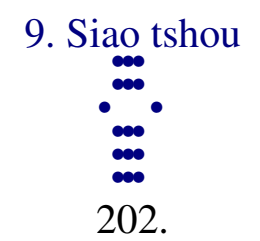

Cinquième trait nonaire : avoir une foi comme inébranlable; riche parle voisinage.

TSHENG TSE. - Le koua siao tshou, «petit arrêt », représente le moment où la foule des positivités est arrêtée par la négativité. Le cinquième trait emploie la justice pour occuper la situation prééminente et il a bonne foi et confiance; il en résulte que ceux qui sont de son genre lui correspondent tous sympathiquement, et c'est pour cela que le texte dit «comme inébranlable »; cela veut dire quills sont attachés à lui et qu'ils se suivent mutuellement. Le cinquième trait doit nécessairement les accueillir pour s'entr'aider mutuellement avec eux, c'est là être riche par le voisinage. Le cinquième trait, par la force naturelle inhérente à l'occupation de la situation prééminente, est comme le riche qui étend le bénéfice de ses richesses et de ses ressources et qui les met en commun avec son entourage. L'homme doué est rendu malheureux par l'homme inférieur ; l'homme droit et intègre es t mis en péril par la foule des méchants ; donc celui qui est dans l’infériorité doit absolument s'attacher au supérieur et le suivre dans le but d'avancer en même temps que lui. Le supérieur, à son tour, doit absolument accueillir et guider l'inférieur, dans le but d'unir leurs forces ; et cela, non pas seulement pour faire profiter les hommes de ses propres forces, mais aussi pour profiter $\mathrm{du}$ concours des inférieurs et pour compléter sa force.

TSHOU HI. - Les trois traits qui forment la substance du koua simple souen, qui exprime l'idée d'humilité, mettent leurs forces en commun pour arrêter l'activité représentée par le koua simple inférieur khien : c'est l'image symbolique de l'association entre voisins et, comme le cinquième trait se maintient dans la justice et est placé au rang prééminent, la force naturelle des choses fait qu'il est capable d'agir pour s'assimiler indistinctement ce qui est au-dessus et au-dessous de lui. C'est pour cela qu'il est considéré comme exprimant l'image symbolique d'avoir confiance et bonne foi inébranlables et d'employer la force qui résulte de la richesse et de la puissance par le concours de son entourage. Dans le texte, le terme yi, antépénultième caractère du texte, est pris dans le même sens que ce même mot dans le Tshouen tsieou «avec tel maître »; il exprime lidée d'assistance et de concours. Si celui qui interprète le sens divinatoire est de bonne foi, il pourra aussi se trouver dans ces mêmes conditions.

Le cinquième caractère du texte exprime particulièrement l'idée d'être attaché, fixé par un lien : choses liées ensemble et s'entraînant mutuellement. 
203.

Avoir une foi comme inébranlable; ne pas être seul riche.

TSHENG TSE. - Avoir une foi qui entraîne ; en effet, ceux qui sont de son genre et ceux de son entourage s'attachent fermement à lui et le suivent. Il est animé des mêmes désirs que la foule, et il ne jouit pas seul de ses richesses. Lorsque l'homme doué est placé dans le péril et le danger, il n'emploie que la plus parfaite sincérité, aussi il jo uit de l'aide des forces de la foule, et il peut régler cette foule en l'assistant. 


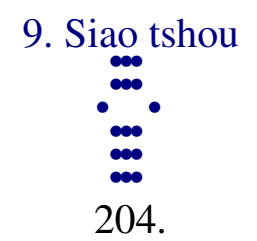

Trait supérieur nonaire : il a déjà plu ; chaque chose est déjà à sa place ; estimer l'accumulation de vertu; danger de la pureté chez la femme; la lune près de son plein ; l'homme doué se met en mouvement, présage malheureux.

TSHENG TSE. - Ce trait est nonaire et il emploie le comble de l'humilité ; il occupe le rang supérieur et est placé à la fin de l'arrêt ; c'est celui qui subit l'arrêt et demeure immo bile; il est arrêté par le quatrième. Il a déjà plu : l'harmon ie règne ; chaque chose est déjà à sa place : donc arrêtée. Dans l'arrêt de la positivité par la négativité, s'il n'y a pas accord harmonique entre les deux éléments, ceux-ci ne peuvent rester immobiles en place; l'harmonie existant et tous deux demeurant en place, la voie de l'arrêt est accomplie. Le koua tae tshou représente la grandeur dans l'arrêt, de sorte quà l'extrême limite il y a dis persion; dans le cas du koua siao tshou il s'agit de la petitesse dans l'arrêt, aussi, à l'extrê me limite, l'arr êt est accompli. Estimer l'accumulation de vertu ; le quatrième trait emploie les vertus de la douceur et de l'humilité ; quand ces vertus sont accumulées et qu’il en est plein, il parvient à la perfection. L'arrêt de la dureté énergique par la douceur malléable n'est pas quelque chose qui peut s'accomplir entre une matinée et une soirée, c'est par une action lente et répétée qu’il survient ; est-il donc possible de ne point $\mathrm{y}$ prendre garde ? Le terme tae, neuvième caractère du texte, signifie accumuler, remplir. Le Shi king dit: «Ce bruit remplit les routes. »Danger de la pureté chez la femme; le terme femme désigne la négativité ; puisqu'il s'agit de la négativité et qu'elle arrête la positivité, puisque la douceur est employée pour dominer la dureté énergique, s'il s'agit d'une femme et qu'elle s'attache avec une perfection inébranlable à maintenir cet état de choses, ce sera une voie rationnelle de péril et de danger. Comment pourrait-il se faire que la femme dominât l'époux, que le sujet imposât sa volonté au prince et qu'en même temps la paix existât ? Lorsque la lune est pleine elle se trouve en opposition avec le soleil ; près de son plein veut dire que sa croissance est sur le point d'atteindre sa perfection. La négativité peut déjà arrêter la positivité et cependant la formule dit: "près du plein », comment cela a-t-il lieu ? Ici, c'est par la douceur et l'humilité que les tendances sont contenues; ce n'est pas la force qui peut les dominer ; mais si l'effet ne cesse point, alors la négativité est sur le point d'arriver à son développement parfait en passant elle-même à l'état de positivité, et c'est alors un présage malheureux. Au sujet du moment où le plein approche, et comme avertissement, on dit : «La femme prête à se poser en adversaire. » Si l'homme doué se meut, se sera un présage malheureux ; l'homme doué désigne la positivité ; le terme employé dans le texte exprime l'ébranlement 
pour entrer en mouvement. Près du plein, c'est le moment où le plein est sur le point de s'achever ; si le plein existait déjà, la positivité serait déjà dissipée et il n'y aurait pas de sujet d'avertissement.

TSHOU HI. - Lorsque l'arrêt est arrivé à son point extrême et accompli, la négativité et la positivité sont en harmonie, c'est pour cela qu'il y a l'imag e symbolique de pluie et de choses à leur place. En effet, c'est en exaltant et en estimant les vertus de la négativité que ces vertus parviennent à s'accumuler jusqu'à être complètes et que le résultat se produit. La négativité influe sur la positivité et c'est pour cela que malgré la droiture, le présage est malheureux. Mais du moment où la négativité est arrivée à son parfait développement et où elle refoule la positivité, l'homme doué, lui-même, ne peut plus être capable d'agir. Tel est le sens divinato ire et l'avertissement est profond.

Il est placé au rang supérieur du koua simple souen.

C'est -à-dire que lorsqu'il s'agit d'une chose peu importante, et qu'elle est réalisée, le résultat peut encore durer, mais s'il s'agit d'une chose très grave, le ré sultat atteint est aussitôt dépassé.

En réalité, ce mot signifie porter, supporter (et aussi année).

Le livre de la poésie.

Le développement parfait d'une chose comporte la positivité ; mais cette positivité n'existe que pendant l'instant insaisissa ble où le développement s'achève. Sitôt achevée, la positivité disparaît et fait place à la négativité qui commence avec la décroissance.

205.

Il a déjà plu, chaque chose est déjà à sa place; la vertu s'accumule complètement. L'homme doué avançant, présage malheureux; il y a lieu de douter.

TSHENG TSE. - Il a déjà plu, les choses sont déjà en leur place ; cela exprime que la voie logique de l'arrêt s'accomplit et s'achève complètement. La négativité est sur le point d'atteindre son maximum de développement; si l'homme doué se meut, il y aura un présage malheureux. La négativité étant en lutte avec la positivité, il doit forcément arriver qu'elle altère et détruit cette positivité; l'homme inférieur attaquant l'homme doué, il doit nécessairement lui nuire : comment celui-ci n'éprouverait-il pas de la défiance et de l'inquiétude ? S'il sait dès l'abord se défier et craindre, en se mettant sur la défensive, et avoir recours aux moyens propres à contenir son ennemi, il n’arrivera pas jusqu'aux conséquen ces malheureuses. 


\section{0. $\mathrm{Li}$}

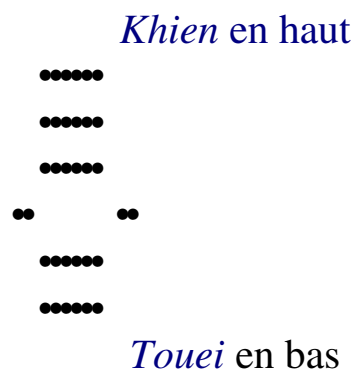

206. Marcher sur la queue du tigre ; il ne mord pas l'homme ; liberté.

[]

211. Premier trait nonaire : simplicité dans la conduite; en agissant, pas de culpabilité.

$*$

213. Deuxième trait nonaire: marcher dans une voie unie et plane ; présage heureux de la pureté de l'homme retiré à l'écart

$*$

215. Troisième trait hexaire : ceil altéré, permettant la vue; claudication permettant de marcher; marcher sur la queue du tigre ; il mord l'homme, présage malheureux; un homme violent est à la place d'un grand prince.

217. Quatrième trait nonaire : marcher sur la queue d'un tigre ; crainte et inquiétude, à la fin, présage heureux.

$*$

219. Cinquième trait nonaire : marcher avec décision ; perfection, péril.

*

221. Trait supérieur nonaire : considérer la démarche, en scruter l’influence ; la perfection dans l'investigation rend le présage absolument heureux.

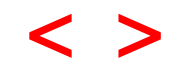


TSHENG TSE. - Li. «L'Ordre des koua » dit : «Aussitôt que les êtres sont réunis ${ }^{1}$, il y a des règles rituelles, aussi le koua siao tshou est suivi du koua $l i$. Car, du rassemblement des êtres ensemble résultent des distinctions de grandeur et de petitesse, des degrés d'élé vation ou d'infériorité, des différences de beauté et de laideur, ce qui fait qu'aussitôt que les êtres se rassemblent il y a des règles rituelles de convenances et de bienséance ; c'est pour cela que le koua $l i$ suit immédiatement le koua tshou; $l i$ équivaut à $l i ; l i$, règles rituelles des bienséances, ce que l'homme suit dans ses actions, ce qui règle ses démarches. Ce koua est constitué par le koua simple du ciel (khien) en haut, et par le koua simple du marais (touei) en bas. Le ciel est ce qui se trouve en haut ; le marais est ce qui est en bas ; c'est la distinction entre ce qui est supérieur et ce qui est inférieur, le devoir entre ce qui est éminent et ce qui est humble, la conséquence naturelle de la raison d'être des choses, l'origine et la base des règles rituelles de convenances et de bienséance, la voie rationnelle des démarches ordinaires de la vie, et c'est pour cela que ce koua est appelé $l i^{2}$; $l i$ veut dire marcher sur, fouler aux pieds. Ce mot désigne encore une natte étendue sur le sol; faire des pas sur quelque chose, c'est marcher, fouler aux pieds ; être foulé aux pieds par quelque chose, c'est lui servir de natte ou tapis. Employer la douceur pour marcher sur la dureté énergique ; le koua exprime donc l'idée rendue par le mot $l i$. On ne dit pas que la dureté marche sur la douceur malléable, mais bien que la douceur malléable marche sur la dureté énergique. La dureté énergique foulant aux pieds la douceur malléable, c'est là le cas ordinaire de la raison d'être des choses, et ce n'est pas une circonstance suffisante pour en tirer une règle morale ; aussi, dans le Yi king, il n'est question que de la douceur qui foule aux pieds la dureté énergique, et il n'est jamais parlé du cas contraire. Lorsqu'on dit qu'elle est foulée aux pieds et sert de natte à la dureté énergique, on voit quil s'agit du devoir de l'inférieur qui se soumet passivement et répond avec joie à l’influence exercée sur l ui.

\footnotetext{
${ }^{1}$ Tshou, c'est le même mot qui, dans l'explication du koua précédent, est traduit par « arrêt ».

2 Cette proposition prise à la lettre ne renferme qu'une affirmation gratuite tout à fait insignifiante. $\mathrm{Si}$, au contraire, on admet un sens ésotérique perdu pour les commentateurs, qui ne font que transmettre traditionnellement des explications plus anciennes, et si on admet, comme moi, que le $\mathrm{Yi}$ king relate des observations faites sur le soleil et la lune, objets des premiers raisonnements de l'homme, on peut apercevoir un sens logique caché sous des erreurs ; relations de position, élévation et infériorité, grandeur et petitesse, etc., entre les objets terrestres, nommées par les sons parlés émis au moment de l'observation de phénomènes astronomiques présentant les mêmes relations.
} 


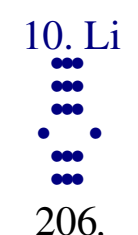

Marcher sur la queue du tigre; il ne mord pas l'homme; liberté.

TSHENG TSE. - $L i$, marcher; ce koua exprime la voie rationnelle suivant laquelle il doit marcher, c'est-à-dire de ce qu'il foule aux pieds. Le ciel est au-dessus et le marais est placé au-dessous ; emploi de la douceur malléable comme natte ou tapis, sous les pas de la dureté énergique; le supérieur et l'inférieur se conformant chacun à leur devoir. C'est l'extrême soumission en toutes choses, l'extrême con séquence normale de la raison d'être des choses. Lorsque les pas et les actions de l'homme sont ainsi réglés, bien qu'il foule sous ses pieds un sol extrêmement périlleux, rien ne peut cependant lui être nuisible ou préjudiciable, de sorte que, marchant sur la queue du tigre, on ne voit cependant point cet animal le mordre, ce qui lui permet une entière liberté d'action.

TSHOU HI. - Touei est encore le nom d'un des koua simples de trois traits. Une négativité est en évidence au-dessus de deux positivités, aussi sa vertu est la satisfaction et son image symbolique est le marais. $L i$, nom du koua parfait, exprime le sens d'avoir quelque chose sur quoi marcher et avancer. Puisque le koua simple touei se rencontre avec le koua simple khien, il y a satisfaction par le fait de marcher sur les traits inférieurs qui expriment la dureté énergique, ce qui constitue liimage symbolique du fait de marcher sur la queue du tigre sans qu'on en voie résulter d'accident. C'est pour cela que le koua est appelé $l i$ et que tel est le sens divinatoire. L'homme qui peut être dans ces conditions sera placé dans le péril et n'en éprouvera aucun dommage.

207.

Le commentaire traditionnel de la formule déterminative dit: Li; la malléabilité foule aux pieds la dureté énergique. 
TSHOU HI. - Explication du sens du nom du koua au moyen des deux substances ${ }^{1}$.

208.

Satisfaction et correspondance sympathique avec l'activité ; c'est à cause de cela que l'homme marchant sur la queue du tigre, celui-ci ne le mord point, et qu'il y a liberté.

TSHENG TSE. - Le koua touei emploi la douceur malléable de la négativité pour servir de natte et de tapis sous la dureté énergique de la positivité du koua simple khien ${ }^{2}$; la douceur malléable est foulée aux pieds par la dureté énergique ${ }^{3}$. Le koua simple touei exprime donc la satisfaction dans la soumission et la sympathie envers la dureté énergique du koua khien, en lui servant de marchepied; l'inférieur passivement soumis au supérieur, la négativité subissant l'action de la positivité, c'est -à-dire l'exacte raison dans les choses de l'univers. Ce qui est foulé aux pieds étant dans ces conditions, c'est l'extrême soumission, l'extrême convenance en toutes choses ; bien quil s'agisse de marcher sur la queue du tigre, on n'en voit point néanmoins résulter de mal et d'accidents. En marchant et en agissant ainsi, la liberté d'action est évidente.

TSHOU HI. - Explication de la formule déterminative au moyen des vertus des deux koua simples.

209.

Dureté énergique, justice, droiture; marcher dans la situation du souverain et sans inconvénient de longue durée ; clarté éclatante ${ }^{4}$.

TSHENG TSE. — Le cinquième trait nonaire emploie la dureté énergique de la positivité, la justice et la droiture, il foule majestueusement aux pieds la

\footnotetext{
1 J'ai traduit en suivant le sens et la valeur ordinaires des mots, mais un commentateur remarque que puisque Tshou Tse parle des deux substances (c'est-à-dire des deux koua simples), c'est que le vrai sens est que «la douceur sert de marchepied à la dureté énergique ».

2 Ce sens est adopté par presque tous les commentateurs.

3 Traduction de la formule $\mathrm{n}^{\circ} 207$ en adoptant le même sens.

${ }^{4}$ Littéralement fouler aux pieds la place qui appartient au souverain ; être le souverain.
} 
situation de souverain ; s'il ne survient aucun inconvénient important, c'est lui qui réussit à atteindre la clarté éclatante de l'extrême excellence dans la voie qu’il doit parcourir. Lé terme du texte tra duit par «inconvénient de longue durée » 1 désigne une cause morbide quelconque, par exemple, un empêchement dans la marche qui cause une démarche irrégulière. Clarté éclatante : vertu parfaite et resplendissante d'éclat.

TSHOU HI. - Nouvel éclaircissement au moyen de la substance même du koua ; il s'agit du cinquième trait nonaire.

210.

Le commentaire traditionnel de la formule symbolique dit : En haut le ciel, en bas le marais: $l i$; lhomme doué en déduit la distinction entre la supériorité et l’infériorité, il détermine les tendances du peuple.

TSHENG TSE. - Le ciel se trouve au-dessus, le marais est au-dessous ; c'est l'exacte rai son d'être de la supériorité et de l'infériorité ; l'homme dans ses démarches doit se régler sur ceci; c'est pourquoi on relève ce sens symbolique et le koua est appelé $l i$. L'homme doué considère cette image symbolique des convenances sociales (li) et il s'en sert pour distinguer les conditions de supériorité et d'infériorité et pour déterminer, en les fixant, les tendances et les aspirations du peuple. Or, lorsque la condition des supérieurs et des inférieurs est clairement marquée, il s'en suit naturellement que les aspirations du peuple sont déterminées ; lorsque les aspirations du peuple sont fixées, il en résulte que l'on peut dire que l'ordre est établi. Si les tendances du peuple ne sont point fixées, l'uni vers ne peut s'accorder et être en ordre. Dans les temps antiques, depuis les dignitaires revêtus des titres de Kong, Khing, Ta fou, et en descendant l'ordre hiérarchique, la situa tion de chacun répondait exactement à ses vertus; chacun l'occupait toute sa vie et se conformait à sa propre condition. Lorsque la situation n'était pas en rapport avec les vertus de l'homme qui l'occupait, le prince le choisissait, en l'élevant, et lui donnait de l'avancement. Les hommes d'étude se perfectionnaient ; quand ces études étaient poussées à leur extrême limite, le prince avait recours aux savants, personne ne se mettait jamais soi-même en avant. Les agriculteurs, les artisans, les marchands et commerçants développaient leur activité dans leur propre sphère, et la liberté d'action dont ils jouissaient avait des limites, de sorte que les tendances de chacun et ses aspirations étaient fixées et déterminées; les cours, dans tout l'univers, pouvaient ne faire qu'un seul cour. Dans les âges post érieurs, depuis les simples personnes adonnées à l'étude jusqu'aux dignitaires revêtus des titres

\footnotetext{
${ }^{1}$ Maladie de longue durée (Tse tien).
} 
de Konget de Khing, chez tous, les aspirations de chaque jour ont été tournées vers l'ambition et la gloire ; chez les agriculteurs, les artisans, les marchands et les commerçants, les tendances de chaque jour ont été tournées vers la richesse et le luxe; dans cette multitude innombrable, le cour de chacun luttait avec activité dans une concurrence générale pour le lucre ; l'univers fut naturellement plongé dans la confusion. Dans ces conditions, comment l'unité de sentiments aurait-elle pu subsister? Vouloir éviter les désordres et les troubles était impossible, et cela résultait de ce que les aspirations des supérieurs et celles des inférieurs n'étaient pas déterminées et limitées. L’homme doué considère l'image symbolique de l'ordre dans la hiérarchie et l'infériori té, afin que chacun mérite sa condition, pour fixer les aspirations et les sentiments du peuple.

TSHOU HI. - Le commentaire traditionnel de Tsheng est parfaitement complet et suffisant. 


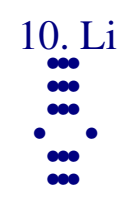

211.

Premier trait nonaire : simplicité dans la conduite ${ }^{1}$; en agissant, pas de culpabilité.

TSHENG TSE. - $L i$, c'est ne pas rester en place, le sens est marcher. Le premier trait est placé au rang le plus bas ; c'est celui qui, avec simplicité, se trouve dans une position inférieure. Mais à cause des capacités de la dureté énergique de la positivité, il lui est possible d'avancer en montant. S’il se contente de la simplicité de son humble position inférieure et s'il agit en conséquence, il ne commettra point de fautes. Mais l'homme est incapable de se contenter de la simplicité d'une situation pauvre et humble, de sorte quil avance, et c'est là se mou voir en se guidant par les passions, et en cherchant à se retirer de la pauvreté et de l'humilité ; ce n'est pas par désir de faire quelque chose. Lorsquil a réussi à avancer, son orgueil déborde infailliblement, de sorte qu'en agissant il commet certai nement des fautes. Le sage, lui, se contentera de parcourir le champ que lui laisse la simplicité de sa condition ; sil reste en place il est joyeux, s'il avance c'est pour pouvoir agir, de sorte qu'ayant réussi à avancer, il a « pu faire » et rien de ce qu’il a fait n'est autrement que bien : c'est là conserver la simplicité dans la conduite.

TSHOU HI. - Puisque la positivité se trouve dans une position inférieure et occupe le premier rang du koua $l i$, qui exprime la convenance dans les démarches et la conduite, elle n'est pas encore altérée par les êtres ; ce trait représente celui qui par sa conduite se maintient dans la simplicité. Si celui qui interprète le sens divinatoire est dans ces conditions, il agira et sera sans culpabilité.

212.

Action de la simplicité dans la conduite ; suivre seulement sa volonté.

TSHENG TSE. - Se contenter de marcher dans la simplicité et agir, sans s'attacher inconsidérément au profit; suivre seulement sa volonté et ses

\footnotetext{
${ }^{1} \mathrm{Li}$, démarche ; manière de marcher, de se conduire dans les actions ; pris tantôt au figuré, tantôt à la lettre.
} 
propres tendances. «Seulement», a la valeur de «exclusivement». Si le sentiment de l'ambition entre en lutte, chez l'homme, avec le sentiment de la voie morale, comment pourrait-il se contenter de marcher dans la simplicité de la condition? 


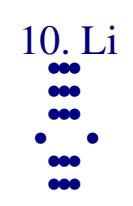

213.

Deuxième trait nonaire : marcher dans une voie unie et plane; présage heureux de la pureté de l'homme retiré à l'écart ${ }^{1}$.

TSHENG TSE. - Le deuxième trait nonaire occupe un rang qui comporte la douceur malléable; il est grand et généreux et possède la justice ; la voie dans laquelle il marche est unie, plane, égale et facile à suivre. Bien quil s'agisse de marcher dans une voie unie et facile, il faut cependant encore que ce soit un homme calme, paisible, froid et retiré ; alors il pourra la suivre avec une perfection inébranlable, et le présage sera heureux. Le second trait nonaire est positif ; ses tendances le portent à avancer en montant; c'est pour cela que la formule comporte l'avertissement relatif à la retraite $\mathrm{d}$ ans l'obscurité.

TSHOU HI. - Dureté énergique et justice dans une situation inférieure, sans correspondance sympathique avec un trait supérieur; aussi ce trait constitue l'image symbolique de marcher dans une voie égale et unie et de conserver la pureté dans la retraite et la solitude. Si un homme retiré et obscur marche en suivant la voie morale et rencontre ce sens divinatoire, ce sera la perfection et un présage heureux.

214.

Présage heureux de la perfection de l'homme obscur ${ }^{2}$; en dedans ne passe troubler soi-même.

TSHENG TSE. - Il marche dans la voie, dans le calme et le recueillement; si dans son for intérieur il est paisible et régi par la droiture, il en résultera que ses démarches seront réglées par le calme et la grandeur d'âme ; si, au contraire, il est agité et précipité, comment ses démarches pourraient-elles être calmes et modérées, et comment se contenterait-il de marcher dans la voie qui lui convient? Aussi il faut qu'il s'agisse d'un homme obscur et retiré, et alors il sera capable d'une fermeté inébranlable et

\footnotetext{
1 Littéralement «obscur», «sombre». Il faut forcer le sens pour arriver à lire « retiré à l'écart ».

${ }^{2}$ C'est le même terme que dans la formule ${ }^{\circ} 213$, traduit littéralement.
} 
le présage sera heureux. En effet, si le cour est calme et tranquille il ne voudra pas se troubler lui-même par le désir d'avantages extérieurs. 


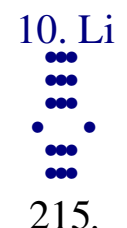

Troisième trait hexaire : ail altéré, permettant la vue ; claudication permettant de marcher ; marcher sur la queue du tigre ; il mord l'homme, présage malheureux; un homme violent est à la place d'un grand prince.

TSHENG TSE. — Le troisième trait emploie la négativité et occupe un rang positif; ses tendances le portent vers la dureté énergique, tandis que sa substance est la douceur malléable de la négativité ; comment pourrait-il se maintenir avec fermeté dans la voie qu'il parcourt ? Aussi, il est comme celui qui voit, bien que l'organe de la vision soit affaibli et altéré ; sa vue n'est pas claire ; il est comme celui qui marche, bien qu'impotent ${ }^{1}$ : il ne va pas loin. Du moment où ses capacités sont insuffisantes, et où, de plus, il se place sans se conformer à la justice, sa démarche et ses pas ne sont pas corrects. C'est parce qu'il est doux et qu'il s'applique à être dur que telle est sa démarche ; c'est fouler aux pieds un ter rain périlleux et c'est pour cela que la formule dit : «Marcher sur la queue du tigre. » Marchant sur un terrain périlleux sans pouvoir assurer sa démarche, il doit certainement en arriver aux malheurs et aux calamités, et c'est pour cela que la formule dit : «Présage malheureux de l'homme mordu. »Un homme violent est à la place d'un grand prince ; par exemple, un homme emporté et violent qui se trouve placé au-dessus des autres hommes, qui donne libre cours à sa violence et à son emportement, sans se soucier de quoi que ce soit; il ne peut pas marcher d'une façon suivie et modérée et parvenir au loin. Étant sans justice ni droiture, et ses tendances le portant vers la dureté, c'est là ce qui fait que la foule des positivités s'accorde avec lui ; c'est employer la dureté énergique et la précipitation pour marcher dans le péril, et arriver ainsi au malheur.

TSHOU HI. - Le troisième trait hexaire est sans justice et sans droiture, il est mou et ses tendances le poussent à la dureté ; dans ces conditions, placé comme un marchepied sous l'activité représentée par le koua simple khien, il est visible qu'il doit en résulter des incon vénients et des souffrances. Aussi, telle est l'image symbolique, et le sens divinatoire est un présage malheureux. Il représente encore l'image symbolique d'un homme dur et vio lent, qui est libre de suivre ses tendances et donne cours à ses emportements comme, par exemple, Tsin Tsheng ou Heang Tsi. Comment, un tel état de choses pourrait-il durer longtemps.

\footnotetext{
${ }^{1} \mathrm{Ne}$ pas pouvoir marcher, expression vague qui ne désigne aucune infirmité particulière. Terme inusité et mal expliqué.
} 
216.

Une vision altérée, permettant de voir un peu, n'est pas suffisante pour avoir une perception claire; une infirmité des jambes, permettant cependant de faire quelques pas ne met pas en état de marcher à volonté. Le présage malheureux de l'homme mordu est l'indication que la situation n'est pas méritée par celui qui l'occupe. Un homme violent à la place d'un grand prince ; dureté énergique dans les tendances.

TSHENG TSE. - Un homme doux et malléable, d'un tempérament négatif ; ses aptitudes sont insuffisantes ; il voit sans discerner clairement, il marche sans pouvoir aller loin. De plus, dans le cas actuel, il s'adonne exclusivement à la dureté énergique : le terrain sur lequel il marche étant tel, est-il donc possible quil puisse éviter le mal ? Puisque, mou et doux, il occupe le troisième rang, le terrain qu'il foule ne lui convient pas, et ses démarches ne sont pas réglées par la droiture, ce qui le conduit jusqu'aux malheurs ; il est donc mordu et le présage est malheureux. Ce qui fait que l'homme violent est pris comme exemple, c'est qu'il occupe un rang positif, que ses facultés sont faibles et que ses tendances sont dures. Ses tendances étant dures et énergiques, il se meut à tort; le terrain quill foule et ses démarches ne sont pas déterminés par la voie rationnelle, il est comme un homme violent et emporté qui tient la place d'un grand prince. 


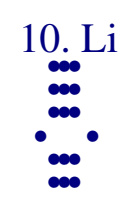

217.

Quatrième trait nonaire : marcher sur la queue d' un tigre ; crainte et inquiétude ${ }^{1}$, à la fin, présage heureux.

TSHENG TSE. - Le quatrième trait nonaire est positif, énergique, et sa substance est celle de l'activité 2 ; bien qu'il occupe le quatrième rang, c'est celui chez qui la dureté énergique l'emporte. Etre placé près du prince, c'est être dans un lieu où il y a beaucoup à craindre ; cette circonstance ne comporte pas le sens que l'un et l'autre s'accordent ensemble et se conviennent mutuellement. Le cinquième trait, à son tour, représente l'excès de dureté énergique dans la décision. C'est pour ces motifs que le quatrième trait est considéré comme ayant l'apparence de fouler aux pieds la queue d'un tigre et d'être at tentif, circonspect et agité par la crainte. S'il peut être circonspect et défiant, il s'en sui vra logiquement que le présage final sera heureux. En effet, bien que dur et énergique, ses tendances naturelles sont la mollesse et la faiblesse de caractère ; bien que le quatrième rang soit proche (de la situation suprême), cependant il ne s'y place pas ; de sorte que pouvant être circonspect et attentif, sachant craindre et se tenir en garde, il en résulte que, finalement il se soustrait au péril et assure son bonheur.

TSHOU HI. - Le quatrième trait nonaire, lui aussi, agit sans justice et sans droiture et il est foulé aux pieds par la dureté énergique du cinquième trait nonaire ; mais, puisque avec sa dureté énergique il accepte un rang qui comporte la douceur et la malléabilité, il peut donc se tenir sur ses gardes et arriver finalement au bonheur.

218.

Crainte et inquiétude, à la fin présage heureux ; tendances vers l'action.

TSHENG TSE. - Souvent être craintif et circonspect, c'est donc celui qui finalement atteint au bonheur. Ses tendances le portent à l'action et il ne reste pas en place ; s'écar tant du péril, il atteindra au bonheur. La dureté énergique

${ }^{1}$ Telle est la lecture adoptée par les Chinois ; mais le sens serait plus correctement : «défaut de correction et de régularité. »

${ }^{2}$ C'est-à-dire, il fait partie du koua simple khien. 
de la positivité, c'est la capaci té d'agir ; sa situation à un rang qui comporte la mollesse, c'est l'indication quill sait se placer avec convenance et soumission. 


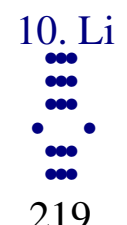

Cinquième trait nonaire : marcher avec décision ; perfection, péril.

TSHENG TSE. - Décision, énergie et dureté dans la décision. Le cinquième trait, avec sa substance positive, énergique et active, occupe la situation prééminente entre toutes; c'est celui qui s'autorise de sa volonté énergique pour se décider et agir. De cette façon, bien qu'il se règle sur la droiture, il sera encore en péril et en danger. Les hommes saints de l'antiquité qui ont occupé le rang suprême dans le monde, étaient, par leur intelligence, capables de juger et, par leur énergie, capables de décision; par la force inhérente à leurs qualités ils étaient capables de diriger toutes choses avec une autorité exclusive. Cependant jamais ils ne négligèrent d'épuiser les moyens de se renseigner. Alors même qu'il ne s'agissait que de choses aussi peu importantes que de brûler des herbes ${ }^{1}$ ils se considéraient encore comme obligés de recueillir tous les avis. C'est par là précisément qu'ils furent saints. Si celui qui est placé sur le trône et possède une intelligence brillante s'autorise de son jugement et de son énergie, agit avec une décision tranchante sans regarder autour de lui, encore qu'on le suppose guidé par la droiture, il n'en est pas moins enga gé dans une voie périlleuse ; comment pourrait-il se maintenir longtemps en sécurité ? Même avec les aptitudes que donnent l'énergie et l'intelligence, une personna lité exclusive, qui ne s'autorise que de son propre jugement, suit une voie périlleuse; combien donc en est-il de même, à plus forte raison, lorsque l'énergie et l'intelligence sont insuffisantes! Dans le Yi king, lorsque l'on rencontre les termes « perfection et péril », le sens diffère selon le cas; ceci peut se voir dans les koua qui suivront.

TSHOU HI. - Le cinquième trait nonaire se sert de l'énergie et de la justice pour se maintenir sur le trône, tandis qu'en bas on lui répond sympathiquement avec satisfaction et plaisir; chaque fois quil doit faire quelque chose, il n'a aucun motif de doute et d'incertitude, aussi le sens symbolique est la décision dans les démarches ; mais bien qu'on le suppose guidé par la droiture, la voie qu’il suit est encore périlleuse, aussi le sens divinatoire est que, même avec droiture, il y a encore péril ; l'avertissement est profond.

\footnotetext{
${ }^{1}$ Citation tirée du Tse tshouen.
} 
Marcher avec décision; perfection, péril, occuper une situation dont on est exactement digne.

TSHENG TSE. - C'est un avertissement à celui qui marche avec décision qu'il doit, par sa droiture, être digne de son rang élevé. Si, occupant le rang suprême, il s'appuie incon sidérément sur la force inhérente à un pouvoir absolu, et s'il s'autorise de sa décision et de son énergie, sans conserver aucun sentiment de circonspection, bien qu'on le suppose guidé par la droiture, il suit néanmoins une voie périlleuse.

TSHOU HI. — Se blesser sur son point d'appui. 


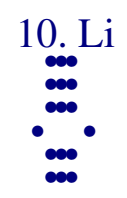

221.

Trait supérieur nonaire : considérer la démarche, en scruter l'influence; la perfection dans l'investigation rend le présage absolument heureux.

TSHENG TSE. - Le trait supérieur se trouve placé à la fin du koua $l i$. Parvenu à la fin d'une entreprise, examiner les actions et la marche suivie, afin d'en scruter les bons et les mauvais côtés et le bien ou le mal qui en peuvent résulter ; si l'investigation est complète, cela constitue le bien, et c'est de plus un présage heureux. Le caractère suen signifie entièrement complet, c'est une action circulaire embrassant tout l'horizon sans rien excepter. Lorsque l'homme scrute et considère la fin, ou résultat de ses démarches, s'il embrasse absolument tout dans cet examen, depuis l'origine jusqu'à la terminaison sans rien omettre, c'est là la limite extrême du bien, et c'est à cause de cela que le présage est absolument heureux. Pour l'homme, le bonheur et le malheur dépendent entièrement de ses démarches; selon le plus ou le moins de bien ou de mal, le bonheur ou le malheur sont plus ou moins grands.

TSHOU HI. - En considérant le résultat probable des démarches afin d'en scruter les présages, si l'investigation embrasse tous les cas possibles, sans exception, on atteindra au bonheur absolu. Le sens divinatoire est que, malheur ou bonheur, tout est le résultat des actions ; il faut d'abord examiner le terrain qu'o n foule, la voie qu'on suit, car rien n'est encore déterminé.

222.

Présage absolument heureux placé au rang supérieur, il y a abondance de félicité.

TSHENG TSE. - Le rang supérieur, c'est la fin du koua $l i$, ou résultat des démarches. Si les démarches, ou actions de l'homme, sont réglées par le bien, le présage sera heureux. Celui qui, parvenu à la fin d'une période ou d'une entreprise, scrute sa conduite en embrassant tous les détails sans rien omettre, est un homme qui jouira d'une très grande félicité. Les actions de l'homme ne sont grandes que par leur résultat.

TSHOU HI. - Si le présage est absolument heureux la félicité sera très grande. 


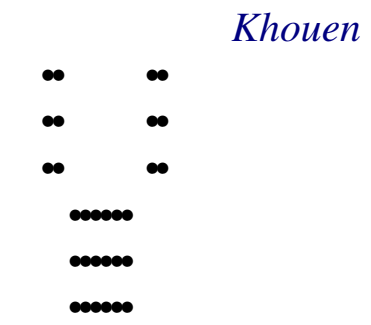

Khien

223. Thae, prospérité ; ce qui est petit s'en va, ce qui est grand vient ; heureuse liberté.

226. Premier trait nonaire : arracher des herbes à racines traçantes; union par le lien commun du genre ; en marchant en avant présage heureux.

$*$

228. Deuxième trait nonaire : tolérer les plantes parasites; aller exposer dans un fleuve, ne rien négliger ; les amis s'oublient ; réussir à égaler le cours de la justice.

$*$

230. Troisième trait nonaire : pas de plaine sans déclivité où convergent les eaux ; pas d'aller sans retour ; perfection dans le péril ; pas de culpabilité ; ne pas regretter la bonne foi ; avoir du bonheur relativement à la nourriture.

232. Quatrième trait hexaire : voltigeant; sans richesse, profiter du voisinage ; sans avertissement, employer la bonne foi.

$*$

234. Cinquième trait hexaire : Ti Yi mariant les princesses; il en résulte le bonheur et un présage absolument heureux.

$*$

236. Trait supérieur hexaire : le rempart retombe dans le fossé ; ne pas employer les armées ; de son propre district appeler et donner mission ; appréhension de la pureté.

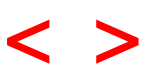

TSHENG TSE. — Thae. «L'Or dre des koua » dit : « Lorsque les hommes sont policés ${ }^{1}$, ils prospèrent ${ }^{1}$; il en résulte qu'ils sont en paix. C'est pour cela

\footnotetext{
${ }^{1} L i$, pris dans le sens de règles rituelles, règles sociales; sens figuré.
} 
que le koua $l i$ est suivi du koua thae. » Lorsque les démarches de l'homme sont ce qu'elles doivent être ${ }^{2}$, la prospérité se développe progressivement ; avec la prospérité vient la paix : c'est pour cette raison que le koua thae suit immédiatement le koua li. Il est formé du koua simple khouen de la négativité, en haut, et du koua simple khien de l'activité, en bas. Les éthers du ciel et de la terre, de la négativité et de la positivité, s'unissent et s'harmonisent, de sorte que tous les êtres naissent et acquièrent leur développement normal, ce qui fait que ce koua est considéré comme exprimant la libre prospérité.

\footnotetext{
${ }^{1}$ Thae, croissance, progrès, pris dans le sens de développement de civilisation.

${ }^{2} \mathrm{Li}$, pris dans le sens de faire des pas; litt. : « en marchant juste dans l'endroit convenable. »
} 


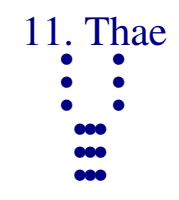

Thae, prospérité ; ce qui est petit s'en va, ce qui est grand vient ; heureuse liberté.

TSHENG TSE. — Le terme «petit» désigne la négativité ; le terme «grand» désigne la positivité. «S'en va », aller de... vers l'extérieur; « vient», vient se placer à l'intérieur. L'éther positif descend et s'abaisse ; l'éther négat if monte et s'unit à lui. La négativité et la positivité se pénètrent harmoniquement, de sorte que tous les êtres naissent et se développent selon la loi de leur organisation : c'est la prospérité du ciel et de la terre. Si on en parle au point de vue des choses humaines, ce qui est grand, c'est le prince, ou le supérieur ; ce qui est petit, c'est le sujet, ou l'inférieur. Le prince s'en tient à la sincérité pour régir les inférieurs; le sujet épuise la sincérité au service du prince; les tendances du supérieur et de l'inférieur concordent librement : c'est la prospérité du gouvernement. La positivité, c'est l'homme doué ; la négativité, c'est l'homme inférieur. L'homme doué vient se placer au -dedans ; l'homme inférieur va se placer à l'extérieur ; donc l'homme doué possède la situation ${ }^{1}$ et l'homme inférieur est au-dessous de lui : c'est la prospérité dans l'empire. La voie rationnelle de la prospérité, est une voie de bonheur et, d'ailleurs, de liberté. Ici le texte ne dit ni « présage absolument heureux », ni «absolue liberté »; selon le temps il y a tendance à décadence ou à illustration ; l'ordre dans le gouvernement est plus ou moins grand ; bien quil s'agisse de prospérité, comment serait-il possible qu'elle ne comportât qu'un seul mode ! En disant « heureuse liberté », cette forme peut tout embrasser.

TSHOU HI. - Thae, libre pénétration. Ce koua est composé des koua simples du ciel et de la terre s'unissant et dont les éthers se pénètrent librement ; c'est pour cela qu'il est considéré comme ex primant la prospérité. C'est le koua du premier mois de l'année. Petit, veut dire négativité ; grand, veut dire positivité ; cela exprime que le koua simple khouen va occuper l'extérieur et que le koua simple khien vient occuper l'intérieur. Sous un autre point de vue, le koua parfait dérive du koua kouei mei dans lequel le trait hexaire va occuper le quatrième rang, tandis que le trait nonaire vient occuper le troisième. Si celui qui consulte le sort possède les vertus de la dureté énergique positive, le présage sera heureux et comportera la liberté d'action.

\footnotetext{
${ }^{1}$ Situation déterminée quelconque, par opposition au simple citoyen dans l'état.
} 
Le commentaire traditionnel de la formule déterminative dit : Prospérité ; ce qui est petit s'en rua, ce qui est grand vient, heureuse liberté; donc c'est le ciel et la terre s'unissant, et le libre développement des êtres et des choses; le supérieur et l'inférieur en contact, et leurs tendances identiques. Au-dedans positivité et au-dehors négativité ; au-dedans force active, et au-dehors l'homme inférieur; la voie morale de l'homme doué prévalant et la voie naturelle de l'homme inférieur s'effaçant.

TSHENG TSE. - Ce qui est petit s'en va, ce qui est grand vient : la négativité s'en va et la positivité vient ; donc les éthers du ciel et de la terre, de la négativité et de la positivité se joignent mutuellement, tous les êtres peuvent suivre les lois de leur développement et prospérer librement. Chez l'homme, ce sont les sentiments du supérieur et de linférieur s'accordant librement et leurs tendances, comme leurs idées, identiques. La positivité vient occuper l'intérieur, la négativité va à l'extérieur ; la positivité avançant et la négativité se reculant. La force active du koua simple khien est au-dedans ; la soumission passive du koua simple khouen est au-dehors, ce qui représente l'activi té intérieure et la soumission extérieure : c'est la voie rationnelle de l'homme doué. L'homme doué est au -dedans; c'est donc la voie rationnelle de l'homme doué. L'homme doué est au -dedans ; l'homme inférieur est au-dehors; c'est donc la voie morale de l'homme doué se développant et la voie naturelle de l'homme inférieur se dissipant, et c'est par là que le koua est considéré comme représentant la prospérité. Après avoir mis en lumière l'union harmonique de la négativité et de la positivité, ce commentaire relève encore le sens de la voie rationnelle de l'homme doué se développant; l'union harmonique de la négativité et de la positivité, c'est précisément le développement de la voie morale de l'homme doué.

225.

Le commentaire traditionnel de la formule symbolique dit: Union du ciel et de la terre, prospérité ; de prince en déduit la détermination et l'accomplissement ${ }^{1}$ de la voie du ciel et de la terre; il concourt à l' exécution du plan naturel tracé par le ciel et la terre ${ }^{2}$; et cela pour assister et aider le peuple.

\footnotetext{
${ }^{1}$ Le sens le plus littéral serait: « Le prince en profite pour tailler (couper) et achever la voie... etc. » Pour l'interprétation de ce passage, on donne aussi au caractère thae, le sens de mesurer.

${ }^{2}$ La lecture des deux caractères traduits par les mots « concourir à l'exécution » dépend essentiellement du sens qu'on donne au caractère $y i$, du texte. Yi signifie «produits naturels » et aussi « conséquences naturelles ». C'est surtout ce sens que les commentateurs chinois adoptent dans ce passage. Questionné sur le sens de ces deux membres de phrase, Tsheng Tse répondait que la voie naturelle du ciel et de la terre ne peut se rétablir d'elle-même, et qu'il faut le concours de l'homme saint venant la déterminer et aider à sa réalisation, par exemple en apprenant aux hommes à profiter successivement des quatre saisons pour les divers travaux
} 
TSHENG TSE. — Le ciel et la terre s'unissent et la négativité s'harmonise avec la positivité ; il en résulte que toutes les choses florissent suivant le cours des lois de leur organisation : c'est par là quill y a prospérité. Le prin ce doit réaliser l’image symbolique de la libre prospérité du ciel et de la terre, et cela pour arriver à la détermination et à l'achèvement de la voie rationnelle du ciel et de la terre, pour concourir et aider à l'exécution du plan naturel du ciel et de la terre, afin d'aider et d'assister le peuple dans la satisfaction des nécessités de la vie. Les caractères traduits par « déterminer » et « accomplir » expriment l'idée de réaliser la voie logique de l'union prospère du ciel et de la terre et de préciser, en la déterminant et en l'accomplissant la règle des effets et de l'action de cette voie. "Concourir à l'exécution du plan naturel tracé par le ciel et la terre » : le libre cours de la prospérité de l'union du ciel et de la terre fait que toutes choses florissent suivant les lois particulières et l'organisation de chacune d'elles ; le prince réalise cette libre prospérité et lui donne un corps en traçant les règles et les lois, afin que le peuple profite des moments marqués par le ciel, quil tire parti des produits de la terre, quil facilite l'auvre de la transformation créatrice et productrice, et quill complète les avantages que lui présente l'abondance. Par exemple, le souffle du printemps donne la vie à toutes choses; de là les règles relatives aux semailles et aux plantations ; le hâle de l'automne ${ }^{1}$ achève et mûrit ${ }^{2}$ toutes choses : de là les règles relatives aux moissons et aux récoltes. C'est là concourir à l'exécution du plan naturel tracé par le ciel et la terre pour aider et assister le peuple. La vie, ou existence, des peuples dépend nécessairement des règles et instructions établies dans ce but par le prince et les supérieurs, pour leur enseigner à les suivre et pour les aider à les pratiquer. Alors ceux-ci peuvent vivre et se substanter suivant les nécessités de leur organisation, c'est là ce qu'il faut entendre par ces mots « aider et assister».

TSHOU HI. - Déterminer et définir, afin de prévenir l'excès ; concourir et aider, afin de suppléer aux lacunes.

\footnotetext{
de l'agriculture. Admettons, à titre de suppositions si on veut, que le « ciel » est l'observation du cours du soleil au-dessus de l'horizon et la « terre » le cours du soleil au-dessous de cet horizon; si « l'homme » est la conjonction de la lune avec le soleil on lira sans difficulté que le phénomène observé au lever du soleil dans l'hémisphère nord permet de déterminer et de compléter le cours entier du soleil autour de la terre, lorsqu'il est visible comme lorsqu'il est invisible. L'observation de Tsheng Tse devient alors compréhensible, car c'est bien la conjonction du soleil avec la lune qui indique les moments de la course annuelle de l'astre, marque les saisons, et prévient l'homme de leurs retours successifs.

${ }^{1}$ Le mot khi, éther, air, souffle, est traduit par « souffle » dans le premier membre de phrase et par «hâle », dans le second; cette traduction, sans être littérale, rend à peu près le sens du texte chinois.

${ }^{2}$ Litt, solidifier, rendre corporel; solidifier la semence.
} 


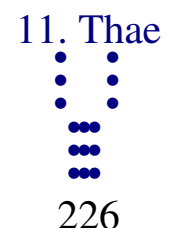

Premier trait nonaire : arracher des herbes à racines traçantes ${ }^{1}$; union par le lien commun du genre ; en marchant en avant présage heureux.

TSHENG TSE. - Au début, un trait positif occupe le rang inférieur : c'est c elui qui possède les aptitudes de la dureté énergique et de l'intelligence, et qui se trouve dans une position inférieure. S’il s'agissait d'un moment de décadence ${ }^{2}$, ce serait l'homme doué se retirant en arrière et se plaçant dans une position infime ; mais, du moment où il s'agit d'un instant de prospérité, c'est que ses tendances le poussent à avancer en s'élevant. Le mouvement en avant de l'homme doué doit être un mouvement d'entraînement mutuel avec ceux qui sont de même nature ; c'est comme le cas de certaines herbes qui repoussent par leurs racines; en en tirant une, toutes suivent et s'enlèvent ensemble. Le terme jou, du texte, exprime des racines traçantes, reliant ensemble des touffes diverses d'une même plante, et c'est pour cela quil est ${ }^{2}$ pris comme symbole. "Genre », catégorie de choses d'une nature analogue. Le sage avance avec ceux de son genre, leurs tendances identiques les portent à suivre sa voie, et c'est par là que le présage est heureux. Le mou vement en avant de l'homme doué doit a voir lieu d'accord avec ceux de son genre ; non seulement, ses tendances, le portent à se trouver au premier rang avec eux, mais ils se plaisent à faire le bien ensemble et, en réalité, c'est là s'appuyer les uns sur les autres pour le bien de l'humanité. Aussi l'homme doué comme l'homme inférieur n'ont jamais pu se maintenir isolément, sans s'appuyer sur l'aide des gens de même nature qu'eux -mêmes. Depuis l'antiquité, lorsqu'un homme doué s'est trouvé au pouvoir, les sages de tout l'empire ont afflué à sa cour, animés des mêmes tendances et unissant leurs forces, afin de compléter la prospérité de l'univers. De même lorsqu'un homme inférieur a occupé une situation importante, les gens déclassés se sont avancés en même temps et il en est résulté que leurs coteries ont prévalu et que l'univers a périclité ; c'est que, en effet, chacun suit ceux de son propre genre.

TSHOU HI. - Les trois positivités sont en bas, elles s'entraînent mutuellement et avancent : c'est l'image symbolique d'arracher des herbes à racines traçantes et le présage heureux du mouvement en avant. Si celui qui consulte le sort possède la dureté énergique, le présage est heureux s'il se

\footnotetext{
${ }^{1}$ Une plante telle que le chiendent, dont les racines donnent naissance à d'autres touffes de tiges, de telle sorte qu'en arrachant un pied on entraîne en même temps les rej etons qui y sont liés et qui en dépendent

${ }^{2}$ La décadence est exprimée par le koua suivant
} 
porte en avant. Kuo Pou Tong Lin lit jusqu'au carac tère qui signifie « genre », et arrête là la phrase ; la même remarque est applicable dans le koua suivant ${ }^{1}$.

Arracher le chiendent, présage heureux du mouvement en avant; tendances vers l'extérieur.

TSHENG TSE. - Le moment de la prospérité approche, aussi la foule des sages veut avancer en montant; les tendances des trois traits positifs les portent d'une façon iden tique à avancer, et c'est pourquoi la formule relève l'image symbolique des « herbes à racines traçantes» et «d'avancer par catégories ». Tendances vers l'extérieur, c'est -à-dire à avancer en montant.

Le nom de « chiendent » est employé au hasard pour éviter la répétition de la périphrase.

\footnotetext{
${ }^{1}$ Kuo Pou, l'annotateur du Yi ya. Cette ponctuation a beaucoup de chances pour être la bonne et, en suivant celle quia prévalu, le sens du deuxième membre de phrase ne peut se trouver qu'en forçant la valeur du caractère $y i$, du texte.
} 


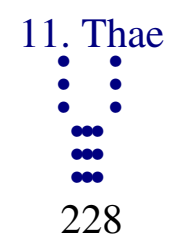

Deuxième trait nonaire : tolérer les plantes parasites ${ }^{1}$; aller exposer dans un fleuve,ne rien négliger ; les amis s'oublient ; réussir à égaler le cours de la justice ${ }^{2}$.

TSHENG TSE. - Le second trait emploie la dureté énergique de la positivité et possède la justice ; en haut, il correspond sympathiquement au cinquième trait. Le cinquième emploie la douceur malléable et la soumission, et il possède la justice ; en bas, il correspond sympathiquement au second. Le prince et le sujet ont les mêmes vertus : c'est à cause de ses aptitudes énergiques et justes que le supérieur investit celui-ci d'une autorité exclusive. Aussi, bien que le second trait occupe la position de sujet, c'est de lui que dépend la prospérité du gouvernement ; c'est ce qu'on exprime en disant que le supérieur et l'inférieur s'allient et que leurs tendances sont identiques, et c'est pour cela, qu'au point de vue de la voie de la prospérité dans le gouvernement, c'est surtout le second trait dont il est question. Tolérer les plantes parasites, aller s'exposer dans un fleuve, ne rien négliger, les amis s'oublient, sont quatre propositions qui expriment la voie à suivre pour se placer dans les conditions de la prospérité. Lorsque les sentiments de l'h omme suivent librement leur cours, les institutions sociales sont progressivement délaissées et les préceptes des règles sont abandonnés et oubliés. Lorsque tout est sans règles, la voie logique pour régulariser doit comporter une juste mesure de tolérance de livraie et des plantes parasites, de façon que son action soit large et magnanime dans la dissipation de l'obscurité en renouvelant la raison d'être de chaque chose ; dans ce cas, les hommes s'en accommodent. Sans cette mesure et cette tolérance, l'es prit est agité par un sentiment de colère et d'emportement, il n'y a plus de vues larges et prévoyantes, il ne reste que les inconvénients de la violence qui fatigue et irrite : les maux profonds ne sont pas extirpés et le péril prochain naît déjà. C'est pour ces raisons que cette voie logique consiste dans la tolérance d'herbes parasites.

«Aller s'exposer dans un fleuve. » Pendant les époques de paix et de prospérité, les sentiments de l'homme shabituent à un calme prolongé ; il se contente de la conservation de l'état actuel et ordinaire de toutes choses, est porté à la routine et à la crainte des nouveautés et du changement. Sans le courage qui permet de s'exposer au courant du fleuve, il est impossible d'aviser aux besoins du temps et d'y subvenir. "S'exposer au cou rant du fleuve » veut dire que l'énergie et la décision sont suffisantes pour traverser la profondeur du courant et franchir l'obstacle. Depuis l'antiquité, pendant les

\footnotetext{
${ }^{1}$ On peut aussi bien lire « envelopper le désert ».

${ }^{2}$ Sens extrêmement vague et douteux, qui n'est guère déterminé que par la tradition.
} 
périodes d'ordre et de prospérité, on en est toujours arrivé peu à pe u à la licence et à la décadence. En effet, par habitude du calme et de l'inactivité, le mal sinsinue et se développe pour finir par éclater brusquement.

Par l'absence d'un prince doué d'énergie et de décision et de ministres habiles et actifs, il y a impossibilité de recourir à des mesures d'exception et d'énergie, pour couper court au mal. C'est pour cela que la formule parle de s'exposer aux dangers d'un fleuve.

On pourrait douter que, puisque, plus haut, il a été question de tolérance des herbes parasites, c'est -à-dire, de tolérance et de longanimité, et qu'ici, il s'agit de s'exposer au cours du fleuve, c'est -à-dire, de recourir à des moyens brusques pour modifier et supprimer en renouvelant, ces deux propositions ne semblent contradictoires. Ce serait ne pas savoir que les tempéraments de la tolérance et l'emploi des effets de l'énergie et de la décision sont précisément le mode d'action propre aux saints et aux sages.

«Ne rien négliger. » Dans les temps de prospérité et de paix, le coar de l'homme s habitue au bien-être, de sorte qu'il s'abandonne au repos de l'oisiveté et de l'impré voyance. Comment serait-il encore capable de pensées profondes et de vues lointaines ? Celui qui gouverne pendant la prospérité, doit embrasser toutes choses dans ses vues; bien qu'il s'agisse de détails et de conséquences encore éloignés, il ne doit rien négliger. Par exemple, les choses en apparence peu importantes et encore dans l'ombre, les capacités du savoir et de la sagesse encore confondues dans les rangs subalternes, sont autant de circonstances dont les conséquences sont encore lointaines et dans les temps de prospérité on les néglige certainement.

«Les amis s'oublient. » Or, puisqu'il s'agit d'un temps de prospérité, les hommes sont certainement habitués au repos de la paix ; leurs sentiments et leurs passions suivent leur libre cours, et ils oublient les prescriptions des lois morales. Pour y mettre un frein et les redresser, si on ne met pas de côté tous les liens privés qui résultent de l'attachement et des relations habituelles, rien ne sera possible, et c'est pour cela que le texte dit « les amis s'oublient ». Depuis l'antiquité, les cas ont été fréquents où en établissant des lois et en statuant sur les faits, dominés par les passions et les sentiments humains, les efforts n'ont finalement pas pu aboutir. Quand il s'agit de lois restrictives ou somptuaires, le changement est gênant et blessant pour des proches et des alliés ; quand il s'agit de lois sur la prospérité agricole et sur l'industrie, elles sont mal vues par les familles riches. Dans de tels cas, lorsqu'on est incapable de décider dans le seul intérêt du bien général et de ne faire qu'en vue du devoir, c'est précisément être retenu par les liens de l'amitié. Gouver ner l'état pendant la prospérité et ne pas être capable d'oublier les liens de l'amitié est une chose bien difficile. Si la voie de celui qui gouvèrne pendant la prospérité est soumise à ces quatre règles, il pourra égaler la vertu du second trait nonaire, et c'est pourquoi la formule dit: "Réussir à égaler le cours de la justice », ce qui exprime la capacité de «s'éga ler au devoir tracé par le cours de la justice ». Le terme traduit par le mot égaler a le sens de «atteindre au niveau de... » 
TSHOU HI. - Le second trait nonaire emploie la dureté énergique et se trouve dans une position qui comporte la douceur; il est au milieu du koua simple inférieur; en haut, il rencontre la sympathie du cinquième trait hexaire ; c'est celui de qui dépend la prospé rité et qui possède la voie de la justice. Si celui qui consulte le sort peut tolérer des plantes parasites, s’il est déterminé et énergique dans ses décisions, s’il ne néglige pas ce qui a rapport à l'avenir, enfin, s’il ne s'attache pas aux coteries qui résultent de l'amitié, alors il pourra se conformer à la voie de la justice dans les actions exprimées par ce trait.

Tolérer les plantes parasites, réussir à égaler le cours de la justice, par l'éclat et la grandeur.

TSHENG TSE. - Le commentaire symbolique relève cette seule phrase : tolérer les plantes parasites, et il explique d'une façon générale le sens des quatre membres de phrase de la formule, en disant, que, de cette façon, il sera possible d'égaler les vertus et la marche de la justice, et que cette voie sera éclatante et brillante, lumineuse et grande. 


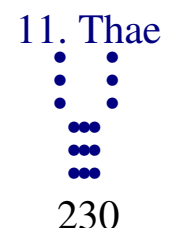

Troisième trait nonaire : pas de plaine sans déclivité où convergent les eaux ${ }^{1}$; pas d'aller sans retour ; perfection dans le péri4 pas de culpabilité ; ne pas regretter la bonne foi ; avoir du bonheur relativement à la nourriture.

TSHENG TSE. - Le troisième trait occupe le milieu du koua thae ; il est en haut de tous les traits positifs; c'est la perfection complète dans la prospérité. La raison d'être des choses est comme la rotation d'un cercle, ce qui est en bas devra remonter et ce qui est au sommet devra nécessairement descendre; lorsque la prospérité dure depuis longtemps, la décadence doit survenir. Aussi, dans la perfection complète de la prospérité, et au moment où la positivité est sur le point d'avancer, la formule prend ce trait comme objet d'un avertissement et dit : il n'y a pas de paix constante et égale sans dangers et accidents ; c'est-à-dire qu'il n'y a pas de prospérité constante. Il n'y a pas de mouvement continu dans un sens, sans retour en sens contraire; c'est-à-dire que la négativité doit revenir. Dans une plaine il y a une pente ; dans l'aller, il y a le retour : c'est ce qui constitue la déca dence exprimée par le koua suivant. Il faut connaître la nécessité fatale de la raison d'être du c iel ; au moment où la prospérité commence, on ne doit pas oser se livrer au repos et à l'inactivité, il faut continuellement prévoir le péril et les difficultés, redresser et affermir la conduite et les actions, et alors il sera possible de ne pas commettre de fautes. Placé dans la voie de la prospérité, du moment où on est capable de perfection dans le péril, on pourra garantir d'une façon permanente cette même prospérité ; ne se tourmentant pas d’inquiétudes pour obtenir ce qu'on désire, n'oubliant point ce qu'on s'est assigné comme objet de confiance, on bénéficiera d'autant plus en traitement et en nourriture. Ces termes «traitement et nourriture » désignent le bien-être matériel. Lorsque les gens vertueux sont dans la prospérité, leur bonheur peut augmenter : en effet, leurs vertus et leur bonté s'augmentant journellement, leur bien -être matériel croît de même façon. Si leurs vertus dépassent la proportion de leur bien-être matériel, c'est que, bien que ces ver tus soient arrivées à la perfection, elles ne sont pas susceptibles d'excès. Depuis les temps antiques, le succès et la grandeur ont toujours été accompagnés de l'oubli de la voie morale et de la ruine.

TSHOU HI. - Il est sur le point de dépasser le juste milieu ; la prospérité approche de son apogée et la décadence commence à poindre. « Regretter», avoir du chagrin; «bonne foi », l'exactitude dans le but assigné. Cette

\footnotetext{
${ }^{1}$ Les commentateurs chinois lisent «pas de paix sans périls». On peut aussi lire «pas de
} plaines sans inégalités »; le sens que nous avons adopté est le plus littéral. 
formule avertit celui qui consulte le sort qu'en conservant sa pureté dans le péril et les difficultés il sera sans culpabilité et possédera le bonheur.

Pas d'aller sans retour; relation entre le ciel et la terre.

TSHENG TSE. — La proposition « pas d'aller sans retour » exprime les circonstances de la relation entre le ciel et la terre, lorsque la positivité descend en bas, elle doit nécessairement revenir en haut; lorsque la négativité monte en haut, elle doit forcément redescendre: c'est la raison d'être permanente de la contraction et de la dilatation, de l'aller et du venir. Cette formule symbolique cite la relation de l'union du ciel et de la terre, pour montrer clairement la raison qui ne permet pas la continuité de la prospérité, ni celle de la décadence, et pour en faire l'objet d'un avertissement.

C'est à dessein que nous maintenons dans la traduction l'équiv alent du terme chinois « en bas ». Le texte chinois peut aussi bien se lire : « descendre dans le dessous », « monter dans le dessus ». «Action et réaction » serait la traduction française du texte chinois ; mais cette traduction plus correcte et moins barbare ferait perdre de vue l'idée primitive des auteurs du texte. 


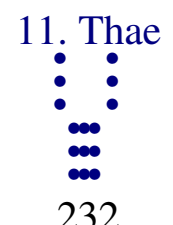

Quatrième trait hexaire : voltigeant; sans richesse, profiter duc voisinage ; sans avertissement, employer la bonne foi.

TSHENG TSE. - Le quatrième trait hexaire se trouve avoir dépassé le milieu du koua qui exprime la prospérité ; comme négativité qui se trouve dans une position élevée, ses tendances le portent à redescendre. Les tendances des deux traits négatifs supérieurs les poussent de même à se rapprocher des rangs inférieurs. Les mots traduits par « voltigeant » expriment l'apparence de voler avec rapidité ; le quatrième trait voltige vers les régions inférieures, de même que ses voisins. "Voisinage» désigne ce qui est de même genre ; c'est -à-dire le cinquième trait et le trait supérieur. Or, lorsqu'un homme est riche et que ceux qui sont du même genre que lui le suivent, c'est à cause du bénéfice que ceuxlà en retirent; mais, s'il n'est pas riche et que ceux-là le suivent néanmoins, c'est à cause de tendances identiques et communes. Les trois négativités sont toutes des objets qui, par leur essence, tiennent à l'infériorité ; occupant les rangs supérieurs, elles sont hors de leur milieu, et leurs tendances les portent également à vouloir rétrograder vers le bas. Aussi, bien que sans richesse, elles se suivent mutuellement, elles n'attendent ni avertisse ment, ni réprimande, et la sincérité de leurs idées est la même. Mais le mouvement ascensionnel ou descendant de la négativité et de la positivité, c'est la prospérité ou la décaden ce dans l'enchaînement des moments ; qu'elles s'unissent ou se séparent, c'est toujours une loi constante. Quand la prospérité dépasse de justes bornes, elle tend à se transformer. L'homme saint, au sujet du troisième trait, développe cette pensée qu'avec la pureté dans les difficultés on arrivera au bonheur. En effet, le troisième trait parvient à la justice (au milieu), s’il connâit l'avertissement il pourra se garantir ; le quatrième a déjà dépassé le juste milieu et la raison naturelle des choses veut qu'il y ait changement ; c'est pour cela qu’il est exclusivement question de la voie rationnelle d'aller et de retour en sens contrai re, du commencement à la Fm. Le cinquième trait est celui de qui dépend la prospérité (le maître du koua thae), de sorte qu'à son sujet il est encore question du sens d'être placé dans la prospérité.

TSHOU HI. - Il a déjà dépassé le juste milieu ; la prospérité est déjà à son apogée ; aussi les trois négativités prennent leur vol et redescendent. Elles ne font pas attention à la richesse, et tout ce qui est du même genre suit ; elles n'attendent pas d'avertissements ni de réprimandes et sont confiantes. Le sens divinatoire est quill y a des hommes inférieurs qui s'unissent pour violer la droite morale ; c'est ce dont l'homme doué doit être averti. La négativité c'est 
le vide, la positivité c'est ce qui est plein ; aussi, toutes les fois qu'il est question d'absence de richesse, c'est toujours au suje t d'un trait négatif.

Voltigeant sans richesse : tous sont hors de leur milieu; sans avertissement employer la bonne foi : aspiration du sentiment de la justice.

TSHENG TSE. - «Voltigeant »; précipitation dans le mouvement vers le bas. « Ne pas s'attacher à la richesse et suivre ceux de son genre »; puisque les traits négatifs sont en haut, il est bien évident que tous sont hors de leur milieu. La négativité est essentiellement une chose qui doit être dans l'infériorité ; ici elle est en haut, c'est bien être hors de son milieu. $\mathrm{Ne}$ pas attendre les avertissements et les réprimandes et s'accorder ensemble avec sincérité d'idée, c'est en effet certainement l'aspiration d'un cour juste. L'expres sion naturelle de la raison d'être et sa conséquence essentielle, c'est le ciel ; ce qui est identique pour tous, c'est le moment (temps).

TSHOU HI. - Essentiellement ${ }^{1}$ la négativité occupe le bas ; occuper le haut, c'est perdre sa condition réelle ou être hors de son milieu.

1 Ou bien : « à l'origine ». 


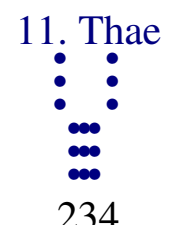

Cinquième trait hexaire : Ti Yi mariant les princesses ${ }^{1}$; il en résulte le bonheur et un présage absolument heureux.

TSHENG TSE. - Les annales rapportent que Theang ${ }^{2}$ régna sous le vocable Thien Yi; après lui, il veut l'empereur Tsou Yi qui fut aussi un roi sage. Plus tard encore, il y eut un souverain connu sous le vocable $\mathrm{Ti} \mathrm{Yi}^{3}$. $\mathrm{La}$ plupart des auteurs disent que, depuis Tsheng Theang jusqu'à Ti Yi, il n'y eut que des princes doués d'intelligence, de vertu, de charité et de piété. On ne sait au juste qui est ici désigné sous le nom de Ti Yi ; à en juger par le sens de cette formule, Ti Yi est celui qui légiféra sur les règles et les cérémonies du mariage des princesses avec des personnes d'un rang inférieur au le ur. Depuis l'antiquité, bien que les filles des souverains aient toujours été mariées avec des personnes d'un rang infé rieur au leur, ce n'est que depuis Ti Yi qu'on aurait établi des règles rituelles pour ces mariages afin de diminuer leur éminence et leur noblesse, pour qu'elles soient soumises et obéissantes à leur époux. Le cinquième trait emploie la douceur malléable de la négativité et occupe la situation du prince; en bas, il sympathise avec le sage doué d'énergie et d'intelligence représenté par le deuxième trait nonaire. Le cinquième trait peut investir de la responsabilité du pouvoir un sage sujet et écouter ses avis avec déférence. C'est absolument comme le mariage des princesses institué par Ti Yi, dans lequel l'éminence du rang est diminuée et la femme soumise à l'époux. De cette façon, le prince est heureux et le présage est absolument heureux. Un présage absolument heureux, c'est-à-dire un grand bonheur et un bien absolu. Cela exprime le mérite qui consiste à gouverner parfaitement dans la prospérité.

TSHOU HI. - Employant la négativité et occupant le rang prééminent, c'est de lui que dépend la prospérité ; il est doux et juste et fait abstraction de sa personnalité. En bas, il correspond sympathiquement avec le deuxième trait nonaire ; c'e st là une voie naturelle de bonheur. Enfin, au moment où Ti Yi s'occupait du mariage des princesses, consultant le sort au sujet de cette institution, il obtint ce trait. Si celui qui consulte le sort est dans ces conditions, il sera heureux et le présage est absolument bon. Toutes les fois que le texte primitif cite un personnage de l'antiquité, par exemple Kao Tsong, Ki Tse, etc., on doit toujours procéder par analogie.

\footnotetext{
${ }^{1}$ Sens très douteux : la traduction est conforme à l'explication traditionnelle, mais le sens littéral serait « épouse sa sœur cadette » ou «marie sa sœur cadette ».

2 Tsheng Theang, fondateur de la dynastie des Sheang.

${ }^{3}$ Littéralement, l'empereur Yi.
} 
Il en résulte le bonheur et un présage absolument heureux; la justice agit suivant ses propres aspirations.

TSHENG TSE. - Ce qui rend possible d'assurer le bonheur et ce qui fait que le présage est absolument heureux, c'est la conformité à la voie de la justice, et c'est que les actions sont le résultat des aspirations de la justice. Il a les vertus de la justice ce qui le rend capable d'investir de l'autorité le sage juste et énergique. Ce quil suit et écoute, ce sont toujours les tendances et les aspirations de celui-là ; si tels n'étaient pas ses propres désirs, pourrait-il les suivre? 


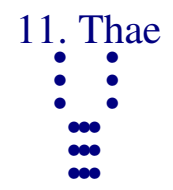

236

Trait supérieur hexaire : le rempart retombe dans le fossé; ne pas employer les armées ; de son propre district appeler et donner mission; appréhension de la pureté.

TSHENG TSE. - En creusant un fossé, la terre s'accumule pour former un rempart. Ainsi, lorsque la voie suivie dans le gouvernement s'est améliorée au point de procurer la prospérité, cette prospérité, parvenue à sa fin, est sur le point de retomber en décadence, comme la terre du rempart qui, en s'écr oulant, retombe dans le fossé. Le trait supérieur, c'est la fin du koua thae, la fin de la prospérité. Puisque le trait est hexaire, c'est qu'il s'agit d'un homme inférieur qui s'y trouve placé ; ses actions tendent à amener la décadence. « Ne pas employer les armées »; ce qui permet au prince de profiter des efforts de la multitude, c'est la communauté de sentiments entre le supérieur et les inférieurs qui fait que le cour de chacun est disposé à le suivre ; dans le moment actuel, à l'instant où la prospérité touche à sa fin, la voie rationnelle de la prospérité se perd et les sentiments du supérieur et de l'inférieur ne s'accordent plus librement ; le cour de la foule est aliéné, il ne tend plus à suivre le supérieur. Comment pourrait-il employer cette foule et profiter de ses efforts ? S’il veut l'employer, il n'en résultera que du désordre. Du moment où il ne peut plus employer la foule, il commence à avoir recours à ses proches, il les appelle et leur confie ses missions. En supposant même que ceux qu'il appelle pour leur confier ses ordres puissent se conformer à la droiture, il est cependant encore possible qu'il éprouve de l'appréhension et qu'il craigne de se tromper. Le terme « hameau » désigne le district où il réside, c'est-à-dire le foyer de ses parents et de ses proches. En général, les appels et les ordres doivent s'adresser en commençant par ce qui est proche ${ }^{1}$. Toutes les fois que les formules emploient les expressions : "présage malheureux de la pureté » ou «appréhension de la pureté », elles ont deux sens : en observant telle chose avec pureté, le présage sera malheureux ou bien il y aura appréhension ; quelquefois, bien qu'en se confor mant à la droiture, le présage est encore malheureux, ou bien il y a lieu à appréhension. Ici, la formule ne dit pas «présage malheureux de la pureté », mais elle emploie les mots «appréhension de la pureté »; au moment où la décadence est proche et sur le point d'appeler à lui et de donner des missions, il y a lieu d'appréhender des méprises et des erreurs ; la décadence ne procède pas du fait de ces appels et de ces ordres.

\footnotetext{
${ }^{1}$ Le commentaire de Tsheng Tse est basé sur une autre lecture ; il faudrait selon lui traduire : « Ne pas employer la foule, commencer par appeler son propre entourage pour confier des missions. »
} 
TSHOU HI. - La prospérité parvenue à son apogée est suivie de la décadence ; c'est là l'image symbolique représentée par le rempart s'écroulant dans le fossé. C'est un avertis sement à celui qui consulte le sort qu'il ne doit pas lutter par la force, mais simplement s'observer et se garder. Bien quil possède la pureté, il ne peut cependant pas se soustraire à l'appréhension de commettre des erreurs.

Le rempart retombe dans le fossé ; trouble dans les ordres souverains.

TSHENG TSE. - Le rempart retombe dans le fossé ; quel que soit le trouble qui existe dans les ordres du souverain, il est impossible d'y mettre un terme.

TSHOU HI. - Trouble dans les prescriptions et, par suite, retour vers la décadence. Il appelle pour donner des ordres, c'est le moyen d'y porter remède. 


\section{2. $\mathrm{P} \mathfrak{i}$}

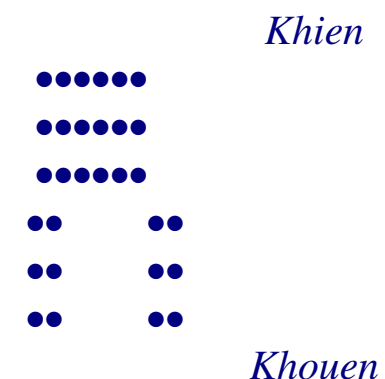

238. Absence d'homme de la décadence ; pas d'avantage pour la pureté de l'homme doué ; ce qui est grand s'en va, ce qui est petit vient.

[]

241. Premier trait hexaire : arracher des herbes à racines traçantes ; employer le genre ; pureté, présage heureux, liberté.

$*$

243. Deuxième trait hexaire : tolérance et soumission; présage heureux pour l'homme inférieur ; adversité et liberté pour le grand homme.

$*$

245. Troisième trait hexaire : supporter la honte.

$*$

247. Quatrième trait nonaire : avoir mandat, pas de culpabilité ; la catégorité entraîne le bonheur.

$*$

249. Cinquième trait nonaire : mettre un terme à la décadence; présage heureux du grand homme ; la perte! la perte! être attaché à un bosquet de mûriers.

$*$

251. Trait supérieur nonaire : déclin de l'adversité ; avant décadence, ensuite joie.

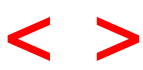


TSHENG TSE. - Pi. «L'Ordre des koua » dit : «Thae, prospérité : liberté ; les choses ne peuvent pas se développer indéfiniment en liberté, aussi le koua thae est immédiatement suivi du koua $p^{\prime} i$, qui exprime la décadence $^{1}$. » Or, la raison d'être des choses est l'aller et le retour ${ }^{2}$; le libre essor de la prospérité parvenu à son apogée doit donc nécessairement être suivi de décadence, et c'est pourquoi le koua pi suit immédiatement le koua thae. Il est constitué par le koua simple du ciel, en haut, et par celui de la terre, en bas. Le ciel et la terre s'unissant, la négativité et la positivité se développant harmoniquement, constituent la prospérité; le ciel placé au-dessus, la terre placée au-dessous, c'est le ciel et la terre séparés d'une façon absolue, ne s'unissant plus librement, ce qui constitue la déca dence.

1 On peut aussi bien lire « adversité ».

2 Action et réaction. 


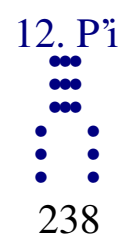

Absence d'homme de la décadence ; pas d'avantage pour la pureté de l'homme doué ; ce qui est grand s'en va, ce qui est petit vient.

TSHENG TSE. - Le ciel et la terre s'unissent et tous les êtres naissent entre eux ; immédiatement les trois causes actives existent. L'homme est la plus spirituelle ${ }^{1}$, aussi il est le premier entre tous les êtres. Tout ce qui naît entre le ciel et la terre suit toujours la voie rationnelle de l'homme. Lorsque le ciel et la terre ne s'uni ssent pas, ils n'engendrent pas les êtres et les choses, la voie de l'homme n'existe donc pas ${ }^{2}$, c'est pourquoi la formule porte les mots fi jen, absence de l'homme, ce qui veut dire que ce n'est pas la voie ration nelle de l'homme. L'extinction ou la croi ssance, la dilatation et la contraction sont réciproquement cause et effet et cette succession n'a point de fin ; la prospérité parvenue à son extrême limite, il doit y avoir réaction ; lorsque la décadence est à son comble, elle doit faire place à la prospérité ; la raison d'être des choses ne comporte pas la durée permanente sans altération; comment la voie rationnelle de l'homme ferait-elle exception ? du moment où il y a décadence, il doit y avoir prospérité. Or, la libre union entre le supérieur et l'inférieur, la coïncidence de la dureté énergique, et de la douceur malléable, constituent la voie rationnelle de l'homme doué. La décadence sera le contraire ; donc il n'y a pas d'avantage pour la pureté de l'homme doué. La vraie voie rationnelle de l'h omme doué est que, dans les cas de décadence et d'obstruction, il n'agit pas. "Ce qui est grand s'en va, ce qui est petit vient. » La positivité s'en va et la négativité vient ; c'est l’image symbolique de la voie de l'homme inférieur s'élargissant et de la voie de l'homme doué diminuant, et c'est pour cela que ce koua exprime la décadence.

TSHOU HI. - $P$ ' $i$, fermer et obstruer ; c'est le koua du septième mois ${ }^{3}$. C'est l'antithèse exacte du koua thae, et c'est pourquoi le texte dit : «absence d'homme ", c'est-à-dire voie rationnelle de l'absence de l'homme. Le sens divinatoire est qu’il n'y a pas avantage pour la vraie voie de l'homme doué ; en effet, le koua simple khien (activité) va occuper l'exté rieur ; le koua simple khouen (passivité) vient occuper le dedans. De plus le koua parfait, lui-même, provient du koua parfait tsien dans lequel le trait nonaire va occuper le quatrième rang tandis que le trait hexaire vient occuper le troisième. On a

\footnotetext{
1 Ling, esprit, âme; l'essence subtile de la négativité. Correspond dans beaucoup de cas au sens figuré de ${ }^{\circ 000}$, raison.

2 Plus littéralement: « C'est donc la voie de l'absence de l'homme. »

3 Équinoxe d'automne. Les conjonctions ont lieu dans l'hémisphère sud et sont invisibles: absence de l'homme.
} 
douté si les deuxième, troisième et quatrième caractères du texte, tshi fi jen, «absence d'homme de », ne seraient pas une superfétation dans le texte, provenant, par erreur, de la formule du troisième trait hexaire du koua pi. Il est d'ailleurs remarquable que le commentaire traditionnel de Tsheng Tse n'en explique pas le sens.

Le commentaire traditionnel de la formule déterminative dit: Absence d'homme de la décadence, pas davantage pour la pureté de l'homme doué ; ce qui est grand s'en va, ce qui est petit vient : C est donc le ciel et la terre ne s'unis sant pas et tous les êtres sans liberté, le supérieur et les inférieurs ne s'unissantpas et l'émpire privé d'états feudataires. Au-dedans négativité et au-dehors positivité ; au-dedans douceur malléable et au-dehors dureté énergique; au-dedans l'homme inférieur et au-dehors l'homme doué ; la voie de l'homme inférieur grandissant et la voie de l'homme doué diminuant.

TSHENG TSE. - Or, les éthers du ciel et de la terre ne s'unissant pas, il en résulte qu'il n'y a aucune raison pour que les êtres et les chos es puissent naître et s'achever; de même, lorsque les devoirs du supérieur et des inférieurs ne s'allient point, il n'y a plus de voie rationnelle reliant, dans l'univers, les états et les royaumes. L'établissement de royaumes et d'états est ce qui assure l'ordre politique ${ }^{1}$. Lorsque le prince promulgue des lois réglant les institutions politiques et sociales, et que les peuples portent le joug du prince $^{2}$ et suivent ses instructions, le supérieur et les inférieurs sont reliés ensemble, et c'est là ce qui co nstitue l'ordre dans le gouvernement, et la paix. Dans le cas actuel, le supérieur et les inférieurs ne se lient point, c'est donc que dans l'univers la voie rationnelle des états et des royaumes n'existe pas. La douceur malléable de la négativité se trouve en dedans; la dureté énergique de la positivité est au-dehors; l'homme doué s'en va résider au-dehors ; l'homme inférieur vient se placer au-dedans; c'est le moment où la voie de l'homme infé rieur grandit et où celle de l'homme doué s'efface.

\footnotetext{
${ }^{1}$ États tributaires dont les chefs relèvent du pouvoir suprême d'un empereur représentant le pouvoir divin.

2 Cette expression tout étrangère ne rend qu'imparfaitement la locution chinoise, qui ne comporte aucune acception fâcheuse, littéralement: «porter le prince sur la tête ».
} 
Le commentaire traditionnel de la formule symbolique dit: Le ciel et la terre ne s'unissent point : décadence, l'homme doué ménage ses vertus ${ }^{1}$, évite les difficultés ; il ne doit pas les faire éclater pour obtenir un traitement public.

TSHENG TSE. - Le ciel et la terre ne s'unissent pas librement, c'est pourquoi le koua exprime la décadence. Dans les moments de décadence, la voie de l'homme doué péricli te ; il doit regarder cette image symbolique de décadence et d'empêchements et travailler à ménager ses v ertus et à éviter le malheur et les difficultés. Il ne doit pas occuper avec éclat une situation officielle rétribuée. La décadence, c'est le temps où les tendances de l'homme inférieur suivent leur libre cours; si l'homme doué occupe une position brillante et en évidence, les malheurs et les périls atteindront forcément sa personne, de sorte qu'il est opportun qu'il se place dans l'ombre et qu'il se confine en se contenant.

TSHOU HI. - Ménager ses vertus, en être économe; ne pas les manifester au-dehors, afin d'éviter le danger que l'homme inférieur cause. L'homme ne doit pas faire éclater ses capacités dans une situation officielle rétribuée.

\footnotetext{
${ }^{1}$ Épargner; ne pas être prodigue de ses vertus en les manifestant par des actes extérieurs.
} 


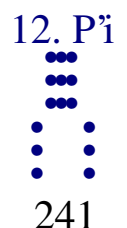

Premier trait hexaire : arracher des herbes à racines traçantes; employer le genre ; pureté, présage heureux, liberté.

TSHENG TSE. - Le koua thae et le koua $p \nmid i$ emploient tous deux l'image symbolique des herbes dont les racines se relient et s'entrecroisent, parce que la foule des positivités ou la foule des négativités s'y trouvent placées en bas d'une façon identique.

Cette expression est un symbole de l'idée de s'entraîner en s'attirant mutuellement. Dans le moment de la prospérité, c'est l'action de marcher ensemble qui constitue le présage heureux; dans le moment de la décadence, ce sera la condition de posséder la même pureté qui procurera la liberté. D'abord, c'est cette circonstance que l'homme inférieur es $\mathrm{t}$ au-dedans et l'homme doué au-dehors, qui est considérée comme exprimant l'idée de décadence ; ensuite, c'est le premier trait hexaire dans la décadence et placé dans l'infé riorité qui est considéré comme exprimant la voie rationnelle de l'homme doué. Dans le Yi king, le sens est choisi selon le moment ${ }^{1}$, il est variable et change sans règles constantes. Dans les moments de décadence, c'est celui qui est dans l'infériorité qui est l'homme doué. Les trois négativités du koua pli ont chacune une correspondance sympathique au-dessus d'elles ; dans un moment de décadence et d'isolement, la séparation est définitive et sans libre communication, aussi le sens de correspondance sympathique n'est pas mentionné. Si le premier trait hexaire peut, avec ceux de son propre genre, se maintenir dans l'observation des principes avec une fermeté pure et inébranlable, il sera dans les meilleures conditions que comporte la décadence et ce sera la liberté possible de sa voie rationnelle. Celui qui se trouve en présence d'un moment de décadence et qui peut néanmoins avancer est un homme inférieur ; l'homme doué redressera sa voie morale et se contentera d'éviter le malheur. L'homme doué, dans ses mouvements en avant ou en arrière, n'agit jamais autrement que ceux de son propre g enre.

TSHOU HI. - Les trois négativités sont en bas; dans les temps de décadence, c'est l'image symbolique des hommes inférieurs s'entraînant mutuellement et avançant ensemble. Cependant, les vices du premier trait ne sont pas encore affichés et manifestés sous une forme visible, aussi la formule avertit qu'avec la pureté, il y aura présage heureux et liberté. En effet, pouvant être dans de telles conditions, il se modifiera et deviendra un homme doué.

\footnotetext{
${ }^{1}$ Exprimé par le koua ou le trait.
} 
Arracher du chiendent, présage heureux de la pureté ; tendances vers le prince.

TSHENG TSE. - Ce trait, avec sa qualité hexaire, s'observant et se contenant dans l'infé riorité, exprime clairement la voie rationnelle de l'homme doué placé dans un rang infé rieur. Le commentaire symbolique éclaire de nouveau le sens en prenant le symbole du coar de l'homme doué. Si l'homme doué maintient rigidement l'observation des prin cipes pour se placer dans l'infériorité, ce n'est pas qu'il se plaise à ne pas avancer et à être seul bon et vertueux ; c'est que sa vo ie commence à être en décadence et qu'il ne doit pas avancer, de sorte quil se contente de cette situation. Son cour, certainement n'est jamais sans s'occuper de l'univers ; ses tendances le portent constamment à s'accorder avec le prince et à s'avancer v ers lui afin de pacifier l'univers et d'y rétablir l'ordre, et c'est pour quoi la formule dit «tendances vers le prince ».

TSHOU HI. - C'est l'homme inférieur qui se modifie peu à peu pour devenir homme doué, de sorte qu'il peut méditer sur l'amour dû au prince, sans tenir compte de ses intérêts privés. 


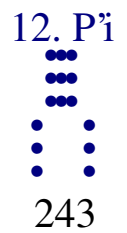

Deuxième trait hexaire : tolérance et soumission ; présage heureux pour l'homme inférieur; adversité et liberté pour le grand homme.

TSHENG TSE. - Le caractère distinctif essentiel du deuxième trait hexaire est la douceur malléable ; ce qui dérive de la position quil occupe, c'est la justice et la droiture. En en parlant au point de vue de la douceur malléable de l'homme inférieur, cela indiquera qu'au moment où l'adversité commence dans les rangs inférieurs, ce qui préoccupe son cour, c'est la soumission et l'obéissance au supérieur ${ }^{1}$, dans le but d'arriver à traverser l'adversité pour son propre bien-être, ce qui constitue le bonheur pour l'homme infé rieur. En présence de l'adversité et de la décadence l'homme doué se placera selon la voie morale ; comment consentirait-il à se courber lui-même en faisant plier les règles morales ? Obéissant et soumis envers le supérieur, il se contente simplement de se préserver lui-même de cette décadence. L'adversité frappant sur sa propre personne, c'est précisément la liberté de la voie morale. On a dit : le supérieur et les inférieurs ne s'unissant point, à quoi se soumèttrait-il ? On doit répondre : Quand la droiture est en décadence, la disposition de l'inférieur à se soumet tre au supérieur existe toujours quelque peu.

TSHOU HI. - Douceur malléable négative, justice et droiture; image symbolique de l'homme inférieur capable de soumission et d'obéissance au supérieur. C'est une voie heureuse pour cet homme inférieur. Aussi, si celui qui consulte le sort est un homme inférieur, et s'il est dans ces conditions, le présage sera heureux; si c'est un grand homme, il devra se contenter de rester dans l'adversité, et plus tard sa voie sera libre. En effet, ce n'est pas parce que tel autre serait porté à la soumission à son égard quill devrait lui même manquer aux règles qu'il observe.

\section{4}

Liberté dans la décadence pour le grand homme : ne pas troubler la foule.

TSHENG TSE. - Dans les temps d'adversité, le grand homme observe et garde les préceptes de la droiture; il ne se confond pas dans la foule des

\footnotetext{
${ }^{1}$ Le commentateur lit: « Être rempli de soumission.
} 
hommes inférieurs ; bien que sa personne soit dans l'adversité, sa voie morale est libre, et c'est pourquoi la formule dit : «adversité et liberté » ou « liberté dans l'adversité ». La liberté du corps, si elle ne provient pas par la voie rationnelle et morale, constitue la décadence de cette voie. La forinule n'emploie plus le terme « homme doué », et elle emploie le terme «grand homme »: c'est que la voie de celui qui peut remplir ces conditions est grande.

TSHOU HI. - Cela veut dire ne pas se mêler et se confondre dans la foule des hommes inférieurs. 


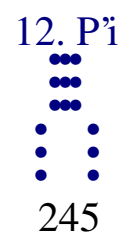

Troisième trait hexaire : supporter la honte ${ }^{1}$.

TSHENG TSE. — Le troisième trait emploie la douceur malléable de la négativité ; il est sans justice ni droiture et se trouve dans l'adversité. De plus, il est le plus voisin des traits supérieurs ; il ne peut conserver la voie morale et se contenter de sa destinée ; finalement il aboutit à l'erreur : ce sont là, au plus haut point, les circonstances et les sentiments qui constituent l'homme inférieur. Ce qu'il couve en lui, ce qu'il médite, le résultat de ses calculs, ne peut jamais manquer d'arriver à constituer le faux, le mal et l'erreur. Il doit arriver à la honte.

TSHOU HI. - Emploi de la négativité dans une position qui comporte la positivité et absence de justice et de droiture ; c'est l'homme inférieur dont les tendances sont tournées au mal, mais qui ne peut encore le commettre, et c'est pour cela que ce trait est limage symbolique de supporter (ou contenir) la honte. Cependant, comme le résultat n'est pas encore manifesté, il s'en suit que la formule ne contient pas d'avertissement de culpabilité et de présage malheureux.

246.

Supporter la honte; situation imméritée.

TSHENG TSE. - Malléabilité négative se trouvant dans l'adversité et dépourvue de justice et de droiture. Ce qui fait qu'il est considéré comme devant éprouver la honte, c'est qu'il est placé dans une situation dont il n'est pas digne. Être placé dans une situation imméritée, c'est agir autrement que la voie morale.

\footnotetext{
${ }^{1}$ On pourrait lire: « contenir la honte ».
} 


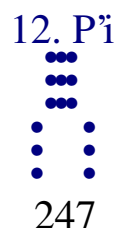

Quatrième trait nonaire : avoir mandat ${ }^{1}$, pas de culpabilité ; la catégorité entraîne le bonheur.

TSHENG TSE. - Le quatrième trait, avec sa substance de dureté énergique positive d'activité, occupe la situation près de celle du prince : c'est donc celui qui a les aptitudes requises pour traverser l'adversité et qui possède une situation élevée. Ces conditions sont suffisantes pour aider le supérieur à remédier à l'adversité de la décadence, mais cependant, en présence d'un moment où la voie morale du prince commence à décliner, étant placé sur un terrain extrêmement voisin de lui, ce qui est à craindre, c'est unique ment de s'attribuer le mérite du bien et de provoquer de l'animosité. S'il peut diriger et imprimer le mouvement, ce doit être en conformité des ordres du prince ; si la poignée de l'arme du pouvoir est exclusivement dans la main du supérieur, alors il sera sans culpabilité et ses tendances prévaudront. Pouvant diriger toutes choses d'après les ordres souve rains, alors il pourra remédier à l'adversité du moment. La catégorie ou le genre, entraîne toujours le bonheur et la félicité. Le terme $l i$, du texte, proche, a le sens d'un caractère $l i$ signifiant être annexé, lié à ${ }^{2}$ Lorsque la voie de l'homme doué agit, il doit avancer de même que ceux de son genre, afin de remédier à l'adversité de l'empire ; « la catégorie entraîne le bonheur ». Dans le mouvement en avant de l'homme inférieur, ce dernier mouvement a également lieu avec ceux du même genre.

TSHOU HI. - L'adversité est traversée plus qu'à moitié ; c'est le moment où la décadence est sur le point d'être enrayée. Le quatrième trait nonaire, avec les qualités de la positivité, occupe un rang qui comporte la négativité ; il ne pousse pas la dureté jusqu'à ses dernières limites, aussi, le sens divinatoire est qu'il est prédestiné et qu’il ne commet point de fautes. Enfin, les trois positivités, réunies par le lien du genre, atteignent toutes le bonheur. Le terme ming du texte, exprime la prédestination ou «mandat céleste $»^{3}$.

248.

Avoir mandat, pas de culpabilité ; tendances prévalant.

\footnotetext{
${ }^{1}$ On peut lire : « avoir mission du prince », ou aussi bien: « être prédestiné ».

${ }^{2}$ Le sens du caractère li du texte, est essentiellement « se séparer de », le sens du passage est très douteux.

${ }^{3}$ Mandat fatal; destinée fixée à l'avance.
} 
TSHENG TSE. - Ayant reçu les ordres du prince, il peut être sans culpabilité ; c'est là ce qui lui permet de traverser l'adversité et d'y remédier ; ses tendances peuvent prévaloir. 


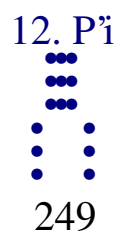

Cinquième trait nonaire : mettre un terme à la décadence ; présage heureux du grand homme; la perte! la perte! être attaché à un bosquet de mûriers.

TSHENG TSE. - Le cinquième trait, avec les vertus de l'énergie positive, de la justice et de la droiture, occupe la situation prééminente, aussi, il peut mettre un terme à la décadence de l'univers ; c'est le présage heureux du grand homme. Le grand homme digne de sa situation peut, au moyen de sa voie morale, arrêter la décadence de l'univers, pour ramener la prospérité ; mais, cependant, il n'est pas encore tiré de l'adversité, de sorte que la formule porte l'avertissement relatif à la perte. Quand la décadence est arrêtée et qu'elle tend peu à peu à laisser la place au retour de la prospérité, on ne doit pas se contenter de se livrer au calme et aux loisirs ; il convient de prévoir de loin, de se tenir en garde contre le retour de l'adversité. La formule dit : La perte ! la perte ! Cette expression : «Être attaché à un bosquet de mûriers », veut dire être dans la voie d'un repos fermement assuré, comme lié à une touffe de mûrier. Comme plante, le mûrier est une de celles qui ont des racines profondes et solides. Touffe ou bosquet, indique une réunion de pieds croissant ensemble. La solidité d'un tel point d'attache est donc des plus grandes. Les avertissements de l'homme saint sont profonds ! Dans la dynastie des Han, le roi Yun, dans celle des Theang, Li Te Yu ne comprirent pas cet avertissement, ce qui les conduisit à leur perte et à la ruine de l'état. Le « grand commentaire des formules annexées » dit : «Le péril, c'est ce qui assure la sécurité de la situation; la perte, c'est ce qui garantit la conservation; le désordre, c'est ce qui contient le germe de l'ordre. Aussi l'homme doué est calme et il n'oublie pas le péril ; il se maintient et n'oublie pas la perte ; il fait régner l'ordre et n'oublie pas le désordre. C'est ainsi que l'état peut être protégé par sa sécuri té personnelle. »

TSHOU HI. - Positivité, énergie, justice, droiture, employées pour se maintenir au rang prééminent ; capacité pour arrêter la décadence du moment : c'est le fait du grand homme. Aussi le sens divinatoire de ce trait, si c'est un grand homme qui le rencontre en consultant le sort, sera un présage heureux. Cependant, il convient encore qu'il se garde et se souvienne des avertissements donnés dans le grand commentaire. 
TSHENG TSE. - Il possède les vertus du grand homme et il possède légitimement la situation de l'éminence suprême, aussi, il peut arrêter la décadence de l'univers, ce qui constitue le présage heureux. S'il n'avait pas cette situation, quand même il aurait la voie morale correspondante, comment atteindrait-il au résultat ? C'est pour cela que la situa tion de l'homme saint est appelée « la grande chose précieuse ». 


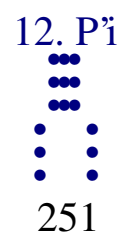

Trait supérieur nonaire : déclin de l'adversité ; avant décadence, ensuite joie.

TSHENG TSE. - Le trait supérieur nonaire représente la fin de l'adversité. Lorsque la raison d'être d'une chose est arrivée à la limite extrême de ses conséquences, elle doit réagir en sens opposé ; c'est pour cela que, quand la prospérité est arrivée à son apogée, elle doit être suivie de décadence ou adversité, et que l'adversité, parvenue à sa limite extrême, doit faire place à la prospérité. Le trait nonaire supérieur exprime l'adversité quand ell e est à son maximum d'intensité ; aussi, la voie de la décadence décline, se renverse et se modifie. D’abord extrême adversité, ensuite inclination vers la joie ; l'adversité déclinant, la prospérité doit apparaître, et ensuite lajoie.

Tsi3ou HI. - Puisque la dureté énergique de la positivité occupe le point limite de l'adversité, elle exprime l'adversité au moment où elle décline ; le sens divinatoire est que d'abord il y a adversité et ensuite joie.

Arrivée à sa fin, l'adversité décline ; comment pourrait-elle durer?

TSHENG TSE. - Lorsque la décadence est à sa fin, elle doit nécessairement décliner; comment la raison d'être des choses pourrait-elle être que l'adversité dût durer éternelle ment? L'apogée atteinte, il y a nécessairement réaction en sens inverse : c'est la loi constante de la raison d'être de toutes choses. Mais, pour ramener la paix après le péril, pour changer le trouble en ordre il est absolument indispensable d'avoir les aptitudes de la dureté énergique positive. C'est pour ces raisons, que le trait supérieur nonaire du koua pli peut détourner l'adversité et la faire plier, tandis que le trait supérieur hexaire du koua tshouen ne peut pas modifier l'état d'empêchement exprimé par ce koua. 


\section{Thong jen}

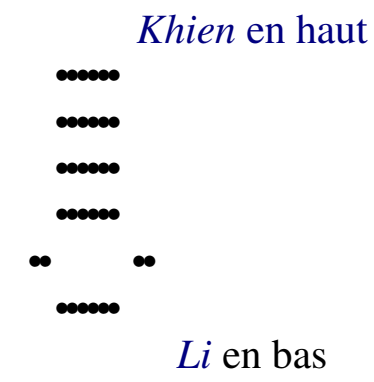

253. Hommes identiques dans le désert ; liberté ; avantage à traverser un grand cours d'eau; avantage pour la pureté de l'homme doué.

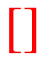

258. Premier trait nonaire: mêmes hommes à la porte; pas de culpabilité.

$*$

260. Deuxième trait hexaire : mêmes hommes dans la souche ; appréhension.

$*$

262. Troisième trait nonaire : cacher des armes dans l'herbe épaisse ; gravir la colline élevée; pendant trois ans ne pas commencer.

$*$

264. Quatrième trait nonaire : monter sur le mur; ne pas pouvoir vaincre; présage heureux.

$*$

266. Cinquième trait nonaire : les mêmes hommes d'abord crient et se lamentent et ensuite ils rient; les grandes armées peuvent se rencontrer ensemble.

$*$

268. Trait supérieur nonaire : mêmes hommes dans la plaine ; pas de regrets.

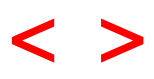


TSHENG TSE. - Thong jen. «L'Ordre des koua » dit : « Les êtres et les choses ne peuvent pas être indéfiniment dans la décadence et l'adversité ; c'est pour cela que le koua pi est suivi du koua thong jen. » Lorsque le ciel et la terre ne s'unissent pas, cela constitue l'adversité ; lorsque le supérieur et l'inférieur sont d'accord ensemble, cela constitue le koua thong jen (dont le nom signifie hommes semblables). Son sens est l'opposé du sens du koua pi, et c'est pour cela que ces deux koua se suivent. De plus, dans les époques où commence la décadence, il faut absolument que les hommes réunissent leurs forces, et c'est seulement ainsi qu'ils peuvent traverser l'adversité, ce qui fait que le koua thong jen suit immédiatement le koua pi. Comme koua, il est constitué par le koua simple khien, en haut, et le koua simple $l i$, en bas. Si on en parle au point de vue des deux symboles, le ciel est ce qui est en haut ; la nature du feu est que la flamme monte : identité de tendances avec le ciel, ce qui fait que ce koua est considéré comme représentant des «hommes semblables ». Si on en parle d'après les deux substanc es, le cinquième trait occupe la situation qui lui convient d'après la droiture ; c'est de lui que dépend l'activité ; le second trait est le maître du koua simple $l i$; ces deux traits se correspondent sympathiquement par la justice et la droiture; le haut et le bas sont identiques, c'est-à-dire que le supérieur et l'inférieur sont d'accord ensemble, ce qui donne le sens d' «hommes semblables». Enfin, l'unique négativité du koua simple $l i$, est l'objet des désirs identiques de la foule des positivités, ce qui donne encore le sens de «hommes semblables». Parmi les autres koua, il y en a certainement qui n'ont qu'un trait négatif ; mais dans le moment de l'accord entre les hommes, et alors que le second et le cinquième trait se correspondent sympathiquement, que le ciel et le feu sont identiques dans leurs tendances, toutes ces raisons font que le sens est plus vaste et plus important. 


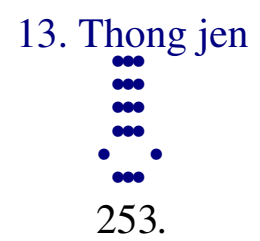

Hommes identiques dans le désert ; liberté ; avantage à traverser un grand cours d'eau; avantage pour la pureté de l'homme doué.

TSHENG TSE. — Le désert, c'est -à-dire les régions vastes et inhabitées ; ce mot est pris dans le sens de lieu extérieur et lointain. Or, ce qui réunit les hommes dans la voie de l'identité de grandeur dans l'univers, ce sera la grandeur du désintéressement du cour des Saints et des Sages ; ce qui réunit les hommes ordinaires sera la concordance de leurs intérêts privés, et c'est bien là un sentiment qui les porte à s'associer. Aussi, il faut que ce soit dans le désert, pour exprimer que leur réunion n'est pas le résultat de sentiments de rapprochement égoïste. Mais sur le terrain des régions lointaines et désertes, dù moment où ils ne sont plus retenus par leurs intérêts privés, c'est donc la voie de l'extrême désin téressement et de l'identité de grandeur qui les dirige, et rien n'est assez loin pour ne pas être entraîné dans le mouvement de réunion. La liberté d'essor de ce penchant est évi dente. Pouvoir s'identifier et s'accorder avec l'univers, c'est être tel que l'univers s’identi fie et s'unit à soi même. Lorsque tout dans l'univers sidentifie, quels obstacles et quels empêchements pourraient être insurmontables ? Quels périls et quelles diffcultés ne pourraient être librement traversés ? C'est pour cela qu'il y a avantage à traverser un grand cours d'eau, et avantage pour la pureté de l'homme doué. Le premier membre de phrase dit : «dans le désert», cela exprime seulement qu'il ne s'agit pas d'une association intime et particulière ; ici, la formule dit de plus qu'il importe que ce soit d'après la droite morale de l'homme doué. La pureté de l'homme doué désigne la voie rationnelle de la grande unité (identification) de l'extrême désintéressement, ce qui fait que bien que placé à mille fi d'éloignement, bien que né après plus de mille ans de distance, en se conformant aux principes fixes ${ }^{1}$, en les analysant et en les pratiquant, dans l'immen sité située entre les quatre mers, la foule des millions des peuples fait sans aucune exception partie de cette unité ou identification. L'homme inférieur ne vise qu'à des intérêts privés ; lorsqu'il s'approche et s'associe, quelle que soit l'immoralité du but, il pratique quand même cette unité de considération pour les choses ou les personnes; lorsquil éprouve de la répulsion, quelles que soient les qualités des objets de son aversion, il les considère néanmoins comme étrangers à lui-même et en dehors de cette unité. Aussi les réunions qu'il forme sont toujours des coteries népotiques, et, en

\footnotetext{
${ }^{1}$ Les deux caractères du texte ont des significations très variables. Le premier exprime l'idée de rapprocher les deux moitiés d'un objet qui sert de signe de reconnaissance, et le second désigne des signaux faits avec des lumières.
} 
effet, son cour manque de droiture. C'est pour ces raisons que dans la voie rationnelle de l'unité de considéra tion des hommes, l'avantage consiste dans la droiture et la pureté de l'homme doué ${ }^{1}$.

TSHOU HI. - $\mathrm{Li}$ est encore le nom d'un koua simple ; une négativité pressée dans l'intervalle entre deux positivités ; c'est pour cela que ses vertus sont la beauté, l'élégance de la forme et de l'apparence, tandis que son image symbolique est le feu, le soleil, l'éclair de la foudre. Les deux caractères du nom de ce koua thong jen signifient " accord avec les hommes ${ }^{2}$. Rencontre des koua simples $l i$ et khien; le feu monte se réunir au ciel. Le second trait hexaire possède une situation, il possède la justice et, vers le haut, il correspond sympathiquement au cinquième trait nonaire. De plus, dans le koua parfait, il n'y a qu'une seule négativité et les cinq positivités se portent également vers elle ; c'est pour cela qu’il est appelé thong jen, et considéré comme exprimant l'accord entre les hommes.

« Dans le désert », veut dire au loin, dans un vaste champ, et sans aucune idée d'intérêt privé ; il y a donc voie de libre expansion. Il y a emploi de l'activité pour l'action, de sorte qu'il est possible de traverser un grand cours d'eau. Le koua parfait est formé, au -dedans, par le koua simple qui exprime la clarté ou distinction de la forme et, au -dehors, par celui qui exprime la dureté énergique et l'activité ; le second trait hexaire possède la justice et la droiture et il rencontre une correspondance sympathique : donc c'est la voie rationnel le de l'homme doué. Si celui qui consulte le sort est dans ces conditions, ce sera un signe de liberté d'action, et de plus, il pou rra traverser les dangers. Toutefois, il faut absolument que ce à quoi il se réunit et s'accorde soit conforme aux voies morales de l'homme doué, et alors seulement ce sera avantageux.

254.

\footnotetext{
${ }^{1}$ Quelle que soit l'élasticité du sens de chaque caractère, la grande facilité que donne dans le style chinois la liberté de prendre, souvent dans une même phrase, le même caractère avec des acceptions extrêmement différentes, enfin le sans-gêne des commentateurs qui ne se piquent pas toujours d'être bien clairs et se contentent de se tenir d'accord ave c l'explication traditionnelle, il est cependant impossible de trouver dans le texte chinois de ce passage un sens déterminé et acceptable. La version française, aussi littérale que possible, laisse paraître le peu de clarté de l'idée que le commentateur s e fait lui-même de l'objet et du sens exprimés parce koua.

${ }^{2} \mathrm{La}$ traduction est conforme au sens adopté par le commentateur; mais, littéralement et grammaticalement, le sens des mots thong jen est «mêmes hommes" ou «identique à l'homme », le caractère thong, bien qu'il reçoive ici l'accep tion de «accord avec » n'a réellement pas ce sens. Si les commentateurs présentaient une explication admissible, un sens plausible, on pourrait admettre que leur lecture est bonne, mais leur lecture n'est ni justifiée, ni justifiable.
} 
Le commentaire traditionnel de la formule déterminative dit: Uniformité des hommes; la douceur malléable possède la situation, elle possède la justice et elle correspond sympathiquement à l'activité ; on l'appelle thong jen : « hommes identiques ».

TSHENG TSE. - Il est question du sens du koua parfait. « La douceur malléable possède la situation », signifie que le second trait, avec ses qualités négatives, occupe un rang négatif; il possède la situation qui lui revient légitimement. Le cinquième trait possède la justice et la droiture, et le second lui correspond sympathiquement par la justice et la droiture ; c'est posséder la justice et correspondre à l'activité. Le cinquième trait est énergique, actif, juste et droit, et le second avec sa douceur, sa soumission, sa justice et sa droiture lui correspond sympathiquement : chacun possède ce qui lui revient légitimement et leurs vertus sont identiques (thong), ce qui fait que le koua est appelé thong jen : « hommes identiques ». Le cinquième trait est celui de qui dépend l'activité, c'est pourquoi la formule dit : «correspond sympathiquement à l'activité ». La formule symbolique est basée sur les images symboliques du ciel et du feu, mais la formule déterminative prend exclusivement le second trait pour thème.

TSHOU HI. - Explication du sens du nom du koua au moyen de la substance des koua simples constitutifs.

255.

TSHENG TSE. - Les trois caractères de cette formule sont une superfétation dans le texte.

TSHOU HI. - Superfétation du texte.

256.

Liberté de l'identité des hommes dans le désert; avantage à traverser un grand cours d'eau: L'activité agit: Clarté de la forme pour l'activité ; justice, droiture et sympathie: droiture de l'homme doué. L'homme doué seul est capable de comprendre les tendances de l'univers.

TSHENG TSE. - Extrême sincérité sans égoïsme ; possibilité de marcher à travers les périls et les dangers : c'est l'action de l'activité. Absence 
d'égoïsme : c'est la vertu du ciel. De plus, si on parle du sens d'après les deux substances, il y a les vertus de l'intelligence dans les actes ext érieurs et dans la forme, et activité énergique ; correspondance sympathique mutuelle par la voie de la justice et de la droiture : c'est donc exactement la voie de l'homme doué. Les tendances de l'univers diffèrent de mille manières ; la raison d'être en est unique; l'homme doué comprend cette loi, de sorte qu'il peut pénétrer et comprendre les tendances de l'univers. L’homme saint regarde le cour de chaque homme, dans les millions de la foule, et il ne voit qu'un seul cour : c'est uniquement parce qu'i 1 a pénétré et compris la raison d'être qui cause les nuances. L'apparence extérieure et la forme éclairées et comprises, il est possible de rendre claire la raison d'être, de sorte qu'il est capable d'accomplir la voie de la grande uniformité. Par la forc e active de l'énergie, il peut se dominer, de sorte qu'il est capable d'accomplir la voie de la grande uniformité, et, par suite, il peut avec justice et droiture se conformer à l'action de l'activité ${ }^{1}$.

TSHOU HI. - Explication de la formule du koua au moyen des vertus et de la substance des koua simples. Comprendre les tendances de l'univers, c'est là ce qui constitue la grande uniformité ; s’il en est autrement, il ne s'agit plus que de l'accord d'intérêts pri vés : comment cela serait-il suffisant pour atteindre à la liberté, et pour qu'il y ait avantage à traverser un grand cours d'eau ?

257.

Le commentaire traditionnel de la formule symbolique dit : Le ciel avec le feu; hommes identiques; l'homme doué classe les êtres et les choses par le genre et la famille.

TSHENG TSE. — Le texte ne dit pas : « Le feu est sous le ciel », ni : «Sous le ciel il y a le feu », mais il dit: « Ciel avec le feu ». Le ciel est ce qui est en haut ; la nature du feu est de monter : le feu est identique au ciel (le feu s'accorde avec le ciel), et c'est pour cela que ce koua constitue le sens d'identité (ou accord) des hommes. L'homme doué regarde l'image symbolique de l'identité des hommes et, d'après le genre et la famille, il classe les êtres et les choses, c'est-à-dire, chacun selon le genre et la famille auxquels il appartient, et en distinguant les rapports communs et différents. Par exemple, les associations entre hommes doués ou entre hommes inférieurs; la raison d'être du bien ou du mal, du vrai ou du faux; le désaccord ou l'union des sentiments des êtres ; la dissemblance ou la simi-

\footnotetext{
${ }^{1} \mathrm{Si}$ on veut se reporter à l'interprétation que je propose, on trouvera facilement un sens
} admissible : identité des phénomènes observés pendant les conjonctions. 
litude de la raison d'être des choses : toutes ces différences ou tous ces rapports communs, l'homme doué peut les distinguer et les comprendre avec clarté, aussi en assignant la place des êtres, il ne perd jamais la mesure.

TSHOU HI. - Le ciel est en haut et la flamme du feu monte : la nature est la même dans les deux cas. Distinguer les êtres par genre et par famille, c'est le moyen de reconnaître les différences et d'arriver à l' uniformité. 


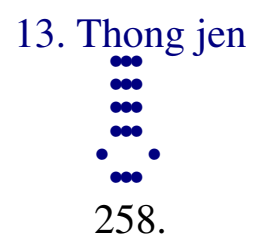

Premier trait nonaire : mêmes hommes à la porte ; pas de culpabilité.

TSHENG TSE. - Un trait nonaire occupe le premier rang du koua thong jen et il n'est retenu par aucu ne correspondance sympathique ; c'est l'absence de partialité et d'égoïs me, le grand désintéressement de l'union des hommes. C'est pour cela que ce trait est considéré comme exprimant l'acte de sortir hors de la porte, pour s'unir aux hommes ${ }^{1}$. Sortir hors de la porte veut dire au-dehors ; puisque c'est au-dehors, il n'y a donc aucune partialité résultant de liens privés : l'accord est large et désintéressé, de cette façon, il n’y aura ni fautes ni culpabilité.

TSHOU HI. - Début de l'accord entre les hommes ; il n'y a pas encore de direction imprimée par les sentiments égoïstes. Puisque la dureté énergique est en bas, qu'en haut elle ne rencontre aucune sympathie, il est possible quil n'en résulte aucune faute, et c'est pourquoi tels sont l'image symboliqu e et le sens divinatoire.

259.

Sortir des portes et s'accorder aux hommes ${ }^{2}$; quelle culpabilité $y$ aurait-il encore?

TSHENG TSE. - Sortir de la porte et s'accorder aux hommes à l'extérieur ; c'est que ce qui est assimilé est vaste ; il n'y a aucun mot if d’intérêt privé et de partialité. La similitude entre les hommes peut être large ou étroite ; elle peut être étendue plus ou moins loin, et de ces distinctions dans la manière de les considérer pour s'allier à eux, naissent les fautes et la culpabilité. Mais du moment où, dans l'accord, il n'y a point de coterie et de partialité, où pourrait être la culpabilité.

\footnotetext{
${ }^{1}$ Thong est pris ici par le commentateur dans le sens de ho, ou huo, s'acc order, se lier à, se mettre d'accord.

${ }^{2}$ On devrait lire « hommes identiques sortant de la porte».
} 


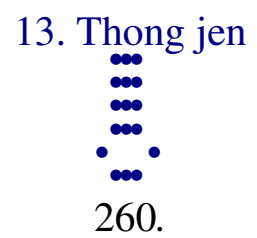

Deuxième trait hexaire : mêmes hommes dans la souche ${ }^{1}$; appréhension.

TSHENG TSE. — Le second et le cinquième trait sont considérés comme se correspondant sympathiquement, et c'est pourquoi la formule dit : «S'accorder aux hommes dans la souche.» La souche désigne le groupe descendant de la même origine. S'accorder à ceux auxquels on est attaché ou lié par la sympathie, c'est être dirigé par la partialité dans ses liaisons ; dans la voie de l'accord avec les hommes, c' est considéré comme un rapprochement égoïste, de sorte qu'il doit en résulter de l'appréhension. Si le second trait était positif, cela constituerait les vertus de la justice et de l'énergie et par suite l'union mutuel le par la voie de la justice ; ce ne serait plus considéré comme une liaison basée sur l'inté rêt et les sentiments privés.

TSHOU HI. - Souche, association, groupe. Bien que le second trait hexaire soit guidé par la justice et par la droiture, cependant il est sympathique à un trait supérieur; il ne peut pratiquer la grande unification et est retenu par les intérêts privés ; c'est une voix logique d'appréhension, et c'est pourquoi tels sont l'image symbolique et le sens divina toire.

261.

S'accorder aux hommes dans la souche; voie logique d'appréhension.

TSHENG TSE. - Dans tous les koua, la correspondance sympathique par la justice et la droiture est considérée comme constituant le bien, tandis que dans le koua thong jen ces mêmes conditions sont considérées comme indiquant qu'il y a lie u à appréhension ; c'est pour cela que le cinquième trait ne prend pas la valeur représentative du prince. En effet, les associations qui ont l'intérêt privé pour mobile, ne constituent pas la voie rationnelle du prince ; l'accord mutuel motivé par l'égoïs me, constitue une cause légitime d'appré hension.

\footnotetext{
${ }^{1}$ Les commentateurs lisent « s'accorder aux hommes dans la souche ». La souche, ou origine commune, en remontant, de toutes les branches d'une même famille.
} 


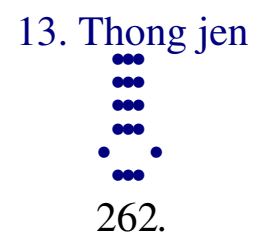

Troisième trait nonaire : cacher des armes dans l'herbe épaisse ; gravir la colline élevée ; pendant trois ans ne pas commencer ${ }^{1}$.

TSHENG TSE. — Le troisième trait, positif, occupe un rang qui comporte la dureté énergique et il n'obéit point à la justice ; c'est un homme énergique et violent. Dans le moment exprimé par le koua thong jen (accord des hommes), les tendances sont portées à l'accord ; dans le koua il n'y a qu'uné seule négativité : les tendances de toutes les positivités les portent également à s'adjoindre et à se réunir à elle. Le troisième trait avec sa force énergique se tient entre le second et le cinquième ; il veut l'enlever de force et se réunir (thong) à ce second trait. Toutefois la raison d'être des choses ne le permet pas ; le devoir ne le tolère pas, de sorte qu'il n'ose pas manifester ouvertement ses sentiments. Il cache ses armes au milieu de l'épaisseur des forêts et des herbes; il nourrit en soi-même ses desseins coupables et illégitimes. C'est pour cela qu'il éprouve encore des craintes ; parfois il monte sur la colline élevée pour observer et surveiller de loin. Cet état de choses dure jusque pendant l'es pace de trois années et, finalement, il n'ose pas commencer l'exécution de ses projets. La formule de ce trait analyse minutieusement la manière d'être et les sentiments de l'homme inférieur, mais, toutefois, elle ne dit pas que le présage soit néfaste. Puisqu'il n'ose pas passer à l'exécution, il n'arrive pas encore jusqu'au malheur.

TSHOU HI. - Dureté énergique et absence de justice ; en haut pas de sympathie; il veut s'accorder avec le second trait et c'est contraire à la droiture ; il craint d'être $\mathrm{vu}$ et combattu par le cinquième trait, et c'est pourquoi il a cette image symbolique.

263.

Cacher des armes dans l'herbe épaisse, résister à l'énergie ${ }^{2}$; pendant trois ans ne pas commencer, agir avec calme.

\footnotetext{
${ }^{1}$ Littéralement « se lever ».

${ }^{2}$ Se poser en ennemi de... comme adversaire de...
} 
TSHENG TSE. - Ce à quoi il résiste, c'est le cinquième trait ; celui-là possédant l'éner gie, et d'ailleurs la droiture, est-il possible qu'il enlève par la force l'objet de ses désirs ? Aussi, il éprouve de la crainte et dissimule en se cachant ; il reste sans agir pendant un temps qui dure jusqu'à trois ans : à la fin comment pourrait-il agir ?

TSHOU HI. - Cela exprime qu'il ne peut agir ${ }^{1}$.

${ }^{1}$ Les deux commentateurs lisent «comment pourrait-il agir », là où nous traduisons, en suivant la forme grammaticale "agir avec calme». Pour que le caractère [], placé en tête d'une proposition prenne la valeur d'une exclamation dubitative, il faut qu'il soit suivi d'un mot exprimant lidée de « pouvoir, être capable de... » et que la proposition se termine par une particule interrogative. 


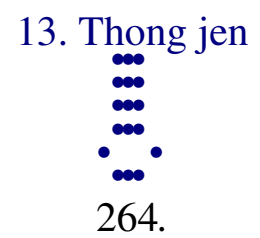

Quatrième trait nonaire : monter sur le mur ; ne pas pouvoir vaincre ;
présage heureux.

TSHENG TSE. - Le quatrième trait est énergique, mais sans justice ni droiture ; ses tendances le portent à se réunir au second trait et c'est aussi celui qui est ennemi du cinquième. Mur, talus ; ce qui sert à limiter et à séparer. Le quatrième trait est extrêmement voisin du cinquième, comme s'il n'en était séparé que par un mur ${ }^{1}$. Il monte sur le mur et veut combattre, mais il reconnaît que ce serait illégitime au point de vue du devoir, et il ne peut s'y résoudre. Puisque de lui-même il peut reconnaître que ce serait illégitime, et ne pas attaquer, cela constitue un présage heureux. S'il donnait libre cours à ses désirs coupables, sans pouvoir faire retour sur lui-même et penser au devoir et à la raison, agissant d'une façon coupable en attaquant et en enlevant de force, alors le présage malheureux serait très grave. Le troisième trait emploie la dureté énergique dans une position qui comporte l'énergie, de sorte qu'il pousse jusqu'à la fin l'exercice de la force, sans pouvoir revenir dans une autre voie ; le quatrième emploie la dureté énergique, mais il occupe un rang qui comporte la douceur malléable, de sorte qu'il exprime le sens d'éprouver des revers et de pouvoir revenir en arrière. Pouvant revenir dans une autre voie, le présage quil indique est heureux. Respecter le devoir et pouvoir se corriger c'est nécessairement un présage heureux.

TSHOU HI. - Dureté énergique sans justice ni droiture ; il est sans sympathies ni alliances, et cependant il veut se réunir au second trait hexaire, mais il en est séparé par le troisième, de sorte qu'il représente l'image symbolique de vouloir monter sur le mur pour enlever d'assaut. Mais, occupant un rang qui comporte la douceur, il a donc aussi l’image symbolique du retour sur soi-même, sans pouvoir se décider à livrer le combat ${ }^{2}$. Si celui qui consulte le sort est dans ces conditions, c'est qu'il pourra se corriger et atteindre au bonheur.

\footnotetext{
${ }^{1} \mathrm{Ou}$ « comme un mur de séparation ».

${ }^{2}$ Les deux commentateurs, comme Khong Tse, lisent les mots comme signifiant « ne pouvoir se décider à combattre ». Le sens naturel est « ne pas pouvoir vaincre le combat ».
} 
Monter sur le mur; le devoir ne le tolère pas ; le présage heureux, c'est de se repentir et de revenir aux principes ${ }^{1}$.

TSHENG TSE. - Ce qui fait qu'il monte sur le mur et ne se décide pas à attaquer, c'est que le devoir ne le souffre point : le devoir ne permet pas l'attaque du droit par l'injustice. Ce qui fait que le présage est heureux, c'est que le devoir ne souffrant pas un tel fait, ses souffrances prennent fin et il revient aux règles. Le second trait est celui auquel toutes les positivités veulent également se réunir et cependant le troisième et le quatrième traits seuls ont le sens de contester et d'enlever par la violence. Ces deux traits sont situés dans l'intervalle entre le second et le cinquième traits ; le premier et le dernier sont trop éloignés, de sorte qu'ils prennent une autre valeur.

TSHOU HI. - Il monte sur le mur ; donc ce n'est pas la force qui lui fait défaut ; c'est uniquement parce que le devoir ne le permet pas qu’il n'attaque point. Pouvant mettre fin au désordre par le devoir, et revenir aux principes réguliers, le présage sera donc heureux.

\footnotetext{
${ }^{1}$ Le caractère kho, antépénultième du texte, signifie : l'emporter sur... pouvoir ... être capable de : ce n'est que dans le commentaire de Khong Tse qu'on lui voit attribuer la valeur de souffrir quelque chose... se résigner à (jen ;... tolérer le mal ;...) cette valeur est arbitraire.
} 


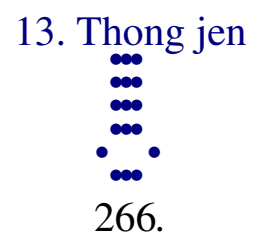

Cinquième trait nonaire : les mêmes hommes d'abord crient et se lamentent et ensuite ils rient; les grandes armées peuvent se rencontrer ensemble.

TSHENG TSE. - Le cinquième trait nonaire s'accorde avec le second et il en est séparé par les deux positivités du troisième et du quatrième trait. Le cinquième se domine par le devoir et la stricte raison, de sorte quil ne supporte pas la présomption insolente qui l'entrave, et cela à un point tel qu'il en crie et verse des larmes. Cependant, l’injustice ne l'emporte pas sur le droit ; bien quîl soit séparé du second trait, finalement, il doit néces sairement réussir à se joindre à lui, de sorte qu'ensuite il rit. "Les grandes armées peuvent se rencontrer ensemble»; le cinquième et le second traits se correspondent sympathiquement avec droiture, tandis que les deux positivités les séparent sans raison et les violentent; il faut donc absolument employer de grandes armées pour pouvoir les réduire, et alors le cinquième et le second peuvent se rencontrer ensemble ${ }^{1}$. Le texte parle de grandes armées; il parle de l'emporter ${ }^{2}$; cela fait voir la force et la violence des deux positivités. Le cinquième trait nonaire occupe la situation du prince et cependant la formule ne choisit pas le sens du prince se joignant aux hommes. En effet, le cinquième trait est exclusivement guidé par un sentiment personnel d'attachement et de sympathie pour le second, et il perd ses vertus de justice et de droiture. Le prince doit pratiquer la grande union avec tout l'univers ; un sentiment unique et personnel pour un seul individu n'est pas la voie morale du prince. De plus, d'abord séparé, il crie et se lamente ; ensuite, après la rencontre, il rit : c'est là un sentiment d'attachement égoiste ; ce ne sont pas là les caractères de la grande union. Le second trait qui est dans une position inférieure, comporte encore un signe d'appréhension à cause de « l'union avec la souche »; à plus forte raison en est-il de même lorsqu'il s'agit du prince ! $\mathrm{Du}$ moment où le cinquième trait ne comporte pas d'acception de la voie morale du prince, la formule n'en parle pas et éclaire seu lement le sens que deux hommes animés des mêmes sentiments ne peuvent pas rester séparés. Les «formules annexées» disent: La voie morale de l'homme doué lui prescrit, tantôt de sortir, tantôt de rester, tantôt de parler, tantôt de rester silencieux. Lorsque deux hommes sont animés des mêmes sentiments, la force

\footnotetext{
${ }^{1}$ Le commentateur lit : «La grande armée l'emporte, ils se rencontrent ensemble. » Ce n'est pas correct et c'est une lecture adoptée pour les besoins de l'explication choisie. Voir $n^{\circ} 267$.

${ }^{2}$ Ce mot kho a bien le sens de " pouvoir », et prend souvent la valeur de «pouvoir l'emporter malgré une résistance ». Tout le commentaire roule sur un contre-sens fait dans la lecture du texte.
} 
de leur action peut trancher le métal ; lorsque la justice et la droiture est ce qui les réunit (thong) ${ }^{1}$, qu'il s'agisse de sortir ou de rester, de parler ou de se taire, tout est identique (thong) en eux et l'univers ne sau rait les disjoindre et les séparer. Le terme thongexprime l'unité ou identité ; ce qui est un ne peut être divisé ; divisé ce serait la dualité. L'unité (union) peut pénétrer librement le métal et la pierre dure; elle peut traverser l'eau et le feu ; il n'est rien qu'elle ne puisse pénétrer. C'est pour cela que le texte dit que son acuité peut trancher le métal. La raison d'être des choses est ce qu’il y a de plus subtil, aussi l'homme saint s'exclame et dit

«L'expression : identité de coar, rappelle le parfum des fleurs les plus suaves. »Cela exprime que liidée et la portée de cette expression sont profondes et vastes.

TSHOU HI. - Le cinquième trait est dur et énergique, juste et droit ; le second.trait par sa douceur malléable, sa justice et sa droiture, lui correspond sympathiquement en bas. Ce sont ceux qui ont un «même (thong) cour ». Mais ils sont séparés par le troisième et le quatrième traits; ils ne peuvent réaliser leur union (thong). Cependant, le devoir et la raison sont ce qui les rend identiques (ou ce quils ont de commun) ; aucun être ne peut réussir à les séparer, et c'est pourquoi il y a ce sens symbolique ${ }^{2}$.

267.

Ce qui précède l'union des hommes, c'est l'emploi de la justice et de la rectitude; les grandes armées se rencontrent ensemble : cela exprime qu'elles peuvent vaincre (kho) ensemble ${ }^{3}$.

TSHENG TSE. - Ce qui fait que d'abord il crie et pleure, c'est la justice, la rectitude, la sincérité et la raison, c'est là ce qui fait qu'il ne peut surmonter son emportement et ce qui l'amène à ce résultat. Bien que l'adversaire soit énergique et fort, au point qu’il en arrive à l'em ploi des grandes armées, cependant, le devoir et la droite raison l'emportent ; à la fin il peut le vaincre, et c'est pourquoi le texte dit quils « peuvent vaincre ensemble». "Vaincre ensemble », veut dire l'emporter sur...; on voit par là la force des deux positivités !

\footnotetext{
1 Pour lire cette partie du commentaire, il faut restituer au mot thong son véritable sens : «identité ». C'est la meilleure réfutation possible du sens arbitraire donné à ce mot thong dans toute l'explication de ce koua.

2 Dans toute l'explication de ce koua, l'équivoque roule sur l'identité de deux choses, considérées séparément, et sur la réunion de deux choses considérées ensemble.

${ }^{3}$ Cette lecture de Khong Tse renverse tout l'échafaudage du contre-sens des commentaires du $\mathrm{n}^{\circ}$ 266. Du reste elle donne encore une lecture arbitraire du texte et n'est pas justifiable.
} 
TSHOU HI. — Rectitude, veut dire rectitude de la raison. 


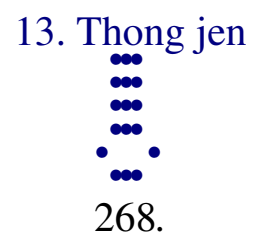

Trait supérieur nonaire : mêmes hommes dans la plaine ${ }^{1}$; pas de regrets.

TSHENG TSE. — La «plaine », un lieu extérieur (à l'état) et éloigné. Ceux qui recherchent l'union, ou accord (thong), doivent nécessairement se rapprocher mutuellement et s'allier ensemble. Le trait supérieur nonaire occupe l'extérieur et est sans correspondan ce sympathique ; c'est celui qui est indéfiniment sans union ni alliance. Lorsqu'il y a union au commencement, il peut arriver qu'à la fin il y ait regret, éloignement et désunion. Aussi, dans le cas actuel, bien qu'il n'y ait pas d'union, il n'y a cependant pas de regrets ; bien qu'il y ait tendance vers l'union, cette tendance ne peut se réaliser et, à la fin, il n'y a pas lieu d'en avoir d es regrets.

TSHOU HI. - Il occupe une position extérieure et est sans correspondance sympathique ; aucun être ne s'unit à lui ; mais cependant il peut être sans aucun regret, et c'est pourquoi tels sont l'image symbolique et le sens divinatoire. La «plaine » est en dedans du « désert » ${ }^{2}$; ce n'est pas encore le désert lointain et vaste, mais cependant c'est une région inculte et abandonnée, où il n'y a d'union possible avec personne.

269.

Union des hommes dans la plaine; tendances pas encore satisfaites.

TSHENG TSE. - Il demeure au loin et sans union, de sorte que finalement il n'a rien à regretter. Mais cependant, étant dans la voie de l'union des hommes, ses tendances qui lui font rechercher l'union ne peuvent pas être satisfaites et, quoique sans regrets, ce n'est pas une bonne situation.

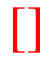

\footnotetext{
${ }^{1} \mathrm{Ou}$ « union des hommes dans la plaine ». D'ailleurs, le mot plaine est tout à fait arbitraire ; il s'agit d'une région, ou zone, entourant l'État à une distance plus ou moins rapprochée.

${ }^{2}$ Voir $n^{\circ} 253$.
} 


\section{Tae yeou}

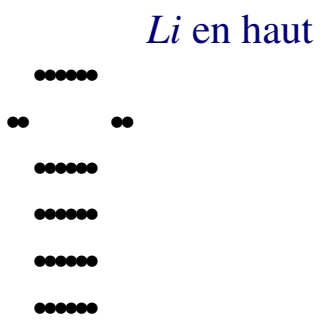

Khien en bas

270. Grand avoir, liberté absolue.

[]

274. Premier trait nonaire : ne pas joindre le malheur; absence de culpabilité ; dans la difficulté il n'y aura pas de culpabilité.

276. Deuxième trait nonaire : grand char pour porter ; il y a lieu d'entreprendre; pas de culpabilité.

$*$

278. Quatrième trait nonaire : ce n'e st pas l'ampleur ; pas de culpabilité.

$*$

280. Quatrième trait nonaire : ce n'est pas l'ampleur ; pas de culpabilité.

$*$

282. Cinquième trait hexaire : la bonne foi relie aussi ; elle en impose aussi ; présage heureux.

$*$

285. Trait supérieur nonaire : par le présage heureux de l'assistance du ciel, rien n'est sans avantage.

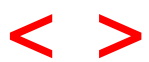


TSHENG TSE. - Tae yeou. «L'Ordre des koua » dit: «Les êtres doivent certainement faire retour vers celui qui s'unit aux hommes ; c'est pourquoi le koua thong jen est suivi du koua tae yeou. »Car celui qui s'unit aux hommes ${ }^{1}$ est celui vers qui les êtres font retour et c'est la raison pour laquelle le koua tae yeou suit immédiatement le koua thong jen. Il est composé du koua simple du feu placé au-dessus de celui du ciel. La position élevée du feu fait que sa clarté atteint au loin; dans la foule innombrable des êtres et des choses, il n'en est aucun qui n'en soit illuminé et qui ne le voie, ce qui constitue l'image symbolique du «grand avoir». Ou bien encore: L'unique trait malléable occupe le rang prééminent ; la foule des positivités lui correspond sympathiquement sans exception; or, occuper le rang prééminent et s'en tenir à la douceur, c'est ce qui ramène les êtres. Au -dessus et au-dessous tous les traits lui sont sympathiques, ce qui constitue le sens de «grand avoir». Le koua tae yeou exprime la perfection complète de la grandeur et la richesse de l'avoir.

\footnotetext{
${ }^{1}$ Je lis comme les commentateurs, mais le sens réel du texte serait « les êtres font retour vers
} ce qui est identique à l'homme ». 


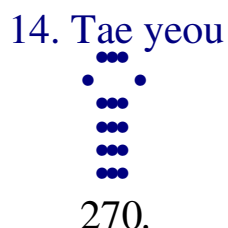

Grand avoir, liberté absolue.

TSHENG TSE. - Les qualités de ce koua permettent une liberté absolue. Toutes les fois que dans l'énoncé des vertus d'un koua ${ }^{1}$, il y a le nom de ce koua, il y a essentiellement le sens de ce nom. Par exemple «présage heureux de l'association $»^{2}$; «liberté de la modestie $»^{3}$. Il y a des cas où, à cause du sens du koua, son nom doit être considéré comme un enseignement et un avertissement. Par exemple : «Perfection du maître »; «présage heureux de l'homme au bâton »; «liberté de l'union des hommes dans le désert » ${ }^{4}$. D'autres fois c'est d'après les aptitudes des koua simples.qu'on l'explique ; c'est le cas de la formu le « liberté absolue du grand avoir ». C'est à cause de l'activité én ergique, des qualités brillantes de l'apparence, de la sympathie du ciel, de l'opportunité du moment d'action, qu'il peut y avoir une liberté absolue.

TSHOU HI. - Tae yeou, " grand avoir»; grandeur de ce qu'il y a. Le koua simple $l i$ se trouve au-dessus du koua simple khien : Le feu est au-dessus du ciel ; il n'est rien qu'il n'éclaire. De plus, le cinquième trait hexaire, unique négativité, occupe le rang prééminent et possède la justice, tandis que les cinq positivités lui sont sympathiques, ce qui fait que le koua est considéré comme exprimant la grandeur de l'avoir. Le koua simple khien exprime l'activité d'action ; le koua simple $l i$ exprime la clarté ; occuper le rang prééminent et être sympathique au ciel, c'est une voie qui comporte la liberté d'ac tion et d'extension. Si celui qui consulte le sort possède ces vertus, ce sera un grand bien et il jouira de sa liberté d'action.

271.

Le commentaire traditionnel de la formule déterminative dit: Grand avoir ; la douceur possède la situation prééminente et une grande justice,

\footnotetext{
${ }^{1}$ Dans la formule déterminative.

$2 \mathrm{~N}^{\circ} 172$. Cette prétendue règle ne résulte que rarement du sens des formules et le commentateur lui-même ne la suit pas dans cet exemple.

${ }^{3} \mathrm{~N}^{\circ} 288$.

${ }^{4} \mathrm{~N}^{\circ} 253$.
} 
tandis qu'en haut et en bas on lui répond sympathiquement : La formule dit : «grand avoir. »

TSHENG TSE. - Il s'agit de ce qui fait que le koua est considéré comme exprimant la grandeur de l'avoir. Le cinquième trait, avec sa nég ativité, occupe la situation du prince; donc la douceur possède la situation prééminente. Il est placé au milieu (d'un koua simple) ; donc il possède la voie de la grande justice. Il représente celui de qui descendent toutes les positivités ${ }^{1}$; donc en haut comme en bas on lui répond sympathiquement. Or, occuper le rang prééminent en s'attachant à la douceur, c'est certainement ce qui rallie la foule. Mais de plus encore il a les vertus, ou propriétés, du vide intérieur, de l'éclat, de l'apparence, et de 1 a grande justice, ce qui fait qu'en haut et en bas il y a identité de tendances sympathiques pour lui, et que le koua est considéré comme exprimant la grandeur de l'avoir.

TSHOU HI. - Explication des sens du nom du koua par la substance de ce koua. «Douceur malléable » désigne le cinquième trait hexaire ; « en haut et en bas » désigne les cinq positivités.

Ses vertus sont la force active dure et énergique et la clarté de l'apparence ${ }^{2}$; correspondance sympathique au ciel et action selon le moment ; c'est par là qu'il y a liberté absolue.

TSHENG TSE. - Les vertus des koua simples sont, pour celui de l'intérieur, la force acti ve dure et énergique, et pour celui de l'extérieur, la clarté de l'apparence. Le prince représenté par le cinquième trait hexaire correspond sympathiquement au second trait nonaire du koua simple khien; le naturel de ce cinquième trait est la douceur malléable, la soumission et la clarté (ou intelligence); il peut correspondre sympathiquement avec soumission au second trait. Or, le second trait est le maître du koua simple khien, celui de qui dépend l'activité ; donc il y a correspondance sympathique à l'activité. Soumission sympathique, activité dans l'action ; soumission au moment indiqué par le ciel, et c'est pourquoi la formule dit: «Correspondance sympathique au ciel et action selon le moment. » Telles étant les vertus exprimées, il en résulte donc une «absolue liberté ». Wang Pi dit : «Sans une grande liberté d'action, comment arriver à un grand avoir ? Avec un grand avoir il doit certainement y avoir une liberté absolue. »C'est là ne pas connaître le sens des koua; les koua simples $l i$ et khien, clarté et

\footnotetext{
${ }^{1}$ Puisque la positivité résulte de la négativité. Trait brisé.

${ }^{2}$ Cette expression désigne aussi l'él égance de la forme.
} 
activité, complètent le sens de « grand avoir»; cela n’indique pas que le sens du terme «grand avoir» doive nécessairement comporter celui de «liberté absolue ». Mais c'est par ses aptitudes, ou propriétés que le koua lui -même possède cette valeur de liberté absolue. Il peut fort bien y avoir des cas de « grand avoir» sans culture du bien, comme aussi des cas où il n'y ait pas de liberté d'action ou d'influence. Dans les divers cas qui réunissent les quatre mentions de grandeur, liberté, avantage et perfection, les formules déterminatives expliquent toujours qu'il s'agit de « grande liberté »; on peut même craindre qu'il faille douter qu'il devrait en être ainsi même dans les cas des koua khien et khouen. Lorsque les deux mots «avantage » et «perfection» n'entrent pas dans la formule, alors les commentaires considèrent les mots «grandeur» et «liberté » comme exprimant «liberté absolue », avec le sens d'absolue grandeur. Le terme yuan ${ }^{1}$, grandeur, a le sens de "grandeur dans le bien ». Ce n'est que dans les quatre koua tae yeou (grand avoir), hou (occupation), sheng (élévation), ting (trépied) qu'on trouve l'expression « liberté absolue »; or, ce n'est que dans le cas du seul koua sheng que la formule déterminative suit par erreur la règle des autres koua et considère ces mots comme si leur valeur était «grandeur et liberté ». On a dit : Comment se fait-il que le mot yuan n'ait pas dans tous les autres koua la même valeur que dans le cas du koua khien? Le mot yuan, grandeur, est considéré comme ayant le sens de «ce qui sort en précédant tous les êtres ». Dans les autres koua, il ne peut évidemment avoir ce sens, et il est considéré comme exprimant le bien, la grandeur, mais rien de plus. On a dit : Le mot yuan peut bien exprimer la grandeur, mais comment peut-il exprimer l'idée de bien ? Voici la réponse : Le mot yuan exprime ce qui est antérieur aux êtres ; comment ce qui précède les êtres ne serait-il pas le bien ? Lorsque le fait est accompli, la chose achevée, ensuite vient la corruption, ou ruine; la corruption ne précède pas l'accomplisse ment; la chose naît, se développe, et ensuite elle dépérit; le dépérissement est certainement postérieur au développement ; il faut posséder pour pouvoir perdre, si on ne possède pas, comment pourrait-il y avoir lieu de perdre ? Enfin, jusques au bien et au mal, à l'ordre et au désordre, au vrai et au faux, dans toute chose de ce monde, il n'en est jamais autrement ; il faut absolument que le bien précède et c'est pour cela que le commentaire de l'expression de la forme dit : Le mot yuan exprime le développement du bien.

TSHOU HI. - Explication de la formule du koua au moyen des vertus des koua simples et de la substance du koua parfait lui-même. «Correspondance sympathique du ciel » est dit par rapport au cinquième trait hexaire.

273.

\footnotetext{
${ }^{1}$ C'est le terme qui se lit, selon les commen taires, grand, ou absolu.
} 
Le commentaire traditionnel de la formule symbolique dit: Le feu est au-dessus du ciel : grand avoir ; l'homme doué agit pour supprimer le mal et étendre le bien ; il se soumet à la destinée bienfaisante du ciel.

TSHENG TSE. - Le feu est si haut qu'il est au-dessus du ciel; il illumine la foule innombrable des êtres et des choses ; c'est pourquoi le koua exprime la grandeur de l'avoir. "Grand avoir» a le sens de multiplicité et diversité. L’homme doué considère l'image symbolique de la grandeur de l'avoir et il en conclut l'obligation de couper court à tout ce qui const itue le mal et de développer, en le rendant clair et visible, tout ce qui constitue le bien, et cela pour se conformer avec soumission aux décrets admirables du ciel. Dans la foule innombrable des êtres, il y a évidemment des différences déterminées par le bien et par le mal; l'homme doué, profitant de la perfection florissante de la grandeur de l'avoir, doit suppléer à l'ouvre du ciel en régissant et en soignant tous les genres. Mais la voie rationnelle pour régir et gouverner la foule, consiste uniquement à supprimer le mal et à étendre le bien. Le mal est corrigé ; le bien est encouragé, et c'est par là qu'il suit avec soumission les décrets du ciel et qu'il assure la paix de tout ce qui vit.

TSHOU HI. — Le feu est au-dessus du ciel ; l'aire qu'il éclai re est vaste, ce qui est considéré comme constituant l'image symbolique du « grand avoir ». Du moment où ce qui constitue l'avoir est grand, s'il n'y a rien pour gouverner cet avoir l'animosité et le mal y germeront. Les décrets du ciel, ou destinée, comportent le bien et non le mal, de sorte que supprimer le mal et étendre le bien, c'est le moyen d'obéir au ciel. Si on reporte ces préceptes à sa propre personnalité il n'en sera encore pas autrement. 


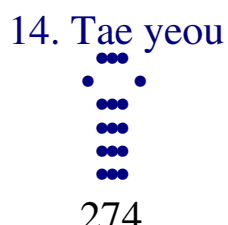

Premier trait nonaire : ne pas joindre le malheur ; absence de culpabilité ; dans la difficulté il n'y aura pas de culpabilité.

TSHENG TSE. - Un trait nonaire occupe le premier rang du koua tae yeou; il n'est pas encore parvenu à son parfait développement, il est placé dans une position humble et il est sans alliance sympathique ; il n'a pas encore les défauts que donne la vanité et la suffisance, de sorte qu’il ne joint pas le malheur, c'est-à-dire qu'il ne s'expose pas encore au mal. Dans la plupart des cas, il est rare que la richesse ne soit pas accompagnée de maux ; même avec la sagesse de Tse Kong, il est difficile de les éviter totalement; à plus forte raison lorsqu'il s'agit de quelqu'un qui est dans l'infériori té. «Ce n'est pas la culpabilité, mais difficile sera d'être sans culpabilité ${ }^{1}$; cela exprime que la richesse et l'avoir ne constituent pas essentiellement la culpabilité, mais que c'est l'homme lui -même qui en arrive, par la possession de la richesse, à commettre des fautes. S'il peut jouir des richesses tout en connaissant la difficulté à laquelle on est exposé par leur possession, il sera naturellement sans aucune culpabilité. Mais si, possédant les richesses, il est incapable de songer aux dangers de leur possession, et de les craindre, les sentiments d'orgueil et de prodigalité naîtront en lui, et c'est par là qu’il deviendra coupable.

TSHOU HI. - Bien que dans un moment de " grand avoir », cependant, occupant un rang inférieur avec les qualités de la positivité, n'étant attaché à personne au-dessus de lui par la sympathie, et se trouvant au début d'une chose, ce trait représente celui qui ne s'expose pas encore au mal. Quelle culpabilité pourrait-il avoir ? Cependant il faut encore quil use de précaution en se plaçant, et alors il sera sans culpabilité. C'est un avertisse ment à celui qui consulte le sort, qu'il doit se mettre dans ces mêmes conditions.

275.

Le premier trait nonaire du koua tae yeou ne joint pas le malheur.

\footnotetext{
${ }^{1}$ C'est une lecture différente du texte, mais elle n'est pas admissible.
} 
TSHENG TSE. — Étant au début du « grand avoir » et capable de méditer sur les dangers et les difficultés, il en résulte que chez lui les sentiments d'orgueil et de vanité n'ont pas de raison de naître, ce qui fait qu'il ne joint pas le malheur, en s'y exposant. 


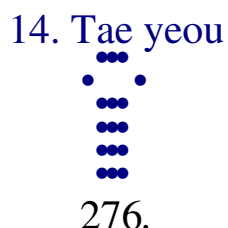

Deuxième trait nonaire : grand char pour porter ; il y a lieu d'entreprendre ; pas de culpabilité.

TSHENG TSE. - Un trait nonaire, avec la dureté énergique de la positivité, occupe le second rang ; c'est à lui que le prince, représenté par le cinquième trait hexaire, confie et délègue l'autorité. Doué de force active et d'énergie, ses capacités l'emportent ; occupant un rang qui comporte la douceur malléable il est modeste et soumis ; possédant la justice il ne commet point d'excès. Ses capacités étant telles, c'est ce qui le met à même de pou voir supporter le fardeau de l'autorité et le poids du « Grand Avoir », comme un grand char dont les matériaux sont forts et solides peut supporter et transporter de lourds fardeaux. Il est capable de supporter un poids considérable, d'aller au loin, de sorte qu'il a lieu d'entreprendre et sera sans culpabilité. Le koua tae yeou indique un moment d'expansion parfaite et florissante. L'avoir existe, mais n'est pas encore arrivé à son extrême limite, de sorte qu'avec les aptitudes du second trait il est possible d'entreprendre et de ne point commettre de fautes. Quand cet avoir est parvenu à la limite extrême de son parfait développement, alors il n'est plus possible d'en profiter pour entreprendre.

TSHOU HI. - Justice et énergie dans les rangs inférieurs, possession de sympathies d'un supérieur, cela constitue l'image symbolique d'un grand char servant à transporter des fardeaux. Si on a quelque chose à entreprendre et si on est dans ces mêmes conditions, il est possible de ne point commettre de fautes. Celui qui consulte le sort doit absolument posséder ces vertus et alors il répondra aux indications du sens divinatoire.

277.

Grand char pour transporter ; accumulation à l'intérieur sans le détruire.

TSHENG TSE. - Char grand et solide, accumulation de poids considérables à l'inté rieur sans qu'il soit abîmé ou détruit. Il en est encore de même de la puissance des forces et des aptitudes du second trait nonaire, pouvant supporter le poids du grand avoir. 


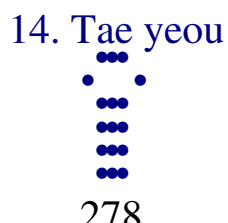

Troisième trait nonaire : le prince (revêtu du titre de Kong) pratique l'offrande au fils du ciel ; l'homme inférieur en est incapable 1.

TSHENG TSE. - Le troisième trait occupe le rang supérieur dans la substance du koua simple inférieur, il est dans un rang inférieur et est placé au-dessus des hommes : c'est

l'image symbolique des divers princes feudataires. Les dignitaires revêtus du titre honorifique de Kong obéissent au Fils du Ciel placé au-dessus d'eux ; le Fils du Ciel occupe le rang prééminent dans l'univers. Être à la tête d'un territoire limité, ce n'est pas autre chose que d'être sujet du roi. Comment celui qui occupe un rang inférieur oserait-il s'attribuer la possession exclusive de ce qu'il a ? Toutes les richesses du sol, la multitude de sa population, tout est l'avoir du roi, et c'est là la conséquence exacte de la raison d'être des choses. Aussi, le troisième trait, dans le moment exprimé par le koua tae yeou, occupe la situation du prince feudataire ; il en possède les richesses complètes et il doit absolument les employer en les faisant parvenir au Fils du Ciel. Tel est le devoir constant de l'homme considéré comme sujet. Si un homme inférieur occupe cette situation, il s'attribue la jouissance exclusive de son avoir et de sa richesse qu'il considère comme constituant son bien privé ; il ignore la voie du désintéressement dans le service du supérieur, et c'est pour cela que la formule dit que l'homme inférieur « en est incapable ».

TSHOU HI. - Le texte porte le mot heng, liberté ; le commentaire traditionnel du Tshouen tsieou porte le caractère heang qui signifie offrir en sacrifice, ou présenter en offrande avec vénération. Dans l'antiquité les deux caractères heng «librement, liberté », heang, "offrir à un supérieur», et peang, "cuire des aliments » s'écrivaient tous comme le premier caractère heng $^{2}$. Le troisième trait occupe le rang supérieur du koua simple inférieur; c'est le symbole des dignitaires revêtus du titre de Kong. Il est énergique et possède la droiture. En haut se trouve le prince, représenté par le cinquième

1 Tel paraît bien le sens de la formule. Cependant pour y arriver, il faut avec tous les commentateurs chinois admettre que le caractère heng (liberté) est là pour le caractère heang (offrir à un supérieur). Si on lit les caractères tels qu’ils existent dans le texte, la traduction deviendra : «Emploi désintéressé parvenant librement au Fils du Ciel. » Ce serait la lecture la plus correcte et on remarquera que le commentaire y revient à la fin.

2 Cette allégation mériterait confirmation ; il s'agirait au contraire de voir si ce n'est pas, comme dans le cas actuel, pour arriver à une lecture déterminée d'avance par la tradition qu'on a identifié ces trois caractères. Le cas serait loin d'être unique. 
trait hexaire, dépourvu de préjugés ${ }^{1}$, et qui s’incline devant la sagesse, de sorte qu'il représente l'image symbolique de l'idée d'offrande au Fils du Ciel. Si celui qui consulte le sort possède ces vertus, tel sera le sens divinatoire. L'homme inférieur est dépourvu des vertus, de l'éner gie et de la droiture, de sorte que quand bien même il rencontrerait cette formule, il ne pourrait pas s'en appliquer le sens.

279.

Emploi désintéressé parvenant librement au Fils du Ciel; l'homme inférieur nuit ${ }^{2}$.

TSHENG TSE. - Le dignitaire revêtu du titre de Kong ${ }^{3}$ doit pratiquer l'offrande au Fils du Ciel. Si un homme inférieur occupe sa position, ce sera nuisible. Dès l'antiquité, tous les divers princes feudataires capables d'observer les préceptes relatifs aux devoirs du sujet, et qui obéissaient avec fidélité et soumission au supérieur, se sont occupés de favoriser l'accroissement de la population, afin d'être à même de servir d'écran (de rempart) et de haie autour du roi, comme aussi de développer les sources de richesse, afin de subvenir aux tributs prélevés par leur supérieur. Lorsque des hommes inférieurs se trouvent investis de telles situations, ils sont incapables de connaître la voie loyale suivant laquelle le sujet sert le supérieur, parce qu'ils considèrent ces biens comme leur propriété privée. Si le peuple est nombreux, si les richesses abondent, ils ne les emploient que suivant leur propre gré et pour l'augmentation de leu r propre puissance et de leur richesse, comme pour aggraver de plus en plus leur insoumission. C'est ainsi que chez l'homme inférieur le « grand avoir » sera nuisible et, de plus, le grand avoir sera en même temps nuisible à l'homme inférieur lui -même.

\footnotetext{
1 Vide à l'intérieur.

2 Je traduis suivant le sens naturel des caractères du texte. Voir au n ${ }^{\circ} 277$ pour l'application du commentaire.

${ }^{3}$ Titre de noblesse dont le sens propre est l'antithèse du mot « égoïsme ».
} 


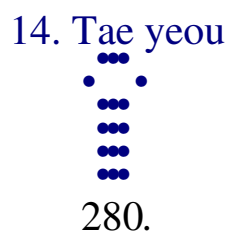

Quatrième trait nonaire : ce n'est pas l'ampleur ${ }^{1}$; pas de culpabilité.

TSHENG TSE. - Le quatrième trait nonaire se trouve dans le moment du « grand avoir »; il a déjà dépassé lé niveau moyen ; il exprime la perfection $\mathrm{du}$ «grand avoir». La perfection dépassée, il en résulte la naissance du présage malheureux. Aussi, la voie à suivre, pour quiconque s'y trouve placé, est qu'en méprisant cette perfection, il n'y aura pas de culpabil ité, c'est-à-dire que si on peut se restreindre avec modestie sans se placer au comble de la perfection dans sa position, on sera sans culpabilité. Le quatrième trait occupe une situation élevée auprès du prince; s'il s'attribue le mérite de la grande perfection, il en arrivera à la culpabilité et au malheur. Le terme du texte exprime l'apparen ce du complet développement de la grandeur; dans le Shi king il est dit : " la rivière Wen roule des flots tumultueux, les voyageurs sont extrêmement nombreux »; ici le même caractère exprime l'abondance. Dans un autre passage il est dit : «Les coursiers noirs et roux sont resplendissants », et ce même caractère y exprime la perfection des chevaux de guerre de Wou Wang.

TSHOU HI. - Le son et le sens du caractère pong, antépénultième du texte, ne sont pas bien connus. Tsheng dit «apparence de perfection »; cela peut être exact. Le cinquième trait hexaire est le prince doux et juste; le quatrième trait nonaire emploie la dureté énergique et s'approche de lui ; il a lajalousie qui naît de la tendance à outrepasser ses droits et à opprimer. Cependant, comme il occupe un rang qui comporte la douceur, il en résulte qu'il a l'image symbolique de ne pas pousser à leur extrême limite les avantages que donne la perfection et il réussit à être sans culpabilité. C'est un avertissement donné à celui qui consulte le sort, afin quill se mette dans ces mêmes conditions.

281.

Ce n'est pas l'ampleur; pas de culpabilité; distinguer clairement et éclaircir.

\footnotetext{
${ }^{1}$ Comme on le verra par les commentateurs le sens est très vague. Je prends le plus simple et
} le plus naturel, en dehors de toute influence traditionnelle. 
TSHENG TSE. - C'est celui qui peut ne pas s'attribuer le mérite du parfait développement de la perfection et qui réussit à être sans culpabilité ; en effet, il possède le jugement qui lui permet de distinguer clairement. Le caractère employé dans le texte indique la clarté de compréhension. Un homme sage et doué de jugement distingue clairement la raison d'être des choses; en présence du développement récent de la richesse, il sait reconnaître que les fautes et les erreurs sont sur le point d'être commises, aussi il doit se restreindre et n'ose pas pousser l'emploi des richesses à son extrême limite et jusqu'à la satiété.

TSHOU HI. - Si. Apparence de clarté de l’intelligence. 


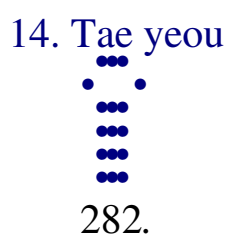

Cinquième trait hexaire : la bonne foi relie aussi ; elle en impose aussi ; présage heureux.

TSHENG TSE. - Le cinquième trait hexaire se trouve en présence d'un moment de grand avoir et occupe la situation du prince; il est sans préventions préconçues et juste ${ }^{1}$; il est considéré comme l'image symbolique de la bonne foi et de la confiance. Si le prince s'en tient à la douceur, observe la justice et emploie la confiance et la bonne foi dans les relations avec les inférieurs, il en résultera que les inférieurs épuiseront la sincérité et la confiance au service du supérieur; la bonne foi et la confiance du supérieur et des inférieurs les relient ensemble. Employant la douceur et occupant la situation prééminente, dans un moment de grand avoir, le coaur des hommes est en repos et tranquille ; mais sill s'attache exclusivement à la douceur et à la condescendance, le manque de considération et de respect pourra naître, de sorte qu’il faut absolument qu’il en impose aussi, et al ors le présage sera heureux. «En imposer aussi » exprime l'autorité et la sévérité. Du moment où il emploie la douceur et la concorde, la bonne foi et la confiance, dans ses relations avec les inférieurs, les tendances de la foule la portent à le suivre et à lui obéir avec joie ; de plus, il a une sévérité imposante pour qu'il y ait lieu de le craindre : c'est bien profiter des avantages possédés, et le présage heureux est évident.

TSHOU HI. - Dans les époques de grand avoir, être doux, condescendant et juste et porter ces qualités dans l'occupation de la situation prééminente, faire abstraction de soi-même par sympathie pour le sage, représenté par le second trait nonaire, de telle façon que les supérieurs et les inférieurs viennent se rallier, c'est là l' union par la bonne foi et la confiance. Cependant, dans la voie rationnelle du prince, l'énergie est précieuse ; trop de condescendance conduit à la négligence, et il convient d'y remédier par l'autorité ; alors le présage est heureux et c'est pourquoi tel s sont l'image symbolique et le sens divinatoire. C'est aussi une formule d'avertissement.

283.

\footnotetext{
${ }^{1}$ Évidé à l’intérieur et occupant le milieu d'un koua simple.
} 
Cette bonne foi relie aussi ; la confiance manifeste les tendances.

TSHOU HI. - La confiance d'un seul homme suffit à faire éclater les tendances du supérieur et des inférieurs.

284.

Présage heureux de l'autorité en imposant aussi; légèreté et pas de complément ${ }^{1}$.

TSHENG TSE. - La tendance de l'inférieur, c'est de suivre le supérieur en lui obéissant. Le supérieur employant la bonne foi et la confiance pour accueillir l'inférieur, il en résul te que celui-ci emploie la confiance et la sincérité en servant le supérieur. C'est pour cela que «la bonne foi relie aussi ». De ce que le supérieur possède la bonne foi et la confiance qui sert à faire manifester les tendances de l'inférieur à la bonne foi et à la confiance, il en résulte que l'obéissance de l'inférieur au supérieur en se conformant aux mêmes voies est comme la réponse de l'écho au son qui le frappe. L'expression « présage heureux de l'autorité en imposant aussi », exprime que sans l'imposante sévérité de l'autorité, l'infé rieur devient irrespectueux et manque de respect et de considération, et qu'alors l'aver tissement manque d'étendue et est incomplet. Cela veut dire que ce ne serait plus une voi e de respect, de crainte et de circonspection envers le supérieur. Le terme du texte pei, «complet», désigne la prévenance qui répond complètement aux réquisitions et aux réprimandes du supérieur.

TSHOU HI. - Par trop de douceur l'homme en arrivera à le traiter avec légèreté et son coar sera sans crainte ni complète circonspection.

\footnotetext{
${ }^{1}$ Le sens est incomplet, les commentateurs sont obligés de sous-entendre ce qui n'est pas
} dans le texte. La lecture la plus rationnelle serait « léger et incomplet ». 


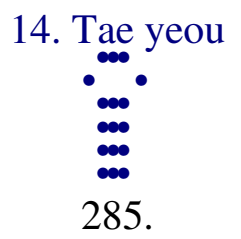

Trait supérieur nonaire : par le présage heureux de l'assistance du ciel, rien n'est sans avantage ${ }^{1 .}$

TSHENG TSE. - Le trait supérieur nonaire est à la fin du koua; il occupe un lieu qui n’indique aucune situation ; c'est celui qui est à la limite extrême du grand avoir et qui ne profite pas de cet avoir. Étant placé au rang supérieur du koua simple $l i$, il indique le comble de la clarté. C'est uniquement à cause de son extrême intelligence ${ }^{2}$ qu'il ne profi te pas de son avoir ; il ne pousse pas les choses jusqu'à l'excès. L'avoir étant extrême, et lui ne s'en prévalant pas, il s'en suit qu'il n'éprouve pas les calamités qui atteignent la suffisance et la présomption ; c'est celui qui peut se conformer à la raison d'être des choses. La bonne foi et la confiance du cinquième trait servant de point d'appui au trait supérieur donnent le sens de marcher dans le chemin de la sincérité et de la confiance. Le cinquième trait a les vertus (propriétés) de la clarté de l'apparence ${ }^{3}$; le trait supérieur est capable d'abaisser le niveau de ses tendances pour lui répondre sympathiquement, ce qui est considéré comme constituant le sens d'estimer la sagesse et d'exalter le bien. Être ainsi placé, c'est être parfaitement d'accord avec la voie morale. Naturellement il mérite de jouir du bonheur et de la félicité : « Naturellement le ciel l'assiste ${ }^{4}$ : Il agit en se soumettant au ciel et il jouit de l'assistance du ciel, de sorte que quoi qu'il entreprenne, le présage est heureux et rien ne peut être sans avantage.

TSHOU HI. - Époque de « grand avoir »; emploi de la dureté énergique pour occuper le rang supérieur, tout en pouvant suivre, en bas, le cinquième trait hexaire ; c'est savoir marcher dans la voie de la confiance, réfléchir avec condescendance, et estimer la sagesse, être comblé des dons de la fortune et ne point s'en prévaloir présomptueusement. C'est pourquoi tel est le sens divinatoire.

286.

\footnotetext{
${ }^{1}$ Plus littéralement encore : «Depuis le présage heureux de... »

${ }^{2}$ Clarté, ou intelligence.

3 Élégance de la forme.

4 Autre lecture du texte : « naturellement le ciel l'assiste ; présage heureux. »
} 

ciel.

Présage heureux du trait supérieur du grand avoir; par l'assistance du

TSHENG TSE. - Rang supérieur dans le koua exprimant le grand avoir ; ce qui est parvenu à la limite extrême du développement doit se modifier. C'est parce que ce quil fait est conforme à la loi du ciel et d'accord avec la voie rationnelle que le ciel l'assi ste dans ce qu'il fait, et c'est par là qu'il y a présage heureux. L'homme doué, bien que comblé, ne devient pas présomptueux : C'est là l'assistance du ciel. Les « Formules annexées » revenant sur ce point disent : "Ce que le ciel aide, c'est la soumission à sa loi ; l'assistance que l'homme prête, c'est la confiance. » Il règle ses actions par la confiance ; il médite sur la soumission, et de plus il estime la sagesse. C'est à cause de cela que le ciel l'assiste, que le présage est heureux, et que rien n'est sans avantage. Régler ses actions par la confiance, c'est-à-dire s'appuyer sur le troisième trait nonaire. Le cinquième trait est sans préventions préconçues, ce qui indique la bonne foi et la confiance. Méditer sur la soumission, c'est se retirer avec modestie et ne pas s'attribuer le mérite ou le bien acquis. Estimer la sagesse ; c'est-à-dire que ses tendances le portent à obéir au cinquième trait et à suivre ses avis. Dans les époques de grand avoir, il ne faut pas se prévaloir de l'abondance et de la richesse et s'en glorifier vainement ; cela ne convient pas. Entre ces six traits, le plus grand nombre se réjouit d'affermir une situation puissante ; le premier trait et le trait supérieur seuls ne se prévalent pas de leur situation, c'est pourquoi le premier trait nonaire est sans culpabilité, et le trait supérieur nonaire jamais sans avantage. Le trait supérieur nonaire occupe le rang le plus élevé; il règle ses actions par la confiance et n'oublie pas la soumis sion ; c'est pour cela que, bien qu 'au point le plus élevé, il jouit du bonheur, car le ciel l'assiste. 


\section{Khien}

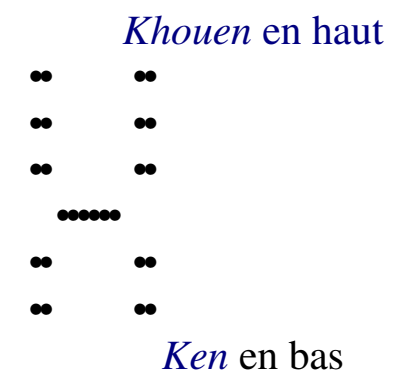

287. Khien, modestie ; liberté ; l'homme doué a une fin.

291. Premier trait hexaire : modeste! modeste! l'homme doué ! l'employer pour traverser un grand cours d'eau ; présage heureux.

$*$

293. Deuxième trait hexaire : modestie renommée; présage heureux de la perfection.

$*$

295. Troisième trait nonaire : l'homme doué méritant et modeste ; il a une fin heureuse.

$*$

297. Quatrième trait hexaire : rien sans avantage ; montrer la modestie.

$*$

299. Cinquième trait hexaire : sans richesse employer le voisinage; avantage à envahir et à réduire par les armes; rien sans avantage.

$*$

301. Trait supérieur hexaire : modestie renommée ; avantage à employer l'action des armées; vaincre le district et les royaumes.

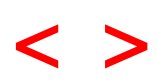


15. Khien.

TSHENG TSE. - nien. «L'Ordre des koua » dit : «Lorsque l'avoir est grand », ce ne doit pas être une cause de suffisance et c'est pourquoi « le koua tae yeoic», grand avoir, «est suivi du koua khien», «modestie ». Quand l'avoir est grand, il ne faut pas en arriver à la suffisance et à l'orgueil ; il faut absolument rester modeste et se restreindre, de sorte qu'après le koua du « grand avoir », vient immédiatement celui de la «modestie ». Il est composé par le koua simple khouen au-dessus et le koua simple ken au-dessous : Dans la terre il y a des montagnes. L'essence de la terre c'est l'humilité et l'infériorité ; la montagne est une chose haute et grande ; or, puisqu'elle est placée sous la terre ${ }^{1}$, c'est l'image symbolique de la modestie. Pratiquer les vertus nobles et élevées et se placer au-dessous des humbles, donne bien le sens de «modestie $»^{2}$.

${ }^{1}$ Khouen, la terre, et ken, la montagne

2 Plus littéralement : «c'est le sens du koua khien. » 


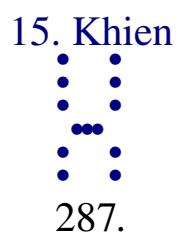

Khien, modestie ; liberté ; l' homme doué a une fin.

TSHENG TSE. - La modestie comporte une voie naturelle de liberté ; posséder une vertu et ne pas s'en prévaloir, c'est ce qu'on exprime par le mot khien, modestie. Lorsque l'homme se place en pratiquant la modestie et l'humilité, que pourrait-il entreprendre qui ne lui présentât pas une voie de liberté ? «L'homme doué a une fin »; les tendances

de l'homme doué le maintiennent dans la modestie et l'humilité ; il pénètre la raison d'être des choses, aussi il met son bonheur dans la loi du ciel et ne se met pas en opposition avec cette loi. Ses qualités intérieures sont solides, de sorte quil se retire modeste ment en laissant les autres se mettre en avant, et ne se vante pas; il se contente de suivre sa voie avec modestie, et jusqu'à la fin de sa vie il ne change pas. Celui qui s'humilie est vanté et exalté par les hommes ; il se place lui-même dans l'ombre et sa vertu n'en devient que plus visible et éclatante : voilà pourquoi la formule dit que l'homme doué a une fin ${ }^{1}$. S'il s'agissait d'un homme inférieur, éprouvant un désir il chercherait à le satisfaire par tous les moyens ; possédant une vertu, il s'en prévaudrait en s'en vantant ; bien que quelquefois il puisse s'appliquer avec efforts à la modestie, il ne pourrait cependant pas se contenter de la pratiquer et de la conserver avec fermeté : Il ne pourrait pas « avoir une fin ».

TSHOU HI. - Khien exprime le sens d'avoir et de ne pas s'en prévaloir. Arrêt au-dedans ${ }^{2}$ et soumission au-dehors ${ }^{3}$ : Représentation de lidée de modestie. La montagne est ce qu'il y a de plus haut et la terre est ce qu'il y a de plus bas : C'est donc que le koua comporte l'idée de se courber et de rester dans l'abaissement : Image symbolique de la modestie. Si celui qui consulte le sort est dans ces conditions, il jouira de liberté d'action et aura une fin. «Avoir une fin » veut dire se courber d'abord et se redresser ensuite.

288.

\footnotetext{
${ }^{1}$ Expression qui dans le Yi king est souvent interprétée comme indiquant qu'une certaine condition est remplie jusqu’à la fin.

${ }^{2}$ Ken, arrêt.

${ }^{3}$ Khouen, soumission ; se contenir et se soumettre.
} 
Le commentaire traditionnel de la formule déterminative dit: Modestie; liberté ; la voie morale du ciel descend assister et elle éclate et brille ; la voie naturelle de la terre est inférieure et monte pour agir ${ }^{1}$.

TSHENG TSE. - Le caractère $t s i$ du texte, traverser, aider, doit être remplacé par le caractère $t s i$, réunir, joindre. Cette formule éclaire le sens de «modestie jointe à la possibilité de liberté », liberté résultant de la modestie. La voie du ciel, par son éther ${ }^{2}$, descend et se communique en bas, de sorte qu'elle peut transformer et e ngendrer tous les êtres. Cette voie est éclatante et brillante. «Descendre se joindre », c'est-à-dire descendre pour s'unir. La voie de la terre étant placée au-dessous, il en résulte que son éther monte agir en s'unissant au ciel. Pour toutes deux, c'est par la condition, d'être inférieure ou de descendre qu'il y a liberté ${ }^{3}$ (d'union et de production d'un résultat).

TSHOU HI. - Il s'agit de la liberté qu'entraîne nécessairement la modestie.

289.

La voie du ciel est l'extinction de la vanité ${ }^{4}$ et l'augmentation de la modestie ; la voie de la terre est la modification de la vanité et l'entraînement de la modestie dans le courant d'action; les génies et les esprits nuisent à ce qui est plein (suffisance) et favorisent ce qui est modeste; la voie de l'homme est la haine de ce qui est plein (orgueil, suffisance) et l'estime de la modestie. La modestie est élevée et éclatante; elle est humble et ne peut être dépassée, c'est la fin de l'homme doué.

TSHENG TSE. - Il s'agit de l'action du ciel ${ }^{5}$; ce qui est plein ${ }^{6}$ s'annihilera ; ce qui est modeste augmentera. C'est par exemple le cas du

\footnotetext{
${ }^{1}$ Le terme du texte est tao, voie, voie morale, rationnelle, naturelle, etc., selon le sens de chaque passage.

2 Essence constitutive.

3 Même en tenant compte des idées développées par les commentateurs chinois, il est impossible d'admettre qu'eux -mêmes aient compris ce qu'ils s'efforçaient d'expliquer. Mais si on applique aux termes « voie du ciel», « voie de la terre », la valeur astronomique que je leur reconnais, il devient facile de comprendre ce qu'il y a de réel sous cette logomachie.

${ }^{4}$ Ce qui est plein.

${ }^{5}$ Litt : « de la marche du ciel. »

${ }^{6}$ Au figuré ce terme désigne la vanité, la suffisance, la satisfaction ; le même terme désigne la lune dans son plein.
} 
soleil et de la lune, de la négativité et de la positivité. Si on en parle au point de vue de la force inhérente à la terre, ce qui est plein et complet s’incline, décline, se modifie et retombe en sens inverse; ce qui est humble et bas s'étend, déborde se répand, et s'accroît. «Génies et esprits » signifie «les traces visibles de la création et de la transformation ». Leur action nuit et altère ce qui est plein et complet; elle favorise et aide ce qui est humble et diminué. Toutes les fois que l'excès conduit à la diminution, que ce qui faisait défaut augmente, c'est l'application de ce prin cipe. Les sentiments de l'homme supportent impatiemment et réprouvent la suffisan ce pleine de soimême ; ils sont portés à l'amour de l'humilité et de la modestie. La modestie est la plus parfaite vertu de l'homme, aussi l'homme saint 1 s'explique clairement, pour avertir l'orgueil et encourager la modestie. La modestie est l'humilité et l'abaissement, et cependant sa voie est éminente et grande, éclatante et visible. Bien qu'elle se place soi-même en s'abaissant et en se déprimant, cependant sa vertu est réellement si haute que rien ne peut y être ajouté pour l'augmenter ; c'est là ne pouvoir être dépassée. L'homme doué est au plus haut point sincère dans sa modestie ; cette modestie est constante et ne s'altère point ; elle est définitive, c'est pourquoi elle est éminente et éclatante.

TSHOU HI. — «Modifier» signifie décliner et détériorer ; « entraînement » veut dire réunion en ramenant vers... Lorsque l'homme peut être modeste, si son rang est éminent, sa vertu éclate davantage ; si son rang est inférieur, personne ne peut encore l'y dépasser. C'est par là que l'homme doué «a une fin ».

290.

Le commentaire traditionnel de la formule symbolique dit: Au milieu de la terre il y a des montagnes modestie; l'homme doué tend à diminuer ce qui est nombreux et à augmenter ce qui est rare; il apprécie les êtres et leur étend équitablement son action.

TSHENG TSE. - L'essence de la terre, c'est l'infériorité et l'abaissement ; la montagne est haute et grande et elle se trouve au milieu de la terre : C'est l'image symbolique de l'humilité et de l'abaissement extérieurs et de l'élévation et de la grandeur concélées

au-dedans. C'est pour cela que le koua est considéré comme exprimant la modestie. La formule ne dit pas «La montagne est au milieu de la terre » mais elle dit : «Au milieu de la terre il y a des montagnes »; cela indique que dans

\footnotetext{
${ }^{1}$ Khong Tse, dans ce cas.
} 
l'humilité et l'abaissement se trouvent concélées l'élévation et la gloire. Si elle disait que la gloire et l'élévation sont concélées dans l'humilité et l'abaissement, le style serait défectueux. Il en est partout ainsi dans les formules symboliques, et cela est visible en faisant attention au style. L'homme doué tend à diminuer ce qui est nombreux et à augmenter ce qui est rare ; il apprécie les êtres à leur juste valeur et leur étend équitablement son action. L’homme doué observe l'image sym bolique du koua khien; une montagne qui se trouve au-dessous de la terre, c'est-à-dire que ce qui est haut est abaissé et que ce qui est bas est élevé ; dans cette image il voit le sens de courber ce qui est élevé, en l'abaissant, et d'élever ce qui est bas, de d iminuer ce qui est en excès et d'augmenter ce qui est insuffisant. En étendant ce principe aux choses, il prend et enlève là où il y a beaucoup et il augmente ce qui est peu abondant. Il apprécie le plus ou le moins dans les choses afin que son action soit équitablement répartie et pour atteindre à l'égalité.

TSHOU HI. - Employer l'humilité pour cacher l'élévation, c'est l’image symbolique de la modestie. Diminuer ce qui est abondant et augmenter ce qui est rare, c'est le moyen par lequel il apprécie les be soins des êtres et leurs convenances et par lequel il égalise équitablement son action sur tous. Il diminue ce qui est élevé et augmente ce qui est humble, pour tendre vers l'égalité, et c'est là encore l'idée de modestie exprimée par le mot khien. 


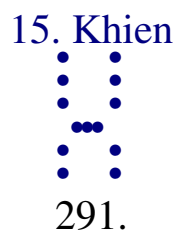

Premier trait hexaire : modeste! modeste! l'homme doué! l'employer pour traverser un grand cours d'eau; présage heureux.

TSHENG TSE. - Le premier trait hexaire emploie la douceur malléable et la soumission, et il se place avec modestie. De plus, il occupe le rang inférieur d'un koua simple, ce qui constitue l'extrême degré dans l'action de se placer avec humilité dans une position inférieure. C'est la modestie dans la modestie, et c'est pourquoi la formule dit: «Modeste ! modeste !» Celui qui peut agir ainsi, c'est l'homme doué. Se plaçant avec la plus extrê me modestie, c'est ce qui attire les sympathies universelles de la foule. Bien quill agisse en s'exposant aux difficultés et aux dangers, il n'en éprouve cependant aucun mal ou dommage, à plus forte raison lorsqu'il se trouve dans des circonstances faciles et ordinaires; en quoi le présage ne serait-il pas heureux ? Le premier trait se place avec modestie et il emploie sa douceur à se maintenir dans l'infériorité ; n'est-ce pas là un excès de modestie ? Il faut répondre: La douceur se maintenant dans linnfériorité, c'est là le cours ordi naire des choses ; mais il faut uniquement regarder qu'il s'agit de l'extrême modestie, ce qui constitue la modestie dans la modestie, et qu'on ne voit encore rien qui pourrait y constituer un inconvénient.

TSHOU HI. - Employer la douceur et se placer dans un rang inférieur, c'est l'extrême degré de la modestie ; c'est la manière d'agir de l'homme doué. En employant cette voie pour traverser un danger, que pourrait-on entreprendre qui ne puisse être accompli ? Aussi, si celui qui consulte le sort est dans ces conditions, il aura avantage à s'exposer à tra verser un grand cours d'eau ${ }^{1}$.

292.

Modeste! modeste! l'homme doué ; il s'abaisse pour se diriger.

${ }^{1}$ À ne pas craindre de s'exposer dans une entreprise dangereuse. 
TSHENG TSE. — «Modeste ! modeste !» extrême degré de la modestie. Cette formule exprime que l'homme doué emploie la voie de la modestie et de l'humilité et se place en conséquence ${ }^{1}$. Les termes du texte tse mou veulent dire «se placer » ${ }^{2}$. Le Shi king dit: Tse mou houei thi : Revenir de soi-même dans l'herbe jaune et fraîche ${ }^{3}$.

${ }^{1}$ Le commentateur lit « se place » là où je traduis « se dirige ».

${ }^{2}$ Le sens du caractère mou est «pasteur» ou «pascere». Tse mou, se conduire soi-même, être son propre pasteur.

${ }^{3}$ Dans cet exemple la traduction littérale est « il se conduit lui-même à revenir ». 


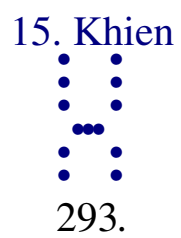

Deuxième trait hexaire : modestie renommée ; présage heureux de la
perfection.

TSHENG TSE. — Le deuxième trait emploie la douceur et la soumission et se maintient dans la justice ; ceci est considéré comme exprimant que la vertu de la modestie s'accroît en lui. La vertu de la modestie le remplit complètement, de sorte qu'elle paraît à l'inté rieur, et qu'elle se manifeste dans son accent et ses paroles comme dans son apparence. C'est pour cela que la formule dit : «modestie renommée ». Occuper le milieu dans un koua simple et posséder la vertu qui correspond exactement à ce rang, c'est posséder les vertus de la justice et de la droiture. C'est pour cela que la formule dit : «présage heureux de la perfection». Lorsqu'une formule porte les deux termes «perfection» et «présage heureux», il y a des cas où ils sont considérés comme exprimant qu'il y a perfection et, d'ailleurs, présage heureux ; il y en a où ils sont considérés comme exprimant que, si on possède la perfection, ce sera un présage heureux. Dans le cas du second trait hexaire, ces deux termes expriment ce qu'il possède essentiellement par lui-même.

TSHOU HI. - Douceur, soumission, justice et droiture : réputation à cause de la modestie ; droiture et d'ailleurs présage heureux. C'est pourquoi tels sont l’image symbo lique et le sens divinatoire.

294.

Modestie renommée; présage heureux de la perfection; le cceur juste le possède 1 .

TSHENG TSE. - La vertu de modestie du second trait provient de l'extrême sincérité accumulée en lui, et par laquelle elle se manifeste dans ses accents et ses paroles. C'est ce qu'un c au r juste possède naturellement et ce n'est pas le résultat d'un effort.

\footnotetext{
${ }^{1}$ Les commentateurs chinois lisent «possédée dans le cour ». Cette lecture est contraire au génie du style chinois.
} 


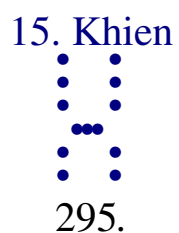

Troisième trait nonaire : l'homme doué méritant et modeste; il a une fin heureuse.

TSHENG TSE. - Le troisième trait emploie les vertus de l'énergie positive et fait partie de la substance du koua simple inférieur ; c'est à lui que la foule des négativités se rattache, et sa démarche est digne de la situation qu'il occupe. Il est le supérieur entre les inférieurs, c'est donc lui qui, d'en haut, est investi de l'autorité par le prince et qui, d'en bas, est suiv i par la foule : c'est celui qui a du mérite par ses efforts et qui s'attache à la vertu de la modestie ; c'est pour cela que la formule dit : «méritant et modeste ». Parmi les anciens, quelques hommes atteignirent à ce niveau : par exemple Tsheou Kong. Personnellement il était à la hauteur des plus hautes charges de l'empire ; il servait un maître jeune et faible ; modeste et digne, sachant se diriger, circonspect et prudent comme maintenu en éveil par une appréhension permanente, on pouvait dire qu'il avait du mérite et savait être modeste. Pouvant être méritant et modeste, il faut encore que l'homme doué pratique cette voie jusqu'à la fin, et alors le présage est heureux. Or, se complaire dans l'élévation, se réjouir du succès, c'est là le sentiment or dinaire chez les hommes ; ceux qui peuvent être modestes dans les circonstances ordinaires et s'affermir dans cette voie sont rares ; combien, à plus forte raison, ceux qui ont pu s'élever à un rang éminent par leur mérite et leurs efforts ! En supposant même qu'ils apprécient la beauté de la modestie, qu'ils s'efforcent et la pratiquent, tant que le principe inné de la vanité et de la présomption n'est pas annihilé en eux, cette modestie factice ne peut être permanente et durable; ils voudraient qu'elle fût définitive et ne peuvent y parvenir. L'homme doué seul se contente de marcher avec modestie et condescendance ; c'est là sa conduite constante ; aussi, chez lui, cet état peut durer sans se modifier, et c'est là ce qu'on appelle « avoir une fin ${ }^{1}$; ayant une fin, c'est un présage heureux. Le troisième trait nonaire emploie la dureté énergique et se maintient dans la droiture; c'est celui qui peut persévérer indéfiniment. Les vertus de ce trait sont parfaitement épanouies, aussi la formule symbolique appuie spécialement.

TSHOU HI. - Dans le koua il n'y a qu'une seule positivité, qui occupe le rang supérieur du koua simple inférieur. Dure, énergique et possédant la droiture, c'est vers elle que ten dent les traits supérieurs et inférieurs. Elle a du

${ }^{1}$ Être définitif. 
mérite par ses efforts et son zèle et elle peut être modeste ; c'est ce qu'il y a de plus difficile pour l'homme, et c'est pour cela que si cette manière d'être est définitive le présage est heureux. Si celui qui consulte le sort est dans ces conditions, l'a pplication de ces indications est acquise.

296.

L'homme doué méritant et modeste; les peuples innombrables se soumettent.

TSHENG TSE. - L'homme doué capable d'efforts et de modestie est celui que la foule des peuples exalte et à qui elle se soumet. Les «formules annexées » disent : «Être capable d'efforts et ne pas s'en vanter, avoir du mérite et ne pas s'en faire une vertu, c'est le comble de la grandeur d'âme »; cela est dit au sujet de celui qui, nonobstant son mérite, s'abaisse devant les hommes. Dans cette citation le terme vertu (s'en faire une vertu) exprime l'idée de s'exalter. Le $\mathrm{Li} k i$ parle de la dignité et de la modestie et dit que celle-ci conduit à la dignité dans l'obser-vation des devoirs de la situation occupée. Avoir du zèle et ne pas s'en prévaloir et s'en vanter, avoir du mérite et ne pas s'en faire une vertu, c'est l'extrême développement de la générosité et de la grandeur de la vertu. Il s'agit, ayant du mérite par le zèle et la grandeur des efforts tentés, de se montrer modeste en s'abaissant soi-même devant les hommes. Dans la vertu on appelle cette qualité la perfection. Dans les actions extérieures on l'appelle dignité. Si on en parle au point de vue de la vertu, c'est son extrême épanouissement ; si on en parle au point de vue des règles rituelles qu'on doit observer en se plaçant, ce sera l'extrême dignité dans la contenance; c'est là ce qu'on appelle la modestie. Mais cette modestie, on dit que c'est elle qui conduit à la digni té dans la contenance pour observer les devoirs de la situation occupée. Observer, c'est maintenir en pratiquant. Produisant la dignité et l'humilité pour observer les devoirs de la situation, il en résulte que malgré l'élévation il n'y a point de péril, que le coa $r$ est plein de cette vertu sans qu'el le dégénère en vanité, et c'est par là que le présage peut être définitivement heureux. Mais si l'homme doué pratique la modestie, c'est parce que telle est sa manière d'être constante ; ce n'est point pour garantir sa situation qu'il agit ainsi ; cependant il est question de conserver une situation (observer les devoirs de la situation); en effet, pouvoir atteindre à la dignité, c'est ce qui permet de conserver la situation et cela exprime que telle est la voie rationnelle de la modestie. Si on disait qu'en faisant le bien on acquiert la renommée, comment supposerait-on que l'homme doit faire le bien pour acquérir la renommée ! Ce serait comme si on disait que la renommée est le mobile qui fait faire le bien. 


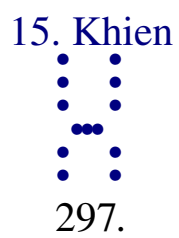

Quatrième trait hexaire : rien sans avantage; montrer la modestie.

TSHENG TSE. - Le quatrième trait fait partie de la substance du koua simple supérieur; il est très rapproché de la situation du prince. Le prince représenté par le cinquième trait hexaire emploie aussi la douceur et la modestie pour se diriger et se placer. Le troisième trait nonaire possède un grand mérite et la vertu ; c'est lui que le supérieur investit de l'autorité ; c'est à lui que la foule se rattache; or, le quatrième se trouvant au-dessus de ce dernier, il convient qu'il soit digne et attentif en servant le prince modeste et vertueux et, en même temps, humble et sans prétention, afin de céder le pas au sujet modeste et actif (représenté par le troisième). S’il se meut, agit, ou fait faire, rien ne sera sans utilité pour montrer et manifester sa propre modestie. Le terme hoei, avant-dernier caractère du texte, exprime l'image d'étendre à quelque chose, comme lorsque l'homme montre ave c la main. Qu'il se meuve ou reste en repos, quil avance ou recule, il doit absolument manifester ${ }^{1}$ sa modestie. En effet, c'est qu'il occupe une position où il a beaucoup à craindre et où, de plus, il se trouve au-dessus d'un sage sujet.

TSHOU HI. - Douceur et possession de la droiture ; élevé et capable de s'abaisser ; le sens divinatoire est que rien n'est sans avantage. Cependant, placé au-dessus du troisième trait nonaire, il était nécessaire que la formule avertît qu'il doit d'autant plus manifester et montrer sa modestie, afin d'enseigner l'idée qu'il ne faut pas oser compter sur soi -même.

298.

Rien sans avantage; montrer ta modestie; ne pas enfreindre les préceptes.

TSHENG TSE. - La modestie, chez chaque homme, doit s'étendre jusqu'à une cer taine limite, et cette limite ne doit pas être dépassée. Ainsi, par

\footnotetext{
${ }^{1}$ En admettant que le mot hoei ait le sens de «montrer, désigner avec le doigt », ce qui est douteux, la formule n'aurait pas encore le sens que lui prête le commentateur.
} 
exemple, le cinquième trait hexaire agit parfois en envahissant et en réduisant par les armes. Pour le quatrième trait, seul, comme il est placé sur un terrain proche du souverain, tenant compte de ce qu'il est au-dessus d'un sujet méritant, il en résulte que quoi quil fasse ou de quelque manière quil se meuve, il n'est jamais sans avantage pour lui de manifester sa modestie. Du moment où il agira ainsi, il sera d'accord avec les règles et les principes, et c'est pourquoi la formule dit : «Ne pas enfreindre les principes »; cela exprime qu'il atteint la mesure convenable.

TSHOU HI. - Cela exprime qu'il ne saurait être trop modeste. 


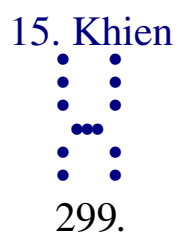

Cinquième trait hexaire : sans richesse employer le voisinage; avantage à envahir et à réduire par les armes; rien sans avantage.

TSHENG TSE. - La richesse est ce qui attire la foule; les richesses seules sont susceptibles de rassembler les hommes. Le cinquième trait, avec l'éminence de la situation du prince, et s'en tenant à la modestie et à la condescendance dans ses relations avec les inférieurs, c'est à lui que la foule se rallie. Aussi, bien que sans richesse, il peut être entouré et disposer de son voisinage. Voisinage, c'est -à-dire ce qui est proche ; bien que sans richesse il jouit du voisinage des hommes qui s'approchent de lui. Être le prince et s'attacher à la modestie et à la condescendance, c'est être celui qui ra llie tous les cou rs dans l'univers. Mais, cependant, la voie rationnelle ne comporte pas exclusivement la modestie et la douceur; il faut absolument que l'autorité et la puissance militaire viennent se joindre à ces premières qualités et ce n'est qu'ensu ite qu'il est possible d'embrasser et de soumettre tout l'univers. C'est pour cela qu'il y a avantage à pratiquer l'envahissement et à réduire par la force des armes. L'autorité et la vertu également manifestées, toutes les conditions de la voie du prince sont remplies, et rien n'est sans avantage. En effet, la modestie et la douceur du cinquième trait doivent être en garde contre l'excès de leurs avantages, et c'est pour cela que la formule développe ce sens.

TSHOU HI. - C'est celui qui emploie la douceur pour occuper un rang éminent, qui est élevé et peut être modeste ; c'est pour cela qu'il est considéré comme le symbole de celui qui est sans richesses et qui peut employer ${ }^{1}$ son voisinage. En effet, ceux qui le suivent et lui obéissent sont la foule ; s'il reste encore des insoumis, il aura avantage à les réduire par la force des armes et, même dans les autres affaires, rien ne sera sans avantage. Si quelqu'un possède ces vertus le sens divinatoire lui sera applicable.

300.

Avantage à envahir et à réduire par les armes ; vaincre les insoumis.

${ }^{1}$ Disposer de... 
TSHENG TSE. - Vaincre par les armes ceux que les actions vertueuses, la modestie et la bienveillance n'ont pu soumettre. Envers ceux que les actions vertueuses ne peuvent soumettre, si on n'emploie pas l'autorit é et la force des armes, comment sera-t-il possible de pacifier et de gouverner l'univers ? Ce ne serait plus là la voie rationnelle du prince; ce ,trait l'excès dans la modestie. 


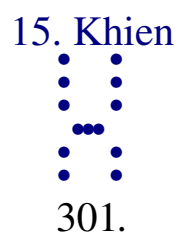

Trait supérieur hexaire : modestie renommée ; avantage à employer l'action des armées ; vaincre le district et les royaumes.

TSHENG TSE. - Un trait hexaire, employant la douceur, est placé à la plus extrême limite de la douceur et de la soumission ${ }^{1}$. De plus il est placé au sommet du koua qui exprime la modestie; c'est le dernier degré de la modestie. Puisqu'il pousse la modestie à son comble et qu'inversement il occupe le plus haut rang, c'est qu’il n'a pas encore pu satisfaire ses tendances vers la modestie, de sorte que cette vertu éclate dans ses accents et ses paroles. De plus, la douceur malléable occupant le point culminant de la modestie, elle doit également être manifeste dans les accents et dans l'apparence, et c'est pourquoi la formule dit : «Modestie renommée. » Bien qu'il occupe un terrain qui ne comporte aucune situation définie, qu'il ne soit investi d'aucune charge publique, cependant, même lorsque l'homme agit pour son propre compte, il faut absolument que la douceur et l'énergie se contrebalancent. Or, le rang supérieur indique le comble de la modestie, de sorte qu'elle est poussée à l'excès et qu'elle devient au contraire un défaut. C'est pour cela que l'avantage consiste à employer l'énergie et la violence pour se corriger soi même. Le royaume du district ${ }^{2}$, c'est ce quill possède en propre : «l'action des armées », c'est -à-dire l'emploi de l'énergie et de la violence. «Vaincre le royaume du district » veut dire réformer soi-même ce qui lui est propre.

TSHOU HI. — La modestie poussée à son extrême limite est renommée ; c'est ce qui rallie les hommes, de sorte qu'il est possible d'agir en maître ${ }^{3}$. Cependant, à cause de ses caractères physiques de malléabilité, et d'ailleurs dépourvu de situation officielle, il lui est seulement possible de dominer le royaume qui constitue son propre district, et rien de plus.

302.

\footnotetext{
${ }^{1}$ Rang pair et dernier rang.

2 Mauvaise lecture.

3 Autre lecture des mots traduits par « l'action des armées ».
} 
Modestie renommée; tendances pas encore satisfaites. Possibilité de l'emploi de l'action des armées ;

vaincre le district et les royaumes.

TSHENG TSE. — La modestie est poussée à l'extrême limite et il occupe le rang supérieur; ses tendances qui lui font désirer d'être modeste ne sont pas encore satisfaites, de sorte qu'il ne peut dominer la force des circonstances et qu'il arrive à la renommée. Bien qu'il ne corre sponde pas à sa situation, du moment où la modestie est excessive, il convient qüil réforme ce qui lui est propre par l'énergie et la violence, et c'est pour cela

que la formule dit: «Avantage à employer les armées (ou à agir en maître), pour vaincre le royaume constitué par son district. »

TSHOU HI. - Douceur malléable négative sans situation; aptitudes et force insuffisantes ; aussi ses tendances ne peuvent pas encore prévaloir, et il en arrive à l'emploi des armées. Mais elles sont cependant juste s uffisantes pour gouverner son propre district, sans rien pouvoir au-delà. 


\section{6. $\mathrm{Yu}$}

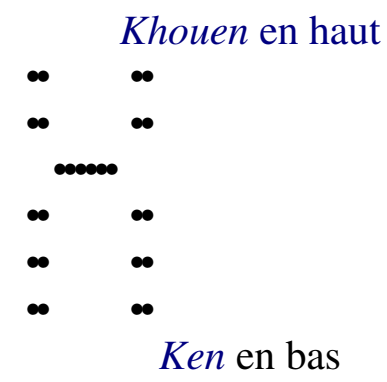

303. Yu, paix, avantage à instituer des feudataires et dans l'action des armées.

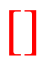

308. Premier trait hexaire : satisfaction bruyante, présage malheureux.

$*$

310. Deuxième trait hexaire : solidité de la pierre ; ne pas attendre au dernier jour, présage heureux de la perfection.

*

312. Troisième trait hexaire : regarder les regrets de la satisfaction ; en tardant il y a regrets.

$*$

314. Quatrième trait nonaire : par la satisfaction; le grand avoir se réalise; point de doute ; les amis pourquoi pas rapidement?

$*$

316. Cinquième trait hexaire : inconvénients de la perfection ; ne jamais mourir.

$*$

318. Trait supérieur hexaire : satisfaction aveugle ; complète elle se modifie ; pas de culpabilité.

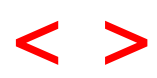


16. $\mathrm{Yu}$.

TSHENG TSE. - YU. «L'Ordre des koua » dit : «L'avoir étant grand, et pouvant être modeste, il y a nécessairement satisfaction, aussi le koua khien est suivi du koua $y u$. » L'ordre est déduit du sens des deux koua précédents. Du moment où l'avoir est déjà grand et qu'on peut cependant être modeste, on doit en éprouver de la satisfaction et du contentement. Ce koua est constitué par le koua simple tshen, en haut, et le koua simple khouen, en bas. C'est l'image symbolique du mouvement avec soumission; se mouvoir avec soumission et concorde, c'est ce qui produit la satisfaction. Le quatrième trait nonaire est celui de qui dépend le mouvement; celui à qui la troupe des négativités supérieures et inférieures correspond avec une égale sympathie. Le koua simple khouen lui est de plus soumis avec obéissance ; c'est employer le mouvement et jouir de la sympathie des supérieurs et des inférieurs, c'est pour cela que ce koua est considéré comme exprimant le sens de concorde et satisfaction. Si on en parle d'après les deux images symboliques, la foudre éclate et sort au-dessus de la terre; la positivité commence par être cachée dans la terre, puis vient le mouvement et elle sort hors de la terre en faisant éclater le bruit, enfin elle s'étend librement avec concorde et tranquillité. C'est pour cela que le koua est appelé $y u{ }^{1}$.

\footnotetext{
${ }^{1} Y u$, calme, paix, repos, satisfaction.
} 


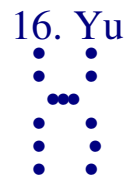

303.

Yu, paix, avantage à instituer des feudataires et dans l'action des armées.

TSHENG TSE. - Paix ; soumission et mouvement : tel est le sens du koua $y u$. L'avantage se rapporte à l'institution de feudataires et à l'action des armées. Or, instituer des feudataires, c'est planter une haie d'arbres autour du trône pour le protéger, et c'est le moyen d'assurer en même temps la paix de l'univers. Si tous les feudataires sont cor diaux et obéissants, il en résultera que la masse des peuples se soumettra volontiers à la levée des troupes et des armées ; tous les cou rs étant pleins de concorde et de soumission, ces armées obéiront avec soumission et il y aura succès. C'est pour cela que la voie de la satisfaction et de la paix est avantageuse pour l'établissement de feudataires et l'action des armées. Ou bien encore, c'est l'image symbolique de en haut mouvement, et en bas soumission : tous les feudataires suivant le roi et des armées se soumettant à leurs ordres. Régir tous les états, rassembler de grandes multitudes, les amener à se soumettre et à obéir, serait impossible sans concorde et satisfaction de toutes parts.

TSHOU HI. - Yu; concorde et joie ; lorsque le cou r de l'homme est disposé à la concorde et à la joie, il est disposé à répondre sympathiquement au supérieur. Le quatrième trait nonaire est runique positivité du toua; les traits supérieurs et inférieurs lui correspondent sympathiquement; ses tendances peuvent prévaloir. De plus, la rencontre du koua simple khouen par le koua simple tshen est considérée comme constituant la soumission dans le mouvement, et c'est pourquoi le koua est appelé $y u$, tandis que le sens divinatoire est qu'il y a avantage à établir des princes et à employer les armées.

304.

Le commentaire traditionnel de la formule déterminative dit: Yu; la dureté énergique correspond sympathiquement et ses tendances agissent; soumission dans le mouvement : paix et satisfaction. 
TSHENG TSE. — «La dureté énergique correspond sympathiquement » est une expression qui indique que le quatrième trait est celui à qui la troupe des traits négatifs est sympathique; la dureté énergique possède les sympathies de la foule ${ }^{1}$. «Les tendances agissent», c'est-à-dire que les tendances de la positivité sont de la faire agir en montant. Elle se meut au-dessus comme au-dessous, tous suivent avec obéissance; ses tendances peuvent agir. "Soumission dans le mouvement, paix et satisfaction »: Le koua simple tshen exprime le mouvement et le koua simple khouen la soumission, cela est considéré comme constituant le mouvement avec soumission à la raison d'être des choses, ou la soumission à cette raison suivie de mouvement. C'est encore considéré comme exprimant le mouvement suivi de la soumission de la foule, et c'est toujours par là qu'i 1 y a paix et satisfaction.

TSHOU HI. - Explication du sens du nom du koua au moyen de la substance de ce koua et des vertus des koua simples qui le composent.

305.

Satisfaction et soumission dans le mouvement; c'est pourquoi le ciel et la terre l'imitent, et à plus forte raison l'établissement des feudataires et l'action des armées.

TSHENG TSE. - Puisqu'il y a satisfaction et soumission dans le mouvement, il en résulte que le ciel et la terre y acquiescent et ne s'y opposent point; à plus forte raison, dans le cas d'établissement de feudataires et d'action des armées, comment la même soumission ne se rencontrerait-elle pas ? La voie du ciel et de la terre, c'est la raison d'être de la foule des êtres et des choses ; ce n'est rien autre chose que l'ext rême soumission. C'est à cause de cela que le grand homme précède le ciel, suit le ciel, sans que celui-ci le démente $^{2}$; ce n'est encore rien autre que la simple soumission à la raison d'être.

TSHOU HI. - Explication de la formule du koua au moyen des vertus des deux koua simples.

\footnotetext{
${ }^{1}$ On peut lire aussi : «sympathie de la dureté énergique », mais en conclure que cela exprime que c'est l'éner gie qui jouit des sympathies est à peu près comme si on disait que les mots « il tue » expriment qu'il est tué.

${ }^{2}$ Citation.
} 
306.

Le ciel et la terre se meuvent avec soumission; c'est pourquoi le soleil et la lune ${ }^{1}$ ne varient point accidentellement et les quatre saisons ne sont jamais interverties. L'homme saint se meut avec soumission, et alors les lois pénales et les châtiments sont clairs et le peuple s'y soumet. Combien sont vastes les moments et le sens indiqués par le koua yu!

TSHENG TSE. - Nouvelles définitions explicatives de la voie du mouvement avec soumission. Dans la translation du ciel et de la terre, c'est parce que ce mouvement est harmonique (parce qu'il a lieu avec soumission), qu'il ne survient ni erreur, ni variation acci dentelle dans la mesure des jours et des mois, et que le cours des quatre saisons n'est jamais altéré, ni troublé 2. L'homme saint se meut avec soumission, aussi, les règles fonda mentales qu'il pose sont correctes, et le peuple s'élève vers le bien ; les lois pénales et les châtiments sont clairs et simples, et la foule du peuple s'y soumet. Ceci définit déjà la voie de la soumission avec satisfaction. Cependant, le thème est inépuisable; les mots sont épuisés que l'idée reste encore indéfinie. C'est pour cela que le texte récapitule le sujet dans une exclamation admirative : «Combien sont vastes les moments et le sens indiqués par le koua $y u$ !»Et cela, pour que l'homme analyse et apprécie la raison d'être du sujet ; pour qu'il s'en imprègne et le connaisse parfaitement. Les mots « moments » et «sens », désignent les moments et les sens indiqués par le koua yu. Dans les

\footnotetext{
${ }^{1}$ On peut aussi bien lire : « le jour et les mois ».- Ou bien il faut reconnaître que Khong Tse connaissait le mouvement propre de la terre ; ou il faut admettre que pour lui, dans ce cas, « ciel» et «terre » ont une valeur particulière propre et ne désignent pas le ciel-firmament et la terre-sol. On pourra objecter que le terme traduit par «mouvoir» ou «mouvement» indique «l'ébranlement qui met en action» et que c'est dans cette acception qu'on doit le prendre dans le membre de phrase suivant. Mais si on veut employer cette acception dans le premier membre de phrase on retombe dans la phraséologie des commentateurs et il n'y a plus de sens intelligible.

${ }^{2}$ Ce commentaire vient renforcer la remarque que j'ai faite au sujet du texte. Qu'on prenne le caractère que je traduis par translation et qu'on recherche quels sont ses acceptions ou sens essentiels, on trouve avant tout «successsion, par transmission de l'un à l'autre » et «mouvement, agitation». Si on admet que Khong Tse ait connu le mouvement propre de la terre, on ne croira certainement pas que ce mouvement ait été encore connu au neuvième et au dixième siècles de notre ère, en Chine. Mais en admettant que Khong Tse ait connu ce mouvement, on serait forcé de reconnaître que, dans ce cas, il savait que le ciel ne se meut pas. Donc, sil parle du mouvement du ciel et de la terre, et si les commentateurs postérieurs en parlent d'après lui et d'après ceux qui l'ont précédé, c'est que ces deux mots « ciel »et «terre » ont, à une certaine époque, exprimé deux mouvements différents et n'avaient pas essentiellement la valeur usuelle qu'on leur a donné depuis. La difficulté cess e si on veut bien reconnaître que le «ciel » est le mouvement du soleil et de la lune au-dessus de l'horizon, et la «terre », le mouvement des mêmes astres sous l'horizon. Je remets à un autre moment une remarque analogue sur les « quatre saisons » ou plutôt « moments ».
} 
divers koua, lorsque le mot «grand» est employé comme qualificatif des mots «sens » et «moment », c'est tou jours une exclamation admirative sur la grandeur de l'étendue et de la portée de ces mots. Tel est le cas pour le koua yu et les dix koua qui suivent : dans les koua yu (16), thouen (33), kieou (44), lu (56), il s'agit du sens et du moment ; dans les koua khan (29), khouei (38), hien (39), il s'agit de l'emploi du moment ; dans les koua yi (27), tae kuo (28), kiae (40), ho (49), il s'agit du moment, et dans chaque cas au sujet de ce qu'ils ont de grand.

TSHOU HI. - Dernières conséquences et exclamation sur la grandeur.

307.

Le commentaire traditionnel de la formule symbolique dit : La foudre sort de terre et éclate; satisfaction; les premiers rois inventèrent la musique pour célébrer la vertu; parfaite elle était offerte au souverain suprême, pour assister leurs ancêtres ${ }^{1}$.

TSHENG TSE. — La foudre, c'est l'éther positif, qui surgit et éclate ; la négativité et la positivité qui se choquent et produisent le bruit. La positivité commence par être ployée et enfermée dans la terre ; survient le mouvement, et elle sort de terre, éclate et ébranle. D'abord cachée et accumulée, survient le moment où elle surgit et éclate en se répandant librement et en produisant la concorde et la paix. C'est pour cela que ce koua est appelé ru et considéré comme exprimant la paix et la satisfaction. Le koua simple khouen exprime la soumission passive; le koua simple tshen exprime le fait de se manifester brusquement au-dehors ; la concorde et la soumission accumulées au-dedans, éclatant en bruit, ou son; c'est l'image symbolique de la musique. Les premiers rois voyant la foudre sortir de terre et éclater et considérant l'image symbolique de l'h armonie, ou accord, se manifestant dans le son, firent de la musique pour célébrer et illustrer le mérite et la vertu; la perfection en était telle qu’ils la consacraient au souverain suprême, et ils la présentaient au nom de leurs ancêtres ${ }^{2}$. Le caractère yin du texte signifie perfection achevée ; dans le $\mathrm{Li}$ king on trouve ce caractère dans une expression qui signifie «parfait développement ».

\footnotetext{
${ }^{1}$ Le sens des deux derniers membres de phrase est inintelligible ; la traduction est basée sur les commentaires, mais ce sens n'est pas complètement compris dans le texte. Littéralement on devrait lire: «Parfaite au point de la consacrer le souverain suprême, pour égaler les ancêtres. »

${ }^{2}$ Pour les faire participer au mérite de l'offrande.
} 
Consacrer au souverain suprême, assister les ancêtres, indique le comble de la perfection.

TSHOU HI. - La foudre sort de la terre et éclate : comble de l'accord. Les premiers rois inventèrent la musique. Outre que le son est symbolisé, le sens est aussi relevé. Yin, perfection achevée. 


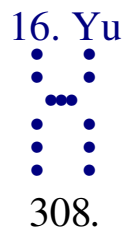

Premier trait hexaire : satisfaction bruyante ${ }^{1}$, présage malheureux.

TSHENG TSE. — Le premier trait hexaire emploie la douceur malléable de la négativité et occupe le rang inférieur; le quatrième est le maître du koua $y u$, c'est-à-dire celui de qui dépend la satisfaction, et lui correspond sympathiquement: donc le troisième est l'homme inférieur sans justice ni droiture, qui se place pour sa propre satisfaction et qui jouit de la faveur du supérieur. Ses tendances et ses idées sont au plus haut point présomptueuses ; il ne sait pas maîtriser sa satisfaction, au point qu'elle éclate dans ses accents et ses paroles. Étant léger et vaniteux à un tel point, il doit en arriver au malheur. Le terme ming, troisième caractère du texte, exprime la manifestation par le son ${ }^{2}$.

TSHOU HI. - Homme inférieur, doux et négatif ; en haut il est accueilli par un soutien puissant ; le moment lui est favorable et il dirige les affaires, aussi il ne domine pas sa satisfaction et il la manifeste lui-même bruyamment. C'est une voie qui conduit au malheur, et c'est pour cela que tel est le sens divinatoire. Le nom que porte le koua exprime essentiellement la paix et la joie ; cependant la formule du koua $\left(\mathrm{n}^{\circ} 303\right)$ donne le sens de joie de la foule et les formules des traits, à l'exception de celle du quatrième trait nonaire qui suit le sens de la formule du koua, donnent toutes le sens de satisfaction personnelle; c'est de là que dépend la différence entre le bonheur et le malheur présagés.

309.

Le premier trait hexaire exprime bruyamment sa satisfaction, ses tendances le conduisent finalement au malheur ${ }^{3}$.

\footnotetext{
${ }^{1}$ D'après les commentateurs, dans la formule $\mathrm{n}^{\circ} 301$, on lit « modestie renommée »; ici, on lit « chanter la satisfaction »; le choix de l'acception donnée au caractère ming est arbitraire 2 Ming, le chant des oiseaux ; émettre un son.

${ }^{3}$ Certains commentateurs lisent : « Tendances exagérées, présage malheureux. »
} 
TSHENG TSE. — Le texte dit : « Le premier trait hexaire » ${ }^{1}$, c'est -à-dire que c'est lui qui est négatif, doux, placé dans un rang inférieur, et dont les tendances et les idées sont absolument excessives; il ne domine pas sa satisfaction, à ce point qu'il la manifeste bruyamment. Il doit nécessairement être orgueilleux, manquer de retenue et finalement arriver au malheur.

TSHOU HI. - Le terme khung, du texte, exprime le comble de la présomption.

\footnotetext{
${ }^{1}$ En général Khong Tse ne répète pas cette indication.
} 


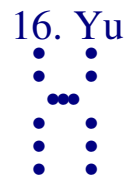

310.

Deuxième trait hexaire : solidité de la pierre ${ }^{1}$; ne pas attendre au dernier jour ${ }^{2}$, présage heureux de la perfection.

TSHENG TSE. — En s'abandonnant à la voie de la satisfaction, l'excès amène la perte de la droiture, et c'est pourquoi parmi les divers traits du koua $y u$, la plupart ne possèdent pas la droiture et leurs aptitudes sont conformes au moment. Un seul trait, le second trait hexaire, est placé selon la justice et la droiture; de plus il est sans correspondance sympathique, ce qui constitue l'image symbolique de l'observation attentive de soi -même. En présence d'un moment de satisfaction, lui seul peut s'observer et se main tenir avec justice et droiture : C'est ce qu'on peut appeler la fermeté da ns l'isolement et cela exprime que ses principes sont fermes comme la solidité de la pierre. «Solidité de la pierre », solidité telle que celle de la pierre ${ }^{3}$. Lorsque l'homme est dans la satisfaction et le plaisir, son c au r s'y complait, de sorte que petit à petit il en arrive, en se laissant aller à suivre ses tendances, jusqu'à la passion des jouissances et sans pouvoir s'arrêter dans cette voie. Le second trait se garde par la justice et la droiture, sa fermeté est comme celle de la pierre ; la rapidité avec laquelle il refoule ses passions est telle qu'il n'attend pas pour cela la fin du jour ; c'est pour cela que sa droiture est parfaite et le présage heureux. Étant placé au milieu des plaisirs, on ne doit pas s'y adonner avec sécurité et pendant longtemps; autrement, au bout d'une durée prolongée, la force morale sera énervée et on sera comme noyé dans les plaisirs. Lorsque les choses se passent comme dans le cas du second trait, c'est ce qu'on peut appeler : «Voir la cause initiale au début de son action et agir. » Fou Tse s'appuie sur cette propriété qu'a le second trait de discerner l'origine, et il développe dans ses derniers détails la voie rationnelle de la connaissance des causes initiales. Il dit: "Connaître les causes initiales, n'est-ce pas là le génie ? L'homme doué s'allie au -dessus de lui sans flatterie ;

\footnotetext{
${ }^{1}$ La traduction suit la version admise, mais le sens naturel, si on ne se préoccupe pas de l'explication tra ditionnelle, serait : «Limite à la pierre » ou « limiter, montrer, par la pierre », étendre une certaine limite jusqu'à une certaine pierre ou borne.

2 De même ici, le sens figuré serait : «pas indéfiniment » et le sens absolu «jour qui ne finit pas ».

3 Même en admettant les acceptions choisies par le commentateur il faudrait lire «solidité dans la pierre ». Le caractère yu, quatrième du texte, exprime essentiellement le mouvement vers.
} 
il s'allie au-dessous de lui ${ }^{1}$ sans mépris ; c'est qu'il connaît la cause initiale, ou origine des effets. Cette cause initiale, c'est la transition insensible du repos au mouvement. C'est voir à l'avance le présage heureux. L'homme doué voit la cause initiale et il agit; il n'attend pas la fin du jour. Le Yi king dit : Solidité de la pierre, ne pas attendre la fin du jour, présage heureux de la pureté ; étant ferme comme la pierre, à quoi bon attendre la fin du jour ? il comprend sur-le-champ et se décide. L’homme doué connaît ce qui est minime et subtil et il connaît aussi ce qui est manifeste ; il connât la douceur, comme il connaît l'énergie. Il est celui sur qui la foule innomb rable a les yeux fixés. Or, voir l'origine des choses dans le moment insaisissable de la naissance de leur cause, c'est avoir le génie transcendant. L'homme doué monte s'allier sans devenir flatteur; il descend s'allier sans devenir méprisant : en effet il connaît la cause initiale, s’il ne la connaissait pas, il se laisserait aller jusqu'à l'excès sans s'arrêter. Les relations avec les supérieurs doivent être basées sur la dignité et la modestie, de sorte que l'excès serait la flatterie. Les relations avec les inférieurs doivent être basées sur l'aménité et la facilité dans les formes, de sorte que l'excès serait le mépris hautain. Mais l'homme doué regarde dans l'imperceptible origi ne de sorte qu'il n'en arrive pas aux excès. Ce qu'on appelle origine insensible des causes, c'est la première naissance du commencement du mouvement; le germe du bonheur ou du malheur y est déjà visible mais n'est pas encore manifesté. Pour ne parler que du présage heureux, si on sait le voir à l'avance, comment pourrait-on craindre le retour du malheur? L'homme doué est intelligent et perspicace; il voit la cause insensible des choses, de sorte quil peut se maintenir ferme comme la pierre. Du moment où il s'observe et se garde avec fermeté, il n'est jamais perplexe et juge clairement; il voit la cause dans son germe et il se meut; pourquoi attendrait-il la fin du jour? » Se décider, c'est distinguer; il est bien évident qu'il distingue, discerne et se décide ensuite. Les expressions «subtil» et «manifeste», «douceur» et «énergie» font opposition entre elles; l'homme doué voit ce qui est subtil de sorte quil connaît ce qui sera manifeste ; il voit la douceur, de sorte quill y reconnaît l'énergie. Connaître ainsi les causes initiales, c'est ce qui fait que la fo ule a les yeux tournés vers lui, aussi c'est pour cela que vient l'exclamation admirative : «Il est celui sur qui la foule des hommes a les regards fixés !»

TSHOU HI. - Bien que la satisfaction $(y u)$ résulte principalement du plaisir, cependant elle perd facilement les hommes, qui se noient dans la volupté ; cet affaiblissement résultant de l'abandon dans l'énivrement du plaisir devient au contraire une cause de chagrin. Dans le koua il n'y a que ce seul trait qui possède la justice et la droiture ; c'est donc que le trait supérieur et les traits inférieurs sont noyés dans la satisfaction et que, seul, il peut se garder et se préserver par la justice et la droiture. Sa fermeté est comme celle de la pierre ; sa vertu est calme et tranquille, mais solide et inébranlable, de

1 Les mots «de lui» sont ajoutés dans le texte, selon la lecture et l'explication des commentateurs ; en réalité le texte dit « en haut » et « en bas ». 
sorte que sa pensée et ses prévisions se résolvent en jugements clairs. Il n'attend pas la fin du jour ${ }^{1}$ et il voit l'origine insaisissable de la cause de chaque chose. La «Grande étude» dit: «Le calme de l'esprit permet la méditation; par la méditation on peut arriver au but»; cette pensée correspond exactement à ce passage. Si celui qui consulte le sort est dans ces conditions, il agira suivant la droiture et le présage sera heureux.

\section{1.}

Ne pas attendre au dernier jour ${ }^{2}$, présage heureux de la perfection par la justice et la droiture.

TSHENG TSE. - Ce qui fait quil peut ne pas attendre la fin du jour, qu'il est parfait, et que d'ailleurs le présage est heureux, c'est qu'il possède les vertus de la justice et de la droiture. Étant juste et droit, sa manière de s'observer est ferme et il peut discerner de bonne heure et écarter rapidement. La formule du trait parle de la voie suivant laquelle le second trait hexaire se place dans la satisfaction; cela constitue la profondeur de l'idée d'enseignement qui y est contenue.

${ }^{1}$ Il faudrait lire « le dernier jour ».

${ }^{2} \mathrm{Ne}$ pas prolonger indéfiniment. 


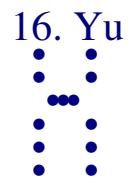

312.

Troisième trait hexaire : regarder les regrets de la satisfaction ; en tardant il y a regrets ${ }^{1}$.

TSHENG TSE. - Le troisième trait hexaire est négatif et il occupe un rang positif ; c'est un homme dénué de justice et de droiture. Étant sans justice ni droiture et se plaçant pour sa satisfation, quoi quill fasse, il en aura des regrets. Le terme hu, troisième caractère du texte, signifie «regarder en haut ». Il regarde au loin, en haut, le quatrième trait, et il attend tout de lui, de sorte que, comme à cause de son manque de droiture et de justice il n'est pas choisi par ce quatrième trait, il éprouve des regrets. Le quatrième trait est.celui de qui dépend la satisfaction ${ }^{2}$; et le troisième est tout à fait proche de lui ; s’il tarde et traîne en longueur sans se mettre en avant, il se verra abandonné et mis de côté, de sorte qu'il aura encore des regrets. En effet, se plaçant luimême sans droiture, qu'il avance ou recule, dans tous les cas il aura des regrets et de l'appréhension. Que convient-il donc qu’il fasse ? Il faut qu'il se réforme et rien de plus. L'homme doué a une voie toute tracée pour se placer ; il contient ses passions par les lois rituelles; bien qu'il se trouve dans un moment de satisfaction, il ne manque ni à la justice ni à la droiture, de sorte qu'il n'éprouve pas de regrets.

TSHOU HI. - Hu, regarder en haut ${ }^{3}$. Négativité, sans justice ni droiture, et de plus placée auprès du quatrième trait. Le quatrième trait est celui de qui dépend la satisfaction, et c'est pour cela que le troisième trait hexaire regarde en haut vers le quatrième en même temps qu'il s'abaisse et se plonge dans les plaisirs : C'est celui qui, no rmalement, doit éprouver des regrets et c'est pour cela que telle est l'image symbolique, tandis que le sens divinatoire est que le fait considéré doit bientôt amener des regrets. Mais si on tarde à en avoir des regrets ${ }^{4}$, alors on le regrettera certainement.

\footnotetext{
1 Les commentateurs lisent «regarder la satisfaction au-dessus de soi, regrets ». Les commentaires se rapportent à cette dernière lecture qu'on peut rendre en français par la phrase suivante : «Envier le bonheur de ceux qui sont au-dessus de soi. »

${ }^{2}$ Le maître du koua $y u$.

3 Voici les principales acceptions de ce mot: «regarder avec de grands yeux » (avec admiration), « élever les yeux ».

${ }^{4}$ Le commentateur adopte une toute autre lecture : «troisième trait hexaire ; regarder en haut la satisfaction ; s'il tarde à avoir de s regrets il en aura du regret », elle est insoutenable.
} 
313.

Regarder la satisfaction et avoir des regrets ; situation imméritée.

TSHENG TSE. - Il se place dans une situation dont il n'est pas digne ; il manque à la justice et à la droiture et c'est à cause de cela que, soit quỉl avance, soit qu'il rec ule, il en éprouvera toujours des regrets. 


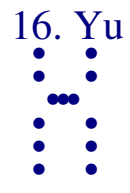

314.

Quatrième trait nonaire : par la satisfaction ; le grand avoir se réalise ; point de doute ; les amis pourquoi pas rapidement ${ }^{1}$ ?

TSHENG TSE. - Ce qui fait que le koua $y u$ est considéré comme exprimant la satisfaction $(y u)$, c'est précisément le quatrième trait nonaire ; c'est lui qui est le maître du mou vement, or, le mouvement qui entraîne la soumission joyeuse de la foule des négativités donne le sens du mot $y u$, satisfaction. Le quatrième rang indique la situation d'un haut dignitaire sujet du prince ; le prince représenté par le cinquième trait hexaire le suit et l'écoute avec soumission et condescendance. Employer la dureté énergique et porter le poids des affaires du supérieur, est la cause dont résulte la satisfaction et c'est pourquoi la formule dit «cause de la satisfaction, il a de grandes facilités d'action », c'est-à-dire qu'il peut largement donner cours à ses tendances, d'o ù résulte la satisfaction de l'univers entier. "Pas de défiance, les amis se groupent rapidement $»$; le quatrième trait occupe la situation du sujet d'un haut rang; il obéit à un prince faible et mou, et il porte le poids du gouvernement de l'empire : c'est un terrain périlleux et suspect. Il est seul apte à supporter le poids de l'autorité que le supérieur délègue, et au-dessous de lui il ne rencontre pas l'aide d'une vertu pareille à la sienne, ce qui lui inspire des craintes. Il doit simplement épuiser la plus extrême sincérité, ne pas avoir de crainte et d'appréhension, et alors ses amis, doués d'un caractère du même genre que le sien, devront naturellement se réunir et se grouper, car pour inspirer confiance au-dessus et au-dessous de soi, il suffit de montrer la plus entière sincérité. S’il épuise la plus complète sincérité, quels malheurs pourraient résulter de ce quil est sans assistance ? Le terme tsan, dernier caractère du texte, signifie rassembler ${ }^{2}$. Le sens de ce mot vient du nom de l'éping le à cheveux, tsan, qui sert à rassembler les cheveux roulés en chignon. On a dit : dans le koua il n'y a qu'une seule positivité ; comment pourrait-il avoir l'assistance de vertus analogues aux siennes ? voici la réponse: Lorsqu'on occupe une situation é levée et qu'on demande assistance avec une

\footnotetext{
${ }^{1}$ Les commentateurs lisent : «Il produit la satisfaction; il a de grandes facilités d'action ; pas de défiance ; les amis se groupent rapidement. » Je pense que traduire ne consiste pas à forcer le sens des mots et à faire abstraction des lois de la langue. Il est probable avant tout qu'il ne faudrait pas couper la phrase après le caractère $y u$. Si le texte a été mutilé ou a subi des altérations, il faut l'étudier tel qu'il est avant de chercher à le corriger. S'il n'en a pas subi et sil ne présente cependant aucun sens correct et intelligible, c'est que les mots avaient une autre valeur que celle qui leur est attribuée aujourd'hui.

${ }^{2}$ Tsan, recouvrir, acception figurée.
} 
parfaite sincérité, la raison d'être naturelle des choses fait qu'on doit arriver à l'obtenir. La formule du cinquième trait nonaire du koua keou dit : «Elle peut tomber du ciel »; c'est précisément le cas. Le quatrième trait emploie la dureté énergique de la positivité ; il est extrêmement rapproché de la situation du prince, et c'est de lui que dépend exclusivement la satisfaction. L'homme saint devait nécessairement en faire l'objet d'un avertissement qui ne se voit pas à priori. La satisfaction, c'est la voie naturelle de l'accord et de la condescendance ; par cette voie d'accord et de soumission, on ne manque pas à la droiture que doit observer celui qui est sujet. Remplir ces conditions et être maître exclusif de la satisfaction, c'est diriger toutes les affaires de l'empire et conduire son époque à la paix et à la satisfaction. C'est pour cela que la formule avertit simplement au sujet de l'absolue sincérité sans appréhension.

TSHOU HI. - Le quatrième trait nonaire est celui à cause duquel le koua est considéré comme exprimant la satisfaction et c'est pour cela que telle est l'image symbolique, tandis que le sens divinatoire est tae yeou te: «grandement avoir succès » 1 . Cependant, il convient encore qu'il possède la plus extrême sincérité et soit sans défiance, et alors les amis du même genre que lui s'assembleront pour le suivre et lui obéir. C'est pour cela que la formule le mentionne et en fait encore l'objet d'un avertissement. Tsan, «se réunir », « rassembler », et aussi, « rapidement $»^{2}$.

315.

Par la satisfaction, le grand avoir est obtenu; tendance agissant largement.

TSHENG TSE. - Il est cause que par sa propre action, l'univers est amené à la satisfaction, et c'est pour cela qu'il est cons idéré comme réunissant largement, c'est-à-dire que ses tendances sont considérées comme pouvant agir largement avec succès.

\footnotetext{
${ }^{1}$ Tshou Hi répète simplement le texte sans l'expliquer.

${ }^{2}$ C'est absolument faux c'est au caractère que les premiers annotateurs ont attribué le sens de « rassembler » et au caractère qüils ont attribué celui de « rapidement ».
} 


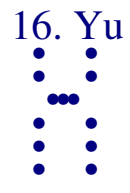

316.

Cinquième trait hexaire : inconvénients de la perfection ; ne jamais mourir.

TSHENG TSE. - Le cinquième trait hexaire emploie la douceur de la négativité pour occuper la situation du prince; en présence d'un moment de paix, il est submergé et noyé dans la satisfaction; c'est celui qui ne peut se maintenir de lui-même. Celui qui est le maître du pouvoir, celui autour de qui vient se ranger la foule, c'est toujours le quatrième trait. La dureté énergique positive du quatrième trait, captive la foule ; il est hors de la puissance du prince faible et mou, troublé par l'abus des plaisirs, de la contenir et de la gouverner. C'est alors que ce prince faible et hors d'état de se maintenir luimême, est dominé par le sujet tout puissant. Occuper dignement la situation du prince, c'est la perfection; être dominé par un inférieur présente des inconvénients pénibles; bien que le cinquième trait hexaire ait déjà laissé échapper le pouvoir qui correspond au rang suprême, cependant, il n'est pas encore entièrement détrôné, et c'est pour cela que la formule dit : «Inconvénients de la perfection, permanence sans mourir »; cela exprime que la perfection a cependant des inconvénients, que ces inconvénients sont permanents, et qu'il ne meurt cependant point : c'est par exemple le cas des derniers princes des dynasties des Han et des Wei. La voie qui conduit les princes aux périls et à leur perte n'est pas unique, mais toutefois c'est généralement celle de l'abus des plaisirs. Dans le cas du quatrième trait, la formule ne dit pas qu'il a perdu la droiture, tandis qu'au sujet du cinquiè me, on commence à voir qu'il opprime par la violence. Essentiellement, le quatrième trait est sans défaut, aussi, à son sujet, il n'est question que du sens d'un haut dignitaire chargé du soin des affaires de l'État ; dans le cas du cinquième, il s'agit du sens de la faiblesse débile occupant le rang suprême, sans pouvoir s'y maintenir elle-même, et à laquelle échappent l'autorité et le pouvoir. Dans chaque cas, le sens est choisi d'après la valeur particulière du trait, et c'est pour cela qu'il n'est pas toujours le même. Si le cinquième trait n'oublie point la voie du prince, et si le quatrième s'attache à le satisfaire, c'est qu'il a investi du pouvoir, l'homme qui était digne de ce choix, l'un jouissant du mérite des ou vres, l'autre attribuant ce mérite au premier, comme par exemple Thae Kia et Sheng Wang. Dans le koua mong aussi, la négativité occupe la situation prééminente, tandis que le second trait, par sa positivité, est considéré comme celui de qui dépend l'enseigne ment. Mais là il y a un présage heureux, et ici il y a des inconvénients ; le moment n'est pas le même. Étre jeune et ignorant et jouir de l'assistance de quelqu'un, c'est p récisément ce qui convient et est opportun ; être noyé dans l'excès des plaisirs et à cause 
de cela être lésé par quelqu'un, c'est au contraire une voie périlleuse qui conduit à la perte. C'est pour cela que dans le koua mong, la délégation de l'autorité a lieu entre ceux qui se correspondent sympathiquement, tandis que dans le koua $y u$, la perte du pouvoir a lieu entre ceux qui se heurtent l'un contre l'autre. De plus, le coa $r$ des supérieurs et des inférieurs est exclusivement porté vers le quatrième trait.

TSHOU HI. - Dans un moment de satisfaction, employer la satisfaction pour occuper le rang suprême et être noyé dans l'excès des plaisirs. De plus, ce trait s'appuie sur l'éner gie du quatrième; la foule ne se joint point à lui, et la force inhérente à sa situation indique le péril, c'est pourquoi ce trait est considéré comme représentant l'image symbo lique des inconvénients de la perfection. Mais cependant, puisquil possède la justice, il est encore considéré comme l'image symbolique de la conservation permanente sans mourir. En saisissant l'image symbolique, on voit forcément le sens divinatoire qui s'y trouve contenu.

317.

Inconvénients de la perfection du cinquième trait hexaire : il s'appuie sur la dureté énergique.

Continuité permanente sans mourir; la justice n'est pas encore perdue.

TSHENG TSE. - Perfection, mais accompagnée d'inconvénients ; s'appuyant sur la dureté énergique, il est meurtri par cette dureté. Continuité permanente sans mourir: la prééminence suprême de la justice n'est pas encore effacée. 


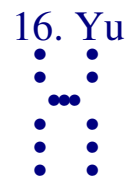

318.

Trait supérieur hexaire : satisfaction aveugle; complète elle se modifie; pas de culpabilité.

TSHENG TSE. - Le trait supérieur hexaire est doux et négatif ; il ne possède point les vertus de la justice et de la droiture ; il emploie la négativité et occupe le rang supérieur, c'est manquer à la droiture. De plus, il est en présence d'un moment où la satisfaction est portée à son extrême limite ; alors même qu'un homme doué se trouve en face d'un tel moment, il convient encore qu'il soit averti et se montre circonspect, combien à plus forte raison sil s'agit de la douceur de la négativité. Ici, il s'agit de celui qui est plongé et noyé dans la satisfaction et les plaisirs, qui est aveuglé et ignorant, et ne sait pas revenir dans la voie opposée. Il est placé à la fin du koua yu et c'est pour cela qu'il est considéré comme exprimant l'aveuglement déjà complet. Mais sil est capable de se réformer en se corrigeant, alors il lui sera possible d'être sans culpabilité. Être à la fin de la satisfaction, comporte le sens de modification ; si l'homme peut lui-même se corriger de ses défauts, il lui est toujours possible d'être sans culpabilité, aussi, bien que l'aveuglemen t dans le plaisir soit déjà complet, pouvant se modifier, il en résultera le bien. L'homme saint développe ce sens pour encourager au bien, de sorte quil ne parle nullement du présage malheureux résultant de l'aveuglement, et quil mentionne exclusivement l'absence de culpabilité qui découle de la transformation.

TSHOU HI. - Employer la malléabilité négative et demeurer dans la plus extrême satisfaction par les plaisirs, est considéré comme constituant l’image symbolique de l'aveugle ment dans la satisfation. Comme ce trait fait partie de la substance du koua simple qui exprime le mouvement, il est donc aussi le symbole des choses qui, bien qu'achevées, peu vent cependant se modifier encore. La formule avertit celui qui consulte le sort, que s'il est $\mathrm{d}$ ans ces conditions, il pourra réparer ses fautes, et sera sans culpabilité.

319.

Satisfaction aveugle chez le supérieur ; comment pourrait-elle durer! 
TSHENG TSE. - Il est aveuglé et étourdi par la satisfaction à un degré définitivement excessif; les calamités causées par sa culpabilité vont l'atteindre ; est-il donc possible que cet état de chose puisse durer longtemps ? Il convient quill se corrige rapidement. 


\section{Souei}

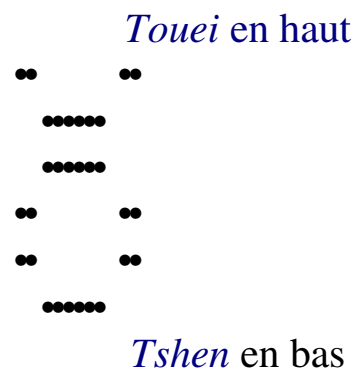

320. Suivre ; grande liberté; perfection avantageuse ; pas de culpabilité.

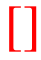

325. Premier trait nonaire : le magistrat se modifie ; présage heureux de la perfection; il sort de la porte se joindre, il a du mérite.

$*$

327. Deuxième trait hexaire : s'attacher au petit enfant ; perdre l'homme au bâton.

$*$

329. Troisième trait hexaire : s'attacher à l'homme au bâton ; pérdre le petit enfant ; en suivant, sil demande il obtiendra ; avantage à demeurer dans la perfection.

331. Quatrième trait nonaire : en suivant, obtenir; présage malheureux de la perfection; avoir foi dans la voie ; employer la clarté ; quelle serait la culpabilité?

$*$

333. Cinquième trait nonaire : foi dans le bien; présage heureux.

$*$

335. Trait supérieur hexaire : s'y attacher avec opiniâtreté ; alors suivre en s'y liant; le roi suit la erratique des offrandes sur les montagnes de l'ouest.

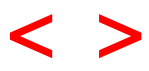




\section{Souei.}

TSHENG TSE. - Souei. «L'Ordre des koua » dit : «La satisfaction fait nécessairement suivre, c'est pourquoi le koua yu est suivi du koua souei. »Or, la voie du plaisir et de la satisfaction est celle que les êtres tendent à suivre, et c'est pour cela que le koua souei suit immédiatement le koua yu. Il est constitué par les koua simples touei en haut, et tshen en bas. Le koua simple touei exprime le contentement ; le koua simple tshen exprime le mouvement ; plaisir et mouvement, mouvement et plaisir, c'est toujours le sens de « suivre». La femme est ce qui suit l'homme $^{1}$; la jeune fille suivant le garçon adulte donne le sens de «suivre ». De plus, tshen exprime la foudre ; touei exprime le marais ; ébranlement de la foudre au milieu du marais: image symbolique du marais qui suit l'ébranlement et du mouvement qui suit l'ébranlement ( ?). Enfin, au point de vue de la transformation des koua, le trait supérieur du koua simple khien vient occuper le rang inférieur du koua simple khouen et le premier trait du koua simple khouen va occuper le rang supérieur du koua khien; la positivité vient s'abaisser devant la négativité. Puisque la positivité se met au-dessous de la négativité, la négativité doit nécessairement la suivre avec joie, ce qui constitue encore le sens de suivre. Dans tout koua parfait, après avoir examiné le sens indiqué par les deux substances ${ }^{2}$, on prend aussi le sens particulier qui résulte de la nature de chaque trait et, enfin, on prend encore le sens de la transformation du koua ${ }^{3}$. Dans le cas du koua souei, le choix des divers sens est absolument clair et complet.

\footnotetext{
${ }^{1}$ Le koua touei représente aussi la «jeune fille» et le koua tshen représente le «garçon adulte $»$.

2 Des deux koua simples.

${ }^{3}$ La transformation des koua, c'est la permutation des traits. Ex. dans le cas actuel (Souei), il est évident que si on remplace le premier par le dernier on obtient le koua $P^{\prime} i$.
} 


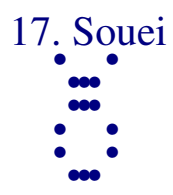

320.

Suivre ; grande liberté ; perfection avantageuse ; pas de culpabilité.

TSHENG TSE. - La voie naturelle de l'action de suivre est susceptible de conduire à une grande liberté. La voie rationnelle de l'homme doué est celle que suit la foule ; qu'il s'agisse de suivre soi -même quelqu'un ou, dans l'occasion, de choisir ce qu'il faut suivre, c'es t toujours l'acte de suivre. Si en suivant on se conforme à la voie rationnelle, il pourra toujours en résulter une entière liberté. Qu'il s'agisse du prince suivant le bien, du sujet ou de l'inférieur suivant ses ordres, de l'homme d'étude qui s'exerce à la pratique du devoir et qui, selon l'occasion, suit le supérieur, c'est toujours l'action de suivre. La voie rationnelle de l'acte de «suivre» est avantageuse par la parfaite droiture. Si en «suivant» on se conforme à ce qui constitue la droiture, il en résulte naturellement qu'on peut jouir d'une grande liberté et rester sans culpabilité ; mais si on manque à la droiture, on sera coupable. Dès lors comment la liberté d'action pourrait -elle exister?

TSHOU HI. - Souei, suivre ; si on en parle d'après la transformation du koua, il vient d'abord du koua khouen dans lequel le trait nonaire vient occuper le premier rang; il vient encore du koua she ho dans lequel le trait nonairé vient occuper le cinquième rang; enfin il provient également du koua wei tsae par la réunion simultanée de ces deux mêmes transformations, ce qui donne, dans tous les cas, le sens de l'énergie venant suivre la dou ceur. Si on en parle au point de vue de la substance particulière de chacun des deux koua simples, alors, l'un représe ntant le mouvement et l'autre le plaisir, il en résulte encore le sens de «suivre». C'est pour cela que le koua est appelé souei. Si on est soi-même capable de suivre les êtres, les êtres viennent et on est suivi par eux ; ceci et cela se suivant mutuellement, la liberté de mouvement est facile et c'est pourquoi le sens divinatoire est « grande liberté ». Cependant, il faut que l'avantage réside dans la perfection, et alors il est pos sible d'être sans culpabilité. Si ce qu'on suit n'est pas conforme à la droiture, alors, bien que jouissant d'une grande liberté, on n'évitera pas d'être coupable, dans le commentaire traditionnel du Tshouen tsieou, Mou Keang dit: «Ce qui possède ces quatre vertus, peut être suivi sans culpabilité ; moi, je n'en possède aucune ; comment me suivrait-on ? » Or il faut observer que bien que les quatre vertus en question soient étrangères au sens primitif, cependant, les paroles qui suivent indiquent une profonde pénétration de l'idée des règles du sens divinatoire. 
321.

Le commentaire traditionnel de la formule déterminative dit: Suivre; la dureté énergique vient et s'abaisse devant la douceur malléable ; mouvement et satisfaction : Suivre.

TSHOU HI. - Explication du sens du nom du koua au moyen de la transformation des koua parfaits et des vertus des koua simples.

322.

Grande liberté, perfection, pas de culpabilité ; et l'univers suit le temps.

TSHENG TSE. - Ce qui fait que le koua est considéré comme exprimant l'action de suivre, c'est que l'énergie vient et s'aba isse devant la douceur et parce qu'il y a mouve ment et satisfaction. Cela veut dire que le trait supérieur nonaire du koua simple khien vient occuper le rang inférieur -du koua simple khouen, tandis que le premier trait hexaire du koua simple khouen va occuper le rang supérieur du koua simple khien. Puisque la dureté énergique positive vient s'abaisser au-dessous de la malléabilité négative, c'est qu'il s'agit du supérieur s'abaissant devant l'inférieur, ou de la noblesse s'abaissant devant ce qui est vil. Pouvoir réaliser ces conditions, c'est ce qui porte les êtres à suivre avec joie ${ }^{1}$. De plus, en bas il y a mouvement et en haut il y a joie ; mouvement qui peut comporter la satisfaction, ce qui conduit à «suivre». Dans ces conditions, il sera possible de jouir d'une grande liberté et de s'accorder à la droiture. Pouvant jouir d'une grande liberté et posséder la droiture, on estime qu’il n'y aura pas de culpabilité. Si la liberté n'est pas possible, si on ne possède pas la droiture, il n'y aura pas de vo ie qui permette de suivre; comment pourrait-on supposer que l'univers suivra ? Ce que l'univers suit, c'est le moment ; c'est pour cela que la formule dit : «l'univers suit le temps ».

TSHOU HI. - Dans le texte donné par Wang Sou : Le dernier caractère, $s h i$, «temps », est remplacé par le caractère tshi (qui indique que l'action de suivre se rapporte à quelque chose énoncée précédemment); on doit maintenant suivre cette leçon ${ }^{2}$. C'est l'explication de la formule

\footnotetext{
1 À suivre celui qui s'abaisse devant les humbles.

${ }^{2}$ Et alors il faudrait lire «grande liberté, perfection, pas de culpabilité, et l'univers le suit ».
} 
déterminative du koua, et elle exprime que si on peut remplir ces conditions, c'est ce qui déterminera l'univers à « suivre ».

323.

Que le sens des mots « suivre le temps » est vaste!

TSHENG TSE. — La voie de l'homme doué consiste à se mouvoir suivant le moment, à suivre l'opportunité qui en découle, et à s'accommoder de ses modifications. On ne doit pas créer des règles invariables. Si on n'a pas sondé la profondeur de la voie, si on ne connaît pas l'origine primitive des causes et si on n'est pas capable de l'apprécier, on est incapable de s e conformer à ces conditions. C'est pour cela que la formule contient cette exclamation admirative : «Que le sens du mot suivre le temps est vaste!» Toutes les fois que le texte porte une telle exclamation, c'est que l'on a voulu que l'homme apprécie la grandeur du sens, qu'il s'en pénètre et en ait conscience. Dans le cas actuel, l'exclama tion sur la grandeur du fait qui consiste à suivre le temps n'a pas la même valeur que celle qu'on trouve dans le cas du koua $y u$ et d'autres koua. Dans tous les autres koua, il s'agit du «moment» et du « sens » considérés comme éléments indépendants l'un de l'autre.

TSHOU HI. - Dans le texte donné par Wang Sou : le caractère shi, est placé après le caractère $t s h i$; actuellement on doit suivre cette leçon.

324.

Le commentaire traditionnel de la formule symbolique dit: Dans le marais il y a la foudre: Suivre; l'homme doué pratique la recherche de l'obscurité et entre dans le repos de l'inactivité ${ }^{1}$.

TSHENG TSE. — La foudre ébranle au milieu du marais ; le marais suit (participe à) cet ébranlement et s'agite : ceci est considéré comme constituant l'image symbolique de l'action de suivre. L'homme doué considère cette image symbolique et il s'applique à suivre le moment pour agir. La nécessité de suivre le moment est la même pour toutes choses. La formule prend comme

\footnotetext{
${ }^{1}$ Les commentateurs lisent : «l'homme doué, quand vient l'obscurité, entre dans le repos de l'inactivité ». Cela peut être une explication critique, mais le texte dit tout autre chose. Cette lecture est contraire à la construction de la phrase.
} 
exemple le cas le plus clair et le plus à portée de l'obser vation : l'homme doué pratiquant la recherche de l'obscurité et entrant dans le repos de l'inactivité. Pendant le jour, l'homme doué s'efforce s ans cesse; vienne le moment où l'obscurité approche, et alors il y entre et y demeure ; il se livre au repos de l'inactivité pour reposer son corps. Il se lève ou reste en place selon le moment et selon l'opportuni té. Le Li king dit que, le jour, l'homme doué ne reste pas dedans et que, la nuit, il ne reste pas à l'extérieur. C'est la voie de l'action suivant le moment.

TSHOU HI. - La foudre est cachée dans le marais ; suivant le moment, repos. 


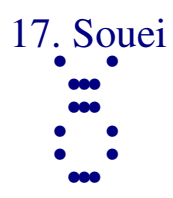

325.

Premier trait nonaire : le magistrat se modifie ${ }^{1}$; présage heureux de la perfection ; il sort de la porte se joindre, il a du mérite ${ }^{2}$.

TSHENG TSE. - Ce trait est nonaire, il se trouve dans un moment où il s'agit de suivre et fait partie de la substance de l'ébranlement ${ }^{3}$; d'ailleurs c'est de lui que dépend le mouve ment: c'est celui qui a quelque chose à suivre. Le terme kouan, troisième caractère du texte, magistrat, désigne celui qui dirige et surveille; du moment qu'il a quelque chose à suivre, c'est que ce qu'il dirige et surveille subit des modifications et change, et c'est pour quoi le texte dit: «Le magistrat se modifie ; présage heureux de la perfection »; si ce qu'il suit est conforme à la droiture, le présage sera heureux. Avoir à modifier et ne pas se conformer à la droiture, c'est se mouvoir à tort. Il sort de la porte se joindre et il a du mérite ; en général ce que le cour de l'homme tend à suivre, c'est ce qui lui est proche et ce qu'il aime. L' homme doué de sentiments ordinaires voit le bien dans ce qu'il aime et le mal dans ce qui lui déplaît; aussi il est très porté à écouter les paroles de sa femme et de ses enfants, même lorsqu'elles conseillent le mal, tandis qu'il lui répugne d'écouter le bien qui lui est conseillé par ceux qu’il n'aime pas. Si quelqu'un suit en se guidant par l'affection ou l'amour, il obéira à un sentiment personnel et égoïste ; comment pourrait-il s'accorder à la seule raison ! donc en sortant hors des portes pour s'all ier, il y aura du mérite. Sortir hors de la porte désigne tout ce qui n'est pas un lien d'affection privée et égoïste ; c'est se lier autrement que par égoïsme, de sorte que ce qui est suivi est digne de l'être et qu'il y a du mérite à le suivre.

TSHOU HI. - Au sujet du koua la formule prend le sens de « Êtres qui suivent »; au sujet du trait le sens est considéré comme exprimant l'idée de «suivre les êtres ». Le premier trait nonaire emploie la positivité et demeure dans l'infériorité ; il est considéré comme celui de qui dépend le mouvement, et c'est à cause de cela que le koua est considéré comme exprimant l'action de suivre. Du moment où il a à suivre quelque chose, c'est qu'il a une tendance particulière pour cette chose. Diriger et modifier, c'est un cas ordinaire ; mais ce n'est que si on agit en se conformant à la droiture que le présage sera heureux. De plus il convient de sortir hors des portes pour s'allier ; ne pas se diriger par l'égoïsme en suivant quelque chose, c'est avoir du mérite et c'est

\footnotetext{
${ }^{1}$ Littéralement : «Le magistrat avoir modifier »; le sens serait donc plutôt : «Le magistrat a à se modifier. »

${ }^{2} \mathrm{Si}$ on suit la ponctuation indiquée on devrait lire : « Se joindre à ceux qui ont du mérite. »

${ }^{3}$ Koua simple tshen.
} 
pourquoi tels sont l'image symbolique et le sens divinatoire, et de plus la formule en fait l'objet d'un avertissement.

326.

Le magistrat se modifie; en suivant ta droiture, présage heureux ; il sort de la porte, se joindre à ceux qui ont du mérite; pas d'inconvénients.

TSHENG TSE. - Du moment où il a à suivre et modifie, il faut absolument que ce qu'il suit soit la droiture, et alors le présage sera heureux. Si ce qu'il suit n'est point conforme à la droiture, il en aura des regrets et de l'appréhen sion. Sortant de la porte ${ }^{1}$ et s'alliant, c'est qu'il n'est point retenu par les liens de l'égoïsme et des sentiments privés, donc son alliance doit être conforme à la droiture ; elle sera sans inconvénients, et il aura du mérite.

\footnotetext{
${ }^{1}$ Porte, porte d'entrée de la maison.
} 


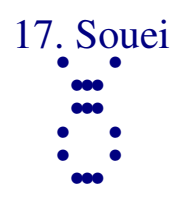

327.

Deuxième trait hexaire : s'attacher au petit enfant; perdre l' homme au bâton ${ }^{1}$.

TSHENG TSE. - Le second trait correspond sympathiquement au cinquième et s'asso cie au premier ; il suit d'abord ce qui e st près. Il est mou et faible et ne peut s'observer avec fermeté, aussi il est considéré comme objet d'avertissement et la formule pose que s'il s'attache au petit enfant, il perdra l'homme au bâton. Le premier trait positif au rang inférieur représente le petit enfant; le cinquième qui lui correspond sympathiquement en haut, selon la droiture, représente l'homme au bâton. Le second trait, si ses tendances l'attachent au premier, perdra la sympathie du cinquième trait nonaire qui lui est acquise par la droiture ; c'est là ce qu'on entend par l'expression « perdre l'homme au bâton ». S'attacher au petit garçon et perdre l'homme au bâton : négliger des sympathies légitimes et suivie ce qui n'est pas correct ; la faute est considérable. Le second trait possède les vertus de la justice et de la droiture ; il ne doit pas nécessairement en arriver à ce point; mais dans le moment où il s'agit de suivre, il était bon d'en faire l'objet d'un avertisse ment.

TSHOU HI. - Le premier trait positif, est au-dessous et proche; le cinquième trait positif correspond sympathiquement suivant la droiture, mais il est loin. Le deuxième est mou et négatif et ne peut s'observer et se préserver en attendant l'action de la sympathie légitime du cinquième, et c'est pourquoi telle est l'image symbolique. Le présage malheureux et l'appréhension de maux futurs sont évidents et il est inutile de les mentionner.

328.

S'attacher au petit enfant; ne pas se donner en même temps à plusieurs.

TSHENG TSE. - Si ce que l'homme suit est co nforme à la droiture, il éloigne le mal; s'il suit le faux, il perd le vrai ; aucune raison ne peut faire

\footnotetext{
${ }^{1}$ Tsheang fou, l'homme au bâton ; un homme âgé. La grammaire de Kaccayana cite souvent comme exemple le mot dandino, dont le sens est exactement celui du terme chinois.
} 
qu'il suive les deux à la fois. Si le second trait s'attache au premier, il perdra le cinquième ; il ne peut se donner à la fois à tous les deux. C'e st le moyen d'avertir l'homme qu'en suivant la droiture il doit s'y adon ner exclusivement. 


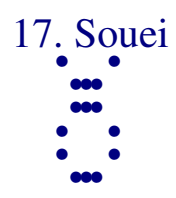

329.

Troisième trait hexaire : s'attacher à l'homme au bâton ; pérdre le petit enfant ; en suivant, sil demande il obtiendra ; avantage à demeurer dans la perfection.

TSHENG TSE. — L'homme au bâton, c'est le quatrième tr ait nonaire ; le petit enfant, c'est le premier trait. La positivité qui occupe le rang supérieur représente l'homme au bâton ; celle qui occupe le rang inférieur représente le petit enfant ; bien que le troisième et le premier trait fassent partie de la même substance, cependant le troisième est le plus rapproché du quatrième, de sorte qu’il s'attache à celui -là. En général, la malléabilité négative ne peut pas se soutenir elle-même ; ordinairement elle s'approche de ce qui est voisin et s'y attache. S'att achant en haut au quatrième, en bas elle perd le premier. Abandonner le premier pour suivre un trait placé plus haut, c'est saisir l'opportunité dans l'action de suivre. Suivre en s'élevant, c'est le bien ; c'est comme l'obscurité suivant la lumière. Suiv re le bien dans les affaires, c'est s'élever en suivant. Tourner le dos au vrai pour suivre le faux, abandonner la clarté et rechercher l'obscurité, c'est suivre en descen dant. Le quatrième trait, lui aussi, est sans correspondance sympathique, c'est celui qui n'est pas suivi ; mais près de lui il réussit à se faire suivre par le troisième; il doit donc nécessairement se montrer bon et affectueux avec lui, de sorte qu'en suivant le quatrième trait, si le troisième a quelque chose à lui demander, il l'obti endra. Pour un homme, suivre le supérieur quand le supérieur répond à ses avances, c'est obtenir ce qu'il recherche. De plus encore, tout ce qu'il recherche, il peut l'obtenir. Mais, bien qu'il en soit ainsi, on ne doit certainement pas aller contre la raison, dans une voie blâmable pour suivre un supérieur. Se servir de l'amour et de l'affection pour arriver à la satisfaction de désirs, serait le cas d'un homme inférieur qui emploie la perversion et la flatterie pour en tirer avantage. Aussi, la formule dit que l'avantage consiste à se maintenir dans la perfection. En se plaçant selon la droiture, ce qui est entendu par les mots : «en recherchant quelque chose, il l'obtiendra certainement », sera quelque chose de correct et telle sera l'action de suivre c hez l'homme doué.

TSHOU HI. — «L'homme au bâton » désigne le quatrième trait nonaire ; de même, le «petit enfant» désigne le premier trait. Le troisième trait tient de près au quatrième et il manque à ce quill doit au premier et le perd. L'image symbolique est exactement le contraire de celle du sixième trait hexaire. Le quatrième trait est momentanément investi de l'autorité et le troisième le suit ; sil recherche quelque chose il l'obtiendra. Toute fois ce n'est pas au quatrième qu'il correspond symp athiquement selon la droiture, de sorte qu'il y a quelque 
chose qui n'est point correct et qu'il est considéré comme expri mant l'intrigue de la basse flatterie. Voilà pourquoi tel est le sens divinatoire et de plus la formule avertit de demeurer dans la perfection.

330.

S'attacher à l'homme au bâton; ses tendances lui font abandonner l'inférieur.

TSHENG TSE. - Du moment où il suit le supérieur, c'est donc que ses tendances lui font abandonner l'inférieur et qu'il ne le suit pas. Abandonner l'inférieur et suivre le supérieur; abandonner ce qui est humble et suivre ce qui est élevé. Dans l'action de suivre cela constitue le bien. 


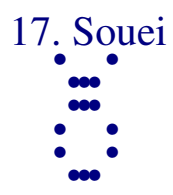

331.

Quatrième trait nonaire : en suivant, obtenir ; présage malheureux de la perfection ; avoir foi dans la voie ; employer la clarté ; quelle serait la culpabilité?

TSHENG TSE. - Le quatrième trait nonaire emploie les aptitudes de la positivité et la dureté énergique ; il est placé au point culminant de la position du sujet; s'il arrive à atteindre ce qu'il suit, il en résultera que, bien que se conformant à la droiture, le présage sera encore malheureux. "Atteindre » 1 , veut dire réussir à se faire suivre par les cours dans tout l'univers. La voie rationnelle de celui qui est sujet est de supposer que les bienfaits comme l'autorité, tout vient également du supérieur, et que le cour de la foule suit toujours le prince. Si le cour des hommes le suit lui -même, c'est une voie périlleuse et pleine d'appréhension, de sorte que le présage est malheureux. Que doit faire celui qui demeure sur ce terrain ? uniquement développer et augmenter en lui-même la sincérité et la bonne foi; ne se mouvoir que d'accord avec la voie rationnelle ; employer l'intelligence et la clarté de l'esprit pour s'y placer, et alors quelle culpabilité pourrait -il avoir ? Parmi les hommes de l'antiquité, quelques -uns ont suivi cette voie : Yi Yuen, Tsheou Kong Ming, par exemple. Chez tous, la vertu frappait le peuple, et le peuple les suivait; ce qui les mit à même de se faire suivre du peuple, c'est qu'ils complétaient l'œuvre de leur prince, et assuraient la paix de l'état ; l'extrême sincérité subsistait en eux : c'est là avoir la bonne foi. Leur action n'était jamais autrement que conforme à la voie rationnelle; c'est là ce qui est exprimé dans la formule par les mots «dans la voie». C'est uniquement par leur intelligence et la clarté de leur esprit qu’ils étaient capables de remplir ces conditions et la formule l'exprime par les mots «employer la clarté ». Quelle aurait pu être leur culpabilité ? C'est ainsi que les inférieurs ont confiance et que le supérieur est sans méfiance. La situation est au plus haut point importante sans qu'il en résulte d'abus contre le supérieur ; la force inhérente à cette situation est considérable sans que la faute, qui consiste à usurper le pouvoir, soit commise ; à moins d'être un homme saint ou un grand sage, ce résultat est impossible à atteindre. Après de tels hommes et à un degré immédiatement inférieur, on peut aussi citer Kuo Tse Yi, de la dynastie des Theang. Son autorité fut suffisante pour «ébranler et diriger en maître » sans que son prince en prît ombrage. Lui aussi agissait pour la justice et était animé par la sincérité et la bonne foi, aussi il ne commit aucune faute grave. Serait-il possible d'atteindre à ce niveau sans intel ligence et clarté d'esprit ?

\footnotetext{
${ }^{1}$ Littéralement le texte porte « avoir atteindre ».
} 
TSHOU HI. - Le quatrième trait nonaire emploie la dureté énergique et occupe le rang inférieur du koua simple supérieur : sa vertu est la même que celle du cinquième, et c'est pourquoi le sens divinatoire est qu'en suivant il arrivera à atteindre. Cependant, la force qui lui est inhérente est gênante pour le cinquième trait, aussi, bien qu'agissant avec droiture, le présage est malheureux. Ce n'est que dans le cas où il aura foi dans la voie, avec l'intelligence, que le supérieur sera tranquille et que les inférieurs le suivront ; alors il pourra être sans aucune culpabilité. Celui qui consulte le sort, dans un moment où il porte le fardeau de la puissance, doit réfléchir attentivement à cet avertissement.

332.

En suivant, obtenir; le sens est un présage malheureux. Avoir foi dans la voie; ceuvre de la clarté ${ }^{1}$.

TSHENG TSE. - Il occupe une situation proche de celle du prince et il atteint son but. Le sens est évidemment un présage malheureux. Pouvant avoir foi et étant dans la voie rationnelle, il sera sans culpabilité; c' est en effet l' ouvre de l' intelligence et de la clarté de l' esprit.

${ }^{1}$ Ou de l' intelligence. 


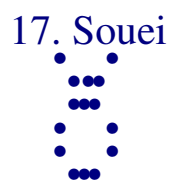

333.

Cinquième trait nonaire :foi dans le bien; présage heureux.

TSHENG TSE. — Le cinquième trait nonaire occupe le rang prééminent, il possède la droiture, et il est plein de sincérité; il exprime donc la justice et la sincérité dans l' acte de suivre le bien, et le présage heureux est évident. Le terme kia, avant-dernier caractère du texte, désigne le bien. Depuis le prince jusqu' à l' homme perdu dans la foule, pour tous également, le présage heureux, en suivant une voie, consiste uniquement à suivre le bien. La sympathie qu' il éprouve pour la justice et la droiture du second trait, placé au-dessous de lui, exprime le sens de suivre le bien.

TSHOU HI. - Dureté énergique positive, justice et droiture; en bas il sympathise à la justice et à la droiture; $c^{\prime}$ est donc celui qui a confiance dans le bien. Si celui qui consulte le sort est dans ces conditions, le présage heureux est naturel.

334.

Présage heureux de la foi dans le bien; occuper une situation avec droiture et justice.

TSHENG TSE. - Se placer dans une situation correcte et juste ; suivre la voie de la droiture et de la justice. Ce que suivent la bonne foi et la sincérité, $c^{\prime}$ est la droiture et la justice, et $c^{\prime}$ est là ce qui est entendu par le mot «bien ». Le présage heureux est évident. Le bien dans lequel il y a foi, $\mathrm{c}^{\prime}$ est ce qui est représenté par le second trait hexaire ; suivre afin d'atteindre la justice constitue le bien ; ce qui est à éviter, en suivant, c'est l'excès ou erreur. En effet, lorsque le coar suit avec pl aisir, il ne sait pas reconnaître ce qui constitue l'excès ou erreur. 


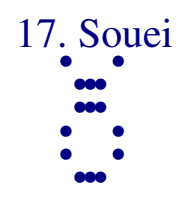

335.

Trait supérieur hexaire : s'y attacher avec opiniâtreté ; alors suivre en s'y liant ; le roi suit la erratique des offrandes sur les montagnes de l'ouest ${ }^{1}$.

TSHENG TSE. - Le trait supérieur hexaire emploie la soumission, possède la douceur malléable, et il occupe le dernier rang dans le koua qui exprime l'action de suivre, souei; c'est celui qui pousse cette action à ses dernières limites. "S'y attacher avec opiniâtreté », c'est-à-dire le dernier degré de l'action de suivre, comme attaché et retenu par un lien. a Alors suivre en s'y liant »: c'est encore suivre comme attaché à ce qu'on suit, et cela exprime que telle est la solidité du lien qui rattache à l'objet suivi. "Le roi suit la pratique des offrandes sur les montagnes de l'ouest »; l'action de suivre est poussée à ses dernières conséquences, comme ceci. Autrefois, Thae Wang pratiqua cette voie, et jouit des prérogatives royales dans les montagnes de l'ouest. Thae Wang pour se soustraire aux dangers qu'il courait du fait de barbares, abandonna le district de Pin et vint dans celui de Khi. Ceux du pays de Pin, vieillards ou jeunes gens, s'entraînaient mutuellement pour le suivre, aussi nombreux que les gens qui convergent vers un marché. En effet, telle est l'énergie de l'attachement quand le cour de l'homme le porte à suivre quelqu'un. En employant cette voie, il put librement ${ }^{2}$ exercer dans toute leur étendue les prérogatives royales dans les montagnes de l'ouest. Les montagnes de l'ouest, sont les montagne s du pays de Khi, le domaine royal de la famille de Tsheou, et c'est là en effet que le royaume fut fondé. Le trait supérieur occupe le rang extrême dans le koua souei, qui exprime l'action de suivre, ce qui constitue certainement l'excès. Cependant, quand il s'agit de réussir à ramener le peuple à suivre, et de la fermeté dans l'action de suivre le bien, c'est cette condition qui est considérée comme constituant le bien. En étendant cette extrême fermeté d'attachement à d'autres sujets, il y aurait excès.

TSHOU HI. - Ce trait occupe le rang extrême dans le koua qui exprime l'action de suivre : c'est celui qui s'attache avec tenacité à ce qu'il suit et ne peut plus s'en détacher. C'est l'extrême degré dans la sincérité de l'idée, qui permet de pénétrer librement l'intel ligence de l'esprit. C'est pour cela que le sens divinatoire est que le roi suit la pratique des offrandes sur les montagnes

\footnotetext{
${ }^{1}$ Cette formule présente un cas de l'emploi du terme heng, douzième caractère du texte, qui semble venir à l'ap pui de ce qui a été dit au $\mathrm{n}^{\circ} 278$. Si on veut conserver à ce caractère son sens propre, il faut lire : «Le roi emploie la liberté (jouit de la liberté), dans les montagnes de l'ouest. »

${ }^{2}$ Ici le commentateur lit : «Le roi emploie la liberté dans les montagnes de l'ouest. »
} 
de l'ouest. Le caractère heng, liberté, doit encore être pris pour le caractère heang de l'expression « offrir des sacrifices ». En parlant par rapport à l'état de Tsheou, les montagnes de Khi étaient situées à l'ouest. Toutes les fois qu'on consulte le sort au sujet de sacrifices à offrir à des montagnes ou à des cours d'eau, et qu'on obtient ce trait, avec une semblable sincérité d'intention, le présage sera heureux.

336.

S y attacher avec opiniâtreté ; limite extrême et finale de la supériorité ${ }^{1}$.

TSHENG TSE. - Si la fermeté dans l'action de suivre est comme celle d'un lien qui attache et retient, c'est le dernier d egré dans la voie de l'acte de suivre. TSHOU HI. — « Final », extrême limite.

\footnotetext{
${ }^{1}$ Les commentateurs lisent : « Dernier degré d'élévation. »
} 


\section{Kou}

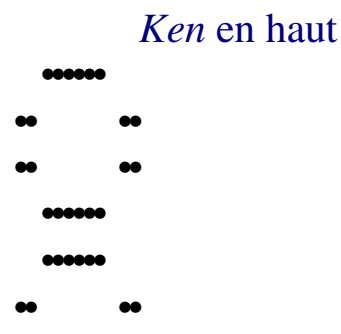

Souen en bas

341. Premier trait hexaire : être capable de supporter le poids des affaires du père; avoir des enfants; le père décédé est sans culpabilité; péril et à la fois présage heureux.

$*$

343. Deuxième trait nonaire : être capable de supporter le poids des allattes de la mère ; impossibilité de la pureté.

345. Troisième trait nonaire : être capable de supporter le poids des affaires du fière ; avoir de légers regrets ; pas de grande oedpabilité.

*

347. Quatrième trait hexaire : négliger les affaires du père ; en avançant voir les causes d'appréhension.

349. Cinquième trait hexaire : supporter le poids des affaires du père ; employer la louange.

$*$

351. Trait supérieur nonaire : ne servir ni roi, ni prince feudataire ; estimer hautement leurs affaires.

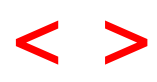




\section{$\mathrm{N}^{\circ} 18$. Kou.}

TSHENG TSE. - Kou. «L'Ordre des koua » dit : «Puisqu'il y a plaisir à suivre un homme, il doit nécessairement y avoir un motif, c'est pourquoi le koua souei est suivi du koua kou. » Cette définition tient compte du sens des deux koua précédents pour déduire les causes dc l'ordre adopté. En effet, lorsqu’il y a plaisir et satisfaction à suivre quelqu'un, il doit nécessairement y avoir un motif; s'il n'y en avait pas, pourquoi y aurait -il satisfaction ? Pourquoi suivrait-on ? C'est pour cela que le koua kou suit immédiatement le koua souei. Kou signifie affaire, chose, motif ; kou n'indique pas l'explic ation d'une affaire, mais l'idée d'avoir une affaire. Il est composé du koua simple de la montagne au-dessous duquel il y a le koua simple du vent. Le vent est au-dessous, ou en bas de la montagne ; il rencontre la montagne et revient en sens opposé, de sorte que toutes choses sont bouleversées, et c'est là ce qui constitue l'image symbolique exprimée par le mot kou Le sens de ce mot est détruire, mettre en désordre. La forme graphique du caractère comprend deux parties, l'une supé rieure, l'autre inférieure ; la première représente un amas d'insectes ; la seconde représente un vase, boîte ou bol ; un vase rempli d'un amas dinsectes, ce qui donne le sens de destruction. 1.e commentaire traditionnel de Tso Shi dit: «Le vent tombe devant la montagne; la fille affole le garçon; puisque la fille adulte s'abaisse devant lejeune garçon, c'est le renversement des sentiments. Le vent rencontre la montagne et revient sur lurntéme; toutes choses sont secouées et bouleversées. Ceci est considéré comme exprimant l'image symbolique d'existence de sujets de trouble ou d'inquiétudes, et c'est pourquoi le koua $\mathrm{Rw}$ exprime les causes d'inquiétudes. » Lorsqu'il y a des causes d'inquiétudes et qu'on pourvoit aux nécessités qui en résultent, c'est encore « une affaire », cause, ou motif. Si on en parle au point de vue de l'image symbolique du koua, c'est le moyen par lequel se produisent les affaires (troubles) ; si on en parle d'après les aptitudes indiquées par les koua simples, c'est le moyen par lequel on répare le trouble. 


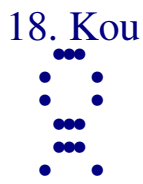

337.

Kou, affaires ; parfaite liberté ; avantage à traverser un grand cours d'eau ; trois jours avant le commencement ; trois jours après le commencement.

TSHENG TSE. - Du moment où il y a cause de trouble, il y a lieu de revenir à l'ordre ; depuis l'antiquité, l'ordre a toujours nécessairement été la conséquence de troubles, parce que les troubles ouvrent la voie au rétablissement de l'ordre : c'est là une loi naturelle. Avec les aptitudes indiquées par les koua simples, appliquées au rétablissement de l'ordre troublé, il sera possible d'arriver à une absolue liberté d'action. Ce qu'il y a de plus important entre toutes les affaires, causes de trouble (kou), c'est de traverser en y remédiant, les dangers et les difficultés, les périls et les obstacles du moment présent; c'est pour cela que le texte dit : avantage à traverser un grand cours d'eau. Le terme kia, neuvième et treizième caractère du texte, exprime le point de départ des nombres ou d'une série; le commencement des choses ; par exemple, comme dans la série des moments $k i a$, yi, etc. ; le degré kia, ou premier degré, expression employée dans la classification aux concours publics; le document, ou l'expédition kia, pour primata. C'est tou jours l'indication du premier terme d'une série, ou l'origine d'une affaire. La voie rationnelle pour remédier aux causes de troubles est de penser et de méditer à ce qui précède ou suit, trois jours avant et trois jours après. En effet, rechercher ce qui précède et déduire les conséquences, c'est la voie logique pour que le remède au mal soit efficace et d'un effet durable. Avant le commencement, c'est-à-dire avant le moment présent, avant telle chose ; il s'agit de l'analyse des causes na turelles du fait. Après le commencement, c'est-à-dire, après ceci, après telle chose ; il s'agit de la méditation sur les conséquences naturelles d'un fait. Un jour, deux jours, jusqu'à trois jours ; cela exprime la profondeur de la méditation, l'étendue de la portée des recherches. En analysant les causes naturelles qui ont amené le fait, on reconnaît la voie à suivre pour y porter remède; en méditant sur les conséquences naturelles du fait, on reconnaît les moyens à employer pour les prévenir et s'en préserver. Si le remède appliqué est bon, les inconvénients primitifs peuvent être supprimés ; si les mesures préventives sont bonnes, leur effet utile peut se faire sentir pendant longtemps. Telle fut la règle des saints rois de l'antiquité pour rénover le monde et léguer leurs exemples aux générations. Ceux qui dans les temps postérieurs eurent à remédier à des causes de trouble, ne saisirent plus clairement le précepte de l'homme saint contenu dans ces mors : avant le commencement, après le commencement; leurs méditations furent superficielles et ne s'appliquèrent qu'aux choses prochaines, aussi, malgré leurs efforts pour le bien de leur époque, le désordre 
ne fut jamais supprimé ; leur œuvre ne put jamais s'accomplir entièrement, et le germe de maux futurs persistait toujours. Le mot kia exprime le commencement, ou point de départ des choses ; le mot kheang exprime le commencement de la modification, de l'altération et du renouvellement. Lorsquil s'agit de documents administratifs ou légis latifs, on emploie le terme $k i$, pour désigner le point de départ; s'il s'agit de lancer des ordonnances ou de promulguer des instructions, on emploie le terme kheang, dans le même cas, kheang veut dire changer, renouveler, et il indique qu'il y a quelque chose à modifier ou à transformer.

TSHOU HI. - Kou, le délabrement est à son comble et il y a des affaires ${ }^{1}$. La dureté énergique du koua simple ken occupe la position supérieure; la douceur malléable du koua simple souen occupe la position inférieure. Le haut et le bas ne s'allient point ; en bas humilité et soumission, et en haut arrêt momentané. C'est pour ces raisons que le koua est appelé kou et quill est considéré comme exprimant les motifs de trouble. On a dit : La dureté énergique qui s'élève et la douceur malléable qui s'abaisse, sont des observations qui se rapportent à la transformation du koua partait, considéré comme provenant du koua pi (22), dans lequel le premier trait monte tandis que le second descend ; ou comme provenant du koua sheng (46), dans lequel le cinquième monte et le trait supérieur descend; ou enfin comme provenant du koua hi tsi (63), dans lequel ces mêmes circonstances se trouvent réunies et où, de même, la dureté énergique monte tandis que la douceur malléable descend. Dans ces trois cas, ce même changement produit toujours le koua kou. Ce mot exprime la dégradation ou la détérioration arrivée à son comble, le moment où le désordre est sur le point de se changer en ordre, et c'est pourquoi le sens divinatoire est « liberté absolue » et «avantage à traverser un grand cours d'eau ». Le mot kia exprime le premier jour dans le temps, l'origine des choses. Trois jours avant lejour kia, c'est le jour sin; troisjours après le jour kia c'est le jour ting. La chose qui précède a dépassé le juste milieu, et entre en décadence, de sorte qu'elle doit se rénover d'elle même en devenant l'origine de la chose qui suivra, sans qu'elle arrive à une extrême des truction. La chose suivante est à son commencement et encore nouvelle. Cependant il convient avant tout d'arriver à l'idée de paix et de repos, afin d'examiner les défauts de la chose précédente et de ne pas s'exposer à retomber rapidement dans une nouvelle période de décadence: tel est l'avertissement profond que donne l'homme saint.

338.

1 En chinois, ce mot comporte toujours, dans de tels cas, un sens fâcheux: Sujet d'inquiétudes, troubles. guerres, etc. 
Le cammeamire traditionnel de la formule déterminative dit: Km, affaires; la dureté énergique en haut, la douceur malléable en bas ; humilité et arrêt : affaires.

TSHENG Tse. - Il est question de la modification du koua et du sens indiqué par les deux substances des doua simples. «Dureté énergique en haut et douceur malléable en bas »; cela exprime que le premier trait romane du koua simple Adieu monte et devient le trait supérieur nmaire du koua, tandis que le trait supérieur hexaire du koua simple khouen descend et devient le premier trait hexaire du koua $\mathrm{km}$. La dureté énergique positive, c'est ce qui est prééminent et élevé ; actuellement, elle va occuper le rang supérieur. La douceur malléable négative, c'est ce qui est humble et inférieur ; or, actuellement, elle occupe le rang inférieur ; le garçon, bien que jeune, occupe la position supérieure ; la fille, bien qu'adulte, est cependant au-dessous de lui. L'éminence et l'humilité sont cor rectement observées ; le haut et le bas sont établis conformément à la raison d'être des choses : c'est la voie pour arriver à régulariser ce qui este, désordre. Parle mouvement ascensionnel de la dureté énergique et le mouvement descendant de la douceur malléable, les deux koua simples se modifient et deviennent les koua simples ken et soucie ; le koua simple ken exprime l'arrêt ; le koua simple souen, la soumission; l'inférieur est sou mis et le supérieur s'arrête : arrêt dans la soumission et l'humilité. Corriger les causes de trouble par lavoie de la soumission et de l'humilité, c'est employer la « liberté absolue ».

TSHOU HI. - Explication du sens du nom du koua au moyen de la substance et de la modification de ce koua et des vertus des koua simples. En effet, dans ces conditions, les fautes s'accumulent et arrivent à produire le désordre (kou).

339.

Liberté absolue dans le trouble et ordre régnant dans l'univers ; avantage à travers un grand cours d'eau ; en entreprenant il y a des difficultés ; trois jours avant le commencement, trois jours après le commencement ; à la fin il y aura commencement; action du ciel.

TSHENG TSE. - Voie rationnelle de régularisation en cas de trouble ; avec les aptitudes exprimées par les koua simples, on jouira d'une liberté absolue et l'univers sera bien gou verné. Or pour régulariser ce qui est en désordre, si on peut faire que le devoir résultant de l'éminence et de l'humilité, de la supériorité et de l'infériorité, soit correctement observé, les inférieurs seront humbles et soumis ; les supérieurs pourront les retenir et les 
organiser, pacifier et établir. Toutes choses étant immobilisées dans la soumission, quelle cause de trouble pourrait encore surgir sans être aussitôt supprimée ? Cette voie est celle de la grandeur dans le bien et de la liberté ; ainsi, l'univers sera en ordre et bien gouverné. À l'instant où survient un moment de trouble et de décadence dans l'univers, il importe de s'exposer à traverser les difficultés et les obstacles pour agir et y remédier ; c'est là entre prendre et avoir à faire ${ }^{1}$. Or, ce qui a un commencement doit nécessairement avoir une fin ; du moment où quelque chose a une fin, elle doit nécessairement avoir un commencement : c'est la voie du ciel ${ }^{2}$. L'homme saint connaît cette voie de fin et de commencement; aussi, il peut remonter à l'origi ne primitive et analyser les causes naturelles d'un fait, comme il peut en préciser la fin nécessaire et prévoir toutes ses conséquences logiques. .Avant le commencement, après le commencement, il en fait l'objet de sa méditation, et est par là qu'il peut régulariser les causes de trouble et arriver à une liberté absolue.

TSHOU HI. - Explication de la formule du koua. Régulariser les causes de trouble et arriver à une liberté absolue, de sorte qu'il y a l'image symbolique du désordre redevenant ordre. la fin du désordre est le commencement de l'ordre : c'est comme le mouve ment de translation di, ciel.

340.

Le commentaire traditionnel de la formule symbolique dit: En bas de la montagne il y a du vent; trouble. $L^{\prime}$ homme doué donne l'impulsion au peuple et nourrit sa vertu.

TSHENG TSE. - En bas de la montagne il y a du vent; le vent rencontre la montagne et revient en sens inverse, de sorte que toutes choses sont dispersées et en désordre, ce qui est considéré comme l' image symbolique de la présence de causes de trouble (kou). L' homme doué considère cette image symbolique de la présence de causes de trouble, et il l' applique à la pratique de l' assistance du peuple en lui donnant l' impulsion et en entretenant ses propres vertus. Par rapport à lui-même, l' actin consiste à développer ses propres vertus; par rapport à l' univers, elle consiste à secourir les peuples. Entre tout ce qui incombe à l' homme doué, il n' y en a pas de plus importantes que les deux choses qui sont mentionnées ici.

TSHOU HI. - En bas de la montagne il y a du vent: les êtres sont bouleversés et il y a des causes de difficultés. Mais parmi toutes les difficultés qui peuvent naître, il n' y en a pas de plus graves que ces deuxà; c' est la voie qui consiste à se gouverner soi-même et à gouverner les hommes.

\footnotetext{
${ }^{1}$ Le texte est dénaturé.

${ }^{2}$ La voie révélée par l'ordre du ciel, des phénomènes célestes.
} 


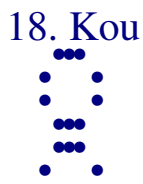

341.

Premier trait hexaire : être capable de supporter le poids des affaires du père; avoir des enfants; le père décédé est sans culpabilité; péril et à la fois présage heureux ${ }^{1}$.

TSHENG TSE. - Le premier trait hexaire, bien qu' il occupe le rang le plus bas, est cependant celui qui détermine la formation du koua ${ }^{2}$; il comporte donc le sens de diriger en qualité de maître. Demeurer à l' intérieur, être dans un rang inférieur, et être le maître, $c^{\prime}$ est être le fils capable a servir de tuteur aux affaires de son père. Dans la voie du fils servant de tuteur aux affaires de son père, être capable de diriger ses affaires, ce sera avoir les qualités d' un fils ${ }^{3}$ et faire que le père puisse être sans culpabilité. Le cas contraire, ce sera faire retomber sur le père la conséquence de sa propre incapacité. $\mathrm{C}^{\prime}$ est pour cela qu' il faut nécessairement la préoccupation des périls éventuels, et alors il sera possible d' atteindre à une fin heureuse. Être placé dans un rang humble et être celui de qui dépendent les affaires d' une personne prééminente, doit naturellement inspirer de l' appréhension et de la crainte; avec les aptitudes inhérentes aux traits hexaires, bien que soumis et humble, comme la substance dont il fait partie exprime la malléabilité négative, comme il est en bas, sans sympathies, et qu' en même temps il dirige et sert de tuteur, il ne comporte pas le sens de capacité de remédier aux difficultés de sa position. Mais, si on en parlait au point de vue de l' inaptitude à servirde tuteur, le sens serait très restreint, et $c^{\prime}$ est pourquoi il est exclusivement question de la voie du fils tuteur des affaires de son père. Il faut absolument qu' il soit capable de traverser les difficultés de la position, et alors il n' est pas cause qudes suites de son incapacité retombent sur son père. Pouvant avoir de l' appréhension, il lui sera possible d' arriver à une fin heureuse. C' est là considéé en entier la grande règle du fils tuteur des affaires du père ${ }^{4}$.

\footnotetext{
${ }^{1}$ Tel est à peu près le sens admis par tous les commentateurs chinois. Cependant, il est très discutable. En admettant le sens du mot kou tel qu' il est déterminé par ce qui précède, et dans l' ignorance de la lecture des cmmentateurs, on lirait certainement: " Être capable de supporter les causes de trouble qui incombent au père; avoir des enfants; vieillir sans culpabilité. »

${ }^{2}$ Au point de vue de la transformation des koua, par permutation des traits.

${ }^{3}$ Littéralement le texte porte « avoir enfant ». Le commentateur emploie les mêmes mots yeou tse et leur donne une toute autre valeur, très arbitraire.

${ }^{4}$ Pour comprendre le commentateur, il faut se reporter à l' importance que les Chinois attachent à la continuation de la lignée. Le fils qui meurt sans enfants est la cause qui fait que le culte dû aux mânes des aïeux est interrompu.
} 
TSHOU HI. — « Tuteur »; comme le tronc de l' arbre auquel sont attachés les rameaux et les feuilles et qui les soutient. Dans ce cas le mot kou désigne la suite des choses relatives à des personnes qui ont existé antérieurement et déjà disparues; c' est pour cela que tous les traits présetent l' image symbolique du père ou de la mère. Si le fils est capable de servir de tronc, ou tuteur, il pourvoit aux soins, améliore et donne l' impulsion qui fait surgir. Dans le cas du premier trait hexaire, les causes de trouble ne sont pas encore profondes et il est aisé d' y remédier, aussi le sens divinatoire est « avoir des enfants », qui pourront remédier aux causes de trouble et le père décédé est à l' abri de toute faute. Mais cepechant, le cas est encore périlleux. La formule avertit donc celui qui consulte le sort qu' il convient qu' il en soit ainsi. De plus, connaître le péril et en être averti, indique une fin heureuse.

342.

Etre capable de supporter le poids des affaires du père; idée de servir le père décédé ${ }^{1}$.

TSHENG TSE. - Dans la voie rationnelle du fils tuteur des affaires du père, l' idée conste à succéder au père dans les devoirs qui lui incombent; c' est pour cela qu' il faut respec attentivement ces devoirs, afin de mettre le père à l' abri de toute faute. Avec une constante apṕchension et circonspection, à la fin on arrivera au bonheur. Épuiser la sincérité attentive au sujet des affaires du père, $c^{\prime}$ est la voie du bonheur.

\footnotetext{
${ }^{1}$ Ici encore, la lecture la plus naturelle serait: «Etre capable de supporter les causes de trouble qui incombent au père. »
} 


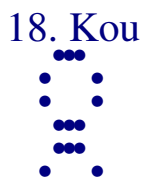

343.

Deuxième traitnonaire : être capable de supporter le poids des a(lattes de la mère ; impossibilité de la pureté.

TSHENG TSE. - Le second trait nonaire est énergique et positif ; il représente l'objet des sympathies du cinquième trait hexaire. Il exprime donc l'emploi des aptitudes de la dureté énergique positive, le séjour dans un rang inférieur, et la capacité de supporter le poids des affaires de la malléabilité négative placée au-dessus de soi. C'est pour cela que la formule choisit le sens du fils servant de tuteur aux affaires de la mère. Le sujet doué d'énergie positive assistant un prince faible et doux, serait encore un sens très voisin. Le second trait fait partie de la substance du koua simple qui exprime l'humilité et il occupe un rang qui comporte la douceur malléable, le sens de soumission domine ; c'est la voie du tuteur des affaires de la mère. Or, lorsqu'il s'agit des devoirs du fils envers la mère, il convient qu'il emploie la douceur et l'humilité pour l'assister et la guider, de telle sorte qu'il agisse conforméme nt au devoir. S’il manque de condescendance et qu'il en résulte la fâcheuse issue de ses affaires, ce sera la faute de ce fils. Mais encore qu'il soit porté à l'indulgence et à une condescendante obéissance, comment serait il possible qu'il n'y eût pas de vuie déterminée dont il ne devrait pas s'écarter ? Si on interprète cette formule au sujet de la femme, il est évident qu'il s'agit de douceur malléable et négative. Or, en se handant soi-même dans la voie de la dureté énergique, en se décidant avec une décision hâtive ne tenant compte que de ses propres impulsions, le fils se montrera ingrat du bienfait quil a reçu; le mal qui en résultera sera grand. Mais alors quelle voie peut-il donc adopter? Simplement celle qui consiste à se plier soi-même en faisant abstraction de ses propres idées, à tendre à se substituer dans le soin de ces affaires avec humilité et conciliation, de telle sorte qu'il soit personnellement correct et que les affaires soient en ordre. C'est pour cela que la formule dit «impossibilité de la pureté », c'est-à-dire qu'il ne doit pas eue abso fument parfait et rigide dans l'observation absolue de la voie de l'énergie et de la rectitta de. En agissant ainsi il suit la voie dujuste milieu. Mais encore, comment serait-il possible que cette voie l'amenât à faire quelque chose d'un ordre très élevé ? Cela ne sera possible que s'il s'agit de suivre la voie du juste milieu au sujet du service d'un prince faible et mort, en épuisant la sincérité et en poussant la fidélité à ses dernières limites. Comment encore serait-il possible de conduire à faire quelque chose de grand ? Seulement en étant un saint tel que Tsheou Kong et en assistant un prince tel que Tsheng Wang ; ce dernier prince n'était pas au plus haut point faible et doux, mais cepend ant il 
sut l'amener à être un roi accompli ${ }^{1}$, il arrêta là son ouvre ; en se maintenant dans le soin de l'accomplissement des devoirs et en ne manquant point à la voie morale cela est possible. Il est bien évident que cette voie ne peut conduire à accomplir les travaux qui illustrèrent Hi, Hoang, Yao, et Shouen. Ie second trait tient de l'humilité par sa substance, et il possède la justice ; il exprime la capacité de soumission et d'humilité et la possession d'une voie de juste milieu; cela est d'accord avec le sens des mot: «impossibilité de la perfection. C'est la conformité à la voie rationnelle à suivre pour servir de tuteur aux affaires de la mère.

TSHOU HI. - Le second trait nonaire exprime la justice énergique et dure ; en haut il sympathise avec le cinquième trait hexaire; c'est l'image symbolique du fils tuteur des affaires de sa mère et qui se conforme à lajustiee. Il emploie la dureté énergique pour se substituer à la douceur malléable, et il en corrige les défauts ; aussi la formule avertit encore quil ne doit pas déployer une parfaite fermeté, c'est-à-dire quil convient qu'il soit humble en s'insinuant dans cette voie.

344.

Etre capable de supporter le poids des affaires de la mère; se conformer à la voie de la justice.

TSHENG TSE. - Le second trait se conforme à la voie de la justice et ne commet point d'excès d'énergie ; c'est le bien dans l'action du tuteur des affaires de la mère.

\footnotetext{
${ }^{1}$ Jeu de mot sur le vocable de ce prince.
} 


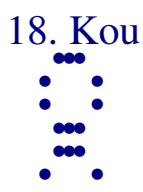

345.

Troisième trait nonaire : être capable de supporter le poids des affaires du fière; avoir de légers regrets ; pas de grande oedpabilité.

TSHENG TSE. - Le troisième trait emploie les aptitudes de la dureté énergique positive ; il occupe le rang supérieur dans le koua simple inférieur ; c'est celui qui agit en maître en servant de tuteur. Lorsque le fils qui sert de tuteur aux affaires de son père emploie les qualités positives, se maintient dans l'usage de la dureté énergique, et manque dejustice, il commet un excès d'énergie. Mais dans ce cas, cependant, il fait partie de la substance de l'humilité ; bien que son énergie soit excessive, il n'est cependant pas dépourvu de soumission. Servir avec soumission c'est la base de l'affection. De plus, lc rang qu'il occupe comporte la droitu re, de sotte qu'il ne commet pas de grandes fautes. Employant les aptitudes de la dureté énergique positive, et capable de supporter le poids des affaires, bien quill commette un excès de dureté énergique, il a fort peu de regrets et, finalement, il ne commet aucune faute grave. Toutefois, il a quelques légers regrets, parce quil n'a pas bien servi ses parents.

TSHOU HI. - Excès dans la dureté énergique, sansjustice; aussi il a un peu de regrets. Mais sa substance est l'humilité et il a un peu de droiture, de sorte quil ne commet pas de grandes fautes.

346.

Etre capable de supporter le poids des affaires du père; à la fin pas de culpabilité.

TSHENG TSE. - Employant les aptitudes du troisième trait pour servir de tuteur des affaires du père, bien qu'il y ait un peu de regrets, finalement il ne commet pas de grandes fautes. En effet, énergique et décidé, il est capable de servir de tuteur ; il ne manque pas à la droiture et il a de la soumission, ce qui fait qu’à la fin il est sans culpabi lité. 


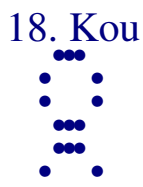

347.

Quatrième trait hexaire : négliger les affaires du père ; en avançant voir les causes d'appréhension.

TSHENG TSE. - Le quatrième trait est négatif et occupe un rang négatif; ses aptitudes sont celles de la mollesse et de la soumission. La manière dont il se place est conforme à la droiture et c'est pour cela qu'il est considéré comme l'image symbolique de celui qui est indulgent et large dans le soin des affaires du père. Or, avec les aptitudes de la douceur et de la soumission, et se plaçant suivant la droiture, c'est tout au plus s'il peut s'observer et se maintenir en suivant le courant des circonstances ordinaires ; sil entreprend de sup porter le poids d'affaires d'une importance dépassant le niveau moyen, il sera incapable d'en venir à bout, et les causes d'appréhension sont évidentes. Employant la douceur négative et dépourvu d'assistance et de sympathie, s'il entreprend quelque chose, com ment pourrait-il en venir à bout?

TSHOU HI. - Employant ses qualités négatives, il occupe un rang négatif; il ne peut pas atteindre à un résultat effectif: c'est l’image symbolique de l'indulgence en dirigeant des affaires. Dans de telles conditions, il arrivera que les affaires en question péricliteront journellement, de sorte qu'en entreprenant quelque chose, les motifs d'appréhension sont visibles. C'est un avertissement à celui qui consulte le sort qu'il ne doit pas agir ainsi ${ }^{1}$.

348.

Négliger les affaires du père ; entreprendre sans réussir.

TSHENG TSE. - En employant les aptitudes du quatrième trait, il sera encore possible de se maintenir au niveau des choses ordinaires et pendant une période d'indulgence.

\footnotetext{
${ }^{1}$ La traduction ne suit pas l'explication des commentateurs chinois. Le terme du texte a aussi le sens de «négliger, ajourner».
} 
Mais s’il veut atteindre le résultat d'une chose qu'il entreprend, il n'y réussira pas. Le poids de la responsabilité augmentant, il ne sera plus capable de la supporter. 


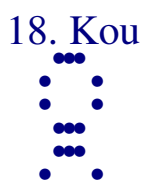

349.

Cinquième trait hexaire : supporter le poids des affaires du père ; employer la louange.

TSHENG TSE. - Le cinquième trait occupe la situation prééminente ; avec les caractères de la douceur malléable négative, il lui incombe d'être le soutien des affaires du prince, et en bas il correspond sympathiquement au second trait nonaire, c'est-à-dire qu'il peut confier le poids de l'autorité à un sujet énergique et positif. Bien que capable de s'abaisser par sympathie vers le sage énergique et actif, et de lui déléguer l'autorité, cependant il est en réalité, lui-même, mou et inactif, de sorte qu'il ne peut rien commen cer ni jeter les fondations de quoi que ce soit ; il est seulement apte à continuer les anciennes traditions. C'est pour cela que ce trait est considéré comme supportant le poids des affaires du père. Or, lorsqu'il s'agit d'affaires telles que de fonder une chose pour léguer à la postérité, il est impossible d'en venir à bout sans les aptitudes de l'énergie et de l'intelligence. Le prince appelé à succéder sur un trône établi, bien que doué de qualités faibles et molles, peut, s'il sait investir de l'autorité un homme sage et énergique, continuer dignement la postérité de ses ancêtres et mériter des louanges. Thae Kia, Tsheng Wang, furent tous les deux loués à cause des actes de leurs ministres.

TSHOU HI. - Mollesse et justice occupant le rang prééminent ; d'ailleurs le second trait nonaire le sert par ses vertus. Dans ces conditions, soutenir le poids des affaires peut conduire à la renommée, et c'est pourquoi tels sont le sens divinatoire et l'image symbo lique.

350.

Supporter le père et employer la louange ; succèder par la vertu ${ }^{1}$.

TSHENG TSE. - Supporter le poids des affaires du père ${ }^{2}$ et agir de façon à avoir des louanges. C'est parce quiil est assisté par le sage placé en bas, qui le sert et le supplée par ses vertus énergiques et justes.

${ }^{1}$ Ou servir par la vertu.

${ }^{2}$ Ce n'est pas dit dans le texte. 


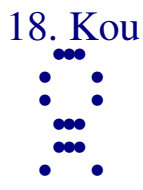

351.

\section{Trait supérieur nonaire : ne servir ni roi, ni prince feudataire ; estimer hautement leurs affaires.}

TSHENG TSE. - Le trait supérieur nonaire est placé à la fin du koua hou ; il est sans attaches sympathiques au-dessous de lui, et placé en dehors des affaires ; c'est un terrain où il n'a point d'affaires à traiter. Employant les aptitudes de l'énergie et de l'intelligen ce, sans assistance ni accueil, et placé sur un terrain où il n'y a pas d'affaires à traiter, c'es $t$ un sage et un homme doué, qui ne se conforme pas aux goûts du moment, estimant surtout la pratique obscure du bien et l'observation de soi-même, sans s'immiscer dans les affaires de son temps. C'est pour cela que la formule dit « ne servir ni roi, ni prince feudataire, estimer hautement ses affaires $»{ }^{1}$. Parmi les hommes de l'antiquité, il y en eut qui agirent ainsi ; tels furent Yi Yuen, Thae Kong, Wang Tshi Shi, Tsang Tse, et Tse Se. Ils ne firent point plier la morale pour s'accommoder aux idées de leur temps, et dès quills ne purent plus faire prévaloir leur influence dans l'empire, ils s'occupèrent de l'améliora tion de leur propre individu, en réservant toute leur estime pour cette occupation, et en ne se préoccupant plus que de suivre leurs propres tendances et les préceptes de la morale. Mais encore, la voie de l'homme cultivé, relative à l'estime de soi-même, n'est pas unique. Elle peut consister à s'attacher à la vertu de la voie morale, sans rien concéder au temps, et en se préservant soimême pour se garder de toute corruption ; elle peut consister à connaître la voie de la modération, à se retirer à l'écart afin de se préserver soi -même ; elle peut consister à mesurer ses aptitudes, à apprécier sa condition, et à s'en contenter sans rechercher la renommée ; elle peut consister à s'observer soi même avec fermeté de sentiments, sans prendre souci des affaires du monde, et en ne s'attachant qu'à se perfectionner personnellement. Quoique le terrain sur lequel chacun se place présente des inégalités par ses avantages ou ses inconvénients, le choix en résulte toujours de la haute importance attachée aux soins de ce qui concerne personnellement ; ce que le commentaire symbolique exprime par les mots «tendances pouvant faire règle». C'est le mouvem ent en avant, ou de recul, réglé par la voie morale.

TSHOU HI. - La dureté énergique occupe le rang le plus élevé ; elle est en dehors des affaires dont il s'agit; c'est pourquoi telle est l'image

\footnotetext{
${ }^{1}$ Le texte porte $k h i$; c'est un démonstra tif, il peut remplacer un possessif, mais il faut que le sens linndique. Ici, tel n'est pas le cas. Le sens le plus naturel est de rapporter khi à ce qui précède, c'est-à-dire $\grave{a}$ « servir roi ou prince ».
} 
symbolique, et le sens divinatoire, comme l'avertissement, s'y trouvent également contenus.

352.

Ne servir ni roi, ni prince feudataire; tendances pouvant faire règle.

TSHENG TSE. - Être, comme le trait supérieur nonaire, placé en dehors des affaires ; ne pas dépendre des événements de son époque; ne point servir comme ministre ni roi, ni prince feudataire ; c'est, en effet, avancer ou reculer toujours selon la voie morale, et employer ou rejeter suivant le moment : cela est-il possible à d'autres qu'au sage ? Les tendances qu'il maintient, peuvent être prises pour règles ou préceptes. 


\section{Lin}

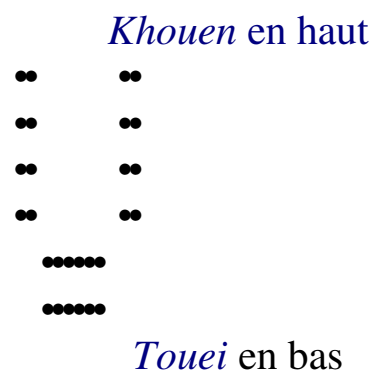

353. Lin surveiller ; grande liberté, perfection avantageuse ; parvenu au huitième mois il y a présage malheureux.

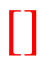

359. Premier trait nonaire : surveiller et émouvoir ; présage heureux de la pureté.

$*$

361. Deuxième trait nonaire : surveiller en émouvant; présage heureux, rien sans avantage.

$*$

363. Troisième trait hexaire : se plaire à surveiller; aucun moyen d'avantage ; en avoir déjà du chagrin ; pas de culpabilité.

$*$

365. Quatrième trait hexaire : extrême surveillance ; pas de culpabilité.

$*$

367. Trait supérieur hexaire : grandeur dans la surveillance ; présage heureux sans culpabilité.

*

369. Trait supérieur hexaire : grandeur dans la surveillance ; présage heureux sans culpabilité.

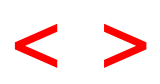


$\mathrm{N}^{\circ}$ 19. Lin.

TSHENG TSE. — Lin. «L'Ordre des koua » dit : « Ce n'est qu'après que les difficultés sont révélées que la grandeur est possible ; aussi le koua kou est suivi du koua lin : lin, grandeur ${ }^{1}$. Le koua kou exprime les affaires, ou causes de trouble ; lorsqu'il y a des affaires, la grandeur peut se révéler ; c'est pourquoi le koua kou est immédiatement suivi du koua lin. Le Han khang po ${ }^{2}$ dit : «La possibilité de la grandeur dans une carrière naît par les difficultés qu'elle présente. » Les deux positivités sont nouvellement développées et ont acquis toute leur grandeur ${ }^{3}$, c'est pour cela que le koua est appelé lin, et considéré comme représentant la croissance, ou grandeur. Il est composé du marais, au-dessus duquel il y a la terre ; la terre qui est au-dessus du marais ; le rivage. Le rivage et l'eau sont mutellement en contact ; regarder de près l'eau ${ }^{4}$, et c'est pourquoi le koua est appelé lin. Parmi les choses ou êtres qui remplissent l'univers, le contact n'est jamais aussi inti me qu'entre l'eau et la terre, de sorte que l'eau sur la terre constituera le sens de « contact», «association » 5 , et que la terre au-dessus du marais donnera le sens de «surveiller de près (lin) ». Lin, surveiller de haut; surveiller le peuple; surveiller des affaires. Le terme est applicable à tout ce qui peut être surveillé. Dans le koua c'est le sens de « surveiller d'en haut ce qui est au-dessous » qui est adopté ; c'est le sens de l'expression lin min, surveiller le peuple (le gouverner).

\footnotetext{
${ }^{1}$ Littéralement « quand il y a des affaires, alors la grandeur est possible ». Le mot « affaires » doit être pris dans l'acception de « difficultés».

${ }^{2}$ Paraît être le titre d'un ouvrage, plutôt qu'un nom propre.

3 «Nouvellement» parce que le koua est supposé se former de bas en haut et que le koua simple touei est considéré comme provenant du koua simple khouen.

${ }^{4}$ Lin signifie aussi surveiller, regarder.

${ }^{5} \mathrm{Pi} ;$ koua $\mathrm{n}^{\circ} 8$.
} 


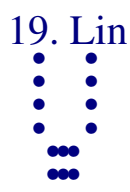

353.

Lin surveiller; grande liberté, perfection avantageuse ; parvenu au huitième mois il y a présage malheureux.

TSHENG TSE. - En parlant au point de vue des aptitudes des koua simples, si la voie à suivre dans l'ac te de la « surveillance » est comme ces aptitudes, cette voie présentera une grande liberté et sera conforme à la droiture. Les deux positivités sont nouvellement développées, en bas ; c'est donc le moment où la voie naturelle de la positivité tend à devenir florissante. L'homme saint en fait à l'avance l'objet d'un avertissement et dit : bien que la positivité vienne seulement de se développer, quand arrivera le huitième mois, sa voie naturelle sera épuisée ; c'est là ce qui rend le présage malheureux. En général, lorsque l'homme saint donne un avertissement, c'est toujours au moment où la prospérité commence à florir ; en prévoyant la décadence au moment où la prospérité est florissante, il sera possible de prévenir les conséquences de son extrême développement, et d'aviser à la rendre permanente et durable. Si l'avertissement venait après que la décadence a commencé, il serait sans portée et il ne s'appliquerait plus. Depuis l'antiquité, la paix et l'ordre dans l'univers n'ont jamais duré longtemps san s que naisse le désordre ; c'est qu'en effet l'avertissement n'avait pas été écouté pendant la prospérité. Au moment où la prospérité devient florissante, on ne sait se mettre en garde contre les retours de la fortune, de sorte qu'on s'habitue au bien-être et à la richesse, et que l'orgueil et la prodi galité naissent ; on donne libre cours aux instincts du plaisir et du dérèglement, et les règles fondamentales de la société se corrompent; oubliant les malheurs et les troubles, les germes de la discorde et des dissensions se développent. Il en est ainsi parce que noyé dans le désordre des passions on ne sait pas reconnaître quel sera le résultat de ce désordre.

TSHOU HI. - Lin exprime l'action d'avancer et d'empiéter en refoulant toutes choses. Les deux positivités croissent et grandissent en refoulant la négativité, c'est pour cela que le koua est appelé lin. C'est le koua du douzième mois. De plus, comme hexagramme, il est formé du koua simple touei, satisfaction, en bas, et du koua simple khouen, passivité, en haut. Le second trait nonaire emploie la dureté énergique et se maintient dans la justice ; en haut il sympathise avec le cinquième trait hexaire, aussi, le sens divinatoire est grande liberté et avantage dans la droiture. Cependant pendant huit mois il devra exister un présage malheureux. L'expression « huit mois », veut dire depuis le mois correspondant au koua fou, qui ne comporte qu'une seule positivité, jusqu'au mois qui correspond au koua thouen, qui n'a que 
deux traits négatifs ${ }^{1}$, c'est-à-dire au temps où la négativité se développe et où la positivité s'évanouit. On a dit aussi que les termes j' fl désignent spéciale ment le huitième mois de l'été, qui correspond au koua kouan et qui est aussi l'antithèse du koua lin. D'ailleurs c'est le sens divinatoire lui-même qui constitue l'avertissement.

354.

Le commentaire traditionnel de la formule déterminative dit: Lin, la dureté énergique s'accroît et grandit.

TSHOU HI. - Explication du sens du nom du koua au moyen de la substance même de ce koua.

355.

Satisfaction et soumission; dureté énergique, justice et correspondance sympathique.

TSHOU HI. - Nouvelle allusion à l'excellence du sens indiqué par les vertus des koua simples, et par la substance du koua parfait.

356.

Grande liberté par la droiture ; voie du ciel $^{2}$.

\footnotetext{
${ }^{1}$ Le commentateur lit « huit mois » au lieu de « huitième mois ». Cette lecture est possible si on ne considère que les deux caractères [] [] pris isolément, mais elle est certainement mauvaise si on considère l'ensemble de la formule. En prenant pour origine du premier mois la conjonction de l'équinoxe du printemps, la conjonction qui précède celle-là sera bien celle du douzième mois. Elle marque bien le moment où le soleil va bientôt passer dans l'hémisphère nord, le commencement de la prospérité, et il est encore évident que la conjonction du huitième mois marquera le moment où le soleil sera descendu assez bas sous l'équateur pour que le froid commence à sévir avec toute sa rigueur dans l'hémisphère nord.

${ }^{2}$ Au figuré, voie morale indiquée par le ciel.
} 
TSHOU HI ${ }^{1}$. - En présence d'un moment où la dureté énergique prévaut, il y a encore un tel degré de bien, et c'est pourquoi tel est le sens divinatoire.

TSHENG TSE. - (Sur les trois paragraphes de la formule déterminative). Le terme Min, du texte, exprime la croissance progressive; les deux positivités grandissent en-dessous et avancent progressivement. En bas, le koua simple touei; en haut, le koua simple khouen; concorde avec satisfaction et soumission. La dureté énergique suit la voie de la justice et elle est soutenue par une assistance sympathique ; c'est par là qu'il peut y avoir une grande liberté, avec observation de la droiture, et conforme à la voie morale indiquée par le ciel. «L'énergie est conforme à la droi ture et s'accorde avec soumission à la voie morale du ciel. » Ce qui fait que l'ouvre de transformation créatrice ne cesse pas, c'est unique ment l'énergie, la droiture, la concorde, et la soumission. Avec ceàqualités, qu'il s'agisse de surveiller (lin) les hommes ${ }^{2}$, de diriger (lin) des affaires, de gouverner (lin) l'univers, on jouira toujours d'une grande liberté d'action tout en se conformant à la droiture. Le koua simple touei exprime la satisfaction; avec la satisfaction vient la concorde et l'harmo nie ; la formule déterminative du koua kouae dit : «Décision et harmonie. »

357.

Parvenue au huitième mois il y a présage malheureux; diminution et manque de durée.

TSHENG TSE. - Dans le koua lin, deux positivités naissent : c'est le moment où la positivité commence à croître progressivement et à devenir florissante. Aussi l'homme saint en fait l'objet d'un avertissement et dit : bien que la positivité commence à se développer, cependant, après huit mois, elle s'évanouira et le présage est malheureux. L'expression huit mois désigne les huit mois pendant lesquels la positivité naît et subsiste; depuis le koua fou, où la positivité commence à naître, jusqu'au koua thouen, il y a huit mois qui correspondent du signe tse jusqu'au signe mei du cycle luna ire annuel ; dans ce dernier koua deux négativités croissent et la positivité s'évanouit. C'est pour cela que le texte dit: «diminution et manque de durée ». Lorsqu’il est question des éthers de la négativité et de la positivité, la disparition et la croissance évoluent comme dans un cycle circulaire, que rien ne peut altérer.

\footnotetext{
${ }^{1}$ Le commentaire de Tshou Hi est donné ici avant celui de Tsheng Tse, pour ne pas séparer la suite du commentaire de TSHOU HI.

${ }^{2}$ Les gouverner.
} 
S’il s'agit des affaires humaines, la positivité représente l'homme doué et la négativité représente l'homme inférieur. Au moment où la voie rationnelle de l'homme doué commence à s'agrandir, l'homme saint en fait le sujet d'un avertissement, afin que chacun sache que, cette voie parvenue à la limite extrême de son développement, il y aura des raisons pour que des malheurs surviennent, mais qu'en y pourvoyant à l'avance, ordinaireme nt ces malheurs n'en arriveront pas à un point extrême, c'est -à-dire que le mal n'arrivera pas.

TSHOU HI. - Cette formule exprime que telles sont les conséquences naturelles du mouvement de translation du ciel, cependant il convient que l'homme doué sache ce qui convient pour les prévenir et être en garde contre elles.

358.

Le commentaire traditionnel de la formule symbolique dit: Au-dessus du marais il y a de la terre; surveiller (lin); l'homme doué applique sans limites sa pensée à l' enseignement; il supporte et protège les peuples sans connaître de frontières.

TSHENG TSE. - Au-dessus du marais il y a de la terre : la berge du marais ; le bord de l'eau. Entre toutes les choses mutuellement en contact et qui se pénètrent en se contenant l'une l'aut re, le contact n'est jamais aussi intime qu'entre l'eau et la terre. C'est pour cela que la terre qui est au -dessus du marais représente le sens du mot lin. L'homme doué considère cette image symbolique du rapprochement (lin) intime de deux choses, dont l'une domine l'autre, et alors il applique sans limites ses idées d'enseignement. Sur veillant le peuple avec affection, il est mû par la pensée de linstruire et de le guider. «Sans limites », c'est-à-dire avec la plus extrême pureté d'intentions, sans la moindre compression. Il regarde l'image symbolique du fait d'entourer et contenir, et alors il a la volonté d'embrasser les peuples dans sa protection sans aucune restriction, c'est-à-dire avec la plus grande ampleur de vues et sans être arrêté par aucune frontière. Cet état, qui consiste à entourer et contenir, comporte une idée d'ampleur et de grandeur qui donne le sens des expressions « sans limites » et « sans frontières ».

TSHOU HI. - La terre domine au-dessus du marais ; ce qui est supérieur domine ce qui est inférieur et le surveille ; dans les deux cas, il s'agit toujours du sens exprimé par le mot lin: surveiller quelque chose au-dessous de soi. L'absence de limites dans l'enseignement est exprimée par le koua simple touei; l'absence de frontières dans la protection est exprimée par le koua simple khouen. 


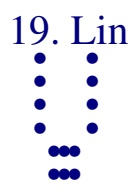

359.
Premier trait nonaire : surveiller et émouvoir ${ }^{1}$; présage heureux de la pureté.

TSHENG TSE. - Le terme hien, troisième caractère du texte, tous, tout, de même, équivaut au mot kan, causer ou ressentir une influence ${ }^{2}$. Au moment où la positivité se développe et grandit, elle influence et émeut la négativité; le quatrième trait correspond sympathiquement au premier, il l'influence. Comparativement à ce qui a lieu dans le cas des autres koua, la correspondance sympathique est de beaucoup plus importante. Le quatrième trait est voisin de la situation du prince ; le premier occupe la situation qui lui convient avec droiture ${ }^{3}$; avec le quatrième, ils sinfluencent sympathiquement. Cela exprime les conditions d'employer la voie de la droiture, être investi de l'autorité par la confiance de quelqu'un digne de sa situation, pouvoir faire prévaloir ses tendances, obtenir du supérieur et réussir à parcourir la droite voie : c'est par là que le présage est heu reux. Dans les autres koua, au sujet du premier trait et du trait supérieur, on ne parle ni de posséder, ni de perdre une situation; en effet, le sens qui résulte de la condition de commencer ou de finir, est plus important. Dans le koua lin, c'est la condition que le premier trait s'accorde à sa situation et se maintient dans la droiture qui est considérée comme importante. Toutes les fois que les formules emploient l'expression «présage heureux de la pureté (ou perfection) », il $\mathrm{y}$ a des cas où elle indique que du moment où il $\mathrm{y}$ a droiture, le présage sera d'ailleurs heureux ; il y a des cas où elle marque qu'en s'accor dant à la droiture, le présage sera heureux; il y en a où elle marque encore qu'en possé dant la pureté et en s'y attachant avec fermeté, le présage sera heureux, dans chaque cas suivant l'objet dont il s'agit.

TSHOU HI. - Dans le koua il n'y a que deux positivités, elles regardent les quatre néga tivités avec une tendance partiale. C'est pour cela que les deux formules rappellent l'image symbolique d'influencer en surveillant. Le premier trait est énergique et il possède la droiture ; c'est pour cela que le sens divinatoire est : «présage heureux de la pureté ».

\footnotetext{
${ }^{1}$ Cette lecture est conforme à l'explication traditionnelle, mais ne résulte pas directement du sens des termes du texte. On devrait lire «tous surveillent» ou « surveiller de même ». Dans le Shi king on trouve ce passage : «Le soleil est en présence de la lune, tous deux illuminent et regardent la terre au-dessous d'eux. »

2 Voir koua 31.

${ }^{3}$ Trait positif et rang impair. jamais rien de pareil ne pourra se déduire du texte considéré seul, et sans jugement préconçu.
} 
360.

Présage heureux de surveiller en émouvant; tendances agissant selon la droiture.

TSHENG TSE. - Ce qu'on entend par le présage heureux de la pureté (ou perfection), c'est que les tendances inhérentes à l'état nonaire consistent à pratiquer la droiture, et cela parce que le trait est nonaire et occupe un rang positif. De plus, la droiture, manifestée par sa sympathie pour le quatrième trait, indique que ses tendances sont portées vers la droiture. 


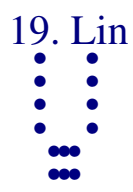

361.

Deuxième trait nonaire : surveiller en émouvant; présage heureux, rien sans avantage ${ }^{1}$.

TSHENG TSE. - Le second trait, au moment où la positivité est nouvellement grandissante et avance peu à peu vers son développement parfait, émeut et agite le prince juste et condescendant représenté par le cinquième trait hexaire, celui auquel il s'allie avec affection. Aussi la confiance, le dépôt de l'autorité sont évidents ; il peut faire prévaloir ses tendances, le présage est heureux pour les intérêts qu’il surveille, et rien n'est sans en retirer avantage. Le présage heureux est inhérent à lui-même ; c'est parce qu'il est ainsi que le présage est heureux. La condition que rien n'est sans avantage est l'effet qu'il prod uit sur les choses auxquelles il étend son influence ; rien qui n'en retire avantage.

TSHOU HI. - Il est énergique, possède la justice, et la force des choses fait qu'il avance en montant, aussi le sens divinatoire est que le présage est heureux et que rien n'est sans en retirer un avantage.

362.

Présage heureux de surveiller en émouvant, rien sans avantage; pas encore soumis à la destinée ${ }^{2}$.

TSHENG TSE. - Le terme wei, du texte, «pas encore », est une expression qui indique l'absence de hâte ou précipitation. Dans les «conversations de Mang Tse » quelqu'un demandant : «Avez-vous, oui ou non, excité l'état de Tsi à détruire l'état de Yien ? » il est répondu «wei, pas encore». Il est encore dit: «Le grain qui a été mangé par Tshong Tse n'était-ce pas celui qui avait été planté par Po Yi, ou bien était-ce encore celui qui avait été planté par Tao Thsi ? c'est ce qu'on né peut pas encore (wei) savoir. » Dans le Li ki il est dit : «ce qui n'est certes pas encore (wei) facile à l'homme de connaître ». Telle fut toujours l'idée des hommes de l'antiquité en

\footnotetext{
${ }^{1}$ Même observation sur la lecture des mots hien lin ; voir $n^{\circ} 359$.

2 Destinée ; ou encore « ordre du prince ».
} 
employant ce caractère (mot) ; actuellement, en général, on l'emploie comme faisant antithèse du caractère $y i$ « déjà fini », de sorte que l'idée contenue dans la formule semble différente, tandis qu'en réalité elle est la même. Le deuxième trait nonaire et le cinquième sinfluencent sympathiquement pour surveiller le trait inférieur. En effet, employant le développement de la dureté énergique, et de plus se conformant à la justice, s’influençant mutue llement par la plus parfaite sincérité, ce n'est donc pas à cause des ordres du supérieur. C'est là ce qui fait que le présage est heureux et que rien n'est sans avantage. Le cinquième trait fait partie de la substance de la soumission ${ }^{1}$, tandis que le second fait partie de la substance de la satisfaction ${ }^{2}$; de plus, la négativité et la positivité sympathisent mutuellement, de sorte que l'image symbolique montre spécialement que ce n'est pas par satisfaction et soumission ${ }^{3}$.

TSHOU HI. - Pas encore interprété ${ }^{4}$.

\footnotetext{
${ }^{1}$ Koua simple khouen.

${ }^{2}$ Koua simple touei.

${ }^{3}$ Tshou Hi, dans son commentaire, est plus franc; il avoue qu'il n'y comprend rien.

4 « Pas encore », wei ; application de la discussion de Tsheng Tse sur ce mot.
} 


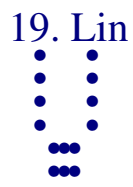

363.

Troisième trait hexaire : se plaire à surveiller ; aucun moyen d'avantage ; en avoir déjà du chagrin ${ }^{1}$; pas de culpabilité.

TSHENG TSE. — Le troisième trait occupe le rang supérieur dans le koua simple inférieur ; c'est celui qui surveille les hommes. Il est mou et négatif et fait partie de la substance du koua simple qui exprime la satisfaction ; de plus il est placé sans justice ni droiture : c'est celui qui s urveille les hommes pour sa propre satisfaction ${ }^{2}$. Être dans un rang supérieur, et diriger les inférieurs pour son agrément et sa satisfaction, c'est gravement man quer de vertu, et aucun avantage ne peut en résulter. La nature indiquée par le koua simple touer étant déjà de rechercher la satisfaction et le plaisir, il est encore appuyé sur deux positivités ; la positivité commence nouvellement à se développer et monte en avançant, de sorte qu'il n'est pas satisfait et qu'il tend à augmenter son propre agrément. Du moment où il sait reconnaître le péril, le craindre, et avoir du regret de sa conduite, s’il peut s'appuyer sur la modestie et observer la droiture, être parfaitement sincère en se plaçant lui-même, alors il sera sans culpabilité. Mal placer sa satisfaction dans l'égoïsme, mais pouvoir s'en repentir et s'en corriger, c'est ne plus laisser place à aucune faute.

TSHOU HI. - Malléabilité négative sans justice ni droiture occupant le rang supérieur entre les inférieurs; cela constitue l'image symbolique de l'action de surveiller les hommes en vue de sa propre satisfaction. Le sens divinatoire est que certainement cette conduite ne peut être avantageuse pour qui que se soit., Cependant pouvant en avoir du chagrin et se corriger, il n'y aura aucune culpabilité. Encourager les hommes à s'amélio rer, constitue la profondeur de l'enseignement.

364.

Se plaire à surveiller; situation imméritée; du moment où il en a du chagrin, la culpabilité ne grandit pas.

\footnotetext{
${ }^{1}$ On pourrait lire : «Du moment où il en a du regret, pas de culpabilité. »
}

${ }^{2} \mathrm{Ce}$ terme surveiller est pris dans l'acception de diriger, gouverner. 
TSHENG TSE. - Un homme doux et sans activité, placé sans justice ni droiture, et occupant le rang supérieur parmi les inférieurs. De plus encore, il s'appuie sur les deux positi vités. C'est être placé dans une situation imméritée. Du moment où il peut en éprouver du chagrin et reconnaître ce qu'il a lieu de craindre, il s'efforcera infailliblement de se corriger, de sorte que ses fautes et sa culpabilité ne grandiront pas. 


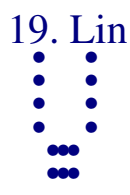

365.

Quatrième trait hexaire : extrême surveillance ${ }^{1}$; pas de culpabilité.

TSHENG TSE. - Le quatrième trait occupe le rang le plus bas parmi les supérieurs ; lui et la substance du koua simple inférieur sont mutuellement en contact; il exprime la surveillance rigoureuse sur les inférieurs et l'extrême surveillance. Dans la voie naturelle de la surveillance (direction), c'est la proximité qui la rend précieuse, de sorte que le contact est considéré comme en indiquant le plus haut degré. Le quatrième trait occupe une situation correcte et il porte ses sympathies en bas, sur le premier trait énergique et positif. Il est placé près de la situation du prince; il observe la droiture et investit le sage du poids de l'autorité, afin de surveiller au-dessous de lui avec sollicitude ; c'est par là qu'il est sans culp abilité, et la position qu’il occupe est méritée.

TSHOU HI. - Il se place dans une situation pour laquelle il est apte ; en bas il sympathise avec le premier trait nonaire, c'est l'extrême degré dans la surveillance mutuelle et il représente celui qui doit naturellement être sans culpabilité.

366.

Extrême surveillance sans culpabilité ; situation méritée.

TSHENG TSE. - Il occupe une situation près du prince et en agissant il se montre au niveau de l'autorité qui lui est confiée. La négativité placée au quatrième rang est considérée comme exprimant quill se conforme à la droiture. Lui et le premier trait sympathisent ensemble, ce qui exprime quil s'abaisse devant le sage, et c'est pour ces raisons qu'il est sans culpabilité. En effet, c'est parce que sa situation est méritée.

\footnotetext{
${ }^{1}$ On peut lire aussi « contact parfait ».
} 


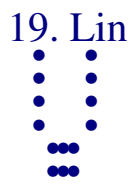

367.

Cinquième trait hexaire : surveiller par le savoir ; ce qui convient à un grand prince ; présage heureux.

TSHENG TSE. - Le cinquième trait avec sa douceur, sa justice, et sa substance passive, accepte la situaton prééminente, et en bas il sympathise avec le sujet énergique et juste représenté par le second trait. C'est donc quiil peut déléguer .le poids de l'autorité au second trait, sans consacrer ses efforts au gouvernement: c'est celui qui surveille les infé rieurs par le savoir. Or quand il faut qu'une seule personne surveille l'immensité du monde, s'il lui fallait se charger elle-même d'entrer dans tous les détails, comment pourrait-elle suffire à embrasser toutes les affaires ! C'est pour cela qu'il se charge lui-même du soin de ce qu'il sait, et qu'il charge des gens compétents de ce qu'il ne sait pas. Il peut seulement choisir les bons parmi tout l'univers, investir de l'autorité tous ceux qui, da ns le monde, brillent par l'intelligence, et alors rien n'échappe à son action. C'est là ne pas s'autoriser de son propre savoir, et c'est précisément ce qui fait la grandeur du savoir. La condescendance du cinquième trait sympathise avec la sagesse du second trait énergique et juste. Il le charge de surveiller les inférieurs, et c'est là surveiller soi-même l'univers par sa propre intelligence et son propre savoir : c'est ce qui convient chez un grand prince. Le présage heureux est évident.

TSHOU HI. - Emploi de la douceur pour se maintenir dans la justice ; en bas sympathie avec le second trait nonaire. Ne pas agir exclusivement par soimême et confier l'autorité à quelqu'un, c'est là un effet du savoir, et ce qui est essentiel chez un grand prince. C'es t une voie rationnelle de présage heureux.

368.

Ce qui convient à un grand prince; désignation de la pratique de la justice.

TSHENG TSE. - La voie rationnelle du prince et celle du sujet concordent ensemble; en effet elles s'appellent mutuellement à cause de l'analogie de leur essence. Le cinquiè me trait possède les vertus de la justice, aussi il est capable d'investir de l'autorité le sage énergique et juste : il 
possède les qualités naturelles requises chez un grand prince, et il accomplit l'œuvre de la surveillance par le savoir, car c'est par la pratique des vertus de la justice. Lorsqu'il s'agit des relations du prince avec les aptitudes de la sagesse, si la voie n'était pas la même, si les vertus ne concordaient pas, comment pourrait-il les employer? 


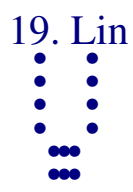

369.

Trait supérieur hexaire : grandeur dans la surveillance ; présage heureux sans culpabilité.

TSHENG TSE. — Le trait supérieur hexaire marque la limite extrême du koua simple khouen : c'est le comble de la soumissi on, ou condescendance. Mais il occupe le dernier rang du koua lin ; il marque donc la magnanimité et la grandeur dans la surveillance. Bien que sa sympathie pour le second trait nonaire ne résulte pas de la droiture, cependant, en général, la négativité appelle la positivité. De plus, à cause de son extrême condescendance, ses tendances le portent à écarter le second trait positif ; il est éminent et il écoute les humbles ; il est élevé et il suit les conseils des inférieurs. Il élève la sagesse et choisit les bons: c'est le comble de la grandeur, et c'est pourquoi la formule dit : «grandeur dans la surveillance ». C'est ce qui fait que le présage est heureux et qu'il n'y a pas de culpabilité. La douceur de la négativité étant dans une position supérieure et incapable de surveiller les inférieurs, il est naturel qu'elle commette des fautes. C'est parce qu'elle montre de la giandeur en écoutant avec soumission les conseils de l'énergie, que le présage est heu reux et qu'elle ne commet pas de fautes. Ce trait hexaire occupe le dernier rang dans le koua lin et cependant le sens de développement extrême poussé à ses dernières limites n'est pas relevé. Dans la surveillance des inférieurs par les supérieurs, il n'y a pas une limi te au-delà de laquelle commence l'excès, et c'est pourquoi ces conditions constituent seulement le sens de grandeur et d'ampleur. Le rang supérieur indique un terrain qui ne comporte aucune situation définie ; il en est seulement question comme de quelqu'un parmi les supérieurs en général.

TSHOU HI. - Ce trait occupe le rang supérieur dans le koua simple ; il est placé à la fin du koua parfait lin ; il exprime la grandeur et l'ampleur dans la surveillance : c'est une voie qui indique un présage heureux et l'absence de culpabilité. C'est pour cela que tels sont l'image symbolique et le sens divinatoire.

370.

Présage heureux de la grandeur dans la surveillance; tendances portées vers l'intérieur. 
TSHENG TSE. — « Tendances portées vers l'intérieur »: sympathie pour le premier et le second trait. Ses tendances le portent à la condescendance envers la dureté énergique positive et à la magnanimité dans le commandement ; le présage heureux est évident. 


\section{Kouan}

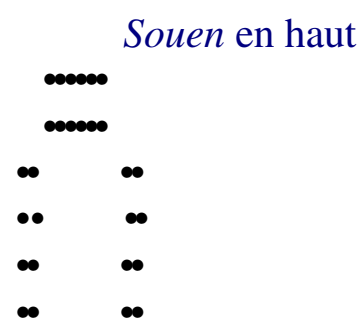

Khouen en bas

371. Kouan, point de mire des regards; se laver les mains avant le sacrifice et ne pas l'offrir ; avoir foi, contenance recueillie.

[]

376. Premier trait hexaire : le jeune garçon regarde; l'homme inférieur est sans culpabilité ; l'homme doué éprouve de l'appréhension.

378. Deuxième trait hexaire : regarder à la dérobée ; avantage de la pureté de la jeune fille.

*

380. Troisième trait hexaire : regarder sa propre vie et ses propres démarches en avant ou en arrière.

*

382. Quatrième trait hexaire : regarder l'éclat du royaume ; avantage à visiter le roi en hôte.

*

384. Cinquième trait nonaire : regarder sa propre vie ; l'homme doué est sans culpabilité.

*

386. Trait supérieur nonaire : regarder sa vie ; l'homme doué est sans culpabilité.

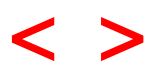




\section{Kouan.}

TSHENG TSE. - Kouan. «L'Ordre des koua » dit: «Le koua lin exprime la surveillance exercée sur les inférieurs. Lorsqu'une chose est grande, il devient possible de la regarder et de la voir, aussi le koua lin est suivi du koua kouan, et celui-ci ne vient qu'après l'autre. » Toutes les fois qu'il s'agit de regarder pour voir quelque chose on emploie le mot kouan. S'il s'agit d'être regardé d'en bas, alors cet état est exprimé par le mot kouan. Ainsi dans l'expression lieou kouan, ce qui motive l'emploi du mot kouan, c'est qu'il s'agit d'une estra de exposée aux regards de ceux placés en dessous ${ }^{1}$. Le prince regarde (kouan) au-dessus de lui la voie rationnelle du ciel ; au-dessous de lui, il regarde les moars du peuple ; tel est l'emploi du mot kouan. En entretenant la vertu, en agissant sur les institutions sociales, il est l'objet des regards des peuples qui ont les yeux levés vers lui avec espérance, et c'est ici le cas de l'emploi du mot kouan. Le vent court sur la terre, atteignant et heurtant toutes choses ; c'est l'image symbolique du regard circulaire embrassant tout l'horizon (kouan). Les deux traits positifs sont en haut ; les quatre négativités, au-dessous. La dureté énergique positive occupe le rang prééminent et c'est sur elle que se portent les regards de la foule des inférieurs, ce qui donne le sens du mot houan, point de mire, objet des regards. Dans les formules des divers traits de ce koua, le sens de kouan, regarder et voir, est seul relevé et précisé, suivant le moment.

${ }^{1}$ Lieou kouan, estrade, tribune, belvédère. Kouan, regarder ; Kouan, être regardé, servir de point de mire aux regards. 


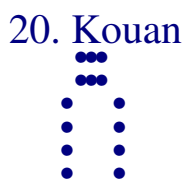

371.

Kouan, point de mire des regards; se laver les mains avant le sacrifice et ne pas l'offrir ; avoir foi, contenance recueillie.

TSHENG TSE. - J'ai entendu rapporter que le vénérable Hou Tshi a dit : «L'homme do ué qui occupe un rang supérieur est comme un phare qui attire l'attention des hommes ; il doit les exciter à la dignité et à la gravité, de sorte que les inférieurs fixent leurs yeux sur lui, le contemplent, et se transforment en s'améliorant. Il est donc le point de mire des regards de l'univers. Il convient donc qu'il soit comme dans le cas de sacrifices dans le temple des ancêtres, au moment où l'officiant commence à se laver les mains, et non pas comme après l'offrande. Alors les inférieurs et le peupl e sont amenés à la plus parfaite sincérité de sentiment, et le contemplent en levant les yeux sur lui avec une attitude recueillie. » Le second caractère de la formule, kouan ${ }^{1}$, indique le commencement de la cérémonie du sacrifice; l'officiant se lave (kouan) les mains et répand une libation de vin parfumé sur le sol ; c'est le moment de l'évocation de l'esprit. «Offrir», se rapporte au moment de la présentation des offrandes en viandes crues ou cuites. Le lavage des mains marque le commencement des cérémonies ; le coar s'absorbe dans la pure té de la pensée ; c'est le moment de l'extrême gravité du recueillement. Puis, après que l'offrande a été faite, les règles rituelles deviennent complexes, et le cour de l'homme est distrait par le soin des détails, de sorte que la pureté de l'attention n'est plus comparable à ce qu'elle était en commençant, au moment du lavage des mains. Celui qui occupe une position élevée rectifie les exemples qu'il donne, afin d'être le modèle et le point de mire ( kouan) des inférieurs et du peuple ; il convient qu'il soit grave et sérieux comme au com mencement de la cérémonie, au moment du lavage des mains, sans laisser, si peu que se soit, distraire la sincère attention de ses idées, comme après le moment des offrandes; alors personne dans l'univers ne peut rester sans être gagné par une foi absolue et sincère; tous seront recueillis et attentifs à ses moindres actes. Le recueillement, c'est l'attente les yeux fixés sur l'objet.

TSHOU HI. - Le mot kouan, exprime ce qui est capable de servir d'exemple et ce que les hommes contemplent. Le cinquième trait nonaire occupe un rang supérieur; les quatre négativités le conteur lent. Ou bien encore, au-dedans soumission et au-dehors humilité, et d'ailleurs le cinième trait nonaire emploie la justice et la droiture pour servir d'exemple à l'univers, ce qui fait qu'il est considéré comme point de mire des regards (kouan). Quant

\footnotetext{
1 Autre caractère et même son, sens mal défini. «Laver les mains avant le sacrifice » et « vase pour laver les mains ».
} 
au caractère kouan, il indique la purification des mains, au moment d'accomplir un sacrifice. Le terme « offrir », exprime l'action de présenter le vin et les mets en accomplissant le sacrifice. «Recueillement», apparence de gravité respectueuse. Il s'agit de l'accomplissement parfait des soins de purification, sans attention aux choses accessoires, de telle sorte que la foi et la sincérité sont en dedans de soi, et que les assistants peuvent contempler avec recueillement. C'est un avertissement adressé à celui qui consulte le sort, qui doit être dans ces mêmes conditions. On dit: Les mots «avoir foi, contenance recueillie », s'appliquent aux inférieurs, qui ont confiance et contemplent.

Dans ce koua les quatre négativités se développent tandis que les deux positivités s'éva nouissent ; c'est donc précisément le koua du huitième mois et cependant le commentaire des «Formules annexées» et le «Nom des koua » relèvent un autre sens ; mais c'est encore l'idée de soutenir la positivité et de déprimer la négativité.

Le commentaire traditionnel de la formule déterminative dit: Le grand objet des regards (kouan) est en haut; soumission et utilité ; justice et droiture en regardant l' Univers.

TSHENG TSE. — Le cinquième trait occupe la situation prééminente ; il emploie les vertus de l'activité énergique, de la justice et de la droiture ; c'est celui que les inférieurs regardent. Ses vertus sont extrêmement grandes, et c'est pour cela que la formule dit : « Le grand objet des regards est en haut. » En bas, le koua simple khouen; en haut le koua simple souen, ce qui indique l'aptitude à la soumission et l'h umilité. Le cinquième trait occupe sa position avec justice et droiture ; il emploie les vertus de l'humilité et de la doci lité, de la droiture et de la justice, pour servir de point de mire et d'exemple à l'univers.

TSHOU HI. - Explication du sens du nom du koua au moyen de la substance du koua parfait et des vertus des koua simples.

373.

Kouan : se laver les mains avant le sacrifice et ne pas l'offrir ; avoir foi, contenance recueillie; les inférieurs regardent (houan) et se transforment. 
TSHENG TSE. - La voie rationnelle, pour celui qui sert de point de mire aux regards (kouan), consiste à être grave et respectueux comme au moment du lavage des mains avant le sacrifice, de telle sorte que les inférieurs ont en lui la confiance la plus sincère, qu'ils le contemplent avec attention et respect, et l'imitent en se transformant. Ne pas offrir, veut dire ne pas laisser le moindre sujet de distraction détourner la parfaite attention de l'esprit.

TSHOU HI. - Explication de la formule du koua.

374.

Regarder la voie spirituelle du ciel, et l'absence d'irrégularité des quatre saisons ${ }^{1}$; l'homme saint emploie la voie spirituelle pour établir son enseignement et l' univers se soumet.

TSHENG TSE. - La voie du ciel est au plus haut point intelligente ${ }^{2}$ c'est pourquoi la formule dit «voie spirituelle» (shen tao). En regardant le mouvement de translation du ciel et l'absence d'irrégularité des quatre saisons, on peut en voir la cause mystérieuse et intelligente. L'homme saint voit l'intelligence manifestée $\mathrm{d}$ ans la voie du ciel; il réalise cette voie intelligente pour établir son enseignement ${ }^{3}$, de sorte qu'il n'est personne dans l'uni vers qui ne se soumette. Or la voie du ciel est ce qu'il y a de plus spirituel (shen), de sorte que dans le mouvement de translation, dans les quatre saisons, dans la production créatrice de toutes choses, il n'y a jamais d'erreur ni d’irrégularités. Cette voie extrêmement intelligente ne peut être désignée par aucun nom ${ }^{4}$. L'homme saint, seul, s'y conforme tacitement ${ }^{5}$; il réalise l'action de la cause mystérieuse, et il s'en sert pour établir ses insti tutions sociales. Aussi, les hommes, dans tout l'univers, s'imprègnent de ses vertus sans connaître son ouvre. Ils se livrent avec ardeur au courant de son action transformatrice sans en calculer l'objet ${ }^{6}$. Ils sont instinctivement portés à élever leurs regards vers lui et à se soumettre à son enseignement. C'est pour

\footnotetext{
${ }^{1}$ Saisons ou « moments ».

2 Shen, génie, esprit, et, par extension, plus rarement, intelligence. Le texte signifie littéralement « voie des génies ».

3 Il donne un corps à cette voie, etc.

${ }^{4}$ Les réminiscences de Lao Tse sont fréquentes dans le commentaire de Tsheng.

5 Expression à remarquer; sa valeur originale paraît être : «fixer mystérieusement par des nouds ».

6 Littéralement il faudrait lire: «Ils représentent par la musique et la danse les transformations, mais sans en apprécier l'utilité. »Ce qui paraît une allusion aux danses mimées représentant les mouvements des astres.
} 
cela que la formule dit «il emploie la voie spirituelle pour établir son enseignement et l'univers se soumet ${ }^{1}$.

TSHOU HI. - Dernière analyse de la considération de la voie. Les quatre saisons sont sans irrégularité ; c'est ce qui fait que le ciel est le point de mire des regards. La voie intelligente (littéralement voie des génies) sert de base à l'enseignement ; ce qui fait que l'homme saint sert également de point de mire aux regards.

375.

Le commentaire traditionnel de la formule symbolique dit: Le vent court sur la terre; regarder. Les premiers rois inspectaient les régions, regardaient le peuple et établissaient leur enseignement.

TSHENG TSE. — Le vent court sur la terre, il enveloppe et atteint toutes choses, ce qui constitue l'image symbolique d'apercevoir de tous côtés. C'est pour cela que les premiers rois réalisaient cette image en posant la règle rituelle de l'inspection des régions, afin de regarder les mours des peuples et d'établir les règles de l'enseignement et des institutions sociales. Le « fils du Ciel » parcourt et inspecte les quatre régions, regarde et considère les mours des peuples et institue les règles sociales et l'enseignement. Ainsi, si le peuple est porté à la prodigalité, il le contient par des préceptes d'économie ; s’il est porté à l'avarice, il l'avertit par les prescriptions rituelles sur les dons. Il inspecte les régions et regarde (kouan) les peuples; il établit ses enseignements et sert de point de mire (kouan) aux peuples.

TSHOU HI. - Inspecter les régions pour regarder (kouan) les peuples ; établir l'ensei gnement pour être regardé (houan) par eux.

\footnotetext{
${ }^{1}$ La plus grande partie de ces commentaires n'est pas l'œuvre propre de leur auteur qui, en général, ne fait que compiler et broder sur des traditions scolastiques plus anciennes. Il n'est donc pas étonnant que certaines expressions figurées, reportent à un sens littéral particulier, plus proche de linterprétation vraie que les explications modernes.
} 


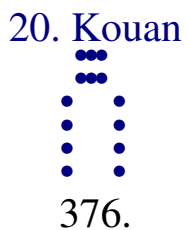

Premier trait hexaire : le jeune garçon regarde ; l'homme inférieur est sans culpabilité ; l'homme doué éprouve de l'appréhension.

TSHENG TSE. - Ce trait hexaire, avec ses caractères de malléabilité négative, se trouve éloigné de la positivité ; c'est celui qui, en regardant, ne voit que superficiellement et pas au loin, précisément comme la jeunesse, et c'est pour cela que la formule dit : le jeune garçon regarde ${ }^{1}$. L'énergie active, la justice et la droiture se trouvent en haut, représentant le prince sage et saint. En s'approchant de lui, la perfection des vertus de la voie qu'il suit devient visible ; la portée de son regard est profonde et lointaine. Le premier trait est éloigné de lui ; ce qu'il voit n'est pas distinct, comme le regard du jeune garçon aveuglé par l'ignorance. «L'homme inférieur » c'est le peuple, ou les inférieurs ; son regard est superficiel et sa vue est confuse ; il est incapable de connaître la voie de l'homme doué. Telle e st sa condition ordinaire, et ce n'est pas suffisant pour dire quill est coupable et que cette infériorité constitue une faute. Mais s’il s'agit d'un homme doué, et qu'il soit dans ces conditions, alors il y a lieu d'en éprouver de l'appréhension.

TSHOU HI. - Lorsqu'il s'agit du koua parfait lui -même, on le considère comme exprimant le sens du mot houan, point de mire des regards, en prenant le cinquième trait nonaire comme le plus important. Dans les formules des traits, on prend le sens du mot kouan, regarder; tous regardent vers le cinquième trait nonaire. Le premier trait hexaire représente la douceur passive dans une position inférieure; il ne peut regarder au loin : c'est l’image symbolique de l'action de regarder chez lejeune garçon. La voie ration nelle de l'homme inférieur ferait la honte de l'homme doué, de sorte que le sens divinatoire sera absence de culpabilité, sîl s'agit de l'homme inférieur, tandis que s'il s'applique à l'homme doué, celui -ci aura lieu d'en éprouver de la honte.

\section{7.}

Premier trait hexaire; le jeune garçon regarde; voie de l'homme inférieur.

\footnotetext{
${ }^{1}$ On peut aussi lire : « action de regarder du jeune garçon ».
} 
TSHENG TSE. - Il ne voit pas clairement ce qüil regarde, comme lorsqu'il s'agit du jeune garçon regardant quelque chose. Telle est la condition naturelle de l'homme infé rieur, et c'est pourquoi la formule dit : voie de l'homme inférieur. 


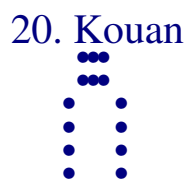

378.

Deuxième trait hexaire : regarder à la dérobée; avantage de la pureté de la jeune fille.

TSHENG TSE. - Le second trait correspond sympathiquement au cinquième ; il regarde vers le cinquième. Le cinquième trait représente la voie de la dureté énergique positive juste et droite, ce n'est donc pas ce que la faiblesse et l'obscurité négative du second puisse regarder et v oir, aussi il ne s'agit que de quelque chose analogue à l'action de regarder à la dérobée en avançant la tête par une porte entrebâillée. En regardant ainsi à la dérobée par une porte entrebâillée, bien qu'il soit possible de voir quelque peu, il n'est cependant pas possible de voir très clairement. Du moment où le second trait ne peut pas voir très clairement la voie de la dureté énergique juste et droite, son avantage consistera dans une pureté analogue à celle de la jeune fille qui, bien qu'incapable de discerner avec une grande clarté, est cependant susceptible de suivre passivement une voie tracée, ce qui est la voie de lajeune fille. Pour une jeune fille, ce trait indique la pureté. Puisque le second trait ne peut pas voir clairement la voie du cinquième trait nonaire, s’il est capable, peut écouter et obéir comme une jeune fille, il ne manquera ni à la justice, ni à la droiture, et c'est en cela que consiste l'avantage.

TSHOU HI. - La douceur malléable se trouve à l'intérieur et elle regarde vers l'ext érieur : image symbolique de regarder à la dérobée, sans se laisser voir, ce qui, chez la jeune fille, constitue la droiture. Voilà pourquoi tel est le sens divinatoire. Si une personne âgée rencontre ce trait, en consultant le sort, il n’indiquera rien d'avantageux.

379.

Perfection de la jeune fille regardant à la dérobée ; ce qui peut cependant encore être honteux.

TSHENG TSE. - L'homme doué ne peut pas regarder et voir la grande voie de la dureté énergique positive de la justice et de la droiture ${ }^{1}$, et c'est à

\footnotetext{
${ }^{1}$ Du cinquième trait dont il est trop éloigné.
} 
peine s'il l'aperçoit à la déro bée sans bien la discerner, bien qu’il puisse la suivre avec soumission. Il en est de sa condition comme de la pureté de la jeune fille, qui peut aussi conduire à un résultat honteux et détestable( ?).

TSHOU HI. - Chez l'homme au bâton ${ }^{1}$ ce sera une chose honteuse.

\footnotetext{
1 Vieillard ; homme d’âge mur qui s'appuie en marchant sur un bâton Dandino.
} 


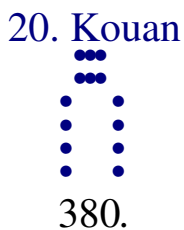

Troisième trait hexaire : regarder sa propre vie et ses propres démarches en avant ou en arrière.

TSHENG TSE. - Le troisième trait occupe une situation qui ne lui convient pas ${ }^{1}$; il est placé au point de l'extrême limite de la soumission ${ }^{2}$; c'est celui qui peut se plier aux nécessités du moment, soit qu'il avance, soit qu'il se recule en arrière. S'il occupait une sit uation qui lui convînt régulièrement, il n’y aurait pas le sens comporté par les termes avancer et reculer. Regarder sa propre vie à soi-même, la vie qu'on mène soi-même, c'est-à-dire les actions qu'on fait soi-même, l'influence qu'on exerce au-dehors et dont l'origi ne est en soi-même. Regarder la vie que l'on mène et, selon l'opportunité, avancer ou reculer, c'est le moyen de faire que, bien que sans droiture, on n'en arrive cependant point à perdre la voie rationnelle. Selon le temps avancer ou reculer, chercher à ne point perdre la voie rationnelle, de sorte qu'il n'y a ni regrets ni culpabilité, en employant la soumission.

TSHOU HI. — «Sa propre vie », les actions que l'on fait soi -même. Le troisième trait hexaire occupe le rang supérieur dans le koua simple inférieur ; il lui est possible d'avan cer, comme de reculer, de sorte qu'il ne regarde pas le cinquième trait nonaire et considère seulement la liberté ou l'empêchement où il se trouve d'agir, afin d'en conclure la convenance d'avancer ou de recul er. Celui qui consulte le sort doit se sur-veiller attentivement.

381.

Regarder sa propre vie et ses propres démarches en avant ou en arrière; ne pas encore avoir perdu la voie.

TSHENG TSE. - Regarder sa propre vie et avancer ou reculer en se conformant à la nécessité. C'est pour cela qu'il n'en arrive pas jusqu'à perdre sa voie.

\footnotetext{
${ }^{1}$ Trait négatif occupant un rang positif.

2 Dernier trait du koua simple khouen.
} 


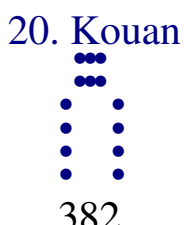

Quatrième trait hexaire : regarder l'éclat du royaume; avantage à visiter le roi en hôte.

TSHENG TSE. - Entre tout ce qu'on regarde, rien n'est plus clairement visible que ce qui est proche; le cinquième trait occupe la situation prééminente avec une énergie active, justice et droiture ; c'est le prince sage et saint. Le quatrième est tout à fait proche de lui, il regarde et voit la voie qu'il suit, et c'est pourquoi la formule dit : regarder l'éclat du royaume, c'est -à-dire regarder et voir l'éclat brillant des vertus florissant dans l'État. Il n'est pas question de la personne du prince, mais de l'État ; en parlant du prince, comment serait-il seulement question de regarder ses seules actions personnelles! Il convient de regarder l'état social et la civilisation de l'empire, de sorte qu'il devient possible de voir et de reconnaître la vertu de la voie suivie par le prince. Bien que le quatrième trait soit doux, malléable et négatif, cependant, sa substance est celle du koua simple qui exprime l'humilité et il demeure dans la droiture. Il est on ne peut plus rapproché du cinquième, donc c'est celui qui regarde, voit, et peut suivre en se conformant avec soumission. «Avantage à visiter le roi comme hôte » car, lorsque l'intelligence et la sainteté occupent le rang supérieur, celui qui possède ces vertus accueille et estime les hommes doués de capacités et de vertus, qui tous aspirent à venir à la cour, l'aider et l'assister pour assurer le bien -être de l'univers. Le quatrième trait voyant et regardant les vertus du prince, l'ordre qui règne dans le gouvernement de l'État, son état de civilisation florissante, ce qui convient qu'il fasse, c'est d'aller en hôte visiter le prince dans sa cour, d'y développer les ressources de son génie en les offrant au prince pour le seconder, afin d'en étendre l'influence bienfaisante à l'univers. C'est pour cela que la formule dit : «Avantage à visiter le prince en hôte. » Dans l'antiquité, les hommes doués de vertu et de sagesse étaient accueillis en hôtes avec distinction par les princes, de sorte que lorsqu'un homme distin gué par son savoir venait à la cour, on l'appelait « hôte ».

TSHOU HI. - Le quatrième trait hexaire est le plus proche du cinquième et c'est pour cela qu'il représente cette image symbolique. Le sens divinatoire est qu'il y a avantage à se rendre à l'audience royale et à entrer dans les affaires publiques.

383. 
Regarder l'éclat du royaume; estimer l'hôte, la qualité d'hôte.

TSHENG TSE. — L'homme doué aime et soutient le talent et la capacité ; ses tendances le portent à améliorer également tout l'univers ; cependant quelquefois il s'attache spécialement à l'observation de soi-même. C'est qu'alors, dans le moment, il n'y a pas un prince intelligent et éclairé et que personne ne serait capable d'utiliser sa voie morale, et quill ne peut pas faire différemment ${ }^{1}$. Mais comment serait-ce là les tendances de l'homme doué ! C'est pour cela que Mang Tse dit : «Il est debout au milieu de l'univers ; il assure le sort des peuples entre les quatre mers; l'homme doué s'en réjouit quand il a regardé et vu l'état florissant et l'éclat de l'état. »C'est ce que les hommes de l'antiquité appelaient une rencontre rare et c'est ce qui fait que ses tendances le portent à désirer venir se présenter à la cour du roi, afin d'y pratiquer la voie morale. C'est pour cela que la formule dit : regarder l'éclat du royaume, estimer l'hôte. " Estimer » veut dire que ses tendances le portent à estimer ; ses tendances et ses idées lui font désirer d'être l'hôte de la cour du roi.

\footnotetext{
${ }^{1}$ Allusion à Khong Tse.
} 


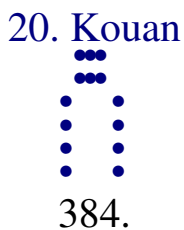

Cinquième tait nonaire : regarder sa propre vie ; l'homme doué est sans culpabilité.

TSHENG TSE. - Le cinquième trait nonaire occupe la situation du prince ; l'ordre ou le trouble qui règnent dans le moment, l'ex cellence ou la corruption des mours, ne dépen dent que de lui seul. Regarder sa propre vie, aussi bien que les mours de l'univers, c'est toujours le fait de l'homme doué, et c'est à cause de cela que les institutions sociales quil établit sont transformatrices et excellentes ; c'est être sans culpabilité. Si les mours, dans l'univers, ne sont pas encore conformes à la voie morale de l'homme doué, c'est que les institutions sociales quil a établies, et son système de gouvernement ne sont pas encore excellents, et il ne peut éviter la culpabilité qui en résulte pour lui.

TSHOU HI. - Le cinquième trait nonaire est énergique, actif, juste et droit, et il emploie ces qualités pour occuper la situation prééminente. Les quatre traits négatifs placés au-dessous de lui, lèvent leurs yeux et le contemplent : il est donc l'image symbolique de l'homme doué. C'est pour cela que la formule avertit que celui qui occupe une telle situation et qui, en consultant le sort, rencontre ce sens divinatoire, doit regarder ses propres actions, et qu’il faut absolument que telles soient également son énergie, son activité, sa justice et sa droiture, et qu'alors il sera sans culpabilité.

385.

Regarder sa propre vie ; regarder le peuple.

TSHENG TSE. — «Sa propre vie », ce qui résulte de ses propres actions. $\mathrm{Si}$ le prince désire regarder si le résultat de son influence et de ses actions est bon, il convient qu'il regar de le peuple. Si les mœurs du peuple sont bonnes, c'est que les institutions sociales sont bonnes et civilisatrices. Wang Pi dit : «Regarder le peuple pour scruter sa propre voie morale »; c'est exactement cela.

TSHOU HI. - Ici, Khong Tse en parle au point de vue du sens ; il montre clairement que le prince, en regardant ses propres actions, ne doit pas se 
borner uniquement à l'exa men de ses qualités et de ses défauts personnels, mais quil doit encore regarder si les vertus du peuple sont bonnes ou non, afin de scruter les siennes propres. 


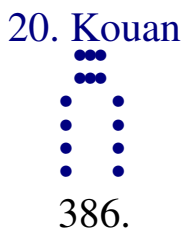

Trait supérieur nonaire : regarder sa vie ; l'homme doué est sans culpabilité.

TSHENG TSE. - Le trait supérieur nonaire emploie les vertus de l'énergie active et se place au-dessus de tous; c'est lui que les inférieurs regardent et il n'est pas digne de la situation : c'est donc un homme sage, ou homme doué qui n'est pas dans une situation définie, mais dont la voie vertueuse est l'objet des regards de l'univers. « Regarder sa vie » ${ }^{1}$, regarder ce qu'il engendre par son action, c'est-à-dire ce qui en résulte, ses vertus et sa pratique du devoir. Du moment où il est le point de mire des regards, il en résulte qu'il regarde lui -même ses actions, et si elles sont toutes dignes d'un homme doué, il sera sans culpabilité ${ }^{2}$, si elles ne sont pas d'un homme doué, comment amènerait-il les hommes à lever les yeux vers lui pour le prendre comme règle et comme modèle ? Ce serait là sa culpabilité.

TSHOU HI. - Le trait supérieur nonaire, énergique et actif, occupe le rang au-dessus de celui qui indique la situation prééminente. Quoiqu'il ne soit chargé présentement du poids d'aucune affaire, il est cependant regardé par les inférieurs, de sorte que l'avertisse ment de la formule est à peu près le même que dans le cas du cinquième trait. La différence consiste uniquement dans le remplacement du terme wo, je, moi, par le terme $k h i$, le, ce... Il y a entre ces deux mots la même légère différence qui existe entre le « maître » et $l^{\prime} \ll$ hôte $»^{3}$.

387.

Regardez la vie; tendances pas encore satisfaites.

TSHENG TSE. - Bien qu'il ne soit dans aucune situation déterminée, cependant, puisque les hommes considèrent ses vertus, et en font la règle de leur conduite, il doit donc s'observer avec une attention circonspecte, regarder

\footnotetext{
${ }^{1}$ Le commentateur lit : « sa vie ».

2 Dans les autres formules le texte porte littéralement: «Regarder ma vie » et ce texte est interprété avec assez de raison comme signifiant: «Regarder sa propre vie. » Ici, le texte porte kouan khi sheang, « regarder la vie » ou « regarder sa vie », mais rien ne peut faire admettre qu'il s'agisse encore de « regarder sa propre vie » à soi-même.

${ }^{3}$ Comparaison très usitée pour marquer ce qui fait ou ce qui reçoit l'action.
} 
le résultat de ses actions, et ne jamais manquer aux devoirs de l'homme doué. Alors les hommes ne seront point déçus dans l'objet de leur attention, et se transformeront. Il ne doit pas, sous prétexte qu'il n'occupe aucune situation, rester dans l'inactivité, s'aband onner à ses propres idées, sans s'occuper de rien. C'est donc que ses idées et ses tendances ne peuvent pas encore être erg repos, et c'est pourquoi la formule dit « pas encore satisfaites », le terme ping, exprime le sens de repos et de tranquillité.

TSHOU HI. - «Tendances pas encore satisfaites », cela exprime que, bien qu'il ne pos sède aucune situation définie, il ne doit cependant pas oublier les avertissements et mépriser toute crainte. 


\section{She ho}

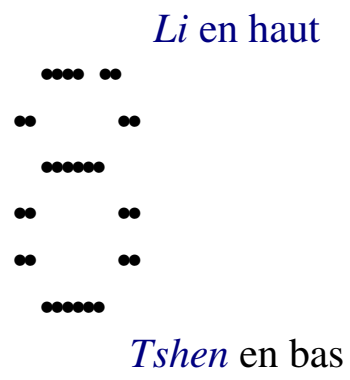

388. She ho, couper avec les dents et joindre les mâchoires ; liberté ; avantage à employer les sentences.

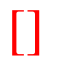

392. Premier trait nonaire : chausser des entraves de bois qui détruisent les orteils; pas de culpabilité.

*

394. Deuxième trait hexaire : mordre la peau, détruire le nez; pas de culpabilité.

$*$

396. Troisième trait hexaire : mordre de la viande séchée et se faire mal fortuitement; légère appréhension ; pas de curabilité.

$*$

398. Quatrième trait nonaire: mordre de la viande sèche; trouver une pointe de flèche en métal; avantage de la perfection difficile; présage heureux.

*

400. Cinquième trait hexaire : mordre de la viande sèche; rencontrer de l'ôr jaune ; inconvénient de la perfection ; pas de culpabilité.

*

402. Trait supérieur nonaire : porter sur les épaules une entrave qui détruit les oreilles; présage malheureux.

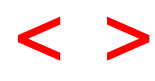




\section{She ho.}

TSHENG TSE. - She ho. «L'Ordre des koua » dit: «Possibilité de regarder et ensuite ligne de conduite à suivre. C'est pourquoi le koua kouan est suivi du koua she ho ; ho équivaut à ho, s'accorder à, s'unir à. » Du moment où il y a quelque chose à regarder, il en résulte aussitôt des adhésions de ceux qui se conforment à l'exemple, et c'est là ce qui fait que le koua she ho suit immédiatement le koua houan. She signifie mordre, ou couper avec les dents : pi ho équivaut à ho, réunir, accorder ensemble. Avoir quelque chose dans la bouche qui la tient ouverte, mordre, couper avec les dents, et la fermer en joignant les mâchoires. Dans le koua, le trait supérieur et le trait inférieur sont tous deux énergiques, tandis que les traits intermédiaires indiquent la douceur malléable; au-dehors dureté énergique, au-dedans vide; c'est l'image symbolique des mâchoires de la bouche de l'homme. Mais au milieu du vide intermédiaire, se trouve encore un trait qui indique la dureté énergique, ce qui constitue l'image symbolique d'avoir quelque chose entre les mâchoires. Lorsqu'il y a quelque chose dans la bouche, cette chose sépare la mâchoire supérieure et la mâchoire inférieure, qui ne peuvent se réunir. Il faut donc mordre cette chose et couper avec les dents, et alors la réunion des mâchoires est possible, et c'est pour cela que le koua est appelé she ho. L'homme saint se sert de l'image symbolique du koua, et il l'applique aux choses de l'univers. Dans la bouche, c'est quelque chose qui sépare les mâchoires et les empêche de se réunir; dans l'univers il s'agira de choses violentes et désordonnées, ou de faussetés insidieuses qui y créent des dissensions et empêchent l'union et l'accord dans les affaires de l'empire. Il convient d'employer les règles pénale s; si les faits répréhensibles sont de peu d'importance, il suffira d'avertissements correctionnels ; sils sont graves, il faudra avoir recours aux supplices et à l'extermination des coupables, afin de supprimer le mal, ce n'est qu'ensui te que l'ordre pourra être parfait dans l'univers. Qu'il s'agisse de l'empire, ou d'un royaume, ou d'une simple famille, ou encore d'une affaire quelconque, ce qui empêche l'union et l'accord, est toujours un obstacle interposé ; sans l'obstacle l'union est partout intime. Enfin, en poussant l'observation jusqu'à l'action créatrice du ciel et de la terre, à la production de toutes choses, c'est toujours après l'union que l'évolution peut suivre son cours naturel. Toutes les fois qu'il n'y a pas encore union et accord, c'est toujours un obstacle qui produit la séparation. Par exemple, entre le prince et le sujet, le père et le fils, les parents et alliés, entre les amis, s'il y a séparation, division, inimitié ou dissension, c'est toujours en effet le résultat de quelque parole insidieuse ou mauvaise. En écartant la cause, l'accord se rétablit et c'est pourquoi la séparation est toujours la grande cause de malheur dans l'univers. L'homme saint regarde l'image symbolique contenue dans le koua she ho et il en tire des déductions relatives aux choses de l'univers, toujours en vue de supprimer les causes de désunion et d'arriver au rapprochement, de sorte que tout soit en harmonie et d'accord. Le koua she ho exprime donc le grand moyen de régir l'univers. La suppression des cause s de désunion dans l'empire consiste donc dans l'emploi des lois pénales et des 
châtiments, et c'est pour cela que la formule du koua est basée sur le sens d'emploi des lois pénales. Dans les deux substances des koua simples, l'intelligence éclaire et l'au torité ébranle : c'est là l'image symbolique de l'emploi des lois pénales. 


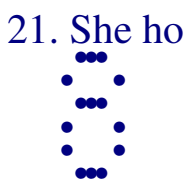

388.

She ho, couper avec les dents et joindre les mâchoires ; liberté ; avantage à employer les sentences ${ }^{1}$.

TSHENG TSE. - «Couper avec les dents et joindre les mâchoires; liberté. » Ce koua comporte essentiellement le sens de liberté. Ce qui fait que les choses de ce monde ne peuvent pas suivre librement leur cours, c'est quil existe des causes de séparation et de désunion; en coupant l'obstacle pour réunir ce qu'il tient écarté, on rétablit la libre com munication. «Avantage à employer les sentences »; dans la voie qui consiste à couper l'obstacle pour réunir, il convient d'employer les lois pénales et le s jugements criminels ; sans les lois pénales et les sentences, comment supprimer les obstacles qui désunissent l'univers ? Le texte ne dit pas : avantage à employer les lois pénales, mais il dit. avantage à employer les sentences. Le koua comporte l'image symbolique d'éclairer par l'intelli gence ${ }^{2}$; c'est ce qui est avantageux dans l'instruction des jugements criminels. Le jugement criminel, c'est l'opération par laquelle on recherche et on corrige les circonstances du fait, les sentiments qui ont guidé l'auteur, et la nature du dol. Ces circonstances et ces sentiments pénétrés, on connaît la voie qui est la cause de l'obstacle à l'union, et par suite il est donc possible d'établir des mesures préventives, comme d'en arriver au châtiment des coupables.

TSHOU HI. - She, mordre et couper avec les dents ; ho, réunir ; lorsque deux choses sont séparées par un obstacle, couper celui-ci et les réunir. Dans ce koua, il y a deux positivités, l'une en haut, l'autre en bas, et entre elles des traits évidés au milieu: c'est l’image symbolique des mâchoires et de la bouche. Un autre trait positif, le quatrième, forme un obstacle entre les autres ; il faut absolument le couper avec les dents pour réunir ensuite les mâchoires. C'est pour cela que ce koua est appelé she ho. Le sens divinatoire est qu'il doit y avoir liberté d'action ; c'est parce qu'il y a obstacle interposé que la libre communication n'existe pas ; en le coupant il y a réunion et, par suite, liberté de communication. De plus, le koua étant composé de trois négativités et trois positivités, la dureté énergique et la douceur malléable sont dans un juste équilibre. En bas mouvement initial, en haut clarté ; en bas l'ébranlement de la foudre, en haut l'éclair. Essentiellement, ce koua provient du koua parfait yi (27), dans lequel la douceur malléable du quatrième trait monte jusqu'au cinquième rang et parvient à la justice ; c'est là savoir employer la

\footnotetext{
${ }^{1}$ Les jugements criminels.

${ }^{2}$ Koua simple $l i$; littéralement, lumière qui éclaire.
} 
négativité dans un rang qui comporte la positivité. Bien que la situation soit imméritée, il y a cependant avantage à employer lesjugements criminels ; en effet, la voie rationnelle pour rendre les jugements consiste uniquement dans l'autorité et l'intelligence, et la confor mité à la justice en fait la noblesse. Aussi, pour celui qui, en consultant le sort, obtient ce koua, le sens divinatoire sera applicable s’il possède les vertus énoncées.

389.

Le commentaire traditionnel de la formule déterminative dit: Entre les mâchoires il y a quelque chose; on dit she ho.

TSHOU HI. - Explication du sens du nom du koua au moyen de la substance du koua lui-même.

390.

Couper avec les dents pour les réunir et ensuite liberté. La dureté énergique et la douceur malléable sont séparées; mouvement et clarté; l'ébranlement de la foudre et l'éclair s'unissent et montrent une image saisissable. La douceur malléable s'accorde à la justice et monte en agissant ; bien que la situation ne soit pas méritée, avantage à employer les jugements criminels.

TSHENG TSE. - Entre les mâchoires il y a quelque chose, c'est pour cela que le koua est appelé she ho. Lorsque quelque chose forme obstacle entre les mâchoires, cela constitue une gêne ; en coupant l'obstacle avec les dents pour réunir les mâchoires, la gêne disparaît, et la liberté d'action et de communication se trouve rétablie. C'est pour cela que la .ormule dit : « Couper avec les dents pour les réunir et ensuite liberté. » «La dureté énerzique et la douceur malléable sont séparées; mouvement et clarté ; l'ébranlement de la :oudre et l'éclair s'unissent et montrent une i mage saisissable. » Tout ceci est dit au sujet des propriétés des koua simples. Les traits qui expriment l'énergie et ceux qui expriment la douceur sont séparés les uns par les autres ; la dureté énergique et la douceur malléable sont séparées, et non groupées ensemble, ce qui constitue l'image symbolique de discer ner avec intelligence et clarté. La clarté dans la distinction des éléments est la base essentielle de l'instruction des jugements criminels. « Mouvement et clarté »; 
en bas le koua simple tshen, en haut le koua simple $l i$; impulsion qui donne la clarté. «L'ébranlement de la foudre et l'éclair s'unissent et montrent une image saisissable »; la foudre ébranle et l'éclair illumine ; les deux effets sont le complément nécessaire l'un de l'autre ; réunis ils forment un phénomène saisissable. La puissance éclairante et l'autorité coercitive agis sent de même : c'est la voie logique de la justice criminelle. Du moment où il est possible d'éclairer, aucune circonstance du fait ne peut rester cachée et obscure ; s'il y a autorité, personne ne peut résister à la crainte. Après avoir déjà précédemment parlé du mouvement et de la clarté d'après les deux images symboliques des koua simples, la formule revient sur l'idée de l'usage simultané de l'intelligen ce et de l'autorité. Le cinquième trait hexaire emploie la douceur et demeure dans la justice, ce qui constitue le sens d'employer la douceur en se conformant à la justice. «Monter en agissant» veut dire occuper la situation prééminente. «Bien que la situation ne soit pas méritée », exprime que le fait d'employer la douceur pour occuper le cinquième rang constitue un état anormal, mais cependant il y a avantage à employer les jugements criminels. Dans la voie rationnelle à suivre pour rendre les jugements, une dureté exclusive constituerait un excès de sévérité et de rigueur; trop de douceur serait un inconvénient consistant en excès d’indulgence. Le cinquième trait représente celui de qui dépend l'emploi de la justice criminelle; il emploie la douceur en se plaçant sur le terrain de l'énergie et se confor me à la justice : il possède donc les qualités requises pour employer les lois pénales. Si l'emploi de la douceur sur le terrain de l'énergie constitue un avantage dans l'emploi de la justice criminelle, employer l'énergie en se tenant sur le terrain de la douceur, sera-ce avantageux ou non ? L'énergie et la douceur sont des caractères personnels ; le choix du terrain est l'effet de ces caractères ; l'emploi de la douceur n'est pas la qualité qui répo nd nécessairement aux nécessités de la justice criminelle.

TSHOU HI. - C'est encore l'explication de la formule du koua au moyen du nom et de la substance du koua parfait, des vertus et des images symboliques des deux koua simples, et enfin de la transformation des koua parfaits.

391.

Le commentaire traditionnel de la formule symbolique dit : Ébranlement de la foudre et éclair; couper avec les dents et réunir les mâchoires; les premierrois employaient des châtiments clairs précisant la règle. 
TSHENG TSE. - Dans les formules symboliques il n'y a jamais d'inversions ; il y a lieu de supposer que l'ordre de ce texte est interverti ${ }^{1}$. L'ébranlement de la foudre et l'éclair sont deux choses nécessairement complémentaires et qui se manifestent en même temps ; il y a donc encore là image symbolique de réunion. L'éclair illumine par sa lumière, et l'ébranlement de la foudre en impose par sa puissance. Les premiers rois regardant l'image symbolique de l'ébranlement de la foudre et de l'éclair, prirent cette clarté et cette puissance d'action comme règles, et s'en servirent pour éclairer les lois pénales et les châtiments, et pour déterminer les prescriptions des règles et des ordonnances. Le mot règle exprime l’idée d'éclairer la raison d'être d'une chose et d'e $\mathrm{n}$ faire un moyen préventif.

TSHOU HI. - Au lieu de «foudre et éclair» il faut lire : «éclair et foudre $»$.

\footnotetext{
${ }^{1}$ Et qu'il faudrait lire : «She ho : ébranlement de la foudre et éclair. »
} 


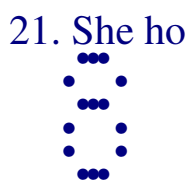

392.

\section{Premier trait nonaire : chausser des entraves de bois qui détruisent les orteils ; pas de culpabilité.}

TSHENG TSE. - Ce trait nonaire occupe le premier rang et est placé tout à fait en bas c'est celui qui est sans situation définie et l'image symbolique des inférieurs et du peuple. Il est considéré comme représentant l'homme qui subit un supplice. Au moment où commence l'emploi des supplices, la faute est peu grave et le supplice léger. Le terme kiao, quatrième caractère du texte, désigne des entraves en bois. La faute est petite, aussi ces entraves sont placées à ses pieds, afin de détruire et blesser les orteils. Un homme a commis une petite faute, on lui met des entraves qui blessent ses orteils, de telle sorte qu'il devra être corrigé par l'avertissement et devenir circonspect, sans oser avancer davantage dans le mal. C'est pour cela qu'il peut être sans culpabilité. Les «formules annexées » disent : «Une petite correction comportant un grand avertissement, tel est le bonheur de l'homme inférieur »; cela exprime qu'il est corrigé au début et dès la première fois, de sorte qu'il peut arriver à être sans culpabilité. Le premier rang et le rang supérieur n’indiquent aucune situation; les traits qui les occupent sont considérés comme représentant l'homme qui subit un supplice; les quatre autres traits représentent tous l'homme qui emploie les peines. Le premier occupe le rang le plus bas ; c'est celui qui est sans situation déterminée ; le trait supérieur est placé plus haut que le rang qui indique la situation prééminente ; or, avoir dépassé la situation prééminente, c'est également être sans aucune situation. Wang Ti considère ce rang comme indiquant une situation qui ne comporte ni la négativité, ni la positivité ; mais la négativité et la positivité dépendent de l'unité ou de la dualité ; comment l'une des deux conditions n'existerait -elle pas ? Cependant, dans les koua, au sujet du premier trait et du trait supérieur, il n'est question ni de situation méritée, ni de situation imméritée ; en effet, le sens d'origine ou de fin, est plus important. Dans le koua lin (19), le premier trait nonaire exprime la droiture au point de vue de la situation, tandis que la fonnule du trait supérieur hexaire du koua su (5) dit qu'il n'est pas digne de la situation qu'il occupe. La formule du trait supérieur nonaire du koua khien (1) dit «point de situation ». Mais l'acception du mot situation n'est pas la même selon qu'il s'agit d'un titre officiel ou de négativité et positi vité.

TSHOU HI. - Le premier rang et le rang supérieur ne comportent pas l'indication d'une situation officielle déterminée ; les traits qui les occupent sont considérés comme représentant l’image symbolique de celui qui subit un supplice. Les quatre traits intermédiaires sont considérés comme représentant 
l'image symbolique de ceux qui emploient les lois pénales. Le premier trait est placé au commencement du koua ; la faute est minime, l'erreur est légère. De plus, étant au bas du koua, il est donc considéré comme représentant l'image symbolique de « chausser des entraves qui détruisent les orteils ». Le mal est arrêté à son début, c'est pourquoi il n'y a point de culpabilité. Celui qui consulte le sort subira un léger mal, mais sera sans culpabilité.

393.

Chausser des entraves de bois qui détruisent les orteils ; ne pas marcher.

TSHENG TSE. - Étant chaussé d'entraves de bois qui détruisent et blessent ses orteils, il en résulte qu'il saura se corriger en profitant de la réprimande et qu'il n'osera pa s aggraver le mal déjà fait ; c'est pour cela que la formule dit «ne pas marcher». Selon les lois pénales établies par les hommes de l'antiquité, les coupables de fautes légères étaient entravés par les orteils. En effet, la condition d'être empêché dans la marche était choisie comme indiquant que le coupable ne doit pas avancer dans la voie du mal.

TSHOU HI. — «Détruire les orteils » exprime encore l'image syinbolique de ne pas avancer dans le mal. 


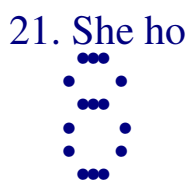

394.

Deuxième trait hexaire : mordre la peau, détruire le nez; pas de culpabilité.

TSHENG TSE. - Le deuxième rang marque la situation correspondant à celle du cinquième ; le trait qui l'occupe est celui qui emploie les lois pénales. Ces quatre traits prennent tous le sens de mordre et couper avec les dents. Le second se maintient dans la justice et possède la droiture ; c'est s'accorder à la justice et la droiture dans l'emploi des peines. L'emploi des peines étant conforme à la justice et au droit, le mal et le crime sont facilement subjugués, et c'est pourquoi l'idée de «mordre la peau » est prise comme image symbolique. La peau et les chairs de l'homme, mordues avec les dents, sont facilement pénétrables ; le terme mie, du texte, veut dire faire disparaître ; la morsure pénètre profondément, jusqu'à enlever le nez. Le second trait emploie la voie de la justice et de la droiture ; la peine est facilement acceptée par le patient. Cependant, il s'appuie sur la dureté énergique du premier trait et le foule à ses pieds; il exprime donc l'emploi des peines contre un homme énergique et violent. En employant les lois pénales contre un homme énergique et violent, il faut absolument lui causer une douleur profonde, et c'est pour cela qu'en p oussant la sévérité jusqu'à détruire le nez, il est encore sans culpabilité. La voie de la justice et de la droiture, qui amène facilement les hommes à se soumettre, et la sévérité des peines infligées à la violence énergique, sont deux sens qui ne s'exclu ent pas.

TSHOU HI. - Dans les sacrifices, il y a le «trépied de peau » ( ?) ; en effet, les chairs molles et tendres sont faciles à couper avec les dents. Le second trait hexaire comporte la justice et la droiture, de sorte que son action s'exerce aussi facilement que lorsque les dents coupent de la peau. Cependant, il emploie la douceur pour dominer l'énergie, de sorte que bien que son action s'exerce très facilement, il ne peut cependant éviter de bles ser et de détruire le nez. Bien que celui qui consulte le sort subisse une souffrance, à la fin, il sera sans culpabilité.

395.

Mordre ta peau, détruire le nez; dominer l'énergie. 
TSHENG TSE. - Profondément au point d'enlever le nez ; c'est parce qu'il agit pour dominer l'énergie. Dominer l'énergie, c' est employer les peines contre un homme énergique et violent; il est impossible de ne pas déployer une sévérité profonde. Avec cette sévérité on atteindra au degré nécessaire et c'est là ce qu'on appelle justice. 


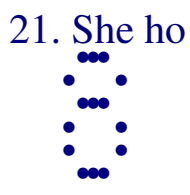

396.

Troisième trait hexaire: mordre de la viande séchée et se faire mal fortuitement ${ }^{1}$; légère appréhension ; pas de curabilité.

TSHENG TSE. — Le troisième trait hexaire occupe le rang supérieur dans le koua simple inférieur ; c'est quelqu'un qui applique les lois pénales. Étant hexaire et occupant le troisième rang, il se place dans une situation imméritée. Se plaçant dans une situation qu'il ne mérite pas et infligeant une peine à quelqu'un, cet homme ne se soumettra po int, éprouvera du ressentiment, et se révoltera. C'est comme si on mord, pour couper avec les dents, quelque chose tel que de la viande séchée souple et résistante, et qu'on rencontre un point dont la saveur est désagréable et empoisonnée ${ }^{2}$, c'est au contr aire la bouche qui se trouve subir le mal. Lorsqu'on applique les lois pénales et que les hommes ne se soumettent pas à la sentence portée contre eux, on éprouve les effets fâcheux de leur ressentiment, et c'est pour cela qu'il y a appréhension. Cependant, dans le moment indiqué par le koua she ho ce qui importe le plus, c'est de couper l'obstacle et de réunir ce qui est séparé. Bien que personnellement placé dans une situation imméritée, et que la violence hardie et criminelle soit difficile à subjuguer, à ce point qu'on peut en éprouver soi même fortuitement quelque inconvénient, cependant, ce n'est pas l'emploi des peines qui n'est pas convenable, de sorte que bien qu'il y ait des motifs d'appréhension, ils ne sont cependant pas graves. En coupant l'obsta cle et en réunissant ce qui est séparé, on sera sans culpabilité.

TSHOU HI. - L'expression « viande séchée» désigne la viande des animaux séchée entière, chair et os ; c'est quelque chose à la fois mou et résistant. Le trait exprime la mollesse négative, sans justice ni droiture : il gouverne et juge les hommes, et ceux-ci ne se soumettent pas à ses sentences : il est considéré comme l'image symbolique de l'action de mordre de la viande séchée et de se faire mal fortuitement. Bien que le sens divinatoire indique une petite appréhension, cependant le moment indique la nécessité de couper et de réunir, et il n'y a point de faute contre le devoir.

397.

\footnotetext{
${ }^{1}$ Les Chinois lisent « rencontrer du poison ».J'ai rendu le sens le plus archaïque et le moins figuré. L'idée est la même.

${ }^{2}$ Cette image n'est réellement pas dans le sens du texte.
} 


\section{Se faire mal fortuitement, situation imméritée.}

TSHENG TSE. - Le troisième trait hexaire emploie la négativité et occupe un rang positif ; il est placé dans une situation imméritée. Se plaçant lui-même dans une situation imméritée, il en résulte que ceux qu'il punit, se soumettent difficilement à ses sentences, et à leur tour lui causent des maux. 


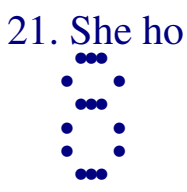

398.

Quatrième trait nonaire : mordre de la viande sèche ${ }^{1}$; trouver une pointe de flèche en métal; avantage de la perfection difficile; présage heureux.

TSHENG TSE. - Le quatrième trait nonaire occupe une situation proche du prince; c'est celui qui est présentement investi de l'autorité nécessaire pour couper et rejoindre. Le quatrième trait a déjà dépassé le juste milieu ${ }^{2}$, ce qui indique que l'obstacle à suppri mer est extrêmement considérable et que l'emploi des peines est extrêmement grave. C'est pour cela que la formule dit : « mordre de la viande sèche ». Le cinquième caractère du texte indique de la viande qui est adhérente à des os ; de la viande séchée avec les os ; c'est ce qu'il y a de plus résistant et de difficile à couper avec les dents. L'objet est excessivement dur à mordre et il s'y rencontre une pointe de flèche en métal. Le mot métal (or) est pris comme synonyme de dureté; le terme flèche indique l'idée de rectitude. Le quatrième trait possède les vertus de la positivité ; il est énergique et rigide ; il est considéré comme indiquant la conformité à la voie de l'énergie et de la rectitude. Bien qu'employant la voie de l'énergie et de la rectitude, l'avantage consiste à surmonter les difficultés que présente l'affaire en question et à se maintenir avec une parfaite fermeté dans la voie adoptée ; c'est donc un présage heureux. Le quatrième trait est dur et énergique et il fait partie de la substance du koua simple qui indique la clarté de l'intelligence. Il est positif et il occupe un rang qui comporte la douceur malléable. La dureté énergique jointe à l'intelligence est dangereuse par trop de décision, de sorte que la formule avertit, afin qu'on sache reconnaître la difficulté ; occupant un rang qui comporte la douceur, il en résultera un manque de fermeté dans l'observation de la voie suivie, de sorte qu'elle avertit aussi au sujet de la fermeté et de la perfection. Il arrive souvent que l'énergie existe sans être unie à la perfection et à la pureté ; quiconque manque d'énergie, n'est jamais parfait. Dans le koua she ho c'est le quatrième trait qui présente les meilleures conditions.

TSHOU HI. - Le terme employé dans le texte, désigne la viande qui entoure des os ; il s'emploie comme synonyme du caractè re qui désigne des lanières de chair séchée ${ }^{3}$. Dans les Rites de Tsheou on trouve ce passage : «Dans les jugements criminels et les procès, il est versé un poids déterminé d'or et un faisceau de flèches ${ }^{4}$, et ensuite l'affaire est entendue. » Le

\footnotetext{
${ }^{1}$ Le terme employé dans le texte est différent de celui qui a été traduit au n 396

2 Du koua.

${ }^{3}$ Ce qui ne correspond nullement à l'explication ci-dessus.

${ }^{4}$ Par les parties ; caution, nantissement.
} 
quatrième trait nonaire emploie la dureté énergique et occupe un rang qui comporte la douceur, il possède la voie rationnelle de l'emploi des lois pénales et c'est pour cela qu'il présente cette image symbolique. Cela exprime que l'objet mordu est des plus résistan ts et que le trait réunit les conditions nécessaires pour entendre et juger les procès. Cependant, il faut absolument qu'il y ait avantage dans les difficultés pendantes et une ferme droiture, et alors le présage est heureux. C'est un avertissement à celu i qui consulte le sort, pour qu'il sache qu’il doit être dans ces mêmes conditions.

399.

Avantage de la perfection difficile, présage heureux, pas encore d'éclat.

TSHENG TSE. - Toutes les fois qu'une formule emploie l'expression «pas encore d'éclat », c'est que la voie à suivre n'est pas encore éclatante et grande. La formule avertit de l'avantage de la perfection difficile ; en effet, ce qui fait défaut, dans le cas de ce trait, c'est certainement qu'il ne possède ni justice ni droiture. 


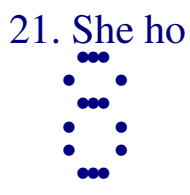

400.

Cinquième trait hexaire : mordre de la viande sèche; rencontrer de l'or jaune ${ }^{1}$; inconvénient de la perfection ; pas de culpabilité.

TSHENG TSE. - Le cinquième trait occupe un rang encore plus élevé et il est considéré comme exprimant l'action de mordre de la viande sèche, ce qui, au contraire, est plus

aisé que l'action du quatrième consistant à couper de la viande séchée sur les os. Le cinquième occupe la situation prééminente ; il s'appuie sur la force et la puissance inhérentes à la supériorité du rang pour infliger des peines aux inférieurs; la force naturelle des choses lui rend cette tâche facile. Dans le koua, il tend vers la limite extrême, ce qui exprime que l'obstacle à trancher est extrêmement considérable et qu'il n'est pas facile de rétablir l'union ; c'est pour cela quil est considéré comme exprimant l'action de mordre de la viande sèche. "Trouver de l'or jaune »; le jaune est la couleur de la justice ; le mot or, ou métal, exprime quelque chose de dur. Le cinquième trait occupant le rang intermédiaire dans un koua simple est considéré comme exprimant la conformité à la voie de la justice; il se place avec énergie et le quatrième l'aide par son énergie : c'est « trouver de l'or jaune ». Le cinquième est sans correspondance sympathique et le quatrième occupe la situation d'un ministre d'un rang élevé ; il jouit de son concours. Inconvénient de la perfection, absence de culpabilité ; le cinquième trait hexaire bien que placé dans un rang qui comporte l'énergie et la justice, est cependant d'une substance essentiellement molle et douce, aussi la formule avertit qu'il faut absolument la droiture et la fermeté, et aussi conserver l'appréhension du péril, et qu'alors il pourra être sans culpabilité. Employant la douceur et occupant un rang prééminent, se trouvant d'ailleurs dans un moment où il faut couper un obstacle pour réunir ce qui est disjoint, comment pourrait-il se passer d'une parfaite fermeté et de conserver l'appréhension du péril ?

TSHOU HI. - Mordre de la chair sèche, c'est -à-dire quelque chose plus difficile à couper que la peau, et plus facile que de la chair séchée avec les os. Le jaune est la couleur intermédiaire, ou couleur de la justice. Le mot métal, du texte, exprime encore un poids déterminé d'or. Le cinquième trait hexaire est doux, condescendant et juste ; puisqu'il occupe la situation prééminente, il applique la loi pénale aux hommes; ceux-ci se soumettent tous à ses sentences, et c'est pour cela qu'il exprime cette image sym bolique. Mais toutefois, il lui faut nécessairement de la pureté et de l'appréhension, et alors

\footnotetext{
${ }^{1}$ Métal jaune, or. Déposé en nantissement parles parties.
} 
il réussit à être sans culpabilité. C'est aussi une formule d'avertissement adressé à celui qui consulte le sort.

401.

Perfection, appréhension, absence de culpabilité; atteindre au point convenable.

TSHENG TSE. - Ce qui fait qu'il peut être sans culpabilité, c'est que ses actions sont conformes à ce qui est convenable. Ce qui est exprimé par le mot convenable, c'est de se maintenir dans la justicé en emplo yant l'énergie, et de pouvoir conserver la droiture en se préoccupant du péril. 


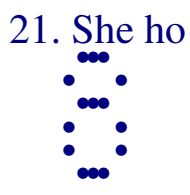

402.

Trait supérieur nonaire: porter sur les épaules une entrave qui détruit les oreilles; présage malheureux.

TSHENG TSE. — Le trait supérieur est encore plus haut que la situation prééminente : il est sans situation, et c'est pour cela qu'il représente quelqu'un qui subit un supplice. Il occupe le rang final dans le koua, c'est donc que l'obstacle cause de séparation est grave ; il s'agit de la dernière période d'intensité dans l'action de couper l'obstacle avec les d ents, ce que les «formules annexées » expriment en disant: «Le mal s'accumule et ne peut plus être caché ; la faute est grande et ne peut plus être absoute. »C'est pour cela qu'on le charge d'une entrave de bois qu'il porte sur les épaules et détruit ses oreilles : le présage malheureux est évident. Le terme employé dans le texte, exprime l'action de porter sur la nuque.

TSHOU HI. - Le terme du texte exprime l'action de porter sur les épaules ; excès final de la positivité. Il est au rang supérieur dans le koua, le mal est extrême, la faute considérable ; c'est une voie logique de présage de malheur, et c'est pourquoi tels sont le sens divinatoire et l'image symbolique.

403.

Porter sur les épaules une entrave de bois qui détruit les oreilles; entendement sans clarté.

TSHENG TSE. - Lorsqu'un homme est sourd et aveugle au point de ne plus rien discerner, il accumule le mal et les fautes jusqu'au point le plus extrême. Selon les règles instituées par les hommes de l'antiquité, lorsque la faute était grave, le coupable était chargé d'une entrave de bois sur les épaules, ce qui était considéré comme exprimant quil ne pouvait plus rien entendre ni connaître. Le mal est complètement accumulé en lui, c'est pour cela qu'on emploie l'entrave de bois et qu'on blesse et détruit ses oreilles, pour le reprendre de son manque de clarté dans l'entendement ${ }^{1}$.

\footnotetext{
${ }^{1}$ Tout ceci est basé sur la croyance que dans la plus haute antiquité, les moyens de châtiment employés étaient symboliques.
} 
TSHOU HI. - Détruire les oreilles, pour punir son défaut d'entendement. S'il peut juger, entendre, et se hâter d'en tirer conclusion, il ne subira pas ce malheur. 


\section{Pi}

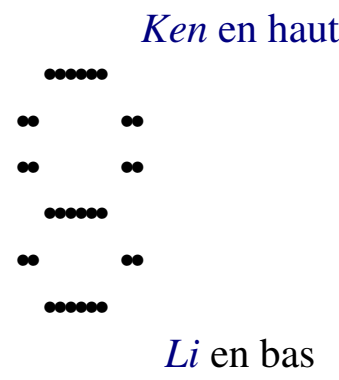

404. Pi, orner ; liberté ; petit avantage dans ce qu'il y a à entreprendre.

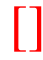

410. Premier trait nonaire : régulariser la position des pieds; quitter la voiture et marcher à pied.

412. Deuxième trait hexaire : arranger la barbe.

*

414. Troisième trait nonaire : comme régularisé, comme imprégné ; présage heureux de la perfection continue.

$*$

416. Quatrième trait hexaire : comme déterminé, comme blanc écru; cheval blanc comme volant; ce ne sont pas des brigands, c'est un mariage.

$*$

418. Cinquième trait hexaire : ordre déterminé dans le jardin enclos sur la colline ; tissus de soie en rouleaux et coupés en petites pièces ; appréhension ; finalement présage heureux.

$*$

420. Trait supérieur nonaire : simplicité de l'arrangement ; pas de culpabilité. 
22. Pi.

TSHENG TSE. - P $i$. «L'Ordre des koua » dit : «Le caractère ho signifie réunion; les êtres ne peuvent pas être seulement réunis accidentellement, aussi le koua she ho est suivi du koua pi; pi, orner et régulariser. » Dans la réunion des êtres, il faut absolument un dessein tracé; ce dessein tracé constitue l'arrangement ou mise en or dre. Ainsi, s’il s'agit de la réunion des hommes se groupant ensemble, il faudra qu'il y ait des liens d'autorité, des principes d'étiquette, des inférieurs et des supérieurs. S’il s'agit de la réunion et du groupement des êtres, il faudra un ordre de classification et de rangement ; la réunion entraîne nécessairement l'existence d'un plan tracé, et c'est pour cela que le koua pi suit immédiatement le koua she ho. Comme koua il représente la montagne sous laquelle il y a du feu. La montagne, c'est le lieu où se trouvent rassemblés les herbes, les arbres et tous les êtres. En bas, il y a du feu qui éclaire donc et rend visible le sommet; les plantes, les arbres, et toutes les catégories et espèces d'êtres ou de choses, tout est illuminé par l'éclat et la lue ur du feu, ce qui donne l'image symbolique de l'ordre et de l'arrange ment, et c'est pour cela que le koua est appelé pi. 


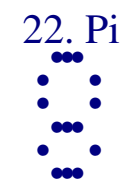

404.

$P i$, orner ; liberté ; petit avantage dans ce qu'il y a à entr eprendre ${ }^{1}$.

TSHENG TSE. - Les êtres étant rangés peuvent ensuite jouir de liberté ; c'est pour cela qu'il est dit : «sans base rien de stable ; sans tracé déterminé, pas d'action. » Lorsqu'il y a une réalité matérielle et qu'on la soumet à un ordre déterminé, il lui devient possible de suivre librement son évolution. La voie rationnelle de la régularisation suivant un plan déterminé peut augmenter la variété de l'éclat, de sorte qu'il est possible qu’il y ait un petit avantage en avançant.

TSHOU HI. - Pi, orner, régulariser. Le koua provient du koua souen (41) dans lequel la douceur malléable vient du troisième rang et donne sa forme au second trait, tandis que la dureté énergique monte du second rang et donne sa forme au troisième. Si on le considère comme provenant du koua ki tsi, la malléabilité vient du rang supérieur et détermine l'apparence du cinquième trait, tandis que la dureté énergique monte du cinquième rang et donne son apparence au trait supérieur. De plus, au-dedans le koua simple $l i$ et au-dehors le koua simple ken; il y a image symbolique de clarté de l'apparence, chaque chose, d'ailleurs, placée selon sa condition. C'est pour cela que le koua est appelé pi. Quant au sens divinatoire, puisque la douceur malléable vient déterminer l'apparen ce de la dureté énergique, que la positivité jouit de l'assistance de la négativité, et que la clarté exprimée par le koua simple li est au-dedans, il est donc considéré comme exprimant la liberté. Puisque la dureté énergique monte donner sa forme à la douceur malléable, et que le koua simple ken est arrêté au-dehors, il en résulte un léger avantage dans ce quill y a à entreprendre.

405.

Le commentaire traditionnel de la formule déterminative dit: $\mathrm{Pi}$, régularisation dans l'arrangement ; liberté.

\footnotetext{
${ }^{1}$ Sens tout à fait conventionnel. La construction ne permet pas une lecture déterminée.
} 
TSHOU HI. - Il est à supposer que le mot liberté est une superfétation dans le texte.

406.

La douceur malléable vient et détermine l'apparence visible de la dureté énergique; donc liberté; une partie de la dureté énergique monte et détermine l'apparence visible de la douceur malléable; c'est pourquoi il y a un petit avantage dans ce qu'il y a à entreprendre ; aspect du ciel.

TSHOU HI. - Explication de la formule du koua au moyen de la transformation des koua parfaits. Jonction de la dureté énergique et de la douceur malléable; image symbolique de l'action naturelle et spontanée. C'est pour cela que la formule dit : «aspect du ciel». Les premiers philosophes expliquent qu'avant les mots « aspect du ciel », il devrait y avoir quatre caractères dont le sens est : «l'énergie et la douceur se joignent et se mélangent ». Cela peut être vrai.

407.

Clarté de l'apparence arrêtant; apparence de l'homme.

TSHENG TSE. - Le koua est considéré comme exprimant l'image symbolique de l'ornementation et de la régula risation. Au point de vue des deux substances des koua simples inférieur et supérieur, la dureté énergique et la douceur malléable s'unissent et constituent ensemble la détermination visible des objets. La substance essentielle du koua simple inférieur était l'activité ${ }^{1}$; la douceur malléable vient donner son apparence au trait du milieu et constitue le koua simple $l i$. La substance primitive du koua simple supérieur était la passivité ${ }^{2}$; la dureté énergique va donner son apparence au trait supérieur (du koua simple khonen) et constitue le koua simple ken (qui exprime l'arrêt). Alors l'ensemble représente la montagne (ken) au bas de laquelle il y a du feu (li), arrêt devant l'éclat de la forme, ce qui produit le koua $p i$, ou réalisation d'un ordre déterminé. Dans les affaires de l'univers, sans ordre déterminé et préétabli, point d'action ; donc par la régularisation

\footnotetext{
${ }^{1}$ Le koua simple khien, et c'est le remplacement d'un trait positif par un trait négatif qui lui donne son apparence actuelle.

${ }^{2}$ Du koua simple khouen.
} 
(pz) la liberté devient possible. La douceur malléable vient et donne son apparence à la dureté énergique, donc liberté ; la douceur malléable vient donner son apparence à la dureté énergique et produit l'image symbolique de clarté de l'apparence. C'est par la clarté de l'apparence qu'il y a ordre déterminé. La voie rationnelle de l'ordre déterminé permet de parvenir à la liberté. En réalité c'es t par l'arra.ngement que la liber té est possible. Une partie de la dureté énergique monte et donne à la malléabilité sa forme apparente ; c'est pour cela qu'il y a un petit avantage dans ce qu'il y a à entre prendre. Le trait intermédiaire du koua khien va donner son apparence particulière au trait supérieur du koua simple hen; c'est par l'arrangement que les choses se perfectionnent; c'est par l'arrangement qu'elles peuvent se produire, et c'est pourquoi un petit avantage peut en survenir. Or, si en entreprenant il peut y avoir avantage, c'est parce qu'il y a une base. La voie rationnelle de l'ordre déterminé et de l'arrangement ne peut par elle -même rien ajouter à la réalité ; elle lui donne uniquement un aspect visible. C'est par leur apparence visible que les choses sont manifestées et parfaites, de sorte qu'il y a un petit avantage dans ce qu'il y a à entreprendre. La liberté dont il s'agit, c'est la liberté de communication et de pénétration ; entreprendre c'est faire avancer. La transformation des deux koua produit également le sens du koua $p i$, arrangement, ordre déterminé, et la formule déterminative traite de chacun des deux koua simples à un point de vue particulier. En effet, la clarté, exprimée par le koua simple $l i$, est suffisante pour faire survenir la liberté ; en donnant son apparence à la douceur malléable elle peut encore avancer un peu ; c'est l'apparence du ciel. « Clarté de l'apparence arrêtant l'apparence de l'homme. » Ceci est dit par rapport au texte qui précède : La négativité et la positivité, la dureté et la douceur se donnant mutuellement leur apparence, c'est l'apparence du ciel ; ce qui arrête dans la clarté de l'apparence, c'est l'apparence de l'homme. Arrêter veut dire se placer dans la clarté de l'apparence. Ce qui a des car actères physiques doit nécessairement avoir une apparence ; c'est là une loi naturelle, ou « raison d'être ». Toute loi naturelle, ou raison d'être doit nécessairement avoir un effet correspondant : c'est là l'origine de la vie et de sa reproduction indéf inie. Du moment où la supériorité existe, l'infériorité existe fatalement ; du moment où on remarque un objet, on établit une distinction entre lui et un autre; du moment où la matière est caractérisée, elle doit avoir une apparence visible : l'unité n'e xiste pas par elle-même, c'est la dualité qui la rend apparente. Comment celui qui ne connaît pas la «voie » pourrait-il le comprendre ? L'apparence du ciel, c'est la «raison d'être » du ciel; l'apparence de l'homme, c'est la « voie rationnelle $\gg$ de l'h omme ${ }^{1}$.

TSHOU HI. - C'est encore dit au point de vue des vertus des koua simples. Arrêter veut dire que chaque chose se conforme à sa propre condition.

\footnotetext{
1 J'ai essayé de traduire aussi littéralement que possible, plu sieurs passages m’échappent, mais je doute que le commentateur ait bien su lui-même ce qu'il voulait dire.
} 
408.

Regarder l'apparence du ciel afin d'examiner les modifications des moments ${ }^{1}$; regarder l'apparence de l'homme afin de transformer et de compléter l'univers ${ }^{2}$.

La lune, des étoiles et des astres ; le remplacement et les modifications du froid et de la chaleur, de la négativité et de la positivité. Regarder leur mouvement de translation, afin de reconnaître les modifications et les changements des quatre saisons. L'apparence de l'homme, c'est -à-dire l'ordre et la nature de la raison d'être des actions de l'homme ; regarder l'apparence de l'homme afin d'instruire et de transformer l'univers. L'univers perfectionnant ses mours et ses règles morales, c'est le résultat de la voie de l'emploi de l'arrangement en ordre déterminé par l'homme saint. L'image symbolique de l'arrange ment en ordre déterminé, vient de ce que le koua représente une montagne au bas de laquelle il y a du feu; ou bien encore de la modification des koua parfaits dans lesquels la douceur malléable vient donner son apparence à la dureté énergique, tandis que la dureté énergique monte donner son apparence à la douceur malléable. Dans les divers koua, quelquefois l'idée est déterminée par le sens des deux substances des koua simples ainsi que par leurs deux images symboliques. Par exemple dans le koua tshonen (3) le sens est basé sur les conditions de «mouvement au milieu du péril » et sur les idées de «vent et de foudre »; dans le koua song (6) on relève les conditions de «dureté énergique en haut et de péril au-dessous » et celle de «l'eau agissant en sens opposé au ciel ». Quelquefois elle est déterminée par un trait particulier; ainsi, la douceur malléable possédant la situation qui lui convient et les traits supérieurs et inférieurs lui correspondant sympathiquement est la cause du nom du koua siao tshou (9); la douceur malléable occupant la situation prééminente avec une grande justice et les traits supérieurs et inférieurs lui correspondant sympathiquement est la cause du nom du koua tae yeou (14). Quelquefois on relève la valeur des deux substances et le sens de diminution ou de croissance ; par exemple : la foudre au milieu de la terre et en même temps la montagne en contact avec la terre, ce

qui donne l'explication du nom du koua po (23). D'autres fois on prend le sens dans les deux images symboliques en même temps que dans la permutation de deux traits ; par exemple : le vent et la foudre, koua yi (42), augmentation, en même temps diminution en haut et augmentation en bas ; le marais au bas de la montagne, diminution ou koua souen (41), en même temps que diminution en bas et augmentation en haut. Quelquefois après avoir défini

\footnotetext{
1 «Des saisons. »

${ }^{2}$ Littéralement : « le dessous du ciel. »
} 
le koua par les deux images symboliques, on prend encore le sens des traits : ainsi, la dureté énergique coupant court à la douceur malléable, dans le koua kouae (43); la douceur malléable rencontrant la dureté énergique, dans le koua keou (44). Quelquefois c'est par l'effet produit que le koua est défini ; ainsi puiser de l'eau et monter cette eau, dans le cas du koua tsing (48); du feu sur du bois, dans celui du koua ting (50). Il faut d'ailleurs noter que le koua ting est ainsi nommé d'après sa for me prise comme image symbolique. Quelquefois l'image symbolique est déduite de la forme ; par exemple : en bas de la montagne il y a du feu, c'est la raison du nom du koua $y i$; mais d'un autre côté, «il y a quelque chose entre les mâchoires » ce qui détermine le nom du koua she ho. Ceci est dit pour le sens des koua parfaits. Mais si on dit : dureté énergique en haut, douceur en bas, ou bien : diminution en haut, augmentation en bas, cela exprime que la dureté occupe un rang plus élevé et la douceur un rang plus bas, que la diminution a lieu en haut et l'augmentation en bas, et cela se rapporte également au koua parfait lui-même ; cela n’indique pas qu'il y a mouvement ascensionnel ou descendant vers le milieu du koua en question. De même, dans le cas du koua song (6) et du koua wou wang (25), les formules disent que la «dureté vient»; comment cela pourrait-il signifier qu'elle provient de la substance du koua simple supérieur ? Toutes les fois que la douceur malléable occupe le cinquième rang, les formules disent toujours que la «douceur avance et agit en montant»; la douceur malléable occupe le rang inférieur, ici elle occupe le rang prééminent, c'est donc avancer et monter; cela ne veut pas dire que c'est une portion de la substance inférieure qui monte. La transformation des koua est toujours observée par rapport aux koua simples khien et khouen; les premiers philosophes confucéens ne l'ont pas compris, de sorte qu'ils ont dit que le koua pi procède du koua thae ; mais comment est-il possible d'admettre que les koua simples khien et khouen superposés formeraient le koua thae? De plus, au sujet de la raison d'être de la transformation du koua thae, le koua simple inférieur $l i$ provient bien du koua simple khien dans lequel le trait intermédiaire se modifie pour former le koua $l i$; le koua simple supérieur ken a pour origine le koua simple khouen, dans lequel le trait supérieur se modifie et donne naissance au koua ken; le koua simple li est en-dedans, et c'est pourquoi la formule dit que «la douceur vient»; le koua simple ken est en haut, et c'est pourquoi la formule dit que la dureté énergique monte : cela ne veut pas dire que c'est une partie de la substance inférieure qui monte. Les koua simples khien et khouen se modifient et donnent naissance aux six autres koua simples; les huit koua simples se combinent et forment les soixante-quatre koua parfaits, et cela, toujours par la modification des deux koua simples khien et khouen ${ }^{1}$.

TSHOU HI. - Dernière dissertation sur la grandeur de la voie indiquée par le koua pi.

\footnotetext{
${ }^{1}$ Lorsqu'il y a, en apparence, autant de règles que de cas, c'est qu'il n'y a vraiment aucune règle, à moins qu'on ne préfère avouer qu'on ne connaît pas celle qui existe.
} 
409.

Le commentaire traditionnel de la formule symbolique dit: En bas de la montagne il y a du feu: détermination de la forme; l'homme doué porte la clarté dans les diverses prescriptions des institutions sociales; il n'ose pas décider dans les jugements criminels.

TSHENG TSE. - La montagne est le lieu où se multiplient les plantes, les arbres et tous les êtres ${ }^{1}$; le feu est au bas et sa lueur éclaire en haut. Les divers genres d'êtres ou de choses reçoivent sa lumière et sa clarté, et qui constitue l'image symbolique de l'ordre et de l'arrangement. L'homme doué regarde la montagne au bas de laquelle il y a du feu et l'image symbolique de lumière qui éclaire et il l'applique au soin de réformer et d'éclairer les diverses institutions sociales, et de compléter un ordre de gouvernement brillant par l'ordre visible qui y règne, mais il n'ose pas assumer la décision dans les jugements criminels. Prononcer une sentence criminelle est l'acte dans lequel le prince apporte la plus extrême circonspection ; comment pourrait-il s'autoriser de son intelligence et la pronon cer légèrement de luimême ? Tel est l'effet des sentiments de l'homme saint, et cela constitue un avertissement profond. Le choix du symbole est uniquement déterminé par cette condition que, en bas de la montagne, il y a du feu qui éclaire et illumine toutes choses ; l'emploi de la clarté, ou intelligence, constitue l'avertissement et le koua pi, ordre régulièrement déterminé, comporte encore essentiellement le sens de ne point oser prononcer une sentence. En prononçant une sentence, on doit exclusivement se baser sur le fait et les circonstances ; s'il y a quelque chose qui constitue un arrangement préconçu ${ }^{2}$ les circonstances du fait et les sentiments de son auteur sont masqués et disparaissent. C'est pour cela qu'on ne doit point oser dénaturer les apparences en prononçant un jugement. TSHOU HI. - En bas de la montagne il y a du feu ; sa clarté n'atteint pas au loin ; porter la clarté dans les diverses institutions sociales ${ }^{3}$. C'est là quelque chose de peu d'importan ce ; au contraire, rendre un jugement criminel est une affaire très grave. Au-dedans, le koua simple $l i$, clarté ; au-dehors, le koua simple ken, arrêt ; c'est pourquoi le symbole est ainsi choisi.

\footnotetext{
${ }^{1}$ Pour les Chinois, comme pour les Annamites, « montagne » est synonyme de « désert », lieu sauvage où l'homme ne vit qu'avec peine.

${ }^{2}$ Ce passage est presque intraduisible en français. La valeur du mot chinois change avec le sens du passage où il est employé. Le terme que le traducteur est obligé de rendre par « arrangement préconçu » est le même qui, ailleurs, signifie aussi «mettre en ordre » et « ornementer», « donner une apparence flatteuse », etc. Il en résulte une concision et une mobilité extraordinaires dans le style.

${ }^{3}$ Le terme rendu par « divers » a aussi le sens de « commun, ordinaire ».
} 


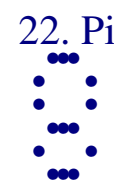

410.

Premier trait nonaire : régulariser la position des pieds; quitter la voiture et marcher à pied.

TSHENG TSE. — Le premier trait nonaire emploie la dureté énergique de l'activité et fait partie de la substance du koua simple qui exprime la clarté. De plus il est placé au rang inférieur ; c'est l'homme doué, possédant les vertus d'énergie et d'intelligence, et qui se trouve dans un rang inférieur. L'homme doué se trouvant placé sur un terrain qui ne comporte aucune situation définie ne peut avoir aucune influence sur l'univers ; il ne peut donc que régulariser et coordonner ses propres actions. Le terme $t s h i^{1}$, cinquième caractère du texte, est choisi parce qu'il rappelle ce qui est bas et ce qui sert à marcher ${ }^{2}$. La voie de l'homme doué qui se réforme et se régularise consiste à rectifier ses actions ou démarches, à maintenir l'observation des préceptes et à se placer suivant le devoir. Les démarches n'ont pas lieu au hasard ; il peut se faire que le devoir l'oblige à modifier sa vie actuelle, et alors il quitte sa voiture ou son char et se contente d'aller à pied. La foule consi dérerait cela comme une humiliation, mais l'homme doué prend cette circonstance comme un moyen de perfectionnement. Le sens de l'expression : "Quitter la voiture et aller à pied » se déduit à la fois du voisinage et de la correspondance sympathique ${ }^{3}$. Le premier trait est en contact avec le second et sympathise avec le quatrième; la sympathie pour le quatrième est le résultat de la droiture ; l'alliance avec le second y est contraire. L'éner gie intelligente du trait nonaire observant la droiture ne s'allie pas, près de lui, avec le second, mais elle sympathise au loin avec le cinquième : elle laisse échapper ce qui est aisé et suit ce qui est difficile, comme lorsqu'il s'agit de quitter un char pour aller à pied. L'observation des préceptes et des devoirs, c'est là la régularisation à laquelle s'astreint l'homme doué. C'est pour cela que ce que l'homme doué considère comme régulier serait une humiliation au point de vue des préjugés des gens de son époque, tandis que ce que ceux-ci considèrent comme régulier est méprisé par lui. La formule emploie les exemples du char et de la marche à pied, et tire le sens des idées de «pieds et de marche ».

TSHOU HI. - Qualités de la dureté énergique ; substance de la clarté ; il se place luimême dans un rang inférieur pour se régulariser en suivant un mode de réforme déterminé, ce qui constitue l'image symbolique de l'id ée d'abandonner le char qui ne suit plus la voie morale, en se contentant de

\footnotetext{
${ }^{1} T$ shi, pieds et aussi les « orteils ».

${ }^{2}$ Hinh, marcher, et aussi : « agir ».

${ }^{3}$ Du second et du quatrième traits.
} 
marcher à pied. Celui qui consulte le sort doit agir de même en choisissant sa position.

411.

Quitter la voiture et marcher à pied; le devoir est de ne pas y rester assis.

TSHENG TSE. - Quitter la voiture et aller à pied parce que le devoir exige de ne point y rester assis. La sympathie du premier pour le quatrième est régulière ; suivre le second serait irrégulier. Il abandonne les facilités que lui offre le second trait placé près de lui, pour suivre le quatrième à travers les difficultés qui se présentent; il quitte la voiture et marche à pied. La règle déterminée et régulière de l'homme doué consiste uniquement dans le devoir.

TSHOU HI. - Le choix de l'homme doué, dans ce qu'il prend ou ce qu'il abandonne, est exclusivement déterminé par le devoir. 


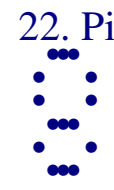

412.

Deuxième trait hexaire : arranger la barbe.

TSHENG TSE. - Bien que ce qui constitue le koua pi, soit le résultat de la modification de deux traits, cependant le sens de clarté d'apparence est le plus important. Le second trait est, en réalité, celui de qui dépendent l'ordre adopté et l'arrangement, de sorte que la formule traite spécialement de la voie rationnelle de la détermination de l'ordre. En arrangeant, ou ornant quelque chose, on ne doit pas en altérer gravement les caractères spéciaux ; c'est selon ces caractères spéciaux que doit être réglé l'arrangement, ou l'orne mentation. C'est pourcela qu'elle c hoisit le sens de « barbe »; la barbe se meut suivant le mouvement des lèvres. Qu'elle remue ou soit immobile, cela dépend uniquement des organes auxquels elle est fixée, de même que le bien ou le mal ne résultent pas de l'ordre ou de l'arrangement des fa its et des actions. La clarté d'apparence exprimée par le second trait indique uniquement l'arrangement et l'ordre adoptés ; le bien ou le mal résulteront des caractères particuliers du fait ou de l'action.

TSHOU HI. - Le second trait emploie la douceur négative et occupe son rang avec justice et droiture ; le troisième emploie la dureté énergique positive et se conforme à la droiture ; tous deux sont sans correspondance sympathique et sans alliance. C'est pour cela que le second s'attache au troisième et se met en mouvement, ce qui présente l'image symbolique d'arrangement de la barbe. Celui qui consulte le sort doit suivre et écouter l'énergie active d'un supérieur et se mouvoir en conséquence.

413.

Arranger la barbe; se lever avec le supérieur.

TSHENG TSE. — La barbe est choisie comme image symbolique : cela veut dire que ce trait et le trait supérieur se lèvent en même temps; il suit le supérieur et se met en mouvement; le mouvement ou l'arrêt dépendent exclusivement de l'objet auquel il est atta ché. Il en est de même en arrangeant, ou ornant un objet ; c'est d'après les caractères particu liers de cet objet qu'on 
l'orne ou qu'on l'arrange ; le bien ou le mal résident dans ses caractères particuliers. 


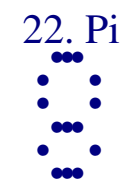

414.

Troisième trait nonaire : comme régularisé, comme imprégné ; présage heureux de la perfection continue.

TSHENG TSE. — Le troisième trait est placé au rang extrême du koua simple qui exprime la clarté de l'apparence. Il est placé entre deux négativités, le second et le quatrième traits, quill sépare, et tous trois déterminent réciproquement leur apparence ; il exprime donc le complet développement de la régularisation et de l'ornementation. C'est pour cela que la form ule dit : «Comme régularisé »; dans ce cas le terme jou, quatrième caractère du texte, est simplement employé comme particule auxiliaire ${ }^{1}$. À l'apogée de la régularisation et de l'ornementation, l'éclat illumine et baigne de son fluide bienfaisant, c'est pourquoi le texte dit: «comme imprégné ». À l'apogée de l'éclat illuminant la forme, il doit y avoir imprégnation par les fluides bienfaisants. Le Shi king dit : «La femelle du chevreuil grasse et luisante » ${ }^{2}$. «Présage heureux de la perfection continue »; entre le troisième trait d'un côté, et les second et quatrième traits de l'autre, la sympathie n'est pas l'effet de la droiture ; ces traits se groupent ensemble et déterminent mutuellement leur apparence respective, de sorte que la formule avertit au sujet de la nécessité de la perfection et de la droiture. Le caractère pi, nom du koua, exprime la même idée que le caractère shi, arranger, orner, prescrire un ordre déterminé. Dans le fait de mettre en ordre, d'arranger et d'orner, la difficulté réside dans l'impossibilité d'en assurer la per manence, de sorte que la perfection continue constitue un présage heureux. Le troisième et le quatrième traits se déterminent mutuellement dans leur apparence ; de plus, le troisième s'abaisse pour se grouper près du second; deux traits indiquant la douceur déterminent l'apparence d'un trait indiquant la dureté énergique. Le trait placé au-dessus et celui placé au-dessous se joignent pour orner et arranger, ce qui constitue le complet développement de l'acti on d'orner et d'arranger.

TSHOU HI. - Une positivité occupe l'intervalle entre deux négativités ; c'est elle qui reçoit l'ornementation et qui imprègne de son influence. Cependant elle ne doit pas s'amollir dans son repos, de sorte quil y a l'avertissemen t relatif à la continuité de la perfection.

\footnotetext{
${ }^{1}$ C'est une explication qu'on rencontre assez fréquemment et qui n'explique pas grand chos e.
}

2 Je ne connais pas le rapport qu'il peut y avoir entre cette citation et l'expression discutée. 
415.

Présage heureux de la perfection continue ; finalement, mépris de nul ${ }^{1}$.

TSHENG TSE. - Lorsque l'arrangement n'est pas constant et que de plus il n'est pas régulier, les hommes le méprisent et le viole nt. Aussi la formule avertit que s'il peut avoir une droiture constante, le présage sera heureux. Du moment où l'arrangement est constant et correct, qui donc pourrait le mépriser?

\footnotetext{
${ }^{1}$ Traduction littérale qui montre le défaut de correction du texte.
} 


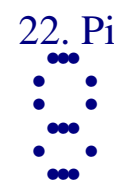

416.

Quatrième trait hexaire : comme déterminé, comme blanc écru; cheval blanc comme volant; ce ne sont pas des brigands, c'est un mariage.

TSHENG TSE. - Le quatrième et le premier traits sont ceux qui se correspondent sympathiquement avec droiture; ce sont ceux qui se déterminent mutuellement. En principe il convient quils soient «comme déterminés »; mais d'ailleurs ils sont séparés par le troi sième, de sorte qu'ils ne réussissent pas à se déterminer l'un l'autre et sont comme blanc écru. Le terme du texte signifie blanc; c'est-à-dire pas encore orné. "Cheval », c'est ce qui est dessous et se meut. Ne pas encore réussir à se déterminer réciproquement; c'est à cause de cela que le texte dit « cheval blanc». Il suit ce qui lui répond sympathiquement avec droiture avec une rapidité semblable au vol des oiseaux, et c'est pour cela que le texte dit « comme volant ». S'ils n'étaient séparés par le brigand ennemi représenté par le troisième trait, ils s'uniraient en mariage ${ }^{1}$ en donnant libre cours à leur affection mutuelle. Ce sur quoi on est monté, et qui entraîne par son mouvement, c'est l’image symbolique du cheval. Le premier et le quatrième traits sont ceux qui sympathisent avec droiture ; finalement ils doivent arriver à se réunir, mais au commencement ils sont séparés par un obstacle.

TSHOU HI. — «Blanc écru », blanc. « Cheval», monture de l'homme. Si l'homme est blanc, le cheval sera également blanc ${ }^{2}$. Le quatrième et le premier traits sont ceux qui s'orne nt, ou se déterminent mutuellement ; mais ici, ils sont séparés par le troisième et ils ne peuvent donner libre cours à leur impulsion naturelle, de sorte qu'ils sont comme blanc écru ${ }^{3}$, et le mouvement de leur cour, qui les porte à se rechercher, égale l' empressement du vol des oiseaux. Mais toutefois, le troisième trait étant nonaire, joint l'énergie à la droiture ; ce n'est pas un brigand, donc c'est quelqu'un qui vient demander en mariage, et c'est pour cela que telle est l’image symbolique.

\footnotetext{
${ }^{1}$ Cette forme conditionnelle est étrangère au texte.

${ }^{2}$ Il est vraiment fâcheux que Tshou Hi ne nous dise pas pourquoi ?

${ }^{3}$ Ils ne sont pas « ornés », pas teints de couleur artificielle.
} 
On a déjà vu cette comparaison et ce pseudo-raisonnement. Il reporte à un état de civilisation dans lequel le fiancé vient à cheval enlever sa future ; ceci, d'ailleurs, dit tout en réservant aucune appréciation sur l'origine d'une telle coutume.

417.

Quatrième trait hexaire; la situation méritée est douteuse ; ce n'est pas un brigand, c'est un mariage ; finalement pas de ressentiment.

TSHENG TSE. — Le quatrième traitest éloigné du premier et le troisième sert de séparation entre eux; la situation qu'il mérite et à laquelle il correspond est douteuse. Bien que séparés par le. brigand ennemi, représenté par le troisième trait, ils ne peuvent pas encore se rapprocher par un mariage, mais toutefois, quant à leur sympathie correcte, la raison d'être des ch oses veut que le strict devoir l'emporte et, finalement, ils doivent for cément réussir à s'unir ; c'est pour cela que la formule dit que finalement il n'y a pas de ressentiment. Le ressentiment, c'est le souvenir du mal et le désir de la vengeance. Finalement ils peuvent se déterminer et s'orner mutuellement ; c'est pour cela qu'il n'y a plus ni ressentiment ni rancune.

TSHOU HI. — «La situation méritée est douteuse »; c'est-à-lire que la situation méritée donne lieu au doute. «Finalement pas de ressentiment», c'est-à-dire qu'en observant la droiture sans se livrer, il n'y aura pas d'autre mal. 


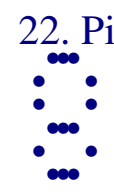

418.

Cinquième trait hexaire : ordre déterminé dans le jardin enclos sur la colline ; tissus de soie en rouleaux et coupés en petites pièces ; appréhension ; finalement présage heureux.

TSHENG TSE. - Le cinquième trait hexaire emploie les caractères distinctifs de la négativité ; il s'associe secrètement ${ }^{1}$ avec le sage énergique et actif représenté par le trait supérieur nonaire. La négativité s'associe à la positivité et n'est plus retenue par aucun lien de sympathie ; elle la suit, et reçoit du trait supérieur son apparence déterminée. Depuis l'antiquité on a considéré les passages dangereux comme constituant la sauvegarde des États, et c'est pour cela que, en général, les forteresses et les remparts sont appuyés sur des élévations et des talus. Le terme khieou, cinquième caractère du texte, désigne quelque chose près et au-dehors, et d'ailleu rs élevé. Le terme yuan, sixième caractère du texte, désigne un jardin enclos où on cultive des légumes, c'est quelque chose de très rap proché des remparts et du district ${ }^{2}$; c'est encore quelque chose de proche et d'extérieur. Les deux mots khieou yuan (jardin enclos sur la colline), désignent ce qui est près et à l'extérieur : ils désignent le trait supérieur nonaire. Bien que le cinquième trait hexaire occupe la situation du prince, cependant, les aptitudes de la douceur malléable négative sont insuffisantes pour qu'il s'observe et se garde lui -même ; lui et la dureté énergique active du trait supérieur se groupent ensemble et ses tendances le portent à écouter et à suivre. Il reçoit l'impulsion déterminante du sage contre lequel il se presse vers l'extérieur: «ordre déterminé par les jardins hors des remparts ${ }^{3}$. S ${ }^{3}$ il peut recevoir du trait supérieur nonaire l'impulsion déterminante, il supporte sa loi et sa décision ; il est comme de la soierie qu'on roule et qu'on coupe en petits morceaux ${ }^{4}$, de sorte que bien que sa faiblesse et sa mollesse ne lui permettent pas de faire par lui-même, il est amené à pouvoir n'éprouver que peu d'appréhension. Cependant, pouvant se plier à l'ouvre de l'homme qui le guide, finalement il atteint au bonheur. L'expression, du texte, exprime une manière de couper, tailler, diviser et déchirer ${ }^{5}$. Lorsqu'un tissu n'est pas encore employé, on le roule ${ }^{6}$, c'est

\footnotetext{
${ }^{1}$ Ou bien : « Il est en contact intime avec... »

2 Culture maraîchère aux abords des remparts des villes.

${ }^{3}$ Cette lecture du texte s'écarte de la traduction et résulte des explications du commentateur ; c'est la lecture qu'il adopte.

${ }^{4}$ La traduction suit sur ce point les explications du commentaire, mais le sens le plus simple serait « rouler de l'étoffe de soie légère ».

${ }^{5}$ C'est très douteux et pas prouvé.

${ }^{6}$ Les soieries chinoises sont en rouleaux.
} 
pourquoi la formule dit «tissus de soie en rouleaux »; mais au moment où on le convertit en vêtements, il faut le tailler en le coupant, le diviser en pièces menues et petites. Rouler des tissus, est une expression figurée employée comme exemple relatif au caractère particulier du cinquième trait hexaire. «Couper en petites pièces » signifie qu'il subit de quelqu'un une action réformatrice qui le rend apte à un emploi usuel. La détermination reçue d'autrui est quelque chose d'analogue à ce qui a lieu dans le cas du koua mong, mais dans celui-ci il n'est pas question d'appréhen sion. En effet, il est naturel qu'un jeun e être encore aveuglé par l'ignorance s'appuie sur quelque autre personne; mais s'il ne s'agit plus d'un jeune garçon et qu'il reçoive d'autrui l'impulsion régulatrice, c'est un motif plausible d'appréhension. Cependant, jouissant de l'œuvre de cette pers onne, finalement le présage est heureux.

TSHOU HI. - La douceur et la justice du cinquième trait hexaire, constituent la force directrice de l'ordre établi ; il affermit la base et estime la réalité ; il se conforme à la voie de la détermination de l'ordre. C'est pour cela qu'il présente limage symbolique du jar din enclos sur une colline. Cependant, la nature de la douceur malléable est portée à la parcimonie, ce qui fait que ce trait a aussi l'image symbolique de l'action de rouler des tis sus de soie taillés en pièces. Rouler des tissus de soie, se rapporte à quelque chose de considérable et d'important, tandis que l'expression couper en petits morceaux, désigne quelque chose de superflu et sans valeur. Lorsque quelqu'un est dans ces conditions, bien qu' il puisse éprouver de la honte et de l'appréhension, cependant, s'il vient à s'agir de questions de dépenses rituelles, comment pourrait-il agir avec parcimonie ? Aussi, à la fin, le présage est heureux.

419.

Présage heureux du cinquième trait hexaire, avoir de la joie.

TSHENG TSE. - Pouvoir écouter et suivre les conseils d'autrui afin d'accomplir une auvre déterminée, et jouir du bonheur et du succès ; c'est là avoir de la joie. 


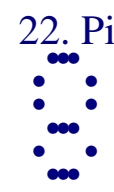

420.

Trait supérieur nonaire : simplicité de l'arrangement ; pas de culpabilité.

TSHENG TSE. - Le trait supérieur nonaire marque la limite extrême de l'arrangement ou ornementation ; or, quand l'ornementation arrive à son extrême limite, elle tombe dans le défaut de fausse élégance et de l'altération. Ce n'est qu'en se montrant capable de s'en tenir aux caractères particuliers en simplifiant l'ornementation, qu'on ne commet tra pas la faute qui consiste dans l'excès. Le terme $p a$, blanc, troisième caractère du texte, désigne ce qui est simple, naturel, écru, sans teinture. En aimant la simplicité naturelle sans aucun fard, on ne perd pas la sincérité primitive ${ }^{1}$. Ce qu'il faut entendre par aimer la simplicité naturelle non altérée, ce n'est pas l'absence complète d'arrangement ; il faut seulement que l'ornementation ne fasse pas perdre de vue la nature réelle.

TSHOU HI. — L'arrangement arrivé à son extrême limite ramène au point de départ primitif ; retour à l'absence de coloration ; le bien compense le mal. C'est pour cela que tels sont le sens divinatoire et l'image symbolique.

421.

Simplicité de l'arrangement sans culpabilité; supériorité réussissant à faire prévaloir ses tendances.

TSHENG TSE. - Simplicité de l'arrangement sans culpabilité ; c'est parce qu'il est au rang supérieur et qu’il peut faire prévaloir ses tendances. Le trait supérieur nonaire est celui qui réussit à faire prévaloir ses tendances, il est au-dessus et détermine l'apparence de la douceur malléable ; il accomplit l'auvre de l'arrangeme nt. De plus, le prince représenté par le cinquième trait hexaire subit l'impulsion qu'il lui imprime, de sorte que, bien qu'il occupe un terr ain qui ne comporte aucune situation, en réalité il est à la tête de l'œuvre de l'arrangement, ce qui est considé ré comme exprimant qu'il réussit à faire prévaloir ses tendances. Ceci est différent de ce qu'exprime, dans d'autres koua, la condition d'occuper le rang extrême ; du moment où il est au rang

\footnotetext{
${ }^{1}$ Terme taoïste.
} 
supérieur et où il réussit à faire prévaloir ses tendances, se plaçant à l'extrême limite de ce qui constitue l'arrangement, il est sur le point de commettre la faute qui consiste à dénaturer la réalité en la masquant par l'ornementation. Ainsi, la formule avertit que, en employant la simplicité naturelle il n'y aura pas de culpabilité. L'arrangement et l'ornementation ne doivent pas être pous sés à l'excès. 


\section{Po}

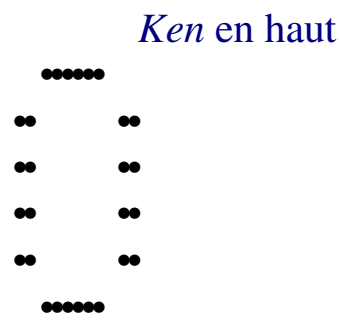

Khouen en bas

422. Po, usure ; sans avantage dans ce qu'il y a à entreprendre.

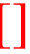

426. Premier trait hexaire: user le lit par les pieds; présage malheureux de la destruction de la pureté.

$*$

428. Deuxième trait hexaire : usure du lit par les traverses; présage malheureux de la destruction de la pureté.

$*$

430. Troisième trait hexaire : l'user ; pas de culpabilité.

*

432. Quatrième trait hexaire : user le lit par la peau ; présage heureux.

*

434. Cinquième trait hexaire : enfiler des poissons par les ouïes; profiter de la faveur des gens du palais; rien sans avantage.

$*$

436. Trait supérieur nonaire : le gros fruit n'est pas mangé ; l'homme doué jouit d'un char ; l'homme inférieur use la maison.

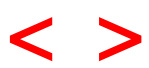




\section{Po.}

TSHENG TSE. - Po. «L'Ordre des koua » dit: «Le koua pi exprime l'arrangement et l'ornementation ; en poussant l'arrangement à l'extrême , il en résulte qu'à la fin la facul té de liberté se trouve épuisée ; c'est pour cela que le koua pi est suivi du koua po. » Or, quand quelque chose parvient à être ornée dans son apparence, cela marque l'extrême limite de la liberté ; l'extrême limite do nne nécessairement lieu à un retour en sens inverse, c'est pourquoi l'arrangement et l'ornementation parvenus à leur fin il y aura « usure », $(\mathrm{po})^{1}$. Dans le koua il y a cinq négativités et une seule positivité ; la négativité commence à naître par en bas ; elle augmente peu à peu jusqu'à son

parfait développement et à son extrême limite: La troupe des négativités dissolvant et usant ( $p o$ ) la positivité, le koua est donc considéré comme exprimant l'action d'user, et est appelé po. Si on en parle d'après les deux substances ${ }^{2}$, la montagne est attenante à la terre. La montagne s'élève haut au-dessus de la terre et, inversement, elle touche à la terre à laquelle elle est attenante : c'est l'image symbolique de la destruction (thouei) et de l'usure $(\text { po })^{3}$.

\footnotetext{
${ }^{1}$ Po, user en frottant peu à peu.

2 Des koua simples.

${ }^{3}$ Thouei; sens assez mal déterminé. Dans le Shi king, on trouve un passage où ce mot est interprété comme désignant une certaine "maladie qui empêche les chevaux de monter et les fait reculer». D'autre part on lui donne aussi diverses valeurs telles que « être pendant ou incliné », « joindre par en bas », etc.
} 


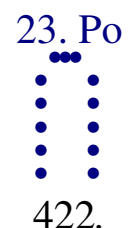

Po, usure; sans avantage dans ce quill y a à entreprendre.

TSHENG TSE. - L'usure, c'est la foule des négativités grandissant et se développant, le moment où elles dissolvent et usent la positivité ; la multitude des hommes inférieurs annihilant l'homme doué; c'est pour cela que l'homme doué est sans avantage dans ce qu'il entreprend. Il lui convient seulement d'être humble dans ses paroles et de cacher ses propres traces, s'effaçant selon le moment, afin d'éviter les injures des hommes infé rieurs.

TSHOU HI. - Po, tomber. Les cinq négativités sont en bas et nouvellement nées; l'unique positivité est au-dessus et sur le point de s'évanouir. La négativité se complète et grandit tandis que la positivité s'évanouit et tombe, c'est le koua du neuvième mois. La négativité fleurit ; la positivité décline; l'homme inférieur est bien portant et l'homme doué est malade. Ou bien encore, au-dedans le koua simple khouen et au-dehors le koua simple ken; image symbolique d'arrêt suivant le moment. Aussi, celui qui, en consultant le sort, obtient ce koua ${ }^{1}$ ne doit rien en conclure pour ce qu’il a à entreprendre.

423.

Le commentaire traditionnel de la formule déterminative dit: Le koua po exprime le sens du mot po, usure; la douceur malléable modifiant la dureté énergique.

TSHOU HI. - Explication du sens du nom du koua au moyen de la substance de ce koua. La formule exprime que la douceur malléable avance vers la positivité et modifie sa dureté énergique, qui devient douceur malléable.

424.

\footnotetext{
${ }^{1}$ Expression technique de divination.
} 
Pas davantage dans ce qu'il y a à entreprendre; l'homme inférieur grandit; suivre avec soumission et s'arrêter ; regarder limage symbolique; l'homme doué estime l'anéantissement et le repos, la plénitude et le vide; mouvement du ciel ${ }^{1}$.

TSHENG TSE. - Le koua po exprime le sens du mot po, c'est-à-dire décroître et tomber. «La douceur malléable modifie la dureté énergique »; la douceur malléable augmente et la dureté énergique se modifie. Au solstice d'été une négativité commence à naître ${ }^{2}$ et grandit peu à peu ; une négativité grandissant, il faut qu'une positivité diminue. Parvenue au moment de la conjonction marquée par le signe sui, la limite extrême est atteinte et elle produit le fait exprimé par le mot po, usure ; c'est là la douceur malléable négative modifiant la dureté énergique positive. La négativité est la voie de l'homme inférieur ; elle est au moment où elle grandit et se développe tout en usant et en dissolvant la positivité, c'est pourquoi l'homme doué est sans avantage dans ce qu'il y a à entreprendre. L’homme doué, en présence du moment marqué par le koua po, sait qu'il ne doit avoir rien à entreprendre ; il se conforme au moment et s'arrête ; c'est là savoir regarder l'image symbolique du déclin marquée par le koua po. Ce koua présente l'image symbolique de se conformer et de s'arrêter, ce qui est la voie rationnelle de se placer pendant une période de déclin ; l'homme doué doit la considérer et la réaliser. "L'homme doué es time l'anéantissement et le repos, la plénitude et le vide; l'action du ciel »; l'homme doué conserve dans son cour le sentiment de la raison d'être de l'anéantissement et du repos, de la plénitude et du vide, et il est capable de s'y soumettre, c'est êt re d'accord avec le mouvement, ou action, du ciel. La raison d'être des choses comporte la dissolution ou évanouissement, la naissance et la croissance, le parfait état de plénitude, comme aussi le vide et l'insuffisance ; en s'y soumettant le présage sera heureux; en allant contre cette loi, le présage sera funeste. L'homme doué suit l'opportunité du moment mais il étend et conserve les facultés par lesquelles il sert le ciel.

TSHOU HI. - Explication de la formule du koua au moyen des substances et des vertus des koua simples.

425.

\footnotetext{
${ }^{1} \mathrm{Ou}:$ « Action du ciel. »

$2 \mathrm{Si}$ on écarte toute origine astronomique du Yi king on peut se demander d'où vient cette explication.
} 
Le commentaire traditionnel de la formule symbolique dit: La montagne jointe à la terre; usure; le supérieur travaille à assurer l'inférieur dans la jouissance paisible de son habitation.

TSHENG TSE. - Le koua simple ken par-dessus le koua simple khouen; la montagne reposant sur la terre. La montagne s'élève haut sur la terre et inversement elle s'appuie sur elle et y est attenante ; c'est l'image symbolique de l'usure (po) et de l'écroulement. Le mot sheang, supérieur, désigne le prince, comme aussi ceux qui sont placés au-dessus des autres hommes; cela donne l’image symbolique de « considérer le déclin, ou usure ». De plus, il affermit et protège les inférieurs, afin d'assurer leur repos dans leur situation. Les inférieurs sont la base du supérieur ${ }^{1}$; il ne peut pas y avoir de base solide et ferme qui, en même temps, soit susceptible d'usure. C'est pour cela que le déclin chez le supérieur doit nécessairement provenir du fait de l'inférieur ; si le dessous s'use, le des sus est en péril, celui qui est supérieur parmi les hommes, et qui sait que telle est la raison d'être, ou loi des choses, assurera la paix du peuple, afin d'affermir sa propre base, et c'est ainsi qu'il affermira sa situation. Le Shou king dit : «Les peuples sont la seule base des États; si la base est ferme, l'État est tranquille. »

1 Telle est bien lidée du commentateur, mais on pourrait lire aussi bien : «Le bas, ou dessous, est l'origine du haut, ou dessus. » Cette absence de limitation dans l'idée représentée par le mot, qu'on reproche à tort au style chinois comme un manque de précision, lui donne, au contraire, une incomparable puissance d'expres sion. Le talent de l'écrivain consiste, par le choix des mots, à préciser, aussi nettement que dans tout autre langue, le sens que le lecteur doit adopter. 


\section{Po

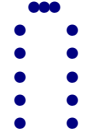 \\ 426.}

Premier trait hexaire : user le lit par les pieds; présage malheureux de la destruction de la pureté.

TSHENG TSE. — L'usure de la positivité par la négativité commence par le bas et agit en montant; le lit est pris comme image symbolique, en le considérant comme l'objet sur lequel le corps est placé. L'usure commence par le bas et petit à petit elle atteint jusqu'au corps. "User le lit par les pieds », user les pieds du lit; l'usure commence par le bas, c'e st pour cela qu'on considère l'usure des pieds. La négativité avance en partant d'en bas ; peu à peu elle détruit la pureté et la droiture : c'est une voie rationnelle de malheur. Le terme mie, septième caractère du texte, a la valeur du caractère wou, absence de ${ }^{1}$; cela veut dire dissolution et oubli de la voie de la droiture. La négativité use la positivité ; la douceur malléable altère progressivement la dureté énergique. C'est le mal et l'erreur envahissant et empiétant sur la droiture; l'homme inférieur supplantant l'homme doué ; le présage malheureux est évident.

TSHOU HI. - L'usure s'élève en partant d'en bas ; elle détruit la droiture, de sorte que le présage est malheureux. C'est pour cela que tel est le sens divinatoire. Le terme mie signifie détruire.

427.

User le lit par les pieds; pour détruire le dessous.

TSHENG TSE. - Les pieds du lit sont pris comme image symbolique, parce que la négativité envahit et détruit la positivité par en bas. Le mot mie de cette formule ${ }^{2}$, veut dire «submerger, supprimer, faire disparaître »; envahissement et suppression de la voie de la droiture ; elle commence par en bas et monte.

\footnotetext{
${ }^{1}$ Traduit par « destruction de $»$.

${ }^{2} \mathrm{Ce}$ caractère n'est pas le même que dans la formule $n^{\circ} 426$.
} 


\section{Po

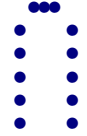 \\ 428.}

Deuxième trait hexaire : usure du lit par les traverses ; présage malheureux de la destruction de la pureté.

TSHENG TSE. - Le terme pien, sixième caractère du texte, désigne ce qui sépare le dessus du dessous, c'est le corps principal du lit. La négativité avance peu à peu et monte ; l'usure atteint les traverses ; accroissement de la destruction de la droiture ; le présage malheureux augmente d'intensité.

TSHOU HI. - Men, le corps du lit; l'usure avance et m onte.

429.

User le lit par les traverses ; il n'y a pas encore alliance.

TSHENG TSE. - L'envahissement et l'usure de la positivité par la négativité réussit et augmente en se complétant. Elle arrive à atteindre les «traverses», parce que la positivité n'a pas encore d'alliance et de correspondance sympathiques. Quand l'homme inférieur gagne et usurpe sur l'homme doué, si ce dernier a des alliés, il est possible qu'il l'emporte sur l'homme inférieur, et il ne peut en subir aucun mal. C'est seulement pa rce qu'il est sans alliance, qu'il est vaincu et que le présage est malheureux. En présence d'un moment de dissolution et de déclin, et sans une seule alliance, comment pourrait-il se préserver par lui-même ? La formule dit : « il n'y a pas encore alliance », lorsque le déclin n'est pas encore complet, avec des alliances, il est encore possible de vaincre. L’idée pré sentée à la réflexion des hommes est profonde!

TSHOU HI. - Cela exprime qu'elle n'est pas encore arrivée à un grand développement. 


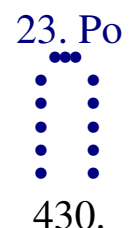

Troisième trait hexaire : l'user ; pas de culpabilité ${ }^{1}$.

TSHENG TSE. - C'est le moment où toutes les négativités usent la positivité et le troisième trait, seul, occupe un rang qui comporte la dureté énergique et correspond sympathiquement à un trait dur et énergique; il diffère en cela des traits négatifs au-dessus et au-dessous de lui. Ses tendances le portent à suivre la droiture, ce qui, dans un moment de déclin constitue l'absence de culpabilité et ce qui fait que le troisième trait exprime ce qu'on peut appeler le bien. Pourquoi la formule ne parle-t-elle pas de présage heureux ? Au moment où la négativité commence à user la positivité, la foule des hommes inférieurs nuit à l'homme doué ; bien que le troisième trait suive la droiture, la force inhérente à sa nature le rend faible et isolé ; ce qui lui est sympathique est placé sur un terrain qui ne comporte aucune situation déterminée ; dans un tel moment, il lui est difficile d'éviter les conséquences de ces conditions. Comment donc le présage serait-il heureux ? Le sens est qu'il n'y a pas de culpabilité. La formule parle de cette absence de culpabilité comme encouragement.

TSHOU HI. - La foule des négativités commence à user la positivité et lui, seul, lui est sympathique ; il s'éloigne de la troupe dont il fait partie et suit la droiture ; c'est une voie rationnelle d'absence de culpabilité. Si celui qui consulte le sort est dans ces conditions, il sera sans culpabilité.

431.

Absence de culpabilité dans l'action d'user; perdre le dessus et le dessous.

TSHENG TSE. - Le troisième trait se trouve parmi ceux qui usent et il est sans culpabilité ; la façon dont il se place n'est pas la même que celle des divers traits négatifs au-dessus et au-dessous de lui, c'est-à-dire que lui et

\footnotetext{
${ }^{1}$ Telle est la lecture si on se conforme à la ponctuation adoptée. Si on supprime le point placé entre les deux derniers membres de phrase, on lit: «Absence de culpabilité de l'usure » (ou du déclin). Remarque : La ponctuation ne fait pas partie du texte, elle est ajoutée, d'après les commentaires traditionnels, par les éditeurs.
} 
ceux qui sont de son genre s'écartent les uns des autres. Quand il s'agit de se placer dans la voie marquée par le koua po, usure, c'est ce qui constitue l'absence de culpabilité. C'est, par exemple, le cas de Lu Keang, sous la dynastie des Han.

TSHOU HI. - Le dessus et le dessous, c'est-à-dire les quatre autres négativités. 


\section{3. $\mathrm{Po}$
$\vdots$
$\vdots$}

432.

Quatrième trait hexaire : user le lit par la peau ${ }^{1}$; présage heureux.

TSHENG TSE. — L'usure commence par les pieds du lit ; peu à peu elle atteint la peau. La peau, c'est-à-dire l'extérieur du corps ; le corps est donc sur le point d'être détruit, et le présage de malheur est évident. La croissance de la négativité est complète ; le déclin de la positivité est extrême ; la voie de la pureté est évanouie. Aussi, la formule ne parle pas davantage «de destruction de la pureté » ${ }^{2}$; elle parle directement du présage malheureux.

Tsllou HI. - Le mal causé par la négativité atteint le corps ; aussi il n'est pas davantage question de la «destruction de la pureté » et il est formellement parlé du présage malheureux.

433.

User le lit par la peau ; calamité extrêmement proche.

TSHENG TSE. - Le cinquième rang indique la situation du prince. L'usure a atteint le quatrième rang ; s'il s'agit de l'homme, c'est que l'usure atteint la peau. L'usure attei gnant la peau, le corps tend à se dissoudre ; il est on ne peut plus rapproché du malheur et des calamités.

\footnotetext{
${ }^{1}$ Le véritable sens est «employer la peau pour user le lit ». Le commentaire part donc d'une fausse interprétation.

${ }^{2}$ Voir les formules 426 et 428.
} 


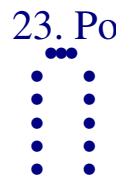

434.

Cinquième trait hexaire : enfiler des poissons par les ouïes ; profiter de la faveur des gens du palais; rien sans avantage.

TSHENG TSE. - La détérioration atteint la situation du prince, c'est la limite extrême de l'usure, et le présage néfaste est évident. Aussi il n'est pas davantage question d'usure, et un nouveau sens est adopté pour ouvrir la porte au retour de l'homme inférieur vers le bien. Le cinquième traitest celui qui dirige la troupe des négativités, le poisson est un être négatif ; c'est pour cela qu'il est pris comme image symbolique. Le cinquième trait peut diriger la foule des négativités de façon à la soumettre à un ordre déterminé ; c'est absolument comme lorsqu'on enfile des poissons par les ouïes. D'un autre côté, il peut arriver à être favorisé de l'amour de la positivité placée au -dessus de lui, comparée à une personne de linntérieur du palais, de sorte que rien ne lui est sans avantage. «Gens du palais », personnes employées dans l'int érieur du palais : épouses, concubines, serviteurs et assistants. Il est question de la négativité, et d'ailleurs c'est le sens de captiver l'affection et la protec tion qui est adopté. Puisqu'il n'y a qu'une seule positivité qui se trouve au rang supérieur, toutes les négativités ont une voie naturelle à suivre avec soumission, et c'est pour cela que la formule met ce sens spécial en évidence.

TSHOU HI. - Le poisson est un être négatif ; les « gens du palais » sont ce qui représente l'élégance et la beaut é dans la négativité et ce qui est soumis à la direction de la positivité. Le cinquième trait représente la négativité devenue grande et ayant acquis toute sa croissance ; il est présentement à la tête de ceux de son propre genre et il subit l'ascen dant de la positivité, c'est pourquoi il présente cette image symbolique. Aussi, si celui qui consulte le sort est dans ces conditions, il en résultera que rien ne sera sans avantage.

435.

Profiter de la faveur des gens du palais ; finalement rien d'étrange ${ }^{1}$.

TSHENG TSE. — La foule des négativités use et dissout la positivité, et il en résulte que cette dissolution arrive à sa plus extrême limite. Si le cinquième trait hexaire peut imposer sa prédominance à la foule des négativités, la

\footnotetext{
${ }^{1}$ Les commentateurs chinois adoptent un sens un peu différent et lisent : «pas d'accident ».
} 
soumettre à un ordre déterminé, en la faisant marcher d'un pas égal, il obtient à son tour la protection et l'affection de la positi vité, de sorte que finalement il ne lui arrive aucun accident fâcheux. Au moment où l'usure arrive vers sa fin, la formule développe encore ce sens, et l'idée de l'homme saint est d'exciter au retour vers le bien, ce qui est le comble de la profondeur de la pensée. 


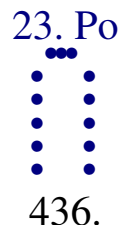

Trait supérieur nonaire : le gros fruit n'est pas mangé ; l'homme doué jouit d'un char ; l'homme inférieur use la maison.

TSHENG TSE. — L'élimination et l'usure des divers traits positifs est absolue ; il ne reste que le trait supérieur nonaire, seul, qui subsiste encore ; il est comme un fruit gros et énorme qu'on ne doit point manger, et qu'on garde dans le but de reproduction ${ }^{1}$. Si le trait supérieur se modifiait aussi, ce serait la pureté absolue de la négativité. Mais la raison d'être des choses ne souffre point que la positivité disparaisse totalement; se modifiant vers le haut, elle doit renaître vers le bas ; aucun accident ne peut faire qu'elle puisse ces ser d'exister. L'homme saint met cette loi en lumière, afin de faire voir que la voie de la positivité et de l'homme doué ne peut jamais périr. On a dit : si l'usure est complète, il en résultera l'unité de substance du koua khouen; comment la positivité subsistera-t-elle encore ? Parce que en établissant la concordance entre les koua et les mois, le koua khouen correspond au dixième mois ; en en parlant au point de vue de l'extinction et de la renais sance des éthers, l'usure de la positivité produit le koua khouen, puis la positivité reparaît et produit le koua fou ; elle n'est jamais complètement épuisée ; quand l'usure est complète par en haut, elle reparaît par en bas. C'est pour cela que le deuxième mois est appelé le mois de la positivité ; de peur qu'on ne doute de la continuité de sa présence. Il en serait de même au sujet de la négativité, et l'homme saint n'en parle pas. Dans le moment du complet développement de la voie de la négativité, le désordre est évident ; le désordre parvenu à son comble, on est naturellement porté à penser à l'ordre, de sorte que tous les cours aspirent à se soumettre à l'autorité de l'homme doué ; «l'homme doué jouit d'un char ». Dans le livre des vers on lit: «La source inférieure sans vent » : ce qui sert à maintenir la fin de la modification du vent ( ?). Du moment où telle est la raison d'être des choses, dans les koua également, toute négativité a la positivité pour origine, ce qui donne l’image symbolique de l'un des deux éthers supportant l'autre. "Lhomme inférieur use la maison »; si l'homme inférieur se trouve en présence de l'extrême déclin, il use la maison, et n'a plus rien pour s'abriter. Il n'est pas davantage question de la négativité et de la positivité ; la formule ne parle plus que de l'homme inférieur placé au comble du déclin, de sorte que l'usure atteint jusquà sa maison. Comme il s'agit du trait supé rieur, c'est l'image de la maison ou hutte en paille qui est choisie. On a dit : lors de la dissolution de la

\footnotetext{
${ }^{1}$ Tel est bien le sens, mais la phrase exprime en même temps que «l'on est sur le point de
} voir la raison d'être de la renaissance de la positivité ». 
négativité ou de la positivité, il faut absolument que l'une soit totalement usée par en haut pour que l'autre renaisse par en bas ; ici, au sujet du trait supérieur, il devrait y avoir le sens de renaissance ? Au sujet du trait supérieur du koua kouae, pourquoi la formule dit-elle que finalement il a un présage malheureux ? Voici la réponse : Le trait supérieur nonaire occupe le rang extrême dans le koua qui marque le déclin et l'usure ; il ne subsiste qu'une seule positivité: or, aucune raison ne peut faire que la positivité soit totalement anéantie et c'est pour cela que la formule développe le sens de renaissance, pour qu'on voie que la voie de l'homme doué ne peut jamais périr. Dans le koua kouae, c'est la positivité qui use la négativité, or la négativité est la voie de l'homme inférieur ; aussi il n'est question que de disparition et d'anéantissement, à quoi servirait-il de dire que, d'ailleurs, la raison d'être veut qu'il y ait également renaissance ?

TSHOU HI. - L'unique positivité est au rang supérieur ; le déclin n'est pas encore absolument complet et il peut encore y avoir renaissance. L’homme doué étant au rang supérieur est celui que toutes les négativités reconnaissent pour maître. Si un homme inférieur occupait cette position, l'usure serait arrivée à son point extrême vers le haut, et lui -même serait cause de la perte de ce qui l'abrite encore ; il n'y aurait d'ailleurs plus les images symboliques du gros fruit et de posséder un char. Dans le choix de l'image symbolique, après que celle-ci est déjà précisée et illustrée par un exemple clair, la formule cite encore l'homm e doué et l'homme inférieur. Il n'en est pas de même dans le sens divinatoire ; l'intention de l'homme saint est on ne peut plus évidente.

437.

L'homme doué jouit d' un char ; c'est lui que le peuple supporte ; l' homme inférieur use la maison ; finalement il ne peut sen servir.

TSHENG TSE. - Quuand l'extinction et la destruction de la voie de la droiture est à son comble, l'homme pense de nouveau à l'ordre et à la régularité ; aussi, l'homme doué, représenté par la dureté énergique positive, est ce que le peuple reconnaît pour autorité dirigeante. Si un homme inférieur était placé à l'extrême limite du déclin et de la des truction, ce serait la misère et la fin de cet homme. «Finalement il ne peut s'en servir »; cela ne veut pas dire que le trait supérieur représente un homme inférieur; il s'agit seule ment de l'extrême limite du déclin, et l'homme inférieur se trouve dans ce cas. 


\section{Fou}

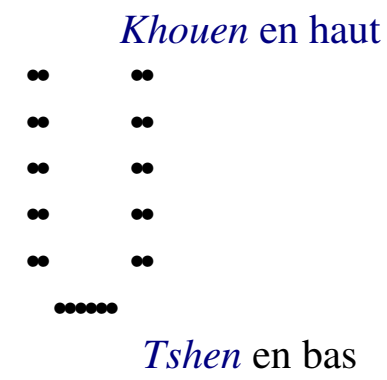

438. Liberté du retour; sortir et entrer sans inconvénient; amis arrivant sans culpabilité ; parcourir la voie en divers sens ; au bout de sept jours venir de retour ; avantage dans ce qu'il y a à entre prendre.

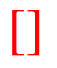

445. Premier trait nonaire : retour pas éloigné ; sans arriver aux regrets ; bonheur absolu.

$*$

447. Deuxième trait hexaire : retour du bien ; présage heureux.

449. Troisième trait hexaire : retours consécutifs ; préoccupation sans culpabilité.

$*$

451. Quatrième trait hexaire : agir au milieu et revenir seul.

*

453. Cinquième trait hexaire : grandeur dans le retour; pas de regrets.

*

455. Trait supérieur hexaire : aveuglement dans le retour, présage de malheur ; il y a des calamités et des maux; emploi de l'action des armées ; à la fin il y a une grande déroute à cause du présage malheureux pour l'État et pour le prince; jusque pendant dix ans ne pas réussir dans la guerre. 


\section{Fou.}

TSHENG TSE. - Fou. «L'Ordre des koua » dit: «Les choses ne peuvent pas finir d'une façon absolue ; lorsque l'usure ou déclin épuise le dessus, le dessous reprend en sens contraire ; c'est pour cela que le koua po est suivi du koua fou. » La raison naturelle des choses ${ }^{1}$ ne comporte pas leur dissolution totale, de sorte que l'usure, ou déclin, parve nue à son extrême limite, il y a retour en sens opposé. La négativité parvenue à son comble, la positivité renaîtra; la positivité arrivée au dernier degré de son déclin par en haut renaît cependant par en bas ; elle finit par en haut et recommence en sens inverse par en bas. C'est pour ce motif que le koua fou suit immédiatement le koua po. Comme koua il est formé d'une seule positivité naissant au-dessous de cinq négativités: la négativité est arrivée à son extrême limite et la positivité réapparaît. Dans le cours de l'année, au dixième mois, le développement de la négativité étant parvenu à son comble, au solstice d'hiver une positivité renaît au milieu de la terre ${ }^{2}$, et c'est pour cela que le koua est appelé fou. La positivité est la voie de l'homme doué. La positivité arrivée à l'extrême limite du déclin revient en sens oppo sé ; la voie de l'homme doué, quand elle atteint la limite extrême de l'effacement, s'élargit de nouveau. C'est pour cela que le koua expri me le sens de retour au bien.

\footnotetext{
1 Wou, choses, êtres.

2 En prenant la conjonction de l'équinoxe du printemps pour origine du premier mois, la conjonction de l'équinoxe d'autom ne marque le septième mois et le dixième mois coïncide bien avec le solstice d'hiver. À ce moment le soleil remonte vers l'horizon. Si, comme je le soutiens, le mot terre a pour premier sens celui de « course du soleil au-dessous de l'horizon » c'est bien au « milieu de la terre » que la positivité renaît.
} 


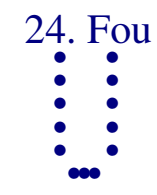

438.

Liberté du retour ; sortir et entrer sans inconvénient ; amis arrivant sans culpabilité ; parcourir la voie en divers sens ; au bout de sept jours venir de retour ; avantage dans ce qu'il y a à entreprendre.

TSHENG TSE. — «Liberté de retour »: du moment où il y a retour c'est qu'il y a liberté. L'éther positif renaît par en bas ; peu à peu il se développe librement et il engendre et produit tous les êtres. Du moment où la voie de l'homme doué refleurit, elle redevient progressivement librement pénétrable, et son influence bienfaisante pénètre et imprègne l'univers. Aussi, le retour comporte nécessairement la raison d'être du libre développement. "Sortir et entrer sans inconvénient »; sortir et entrer, c'est-à-dire naître et grandir. Renaître au-dedans, c'est entrer ; grandir et avancer au-dehors, c'est sortir. Le mot sortir est énoncé le premier par euphonie. Lorsque la positivité naît, ce n'est pas par l'extérieur qu'elle commence à exister ; elle vient à l'intérieur, et c'est pour cela que la formule emploie le mot entrer. Lorsque les choses ou les êtres commencent à naître, leur éther constitutif est extrêmement subtil et c'est pour cela que leur naissance présente beaucoup d'empêchements et de difficultés. Lorsque la positivité commence à naître, son éther est aussi extrêmement subtil, et c'est pour cela que sa naissance présente beau coup d'accidents. Le printemps est l'éclosion de la positivité ; il présente les accidents causés par le froid de la négativité : si on examine les arbres et les végétaux au point du jour, on en voit de nombreux exemples ${ }^{1}$. "Sortir et entrer sans inconvénient » exprime que la positivité, encore ténue et subtile, naît et grandit, sans causer de mal. Du moment où elle ne cause pas de mal et où tout ce qui est de son genre avance peu à peu et vient, c'est qu'elle est sur le point de parvenir à son libre développement, et c'est pour cela qu'il n'y a point de culpabilité. Ce quil faut entendre par ce mot « culpabilité », ce sera, au sujet de l'éther, l'irrégularité ou inversion dans les moments ; au sujet de l'homme doué, un empêchement ou obstacle à son action ; on ne peut pas citer tous les exemples. Le mouvement de retour de la positivité, bien qu'on puisse le supposer entravé par des difficultés, ne peut cependant certainement jamais être arrêté par elles; celles-ci ne peuvent jamais constituer que des obstacles. D'ailleurs, les aptitudes exprimées par le koua donnent le sens d'absence d'inconvénients ; c'est là le bien lorsqu'il s'agit du retour à la voie rationnelle. Une seule positivité commence à naître et est encore extrêmement ténue et subtile ; assurément elle ne peut pas encore dominer la foule des négativités,

${ }^{1}$ Le terme du texte, qui est rendu par le mot «accident», signifie spécialement «casser, briser ». Le commentateur fait allusion aux jeunes pousses rendues cassantes par la gelée. 
et pour donner naissance à toutes choses, il lui faut nécessairement attendre l'arrivée de toutes les autres positivités ; après leur venue, elle peut produire l'œuvre de la naissance des êtres, sans aucune irrégularité ou erreur ; c'est employer la venue des amis sans commettre d'erreur. L'éther des trois positivités tse, tsheou et yin engendre et produit tous les êtres, c'est l'ouvre de la foule des positivités. S'il s'agit de la voie de l'homme doué, du moment où elle reparaît après son déclin, comment pourrait-elle de prime abord l'emporter sur l'homme inféri eur? Il faut attendre la venue des amis et son développement progressif, et alors le concours de leurs forces réunies leur permettra de vaincre. "Parcourir la voie en divers sens », exprime la voie de l'extinction et la croissance ; revenir de nouveau et à diverses reprises. L'évanouissement de la positivité dure sept jours, après lesquels elle revient de nouveau. Le koua keou exprime le commencement de son évanouissement ; après sept modifications ce même koua devient le koua fou ; c'est pour cela que la formule parle de sept jours, c'est-à-dire sept changements. Dans le cas du koua lin la formule dit qu'après huit mois il y a un présage malheureux; cela exprime que la positivité grandit jusqu'au moment où la négativité croît, pendant huit mois. Lorsque la positivité avance, la négativité se retire; lorsque la voie de l'homme doué grandit, celle de l'homme infé rieur s'évanouit, de sorte qu'il y a avantage dans ce qui est à entreprendre.

TSHOU HI. - Fou, renaissance de la positivité par en bas. Lorsque sa disparition, par suite de son déclin, est totale, elle produit la pureté de substance du koua khouen, et au dixième mois l'éther de la positivité est déjà né par en bas. Cet état dure un mois entier et, ensuite, la substance d'une nouvelle positivité se forme et vient à son tour de nouveau, ce qui fait onze mois. Le koua est appelé fou, retour, parce que la positivité est déjà partie et de nouveau reparaît en sens inverse. C'est pour cela quil exprime une voie de liberté. De plus, il est formé par le koua simple tshen au-dedans et le koua simple khouen au-dehors ; il y a agitation de la positivité en bas, et image symbolique d'emploi de la positivité pour agir en montant. C'est pour cela que le sens divinatoire exprime encore sa sortie et son entrée; du moment qu'il n'éprouve pas d'inconvénients, l'arrivée des amis qui sont de son propre genre peut aussi avoir lieu sans aucune erreur ou culpabilité. Ou bien encore, depuis le koua keou du cinquième mois, dans lequel une seule négativité commence à naître, jusqu'à celui -ci, il y a un mouvement de sept traits, et ensuite une seule positivité vient de nouveau. C'est là la spontanéité naturelle du mouvement de translation du ciel, et c'est pour cela que le sens divinatoire est «parcourir la voie en divers sens ». Ce mouvement dure jusqu'à sept jours après lesquels la positivité doit être de retour. Ou bien encore : puisque la vertu de dureté énergique commence à grandir, il en résulte que le sens divinatoire exprime encore qu'il y a avantage dans ce qu'il y a à entreprendre. « Parcourir la voie en divers sens », c'est -à-dire s'en aller et ensuite revenir de nouveau, revenir pour recommencer à partir. "Sept jours », c'est le délai dans lequel adviendra le fait au sujet duquel on consulte le sort. 
439.

Le commentaire traditionnel de la formule déterminative dit: Liberté du retour ; la dureté énergique revient.

TSHOU HI. — La dureté énergique revient, donc, liberté de circulation.

440.

Elle se meut et agit par la soumission; donc elle sort et entre sans inconvénients, et les amis arrivent sans culpabilité.

TSHOU HI. - Ceci est dit d'après les vertus des koua simples.

441.

Elle parcourt la voie en divers sens ${ }^{1}$; au bout de sept jours venir de retour : mouvement du ciel.

TSHOU HI. - L'évanouissement e t la renaissance de la négativité et de la positivité ont lieu absolument comme le mouvement de translation du ciel ${ }^{2}$.

442.

Avantage dans ce qu'il y a à entreprendre ; la dureté énergique grandit.

TSHOU HI. - En en parlant d'après la substance du koua, après qu'elle est née, elle grandit peu à peu.

\footnotetext{
${ }^{1}$ La traduction la plus littérale serait : «Elle va et revient suivant cette voie. »

2 Telle est bien l'idée du commentateur, qui répète un axiome plus vieux, mais on peut lire et traduire : «Sont l'effet spontané du mou vement du ciel.
} 
443.

Le koua fou ne rend-il pas visible le cour du ciel et de la terre?

TSHENG TSE. — «Liberté de retour»; c'est-à-dire que la dureté énergique revient en sens opposé et librement. La dureté énergique positive, parvenue à la limite extrême de son évanouissement, revient dans la direction opposée; puisqu'elle revient en sens opposé, elle se développe progressivement et jouit de la liberté de circulation. Elle se meut et emploie la soumission pour agir, donc elle sort et entre sans inconvénients, et les amis viennent sans commettre de faute. Ces formules expriment comment il se fait qu'il en est ainsi, en s'appuyant sur les aptitudes exprimées par les koua simples. En bas mouvement et en haut soumission : c'est se mouvoir et employer la soumission en agissant. La dureté énergique revient en sens opposé et se meut avec soumission, c'est là pouvoir sortir et entrer sans inconvénients, et les amis viennent sans culpabilité. La venue des amis est également un mouvement avec soumission. Cette voie comporte une suite de départs et de retours en sens opposé, une succession d'évanouissements et de renaissances. «Après sept jours venir de retour», tel est le mouvement de translation du ciel et de la terre. L'év anouissement et la croissance sont réciproquement cause et effet: telle est la raison d'être du ciel. La dureté énergique positive, voie de l'homme doué, grandit ; donc l'avan tage a le moyen de se produire ${ }^{1}$. Une seule positivité revient par en bas; c'est la volonté (cœur) du ciel et de la terre donnant naissance aux êtres. Tous les premiers philosophes confucéens ont toujours considéré le repos comme constituant le fait de regarder le cour du ciel et de la terre. En effet, c'est qu'ils ne comprenaient $\mathrm{p}$ as que l'origine du mouve ment est précisément cette volonté du ciel et de la terre. Qui donc pourrait le comprendre sans connaître la voie ${ }^{2}$ ?

TSHOU HI. - Au-dessous de l'amas des négativités, une positivité renaît ; la volonté (cour) du ciel et de la te rre, créatrice des êtres, était sur le point d'expirer et, parvenue à ce point, elle redevient de nouveau visible. Chez l'homme ce sera l'extrême limite du repos suivie du mouvement, ou l'extrême limite du mal suivie du bien. Le cour originel ${ }^{3}$ sur le point d'être annihilé et commençant à se manifester de nouveau. Les explications de Tsheng Tse sont parfaites et claires. D'ailleurs les poésies de Shiao Tse disent : «Le solstice d'hiver marque le milieu du moment appelé tse ; - la volonté du ciel est

\footnotetext{
${ }^{1}$ Traduction plus littérale de l'ex pression traduite dans la formule par les mots : «avantage dans ce qu'il y a à entreprendre ».

2 Allusion à la doctrine de Lao Tse, dont Tsheng Tse est très imbu.

${ }^{3}$ La nature originelle, essentiellement parfaite et bonne.
} 
immuable. - Une positivité commence à donner l'impulsion déterminante ; - c'est le moment où tous les êtres ne sont pas encore nés. — Le vin sombre et coloré est encore sans saveur; -le grand son et le ton musical changent précisément ( ?). - Si vous ne croyez à ces paroles, — demandez plutôt à Pao Hi et questionnez-le. » Que ces observations sont justes ! L'étudiant doit y appliquer toutes ses facultés ${ }^{1}$.

444.

Le commentaire traditionnel de la formule symbolique dit : La foudre est dans la terre : retour. Les premiers rois profitaient du jour du solstice pour fermer les portes ${ }^{2}$. Les marchands et les voyageurs ne circulaient pas; le prince n'inspectait pas les régions.

TSHENG TSE. - La foudre, c'est la négativité et la positivité se choquant ensemble et produisant le son ; au moment où la positivité est encore à l'état rudimentaire, elle ne peut pas encore éclater. "La foudre est dans la terre », c'est le moment où la positivité commence à revenir ; la positivité commence à naître par en bas et est encore extrêmement ténue, calme et tranquille ; elle croîtra plus tard. Les premier rois, se conformant à la voie du ciel, avaient soin le jour du solstice, quand la positivité commence à naître, de rester dans le calme et le repos afin de la développer. C'est pour cela qu'ils fermaient les portes des passages frontières, afin que les voyageurs et les marchands ne pussent pas circuler et que les princes n'allassent pas inspecter les quatre régions ${ }^{3}$; ils observaient l'image symbolique de ce retour et se conformaient à la voie du ciel. Il en est encore de même de la personne d'un individu ; il convient d'observer le repos afin de permettre à la positivité de se développer.

TSHOU HI. - Le calme et le repos servent à développer la positivité encore rudimentaire; dans le Yue ling ${ }^{4}$, c'est le motif de la prescription relative aux purifications mensuelles, c'est un avertissement prescrivant de cacher le corps, afin d'attendre ce qui sera déterminé par la négativité et la positivité.

\footnotetext{
${ }^{1}$ Il est difficile de bien préciser le sens de ces vers détachés, sans connaître le reste de l'ouvrage, ou tout au moins du passage.

2 Portes des frontières donnant accès dans l'intérieur du pays.

${ }^{3}$ Ce passage est intéressant, en ce quill donne l'origine de la coutume de la « fermeture des sceaux » à la fin de l'année, et du repos du jour de l'an. Ce terme est encore usité aujourd'hui pour exprimer la «fermeture des sceaux».

${ }^{4}$ Chapitre du Li ki.
} 


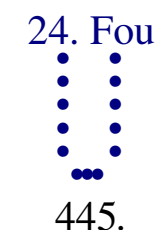

\section{Premier trait nonaire : retour pas éloigné ; sans arriver aux regrets ${ }^{1}$; bonheur absolu.}

TSHENG TSE. - Le retour en question, c'est le retour de la positivité revenant en sens opposé. La positivité c'e st la voie de l'homme doué, de sorte que le « retour» est considéré comme exprimant le sens de revenir au bien. Dans le premier trait, la dureté énergique positive revient; elle est placée au premier rang dans le koua; ce trait représente donc celui qui revient le premier, donc c'est ne pas être éloigné et revenir. La positivité était perdue, et ensuite elle réapparaît ; si elle n'avait pas été perdue, comment pourrait-il y avoir retour? mais seulement, la perte n'était pas ancienne quand elle revient déjà, de sorte qu’il n'en résulte pas de regrets : c'est un grand bien et le présage est heureux. Le septième caractère du texte, $t s h i$, « respect », doit être lu avec le son du caractère $t i$, « racine d'arbre », et prendre le sens du mot $t i$, «jusqu’à... a rriver à». Le dictionnaire $Y u$ pien lui donne le sens de « équivaloir à... » et le sens est encore le même. «Sans arriver aux regrets »; ne pas arriver jusqu’à avoir des regrets. Au sujet du koua khan il est dit: «Jusqu'au moment ( $t s h a)$ où la tranquillité est déjà établie; pas de culpabilité »; c'est-à-dire «parvenue au moment où la paix est déjà établie ». Au sujet de l'erreur de Yien Tse, alors qu'elle n'a pas encore pris une apparence et une forme manifestes, Khong Tse dit qu'elle est « sur le point d'être »; c'est précisément là un cas de l'application de l'expres sion «sans arriver aux regrets ». Tant que l'erreur n'a pas encore pris une forme visible, si on vient à se corriger, quels regrets pourrait-on en avoir ${ }^{2}$ ? Du moment où on ne s'est pa s laissé aller à commettre la faute sans résister au désir de la commettre, ce désir n'a pas dépassé les proportions d'une tendance ou d'un projet; c'est bien là une faute. Cependant, avec de l'intelligence et de l'énergie, on n'est jamais sans reconnaît re ce qui n'est pas bien ; du moment où on le reconnaît, on n'est jamais sans empressement pour s'en corriger, de sorte qu'on n'en arrive pas aux remords et aux regrets, et c'est là le retour peu éloigné dont parle la formule ${ }^{3}$.

\footnotetext{
$1 \mathrm{Si}$ on lit le texte on traduira «regret du manque de respect». Il faut, comme les commentateurs, donner au mot $t s h i$, « respect », la valeur de $t i$, «arriver à », qu'il n'a pas, pour lire comme eux «ne pas arriver aux regrets ».

${ }^{2}$ Il est bien certain qu'il ne s'agit que d'une « pensée coupable qui n’a pas encore été mise à exécution ».

${ }^{3}$ Le commentaire revient encore en quelques mots sur les divers sens qui ont été attribués au même caractère tshi.
} 
TSHOU HI. - Une positivité recommence à naître par en bas ; c'est d'elle que dépend le retour. Le caractère $t s h i$ a la valeur du caractère $t i$, « il convient; il est nécessaire; équivalent à...». De plus, puisque le trait représente quelque chose placé au commencement de l'action exprimée par le koua, c'est que la faute n'est pas encore éloignée dans le passé, que le retour au bien est encore possible, sans en arriver jusqu'aux remords ; c'est une voie largement ouverte vers le bien et un présage heureux, et c'est pourquoi tels sont le sens divinatoire et limage symbolique.

446.

Retour pas éloigné, pour corriger sa propre personne.

TSHENG TSE. - Ne pas être éloigné et revenir ; c'est la voie que l'homme doué emploie pour corriger sa propre personne, et la voie logique de l'étud e n'est pas autre ; elle consiste uniquement à s'empresser de se corriger pour revenir au bien dès qu'on s'est aperçu qu'on s'en écarte. 


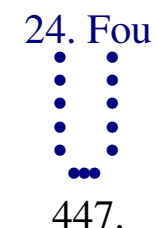

447.

Deuxième trait hexaire : retour du bien ${ }^{1}$; présage heureux.

TSHENG TSE. - Bien que le second rang soit occupé par un trait négatif comme sa position comporte la justice et la droiture et qu'il est tout à fait rapproché du premier, les tendances du second trait le portent à suivre la positivité et il est capable de s'abaisser devant la vertu ; c'est la beauté et le bien dans le retour. Le retour, c'est le retour aux règles rituelles, et le retour aux règles rituelles constitue la vertu ${ }^{2}$. Le trait représente le retour de la positivité ; c'est le retour à la vertu ; le second s'associe à lui et s'abaisse devant lui, ce qui constitue la beauté du retour et le présage heureux.

TSHOU HI. - Douceur malléable, soumission, justice et droiture ; proximité du premier trait nonaire, et capacité de s'abaisser devant lui : beauté et bien dans l'action du retour ; voie rationnelle de bonheur.

448.

Présage heureux du retour du bien, par l'abaissement devant la vertu.

TSHENG TSE. - C'est lui qui exprime le beau et le bien dans le retour et le présage de bonheur, parce qu'il peut s'abaisser devant la vertu. Ce caractère jen, humanité, vertu, exprime l'amour désintéressé de l'univers ; c'est la base essentielle du bien. Le premier trait exprime le retour à l'humanité ; le second est capable de se rapprocher de lui et de s'abaisser vers lui, c'est pour cela qu'il comporte un présage heureux.

\footnotetext{
${ }^{1}$ Le sens est plutôt « retour du beau ».

${ }^{2}$ Jen, vertu, et plus spécialement « humanité ».
} 


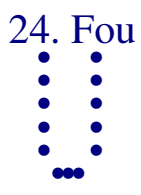

449.

Troisième trait hexaire : retours consécutifs; préoccupation sans culpabilité.

TSHENG TSE. - Le troisième trait emploie la négativité et s'empresse de se placer au comble du mouvement ; c'est la multiplicité des retours ${ }^{1}$ sans pouvoir en assurer la fermeté. Le retour est précieux par la fermeté et le calme ; sil y a fréquence dans les retours, il y a fréquence dans les chutes, et le retour manque de calme et de sérénité. Revenir au bien et retomber fréquemment dans les mêmes errements est une voie périlleuse. L'homme saint ouvre une voie d'amélioration et d'amendement ; il loue le retour et montre le péril de la fréquence des rechutes, c'est pourquoi la formule dit : préoccupation sans culpabilité. Il n'est pas possible de se servir de la fréquence des rechutes pour avertir au sujet du retour. La fréquence des rechutes constitue un péril, tandis que dans la multiplicité des retours il n'y a aucune culpabilité ; la faute est dans la chute et n'est pas dans le retour.

TSHOU HI. - Emploi de la négativité dans l'occ upation d'un rang qui comporte la positivité ; absence de justice et de droiture ; de plus, le trait est placé à l'extrême limite du mouvement. Retour sans fermeté; image symbolique de la fréquence des chutes et de la multiplicité des retours. La fréquence des chutes est certainement périlleuse tandis que le retour ne présente jamais de culpabilité, et c'est pour cela que le sens divinatoire est encore ainsi.

450.

Appréhension de la fréquence des retours; le sens est l'absence de culpabilité.

TSHENG TSE. - Fréquence des retours, fréquence des rechutes ; bien que ce soit une cause d'appréhension et de péril, cependant le sens du retour au bien exclut l’idée de culpabilité.

${ }^{1}$ Le caractère employé dans le texte a le sens de «précipitation» plutôt que celui de « fréquence ». 


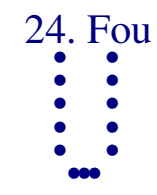

451.

Quatrième trait hexaire : agir au milieu et revenir seul.

TSHENG TSE. - Le sens de la formule de ce trait doit surtout être particulièrement compris et pénétré. Le quatrième trait marche au milieu de la troupe des négativités et, seul, il est capable de retour. Cela vient de ce qu'il se place d'après la droiture, qu'il se baisse pour répondre sympathiquement à la dureté énergique de la positivité; ses tendances peuvent être qualifiées bonnes. S'il n'est pas question de présage heureux ou mal heureux, c'est qu'en effet, le quatrième trait emploie la douceur et se maintient parmi la troupe des négativités ; le premier trait est encore extrêmement faible et ces conditions sont insuffisantes pour que tous deux puissent s'entraider. Il n'y a aucune chance de remédier à cet état de choses, aussi l'homme saint mentionne uniquement la capacité de retour, sans vouloir exprimer qu'un retour isolé à la voie morale comporte nécessairement un présage de malheur. On a dit : s'il en est ainsi, pourquoi ne pas mentionner l'absence de culpabilité ? Puisque la négativité occupe un rang négatif, elle exprime l'extrême faiblesse et la mollesse ; bien que ses tendances la portent à suivre la positivité, finalement rien ne peut remédier à ce défaut, donc il n'y a pas absence de culpabilité.

TSHOU HI. - Le quatrième trait est placé au milieu du groupe des négativités et, seul, il sympathise avec le premier; cela constitue l'image symbolique d'agir avec la foule et d'être seul capable de suivre le bien. En présence d'un tel mom ent, où l'éther de la posi tivité est encore extrêmement ténu et pas encore suffisant pour agir, il n'est pas question de présage heureux, mais toutefois, au point de vue des conséquences logiques, il n'y a pas lieu de discuter le présage heureux ou malheureux. Tong Tse dit : "L'homme humain s'applique exactement au devoir ; il ne spécule pas sur l'utilité. Il projette la lumière sur la voie morale, et ne calcule pas le mérite qu'il peut avoir à la suivre »; cela est visible au sujet du troisième trait hexaire du koua po et dans le cas présent.

452.

Agir au milieu et revenir seul; en suivant la voie. 
TSHENG TSE. - La formule le désigne comme retournant seul, parce qu'il suit la voie du bien tracée par l'homme doué de l'énergie active positive. 


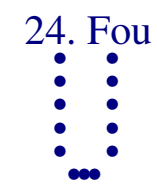

453.

Cinquième trait hexaire : grandeur dans le retour; pas de regrets.

TSHENG TSE. - Le cinquième trait hexaire emploie les vertus de la justice et de la condescendance et se place dans la situation du prince; c'est celui qui peut guider avec grandeur dans la voie du retour au bien. C'est pour cela quil est sans regrets. Bien que ceci constitue essentiellement le bien, il s'y trouve cependant encore un sujet d'aver tissement. Au moment où la positivité, sur son retour est encore à peine sensible, en employant la douceur pour se maintenir au rang prééminent, et n'ayant de plus aucune assistance au-dessous de soi, il ne peut pas encore atteindre librement au bonheur; il ne peut qu'éviter les regrets.

TSHOU HI. - Employant la justice et la condescendance pour occuper le rang prééminent, et en présence d'un moment de retour, il présente l'image symbolique de la grandeur dans le retour, et c'est une voie qui ne conduit pas aux regrets.

454.

Absence de regrets dans la grandeur du retour; justice appliquée à son propre perfectionnement.

TSHENG TSE. - Emploi de la voie de la justice pour se perfectionner. Le cinquième trait emploie les qualités de la négativité pour occuper le rang prééminent; il se place avec justice et sa substance comporte la condescendance; il est capable de diriger ses tendances avec grandeur. Employant la voie de la justice pour se perfectionner, il lui sera donc possible d'être sans regrets. Se perfectionner, c'est -à-dire perfectionner ses vertus de justice et de soumission.

TSHOU HI. - Le terme khao, du texte, équivaut à tsheng, achever, perfectionner ${ }^{1}$.

\footnotetext{
${ }^{1}$ Khao signifie : «rechercher, investigation ». C'est arbitrairement que les commentateurs lui donnent cette nouvelle valeur.
} 


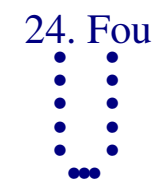

455.

Trait supérieur hexaire : aveuglement dans le retour, présage de malheur; il y a des calamités et des maux; emploi de l'action des armées; à la fin il y a une grande déroute à cause du présage malheureux pour l'État et pour le prince; jusque pendant dix ans ne pas réussir dans la guerre.

TSHENG TSE. - Employant la mollesse de la négativité et occupant le dernier rang dans le koua qui exprime le retour, c'est celui dont l'aveuglement est final et qui ne revient pas dans la bonne voie; aveuglé et ne retournant point, le présage malheureux est évident. « Il y a des calamités et des maux »; calamités, c'est-à-dire calamités envoyées par le ciel ; qui ne proviennent pas de son fait personnel. "Maux », résultat d'erreurs person nelles, de son propre fait. Du moment où il est aveuglé et ne revient pas au bien, toutes les fois qu'il agira, il commettra toujours quelque faute ou erreur ; les calamités et les malheurs surviennent également de causes étrangères, et en effet sa conduite les appelle. Il a perdu sa voie et ne retourne pas en arrière, aucune de ses entreprises ne peut réussir. S’il lui est nécessaire d'employer les armées, il aboutira finalement à une grande défaite; s’il s'agit d'un État, ce sera un présage malheureux pour le prince. «Dix ans », c'est la limite des nombres ${ }^{1}$; «jusque pendant dix ans ne pas réussir dans la guerre », c'est-à-dire que finalement, il ne peut plus agir. Puisqu'il est aveuglé sur le choix de sa voie, à quel moment pourrait-il bien agir?

TSHOU HI. - Puisque ce trait emploie la mollesse de la négativité et se maintient au dernier rang du koua qui indique le retour, il présente l’image symbolique de l'aveugle ment final sans retour ; c'est une voie qui indique un présage de malheur. C'est pour cela que tel est le sens divinatoire. Le caractère $y i \mathrm{du}$ texte, «employer, à cause de... » équivaut au caractère ki «atteindre quelque chose $»^{2}$.

456.

Présage malheureux de l'aveuglement dans le retour; aller contre la voie rationnelle du prince.

${ }^{1}$ Cette explication revient souvent dans les commentaires. Dix est la base de la numération chinoise.

${ }^{2}$ Le commentateur lit : « Appliqué à l'État et au prince, présage de malheur. » 
TSHENG TSE. — Le retour constitue l'accord à la voie rationnelle ; diU moment où l'aveuglement fait perdre de vue le retour, c'est aller à l'encontre de la voie rationnelle. Le présage malheureux est évident. L'expression : «à cause du malheur du prince et de l'État » exprime qu'il va contre la voie rationnelle du prince. Le prince occupe le rang supérieur et gouverne la foule ; il doit rechercher le bien de l'univers, tandis que dans le cas actuel il s'agit d'aveuglement au sujet du retou r, ce qui est opposé à la voie rationnelle du prince. Ceci n'est pas seulement applicable au prince ; quiconque est aveuglé au sujet du retour va toujours contre la voie rationnelle, et le présage est malheureux. 


\section{Wou wang}

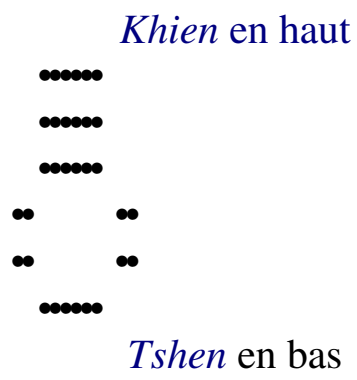

457. Premier trait nonaire : absence de désordre; présage heureux de l'entreprise.

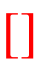

460. Premier trait nonaire : absence de désordre; présage heureux de l'entreprise.

*

462. Deuxième trait hexaire : récolter sans labourer; rizière en rapport sans rizière en friche; de sorte que l'avantage pourra en résulter.

$*$

464. Troisième trait hexaire : calamité de l'absence de désordre ; parfois le bouf attaché ; capture du passant ; calamité de l'homme du district.

$*$

466. Quatrième trait nonaire : possibilité de perfection ; pas de culpabilité.

$*$

468. Cinquième trait nonaire : inconvénient de l'absence de désordre ; sans remède il y a joie.

*

470. Trait supérieur nonaire : absence de désordre ; en marchant il y a malheur ; aucun moyen davantage. 
25. Wou wang.

TSHENG TSE. - Wou Wang. «L'Ordre des koua » dit : «Puisqu'il y a retour, il n'y a donc point de désordre ; c'est pourquoi le koua fou est suivi du koua wou wang. » Le koua fou exprime le retour à la voie rationnelle, il y a accord et conformité à la droite raison d'être des choses et pas de désordre. C'est pour cela qu'après le koua fou vient immédiatement le koua wou wang. Il est constitué par le koua simple khien en haut et le koua simple tshen en bas. Le koua simple tshen exprime le mouvement ; le mouvement d'après le ciel est considéré comme l'absence de désordre, tandis que le mouvement causé par les désirs humains indiquera le désordre. Que le sens exprimé par le koua wou wang est grand! 


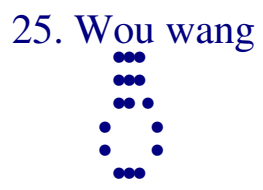

457.

Pas de désordre; liberté absolue; perfection de l'avantage ; ce qui n'est pas correct entraîne le malheur; pas davantage dans ce qu'il y a à entreprendre.

TSHENG TSE. - L'absence de désordre, c'est l'extrême sincérité ; l'ext rême sincérité, c'est la voie du ciel. Dans l'action du ciel produisant et transformant tous les êtres, et dans la suite indéfinie de leur succession dans la vie, chacun suit exactement sa destinée tracée par la nature : c'est l'absence de désordre. Lorsque l'homme peut se conformer à cette voie exclusive de tout désordre, c'est ce qu'on peut appeler conformer ses factultés à l'action du ciel et de la terre. L'absence de désordre comporte la raison d'être d'une grande liberté. L'homme doué parcourt cette vo ie d'absence de désordre, de sorte qu'il peut arriver à cette grande liberté. L'absence de désordre, c'est la voie du ciel. Le koua exprime le passage de l'homme par cette voie d'absence de désordre; la perfection de l'avantage, consiste à se régler sur cette voie d'absence de désordre; l'avantage réside dans la parfaite droiture; en manquant à cette parfaite droiture on cause le désordre. Bien que le cœur ne soit pas vicieux, s'il ne se conforme pas à la droite raison d'être le désordre surgit. Dans le cas actuel, il s'agit d'un coar vicieux ; aussi il est question de ce qui n'est point correct, ce qui produira le malheur et l'erreur. Du moment où il n'y a point de désordre, il ne convient pas d'entreprendre quoi que ce soit ; entreprendre constituerait le désordre.

TSHOU HI. - «Absence de désordre » est une expression qui marque la véritable raison d'être spontanée. Dans les « annales », cette expression est rendue par les caractères dont le second, avec le même son wang, a le sens d'espoir, c'est -à-dire qu'il n'y a rien à espérer et que la possession est déjà obtenue; le sens est encore intelligible. Ce koua provient du koua song par une modification progressive ; le trait nonaire vient du second rang et occupe le premier; de plus, c'est de lui que dépend l'existence du koua simple tshen, c'est le mouvement sans désordre et c'est pourquoi le koua parfait est consi déré comme exprimant l'absence de désordre. Ou bien encore, les deux substances (des koua simples) sont le mouvement du koua tshen et l'activité du koua khien; le cinquième trait nonaire possède l'énergie et la justice et sympathise avec le second trait hexaire, de sorte que le sens divinatoire est « grande liberté » et « avantage dans la droiture ». S'il s'agit de ce qui n'est pas conforme à la droiture, il surviendra des malheurs et il n'y aura aucun avantage dans ce quỉl y aura à entreprendre. 
458.

Le commentaire traditionnel de la formule déterminative dit : Absence de désordre; la dureté énergique vient de l'extérieur et devient maîtresse à l'intérieur. Mouvement et activité ; dureté énergique, justice et sympathie; grande liberté par la droiture; destinée imposée par le ciel. Ce qui n'est pas correct entraîne le malheur; pas davantage dans ce qu'il y a à entreprendre. Comment le pourrait-on? Ce que le ciel ne favorise pas pourrait-il donc être fait?

TSHENG TSE. - Il est question du premier trait nonaire. Le premier trait du koua simple khouen se modifie et produit le koua simple tshen; la dureté énergique vient par l'extérieur. Dans le koua simple tshen, c'est le premier trait qui domine et c'est aussi par lui que le koua parfait se produit, c'est pour cela que ce premier trait est le maître du koua wou wang. Se mouvoir d'après le ciel constitue précisément l'absence de désordre ; il y a mouvement, et ce mouvement est conforme à l'impulsion du ciel ; c'est le mouvement qui est l'action dominante. Employer la dureté énergique pour modifier la douceur malléable, constitue l'image symbolique de l'emploi de la droiture pour écarter le désordre. D'un autre côté, la droiture énergique domine à l'intérieur ; cela donne encore le sens d'absence de désordre. Le trait nonaire, occupant le premier rang, indique la droiture.

En bas, mouvement, et en haut activité ; c'est donc que le mouveme nt est énergique et actif; or l'activité énergique est l'essence de l'absence de désordre. Dureté énergique, justice et correspondance sympathique; le cinquième trait emploie la dureté énergique et se maintient dans la justice et la droiture et tous deux sympathisent ensemble: c'est l'indication de la soumission à la raison d'être des choses, et de l'absence de désordre. C'est pour cela que la voie suivie présente une grande liberté de circulation et une parfaite droiture et c'est là la destinée marquée par le ciel. «Destinée tracée par le ciel » veut dire « voie du ciel »; c'est ce qu'on entend par l'expression : «absence de désordre. » Ce qu'on appelle l'absence de désordre n'est pas autre chose que la droiture, ou régularité. Si peu qu'il y ait déviation de la droiture, cela constitue une faute ou irrégularité, et c'est là le désordre. Ce qui, dans le texte, est entendu par les mots : «pas correct», provient en effet de ce qu'il y a à entreprendre ; s'il y a absence de désordre et qu'on n'entrepre nne rien, d'où résulterait l'absence de rectitude ? L'absence de désordre, c'est la rectitude déterminée par la raison d'être des choses ; que chercherait-on de plus en entreprenant quoi que ce soit ? Ce serait précisément se jeter dans le désordre. En entreprenant, on irait contre la raison d'être émanée du ciel ; ce que la voie du ciel ne favorise point pourrait-il donc être fait ? 
TSHOU HI. - La formule se base sur la modification progressive du koua, sur les vertus des koua simples et sur leur substance pour établir que telle est la valeur de ce même koua. C'est pour cela que celui qui consulte le sort devra arriver à jouir d'une grande liberté et retirer un avantage de sa droiture : c'est la conséquence naturelle de la destinée tracée par le ciel. Ce qui ne sera pas conforme à la droiture ne pourra pas être avantageux dans ce qu'il aura à entreprendre : mais que pourrait-il vouloir entreprendre ? Le ciel ne favorise point ce qui est contraire à la destinée qu’il a marquée, de sorte que cela ne doit point être entrepris.

459.

Le commentaire traditionnel de la formule symbolique dit : Au-dessous du ciel la foudre agit, les êtres s'y conforment sans désordre; les premiers rois employaient la parfaite concordance aux moments ${ }^{1}$ pour nourrir tous les êtres.

TSHENG TSE. - L'ébranlement de la foudre agit et se propage dans tout l'univers ; la négativité et la positivité s'unissent harmoniquement ; elles se choquent ensemble et produisent le son ; à ce moment elles tirent la larve de son état de repos, elles donnent l'impulsion au germe végétal, et amènent tous les êtres à la vie. Ce qui est ainsi départi à chacun, quelle que soit son énormité ou sa ténuité, son élévation ou sa petitesse, correspond dans chaque cas exactement à la raison d'être de sa destiné e, sans qu'il y ait jamais aucune erreur: «les êtres s'y conforment sans désordre ». Les premiers rois, considérant l'image symbolique de la foudre agissant dans l'univers, appelant à la vie et douant chaque être selon sa destinée, s'appliquaient ensuite d'une façon parfaite à se conformer aux saisons pour nourrir et entretenir tous les êtres et toutes choses, de telle sorte que chacun pût jouir de ce qui lui était nécessaire, comme dans «l'absence de désordre » des dons du ciel. Le caractère [] du texte, exprime l'idée d'exubérance des végétaux et il est employé ici dans le sens de perfection achevée. «Se conformer parfaitement » ou « concordance parfaite » équivaut comme expression à « action parfaite et continue $»$.

«Concordance aux moments » veut dire conformité aux saisons marquées par le ciel. La «voie du ciel » donne la vie à tous les êtres; chacun se conforme à sa nature particulière et à sa destinée, sans aucun désordre. Les rois réalisent la voie du ciel en la mettant en pratique; ils nourrissent et entretiennent les peuples et jusqu'aux insectes, aux plantes et aux végétaux,

\footnotetext{
${ }^{1}$ Saisons
} 
faisant en sorte que chacun jouisse de ce qui lui est nécessaire. Telle est la voie de la concordance aux saisons pour entretenir les êtres.

TSHOU HI. - Au-dessous du ciel la foudre agit; elle ébranle, met en mouvement, et appelle à la vie ; chaque être reçoit sa nature et sa destinée, de telle sorte que tout être s'y conforme sans aucun désordre. Les premiers rois prenant cet exemple pour règle, s'appliquaient à agir av ec concordance aux saisons pour entretenir tous les êtres; ils écoutaient leur propre nature sans cependant agir avec égoïsme. 


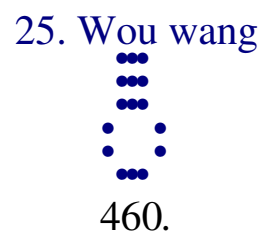

Premier trait nonaire : absence de désordre ; présage heureux de l'entreprise.

TSHENG TSE. - Ce trait nonaire emploie la dureté énergique pour dominer à l'inté rieur ${ }^{1}$; c'est l'image symbolique de l'absence de désordre. Employant la réalité ${ }^{2}$ pour modifier la malléabilité négative et se maintenant à l'i ntérieur ${ }^{3}$; c'est celui qui est juste, sincère et sans mauvaises intentions. Entreprenant et s'abstenant de toute mauvaise intention, en quoi pourrait-il ne pas être heureux ? La formule du koua parle d'absence d'avantage dans ce qu'il y a à entreprendre, c'est-à-dire que, du moment où il y a absence de désordre, on ne doit rien entreprendre pour innover; dépasser ce point serait causer le désordre. La formule spéciale du trait dit "présage heureux de l'entreprise », c'est -à-dire qu'en agissant, en emplo yant l'absence de désordre, le présage sera heureux.

TSHOU HI. - Employant la dureté énergique au-dedans, c'est de lui que dépend la sincérité. Dans de telles conditions, entreprenant quelque chose, le présage heureux est évident, et c'est pour cela que tels sont le sens divinatoire et l'image symbolique.

461.

Entreprise dans l'absence de désordre ; réussir selon ses tendances.

TSHENG TSE. - Agissant sans aucune mauvaise intention et entreprenant quelque chose, rien ne peut l'empêcher de faire prévaloir ses tendances. En effet, il n'est rien que la sincérité employée vis -à-vis des êtres ne puisse émouvoir. En l'employant au redressement de sa propre personnalité, celle-ci devient correcte ; en l'employant au soin des affaires, celles-ci sont expédiées conformément à la raison; en l'employant au gouvernement des hommes, ces derniers en ressentent l'influence et se transforment : rien n'est entrepris sans que de telles tendances ne prévalent.

\footnotetext{
${ }^{1}$ Pour être le maître dans le koua simple inférieur.

2 Parce que c'est un trait plein ; pas évidé au milieu.

${ }^{3}$ Du koua simple inférieur.
} 


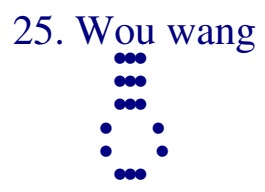

462.

Deuxième trait hexaire : récolter sans labourer; rizière en rapport sans rizière en friche; de sorte que l'avantage pourra en résulter.

TSHENG TSE. - Tout ce qui est le résultat naturel de la raison d'être des choses est l'opposé du désordre, ou déception. La création du vouloir de l'homme constitue ce désordre ou cette déception. Voilà pourquoi la formule cite en exemples les cas de labour et de récolte, de rizières d'un an et de rizières de trois ans ${ }^{1}$. Le second trait hexaire se maintient dans la justice et possède la droiture; de plus il sympathise avec la justice et la droiture du cinquième. Il fait partie de la substance du mouvement ${ }^{2}$ et il se soumet passivement à l'impulsion reçue ; il représente le mouvement avec possibilité de soumission à la justice et la droiture ; c'est ce qui constitue l'absence de désordre et de fausseté dans les intentions, et c'est pour cela qu'il est, avant tout, question du sens d'absence de désordre. Le labourage est la première opération de l'agriculture ; la récolte en est le résultat et la fin. Un marais converti en rizière est, la première année, désigné par le caractère [], la troisième année il est désigné par le caractère []. Ne pas labourer et récolter, ne pas défricher la première année et avoir une rizière à sa troisième année de défrichement, c'est -à-dire ne pas faire quelque chose a priori et s'en tenir à la conséquence naturelle de la raison d'être. Poser le fait a priori c'est créer par la propre volonté de l'homme ; c'est le désordre ou la déception, le fait arbitraire. S'en tenir à la conséquence naturelle, c'est se conformer à la raison d'être du fait et en accueillir le résultat : c'est l'absence de désordre. Tel est le cas lorsqu'il s'agit de récolter et de rizière arrivée à sa troisième année de défrichement. En effet, en labourant, il y aura certainement lieu de récolter ; en défrichant une rizière à sa première année, il y aura nécessairement une rizière arrivant à sa troisième année de défrichement: telle est la certitude naturelle qui découle de la raison d'être des choses, ce n'est pas le résultat de la création, de la volonté et de l'intention, et cela constituera l'absence de désordre. Du moment où il n'y a point de désordre, ce qui sera entrepris sera avantageux et n'entraînera aucun mal. On a dit: Ce que l'homme saint imagine pour le bien de l'univers est toujours une « invention »; comment n'est-ce point là un désordre, ou une déception ? C'est que l'homme saint

\footnotetext{
${ }^{1}$ Dans la formule, les commentateurs lisent : «rizières de trois ans sans rizière d'un an ». Le sens des mots employés est mal défini, mais lïdée est compréhensible.

${ }^{2}$ Cette forme est évidemment vicieuse en français. Cependant elle est souvent employée dans la traduction, faute d'un terme qui puisse correspondre au caractère chinois qui a à la fois la valeur de «corps », « essence », «substance » et qui répond à une idée mal définie et pas logiquement déterminée.
} 
règle ses créations sur les saisons, qu'il s'accorde aux nécess ités de l'état de l'atmosphère, et que jamais il n'entre prend avant le moment opportun. S'il n'attendait pas le moment, un seul homme saint aurait suffi à l'accomplissement de l'auvre. Quelle nécessité y eût -il eu à ce que plusieurs hommes saints se succédassent dans leur auvre ? Le temps est l'origine des choses; l'homme saint suit le temps et agit.

TSHOU HI. - Douceur malléable, soumission, justice et droiture ; selon le moment se plier à la raison d'être des choses, sans aucun calcul d'intérêt privé; aussi, il y a image symbolique de récoltes sans avoir labouré et d'obtenir des rizières en culture sans passer par le défrichement ; cela exprime qu'il n'y a rien à faire à l'avance, ni rien à désirer dans l'avenir. Si celui qui consulte le sort est dans ces conditions, il y aura avantage dans ce qu’il aura à entreprendre.

463.

Récolter sans labourer; pas encore riche.

TSHENG TSE. - Wei, pas encore, terme qui comporte l'incertitude. Au sujet du koua lin, il est dit: «Pas encore soumis à la destinée »; c'est exactement ce sens. Ne pas labourer et récolter, ne point défricher et avoir des rizières en culture, c'est profiter de la spon tanéité des choses. Du moment où on a labouré, il y a nécessairement lieu de récolter; quand on a défriché des rizières d'une année, elles doivent nécessairement devenir rizières à la troisième année de culture. Ce n'est pas expressément pour arriver à la riches se représentée par la récolte et par les rizières en culture depuis trois ans que l'action est faite ; celui qui commence par labourer et par défricher des rizières d'un an, manifeste sa volonté au sujet de ce qu'il veut arriver à obtenir comme récolte ou comme rizières de trois ans ; c'est parce qu'il a le désir des richesses qu'il agit, donc il y a désordre et déception.

TSHOU HI. - Le mot fou, richesse, doit être pris avec l'acception qu'il a dans le passage : ne pas être riche de la richesse de l'univers ${ }^{1}$, c'est-à-dire ne pas spéculer sur les avantages matériels de l'action.

\footnotetext{
${ }^{1}$ D'après ce commentaire, il faudrait lire le mot fou en lui donnant un sens verbal : « ne pas s'enrichir ».
} 


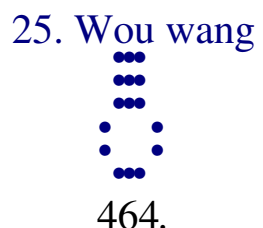

Troisième trait hexaire : calamité de l'absence de désordre ; parfois le bouf attaché ; capture du passant ; calamité de l'homme du district.

TSHENG TSE. - Le troisième trait emploie la douceur malléable négative, et il est sans justice ni droiture ; c'est celui qui fait le désordre. De plus, ses tendances sympathisent avec le trait supérieur, ce qui marque le « désir », autre cause de désordre ; dans la voie de l'absence de désordre, cela constitue la calamité et le mal. Lorsque l'homme se meut à tort, c'est qu'il est poussé par le désir ; lorsqu'il se meut mal à propos et qu’il réussit à obtenir, il doit nécessairement reperdre ; bien qu'on suppose qu'il obtienne ce qui lui est avantageux, son mouvement est cependant illégitime ; la perte qu'il en subit est grande par cela même. Combien donc, à plus forte raison, lorsque le malheur revient et que les remords suivent! Celui qui le sait considère l'acquisition illégitime et connaît qu'elle constitue une perte ; le résultat doit nécessairement être en rapport avec ce que mérite l'ouvre. Aussi l'homme saint argue de l'image symbolique de désordre inhérente au troi sième trait hexaire et il en fait éclater la raison d'être. La calamité de l'absence de désordre : parfois le bouf attaché est la capture du passant et la calamité de l'homme du district ; cela exprime que le désordre commis par le troisième trait est précisément la calamité et le malheur causés par l'absence de désordre. Par exemple, s’il obtient, la perte suit im médiatement. Ainsi, dans l'expression « parfois le bouf attaché », le mot parfois indique la supposition et l'incertitude. Parfois il attache et capture le bœuf ; le passant le capture et le considère comme son butin. L'homme du district perd le bouf, c'e st la calamité. Qu'on suppose que l'homme du district attache et capture le cheval, c'est que le passant perd le cheval, et là est la calamité : cela exprime que s'il y a obtention, il doit nécessairement y avoir perte ; ce n'est donc pas suffisant pour qu'on suppose que la chose considérée est obtenue. Les expressions « le passant », «l'homme du district », servent simplement à marquer que s'il y a capture, l'autre face de la question est une perte ; il ne s'agit pas de deux personnes spécialement considérées. La chance qui résulte de l'obten tion illégitime, est cependant encore suivie d'une calamité ; l'obtention d'une capture illégitime est encore récompensée par une perte équivalente, de sorte qu'elle ne peut certainement pas être considérée comme une chance, ou une acquisition. L'homme qui peut se rendre compte de ceci n'agira pas injustement.

TSHOU HI. - Les six formules des traits de ce koua mentionnent toutes l'absence de désordre. Le troisième trait hexaire se place sans se conformer à 
la droiture, aussi, celui qui obtient ${ }^{1}$ ce sens divinatoire, doit éprouver quelque calamité non motivée ; telle que dans le cas du passant qui tire le bouf par une corde pour l'emmener, tandis que l'habi tant, qui reste sur les lieux, subit au contraire les ennuis d'une accusation de vol.

465.

Le passant a la possession du bœuf; l'homme du district subit la calamité.

TSHENG TSE. - Le passant a la possession du bøaf ; c'est précisément là la calamité de l'homme du district. Il y a obtention d'un côté, donc il y a perte de l'autre ; comment cela serait-il suffisant pour justifier le terme possession?

\footnotetext{
${ }^{1}$ En consultant le sort.
} 


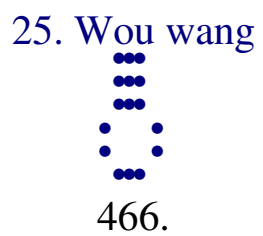

Quatrième trait nonaire : possibilité de perfection ; pas de culpabilité.

TSHENG TSE. - Le quatrième trait est énergique et positif et il fait partie de la substance de l'activité ${ }^{1}$; il est en outre sans correspondance sympathique; c'est celui qui ne com met point de désordre. Énergique et sans égoïsme, comment agirait-il à tort ? Pouvant observer cette règle de conduite avec une parfaite fermeté, il est naturellement sans culpabilité. Un trait nonaire occupant un rang négatif peut-il donc être considéré comme comportant le sens de droiture ? Réponse : puisque ce trait est positif et fait partie de la substance de l'activité exprimée par le koua simple khien, s'il occupait en outre un rang comportant le sens de dureté énergique, cela constituerait un excès de dureté ; or l'excès sous -entend le désordre. Occupant le quatrième rang, ses tendances ne sont pas exclusivement portées vers la dureté énergique ${ }^{2}$. Les expressions «possibilité de perfection» et «avantage de la perfection » ne sont pas équivalentes. Possibilité de perfection exprime qu'il peut se maintenir avec une parfaite fermeté $d$ ans la position où il se place; avantage de la perfection veut dire quil y a avantage dans cette perfection.

TSHOU HI. - Dureté énergique positive, substance de l'activité ; en bas pas de sympathies : possibilité de se maintenir en s'observant avec ferm eté et perfection, impossibilité de considérer cette condition comme suffisante pour rien entreprendre ; tel est le sens divinatoire.

467.

Possibilité de perfection sans culpabilité ; cela existe certainement.

TSHENG TSE. - En s'y maintenant avec une p arfaite fermeté, il n'y aura point de culpabilité.

\footnotetext{
${ }^{1}$ Koua simple khien.

${ }^{2}$ Un rang positif.
} 
TSHOU HI. - Le caractère yeou, du texte, avoir, équivaut au mot sheou, garder, maintenir ${ }^{1}$.

${ }^{1}$ Allégation absolument arbitraire. 


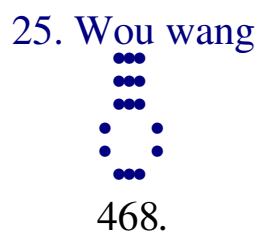
Cinquième trait nonaire : inconvénient de l'absence de désordre; sans remède il y a joie.

TSHENG TSE. - Ce trait nonaire emploie la justice et la droiture et il se trouve occuper le rang suprême; en bas, de plus, on lui répond sympathiquement avec soumission par la justice et la droiture : c'est là ce qu'on peut appeler la plus parfaite absence de désordre. Rien ne peut plus être ajouté dans cette voie. Le terme traduit par «inconvénient» est pris dans le sens de mal, incommodité; dans l'absence de désordre du cinquième trait nonaire, s’il arrive qu’il en résulte quelque « inconvénient » sans traiter cette incommodité par les médicaments, la joie reviendra d'elle-même ${ }^{1}$. Quand l'homme est malade, il emploie les remèdes végétaux et minéraux pour combattre et chasser le mal et pour nourrir le principe vital ; si les substances de son éther constitutif sont en équilibre harmonique, sans maladie ni indisposition, en se médicamentant, il nuira au contraire à ce principe vital. C'est pour cela que sans remède il y aura de la joie. "Yavoir de la joie », exprime que la maladie, ou inconvénient se dissipera naturellement. Ce qui est appelé inconvénient de l'absence de désordre, c'est, par exemple, régir sans réussir à faire régner l'ordre, se mettre en avant et n'être pas suivi, travailler à améliorer les hommes sans réussir à supprimer leurs vices; dans tous ces cas le mal qui subsiste, ou désordre, est considéré comme l'inconvénient de l'absence de désordre. Par exemple, c'est la présence du peuple Miao dans l'empire de Shouen ; c'est Kouan Tsai pendant la carrière de Tsheou Kong, ou enfin Sou Souen et Wou Sou dans celle de Khong Tse. Du moment où -il y a absence de désordre et où cependant il existe quelque chose qui y constitue un inconvénient, il convient de ne point s'en occuper ; l'inconvénient dans l'absence de désordre n'est pas quelque chose qui puisse constituer un malheur. Si on se laisse aller à le combattre et à vouloir y remédier, c'est dépasser le niveau de l'absence de désordre et la transformer en désordre. Puisque le cinquième trait est placé au point de l'extrême absence de désordre, l'avertissement est seulement relatif au fait de se mouvoir ; s’il se meut, c'est le désordre.

TSHOU HI. - Dureté énergique, justice et droiture. Puisque ce trait occupe la situation prééminente et qu'en bas les sympathies qu'il rencontre sont égalementjustes et droites, il exprime le parfait état d'absence de désordre. Si dans de telles conditions il existe quelque inconvénient on ne doit

\footnotetext{
${ }^{1}$ Sans médicaments cette maladie guérira et la joie reviendra d'elle -même.
} 
pas y remédier, et le mal guérira naturellement. C'est pourquoi tels sont l'image symbolique et le sens divinatoire.

469.

Remède de l'absence de désordre; on ne doit pas l'essayer.

TSHENG TSE. - Lorsquil y a du mal dans l'homme, la raison prescrit qu'il faut y remé dier et le corriger ; mais du moment où il y a absence de mal, si cependant on emploie une médicamentation pour le soigner, c'est au contraire travailler à créer le désordre. Où pourrait en être l'utilité ? C'est pour cela que la formule défend d'en faire l'essai. Ce mot essayer, signifie employer temporairement, ou, comme on dit aussi, à titre provisoire.

TSHOU HI. - Du moment où il n'y a pas de désordre, et où on emploie cependant encore les remèdes, on sera au contraire la cause d'un désordre et le mal naîtra. Essayer, veut dire pratiquer à titre temporaire. 


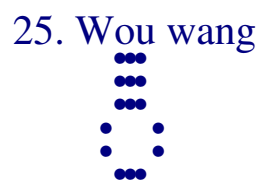

470.

Trait supérieur nonaire : absence de désordre ; en marchant il y a malheur ; aucun moyen davantage.

TSHENG TSE. - Le trait supérieur nonaire occupe le dernier rang dans le koua; il exprime le comble d'absence de désordre. Arrivé à l'extrême limite, si on marche encore, on dépasse le point indiqué par la raison d'être des choses; dépasser la raison, c'est le désordre. Aussi, si le trait supérieur nonaire marche encore, il commet une faute, attire les calamités et rien ne peut lui être avantageux.

TSHOU HI. - Le trait supérieur nonaire ne renferme en lui-même aucun mal ; mais étant arrivé à la dernière et extrême limite, il ne doit plus marcher ni agir, et c'est pour cela que tels sont l'image symbolique et le sens divinatoire.

471.

Agir dans l'absence de désordre ; calamité résultant de la fin ${ }^{1}$.

TSHENG TSE. - Du moment où l'absence de désordre est arrivée à sa plus extrême limite, et où cependant on avance encore, cela constitue le désordre ; c'est l'extrême limi te constituant les calamités et le malheur.

\footnotetext{
${ }^{1}$ On pourrait lire « de l'épuisement final ».
} 


\section{Tae tshou}

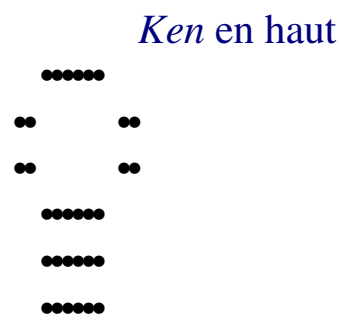

Khien en bas

472. Grand arrêt ; avantage de la perfection ; présage heureux de ne pas vivre aux dépens de la famille; avantage à traverser un grand cours d'eau.

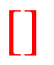

480. Premier trait nonaire : il y a danger; avantage à cesser.

$*$

482. Deuxième trait nonaire : le char manque d'essieu.

$*$

484. Troisième trait nonaire : un excellent cheval poursuit; avantage de la perfection difficile; journellement préparer les chars et les enceintes ; avantage dans ce qu'il y a à entreprendre.

$*$

486. Quatrième trait hexaire : la planche placée sur le front du jeune taureau; grandeur du présage heureux.

$*$

488. Cinquième trait hexaire : les défenses du parc châtré ; présage heureux.

$*$

490. Trait supérieur nonaire : quelle est la liberté d'action de l'influence du ciel ? Liberté.

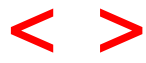


26. Tae tshou.

TSHENG TSE. - Tae tshou. «L'Ordre des koua » dit : «Aussitôt qu’il y a absence de désordre, il peut y avoir groupement, c'est pourquoi le koua wou wang est suivi du koua tae tshou. » L'absence du mal ${ }^{1}$ constitue la réalité ou sincérité, de sorte qu'il peut y avoir grou pement et rassemblement ; c'est ce qui fait que le koua wou wang est immédiatement suivi du koua tae tshou. Comme diagramme, il est formé du trigramme ken en haut et du trigramme khien en bas ; c'est le ciel (khien), mais placé au milieu de montagnes ; image symbolique indiquant que ce qui est rassemblé est ce quill y a de plus grand. Le mot tshou est pris dans le sens de « rassembler en arrêtant». Il a aussi le sens de «réunir en rassemblant»; du moment où il y a arrêt, il y a rassemblement. Si on considère l’image symbolique du ciel entre des montagnes, cela donnera le sens d'accumulation ; si on ne considère que l'arrêt du koua simple khien par le koua simple ken, cela donnera le sens de rassemblement par arrêt. Le groupement commence par l'arrêt, et c'est pour cela que l'idée d'arrêt conduit au rassembl ement exprimé par le caractère tshou.

${ }^{1}$ Désordre ; erreur ; mensonge. 


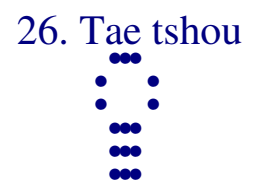

472.

Grand arrêt ; avantage de la perfection; présage heureux de ne pas vivre aux dépens de la famille ${ }^{1}$; avantage à traverser un grand cours d'eau.

TSHENG TSE. - Rien n'est plus grand que le ciel, et cependant il est compris entre les montagnes. Le koua simple ken est au-dessus et arrête le koua simple khien (activité), en bas ; cela donne toujours l'image symbolique de l'extrême grandeur dans l'accumulation, le groupement et l'arrêt. Chez l'homme, ce sera l'étude des systèmes basés sur la vertu de la voie, dont il se pénètre et se remplit; c'est ce qu'il peut accumuler et rassem bler de plus grand et de plus important. Cela s'applique égalem ent à tout ce qui est accumulation; pour ne parler que de ce qu'il y a de plus grand, dans le groupement des hommes, il convient d'observer la voie de la droiture, c'est pourquoi la formule dit: «avantage de la perfection ». En effet, sill s'agit de principes étranges, d'études particulières, les résultats peuvent être très nombreux et divers, et dans le nombre il y en a certainement qui ne sont point corrects. Du moment où les vertus de la voie pénètrent et remplissent l'homme, il mérite une situation supérieure, pour jouir des faveurs du ciel et les répandre sur l'univers, et alors ce ne sera plus le bonheur d'un seul, mais le bonheur de l'univers. Si cet homme est misérablement placé, et se nourrit aux dépens de sa famille, cela indique la décadence de la voie rationnelle. Aussi, sil ne vit point aux dépens de sa famille, ce sera un présage heureux. Du moment où ce qui est réuni et accumulé est grand, il convient que l'effet en soit étendu à tous ceux qui vivent dans le même temps, afin de remédier aux difficultés et aux périls de l'univers, et c'est là l'usage et l'effet du « grand rassemblement ». Aussi, il y a avantage à traverser un grand cours d'eau. Tout ceci se rapporte uniquement au sens du terme «grand rassemblement »; la formule déterminative en parle autrement, au point de vue des vertus et des aptitudes des koua simples. Tous les divers traits ont seulement le sens « d'arrêter en rassemblant ». En effet, dans le Yi king, la voie rationnelle est présentée sous une forme palpable, selon le cas, et l'exemple choisi est toujours le plus clair et le plus à portée.

TSHOU HI. - Le mot tae, grand, exprime la positivité ; emploi du koua simple ken pour arrêter le koua simple khien; ou bien encore : grandeur dans l'arrêt. Enfin puisque, au-dedans il y a la dureté énergique de la force active, et au-dehors l'éclat resplendissant et certain exprimé par le terme hen, c'est l'indice de l'aptitude à la rénovation journalière de la vertu et c'est considéré

\footnotetext{
${ }^{1}$ Cette lecture est arbitraire. Le vrai sens est mal défini.
} 
comme exprimant la grandeur de ce qui est accumulé. Si on en parle d'après les modifications progressives du koua lui-même, celui-ci provient du koua parfait su, dans lequel le trait nonaire monte du cinquième rang au rang supérieur ; si on en parle d'après la substance, le cinquième trait hexaire es $t$ prééminent et en fait la noblesse ; si on en parle au point de vue des vertus exprimées par les koua simples, elles marquent aussi la faculté d'arrêter la force active. Dans tous les cas l'action indiquée est toujours impossible sans une grande droiture, de sorte que le sens divinatoire est: "avantage de la perfection et présage heureux de ne point se nourrir aux dépens de la famille ». De plus encore, le cinquième trait hexaire sympathise avec l'activité, ce qui est considéré comme exprimant la correspondance sympathique aux lois célestes, de sorte que le sens divinatoire est aussi qu'il y a avantage à traverser un grand cours d'eau. Ne pas vivre aux dépens de la famille, c'est-à-dire vivre d'émoluments payés par le gouvernement, sans se nourrir des biens de la famille.

473.

Le commentaire traditionnel de la formule déterminative dit: Tae tshou, force active énergique,réalité absolue, éclat resplendissant; rénover journellement la vertu.

TSHENG TSE. - Ceci est dit au sujet des vertus et des propriétés des koua simples. La substance du koua simple khien est la force active énergique; la substance du koua simple ken est la réalité absolue. Si les aptitudes d'un homme sont l'activité énergique et la sincé rité absolue, ce qu'il arrêtera ou accumulera pourra être grand; réalité complète et en outre éclat resplendissant ; l'accumulation ne cessant point, il en résulte que les vertus se rénovent journellement.

TSHOU HI. - Explication du sens du nom du koua au moyen des vertus des deux koua simples.

474.

La dureté énergique occupe le rang supérieur et estime la sagesse; elle peut arrêter la force active; grande droiture. 
TSHENG TSE. - La dureté énergique au-dessus, c'est -à-dire qu'un trait positif occupe le rang supérieur ; la dureté énergique de la positivité est placée au-dessus de la situation prééminente, ce qui donne le sens d'estime de la sagesse. L'arrêt est au-dessus de l'activi té, ce qui donne le sens de pouvoir arrêter la force active. Comment l'arrêt de l'activité serait -il possible sans une grande droiture? Puisque la dureté énergique positive est au-dessus, puisqu'elle s'allie à la prééminence et qu'elle estime la sagesse, qu'elle est capable d'arrêter la force la plus active, c'est donc toujours la voie d'une grande droiture.

TSHOU HI. - Explication de la formule du koua au moyen de la formation du koua parfait et de la substance des koua simples.

475.

Présage heureux de ne pas vivre aux dépens de la famille; entretenir la sagesse.

TSHOU HI. - C'est encore l'image symbolique d'es timer la sagesse qui est mise en relief.

476.

Avantage à traverser un grand cours d'eau; répondre au ciel.

TSHENG TSE. - Lorsqu'un homme accumule ce qui est grand, ce quil doit répandre et communiquer est justement ce qu'il accumule, afin d'assister l'univers, de sorte quil ne vit pas aux dépens du bien de sa famille, le présage est heureux; cela indique quil tient la situation qui représente le ciel, et qu'iljouit des bienfaits célestes. Si l'état entre tient les sages, ceux-ci peuvent suivre leur voie. "Avantage à traverser un grand cours d'eau » veut dire que l'homme qui a beaucoup acquis et accumulé doit remédier aux difficultés de l'univers. La formule déterminative insiste sur l'explication des aptitudes des koua simples ; ce qui est exprimé par les mots «pouvoir traverser un grand cours d'eau », c'est la correspondance sympathique au ciel. Le cinquième trait hexaire, c'est le prince ; au-dessous, il correspond sympathiquement au trait intermédiaire du koua simple khien ; c'est le prince qui a beaucoup recueilli et accumulé ; il sympathise à l'activité et agit ; ce qu'il fait peut correspondre à 
l'action du ciel ; il n'est aucune difficulté dont il ne puisse venir à bout, combien à plus forte raison lorsqu'il s'agit de choses moins importante $\mathrm{s} 1$

TSHOU HI. - C'est encore dit au sujet de la substance du koua lui même.

477.

Le commentaire de la formule symbolique dit: Le ciel est au milieu des montagnes; grandeur de l'arrêt; l'homme doué pratique la grande connaissance des paroles et des entreprises antérieures, afin d'accumuler les vertus.

TSHENG TSE. - Le ciel est ce qu'il y a de plus grand, et cependant il est compris entre les montagnes : ce qui est réuni et rassemblé est ce qu’il y a de plus grand. L'homme doué regarde l'image symboliq ue et l'applique à l'agrandissement de ce qu'il accumule. Les connaissances acquises par l'homme s'agrandissent par l'étude ; c'est en écoutant attenti vement le récit des paroles et des actions des sages et des saints de l'antiquité, en en recherchant les traces pour voir quels en ont été les effets, en scrutant les paroles pour découvrir les intentions, quil sait et peut profiter, afin de compléter ses vertus et de les augmenter. Tel est le sens du koua tae tshou.

TSHOU HI. - Le ciel est entre les montagnes ; ce n'est pas à dire qu'une telle circonstance existe en réalité, mais il en est seulement question comme d'un symbole.

478.

Il y a péril, avantage à cesser ; ne pas braver les calamités.

TSHENG TSE. - Il y a péril, il est donc opportun de cesser; on ne doit pas braver les calamités et le danger en agissant. Si on avance sans tenir compte de la force naturelle des circonstances, il s'en suivra certainement des calamités. 
Le char manque d'essieu; la justice ne commet point de fautes.

TSHENG TSE. - C'est le char dépourvu d'essieu et qui ne progresse pas. En effet, il se place dans une voie conforme à la justice; s'il se meut il ne manque point aux convenances, de sorte quil n'y a ni faute, ni erreur. Dans le bien, rien n'est meilleur que la justice de l'énergie. La justice de la mollesse, n'est rien de plus que l'excès dans la douceur. La justice de l'énergie, c'est la justice jointe à la capacité. Le premier trait nonaire se place sans observer la justice ; aussi la formule avertit qu'il y a péril et qu'il convient de cesser. Le second possède la justice ; quill avance ou recule, il ne commet naturellement ni faute ni erreur, aussi la formule parle seulement du char qui manque d'essieu, c'est -à-dire qui peut ne pas avancer ; en n'avança nt pas, il n'y aura point de fautes commises. Le premier et le second traits font partie de la substance de l'activité, leur activité est énergique, et elle est cependant insuffisante pour leur permettre d'avancer. Le quatrième et le cinquiè me sont faibles et mous et ils peuvent s'arrêter. Cette opposition marque l'état florissant ou de décadence du moment, le plus ou moins d'énergie de la force naturelle des événements; c'est ce que ceux qui étudient le Yi kingdoivent particulièrement approfondir. 


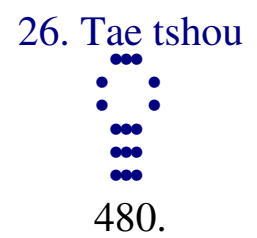

Premier trait nonaire : il y a danger; avantage à cesser.

TSHENG TSE. — Grandeur de l'arrêt ; le koua simple ken arrête le koua simple khien, aussi les formules des trois traits du koua simple Khien donnent toutes le sens d'être arrêté, tandis que celles des trois traits du koua simple ken donnent le sens d'arrêter. Le premier trait emploie la dureté énergique de la positivité ; de plus il fait partie de la substance de la force active, et il demeure dans l’infériorité ; c'est celui qui doit nécessairement monter en avançant, le quatrième trait hexaire est en haut, qui arrête et contient le premier ; comment celui-ci pourrait-il lutter contre la force inhérente à une situation acquise et à la supériorité ? S'il la brave et avance, il courra des dangers et sera en péril, aussi il a avantage à cesser, et à ne pas avancer. Dans d'autres koua, la relation entre le quatrième et le premier trait exprimerait la sympathie correcte et légitime, et ces deux traits figureraient ceux qui s'accueillent et vont au-devant l'un de l'autre ; dans le koua tae tshou la correspondance mutuelle est considérée comme indiquant qu’ils s'arrêtent l'un l'autre. Le trait supérieur et le troisième sont également positifs, donc ils sont considérés comme ayant les mêmes tendances, en effet tout ce qui est positif est toujours d'une nature qui tend à monter en avançant, de sorte quils ont l'image symbolique de communauté de tendances, sans avoir le sens de s'arrêter mutuellement.

TSHOU HI. - Les trois traits positifs du koua simple khien, représentent ce qui est arrêté par le koua simple ken, aussi chacun des deux koua simples prend son sens de la position intérieure ou extérieure qu'il occupe. Le premier trait nonaire est arrêté par le quatrième trait hexaire, aussi le sens divinatoire est que, en continuant en avant, il y aura péril, et que l'avantage consiste à s'arrêter.

481.

Il y a péril, avantage à cesser; ne pas braver les calamités. 
TSHENG TSE. Il y a péril, donc il y a avantage à cesser; on ne doit pas braver les calamités et agir ; si on ne mesure pas la force naturelle des choses, il est certain qu'on tombe dans le péril. 


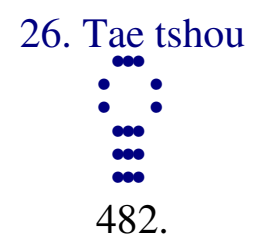

Deuxième trait nonaire : le char manque d'essieu.

TSHENG TSE. - Le second trait est arrêté par le cinquième trait hexaire ; la force des choses est telle quil ne doit pas avancer. Le cinquième s'autorise de sa supério rité : comment le braver ? Bien que le second fasse partie de la substance de la force active énergique, cependant sa position est conforme à la voie de la justice, de sorte que, soit qu'il avance, soit quill recule, il ne commet pas d'erreurs. Bien que ses tendances le portent à avancer, il sait apprécier que la force naturelle des choses ne le lui permet pas, de sorte qu'il s'arrête, sans continuer à agir, comme le char dont on a enlevé l'essieu, c'est -à-dire, qui ne peut rouler.

TSHOU HI. - Le deuxième trait nonaire est, d'une façon analogue, celui qui est arrêté par le cinquième trait hexaire ; c'est parce qu'il est placé avec justice qu'il peut se conte nir lui-même sans avancer. Telle est l'image symbolique.

483.

Le char manque d'essieu ; avec la justice pas d'erreurs.

TSHENG TSE. - C'est le char qui manque d'essieu et qui ne peut progresser ; en effet, sa position est conforme à la voie de la justice ; en se mouvant il ne manque point aux convenances, de sorte qu'il ne commet ni fautes, ni erreurs. Dans le bien, rien n'est meilleur que la justice dans l'énergie ; celui qui joint la justice à la douceur malléable n'atteint qu'à une excessive mollesse ; avec la justice et l'énergie, l'on dispose de la justice et de toutes les capacités. Le premier trait nonaire est placé sans tenir compte de la justice, aussi la formule avertit qu'il y a péril et qu’il y a lieu de cesser et d'arrêter ; le second se conforme à la justice, qu'il avance ou s'arrête, il n'y a essentiellement ni faute, ni erreurs ; aussi la formule ne parle que du char qui manque d'essieu, c'est -à-dire qui peut ne pas rouler. N'agissant pas, il n'y a point d'erreurs commises. Le premier et le second traits font également partie de la substance du koua simple khien, leur activité est énergique, et elle est cependant insuffisante pour qu'ils avancent. Le quatrième et le cinquième sont 
également malléables et négatifs et ils sont toutefois capables d'arrêter. L'essor florissant ou la décadence du moment, le degré de puissance ou de faiblesse de la force naturelle des choses, sont essentiellement ce qui doit faire l'objet de l'attention de ceux qui étu dient le Yi king. 


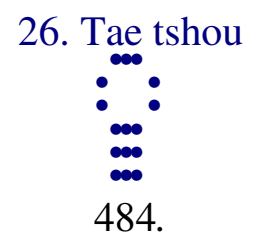

Troisième trait nonaire : un excellent cheval poursuit; avantage de la perfection difficile ; journellement ${ }^{1}$ préparer les chars et les enceintes ; avantage dans ce qu'il y a à entreprendre.

TSHENG TSE. — Le troisième trait exprime l'extrême limite de l'activité énergique, et la positivité représentée par le trait supérieur nonaire est aussi quelque chose qui avance en montant. De plus, ce trait supérieur est à l'extrême limite de l'arrêt, et il s'apprête à se modifier ; lui et le troisième, sont ceux qui ne s'arrêtent pas mutuellement et dont les ten dances sont identiques; ce sont donc ceux qui sympathisent ensemble pour avancer. Le troisième trait emploie les aptitudes de l'activité énergique, et celui qui est au rang supérieur conforme ses tendances aux siennes et avance; leur mouvement de progression en avant est comme celui d'excellents chevaux poursuivant avec ardeur; cela exprime la vitesse et la rapidité. Bien que la force inhérente à leur mouvement de progression en avant soit rapide, il ne doit pas se fier à l'activité de ses aptitudes et à la sympathie du supérieur, en oubliant les précautions et la prudence. Aussi il importe qu'il apprécie la diffi culté des choses et qu'il suive la voie de la parfaite droiture. Le char est ce qui sert à transporter; les enceintes sont des moyens de protection; il convient qu'il exerce journellement ses chars et qu'il entretienne ses défenses et enceintes, et alors il aura avantage dans ce quill aura à entreprendre. Le troisième trait fait partie de la substance du koua simple khien et il occupe son rang avec droiture ; c'est celui qui est capable de perfection. C'est le moment où il avance activement, aussi la formule avertit, afin qu'on reconnaisse le danger et qu'on ne manque pas à la perfection. Du moment où les tendances portent activement à avancer, malgré l'énergie et l'intelligence, il y a parfois des erreurs commises; il est impossible de ne point en faire le sujet d'un avertissement.

TSHOU HI. - Le troisième trait emploie la positivité et occupe le rang extrême dans le koua simple qui indique la force active; le trait supérieur emploie la positivité et occupe le dernier rang dans le koua qui exprime le groupement, ou arrêt: C'est le moment où les choses sont poussées à l'extrême et qui comporte en même temps la liberté d'action. De plus tous deux sont des traits positifs, de sorte quils ne s'arrêtent pas mutuellement et qu'ils avancent ensemble ; ils présentent l'image symbolique de chevaux ardents qui se poursuivent. Toutefois, il y a excès dans la dureté énergique et

\footnotetext{
${ }^{1}$ Le caractère est douteux.
} 
plaisir à avancer; de sorte que le sens divinatoire doit avertir des dangers de la perfection et de la nécessité de se préparer à la défense. C'est par là qu'il y aura avantage dans les entreprises. Le neuvième caractère du texte, doit être remplacé par le caractère dont le sens est « soleil », ou « jour ».

485.

Avantage dans ce qu'il y a à entreprendre ; le supérieur s'accorde à ses tendances.

TSHENG TSE. - Ce qui fait qu'il y a avantage dans ce qu'il y a à entreprendre, c'est que lui et celui qui est au rang supérieur on t des tendances qui s'accordent. La nature du trait supérieur nonaire est positive, il avance en montant; d'ailleurs l'arrêt est arrivé à sa plus extrême limite, de sorte qu'il ne s'abaisse pas pour arrêter le troisième, mais quils unis sent mutuellement leurs tendances pour avancer ensemble en montant. 


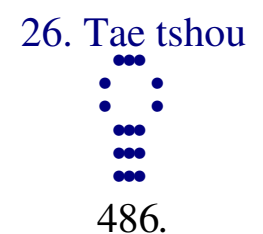

Quatrième trait hexaire : la planche placée sur le front du jeune taureau; grandeur du présage heureux.

TSHENG TSE. - En en parlant au point de vue de la situation, le quatrième se baisse pour sympathiser avec le premier; c'est lui qui arrête le premier trait. Le premier demeure au rang le plus inférieur, c'est le degré le plus minime de la positivité. Puisque la positivité est minime et qu'il l'arrête, il lui est facile de la dominer. C'est comme lorsqu'il s'agit d 'un jeune taureau auquel on a placé une planchette sur le front ; l'effet est excellent et le présage heureux. Dans ces formules il est toujours question de la voie de l'arrêt, donc, le quatrième trait faisant partie de la substance du koua simple ken, occupant une situation supérieure et possédant la droiture, c'est qu'il emploie la vertu de la droiture pour occuper la situation d'un sujet de rang élevé ; c'est celui qui porte le poids de la responsabilité pendant l'arrêt. L'autorité dévolue au ministre d'un rang élevé lui impose le devoir d'arrêter, en haut, les mauvaises pensées du prince et, en bas, de contenir les vices des hommes. Il est relativement facile d'arrêter les penchants vicieux des hommes dès leur début; mais lorsque ces penchants sont complètement développés et qu'ensuite on les entrave, ils résistent et il est difficile d'en venir à bout. Aussi, lorsque le mal est déjà considérable chez le supérieur, bien qu'un homme saint lui-même travaille à y remédier, il est impossible d'éviter les résistances et la révolte ; lorsque le mal est déjà invétéré chez les inférieurs bien que ce soit un homme saint qui y mette ordre, il ne peut se dispenser de recourir aux peines et aux supplices. Il vaut mieux agir, comme lorsqu’il s'agit d'un jeune taurea et qu'on lui place une planche sur le front, ce qui constitue un présage parfaitement heureux. Le naturel du taureau le porte à frapper avec les cornes, aussi on l'entrave, afin de le dominer ; si, pendant qu'il est encore jeune et au moment où ses cornes commencent à croître, on lui applique une planche sur le front pour prévenir les manifestations de son naturel qui le porte à frapper avec les cornes, le résultat sera facile à obtenir, sans accidents. Combien, à plus forte raison, sera grand le présage heureux de la perfection du bien si le quatrième trait hexaire peut contenir le mal chez le supérieur et les inférieurs, avant que ce mal ne soit manifesté !

TSHOU HI. - jeune, signifie qu'il n'a pas encore de cornes ; le sixième caractère du texte désigne une planche placée sur les cornes du taureau pour l'empêcher de frapper; dans le Shi king cette planche est désignée par l'expression «traverse favorable». En prévenant à l'époque où il n'a pas encore de cornes, la tâche sera rendue facile, et c'est un présage de grand bien. 
C'est pourquoi tels sont l'image symbolique et le sens divinatoire. Le livre $H o$ $k i$ dit : «Prévenir, c'est arrêter à l'avance » : c'est précisément là l’idée expri mée.

487.

Grandeur du présage heureux du quatrième trait hexaire; il y a de la joie.

TSHENG TSE. - Pour arrêter le mal dans l'univers après qu'il est déjà développé, il faut que le supérieur déploie tout son effort afin de le prohiber et de le contenir, tandis que les inférieurs pâtiront sous le poids des peines et des supplices. Aussi, en l'arrêtant à l'avance, alors qu'il ne sera encore que très minime, ce sera un grand bien et un présage heureux ; pas d'efforts pénibles, pas de souffrances, de sorte que la joie est possible. Tel est le cas de l'arrêt du premier trait par le quatrième, et il erg est de même dans le cas rie l'arrêt du trait supérieur. 


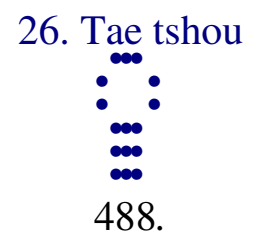

Cinquième trait hexaire : les défenses du porc châtré ${ }^{1}$; présage heureux.

TSHENG TSE. - Le cinquième trait hexaire occupe la situation du prince, il arrête et contient les mauvaises passions dans l'univers entier. Or, lorsqu'il s'agit d'une multitude qui se compte par millions et dizaines de millions, et dont les passions vicieuses se manifestent et éclatent, si le prince veut employer la force pour contenir ces passions, quelle que soit la sévérité des règles et la dureté des peines, il ne peut y réussir. Mais les êtres sont reliés par un lien commun sous une dépendance unique; les choses ont un mécanisme général; l'homme saint s'empresse de se conformer aux nécessités du cas considéré, de sorte qu'il regarde tous les cours de cette multitude comme ne constituant qu'un seul cour ; il la moralise par sa voie et elle s'y soumet, il l'arrête et elle s'incline, de sorte que, sans efforts, il la domine. Son action est semblable, par ses effets, à celle qui consiste à rendre inoffensives les défenses du porc, par la castration. Le porc est un animal brusque et emporté et ses défenses constituent sa puissance d'action. Si on veut employer la force pour le contenir, on consume inutilement ses forces sans réussir à le mettre hors d'état de nuire ; bien qu'on l'attache et qu'on le lie, on ne peut l'amener à se transformer. Mais si on le châtre pour lui enlever sa puissance, alors, bien que ses défenses soient intactes, la violence de sa nature cesse. C'est d'en user d'une façon analogue que résulte le présage heureux. L'homme doué applique l'idée de la castration du porc, et sait que le mal, dans l'univers, ne peut pas être dominé par la force. Il en résulte qu'il recherche le moyen d'action approprié, qu’il se base sur les néces sités spéciales du moment, en tarissant la source originelle d'où dérive ce mal, de sorte que, sans avoir recours aux peines et aux lois sévères, il fait que le mal cesse de lui-même. Par exemple, s’il s'agit de remédier au penchant au vol, il sait que le peuple est poussé par ses désirs et qu'en lui montrant un avantage il se tournera vers ce nouveau but. Si, au lieu de savoir instruire le peuple, on le comprime dans sa misère et ses souffrances, bien que les supplices soient prodigués journellement, est-il possible de contenir les passions et les désirs d'une multitude innombrable qui ne recherche que la satisfaction de ses besoins ? Lhomme saint, reconnaîtra la voie à suivre pour contenir cette multitude ; au lieu de s'appuyer de préférence sur les pei nes et sur l'autorité

\footnotetext{
${ }^{1}$ Telle est la lecture admise et le caractère du texte est traduit pour « limer les dents du porc » ou «châtrer le porc », mais il faut tenir compte que ces valeurs sont, dans les dictionnaires, attribuées à ce mot d'après l'explication admise de ce passage. Le terme fers, d'après les plus anciens dictionnaires, désigne un animal sauvage très farouche et pourvu d'un seul oall. La traduction la plus naturelle serait donc : «Les défenses du porc farouche.»
} 
que donne le pouvoir, il réformera les institutions sociales et l'enseignement, afin de la pousser vers l'agriculture et le commerce ; il lui apprendra à connaître la honte jusqu'à ce point que, même à prix de récompense, personne ne voudrait commettre un larcin. Aussi la voie morale pour arrêter le mal consiste uniquement à en connaître l'origine et à se conformer simplement aux nécessités des circonstances considérées. D’un côté ne pas avoir recours aux châtiments sévères; de l'aut re, réformer les institutions sociales, c'est encore le même moyen constaté au sujet du danger présenté par les défenses du porc : ne pas chercher à dominer ses défenses et le châtrer pour supprimer leur puissance.

TSHOU HI. - La positivité a déjà avancé et on l'arrête : ce n'est jamais aussi facile qu'au début. Toutefois, employant la douceur et se maintenant dans la justice pendant qu'on est placé dans la situation supérieure, c'est réellement agir conformément aux moyens d'action appropriés, et être cap able de dominer. C'est pour cela que telle est l'image sym bolique. Bien que le sens divinatoire exprime un présage heureux, cependant ce présage n'est pas qualifié par le mot grand.

489.

Présage heureux du cinquième trait hexaire ; il y a des félicitations.

TSHENG TSE. - Si celui qui occupe un rang supérieur ne connaît pas les règles à suivre pour arrêter le mal et le vice, s’il punit avec sévérité, pour résister aux passions du peuple, les souffrances qui en résulteront seront considérables et n'auron t point d'effet utile. S'il connaît l'origine du mal, il aura une voie morale à suivre pour l'arrêter, et alors, sans efforts, sans souffrances, les moaurs se renouvelleront, et cela constituera le bonheur et la félicité de l'univers. 


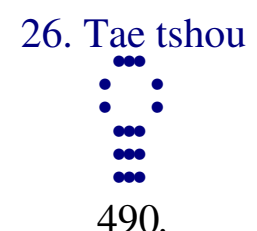

Trait supérieur nonaire : quelle est la liberté d'action de l'influence du ciel? Liberté.

TSHENG TSE. - J'ai entendu dire au philosophe Hou que le texte est : «liberté d'action de l'influence du cie 1 » et que l'adjonction du caractère interrogatif «quelle » est une erreur. Les choses poussées à leur plus extrême limite doivent provoquer une réaction en sens opposé; c'est là une loi constante. Aussi, l'arrêt parvenu à sa limite extrême, il y a liberté. Le koua siao tshou exprime la petitesse dans l'arrêt, aussi, quand il est arrivé à sa limite, il est complet; le koua tae tshou exprime la grandeur de l'arrêt, aussi, quand il est arrivé à sa limite, il est suivi de dispersion. Puisque le point extrême correspond à une modification, et que de plus le naturel de la positivité est d'agir en montant, elle suit son impulsion naturelle et se disperse. La liberté d'action de l'influence du ciel, c'est la voie du ciel, c'est-à-dire le milieu vide dans le néant. Les vapeurs et les nuages, les oiseaux dans leur vol, le parcourent en tous sens, et c'est pour cela que le texte parle de la libre circulation du ciel ${ }^{1}$. Liberté de la pénétrabilité du ciel, c'est-à-dire libre pénétrabilité en tous sens et indéfiniment, sans qu'il y ait aucun obstacle limitatif. Dans la voie de l'arrêt ce sera donc une modification; cette modification sera la liberté, il ne s'agit point de liberté dans la voie de l'arrêt.

TSHOU HI. - «Quelle est la liberté du ciel!» Cette exclamation exprime l'immensité de la pénétrabilité du ciel. Lorsque l'arrêt est extrême il est suivi de liberté dans la circulation; pénétration libre et sans obstacle. C'est pour cela que tels sont limage symbolique et le sens divinatoire.

491.

Quelle est la liberté de l'influence du ciel! Grande action de la voie morale.

TSHENG TSE. - Pourquoi est-il question de la liberté, ou libre pénétration du ciel ? Parce qu'elle ne comporte aucun obstacle qui arrête ;

\footnotetext{
${ }^{1}$ Autre lecture du texte.
} 
c'est une route largement ouverte à la circulati on. C'est parce que l'expression de «pénétrabilité du ciel » n'est pas une locution ordinaire que la formule symbolique affecte spécialement la forme d'une interrogation. Que veut dire l'expression « pénétrabilité du ciel »? C'est que la libre circulation, ou viabilité d'une route, est prise comme emblème du vide libre et pénétrable. C'est parce que la formule symbolique emploie le caractère ho, qui marque l'interrogation, que la formule du trait ne l'ajoute pas encore, ce qui serait une superfétation. 


\section{Yi}

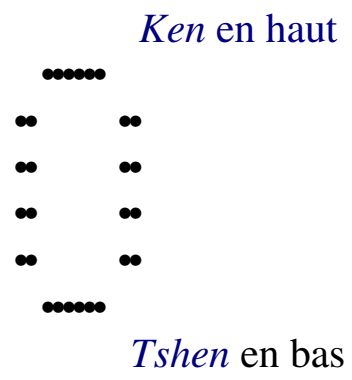

492. Yi ; présage heureux de la perfection ; regarder la bouche ; de là, chercher à se remplir la bouche.

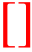

496. Premier trait nonaire : écartez votre tortue spirituelle; regardez le mouvement de mes mâchoires; présage funeste.

$*$

498. Deuxième trait hexaire : subversion dans l'acte de se nourrir ; lier les lois fondamentales; manger sur une hauteur ; en agissant, présage malheureux.

500. Troisième trait hexaire : transgression dans la nourriture ; présage malheureux de la perfection ; pendant dix ans ne pas agir ; aucun moyen davantage.

502. Quatrième trait hexaire : présage heureux de la subversion dans l'acte de la nutrition; le tigre guette en baissant la tête sans regarder; ce qu'il veut, c'est atteindre brusquement par bonds ; pas de culpabilité.

$*$

504. Cinquième trait hexaire : nier les principes fondamentaux; présage heureux du maintien dans la perfection; on ne doit pas traverser un grand cours d'eau.

506. Trait supérieur nonaire : cause de sustentation ; présage heureux du péril ; avantage à traverser un grand cours d'eau.

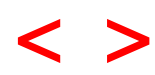


27. Yi.

TSHENG TSE. — Yi. «L'Ordre des koua » dit : «Aussitôt que les êtres sont arrêtés il faut les nourrir, aussi le koua tae tshou est suivi du koua yi. » Or, aussitôt après que les êtres sont rassemblés et arrêtés, il devient indispensable d'avoir de quoi les nourrir ; sans nourriture, ils ne peuvent subsister, et c'est là ce qui fait que le koua yi suit immédiatement le koua tae tshou ${ }^{1}$. Le koua est composé du koua simple ken en haut et du koua simple tshen en bas ; le trait inférieur et le trait supérieur sont les deux seuls traits positifs du koua et ils embrassent entre eux quatre négativités. Le haut est fixe et le bas se meut; l'extérieur est solide, et le milieu est vide ; c'est l'image symbolique des mâchoires et de la bouche de l'homme. Yi signifie nourrir ; introduire dans la bouche afin de boire et de manger; nourrir le corps de l'homme. C'est pour cela que le nom du koua est $y i$. L'homme saint, en établissant les koua, examine le sens du mot nourrir; dans son acception la plus large, et appliqué au ciel et à la terre, c'est la production et la sustentation de tous les êtres. L'homme saint nourrit les sages pour que leur influence atteigne tous les peuples; cette action est celle de l'homme entretenant la vie, nourrissant la forme, développant la vertu, nourrissant d'autres hommes; toutes ces circonstances sont des applications de la voie exprimée par le terme $y i$, pris avec l'acception de nourrir. Les règles convenables pour le mouvement et l'immobilité naissent du sens du mot nourrir ; les prescriptions sur les boissons et les aliments, sur les vêtements, résultent de l'entretien de la forme; les règles d'étiquette qui manifestent l'autorité, et la pratique des devoirs, résultent du « développement de la vertu »; dériver de l'observation de soi-même pour conclure au sujet des êtres, provient de ce qui est exprimé par le terme «nourrir les hommes ».

\footnotetext{
${ }^{1}$ Ici, le caractère tshou est pris dans une nouvelle acception : «troupeaux, bétail », qưil
} comporte également. 


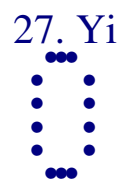

492.

Yi ; présage heureux de la perfection ; regarder la bouche ; de là, chercher à se remplir la bouche.

TSHENG TSE. - La voie indiquée par le koua yi est telle que, par la droiture, le présage sera heureux. L'homme, quand il nourrit son corps, quand il développe ses vertus, quand il nourrit d'autres hommes ou qu'il nourrit pour quelqu'un, trouvera toujours un présage heureux par l'emploi de la voie de la droiture. Le ciel et la terre créent et transforment; ils nourrissent et développent tous les êtres ; chacun de ceux-ci possède les moyens de satisfaire à ses besoins résultant des lois de la nature ; cela n'est jamais rien autre que la droiture. Regarder la bouche et, de là, chercher à se remplir la bouche, c'est regarder de quoi l'homme se nourrit et la voie qu'il suit pour arriver à se nourrir; il en résulte que le bien et le mal, les présages heureux ou malheureux, dans cette voie, peuvent être observés.

TSHOU HI. - Yi ; les commissures des lèvres. La bouche mange pour nourrir l'individu, c'est ce qui fait que le sens de nourriture est considéré. Comme koua, il est composé de deux positivités, dont l'une en haut et l'autre en bas, qui embrassent entre elles quatre négativités. L'extérieur est matériel et solide, l'intérieur est vide ; le haut est immobile, le bas se meut; cela constitue l'image symbolique de la bouche et le sens de nourrir. L'expression «présage heureux de la perfection » indique que, si celui qui consulte le sort possède la droiture, le présage sera heureux. "Regarder la bouche", c'est-à-dire regarder la voie suivie pour nourrir. «Chercher à se remplir la bouche », c'est regarder le système suivi pour nourrir le corps. Dans tous les cas, en se conformant à la droiture, le présage sera heureux.

493.

Le commentaire traditionnel de la formule déterminative dit: Yi, présage heureux de la perfection; nourrir la droiture sera un présage heureux. Regarder la bouche, regarder comment on nourrit; chercher à se remplir la bouche, regarder à sa propre nourriture ${ }^{1}$.

\footnotetext{
${ }^{1}$ Le mot « nourrir » se prend aussi bien au figuré avec le sens d'entretenir, développer.
} 
TSHENG TSE. - Présage heureux de la perfection ; si ce qui est nourri et entretenu est la droiture, le présage sera heureux. "Comment on nourrit», c'est-à-dire quels sont les hommes qu'on nourrit et la voie suivie pour les nourrir. Chercher à se remplir la bouche, c'est-à-dire chercher soi-même la voie à suivre pour soigner son corps. Si tout cela a lieu conformément à la droiture, le présage sera heureux.

TSHOU HI. - Explication de la formule du koua.

494.

Le ciel et la terre nourrissent tous les êtres; l'homme saint nourrit les sages afin que l'effet atteigne la foule des peuples. Que le moment exprimé par le koua yi est grand!

TSHENG TSE. — L'homme saint ${ }^{1}$ résume dans sa plus haute acception la voie rationnelle exprimée par le koua yi et s'exclame sur sa grandeur. Dans la voie rationnelle du ciel et de la terre, ce sera l'action d'engendrer et de nourrir tous les êtres ; or, la voie qui produit la génération et le développement de tous les êtres n'est pas autre chose que la droiture. L'homme saint, de son côté, pourvoira aux besoins des hommes sages et capables; par rapport à ceux-là, il occupe la situation du $\operatorname{ciel}^{2}$; il les admet à jouir des faveurs qu'il tient du ciel, afin qu'ils étendent l'action de son influence bienfaisante à tout l'univers ; c'est là nourrir les sages pour atteindre la foule des peuples. Nourrir les sages, c'est le moyen de nourrir la foule innombrable des peuples. Or, entre le ciel et la terre, tous les êtres, de quelque genre quils soient, ne peuvent vivre sans nourriture. L'homme accomplit la voie tracée par le ciel et la terre, il aide à la réalisation des conditions naturelles imposées par le ciel et la terre, afin de nourrir l'univers, à tel point que, jusqu'aux oiseaux et aux ani maux, tout a des moyens de subsistance résultant des institutions quil établit. La voie qu'il suit égale celle du ciel et de la terre, de sorte que Fou Tse analysant la voie rationnelle exprimée par le koua yi, admirant l'ouvre du ciel et de la terre ainsi que celle de l'homme saint, s'écrie : que le moment indiqué par le koua yi est grand! Parfois, dans ses formules, il s'attache au sens ; d'autres fois aux effets produits; d'autres fois encore au moment indiqué, selon l'importance relative de ces diverses données. Lorsqu'il s'agit de la vie et de la subsistance de tous les êtres, c'est le moment qui est la donnée la plus grande, et voilà pourquoi il parle du moment.

TSHOU HI. - Dernière analyse de la voie morale de l'entretien de l'être vivant, et exclamation admirative sur cette voie.

\footnotetext{
${ }^{1}$ Khong Tse, l'auteur de ce commentaire.

${ }^{2}$ Il joue le rôle du ciel.
} 
495.

Le commentaire traditionnel de la formule symbolique dit: Au-dessous de la montagne il y a la foudre: bouche. L'homme doué emploie la prudence dans les paroles, il fixe les préceptes relatifs à la boisson et à la nourriture.

TSHENG TSE. - En parlant d'après les substances des deux koua simples, au bas de la montagne il y a la foudre; la foudre ébranle au bas de la montagne. Les choses qu'engendre la montagne ${ }^{1}$ commencent toutes à pousser par des racines, puis émettent des germes, ce qui est considéré comme l'image symbolique de liidée de développement et de nourriture. Si on en parle au point de vue du sens qui résulte de la situation relative de dessus et de dessous, le koua simple ken exprimant l'arrêt, et le koua simple tshen le mouvement, le dessus arrête ou est arrêté et le dessous se meut, ce qui donne l'image symbolique de la bouche dans l'action de manger. Si on en parle d'après la forme, ou figure du koua, en haut et en bas se trouvent deux positivités qui enveloppent entre elles quatre négativités ; l'extérieur est plein et solide, le milieu est évidé ; donc image symbolique des mâchoires de la bouche. La bouche est l'organe qui sert à la nutrition du corps, aussi l'homme doué considère ces images symboliques au point de vue de la nourriture du corps et des soins de la conservation de la personne: prudence et circonspection dans les paroles, pour entretenir les vertus ; règles concernant la boisson et la nourriture, afin d'entretenir et de conserver le corps. Non seulement c'est prendre le sens du mot nourrir en ce qui se rapporte à la bouche, mais son application est étendue et généralisée du plus proche au plus éloigné ; or dans ces deux ordres de conditions rien ne surpasse en importance la fonction de la nutrition et celle de la parole. Par rapport à l'individu il s'agit de la parole ou conversation ; par rapport à l'univers, il s'agit de tout ce qui émane de la personne du prince, comme les lois, règlements, institutions, ordres, décrets, etc. En y portant la circonspection, chacun de ces détails sera ce qu'il convient qu'il soit et il n'y aura point de méprises. Par rapport à l'individu, il s' agit de la boisson et de la nourriture ; par rapport à l'univers il s'agira du commerce, des denrées, des richesses et de leur emploi ou effet ; tout ce qui sert à l'entretien des besoins des hommes rentre dans cet ordre d’idées. Avec des principes réguliers, chaque chose répondra aux besoins et aux nécessités communes dans une mesure justement appropriée, sans qu'il en résulte de souffrances. En analysant la voie rationnelle qui se déduit de l’idée d'entretenir en nourrissant, qu'il s'agisse d'entretenir la vertu, ou d'entretenir l'univers, rien n'échappe à cette loi géné rale.

\footnotetext{
${ }^{1}$ Montagne est souvent pris comme synonyme de forêt.
} 
TSHOU HI. - Ces deux côtés de la question sont les plus importants pour l'entretien des facultés, ou vertus, et pour celui du corps. 


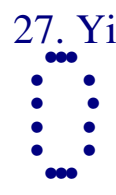

496.

Premier trait nonaire : écartez votre tortue spirituelle; regardez le mouvement de mes mâchoires; présage funeste.

TSHENG TSE. - Le premier trait hexaire du koua mong exprime l'aveuglement de l'ignorance ; la formule s'oc cupe surtout de la dissipation de l'aveuglement; le premier trait nonaire du koua $y i$ emprunte aussi une personnalité étrangère. Le caractère $y i$, votre, se rapporte au premier trait; écartez votre tortue spirituelle, ensuite, regardez-moi et remuez les mâchoires ${ }^{1}$; le mot «moi » est employé pour faire opposition au mot « votre » (vous) ${ }^{2}$. Ce qui fait que le premier trait agite les mâchoires, c'est la vue du quatrième trait, cependant cela n'est pas dit au sujet du quatrième, c'est sous forme de suppositi on explicative par un exemple. La condition que le trait est nonaire exprime que sa substance est la positivité, et que son intelligence est énergique: ses aptitudes et ses facultés mentales sont suffisantes pour entretenir la droiture. La tortue peut avaler de l'eau et respirer, sans manger. «Tortue spirituelle » est une expression qui indique la clarté de ses facultés intellectuelles, et, en même temps, qu'elle n'est pas obligée de chercher sa nourriture et le développement de ses facultés au-dehors d'elle-même. Bien que telles soient ses aptitudes, cependant la condition de positivité faisant partie de la substance du mouvement ${ }^{3}$, et se trouvant dans le moment de l'entretien, ou sustentation, marqué par le koua $y i$, fait que ce trait exprime l'action de rechercher la nourriture ou l'entretien, ce qui est le mobile des passions humaines. En haut il répond sympathiquement au quatrième trait; il ne peut se préserver et se conserver de lui-même ; ses tendances le portent à progresser en montant ; c'est cel ui qui se complaît dans ses passions et qui agite les mâchoires. Du moment où son cour est agité, il est certain qu'il sera lui -même cause de sa propre perte. S'aveuglant par la passion et s'oubliant, étant positif et suivant la négativité, jusqu'où n'en arriverait-il pas ? C'est à cause de cela que le présage est malheureux. Remuer les mâchoires, c'est remuer et agiter les parois de la bouche ; lorsqu'un homme voit de la nourriture et en a envie, il remue les lèvres et l'eau lui vient à la bouche, c'est pourquoi cette expression est prise comme image symbolique.

TSHOU HI. - La tortue spirituelle est un être qui ne mange pas. Le terme toa, antépénultième caractère du texte, veut dire suspendu, ou étendre ; toa yi

\footnotetext{
${ }^{1}$ Cette lecture fausse arbitrairement le sens tel quill résulte de la construction.

${ }^{2}$ Le même caractère $y i$ a aussi le sens de «près de, approcher ».

${ }^{3}$ Du koua simple inférieur tshen.
} 
exprime l'apparence du désir de manger. Le premier trait nonaire est dur, énergique, et se trouve dans une position inférieure ; c'est suffisant pour quil ne mange point. Ensuite, il monte sympathiser avec la négativité du quatrième trait hexaire, et se meut vers ce qu'il désire : c'est une voie logique de malheur, et c'est pourquoi tels sont l'image et le sens divinatoire.

497.

Regarder le mouvement de mes mâchoires; ce n'est encore pas suffisamment estimable.

TSHENG TSE. - Ce trait nonaire fait partie de la substance du koua simple qui symbolise le mouvement ; « remuer les mâchoires » veut dire qu'il se plàît avec la négativité et que ses tendances le portent au mouvement. Du moment où il est agité par ses passions, il en résultera que, bien que possédant les aptitudes et les capacités de l'activité énergique et d'une intelligence lucide, à la fin il se perdra, de sorte que ses aptitudes ne sont encore pas suffisamment précieuses. Ce que l'homme estime dans l'énergie, c'est la faculté qu'elle donne pour se maintenir droit sans se laisser ent raîner par les passions; ce qu'il estime dans l'intelligence, c'est qu'elle permet de tout élucider et de ne point s'écarter de la droi ture. Du moment où il est troublé par les passions et perd la droiture, à quoi lui servent et son énergie et son intelligence? C'est pour cela qu'il est considéré comme méprisable. 


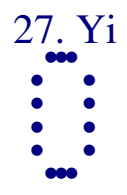

498.

Deuxième trait hexaire : subversion dans l'acte de se nourrir ${ }^{1}$; lier les lois fondamentales ; manger sur une hauteur ; en agissant, présage malheureux.

TSHENG TSE. — La jeune fille ne peut se placer d'elle-même; il faut qu'elle suive le garçon ; la négativité ne peut se maintenir seule, il faut qu'elle suive la positivité. Le second trait est négatif et doux ; il ne peut se nourrir luimême : c'est celui qui attend sa nourritu re d'autrui. Le représentant du ciel 2 prend soin de (nourrit) l'univers ; chacun des grands feudataires nourrit un royaume; les ministres d'un prince sont nourris par la faveur de ce prince ; le peuple compte sur les soins et la nourriture de ses pasteurs : c'est toujours par ce qui lui est supérieur que chacun est nourri et c'est la conséquence stricte de la raison d'être des choses. Du moment où le second trait ne peut pas se nourrir lui-même, il est obligé de demander sa nourriture à l'énergie de la positivité ; si contrairement à l'ordre logique il s'abaisse et cherche à obtenir du premier trait, ce sera considéré comme un cas de subversion de l'ordre logique, et c'est pour cela que la formu le dit : «subversion dans l'acte de se nourrir ». S’il y a subversion, c'est la négation des principes fondamentaux et immuables, et cela ne doit pas avoir lieu. S'il cherche à obtenir sa nourriture sur la hauteur, alors, en y allant, il éprouvera nécessairement des malheurs. Le caractère qui est traduit par le mot hauteur exprime quelque chose d'extérieur et d'élevé ; il désigne le trait supérieur nonaire. Dans le koua, il n'y a que deux positivités; du moment où déjà il ne doit point subversivement se faire nourrir par le premier trait, s'il demande sa nourriture au trait supérieur nonaire, en y allant, il éprouvera des malheurs. Dans le moment marqué par le koua $y i$, ceux qui sympathisent ensemble sont ceux qui se nourrissent mutuellement. Le trait supérieur n'est pas celui qui lui correspond sympathiquement et il va lui demander sa nourriture ; ce n'est pas la voie rationnelle, c'est un mouvement inopportun, et c'est à cause de cela que le présage est malheureux. La subversion comporte la négation des principes fondamentaux ; il n'obtient pas d'être soigné et nourri ; il demande à tort au trait supérieur, donc en y allant il rencontre le malheur. Dans le cas actuel, s'il y a un homme dont les capacités sont insuffisantes pour qu'il se nourrisse lui-même, sill voit que celui qui est dans une position élevée est, par la force naturelle des choses et par sa propre puissance, en état de nourrir les hommes, mais est en même temps d'un autre genre que lui, et si cependant il va à tort lui demander

\footnotetext{
${ }^{1}$ Cette lecture est conforme à l'explication traditionnelle, mais la lecture naturelle du texte conduit difficilement à ce sens.

${ }^{2}$ L'em pereur.
} 
quelque chose, il recueillera de la honte et éprouvera des malheurs ; cela est certain. Le deuxième trait hexaire possède la justice et la droiture; dans d'autres koua c'est le plus généralement un présage heureux et cependant, ici, le présage est néfaste ; comment cela peut-il se faire ? C'est la consé quence spontanée et naturelle du moment. Doux, malléable et négatif, puisqu’il est incapable de se nourrir lui-même, et que le premier trait et le trait supérieur ne s'accordent ni l'un ni l'autre avec lui, il en résulte donc qu'en allant leur demander il viole la raison d'être des choses et rencontre le malheur.

TSHOU HI. - Chercher à obtenir sa nourriture du premier trait serait subversif et contraire aux principes immuables et à la raison d'être des choses ; chercher à l'obtenir du trait supérieur, sera entreprendre et rencontrer le malheur. La hauteur, c'est une élé vation de terrain; c'est l'image symbolique du trait supérieur.

499.

Présage malheureux de l'entreprise du second trait hexaire ; en agissant il perd son propre genre.

TSHENG TSE. - S’il se met en marche et suit le trait supérieur le présage sera malheureux ; c'est parce qu'il n'est pas du même genre. Allant solliciter et s'écartant de son genre, il est naturel qu'il rencontre le malheur. Les mots agir, aller, ou entreprendre sont synonymes.

TSHOU HI. - Le premier trait et le trait supérieur ne sont ni l'un ni l'autre de son genre. 


\section{$27 . \mathrm{Yi}$
$\vdots \vdots$
$\vdots$ \\ 500 .}

Troisième trait hexaire : transgression dans la nourriture; présage malheureux de la perfection ; pendant dix ans ne pas agir ; aucun moyen davantage.

TSHENG TSE. - Dans la voie de la sustentation, ce n'est que par la droiture que le présage est heureux. Le troisième trait, avec ses caractères de malléabilité négative, se place sans justice ni droiture; en outre, il est à l'extrême limite du mouvement ; c'est donc celui qui est mou, pervers, sans droiture, et qui s'agite. Dans de telles conditions, en se nourris sant, il s'écarte de la droite voie rationnelle de l'acte de se nourrir et la transgresse, ce qui rend le présage malheureux. S’il s'accordait à la droiture dans l'acte de se nourrir, le présage serait heureux dans tout ce qu'il nourrirait. Demandant sa nourriture ou nourrissant autrui, ce serait toujours d'accord avec le devoir et, se nourrissant lui-même, il achèverait le développement de ses vertus. Mais, dans ce cas, le troisième trait viole et transgresse la droite voie rationnelle, aussi la formule avertit de rester dix ans sans agir. Dix est la fin du nombre; cela exprime donc qu'on doit rester définitivement sans agir ; rien de ce qui pourrait être entrepris ne serait avantageux.

TSHOU HI. - Malléabilité négative sans droiture; ces qualités étant employées pour se placer au comble du mouvement, il s'agit de la transgression dans l'acte de se nourrir. Puisqu'il y a transgression dans l'acte de se nourrir, même en admettant la droiture, le présage est néfaste, et c'est pourquoi tels sont le sens divinatoire et l'image symbolique.

501.

Pendant dix ans ne pas agir ; voie très subversive.

TSHENG TSE. - Ce qui fait que la formule avertit de rester définitivement sans agir, c'est la voie par laque lle le fait survient ; c'est une profonde subversion du devoir et de la raison d'être des choses. 


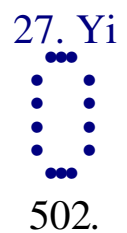

Quatrième trait hexaire : présage heureux de la subversion dans l'acte de la nutrition; le tigre guette en baissant la tête sans regarder; ce qu'il veut, c'est atteindre brusquement par bonds ; pas de culpabilité.

TSHENG TSE. — Le quatrième rang est au-dessus de l'homme ${ }^{1}$; c'est la situation d'un haut dignitaire ; il est occupé avec mollesse par un trait hexaire. La douceur malléable négative est incapable de se nourrir elle-même, à plus forte raison, comment serait-elle capable de nourrir et d'entretenir l'univers ! Le premier trait nonaire emploie sa dureté énergique, positive, et occupe le rang inférieur; c'est le sage placé dans l'infériorité, et il correspond sympathiquement au quatrième. Le quatrième trait, également, est doux, soumis et possède la droiture; il peut donc se soumettre passivement au premier et compte sur lui pour en recevoir sa nourriture. Lorsqu'un supérieur nourrit un inférieur, cela est considéré comme constituant l'ordre régulier et convenable; dans le cas actuel, au contraire, il attend sa nourriture d'un inférieur : c'est l'ordre renversé, ou subversion, c'est pour cela que la formule dit: subversion dans l'acte de la nutrition. Cependant, étant soi-même au-dessous de ses propres attributions, il a recours au sage placé dans l'infériorité et l'écoute avec déférence, pour remédier aux embarras actuels, $\mathrm{d}$ e sorte que l'univers jouit néanmoins des soins qui lui sont nécessaires (nourriture, etc.) et lui-même est exempt de toute culpabilité provenant de la ruine et de la famine des populations, de sorte que le présage est considéré comme heureux. Or, celui qui occupe une situation élevée doit absolument posséder des capacités et des vertus imposantes, afin d'être l'objet de la vénération et de la crainte du bas peuple ; alors les affaires suivent leur cours et tous les cours se soumettent. S'il arrive parfois que l'inférieur méprise le supérieur, il en résulte que les règlements relatifs aux institutions sociales, bien que promulgués, sont violés par les hommes; les lois pénales sont appliquées et les ressentiments prennent naissance. La tendance à la transgression est le canal par où vient le désordre. Bien que le quatrième trait hexaire puisse écouter avec soumission la dureté énergique positive, il n'abandonne cependant point sa propre dignité, mais son caractère étant essentiellement la mollesse négative, il compte sur autrui pour aviser aux soins qu'elle comporte, et c'est là ce que les hommes méprisent facilement, de sorte qu'il doit veiller aux soins de son prestige. En observant attentivement sans en avoir l'air, comme le tigre qui guette, il pourra don ner plus d’importance à sa contenance, de façon que les inférieurs n'osent point le

\footnotetext{
${ }^{1}$ Le troisième rang marque la situation de l'homme, troisième cause efficiente.
} 
traiter avec légèreté. De plus, celui qui suit les conseils d'autrui, doit le faire avec continuité et d'une façon suivie ; s'il n'écoute ces conseils que d'une façon intermittente et sans continuité, les institutions sociales péricliteront. $\mathrm{Ce}$ qu'il veut, c'est -à-lire ce dont il a besoin, ou ce qu'il lui faut employer, c'est nécessairement de poursuivre sans cesse, avec continuité et sans défaillance, et alors les affaires peuvent être réglées et maintenues en ordre. S’il emprunte à autrui, mais sans continuité, il en résulte une ruine finale. Du moment où il sait être imposant, et où, de plus, l'effet de son action ne cesse point, il peut alors être sans culpabilité. La subversion dans l'acte de la nutrition, dans le cas du second trait, comporte la négation des principes fondamentaux ; dans le cas du quatrième, le présage est heureux ; comment cela peut-il être ? Le second trait bien que placé plus haut demande néanmoins sa nourriture à un inférieur ; cet inférieur, par son genre, ne lui est pas sympathique, et c'est pour cela qu'il y a négation des principes fondamentaux. Le quatrième trait occupe une situation élevée ; puisque, quoique noble, il s'abaisse devant l'humi lité, afin que le sage placé dans un rang inférieur agisse à sa place et pratique sa voie, les tendances du supérieur et de l'inférieur se correspondent sympathiquement et étendent leur effet sur les hommes. Qu'y a-t-il d'aussi heureux que ce résultat? Depuis le troisième trait et en descendant, il s'agit de la nourriture du corps au moyen de la bouche; depuis le quatrième et en montant, il s'agit du sens de nourrir et entretenir la vertu. Quand il s'agit d'un prince qui reçoit la nourriture et le secours d'un sujet, d'un homme placé dans une situation élevée qui compte sur les soins d'un inférieur, il est toujours question de l'entre tien (nourriture) et du développement des vertus.

TSHOU HI. - Douceur malléable occupant un rang élevé et possédant la droiture ; celui qui lui est sympathique possède également la droiture, et il compte sur son assistance pour étendre son influence aux inférieurs ; aussi, bien qu'il y ait subversion, le présage est cependant heureux. Le tigre guette et regarde en baissant la tête : infériorité et concentration exclusive du pouvoir ; ce qu'il veut c'est poursuivre en atteignant par bonds : désir d'obtenir et persistance continue. Enfin, s'il est possible de réunir ces conditions, il n'y aura point de culpabilité.

503.

Présage heureux de la subversion dans l'acte de la nutrition ; le supérieur répand l'éclat.

TSHENC TSE. - Il y a subversion et interversion dans l'acte de chercher à obtenir la sustentation, c'est cette subversion qui fait le présage heureux. En effet, il possède les sympathies de l'énergie positive pour veiller au soin des affaires, d'où résulte qu'à lui -même estattribuée la vertu dont il communique 
l'influence, et que son éclat s'étend à tout l'uni vers, c'est ce qu'il y a de plus heureux. 


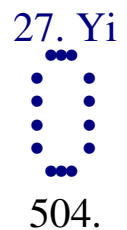

Cinquième trait hexaire : nier les principes fondamentaux; présage heureux du maintien dans la perfection; on ne doit pas traverser un grand cours d'eau.

TSHENG TSE. - Le cinquième trait hexaire, pendant le moment exprimé par le koua yi, occupe la situation du prince ; c'est celui qui soigne et nourrit l'univers. Toutefois, par ses caractères physiques de malléabilité négative, ses aptitudes sont insuffisantes pour qu'il nourrisse et soigne l'u nivers. Au-dessus, se trouve un sage doué de l'énergie positive, aussi il écoute et suit ses conseils, et compte sur lui pour être mis, pas ses soins, en état de remédier aux maux de l'univers. Le prince, est celui qui prend soin des hommes (qui les nourrit) ; c'est donc la négation des principes fondamentaux et l'abandon des règles éternelles. À cause de sa propre insuffisance, il écoute avec docilité les conseils d'un maître doué de sagesse. Le rang supérieur marque la situation du précepteur, ou maître. Il faut nécessairement qu’il se maintienne avec une parfaite fermeté, qu'il s'applique à la conci liation et à la confiance, et alors il pourra, en garantissant sa propre personne, étendre à l'univers son influence bienfaisante, ce qui constitue le présage heureux. Les caractères physiques qui résultent de la malléabilité négative ne comportent pas un naturel énergique dans la perfection, aussi la formule avertit que c'est par là possibilité de se maintenir dans la perfection que le présage sera heureux. Employant les aptitudes de la malléabilité négative, bien quil compte sur l'énergie et la sagesse d'autrui, il peut bien se main tenir dans les temps ordinaires, mais il ne doit pas se placer dans des circonstances difficiles et au milieu de causes de modifications ${ }^{1}$; c'est pour cela que la formule dit qu'il ne doit pas traverser un grand cours d'eau. Les aptitudes de Tsheng Wang n'étaient pas des plus faibles et débiles; cependant, au moment des troubles suscités par Kouan Tsae, il venait précisément d'être privé de la protection de Tsheou Kong. Combien à plus forte raison en est-il de même lorsquil s'agit de gens inférieurs ! Aussi le Shou king dit : «Le roi n'ose pas non plus encore décliner les services de Kong ; il s'appuie sur les deux Kong et leur témoigne jusqu’à la fin sa confiance. » En effet, dans les circonstances difficiles et périlleuses, sans un maître intelligent et énergique, il est impossible de répondre de l'ave nir. Il y a aussi des cas où, sous l'empire d'une nécessité absolue on $\mathrm{p}$ eut remédier à des causes de danger ; développer ce sens, c'est donc donner aux princes un avertissement plein de profondeur. Au sujet du trait supérieur nonaire, il est question de la voie morale du sujet qui pousse jusqu'au sacrifice

\footnotetext{
${ }^{1}$ Les révolutions.
} 
de soi-même son dévouement et sa fidélité, de sorte que le cas considéré est différent.

505.

Présage heureux du maintien dans la perfection; soumission pour écouter le supérieur.

TSHENG TSE. - Présage heureux du maintien dans la perfection, c'est-à-dire de pouvoir, avec fermeté et continuité, écouter le sage représenté par le trait supérieur nonaire, afin de pouvoir veiller aux besoins de l'univers. 


\section{$27.0 \times 1$
$\vdots$
$\vdots$ \\ 506.}

Trait supérieur nonaire : cause de sustentation ; présage heureux du péril ; avantage à traverser un grand cours d'eau.

TSHENG TSE. - Le trait supérieur nonaire emploie les vertus de la dureté énergique, il remplit les fonctions du précepteur qui enseigne. Le prince représenté par le cinquième trait hexaire est doux et déférent et il l'écoute avec soumission ; il attend de lui le soin de sa conservation. C'est là être investi du pouvoir sur l'univers entier et être celui par qui l'univers est soigné. Étant sujet et investi d'une telle fonction, il y a nécessairement lieu d'être dans une appréhension permanente de péril et c'est cette appréhension qui rendra le présage heureux. Ainsi, comment Yi Yin et Tsheou Kong n'auraient-ils pas été constamment préoccupés et actifs à prévenir le danger ? C'est ce qui fit que finalement ils furent heureux. Mais, puisque les aptitudes du prince sont insuffisantes et que celui-là compte sur lui, il est personnellement investi de l'autorité sur l'univers ; il a lieu d'exercer toutes ses facultés et d'y consacrer toute sa force, afin de remédier aux difficultés que traverse l'univers, et d'achever l'établissement du règne de l'ordre et de la paix. C'est pour cela que la formule dit : avantage à traverser un grand cours d'eau. Possédant ainsi la toute-puissance, investi d'une fonction aussi importante, s'il ne pare pas aux périls qui menacent l'univers, comment serait-il digne de la confiance qui lui est accordée et comment pourrait-il être appelé sage ? Il convient qu’il épuise la sincérité, quill développe tous ses efforts, sans regarder en arrière et sans être retenu par l'appréhension, mais en pensant constamment au péril ; c'est ainsi qu'il pourra ne pas succomber.

TSHOU HI. - Le cinquième trait hexaire compte sur les soins du trait supérieur nonaire, afin de pourvoir aux soins que réclament les hommes ; c'est donc par le trait supérieur nonaire que tous les êtres sont soignés et conservés. La situation est élevée, la charge est grave, ainsi la préoccupation du péril est un présage heureux. La dureté énergique est au rang supérieur, aussi il y a avantage à traverser un grand cours d'eau.

507.

Présage heureux de la crainte du péril chez celui qui pourvoit à la sustentation; il y a grande félicité. 
TSHENG TSE. - Si celui qui, comme le trait supérieur nonaire, est chargé d'une grave responsabilité peut être attentif au danger et craindre le péril, comme lui, l'univers entier est imprégné par l'influence de ses vertus, ce qui constitue le bonheur et la félicité. 


\section{Tae kuo}

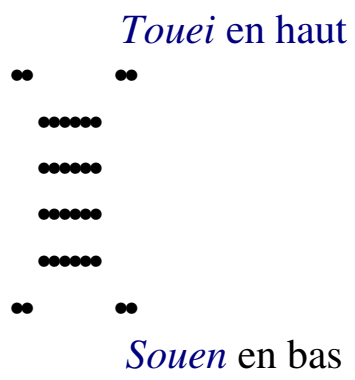

508. Excès de grandeur; L'arête du toit faiblit ; avantage dans ce qu'il y a à entreprendre ; liberté d'action.

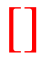

514. Premier trait hexaire : tapisser en employant du chaume blanc; pas de culpabilité.

$*$

516. Deuxième trait nonaire : le saule sec pousse des bourgeons; le vieillard possède ses filles et son épouse ; rien sans avantage.

$*$

518. Troisième trait nonaire : arête recourbée du toit ; Présage de malheur. $*$

520. Quatrième trait nonaire : présage heureux de l'éclat du faîte ; il y a d'autres appréhensions.

$*$

522. Cinquième trait nonaire : le saule desséché pousse des fleurs; la vieille épouse possède le jeune époux; pas de culpabilité, pas de louanges.

$*$

524. Trait supérieur hexaire : traverser en nageant et submerger le sommet du crâne ; présage malheureux; pas de culpabilité. 
28. Tae kuo.

TSHENG TSE. - Tae kuo. «L'Ordre des koua» dit: «Le koua yi exprime la nutrition; sans nutrition le mouvement est impossible, aussi est-il suivi du koua tae kuo.»Ce n'est que par la nutrition que tous les êtres peuvent se développer et s'achever; une fois développés, ils peuvent se mouvoir ; quand ils se meuvent il doit y avoir excès ${ }^{1}$ : c'est là ce qui fait que le koua tae kuo suit immédiatement le koua yi. Comme koua, il est formé du koua simple touei, en haut, et du koua simple souen, en bas. Le marais (touei) est placé au-dessus de l'arbre (souen); il détruit l'arbre. Le marais arrose et nourrit l'arbre ; ici, son effet va jusqu'à détruire cet arbre, qu'il submerge, ce qui constitue le sens de « grand excès » exprimé par les mots tae kuo. L'excès dans la grandeur, c'est l'excès dans la positivité, c'est pourquoi ce koua exprime l'excès dans la grandeur, la grandeur de l'excès, et l'excès de grandes choses. La vertu de la voie, ou vertu morale, les capacités dépassant en grandeur ces mêmes capacités chez les autres hommes, toute chose qui dépasse en grandeur la moyenne ordinaire se trouve toujours comprise dans la portée de l'expression tae kuo. Or, l'homme saint épuise la voie morale de l'homme, mais sans en dépasser la limite raisonnable. Quand il statue sur quelque chose, c'est d'après l'exacte raison d'être de l'univers. L'usage mal à propos, ou dépassant quelque peu lajuste mesure, est un cas qui peut se présenter ; ainsi l'excès de respect dans la contenance, l'excès de dou leur dans le deuil, l'excès d'économie en sont autan $t$ d'exemples. En effet, le léger excès, ou abus, qui peut ensuite être ramené à la juste mesure, constitue précisément l'effet de la recherche du juste milieu. Ce qu'on appelle grand excès, c'est la grandeur dans les choses ordinaires, sans qu'il y ait exc ès par rapport à la raison d'être de l'objet. C'est simplement parce que ces choses sont grandes qu'elles ne sont plus considérées comme ordinaires, c'est parce que, comparées aux choses ordinaires, on les voit plus grandes, qu'on les appelle trop grandes. Ainsi, l'abdication de Yao et la récusation de Shouen, Wou abandonnant et Sheang repoussant le trône, sont des exemples également inspirés par la morale ${ }^{2}$. La voie morale (tao) a un juste milieu; elle est constante ; c'est parce que les hommes d'une épo que ${ }^{3}$ ne la voient point constamment qu'ils disent qu'elle est plus grande, que la limite ordinaire.

\footnotetext{
1 Le sens de «trop, excès », donné au caractère kuo, est un sens dérivé. Kuo signifie essentiellement «traverser, passer, dépasser ». En se référant au sens propre des mots on lira « les êtres se mouvant doivent passer, ou traverser». Ce qui selon moi, se rapporte à la lune, nouvelle d'abord, croissant ensuite, et passant enfin dans l'autre hémisphère.

${ }^{2}$ Littéralement : « résultant de la voie ». Dans l'hypothèse que j'avance, on peut lire « sont des symboles de phénomènes de la marche du soleil et de la lune autour de la terre. »

${ }^{3}$ Littéralement « de l'époque, ou période actuelle ».
} 


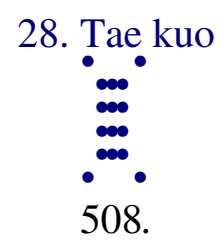

Excès de grandeur ; L'arête du toit faiblit ${ }^{1}$; avantage dans ce qu'il y a à entreprendre ; liberté d'action.

TSHENG TSE. - Dans le koua siao kuo, la négativité perce en haut et en bas ; dans le koua tae kuo, la positivité est en excès au milieu. La positivité est en excès au milieu, tandis que le haut et le bas sont faibles. C'est ce qui constitue l'image symbolique de l'arête du toit qui faiblit. Le chevron du faite du toit est pris comme représentation de quelque chose qui supporte un poids considérable. Les quatre positivités se groupent au centre

c'est là ce qu'on peut appeler un poids considérable. Chacun des troisième et quatrième traits nonaires prend également l'image symbolique de l'arête du toit, c'est-à-dire qu'ils supportent un fardeau considérable. Dans le terme « faiblir » on dépeint la condition que les extrémités sont faibles, le milieu est fort mais les extrémités sont faibles, c'est pour cela qu'elle faiblit. La négativité est faible et la positivité est forte ; l'homme doué florit, l'homme inférieur décline, de sorte qu'il y a avantage dans ce qui est à entreprendre, et liberté d'action. Le caractère ting, du texte, exprime ce qu'on appelle aujourd'hui lin : «pièce de bois horizontale sur le faite de la maison » ${ }^{2}$.

TSHOU HI. - La grandeur, c'est la positivité ; quatre positivités occupent le centre et l'expansion de leur développement est excessif, de sorte que le koua est considéré comme exprimant l'excès de grandeur. En haut et en bas, deux négativités ne peuvent supporter l'effort de ce poids considérable, de sorte qu'il y a l'image symbolique de l'arête du toit faiblissant. De plus, puisque, bien que les quatre postivités soient en excès, le second et le cinquième trait possèdent la justice, qu'au-dedans il y a humilité (souen), et au-dehors satisfaction (touei), il y a cependant une voie praticable, de sorte qu'il y a avan tage dans ce qui est à entreprendre et qu'on jouit de la liberté d'action.

509.

\footnotetext{
${ }^{1}$ On peut lire aussi : « l'arête du toit est déjetée, ou faible ».

${ }^{2}$ Tse tien.
} 
Le commentaire traditionnel de la formule déterminative dit : Excès de grandeur; ce qui est grand est en excès ${ }^{1}$.

TSHENG TSE. - Ce qui est grand est en excès, dans les choses c'est leur grandeur dépassant la limite ordinaire, ainsi que la grandeur dans l'excès.

TSHOU HI. - Explication du sens du nom du koua au moyen de la substance du koua.

510.

L'arête du toit faiblit ; l'origine et l'extrémité sont faibles.

TSHENG TSE. - C'est -à-dire que les deux négativités, en haut et en bas, sont faibles et débiles. La positivité s'achevant, la négativité dé cline, de sorte que c'est considéré comme exprimant que la grandeur est en excès. Dans le koua siao kuo la formule dit: «ce qui est petit est en excès »; excès de négativité.

TSHOU HI. - Nouvelle explication de la formule du koua au moyen de la substance de ce koua. L'origine, c'est le premier trait ; l'extrémité, c'est le trait supérieur. La faiblesse désigne la négativité.

511.

Excès dans la dureté énergique et justice; humilité agissant avec satisfaction. Avantage dans ce qu'il y a à entreprendre, donc liberté d'action.

TSHENG TSE. - Il s'agit du bien dans les aptitudes exprimées par ce koua. Bien que la dureté énergique soit en excès, cependant, chacun des second et cinquième traits possède la justice : c'est donc quils se placent sans s'écarter de la voie morale (tao). En bas humilité, en haut satisfaction : c'est donc agir en suivant la voie de l'humilité, de l'accord, et de la satisfaction. Dans le moment exprimé par le koua tae kuo, employer la voie de la justice et agir avec humilité et satisfaction ; c'est pour cela qu'il y a avantage dans ce qui peut être à entreprendre, et c'est par là qu'il y a liberté d'action.

\footnotetext{
1 Ou : « grand, il traverse ».
} 
TSHOU HI. - Encore une explication de la formule du koua au moyen de la substance du koua lui-même, et des vertus des koua simples.

512.

Que le moment de l'excès de la grandeur est grand!

TSHENG TSE. - Dans le moment de l'excès de la grandeur, les choses sont extrêmement grandes, aussi la formule est une exclamation : "Qu'il est grand! » Par exemple lorsqu'il s'agit d'étab lir quelque chose d'important et qui n'est pas ordinaire, de poser les fondements d'une auvre qui doit durer des siècles, d'acquérir une grande vertu qui dépasse le niveau actuel des moaurs, ce sont toujours des cas d'excès dans la grandeur.

TSHOU HI. - Dans les moments d'excès de la grandeur, sans des aptitudes plus grandes que celles des hommes ordinaires, il est impossible de parer aux événements. C'est pour cela que la formule s'exclame admirativement sur la grandeur.

513.

Le commentaire de la formule symbolique dit: Le marais détruit l'arbre; excès de grandeur. L'homme doué demeure seul sans craindre l'isolement ; il fuit le monde sans chagrin.

TSHENG TSE. - Le marais est ce qui nourrit et abreuve les arbres. Dans le cas actuel, son action va jusqu'à détruire et à submerger l'arbre ; donc l'excès est considérable. Aussi, ce cas est considéré comme exprimant l'excès dans la grandeur. L'homme doué regarde l'image symbolique de l'excès de grandeur; il agit dans ses actions en se montrant plus grand que le reste des hommes. Ce qui fait que l'homme doué est plus grand que les autres hommes, c'est qu'il peut se maintenir seul sans crainte, et fuir le monde sans chagrin. L'univers ne fait aucun cas de lui et le traite sans considération : il se maintient seul et sans rien craindre ; il sait que son siècle ne le remarque point, ne le connaît point, et il n'en a pas de regrets, il fuit le monde sans chagrin. Étant ainsi, il peut par suite s'observer, et c'est par là qu'il est plus grand que le reste des hommes. 
TSHOU HI. - Le marais détruit l'arbre : image symbolique de l'excès dans la grandeur. Ne pas craindre, ne pas éprouver de chagrin, c'est l'effet de l'excès de grandeur. 


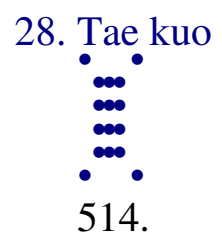

Premier trait hexaire : tapisser en employant du chaume blanc; pas de
culpabilité.

TSHENG TSE. — Le premier trait emploie la douceur de la négativité ; sa substance est l'humilité, et il est placé dans l'infériorité, c'est celui qui est trop circonspect et trop craintif. Employant la négativité et étant dans l'infériorité, il donne l'image symbolique de l'emploi du chaume pour tapisser quelque chose. Ne pas reposer sur la terre et la tapisser de chaume c'est un excès de précaution et c'est à cause de cela qu'il est sans culpabilité.

Bien que le chaume soit une matière commune, il peut cependant devenir précieux par son emploi ; en l'employant, il est possible d'accomplir la voie de la circonspection et du respect. En observant scrupuleusement cette manière d'agir, comment manquerait-on à aucun devoir ? Tel est l'effet de l'excès dans la grandeur. Les «Formules annexées» disent: "Sil est possible de placer sur la terre, et qu'on la garnisse cependant avec du chaume, où serait la faute? C'est le comble de la circonspection. Mais le chaume est une matière grossière et son emploi peut être important; en observant scrupuleusement cette manière d'agir en toute occasion elle ne peut constituer un manquement. » Cela exprime l'extrême circonspection respectueuse. Bien que le chaume soit une matière des plus communes, son emploi peut être de la plus grave importance. En s'en servant pour garnir les objets présentés en offrande, son emploi constituera la voie de la considération circonspecte et attentive, et c'est là l'importance de son emploi. L'excès dans le respect attentif n'est pas, pour l'homme, une chose difficile et cependant il peut assurer son repos et lui faire éviter les erreurs. Si on peut apporter de l'attention à suivre cette voie, l'étudier et l'appliquer aux actions, il n'y aura jamais de fautes ni d'erreurs commises.

TSHOU HI. - Au moment de l'excès dans la grandeur, employant la douceur malléable de la négativité et demeurant dans l'humilité et l'infériorité, c'est un excès de circonspection respectueuse sans fautes ni erreurs. C'est pour cela que tels sont l'image sym bolique et le sens divinatoire. Le chaume blanc est une matière propre et nette. 
Tapisser en employant du chaume blanc; douceur malléable dans l'infériorité.

TSHENG TSE. - Dans la voie de l'emploi de la douceur de la négativité et dans une situation humble et inférieure, il convient uniquement de dépasser les bornes ordinaires de la considération attentive et respectueuse. Puisqu'il s'agit de douceur malléable pla cée dans l'infériorité, cela constitue liimage symbolique de l'emploi du chaume pour garnir et tapisser, voie de circonspection respectueuse et attentive. 


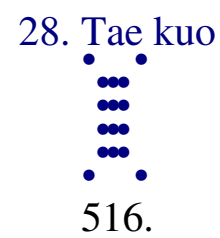

Deuxième trait nonaire : le saule sec ${ }^{1}$ pousse des bourgeons ${ }^{2}$; le vieillard possède ses filles et son épouse ${ }^{3}$; rien sans avantage.

TSHENG TSE. - L'excès de la grandeur dans la positivité étant en contact avec la négativité, il y aura union ; aussi le second trait et le cinquième ont tous deux le sens symbolique d'engendrer. Le second trait nonaire en présence du commencement de l'excès dans la grandeur, se conforme à la justice et se maintient dans la douceur; il se rapproche secrètement du premier trait et tous deux s'accordent et s'allient. Du moment où le premier trait est extrêmement serré contre le second, celui-ci ne conserve plus de sympathie pour le trait supérieur ; l'alliance entre eux est évidente ; c'e st l'homme doué d'une énergie excessive, qui peut se placer d'après la justice, employer la douceur, et ces qualités diverses se contrebalancent. Par l'excès d'énergie il serait impossible d'arriver à aucun résultat ; c'est le cas du troisième trait. Possédant la justice et employant la douceur, il est possible d'accomplir l'ouvre de l'excès dans la grandeur : c'est le cas du second. L'arbre yeang (sorte de saule ?) est une chose facilement influencée par l'éther positif ${ }^{4}$; par un excès de positivité, il se dessèche ${ }^{5}$. Un saule (yeang) desséché, qui pousse de nouveau des racines ; la positivité est excessive, mais elle n'est pas encore arrivée à la dernière limite. Chez le second trait nonaire, la positivité est excessive et il s'allie avec le premier; c'est l'image symbolique du vieillard qui possède une jeune épouse. Le vieillard qui possède une jeune épouse pourra accomplir l'ouvre de procréation. Le second trait se conforme à la justice, il se maintient dans la douceur, et il s'allie avec le premier, aussi il peut repousser de nouvelles racines, sans inconvénient résultant de l'extrême excès ; rien n'est sans avantage. Dans l'excès de la grandeur un trait positif se maintenant dans la douceur ${ }^{6}$ représente le bien ; c'est le cas du second et du quatrième formule annonce que rien n'est sans avantage, il n'y a pas encore lieu de parler hâtive ment de présage heureux. Le terme $t h i$, sixième caractère du texte, désigne les racines ${ }^{7}$; Lieou Kouen dans

\footnotetext{
${ }^{1}$ Yeang ; nom d'un arbre que le Tse tien décrit en le comparant au saule, branches longues, flexibles, agitées par le moindre vent. (Quamoclit, Taberd).

${ }^{2}$ Les commentateurs lisent « racines »; le terme est vague, mais il exprime la croissance des tiges et non celle des racines.

${ }^{3}$ Les commentateurs lisent : «La jeune épouse »; cela n'est pas la traduction du texte.

${ }^{4}$ Les premières effluves du printemps.

${ }^{5}$ Par les sécheresses de l'été.

${ }^{6}$ Dans un rang négatif.

${ }^{7}$ Thi, le chaume nouvellement poussé (Wou feang).
} 
son «Guide de l'exhortation au progrès» dit: «faire naître des fleurs abondantes sur des herbes sèches (thi)», c'est-à-dire sur des racines desséchées. Tsheng Kang Tsheng, dans son commentaire sur le Yi king emploie aussi l'autre caractère thi, ou celui du texte, l'un pour l'autre.

TSHOU HI. - Commencement de l'excès dans la positivité et contact avec la négativité du premier trait, de sorte que tels sont l'image symbolique et le sens divinatoire. Thi, racines; exubérance de sève par en bas. Se développant par en bas, la plante renaîtra par en haut. L'époux quoique vieux, possède une jeune épouse, il peut encore engendrer.

517.

Vieil époux, jeune épouse ; excès dans leur alliance.

TSHENG TSE. — L'amour du vieillard pour la jeune fille, la soumission de la jeune fille au vieillard, leur accord mutuel dépassent les bornes ordinaires comportées par leurs conditions respectives. Cela veut dire que l'harmonie qui existe dans l'accord mutuel entre le second trait nonaire et le premier trait hexaire, entre la négativité et la positivité, est plus grande que la mesure ordinaire. 


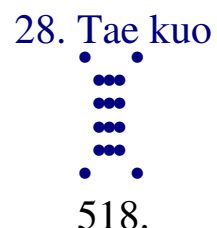

Troisième trait nonaire : arête recourbée du toit ; Présage de malheur.

TSHENG TSE. - Or, se trouver dans un moment d'excès dans la grandeur, fonder une ouvre d'une grandeur extraordinaire, instituer quelque chose d'une importance dépas sant le degré commun, c'est toujours une impossibilité sans un juste équilibre entre l'énergie et la douceur et sans emprunter l'alliance et l'aide d'autrui. S'il y a excès dans la fo rce dure et énergique, il est impossible d'agir ensemble avec d'autres hommes ; même lorsqu'il s'agirait de choses très ordinaires, il serait encore impossible d'en venir à bout seul et de les mettre à exécution; combien donc, à plus forte raison, lorsqu'il s'agit d'affaires d'une importance plus qu'ordinaire ! Bien que possédant les aptitudes de la sainteté, alors même qu'il ne s'agit que de petites choses, il faut encore emprunter à autrui ; en présence du grand fardeau de l'univers, c'est donc encore pl us évident. Le troisième trait nonaire emploie la positivité comportée par l'excès dans la grandeur ; en outre, il emploie la dureté énergique pour se maintenir et il ne se conforme pas à la justice : cela indique l'extrême degré dans l'excès de l'énergie. Employant avec un tel excès la dureté énergique, en se mouvant, il ira contre la concorde et la justice et il mécontentera le cour de la foule ; comment pourrait-il soutenir le fardeau de l'excès de sa grandeur ? Aussi, il n'est pas capable de supporter le poids de ce fardeau; il est comme l'arête d'un toit qui s’incurve et entraîne la déviation et la ruine de la maison; c'est à cause de cela que le présage est malheureux. Ce qui fait que l'arête du toit est prise pour symbole, c'est qu'il est sans assistance et qu'il ne peut supporter le lourd fardeau dont il est chargé. On a dit : le troisième trait fait partie de la substance de l'humilité ${ }^{1}$ et il correspond sympathiquement au trait supérieur; pourquoi ne pas relever l’image symbolique de douceur ? Lorsqu'on raisonne sur le Yi king, il importe avant tout de savoir reconnaître le plus ou moins dimportance des conditions indiquées, et les modifications ou changements comportés par le moment. Le troisième trait comporte le sens d'excès par la position qu'il occupe ${ }^{2}$ et il remplace la dureté énergique; l'humilité étant à sa fin ${ }^{3}$ et d'ailleurs se modifiant, comment pourrait-on encore employer le sens de douceur malléable? La correspondance sympathique ou sympathie, indique la communauté de tendances ; or, le troisième trait représente le début de l'excès

\footnotetext{
${ }^{1}$ Koua simple souen.

2 Trait nonaire, rang impair ; excès de positivité.

${ }^{3}$ Dernier rang du koua simple souen.
} 
dans l'énergie ; le trait supérieur est-il donc en état de contenir cette tendance ?

TSHOU HI. - Le troisième et le quatrième traits occupent à eux deux le milieu du koua ; image symbolique du chevron de l'arête du toit. Le troisième trait nonaire emploie la dureté énergique et se tient dans une position qui comporte la dureté énergique; il est incapable de supporter le poids de son fardeau, aussi le symbole est la courbure, ou dépression, et le sens divinatoire est néfaste.

519.

Présage malheureux de la courbure de l'arête du faîte; impossibilité d'obtenir assistance.

TSHENG TSE. - Excès de dureté énergique et de violence, donc, impossibilité d'obte nir quoi que ce soit d'autrui ; inversement personne ne peut se rapprocher de lui pour l'aider. Il est le chevron du faîtage plié et craqué, et impossible à réparer. Le chevron du faîtage se trouve juste au milieu de la maison; il est impossible de le réparer; cela exprime donc l’impossibilité de rec evoir de l'assistance. 


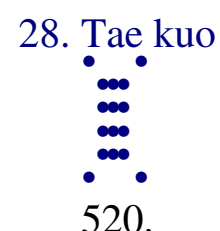

Quatrième trait nonaire : présage heureux de l'éclat du faîte ${ }^{1}$; il y a d'autres appréhensions.

TSHENG TSE. — Le quatrième trait est proche de la situation du prince ; c'est celui qui se trouve présentement chargé du poids de l'excès de grandeur. L'occupation d'un rang qui comporte la douceur est considérée comme constituant l'aptitude à employer la douceur de telle sorte que les deux qualités s'équilibrent entre elles. Puisqu'il n'y a pas excès de dureté énergique, il y aura possibilité d'être à la hauteur de la tâche qui lui incombe, comme lorsqu'un pilier est dressé debout ; c'est par là qu’il y a un présage heureux. Le terme long, quatrième caractère du texte, signifie «dresser, mettre debout »; la formule choisit le sens de «ne pas plier en sincurvant ». Dans le moment de l'excès de grandeur, sans la dureté énergique positive, il est impossible d'être à la hauteur des circonstances ; employer la dureté énergique en se plaçant sur le terrain de la douceur est considéré comme atteindre au niveau convenable. Si, de plus, il sympathisait encore avec la négativité du premier trait hexaire, ce serait un excès. Puisque l'énergie et la douceur s'allient au degré convenable, et que de plus ses tendances sympathisent encore avec la négativité, c'est ce qu'on appelle avoir des arrière-pensées. Ayant des arrière-pensées, c'est qu'il est porté à la dureté énergique; bien qu'il n'en résulte pas encore un mal considérable, c'est cependant un sujet d'appréhensions ${ }^{2}$. En effet, dans le moment de l'excès dans la grandeur, le mouvement constituera un excès. Les caractères [.] signifient «avoir encore d'autres tendances $»^{3}$. Le dernier caractère du texte est considéré comme exprimant le sens d'insuffisance, c'est-à-dire «possibilité d'insuffisance, ou de diminution ». On a dit: Le second trait s'associant au premier, il en résulte que rien n'est sans avantage ; ici le quatrième sympathise avec le premier, c'est considéré comme constituant un motif d'appréhension ; comment cela peut-il être ? Le second se conforme à la justice et s'asso cie au premier, cela constitue le sens d'employer la douceur pour équilibrer une qualité opposée ; le quatrième et le premier sont ceux qui se correspondent sympathiquement par la droiture; ce sont ceux que leurs tendances relient et attachent l'un à l'autre. Un trait nonaire occupant le

\footnotetext{
${ }^{1}$ Les commentateurs lisent « pilier dressé »; pour arriver à ce sens il faut donner au caractère long un sens qu'il n’a pas et lui faire jouer le rôle de qualificatif du mot précédent, ce qui est incorrect.

${ }^{2}$ Le commentateur scinde le dernier membre de phrase en deux.

${ }^{3}$ C'est absolument arbitraire, ces deux caractères, lus séparé ment, signifient « avoir autre » et ne donnent pas un sens complet.
} 
quatrième rang, la dureté énergique et la douceur sont en proportion convenable; si, par surcroît il est retenu et attiré vers la négativité au détriment de la dureté énergique, il y aura lieu d'avoir de l'appréhension.

TSHOU HI. - Employer la positivité pour se maintenir dans la négativité, c'est dépas ser le niveau ordinaire sans commettre d'excès, c'est pour cela que le symbole est la splendeur florissante et que le sens divinatoire est un présage heureux. Toutefois, en bas, il sympathise avec le premier trait, il emploie la douceur pour contrebalancer sa propre qualité, donc il y a excès de douceur. C'est pour cela que la formule avertit encore que s'il y a d'autres arrière-pensées, ce sera un motif d'appréhension.

521.

Présage heureux de l'éclat du faîte, il ne s incurve pas vers le bas.

TSHENG TSE. - Ce faîte s'élevant resplendissant, le présage est heureux ; il ne se plie et ne se déprime pas vers le bas, c'est-à-dire qu'il n'est pas attiré et retenu par le premier trait. 


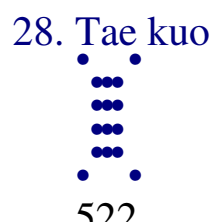

522.

Cinquième trait nonaire : le saule desséché pousse des fleurs; la vieille épouse possède le jeune époux; pas de culpabilité, pas de louanges.

TSHENG TSE. - Le cinquième trait en présence du moment de l'excès dans la grandeur emploie essentiellement la justice et la droiture pour se maintenir dans la situation prééminente. Cependant, en bas, il ne rencontre ni aide, ni sympathie ; il ne pourra certainement pas achever l'ouvre de l'excès de la grandeur, et, au-dessus de lui, il s'associe à la négativité poussée au plus extrême excès, avec laquelle ses propres facultés s'équili brent. Il en est de lui comme de l'arbre yeang, desséché, engendrant des fleurs. L'arbre yeangdesséché émet des racines par en bas, de sorte qu'il peut revivre. Il en est de même de la positivité dans l'excès de la grandeur ; elle commence et accomplit son auvre. Si le haut de l'arbre éme t des fleurs et reverdit, bien que ces bourgeons puissent éclore, cela ne remédie point à son état de dessèchement. Le trait supérieur hexaire exprime la négativité à l'excès le plus extrême ; c'est la vieille épouse. Le cinquième trait, bien que n'exprim ant pas la jeunesse, comparé à la vieille épouse est néanmoins considéré comme dans la force de l'âge. Il n'a rien à attendre du cinquième trait, aussi, la formule dit, au contraire, que c'est l'épouse qui possède. La négativité parvenue à l'extrême limite de l'excès, jouit de l'effet compensateur de la positivité ; cela ne peut être considéré comme sans avantage. Pour un époux dans la force de l'âge, bien que posséder une vieille épouse ne constitue pas un cas de culpabilité et une faute, c'est encore un c as étrange et qui n'a rien de beau, aussi la formule dit: «pas de culpabilité, pas de louanges ». Le commentaire symbolique revient sur le côté blâmable de cette situation insolite.

TSHOU HI. - Le cinquième trait nonaire exprime le plus extrême degré de l'excès de la positivité, de plus il s'associe à la négativité également arrivée à la plus extrême limite de l'excès, c'est pourquoi le symbole et le sens divinatoire sont tous deux inverses de ceux du deuxième trait.

523.

Le saule desséché pousse des fleurs; comment cela pourrait-il durer ?Vieille épouse, époux dans la force de l'âge; c'est encore méprisable. 
TSHENG TSE. - L'arbre yeang desséché ne pousse pas de racines et pousse des fleurs ; il se desséchera de nouveau ; comment pourrait-il durer ? Une vieille épouse possédant un époux dans la vigueur de l'âge ; comment pourrait-elle accomplir l'auvre de la procréa tion? De plus, c'est encore quelque chose de méprisable. 


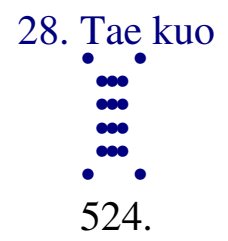

Trait supérieur hexaire : traverser en nageant et submerger le sommet du crâne ; présage malheureux; pas de culpabilité.

TSHENG TSE. — Le trait supérieur hexaire emploie la douceur malléable négative et se place au point culminant; c'est l'homme inférieur atteignant l'extrême limite, en dépas sant les règles ordinaires. Ce qu'on appelle l'excès de la grandeur chez l'homme inférieur, ce n'est pas de pouvoir faire des choses plus grandes que les autres hommes ; c'est s implement dépasser les règles ordinaires et outrepasser les bornes de la raison d'être des choses. Il ne se soucie pas du péril et de la perte finale; il ne fait que fouler aux pieds les dangers et s'aventurer sur un sol miné par le malheur. C'est comme s'aventurer trop en marchant dans l'eau, jusqu'à en arriver à la submersion et à la disparition du sommet du crâne. Le présage malheureux est évident. L'homme inférieur, par son ardeur irréfléchie est la cause de son propre malheur, et c'est en effet nature 1 ; à qui pourrait-il encore s'en prendre ? C'est pour cela que la formule dit : «pas de culpabilité »; cela exprime qu'il est lui même l'auteur de son mal, et il ne peut en imputer la faute et la culpabilité à personne ${ }^{1}$. C'est à cause de l'image symbo lique du marais que la formule choisit le sens de marcher dans l'eau ${ }^{2}$.

TSHOU HI. - Il est placé sur le terrain de l'extrême limite de l'excès ; ses aptitudes sont faibles, insuffisantes pour remédier aux dangers de la position, mais toutefois, pour le sens, c'est considéré comme exprimant l'absence de culpabilité. En effet, c'est perdre la voie pour accomplir un acte d'humanité, et c'est pourquoi tels sont l'image symbolique et le sens divinatoire.

525.

Présage malheureux de marcher trop loin dans l'eau; ne paspouvoir en faire un sujet de culpabilité.

\footnotetext{
${ }^{1}$ Singulier moyen de faire cadrer le texte avec le raisonnement adopté !

${ }^{2}$ Souen, marais.
} 
TSHENG TSE. - Marcher trop loin dans l'eau jusqu'à se noyer ; c'est le résultat de son propre fait, on ne doit pas considérer ce cas comme un crime ; cela veut dire qu'il ne peut en vouloir à personne. 


\section{Khan}

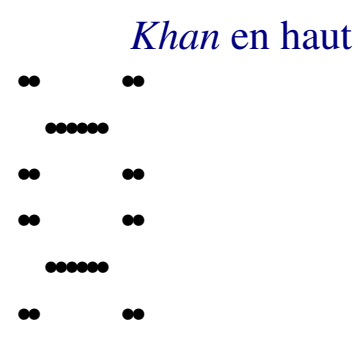

Khan en bas

526. Répétition de la chute ; avoir foi ; le cour seul jouit de liberté ; l'action est estimable.

532. Premier trait hexaire : répétition du péril ; entrer dans la fosse de l'abîme ; présage malheureux.

$*$

534. Deuxième trait nonaire : la chute est périlleuse ; demander et obtenir un peu.

$*$

536. Troisième trait hexaire : en venant ou en allant, nombreux périls ; être dans l'embarras et s'envelopper la tête ; entrer dans le gouffre de l'abîme; ne pas agir.

*

538. Quatrième trait hexaire : urne, vin, plateau à offrandes; dans les deux cas employer la poterie; venir se joindre par la fenêtre ; finalement pas de culpabilité.

$*$

540. Cinquième trait nonaire : l'abîme ne se remplit pas ; quand le fond est nivelé, pas de culpabilité.

542. Trait supérieur hexaire : attacher en employant une bonne corde ; placer dans un buisson d'épines ; trois ans sans réussir ; présage malheureux.

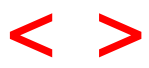




\section{Khan.}

TSHENG TSE. - Répétition du koua simple khan. «L'Ordre des koua » dit : «Les êtres ne peuvent pas indéfiniment pousser les choses à l'excès, c'est pourquoi le koua tae kuo est suivi du koua khan ; khan, tomber, chute. » La raison d'être naturelle n'admet pas l'excès sans une limite inévitable ; l'excès poussé à un point extrême, il y a nécessairement chute, et c'est la raison qui fait que le koua khan suit le koua tae kuo. Répétition indique l'usage réitéré ; dans les autres koua, alors même qu’il y a répétition, elle n'est pas men tionnée; ce n'est que dans le cas du koua khan qu'elle est relevée, afin de manifester la répétition du péril. Dans le péril lui-même il y a un autre péril ; le sens est vaste. Au milieu du koua simple il y a une positivité, au-dessus et au-dessous, deux négativités. La positivité indique la réalité, la négativité marque le vide, le néant, l'incertain. En haut comme en bas, sans distinction, une positivité unique tombe entre deux négativités, ce qui est considéré comme exprimant le sens de péril et de chute. Lorsque la positivité demeure au milieu de négativités, ce doit être considéré comme exprimant la chute ; lorsque la négativité se trouve placée au milieu de positivités, ce doit être considéré comme indiquant l'adjonction et le rapprochement. Toutes les fois que c'est la positivité qui est au-dessus, il y a l'image symbolique d'arrêt ; si elle est au milieu, c'est l'image symbolique d'une chute ; si elle est au-dessous, c'est l'image symbolique de l'ébranlement ou mouvement. Si la négativité est au-dessus, il y a image symbolique de séduction ou satisfaction ; si elle est au milieu, c'est le symbole du rapprochement, enfin, si elle est au-dessous, il y a image symbolique de l'humilité. S’il y a chute, il doit y avoir péril. La répétition c'est la pratique réitérée d'un même acte, comme lorsqu'il s'agit de l'exercice répété dans l'étude ; être rompu à un exercice répété ; c'est toujours le sens de renouvellement fréquent d'un même acte. Khan exprime la chute; ce qu'exprime le koua, c'est la voie rationnelle à suivre lorsqu'on est placé dans le danger et les difficultés. Le koua simple khan représente l'eau ; l'unité commence au milieu, c'est la première origine de la naissance et de la vie, et c'est pour cela quill est considéré comme représentant l'eau ; la chute c'est l'état inhé rent à l'eau. 


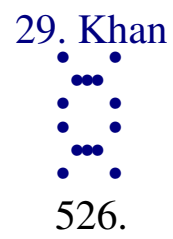

Répétition de la chute ; avoir foi ; le cour seul jouit de liberté ; l'action est estimable.

TSHENG TSE. — La réalité positive est au milieu, c'est considéré comme exprimant qu'au-dedans il y a bonne foi et confiance. Le cour seul jouit de liberté, le coaur seul est parfaitement sincère, de sorte qu'il peut être libre. La parfaite sincérité est capable de traverser le métal et la pierre, de marcher à travers l'eau et le feu ; quelle difficulté, quel péril ne traverserait-elle pas librement ? L'action est estimable, c'est -à-dire que c'est par l'emploi de la plus parfaite sincérité que l'action est dirigée, il y aura donc possibilité de sortir du péril, donc il y a lieu de louer et d'estimer, c'est -à-dire qu'il y a mérite. Sans l'action il y aurait permanence dans le péril.

TSHOU HI. - Répétition, renouvellement fréquent et habituel ; khan, péril et chute. L'image symbolique est l'eau. La positivité tombe au milieu de négativités ; au-dehors vide et incertitude, au-dedans réalité. Dans ce koua, le koua simple khan est répété en haut et en bas; c'est considéré comme exprimant un péril répété. La réalité de lintérieur est considérée comme exprimant l’image symbolique d'avoir foi, et de liberté de cour. En agissant dans ces conditions, il doit nécessairement y avoir du mérite, et c'est pour cela que telle est la formule divinatoire.

527.

Le commentaire traditionnel de la formule déterminative dit: Fréquence de la chute, répétition du péril.

TSHOU HI. - Explication du sens du nom du koua. 
L'eau coule et ne remplit pas; agir dans le péril et ne pas perdre confiance.

TSHOU HI. - Répétition du koua simple khan, c'est-à-dire répétition du péril; deux périls superposés. La formule du premier trait hexaire dit: " fissure dans l'abîme », c'est un abîme dans un autre abîme, un redoublement de périls. L'eau coule et ne remplit pas ; la positivité s'agite au milieu du péril et n'en sort pas encore ; c'est le flux ou courant inhé rent à la nature de l'eau, et qui cependant ne remplit pas encore l'abîme; s’il était plein, elle déborderait du gouffre. Agir dans le péril et ne pas manquer. à la confiance, l'énergie positive, la justice et la réalité demeurent au milieu du péril : c'est donc celui qui agit sans manquer à la bonne foi. Le gouffre est au milieu, l'eau coule vers le bas, c'est toujours considéré comme comportant le sens de confiance et d'avoir foi.

TSHOU HI. - Explication du sens des mots «bonne foi » au moyen des images symboliques du koua simple. Cela exprime que l'intérieur est réel et solide et que les actions extérieures en portent la trace constante.

529.

Le cœur seul jouit de liberté, et cela à cause de la justice énergique ; l'action est estimable, en continuant il y aura du mérite.

TSHENG TSE. - C'est le cour seul qui peut jouir de liberté, et cela à cause de sa justice énergique; le trait plein du milieu est considéré comme le symbole de la foi, ou confiance. En suivant la voie de l'extrême sincérité, où ne parviendrait-on pas ? En agissant par la voie de la justice et de l'énergie, il deviendra possible de parer aux dangers et aux périls, et de les traverser librement; en employant les aptitudes de la justice énergique pour entreprendre quelque chose, on acquerra du mérite, de sorte que l'ac tion sera louable et estimable. Mais si on s'arrête, sans agir, on demeurera d'une façon permanente dans le danger. Quand il s'agit du koua khan, qui exprime la chute et le péril, c'est la condition d'être capable d'agir qui constitue le mérite.

TSHOU HI. - La condition de l'énergie placée au milieu est considérée comme l'image symbolique de la liberté du cour ; cette condition remplie, en agissant il y aura du mérite. 
L'altitude (péril) du ciel ne peut être franchie; les obstacles (périls) de la terre sont les montagnes, les torrents, les éminences et les collines. Les rois et les princes établissent des obstacles (périls) pour préserver le royaume; que le moment et les effets du péril sont vastes

TSHENG TSE. - L'élévation qui ne peut être attein te, c'est le péril, ou obstacle du ciel. Les montagnes, les torrents, les éminences et les collines sont les périls de la terre. Les rois et les princes sont les souverains des États. Considérant l'image symbolique du koua khan, ils reconnaissent que le péril ne doit pas être méprisé, aussi ils établissent des remparts et des fossés, comme obstacles, pour préserver les États et protéger les populations ; c'est là le moment de l'emploi des obstacles. Cet emploi est très vaste, aussi la formule comporte une exclamation admirative sur sa grandeur. Les montagnes, les fleuves, les remparts et les fossés sont pris comme exemples de ce qu'il y a de plus grand parmi les obstacles. S'il s'agit de distinguer entre l'éminence et l'infériorité, entre ce qui est noble et ce qui est vil, d'éclairer les degrés dans l'autorité, de préciser la couleur des objets et leur apparence, ce qui est toujours destiné à couper court aux usurpations de prérogatives et à séparer les rangs de diverses classes, il s'agit toujours de l'appl ication de «l'usage des obstacles ».

TSHOU HI. - Dernière analyse et exclamation admirative sur la grandeur.

531.

Le commentaire traditionnel de la formule symbolique dit : Débordement de l'eau qui coule; répétition du péril ; l'homme doué déploie une vertu constante dans son action; il répète l'enseignement des choses.

TSHENG TSE. - Le koua simple khan représente l'eau ; le courant de l'eau, c'est son écoutement et son renouvellement. Deux koua simples khan sont répétés l'un au -dessus de l'autre : c'est l'image symbolique du courant de l'eau débordant. L'eau s'écoule par des ruisselets qui s'élargissent de plus en plus, jusqu'à former des fleuves, et enfin l'océan : c'est la répétition incessante du courant, ou afflux, sans arrêt. Par sa force naturelle elle tend à descendre ; c'est un effet assuré qu'on peut considérer avec confiance comme permanent, aussi l'homme doué regarde l'image symbolique de l'eau, dans le koua simple khan, il en extrait le concept de régularité permanente, de sorte qu'il ass ure la durée constante de sa conduite vertueuse. Si la conduite vertueuse de l'homme n'est pas permanente, la fausseté prend la place de la 
vertu ; aussi il convient qu'elle soit comme la continuité incessante du courant de l'eau. L'homme doué relève le se ns de succession ininterrompue dans la continuité du courant, et il mûrit et répète l'objet de son enseignement. Or, l'établissement des institutions civiles et la pratique de l'enseigne ment doivent nécessairement pousser le peuple à s'en pénétrer en les écoutant; il en résulte immédiatement qu'il peut les suivre et s'y conformer. C'est dans ce but que l'homme doué « répète trois fois l'ordre et en surveille l'exécution »; s’il avertissait à la hâte, sans avoir déjà appuyé le précepte par son exemple, imposant brusquement l'obéis sance, même avec la sanction de peines sévères pour y contraindre, le résultat serait impossible à atteindre ; il convient donc d'imiter la persistance continue du courant de l'eau.

TSHOU HI. - Qu'il s'agisse de se gouverner soi -même, ou de gouverner les hommes, il faut toujours procéder par la répétition de l'exercice et de la pratique ; ce n'est qu'ensui te que la parfaite habitude assure la tranquillité. 


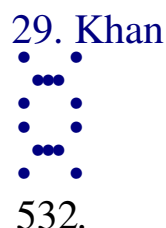

Premier trait hexaire : répétition du péril ; entrer dans la fosse de l'abîme ; présage malheureux.

TSHENG TSE. - Le premier trait emploie la douceur malléable de la négativité et se tient au bas du koua khan qui représente le péril ; il est doux et faible, sans personne pour lui tendre la main, et placé sans répondre aux nécessités de sa situation : il ne peut sortir du péril. Il ne fait que s'enfoncer de plus en plus dans un péril profond. L'avant-dernier caractère désigne une fosse ou fissure, gouffre dans un gouffre. Étant déjà au milieu de périls répétés, il entre encore dans un gouffre au fond du précipice; le présage néfaste est évident.

TSHOU HI. - Employant la douceur malléable de la négativité et se retenant au bas du koua qui indique la répétition du péril, et le gouffre se creusant de plus en plus, tels sont donc l'image symbolique et le sens divinatoire.

533.

Répétition de la chute pour entrer dans le gouffre; présage funeste de la perte de la voie.

TSHENG TSE. - Par suite de la répétition du péril entrer encore dans un gouffre situé au fond d'un abîme ; c'est la perte de la voie, et c'est par là qu'il y a présage malheureux. Pouvoir sortir du péril, c'est ne pas perdre la voie. 


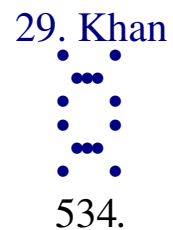

Deuxième trait nonaire : la chute est périlleuse ; demander et obtenir un peu.

TSHENG TSE. - Le second trait se trouve en présence d'un moment de chute périlleuse, il tombe entre les deux négativités placées au-dessus et au-dessous de lui; c'est un ter rain extrêmement périlleux, il y a bien réellement danger. Cependant ses aptitudes sont la justice et l'énergie, bien qu'il ne puisse pas encore sortir du milieu du péril, il peut néanmoins y remédier quelque peu par lui-même, et il n'en arrive pas, comme le pre mier, à s'enfoncer de plus en plus dans des périls profonds : c'est ce qu'on entend par les mots «obtenir un peu ${ }^{1}$. C'est l'homme doué placé dans le péril et le danger et qui peut se protéger par lui-même. Cela tient uniquement à la justice et à l'énergie ; étant énergique, ses aptitudes sont suffisantes pour sa propre défense : étant juste, en se mouvant, il ne manque point le but convenable.

TSHOU HI. - Il est placé au milieu d'obstacles et ne peut pas encore en sortir luimême, de sorte qu'il est considéré comme exprimant liimage symbolique de la rencontre du péril. Toutefois il est énergique et se conforme à la justice, de sorte que le sens divinatoire est qu'il est possible de demande $r$ et d'obtenir un peu.

535.

Demander et obtenir un peu; ne pas encore sortir dedans.

TSHENG TSE. - Au moment où il se trouve embarrassé par deux négativités, dans un lieu périlleux, à cause de ses aptitudes de justice et d'énergie, il n'en vient pas à tomber dans des périls très profonds ; c'est ce qui est exprimé par les mots «demander et réussir» (ou obtenir) un peu. Cependant il ne peut pas encore sortir des périls qui sont dans le gouffre.

On lit « sortir de dedans » mais tel est bien le texte.

\footnotetext{
${ }^{1} \mathrm{Ou} \ll$ réussir un peu ».
} 


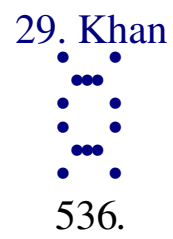

Troisième trait hexaire : en venant ou en allant, nombreux périls; être dans l'embarras et s' envelopper la tête ${ }^{1}$; entrer dans le gouffre de l'abîme ; ne pas agir.

TSHENG TSE. - Le troisième trait hexaire se trouve dans un moment de chute périlleuse ; il emploie la douceur molle de la négativité et se maintient sans justice ni droiture; la position n'est pas bonne, c'est celui qui ne peut ni avancer, ni reculer, ni demeurer en place. S’il vient vers le bas, il entrera au milieu des obstacles; s’il monte, il y aura répéti tion de périls ; quil revienne en arrière ou progresse en avant il y a toujours péril, et c'est pourquoi la formule dit: en venant ou en allant, nombreux périls. Il lui est également périlleux d'avancer ou de reculer, et il y a aussi péril à demeurer. Le neuvième caractère du texte, tshen, signifie s'appuyer pour se soutenir ; il se maintient au milieu des périls et il s'appuie pour se retenir en place ; c'e st, au plus haut point, être mal à l'aise. Étant ainsi placé, il ne réussit qu'à s'enfoncer de plus en plus dans des périls profonds, et c'est pourquoi la formule dit: « entrer dans le gouffre de l'abîme ». La voie suivie par le troisième trait pour se placer ne doit pas être employée, aussi la formule avertit de ne pas agir.

TSHOU HI. - Employant la mollesse de la négativité, sans droit ni justice, et circulant entre des obstacles accumulés, il est aussi périlleux d'avancer que de reculer. En avant des obstacles, et en arrière la nécessité de se retenir en s'appuyant ; la chute est de plus en plus profonde ; il ne sait pas nager. Aussi tels sont l'image symbolique et le sens divinatoire. Le terme tshen, du texte, exprime l'idée de s'appuyer sans pouvoir trouver une position commode.

537.

En venant et en allant, nombreux périls ; finalement pas de succès.

\footnotetext{
${ }^{1}$ Les commentateurs chinois lisent : « s'appuyer sur un coussin ».
} 
TSHENG TSE. - Il lui est également périlleux d'avancer ou de reculer, et de plus il n'est pas tranquille dans la situation où il est placé. En emplo yant cette voie on doit naturellement s'enfoncer de plus en plus dans le péril ; finalement comment pourrait-il y avoir du succès ? En employant la douceur malléable de la négativité, et se plaçant sans justice ni droiture, même sur un terrain uni et facile, il en résulte ordinairement des regrets et des fautes, combien à plus forte raison lorsqu'on se trouve placé dans le péril ! L'homme aspire à sortir du péril ; il faut absolument qu'il se conforme à la voie morale, alors il peut s'en éloigner ; si, cherchant à s'en éloigner, il manque à cette voie morale, il ne fait qu'aggra ver de plus en plus sa misère et ses souffrances. Aussi l'homme saint avertit que, placé comme le troisième trait, on ne doit pas employer sa voie. 


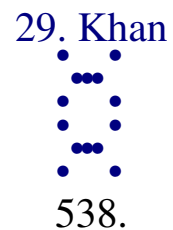

Quatrième trait hexaire: urne, vin, plateau à offrandes; dans les deux cas employer la poterie ; venir se joindre par la fenêtre ${ }^{1}$; finalement pas de culpabilité.

TSHENG TSE. - Le quatrième trait hexaire est malléable et négatif et au-dessous de lui il ne trouve aucune assistance; c'est celui qui n'est pas capable de remédier aux périls de l'univers ; c'est parce qu'il est dans une situation élevée que la formule parle de la voie du ministre placé dans le péril. Un sujet de haut rang, en présence d'un moment de difficul tés et d'obstacles doit uniquement pousser la sincérité à l'extrême limite et manifester sa bonne foi envers le prince ; alors leur liaison est solide et sans solution de continuité. De plus, s'il peut ouvrir et éclairer le cœur (intelligence) du prince, il pourra par là se protéger contre toute faute. Or, voulant jouir de l'entière confiance du supérieur, il sait simplement épuiser la simplicité naturelle sans aller au-delà. La multiplication des règles d'étiquette, la recherche dans les apparences extérieures, ne valent pas les rites des offrandes de Yen et c'est pour cela que l'offrande de Yen est citée comme exemple. Cela exprime quil convient de ne point estimer la surabondance d'appareil extérieur, de n'employer que la simplicité naturelle. Ce qui est employé c'est le vin d'une seule jarre et les aliments présentés sur deux plateaux; de plus les vases sont en poterie ; c'est le comble de la simplicité. Quand la simplicité naturelle est poussée à ce point, il convient encore de «venir se joindre par la fenêtre ». Venir joindre ${ }^{2}$, c'est-à-dire avancer et se lier à la voie du prince. Le mot fenêtre comporte le sens d'ouverture donnant liberté d'accès ; à cause de l'obscurité intérieure des habitations, on établit des ouvertures qui donnent accès à la lumière. "Par la fenêtre », c'est-à-dire d'un lieu situé en pleine clarté, pour augmenter encore la clarté des décisions du prince.

Le Shi king dit: "Le ciel en guidant ${ }^{3}$ le peuple agit comme le shi ou comme le huien ${ }^{4}$. » Mao Kong explique le caractère yeou de cette citation ${ }^{5}$ en lui donnant le sens de moraliser en éclairant, instruire, guider ; c'est encore une expression analogue au terme « ouvrir l'intelligence ».

\footnotetext{
1 Version tout à fait arbitraire.

${ }^{2}$ Littéralement : « apporter et lier »,

${ }^{3}$ En éclairant.

${ }^{4}$ Noms de deux instruments de musique.

5 Traduit par fenêtre dans la formule.
} 
Le sujet emploie la fidélité et la confiance, il suit la voie du bien, et se lie au cour du prince ; il faut absolument que ce soit en venant d'une position prise avec intelligence, et alors il peut pénétrer. Le cour de l'homme a des replis secrets et des côtés accessibles et pénétrables; les replis secrets en sont les parties obscures; les côtés pénétrables sont les parties intelligentes et éclairées. Il convient de s'adresser aux parties intelligente et éclai rées et de les avertir, alors il est facile de provoquer la confiance ; c'est pour cela que la for mule emploie l'expression « venir se joindre par la fenêtre ». Pouvant agir ainsi, il en résultera que bien que dans un moment de difficultés et de périls, finalement il est possible d'éviter toute culpabilité.

D'ailleurs, si le cour du prince est obscurci par l'abus des plaisirs, et que cette cause d'obscurcissement soit la seule, bien qu'on lui représente énergiquement les inconvénients de la dissolution et des plaisirs, alors même qu'il n'y prendrait pas garde, il arriv era nécessairement qu’il en tirera des conséquences relatives aux points sur lesquels il n'est pas aveuglé, ce qui réveillera son cour. Depuis l'antiquité, ceux qui se sont montrés capables d'adresser des remontrances aux princes n'ont jamais agi qu'en se servant des côtés demeurés libres dans l'intelligence de ceux -ci ; ceux qui leur ont adressé des reproches directs et brusques n'ont, en général, recueilli que de l'antipathie, tandis que les paroles de ceux qui ont su discuter avec modération et ampleur ont le plus souvent produit leur effet. Par exemple, le fondateur de la dynastie des Han, amoureux de l'une de ses concubines, était sur le point de changer l'ordre d'hérédité en faveur du fils de cette femme ; c'était là le côté par où il était aveuglé. Dans la foule des ministres et des serviteurs du prince, grand était le nombre de ceux qui cherchaient à s'opposer à cette mesure ; les droits qui résultent de la naissance, l'ordre de l'âge, tout était parfaitement clair, d'où pouvaient provenir cet aveuglement et ce manque de discernement? Mais quant aux «quatre vieillards », l'empereur connaissait bien leur sagesse et il les estimait, c'était là le côté non obscurci de son intelligence ; aussi sa raison, réveillée par ce côté accessible, modifia enfin sa décision et ce résultat fut obtenu sans aucune difficulté. La force des quatre vieillards était-elle donc plus grande que celle de tous les grands et de la foule des docteurs de la cour? L'instance de leurs discours était-elle donc plus pénétrante que celle des paroles de Tsheou Tsheang ou de Sou Souen? Cependant l'empereur n'écouta pas ceux -ci et se rendit aux conseils de ceux-là, parce que les uns combattirent son aveuglement, tandis que les autres s'adressèrent à son intelligence.

Il en est encore de même dans l'exemple de Thai Heou, reine de l'état de Tshieou ; elle aimait son plus jeune fils, Tsheang Ngan Kiun, et ne voulait pas l'envoyer dans l'état de Tsi ; ici, l'aveuglement était causé par l'affection. Bien que les grands dignitaires lui adressassent des remontrances énergiques, étant de jour en jour plus aveuglée, comment aurait-elle pu les écouter? L'amour de ses enfants et le désir de les rendre illustres et puis sants était le côté intelligent de son cour, aussi, Tso Shi et Tshou Long se se rvirent de la partie éclairée de son esprit, ils surent la diriger par le calcul de la longue durée de sa postérité, et elle les écouta comme un écho. Ce n'est pas 
seulement en avertissant les princes qu'il faut suivre cette voie ; quiconque enseigne, ou dirige, doit s'y conformer. Pour ins truire, il faut s'appuyer sur les inclinaisons prédominantes de celui qu'on instruit ; or ce qui prédomine, c'est ce que le coar comprend ; en s'appuyant sur ce que le cour com prend, pour pénétrer dans l'esprit, on att eint peu à peu toutes les autres questions. C'est ce que Mang Tse exprime par les mots : «compléter la vertu et atteindre aux aptitudes $\gg$.

TSHOU HI. - Tshao Shi dit : Les anciens disciples de la doctrine lisaient les trois caractères «jarre, vin, plateau» comme formant une phrase, et les mots «redoubler l'emploi de la poterie ${ }^{1}$ comme une autre phrase ; actuellement, on suit cette leçon. Le terme ji, deux, ou doubler, veut dire augmenter. Dans les Rites de Tsheou on trouve les mots : " Dans les grands sacrifices, tripler et doubler. Les cadets et les enfants assistent; ceux de gauche tiennent un plat à viande vide; ceux de droite tiennent la louche; ils font un tour complet et reviennent une seconde fois (doublent)»; c'est précisément le sens. Le cinquième trait nonaire représente la situation prééminente ; le quatrième trait hexaire est proche de lui ; dans un moment de péril, la dureté et la douceur s'assistant mutuellement, il y a donc l’image symbolique de l'emploi unique de rites simples, de les répéter avec pureté de coaur, et de venir se joindre par la fenêtre. La fenêtre n'est pas l'ouverture convenable pour pénétrer, et c'est par elle que l'habitation reçoit la lumière. Bien que les commentateurs soient pénibles et difficiles, à la fin, cependant, il est possible d'éviter les fautes, et c'est pourquoi tel est le sens divinatoire.

539.

Jarre, vin, plateau, doubler ${ }^{2}$; la dureté énergique et la douceur malléable sont en contact.

TSHENG TSE. - Le commentaire de la formule symbolique ne relève que le premier membre de phrase ; il en est souvent ainsi. Une jarre de vin et deux plateaux de mets, c'est la plus extrême simplicité (pour une offrande). Si telle peut être la voie suivie lorsque la dureté énergique et la douceur malléable se rencontrent et s'accueillent, il sera possible d'affirmer que, finalement, aucune faute ne sera commise. Si les relations entre le prince et le sujet peuvent être fermes et permanentes, cela tient exclusivement à la sincérité. Les expressions dureté énergique, et malléabilité, désignent le

\footnotetext{
${ }^{1}$ Ce n'est pas là le sens naturel du texte.

2 On lit ordinairement : «Jarre de vin, plateau de mets, répétition. » Mais la construction n'autorise pas cette lecture.
} 
quatrième et le cinquième traits et font allusion aux relations entre le prince et le sujet.

TSHOU HI. - Tshao Shi dit: Dans le texte des explications deTsou Shi, il n'y avait pri mitivement pas le caractère ji, deux, ou doubler ; actuellement on suit cette lecture. 


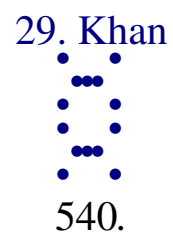

Cinquième trait nonaire : l'abîme ne se remplit pas ; quand le fond est nivelé, pas de culpabilité.

TSHENG TSE. - Le cinquième trait nonaire est au milieu de l'abîme ${ }^{1}$, celui-ci n'est donc pas rempli ; s’il était rempli, il serait nivelé et le cinquième trait en sortirait. Le caractère $t s h i$, du texte, respecter, doit être lu avec le son $t i$ (racine d'arbre), et en prenant le sens du mot $t i$, parvenir à. Dans le koua fou il est dit: mou tshi houei, ne pas en arriver jusqu'aux remords. Il faut absolument ajourner jusqu'à ce que l'obstacle soit nivelé, et alors il n'y aura aucune culpabilité. Puisque la formule dit : « ne se remplit pas », ou pas plein, c'est donc que l'obstacle n'est pas encore nivelé, que le cinquième trait n'est pas encore en paix, et qu'il continue à être placé au milieu du péril ; il n'est pas encore parvenu à être sans culpabilité. Puisque le cinquième trait nonaire possède les aptitudes de la justice et de l'énergie et qu'il s'en sert en occupant la situation prééminente, il en résulte naturellement qu'il doit lui être possible de traverser les obstacles ; cependant, au-dessus de lui, il ne rencontre aucune assistance. Le second trait tombe dans l'abîme et ne peut pas encore en sortir ; tous les autres sont mous et négatifs et n'ont pas les aptitudes néces saires pour traverser le péril ; le prince, bien que doué de ces aptitudes, pourrait-il donc, à lui seul, remédier aux périls de tout l'univers ? Occuper la situation du prince, et ne pas être capable de diriger l'univers en le faisant sortir du péril, c'est considéré comme être coupable; il faut absolument en arriver à ce que les obstacles soient aplanis, et alors c'es t atteindre à l'absence de culpabilité.

TSHOU HI. - Bien que le cinquième trait soit plongé dans l'abîme, cependant, puisqu'il est énergique, juste et droit, et qu'il occupe la situation prééminente, le temps approche cependant où il pourra sortir. C'est pourquoi tels sont l'image symbolique et le sens divinatoire.

541.

L'abîme n'est pas plein ; la justice n'est pas encore grande.

\footnotetext{
${ }^{1}$ Au milieu du koua simple khan.
} 
TSHENG TSE. - Le cinquième trait nonaire présente les qualités de la justice et de l'énergie, et il possède la situation prééminente ; il est en train de remédier aux difficultés et aux périls qui menacent l'empire, mais cependant l'abîme n'est pas encore rempli et comblé ; c'est donc qu'il ne peut pas encore aplanir les obstacles et les difficultés, et cela tient à ce que lavoie de justice et d'énergie qu'il ouvre n'est pas encore resplendissante et grande. Dans un moment de difficultés et de périls, si le prince et les sujets n'unissent point leurs forces, leur serait-il donc possible d'y remédier ? La voie du cinquième trait n'est pas encore grande ; c'est parce qu'il n'a pas de ministres. Si la voie $\mathrm{du}$ prince ne peut pas encore remédier aux périls et aux obstacles, elle est considérée comme n'étant pas encore grande, et le prince lui -même n'est pas à la hauteur de la situation.

TSHOU HI. - Il possède les vertus de la justice, mais elles ne sont pas encore largement développées. 


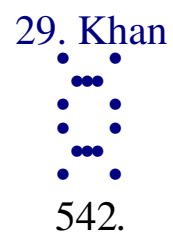

Trait supérieur hexaire : attacher en employant une bonne corde ; placer dans un buisson d'épines ; trois ans sans réussir ; présage malheureux.

TSHENG TSE. — Le trait supérieur hexaire emploie la douceur malléable de la négativité, et il est placé au comble du péril ; il exprime la profondeur de la chute, la captivité est prise comme exemple, comme lorsqu'on attache avec une corde à trois brins et qu'on retient emprisonné au milieu d'un buisson d'épines. Malléabilité négative et profondeur de la chute ; impossibilité d'en sortir. C'est pour cela que la formule mentionne une durée qui s'étend jusqu'à trois ans ; il ne réussit pas à s'y soustraire ; le présage malheureux est évident.

TSHOU HI. - Avec sa malléabilité négative il est placé au comble du péril, et c'est pour quoi tels sont l'image symbolique et le sens divinatoire.

543.

Le trait supérieur hexaire perd la voie morale ; présage malheureux pour trois ans.

TSHENG TSE. - Puisquil emploie la malléabilité négative et qu'il se place lui-même sur le terrain le plus périlleux, c'est la perte de la voie, aussi le malheur dure jusque pendant trois ans. Pendant toute cette durée de trois ans, il ne peut s'y soustraire ; c'est une locu tion qui comporte le sens de malheur définitif. Pour désigner une longue durée, quelquefois les formules emploient l'expression « dix jours », quelquefois l'expression «trois jours », selon le cas. Tomber dans une prison, jusque pendant trois ans, c'est le comble de la durée ${ }^{1}$. Dans d'autres koua, lorsqu'il s'agit d'un nombre d'années, c'est encore dans chaque cas selon le sujet traité ; par exemple : «Pendant trois ans ne pas commencer $»^{2}$; « après dix ans, alors elle est accordée $»^{3}$.

\footnotetext{
${ }^{1}$ D'après les lois chinoises la durée de l'emprisonnement, ou plutôt du travail pénible, ne dépasse jamais trois ans.

${ }^{2} \mathrm{~N}^{\circ} 262$.

${ }^{3} \mathrm{~N}^{\circ} 98$.
} 


\section{0. $\mathrm{Li}$}

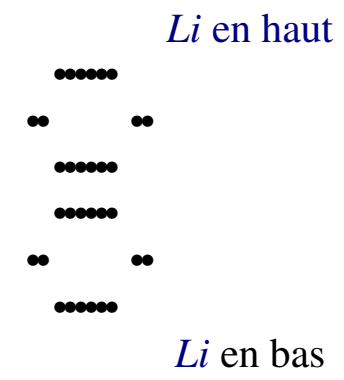

544. Li, avantage de la perfection ; liberté, réunir des vaches, présage heureux.

[]

548. Premier trait nonaire : démarche hésitante; absence de culpabilité résultant du respect.

*

550. Deuxième trait hexaire : clarté jaune ; grandeur du présage heureux.

*

552. Troisième trait nonaire : clarté du soleil qui décline; ne pas frapper sur la terre cuite et chanter; formuler des lamentations sur la grande vieillesse; présage malheureux.

$*$

554. Quatrième trait nonaire : comme un courant rapide, de même il vient; comme brûlant ; comme mort ; comme abandonné.

$*$

556. Cinquième trait hexaire : comme versant des larmes qui coulent; comme triste et se lamentant; présage heureux.

$*$

558. Trait supérieur nonaire : le roi se dispose à partir en guerre; il a des succès, il choisit des chefs ; ce qu'il capture n'est pas le vulgaire ; pas de culpabilité.

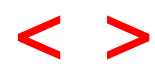


30. Li.

TSHENG TSE. - Li. «L'Ordre des koua » dit : «Khan, chute; dans la chute il y a nécessairement un point d'arrivée, c'est pourquoi le koua khan est immédiatement suivi du koua $l i$; $l i$, venir se joindre à. » Tombant au milieu des obstacles et des difficultés, il faut nécessairement quil y ait un point auquel on arrive à se joindre et s'annexer ; c'est là une loi naturelle, et c'est pour cette raison que le koua $l i$ suit immédiatement le koua khan. Li signifie se joindre, s'annexer à, et a ussi, clarté, lumière. Si on relève la condition de la négativité se joignant aux positivités placées au-dessus et au-dessous d'elle, cela constituera le sens de s'attacher, se lier en s'annexant ; si on relève la condition de vide intérieur ${ }^{1}$, cela constituera le sens de clarté. Le koua simple $l i$ est considéré comme représentant le feu ; par sa nature, la substance du feu est incorporelle, c'est ce qui s'ajoute aux corps et les rend éclairants. Il est encore considéré comme exprimant le jour, également à cause de l’image symbolique de vide impalpable et de clarté ${ }^{2}$.

\footnotetext{
${ }^{1}$ Le trait brisé entre deux traits pleins.

${ }^{2}$ Le véritable sens du caractère li est : « Jonction suivie de séparation »; « se quitter ».
} 


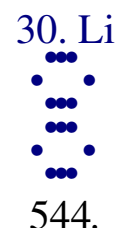

Li, avantage de la perfection ; liberté, réunir des vaches, présage heureux.

TSHENG TSE. - $L i$, adjonction, annexion à ; tous les êtres, sans exception, ont quelque chose à quoi ils se joignent; du moment où il y a forme, c'est qu'il y a jonction ${ }^{1}$. Pour l'homme ce sera les personnes qu'il affectionne et auxquelles il se joint, la voie par laquelle il procède, les choses qui dépendent de lui; tout cela constitue également les objets de son attachement. L'objet de l'attachement des hommes est avantageux par la perfection de la droiture ; en s'accordant à la droiture, il devient possible de jouir de liberté, et c'est pourq uoi la formule dit : «attachement (la), avantage de la perfection, liberté. » Présage heureux de la réunion des vaches en troupeau; le naturel du bouf est placide et soumis et, de plus, il s'agit d'animaux femelles ; cela exprime donc l'extrême soumissio n. Du moment où on s'attache à la droiture, il faut pouvoir se soumettre à la voie rationnelle du droit, si on le fait avec une soumission analogue à celle de la vache, ce sera un présage heureux. Réunir des vaches en troupeau, ou nourrir et élever des vaches, signifie nourrir et développer la vertu de soumission; chez l'homme, la vertu de la soumission se développe et s'achève par le soin et la culture ; du moment où il s'attache à la droiture, il doit développer et exercer cette vertu de soumission, afin de la compléter.

TSHOU HI. - $L i$, adjoindre, s'attacher à. La négativité s'attache à la positivité ; l'image symbolique est le feu, dont la substance est négative et l'effet positif. L'objet de l'attache ment des êtres n'est noble que par l'accord avec la droiture. La vache est un animal doux et soumis, aussi, si celui qui consulte le sort peut se maintenir dans la droiture, il jouira de liberté, et le fait de « nourrir la vache $»^{2}$ sera un présage heureux.

545.

Le commentaire traditionnel de la formule déterminative dit : Li, contact; le soleil et la lune sont en contact avec le ciel ; les cent sortes de grains ${ }^{3}$, les

\footnotetext{
${ }^{1}$ Contact limitant dans l'espace.

2 S'exercer à la soumission.

${ }^{3}$ Les céréales et les grains alimentaires.
} 
plantes et les arbres sont en contact avec la terre; redoubler l'intelligence afin de s'attacher à la droiture ; c'est là transformer et civiliser l' univers.

TSHENG TSE. - $L i$, être en contact, s'attacher, se joindre à quelque chose ; par exemple, comme le soleil et la lune sont attachés au ciel ${ }^{1}$, comme les cent sortes de grains, les plantes et les arbres sont attachés à la terre. Tous les êtres sans exception ont quelque chose à quoi ils s'attachent ; entre le ciel et la terre il n'y a rien qui ne soit attaché à quelque chose ${ }^{2}$. Lorsqu'il s'agit de l'homme, il convient d'examiner l'objet de son atta chement; si cet objet est conforme à la droiture, il pourra jouir de liberté. «Redoubler l'intelligence afin de s'attacher à la droiture » est dit au sujet des aptitudes exprimées par le koua; il est formé, en haut et en bas, par le koua simple li, c'est là la répétition de clarté. Le cinquième trait et le second sont tous deux placés selon la justice et la droiture, ils expriment l'attachement à la droiture ; le prince et le sujet, le supérieur et l'inférieur, possèdent tous les facultés de l'intelligence et se placent suivant la justice et la droiture : il leur est possible de transformer l'univers et de perfectionner l'élégance des mours.

TSHOU HI. - Explication du sens du nom du koua.

546.

La douceur est jointe à la justice et à la droiture ; donc, liberté ; c'est ce qui produit le présage heureux de l'action de nourrir la vache.

TSHENG TSE. — Le second et le cinquième traits emploient la douceur et la soumission et s'attachent à la justice et à la droiture, d'où résulte la liberté d'action. Si l'homme peut développer la plus grande s oumission pour s'attacher à la justice et à la droiture, le présa ge sera heureux, et c'est pour cela que la formule dit: «Présage heureux de l'action de nourrir la vache. » On a dit : Le second trait se conforme strictement à la justice et à la droiture, mais le cinquième, employant la négativité pour se maintenir à un rang positif, peut-il être considéré comme se conformant à la droiture ? Dans le koua $l i$, tout dépend de l'objet de l'attachement ; le cinquième rang est une situation qui comporte la justice et la droiture ; c'est un trait hexaire qui s'attache à une situation qui lui convient correctement ; c'est cela qui est considéré comme constituant la droiture. Lorsque l'étudiant comprend le sens indiqué par le

\footnotetext{
${ }^{1}$ On peut remarquer que la lecture la plus naturelle serait : « le soleil et la lune sont en contact dans le ciel ».

${ }^{2}$ La véritable idée, développée mais altérée par la tradition, serait que toute chose, dans la nature, n'existe que par rapport avec d'autres sujets. Li, clarté, lumière, intelligence, feu.
} 
moment et ne se méprend pas sur l’importance relative des diverses circonstances, alors il peut se permettre de raisonner sur le Yi king.

TSHOU HI. - Explication de la formule du koua au moyen de la substance même de ce koua.

547.

Le commentaire traditionnel de la formule symbolique dit: La clarté jaillit deux fois, $l{ }^{1}$, le grand homme agit pour éclairer successivement les quatre régions.

TSHENG TSE. - Si la formule portait « deux clartés », il s'agirait de deux clartés différentes et on ne comprendrait pas le sens des mots « éclairer successivement»; c'est pour cela que le texte porte « clartés deux », il s'agit d'une clarté qui se répète et se redouble, c'est -à-dire que les deux clartés se succèdent réciproquement. Les deux caractères suivants, «faire la séparation $»^{2}$; la clarté est double et produit le koua $l i$, séparation, cela donne le sens de "succession de clarté ». Les koua tshen, souen, et autres du même genre ${ }^{3}$ prennent aussi le sens de suite, succession continue, comme dans le cas d'un courant; toutefois, le sens du koua $l i$ est de beaucoup le plus important. Lorsqu'il s'agit de la gran deur de la vertu on emploie l'expression «homme saint»; s’il s'agit de la grandeur de la situation on dit : le « prince », dans les deux cas il s'agit d'un « grand homme ». Le grand homme regarde limage symbolique de clartés se succédant réciproquement, et il applique cette conception à la transmission de la faculté dintelligence de génération en génération ${ }^{4}$, pour éclairer, en les régissant, les quatre régions. Dans la majorité des cas, lorsque les clartés se succèdent, c'est toujours une succession de clarté ; la formule relève le cas le plus important, et elle en parle au point de vue de la transmission successive de la clarté, de génération en génération.

TSHOU HI. — Le terme tso, du texte, faire, produire, signifie commencer.

\footnotetext{
${ }^{1}$ Le texte est très obscur ; la lecture la plus naturelle et la plus correcte serait : « La clarté agit de deux côtés, séparation. »

2 Tsheng Tse change la ponctuation; il lit : «clartés deux, faire ou produire la séparation » (la).

${ }^{3}$ Formés par la répétition d'un même koua simple.

${ }^{4}$ Ce passage est surtout remarquable si on observe que les commentaires modernes sont toujours des compilations d'explications plus traditionnelles, plus anciennes. La seule idée rationnelle qui peut se distinguer dans cette obscurité serait relative à la transmission de la mémoire des faits par des signes permanents, tels qu'une écriture.
} 


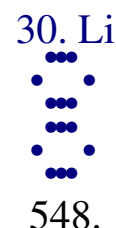

Premier trait nonaire : démarche hésitante ${ }^{1}$; absence de culpabilité résultant du respect.

TSHENG TSE. - La positivité est certainement portée au mouvement ; de plus elle occupe le rang inférieur et fait partie de la substance du koua simple $l i$. Un trait positif occupant un rang inférieur veut certainement avancer, et la nature propre du koua $l i$ est la tendance ascendante de la flamme; sa volonté est donc de monter se joindre, il est sur le point de se mouvoir avec précipitation, sa démarche n'est pas encore nettement dessi née et elle semble hésitante. Bien qu'il n'avance pas encore, cependant les symptômes de son mouvement sont déjà visibles. En se mouvant, il perd sa condition d'occuper un rang inférieur et il assume une culpabilité. Cependant ses aptitudes sont la dureté énergique et l'intelligence, s'il connaît le devoir et sil s'y conforme avec respect, il n'en arrivera pas à cette culp abilité. Le premier trait est celui qui est dans un rang inférieur et sans situation ; porter l'intelligence dans le mouvement de sa propre personne pour avancer ou reculer, telle est la voie rationnelle à laquelle il s'attache. Mais du moment où ses tendances lui donnent l'impulsion, il ne peut plus être attentif et contenu, alors il se meut mal à propos et c'est là manquer de discernement dans le choix de l'objet de son attache ment, et être coupable.

TSHOU HI. - Employer la dureté énergique, occuper un rang inférieur, et être placé dans la substance de la clarté ; les tendances portent à avancer en montant, de sorte qu'il y a l'image symbolique d'une démarche ambiguë et hésitante. Avec le respect il n'y a pas de culpabilité. C'est un avertissement à celui qui consulte le sort, afin qu'il se mette dans ces conditions.

549.

Prudence de la démarche hésitante ; elle sert à éviter la culpabilité ${ }^{2}$.

TSHENG TSE. — La démarche est encore mal dessinée et il y a volonté de se mouvoir, mais connaissant le respect et la circonspection, il n'ose pas

\footnotetext{
${ }^{1}$ Confuse, difficile à discerner exactement.

${ }^{2}$ Le texte du $n^{\circ} 548$ est déjà modifié et interprété.
} 
encore avancer ; c'est par là qu'il cherche à éviter les fautes et la culpabilité. Il s'en tient à l'intelligence et il est éner gique, de sorte qu'il comprend et sait se garer ; s’il n'était ni intelligent, ni énergiq ue, il se mettrait en mouvement mal à propos. 


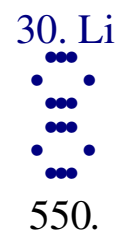

Deuxième trait hexaire : clarté jaune ; grandeur du présage heureux.

TSHENG TSE. — Le second trait se maintient dans la justice et s'acco rde à la droiture ; il s'attache à la justice et à la droiture. Le jaune est la couleur de la justice ; c'est la plus belle des apparences visibles. Élégance de la forme et de l'apparence, justice et droiture : achèvement de la beauté. Aussi la formule dit : «clarté jaune ». Il emploie les qualités de l'élé gance de la forme et de l'apparence et celles de la justice et de la droiture ; il monte s’identifier à l'intelligence brillante et à la justice condescendante du prince ; étant intelligent à ce point, et l'objet de son attachement étant tel, c'est donc le présage heureux d'un grand bien.

TSHOU HI. - Le jaune est la couleur de la justice, la douceur malléable s'attache à la justice et possède la droiture, et c'est pourquoi tels sont l'image symbolique et le sens divinatoire.

551.

Grandeur du présage heureux de la clarté jaune; se conformer à la voie de la justice.

TSHENG TSE. - Ce qui fait la grandeur du présage heureux, c'est qu'il possède la voie de la justice. Il n'est pas question de la droitu re ; dans le rapprochement, c'est la justice qui est considérée comme importante ; c'est par la justice que s'achève la perfection de la forme et de l'intelligence ; la droiture s'y trouve sous -entendue. 


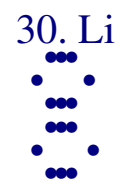

552.

Troisième trait nonaire : clarté du soleil qui décline; ne pas frapper ${ }^{1}$ sur la terre cuite et chanter; formuler des lamentations sur la grande vieillesse ; présage malheureux.

TSHENG TSE. - Les huit koua dont la substance est immélangée ont tous le sens indiqué par cette double substance. Ainsi, dans le koua khien, le koua simple intérieur et le koua simple extérieur expriment également l'activité ; dans le koua khouen, les koua simples supérieur et inférieur expriment tous deux la soumission passive ; dans le koua tshen, la majesté et la puissance qui ébranle se succèdent; dans le koua souen, en haut et en bas il y a docilité dans l'action de suivre ; dans le koua khan les périls sont répétés et accumulés; dans le koua $l i$, les clartés se succèdent et illuminent ; dans le koua ken, l'intérieur et l'extérieur expriment également l'arrêt ; dans le koua touei, l'un et l'autre indiquent la même satisfaction. Mais dans le cas du koua $l i$, le sens, étant relatif aux choses de l'homme, est pl us important que dans tout autre. Le troisième trait nonaire occupe le dernier rang dans la substance du koua simple inférieur ; c'est le moment où la clarté pré cédente étant sur le point d'être épuisée, la clarté suivante est prête à lui succéder ; le commencement et la fin de l'homme, le renouvellement des saisons. C'est pour cela que ce trait est considéré comme exprimant la clarté du soleil déclinant, la lumière du soleil descendant et déclinant ; puisqu'il décline, il est sur le point de disparaître. En en parlant au point de vue de la raison d'être des choses, ce qui a fleuri doit dépérir ; ce qui a commencé doit avoir une fin ; c'est là une voie immuable. Celui qui la comprend se conforme à la raison des choses et c'est en cela qu'il fait consister son plaisir. Poterie, objet d'usage commun et ordinaire; frapper sur une terre cuite ${ }^{2}$ et chanter, c'est se contenter avec quelque chose de très ordinaire ; celui qui ne peut agir ainsi, considérera la vieillesse comme un sujet de lamentations et de chagrin, et c'est là ce qui constituera le présage malheureux. Telle est la voie rationnelle pour se placer soit pendant la vie, soit au moment de la mort. Le quatorzième caractère, tie, qui désigne la vieillesse à partir de l'âge de quatre-vingts ans, équivaut au caractère tie, qui désigne le déclin du soleil.

TSHOU HI. - Intervalle entre le renouvellement des clartés ; la clarté précédente est sur le point d'être épuisée, donc image symbolique du déclin du soleil. Si on ne sait se contenter des choses ordinaires dans les moyens de

\footnotetext{
1 Battre la mesure.

2 À défaut de vrai tambour.
} 
plaisir, on ne saura se tenir en place, et le présage sera malheureux. La formule avertit celui qui consulte le sort quil doit être dans ces conditions.

553.

Clarté du soleil à son déclin ; comment pourrait-elle durer?

TSHENG TSE. - Du moment où le soleil décline, sa clarté peut-elle durer longtemps? Celui qui est intelligent le comprend; aussi il appelle quelqu'un pour lui transmettre les affaires; il se retire et se place dans la retraite pour reposer son corps, se contente de la médiocrité et se soumet aux conditions de sa position; comment cela pourrait-il constituer un présage malheureux? 


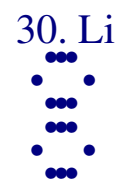

554.

Quatrième trait nonaire : comme un courant rapide ${ }^{1}$, de même il vient ; comme brûlant ; comme mort ; comme abandonné ${ }^{2}$.

TSHENG TSE. - Le quatrième trait nonaire quitte la substance inférieure et monte faire partie de la substance supérieure ; c'est l'origine de la clarté qui succède à la précédente, et c'est pourquoi il est question du sens de succession immédiate. Être dans un rang supérieur et près du prince, c'est être sur un terrain où l'on prend rang immédiate ment après lui ${ }^{3}$. Ce trait emploie l'énergie active de la positivité, il fait partie de la sub stance du koua simple $l i$, et il se place au quatrième rang, ce qui indique la violence, la précipitation et l'absence de justice et de droiture. D'ailleurs, il y a répétition de dureté énergique sans emploi de la droiture, et les conséquences logiques du développement complet de la dureté énergique surviennent comme un courant rapide; ce n'est pas celui qui succède honnêtement. Or, celui qui succède légitimement et suivant ce qui est le bien, doit nécessairement posséder la sincérité de la modestie et de l'humilité, et suivre la voie de la soumission aux lois naturelles de la succession, exactement comme Shouen et comme Khae. Dans le cas présent, le quatrième trait survient comme un courant rapide et brusquement, et il manque à la voie normale de la succession légitime. De plus, il est subordonné au prince mou et négatif représenté par le cinquième trait hexaire; la force naturelle inhérente au complet développement de la dureté énergique envahissante et dévorante fait que son éther brûle comme la flamme, et c'est pourquoi la formule dit : « comme brûlant». L'action du quatrième trait étant à ce point mauvaise, il doit nécessairement en éprouver lui-même les effets funestes, et c'est pour cela que la formule dit : «comme mort ». Manquer au devoir au sujet de la succession et de l'héritage des supé rieurs, c'est toujours une propension à la révolte, et c'est ce qui fait que la foule le délaisse, ainsi la formule dit : « comme abandonné ». Lorsqu'il s'agit de «mort » et « d'aban don », c'est l'extrême limite du malheur, de sorte qu'il n'est pas nécessaire de mention ner le présage malheureux.

TSHOU HI. - C'est le moment où la clarté suivante est sur le point de succéder et le quatrième trait nonaire emploie la dureté énergique pour précipiter cet instant marqué pour la succession, ce qui fait que telle est l’image symbolique.

\footnotetext{
${ }^{1}$ Le sens n'est explicable que parla tradition et parla valeur qu'elle attribue à ces mots, en apparence sans suite.

${ }^{2}$ Les commentateurs chinois disent : « comme rapidement ».

${ }^{3}$ Littéralement : « sur le terrain de la succession ».
} 
555.

Comme un courant rapide, de même il vient ; rien n' est supportable ${ }^{1}$.

TSHENG TSE. - Il monte, bravant le prince, sans soumission à l'autorité qu'il doit subir ; les hommes le baissent, la foule l'abandonne ; c'est lui que l'univers ne peut tolé rer.

TSHOU HI. - Rien n'est supportable ; allusion aux termes «brûlant, mort, abandonné », du texte.

\footnotetext{
${ }^{1}$ Les commentateurs chinois lisent « personne ne le tolère ».
} 


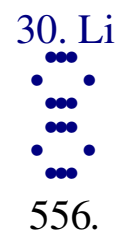

Cinquième trait hexaire : comme versant des larmes qui coulent; comme triste et se lamentant; présage heureux.

TSHENG TSE. - Le cinquième trait hexaire occupe la situation prééminente et observe la justice ; il a les qualités de l’intelligence manifestée dans l'apparence ; on peut dire qu'il représente le bien. Cependant, employant la douceur malléable et occupant un rang supérieur, n'ayant aucune assistance au-dessous de lui, il se trouve seul et isolé entre la dureté énergique et la violence avec lesquelles il est en contact; c'est une condition périlleuse et redoutable. Ce n'est que par son intelligence qu'il peut scruter la profon deur du danger et le craindre au point que ses larmes coulent, et qu'il peut être profondément triste et préoccupé au point qu'il est morne et se lamente, ce qui fait qu'il peut assurer le présage heureux de l'avenir. Verser des larmes, être triste et se lamenter, sont des expressions qui indiquent l'intensité de la tristesse et de la crainte; c'est une conséquence naturelle du moment. Occupant la situation prééminente et possédant l'intelligence qui se révèle dans les actes extérieurs, sachant craindre, et susceptible de se préoccuper à un tel point, le présage est donc heureux pour lui. S’il se fiait à ses facultés intelligentes, comme à cette condition qu'il s'attache à la justice et à la droiture, jouissant de la prospérité sans rien prévoir, comment pourrait-il garantir ce présage heureux !

TSHOU HI. - Il emploie la douceur malléable et occupe le rang prééminent; doux et pliant, il s'attache à la justice. Cependant, il ne se conforme pas à la droiture ${ }^{1}$ et il est pressé en dessus et en dessous par des traits positifs, ce qui le rend triste et préoccupé à ce point. Cependant, par la suite, le présage devient heureux. C'est un avertissement donné à celui qui consulte le sort, qui doit être dans ces mêmes conditions.

557.

Présage heureux du cinquième trait hexaire; s'attacher au roi et au prince.

\footnotetext{
${ }^{1}$ Trait négatif, rang positif.
} 
TSHENG TSE. - Le présage heureux du cinquième trait hexaire, c'est que ce à quoi il s'attache est la situation légitime du roi ou du prince. Il subit la condition naturelle de la supériorité et il examine avec lucidité la raison d'être des choses; il sait craindre et prévoir, afin de faire face aux événements, ce qui fait qu’il peut avoir un présage heureux. S’il n'était pas ainsi, comment pourrait-il être tranquille ? 


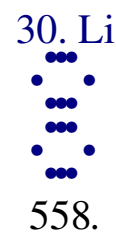

Trait supérieur nonaire : le roi se dispose à partir en guerre; il a des succès, il choisit des chefs, ce qu'il capture n'est pas le vulgaire ; pas de culpabilité.

TSHENG TSE. - Ce trait nonaire emploie la positivité et occupe le rang supérieur, il est au dernier rang du koua $l i^{1}$; c'est celui qui représente l'extrême degré de l'énergie et de l'intelligence. Intelligent, il est capable d'élucider ; énergique, il est capable de décision. Être capable d'élucider suffit pour reconnaître le mal ; être capable de décision suffit pour agir avec autorité et punir. Aussi, les rois doivent-ils employer une telle énergie et une telle intelligence, pour remédier au mal dans l'univers et procéder à sa destruction. $\mathrm{Ce}$ sera accomplir une auvre grande et louable. La coerc ition par la destruction est le cas le plus grave de l'emploi des châtiments ; or, si l'intelligence est extrême, il n'est pas de détail, quelque minime qu'il soit, qui ne soit scruté et éclairé ; si la décision est extrême, il n'y aura ni indulgence, ni pardon ; si ces qualités ne sont contenues par la justice, il y aura excès de sévérité dans le jugement. Extirpant le vice de l'univers, si on recherche jusqu'au bout tout ce qui, à un degré quelconque, peut être imprégné de fausseté ou d'erreur, comment ré ussir à punir tout ce qui est coupable ? Le mal résultant de cet excès de sévérité sera lui-même considérable. Aussi il convient seulement de choisir et de prendre les principaux chefs ${ }^{2}$; ce qui est pris n'étant pas le vulgaire, il n'y aura pas culpabili té par excès de sévérité cruelle. Le Shou king dit: «Exécuter les chefs des brigands; ne pas punir leurs complices. »

TSHOU HI. — L'énergie et l'intelligence atteignent au loin, l'autorité magistrale ébranle et punit sans erreur ; c'est une voie qui ne comporte aucune culpabilité, aussi tels sont l'image symbolique et le sens divinatoire.

559.

Le roi se prépare à la guerre, pour redresser les États.

\footnotetext{
1 À la fin de l'attachement.

${ }^{2}$ Les principaux coupables.
} 
TSHENG TSE. - Le roi déploie les facultés indiquées par ce trait supérieur nonaire ; il élucide avec intelligence et prononce avec décision, afin de rechercher et d'extirper le mal dans l'univers. C'est par là qu'il redresse et corrige les États. L'énergie et l'intelligen ce constituent la voie rationnelle de celui qui occupe un rang supérieur. 


\section{E U X I È M E P A R T I E}




\section{Hien}

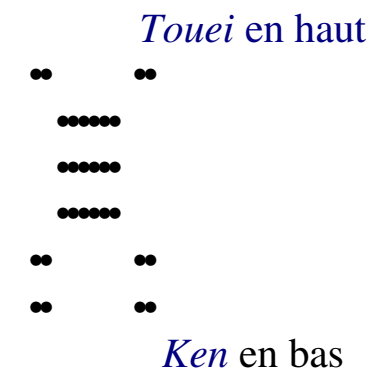

560. Hien, liberté, avantage de la perfection, Présage heureux en épousant une jeune fille.

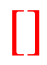

565. Premier trait hexaire: influencer l'orteil.

$*$

567. Deuxième trait hexaire : influencer le mollet ; présage malheureux; présage heureux de la station.

$*$

569. Troisième trait nonaire : influencer la jambe; se tenir à ce qu'on suit ; en entreprenant, appréhension.

*

571. Quatrième trait nonaire : présage heureux de la perfection, oubli des regrets; aller et venir avec incertitude; les amis suivent la pensée.

*

573. Cinquième trait nonaire : influencer les muscles de la colonne vertébrale; pas de regrets.

*

575. Trait supérieur hexaire: influencer les os des joues et la langue.

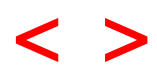




\section{Hien.}

TSHENG TSE. - Hien. «L'Ordre des koua » dit : « Le ciel et la terre existant, l'existence de toutes choses en résulte immédiatement ; de là la distinction des sexes, et de celle-ci la relation d'époux et d'épouse. De la relation d'époux et d'épouse résulte nécessaire ment à son tour celle de père et d'enfant, et de même, de celle-ci, celle de prince et de sujet. De cette dernière, résulte encore la relation de supériorité et d'infériorité, d'où découle, comme conséquence, que les règles rituelles et les devoirs réciproques peuvent être définis. Le ciel et la terre sont l'origine de toutes choses ; la relation d'époux et d'épouse est le commencement de tou s les rapports sociaux : c'est là ce qui fait que la première partie du king débute par les koua khien et khouen, tandis que la seconde partie débute par le koua hien et continue par le koua heng. Le ciel et la terre sont deux choses distinctes, aussi ces deux koua posent séparément les voies rationnelles du ciel et de la terre. Le jeune homme et la jeune fille s'unissent et deviennent époux et épouse, aussi le koua hien et le koua heng, tous deux également composés des deux substances réunies, posent ensemble le devoir entre les époux. » Hien équivaut à kan, « ressentir l'influence de quelque chose ; être ému »; c'est le plaisir qui dirige. Heng équivaut à sheang, " persistance, durée permanente »; c'est la droiture qui sert de base, et ainsi la voie naturelle du plaisir est réglée par la droiture et le devoir. La voie naturelle et la droiture comportent certainement le plaisir. Humilité et mouvement ${ }^{1}$; dureté énergique et douceur malléable se correspondant toujours sympathiquement : donc plaisir et satisfaction. Ce qui constitue le koua complet hien, c'est le koua simple touei, en haut, et le koua simple hen en bas la jeune fille et le jeune garçon. L’intensité de l'influence réciproque du sexe mâle et du sexe féminin l'un sur l'autre n'est jamais aussi grande que dans la jeunesse, et c'est pour ,.cela que les deux koua simples qui expriment le jeune âge chez les deux sexes constituent ensemble le koua hien. La substance du koua simple ken est essentiellement la fermeté ; l'arrêt, exprimé par ce koua simple, constitue le sens de vérité sincère. Les tendances de l'être mâle sont la fermeté et la sincérité et elles l'entraînent pour descendre, pour s'unir ${ }^{2}$; le coar de la femme en est satisfait et elle monte sympathiquement vers lui : c'est là le prélude de l’influence du mâle. Le jeune garçon emploie d'abord la sincérité pour faire sentir son influence, de sorte que la jeune fille en éprouve du plaisir et lui répond sympathiquement.

\footnotetext{
${ }^{1}$ Indiqués par les deux koua simples.

2 Les diverses acceptions des caractères employés permettraient de lire : «Ses tendances portent à diriger la génération. »
} 


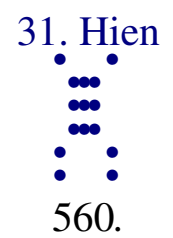

Hien, liberté, avantage de la perfection, Présage heureux en épousant une jeune fille.

TSHENG TSE. - Hien équivaut à kan : impression, influence. Le texte n'emploie pas le caractère kan, mais le caractère hien réunit les mêmes sens. Le sexe masculin et le sexe féminin s'unissent et s’influencent réciproquement. L'influence réciproque des êtres n'est jamais comparable à celle que les deux sexes exercent l'un sur l'autre, et dans le jeune âge elle est spécialement forte. Le prince et le sujet, le supérieur et l'inférieur, et jusqu'aux êtres quels qu'ils soient, subissent tous une loi d'influence réciproque. L'influence réciproque des êtres doit nécessairement avoir une raison d'être de liberté et de pénétration. Si le prince et le sujet peuvent sinfluencer mutuellement, c'est que la voie rationnelle du prince et du sujet est libre et ouverte; si le supérieur et l’inférieur peuvent s’influencer réciproquement, alors les tendances du supérieur et de l'inférieur se développent librement. En continuant jusqu'au père et à l'enfant, à l'époux et l'épouse, aux parents et alliés, aux amis et aux compagnons, si pour chacun les sentiments et les idées exercent une influence réciproque, alors la paix et l'harmonie prévalent libre ment; pour les choses et les êtres, il en est toujours de même, et c'est pour cela que l'influence mutuelle exprimée par le koua hien a une raison d'être de liberté. "Avantage de la perfection », dans la voie rationnelle de l'influence réciproque, l'ava ntage consiste dans la droiture et le devoir. Si cette influence ne dérive pas de la droiture, elle tombe dans ce qu'on appelle le mal. Ainsi, si entre l'époux et l'épouse elle devient licence et volupté, si entre le prince et le sujet elle devient séduction et flatterie, si entre le supérieur et l'inférieur elle devient corruption et bassesse, dans tous les cas, l’influence mutuelle ne dérive plus de la droiture. "Présage heureux en épousant une jeune fille » est dit au sujet des aptitudes exprimées par les koua simples. Dans ce koua, il y a douceur malléable en haut et dureté énergique en bas; les deux éthers s’influencent sympathiquement et s'allient ensemble. Arrêt et satisfaction ; c'est le sens de l'homme s'abaissant devant la femme. À cause de ce sens, sil s'agit d'épouser une jeune fille, il y a conformité à la droiture et présage heureux.

TSHOU HI. - Hien, joindre et émouvoir ; la douceur malléable du koua simple touei en haut, la dureté énergique du koua simple ken en bas, et elles se joignent mutuellement s'influençant réciproquement. Ou bien encore, l'arrêt, du koua ken, exprimera l'influen ce exclusive, et la satisfaction, du koua touei, exprimera le plus haut degré de sympathie correspondante. Ou encore, le 
jeune garçon représenté par le koua simple ken s’inclinant devant la jeune fille représentée par le koua simple touei; le jeune garçon en avant de la jeune fille, la conformité à la droiture (au devoir) entre jeune garçon et jeune fille, le moment de l'union matrimoniale, de sorte que le koua complet exprime le sens du mot hien: jonction et influence réciproque. Le sens divinatoire est «liberté et avantage de la droiture ; présage heureux en épousant une jeune fille »; en effet, l'influence mutuelle comporte une raison d'être qui nécess ite la liberté de sentiment; cependant, si cette influence ne s'exerce pas conformément à la droiture, elle perd cette voie de liberté et ses résultats sont tous malheureux.

561.

Le commentaire traditionnel de la formule déterminative dit: Hien, influence.

TSHOU HI. — Explication du sens du nom du koua.

562.

Douceur malléable en haut et énergie en bas, les deux éthers s'influencent sympathiquement et ils s'unissent ensemble; joie et arrêt. Le sexe masculin s'abaisse devant le sexe féminin; c'est jouir de la liberté et de l'avantage de la perfection: présage heureux en épousant une jeune fille.

TSHENG TSE. - Le sens du mot hien est han, «influencer». Dans le koua, le trait qui exprime la douceur malléable est au-dessus et les traits qui indiquent l'énergie sont au-dessous : la douceur malléable monte modifier la dureté énergique et produit la satisfaction en donnant naissance au koua simple touei ; la dureté énergique descend modifier la douceur malléable et produit le koua ken, arrêt. La négativité et la positivité s'unissent ensemble, ce qui constitue le sens du garçon et de la fille s'alliant et échangeant leurs influences. De plus, le koua touei, qui représente le sexe féminin, la fille, est au-dessus ; le koua simple ken, qui représente le sexe masculin, le garçon, est au-dessous; c'est encore la douceur malléable au-dessus et la dureté énergique au-dessous. La négativité et la positivité, les deux éthers s’influencent réciproquement, se correspondent mutuellement avec sympathie, et ils s'accordent harmoniquement : c'est l'alliance mutuelle. Arrêt et satisfac - 
tion ; l'arrêt dans la satisfaction exprime l'idée de fermeté dans la sincérité. Le koua simple hen, qui exprime l'arrêt, est en dessous ; sincérité exclusive s'alliant au -dessous de soi. Le koua simple touei, qui exprime la satisfaction, est au-dessus ; harmonie, satisfaction et sympathie mutuelle. Quand il s'agit de l'homme et qu'il s'incline devant la femme, c'est le plus haut degré de la concorde. Telle est la voie rationnelle de l'influence réciproque, et c'est ainsi qu'il peut y avoir liberté avec conformité à la droiture ; prendre une épouse dans de telles conditions est un présage heureux. Telles sont les aptitudes exprimées par les koua simples. Dans la grande majorité des cas, la voie de l'influence est avan tageuse par la droiture.

TSHOU HI. - Explication de la formule du koua au moyen de la substance, des vertus et des images symboliques des koua simples qui le composent, on peut aussi en parler au sujet de la transformation du koua : la douceur malléable monte ; la dureté énergique descend; le sens de ces mots exprime que le koua hien provient du koua lou, dans lequel le trait qui représente la douceur monte occuper le sixième rang, tandis que le trait qui exprime l'énergie descend occuper le cinquième. Cela est encore admissible.

563.

Le ciel et la terre s'influencent ${ }^{1}$ et toutes choses se transforment et naissent. L'homme saint influence le cour des hommes et l'univers est dans la concorde et dans la paix. En regardant ce qui influence, les sentiments ${ }^{2} d u$ ciel, de la terre et de toutes choses sont visibles.

TSHENG TSE. - Après avoir parlé du sens de l’influence mutuelle que les deux sexes exercent l'un sur l'autre, le commentaire revient à une dernière analyse de la voie rationnelle de l'influence, afin d'épuiser la raison d'être qui dérive du ciel, de la terre et de l'action de l'homme saint. Les deux éthers du ciel et de la terre s'unissent et s'influencent, ils transforment et font naître tous les êtres; l'homme saint pousse la sincérité à l'extrême pour influencer le cour des êtres innombrables qui composent la foule, et l'univers jouit de la concorde et de la paix. Ce qui fait que tous les cours, dans l'univers, jouissent du repos et de la paix, c'est linfluence que l'homme saint exerce sur eux. En regardant la raison d'être suivant laquelle le ciel et la terre s'unissent et sinfluencent et engendrent tous les êtres, et la voie rationnelle suivant laquelle l'homme saint influence le cœur des hommes pour amener 1 harmonie et la concorde, les sentiments ${ }^{3}$ du ciel, de la terre et de tous les êtres

\footnotetext{
${ }^{1}$ Ou s'unissent.

${ }^{2}$ Le naturel.

${ }^{3}$ La nature.
} 
deviennent évidents. Quant à la raison d'être de cette liberté d'influence, ceux qui connaissent la voie rationnelle peuvent la contempler en gardant le silence.

TSHOU HI. - Dernière dissertation sur la raison d'être de la liberté de l’influence.

564.

Le commentaire traditionnel de la formule symbolique dit: Sur la montagne il y a le marais : union. L'homme doué pratique l'annihilation pour subir l'ascendant de l'homme.

TSHENG TSE. - La nature du marais, ou de l'humidité, est d'imbiber en descendant, la nature de la terre est de subir l'imbibition. Le marais est sur la montagne et son humidité imbibe peu à peu et pénètre sans obstacles ; c'est que les éthers des deux substances sinfluencent et se pénètrent librement. L’homme doué, considère l’image symbolique de la pénétration des éthers de la montagne et du marais et il annihile sa propre initiative pour subir celle d'autrui, car quand l'homme est dégagé de préventions intérieures, il peut recevoir une influence extérieure ; s'il en est rempli, rien ne peut pénétrer. L'anni hilation intérieure, c'est la suppression du moi ; l'intérieur ne subissant plus une domination personnelle, il n'est plus aucune influence qui ne puisse pénétrer librement, pour s'y développer et s'y implanter. L'influence s'accommode à ce milieu et elle y est reçue ; ce n'est pas la voie rationnelle de l'influence, nécessairement pénétrante de l'homme saint ${ }^{1}$.

TSHOU HI. - Sur la montagne il y a le marais ; employer l'annihilation et percevoir.

${ }^{1}$ Ce passage obscur est une allusion aux deux doctrines différentes de Lao Tse et de Khong Tse. 


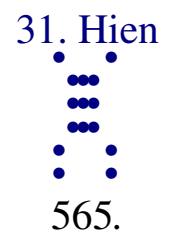

Premier trait hexaire : influencer l'orteil.

TSHENG TSE. - Le premier trait hexaire se trouve en bas du koua simple inférieur; lui et le quatrième sinfluencent réciproquement. Puisquil s'agit de quelque chose de minime, placé au premier rang, son influence n'est pas encore considérable ; comment pourrait-elle agiter l'homme lui -même ! Aussi, c'est comme le mouvement de l'orteil de l'homme, encore insuffisant pour déterminer la progression en avant. Le caractère meou, du texte, désigne le gros doigt du pied. Dans l'influence mutuelle que les hommes exercent réciproquement les uns sur les autres, il y a des différences d'intensité ou de faiblesse ; cette influence peut être profonde ou superficielle ; si on connaît la force inhérente au moment considéré, ce qui sera fait ne sera jamais autrement qu'approprié aux circonstances.

TSHOU HI. - Meou, gros doigt du pied. Dans le koua hien, les images symboliques sont empruntées au corps de l'homme. Influencer dans les choses les plus inférieures; c'est l'image symbolique d'influencer l'orteil. Limpression étant encore superficielle, il y a désir d'avancer et pas encore capacité de progresser. Aussi, la formule ne parle ni de présage heureux, ni de présage malheureux. Dans ce koua, bien qu'il s'agisse spécialement de l'influence et que tout en dépend, cependant les six formules des traits comportent toutes la convenance du calme et jamais celle du mouvement.

566.

Influencer l'orteil ; tendance vers l'extérieur.

TSHENG TSE. - Mouvement des tendances du premier trait, produit par l'influence du quatrième, de sorte que la formule dit : «vers l'extérieur ». Bien que les tendances soient éveillées, l’impression est encore superficielle ; c'est comme le mouvement du gros orteil, insuffisant pour faire avancer. 


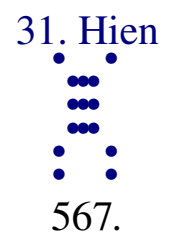

Deuxième trait hexaire : influencer le mollet ; présage malheureux; présage heureux de la station.

TSHENG TSE. - Le deuxième trait, à cause de sa douceur malléable placée dans un rang inférieur, correspond sympathiquement au cinquième, aussi la formule pose l'avertissement au sujet de l'impression ressentie au mollet ${ }^{1}$. Le mollet est le muscle moteur du pied; pour marcher, il faut d'abord le mettre en mouvement, et alors le pied se soulève. C'est surtout dans le mollet que réside l'initiative du mouvement dans la marche. Si le second trait n'observe pas la voie ra tionnelle en attendant l'appel du supé rieur, et sil provoque le mouvement comme le muscle du mollet cause le mouvement de la marche, il se perdra lui-même par sa précipitation inconsidérée, et c'est par là que le présage est malheureux. En se contentant de la position qu'il occupe et en ne se remuant pas, pour attendre l'ordre du supérieur, il se conformera à la voie rationnelle du mouvement en avant ou en arrière, et le présage sera heureux. Le second trait représente l'homme doué de justice et de droiture, c'est parce qu'il fait partie du koua hien et qu'il correspond sympathiquement au cinquième trait, que la formule comporte cet avertissement; elle revient ensuite sur le même sujet et ajoute : "Présage heureux de la station »; s'il se contente de s a condition et ne se meut pas de lui-même, le présage sera heureux.

TSHOU HI. - Mollet, muscle moteur du pied ; pour marcher il faut que le muscle commence à remuer ; cela exprime l'empressement prématuré sans savoir se contenir avec fermeté. Le second trait correspond précisément à cette situation ; de plus, à cause de sa douceur malléable, il est incapable de se contenir avec fermeté, c'est pourquoi il prend cette image symbolique. Cependant il possède les vertus de justice et de droiture ; il est capable de garder la position quil occupe, aussi le sens divinatoire est que le mouvement est un présage malheureux et que le calme est un présage heureux.

568.

\footnotetext{
${ }^{1}$ Ou influence exercée sur le mollet.
} 
Bien que le présage soit malheureux, en restant en place il devient heureux; avec la soumission, point de mal.

TSHENG TSE. - Le second trait se maintient dans la justice et se conforme à la droiture ; le trait auquel il correspond sympathiquement est aussi juste et droit; ses aptitudes sont essentiellement bonnes. C'est parce qu'il se trouve dans le moment exprimé par le koua hien, parce que son caractère physique est la douceur malléable et qu'il sympathise à ce qui est au-dessus de lui, que la formule avertit au sujet du présage malheureux qui résulterait de son mouvement s'il prenait l'initiativ e de s'adresser au prince ; sil demeure dans la position et s'observe lui -même, le présage sera heureux. Le commentaire de la formule symbolique revient sur ce point, pour l'éclairer, en disant que ce n'est pas un avertissement «qu'ils ne peuvent pas sinfluencer réciproquement», mais seulement qu'en se soumettant passivement à la raison d'être des choses il ne lui arrivera pas de mal ; cela veut dire observer la voie rationnelle et ne pas prendre l'initiative du mouvement. 


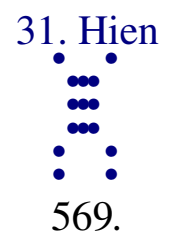

Troisième trait nonaire : influencer la jambe ; se tenir à ce qu'on suit ; en entreprenant, appréhension.

TSHENG TSE. — Le troisième trait nonaire emploie la positivité pour se maintenir dans une position qui comporte l'énergie ; il a les aptitudes de la dureté positive énergique, et c'est de lui que tout dépend à l'intérieur ${ }^{1}$. Il occupe le rang supérieur dans le koua simple inférieur. Cela exprime donc qu'il convient qu'il s'attache à la droite voie rationnel le pour étendre son influence à toutes choses; cependant, dans ce cas, il correspond sympathiquement au trait supérieur hexaire. La positivité aime à monter et se plaît au contact de la négativité ; le trait supérieur occupe l'extrême limite du koua qui exprime linnfluence de la satisfaction, aussi le troisième subit son influence et le suit. La jambe est au-dessous du corps et au-dessus du pied; par elle-même elle est incapable de produire aucun effet ; elle suit l'impulsion du corps et se meut, c'est c e qui motive le choix de cette image symbolique. Cela exprime que le troisième trait nonaire ne peut se diriger de lui-même ; il suit quelque chose et se meut, exactement comme la jambe. Ce quil tient et conserve, c'est la condition de suivre quelque chos e. Les aptitudes de la dureté énergique positive lui font ressentir l'influence de ce qui lui plaît et le suivre ; être dans de telles conditions et entreprendre quelque chose doit conduire à des méprises et à des regrets.

TSHOU HI. - La jambe suit l'impul sion du pied et se meut ; elle ne peut rien faire par elle-même. "Tenir », comporte l'idée de nécessité de tenir et de conserver. Les deux inférieurs veulent tous deux s'agiter ; le troisième, lui aussi, ne peut pas se contenir lui-même et les suit; en entreprenant quelque chose, il y aura appréhension ; c'est pourquoi tels sont le sens divinatoire et l’image symbolique.

570.

Influencer la jambe; il ne reste pas non plus en place; ses tendances le portent à suivre quelqu'un; ce qu'il tient est ce qui est au-dessous.

${ }^{1}$ Il est le maître du koua simple intérieur. Dans un autre ordre didées, il représente un magistrat investi d'un gouvernement territorial et qui règle les affaires de politique intérieure. 
TSHENG TSE. - Le texte porte le caractère $y i$, qui exprime la comparaison et l'analogie; en effet, la formule symbolique ${ }^{1}$ n'est essentiellement pas de même nature que celle du Yi king ; par elle-même, elle forme un tout séparé, de sorte que dans toutes les formules du commentaire symbolique l'idée contient un certain lien de connexité. Ici la formule emploie le caractère $y i$, « encore », « de la même manière », «aussi », et c'est par rapport à la formule précédente ${ }^{2}$. D'abord elle dit ${ }^{3}:$ " Influencer l'orteil ; tendances vers l'ex térieur», puis elle ajoute ${ }^{4}$ : «Bien que le présage soit malheureux, en restant en place il devient heureux ; avec la soumission, point de mal », et enfin: Influencer la jambe, elle ne reste pas non plus (yi) en place. D'abord les deux traits négatifs sont influencés et se meu vent, et bien que le troisième soit positif, il est encore dans le même cas ; c'est pour cela que la formule dit qu'il ne reste pas non plus en place. Ne pas rester en place, c'est-à-dire, se mouvoir. Il a les caractères physiques extérieurs de l'énergie positive et ne peut se diriger et se maîtriser lui-même; au contraire ses tendances le portent à suivre autrui ; c'est donc que la chose à laquelle il se tient et s'attache est extrê mement basse et inférieure.

TSHOU HI. - La formule emploie le mot $y i$, « encore, de même », parce que les deux traits précédents veulent aussi se mouvoir. Ces deux traits expriment tous deux l'impa tience de la négativité et le mouvement en est la conséquence naturelle ; mais le troisième trait nonaire est doué de la dureté énergique positive, il occupe le plus haut rang dans le koua simple qui exprime l'arrêt, il conviendrait qu'il fût calme, et cependant il se meut : il y a, au plus haut point, motif d'app réhension et de regrets.

\footnotetext{
${ }^{1}$ Le commentaire de Khong Tse.

$2 \mathrm{~N}^{\circ} 568$.

${ }^{3} \mathrm{~N}^{\circ} 566$.

${ }^{4} \mathrm{~N}^{\circ} 568$.
} 


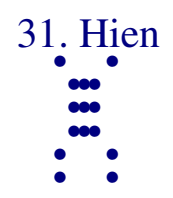

571.

Quatrième trait nonaire : présage heureux de la perfection, oubli des regrets ; aller et venir avec incertitude; les amis suivent la pensée.

TSHENG TSE. - Linfluence, ou impression, est ce qui détermine le mouvement de l'homme, aussi toutes les formules empruntent leur symbole au corps de l'homme. Au sujet de l'orteil, c'est la condition d'être en bas et de donner le mouvement initial qui est relevée ; au sujet du mollet, c'est celle de commencer le mouvement; dans le cas de la jambe, c'est la condition de suivre l'impulsion qui est considérée. Dans le cas du quatriè me trait, il n'y a rien à remarquer, et la formule parle directement de la voie rationnelle de l'influence. La formule ne parle point «d'influencer le cour »; l'influence, ou l'impression, c'est le coaur lui -même. Le quatrième trait est au milieu du koua et dans la partie supérieure ; il occupe la position du coar, aussi c'est de lui que dépend l'influence et la formule parle de la voie rationnelle de l'influence. Avec la perfection de la droiture, le présage sera heureux et les regrets seront oubliés; si l'influence ne s'exerce pas suivant la droiture, il y aura des regrets. De plus, le quatrième trait fait partie de la substance du koua simple qui exprime la satisfaction; il occupe un rang négatif et sympathise avec le premier, aussi la formule avertit au sujet de la perfection, ou pureté. La voie de l'influen ce pénètre partout, sans exceptions ; si l'on est retenu par des considérations égoïstes, cela nuira à la liberté de l'influence, et c'est alors qu'il y aura des regrets. L'homme saint influence le cour de tout l'univers, comme le froid et le chaud, comme la pluie et le soleil ${ }^{1}$; son influence pénètre tout, rien n'y reste insensible : c'est encore uniquement à cause de sa perfection. Perfection, est une expression qui désigne l'absence absolue de préjugés et l'annihilation des préférences personnelles. "Aller et venir avec certitude ; les amis suivent sa pensée »; en effet, si la perfection est absolue, son influence pénètre librement partout; mais lorsqu'il s'agit d'un mouvement de va-et-vient irrésolu et que les sentiment privés et égoïstes sont mis en usage pour influencer quelque chose, alors ceux à qui ces sentiments conviendront pourront parfois être influencés et se mettre en mouvement, tandis que ceux que ces sentiments n'atteindront pas ne pourront pas être influencés. C'est ce que la formule exprime en disant que les ami s et ceux du même genre suivent la pensée. Puisqu'il s'agit d'être retenu par les affections égoïstes, en gouvernant une région ou en dirigeant quelque chose, comment serait-il possible que l'action s'exerçât avec ampleur et qu'elle pût s'étendre librement à tout sans exception ? Les «Formules annexées » disent : «Quelles

\footnotetext{
${ }^{1}$ La pluie amollit, le soleil durcit (Tse tien).
} 
sont les pensées et les préoccupations de l'univers ? Tout aboutit au même point quoique par des voies diverses. Un seul fait qui survient soulève mille préoccupations. Quelles sont donc les pensées et les préoccupations de l'univers ? » Dans ce passage, Khong Tse vise le koua hien et analyse la voie rationnelle de la liberté de l'influence. Or, lorsqu'on influence les êtres en se guidant sur des motifs égoïstes nés de préoccupations particulières, le champ de cette influence se trouve rétréci. Dans l'uni vers, la raison d'être des choses est unique ; bien que les voies soient différentes, elles tendent toutes au même résultat; bien que les sujets de préoccupation soient innombrables, leur résultat est invariable ; bien que les êtres diffèrent entre eux de mille façons, bien que les choses puissent se modifier à l'infini, tout revient à un centre unique: il est donc impossible d'y résister et d'y contrevenir. Ainsi, en purifiant l'idée, l'univers entier sera librement pénétré par l’influence ; c'est pour cela que le passage cité plus haut dit: «Quelles sont les pensées et les préoccupations de l'univers ?» En employant les pensées égoïstes, comment l'influence pénétrerait-elle partout? Lorsque le soleil part, la lune vient; lorsque la lune s'en va, le soleil arrive ; le soleil et la lune s'entraînent mutuellement et la lumière naît. Si le froid s'en va, la chaleur arrive ; si la chaleur passe, le froid survient; le froid et le chaud se rencontrent réciproquement et par leurs périodes l'année s'achève. S'en aller, c'est le mouvement de contraction; venir, c'est le mouvement de dilatation; la contraction et la dilatation se provoquent mutuellement et le bien en résulte. Ceci explique la raison d'être de l’influence et de la sympathie par les phéno mènes de l'aller et du venir, de la contraction et de la dilatation ; s'il y a contraction, il y aura dilatation, et s'il y a dilatation, il y aura contraction : c'est là ce qu'on appelle «influence et sympathie » 1 . C'est à cause de cette loi que le soleil et la lune produisent leur clarté par leur double action réciproque ; que l'année se complète par les périodes marquées par la double action du froid et de la chaleur; l'action, ou cau se, et l'effet se produisent par la même loi, et c'est pour cela que la formule dit que la contraction et la dilatation se déterminent mutuellement et que le bien en résulte. Influencer, c'est faire naître le mouvement ; s'il y a influence, il y a nécessai rement sympathie correspondante ; tout mouvement est causé par une influence et toute influence doit avoir un effet sympathique correspondant; ce qui est effet sympathique devient à son tour influence et cause et toute influence cause redevient effet, de sorte que l'enchaînement n'a pas de fin. La chenille tshi hиo se contracte pour s'étendre ensuite ; le dragon et le serpent se cachent sous terre pour préserver leur corps; le pur devoir pénètre l'esprit pour produire ses effets ; le bien assure le repos du corps, pour y compléter toutes les vertus ; passé ce point et en continuant, on ne connaît rien encore. Ce qui précède explique la raison d'être de la contraction et de la dilatation ${ }^{2}$. Si on revient encore aux êtres pour éclairer le sujet, la marche de la chenille tshi huo

\footnotetext{
${ }^{1}$ Cause et effet.

${ }^{2}$ Cette expression, mal définie, même pour les Chinois, une des formes sous lesquelles ils expriment les idées de cause et d'effet, est visiblement déri vée des termes qui désignent les phases de la lune.
} 
consiste d'abord dans un mouvement de contraction suivi d'un mouvement de distension; en effet, sans contraction, il n'y aurait pas allongement ; après l'allongement, il y a de nouveau contraction ; en considérant cet insecte on comprend la raison d'être de l'influence et de l'effet sympathique correspondant. Le concèlement du dragon et du serpent dans la terre est le moyen par lequel ils assurent le repos de leur corps pour pouvoir ensuite s'élancer avec impétuosité ; s’ils ne se repliaient sur eux-mêmes, ils ne pourraient bondir ${ }^{1}$; le mouvement, ou impulsion, et le repos se provoquent réciproquement : c'est là la contraction et la dilatation.

L'homme doué porte caché dans son cour la notion du devoir subtile et pure ; cette notion passe dans les spéculations de son esprit, et elle produit ainsi ses effets. La pureté subtile du sentiment inné dans le cour, c'est «l'accumulation »: l'effet qui en résulte, c'est l'extension générale de ce sentiment : l'accumulation et l'extension générale sont encore une forme de la contraction et de la dilatation. Le bien ${ }^{2}$ a pour effet d'assurer le repos du corps, pour développer et perfectionner ses aptitudes ou facultés ${ }^{3}$; en se reportant à ce qui précède et en parlant des effets produits, bénéficier de ses conséquences, assurer le repos matériel du corps, c'est le moyen par lequel les aptitudes et les facultés sont agrandies et rendues brillantes; la cause productive étant conforme à la raison d'être, chaque chose est correcte et le corps est en repos: l'aptitude de l'homme saint à toutes choses réside entièrement en ceci. C'est pour cela qu'il est dit qu'au -delà de ce point et en continuant personne n'est jamais arrivé à connaître plus. Élucider à fond la cause et connaître la transformation, c'est la perfection des facultés ; du moment où il est dit qu'au-delà de ce point et en continuant on n'est jamais arrivé à rien connaître, on peut encore terminer par cette autre proposition : épuiser l'analyse la plus profonde de la cause mystérieuse des phénomènes, connaître la voie rationnelle de la transformation et de la génération, c'est l'extrême perfection des facultés. Il n'y a rien à ajouter à ceci.

TSHOU HI. - Le quatrième trait nonaire est placé au-dessus de la jambe et au-dessous des muscles attachés à l'épine dorsale ; de plus, il se trouve être au milieu des trois positivités : c'est l'image symbolique du coar et c'est de lui que dépend l'influence. Lorsque le cour influence, il doit être droit et ferme, et alors tout est conforme à la raison d'être ; actuellement, le quatrième trait nonaire, positif, occupe un rang négatif; c'est considéré comme exprimant qu'il perd la droiture et ne peut posséder la fermeté, aussi l'avertisse ment est posé dans le sens divinatoire. Par la même raison, pouvoir posséder la droiture et la fermeté constituera un présage heureux et les regrets seront oubliés. Mais si l'on va et vient avec hésitation, sans pouvoir être ferme et droit, en étant retenu par des influences particulières, il n'y aura que les

\footnotetext{
${ }^{1}$ Il n'est pas impossible quill y ait ici une légère erreur de traduction et que les deux caractères que je traduis séparément par les mots dragon et serpent ne forment ensemble le nom d'un insecte ou d'un reptile particulier.

${ }^{2}$ Le bien matériel ; l'abondance des récoltes, etc.

${ }^{3}$ Les vertus.
} 
amis et ceux du même genre qui suivront et il ne sera pas davantage possible d'étendre l'influence au loin.

572.

Présage heureux de la perfection, oubli des regrets; ne pas encore être influencé par le mal. Aller et venir avec incertitude: pas encore d'éclat ni de grandeur.

TSHENG TSE. - Par la perfection le présage sera heureux et les regrets seront oubliés ne pas encore subir le mal causé par les influences particulières et égoïstes; si on est retenu par des sympathies égoïstes, l'influence sera diminuée. Aller et venir avec incertitude ; lorsque l'influence mutuelle dépend de considérations égoïstes, la voie de l’influence est rétrécie, aussi la formule dit qu'elle n'est encore ni grande, ni brillante.

TSHOU HI. — L'influence nuit ; c'est-à-dire influencer sans droiture ; il en résultera un mal. 


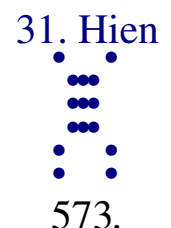

Cinquième trait nonaire : influencer les muscles de la colonne vertébrale; pas de regrets.

TSHENG TSE. - Ce trait nonaire occupe la situation prééminente ; il convient qu'il emploie l'extrême sincérité pour influencer l'univers. Cependant, il sympathise avec le second et se presse contre le trait supérieur. S'il est retenu par le second et s'il se plaît avec le trait supérieur, la partial ité égoïste l'envahit peu à peu : ce n'est pas là la voie rationnelle du prince ; comment pourrait-il influencer tout l'univers ! Le cinquième terme du texte, désigne les muscles de la colonne vertébrale ; ils sont opposés au cour et ils ne se voient pas mutuellement. Cela veut dire que sil peut aller contre ses propres tendances égoïstes, influencer d'autres que ceux qu'il voit et quil chérit, il possédera la droiture du prince influençant l'univers et il n'aura pas de regrets.

TSHOU HI. - Le cinquième caractère du texte désigne les muscles du dos, qui sont au-dessus du cœur, et qui sont inversement placés avec lui par rapport à la colonne vertébrale. Ces muscles ne peuvent imprimer l'impulsion à l'être et n'ont point d'attache. Le cin quième trait nonaire se trouve précisément dans une situation analogue, et c'est pour cela que cette image symbolique est choisie et que la formule avertit celui qui consulte le sort que, pouvant être dans de telles conditions, il en résultera que bien qu’incapable d'influe ncer les êtres, il pourra cependant ne pas avoir de regrets.

574.

Influencer les muscles de la colonne vertébrale, tendances émoussées.

TSHENG TSE. - Avertissement d'avoir à lutter contre son propre cœur et à donner l’impulsion aux muscles de la colonn e vertébrale ; pour contribuer à préserver le coaur. Il est attaché au second trait et se plaît avec le trait supérieur : c'est être influencé par les désirs privés.

TSHOU HI. - Tendances émoussées, c'est-à-dire «incapable d'influencer les êtres ». 


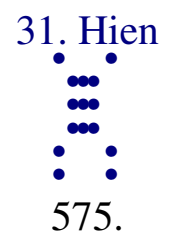

Trait supérieur hexaire : influencer les os des joues et la langue.

TSHENG TSE. — Le trait supérieur est mou, malléable et négatif, et il fait partie de la substance du koua simple qui exprime la satisfaction ; c'es t de lui que dépend la satisfaction. De plus, il occupe le rang extrême dans le koua qui exprime l'influence, c'est donc qu'il désire, au plus haut point, influencer les êtres. Aussi, il ne peut se borner à employer la plus extrême sincérité pour les influencer, et il manifeste son désir par la bouche, au moyen de la parole : telle est la manière d'agir habituelle des hommes inférieurs et des femmes. Comment serait-il capable de mettre les hommes en mouvement ! La formule ne désigne pas directement la bouche, mais elle parle des os des joues et de la langue ; c'est une locution analogue à l'expression moderne « commissure des lèvres » pour désigner l'excès de paroles. La formule dit : «os des joues, et langue »; les os des joues et la langue sont également les instruments de la parole.

TSHOU HI. - Les os des joues et la langue sont également ce qui sert pour parler; de plus, ce sont des parties supérieures du corps. Le trait supérieur hexaire emploie la mollesse négative et se tient au dernier rang du koua simple qui exprime la satisfaction ; il est placé au dernier rang du koua qui exprime l'action d'influencer : il influence les hommes au moyen de la parole et non par la réalité. De plus encore, le koua simple touei représente aussi la bouche et la langue, de sorte que telle est bien limage symbolique. Le malheur et les regrets sont évidents.

576.

Influencer les os des joues et la langue; parler avec une bouche écumante.

TSHENG TSE. — L'extrême sincérité seule peut influencer les hommes ; ici puisque la mollesse et la satisfaction sensuelle sont manifestées par le bouillonnement de la bouche, comment pourrait-il influencer les hommes ? 
TSHOU HI. - Le terme traduit par «écumant», ou «bouillonnant», équivaut à un autre caractère dont le sens exprime la rapidité et la passion dans le mouvement. 


\section{Heng}

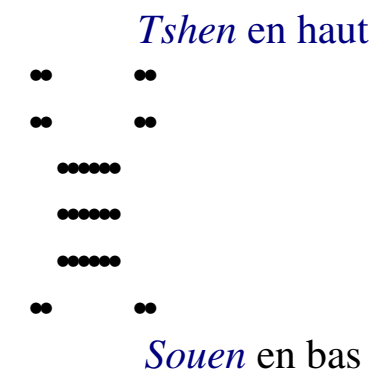

577. Heng ; liberté ; pas de culpabilité ; avantage de la perfection ; avantage dans ce qu'il y a à entreprendre.

583. Premier trait hexaire : approfondir la persistance ; présage malheureux de la perfection; aucun moyen davantage.

*

585. Deuxième trait nonaire : les regrets se dissipent.

$*$

587. Troisième trait nonaire : ne pas persister dans sa vertu; parfois en recueillir de la confusion ; appréhension résultant de la perfection.

$*$

589. Quatrième trait nonaire : chasser sans oiseaux.

$*$

591. Cinquième trait hexaire : persister dans la perfection de la vertu; présage heureux pour l'épouse ; présage malheureux de l'époux et des enfants.

$*$

593. Trait supérieur hexaire : ébranler la persévérance ; présage malheureux.

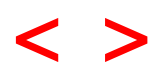




\section{Heng.}

TSHENG TSE. - Heng. «L'Ordre des koua » dit : «La voie morale de l'époux et de l'épouse comporte nécessa irement la durée ; c'est pour cela que le koua hien est suivi du koua heng : heng exprime la longueur de la durée. » Le koua hien exprime la voie morale de l'époux et de l'épouse ; l'époux et l'épouse sont unis pour toute la durée de leur vie sans modification ; aussi, après le koua hien vient le koua heng. Dans le koua hien le jeune garçon est au-dessous de la jeune fille; puisque le garçon s’incline devant la fille, il s'agit donc du sens d'échange mutuel d’influences entre le garçon et la fille. Dans le koua heng, le garçon adulte est placé au-dessus de la jeune fille adulte ; le mâle occupe la position prééminente, la fille le rang inférieur ; c'est la voie rationnelle de l'époux et de l'épouse dans l'intérieur du foyer. Si on discute la nature des influences échangées, le jeune âge indique le rapprochement intime; si on discute l'ordre de prééminence, l'âge mûr commande la circonspection et la droiture. C'est pour cette raison que les koua simples touei et ken constituent le koua hien, tandis que les koua simples tshen et souen constituent le koua heng. L'élément mâle est au-dessus de l'élément femelle ; le mâle se meut au-dehors et donne l'impulsion, la femelle la subit au-dedans : c'est la raison d'être constante entre les hommes, aussi le koua est appelé heng. Enfin la dureté énergique est en haut et la douceur malléable en bas ; la foudre et le vent se rencontrent ensemble humilité et mouvement; la dureté énergique et la douceur malléable se correspondent sympathiquement, c'est toujours le sens du mot heng ${ }^{1}$.

\footnotetext{
${ }^{1}$ Heng : «permanence, ordre constant et durable »; synonyme de sheang.
} 


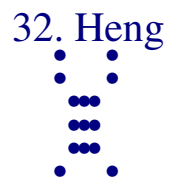

577.

Heng ; liberté ; pas de culpabilité ; avantage de la perfection ; avantage dans ce qu'il y a à entreprendre.

TSHENG TSE. - Heng : durée constante, permanence. La voie de la permanence doit être librement pénétrable. La permanence qui peut être accompagnée de la liberté d'action constitue l'absence de culpabilité ; sil y a permanence sans qu'elle puisse exister par la liberté, ce n'est plus la voie rationnelle de la possibilité de la permanence, et cela constitue l'existence de la culpabilité. Par exemple, la constance de l'homme doué dans la pratique du bien, c'est la voie rationnelle qui comporte la permanence ; la persistance de l'homme inférieur dans le mal, c'est la voie rationnelle qui fait perdre la possibilité de la permanence. Ce qui rend possible la liberté de la permanence, c'est la perfection et la droiture ; aussi la formule parle de l'avantage de la perfection. Car, ce qu'on appelle per manence, c'est la voi e rationnelle qui permet la durée permanente ; ce n'est pas l'obsti nation ${ }^{1}$. Aussi il y a avantage à entreprendre ${ }^{2}$; c'est seulement parce qu'il y a à entre prendre qu'il peut $\mathrm{y}$ avoir permanence : ce qui est déterminé et fixé une fois pour toutes ne peut pas constituer la permanence ${ }^{3}$. Enfin, la voie rationnelle de la durée permanente ne peut exister sans être avantageuse.

TSHOU HI. - Heng : durée permanente. Le koua est constitué par le koua simple tshen, actif, placé au-dessus, et par le koua simple souen, passif, placé au-dessous. Le koua simple tshen représente l'ébranlement de la foudre; le koua simple souen représente le vent; les deux choses se rencontrent et s'accordent. Le koua simple souen exprime la soumission passive ; le koua simple tshen exprime le mouvement impulsif : cela exprime la soumission dans l'action. Dans les deux substances ${ }^{4}$ et dans les six traits, la négativité et la positivité se correspondent sympathiquement; ces quatre conditions expriment toutes la permanence de la raison d'être des choses, aussi le koua est considéré comme exprimant le sens du caractère heng. Le sens divinatoire est que s'il y a possibilité de persistance dans la voie rationnelle, il y aura liberté et absence de culpabilité. Toutefois, il faut encore qu'il y ait avantage dans l'observation de la droiture, et alors cela constitue l'accord à la voie ration nelle de ce qui est persistant, et l'avantage existe dans ce qu'il y a à entreprendre.

\footnotetext{
${ }^{1}$ Littéralement : «Ce n'est pas savoir rester dans un coin sans savoir se modifier. »

$2 \mathrm{Ou}$ : l'avantage consiste à entreprendre.

${ }^{3}$ L'absolu, ce qui est en dehors de to ute condition d'existence n'a pas d'existence.

${ }^{4}$ Des deux koua simples.
} 
578.

Le commentaire de la formule déterminative dit : Heng, longue durée ; la dureté énergique est au-dessus et la douceur malléable au-dessous; l'ébranlement de la foudre et le vent s'allient ensemble; soumission et mouvement; la dureté énergique et la douceur malléable se correspondent toujours sympathiquement : permanence.

TSHENG TSE. - Heng : longueur de la durée. Les aptitudes exprimées par le koua expriment ces quatre conditions : sens de parfaite permanence. Dureté énergique et douceur malléable au-dessous : cela veut dire que le premier trait du koua simple khien monte occuper le quatrième rang, tandis que le premier trait du koua simple khouen descend occuper le premier : le trait qui exprime la dureté énergique monte et celui qui exprime la douceur malléable descend. Les deux traits changeant de position constituent les koua simples tshen et souen. Le koua simple tshen est au-dessus et le koua simple souen au-dessous : c'est encore la dureté énergique au-dessus et la douceur malléable au-dessous: c'est la voie rationnelle de la permanence. L'ébranlement de la foudre et le vent se rencontrent et s'allient : la foudre ébranlant, le vent s'élève ; ces deux phénomènes dérivent nécessairement l'un de l'autre et fortifient mutuellement leur puissance naturelle d'action : c'est pour cela que la formule emploie l'expression d'alliance mutuelle, et c'est en cela que consiste la permanence. Soumission et mouvement; en bas humilité et soumission passive, en haut ébranlement et mouvement impulsif, ce qui représente l'emploi de la soumission dans l'action. La créatio $\mathrm{n}$ et la transformation par le ciel et la terre sont permanentes et sans fin : ce n'est là que la soumission dans le mouvement; la soumission passive dans le mouvement, c'est la voie rationnelle de la permanence dans la durée. Comment l'impulsion sans soumission pourrait-elle conduire à la permanence ? La dureté énergique et la douceur malléable se correspondent toujours sympathiquement; les traits qui expriment la dureté énergique et la douceur malléable dans chaque koua se correspondent tous sympathiquement; la correspondance sympathique mutuelle entre la dureté énergique et la douceur malléable constitue la voie constante de la raison d'être naturelle des choses. Ces quatre conditions constituent la voie rationnelle de la permanence, et c'est à cause de cela que le koua est appelé heng.

TSHOU HI. - Explication du sens du nom du koua au moyen des substances des koua simples, de leurs images symboliques, et de leurs propriétés. Peut-être est-il aussi question de la transformation des koua; le sens des mots : la dureté énergique monte, la douceur malléable descend, peut exprimer que le koua heng provient du koua fong, dans lequel le trait qui 
exprime la dureté énergique monte occuper le second rang, tandis que celui qui exprime la douceur malléable descend occuper le premier ; cela est encore admissible.

579.

Permanence, liberté, absence de culpabilité; avantage de la pureté; persistance dans la voie rationnelle. La voie rationnelle du ciel et de la terre est la permanence et la durée infinie.

TSHENG TSE. - La voie rationnelle de la permanence peut produire la liberté, mais sans fautes et sans erreurs ; toutefois, ce qui est permanent doit être conforme à la droiture ; manquant à la droiture, ce ne sera plus la voie rationnelle de la possibilité de la permanence ; c'est pour cela que la formule dit : «persistance dans la voie rationnelle». Cette voie rationnelle, c'est la droite voie rationnelle de la permanence possible; si les vertus ne sont pas rendues permanentes, ou aussi bien s'il s'agit de $\mathrm{p}$ ermanence dans ce qui n'est point la droiture, dans tous les cas, la liberté n'est pas possible et il y a culpabilité. C'est en cela que consiste l'éternité du ciel et de la terre, et en effet le ciel et la terre ont une voie rationnelle de permanence indéfinie. Si l'homme peut persister dans une voie rationnelle qui com porte la permanence, il se conforme à la raison d'être du ciel et de la terre.

TSHOU HI. - La permanence peut certainement être libre et, d'ailleurs, sans culpabilité. Toutefois il faut nécessairement qu'elle soit avantageuse pour la droiture, et alors elle constitue la persistance durable dans la voie rationnelle. Sans la droiture, elle entraîne la durée de ce qui est contraire à la voie rationnelle. Si la voie rationnelle du ciel et de la terre est permanente et durable, c'est encore uniquement parce qu'elle est correcte et conforme à la droiture.

580.

Le bien a le moyen de se produire; ce qui a une fin aura un commencement.

TSHENG TSE. - La raison d'être du ciel et de la terre est que rien ne peut être permanent sans mouvement; ce qui se meut aura une fin et recommencera, ce qui assure la permanence et la durée infinie. Toute chose 
engendrée par le ciel et la terre, même aussi matériellement solide et vaste qu'un massif d'une chaî ne de montagnes, ne peut exister sans se modifier. Aussi le terme heng n'est pas une expression qui comporte le sens de fixi té déterminée. Ce qui est une fois fixé ne peut être permanent, mais le changement et la modification suivant le moment constituent la voie rationnelle de la permanence ordinaire. Aussi, la formule dit : avantage dans ce qu'il y a à entreprendre (ou ce qui revient au même : le bien a le moyen de se produire). Elle montre clairement que telle est la raison d'être des choses, de peur que les hommes ne s'endorment dans la routine de ce qui leur est habituel.

TSHOU HI. - Persistance dans la voie rationnelle : persistance indéfinie ; le bien a le moyen de se produire : commencement. Le mouvement et le repos s'engendrent réci proquement : c'est la raison d'être du mouvement circulaire. Toutefois, c'est le repos qui est la cause déterminante.

581.

Le soleil et la lune suivent le ciel et peuvent éclairer dune façon permanente et durable; les quatre saisons se modifient, se transforment et peuvent indéfiniment se produire; l'homme saint persiste indéfiniment dans sa voie morale et l'univers se transforme et se perfectionne. En regardant ce qui est permanent on peut voir la nature du ciel, de la terre et de tous les êtres.

TSHENG TSE. - Ceci est une dernière analyse de la raison d'être permanente. Le soleil et la lune sont, respectivement, la pureté absolue de l'éther positif ou négatif ; c'est uniquement par la soumission passive à la voie rationnelle du ciel et de la terre que chacun va et vient, croît ou décroît, de sorte que tous deux peuvent éclairer d'une façon permanente, et sans fin ; ils suivent le ciel : c'est-à-dire qu'ils suivent passivement la raison d'être du ciel. Les quatre saisons représentent l'éther positif et l'éther négat if ; elles passent et reviennent, modifient et transforment, produisent et achèvent toutes choses ; c'est encore parce qu'elles suivent le ciel, et c'est pour cela qu'elles peuvent durer éternellement. Lhomme saint emploie la voie rationnelle de la persistance continue; il la pratique d'une façon régulière, et l'univers se transforme sous son influence, les moars s'améliorant. En regardant sur quoi s'exerce la permanence, c'est -à-dire en regardant l'action du soleil et de la lune éclairant indéfiniment le renouvellement constant des quatre saisons, la voie morale de l'homme saint et la raison d'être qui en rend possible la durée permanente, en regardant tout cela, il devient possible de voir la nature et la raison d'être du ciel, de la terre, et de tout es choses. La voie rationnelle de permanence durable du ciel et de la terre, c'est la raison d'être qui assure la 
permanence de l'univers. Comment celui qui ne connaît pas la voie rationnelle ${ }^{1}$ comprendrait-il ceci ?

TSHOU HI. - Dernière analyse de la voie rationnelle de la permanence continue.

582.

Le commentaire traditionnel de la formule symbolique dit : Ébranlement de la foudre et du vent, persistance ; l'homme doué s' habitue à rester en place sans changer de région.

TSHENG TSE. — L'homme doué cons idère l'ébranlement de la foudre et le vent s'accompagnant mutuellement et produisant l'image symbolique de la permanence ; il en conclut à la persistance continue dans la vertu, se place dans la voie rationnelle de la grande justice constante et permanente, sans changement de lieu ou de région.

\footnotetext{
${ }^{1}$ La doctrine de Lao Tse.
} 


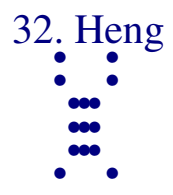

583.

Premier trait hexaire : approfondir la persistance; présage malheureux de la perfection; aucun moyen davantage.

TSHENG TSE. — Le premier trait occupe le rang inférieur et le quatrième est considéré comme lui correspondant sympathiquement avec droiture ; c'est l'homme mou et peu éclairé, apte à observer les règles ordinaires, mais incapable de mesurer le degré de la force inhérente à chaque chose. Le quatrième fait partie de la substance du koua simple tshen, et sa nature est positive ; il emploie la dureté énergique et occupe une position élevée ; ses tendances le portent à s'élever et non pas à descendre. De plus, il est entravé dans ses tendances sympathiques pour le premier par le second et le troisième traits, qui les séparent : c'est un cas différent de la règle ordinaire. Cependant, ici, le premier trait exprime la profondeur de la convoitise et de l'espérance, ce qui marque bien qu'il ne sait que suivre la voie ordinaire sans savoir la modifier suivant les circonstances. Le troisième caractère du texte a le même sens que le caractère [], approfondir, creuser, profond. Approfondir la persistance, c'est-à-dire : «profondeur du désir de persister ». S'en tenir à la règle ordinaire et être incapable de mesurer le degré de la force inhérente aux choses, exprime la profondeur dans l'attente et dans l'espérance dans le supérieur ; s'en tenir obstinément à cette espérance est une voie logique dont le présage est malheureux. En s'attachant ainsi d'une façon inébranl able à la voie ordinaire ${ }^{1}$, rien de ce qu'il peut entreprendre ne peut être avantageux. Dans ce monde, l'attachement à d'anciennes liaisons, d'où résultent les regrets et les fautes, provient toujours de la «profondeur de la persistance». Du moment où ses tendances sont profondément tournées vers le désir d'obtenir quelque chose d'un supérieur, c'est donc évidemment qu'il ne sait pas se contenter de la permanence en la situation actuelle. Étant minime et mou et ne sachant se contenter de la permanence dans sa situation actuelle, c'est donc encore une voie logique de présage malheureux. Dans tous les koua, le premier et le dernier traits expriment la superficie et la profondeur, le début insaisissable et le parfait développement. Être dans l'infériorité et se laisser aller à la profondeur des désirs, c'est encore ne pas savoir connaître l'opportunité du moment.

TSHOU HI. - Le premier et le quatrième traits sont considérés comme se correspondant sympathiquement avec droiture ; telle est la règle habituelle. Toutefois, le premier occupe le rang inférieur et se trouve au début, il ne doit

\footnotetext{
${ }^{1}$ Routine.
} 
pas encore se laisser aller à désirer profondément quoi que ce soit. Le quatrième fait partie de la substance du koua simple tshen et sa nature est positive ; il monte et ne descend pas; de plus il est entravé dans ses idées sympathiques pour le premier par la présence du second et du troisième traits interposés entre eux ; cela exprime un cas différent de la règle habituelle. La mollesse et le manque de discernement du premier ne lui permettent pas de mesurer la force inhérente à cette situation anormale; de plus, puisque malléable et négatif, il occupe le rang inférieur du koua simple souen, qui exprime l'humilité, c'est de lui que dépend cette humilité. Son naturel le po rte essentiellement à pénétrer, à se renfermer, aussi il s'attache profondément aux moyens ordinaires pour obtenir ce qu'il convoite, ce qui donne l'image symbolique d'approfondir la permanence, ou la profondeur de l'attache ment à la permanence. Si celui qui consulte le sort est dans ces conditions, bien qu'il puisse posséder la droiture, le présage sera malheureux et rien ne peut lui être avantageux.

584.

Présage malheureux d'approfondir la persistance ; commencer à désirer profondément.

TSHENG TSE. - Ce trait est placé au commencement du koua heng et il convoite profondément d'obtenir quelque chose du supérieur ; c'est là savoir suivre la voie ordinaire et surtout ne pas savoir mesurer la force inhérente aux conditions du moment. Ce qui fait le présage malheureux, c'est le manque de discernement ${ }^{1}$ et la faiblesse négative ne s'accordant pas aux nécessités de la permanence.

\footnotetext{
${ }^{1}$ L'obscurité.
} 


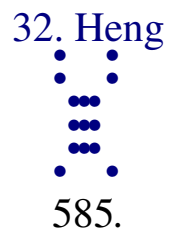

Deuxième trait nonaire : les regrets se dissipent.

TSHENG TSE. - Dans le sens que comporte le koua heng, demeurer dans une position conforme à la droiture constituera la voie rationnelle ordinaire. Les traits nonaires sont positifs ; lorsquils occupent une situation qui comporte la négativité ${ }^{1}$, ils ne sont plus dans les conditions régulières de la loi générale; se plaçant sans se conformer à la règle ordinaire, ils doivent naturellement avoir des regrets. Cependant, le second trait nonaire emploie la vertu de la justice et sympathise avec le cinquième ; le cinquième, à son tour, se maintient dans la justice; employant la justice et sympathisant avec la justice, la position où il se place, comme l'impulsion quill s e donne en se mouvant, sont également justes ; cela exprime qu'il peut persister d'une façon durable dans la justice. Pouvant persister d'une façon durable dans la justice, il ne manquera donc point à la droiture. La justice est plus importante que la droiture ; ce qui est juste est le droit, tandis que le droit ne comporte pas nécessairement la justice. Le second trait nonaire emploie les vertus de la dureté énergique et de la justice et il sympathise avec la justice: la prépondérance de la vertu est suffisante pour dissiper les regrets. Lorsque l'homme sait discerner le degré de gravité de la force inhérente à chaque condition, alors il est capable de parler du Yi king.

TSHOU HI. - Il emploie la positivité et occupe un rang qui comporte la négativité ; essentiellement il doit avoir des regrets. Mais puisqu'il persiste dans la justice, ces regrets peuvent donc être dissipés.

586.

Les regrets du second trait nonaire se dissipent; il peut persister dans ta justice.

TSHENG TSE. - Ce qui fait que ses regrets peuvent se dissiper, c'est qu'il est capable de persister indéfiniment dans la justice. Lorsque l'homme sait persister indéfiniment dans la justice, qu'est-ce qui pourrait empêcher ses regrets de se dissiper? Tel est le bien de la vertu.

\footnotetext{
${ }^{1}$ Un rang impair.
} 


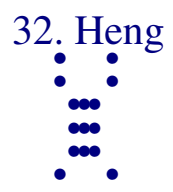

587.

Troisième trait nonaire : ne pas persister dans sa vertu ; parfois en recueillir de la confusion ; appréhension résultant de la perfection.

TSHENG TSE. - Le troisième trait est positif ; il occupe une situation positive; donc il se place dans une situation qui lui convient et c'est la position qui résulte pour lui des règles fondamentales ordinaires. Dans le cas actuel, ses tendances le portent à suivre le trait supérieur hexaire; non seulement la positivité et la négativité se correspondent mutuellement avec sympathie, mais, de plus encore, le vent suit l'ébranlement de la foudre ${ }^{1}$; il est placé dans un lieu qui comporte la persistance et il ne s'y établit point ; donc c'est un homme sans persistance. Ses vertus ne sont pas persistantes, de sorte que parfois il en recueille de la confusion et des affronts. L'expression : «parfois recueillir », veut dire qu'il y a des moments où le fait se produit. « Appréhension résultant de la perfection »; faisant consister la persévérance à s'attacher à l'inconstance, comment serait -il possible qu'il pût ne pas appréhender des affronts?

TSHOU HI. - Bien que sa situation soit conforme à la droiture, cependant elle comporte l'excès dans la dureté énergique et le manque de justice; ses tendances le portent vers le trait supérieur; il ne peut rester longtemps dans le même lieu, aussi il est considéré comme manquant de persistance dans ses vertus. Pour quiconque le sert, confusion ${ }^{2}$; quiconque est une expression qui indique le doute sur la personne; le huitième caractère équivaut à et cela veut dire que tout le monde peut se mettre en avant et le servir, sans qu'on sache d'où chacun provient. "Appréhension de la perfection »; la droiture sans constance est une cause probable de confusion ; c'est un nouvel avertissement à celui qui consulte le sort ${ }^{3}$.

588.

Il ne persévère pas dans sa vertu; rien à supporter.

\footnotetext{
${ }^{1}$ Allusion aux deux koua simples.

${ }^{2}$ Le commentateur lit tout autrement que Tsheng Tse : «Pour quiconque le sert, confusion. »

${ }^{3}$ Le commentaire est un exemple frappant de la divergence des interprétations du texte par les plus célèbres philosophes chinois. En réalité : aucun ne comprend le texte dont la vraie signification est perdue.
} 
TSHENG TSE. - Quand l'homme est sans persévérance, comment supporterait-il aucune position? Du moment où il ne peut supporter le lieu qu'il occupe et où il se place sans empressement ${ }^{1}$, comment pourrait-il y persister avec persévérance ? C'est pour cela que l'homme sans persévérance ne peut supporter aucun lieu et s'y placer à demeure.

\footnotetext{
${ }^{1}$ Sans satisfaction.
} 


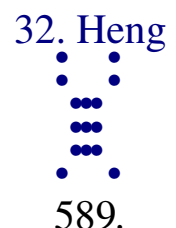

Quatrième trait nonaire : chasser ${ }^{1}$ sans oiseaux.

TSHENG TSE. - Employant la positivité et occupant un rang qui comporte la négativité, il se place dans une situation impropre, la situation ne lui convient point; bien qu'il y reste d'une façon permanente, où serait l'avantage ? Si les actions de l'homme concor dent avec la voie morale, son œuvre sera durable et produira un résultat méritoire ; si elles ne sont pas conformes à la voie morale, même avec de la persévérance, où serait l'avan tage ? C'est pour cela que l'action de chasser, $M$ (rizière), est prise comme exemple. La formule exprime que l'occupation du quatrième rang par un trait nonaire, bien qu'on la suppose permanente et durable, est comme l'action de chasser sans quil y ait capture de gibier; cela veut dire qu'il dépense inutilement son action et ses forces, sans résultat.

TSHOU HI. - Il emploie les qualités de la dureté énergique positive et occupe un rang qui comporte la négativité ; il persiste dans une situation qui ne lui convient point, ce qui est considéré comme donnant cette image symbolique. Celui qui consulte le sort chasse sans avoir rien à capturer, et, en toutes choses, il ne peut également pas arriver à obtenir ce qu'il convoite.

590.

Rester indéfiniment dans une situation impropre: comment capturer le gibier?

TSHENG TSE. - Se plaçant dans une situation impropre, bien qu'il y persiste, que pourrait-il en retirer ? L'action de chasser est prise comme exemple ; c'est pour cela que la formule dit : Comment capturer le gibier ?

\footnotetext{
${ }^{1}$ Le sens naturel serait « rizières sans oiseaux ».
} 


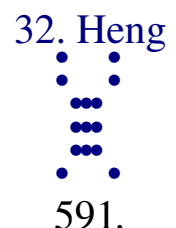

Cinquième trait hexaire : persister dans la perfection de la vertu ; présage heureux pour l'épouse ; présage malheureux de l'époux et des enfants.

TSHENG TSE. - Le cinquième trait correspond sympathiquement au second; il emploie la douceur malléable négative pour correspondre à la dureté énergique positive ; il se maintient dans la justice et ce à quoi il correspond possède aussi la justice ; pour la douceur malléable négative, cela constitue la droiture. Aussi, s'il persiste d'une façon durable dans ses vertus, cela constituera la perfection. Or, appliquer la persévérance à suivre passivement, c'est la voie rationnelle de l'épouse, de sorte que chez celle -ci, cela sera considéré comme constituant la perfection, aussi le présage est heureux. S’il s'agit d'un homme adulte, et s’il fait consister la persévérance dans le fait de suivre et d'écouter autrui, il manquera au devoir (droiture) de sa dureté énergique positive, et c'est en cela que consiste le présage malheureu x. Le cinquième rang indique la situation du prince et, cependant, la formule ne parle point de la voie rationnelle du prince; or, si le sens indiqué par le cinquième trait hexaire est déjà néfaste pour l'homme adulte, com bien à plus forte raison doit-il en être de même pour la voie rationnelle du prince ! Dans d'autres koua, lorsqu'un trait hexaire occupe la situation du prince et correspond à la dureté énergique, cela ne constitue pas encore un inconvénient, mais dans le koua heng il ne peut certainement pas en être de même. Comment la voie du prince pourrait-elle comporter la persistance dans la mollesse et la soumission?

TSHOU HI. - Il emploie la douceur malléable et la justice et sympathise avec la justice énergique ; il persévère indéfiniment sans changer ; droiture et fermeté. Mais cela est la voie rationnelle de l'épouse et ce qui ne saurait convenir à l'homme, aussi tels sont l’image symbolique et le sens divinatoire.

592.

Présage heureux de la perfection de l'épouse; suivre un seul jusqu'à la fin. L'homme se règle sur le devoir; s'il obéit à l'épouse c'est un présage malheureux. 
TSHENG TSE. - Suivre, comme le cinquième trait suit le second, constituera la droiture chez l'épouse et ce sera un présage heureux. Pour l'épouse c'est la soumissio n qui fait sa vertu et elle doit attacher son existence entière à un seul ${ }^{1}$. Pour l'homme, c'est sur le devoir qu'il se règle. ; la voie qui consiste à se soumettre à l'épouse sera pour lui un présa ge malheureux.

1 Époux. 


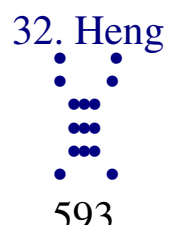

Trait supérieur hexaire : ébranler la persévérance ; présage malheureux.

TSHENG TSE. - Un trait hexaire occupe le rang extrême du koua tshen ; si la persévérance est poussée à son extrême limite, elle sort de ce qui est ordinaire. Le dernier rang du koua simple tshen exprime le comble du mouvement; occuper le rang supérieur en employant la douceur négative n'est pas une position naturelle. De plus, la douceur mal léable négative ne peut pas mettre une fermeté durable dans l'observation des règle qu'elle suit : tout ceci comporte le sens de contradiction à ce qui est normal et ordinaire, aussi est-ce considéré comme comportant l'idée d'ébranlement de la persévérance ou l'idée de persévérer dans l'ébranlement. Ébranler, i ndique la rapidité dans le mouvement d'agitation, comme dans les expressions « secouer un habit», «feuilleter un livre»; il implique l'idée d'agiter rapidement en remuant. Étant dans un rang supérieur, et ses mouvements étant désordonnés, faisant consister sa persévérance dans cet état, le présage malheureux est naturel.

TSHOU HI. - Ébranler, agiter avec rapidité. Le trait supérieur hexaire occupe le rang extrême dans le koua heng; il est placé au dernier rang du koua simple tshen ${ }^{1}$. La persistance poussée à l'excès dépasse le niveau ordinaire ; l'ébranlement arrivé à sa fin dépasse le mouvement et, de plus, la douceur malléable négative ne peut se tenir avec fermeté. L'occupation du rang supérieur ne peut assurer sa satisfaction, aussi ce trait comporte. l'image symbolique d'ébranler avec persistance, et le sens divinatoire est un présage malheureux.

594.

L'ébranlement de la persistance est au-dessus; grand manque de mérite.

TSHENG TSE. - La voie rationnelle, pour se maintenir au rang supérieur, comporte nécessairement la possession de la vertu de persévérance; alors le mérite est possible. S’il s'agite impatiemment sans

\footnotetext{
${ }^{1}$ Qui exprime le mouvement.
} 
suivre une ligne fixe, comment pourrait-il arriver à accomplir quoi que ce soit ? Occupant le rang supérieur et manquant de persévérance le présage malheureux est grave. La formule symbolique ${ }^{1}$ parle encore de l'impossibilité d'accomplir quoi que ce soit, et c'est pour cela qu'elle ajoute : "grand manque de mérite ».

\footnotetext{
${ }^{1}$ Mérite, ou résultat de l'ouvre. $\mathrm{N}^{\circ} 594$.
} 


\section{Thouen}

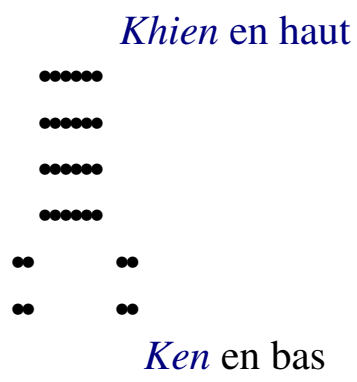

595. Retraite ; liberté ; petit avantage de la perfection.

600. Premier trait hexaire : retirer la queue dans le péril ; ne pas agir dans ce qu'il y a à entreprendre.

$*$

602. Deuxième trait hexaire : tenir en employant le cuir du bouf jaune ; impossibilité de réussir à échapper.

$*$

604. Troisième trait nonaire : retraite contrariée par des attachements; il y a des inconvénients et des périls; présage heureux pour le fait de grouper des serviteurs et des concubines.

$*$

606. Quatrième trait nonaire : aimer la retraite ; pour l'homme doué, présage heureux; pour l'homme inférieur, ruine.

$*$

608. Cinquième trait nonaire : se plaire dans la retraite; présage heureux de la perfection.

$*$

610. Trait supérieur nonaire : profiter dans la retraite; rien sans avantage.

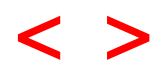




\section{Thouen.}

TSHENG TSE. - Thouen. «L'Ordre des koua » dit : «Le koua heng exprime la permanence; les êtres ne peuvent pas occuper leur situation avec une persistance durable, aussi ce koua est suivi du koua thouen; thouen a le sens de se retirer en arrière. » Or, par suite de la durée du temps, il doit y avoir disparition ; ces deux termes s'entraînent mutuellement, et c'est pour cela que le koua thouen suit immédiatement le koua heng. Thouen, se retirer en arrière, se garer ; terme qui exprime l'action de se mettre à l'écart en s'en allant. Le koua est constitué par la «montagne » placée sous le « ciel » ${ }^{1}$; le ciel, par sa nature, est quelque chose qui est au-dessus ; cette nature est positive, et le porte à avancer en montant. La montagne est une chose qui s'élève haut ; bien que sa forme soit haute et élevée, par sa substance c'est une chose stable : il y a donc l'image symbolique du mouvement ascensionnel dominant tout et d'arrêt sans propulsion en avant. Le ciel est au-dessus, il avance en montant et s'en va ; ce qui est inférieur poursuit en s'élevant et ce qui est supé rieur s'en va, il y a donc contradiction et opposition d'un côté, et retraite de l'autre, de sorte que le koua est considéré comme exprimant le sens de «se retirer en se garant ». Deux négativités sont nées en dessous; la négativité grandit et est sur le point de s'épa nouir complètement; la positivité diminue et se retire ; l'homme inférieur acquiert peu à peu la prépondérance, l'homme doué se retire et se gare, et c'est pour cela que le koua est appelé thouen.

\footnotetext{
${ }^{1}$ Khien, le ciel ; ken, la montagne.
} 


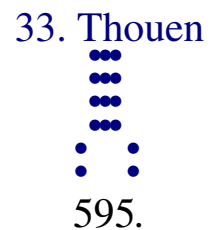

Retraite ; liberté ; petit avantage de la perfection.

TSHENG TSE. - Thouen, retraite : la négativité grandit et la positivité diminue; c'est le moment où l'homme doué se retire et se cache dans la retraite. L'homme do ué se retire et se cache dans la retraite pour redresser sa voie morale ; la voie morale n'étant pas dépri mée et violée constitue la liberté, et c'est par là que la retraite comporte la liberté. Dans les affaires il y a aussi des cas où la retraite procure la liberté. Bien que ce soit le moment où la voie de l'homme inférieur grandit, l'homme doué sait en reconnaître les premiers indices, se retirer à l'écart et rester ferme dans le bien. Cependant, les affaires ${ }^{1}$ suivent un cours variable et, suivant le temps, s'apaisent ou s'élèvent ; il en est absolument de même lorsqu'il s'agit du ciel. La mollesse négative arrivant seulement à sa période de croissance et n'étant pas encore parvenue à son extrême développement, l'homme doué estime qu'il y a une voie rationnelle d'ajourner l'emploi de la force ; il ne doit pas étaler une grande perfection et il estime qu'il y a avantage dans une petite perfection.

TSHOU HI. - Thouen, se retirer en arrière en se garant. Le koua est constitué par deux négativités qui croissent et augmentent en se développant, tandis que la positivité se retire et se met à l'abri ; c'est pour cela qu'il est appelé thouen. C'est le koua du sixième mois. Bien que la positivité soit sur le point de se retirer, cependant, le cinquième trait nonaire mérite la situation qu'il occupe et, en bas, il jouit de la sympathie du second trait hexaire : c'est encore comme s’il avait le moyen de faire. Toutefois, les deux négativités croissent peu à peu par en bas, la force naturelle des choses est telle qu'il ne peut faire autrement que de se retirer, de sorte que le sens divinatoire exprime que, si l'homme doué est capable de se retirer à l'écart, bien que sa personne soit dans la retraite, sa voie morale est libre. Pour l'homme inférieur, l'avantage sera dans l'observation de la droiture ; il ne doit pas, sous le prétexte que sa voie grandit et se développe, empiéter et envahir sur la positivité, en l'opprimant. Le terme siao, troisième caractère du texte, désigne l'homme infé rieur mou et négatif. Le sens divinatoire de ce koua et celui des formules du premier et du second trait du koua $p i$ sont du même genre ${ }^{2}$.

\footnotetext{
1 Toutes les causes de trouble.

2 Tshou Hi lit : «pour le petit, avantage dans la perfection. »
} 
596.

Le commentaire traditionnel de la formule déterminative dit: Liberté de la retraite; se retirer et jouir de la liberté. La dureté énergique occupe la situation qui lui convient et lui correspond; elle agit suivant le moment.

TSHENG TSE. - Dans le moment où la voie de l'homme inférieur grandit, l'homme doué se retire à l'écart, c'est là la liberté de la voie rationnelle; c'est en se retir ant à l'écart que l'homme doué redresse la voie morale. Ceci traite de la voie rationnelle pour se placer à l'écart dans la retraite. Depuis les mots «La dureté énergique occupe la situation qui lui convient et lui correspond » et en continuant, il est question du moment ainsi que des aptitudes exprimées par les koua simples; il y a encore une raison d'être de la possibilité de faire. Bien que ce soit le moment de la retraite, et que l'homme doué s'y place, il n'y a cependant pas encore le sens d'obligation de se retirer à l'écart. Le cinquième trait emploie les vertus de la dureté énergique, il se place dans une situation qui comporte la justice et la droiture. Bien que ce soit le moment où la négativité grandit, à cause des aptitudes exprimées par les koua simples, il doit encore y avoir, suivant le moment, des alternatives d'expansion et d'évanouissement. S'il lui est possible de développer ses forces, et de ne jamais se placer autrement qu'en s'appliquant entièrement à la plus extrême sincérité pour maintenir et consolider la voie morale, il n'est pas absolument réduit à se cacher dans la retraite sans pouvoir produire aucun effet; c'est pour cela que la formule ajoute « agir suivant le temps ».

TSHOU HI. - Explication du sens du mot liberté, au sujet du seul cinquième trait nonaire.

597.

Petit avantage de la perfection ${ }^{1}$; croître petit à petit et grandir.

TSHOU HI. - Explication des mots « petit avantage de la perfection » au moyen des deux traits inférieurs négatifs.

598.

\footnotetext{
${ }^{1} \mathrm{Ou}$, suivant Tshou $\mathrm{Hi}$, « ce qui est petit tire avantage de la perfection ».
} 
Que le moment et le sens du koua thouen sont grands !

TSHENG TSE. - Dans le temps où la négativité grandit, on ne doit pas viser à une grande perfection et il convient d'estimer les petits avantages de la perfection. En effet, la négativité grandissant, sa croissance se produit progressivement et petit à petit ; il est impossible qu'elle arrive brusquement à son parfait épanouissement. L'homme doué perfectionne peu à peu sa voie morale, et c'est ce qui est exprimé par les mots. "petit avantage de la perfection »; c'est la maintenir en la soutenant pour qu'elle ne s'effondre pas sans résistance. Le koua thouen représente le commencement de la croissance de la négativité ; l'homme doué sait reconnaître les moindres indices, aussi il convient d'avertir énergiquement, et l'id ée de l'homme saint est qu'il ne faut pas brusquement cesser d'agir ; c'est pour cela que la formule comporte les enseignements «d'agir pendant le moment » et du «petit avantage de la perfection ». Pendant le séjour des saints et des sages dans ce monde, bien qu'ils sachent que la voie morale est sur le point de péricliter et de s'évanouir, comment serait-il possible quills consentissent à assister tranquille ment au désordre sans y porter remède ? Ils doivent certainement exercer discrètement leurs forces au sujet de tout ce qui n'est pas arrivé à un point extrême et irrémédiable, résister à la décadence d'une part, entraver la progression du mal, de l'autre, viser à un répit temporaire, et agir dans ce but toutes les fois que leur action est possible. Telle fut l'action insensible de Khong et de Ming; telle fut encore celle de Wang Yuen et de Tsie Ngan sous les Han et sous les Tsin. S'il peut survenir quelque moyen pour modifier, quelque raison d'être de liberté d'action, il est inutile de dire qu'ils sa vent en profiter. Telle est la voie rationnelle du moment de la retraite à l'écart, aussi l'homme saint s'exclame admirativement sur la grandeur du moment et sur l'ampleur du sens ; qu'il s'agisse d'un fait de longue durée ou d'un événement brusque, le sens comporté est toujours vaste.

TSHOU HI. - La négativité commence seulement à croître peu à peu; se placer est difficile, aussi le moment et le sens sont également très vastes et larges.

599.

Le commentaire traditionnel de la formule symbolique dit: Sous le ciel il y a la montagne : retraite.

L'homme doué s'applique à s'éloigner des hommes inférieurs sans haine, mais avec sévérité. 
TSHENG TSE. - Sous le ciel il y a la montagne; la montagne s'élève d'en bas et s'arrête, le ciel avance en montant et les deux termes sont en opposition : c'est là l'image symbo lique de l'action de se retirer à l'écart dans la retraite. L'homme doué contemple ce symbole et il l'applique en s'éloignant de l'homme inférieur. Dans la voie rationnelle de l'opposition à l'hom me inférieur, l'emploi de mots dédaigneux et de gestes de réprobation est propre à faire naître le ressentiment et la colère; ce n'est que par une attitude correcte et sévèrement imposante, susceptible de produire un sentiment de crainte et de respect, qu'il sera naturellement amené à s'écarter et à s'éloigner de lui -même.

TSHOU HI. - La substance du ciel est illimitée, la hauteur des montagnes a des bornes; image symbolique de se maintenir en arrière. La sévérité est la manière d'être habituelle de l'h omme doué qui s'observe luimême et, à cause de cette manière d'être, l'homme inférieur ne peut s'approcher. 


\section{Thouen}

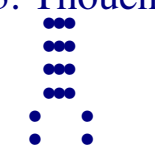

600.

Premier trait hexaire : retirer la queue dans le péril ; ne pas agir dans ce qu'il y a à entreprendre.

TSHENG TSE. - Dans les autres koua, le trait inférieur est considéré comme exprimant le début; dans le koua thouen, il exprime l'action de s'en aller en se garant. Ce qui est en avant marche et avance d'abord, aussi le premier trait est considéré comme représentant la queue ${ }^{1}$. La queue est ce qui est en arrière. S'il n'atteint pas au but, c'est à cause d'un péril ; le premier trait emploie la douceur malléable et il se trouve placé dans une position infime ; du moment où il est en arrière, il ne doit rien entreprendre ; l'entreprise consti tue un péril. Ce qui est minime est facile à cacher et à mettre à l'abri ; du moment où il y aurait péril à entreprendre, l'abîme de calamités résultant du fait de ne rien entreprendre est préférable.

TSHOU HI. - Il se retire et se trouve le dernier en arrière ; c'est l'image symbolique de la queue et elle indique une voie périlleuse. Celui qui consulte le sort ne doit rien essayer d'entreprendre, mais uniquement se tenir dans l'ombre et s'y abriter ; il pourra éviter les calamités.

601.

Péril de la queue pendant la retraite; en n'entreprenant rien, quelle calamité?

TSHENG TSE. - Il voit le fait dans la première manifestation de son germe et se retire le premier ; c'est certainement ce qui constitue le bien. Etre à la queue pendant la retraite est une circonstance périlleuse ; du moment où il $\mathrm{y}$ a péril à entreprendre, il vaut mieux ne rien entreprendre et se cacher à l'abri ; il sera possible d'éviter les calamités, et cela parce qu'il s'agit d'être déjà placé dans le péril. Les hommes de l'antiquité qui se placè rent dans une position infime, pour se cacher pendant les périodes troublées, et qui ne quittèrent point leur retraite, ont été nombreux.

\footnotetext{
${ }^{1}$ En avant. ou devant, quand on marche à reculons.
} 


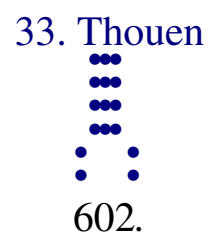

Deuxième trait hexaire : tenir en employant le cuir du bouf jaune ; impossibilité de réussir à échapper.

TSHENG TSE. - Le second et le cinquième sont considérés comme se correspondant sympathiquement suivant la droiture; bien que dans un moment d'éloignement dans des directions opposées et de retraite à l'écart, le second trait emploie la justice et la droiture et il sympathise passivement avec le cinquième ; le cinquième emploie la justice et la droiture pour se rapprocher du second et se joindre à lui ; la liaison est essentiellement solide. Le jaune est la couleur intermédiaire, ou couleur de la justice ; le bœuf est un animal passif et soumis; le cuir est une matière solide et résistante. Le second et le cinquième traits employant la justice et la droiture pour suivre avec soumission la voie rationnelle et s'allier ensemble, la solidité de leur attachement est aussi grande que s’ils étaient unis par un lien de cuir ; «il est impossible de parvenir à s'en échapper » est une phrase qui exprime la solidité de leur liaison, si grande qu'il est impossible de ne pas la mentionner. Il s'agit du moment de la retraite à l'écart, aussi il est indispensable de le mentionner avec un soin extrême.

TSHOU HI. - Employant la justice et la soumission et s'observant soi même, aucun homme ne peut échapper à son influence et aux tendances qui le portent nécessairement vers la retraite. La fermeté et la réserve de celui qui consulte le sort doivent être telles.

603.

Retenir en employant le bœuf jaune, fermeté des tendances.

TSHENG TSE. - Le supérieur et l'inférieur emploient la voie de la justice et de la soumission et se lient fortement ensemble; leurs tendances et leurs volontés sont extrêmement fermes : solidité analogue à celle d'un lien de cuir de bouf. 


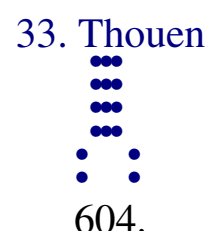

Troisième trait nonaire : retraite contrariée par des attachements; il y a des inconvénients et des périls; présage heureux pour le fait de grouper des serviteurs et des concubines.

TSHENG TSE. - Les tendances de la positivité lui rendent la négativité agréable ; le troisième et le second trait sont en contact intime ; le troisième représente donc celui qui est attaché au second. Ce qui rend la retraite méritoire, c'est sa promptitude et l'éloigne ment; étant retenu et attaché, comment serait-il possible qu'il s'éloignât rapidement et à une gra nde distance ? C'est là ce qui nuit à la retraite, et aussi est-ce considéré comme exprimant l'inconvénient. Se retirer sans y mettre de l'empressement, c'est jouer avec le péril. Les termes «serviteurs » et «concubines » désignent, d'une façon générale, les personnes peu importantes et les enfants. Celles-là s'attachent par l'affection et la reconnais sance et ne connaissent point le devoir; les aimer et les chérir, c'est précisément le rôle du supérieur ; être attaché par la reconnaissance des bienfaits particuliers, telle est la voie rationnelle de l'affection des concubines, des serviteurs et des enfants. Aussi, s'il s'agit de réunir et d'entretenir des serviteurs et des concubines, ce sera satisfaire aux besoins du cour, et cela constituera un présag e heureux. Toutefois, même dans son attachement pour les petites gens de son entourage, l'homme doué n'agit pas ainsi. Le troisième et le second traits ne sont pas ceux qui se correspondent sympathiquement suivant la droiture; ils se rapprochent par une liaison cachée et secrète, et ne s'en tiennent pas à la voie de l'homme doué. Si cet attachement était conforme à la droiture, bien quill y ait attachement, cela ne pourrait pas être considéré comme constituant un danger ou un inconvénient. Tel est, par exemple, le cas de Tou Sien Tshou, ne pouvant se décider à abandonner ses soldats et son peuple ; bien qu'il puisse y avoir péril, il n'y a pas de culpa bilité.

TSHOU HI. - En bas, il est en contact avec deux négativités, il présente l'image symbolique de devoir se retirer dans la retraite et d'être retenu par quelque lien; c'est une voie rationnelle d'inconvénients et de périls. Toutefois, suivie à l'égard des serviteurs et des concubines, elle présente un présage heureux. En effet, de la part de l'homme do ué, et par rapport aux petites gens de son entourage, ce n'est que dans le cas où il s'agit des ser viteurs intimes et des concubines qu'il n'est pas absolument indispensable de ne tenir compte que du degré de sagesse en les groupant autour de soi. C'est pour cela que tel est le sens divinatoire. 
605.

Péril de la retraite contrariée par des attachements; il y a des inconvénients et de la honte; présage heureux s'il s'agit de grouper et d'entretenir des serviteurs et des concubines; incapacité dans les grandes choses.

TSHENG TSE - Se retirer dans la retraite et être retenu par des considérations d'atta chements doit forcément amener des maux, de la honte, et faire survenir des périls. Toutes les fois qu’il y a inconvénient à faire quelque chose, il en résulte de la honte. En effet, la force manque. Si ce sentiment d'affection intime a pour objet le soin de la conservation et de la protection des serviteurs et des concubines, alors le présage est heureux; mais, dans ce cas, comment serait-on capable de se montrer à la hauteur de grandes choses? 


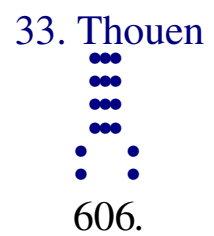

Quatrième trait nonaire : aimer la retraite; pour l'homme doué, présage heureux; pour l'homme inférieur, ruine.

TSHENG TSE — Le quatrième et le second traits sont considérés comme se correspondant sympathiquement suivant la droiture; ce sont ceux qui s'aiment et s'affectionnent. Bien que l'homme doué possède des êtres à aimer et à chérir, lorsque le devoir lui commande de se retirer dans la retraite, il part sans hésitation; c'est ce qu'on appelle « se dominer pour revenir aux convenances ». Puisqu'il se sert de la voie rationnelle pour dominer ses propres désirs, c'est par là que le présage est heureux. S'il s'agit d'un homme inférieur, il ne pourra se placer d'après le devoir ; il s'attachera par faiblesse à l'ob jet de son affection ; il sera retenu par son égoïsme, jusqu'à ce qu’il tombe dans la honte, sans pouvoir s'en relever ; aussi pour l'homme inférieur, c'est un présage de ruine et de perdition. Le dernier caractère du texte exprime le contraire du bien. Le quatrième trait fait partie de la substance du koua simple khien ; c'est celui qui est capable d'énergie et de décision. C'est parce qu'il est placé dans un rang négatif et quiil est retenu par des attachements, que l'homme saint pose l'avertissement re latif au cas de l'homme inférieur, de peur qu'il ne perde sa droiture.

TSHOU HI - En bas il sympathise avec le premier trait hexaire, tandis que sa substance est l'activité énergique ; il présente l'image symbolique d'avoir des sentiments d'affection et d' être capable de les briser pour se retirer dans la retraite. L'homme doué qui sait se vaincre lui-même, seul, en est capable, tandis que l'homme inférieur ne le peut pas ; aussi, si celui qui consulte le sort est un homme doué, le présage sera heureux.

607.

L'homme doué aime la retraite; pour l'homme inférieur, c'est un malheur.

TSHENG TSE - Bien que l'homme doué éprouve des sentiments d'affection, cepen dant il est capable de se retirer dans la retraite, sans faillir au 
devoir. L'homme inférieur serait incapable de surmonter ses sentiments personnels et il en résulterait des maux pour lui. 


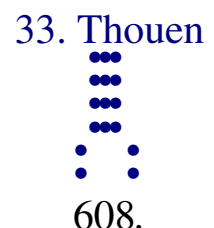

Cinquième trait nonaire : se plaire dans la retraite; présage heureux de la perfection.

TSHENG TSE - Le cinquième trait nonaire possède la justice et la droiture, cela constitue la beauté de la retraite ${ }^{1}$; il se place conformément à la justice et à la droiture ; parfois il s'arrête, parfois il agit : c'est là ce qu'on entend par «beauté de la retraite », et c'est pourquoi il est considéré comme exprimant la perfection de la droiture et comme comportant un présage heureux. Ce n'est pas que le cinquième trait nonaire ne soit retenu et attaché par la sympathie, mais comme lui et le second trait se placent tous deux d'après la justice et la droiture, sa volonté et ses tendances se conforment à la nécessité du mouvement ou de l'arrêt. N'être point dépourvu de justice et de droiture, et ne commettre aucune faute par attachement égoïste, c'est ce qui est considéré comme constituant la beauté digne de louanges. Dans le commentaire de la formule déterminative, après qu'il a été question du moment indiqué par le koua thouen, c'est pour le même motif qu'il est encore dit : Agir avec le temps, petit avantage de la perfection ; c'est qu'il y a encore l'idée d'atténuer les conséquences de cette retraite. Dans les formules des traits, lorsqu'il s'agit du cinquième, la retraite est sur le point d'être arrivée à la limite extrême de son développement, aussi il est seulement question de l'emploi de la justice et de la droiture pour s'y placer. La retraite à l'écart n'est pas le fait de l'homme doué ; c'est pour cela que la for mule ne s'attache pas spécialement à la considération de la situation du prince. Toutefois, lorsque le prince s'écarte et s'éloigne dans la retraite, c'est encore uniquement le résultat de la justice et de la droiture.

TSHOU HI. - Dureté énergique positive, justice et droiture ; en bas il sympathise avec le second trait hexaire, qui, lui aussi, est doué de douceur, de soumission, de justice et de droiture ; c'est celui qui exprime la beauté de la retraite. Si celui qui consulte le sort est dans ces conditions, et sîl possède la droiture, le présage sera heureux.

609.

\footnotetext{
${ }^{1}$ Les commentateurs chinois lisent dans le texte : «Beauté de la retraite. »
} 
Présage heureux de la perfection de la beauté de la retraite : à cause de la droiture des tendances.

TSHENG TSE — Lorsque les tendances sont dirigées par la droiture, le mouvement ${ }^{1}$ résulte nécessairement de la droiture: c'est par là qu'est constituée la beauté de la retraite. Occupant une position qui comporte la justice, possédant la droiture et sympathisant à la justice et à la droiture, c'es t donc que ses tendances sont droites et correctes, et c'est par là que le présage est heureux. La retraite, pour l'homme, c'est l'arrêt dans l'action ; elle a uniquement pour but le redressement de ses tendances.

${ }^{1}$ Les actions. 


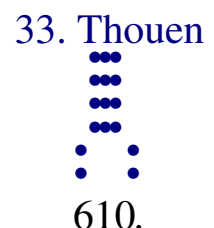

Trait supérieur nonaire : profiter dans la retraite ; rien sans avantage.

TSCHENG TSE — Le troisième caractère du texte, fei, comporte l'idée d'augmenter et de grandir, d'ampleur et d'éclat. La retraite, c'est simpleme nt le fait d'être emporté au loin, roulé par le vent ; n'être arrêté ni retenu par rien en constitue la beauté. Le trait supérieur nonaire fait partie de la substance du koua simple khien, il est dur, énergique, et plein de décision; il est placé à l'extérieur du koua, et enfin, en bas il n'est retenu par rien ; il exprime donc l'éloignement dans la retraite, sans être retenu par aucun lien, c'est là ce qu'on peut appeler un excès de grandeur et de magnanimité. La retraite est un moment de misère et de malheur; s'y placer suivant le bien est considéré comme exprimant l'idée d'en profiter. Lorsque la retraite a lieu dans ces conditions, en quoi ne serait-elle pas avantageuse ?

TSHOU HI. - Employant la dureté énergique positive et occupant le rang extérieur du koua, en bas, n'étant retenu par aucune sympathie, c'est celui qui exprime l'éloignement de la retraite et l'action de se placer avec grandeur d'âme. C'est pour cela que tels sont l'image symbolique et le sens divinatoire. Le caractère fei exprime l'idée de grande magnanimité se dominant elle-même.

611.

Profiter dans la retraite, rien sans avantage; aucun sujet de doute.

TSCHENG TSE - C'est l'éloignement dans la retraite sans aucun sujet d'hésitation ou de retard. En effet, étant à l'extérieur, il es t déjà éloigné ; étant sans correspondance sympathique, il n'est retenu par aucun attachement, aussi il exprime la décision énergique, sans aucune hésitation. 


\section{Ta tshang}

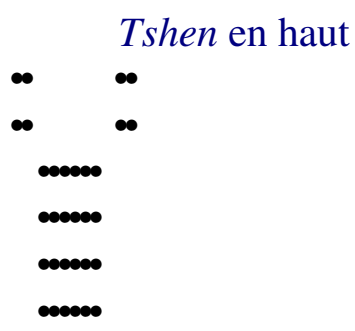

Khien en bas

612. Grande force ; avantage de la perfection.

[]

616. Premier trait nonaire : épanouissement de la vigueur dans le gros orteil ; en agissant, présage malheureux; il y a bonne foi.

$*$

618. Deuxième trait nonaire : présage heureux de la perfection.

*

620. Troisième trait nonaire : l'homme inférieur emploie la force ; l'homme doué la méprise ; danger de la perfection ; le jeune bélier heurte la cloison avec sa tête; il use ses cornes.

*

622. Quatrième trait nonaire : présage heureux de la perfection, les regrets se dissipent; la palissade se fend sans user les cornes; épanouissement de la vigueur dans le moyeu de la roue du grand char.

$*$

624. Cinquième trait hexaire :faire disparaître le bélier dans le changement ; pas de regrets.

$*$

626. Trait supérieur hexaire : le jeune bélier frappe de la tête contre la palissade; il est incapable de se retirer en arrière; il ne peut réussir dans son dessein; aucun moyen d'avantage ; la difficulté sera un présage heureux.

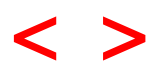


34. Ta tshang.

TSHENG TSE. — Ta tshang. «L'Ordre des koua » dit : «Le koua thouen exprime la retraite à l'écart ; les êtres ne peuvent pas rester indéfiniment dans la retraite, aussi ce koua est suivi du koua ta tshang. » Thouen exprime le sens d'opposition et d'éloignement ; tshang celui de progression en avant et d'expansion florissante. Dans le koua thouen, la négativité grandit et la positivité se retire à l'abri; le koua ta tshang représente le parfait épanouissement de la positivité. La dissolution doit nécessairement donner lieu à l'expansion de la croissance, l'extinction et la renaissance s'entraînent mutuellement ${ }^{1}$; aussi après qu'il $\mathrm{y}$ a eu retraite à l'écart, il faut nécessairement qu'il y ait développement florissant, et c'est pour cette raison que le koua ta tshang suit immédiatement le koua thouen. Il est composé du koua simple tshen, en haut, et du koua simple khien, en bas ; le koua simple khien exprime l'activité énergique et le koua simple tshen exprime le mouvement ; emploi de l'activité énergique et mouvement, tel est le sens du koua ta tshang. L'énergie active positive grandit ; la croissance de la positivité a déjà dépassé le milieu ${ }^{2}$ ordinaire. Ce qui est grand est vigoureux et florissant. Enfin, l'ébranlement majestueux de la foudre au-dessus du ciel, ce qui donne encore le sens de « grande force d'expansion florissante ».

${ }^{1}$ On peut aussi lire : « aspiration » et « expiration ».

2 Du koua. 


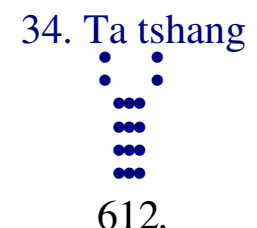

Grande force $^{1}$; avantage de la perfection.

TSHENG TSE. — La voie de la grandeur de l'éclat est avantageuse par la perfection de la droiture ; une grande force sans la droiture n'est que de la violence, ce n'est pas la voie florissante et éclatante de l'homme doué.

TSHOU HI. - Le caractère $t a$, grand, grandeur, désigne la positivité ; les quatre traits positifs achèvent l'épanouissement de leur croissance, aussi le koua est appelé ta tshang, grande force, grand éclat. C'est le koua du second mois. La positivité étant dans l'éclat de sa force, il est inutile de mentionner que cette condition comporte un présage heureux et la liberté d'action ; l'avantage n'existe rien que par la droiture et la fermeté.

613.

Le commentaire traditionnel de la formule déterminative dit: Ta tshang; ce qui est grand est épanoui; énergie dans l'impulsion, donc force et épanouissement.

TSHENG TSE. - Ce qui fait que le koua est appelé ta tshang, c'est que ces mots expriment que ce qui est grand est épanoui et florissant. La négativité est considérée comme exprimant la petitesse; la positivité exprime la grandeur. La positivité grandit et se développe pour fleurir, c'est donc que la grandeur est épanouie. En bas dureté énergique active ; en haut mouvement, l'emploi de l'extrême énergie causant le mouvement, ce qui est bien exprimé par le terme ta tshang. Ce koua est considéré comme exprimant que ce qui est grand est fort et florissant et, en même temps, la grandeur de l'éclat ou de la force.

TSHOU HI. - Explication du sens du nom du koua. En en parlant au point de vue de la substance du koua lui-même, la positivité grandissant et dépassant le milieu, c'est la force de l'épanouissement de la grandeur ; en en parlant au point de vue des vertus exprimées par les koua simples, alors

\footnotetext{
${ }^{1}$ Tshang, ou peut-être tsheang, force; grandeur ; éclat parfait, désigne la huitième lune. Le
} commentateur lit plutôt : « épanouissement de la grandeur ». 
l'activité énergique du koua khien ébranle et agite, d'où résulte la force de la grandeur.

614.

La force de la grandeur est avantageuse par la perfection; ce qui est grand est droit. En conformant la grandeur à la droiture ${ }^{1}$, les sentiments du ciel et de la terre sont visibles.

TSHENG TSE. - Du moment où la grandeur est florissante, elle sera avantageuse par la perfection de la droiture; la droiture et la grandeur constituent la voie rationnelle. En analysant jusqu'au bout la raison d'être de la droiture et de la grandeur, il devient possible, de voir le sentiment du ciel et de la terre. La voie rationnelle du ciel et de la terre c'est la durée permanente et indéfinie; c'est l'extrême grandeur et l'ex trême droiture. Celui qui étudie peut, en méditant, pénétrer la raison d'être de la droiture et de la gran deur. Le texte ne porte pas «grande droiture », mais «droiture et grandeur» ou «droiture de la grandeur »; l'ordre des deux mots a été adopté p our qu'on ne puisse douter qu'il s'agit de deux choses distinctes ${ }^{2}$.

TSHOU HI. - Dernière et extrême analyse et explication du sens des mots « avantage de la perfection ».

615.

Le commentaire traditionnel de la formule symbolique dit: La foudre est au-dessus du ciel : grande force. L'homme doué s'applique à ne faire aucune démarche contraire aux rites.

TSHENG TSE. - L'ébranlement de la foudre agite au-dessus du ciel : grandeur et force. L'homme doué considère l'image symbolique de la grandeur de la force et il en applique le sens à l'austérité de sa démarche. La grandeur de l'austérité chez l'homme doué n'est jamais parfaite que par la puissance qu'il a de se vaincre et de revenir aux règles rituelles. Les anciens disaient : «Se dominer, c'est se viole nter par la force. » Le Tshong yong, au sujet de l'affabilité sans laisser -aller et du maintien correct sans affaissement

\footnotetext{
${ }^{1}$ Littéralement « droit et grand, et il est possible devoir la nature réelle du ciel et de la terre ».

${ }^{2}$ C'est-à-dire que $t a$, grandeur, n'est pas pris adjectivement comme qualificatif de droiture.
} 
sur un point d'appui, dit également : «Quel effort ! Se jeter hardiment dans l'eau bouillante ou le feu, marcher sur un tranchant éti ncelant, sont des actions qui peuvent être accomplies par le courage du guerrier. Mais se vaincre soimême et revenir aux règles rituelles est impossible sans la grandeur de la force ${ }^{1}$ de l'homme doué. » C'est pour cela que la formule dit que l'homme doué s'applique à ne faire aucune démarche contraire aux rites.

TSHOU HI. - Se vaincre soi-même, c'est la force.

\footnotetext{
${ }^{1}$ Ta tshang
} 


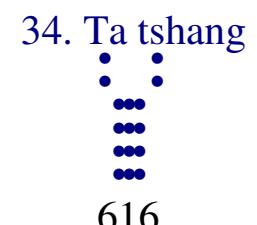

Premier trait nonaire : épanouissement de la vigueur dans le gros orteil ; en agissant, présage malheureux; il y a bonne foi.

TSHENG TSE. - Le premier trait possède l'énergie active de la positivité, il fait partie de la substance du koua simple khien et est placé dans l'infériorité ; c'est celui qui applique sa force à se porter en avant. Étant dans l'infériorité et employant la force, il représente la force se développant dans le gros orteil. L'orteil est une partie inférieure du corps et un instrument de la marche en avant et du mouvement. Ce trait est nonaire, il est dans l'infé riorité, il emploie la force et il ne possède pas la justice ; or employer la dureté énergique et se placer sur le terrain de la force est une voie qui ne doit pas être suivie, alors même qu'on occupe une position supérieure : combien à plus forte raison lorsqu'on est dans l'infériorité ! Aussi, sill avance, le présage est malheureux. «Il y a bonne foi »; bonne foi, confiance : cela veut dire quil est certain qu'en employant la force et en avançant le pré sage sera malheureux ${ }^{1}$.

TSHOU HI. - Le gros orteil est une extrémité inférieure du corps et un des instruments de la marche et du mouvement. Dureté énergique placée dans l'infériorité et en présence d'un moment d'épanouissement de la force ; cela exprime la violence dans l'action de se porter en avant, et c'est pourquoi le trait présente cette image symbolique. Étant dans l'infériorité et employant la violence pour se porter en avant, le présage malheureux est certain, de sorte que tel est aussi le sens divinatoire.

617.

Vigueur dans l'orteil ; la bonne foi est épuisée ${ }^{1 .}$

TSHENG TSE. — Étant au niveau le plus bas et employant la force pour agir, on doit certainement s'attendre avec conf iance au malheur et à la misère, et le présage est malheureux.

TSHOU HI. - Cela exprime quill y aura nécessairement de la misère.

${ }^{1}$ Le commentateur dénature le sens des mots «avoir foi » et lit: «Il y a lieu d'en être persuadé ». 


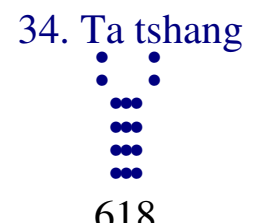

618.

Deuxième trait nonaire : présage heureux de la perfection.

TSHENG TSE. - Bien que le deuxième trait emploie la dureté énergique dans un moment d'épanouissement et de grandeur, cependant, il reste dans une situation qui comporte la douceur malléable et se place suivant la justice : la dureté énergique et la douceur s'accordent donc dans une juste mesure, il n'y a pas excès dans la force, il possède la parfaite droiture, et le présage est heureux. On a dit: N'est-ce pas parce qu'un trait nonai re occupe le second rang que la perfection est prise pour sujet d'avertissement ? Dans le Yi king, c'est le sens le plus important qui est choisi ; puisque ce trait, par sa substance ${ }^{2}$, possède la dureté énergique positive et l'activité, quill se trouve en face d'un moment d'épanouissement de la force, et qu'il se c onforme à la justice en se plaçant, la voie rationnelle qu'il indique n'est dans aucun cas contraire à la droiture. Dans le cas du quatrième trait, il y aurait avertissement au sujet du manque de droiture. Lhomme qui est capable de discerner l'importance relative du sens à relever est seul capable d'étudier le Yi king.

TSHOU HI. - Ce trait emploie la positivité et occupe un rang négatif ; par lui-même, il ne s'attache pas à la droiture. Cependant la position dans laquelle il se place est conforme à la justice, de sorte qu'il peut encore en résulter qu'il ne manque pas à cette droiture. Aussi la formule avertit celui qui consulte le sort, afin qu'il s'appuie sur la justice pour rechercher la droiture ; ce n'est qu'après cette condition remplie que le présag e pourra être heureux.

619.

Présage heureux de la perfection du second trait nonaire, à cause de la justice.

TSHENG TSE. - Ce qui rend le présage heureux et ce qui indique la perfection de la droiture, c'est la conformité à la voie de la justice. Avec la justice on ne manquera pas à la droiture ; à plus forte raison si on possède déjà la dureté énergique positive et si la substance du sujet est l'activité.

\footnotetext{
${ }^{1}$ Les commentateurs lisent : «On doit croire avec confiance que la misère surviendra »; ce n'est pas dans le texte.

2 Par le koua simple dont il fait partie.
} 


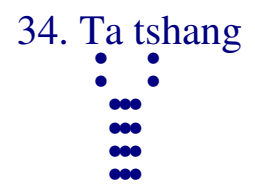

620.

Troisième trait nonaire : l'homme inférieur emploie la force ; l'homme doué la méprise ${ }^{1}$; danger de la perfection ; le jeune bélier heurte la cloison avec sa tête; il use ses cornes ${ }^{2}$.

TSHENG TSE. - Le troisième trait emploie la dureté énergique, il occupe un rang positif et se place dans l'épanouissement de la grandeur ; de plus, il est à la fin de la substance de l'activité, il exprime l'extrême limite de la parfaite vigueur. Pousser ainsi l'épanouisse ment de la vigueur à sa plus extrême limite exprimera, chez l'homme inférieur, l'emploi de la force physique, et chez l'homme doué, le mépris et l'annihilation de cette même force. L'homme inférieur estime et prise la force, aussi il fait usage de sa vigueur et de son courage guerrier; mais les tendances ${ }^{3}$ de l'homme doué sont énergiques et puissantes, de sorte quil ne fait nul cas de cette force physique ; il l'annihile, ou la méprise, il la brise par sa force morale. C'est parce qu'il est extrêmement énergique, qu' il regarde tout d'un point de vue élevé sans éprouver aucune crainte ni appréhension. L’homme inférieur et l'homme doué sont des expressions qui se rapportent à la terre ${ }^{4}$. Par exemple, l'homme doué, animé du courage guerrier, sans la notion du devoir, commettrait des désordres ${ }^{5}$; mais l'énergie et la douceur se contrebalançant dans de justes proportions, il sait se maintenir sans arrogance comme sans bassesse; il traite l'univers entier d'après le même prin cipe et jamais autrement que selon l'exacte convenance, dans chaque cas. S'il poussait trop loin la dureté énergique, il ne posséderait point les vertus de la concorde et de la mansuétude et rien ne serait propre à causer plus de blessures; se maintenir avec une parfaite fermeté dans une telle voie, serait suivre une voie périlleuse. Aucun être n'existe sans employer sa vigueur ; les dents servent à mordre, les cornes à frapper avec la tête, les pieds armés de corne servent à ruer. La vigueur du bélier réside dans sa tête ; le jeune bélier se plaît à heurter avec son front, et c'est pour cela qu'il est choisi comme image symbolique. Le bélier aime à frapper la cloison avec ses cornes, uniquement parce que les cloisons se trouvent devant lui ; en effet, il faut qu'il heurte tout ce qui se présente

\footnotetext{
${ }^{1}$ L'annihile.

${ }^{2}$ Lucrèce, De natura : «De même que le chevreau sent ses cornes et veut s'en servir... » Voir Lange, Hist. du matérialisme, t. I, p. 144, dernière ligne.

${ }^{3}$ La volonté.

${ }^{4}$ Dans la pensée du commentateur cette phrase signifie probablement «ces expressions se rapportent aux choses de la terre ».

${ }^{5}$ Se sentant digne du plus haut rang, il chercherait à l'occuper par la force en renversant le souverain.
} 
devant lui. Se plaisant ainsi à déployer sa vigueur, il doit nécessairement arriver qu’il use et détériore ses cornes. Il en est encore de même pour l'homme qui se plaît à montrer son énergie et sa vigueur ; il emploie ces dons dans toutes les circonstances qui se présentent, et il doit nécessairement en résulter pour lui des maux et des soucis. La vigueur du troisième trait étant telle, comment n'en résulte-t-il aucun présage malheureux ? Dans les conditions où se trouve le troisième trait, toute entreprise suffit à entraîner un présage malheureux, mais la formule commence précisément à annoncer le péril, de sorte que le présage malheureux n'est pas encore manifesté ; toutes les fois qu'une circonstance quelconque est susceptible d'entraîner un présage malheureux, mais que ce présage n'est pas encore manifesté, les formules ne parlent que d'appréhension du péril, li.

TSHOU HI. - Excès d'énergie sans justice, dans un moment d'épanouissement de la vigueur ; c'est le cas où l'homme inférieur emploie la vigueur et où l'homme doué agit pour la dominer et l'annihiler. Le dixième caractère du texte est une négation et signifie « regarder comme n'existant pas »; cela constitue l'excès de courage chez l'homme doué, et, de cette façon, même avec la droiture, il y a encore péril. Le jeune bélier est un animal vigoureux qui se plaît à heurter avec le front. Le terme cloison désigne les cloisons en branches, ou lattes, entrelacées. User, détériorer, abîmer les cornes, sens divinatoire exprimant l'appréhension d'un péril ré sultant de la perfection ${ }^{1}$; telle est l'image symbolique ${ }^{2}$.

621.

L'homme inférieur emploie la force ; l'homme doué la méconnaît.

TSHENG TSE. - Chez l'homme inférieur cela constituera l'emploi de la vigueur et de la force physique ; chez l'homme doué, la méconnaissance de cette vigueur; ses tendances et sa volonté étant énergiques et fortes, il méconnaîtra cette même force dans les événements, il n'éprouvera aucune crainte et manquera de circonspection.

TSHOU HI. - L'homme inférieur arrive à sa perte à cause de sa vigueur, l'homme doué souffre pour avoir méconnu cette même vigueur.

\footnotetext{
${ }^{1}$ D'une qualité poussée trop loin.

${ }^{2}$ Dans les anciennes poésies, le bélier est souvent pris comme symbole de la lune et de son croissant.
} 


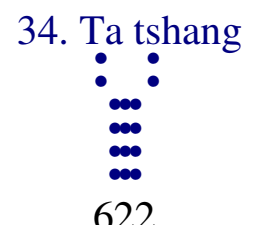

Quatrième trait nonaire : présage heureux de la perfection, les regrets se dissipent; la palissade se fend sans user les cornes; épanouissement de la vigueur dans le moyeu de la roue du grand char.

TSHENG TSE. - Dans le quatrième trait, la dureté énergique positive grandit et se développe florissante; la vigueur dépasse déjà la juste proportion; c'est l'extrême développe ment de la vigueur. Cependant, l'occupation du quatrième rang exprime le manque de droiture ${ }^{1}$; au moment où la voie morale de l'homme doué se développe et grandit, comment l'absence de droiture serait-elle possible ? Aussi, la formule avertit que l'emploi de la perfection sera un présage heureux et que les regrets seront dissipés. En effet, au moment où cette voie grandit et se développe, la plus légère imperfection nuira à la force naturelle de son libre essor ; donc il y aura des regrets. Dans un autre koua la condition de l'énergie active occupant un rang qui comporte la douceur malléable serait la plus importante, il ne serait pas absolument forcé que cette condition exprimât autre chose que le bien ; c'est le cas dans le k oua tae kuo. La palissade de piquets s'entrouvre et se fend sans abîmer d'avantage les cornes et sans causer d'autres inconvénients à la vigueur. Dans un char grand et élevé, si l'essieu et les moyeux sont forts et puissants, il en résulte évidemment un avantage pour le mouvement de la marche ; c'est pour cela que la formule dit : «Épanouissement de la vigueur dans le moyeu de la roue du grand char. » Le moyeu est la partie la plus essentielle de la roue; la destruction d'un char provient ordinairement de la rupture du moyeu ; si le moyeu est solide, le char est fort. Le texte, en disant « épanouissement de la vigueur dans le moyeu », exprime l'épanouissement de la vigueur dans le mouvement de progression en avant.

TSHOU HI. - Présage heureux de la perfection, les regrets se dissipent ; le sens divinatoire est le même que pour le quatrième trait nonaire du koua hien ${ }^{2}$. La «palissade se fend sans dommage pour les cornes »; cette phrase se rapporte au texte qui précède ${ }^{3}$. Le terme kuie signifie ouvrir, entrouvrir. En avant du troisième trait se trouve le quatrième. c'est comme s'il existait une palissade, ou cloison ; en avant du quatrième se trouvent deux négativités, qui, elles, représentent une palissade entrouverte. «Épanouissement de la vigueur dans le moyeu de la roue du grand char »; c'est de même une image symbo lique de la possibilité du mouvement de progression en avant. Puisque la

\footnotetext{
${ }^{1}$ Trait positif, rang négatif.

${ }^{2} \mathrm{~N}^{\circ} 571$.

${ }^{3} \mathrm{~N}^{\circ} 620$.
} 
dureté énergique occupe un rang négatif, c'est qu'elle n'est pas poussée à sa dernière et plus extrême limite, et c'est pour cela que tels sont le sens divinatoire et limage symbolique.

623.

La palissade se fend sans dommage pour les cornes; progression continue en avant.

TSHENG TSE. - La croissance de la positivité énergique doit nécessairement arriver à une limite extrême; dans le quatrième trait, bien qu'elle soit arrivée à son parfait déve loppement, cependant son mouvement en avant n'est pas encore arrêté. Ce trait emploie le parfait développement de la positivité, il se sert de sa vigueur, et il avance, aussi rien ne s'oppose à son mouvement; la palissade s'entrouvre sans dommage pour ses cornes et sans nuire à sa force. Progression continue en avant; son mouvement en avant ne cesse point. 


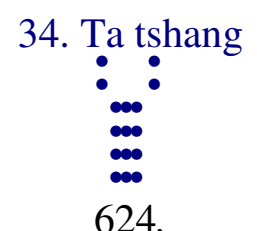

Cinquième trait hexaire :faire disparaître le bélier dans le changement ${ }^{1}$; pas de regrets.

TSHENG TSE. - Les béliers marchent en troupeaux et se plaisent à se heurter avec leurs cornes ; cette image est employée comme symbole de toutes les positivités qui avancent également ensemble. Les quatre positivités sont nouvellement développées et elles avancent toutes également; le cinquième trait emploie la douceur et se maintient au-dessus ; s'il les contenait par la force, il lui serait impossible de les dominer et il en aurait des regrets. Ce n'est qu'en la traitant par la tolérance et la concorde quil amène cette troupe de positivités à n'avoir pas à employer la force énergique, et c'est là « noyer la vigueur dans l'harmonie et la concorde », de cette façon il devient possible qu'il n'ait point de regrets. Le cinquième trait, considéré au point de vue de sa situation, exprime la droiture ; au point de vue de ses propriétés, ou vertus, il exprime la justice ; c'est pour ces raisons qu'il est apte à employer la voie de l'harmonie et de la douceur, de façon que la troupe des négativités, bien que pleine de vigueur, n'ait point l'occasion de la déployer et de s'en servir.

TSHOU HI. - La substance du koua semble se prêter au symbolisme du bélier; au-dehors douceur malléable et, au-dedans, dureté énergique. Le cinquième trait hexaire, seul, emploie la douceur et se maintient dans la justice ; il ne peut pas frapper avec les cornes. Bien qu'il manque de vigueur, cependant il n'en résulte pour lui aucun motif de regrets, aussi tels sont l'image symbolique et le sens divinatoire. Le sixième caractère du texte doit être pris dans l'acception de «tolérance facile », et exprime une action subite sans qu'on aperçoive la disparition. On a aussi lu ce caractèr e avec le sens de [] , vigueur énergique ; le sens est encore intelligible.

625.

Faire disparaître ${ }^{2}$ le bélier dans le changement ; la situation ne convient pas.

\footnotetext{
1 Traduction littérale. Les commentateurs chinois lisent «perte de vigueur par l'harmonie ».

2 Enterrer ; recouvrir, cacher ; faire disparaître.
} 
TSHENG TSE. - Ce qui fait la nécessité absolue de l'emploi de la douceur et de la concorde ${ }^{1}$, c'est que la douceur malléable négative occupe la situation prééminente. Si le trait comportait l'emploi de la justice, de la droiture et de la dureté énergique positive, en profitant de cette situation prééminente, au-dessous, il n'y aurait pas d'épa nouissement de la vigueur. Puisque le cinquième trait est hexaire, la situation (prééminente) ne lui convient pas, aussi la formule donne le sens de «disparition du bélier dans l'accord $»^{2}$. Toutefois, dans la plupart des cas, pour réfréner la vigueur, on ne doit point employer la dureté énergique. La force inhérente à la condition du prince ou à celle du sujet, à une situation supérieure ou inférieure, n'est pas identique ; si le pouvoir du prince est suffisant pour régir les inférieurs, alors, bien qu'il puisse exister quelques individus énergiques et vigoureux, capables de passer par-dessus tous les obstacles, ces cas isolés ne sont pas assez nombreux pour constituer le «développement de la vigueur »; il faut que la force inhérente à la condition du prince soit insuffisante en quelque point, et alors on peut dire qu'il s'agit de « réfréner l'explosion de la vigueur ». La voie rationnelle de la répression de la vigueur ne doit pas consister dans l'emploi de la dureté énergique.

\footnotetext{
${ }^{1}$ Les commentateurs donnent au caractère $y i$ le sens de «facilité, accord ».

${ }^{2}$ Lecture qui dénature le texte.
} 


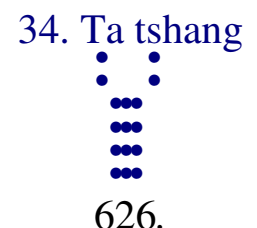

Trait supérieur hexaire : le jeune bélier frappe de la tête contre la palissade; il est incapable de se retirer en arrière ; il ne peut réussir dans son dessein ; aucun moyen d'avantage ; la difficulté sera un présage heureux.

TSHENG TSE. - Le jeune bélier est choisi simplement à cause de l'emploi d'exubéran ce de vigueur, de sorte que cette figure est applicable même à un trait négatif. Ce trait hexaire emploie la négativité et se place au dernier rang du koua simple tshen, qui exprime l'impulsion, ou ébranlement, et il se trouve en présence de l'extrême développement de la vigueur ${ }^{1}$; l'excès est évident. C'est comme un jeune bélier qui, en frappant du front contre les palissades, en se lançant en avant, doit nécessairement se blesser lui-même, tandis qu'en se retirant en arrière il blesse ses cornes ; il ne peut donc ni avancer ni reculer. Ses qualités essentielles sont la douceur malléable négative, de sorte quil est incapable de se dominer lui-même pour obéir au devoir ; c'est là ne pas être capable de se retirer en arrière. L'homme mou et négatif, bien qu'absolument décidé à employer sa vigueur, ne peut néanmoins pas l'employer jusqu'à la fin ; s'il rencontre la résistance, il recule ; c'es t là ne point pouvoir réussir dans ses desseins. Agissant de cette manière, il ne peut rien entreprendre qui puisse être avantageux. La malléabilité négative, placée dans une position qui comporte l'exubérance de la vigueur, n'est pas capable d'une fermeté assurée ; si elle rencontre des difficultés pénibles, elle perdra sa vigueur $^{2}$; perdant sa vigueur, elle retombera dans son état de faiblesse ; c'est en cela que la difficulté sera pour elle un présage heureux. En employant la vigueur, elle n'a aucun a vantage, de sorte qu'en recon naissant la difficulté de sa tâche et en s'en tenant à la douceur dans les moyens qu'elle emploie, le présage sera heureux. Occupant le dernier rang dans le koua qui exprime l'épanouissement de la vigueur, ce trait comporte le sens de modification et de changement.

TSHOU HI. - L'épanouissement de la vigueur tire à sa fin ; le mouvement arrive à son extrême limite, aussi il heurte contre la cloison et est incapable de s'en retirer. Toutefois son caractère physique est essentiel lement la mollesse, de sorte que, de plus, il ne peut pas non plus réussir à se porter en avant. L’image symbolique étant telle, le sens divinatoi re est évident. Cependant son manque d'énergie est encore une chance heureuse, de sorte que

\footnotetext{
${ }^{1}$ Dernier rang du koua ta tshang.

${ }^{2}$ Factice.
} 
sil peut rencont rer de la difficulté à se placer, il peut encore résulter pour lui un présage heureux.

627.

Incapable de se retirer en arrière, incapable de réussir, manquer de clarté dans le jugement. La difficulté sera un présage heureux; l'erreur ne dure pas longtemps.

TSHENG TSE. - Ce n'est pas la position qui lui convient et il s'y place, de sorte qu'il ne peut ni reculer, ni avancer ; donc, en se plaçant il manque de clarté dans le jugement et de prévoyance. «La difficulté sera un présage heureux », la douceur malléable rencontrant une difficulté, et de plus occupant le dernier rang dans le déploiement de la vigueur, doit naturellement se modifier. En se modifiant elle se mettra en rapport avec sa propre condition ; son erreur et sa culpabilité ne sont pas de longue durée, c'est en cela que le présage est heureux. 


\section{5. $T \sin$}

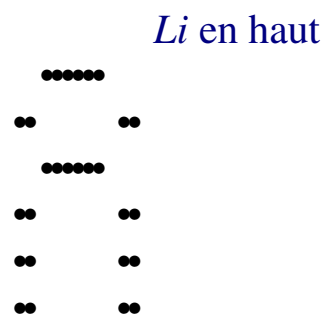

Khouen en bas

628. Tsin, progression en avant; pacifier les feudataires en employant le don de nombreux chevaux; pendant la clarté du jour, trois entrevues.

632. Premier trait hexaire : comme avançant, comme se retirant; présage heureux de la perfection; absence de foi ; magnanimité sans culpabilité.

634. Deuxième trait hexaire : comme avançant, comme triste ; présage heureux de la perfection; il reçoit ce grand bonheur; vers la mère du roi.

$*$

636. Troisième trait hexaire : confiance universelle; les regrets se dissipent.

$*$

638. Quatrième trait nonaire : avancer comme le rat des champs ; inconvénients de la perfection.

640. Cinquième trait hexaire: Les regrets se dissipent; ne pas s’inquiéter de perdre ou d'obtenir ; en agissant présage heureux, rien sans avantage.

*

642. Trait supérieur nonaire : avancer la corne ; seulement l'employer à détruire le district ; appréhension, présage heureux, pas de culpabilité. Perfection cause d'inquiétude.

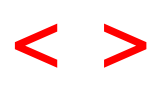




\section{Tsin.}

TSHENG TSE. - T Sin. «L'Ordre des koua » dit : «Les êtres ne peuvent pas indéfiniment jouir de l'épanouissement de la vigueur et c'est pourquoi le koua ta tshang est suivi du koua $t \sin$; tsin, avancer. » Aucune raison n'existe qui fasse que les êtres soient indéfiniment dans l'épanouissement de la vigueur et qu'ils s'y maintiennent arrêtés ; du moment où leur vigueur est arrivée à son parfait développement, ils doivent nécessairement avancer, et c'est ce qui fait que le koua tsin suit immédiatement le koua ta tshang. Il est formé par le koua simple li au-dessus du koua simple khouen : la clarté sort au-dessus de la terre. Le soleil sort sur la terre ${ }^{1}$, il s'élève et devient de plus en plus brillant, ce qui est considéré comme son mouvement de progression en avant. Ce caractère tsin comporte lidée de mouvement en avant et de grandeur de développement de l'éclat. Toute chose qui se déve loppe et se perfectionne peu à peu est considérée comme «avançant», aussi le commentaire de la formule déterminative dit : «Tsin, mouvement de progression en avant. » Parmi les koua, les uns possèdent des vertus, ou propriétés, les autres n'en possèdent point ${ }^{2}$, suivant l'opportunité. En dehors des koua khien et khouen, il s'en trouve certainement au sujet desquels la formule dit : « grande liberté », ou : «avantage de la perfection »; ce qui manque pour qu’il soit susceptible d'être avantageux n'est pas toujours la même condition ; tel est le cas des koua ko et khien et cela peut se voir selon le koua dont il s'agit. Ce qui fait que le koua $t \sin$, bien qu'exprimant un état de développement florissant, ne possède cependant point ces vertus, c'est qu'il serait inutile qu'il les possédât. La clarté exprimée par le koua min est parfaitement grande, de sorte qu'il est inutile d'ajouter qu'e lle jouit de liberté ; puisqu'elle s'accorde à la grande clarté, il est inutile d'avertir au sujet de la nécessité de la droiture.

\footnotetext{
${ }^{1}$ S'élève au -dessus de l'horizon.

2 Il s'agit des quatre vertus fondamentales du koua khien : «Grandeur, liberté, bien, perfection.
} 


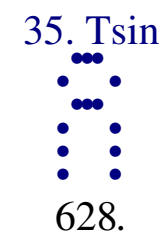

Tsin, progression en avant; pacifier les feudataires en employant le don de nombreux chevaux; pendant la clarté du jour, trois entrevues.

TSHENG TSE. - Le koua tsin exprime le moment du parfait épanouissement de la progression en avant ; la grande clarté est au-dessus et la substance inférieure s'annexe passi vement; c'est l'image symbolique de tous les feudataires se soumettant au roi, et c'est pour cela que la formule le considère comme indiquant l'action de pacifier les feudataires. "Les feudataires », c'est-à-dire les feudataires de régions en paix et en ordre. Grande clarté du supérieur et capacité de développer des vertus équivalentes pour s'annexer à lui et le suivre, exprime bien la condition des feudataires soumis et réguliers dans leurs devoirs, qui, par suite, sont comblés de faveurs répétées; les chevaux qui leur sont donnés sont en très grand nombre. Les chars et les chevaux sont les cadeaux les plus importants. Les septième et huitième caractères signifient «grand nombre ». Non seulement il s'agit de cadeaux très considérables, mais, de plus, il s'agit de la cérémonie des audiences personnelles ${ }^{1}$; pendant la durée de la clarté du jour, il y a jusqu’à trois entrevues, ce qui exprime le plus haut degré de la faveur. Le koua $t$ sin exprime le moment de l'épanouisse ment de la progression en avant; le supérieur éclaire, l'inférieur reçoit la clarté et suit. Le prince et le sujet s'accordent ensemble. Appliquée au supérieur, cette formule expliquera l'action d'avancer dans l'illustration en s'élevant de plus en plus, en recevant des faveurs signalées.

TSHOU HI. - Tsin, progresser, avancer. « Pacifier les feudataires », apaiser et purifier les feudataires qui gouvernent les divers royaumes. «Don de nombreux chevaux ; pendant la clarté du jour, trois entrevues », exprime la concession des grands cadeaux et l'admission à la cérémonie de l'audience. En effet, ce qui constitue le koua, c'est le koua simple $l i$, clarté, en haut, et le koua simple khouen, passivité, en bas; l'image symbolique du soleil émergeant sur la terre s'y trouve virt uellement; soumission s'adjoignant à la vertu d'une grande intelligence. De plus, par modification, le koua provient du koua kouan ${ }^{2}$ dans lequel la douceur malléable représentée par le quatrième trait avance et progresse en montant pour parvenir jusqu'au cinquième rang. Si celui qui consulte le sort réunit ces trois conditions, il devra, de même, jouir de faveurs analogues.

\footnotetext{
${ }^{1}$ Accordées par le souverain.

${ }^{2}$ Koua ${ }^{\circ} 20$.
} 
629.

Le commentaire traditionnel de la formule déterminative dit: Tsin, progresser en avant.

TSHOU HI. - Explication du sens du nom du koua.

630.

La clarté sort au-dessus de la terre : soumission et annexion à la grande clarté $^{1}$; la douceur malléable avance et monte en progressant ${ }^{2}$; c'est parce qu'en pacifiant les feudataires il emploie le don de nombreux chevaux et trois audiences pendant la clarté du jour.

TSHENG TSE. - T Sin, avancer ; la clarté avance et s'épanouit ; la clarté sort au-dessus de la terre, elle avance, augmente et se développe, de sorte que le koua est appelé $t \sin$. Ce qui fait que ce n'est pas le caractère $t \sin$, qui est employé ${ }^{3}$, c'est que ce dernier caractère désigne l'action de progresser en avant, mais ne peut comporter le sens de «clarté qui se développe ». La clarté sort au-dessus de la terre; c'est-à-dire que le koua simple li est au-dessus du koua simple khouen; le koua simple khouen s'attache au koua simple $l i$, pour se conformer à la grande clarté ${ }^{4}$; c'est le sujet vertueux et soumis qui monte se joindre au prince doué d'une grande intelligence. « La douceur malléable avance et monte en progressant »; toutes les fois que le koua simple li est au-dessus, la douceur malléable occupe la situation du prince, et le plus souvent la formule dit que la douceur malléable avance et monte en progressant ; c'est le cas, par exemple, dans les koua she ho ${ }^{5}$, khouei ${ }^{6}$, ting ${ }^{7}$. Le cinquième trait hexaire emploie la douceur pour se maintenir dans la situation du prince; il est intelligent et s'unit avec soumission, ce qui est considéré comme exprimant le sens de pouvoir traiter ses inférieurs avec

\footnotetext{
1 «Clarté », et « intelligence », sont synonymes.

2 En marchant.

${ }^{3}$ Mot à peu près synonyme et plus usuel.

${ }^{4}$ Le commentateur lit évidemment « à la grande intelligence ».

5 Koua n ${ }^{\circ} 21$

${ }^{6}$ Koua $n^{\circ} 38$.

${ }^{7}$ Koua $\mathrm{n}^{\circ} 50$.
} 
faveur et de leur accorder des audiences privées. C'est à cause de cela que le koua est considéré comme exprimant l'action de pacifier les feudataires en employant le don de nombreux chevaux et d'accorder trois audiences pendant la durée de la clarté du jour. Un prince doué d'une grande intelligence est celui qui pacifie l'univers ; les divers feudataires capables de suivre avec déférence l'ascendant de la grande intelligence du souverain suprême, sont les feudataires qui savent assurer la paix des peuples des divers royaumes, et c'est pour cela que la formule emploie les mots «feudataires pacifiés ». Ce résultat est obtenu par la libéralité de grandes faveurs et par des audiences personnelles, telles que dans la durée de la clarté d'un même jour les feudataires sont admis trois fois à voir le souverain suprême ${ }^{1}$. La formule ne parle pas des dignitaires revêtus des titres de kong et de khing ; elle parle des «feudataires ». Le souverain suprême est celui qui gouverne au-dessus de tous ; les divers feudataires sont ceux qui gouvernent au-dessous de lui ; être dans un rang secondaire et se joindre avec déférence au prince doué d'une grande intelligence donne l'image symbolique des divers feudataires.

TSHOU HI. - Explication de la formule du koua au moyen des images symboliques et des vertus des koua simples et au moyen des modifications progressives du koua lui même.

631.

Le commentaire traditionnel de la formule symbolique dit : La clarté sort au-dessus de la terre progression en avant; l'homme doué s'applique à éclaircir lui-même sa brillante vertu ${ }^{2}$.

TSHENG TSE. - Éclaircir, éclairer, rendre plus brillantes. Le commentaire traditionnel ${ }^{3}$ dit: «Éclairer la vertu, prévenir les transgressions »; éclairer les préceptes. L’homme doué contemple l’image symbolique de la clarté sortant au-dessus de la terre, du développement et de l'accroissement de l'éclat de cette clarté, et il s'emploie à éclairer lui -même sa propre faculté d’intelligence, pour écarter ce qui la voile et étendre son savoir. Éclairer sa propre vertu d'intelligence, c'est éclairer la faculté d'intelligence de tout l'univers ; éclairer l'intelligence hors de soi-même, c'est éclairer sa propre intelligence, et c'est pour cela que la formule dit : «éclairer luimême ».

TSHOU HI. - Le terme tshiao du texte a le sens de ming, éclairer.

\footnotetext{
${ }^{1}$ Littéralement « fils du Ciel ».

${ }^{2}$ L'âme ; les facultés de discernement du bien, du juste et du vrai.

${ }^{3}$ Le grand commentaire, c'est -à-dire les « formules annexées » de Khong Tse.
} 


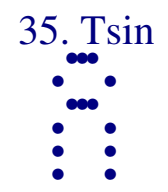

632.

Premier trait hexaire : comme avançant, comme se retirant; présage heureux de la perfection ; absence de foi ; magnanimité sans culpabilité.

TSHENG TSE. - Le premier trait occupe le rang inférieur dans le koua qui marque le mouvement en avant; il indique le début du mouvement de progression. "Comme avançant», montant et avançant; «comme se retirant », se retirant en arrière. Au début du mouvement de progression, la formule exprime que, soit qu'on donne libre cours au mouvement de progression en avant, soit qu'on continue ce mouvement, ce n'est qu'en se conformant à la droiture que le présage sera heureux. " Absence de foi », ou de confiance, exprime que celui qui est en bas et qui commence le mouvement en avant ne saurait avoir subitement une profonde confiance dans celui qui est au-dessus de lui; si le supérieur ne manifeste pas encore sa bonne foi, l'inférieur doit se contenter de se main tenir et de s'observer lui -même, avec de la douceur et de la patience, de la grandeur et de la magnanimité, sans mettre d'empressement prématuré à appeler la confiance du supé rieur. Si le désir d’inspirer la confiance est ardent, en admettant même qu'il ne s'agisse pas d'une agitation d'esprit propre à faire manquer à l'o bservation de soi-même, il y aura néanmoins une excitation propre à conduire au manquement au devoir ; aussi avec de la grandeur et de la magnanimité, il n'y aura point de culpabilité : c'est la voie suivant laquelle l'homme doué se porte en avant ou se retire en arrière.

TSHOU HI. - Employant la négativité et se maintenant au rang inférieur, ses sympathies n'étant conformes ni à la justice, ni à la droiture, il donne l'image symbolique de vouloir avancer et d'apercevoir un obstacle qui arrête. Si celui qui consulte le sort est dans ces conditions, et s'il peut observer la droiture, le présage sera heureux. En supposant que les hommes n'aient point confiance en lui, il doit néanmoins se placer avec grandeur et générosité, et il évitera toute culpabilité.

633. 
Comme avançant, comme se retirant, seul il marche avec droiture. Magnanimité sans culpabilité ; il n'a pas encore reçu de mandat ${ }^{1}$.

TSHENG TSE. - Sans avancer, sans reculer; lui seul marche dans la voie de la droiture; avec la grandeur et la générosité il sera sans culpabilité, parce qu'il commence à vouloir avancer et qu’il ne mérite pas encore la situation qu’il désire. L’homme doué, lorsqu'il s'agit d'avancer ou de se retirer, agit tantôt avec empressement et tantôt avec lenteur, uniquement selon que le devoir le comporte, mais il n'agit jamais sans grandeur d'âme. L'homme saint ${ }^{2}$, craignant que la postérité ne comprit pas le sens des mots grandeur et générosité et que ceux qui occuperaient quelque situation importante n'abandonnassent leurs fon ctions et manquassent à leurs devoirs en croyant montrer de la grandeur d'âme, mentionne expressément, et d'une manière toute spéciale, que la magnanimité du premier trait hexaire sera sans culpabilité : parce qu'il commence son mouvement en avant, qu’il n'a pas encore été investi d'un mandat par l'autorité suprême et qu'il n'est encore revêtu d'aucune charge ou dignité. S'il était investi d'une magistrature, s'il manquait de bonne foi envers le supérieur et s’il manquait aux devoirs de sa charge, il ne devrait pas y rester un seul jour. Mais tous les cas ne sont pas identiques ; l'empressement ou l'hésita tion conviennent selon le moment et dépendent aussi de l’infinie diversité des affaires en jeu.

TSHOU HI. - Le premier trait occupe la situation inférieure, il n'a pas encore reçu de mandat souverain conférant une magistrature.

\footnotetext{
${ }^{1}$ Mandat céleste, ou destin ; charge conférée par l'empereur.

${ }^{2}$ Khong Tse.
} 


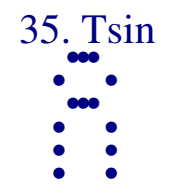

634.

Deuxième trait hexaire : comme avançant, comme triste; présage heureux de la perfection ; il reçoit ce grand bonheur ; vers la mère du roi.

TSHENG TSE. - Le second trait hexaire est dans une position inférieure ; au-dessus de lui, il ne rencontre aucun accueil sympathique ; c'est celui qui emploie les vertus de la justice et de la droiture, de la douceur et de l'aménité sans essayer d'avancer par la force. Aussi, dans l'action d'avancer, cela peut causer de la tristesse et du chagrin, ce qui exprime la difficulté qu'il éprouve pour avancer. Cependant, conservant sa parfaite droiture, il méritera d'atteindre le bonheur, de sorte que la formule dit : " comme avançant, comme triste, présage heureux de la perfection ». L'expression wang mou, composée des deux derniers caractères du texte, désigne une aïeule, c'est-à-dire une personne de rang prééminent dans le genre négatif ${ }^{1}$, et elle se rapporte au cinquième trait hexaire. Le second emploie la voie de la justice et de la droiture et s'observe lui -même ; bien qu'en haut il ne rencontre aucun accueil ou correspondance sympathique, et qu'il ne puisse avancer par luimême ${ }^{2}$, cependant ses vertus de justice et de droiture doivent à la longue finir par briller et les hommes du rang supérieur devront nécessairement avoir recours à lui. En effet, le prince doué d'une grande intelligence, représenté par le cinquième trait hexaire, possède les mêmes vertus, il devra donc certainement l'appeler à lui, le combler de faveurs et d honneurs : «il reçoit ce grand bonheur par l'aïeule royale ». Le terme kiae, onzième caractère du texte, a le sens de $t a$, grand.

TSHOU HI. - Le second trait hexaire possède la justice et la droiture ; au-dessus de lui, il ne rencontre aucun accueil sympathique, aussi, il voudrait avancer et il est triste. Si celui qui consulte le sort est dans ces conditions, et sil est capable d'observer la droiture, le pré sage sera heureux, et il recevra des honneurs conférés par l'aïeule royale. L'expression wang mou, aïeul royal, désigne le cinquième trait hexaire. En effet il bénéficiera du sens divinatoire propice qui s'attache à l'idée d'une aïeule des générations passées et à tout ce qui, étant négatif, occupe un rang prééminent.

\footnotetext{
1 Sexe féminin.

2 Par ses seules forces.
} 
635.

Il reçoit ce grand bonheur à cause de la justice et de la droiture.

TSHENG TSE. - Il reçoit ce grand bonheur à cause de la voie rationnelle de la justice et de la droiture. L'homme qui peut conserver la voie de la justice et de la droiture doit nécessairement, à la longue, jouir de la liberté ; à plus forte raison encore, lorsqu'une grande intelligence occupe le rang supérieur et possède les mêmes vertus, il doit certainement en recevoir de grands honneurs. 


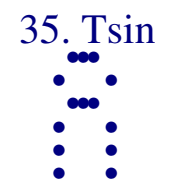

636.

Troisième trait hexaire : confiance universelle; les regrets se dissipent.

TSHENG TSE. - Puisque c'est un trait hexaire qui occupe le troisième rang, il ne possède ni la justice, ni la droiture ; il doit nécessairement éprouver des regrets et commettre des fautes. D'ailleurs, le troisième rang est le dessus de la substance de la passivité ${ }^{1}$; il exprime le degré le plus extrême de la soumission. Les trois traits négatifs se soumettent tous également au supérieur ; donc la soumission du troisième, envers le supérieur, marque la communauté de tendances de la foule, celui que la foule suit avec une entière confiance, et c'est à cause de cela que ses regrets se dissipent. Ses tendances le portant à la soumission au supérieur et à se tourner vers l'intelligence ${ }^{2}$, et la foule le suivant avec une entière confiance, qu'est-ce qui ne lui serait pas avantageux ? On a dit: Ne procédant ni par la justice, ni par la droiture, et étant de même nature que la foule, cela peut-il être considéré comme exprimant le bien ? Celui en qui la foule a confiance doit nécessairement en être on ne peut plus digne ; à plus forte raison comment la soumission à la grande intelligence du supérieur aurait-elle de mauvais côtés ? C'est à cause de cela que les regrets se dissipent, car il efface les fautes résultant du manque de justice et de droiture. Les anciens disaient: "Quiconque suit la foule s'associe à la volonté du ciel ${ }^{3}$. »

TSHOU HI. - Le troisième trait est sans justice, ni droiture ; c'est celui qui doit naturellement éprouver des regrets. Puisque avec les deux traits négatifs inférieurs, ils veulent tous ensemble avancer en montant, il est considéré comme représentant celui en qui la foule a confiance, et dont les regrets se dissipent.

637.

La foule a confiance en lui ; ses tendances le portent à monter.

\footnotetext{
1 Trait supérieur du koua simple kkouen.

${ }^{2}$ Exprimée par le koua simple $l i$.

${ }^{3}$ Vox populi, vox Dei.
} 
TSHENG TSE. — «Agir en montant», c'est monter avec déférence pour s'associer à la grande intelligence ${ }^{1}$. Il monte suivre le prince doué d'une grande intelligence, c'est en cel a que ses tendances sont identiques à celles de la foule.

${ }^{1}$ Grande clarté. 


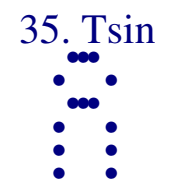

638.

Quatrième trait nonaire : avancer comme le rat des champs ${ }^{1}$; inconvénients de la perfection.

TSHENG TSE. - Puisqu'il est nonaire et qu’il occupe le quatrième rang, ce n'est point la situation qui lui convient ; ce n'est point la position qui lui convient et il l'occupe, c'est donc celui qui ambitionne prématurément une situation. Ayant l'ambition de se placer dans une situation élevée, n'ayant pas ce qui le satisferait, et d'ailleurs possédant les mêmes vertus que le trait supérieur, il s'associe avec soumission au supérieur. Les trois négativités sont toutes au-dessus de lui, la force naturelle des choses fait qu'il doit néces sairement avancer en montant, aussi son cour est agité par la crainte et le soupçon, c'est un homme ambitieux et craintif. Le rat des champs est avide et craint l'homme, aussi la formule dit : «Avancer comme le rat des champs. » Il est avide de ce qui n'est pas à sa portée et conserve son cour craintif et soupçonneux; en maintenant ces dispositions avec une parfaite fermeté, la possibilité du péril devient évidente, ce que la formule exprime par les mots inconvénients de la perfection. Elle ouvre une voie à la réforme.

TSHOU HI. - Sans justice, sans droiture, agissant pour s'approprier clandestinement une situation élevée, ambitieux et craintif : c'est en effet une voie de périls, aussi ce trait est considéré comme comportant l'image symbolique du rat des champs. Si celui qui consulte le sort est dans ces conditions, bien qu'il ait le droit pour lui, il court cependant des périls.

639.

Inconvénients de la perfection pour le rat des champs; situation imméritée.

TSHENG TSE. - Le sage, employant les vertus de la droiture, doit naturellement occuper une situation élevée; être sans droiture et se placer dans une situation élevée constituera ce qu'on entend par chercher à obtenir ce qui n'est pas à portée. Avide, et craignant de perdre cette situation, il a peur des hommes ; il se maintient fermement sur ce terrain, et le péril est évident.

\footnotetext{
${ }^{1}$ Il s'agit d'un rongeur à queue poilue qui dévore les récoltes.
} 


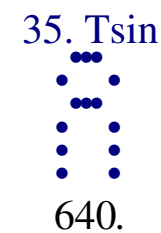

Cinquième trait hexaire : Les regrets se dissipent; ne pas s'inquiéter de perdre ou d'obtenir ; en agissant présage heureux, rien sans avantage.

TSHENG TSE. - Ce trait hexaire emploie la douceur pour se maintenir dans la situation prééminente; d'abord il est naturel quil éprouve des regrets ${ }^{1}$, c'est à cause de sa grande intelligence et parce que tous les inférieurs se joignent à lui avec soumission que ses regrets peuvent ensuite se dissiper. Puisque les inférieurs possèdent les mêmes vertus et se joignent à lui avec soumission, il convient qu'il examine leur sincérité pour leur délé guer l'autorité ${ }^{2}$, qu'il utilise les aptitudes de tous, qu’il se rende compte des tendances de l'univers, sans continuer plus longtemps à s'autoriser de sa propre intelligence et à s'inquiéter de réussir ou d'échouer dans ses projets. Dans de telles conditions, sîl entre prend quelque chose, le présage sera heureux et rien ne sera sans avantage. Le cinquième trait hexaire est celui de qui dépend la grande intelligence ${ }^{3}$; il ne déplore pas de ne pouvoir éclairer et illuminer, il déplore les erreurs qu'il peut commettre dans l'emploi de son intelligence, d'où résulte que dans l'examen du mérite individuel de chacun il en vient à manquer à la voie rationnelle quiil devrait observer en déléguant son autorité. C'est pour cela que la formule avertit au sujet de la préoccupation de perdre ou d'obte nir ${ }^{4}$, car la partialité dans la délégation du fardeau de l'autorité le conduira, sỉl n'y fait attention, à aliéner et à éteindre dans l'univers le sentiment du dévouement au bien public. Comment donc pourrait-on encore baser ses choix sur des motifs d'intérêt privé !

TSHOU HI. - Employant la douceur malléable et occupant une situation qui comporte la dureté énergique, il est naturel quill en éprouve des regrets. C'est parce que la gran de intelligence (clarté) est en haut, et qu'en bas tous suivent avec soumission, que le sens divinatoire pour celui qui obtient ce trait en consultant le sort, sera que les regrets se dissiperont. De plus, en dépouillant tout sentiment de calcul d'intérêt privé, le présage sera heureux pour ses entreprises, et rien ne sera sans avantage. Mais toutefois, il faut absolument qu'il possède ces vertus pour être dign e de ce sens divinatoire.

\footnotetext{
${ }^{1}$ Ou des inquiétudes.

${ }^{2}$ Qu'il s'assure des qualités de ceux qu'ill nomme aux emplois publics.

${ }^{3}$ Ou clarté.

${ }^{4}$ De réussir ou d'échou er.
} 
641.

Ne se préoccupant pas de réussir ou d'échouer, en entreprenant il y a succès.

TSHENG TSE. - Employant les vertus de la grande intelligence, jouissant du concours des inférieurs, recherchant la sincérité du mérite pour lui confier le poids de l'autorité, il pourra réussir et achever la grande auvre de l'univers ; c'est là avoir du succès dans ses entreprises. 


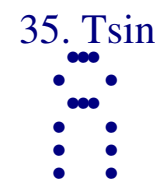

642.

Trait supérieur nonaire : avancer la corne ; seulement l' employer à détruire le district ; appréhension, présage heureux, pas de culpabilité. Perfection cause d'inquiétude.

TSHENG TSE. - La corne est quelque chose de dur et placée haut. Le trait supérieur nonaire emploie la dureté énergique et occupe le dernier rang dans le koua, aussi la formule prend la corne comme image symbolique. Un trait positif occupant le rang supérieur, c'est le comble de la dureté éner gique ; étant au rang supérieur du koua $t$ sin (avancer), il indique le degré extrême dans le mouvement en avant. La dureté énergique étant à son comble, il doit nécessairement y avoir excès de violence et de brutalité ; le mouvement de progression en avant étant arrivé à son plus extrême degré, il y a nécessairement des fautes commises par précipitation et hâte excessive. Employer la dureté énergique et pousser le mouvement de progression en avant jusqu'à l'excès, c'est un manque complet de modération ; une telle voie n'est jamais licite, quoi qu'il s'agisse d'entreprendre ; elle ne peut s'employer que pour combattre et détruire un pays, car alors, bien qu'il y ait des motifs d'appréhension, cependant c'est un présage heureux de succès, et d'ailleurs cela ne constitue plus un fait de culpabilité. L'expression « détruire les quatre régions » indique qu'il s'agit de dominer et de soumettre à l'extérieur de l'Etat, l'expression « détruire le district » indique qu'il s'agit de dominer à l'intérieur des limites de l'État. La formule emploie l'expression détruire le district ; cela exprime se dominer soi-même intérieurement. L'homme qui cherche à se dominer soi-même, et qui pousse l'énergie à l'excès dans cette voie ne manque pas à la voie rationnelle et s'y attache au contraire avec fermeté ; en poussant le mouvement de progression en avant à son extrême limite, il se convertira au bien plus rapidement. Ainsi, lorsqu’il s'agit du trait supérieur nonaire, s'appliquant à se dominer, bien qu'il puisse souffrir d'appréhension, cependant le présage est heureux, et d'ailleurs il n'y a point de culpabilité. La sévérité qui inspire la crainte et l'appréhension n'est pas une voie rationnelle de paix et de calme, cependant dans le cas unique où il s'agit de se corriger soi-même, cette voie a son mérite. La formule ajoute «perfection cause d'inquiétude »; c'est pour épuiser le sens. Bien que, lorsqu'il s'agit de se dominer soi -même, l'extrême limite dans l'énergie et dans le mouvement de progression ait ses avantages, cependant ce n'est jamais une vertu qui comporte la modération et la justice ; aussi, même dans une voie suivie avec une parfaite droiture, cela peut encore être une cause d’inquiétude. La «perfection», c'est l'absence de tout manquement à la justice et à la droiture. 
TSHOU HI. - La corne est dure et placée haut ; le trait supérieur nonaire exprime l'extrême limite de la dureté énergique dans le mouvement de progression en avant, donc il a cette image symbolique. Si celui qui consulte le sort obtient ce trait, et s'il tra vaille à se vaincre lui-même ${ }^{1}$, bien que la voie soit périlleuse, le présage est heureux et d'ailleurs il n'y a pas de culpabilité. Toutefois, employant une extrême dureté pour dominer un petit district ${ }^{2}$, bien qu'il s'accorde à la droiture, c 'est cependant une cause d’inquiétude.

643.

Seulement l'employer à détruire le district; la voie n'est pas encore brillante.

TSHENG TSE. - Seulement l'employer à détruire le district ; outre le présage heureux et l'absence de culpabilité, la formule mentionne encore la perfection cause d'inquié tudes. La voie n'est pas encore grande et brillante. En en parlant au sujet de la droite raison d'être, il y a essentiellement une cause d'inquiétude. Or, lorsque la voie est grande et brillante, rien n'est évidemment dépourvu de justice et de droiture ; comment y aurait-il donc un inconvénient? Dans le cas actuel, il s'agit de se corriger avec un excès de dureté ; bien que cette aure comporte un certain mérite, cependant la voie de celui qui l'accom plit n'es t pas encore grande et brillante (puisquil se corrige), de sorte qu'il y a encore une cause d'inquiétude. L'homme saint parle de la voie rationnelle du bien absolu.

\footnotetext{
1 «Détruire son propre district. »
}

${ }^{2}$ Pour un petit motif. 


\section{Ming yi}

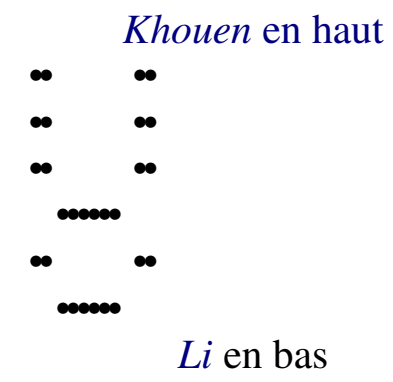

643. Ming yi ; avantage de la défiance ; perfection.

648. Premier trait nonaire : la lumière blesse le vol; laisser pendre l'aile. L'homme doué, sur le point d'agir, reste trois jours sans manger ; il y a lieu d'agir ; l'hôte fait des observations.

*

650. Deuxième trait hexaire: ming yi ; blessure dans la cuisse gauche; employer le secours d'un cheval vigoureux ; présage heureux.

652. Troisième trait nonaire : la lumière s'obscurcit vers la limite méridionale; capturer le grand chef; on ne doit pas tendre hâtivement vers la perfection.

654. Quatrième trait hexaire : pénétrer dans les viscères de gauche; saisir l'intention d'obscurcir la clarté ; au sortir de la porte et du parvis.

$*$

656. Cinquième trait hexaire : obscurcissement de la clarté par Ki Tse ; avantage de la perfection.

*

658. Trait supérieur hexaire : il n'éclaire pas l'obscurité ; d'abord il s'élève au ciel ; ensuite il rentre dans la terre.

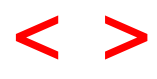




\section{Ming yi.}

TSHENG TSE. — Ming yi. «L'Ordre des koua » dit : « Tsin, avancer; en avançant il y a nécessairement des causes de blessures, et c'est pour cela que ce dernier koua est suivi du koua ming yi. Yi, blessures, accidents nuisibles. » Or, lorsque le mouvement de progression en avant ne cesse point, il doit nécessairement être nuisible à certaines choses; la raison d'être de cette assertion est évidente par elle-même, et c'est pour cela que le koua ming yi suit immédiatement le koua tsin. Il est constitué par le koua simple khouen en haut et par le koua simple $l i$, en bas ; la clarté entre dans l'intérieur de la terre ${ }^{1}$; en renversant le koua $t \sin$ on obtient le koua ming $y i$, aussi le sens exprimé et celui qui est relatif au koua $t$ sin sont diamétralement opposés. Le koua $t \sin$ est l'emblème de la clarté parfaite ; un prince intelligent occupe le rang supérieur ; c'est le moment où la foule des sages avance d'un mê me pas. Le koua ming yi est l'emblème de l'obscurité ; c'est un prince dénué d'intelligence qui est au rang supérieur, et c'est le moment où la clarté s'affaiblit. Le soleil rentre dans la terre ; la lumière s'altère ${ }^{2}$, aussi le koua est appelé ming yi.

\footnotetext{
${ }^{1}$ Ming, clarté ; khouen, la terre.
}

${ }^{2}$ Littéralement : est blessée. 


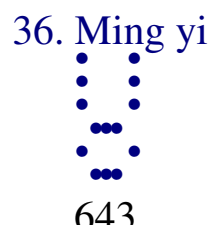

Ming yi ; avantage de la défiance ; perfection.

TSHENG TSE. - Pour l'homme doué qui se trouve en présence du moment exprimé par le koua ming yi, l'avantage consiste dans la connaissance du danger ; il consiste à savoir le craindre et à ne pas manquer à la droiture. C'est là ce qui constitue l'intelligence de l'homme doué.

TSHOU HI. — Le mot yi signifie « blesser », blessure, dommage, mal. Le koua est constitué par le koua simple $l i$, en bas, et le koua simple khouen, en haut ; le soleil rentrant dans la terre ; c'est le symbole de la clarté sur le point de s'affaiblir, aussi ce koua est appelé ming yi. De plus, le trait supérieur hexaire est celui de qui dépend l'obscurité ; le cinquième trait hexaire s'approche e t se serre contre lui, aussi, pour celui qui consulte le sort, le bien consiste dans la défiance du péril, pour conserver la droiture, et il consiste aussi à masquer soi-même, en la cachant, sa propre intelligence.

644.

Le commentaire traditionnel de la formule déterminative dit : La lumière entre dans la terre; ming yi.

TSHOU HI. - Explication du nom du koua au moyen de l’image symbolique du koua.

645.

Au-dedans clarté de la forme et au-dehors malléabilité passive; par l'obscurité naissante grande difficulté ; Wen Wang s'en servit.

TSHENG TSE. - La lumière entre dans la terre ; sa clarté s'éteint, aussi, le koua est appelé ming yi. Au-dedans, le koua simple $l i$; c'est le symbole de 
la clarté de la forme. Au-dehors, le koua simple khouen; c'est le symbole de la malléabilité passive. Au sujet de l'homme, il indique les vertus intérieures de la clarté de l'intelligence, avec la faculté de se soumettre avec flexibilité aux circonstances extérieures. Tel, autrefois, fut Wen Wang, aussi la formule dit : «Wen Wang s'en servit. » En présence de l'aveuglement de l'empe reur Tsheou, ce qui constituait bien le moment désigné par le koua ming yi, Wen Wang possédait en lui-même les vertus de l'intelligence de la forme ${ }^{1}$, tandis qu'à l'extérieur, il se montra it soumis envers Tsheou et le servait. Le péril causé par l'obscurcissement cou pable était grand et cependant il ne s'écarta pas, dans sa conduite personnelle, de la sainteté de l'intelligence, tandis qu'au -dehors il savait détourner le malheur et le conjurer. Telle fut la voie que suivit Wen Wang, et c'est pour cette raison que la formule dit : «Wen Wang l'employa. »

TSHOU HI. - Explication du sens du koua au moyen des propriétés des koua simples. «Grand péril de l'obscurité » c'est-à-dire se trouver exposé aux perversités de Tsheou et subir une captivité ${ }^{2}$.

646.

Avantage par le danger et la perfection ; voiler la clarté ; au-dedans, péril et être capable de rectifier le jugement; c'est ce que fit Ki Tse.

TSHENG TSE. - Dans le moment exprimé par le koua ming yi, l'avantage consiste à être placé dans le danger sans cependant manquer à la plus parfaite droiture, c'est-à-dire à savoir voiler et cacher l'éclat de son propre mérite. Si on ne savait pas cacher l'éclat de sa propre valeur, on serait atteint par le malheur; d'un autre côté, si on ne maintenait pas intacte sa propre droiture, ce ne serait plus posséder l'éclat de la sagesse. Ki Tse, au temps de Tsheou, se trouvait à l'intérieur du royaume ${ }^{3}$; c'était bien être placé au plus fort du péril, et aussi la formule dit : «au-dedans péril » ${ }^{4}$. Cependant, $\mathrm{Ki}$ Tse sut voiler l'éclat de sa propre intelligence, et conserver en même temps la droiture de ses desseins. Telle fut la voie suivie par Ki Tse, et c'est pour cela que la formule dit : «Ki Tse s'en servit. »

\footnotetext{
${ }^{1}$ Expression figurée dont le sens est conventionnel et mal défini, les qualités de lintelligence et de l'âme.

2 Wen Wang.

${ }^{3}$ C'est-à-dire qu'au lieu d'être dans son fief, il résidait dans le district royal.

${ }^{4}$ Le commentateur lit évidemment «dans le péril », mais alors il renverse l'ordre de la construction.
} 
TSHOU HI. - Explication de la formule du koua au moyen du sens de la seule formule du cinquième trait hexaire. «Au-dedans péril » ${ }^{1}$, c'est-à-dire être à proximité de Tsheou, être à l'intérieur de son royaume, comme le cinquième trait hexaire se trouve proche du trait supérieur, aussi négatif.

647.

Le commentaire traditionnel de la formule symbolique dit: La lumière entre dans la terre : ming yi. L'homme doué en surveillant ${ }^{2}$ la foule se sert de l'obscurité et éclaire.

TSHENG TSE. — La lumière est ce qui éclaire ; il n'est rien que l'homme doué n'éclaire, mais en poussant trop loin l'usage de l'intelligence, en projetant trop de lumière, il blesserait par la sévérité de son examen et la rigidité de ses investigations. L'excès dans l'invest igation ne laisse échapper aucun détail et ne comporte aucune mesure de tolérance et de modération. Aussi, l'homme doué considère l'image symbolique de la clarté entrant dans la terre, et, en gouvernant la foule, il ne pousse pas à son extrême limite la clarté de son examen et il sait se servir de son obscurité. Il en résulte naturellement qu'il sait être tolérant envers les êtres et qu'il montre de la mansuétude envers les foules. Tous s'approchent de lui et vivent en paix. C'est ainsi qu'il sait se servi $r$ de l'obscurité et qu'il la convertit en clarté. S'il s'autorisait de sa propre intelligence et s’il ne laissait rien échap per à l'investigation de sa surveillance, il ne saurait dominer son mécontentement et ne posséderait pas la vertu de la tolérance et de la mansuétude. Les sentiments de défiance et de crainte seraient tenus en éveil chez les hommes qui ne pourraient être en paix; ce serait là manquer à la voie rationnelle du gouvernement de la foule, et précisément ce qui ferait qu’il manquerait d'in telligence. Les hommes saints de l'antiquité, en établis sant un écran d'arbres protecteurs, voulaient éviter que la lumière supprimât toute espèce d'ombre.

\footnotetext{
${ }^{1}$ Ou péril intérieur.

${ }^{2}$ Gouvernant.
} 


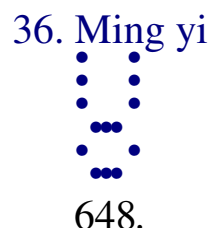

Premier trait nonaire : la lumière blesse le vol; laisser pendre l'aile. L'homme doué, sur le point d'agir, reste trois jours sans manger; il y a lieu d'agir ; l' hôte fait des observations.

TSHENG TSE. — Le premier trait nonaire fait partie de la substance de la clarté ${ }^{1}$ et il occupe le premier rang dans le koua ming yi; il voit le début du mal. Le terme nonaire indique ce qui est positif, ce qui est intelligent, ou brillant, ce qui monte et s'élève, aussi ce trait reçoit l'image symbolique du vol. Les ténèbres de l'ob scurité sont au-dessus ${ }^{2}$, et nuisent à l'éclat (clarté) de la positivité, pour que sa lumière ne puisse monter en avançant

c'est bien blesser l'aile pendant le vol. L'aile sent la blessure, aussi elle pend inerte. Toutes les fois que l'homme inférieur nuit à l'homme doué, il le blesse dans ses moyens d'action. L'homme doué sur le point d'agir reste trois jours sans manger. L'homme doué est intelligent et il éclaire ; il voit les choses dans leur germe ; bien qu'au début il voie la cause du mal, alors qu'el le n'est pas encore bien distincte, il peut cependant la voir et la reconnaître, de sorte qu'il agit pour s'en garder et l'éviter. " L'homme doué sur le point d'agir », c'est-à-dire sur le point d'abandonner sa situation, ses fonctions et son traitement, pour se retirer à l'écart et à l'abri. « Trois jours sans manger », exprime le dernier degré de la misère et de la souffrance. Le fait n'est pas encore manifesté et le lieu est extrêmement dangereux ; sans l'intelligence qui permet de distinguer le mal dans son germe, il serait impossible de l'éviter. Or, c'est l'homme doué seul qui peut voir cette ori gine ; sa connaissance n'est pas à la portée de la foule ; aussi au début du mal causé à ce qui est lumineux (ming yi), il évite ce mal avant qu'il ne soit déjà manifesté, de sorte que les gens de son époque, imbus des erreurs de leur temps, s'étonnent de ce quill fait, et il en résulte que lorsqu'il a à entreprendre quelque chose, ceux à qui il a affaire font des observations. Mais l'homme doué ne se laisse $\mathrm{p}$ as retarder dans son action par la mesquine considération de l'étonnement qu'il cause au vulgaire; s'il attendait jusqu'au moment où tous les hommes auront reconnu la présence du danger, le mal l'aurait déjà atteint et il ne serait plus à temps pour l'éviter. C'est en ceci que Pi Fang se montra intelligent, et c'est aussi ce qui fit que Yeang Hung ne sut pas assurer sa fuite. On a dit : Si le mal atteint déjà un point tel que les ailes pendent, ce mal est bien déjà devenu évident ; comment pourrait-il se faire que tous les hommes ne le vissent pas? Le

\footnotetext{
${ }^{1}$ Du koua simple $l$.

${ }^{2}$ Le koua simple khouen, qui exprime les ténèbres.
} 
premier trait indique le début du mal; la formule emploie l'expression «laisser pendre les ailes », c'est pour indiquer que ce qui est blessé est précisément l'instrument du vol ; mais le fait même n'est pas encore manifesté. L'homme doué voit l'origine du mal dans sa cause, de sorte qu'il est à temps pour l'éviter ; le vulgaire des hommes de son époque est incapable de voir cette origine, de sorte que chacun s'étonne de ce qu’il fait et le blâme. Ainsi, lorsque Mou Sheng quitta l'état de Tshou, Shen Kong, Pa Kong le blâmèrent inconsidérément; à plus forte raison fut-il aussi blâmé par le vulgaire. Mais ils critiquaient les remontrances qu'il avait adressées au sujet d'une cérémonie de peu d'importance, ta ndis qu'ils ignoraient que Mou Sheng évitait ainsi le malheur d'un supplice cruel ; en en parlant celui-ci disait : « Si je n'étais parti à temps, les gens de Tshou étaient sur le point de me traîner sur la place d'un marché ${ }^{1}$. » Bien que Shen et $\mathrm{Pa}$ fussent deux philosophes instruits, ils considéraient ces paroles comme une exagération. On peut citer encore, comme un autre exemple le cas de Yuen Hong avant l'époque de la conspiration; des lettrés de renom et de mérite se heurtèrent à la pointe du glaive ${ }^{2}$, et lui seul se cachait dans une hutte de terre, de telle sorte que les hommes le considéraient comme un être sauvage et idiot alors que, seul, il évitait les maux d'une incarcération pour fait de conspiration politique. Lorsqu'on entreprend quelque chose, y a-t-il de quoi s'étonner si quelqu'un blâme l'entreprise ?

TSHOU HI. - Voler et laisser pendre les ailes; image symbolique de blessure. Le sens divinatoire est : «agir et ne pas manger »; si le résultat de l'entreprise n'est pas favorable, c'est la conséquence du moment et des circonstances ; il est impossible de l'éviter.

649.

L'homme doué sur le point d'agir ; le devoir ne nourrit point.

TSHENG TSE. - L'homme doué se retire à écart, se cache et vit misérable ; c'est que le devoir l'exige ainsi. C'est uniquement la conséquence naturelle du devoir, aussi il se place ainsi calme, sans tristesse et sans colère, bien qu'il soit possible qu'il se prive de nourriture.

TSHOU HI. - Seulement à cause du devoir, cependant il est possible qu'il en résulte p our lui une privation.

\footnotetext{
${ }^{1}$ Au supplice.

2 Périrent par le glaive.
} 


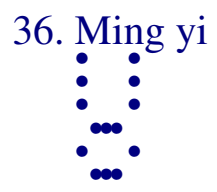

650.

Deuxième trait hexaire : ming yi; blessure dans la cuisse gauche ; employer le secours d'un cheval vigoureux; présage heureux.

TSHENG TSE. — Le second trait hexaire déploie les aptitudes de la plus brillante intelligence; il se conforme à la justice et à la droiture, et sa substance est la passivité. Il se place en se pliant aux exigences du moment : c'est le bien absolu dans la manière de se placer. Bien que l'homme doué se place en se conformant au bien, cependant il se trouve en présence d'un moment où l'ombre obscure de l'homme inférieur nuit à ce qui est brillant, et il ne peut pas non plus éviter d'en être blessé. Mais, en se plaçant, l'homme doué suit une voie rationnelle, de sorte qu'il ne peut en être profondément blessé et, finalement, il peut se soustraire au mal et l'éviter. Le pied est l'instrument de la marche, la cuisse est au -dessus de la jambe et du pied; son usage n'est que secondaire dans l'action de la marche ; de plus le côté gauche n'est pas celui qui doit être employé de préférence ; dans l'emploi de la main et du pied, il n'y a que l'action de tendre l'arc où le côté gauche a le rôle prépondérant, et en effet, dans ce cas, le côté droit est fixé et sert de point d'appui. "Blessure dans la cuisse gauche », veut dire blesser en nuisant à la marche sans que le mal soit très considérable. (Quoi qu’il en soit, il faut encore qu'il existe une voie pour se soustraire à ce mal ; en montant un cheval vigoureux et rapide on parviendra vite à s'écarter du danger et le présage devient heureux. L'homme doué, étant celui qui est blessé par l'ombre et l'obscurité, a une voie rationnelle à suivre pour se placer, aussi sa blessure n'est pas très grave ; il emploie cette voie et parvient vite à éviter le mal. Si en suivant cette voie il ne déployait pas d'énergie, il serait très gravement blessé, aussi la formule dit : «Avec un cheval vigoureux le présage sera heureux. » Le second trait, avec son intelligence brillante, occupe le rang inférieur dans l'obscurité ; ce qu'on entend par « présage heureux », c'est donc uniquement de pouvoir se soustraire au mal ; cela n'indique nullement qu'il pourra agir et prévaloir dans le même temps.

TSHOU HI. - Il y a blessure mais pas encore grave; en y remédiant rapidement le mal sera évité ; c'est pour cela que tels sont le sens divinatoire et l'image symbolique.

651. 
Présage heureux du second trait hexaire ; obéissance aux préceptes.

TSHENG TSE. - Ce qui fait que le second trait hexaire comporte un présage heureux, c'est qu'il se place avec déférence et soumission et qu'il se conforme aux règles et aux préceptes de la morale. Le mot précepte désigne la voie rationnelle de la justice et de la droiture. Étant capable de soumission et possédant la justice et la droiture, c'est ainsi qu’il peut se placer pendant le moment exprimé par le koua coing yi et quil peut assurer un présage heureux pour l'avenir. 


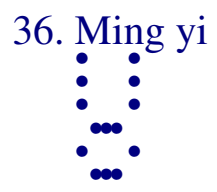

652.

Troisième trait nonaire : la lumière s'obscurcit vers la limite méridionale ${ }^{1}$; capturer le grand chef; on ne doit pas tendre hâtivement vers la perfection.

TSHENG TSE. - Le troisième trait nonaire est le trait supérieur du koua simple $l i$; il représente l'extrême degré de la clarté, ou de l'intelligence. De plus il se place dans un rang qui comporte l'énergie et il progresse en avant. Le trait supérieur hexaire, qui est aussi le trait supérieur du koua simple khouen, exprime le comble de l'obscurité. L'extrê me clarté est placée en dessous et est la partie supérieure de ce qui est en bas ${ }^{2}$, tandis que l'extrême obscurité est en haut et y occupe la position extrême ; il y a correspondance exacte entre les deux, ce qui exprime que la lumière est sur le point de dissiper les ténèbres. Ce sens ne se rapporte-t-il pas exactement au cas de Wou de la dynastie des Theang ? Le Midi est en avant et c'est la région de la lumière. Le septième caractère du texte indique quelque chose qui garantit et évite le mal ${ }^{3}$; l'expression « limite méridionale » signifie «progresser en avant et écarter le mal ». Il convient de capturer le grand chef; le «grand chef», c'est-à-dire le chef des rebelles représentés par l'obscurité, le trait supérieur hexaire. Le troisième trait et le trait supérieur se correspondent exactement et constituent l'image symbolique de l'extrême clarté l'emportant sur l'extrême obscurité. On ne doit pas tendre hâtivement vers la perfection; c'est-à-dire exterminer radicalement le mal; lorsque la cause du mal produit depuis longtemps son effet délétère et que les mours en sont imprégnées et corrompues, on ne doit pas l'extirper brusquement et il faut procéder peu à peu et progressivement. En supprimant brusquement le mal il y aurait des causes de crainte et de troubles; aussi le Shou king dit: "C'est seulement l'œuvre de l'exemple donné par Yin à tous ses sujets d'où est résulté la licence et l'abus du vin. Ne mettez pas légèrement à mort, mais bornez-vous à instruire, et cela même pendant une longue durée. » Le même livre dit encore : «Les autres coutumes ne sont pas encore abolies; ce sont les produits d'un effet lent et répété sur les moars ; on ne doit pas les supprimer radicalement à la hâte. » C'est ainsi que la formule dit qu'on ne doit pas tendre hâti vement vers la perfection; la droiture ne doit pas comporter la précipitation. Bien que le trait supérieur hexaire n'occupe pas la situation du prince ${ }^{4}$, comme il est

\footnotetext{
${ }^{1}$ Le sens de ce passage est très obscur et très diversement interprété.

${ }^{2}$ Le trait supérieur du koua simple inférieur ; telle est l'interprétation du commentateur, mais le sens littéral serait : «Le dessus du dessous. »

3 On traduit aussi ce caractère par « gibier » ou « chasse ».

${ }^{4}$ Le cinquième rang.
} 
placé au rang le plus élevé et comme il exprime le comble de l'obscurité, il est considéré comme représentant celui de qui dépend l'obscurcissement, et désigné par l'expression « grand chef » ${ }^{1}$.

TSHOU HI. - Avec sa dureté énergique il occupe un rang qui comporte l'énergie et la dureté, de plus il est au-dessus de la substance qui exprime la clarté ${ }^{2}$; cependant il plie sous la plus extrême obscurité et correspond exactement au trait supérieur hexaire de qui dépend l'obscurcissement, aussi il comporte l'image symbolique de se tourner vers la clarté, d'écarter le mal, et de capturer le principal auteur du mal. Toutefois, il ne doit pas agir avec précipitation, ce qui fait que la formule comporte l'avertissement de ne pas tendre hâtivement à la perfection. Tsheng fondant la dynastie des Theang à Hia tae, Wen Wang s’insurgeant à Keang li, offrent précisément des exemples de l'application du sens de cette formule, et il en est encore de même dans les choses de moindre importance.

653.

Tendances de la limite méridionale ${ }^{3}$; c'est alors un grand succès.

TSHENG TSE. - En effet, c'est employer la clarté d'en bas pour dis siper les ténèbres d'en haut ; la tendance consiste uniquement à écarter le mal. Par exemple Theang de la famille de Sheang et Wou de la famille de Tsheou pouvaient-il avoir en vue de s'emparer de l'empire ? S'emparer du grand chef, c'est pouvoir écarter le mal et réussir largement dans ses desseins. Si telles n'étaient pas les tendances, il n'y aurait plus qu'un fait de vul gaire ambition.

\footnotetext{
${ }^{1}$ Expression analogue à celle de « principal coupable ».

${ }^{2}$ Koua simple $l$.

${ }^{3}$ En adoptant le sens que l'on donne souvent à ce passag e, on lirait : « but de la chasse dans le midi ».
} 


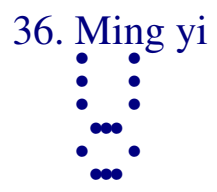

654.

Quatrième trait hexaire : pénétrer dans les viscères de gauche; saisir l'intention d'obscurcir la clarté ; au sortir de la porte et du parvis.

TSHENG TSE. - Le quatrième trait hexaire emploie les qualités de la négativité et occupe un rang qui comporte ces mêmes qualités; de plus encore, il fait partie de la substance de la malléabilité négative ${ }^{1}$; il est placé près de la situation du prince : c'est un homme inférieur et vicieux qui occupe une haute situation et qui emploie la bassesse et la flatterie pour complaire au prince. Le cinquième trait hexaire occupe la situation du prince dans le koua ming yi ; c'est de lui que dépend le mal causé à la lumière. Le quatrième le suit passivement en se prêtant bassement à ses mauvais desseins, pour affermir leur liaison. Or, lorsqu'un homme inférieur sert le prince, il n'agit jamais en suivant une voie claire et manifeste ; c'est nécessairement par la voie des inclinations secrètes qu'il se lie au supé rieur. Le côté droit est celui qu'il convient d'employer, aussi il est considéré comme le lieu de la clarté ; le côté gauche ne doit pas être employé, aussi il est considéré comme le côté des inclinations secrètes. L'homme, en se servant de ses mains et de ses pieds emploie toujours le membre droit. On appelle communément «inclination partiale » ce qui est considéré comme constituant une coupable inclination, ou inclination à gauche. parce que la gauche est le côté des inclinations secrètes. Le quatrième trait emploie la voie des inclinations secrètes; il s’insinue profondément dans l'esprit du prince, aussi la formule dit : «pénétrer dans les viscères gauches »; pénétrer les viscères, c'est-à-dire que la liaison est très intime. Comme la liaison est très intime, il pénètre les intentions et les pensées. Toutes les fois que des gens pervers et mal intentionnés obtiennent la confiance du prince, c'est toujours parce qu'ils s'emparent de son cour ; s'ils ne s'emparaient pas de son coar, le prince ne reconnaîtrait -il pas leurs intentions perverses ? «Au sortir de la porte et du parvis »; outre quil jouit de la confiance du prince et agit sur son esprit, il agit encore à l'extérieur ; les sujets pervers qui servent un prince borné corrompent nécessairement d'abord son cour, et après cela ils peuvent agir directement à l'extérieur ${ }^{2}$.

TSHOU HI. - Le sens de la formule de ce trait n'est pas encore bien élucidé. Je me permets de supposer que l'expression « viscères de gauche » désigne un lieu sombre et caché, et que la phrase «saisir l'intention d'obscurcir la clarté, au sortir de la porte et du parvis », exprime le sens de

\footnotetext{
${ }^{1}$ Koua simple khouen.

${ }^{2}$ Agir ouvertement et d'eux -mêmes, leurs actes étant tolérés par la faiblesse du prince qu'ils ont capté.
} 
saisir de loin les intentions de quelqu'un. Cela exprimerait que celui qui consulte le sort, et qui obtient ce trait comme réponse, doit en se plaçant, suivre cet exemple. En effet, la substance du koua simple li est considérée comme exprimant les qualités de l'extrême clarté, ou intelligence ; la substance du koua simple khouen exprime le lieu de l'extrême obscurité. Les trois traits inférieurs expriment la clarté au-dehors des ténèbres, de sorte que suivant leur degré d'éloignement ou de proximité, d'élévation ou d'infériorité, chacun d'eux se place d'une façon différente. Le quatrième trait hexaire emploie la douceur malléable, il occupe légitimement un lieu obscur ${ }^{1}$, mais où les ténèbres ne sont pas encore épaisses, de sorte qu'il lui est encore possible d'étendre ses idées à ce qui est éloigné. Le cinquième emploie aussi les qualités de la mollesse et de la négativité ; il est juste au milieu du lieu des ténèbres et il y est entièrement plongé, aussi il est considéré comme exprimant l'image symbolique de comprimer au-dedans ses tendances légitimes pour obscurcir et masquer sa propre clarté ou intelligence. Quant au trait supérieur, il est au comble des ténèbres, de sorte quill est considéré comme oblité rant lui-même sa propre intelligence jusqu'au plus complet obscurcissement et comme étant même capable de nuire à l'intelligence d'autrui. C'est qu'en effet les cinq traits inférieurs sont considérés comme représentant des hommes doués, tandis que le trait supérieur, seul, est considéré comme représentant un prince sans intelligence et sans lumières.

655.

Pénétrer dans les viscères gauches, saisir l’idée du cour.

TSHENG TSE. - Pénétrer dans les viscères gauches, c'est-à-dire suivre une voie d'inclinations partiales, s’introduire dans l'esprit du prince et posséder ses intentions et ses idées. Posséder son cœur, de sorte que le prince ne peut plus jamais s'apercevoir qu'il est circonvenu.

${ }^{1}$ Quatrième rang négatif. 


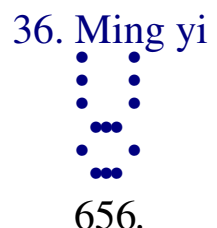

Cinquième trait hexaire : obscurcissement de la clarté par K Tse ; avantage de la perfection.

TSHENG TSE. - Le cinquième rang représente la situation du prince ; telle est l'inter prétation ordinaire. Cependant dans le $Y i$ king, le choix du sens varie et change selon le moment. Le trait supérieur hexaire est placé au rang supérieur du koua simple khouen et au rang le plus élevé du koua ming yi; c'est celui qui indique le comble de l'obscurcisse ment de la lumière. Le cinquième est le plus rapproché de lui ; l'homme saint ${ }^{1}$ en déduit que le cinquième trait représente l'homme très rapproché de l'obscurité absolue. C'est parce qu'il voit comment le devoir lui commande de se placer, que la formule ne le considère pas exclusivement au point de vue de l'occupation de la situation du prince. Le trait supérieur exprime le comble de l'obscurité et de l'effacement de la lumière, de sorte qu'il est considéré comme représentant celui de qui dépend cet obscurcissement ; le cinquième est très rapproché de celui de qui dépend l'obscurcissement de la lumière ; s’il manifestait son intelligence (sa clarté), il serait infailliblement et sur-le-champ blessé et atteint par le mal. Aussi, il convient qu'il agisse comme Ki Tse ${ }^{2}$ se cachant luimême dans l'obscurité, et alors il pourra éviter le péril. Ki Tse était un ancien serviteur des Sheang et un parent portant le nom de la famille impériale ; c'est bien ce qu'on peut appeler être au plus haut point rapproché de Tsheou. Sill n'eût pas lui-même masqué son intelligence, il eût infailliblement été atteint par le malheur ; aussi il simula la folie et la dégradation pour se soustraire au malheur. Bien quill cachât son intelligence, cependant, au-dedans de luimême, il conservait sa droiture, c'est ce qu'on doit entendre par l'expression « souffrir intérieurement et pouvoir rectifier ses tendances », et ce qu'on peut appeler « humanité dans l'intelligence » ${ }^{3}$. Quant à la conduite de Ki Tse, c'est ce qu'on peut appeler la «perfection» dans la voie suivie. Comme le cinquième trait est négatif et faible, la formule en fait l'objet d'un avertissement et dit : «avantage de la perfection », ce qui exprime quil faut pousser la fermeté au même point de perfection que Ki Tse. Si on en parlait au point de vue de la voie rationnelle du prince, le sens serait encore le même ; il $\mathrm{y}$ a aussi des moments où le prince doit supporter l'obscurcissement et même

\footnotetext{
${ }^{1}$ Wen Wang, auteur de la formule du koua ou son fils, auteur de la formule des traits.

${ }^{2}$ Voir $\mathrm{n}^{\circ} 646$

${ }^{3}$ Le terme jen, traduit par humanité, désigne l'ensemble des plus hautes qualités ou aptitudes de l'âme humaine.
} 
obscurcir et cacher sa propre intelligence, tout en redressant intérieurement ses tendances.

TSHOU HI. - Il occupe un lieu de ténèbres absolues, il est près du prince complètement dépourvu de lumières, et cependant il est capable de rectifier ses propres tendances : c'est l'image symbolique de Ki Tse, et le comble de la perfection. «Avantage de la perfection », c'est pour avertir celui qui consulte le sort.

657.

Perfection de K Tse ; la clarté ne peut pas s'éteindre.

TSHENG TSE. - Ki Tse se cacha dans l'ombre et ne manqua pas à la perfection de la fermeté ; bien qu'en présence de calamités et de périls, il conserva son intelligence intacte et se préserva lui-même. Lorsque les alternatives de bonheur, de chagrin et, de périls réussissent tour à tour à faire dévier de la voie suivie, c'est ce qu'on peut appeler perte de l'intel ligence, et alors elle est bien détruite; c'est, par exemple, dans l'antiquité, le cas de Yiang Hung. 


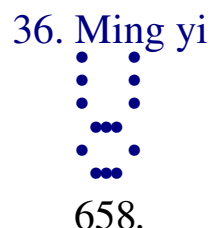

Trait supérieur hexaire : il n'éclaire pas l'obscurité ; d'abord il s'élève au ciel; ensuite il rentre dans la terre.

TSHENG TSE. — Le trait supérieur occupe le dernier rang du koua, c'est de lui que dépend l'obscurcissement de la lumière et, de plus, il exprime le comble de cet obscurcissement. Le rang supérieur est le lieu le plus élevé. Lorsque la lumière est au point le plus élevé, elle doit naturellement éclairer au loin ; du moment où la lumière est oblitérée, il n'y a plus de clarté, mais au contraire l'obscurité et les ténèbres. Essentiellement, puisque ce trait occupe une position élevée, son intelligence devrait atteindre au loin : «d'abord il monte au ciel »; mais, au contraire, il détruit la lumière et l'obscurcit : «ensuite il retombe sur la terre ». Le trait supérieur est le dernier du koua ming yi ; c'est aussi le dernier trait du koua simple khouen : c'est celui qui indique le comble de l'obscurcissement de la lumière.

TSHOU HI. - Il occupe le sommet du koua khouen avec ses qualités négatives ; il ne développe point ses vertus, et il arrive à l'obscurcissement de ses facultés. D'abord il se place dans une situation élevée, pour éteindre l'intelligence des hommes, et, à la fin, il en arrivera infailliblement à se détruire lui-même et à mettre fin à sa propre destinée. c'est pourquoi telle est l'image symbolique, tandis que le sens divinatoire s'y trouve inclus.

659.

D'abord il s'élève au ciel ; il éclaire les quatre royaumes ${ }^{1}$; ensuite il entre dans la terre; il manque aux préceptes.

TSHENG TSE. - D'abord il s'élève au ciel : il est placé haut et brillant, de sorte que sa clarté atteint nécessairement aux quatre points cardinaux. Mais ensuite elle est détruite et il devient obscur, c'est là rentrer ensuite dans la terre et c'est la voie qui lui fait perdre son intelligence. Manquer aux préceptes, c'est perdre la voie ratio nnelle.

TSHOU HI. - Éclairer les quatre régions est dit à cause de la situation qu'il occupe.

\footnotetext{
${ }^{1}$ Régions.
} 


\section{Kia jen}

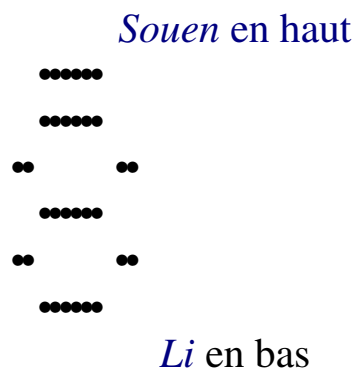

660. Kian jen; personnes de la famille ; avantage de la pureté de la jeune fille.

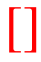

665. Premier trait nonaire : régler la famille : les regrets se dissipent.

667. Deuxième trait hexaire : pas de moyen de réussir ; être dans la marmite ; présage heureux de la perfection.

$*$

669. Troisième trait nonaire : gronderie entre les personnes de la famille; regrets, préoccupations, présage heureux; folle dissipation de la femme et des enfants; à la fin, appréhension d'un mal futur.

$*$

671. Cinquième trait nonaire : le roi vient à avoir une famille; il ne s'en inquiète pas, présage heureux.

$*$

673. Cinquième trait nonaire : le roi vient à avoir une famille; il ne s'en inquiète pas, présage heureux.

*

675. Trait supérieur nonaire : avoir de la foi et être imposant ; finalement présage heureux.

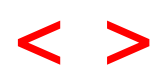


37. Kia jen.

TSHENG TSE. — Kia jen. «L'Ordre des kou a » dit : «Y $Y i^{1}$, endommager, blesser; la blessure faite à autrui ${ }^{2}$ fera souffrir son auteur ${ }^{3}$; aussi le koua ming yi est suivi du koua kia jen. »Car le mal et la misère sévissant à l'extérieur doivent nécessairement retomber à l'intérieur, c'est là la raison qui fait que le koua kia jen suit immédiatement le koua ming yi. Le koua kia jen 4 exprime la voie rationnelle de l'intérieur de la famille (maison). L'affec tion entre le père et les enfants ; le devoir entre l'époux et l'épouse ; l'ordre de préséance entre les personnes de rang prééminent et celles de rang inférieur ${ }^{5}$, entre les aînés et les cadets ; la raison d'être des liens sociaux ; la démarcation entre les devoirs réciproques, affection et déférence, bienfait et reconnaissance; telle est la voie rationnelle exprimée par le koua kia jen. Comme koua ${ }^{6}$, il est constitué par le koua souen à l'extérieur et le koua $l i$ à l'intérieur ; il représente le vent résultant du feu. Lorsque le feu brûle, le vent naît ${ }^{7}$, le vent naît du feu, il sort de ce qui est intérieur. Sortir de l'intérieur, image symbolique de ce qui procède de la famille et gagne au-dehors. Le second et le cinquième rangs représentent exactement la situation du garçon et de la fille, à l'intérieur et à l'extérieur ; cela exprime que la voie rationnelle des personnes d'une même famille ( kia jen) comporte l'intelligence au-dehors et l'humilité au-dedans ; telle est la voie qui doit régir la famille. Or, lorsque l'homme possède quelque qualité en lui -même, il est capable de développer cette même qualité dans sa famille; en la pratiquant dans sa famille, il pourra la développer dans son pays, et, de degré en degré, l'étendre au gouvernement de l'univers ${ }^{8}$ et en effet, la voie rationnelle pour régir l'univers n'est autre que la voie rationnelle pour diriger la famille, étendue et pratiquée en dehors des limites du foyer. C'est pour cela que la formule choisit l'image symbolique d'étendre au-dehors ce qui est à l'intérieur, et cela constitue le sens exprimé par le koua kia jen. Le Wen tshong tse ${ }^{9}$ choisit le sens de la clarté intérieure portant l'ordre à l'extérieur. Dans l'antiquité comme dans les temps modernes, on a loué ce passage sans saisir l'idée enveloppée dans l'image symbolique. Ce qu'on entend par l'expression « régulariser l'humil ité », exprime que tous les êtres sont rangés successivement en ordre dans la région désignée par le

\footnotetext{
${ }^{1}$ Dans l'expression ming yi; koua $\mathrm{n}^{\circ} 36$.

2 À l'extérieur.

${ }^{3}$ Blessera réciproquement la famille de l'auteur de la blessure.

${ }^{4}$ Littéralement « homme de la famille ».

5 Voir les degrés de la parenté dans le code chinois. Traduction de P. Philastre, E. Leroux, Paris.

${ }^{6}$ Comme forme ou diagramme.

7 Le courant d'air ascensionnel causé par la différence de densité entre l'air froid et l'air échauffé.

8 Définition de la «Grande Étude». Voir Annales du Musée Guimet, tome 1. Exégèse chinoise, P. Philastre.

9 Titre d'un ouvrage sur le sens des caractères interprétés par leur forme graphique. Le sud-ouest.
} 
nom du koua simple souen ${ }^{1}$; cela n'implique pas que le caractère souen comporte le sens de «mettre en ordre». C'est comme l'expression «combattre » employée au sujet du koua khien, ce qui n'implique pas que le koua khien comporte le sens de combattre ".

\footnotetext{
${ }^{1}$ Voir $n^{\circ} 75$
} 


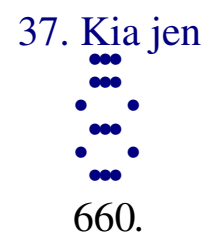

Kian jen ; personnes de la famille ; avantage de la pureté de la jeune fille.

TSHENG TSE. - Dans la voie rationnelle des personnes de la famille, l'avantage consis te dans la droiture des personnes du sexe féminin; si la femme possède la droiture, la voie rationnelle de la famille sera droite et correcte. L'époux se condui sant en époux, l'épou se en épouse, la voie rationnelle de la famille est droite et correcte. La formule ne parle que de l'avantage de la perfection de la femme ; or, la rectitude chez l'époux, n'est que la rectitude de sa personne et de ses actions ; la rectitude chez la femme, c'est la rectitude de toute la famille ; donc si la femme possède la droiture, les hommes la posséderont évidemment.

TSHOU HI. - Kia jen, personne de la famille. Dans le koua, le cinquième trait nonaire et le second trait hexaire, l'extérieur et l'intérieur ${ }^{1}$ sont l'un et l'autre placés conformé ment à la droiture. C'est pour cela que le koua est considéré comme exprimant les personnes de la famille. «Avantage de la pureté de la femme »: marque qu'il faut avant tout la rectitude à l'intérieur ; si la droiture et la rectitude prévalent à l'intérieur, rien, à l'exté rieur, ne sera dépourvu de ces qualités.

661.

Le commentaire de la formule déterminative dit: Personnes de la famille; la situation correcte de la femme ${ }^{2}$ est à l'intérieur; la situation correcte pour l'homme est à l'extérieur. L' homme et la femme possédant la droiture, c'est le grand devoir du ciel et de la terre ${ }^{3}$.

TSHENG TSE. - La formule déterminative traite des aptitudes exprimées par le koua. La positivité occupe le cinquième rang, et demeure à l'extérieur ; la négativité est placée au second rang et se trouve à l'intérieur :

\footnotetext{
${ }^{1}$ Les traits centraux des koua simples extérieur et intérieur.

2 Ou de la jeune fille.

3 C'est la lecture admise. Devoir, règle tracée par les préceptes fondamentaux de la morale, mais le caractère traduit par devoir a aussi la signification de «sens, interprétation » et on pourrait lire : «c'est le sens fondamental des mots ciel et terre ».
} 
l'homme et la femme occupent chacun la situation qui leur convient exactement. La voie rationnelle de la prééminence et de l'infériorité, de l'intérieur et de l'extérieur, étant correctement observée, c'est la conformité exacte au grand devoir indiqué par le ciel et la terre, la positivité et la négativité.

TSHOU HI. - Explication du sens des mots « avantage de la pureté de la femme » au moyen des substances des koua simples, du cinquième trait nonaire et du second trait hexaire.

662.

Les personnes de la famille ont un maître sévère : c'est la désignation du père et de la mère.

TSHENG TSE. — La voie rationnelle des personnes de la famille exige une autorité prééminente et grave pour la gouverner : cela désigne le père et la mère. Bien qu'il ne s'agis se que d'un groupe aussi minime qu'une famille, s'il n'y a aucun ordre de prééminence, aucune autorité qui simpose, la piété filiale et le respect déclineront ; s'il n'y a ni maître, ni chef, les règles seront abandonnées. Pour que la voie rationnelle de la famille soit droite et correcte, il faut un chef sévère : or, la famille est l'image réduite de l'État 1.

TSHOU HI. - Il s'agit encor e du second et du cinquième traits.

663.

Si le père agit en père, le fils en fils, l'aîné en aîné, l'époux en époux, l'épouse en épouse, la voie rationnelle de la famille sera correcte et droite. En rectifiant la famille on établit la stabilité de l'univers.

TSHENG TSE. - Si le père, le fils, l'aîné et le cadet, l'époux et l'épouse, se conforment chacun à la voie rationnelle qui leur est départie, la voie morale de la famille sera correcte et droite. De la voie rationnelle de la famille, cette règle peut être étendue à l'univers, de sorte que, si la famille est établie sur des bases correctes et droites, l'univers sera stable et fixe.

\footnotetext{
${ }^{1}$ Littéralement « la mesure à laquelle se compare l’Etat ».
} 
TSHOU HI. - Le trait supérieur représente le père, le premier représente le fils, le cinquième et le troisième représentent l'époux ; le quatrième et le second représentent l'épouse. D'un autre côté, le cinquième trait représente l'aîné et le troisième représente le frère cadet, l’image symbolique peut donc encore se déduire de la considération des traits qui composent le koua.

664.

Le commentaire de la formule symbolique dit: Le vent résulte du feu: personnes de la famille. L'homme doué fait ce qu'il dit et son action est persistante ${ }^{1}$.

TSHENG TSE. - La base essentielle de la régularité dans la famille consiste dans la réforme de sa propre personne; la voie rationnelle, pour réformer sa propre individualité, est qu'une parole, un mouvement, ne doivent pas être altérés. L’homme doué consi dère l'image symbolique du vent résultant du feu et il reconnaît que toute chose procède de l'intérieur vers l'extérieur ; aussi, ce quil dit doit avoir un corps ${ }^{2}$, son action doit être persistante. Le caractère wou du texte désigne une chose réelle; le caractère heng (koua $\mathrm{n}^{\circ} 32$ ) désigne une règle fixe constante et permanente. La manifestation extérieure des qualités se traduit par la circonspection intérieure apportée aux paroles et aux actions ; si la parole est sincère et l'action retenue, l'individu correct dans sa personne, la famille sera régulière.

TSHOU HI. - La personne réformée, la famille sera en ordre.

\footnotetext{
${ }^{1}$ Telle est bien la lecture admise, mais c'est précisément de l'interprétation de ce passage que vient l'acception donnée aux caractères qui le composent. En réalité, il y a écrit : «L'homme doué emploie la parole pour réaliser les êtres (exprimer les choses), et l'expression est persistante. »

${ }^{2}$ Sa parole doit être appuyée sur le fait. Méthodique ; conforme à une règle fixe.
} 


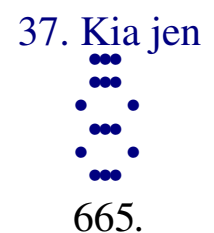

Premier trait nonaire : régler la famille ${ }^{1}$ : les regrets se dissipent.

TSHENG TSE. - Le premier trait, exprime le commencement de la voie rationnelle de la famille. Le troisième caractère du texte signifie établir des défenses protectrices, des règles de direction et de préservation. Régler les commencements dans la possession de la famille, pouvoir employer les préceptes et les mesures d'ordre comme défense protectrice et comme préservation, conduira à éviter les regrets. Gouverner la famille, c'est gou verner la foule des hommes. Si on ne l'enserre pas par des mesures limitatives, les sentiments humains se donneront libre cours, et il en résultera infailliblement des regrets. Il n'y a pas de conséquences, si graves qu'elles soient, où ne puissent conduire l'oubli de l'ordre de préséance entre les aînés et ceux qui sont plus jeunes et de la séparation des sexes, les offenses aux liens de devoir et de reconnaissance, la perturbation dans les relations de famille. Si on peut dès le commencement employer les règles et les mesures restrictives pour la préserver et la garantir, toutes ces conséquences ne se présenteront pas, de sorte que les « regrets se dissipent». La qualité de trait nonaire comporte les aptitudes de l'intelligence et de la fermeté énergique, elle désigne celui qui peut préserver la famille. La formule ne dit pas : «pas de regrets »; lorsque plusieurs personnes sont groupées en troupe, il y a nécessairement des causes de chagrin et de regrets ; c'est parce qu'il y a des mesures de préservation que ces regrets se dissipent.

TSHOU HI. - Le premier trait nonaire emploie la dureté énergique de la positivité pour poser les principes de la possession de la famille ; pouvant la préserver par des mesures restrictives et préservatrices, les regrets se dissiperont. C'est un avertissement à celui qui consulte le sort, qu'il doit être dans de telles conditions.

666.

\footnotetext{
${ }^{1}$ C'est la lecture admise, mais elle est insoutenable. La seule autorité à laquelle on puisse avoir recours pour expliquer ce caractère serait peut-être le Yi ya qui donne le sens de « répétition d'un même fait, ex ercice ». Ce même caractère est encore assimilé à un autre dont le sens, très vague, est « extinction ; mutation, disparition », et aussi «parc où on enferme les bêtes à cornes ».
} 


\section{L'enceinte à la maison ${ }^{1}$; tendances pas encore altérées.}

TSHENG TSE. — Entourer de barrières au début, avant que les tendances et les idées des personnes de la famille soient agitées et altérées. La droiture et la rectitude des tendances n'étant pas encore altérées et troublées, en les préservant par des barrières restrictives, la reconnaissance ne sera point violée, le devoir ne sera pas transgressé ; c'est le bien dans l'établissement de la famille, et c'est à cause de cela que les regrets se dissipent. Si on attend pour corriger et régler jusqu'après que les tendances sont altérées, le mal causé s era grave, et alors il y aura des regrets.

TSHOU HI. - Les tendances ne sont pas encore altérées et on prévient le mal par avance.

${ }^{1}$ Ce n'est pas l'interprétation admise, mais c'est la seule lecture possib le, si l'on respecte l'ordre de construction. Le terme du texte qu'on traduit généralement par « tendance de l'esprit, desseins » désigne aussi le siège de la volonté et du sentiment. 


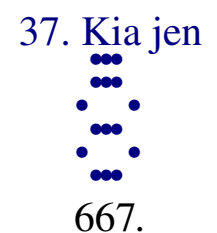

Deuxième trait hexaire : pas de moyen de réussir ; être dans la marmite ${ }^{1}$; présage heureux de la perfection.

TSHENG TSE. — La famille de l'homme. Entre les os et la chair, entre le père et les enfants, le plus souvent, la force du sentiment prévaut sur la règle rituelle de l'étiquette, la reconnaissance prévaut sur le devoir. L'homme qui se maintient avec rigidité, seul, peut ne pas se laisser aller à manquer à la droite raison à cause de ses amitiés privées. Aussi dans le koua kia jen, dans le plus grand nombre des cas, c'est la dureté énergique qui est considérée comme constituant le bien ; c'est, par exemple, le cas du troisième trait supé rieur. Le second trait hexaire emploie les aptitudes de la malléabilité négative et il occupe un rang qui comporte la même qualité : c'est celui qui est incapable de faire régner l'ordre dans la famille et c'est à cause de cela qu'il n'a aucun moyen de réussir ; rien de ce qu'il fait n'est possible. Or, avec les aptitudes de l'intel ligence et de la sévérité, il y a encore des hommes qui se laissent entraîner par le courant de leurs affections ou de leur amour et qui sont incapables de se contenir et de se préserver; à plus forte raison, comment l'homme faible et mou pourrait-il dominer les sentiments de son épouse et de ses enfants pour leur imposer une règle de conduite ? Les aptitudes du second trait, s'il s'agit de suivre la voie rationnelle de l'épouse, constitueront bien la droiture, car employer la douceur et l'obéissance pour se placer dans la justice et dans la droiture est bien la voie rationnelle de l'épouse; aussi, dans l'intérieur du ménage, elle se conformera à la droitu re et le présage sera heureux. La femme demeure à l'intérieur et préside aux soins du ménage, c'est pour cela que la formule dit : «dans le ménage ».

TSHOU HI. - Le second trait hexaire est mou, soumis, juste et droit ; la situation convenable pour la femme est à l'intérieur. C'est pourquoi tels sont l'image symbolique et le sens divinatoire.

668.

Présage heureux du second trait hexaire ; soumission par humilité.

\footnotetext{
${ }^{1}$ Douteux, peut-être « cuisine ».
} 
TSHENG TSE. - Le second trait emploie la douceur de la négativité et demeure dans la justice et la droiture ${ }^{1}$; il est capable d'obéir avec soumission et de s'effacer avec humilité, de sor te qu'il est considéré comme exprimant le présage heureux de la perfection des qualités de la femme.

\footnotetext{
${ }^{1}$ Rang pair au milieu d'un koua simple.
} 


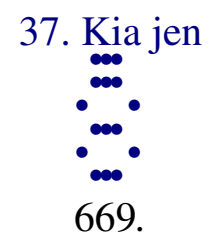

Troisième trait nonaire : gronderie entre les personnes de la famille ; regrets, préoccupations, présage heureux ; folle dissipation de la femme et des enfants ; à la fin, appréhension d'un mal futur ${ }^{1}$.

TSHENG TSE. - Le sens des cinquième et sixième caractères n'est pas encore bien élucidé, toutefois, en tenant compte du sens du texte, ainsi que du son et de l'idée ${ }^{2}$, on peut assimiler l'expression à « toutes les bouches récriminant », «morigéner »; de plus la forme de ces caractères comporte l'idée de précipitation hâtive. Le troisième trait nonai re se trouve placé au rang supérieur du koua simple intérieur, il représente celui qui dirige et gouverne à l'intérieur. Puisque avec sa nature positive, il occupe un rang qui comporte la dureté et l'absence de justice, bien qu'il se conforme à la droiture, c'est celui qui déploie un e xcès de dureté. Dirigeant l'intérieur avec trop de dureté, il blesse par sa sévérité trop hâtive, de sorte que les personnes de la famille murmurent et grondent. Mais la famille étant gouvernée avec trop de sévérité, il n'est pas possible qu'il n'en résul te pas d'inconvénients, de sorte qu'il doit y avoir appréhension de regrets au sujet de la sévérité. Entre la chair et les os, c'est l'affection qui domine les autres sentiments, la sévérité est excessive, d'où résultent les regrets. Malgré l'appréhension des regrets au sujet de la sévérité, bien qu'il manque de justice et de tolérance, cependant, la voie rationnelle de la famille comporte la gravité et le décorum; les sentiments de chacun des membres de la famille étant dominés par la crainte et le respect, c'est encore quelque chose qui consti tue un présage heureux pour la famille. Si la femme et les enfants sont dissipés et adonnés aux amusements frivoles, il en résultera finalement de la honte et il y a appréhension d'un mal futur. Dans le koua, il n'y a pas l'image symbolique de cette folle gaieté ; c'est qu'en effet cette expression est empruntée pour faire contraste avec l'expression «gronderie» et elle marque qu'avec de tels manquements résultant de trop de laisser-aller il ne peut pas y avoir excès dans la sévérité. Les caractères du texte signifient « rires et gaieté sans mesure ». Le laisser-aller sans aucune retenue conduira finalement à la désorganisation de la famille, c'est une cause légitime de honte et d'appréhension pour l'avenir. Bien que l'excès de sévérité et de circonspection ne puisse pas manquer d'entraîner des froissements de sentiments, cependant les règles sont maintenues et l'ordre des relations dans

\footnotetext{
${ }^{1}$ Le sens de plusieurs caractères est à peu près inconnu, aussi la traduction est simplement la reproduction des commentaires.

${ }^{2}$ Représentés par la forme graphique.
} 
la famille est respecté, ce qui préserve la loi du devoir entre les personnes de la famille ; si au contraire il s'agit de la dissipation sans règles ni retenue, c'est par là que les préceptes sont transgressés et méprisés, que les relations sont bouleversées ; comment la famille pourrait-elle échapper aux conséquences de ses désordres ? Si la dissipation est poussée à un point extrême, elle conduira jusqu’à la ruine de la famille. La formule dit : «appréhension d'un mal futur », c'est certainement une des plus grandes causes d'appréhension ; mais comme le malheur de la famille est la conséquence extrême où conduit cette dissipation, la formule n'emploie pas prématurément le terme «présage malheureux ».

TSHOU HI. - Avec la dureté énergique, il occupe un rang qui comporte la dureté et il est dépourvu de justice : c'est celui qui abuse de la dureté, aussi il y a l'image symbolique de gronderie et d'appréhension résultant de l'excès de sévérité. Dans ces conditions, bien quil y ait appréhension de regrets, cependant le présage est heureux. La «folle gaieté », c'est le contraire des «murmures» exprimés précédemment; c'est une voie rationnelle d’inquiétude et d'appréhension. L'interprétation divinatoire répondra selon le cas, aux qualités de celui qui consulte le sort, et c'est pour cela que la formule les prévoit tous les deux.

670.

Les personnes de la famille murmurant, rien n'est encore perdu. Les femmes et les enfants rient follement, perte des principes de la famille.

TSHENG TSE. - Bien qu'il y ait des murmures au sujet de la voie suivie dans le gouvernement de la famille, cela ne constitue pas encore un grand inconvénient. Si les femmes et les enfants font entendre les éclats de leur dissipation joyeuse, il n'y a plus ni gouverne ment, ni règles rituelles de bienséance, c'est la perte des préceptes de la famille, qui par suite tombera dans le désordre. 


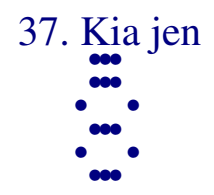

671.

Quatrième trait hexaire : grand présage heureux de la famille riche.

TSHENG TSE. - Le trait est hexaire et fait partie de la substance du koua simple souen qui exprime l'humilité ; il occupe le quatrième rang, et se conforme exactement avec droiture à la situation qui lui convient. La rectitude dans la position occupée est considérée comme exprimant le sens de savoir se contenter de sa situation. Se soumettant avec humilité aux circonstances et suivant la voie de la droiture, on peut garantir qu’il aura la richesse. Dans la voie qui consiste à rester dans le cercle des occupations de la famille, pouvoir garantir l'obtention de la richesse sera considéré comme constituant la grandeur du présage heureux. Le quatrième rang est une situation élevée, et il n'est question que de la richesse ; c'est que, lorsqu'il s'agit de la famille, la situation élevée, c'est celle de la personne de rang prééminent ; si elle peut avoir la richesse, elle peut donc garantir l'exis tence de sa famille, c'est un présage grandement heureux.

TSHOU HI. - Le devoir relève de la positivité ; les avantages matériels sont du genre négatif. Puisque ce trait est négatif et occupe un rang négatif, que de plus il occupe un rang supérieur, il représente celui qui peut enrichir sa famille.

672.

Grand présage heureux de la famille riche; l'obéissance est dans la situation.

TSHENG TSE. - Il emploie l'humilité et la soumission et il se maintient dans la droiture. La situation est correcte, la soumission est humble, c'est celui qui peut garantir qu’il possédera la richesse. Grand présage heureux de la famille riche. 


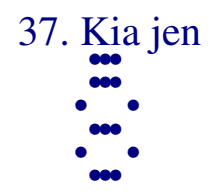

673.

Cinquième trait nonaire : le roi vient à avoir une famille; il ne s'en inquiète pas, présage heureux.

TSHENG TSE. - Le cinquième trait nonaire représente le sexe masculin et il se trouve à l'extérieur ; il est énergique et se place dans un rang positif, il occupe une position prééminente avec justice et droiture. De plus il sympathise ${ }^{1}$ à la droiture et à la soumission placées à l'intérieur ; c'est celui qui représente l'extrême excellence et l'extrême droiture dans le gouvernement de la famille. «Le roi vient à avoir une famille », le cinquième rang marque la situation du prince, c'est pour cela qu'il est question du roi. Le terme traduit par « il vient à avoir » exprime qu'il « arrive » que... Il s'agit du cas le plus important dans la voie rationnelle de la famille. Or, la voie rationnelle de celui qui est le roi consiste à réformer sa propre personne pour régulariser sa famille et, lorsque la famille est correctement régularisée, l'univers est en ordre. Depuis l' antiquité, il n'est pas un saint roi qui n'ait essentiellement fait consister sa règle de conduite à se montrer respectueux de ses propres devoirs et à faire régner la droiture dans sa famille. Aussi, la voie pour ceux qui possèdent une famille étant parvenue à son degré de plus haute importance et de parfaite régularisation, il n’y aura ni chagrin, ni soucis pénibles et l'univers sera en ordre. Il ne s'en inquiète pas et le présage est heureux. Le cinquième trait exprime le respect de soi-même à l'exté rieur et le second exprime le redressement de la famille, à l'intérieur ; les mêmes vertus régnant à l'intérieur et à l'extérieur, c'est ce qu'on peut appeler parfait développement.

TSHOU HI. — «Venir à », arriver à, atteindre à ; c'est le même terme qui est employé dans l'expression « ver au grand autel ». " Avoir une famille», c'est comme si on disait « avoir un royaume ». Le cinquième trait nonaire est énergique, actif, juste et droit ; en bas, il correspond sympathiquement à la justice, à la droiture et à la douce soumission du second trait hexaire. Celui qui est roi, et qui porte ces qualités dans le sein de sa famille, n'aura pas de préoccupations chagrines et le présage heureux existe nécessairement. En effet, le sens divinatoire montre le présage heureux dans le cas où il s'agit de conduire au roi sa fiancée, mais pour quiconque possédera les mêmes vertus, et qui rencontrera ce trait en consultant le sort, le présage sera également heureux.

\footnotetext{
${ }^{1}$ Il correspond.
} 
674.

Le roi vient à avoir sine famille ; amour mutuel entre ceux qui sont unis.

TSHENG TSE. - Il s'agit de la voie rationnelle du roi qui vient à avoir une famille; non seulement il peut la diriger en la faisant obéir avec soumission, mais encore il arrive à ce point que les cours se transforment et s'unissent dans la sincérité ; l'époux aime son aide intérieur ${ }^{1}$, l'épouse aime le justicier de la famille : amour mutuel entre ceux qui sont unis. Ceux qui sont dans ce cas ne sont-ils point comme Wen Wang et son épouse ? Si l'individu est réformé, si les règles son t observées, mais que la famille ne soit pas encore transformée, on ne peut pas encore dire que la voie d'arriver à posséder une famille est entièrement accomplie.

TSHOU HI. -2 .

${ }^{1}$ Son épouse.

${ }^{2}$ Cite simplement Tsheng Tse. 


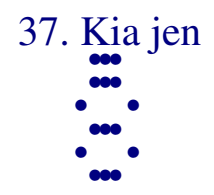

675.

Trait supérieur nonaire : avoir de la foi et être imposant; finalement présage heureux.

TSHENG TSE. - Le trait supérieur occupe le dernier rang dans le koua ; il indique l'achèvement dans la voie rationnelle de la fam ille ; aussi la formule définit une dernière fois la base essentielle du gouvernement de la famille. La voie rationnelle du gouvernement de la famille est impossible à suivre sans la plus parfaite sincérité de sentiments ; il faut donc nécessairement posséder en soi la confiance et la bonne foi, pour que cette voie puisse être permanente et durable et pour que chacun en soit influencé et se transforme. Lorsque l'amélioration ${ }^{1}$ ne provient pas de la plus parfaite sincérité de sentiments, elle ne peut durer; à plus forte raison en est-il ainsi quand il s'agit d'amener autrui à se transformer. C'est pour cela que, dans la voie rationnelle du gouvernement de la famille, la. base essentielle est d'avoir de la foi. Chez celui qui gouverne une famille, trop d'af fection et de sentiments d'amour pour l'épouse et les enfants produira le manque de sévérité ; si l'affection l'emporte, le sentiment du devoir s'efface et la ruine de la famille provient toujours de l'insuffisance des règles et du relâchement progressif dans l'observation des pres criptions. Le supérieur perdant la dignité grave qui convient à la prééminence, les inférieurs oublient la condescendance respectueuse et il est impossible que le désordre ne sintroduise pas dans la famille. Il faut donc nécessairement posséder une gravité sévère et imposante pour que le résultat final puisse être heureux. La garantie du sort final de la famille repose uniquement dans la possession de la foi et de la dignité imposante, et c'est pour cela qu'il en est question à la fin du koua.

TSHOU HI. - Le trait supérieur nonaire emploie la dureté énergique et occupe le rang supérieur; il est placé à la fin du koua de sorte que la formule traite de la voie du redressement de la famille, durable et pouvant s'étendre au loin. Celui qui consulte le sort doit être sincère et confiant, sévère et digne, alors le présage final est heureux.

676.

\footnotetext{
${ }^{1}$ La transformation en bien.
} 
Présage heureux de la gravité imposante; expression qui marque le retour de la personne ${ }^{1}$.

TSHENG TSE. - La voie rationnelle du gouvernement de la famille est basée sur le redressement de la propre personne de son chef. Aussi la formule dit : expression qui marque «l'application inverse à la personne ». La formule du trait ${ }^{2}$ dit que pour gouverner la famille il faut posséder une gravité sévère et imposante ; Khong Tse formule un nouvel avertissement : il faut d'abord appliquer cette sévérité à sa propre personne. Si la sévérité n'est pas avant tout appliquée à soi-même, elle provoquera chez autrui le ressentiment et la révolte ; c'est po ur cela quill dit : « le présage heureux de la gravité imposante » c'est de pouvoir l'appliquer à sa propre personne ${ }^{3}$. C'est ce qu'exprime Mang Tse en disant que, si on ne pratique pas soi-même une voie, on ne peut la faire suivre à son épouse et à ses enfants.

TSHOU HI. - Exprime que sans user de sévérité, et en se redressant soimême, chacun éprouvera une crainte respectueuse et se soumettra.

\footnotetext{
${ }^{1}$ Les commentateurs lisent «qui marque l'application inverse de ces principes à la personne même de celui qui doit les faire observer aux autres ».

${ }^{2} \mathrm{~N}^{\circ} 675$.

${ }^{3}$ Le texte est dénaturé.
} 


\section{Khouei}

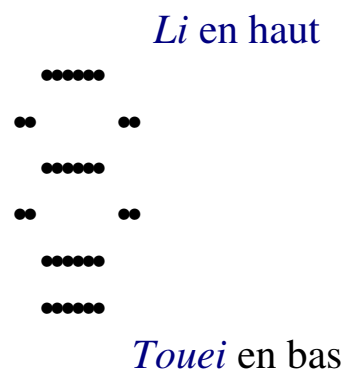

677. Désaccord ; présage heureux pour les petites choses.

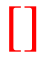

682. Premier trait nonaire: les regrets se dissipent; perdre le cheval, ne pas le poursuivre, il revient de lui-même ; voir les méchants, pas de culpabilité.

$*$

684. Deuxième trait nonaire : rencontrer le maître dans la ruelle; pas de culpabilité.

$*$

686. Troisième trait hexaire : le char conduit; le bauf traîne ; l'homme a la tête rasée et le nez coupé; soins origine avoir une fin.

$*$

688. Quatrième trait nonaire : séparé et isolé ; rencontrer un grand personnage ; union et foi ; péril sans culpabilité.

*

690. Cinquième trait hexaire : regrets dissipés; cette souche mord la peau; en agissant quelle culpabilité?

$*$

692. Trait supérieur nonaire : séparé et isolé ; voir le porc au dos enduit de boue; transporter une charretée d'esprits ; d'abord tendre l'arc, ensuite abandonner l'arc ; s'il ne s'agit pas de brigands, c'est un mariage ; rencontrer la pluie en entreprenant sera un présage heureux.

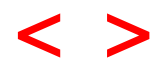




\section{Khouei.}

TSHENG TSE. - Khouei. «L'Ordre des koua » dit: «La voie rationnelle de la famille épuisée, il y a nécessairement opposition ; khouei signifie opposition ${ }^{1}$. Lorsque la voie de la famille décline il doit y avoir éloignement, et séparation; telle est la conséquence logique de la raison d'être naturelle des choses. Aussi, immédiatement après le koua kia jen vient le koua khouei. Il est composé du koua simple $l i$, au-dessus, et du koua simple touei, au-dessous. Le koua simple $l i$ représente le feu et la flamme qui monte; le koua simple touei représente le marais et l'humidité qui sinfiltre en descendant ; les deux essences sont opposées dans leur nature, et c'est là le sens du mot khouei. De plus les deux jeunes filles, la moyenne et la petite ${ }^{2}$, bien qu'elles habitent ensemble ${ }^{3}$, se marient dans deux familles distinctes, donc leurs tendances ne les portent pas d'un même côté, ce qui constitue encore le sens de séparation et d'opposition.

\footnotetext{
${ }^{1}$ Littéralement : «Loucher, regarder de deux côtés à la fois. »

2 Représentées par deux traits appartenant aux deux koua simples.

${ }^{3}$ Que ces deux traits fassent partie du koua khouei.
} 


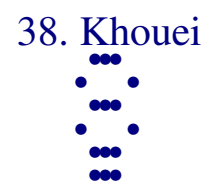

677.

Désaccord ; présage heureux pour les petites choses.

TSHENG TSE. - Le terme khouei indique un moment de séparation et d'opposition ; ce n'est pas une voie qui comporte un présage heureux, mais à cause de l'excellence des qualités spéciales de chaque koua simple, bien que, dans un moment de séparation et d'opposition, cependant, pour les choses de peu d'importance, le présage est heureux.

TSHOU HI. - Khouei, opposition, différence. Ce koua est composé des koua simples du feu, en dessus, et du marais, en dessous : leurs natures sont opposées et différentes. Ils représentent la jeune fille d'âge moyen, et la petite fille ; leurs tendances ne les portent pas vers le même foyer, et c'est pour ces motifs que le koua est appelé khouei. Toutefois, en en parlant au point de vue des qualités propres aux koua simples, au-dedans satisfaction ${ }^{1}$, et au-dehors clarté $^{2}$. Si on en parle au point de vue de la transformation des koua, si on le considère comme provenant du koua $l i$, le trait qui exprime la douceur malléable avance pour occuper le troisième rang; si on le considère comme provenant du koua tshong fou, le trait qui exprime la douceur malléable vient occuper le cinquième rang; si on le considère comme provenant du koua kia jen, ces deux conditions sont réunies. Si on en parle d'après la substance du koua, alors, le cinquième trait hexaire possède la justice et il s'abaisse sympathiquement vers la dureté énergique du second trait nonaire ; c'est à cause de cela que le sens divinatoire exprime l'impossibilité des grandes choses tandis que, logiquement, le présage est encore heureux pour les choses de moindre importance.

678.

Le commentaire traditionnel de la formule déterminative dit : Opposition ; le feu s'agite et s'élève, l'eau s'agite et descend; les deux jeunes filles demeurent ensemble, leurs tendances ne les portent pas à agir ensemble.

\footnotetext{
${ }^{1}$ Touei.
}

${ }^{2} \mathrm{Li}$. 
TSHENG TSE. - Le commentaire de la formule déterminative explique d'abord le sens du koua khouei, ensuite il traite des aptitudes qu'il exprime ; enfin, il parle de la voie rationnelle de l'union et de la séparation et s'exclame admirativement sur la grandeur du moment et de l'effet. La nature du feu est de monter aussitôt qu'il se produit, la nature de l'eau est de s'écouler en descendant aussitôt qu'elle est en mouvement. Les natures de ces deux choses sont différentes et opposées et cela constitue le sens «d'opposition ». Les deux jeunes filles, la moyenne et la plus jeune, bien qu'elles habitent ensemble, ne sont pas portées par leurs tendances à agir d'une façon identique, ce qui constitue encore le même sens. Pendant leur jeunesse, les jeunes filles sont placées ensemble; devenues adultes, chacune suit une voie différente avec son propre époux, leurs tendances sont différentes. Puisqu'il est question de séparation, c'est que primitivement il y a eu réunion ; si originellement, il n’y avait pas unité, il ne pourrait pas y avoir diversité.

TSHOU HI. - Explication du sens du nom du koua au moyen du symbolisme des koua simples.

679.

Satisfaction et attachement à la clarté; la douceur malléable avance et monte pour agir; elle possède la justice et sympathise avec la dureté énergique; c'est à cause de cela que le présage est heureux pour les petites choses.

TSHENG TSE. - Les qualités du koua étant telles, il en résulte un présage heureux pour les petites choses. Le koua simple touei exprime la satisfaction ; le koua simple li exprime la séparation et aussi l'attachement ; il est encore considéré comme symbole de la clarté, de sorte que le koua complet exprime la satisfaction et la soumission avec attachement à la clarté de l'intelligence. Toutes les fois que le koua simple $l i$ est au-dessus et que le commentaire traditionnel de la formule déterminative veut appeler l'attention sur l'occupa tion du rang prééminent par la douceur malléable négative, elle dit : «la douceur malléable avance et monte en agissant»; c'est le cas, par exemple, dans les koua tsin et ting. Au moment où commencent la séparation et l'opposition, le cinquième trait hexaire emploie la douceur et occupe la situation prééminente ; il présente l'avantage qui consis te dans la satisfaction, la soumission et l'att achement à la clarté (intelligence). De plus il se conforme à la voie de la justice et sympathise avec la dureté énergique ; bien qu'il ne puisse rétablir le contact du ciel et de la terre ${ }^{1}$, ni accomplir de grandes choses

\footnotetext{
${ }^{1}$ Au figuré et dans le sens que lui donnent les Chinois, cette phrase signifie « rétablir l'accord entre le souverain et le peuple », ou « entre la morale et les nécessités du gouvernement »,
} 
dans l'uni vers, il est cependant capable de petites améliorations ; c'est ainsi que le présage est heureux sil s'agit de choses de peu d'importance. Le cinquième trait emploie l'intelligence et sympathise avec la dureté énergique ; cependant il ne peut atteindre à la grandeur du présage heureux, comment cela peut-il être concilié ? Réponse : le cinquième trait est malléable et négatif ; bien qu'il sympathise avec le second, cependant, c'est dans un moment de diversité de vues; la voie rationnelle de leur alliance mutuelle ne peut pas encore être ferme et profonde ; aussi le second doit « rencontrer le maître dans la ruelle », et si le cinquième "mord la peau » ${ }^{1}$ il n'y aura aucune culpabilité. Dans l'univers, pendant le moment de séparation et d'opposition, il faut absolument que le prince et ses ministres possèdent la droiture et la justice avec la dureté énergique positive et ensuite qu'ils unissent leurs efforts avec une sincérité absolue ; ce n'est qu’à ce prix qu'ils peuvent « réussir » ${ }^{2}$.

TSHOU HI. - Explication de la formule du koua au moyen des vertus des koua simples, de la transformation, et de la substance du koua.

680.

Le ciel et la terre sont séparés et la chose est identique; le garçon et la fille sont séparés et leurs tendances se comprennent librement; toutes choses sont séparées et les genres sont définis : qu'ils sont grands le moment et l'effet de la séparation!

TSHENG TSE. - Examen de l'identité de la raison d'être des choses pour éclairer les effets et le moment indiqué par le koua khouei : telle est la voie que suit l'homme saint pour réunir ce qui est séparé. Voir dans l'identité ce qui constitue cette identité, c'est la science ordinaire dans le monde ; mais l'homme saint comprendra l'identité essentielle et primordiale de la raison d'être des choses, ce qui lui permettra d'unifier l'univers et de relier harmoniquement tous les genres entre eux. En l'éclairant au moyen du ciel et de la terre, au moyen des deux sexes, et de tous les êtres, il voit que le ciel est élevé et la terre placée au-dessous ; leurs substances sont opposées entre elles. Mais la positivité descend, la négativité monte ; elles se réunissent ensemble et la création et la génération des choses sont identiques. Les sexes masculin et féminin ont des caractères physiques différents : c'est l'op position ; mais leurs attractions réciproques se comprennent librement et les réunissent. Le mode d'enfantement des êtres est différent pour chacun : c'est l'opposition ; mais toutefois cet enfantement est toujours la conséquence de l'accord entre le

\footnotetext{
${ }^{1}$ La première expression indique une action secrète qui n'ose s'exercer en plein jour ; pour le sens de la seconde, voir koua $\mathrm{n}^{\circ} 21$. Voir $\mathrm{n}^{\circ} 685$.

${ }^{2}$ Koua $n^{\circ} 21$.
} 
ciel et la terre, et le don des deux éthers positif et négatif produit l'être semblable à son propre genre. Bien que les êtres soient différents entre eux, cependant, leur raison d'être est identique, aussi, qu'il s'agisse de l'immensité du ciel et de la terre, de la foule des êtres vivants, des variétés infinies qui les distinguent et les séparent, l'homme saint est capable de les identifier. Le moment de la séparation, l'effet de la réunion de ce qui est opposé, sont deux choses de la plus haute importance et c'est pour cela que la formule se termine par une exclamation admirative.

TSHOU HI. - Dernière analyse de la raison d'être du fait et exclamation admirative.

681.

Le commentaire traditionnel de la formule symbolique dit: En haut le feu, en bas le marais opposition; l'homme doué en conclut la distinction entre ce qui est identique.

TSHENG TSE. - En haut le feu, en bas l'humidité ; la nature de chacune de ces deux choses est opposée et différente et c'est par là qu'est constituée l'image symbolique de s éparation et de désunion. L'homme doué contemple le sens symbolique de l'opposition et de la distinction et, au milieu de la grande unité, il sait reconnaître ce qui doit être distingué. Or le saint ou le sage, par rapport à la moyenne de la raison humaine dans leur génération, sont, d'une façon générale, identiques aux autres hommes ; mais, dans ce que la masse des hommes unifie et identifie, par moments ils sont seuls à distinguer. En effet, ils s'appliqueront comme les autres à maintenir les grandes liaisons ordinaires de la société, tandis qu'ils se distingueront de la foule en ne sacrifiant pas aux préjugés et aux travers de leur époque. Ils ne pourront pas identifier leur action avec celle des hommes qui corrompent les mours et bouleversent les espr its, ils se sépareront de ceux qui par leurs pratiques détruisent les liens de la société. Il faut être dans l'unité et savoir distinguer et séparer; le Tshong yong dit: "s'accorder sans se laisser entraîner », tel est précisément le cas.

TSHOU HI. - Les deux koua simples sont identiques par leur substance et différents par leur nature. 


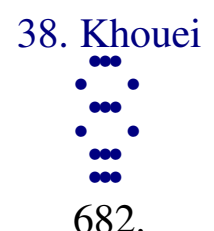

Premier trait nonaire : les regrets se dissipent; perdre le cheval, ne pas le poursuivre, il revient de lui-même; voir les méchants, pas de culpabilité.

TSHENG TSE. - Un trait nonaire occupe le premier rang du koua ; c'est le commencement du désaccord. Dans un moment d'opposition, employer la dureté énergique et agiter dans les rangs inférieurs, c'est ce qui entraîne évidemment les regrets; ce qui fait qu'ils pourront se dissiper, c'est que le quatrième trait nonaire, placé dans une position élevée, emploie aussi la dureté énergique positive, s'écarte et se sépare, sans alliance ; naturellement étant de même genre, ils s'accordent ensemble. Tous deux sont de même positifs ; tous deux occupent de même le rang inférieur d'un koua simple ; enfin ils occupent des situations qui comportent le sens nécessaire de sympathie. Deux positivités ne sont essentiellement pas deux traits qui comportent la sympathie, mais c'est à cause de «l'opposition » qu'elles s'unissent ${ }^{1}$. Le supérieur et l'inférieur s'alliant ensemble, il est donc possible que les regrets se dissipent. Dans le koua khouei, tous les traits ont une correspondance sympathique ; or ce qui s'unit doit nécessairement se séparer ; si essentiellement deux objets sont distincts, comment cesseraient-ils d'être confondus ? Il n'y a que le premier et le quatrième traits, lesquels, bien que sans correspondance sympathique, s'allient néanmoins à cause de l'identité de leurs qualités, et c'est pour cela quils s'accueillent mutuellement. Le cheval exprime ici le moyen de locomotion, c'est la positi vité qui monte. Séparé, isolé, sans alliance, il n'est pas possible de marcher et de progres ser ; c'est ce qui est exprimé par les mots «perdre le cheval». Puisque le quatrième se réunit à lui, il pourra donc marcher et progresser ; c'est ce qui est exprimé par les mots «ne pas le poursuivre, le cheval revient de lui-même». Les méchants, c'est-à-dire ceux qui diffèrent de lui-même. Voir, c'est-à-dire s'apercevoir mutuellement. En présence d'un moment de séparation et d'opposition, bien que ceux qui possèdent les mêmes vertus s'allient ensemble, cependant les hommes inférieurs, qui sont ceux qui se séparent, sont extrêmement nombreux ; sill les abandonne et se sépare d'eux, ne s era-ce pas presque l'universalité de l'empire en état d'hostilité contre l'homme doué ? S'il en était ainsi, ce serait un manquement au devoir de la tolérance et de la clémence et une voie logique de misère et de maux. De plus, comment alors pourrait-il transformer et améliorer en dirigeant vers l'union et la concorde ? Aussi, il faut absolument qu'il, sache regarder les méchants et cela

\footnotetext{
${ }^{1}$ Opposition ; sens général du koua ; ils ne doivent pas s'unir, mais par opposition à ce qui est ordinaire, ils s'unissent.
} 
ne constituera pas un fait coupable. Si les saints rois de l'antiquité ont pu transformer le vice et le changer en vertu, changer leurs ennemis en sujets fidèles et en serviteurs dévoués, c'est parce qu'ils n'ont pas brisé toutes relations avec eux.

TSHOU HI. - En haut il n'a pas de correspondance sympathique conforme à la droiture : donc il a des regrets ; d'ailleurs, il se trouve dans un moment d'opposition et de sépa ration; les vertus identiques sympathisent et les regrets se dissipent. Aussi, il y a l'image symbolique de perdre le cheval, de ne pas le poursuivre, et du cheval revenant de luimême. Cependant il faut aussi qu’il voie les méchants, et alors seulement il pourra éviter les fautes, comme Khong Tse vis-à-vis de Yeang Huo.

683.

Regarder les méchants, pour éviter la culpabilité.

TSHENG TSE. - Dans un moment de séparation et d'opposition, les sentiments des hommes sont en hostilité et contraires; il cherche à ramener l'union et la concorde et déplore de ne pouvoir y réussir. S'il repoussait les méchants et cessait toute relation avec eux, ce serait la presque totalité des hommes en lutte contre l'homme doué, et il en résulterait des maux et des fautes. Aussi, il faut qu’il les considère, ce qui lui fera éviter leurs ressentiments ; alors, à cause de l'absence de ressentiment, il pourra y avoir une voie d'union et de paix. 


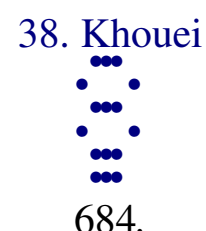

Deuxième trait nonaire : rencontrer le maître dans la ruelle; pas de culpabilité.

TSHENG TSE. - Le second et le cinquième traits sympathisent avec droiture; ce sont ceux qui s'allient ensemble. Mais dans un moment d'opposition la voie rationnelle de sympathie mutuelle de la positivité et de la négativité s'efface, tandis que l'idée de lutte entre l'énergie et la douceur prévaut. Si celui qui étudie le $Y i$ king sait cela, il comprendra la transformation. Aussi, bien que le second et le cinquième traits sympathisent suivant la droiture, ils doivent biaiser et suivre des détours pour se rechercher et s'appeler. Le second trait, avec les vertus de la dureté énergique et de la justice occupe un rang inférieur; il s'élève a vec sympathie vers le prince représenté par le cinquième trait hexaire; leurs voies s'accordant, leurs tendances peuvent agir et prévaloir et l'œuvre de l'apaise ment de l'opposition s'achève. Mais dans un moment d'opposition et de séparation, l'alliance n'est pas solide; le second trait doit se plier, biaiser et suivre des voies détournées pour parvenir à se rencontrer avec le cinquième en épiant les occasions favorables. C'est pour cela que la formule dit : « rencontrer le maître dans la ruelle ». Il faut absolument quill puisse se réunir et ensuite il ne commettra pas de fautes. Lorsque le prince et le sujet sont désunis et séparés, la culpabilité est grande. « Ruelle », sentier tortueux ; « rencontrer », expression qui comporte le sens de hasard. Il doit suivre des voies détournées en cherchant à se réunir, c'est-à-dire dans le but de se rencontrer avec le cinquième trait, et de s'unir avec lui. Ce qu'on entend par l'expression « voie détournée », c'est uni quement employer la voie du bien en procédant avec lenteur et prudence pour se réunir ; il ne s'agit pas de voies criminelles suivies en s'abaissant moralement.

TSHOU HI. - Le second et le cinquième traits : positivité et négativité, correspondance sympathique correcte ; dans un moment de séparation, ils sont opposés, désunis et en hostilité ; il faut quills suivent des voies détournées en cherchant l'occasion de se rencon trer et de se réunir, et c'est là l'absence de culpabilité. C'est pour cela que tels sont le sens divinatoire et l'image symbolique.

685. 
Rencontrer le maître dans la ruelle; ne pas encore perdre la voie rationnelle.

TSHENG TSE. - Dans le moment de la séparation, le cour du prince n'est pas encore en paix ; le sage sujet est en bas, il épuise ses forces et la sincérité de son coar uniquement pour amener le prince à avoir confiance et à s'unir à lui. Il déploie la sincérité la plus absolue pour l'émouvoir et l'influencer ; il épuise ses forces pour le soutenir et l'affermir ; il éclaire le devoir et la raison pour les lui faire connaître ; il écarte l'erreur et la voile pour arriver à la sincérité de l'idée. Il agit ainsi et cherche à opérer le rapprochement; s'il rencontre une voie illicite, il la suit dans ses méandres. Ruelle ne signifie pas une voie mauvaise et contraire à la morale. Aussi Khong Tse mentionne spécialement quill rencontre le maître dans la ruelle mais sans manquer à la voie rationnelle. La négation employée ne comporte pas le sens de nécessité absolue ; cela veut dire qu'il est possible que cette voie vienne à être perdue.

TSHOU HI. - Comme essentiellement il y a correspondance sympathique conforme à la droiture, il n'y a pas de mal commis. 


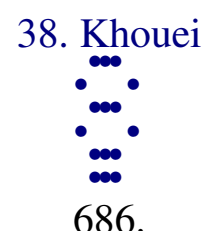

Troisième trait hexaire : le char conduit ; le bauf traîne ; l'homme a la tête rasée et le nez coupé ; soins origine avoir une fin.

TSHENG TSE. - En temps ordinaire, la malléabilité négative est encore incapable de se maintenir par elle-même ; à plus forte raison en est-il de même lorsqu'elle est en présence d'occasions de désunion et de séparation. Le troisième trait occupe l'intervalle entre deux traits énergiques ; il est placé sans pouvoir se satisfaire ; il est évident quil regarde avec envie. Puisquil correspond sympathiquement avec un trait placé au-dessus de lui, il veut avancer pour unir ses tendances à celles du supérieur, mais le quatrième l'en empêche, en avant, tandis que le second le tire en arrière. La voiture et le bouf sont des moyens de transport ; les deux caractères [] [] expriment l'idée d'être tiré en arrière ; les deux caractères [] [] expriment l'idée de barrer le chemin en avant. Ceux qui sont en arrière le tirant et le retenant, tandis que ceux qui sont en avant ou qui avancent le blessent par leur force, il est encore blessé par ceux qui sont au-dessus de lui et par le quatrième trait. Le caractère thien ${ }^{1}$ signifie «raser la tête» et le caractère [] signifie «couper le nez ${ }^{2}$. Le troisième trait suit sa sympathie correcte, tandis que le quatrième l'en empêche et l'arrête ; bien que le troisième trait exprime la malléabilité négative, il est néanmoins placé dans un rang qui comporte la dureté énergique et ses tendances agissent ; aussi, il avance par force pour braver le quatrième, ce qui fait qu'il est blessé. La tête rasée et le nez coupé expriment la répétition de la blessure. Le troisième trait ne s'accor de ni avec le second ni avec le quatrième ; or le moment exprimé par le koua khouei comporte, par lui-même, le sens de l'absence d'accord ; c'est précisément la voie r ationnelle de répondre au sentiment d'union de se maintenir avec énergie en conservant la droiture. En ce qui a rapport à la sympathie conforme à la droiture, la raison d'être des choses indique que la séparation étant parvenue à son extrême limite il y aura finalement réunion et accord ; mais au commencement, comme il est empêché et isolé par les deux traits positifs, il est «sans origine», comme plus tard il réussira à s'unir, il aura une fin. Le caractère formé de deux autres qui représentent, l'un l'idée de dominer, l'autre la main, comporte l'idée de saisir et d'arrêter en retenant.

TSHOU HI. - Le troisième trait hexaire et le trait supérieur nonaire se correspondent sympathiquement et avec droiture, mais le troisième est placé

\footnotetext{
${ }^{1}$ Les caractères du texte n'ont aucun rapport avec ces idées.

${ }^{2}$ Deux supplices de l'antiquité.
} 
dans l'intervalle ent re deux positivités ; derrière lui il est tiré en arrière par le second trait, en avant il est retenu et arrêté par le quatrième, enfin il est dans un moment de séparation et d'opposition. Le caractère du trait supérieur nonaire devient profondément intraitable, de sorte qu'il subit encore les blessures exprimées par les mots « raser la tête et couper le nez ». Cependant la perversité ne peut l'emporter sur la droiture, à la fin il réussira à s'unir, aussi tels sont l'image symbolique et le sens divinatoire.

687.

Voir le char retenu; situation imméritée. Sans origine, avoir une fin; rencontrer la dureté énergique.

TSHENG TSE. - Puisqu'un trait hexaire occupe le troisième rang, il y a absence de droiture; sans droiture, pas de satisfaction. De plus il est dans l'intervalle entre deux

positivités. Ce qui cause un tel état de péril et de gêne, c'est que la situation est imméritée. "Sans origine avoir une fin »; à la fin il doit arriver à se rencontrer avec le trait supérieur nonaire et à s'unir à lui, c'est « rencontrer la dureté énergique ». Lorsqu'il y a réunion sans qu'elle soit conforme à la droiture, elle ne peut jamais durer longtemps sans séparation. Si la réunion a lieu par la voie de la droiture ${ }^{1}$, il n'y a essentiellement aucune raison d'être de séparation finale, aussi, le sage obéit à la raison d'être et agit tranquillement ; savoir, c'est connaître la cause dans son germe et se garder avec fermeté.

\footnotetext{
${ }^{1}$ Une voie correcte.
} 


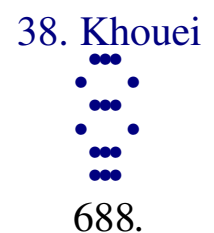

Quatrième trait nonaire : séparé et isolé ; rencontrer un grand personnage ; union et foi ; péril sans culpabilité.

TSHENG TSE. - Le quatrième trait nonaire, pendant le moment de la séparation, occupe une position dont il n'est pas satisfait, il est sans sympathie et se trouve dans l'intervalle entre deux négativités, c'est celui qui est séparé et isolé. Déployant les vertus de la dureté énergique positive, et se trouvant dans un moment de séparation et d'opposition, i solé et sans alliance, il doit, avec ceux qui sont d'un même genre et d'un même éther que lui même, chercher à s'appeler et à se réunir ; c'est à cause de cela qu'il y a rencontre d'un grand personnage. Ici, le mot personnage est employé comme désignation de la positivité, et le mot grand exprime le bien dans les qualités. Le premier trait nonaire est en présence du début de la séparation, il peut réussir à se réunir et à s'allier avec ceux de mêmes vertus, et oublier les regrets de la séparation; c'est la meilleure manière d'être placé dans le moment de l'opposition et c'est pour cela qu'on désigne aussi ce trait en l'appelant le « grand personnage », ou bien encore le «grand docteur». Le quatrième trait a déjà dépassé le juste milieu, il exprime que la séparation est déjà profonde, ce qui n'est pas comparable aux avantages indiqués dans le cas du premier. Le quatrième, comme le premier, occupent tous les deux, avec leur positivité, le rang inférieur dans un koua simple; ils conservent les situations qui se correspondent sympathiquement. Dans le moment du désaccord et de la séparation, chacun d'eux est sans accueil et sans correspondance sympathique. Il est naturel que ceux qui possèdent les mêmes vertus se rapprochent mutuellement, c'est pourquoi ils se rencontrent et se rassemblent. Ayant les mêmes vertus et se rencontrant, il faut nécessairement qu'ils s'allient avec la plus parfaite sincérité ; union et foi : c'est-à-dire qu'ils sont sincères et de bonne foi. Deux positivités, l'une supérieure, l'autre inférieure s'unissent ensemble avec la plus parfaite sincérité ; dans quel moment ne pourraient-elles pas agir ? À quels périls ne pourraient-elles pas remédier ? Aussi, bien que placées dans le péril, il n'y a point de culpabilité. Dans un moment de séparation, être isolé entre deux négativités, placé dans une situation imméritée, indique à la fois et le péril et la culpabilité ; c'est à cause de la rencontre d'un grand personnage, et aussi de l'union et de la bonne foi, qu'il peut ne pas y avoir de culpabilité.

TSHOU HI. - «Séparé et isolé », c'est-à-dire sans sympathies ; « rencontrer un grand personnage », c'est-à-dire rencontrer le premier trait nonaire. «Union et foi », c'est-à-dire que ceux qui ont les mêmes vertus sont 
animés d'une mutuelle confiance. Toutefois, il s'agit d'un moment de séparation, c'est pourquoi il y a nécessairement péril et en même temps possibilité de ne point commettre de fautes. Il en sera de même pour celui qui consulte le sort.

689.

Alliance de bonne foi sans culpabilité ; action des tendances.

TSHENG TSE. - Le premier et le quatrième traits sont tous deux des hommes doués de l'énergie active positive; en présence d'un moment d'opposition et de séparation, le supérieur et l'inférieur s'allient ensemble avec la plus parfaite sincérité, ils identifient leurs tendances, réunissent leurs forces, d'où résulte que leurs tendances pourront préva loir et agir : il n'y a donc pas seulement absence de culpabilité. La formule du trait ne parle que de l'absence de culpabilité ; Khong Tse l'imite en l'éclairant et dit qu'il leur sera possible d'agir suivant leurs tendances et de remédier à l'état de séparation du moment. En effet, avec les aptitudes d'énergie positive de l'homme doué, et s'assistant mutuellement avec la plus parfaite sincérité, à quels maux ne pourraient-ils pas remédier? Il n'y a que l'homme doué dont les tendances peuvent agir. 


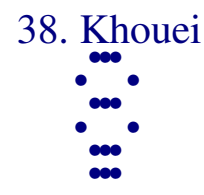

690.

Cinquième trait hexaire : regrets dissipés; cette souche ${ }^{1}$ mord la peau ; en agissant quelle culpabilité ?

TSHENG TSE. - Un trait hexaire qui, avec sa malléabilité négative, se trouve en présence d'un moment d'opposition et de séparation, et qui occupe le rang prééminent : il est évident qu'il a des regrets. Cepe ndant, comme en dessous se trouve le sage doué de l'énergie positive représenté par le second trait nonaire, qui sympathise avec lui pour l'aider et l'assister, les regrets peuvent donc se dissiper. "Cette souche », cette tribu, bande, agrégation; désigne le second trait nonaire correspondant sympathiquement avec droiture. «Mordre la peau » ${ }^{2}$, mordre avec les dents dans la peau et les tissus mous de sorte que la morsure pénètre profondément. Dans un moment d'opposition, si la pénétration n'est pas profo nde, comme réunir ${ }^{3}$ ? Bien que le cinquième trait possède les aptitudes de la malléabilité négative, le second l'assiste en suivant la voie rationnelle de la dureté énergique positive et ${ }^{4}$ pénètre profondément, de sorte que le cinquième pourra entreprendre et avoir des succès; quelles fautes subsisteraient encore ? C'est pendant l'enfance de Tsheng, de la dynastie des Tsheou, que s'érigea le gouvernement de Tsheng Wang ; c'est pendant la faiblesse et le manque d'intelligence de Lieou que se développa la puissance de Tshong Hing, dans les deux cas par l'aide de sages ou de saints investis de l'autorité, et c'est aussi ce qui fit que l'influence de Ki Kong et de Khong Mang pénétra profondément.

TSHOU HI. - L'occupation d'un rang positif par un trait négatif comporte le regret ; c'est parce qu'il demeure dans la justice et rencontre de la sympathie, qu'il peut les dissi per. L'expression «cette souche » désigne le second trait nonaire. «Mordre la peau » exprime que l'action pénètre facilement. Le cinquième trait hexaire possède les vertus de la douceur et de la justice, et c'est pourquoi tels sont l'image symbolique et le sens divina toire.

691.

\footnotetext{
1 Tribu.

${ }^{2} \mathrm{~N}^{\circ} 394$.

${ }^{3}$ Voir le koua $n^{\circ} 21$.

${ }^{4}$ Son action.
} 
Cette souche mord la peau ; en agissant, il y a succès.

TSHENG TSE. — La formule du trait dit seulement : cette souche mord la peau de sorte qu'il devient possible d'agir et d'éviter la culpabilité. La formule symbolique discute et éclaire de nouveau le sens, et elle explique que, bien que les aptitudes personnelles du prince soient insuffisantes, s'il peut cependant avoir confiance dans le sage investi du pouvoir et qui l'assiste en faisant pénétrer profondément en lui l'influence de la voie rationnelle, il lui deviendra possible de réussir à faire quelque chose, et c'est là entre prendre et avoir du succès. 


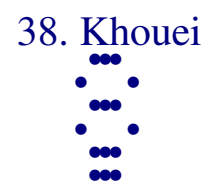

692.

Trait supérieur nonaire : séparé et isolé; voir le porc au dos enduit de boue; transporter une charretée d'esprits ${ }^{1}$; d'abord tendre l'arc, ensuite abandonner l'arc; s'il ne s'agit pas de brigands, c'est un mariage ${ }^{2}$; rencontrer la pluie en entreprenant sera un présage heureux.

TSHENG TSE. - Le trait supérieur occupe le dernier rang du koua et exprime le comble de l'opposition ; l'énergie positive occupant le rang supérieur exprime le comble de l'énergie ; ce trait étant au rang supérieur du koua simple li exprime le comble dans l'emploi de la clarté de l'intelligence ${ }^{3}$. Comble de la séparation, donc opposition et difficulté de réunir ; comble de la dureté énergique, donc précipitation, violence et manque de discernement; comble de l'intelligence, donc excès dans l'investigation et beaucoup de soupçons. Le trait supérieur nonaire jouit de la sympathie, correcte suivant la droiture, du troisième trait hexaire ; en réalité il n'est pas délaissé et isolé et telles sont ses aptitudes et sa nature; c'est par son propre fait que vient sa séparation et son isolement. Tel un homme qui, bien qu'ayant des parents et des alliés, est cependant plein de soupçon et de défiance brusque et sauvage, qui fait naître hors de propos des motifs de désunion, et qui, enfin, bien qu'entouré de ses proches, est toujours seul et isolé. Du trait supérieur au troi sième, bien qu'il existe une correspondance sympathique conforme à la droiture, cependant, comme il se trouve au comble de l'opposition, il doute de tout, et il regarde le troisième comme aussi sale qu'un porc dont le dos serait en outre enduit de boue et de fange, c'est-à-dire comme ce quill y a de plus odieux à voir, du moment où il déteste excessivement, le déchaînement de sa violence est complet et sa faute est atroce, comme de voir transporter une pleine charretée de démons. Les démons, ou esprits, sont essentiellement dépourvus de forme matérielle et l'expression «voir transporter une pleine charretée » exprime l'idée de considérer comme existant quelque chose qui n'existe réellement pas, ce qui est le comble de l'erreur. Lorsque la raison d'être des choses est arrivée à sa limite extrême il doit y avoir nécessairement retour ou renversement en sens inverse. Pour l'expliquer par un exemple à la portée de tous, si un homme se dirige vers l'est, par venu à l'extrême limite de l'est, s'il se meut, ce sera vers l'ouest, s’il s'élève haut, lorsqu'il sera parvenu à la limite extrême de la hauteur, s'il se meut, il descend ; d'une façon géné rale la limite extrême atteinte, tout mouvement doit nécessairement avoir lieu

\footnotetext{
1 Démons.

$2 \mathrm{~N}^{\circ} 98$.

${ }^{3}$ Clarté, lumière.
} 
en sens inverse. La séparation et l'opposition du trait supérieur sont arrivées à leur comble ; la position occupée par le troisième est conforme à la raison d'être des choses. Dans la majo rité des cas, si la perte de la voie rationnelle est poussée à son comble, il doit nécessairement y avoir retour à la droite raison ; aussi, le trait supérieur et le troisième, qui commencent par être séparés par le doute et le soupçon, doivent finalement se réunir. D'abord il tend l'arc, il commence par soupçonner et se méfier et il veut lui lancer une flèche. Ce doute est mal placé et résulte d'une erreur ; l'erreur ne peut pas être conti nuelle, de sorte que finalement il doit nécessairement revenir à la droiture. Le troisième trait n'a en réalité aucun vice ; aussi, ensuite, il abandonne l'arc et ne tire pas ; le désaccord est arrivé à son comble et il y a retour inverse, de sorte qu'ils ne sont plus, lui et le troisième, en état d'hostilité : c'est le mariage. Ici, cette expression : "S'il ne s'agit pas de brigands, c'est un mariage », est la même que dans d'autres koua, mais le sens en est diffé rent. Lorsque la positivité et la négativité s'unis sent et s'étendent harmoniquement, cela constitue et produit la pluie ; le trait supérieur et le troisième commencent par être séparés par la défiance; cette défiance arrivée à son comble ne subsiste plus et ils se réunissent : la positivité et la négativité s'unissent et leur concorde augmente, de sorte qu'elles produisent la pluie, et c'est pour cela que la formule dit: en entreprenant, rencontrer la pluie sera un présage heureux. Entreprendre, c'est partir d'ici pour aller ailleurs, et cela veut dire que, du moment où ils sont réunis et où leur concorde augmente, le présage sera heureux.

TSHOU HI. — «Séparé et isolé » se rapporte au troisième trait hexaire, dominé par deux positivités, tandis que lui-même il se place par son énergie active dans un lieu qui est à la fois le comble de la clarté et le comble de l'isolement. De plus, à cause de la rudesse de son naturel, il est maintenu dans l'isolement. «Voir le porc au dos enduit de boue »: c'est voir l'impureté. «Transporter une charretée d'esprits », imaginer quelque chose qui n'existe pas réellement. "Tendre l'arc », vouloir lancer un trait ; « abandonner l'arc », douter et changer peu à peu d’intention. "S Sil ne s'agit de brigands, c'est un mariage », il sait que ce ne sont pas des brigands, mais que ce sont certainement des amis qui s'appro chent. «Rencontrer la pluie en entreprenant sera un présage heureux »; le doute est complètement éclairé et ce qui était séparé est réuni. Considéré par rapport au troisième trait hexaire, le trait supérieur nonaire s'en trouve d'abord séparé et plus tard il lui est uni et c'est pourquoi tels sont l'image symbolique et le sens divinatoire.

693.

Présage heureux de la rencontre de la pluie ; la foule des doutes dissipée. 
TSHENG TSE. - La pluie c'est le rés ultat de l'harmonie entre la négativité et la positivité ; les deux principes sont d'abord séparés, mais peuvent finalement se réunir, de sorte que le présage est heureux. Ce qui fait qu'ils peuvent se réunir, c'est que tous les doutes sont totalement dis sipés. Au commencement, séparation et isolement; tout est sujet de doute et de soupçon ; aussi le texte dit-il «la foule des doutes ». La séparation parvenue à son comble, survient la réunion, de sorte que tous ces doutes sont donc dissipés. 


\section{Kien}

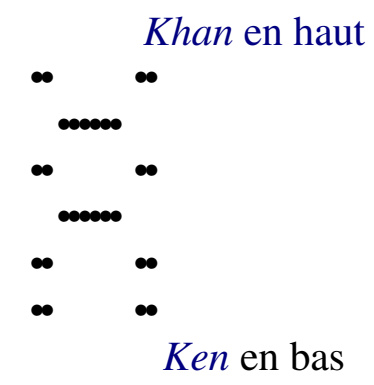

694. Kien, avantage du côté du Sud-Ouest; pas davantage au Nord Est ; avantage à voir un grand homme ; présage heureux de la perfection.

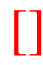

698. Premier trait hexaire : en allant péril ; en venant louanges.

$*$

700. Deuxième trait hexaire : roi et sujet, difficulté ; Pas gardes causes personnelles.

*

702. Troisième trait nonaire : en allant péril ; en venant retour.

$*$

704. Quatrième trait hexaire : en allant, péril ; en venant, entraînement. *

706. Cinquième trait nonaire : grande difficulté, les amis viennent. *

708. Trait supérieur hexaire : en allant péril, en venant grandeur ; présage heureux; avantage à voir un grand homme.

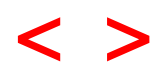


39. Kien.

TSHENG TSE. - Kien. «L'Ordre des koua » dit : « Le koua khouei ${ }^{1}$ exprime l'opposition ou animadversion ; l'opposition comporte nécessairement le danger, de sorte que ce koua est immédiatement suivi du koua kien : kien exprime la difficulté. » Le moment de l'opposition et de la discorde doit nécessairement présenter des difficultés, et c'est ce qui fait que le koua kien suit immédiatement le koua khouei. Le mot kien a le sens de danger et d'empêchement, de sorte que le koua est considéré comme exprimant le danger. Comme koua, il est constitué par le koua simple khan en haut et le koua simple ken, en bas. Khan, péril ; ken, arrêt : en présence du péril, arrêt; il n'est pas possible d'avancer. En av ant péril et danger; en arrière, de hautes montagnes qui forment une barrière redoutable. C'est pour cela que le koua est considéré comme exprimant la difficulté.

\footnotetext{
${ }^{1}$ Koua ${ }^{\circ} 38$.
} 


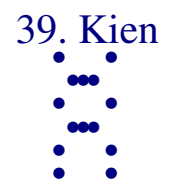

694.

Kien, avantage du côté du Sud-Ouest; pas davantage au Nord Est; avantage à voir un grand homme; présage heureux de la perfection.

TSHENG TSE. - Le Sud-Ouest est la région du koua khouen; le koua khouen exprime la terre, sa substance est la passivité et le changement. Le Nord-Est est la région du koua simple ken, qui représente la montagne et dont la substance est l'arrêt et l'obstacle. Dans le moment de difficulté exprimé par le koua kien, l'avantage consiste à se placer sur un terrain uni et plat ; il n'y a pas d'avantage à s'arrêter au milieu des périls et des obstacles. En se plaçant commodément, la difficulté peut être aplanie ; en s'arrêtant dans le péril, les difficultés croîtront en s'exagérant. Dans les temps difficiles il faut des hommes saints et des sages, et alors il devient possible de parer aux difficultés de l'univers, de sorte que l'avantage consiste à voir un grand homme. Pour remédier aux difficultés, il faut employer la voie rationnelle de la grande droiture et s'affermir dans l'observation de ce tte voie, de sorte que la perfection constituera un présage heureux. Celui qui se trouve placé dans la difficulté doit s'en tenir à l'observation de la parfaite droiture. Si on suppose que les difficultés ne se dissipent pas, il ne perd cependant point la vertu de la droiture, c'est là ce qui constitue le présage heureux. Mais, si rencontrant la difficulté on n'est pas capable de conserver fermement cette droiture, si on se laisse aller au flot débordant de l'erreur et du mal, alors même qu'on suppose s'y s oustraire subrepticement, c'est encore par un artifice pervers et coupable ; celui qui connaît le devoir et la destinée ${ }^{1}$ n'emploie pas de tels moyens.

TSHOU HI. - Kien, difficulté, danger; les pieds ne peuvent avancer, difficulté dans l'action. Il est co nstitué par les koua simples ken, en dessous, et khan, en haut; voir le péril et s'arrêter. C'est pour cela que le koua est considéré comme exprimant la difficulté. Le Sud-Ouest désigne ce qui est plan et uni, facile et aisé ; le Nord-Est indique l'obstac le et l'empêchement et, de plus, c'est la région du koua simple ken. Au moment où on commence à se trouver au milieu du danger, il ne convient pas de courir au devant du péril. D'ailleurs, le koua provient du koua siao kuo dans lequel la positivité avançant viendra occuper le cinquième rang et l'occupera avec justice, ou bien reculant entrera dans le koua simple ken et n'avancera pas. Aussi, le sens divinatoire dit: avantage dans le Sud-Ouest et pas d'avantage dans le Nord-Est. En présence d'un moment d'opposition, il faut nécessairement voir un grand homme et il devient ensuite possible de remédier aux difficultés ; de

\footnotetext{
${ }^{1}$ La destinée providentielle.
} 
plus, il faut observer la droiture et, alors seulement, le présage devient heureux. Cependant, le cinquième trait nonaire du koua possède la dureté énergique, la justice et la droiture ; il a l’image symbolique du grand homme. Depuis le second trait et en montant, les cinq traits occupent tous la situation qui leur correspond exactement ${ }^{1}$, ce qui comporte encore le sens de perfection, de sorte que le sens divinatoire ajoute encore «avantage à voir un grand homme ; présage heureux de la perfection ». En effet, pour celui qui voit le péril, ce qui est précieux, c'est de savoir s'arrêter et, de plus, il ne doit pas s'arrêter indéfiniment ; pour celui qui est placé dans le péril, l'avantage consiste à avancer, mais il ne doit pas manquer à la droiture.

695.

Le commentaire de la formule déterminative dit: Kien, difficulté ; le péril est en avant; voir le péril et pouvoir s'arrêter: qu'elle est grande cette science!

TSHENG TSE. - Kien, difficulté ; ce qui constitue la difficulté dans le koua kien est comme ce qui constitue l'activité dans le koua khien. Quant à la valeur du mot difficulté dans le Yi king, le sens est souvent insuffisant. Le mot kien comporte le sens d'empêchement et d'obstacle. Mais le koua tshouen comporte aussi la difficulté ; de même encore pour le koua khouen ; dans tous, également, il est question de «difficulté » et cependant le sens est différent. Dans le koua tshouen le commencement est difficile et la liberté d'essor n'existe pas encore ; dans le koua khouen, il s'agit de l'épuisement de la force ; dans le koua kien, il est question de la difficulté des empêchements et des obstacles, chaque cas comporte donc un sens particulier. L'empêchement ou péril est en avant, le péril exprimé par le koua simple khan est en avant ; le koua simple inférieur est arrêté et ne peut avancer, de sorte que le koua est considéré comme exprimant la difficulté; voir la difficulté et pouvoir s'arrêter. En en parlant au sujet des aptitudes exprimées par le koua, il s'agit de la voie rationnelle de se placer dans les cas de difficulté. En haut péril et en bas arrêt : voir le péril et pouvoir s'arrêter ; si on brave le péril, en avançant quand même, on en éprouvera des regrets, aussi le texte admire le fait de pouvoir s'arrêter et le considère comme une science. Au moment où surviennent les difficultés, c'est seulement la capaci té de s'arrêter qui constitue le bien, aussi, les formules de tous les traits, excepté celle du cinquième et du second, considèrent le fait d'entreprendre comme constituant une faute, et le fait de revenir sur ses pas comme convenable.

\footnotetext{
1 Traits pleins, rangs impairs ; traits évidés, rangs pairs.
} 
TSHOU HI. - Explication du sens du nom du koua au moyen des propriétés particulières des koua simples et exclamation admirative.

696.

La difficulté avantage le Sud-Ouest; en allant conformément à la justice. Elle n'avantage pas le Nord-Est, cette voie est épuisée. Avantage à voir un grand homme, en allant il y a du mérite, présage heureux de la perfection de la situation méritée; en redressant les Etats. Que le moment exprimé parle koua kien est vaste!

TSHENG TSE. - Dans les temps de difficulté, l'avantage consiste à se placer à l'aise et sur un terrain uni ; or le Sud-Ouest, région du koua khouen exprime la commodité aisée ; le Nord-Est, région du koua simple ken, exprime l'empêchement et le péril. Un trait nonaire monte occuper le cinquième rang et il possède une situation conforme à la justice et à la droiture : c'est là entreprendre, aller, et réussir à trouver un terrain uni et facile, ce qui est considéré comme constituant l'avantage. Le cinquième trait occupe le milieu du koua simple qui exprime le péril et, cependant, il est parlé de celui-ci comme d'un terrain plan et aisé. En effet, le koua simple primitif est le koua khouen; c'est parce que le cinquième avance que ce koua devient le koua simple khan, aussi, la formule relève seulement cette circonstance d'entreprendre et de se conformer à la justice, sans relever le sens qui résulterait de la formation du koua khan. Lorsque le péril survient, si par surcroît on s'arrête sur un terrain périlleux et difficile, les difficultés augmenteront indéfiniment; aussi, il n'y a pas avantage dans le Nord-Est. « Cette voie est épuisée », signifie que la difficulté est à son comble. Dans un temps de difficultés, sans le secours de saints et de sages il sera impossible de remédier aux difficultés de l'univers, de sorte que l'avantage consiste à voir un grand homme. Un grand homme occupant la situation, il parviendra au mérite qui résulte de l'aplanissement des difficultés. Entreprendre et avoir du mérite ${ }^{1}$, c'est-à-dire pouvoir remédier aux difficultés de l'univers ; c'est uniquement la voie rationnelle de la grande droiture. Khong Tse relève encore les aptitudes exprimées par le koua simple, et dit, les divers traits du koua kien, à l'exception du premier, occupent tous une situation méritée, ou dont ils sont dignes, de sorte que ceci constitue la perfection de la droiture et un présage heureux. Bien que le premier trait soit négatif et occupe néanmoins un rang positif, cependant, il se place dans l'infériorité, ce qui constitue encore la droiture dans la négativité. En suivant une telle voie rationnelle de droiture pour redresser les états, il devient possible de remédier aux difficultés. Placé dans un moment de difficulté, suivant la voie rationnelle pour y remédier,

\footnotetext{
${ }^{1}$ C'est la phrase traduite dans le texte, par les mots : « en allant il y a du mérite ».
} 
l'effet accompli est extrêmement grand, aussi la formule dit : qu'il est vaste 1 Les difficultés de l'univers ne sont pas des choses faciles à surmonter; à moins d'être un saint ou un sage, une telle tâche est impos sible ; l'effet peut donc à juste titre être qualifié de grand. Se plier aux nécessités du moment en se plaçant, mesurer le péril et agir, suivre une voie aisée et plane en se conformant à la raison d'être de la plus extrême droiture, tel est l'emploi convenable d'un moment de difficulté.

TSHOU HI. - Explication des termes de la formule du koua au moyen de la transformation du koua et des substances des koua simples, et exaltation de l'importance et de la grandeur de l'emploi et de l'effet de ce moment.

697.

Le commentaire traditionnel de la formule symbolique dit: Sur la montagne il y a de l'eau difficulté ; l'homme doué fait un retour sur lui-même et améliore sa vertu.

TSHENG TSE. - En outre de l'obstacle élevé, présenté par la montagne, et par-dessus, il y a de l'eau. Le koua simple khan représente l'eau et est considéré comme exprimant symboliquement l'empêchement et la difficulté. Au-dessus comme au-dessous, empêchement et obstacle, c'est pourquoi le koua exprime la difficulté. L’homme doué considé rant l'image symbolique de difficultés, en profite pour faire un retour sur lui-même et pour réformer ses aptitudes et ses vertus. L'homme doué, se trouvant en présence de difficultés et d'obstacles doit absolument avoir recours à lui-même et accélérer la réforme de ses aptitudes. Mang Tse dit: «Quiconque, agissant, rencontre un insuccès, doit toujours avoir recours à soi-même »; aussi rencontrant des difficultés et des obstacles, on doit absolument en rechercher les causes dans les imperfections de sa propre personne : c'est là ce qu'on entend par « retour sur soi-même ». Si on découvre en soi quelque chose de mal, on doit le corriger; si le cour est sans perfection, il faut redoubler d'efforts : c'est là ce qu'on entend par l'expression « améliorer ses vertus». L'homme doué améliore ses vertus uniquement dans l'attente du moment favorable. 


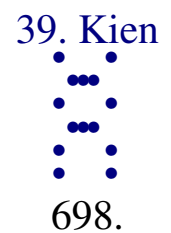

Premier trait hexaire : en allant péril ; en venant louanges.

TSHENG TSE. - Un trait hexaire occupe le premier rang dans le koua kien qui exprime la difficulté ; sil entreprend et avance, il entrera de plus en plus dans les difficultés ${ }^{1}$ : «en entreprenant, difficultés ». En présence d'un moment de difficulté, employant la malléabilité négative, dépourvu d'assistance, et se portant en avant, la difficulté est évidente. Venir, est un terme opposé au mot «entreprendre, aller»; monter en avançant sera «aller», ne pas avancer sera venir. S'arrêter et ne pas avancer, c'est l'avantage qui résulte de la connaissance du moment et de la perception de la cause dans le début de ses effets ; «en venant, louanges ».

TSHOU HI. - En allant on rencontre la difficulté, en venant on rencontre des louanges.

699.

En allant péril, en venant louanges ; il convient d'attendre.

TSHENG TSE. - Au moment du début de la difficulté qui survient, en avançant la difficulté croîtra; c'est un moment où on ne doit pas encore avancer, aussi il convient d'exa miner les causes dans le germe de leurs effets, et de s'arrêter, pour attendre le moment d'agir. Dans toutes les formules des traits il est question de difficulté en allant et d'ava ntage en venant ; cependant cela ne comporte-t-il pas le sens de sortir de la difficulté ? On a répondu: Étant dans la difficulté, entreprendre quelque chose, serait encore une difficulté ; à la fin la difficulté se transformera, et c'est pour cela que le trait supérieur prend le sens de grandeur.

\footnotetext{
${ }^{1}$ Ce sens est tout à fait opposé à ce qu'on lit dans le texte.
} 


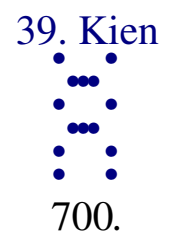

Deuxième trait hexaire : roi et sujet, difficulté ${ }^{1}$; Pas gardes causes
personnelles.

TSHENG TSE. - Le second trait, avec les vertus de la justice et de la droiture, fait partie de la substance du koua simple ken : c'est celui qui s'en tient à la justice et à la droiture, lui et le cinquième se correspondent sympathiquement, c'est un homme juste et droit investi de l'autorité par la confiance du prince juste et droit, et c'est pourquoi la formule emploie l'expression «prince et sujet». Bien que le supérieur et l'inférieur soient doués des mêmes vertus, cependant, le cinquième vient à se trouver au milieu de grandes difficultés, et il développe toute son énergie dans un moment de péril ; les embarras et les dangers sont extrêmes, de sorte que cela constitue un péril dans le péril. Bien que le second soit doué de justice et de droiture, comment, avec ses aptitudes nulles et négatives, viendrait-il aisément à bout du fardeau dont il est chargé ! C'est donc ce qui constitue encore la difficulté dans la difficulté. Ses tendances le portent à secourir le prince placé au milieu des difficultés, ces difficultés dans les difficultés ne sont pas causées par sa propre personne. Bien quill soit possible de supposer qu'il ne parvient pas à les surmonter, ses tendances et son sentiment du devoir sont louables, aussi la formule mentionne son extrême dévouement et son abnégation. Toutefois, ses aptitudes étant insuffisantes pour remédier aux difficultés, sîl lui est possible d'y remédier quelque peu, l'homme saint doit le mentionner formellement comme encouragement au bien.

TSHOU HI. - Malléabilité suivant la justice et la droiture, correspondance sympathique et conforme à la droiture au-dessus ; mais étant placée au milieu des empêchements, il y a donc difficulté, et encore difficulté. Sil cherche à y porter remède, ce n'est pas que sa propre personne en soit la cause, et ce n'est pas non plus dans son propre intérêt. La formule ne mentionne aucun présage heureux ou malheureux; celui qui consulte le sort doit donc uniquement consacrer toutes ses forces et toute son énergie à son entreprise. Quant au succès ou au revers, à la réussite ou à la défaite, ce n'est pas de cela dont il est question.

\footnotetext{
${ }^{1}$ Dans le texte, le mot difficulté est simplement répété deux fois.
} 
701.

Roi et sujet, difficultés! Finalement pas de faute.

TSHENG TSE. - Bien que gêné et empêché dans un moment de difficultés, ses tendances le portent uniquement à remédier aux embarras dont souffre le prince; bien qu'il ne puisse pas encore achever cette auvre, cependant, finalement, il est sans faute et sans culpabilité. L'homme saint relève les tendances et le sentiment du devoir et marque l'absence de culpabilité, afin d'encourager à la fidélité et au dévouement. 


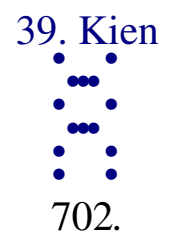

Troisième trait nonaire : en allant péril ; en venant retour.

TSHENG TSE. - Le troisième trait nonaire emploie l'énergie et se maintient dans la droiture ; il est placé au rang supérieur dans la substance du koua simple inférieur. En présence d'un moment de difficulté, ceux qui sont au-dessous sont tous malléables et négatifs, et ils doivent se modeler sur le troisième: c'est donc celui autour de qui les inférieurs se groupent. Le troisième trait et le trait supérieur se correspondent sympathiquement avec droiture ; le trait supérieur est malléable et négatif et il est dépourvu de situation ; cela est insuffisant pour qu'on puisse le considérer comme un auxiliaire, aussi, si le troisième va en avant et monte, il rencontrera les difficultés. Venir, c'est-à-dire venir en descendant ; retourner, revenir. Le troisième trait est ce qui réjouit les deux négativités inférieures et ce qui leur plaît, de sorte que venir en redescendant est considéré comme revenir à la place convenable, dans un lieu de paix et de repos.

TSHOU HI. - En retournant vers les deux négativités, il obtient ce qui lui donne le repos et le calme.

703.

En allant, difficulté ; en venant, retour ; L'intérieur s'en réjouit.

TSHENG TSE. — Lintérieur: les négativités placées en dessous. En présence d'un moment de difficultés, ce qui est négatif et mou ne peut se maintenir debout de soi-même; aussi tous les traits inférieurs se groupent autour de la positivité du troisième et l'entourent de leur affection. La situation d'un trait nonaire occupant le troisième rang, pendant les difficultés, est considérée comme convenable ; étant placé dans la difficulté et possédant le cour des inférieurs, il lui est possible d'obtenir la paix et le repos, aussi revenir est considéré comme un retour; c'est encore ce que le Tshouen tsieou appelle « retour $\gg 1$.

${ }^{1}$ Le Tshouen tsieou est une des auvres de Khong Tse ; le titre signifie «printemps et automne ». 


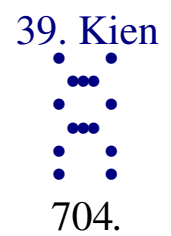

Quatrième trait hexaire : en allant, péril ; en venant, entraînement.

TSHENG TSE. - En allant, il entrera de plus en plus dans la profondeur de l'abîme, donc, en allant, péril. Dans un moment de difficulté, ceux qui se trouvent placés dans les mêmes alternatives périlleuses, obéissent aux mêmes tendances instinctives, sans s'être concertés. De plus, le quatrième rang occupe une situation élevée et, comme ceux qui sont au-dessous de lui, il possède de même la droiture qui résulte de la situation occupée ${ }^{1}$. De plus encore, lui et le troisième se rapprochent, se pressent et se groupent ensemble. Le second et le premier traits sont du même genre : ce sont ceux qui s'allient ensemble; celui-ci a les mêmes tendances que ceux qui sont au-dessous de lui : la foule se groupe autour de. lui, et c'est pourquoi la formule dit : "venir avec entraînement »; en venant, ils s'entraîneront mutuellement avec ceux qui sont au-dessus. Pouvoir s'accorder avec la foule, c'est posséder la voie rationnelle pour se placer pendant les difficultés.

TSHOU HI. - Ils s'entraînent mutuellement, lui et le troisième, réunissent leur force, et remédient aux dangers en les traversant.

705.

En allant, péril ; en venant, entraînement ; mériter réellement la situation.

TSHENG TSE. - Le quatrième trait est en présence d'un moment de difficulté ; il occupe une situation élevée, il ne s'en va pas et il vient. Il a les mêmes tendances que ceux au-dessous de lui, c'est certainement suffisant pour posséder le concours de la foule. De plus, négatif, il occupe un rang négatif ; c'est posséder la sincérité. Puisqu'il est compl ètement sincère avec les inférieurs, il peut les entraîner, en descendant avec eux. Le second et le troisième possèdent aussi chacun la sincérité ; le premier, avec sa négativité, occupe le rang inférieur, c'est encore la sincérité. En présence d'un moment de malheur commun, il est évident qu'ils s'allient ensemble pour rendre leur union sincère, de sorte qu'en venant, ils s'entraînent mutuellement ; il mérite

\footnotetext{
${ }^{1}$ Trait négatif, rang pair.
} 
sa situation par sa sincérité. Étant placé dans les difficultés et les périls, comment pourrait-on traverser sans une parfaite sincérité d'intention ? Quand il s'agit de la situation méritée, la formule n'emploie plus le terme droiture, et prend le terme sincérité ; dans l'alliance entre le supérieur et les inférieurs, tout dépend d'une parfaite sincérité ; le terme employé correspond à chaque cas. 


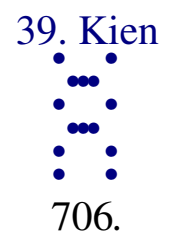

Cinquième trait nonaire : grande difficulté, les amis viennent.

TSHENG TSE. - Le cinquième trait occupe la situation du prince et il se trouve au milieu des difficultés; c'est là la grande difficulté de l'univer s. Il est en présence de difficultés et, de plus, il est au milieu de périls, ce qui constitue encore une grande difficulté. C'est un moment de grande difficulté, et, avec le second trait placé au-dessous de lui, ils sympathisent par la justice et la droiture : c'est là l'assistance qui lui arrive. Au moment où l'univers se trouve plongé dans les difficultés, ce n'est pas un mince avantage que de posséder l'aide et le concours d'un sujet juste et droit ; comment n'y a-t-il pas un présage heureux, puisqu'il jouit de l'avantage de la venue d'amis dévoués ? On répond à cette objection, que cette dernière condition n'est pas suffisante pour traverser le péril et le conjurer; puisqu'il s'agit d'un prince doué d'une énergie active positive, de la justice et de la droiture, et qu'il vient précisément à se trouver au milieu de grands périls, sans l'aide et l'assistance d'un sujet énergique et positif, possédant la justice et la droiture, il lui sera impossible de conjurer les périls et les difficultés de l'univers. La justice et la droiture du second trait constituent certainement une aide ; mais, vouloir avec l'aide de la douceur faible et malléable de la négativité remédier aux difficultés de l'univers serait une chose impossible. Depuis l'antiquité, lorsque les saints rois ont remédié aux difficultés de l'univers, cela n'a jamais été autrement que par le concours de sujets sages et saints; tels sont les exemples de Theang et de Wou arrivant à posséder les états de Lu et de Yi. Il est rare qu'un prince d'un mér ite modeste et ordinaire, aidé par des ministres énergiques et éclairés, puisse remédier aux difficultés de l'univers, cependant, le cas peut certainement se présenter ; tels sont les exemples de Lieou Thien avec Khong Ming, de Sou Tsong, de la dynastie des Theang avec Kuo Tse Yi, ou encore de Ti Tsong et de Li Sheng. Alors même qu'il s'agirait d'un prince éclairé, s'il n'avait pas la disposition d'un sujet doué d'aptitudes convenables, il ne pourrait pas remédier aux difficultés, aussi, toutes les fois qu'un trait hexaire occupe le cinquième rang tandis qu'un trait nonaire occupe le second, c'est surtout à cause de l'assistance dont il jouit que le prince par vient à accomplir son auvre ; tel est le cas lorsqu'il s'agit des koua mong $^{1}$ et thae ${ }^{2}$. $\mathrm{Si}$, au contraire, un trait nonaire occupe le cinquième rang tandis qu'un trait hexaire occupe le second, en général, l'ouvre est imparfaite, et c'est le cas des

\footnotetext{
${ }^{1}$ Koua $n^{\circ} 4$.

2 Koua $n^{\circ} 11$.
} 
koua $t$ shouen ${ }^{1}$ et $p i^{2}$. Et en effet, si le sujet est plus sage que le prince, il aide le prince en ce que celui-ci serait incapable de faire; si le sujet n'atteint pas au niveau du prince, il l'aide seulement dans la limite de ses propres moyens, de sorte que l'ouvre accomplie ne peut pas être bien grande.

TSHOU HI. - Une grande difficulté est une difficulté qui dépasse le niveau ordinaire. Le cinquième trait nonaire occupe le rang prééminent et il possède les vertus de l'activité énergique de la justice et de la droiture ; il doit jouir du concours d'amis qui viennent le rejoindre et l'assister. Le sens divinatoire exprime que, possédant de telles vertus, on obtiendra ce même concours.

707.

Grandes difficultés; les amis viennent à cause des préceptes de la justice.

TSHENG TSE. - Amis, amis et personnes de même genre. Le cinquième trait possède les vertus de la justice et de la droiture, et le second est aussi juste et droit ; bien que dans un moment de grande difficulté, ils ne manquent point à l'observation de ces vertus. Ils luttent contre la difficulté en s'entraidant mutuellement, et c'est là l'appl ication des préceptes de la justice et de la droiture. Le supérieur et l'inférieur possèdent la justice et la droiture et ne peuvent remédier aux périls du moment : c'est donc que les aptitudes du sujet sont insuffisantes pour la tâche qui lui incombe. Depuis l'antiquité, l'observation des préceptes, le respect des devoirs, et, en même temps, l'insuffisance des aptitudes pour remédier aux difficultés pendantes sont un cas qui est loin d'avoir été rare ; sous les Han, on trouve l'exemple de Li Kou et de Wang Yin; sous la dynastie des Tsin on rencontre des gens tels que Tsheou Yi et Wang Tao.

\footnotetext{
${ }^{1}$ Koua $^{\circ} 3$.

${ }^{2}$ Koua ${ }^{\circ} 12$.
} 


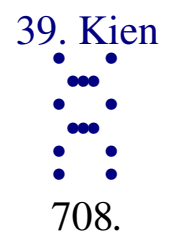

Trait supérieur hexaire : en allant péril, en venant grandeur; présage heureux; avantage à voir un grand homme.

TSHENG TSE. - Ce trait hexaire, avec sa douceur malléable négative, est placé au comble de la difficulté ; il brave cet extrême danger et avance, d'où résulte pour lui le péril ; si au lieu d'avancer il revient, suivant le cinquième et appelant le troisième à son aide, possédant l'aide de la dureté énergique, il peut grandir. La voie rationnelle, en cas de difficulté, c'est d'arrêter les progrès du mal et du danger. Grandeur est un terme qui exprime ce qui est élevé et grand ${ }^{1}$, elle comporte l'idée de magnanimité, un sens avantageux. En revenant, il sera magnanime et grand; la difficulté s'évanouira. Lorsque la diffi culté est à son comble, il y a une voie rationnelle de sortie hors de cette difficulté; le trait supérieur emploie la douceur malléable négative, aussi, lui ne peut en sortir ; mais sỉl jouit de l'assistance de la dureté énergique positive, alors seulement il pourra se soustraire au danger. Dans un moment où la difficulté est à son comble, pouvoir l'éviter constitue un présage heureux; sans la dureté énergique positive, la justice et la droiture, comment pourrait-il sortir des difficultés où il se trouve ? La formule dit encore : avantage à voir un grand homme; dans un moment de grande difficulté, voir un homme doué de grandes vertus, ce sera pouvoir remédier aux difficultés pendantes. Grand homme, désigne le cinquième trait ; c'est la comparaison des aptitudes relatives des deux traits qui révèle ce sens. Le cinquième possède l'énergie active et positive, il est ju ste et droit, et enfin il occupe la situation du prince; c'est le grand homme. Lorsqu'il s'est agi du cinquième trait, il n'a pas été question qu'il pût avoir le mérite de l'apaisement des difficultés, et, cependant, le trait supérieur hexaire a avantage à le voir : comment cela peut-il s'accorder ? Voici la réponse : au sujet du cinquième, il n'en est point parlé parce qu'il est au milieu du péril ${ }^{2}$, et que, étant dénué de l'assistance de la dureté énergique positive, il ne comporte pas le sens de possibilité de remédier au péril. Mais lorsqu'il s'agit du trait supérieur hexaire, alors que les difficultés sont arrivées à leur comble, comme voir un homme doué de grandes vertus c'est pouvoir remédier au danger, cela constitue donc un avantage. Au sujet de chaque trait, le sens est choisi d'une façon particulière et qui n'est pas uniforme ; ainsi, le premier trait nonaire du koua tshouen $^{3}$, dont les tendances sont droites et correctes, est, au

\footnotetext{
${ }^{1}$ Synonyme de ta, grand.

${ }^{2}$ Koua simple khan

${ }^{3} N^{\circ} 96$.
} 
sujet du second trait hexaire, désigné comme représentant un brigand. Au sujet des divers traits du koua kien, il n'est jamais parlé de présage heureux, excepté dans le cas du trait supérieur seul. Tous les autres traits possèdent la droiture, et chacun d'eux présente quelque chose qui constitue le bien, mais cependant aucun d'eux ne peut enco re sortir des difficultés, de sorte que les conditions représentées sont encore insuffisantes pour constituer un présage heureux. Le trait supérieur seul, placé au comble de la difficulté, possède cependant la grandeur et la magnanimité ; c'est là ce qui constitue le pré sage heureux.

TSHOU HI. - Il est arrivé au sommet extrême du koua et il n'a plus la possibilité d'avancer ; l'accroissement de la difficulté est arrivé à son terme ; en revenant vers le cinquième, ils résistent ensemble aux difficultés pendantes, de sorte qu'il aura le mérite de la grandeur et de l'illustration. L'expression grand homme désigne le cinquième trait nonaire ; celui qui comprend le sens divinatoire doit agir ainsi.

709.

En allant, difficulté ; en venant, grandeur: ses tendances le portent vers l'intérieur. Avantage à voir un grand homme, pour suivre ce qui est noble.

TSHENG TSE. - Le trait supérieur hexaire sympathise avec le troisième et suit le cinquième trait, ses tendances sont tournées vers l'i ntérieur. Le péril étant arrivé à sa plus extrême limite, comme le trait supérieur possède une aide, il peut donc arriver à la grandeur, et c'est là ce qui constitue le présage heureux. Étant hexaire et employant ses qualités de douceur négative en présence d'une extrême difficulté, étant très rapproché du prince dur et énergique, doué de justice et de droiture, ses tendances le portent spontanément à le suivre et à se joindre à lui afin de chercher à remédier aux difficultés pendantes, ce qui fait qu'il a avantage à voir un grand homme, c'est-à-dire à suivre la noblesse du cinquième trait nonaire. Ce qui fait que Khong Tse ajoute les mots « suivre ce qui est noble », c'est qu'il craint qu'on ne comprenne pas que les mots « grand homme » désignent le cinquième trait. 


\section{Kiae}

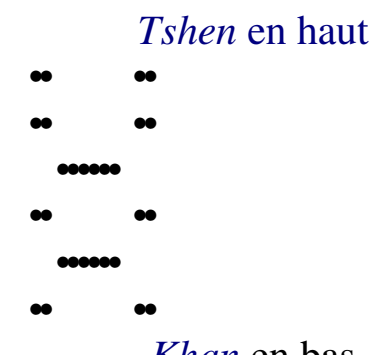

Khan en bas

710. Kiae ; avantage au Sud-Ouest ; s'il n'y a rien à entreprendre, le re tour est un présage heureux; s'il y a à entreprendre, agir de bonne heure est un présage heureux.

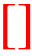

715. Premier trait hexaire : pas de culpabilité.

*

717. Deuxième trait nonaire : chasser et capturer trois renards, posséder une flèche jaune ; perfection, présage heureux.

$*$

719. Troisième trait hexaire : porter sur les épaules et d'ailleurs en voiture ; il en résulte que les brigands surviennent ; pureté, appréhension.

$*$

721. Quatrième trait nonaire : délivrance et gros orteil ; les amis surviennent avec confiance.

$*$

723. Cinquième trait hexaire : l'homme doué pense à la délivrance ; présage heureux ; il a confiance dans l'homme inférieur.

*

725. Trait supérieur hexaire : le dignitaire revêtu du titre de kong emploie la flèche ; l'oiseau sin est posé sur une muraille élevée ; il le capture ; rien n'est sans avantage.

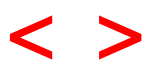


40. Kiae.

TSHENG TSE. - Kiae. «L'Ordre des koua » dit: «Le koua kien exprime la difficulté ; les êtres ne peuvent pas être indéfiniment plongés dans les difficultés, aussi ce koua est-il immédiatement suivi du koua kiae. » Il n'existe aucune raison pour laquelle les êtres res teraient indéfiniment plongés dans les difficultés; quand la difficulté est parvenue à son extrême développement, elle doit se dissiper; kiae signifie dispersion, délivrance; c'est pour cela que ce koua suit immédiatement le koua kien. Comme koua, il est constitué par le koua simple tshen au-dessus, et par le koua simple khan au-dessous, le premier exprime le mouvement, l'ébranlement, et le second exprime le danger, l'abîme ; mouvement à l'extérieur de l'abîme, c'est -à-dire sortir de l'embarras, de sorte qu'il est considéré comme représentant l'image symbolique de l'évanouissement des difficultés. De plus, le koua simple tshen représente la foudre ; le koua simple khan représente la pluie : cela exprime l'action de la foudre et de la pluie. En effet, la positivité et la négativité s'unissant et s'influençant mutuellement, l'harmonie pénètre et se répand graduellement, ce qui fait que le koua est appelé kiae; kiae exprime le moment où les difficultés et les malheurs de l'univers s'aplanissent et s’évanouissent. 


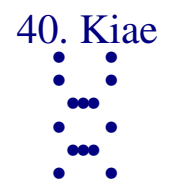

710.

Kiae; avantage au Sud-Ouest; s'il n'y a rien à entreprendre, le retour est un présage heureux ; s'il y a à entreprendre, agir de bonne heure est un présage heureux.

TSHENG TSE — Le Sud-Ouest est la région du koua simple khouen; la substance de ce koua khouen est l'immensité, plane et unie ; au moment où les difficultés de l'univers sont sur le point de s'aplanir, l'homme commence à être délivré des périls et des souffrances; il ne convient pas de le soumettre de nouveau à des tracas fastidieux et à un régime hâtivement sévère ; on doit le traiter avec indulgence et le soumettre à un régime indulgent et aisé à supporter, telle est la marche qu'il convient d'adopter. De la sorte, le coaur de l'homme, en éprouvant de la reconnaissance, se calmera et demeurera en paix, de sorte quil y a avantage dans le Sud-Ouest. Theang abolit les lois cruelles édictées par Kie ${ }^{1}$ et gouverna avec magnanimité ; Wou Wang mit fin aux violences de Tsheou et renversa les institutions politiques des Sheang; tous deux suivirent la voie de la générosité et de la tolérance. "S'il n’y a rien à entreprendre, le retour est un présage heureux; s'il y a à entreprendre, agir de bonne heure est un présage heureux. » S'il n'y a rien à entre prendre, c'est-à-dire si les difficultés dont souffrait l'univers sont déjà aplanies et écartées ; il n'y a rien à faire. S'il y a à entreprendre, c'est -à-dire s'il y a encore des choses à faire disparaître. Or, dans l'univers, dans un État, c'est certainement par suite de l'aban don et du renversement des règles et des lois que les malheurs et les calamités surviennent. Quand les hommes saints ont déjà aplani les difficultés de l'univers, qu'ils ont fait régner le calme et rétabli la paix, on peut dire qu'il n'y a rien à entreprendre ; alors, il convient d'améliorer et de restaurer la voie rationnelle du gouvernement, de rectifier les institutions fondamentales, d'éclairer les lois, et d'avancer la voie du retour aux institutions des premiers âges et des rois illustres de l'antiquité : c'est là le « retour », et cette expression signifie revenir à la droite raison d'être de toutes choses, ce qui constitue un présage heureux pour l'univers. Le mot traduit dans ce passage par «le » est une particule auxiliaire du langage ${ }^{2}$. Depuis l'antiquité, les saints rois, en portant remède aux diffi cultés et en réprimant les désordres, n'ont jamais mis de précipitation au début de leur action ; lorsque la paix et la tranquillité étaient bien assurées, alors ils établissaient des institutions durables et permanentes. Depuis la dynastie des Han et en suivant, les

\footnotetext{
${ }^{1}$ Dynastie des Hia.

$2 K h i$; ce, le; démonstratif et article. Le commentateur veut préciser qu’ici ce mot ne détermine pas un retour particulier, mais exprime l'idée générale de retour.
} 
troubles une fois apaisés, il ne fût plus apporté d'innovations et de changements dans les institutions de l'État, et on se contenta simplement d'aviser, à mesure qu'elles surgirent, aux nécessités de chaque moment ${ }^{1}$, de sorte que cela ne pût jamais constituer un mode de gouvernement parfait : en effet, c'était ignorer le sens du mot $«$ retour $»^{2}$. Sỉl y a à entre prendre, agir de bonne heure est un présage heureux ; c'est-à-dire que, tant qu'il y a enco re quelque difficulté à faire disparaître, c'est la hâte qu'on apporte à y remédier qui constitue le présage heureux. Pendant qu'on écarte ces difficultés, et qu'elles ne sont pas encore absolument toutes supprimées, si on ne se hâte pas de les aplanir, elles reprendront une nouvelle importance ; ce qui fait renaître les troubles, c'est de ne pas y apporter de bonne heure un remède ; alors de petits ferments se développent de nouveau peu à peu et grandissent, de sorte qu'agir de bonne heure est un présage heureux.

TSHOU HI. - Kiae, dissipation, ou évanouissement de la difficulté ; être dans le péril et pouvoir se mouvoir, c'est sortir des difficultés : c'est l’image symbolique de la délivrance. Du moment où les difficultés sont aplanies, l'avantage consiste à demeurer en paix, dans le repos et la tranquillité, sans vouloir plus longtemps prolonger les causes de fatigue et d'ennui ${ }^{3}$. D'ailleurs, ce koua provient du koua sheng dans lequel le troisième trait va occuper le quatrième rang et entre dans la substance du koua simple khouen, tandis que le second conserve sa position et, de plus, possède la justice, de sorte que l'avantage est dans le terrain uni et facile de la région du Sud-Ouest. S'il n'y a rien à entreprendre, il convient alors de revenir à la place primitive et de rester dans le calme et le repos. S’il y a encore quelque chose à entreprendre, il convient de l'entreprendre de bonne heure, sans prolonger indéfiniment les mesures rigoureuses et pénibles à supporter.

711.

Le commentaire traditionnel de la formule déterminative dit: Kiae, étant dans le péril se mouvoir ${ }^{4}$; se mouvoir et se soustraire au péril ; délivrance.

TSHENG TSE. - Le koua simple khan exprime le péril et le koua simple tshen le mouvement; dans le péril, employer le mouvement. Sans péril, il n'y aurait pas difficulté ; sans le mouvement il ne serait pas possible de sortir des difficultés, tandis que par le mouvement on en sort. C'est là se soustraire au

\footnotetext{
${ }^{1}$ Le commentateur fait allusion à tous les changements de dynasties qui ont eu lieu en Chine : période de guerres civiles, triomphe définitif d'un parti, renouvellement en bloc de toutes les institutions et de toutes les lois, le nouveau régime étant proclamé définitif et éternel.

${ }^{2}$ Aux institutions antiques.

${ }^{3}$ Les mesures rigoureuses prises pendant la guerre pour terrifier les opposants.

${ }^{4}$ Le sens serait même plutôt « péril à cause du mouvement »; « se mouvoir et... »
} 
péril et à la difficulté, de sorte que le koua est considéré comme exprimant la délivrance.

TSHOU HI. - Explication du sens du nom du koua au moyen des vertus des koua simples qui le composent.

712.

La délivrance est avantageuse dans le Sud-Ouest: en entreprenant on jouira du concours de la foule ; le retour est un présage heureux: c'est se conformer à la justice; s'il y a à entreprendre, agir de bonne heure sera un présage heureux, en entreprenant il y aura du mérite.

TSHENG TSE. - Dans la voie rationnelle de la délivrance des difficultés, l'avantage consiste à employer la grandeur d'âme et la tolérance facile ; suivant cette voie en entreprenant d'aplanir les difficultés, on aura l'avantage de jouir du concours de la foule, dont le cœur sera gagné par ces procédés. Le texte ${ }^{1}$ ne parle pas du cas où il n'y a rien à entre prendre ; c'est uniquement pour abréger. Remédier aux désordres, écarter les difficultés, sont deux choses simultanées. Tant qu'on n'a pas encore réussi à compléter un mode de gouvernement, il faut attendre le moment où les difficultés seront aplanies et où il n'y aura plus rien à entreprendre ; aussitôt après, il conviendra de faire retour aux modes de gouvernement des premiers rois. C'est là posséder la voie rationnelle de la justice, c'est-à-dire se conformer à ce qui est opportun. Sill y a quelque chose à faire, agir de bonne heure sera le présage heureux ; en agissant de bonne heure on acquerra du mérite par l'ouvre entreprise ; en tardant, les vices augmentent peu à peu, le mal deviendra profond.

TSHOU HI. - Explication de la formule du koua au moyen des transformations du koua. Le koua simple khouen représente la foule; «posséder la foule» est une expression qui se rapporte au quatrième trait nonaire pénétrant dans la substance du koua simple khouen; "posséder la justice et avoir du mérite » se rapporte également au second trait nonaire.

713.

${ }^{1} \mathrm{~N}^{\circ} 712$. 
Le ciel et la terre séparés, la foudre et la pluie agissent; La foudre et la pluie agissant, les cent fruits, les herbes et les arbres ${ }^{1}$ brisent tous leur enveloppe ${ }^{2}$. Que le moment exprimé par le koua kiae est grand!

TSHENG TSE. - Après avoir clairement tracé la voie de la délivrance, il est encore question de la séparation du ciel et de la terre, afin de faire voir l'importance du moment de la délivrance ${ }^{3}$. Les éthers du ciel et de la terre se dispersent, s'unissent et sinfluencent, ils se répandent harmoniquement, de sorte qu'ils produisent et la foudre et la pluie. La foudre et la pluie agissent et toutes choses germent, brisent leur enveloppe et naissent. L'œavre du ciel et de la terre s'accomplit par la délivrance (séparation), de sorte que Khong Tse s'exclame admirativement sur la grandeur du moment de la délivrance (séparation). Les rois se règlent sur la voie du ciel; ils pratiquent la magnanimité et le pardon; ils répandent leurs bienfaits et leurs grâces, prennent soin de la multitude, et leur action s'étend même aux insectes et aux végétaux. C'est là se conformer au moment de la délivrance et unir sa vertu à celle du ciel et de la terre.

TSHOU HI. - Dernière analyse et exclamation admirative sur la grandeur.

714.

Le commentaire de la formule symbolique dit: La foudre et la pluie agissent: délivrance; l'homme doué en conçoit l'idée de l'amnistie et du pardon des fautes ${ }^{4}$.

TSHENG TSE. - Le ciel et la terre séparés et devenus distincts produisent la foudre et la pluie, de sorte que la foudre et la pluie agissent et délient ce qui est entravé. Ces mots n'ont pas la même valeur que dans l'expression « la lumière est double et agit». Amnistier, c'est renvoyer absous; pardonner, ou commuer, c'est user d'indulgence; les fautes ou manquements commis par erreur peuvent être amnistiés, mais l'amnistie des fautes et du vice serait la négation de la justice, aussi, dans ce dernier cas, il s'agit simplement d'indulgence. L’homme doué considère l'image symbolique de la foudre et la pluie agissant pour délivrer, il réalise cette conception d'une action fécondante en répandant les bienfaits et la charité ; il

\footnotetext{
${ }^{1}$ Les végétaux.

${ }^{2}$ L'enveloppe de leur germe.

3 Séparation.

${ }^{4} \mathrm{Ou}$ « commutation des peines »; c'est le même sens.
} 
réalise cette délivrance de l'obstacle et il emploie la magnanimité et la clémence. 


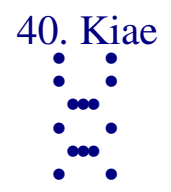

715.

Premier trait hexaire : pas de culpabilité.

TSHENG TSE. - C'est un trait hexaire qui occupe le premier rang du koua kiae; au moment où les malheurs et les difficultés se dissipent et disparaissent, occuper avec les qualités de la douceur malléable un rang qui comporte la dureté énergique donne le sens de la douceur capable d'énergie. Il n'y a plus de malheurs et de difficultés, et il se place suivant ce qui convient au point de vue de la douceur et de l'énergie. Quoique les maux et les difficultés soient dissipés, il s'en faut bien qu'on soit déjà dans le calme et à l'abri des soucis ; mais comme il se place de lui-même suivant l'opportunité, c'est ce qui constitue l'absence de culpabilité ou de danger. Au début d u temps de la délivrance, il convient d'être calme et recueilli, pour prendre des forces et du repos ; la formule qui accompagne le trait est fort brève et c'est précisément pour marquer cette idée.

TSHOU HI. - La difficulté étant déjà évanouie, il emploie la douceur dans l'infériorité; en haut il rencontre une correspondance sympathique. Quelle faute commettrait-il ? C'est pour cela que tel est le sens divinatoire.

716.

Coüncidence de dureté énergique et de douceur malléable; sens d'absence de culpabilité.

TSHENG TSE. - Le premier et le quatrième traits se correspondent mutuellement: cela indique la rencontre et la coïncidence de la dureté énergique et de la douceur malléable. La coexistence de cette dureté énergique avec la douceur malléable constitue la conformité à l'opportunité. Puisque les difficultés sont évanouies et que la douceur et l'énergie se placent d'une façon convenable, le sens est l'absence d'erreurs ou de culpa bilité. 


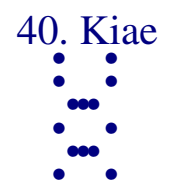

717.

Deuxième trait nonaire : chasser et capturer trois renards, posséder une flèche jaune; perfection, présage heureux.

TSHENG TSE. — Le second trait nonaire, avec les aptitudes de l'énergie positive et de la justice, s'élève sympathiquement vers le prince représenté par le cinquième trait hexaire

C'est celui qui répond aux besoins du moment. Dans l'univers, les hommes inférieurs sont toujours en foule; si c'est un prince énergique et intelligent qui règne, son intelligence suffira pour les éclairer, son autorité sera suffisante pour leur inspirer la crainte, enfin son énergie sera suffisante pour les contenir, de sorte que ces hommes inférieurs n'oseront point donner cours à leurs sentiments ; cependant ils subsistent toujours comme un avertissement et une menace, et le prince se préoccupe de la possibilité quills puissent trouver quelque moyen détourné de nuire au droit. Le cinquième trait hexaire occupe la situation prééminente, mais avec les aptitudes qui résultent de la malléabilité négative, son intelligence s'obscurcit facilement ; son autorité est aisément bravée; sa décision manque d'énergie et devient facilement flottante; si quelque homme inférieur parvient une fois à l'approcher, il changera son cour en le corrompant. À plus forte raison, au moment où les difficultés viennent de se dissiper et où l'ordre commence seulement à s'établir, un changement en sens contraire est encore particulièrement facile. Mais si le second trait est employé dans un tel moment, il sera certainement capable d'écarter les hommes inférieurs, de sorte quil pourra redresser le cour du prince et suivre la voie de la justice énergique et ferme. La chasse a pour objet d'écarter ce qui est nuisible ; le renard est un animal méchant et rusé. L'expression " trois renards » désigne les trois traits négatifs du koua: ce sont les hommes inférieurs du moment actuel. Le mot capturer veut dire pouvoir les transformer et les écarter, comme on capture des renards à la chasse. Les capturer sera suivre la voie de la justice et du droit et c'est en cela que consiste la perfection de la droiture et le présage heureux. Le jaune est la couleur de la justice ; la flèche est un objet rectiligne ; «flèche jaune » est une expression qui indique la justice et le droit. Si la foule des méchants n'est pas tenue à l'écart, elle s'emparera infaillible ment du coar du prince ; alors la voie de la justice et de la droiture ne pourra plus être suivie. Tel fut le cas de Hoan King négligeant d'écarter Wou San Se ${ }^{1}$.

\footnotetext{
${ }^{1}$ Citation historique.
} 
TSHOU HI. - Lidée qui a conduit au choix de l'image symbolique de ce trait n'est pas encore bien expliquée. On a dit : dans ce koua il y a en tout quatre traits négatifs; en retranchant le cinquième qui occupe la situation du prince, il en reste encore trois ; ce serait là l’image symbolique des trois renards. En général ce trait est considéré comme ayant un sens augural favorable lorsque l'on consulte le sort au sujet de la chasse ; il est encore considéré comme ayant l'image symbolique d'éloigner le mal et la ruse et de posséder la justice et la droiture. Pouvant conserver la droiture, alors rien ne sera sans présage heureux.

718.

Deuxième trait nonaire, pureté, présage heureux : posséder la voie de la justice.

TSHENG TSE. - Ce qui est exprimé par les mots «pureté, présage heureux », c'est la possession de la voie de la justice. Supprimer et chasser le vice, de façon que la voie de la justice et de la droiture soit praticable, c'est là la pureté et le présage heureux. 


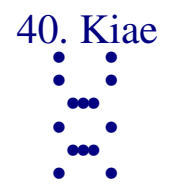

719.

Troisième trait hexaire : porter sur les épaules et d'ailleurs en voiture; il en résulte que les brigands surviennent ; pureté, appréhension.

TSHENG TSE. — Le troisième trait hexaire, c'est la malléabilité négat ive occupant le rang supérieur dans le koua simple inférieur; il est placé dans une situation qui ne lui revient pas ; c'est comme un homme inférieur qui devrait être placé dans une position humble et basse pour porter des fardeaux sur sa tête et ses épaules et qui ; cependant, monte dans une voiture; ce n'est pas sa place. Il doit nécessairement en résulter que des brigands surviendront et la lui enlèveront. En supposant même que ce qu'il fait puisse être conforme au droit, il y aurait encore des motifs de crainte et d'appréhension. Si un homme inférieur usurpe une situation distinguée, bien qu'il s'efforce d'agir avec droiture, ce n'est cependant pas, à cause de son essence vile et grossière, quelqu'un propre à une position élevée : finalement, il a lieu d'éprouver de l'appréhension. S’il peut cepen dant être capable d'une grande droiture, qu'arrivera-t-il ? Réponse : La grande droiture n'est pas à la portée de la malléabilité négative ; donc s'il est dans un tel cas, c'est qu'il s'est transformé et est devenu homme doué. Le troisième trait représente un homme inférieur, malléable et négatif ; il devrait rationnellement être dans l'infériorité et au contraire il est au-dessus de ce qui est inférieur: c'est comme un homme inférieur qui devrait porter des fardeaux et qui, au contraire, marche en voiture ; il doit survenir des brigands qui le violenteront. Dans le moment où les difficultés se dissipent, si un homme inférieur usurpe le pouvoir, il ramène le désordre et la violence.

TSHOU HI. - Les formules annexées sont suffisantes. Les mots pureté et appréhension expriment que, bien que possédant légitimement la situation, on éprouvera encore de la honte ; ce n'est qu'en se retirant et en s'en allant qu'il sera possible de l'éviter.

720.

Porter sur les épaules et aller en voiture ; cela doit encore être mauvais; c'est par mon propre fait que survient l' ennemi : qui donc est responsable de la faute 
TSHENG TSE. - Lorsqu'un homme bon à porter des fardeaux sur la tête et sur les épaules, se fait au contraire transporter en voiture, c'est un fait blâmable et mauvais. Il se place autrement qu’il ne convient ; son mérite ne répond pas à sa fortune, de sorte que l'arrivée de brigands et d'ennemis qui surviennent est comme le résultat de sa propre action qui les appelle et les attire. Qui pourrait encore être rendu responsable de la faute? Lhomme saint ${ }^{1}$ a encore éclairci dans les formules annexées la voie qui fait survenir le mal (les brigands). Il explique comment les auteurs du Yi king peuvent prévoir les faits du vol. Les brigands ${ }^{2}$ n'existent que par suite du désir de représailles et de revanche ; s'il n'y a aucun motif d'animosité et de représailles, pourquoi les brigands attaqueraient-ils ? Porter sur la tête, c'est le sort d'un homme inférieur ; aller en char, c'est la manière de voyager de l'homme doué. Etre homme inférieur et se servir d'un objet destiné à l'homme doué, c'est ce qui ne peut être supporté tranquillement, aussi les brigands profitent de ce prétexte pour le lui enlever. Pour l'homme inf érieur, occuper la situation de l'homme doué est quelque chose qui dépasse ses capacités ; il en devient orgueilleux et vain, arrogant, hautain envers ses supérieurs, comme aussi méprisant et dur envers ses inférieurs : les brigands, c'est-à-dire ses ennemis, saisiront ces motifs de mécontentement et de haine et le détruiront. Détruire, dans ce cas, c'est répandre le bruit et la renommée de ses fautes. Les brigands sont ceux qui se lèvent et renversent les pouvoirs établis. Posséder des marchandises précieuses et des richesses, et les serrer avec négligence, c'est avertir les voleurs et les tenter pour qu'ils viennent s'en emparer ; une jeune fille qui se rend séduisante et jolie excite les libertins pour qu'ils la violentent. L'homme inférieur qui se sert des attributs de l'homme doué, appelle les brigands pour que ceux-ci les lui enlèvent. C'est toujours le sens de l'expression être soi même la cause du mal que l'on s'attire.

\footnotetext{
${ }^{1}$ Khong Tse, auteur des commentaires appelés « sens symbolique » et « formules annexées ».

2 Pour les Chinois, les mots brigands, rebelles, ennemis, sont presque synonymes.
} 


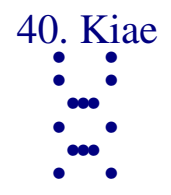

721.

Quatrième trait nonaire : délivrance et gros orteil ; les amis surviennent avec confiance.

TSHENG TSE. - Le quatrième trait, avec ses aptitudes de positivité énergique, occupe une situation élevée; il sert le prince représenté par le cinquième trait hexaire; il représente un sujet d'un rang très élevé et au-dessous de lui il correspond sympathiquement à la négativité du premier trait hexaire. Le terme gros orteil désigne ce qui est bas et menu ; c'est-à-dire le premier trait. Occupant une situation élevée et cultivant la société des hommes inférieurs, il en résultera que les sages et les docteurs, animés par la droiture, s'éloigneront de lui et se mettront à l'écart. Si, au contraire, il chasse et écarte les hommes inférieurs, la foule des hommes doués s'avancera et les sentiments de cordiale sincérité régneront entre eux. Si le quatrième trait est capable de dissiper et d'écarter la malléabilité négative du premier trait hexaire, ses amis, les hommes doués des vertus de l'énergie positive, surviendront et s'uniront sincèrement à lui. S'il n'écarte pas les hommes infé rieurs, c'est que sa propre sincérité de sentiment n'est pas complète : comment jouirait-il de la confiance des hommes ! C'est le premier trait hex aire qui lui est sympathique, et c'est pour cela que la formule exprime que son éloignement constituera la délivrance.

TSHOU HI. — Le terme gros orteil désigne le premier trait, le début; le premier et le quatrième traits ne méritent ni l'un ni l'autre le ur propre situation et, de plus, ils se correspondent sympathiquement; ils représentent ceux qui sympathisent autrement que par la droiture. Mais toutefois, le quatrième est positif, le premier est négatif; ils sont de genres différents; sils peuvent se séparer et s'écarter, alors les amis de l'homme doué sur viennent et la confiance est réciproque.

722.

Délivrance et orteil ; ne pas encore être digne de la situation.

TSHENG TSE. - Bien que le quatrième trait soit énergique et positif, cependant il occupe un rang négatif; il y a lieu de craindre qu'il n'ait pas 
assez de droiture. Si, en outre, il s'associe à des hommes inférieurs et recherche leur société et leur concours, il est évident quil perdra définitivement sa droiture, aussi la formule avertit qu'il faut absolument quil se délivre de son orteil ${ }^{1}$. Ensuite les hommes doués pourront venir. Cela est ainsi parce qu'il n'est pas encore digne de sa situation. Ici le mot kiae, nom de ce koua, veut dire que d'abord il y a union et ensuite séparation; il faut absolument qu'il se sépare de ses orteils et ensuite ses amis auront confiance. En effet, si l'homme doué supporte l'immixtion de quelque homme inférieur dans ses alliances, il manque évidemment de sincérité et de confiance envers les hommes doués.

\footnotetext{
${ }^{1}$ De ce qui est à ses pieds.
} 


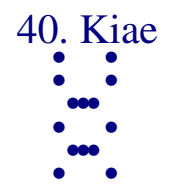

723.

Cinquième trait hexaire : l'homme doué pense à la délivrance ; présage heureux; il a confiance dans l'homme inférieur.

TSHENG TSE. - Le cinquième trait hexaire occupe la situation prééminente, c'est de lui que dépend la délivrance : c'est la délivrance du prince. En parlant d'une façon géné rale au point de vue de l'homme doué, la société qu'il doit rechercher est celle des hommes doués ; ceux quill doit écarter, sont évidemment les hommes inférieurs. Aussi, l'homme doué songeant à la délivrance constituera un présage heureux; lorsque l'homme inférieur s'éloigne, l'homme doué avance. Quel présage serait plus important ? Avoir confiance, est une expression analogue à l'e xpression courante «vérifier ce qui est manifeste». On peut le vérifier au sujet des hommes inférieurs ; si leur troupe s'écarte, c'est que l'homme doué peut s'en délivrer, l'homme inférieur éloigné, l'homme doué s'avance ; la voie de la droiture prévaut naturellement, et l'univers n'a plus besoin d'être régi ${ }^{1}$.

TSHOU HI. - Le koua contient en tout quatre traits négatifs; le cinquième trait hexaire occupe la situation du prince; il est de même nature que les trois autres traits négatifs. Il faut qu'il s'en délivre et qu'il les écarte, et alors le présage sera heureux. Le dixième caractère du texte, confiance, a le sens de vérifier, rendre manifeste. Si l'homme doué est délivré, cela devient manifeste par la retraite de l'homme inférieur relégué à l'écar t.

724.

L'homme doué est délivré ; l' homme inférieur se retire à l'écart.

TSHENG TSE. - La délivrance de l'homme doué exprime le fait d'écarter et de chasser l'homme inférieur. Si l'homme inférieur s'en va, la voie de l'homme doué prévaut, et c'est en cela qu'est le présage heureux.

\footnotetext{
1 Toute chose suivant son cours régulier.
} 


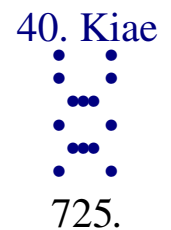

Trait supérieur hexaire : le dignitaire revêtu du titre de kong ${ }^{1}$ emploie la flèche; l'oiseau sin est posé sur une muraille élevée; il le capture ; rien n'est sans avantage.

TSHENG TSE. - Le rang supérieur hexaire, indique un lieu ou une position prééminente et élevée, mais ce n'est point la situation du prince, aussi le texte dit : le dignitaire revêtu du titre de kong. Il s'agit exclusivement de ce qui a rapport à la fin de la dissipation des difficultés. Sin est le nom d'un oiseau, c'est un animal méchant et nuisible ; il symbolise l'homme inférieur, cause du mal. "Muraille», mur d'enceinte, limite entre l'intérieur et l'extérieur. Si le mal était à l'intérieur, ce ne serait pas encore le moment de la délivrance ; si le mal est sorti et passé au-dehors, c'est qu'il n'y a plus aucun mal ; que resterait-il encore à dissiper ? C'est pour cela que la formule dit qu'il est sur l'enceinte quittan t l'intérieur, mais pas encore éloigné. Le texte mentionne que l'enceinte est élevée ; cela indique l’importance du rempart protecteur que le mal n'a pas encore abandonné. Le trait supérieur marque le plus haut degré de délivrance ; au moment où la délivrance est à son comble, ce trait, seul, comporte cependant le sens de délivrance pas encore accomplie : Cela est ainsi pour marquer la ténacité et la force du mal. Le trait supérieur occupe le rang le plus élevé dans le koua kiae; la voie logique de la délivrance est arrivée à son plus haut point d'expansion, l'instrument en est déjà prêt; aussi il peut lancer sa flèche et il atteint le but; du moment où il aura atteint son but, les maux dont souffre l'univers seront absolument dissipés. Que resterait-il qui ne soit avantageux ? Fou Tse développe encore ce sens dans les «formules annexées ${ }^{2}$ en disant : Le sin est le gibier ailé ; l'arc et la flèche sont les instruments ; celui qui lance la flèche, c'est l'homme. L'homme doué cache les instruments ${ }^{3}$ sur sa personne ; il attend le moment opportun il agit: Qu'est-ce qui serait sans avantage ? S'il se meut, il ne les attache pas ${ }^{4}$; c'est pour cela qu’il sort et peut capturer, « cela exprime que les instruments sont prêts et qu'il se meut ». - Un animal nuisible et cruel est sur le rempart, s'il n'avait pas les instruments, ou bien s'il n'atten dait pas le moment pour tirer, alors, comment pourrait-il atteindre le but et capturer ?

\footnotetext{
${ }^{1}$ Titre honorifique d'un ordre élevé. " Emploie la flèche », c'est-à-dire lance une flèche ; on peut lire «lance une flèche à l'oise au sin... »

${ }^{2}$ Le « Grand Commentaire ».

${ }^{3}$ Objets, instruments ; tout ce qui est manufacturé ou approprié à un usage spécial par la main de l'homme ; cause, moyen.

${ }^{4}$ Ou ne les serre pas.
} 
C'est à cause de cela que la voie rationnelle de la délivrance est la prépar ation de l'instrument. S'il y a lieu de se délivrer de quelque chose, la voie rationnelle de s'en délivrer soi-même, c'est d'attendre que le moment opportun survienne. Le trait supérieur est dans ces conditions et il agit; aussi, il n'attache et ne serre pas ses armes; il tire et jamais sans succès. Les mots serrer et lier expriment un danger et un obstacle. Dans ce passage, l'homme saint ${ }^{1}$ explique le sens des expressions « serrer l'instrument » et «attendre le moment». Or, lorsqu'une seule personne, en agissant, étend les effets de son action à toutes les choses de l'univers, si elle n'a pas préparé l'instrument, comme aussi si elle se meut sans attendre le moment, le moins qui puisse en résulter sera quelque empêchement ou quelque embarras, et s'il s'agit de choses importantes, les conséquences pourront aller jusqu'à la ruine et à la destruction ${ }^{2}$. Depuis l'antiquité beaucoup se sont plu à préparer leur auvre et n'en ont point eu le mérite, ou même l'ont vu s'écrouler, et cela a toujours été par ces mêmes causes.

TSHOU HI. — Les « formules annexées » suffisent.

726.

Le dignitaire revêtu du titre de kong lance une f lèche à l'oiseau sin, afin de dissiper la révolte.

TSHENG TSE. - Il est parvenu à la fin de la délivrance et n'est pas encore délivré. Le mot révolte a la valeur de «troubles très graves ». C'est en lançant des flèches avec un arc qu'il dissipe la révolte et s'en délivre. Après la délivrance, l'univers sera en paix.

\footnotetext{
${ }^{1}$ Khong Tse.

${ }^{2}$ De l'État et du prince imprévoyant.
} 


\section{Souen}

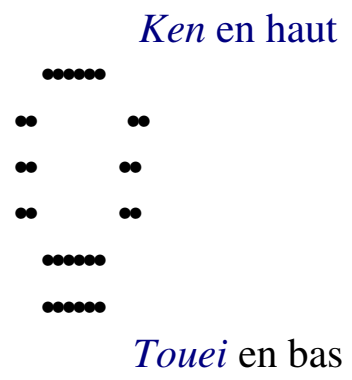

727. Souen, avoir confiance ; grandeur du présage heureux ; pas de culpabilité ; possibilité de la pureté ; avantage dans ce qu'il y a à entreprendre.

728. Qu'employer ?Deux plateaux chargés de mets peuvent être offerts en sacrifice.

732. Premier trait nonaire : l'affaire finie se presser d'aller ailleurs; pas de culpabilité ; apprécier et diminuer.

$*$

734. Deuxième trait nonaire : avantage de la pureté ; en avançant présage malheureux; ne pas diminuer ni augmenter.

*

736. Troisième trait hexaire : trois hommes marchant, donc diminuer un homme ; un homme marche, donc il possède des amis.

*

738. Quatrième trait hexaire : diminuer l'inconvénient, pour avoir promptement la joie ; pas de culpabilité.

$*$

740. Cinquième trait hexaire : parfois l'augmenter ; la tortue de dix amis n'a pas la puissance de contrevenir; grandeur du présage heureux.

$*$

742. Trait supérieur nonaire : ne pas diminuer ni augmenter; pas de culpabilité ; présage heureux de la pureté ; avantage dans ce qu'il y a à entreprendre : avoir des serviteurs et pas de famille.

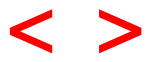


41. Souen.

TSHENG TSE. - Souen. «L'Ordre des koua » dit : «Kiae, ajourner; en ajournant il doit y avoir des inconvénients ; c'est pourquoi le koua kiae est suivi du koua souen. » En agissant avec lenteur, il doit nécessairement y avoir perte ; la perte entraîne la diminution, la décroissance, c'est pour cela que ce koua suit immédiatement le koua kiae. Il est constitué par le koua simple ken en haut et le koua simple touei au-dessous. La matière de la montagne est élevée, celle du marais est humide et profonde. Le dessous étant profond, il en résulte que le dessus est d'autant plus élevé, ce qui donne le sens de diminuer le dessous et d'augmenter le dessus. Ou bien encore, le marais est en bas de la montagne, son éther ${ }^{1}$ monte librement; l'humidité atteint les plantes et les arbres, ainsi que tous les êtres ; c'est encore diminuer le bas et augmenter le haut. De plus, le koua simple inférieur exprime la satisfaction; les trois traits qui le composent correspondent tous sympathiquement avec les traits supérieurs ; cela exprime la satisfaction éprouvée en servant le supérieur, ce qui constitue encore le sens de diminuer le bas et d'augmenter le haut. Autrement ; en bas, satisfaction complète exprimée par le koua simple touei provenant de la transformation du troisième trait hexaire; en haut, obstacle complet exprimé par le koua simple ken provenant de la transformation du trait supérieur nonaire. Le troisième trait qui originellement exprimait la dureté énergique ${ }^{2}$ est devenu malléabilité négative; le trait supérieur, primitivement malléabilité négative ${ }^{3}$, est devenu dureté énergique, ce qui donne toujours le sens de diminuer en bas pour augmenter en haut. La diminution en haut avec augmentation en bas constituent le koua $y i^{4}$, au contraire, prendre en bas pour avantager en haut constituent le koua souen. Pour l'homme placé dans une position élevée, étendre son influence pour qu'elle atteigne les inférieurs sera ce qui constitue l'avantage ( yi); prendre aux inférieurs pour s'enrichir lui-même sera le dommage (souen). Par exemple, s'il s'agit d'un rempart en terre, diminuer la hauteur pour élargir et consolider la base, sera le moyen d'assurer la solidité et la fixité et du haut et du bas ; cela pourrait-il ne pas être avantageux ? Au contraire, prendre en bas pour en accroître l'élévation sera rendre l'écroulement imminent ; comment cela ne serait-il pas nuisible ? C'est pour cela que le koua souen comporte le sens de diminution en bas et d'augmentation en haut ; dans le koua yi, ce sera le sens opposé.

\footnotetext{
${ }^{1}$ Ce mot khi, vapeur, air, souffle, éther, essence, est pris tantôt pour exprimer une matière réelle, tantôt pour indiquer un principe, invisible et insaisissable.

${ }^{2}$ Dans le koua simple khien.

${ }^{3}$ Dans le koua simple khouen.

${ }^{4} \mathrm{~N}^{\circ} 42$.
} 


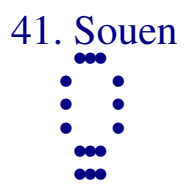

727.

Souen, avoir confiance ; grandeur du présage heureux ; pas de culpabilité ; possibilité de la pureté ; avantage dans ce qu'il y a à entreprendre.

TSHENG TSE. - Souen, diminuer, décroître. Toutes les fois qu'on diminue et qu'on réduit ce qui est en excès, pour se rapprocher de ce qui doit être conformément à la raison, c'est toujours suivre la voie rationnelle de la diminution. La voie rationnelle de la diminution doit nécessairement comporter la confiance et la sincérité ; c'est-à-dire qu'il faut agir avec la plus sincère soumission à la raison d'être des actions que l'on fait. En diminuant conformément à la raison d'être des choses, ce sera un grand bien et le présa ge sera heureux; la diminution ne sera entachée ni d'excès, ni d'erreur ; l'action pourra se continuer avec fermeté et pureté d'une manière permanente et réglée, de sorte que ses bons efforts pourront se produire. Quand les hommes font des diminutions, parfois la diminution est insuffisante, parfois elle est excessive, parfois elle n'est pas régulière et constante, ce qui fait qu'elle est toujours non conforme à la droite raison ; elle n’inspire pas la confiance. S’il n'y a point de confiance et de sincérité il n'y aura point de présage heureux et les fautes surviendront; toute voie qui n'est pas susceptible de pureté ne doit pas être suivie.

TSHOU HI. - Souen, diminuer l'excès. Ce koua est formé en diminuant la positivité du trait supérieur du koua inférieur et en augmentant la négativité du trait supérieur du koua simple supérieur : diminution de la profondeur du marais représenté par le koua simple touei et augmentation de la hauteur de la montagne représentée par le koua simple ken. Diminution de l'intérieur et augmentation de l'extérieur: c'est l'ima ge symbolique de l'action de pressurer le peuple pour servir le prince, et c'est pour cela que le koua est appelé souen. Lorsqu'on diminue ce qu'il convient de diminuer et que l'on agit avec bonne foi, alors, le sens divinatoire devra être d'accord avec les quatre dernières sentences de la formule. 


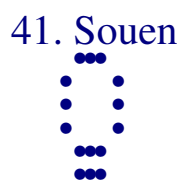

728.

\section{Qu' employer ?Deux plateaux chargés de mets peuvent être offerts en sacrifice.}

TSHENG TSE. - Le koua souen exprime la diminution de l'excès pour se rapprocher de ce qui est juste : c'est diminuer les détails superflu s et se rapprocher de la simplicité primitive. L'homme saint considère la simplicité et l'économie comme constituant la base fondamentale des règles rituelles, et c'est pour cela quill se sert du koua souen pour éclairer et développer ce sens en parlant au point de vue des offrandes dans les sacrifices. Les règles rituelles relatives aux offrandes dans les sacrifices sont extrêmement multiples comme détails, mais toutefois, elles sont essentiellement fondées sur la sincérité du respect. Le grand nombre des cérémonies, la multiplicité des objets servent à parer la sincérité du respect que ressent le coar. Mais si on masque trop cette sincérité sous la quantité des démonstrations, elle devient de la fausseté. Dans ce cas, diminuer le luxe des démonstrations constitue la préservation de la sincérité. C'est pour cela que le texte dit : Qu'employer ? Deux plateaux chargés de mets peuvent servir d'offrande. C'est limiter à deux plateaux de mets ce qui peut servir d'offrande dans les sacrifices, et cela exprime qu'il faut seulement s'en tenir à la sincérité. La sincérité est la seule vraie base ; les maux dont souffre l'univers ne surviennent jamais que parce que les détails finissent par prévaloir sur le fond. Les hauts pignons et les murs recouverts d'ornemen tations ${ }^{1}$ n'ont d'autre origine que l'abri servant de demeure ; les ruisseaux de vin, les montagnes de viande n'ont d'autre raison d'être que de rappeler le besoin de boire et de manger ${ }^{2}$; la licence des mours, l'ivrognerie, la cruauté, le manque de piété ont leur origine dans l'emploi des punitions ; l'emploi abusif des armes, l'habitude des procédés violents ont leur origine dans les expéditions guerrières. Tous les excès dans les désirs des hommes, ont toujours leur origine dans la recherche de la satisfaction de leurs besoins et c'est le trop d'expansion qui leur est donné qui constitue le mal. Les premiers rois, en définissant les principes, se guidèrent sur la raison d'être tracée par le ciel ; plus tard, leurs successeurs se laissèrent aller à la multiplication des détails superflus, en se guidant sur les désirs humains. Le sens du koua souen est simplement la diminution des désirs humains pour revenir à la raison d'être tracée par le ciel.

\footnotetext{
${ }^{1}$ Caractérisant l'ornementation des toitur es et des murs des temples et des lieux de sacrifices.

${ }^{2}$ Le commentateur, en écrivant, a en vue les offrandes faites aux mânes des défunts.
} 
TSHOU HI. - Cela exprime que, dans les moments de diminution, la plus extrême simplicité sera sans inconvénients.

729.

Le commentaire traditionnel de la formule déterminative dit: Souen, diminuer en bas, augmenter en haut : la voie monte et agit.

TSHENG TSE. - Ce qui fait que ce koua est considéré comme exprimant la diminution, c'est qu'il diminue dans sa partie inférieure et augmente dans sa partie supérieure ${ }^{1}$. Prendre en bas pour ajouter en haut ; c'est pour cela que la formule dit que la voie rationnelle est de monter pour agir. Or, diminuer ce qui est élevé pour augmenter ce qui est bas constituera le koua yi; diminuer en bas pour augmenter en haut constitue le koua souen. Dans celui-ci il s'agit de diminuer la base pour augmenter l'élévation : comment cela pourrait-il être considéré comme avantageux ${ }^{2}$.

TSHOU HI. - Explication du nom du koua, au moyen de sa propre substance ${ }^{3}$.

730.

Diminuer et avoir confiance, grandeur du présage heureux, pas de culpabilité, possibilité de la pureté, avantage dans ce qu'il y a à entreprendre. Qu'employer?Deux plateaux chargés de mets peuvent servir d'offrande. Deux plateaux correspondent au moment présent ; la diminution de la dureté énergique et l'augmentation de la malléabilité négative ont lieu à des moments fixes: la diminution et l'augmentation, le plein et le vide, se succèdent toujours avec le temps.

TSHENG TSE. - Cela veut dire que, en diminuant ${ }^{4}$, mais avec la plus extrême sincérité de sentiments, il y aura le grand présage heureux et les autres avantages exprimés dans les quatre dernières sentences; c'est là la parfaite excellence dans la voie de la diminution marquée par le koua souen.

\footnotetext{
${ }^{1}$ Par transformation d'un trait dans chacun des koua simples.

${ }^{2}$ Ce mot « avantageux » a aussi le sens de « augmenter » et c'est le nom du koua yin 42.

${ }^{3}$ Des koua simples dont il est formé.

${ }^{4}$ Ce qui est superflu.
} 
Fou Tse explique spécialement les mots traduits par l'expression « qu'employer ? Deux plateaux chargés de mets peuvent servir d'offrande ». La formule du koua est brève et tranchante; elle exprime qu'il convient de diminuer les enjolivements superflus ; elle dit: Que faut-il donc employer ? Deux plateaux peuvent suffire en offrande. C'est une expression qui indique qu'il faut affermir et augmenter la base et diminuer les détails extérieurs ${ }^{1}$. Fou Tse, craignant que plus tard les hommes ne comprissent pas sa pensée, qu'ils n'en puissent conclure la convenance de la suppression absolue de toute ornementation ou détail de luxe, explique ses paroles. Ce qui a un tronc a nécessairement des rameaux ; c'est-à-dire que ce qui a une origine primitive conduit nécessairement à des détails accessoires, ce qui a un fond réel doit aussi avoir des apparences extérieures; dans l'univers, aucune chose n'échappe à cette loi. Sans base, rien ne tient debout; sans règle tracée, pas de pratique. Le père et le fils sont principalement dirigés par l'affection, donc il faut absolument des règles rituelles au sujet de la gravité sérieuse et de la soumission obéissante ; le prince et le sujet, dans leurs relations, ont pour loi le respect, donc il faut nécessairement des règles d'étiquette pour régler leurs relations. Par les règles rituelles, la modestie et l'humilité persistent dans le cœar ; les règles d'étiquette sont alors observées dans toutes les actions. Dans la prééminence ou l'infériorité de rang, il y a un ordre de préséance ; même dans ce qui a trait aux objets et aux choses d'usage journalier, il ne doit pas en être autrement. L'image et la réalité doivent s'accorder ensemble, sans aucune dissonance, mais si elle arrive jusqu'à la prédominance de la forme, au débordement des détails, à l'oubli de l'origine et à la disparition de la réalité, c'est alors le moment opportun pour faire des diminutions. C'est pour cela que le texte dit: Que faut-il donc employer? Deux plateaux suffisent pour offrande témoignant la sincérité des sentiments, c'est-à-dire qu'il convient d'attacher de l'importance au fait réel et de diminuer ce qui constitue l'ornementation et la décoration. Fou Tse craignant que les hommes ne s'attachent trop à la lettre de ses paroles, précise encore et dit : Quant à la composition de ces plateaux, il faut qu'elle soit d'accord avec le moment où on les emploie ; on ne doit pas employer ce quil convient de ne pas employer dans chaque circonstance. Cela signifie que, si les détails d'ornementation et de luxe ne sont pas excessifs et si néanmoins on les diminue, comme aussi si la diminution est trop considérable, dans chaque cas cela ne sera pas convenable. La diminution de la dureté énergique et l'aug mentation de la douceur malléable ont un moment indiqué; si la dureté énergique est en excès, la douceur malléable fait défaut; les deux koua souen et yi traitent également de la diminution de la dureté énergique et de l'aug mentation de la douceur malléable, c'est toujours en se conformant au temps qu'elles se produisent. Si ce n'était pas au moment convenable, l'augmentation et la diminution seraient irrégulières; quill s'agisse de dimi nution ou d'augmentation, qu'il s'agi sse de remplir ou de vider ${ }^{2}$, rien ne doit jamais avoir lieu que conformément au temps. Ce qui est en excès est diminué, ce qui

\footnotetext{
${ }^{1}$ Élargir le tronc et diminuer les rameaux.

${ }^{2}$ On peut lire de plein et de vide. Ce mot plein signifie essentiellement le plein de la lune.
} 
fait défaut est augmenté ; après la décroissance vient la plénitude, ce qui est plein se vide : tout agit toujours suivant le moment.

TSHOU HI. - C'est l'explication de la formule du koua. Le moment, c'est-à-dire le moment opportun pour diminuer ${ }^{1}$.

731.

Le commentaire traditionnel de la formule symbolique dit: En bas de la montagne il y a un marais: Diminution (souen); l'homme doué se met en garde contre la colère et limite ses désirs.

TSHENG TSE. - En bas de la montagne il y a un marais, ses vapeurs montent librement et imprègnent; c'est comme si on creusait en bas pour augmenter l'élévation, et il y a tou jours le symbole de diminution. L'homme doué contemplant l'image symbolique de la diminution, l'applique à sa propre diminution ${ }^{2}$. Dans la voie rationnelle de l'amende ment de soi-même, ce qu'il convient de diminuer, c'est surtout la colère et les passions, c'est pour cel a qu'il s'applique à réfréner ses sentiments de colère et à éteindre ses désirs et ses passions.

TSHOU HI. - En amendant sa propre personne, ce que l'homme doué doit diminuer n'est jamais aussi important que cela.

${ }^{1}$ Ce fait que le commentateur croit devoir préciser le sens du mot «temps » qu'il traduit par «moment» montre que le texte chinois peut se lire tout autrement. En fait le texte semble énoncer des phénomènes qui ont lieu avec le temps et dans un ordre fixé.

2 À son propre amendement. 


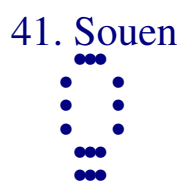

732.

Premier trait nonaire : l'affaire finie se presser d'aller ailleurs ; pas de culpabilité ; apprécier et diminuer.

TSHENG TSE. - Le sens du mot souen est diminution de la dureté énergique et augmentation de la douceur malléable; diminuer en bas et augmenter en haut. Le premier trait, par son énergie positive, correspond sympathiquement au quatrième ; le quatrième, avec sa malléabilité négative, occupe une situation élevée ; il compte sur l'avantage qu'il retire du premi er. Linférieur, en agissant pour le bien du supérieur, doit se diminuer lui même et ne pas s'en attribuer le mérite. Quand ce qu'il fait pour l'avantage du supérieur est fini, il doit vivement se retirer, et ne pas demeurer en s'en attribuant le mérite; c'est ainsi qu'il ne commettra pas de faute. $\mathrm{Si}$, au contraire, il se complaît à jouir du mérite et de la beauté de l'oavre accomplie, ce n'est plus se diminuer lui -même pour avantager le supérieur et, dans la voie rationnelle de l'inférieur, c'est ce qui c onstitue la faute ou culpabilité. La douceur malléable du quatrième trait compte sur l'appui du premier ; aussi ce trait écoute le premier; le premier, de son côté, doit donc apprécier la convenance et l'opportunité et se diminuer afin de l'avantager. Il ne faut jamais qu'il aille trop avant ou qu'il reste en deçà de ce qui convient.

TSHOU HI. - Le premier trait nonaire se trouve en présence d'un moment qui comporte la diminution de ce qui est bas pour augmenter ce qui est haut ; en haut, il correspond sympathiquement à la négativité du quatrième trait hexaire ; il quitte la chose qu'il faisait et se presse d'aller rendre service au quatrième. C'est une voie rationnelle d'absen ce de culpabilité, et c'est pourquoi tels sont le sens divinatoire et l'image symbolique. Toutefois, occupant le rang inférieur et contribuant à l'avantage du supérieur, il doit encore apprécier et mesurer, le plus ou moins de gravité de son action.

733.

L'affaire terminée se presser daller ailleurs; tendances concordant en haut. 
TSHENG TSE. - Le caractère [] désigne ici ce qui est élevé, le supérieur ; ce que l'on révère comme étant supérieur dans ce moment, c'est ce que le premier trait révère comme supérieur. Lui et le supérieur accordent leurs tendances. Le quatrième trait compte sur le premier, le premier agit pour l'avantage du quatrième, il accorde ses ten dances avec celles du supérieur.

TSHOU HI. - [] supérieur, élevé, en haut. 


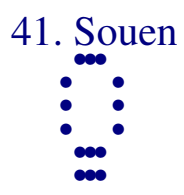

734.
Deuxième trait nonaire : avantage de la pureté ; en avançant présage malheureux ; ne pas diminuer ni augmenter.

TSHENG TSE. — Le second trait avec sa dureté énergique et sa justice se trouve en présence d'un moment de diminution de la dureté énergique ; il occupe un rang qui comporte la douceur malléable et fait partie de la substance de la satisfaction ${ }^{1}$; en haut, il correspond sympathiquement au prince doué de malléabilité négative représenté par le cinquième trait hexaire, puisqu'il correspond sympathiquement au su périeur à cause de sa mollesse et par recherche de sa satisfaction, il doit nécessairement perdre ses vertus d'énergie et de justice, aussi la formule contient l'avertissement que ce qui est avantageux consiste dans la pureté. Le cinquième caractère du texte signifie avancer, agir ${ }^{2}$. S'écartant de la justice, il perdra sa pureté et sa droiture et le présage sera malheureux. Conserver la justice, c'est la pureté. « Ne pas diminuer ni augmenter », en ne diminuant pas sa propre énergie et sa pureté, il pourra être avantageux pour le supérieur, c'est là ce qu'on entend par l'augmenter $(y i)$; s'il perd son énergie et sa pureté, sỉl s'adonne à la mollesse et aux plaisirs, c'est uniquement propre à lui nuire ; ce n'est pas là ce qu'on entend par diminution de soi-même pour l'avantage ${ }^{3}$ du supérieur. Dans ce monde, les gens grossiers et ignorants, quoiqu'ils n'aient pas toujours le cour pervers, considèrent seulement comme constituant la fidélité au prince le fait d'appliquer toutes leurs forces à lui obéir ; c'est que, en effet, ils ignorent le sens de cette expression «.ne pas diminuer, ni augmenter».

TSHOU HI. - Le second trait nonaire, énergique et juste, tend à se préserver luimême; il ne veut pas avancer mal à propos, aussi le sens divinatoire est «avantage de la pureté » et «en avançant, présage malheureux ». Ne pas diminuer ni augmenter, veut dire ne pas modifier ce qu'il conserve, c'est ainsi qu'il pourra être avantageux pour le supérieur.

735.

\footnotetext{
${ }^{1}$ Koua simple touei.

${ }^{2}$ Ce caractère a aussi le sens de « détruire par les armes ».

3 Augmentation.
} 
Deuxième trait nonaire, avantage de la pureté; la justice considérée comme tendance.

TSHENG TSE. — Le second trait nonaire ne possède pas la droiture ; il se place pour sa satisfaction. Ne pas être dur et énergique et posséder la justice constituerait le bien. S’il exerce ses vertus et sa justice, en quoi dé rogerait-il à la loi du bien? Comment peut-on posséder la justice sans la droiture ? Comment peut-on posséder la justice et commettre des excès ? Dans le cas du second trait, ce qu'on entend par les mots « avantage de la pureté » c'est avoir la justice en vue comme but de ses tendances ; si ses tendances sont concentrées vers la justice, il se redressera lui-même. Dans la plupart des cas, la justice est plus importante que la droiture, avec la justice viendra la droiture. On peut posséder la droiture sans être nécessairement juste. En pouvant conserver la justice, il sera utile et avantageux au supérieur. 


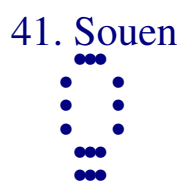

736.

Troisième trait hexaire : trois hommes marchant, donc diminuer un homme ; un homme marche, donc il possède des amis.

TSHENG TSE. - Souen, diminution de l'excès, yi, augmentation de ce qui est insuffisant. Trois désigne les trois positivités du koua simple inférieur et les trois négativités du koua simple supérieur ${ }^{1}$; les trois positivités agissent ensemble, alors survient la diminution du troisième trait hexaire afin d'augmenter le koua simple supérieur ; trois négativités agissent ensemble, alors survient la diminution du sixième trait hexaire qui devient le troisième trait du nouveau koua (souen). C'est ainsi que « trois hommes marchant il y aura diminution d'un homme. » Au sujet du trait supérieur, le changement de la douceur malléable en dureté énergique est désigné comme une diminution, mais c'est uniquement qu'il est question de la diminution d'un des traits. Bien que le trait supérieur et le troisième trait se correspondent sympathiquement par leur origine, comme les deux traits montent ou descendent et contribuent également à constituer le nouveau koua, ils s'accor dent ensemble dans leur action. Le premier et le second traits sont deux positivités, le quatrième et le cinquième sont deux négativités ; les mêmes vertus se pressent ensemble ; le troisième et le trait supérieur sympathisent; c'est toujours l'image d'un groupe de deux choses qui s'accordent ensemble, de sorte que la tendance est unique, et dans chaque cas chacun s'accorde avec son associé. Bi en que le troisième trait et le quatrième s'associent entre eux, toutefois ils font chacun partie d'une substance différente, et le troisième trait correspond au trait supérieur ; ils n'agissent pas ensemble. Sur trois hommes, il faut diminuer un homme et alors chaque homme possédera son associé. En effet, dans l'univers, tout existe par couple ; un et deux, par leur contraste, sont l'origine de la naissance de toutes choses ; trois est donc en trop et doit être diminué : telle est la haute signification des koua souen et $y i$ (diminution et augmentation). Fou Tse, dans les formules annexées, complète le sens et dit : «Le ciel et la terre par leur union mystérieuse créent et développent tous les êtres ; le principe mâle et le principe femelle par leur union intime donnent naissance à tous les êtres. Le Yi king dit: trois hommes marchent, donc, diminuer un homme; un homme marche, donc il possède un ami; cela exprime l'unité exclusive. » Les mots traduits par «union mystérieuse » expriment l'apparence de l'u nion intime; les éthers du ciel et de la terre s'alliant et se matérialisant, de façon à don ner naissance à la création de tous les êtres et à leur croissance. L'essence du principe mâle et du principe

\footnotetext{
${ }^{1}$ Des koua simples khien et khouen avant la transformation.
} 
femelle s'unissant engendrera tous les êtres ; c'est uniquement par l'unité absolue du principe qu'il peut y avoir naissance. Comment la négativité unique et la positivité unique pourraient-elles constituer la dualité ${ }^{1}$ ? Aussi lorsque le nombre atteint trois, il convient de diminuer, c'est-à-dire de s'en tenir exclusivement à l'unité absolue. Entre le ciel et la terre, ce qu'il y a de plus clair et de plus grand dans la nécessité de diminuer ou d'augmenter, n'est jamais plus important que ceci.

TSHOU HI. - Primitivement, le koua inférieur était le koua simple khien $\left(\mathrm{n}^{\circ} 1\right)$, mais la diminution de son trait supérieur a servi à augmenter le koua simple khouen $\left(\mathrm{n}^{\circ} 2\right)$. Trois hommes marchent et il y a réduction d'un homme ; une positivité monte et une négativité descend ; un homme marche et possède un ami ; tous deux par leur accord mutuel constituent la totalité ou unité absolue. Le nombre trois constitue le mélange et le désordre. Le koua présente cette image symbolique, et c'est pour cela que la formule avertit celui qui consulte le sort qu'il convient de tendre vers l'unité absolue ${ }^{2}$.

737.

Un homme marche; s'il y en a trois il y aura défiance.

TSHENG TSE. - Un homme marche ou agit et il est d'accord avec un seul homme, c'est là ce qu'il faut entendre par posséder un ami. Si trois hommes agissent, il y aura défiance sur leurs alliances mutuelles. La raison d'être des choses commande d'éloigner le pre mier homme pour diminuer ce qui est en trop.

${ }^{1}$ La seule idée à saisir est quiil s'agit d'une chose unique, considérée sous deux aspe cts différents et que la réunion des deux aspects, constitue le phénomène étudié.

2 De but, de sentiment. 


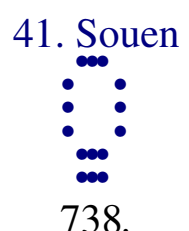

Quatrième trait hexaire : diminuer l'inconvénient, pour avoir promptement la joie ; pas de culpabilité.

TSHENG TSE. - Le quatrième trait avec sa malléabilité négative, occupe un rang élevé, les qualités et la dureté énergique positive du premier trait s'accordent et se correspon dent. Dans un moment de diminution, la sympathie pour la dureté énergique rend possible la diminution de soi-même pour suivre la dureté énergique positive ; suppression de ce qui n'est pas le bien pour se conformer au bien. Le premier trait en agissant pour le bien (augmentation) du quatrième, diminue sa malléabilité et l'améliore par sa dureté énergique, il diminue ce qui n'est pas le bien, aussi la formule dit: diminuer le mal (inconvénient); mal signifie maladie; c'est ce qui n'est pas le bien. En diminuant le mal et en visant exclusivement à accomplir cette tâche avec promptitude et rapidité, il éprouvera de la satisfaction et sera sans culpabilité. Quand l'homme diminue ce qui est en excès, la seule crainte à éprouver est qu'il n'agisse pas avec assez de rapidité ; en agissant avec rapidité, le mal n’ira pas jusqu'à l'excès, c'est là ce qui est considéré comme motif de réjouissance.

TSHOU HI. - S'augmentant par la dureté énergique et positive du premier trait nonaire et diminuant les inconvénients de la malléabilité négative, c'est exclusivement la rapi dité de l'action qui constituera le bien. C'est un avertissement à celui qui consulte le sort, que s'il est dans ces mêmes conditions, il sera sans culpabilité.

739.

Diminuer l'inconvénient, il y a encore possibilité de s'en réjouir.

TSHENG TSE. - Diminuer ce qui constitue un inconvénient est certainement un motif de se réjouir; le texte emploie l'expression « il y a encore $»$, c'est un terme auxiliaire au discours ${ }^{1}$.

\footnotetext{
${ }^{1}$ Terme grammatical pour désigner certains mots qui n'ont pas un sens propre et bien défini par eux-mêmes.
} 


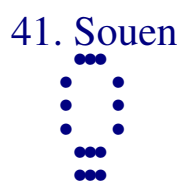

740.

Cinquième trait hexaire : parfois l'augmenter ; la tortue de dix amis n'a pas la puissance de contrevenir ${ }^{1}$; grandeur du présage heureux.

TSHENG TSE. - Dans le moment de la diminution, le cinquième trait hexaire pratique la justice et la déférence pour se maintenir dans la situation prééminente; il annihile ses propres sentiments ${ }^{2}$ pour correspondre à la positivité énergique du second. C'est le prince capable de faire abstraction de ses propres volontés, de se diminuer lui-même, afin de suivre passivement les conseils d'un sage placé au-dessus de lui. S’il peut être ainsi, qui donc, dans l'univers, ne se diminuerait soi-même et ne s'épuiserait pour l'avantager (l'augmenter) ? Aussi, sill se présente une occasion de l'avantager, dix amis l'aideront. Dix est une expression qui comporte l'idée de multitude, la tortue est un être qui indique ce qui est bien ou mal, ce qui est faste ou néfaste. Ce que la foule des hommes décide en commun (vox populi) est nécessairement d'accord avec la droiture et la raison d'être des choses ; l'augure de la tortue même ne peut prévaloir contre cette volonté de la foule. Dans ces conditions, on peut dire que le présage heureux est celui d'un grand bien. Les anciens disaient: "Baser ses desseins sur la volonté de la foule, c'est se mettre d'accord avec la volonté du ciel. »

TSHOU HI. - Malléable, soumis, vide à l'intérieur ${ }^{3}$, puisqu'il occupe la situation prééminente en présence d'un moment de diminution, il représente celui qui reçoit avantage de tout l'univers. Deux tortues font une paire ; les tortues de dix paires, représentent quelque chose de très précieux ${ }^{4}$. Parfois, il reçoit un avantage analogue sans pouvoir s'en défendre ; le présage heureux est évident. Si celui qui consulte le sort possède cette vertu, il bénéficiera du présage qui y correspond.

741.

\footnotetext{
${ }^{1}$ Ce passage ne présente aucun sens saisissable ; voir les commentaires.

${ }^{2}$ Littéralement, il évide son intérieur ; c'est-à-dire devient trait interrompu au milieu.

${ }^{3}$ Sans parti pris.

${ }^{4}$ Le commentateur lit le texte autrement ; il traduit le mot amis par « paire ».
} 
Grandeur du présage heureux du cinquième trait hexaire: assistance venant d'en haut.

TSHENG TSE. - Ce qui fait qu'il jouit d'un grand présage heureux, c'est qu'il est capable de satisfaire complètement aux vieux de la foule des hommes et de s'accorder à la raison d'être du ciel et de la terre. C'est pourquoi les faveurs et l'assistance du ciel des cendent sur lui. 


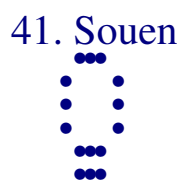

742.

Trait supérieur nonaire : ne pas diminuer ni augmenter ; pas de culpabilité ; présage heureux de la pureté ; avantage dans ce qu'il y a à entreprendre : avoir des serviteurs et pas de famille.

TSHENG TSE. - La diminution, représentée par le koua souen comporte trois sens : se diminuer pour obéir à autrui ; se diminuer soi-même pour le bien (augmentation) d'autrui ; pratiquer la voie rationnelle de la diminution pour diminuer dans autrui. Se diminuer par obéissance à quelqu'un, c'est se conformer au devoir ; se diminuer soi-même pour augmenter quelqu'un, c'est étendre son action aux êtres ; pratiquer la voie de la diminution pour diminuer chez autrui, c'est pratiquer la voi e du devoir. Chaque sens correspond à un moment particulier. Pour en parler au point de vue de ce quil y a de plus important, le quatrième trait et le second prennent tous deux le sens de se diminuer pour obéir à quelqu'un; les trois traits de la substance du koua simple inférieur prennent le sens de se diminuer pour avantager autrui. L'effet du moment de la diminution, c'est de pratiquer la voie rationnelle de la diminution afin de diminuer ce qui, dans l'univers, doit être diminué. Quant au trait supérieur nonaire, il prend le sens de ne pas pratiquer la diminution. Il est nonaire et occupe le rang final dans le koua souen; la diminution est à son comble et il doit y avoir une transformation. Occupant le rang supérieur avec la dureté énergique positive, sỉl emploie son énergie à diminuer et à amoindrir ce qui est au-dessous de lui, ce ne sera pas la voie rationnelle du supérieur, et la culpabilité sera grande. S’il ne pratique pas la diminution, sỉl transforme son action et emploie la voie de la dureté énergique positive pour avantager ce qui est au-dessous de lui, il sera sans culpabilité et sera d'accord avec la droiture; d'ailleurs le présage sera heureux. Étant dans de telles conditions, il conviendra qu'il ait quelque chose à entreprendre, car en entreprenant, il réussira. Étant au rang supérieur, pouvant ne pas diminuer ce qui est en bas et, au contraire, l'avantageant, qui donc, dans l'univers, ne le suivrait avec empressement et soumission? Pour la foule qui se soumet avec empressement, il n'y a ni intérieur ni extérieur ${ }^{1}$; aussi, le texte dit avoir des serviteurs et pas de famille. Avoir des serviteurs, cela exprime qu'il possède le coar des hommes et que ceux -ci viennent à lui et se soumettent; pas de famille veut dire qu'il n'y a aucun e démarcation entre ce qui est loin ou près, ce qui est intérieur ou extérieur.

\footnotetext{
${ }^{1}$ Il n'y a pas de frontières.
} 
TSHOU HI. - Le trait supérieur est en présence d'un moment de diminution en bas et d'augmentation en haut ; il occupe le sommet du koua ; il touche à la limite de l'augmen tation ${ }^{1}$ et il désire se diminuer pour l'avantage et le bien des hommes. Cependant, occuper un rang supérieur et avantager les inférieurs, c'est posséder ce qu'on appelle la man suétude et ne pas abuser de sa situation. Il ne sera pas nécessaire quil aille j usqu'à se dimi nuer, pour qu'il puisse ensuite avantager les hommes. Étant dans ces conditions, il est sans culpabilité, mais cependant il faut encore qu'il agisse d'après la droiture, et alors le présage sera heureux et l'avantage pourra se produire. Mans uétude sans abus de la situation, c'est-à-dire grande mansuétude ; c'est pour cela que la formule dit encore « avoir des serviteurs et pas de famille».

743.

Ne pas diminuer ni augmenter ; réussir grandement dans ses tendances.

TSHENG TSE. - Il occupe le rang supérieur, ne diminue pas ce qui est au-dessous de lui, et, au contraire, l'augmente ; c'est l'homme doué qui peut grandement faire prévaloir ses tendances. Les tendances de l'homme doué sont uniquement tournées vers le bien des hommes.

\footnotetext{
${ }^{1}$ C'est-à-dire qu'après lui vient le koua $y i$.
} 


\section{2. $\mathrm{Yi}$}

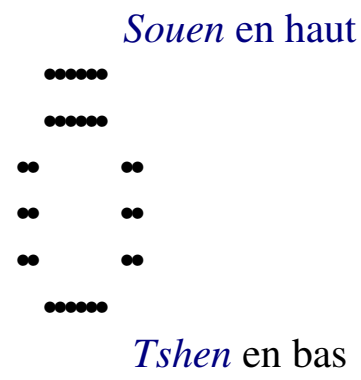

744. Yi : avantage dans ce qu'il y a à entreprendre ; avantage à traverser un grand cours d'eau.

749. Premier trait nonaire : avantage à agir pour ce qu'il importe de faire ; grandeur du présage heureux; pas de culpabilité.

*

751. Deuxième trait hexaire : parfois il augmente; dix paires de tortues ne peuvent aller contre; présage heureux d'éternelle pureté ; le roi pratique l'offrande à l'être suprême ; présage heureux.

753. Troisième trait hexaire : augmenter en employant des choses mauvaises; pas de culpabilité ; avoir, confiance, agir avec justice ; avertir le dignitaire revêtu du titre de kong, employer l'insigne secret.

$*$

755. Quatrième trait hexaire : agir avec justice ; avertir le dignitaire revêtu du titre de kong, suivre ; avantage à employer l'action conforme et changer le royaume.

$*$

757. Cinquième trait nonaire : avoir confiance, caur bienveillant ; ne pas demander ; grandeur du présage heureux; avoir foi et bienveillance au sujet de mes vertus.

$*$

759. Trait supérieur nonaire : ne pas augmenter; parfois frapper ; affermir le cœur sans permanence ; présage malheureux.

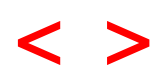


42. Yï.

TSHENG TSE. - Yi. «L'Ordre des koua » dit: «La diminution ne cessant pas, il doit y avoir nécessairement augmentation ; c'est pourquoi le koua souen est suivi du koua $Y i$. » La perfection et la décadence, la diminution et l'augmentation se suivent comme dans un cercle ; la diminution parvenue à son extrême limite, il y a nécessairement augmentation ; c'est la nature même de la raison d'être des choses, et ce qui fait que le koua $Y i$ succède au koua souen. Il est constitué par le koua simple souen en haut et le koua simple tshen en bas : la foudre et le vent qui s'augmentent mutuellement. Si le vent est violent, la foudre sera plus impétueuse; si la foudre éclate, le vent se déchaîne ; les deux phénomènes s'augmentent mutuellement l'un par l'autre, ce qui produit leur accroissement. Ceci est dit au sujet de l'image symbolique des koua simples. Les deux koua simples souen et tshen commencent et s'achèvent tous deux par la transformation du trait inf érieur ${ }^{1}$. La positivité se transforme et devient négativité : c'est la diminution ; la négativité se transforme et devient positivité : c'est l'augmentation. Le koua simple supérieur diminue, le koua simple inférieur augmente; diminution en haut, augmentation en bas, ce qui constitue l'augmentation symbolisée par le koua $y i$. Ceci est dit au sujet du sens. Quand l'inférieur est à son aise, le supérieur est tranquille, aussi augmenter ce qui est inférieur constitue le progrès ( $y i$, augmentation, avantage).

\footnotetext{
${ }^{1}$ Par la transformation du trait inférieur de chacun des koua simples khien et khauen.
} 


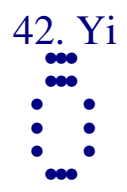

744.

$Y i$ : avantage dans ce qu'il y a à entreprendre; avantage à traverser un grand cours d'eau.

TSHENG TSE. - Le koua yi exprime la voie rationnelle de ce qui est avantageux à l'uni vers, aussi il y a avantage dans ce qui est à entreprendre. La voie rationnelle de l'avantage, ou augmentation, est capable d'aider à traverser les obstacles et les périls : avantage à traverser un grand cours d'eau.

TSHOU HI. - Yi, augmenter. Ce koua est constitué par la diminution du premier trait positif du koua simple supérieur et par l'augmentation de la négativité du premier trait du koua simple inférieur; elle descend du koua simple supérieur vers le bas du koua simple inférieur, et c'est pour cela que le koua parfait est appelé $y i$, et symbolise l'aug mentation. Le cinquième trait nonaire et le second trait hexaire de ce koua possèdent tous deux la justice et la droiture. En bas le koua simple tshen, en haut le koua simple souen; ils ont tous deux l'image symbolique du bois, de l'arbr e, aussi le sens divinatoire exprime qu’il y a avantage à entreprendre et à traverser un grand cours d'eau.

745.

Le commentaire traditionnel de la formule déterminative dit: Yi, diminuer en haut et augmenter en bas; la satisfaction du peuple est sans bornes; de haut, descendre en bas ; la voie rationnelle est très brillante.

TSHENG TSE. - - Il s'agit du sens et des aptitudes exprimées par le koua. Ce qui fait que le koua exprime l'avantage et l'augmentation, c'est qu'il diminue ce qui est haut et augmente ce qui est bas. Diminuant le haut et augmentant le bas, le peuple s'en réjouit et sa satisfaction est sans bornes. D'une position élevée s'abaisser soi -même au-dessous de ce qui est bas ; le grand éclat de la voie rationnelle est manifeste et visible. La positivité descend occuper le premier rang, la négativité monte occuper le quatrième, ce qui donne le sens de descendre du haut vers le bas.

TSHOU HI. - Explication du nom du koua au moyen de la substance de ce koua. 
746.

Avantage à entreprendre; la justice et la droiture reçoivent des louanges ; avantage à traverser un grand cours d'eau. La voie rationnelle du bois prévaut.

TSHENG TSE. - Le cinquième trait pratique la dureté énergique positive, la justice et la droiture, et occupe la situation prééminente; le second, à son tour, emploie la justice et la droiture et lui répond sympathiquement: c'est avantager l'univers par l'emploi de la voie rationnelle de la justice et de la droiture ; l'univers en reçoit le bonheur. C'est ce qui constitue la voie rationnelle de l'augmentation dans les circonstances ordinaires de paix et de tranquillité. L'augmentation est encore faible ; mais en présence de périls et de difficultés ${ }^{1}$, ce qui constitue un avantage ou augmentation, devient extrêmement grave, aussi il y a avantage à traverser un grand cours d'eau ${ }^{2}$, pour remédier aux difficultés présentes, car c'est le moment de la grandeur de l'action de la voie rationnelle de l'augmen tation. Le mot «bois » est mis par erreur à la place du mot « augmentation »; on a cru aussi que c'est parce que le koua simple souen est en haut et le koua simple tshen en bas que le texte porte les mots « voie rationnelle du bois »; c'est une erreur.

TSHOU HI. - Explication de la formule du koua au moyen de sa substance et de son image symbolique.

747.

$Y i$, mouvement et humilité ; journellement avancer sans limite ; le ciel féconde, la terre engendre; l'augmentation est sans région spéciale; tout ce qui tient à la voie rationnelle de l'augmentation se produit avec le temps.

TSHENG TSE. - Enfin, en en parlant d'après la substance des deux koua simples qui le composent, les qualités de ces koua sont, en bas, le mouvement, et en haut l'humilité. Le mouvement et l'humilité constituent la voie rationnelle de l'augmentation. Le mouvement conforme avec humilité aux lois

${ }^{1}$ Dans les temps troublés.

2 À s'exposer dans une entreprise périlleuse. 
de la raison d'être des choses, l'augmentation avance journellement, grande, ample, sans aucune limite restrictive ${ }^{1}$. Comment le mouvement qui ne serait pas conforme à la raison d'être des choses pourrait-il produire une grande augmentation (un grand avantage) ? Par l'ouvre du ciel et de la terre, le texte exprime la grandeur de la voie rationnelle de l'augmentation que l'homme saint réalise pour le bien (l'augmentation) de l'univers. La voie du ciel donne le commencement (crée), la voie de la terre engendre les êtres; le ciel féconde, la terre donne la vie ; tous les êtres sont transformés et créés, chacun accomplit sa destinée en suivant sa propre nature et on peut dire que l'augmentation n'est pas limitée à auc un lieu particulier. Le caractère du texte a ici le sens de lieu, région; si l'augmentation était relative à un lieu parti culier, on pourrait dire qu'elle est mesurée et bornée ; sans région veut dire qu'elle est immense et sans aucune limite. Comment d' ailleurs l'augmentation (le bien) de tous les êtres par l'action du ciel et de la terre, aurait-elle une limite et des bornes ou tiendrait-elle à des circonstances spéciales de lieu ? L'augmentation produite par le ciel et la terre est ce qu'il y a d'infin i ; c'est la raison d'être et rien de plus. L'homme saint loue la voie ration nelle de l'augmentation de l'univers ; elle répond au moment et est conforme à la raison d'être des choses; elle s'accorde avec le ciel et la terre, elle agit toujours conformément au moment.

TSHOU HI. - Mouvement et utilité, vertus des deux koua simples; en haut, le koua simple khien descend et féconde, en bas, le koua khouen monte et engendre, ce qui donne bien encore le sens du texte ci-dessus. De plus, par ces paroles, l'hom me saint exprime la plus grande admiration pour la grandeur de l'augmentation.

748.

Le commentaire de la formule symbolique dit: Le vent et la foudre augmentent; l'homme doué s'amende en considérant le bien ; s'il se trompe, il se corrige.

TSHENG TSE. - Si le vent est violent, la foudre précipite ses éclats; si la foudre éclate, le vent se déchaîne: ces deux choses s'accroissent et s'augmentent mutuellement. L'homme doué regarde l'image symbolique du vent et de la foudre s'augmentant mutuellement et cherche à s'accroître (s'améliorer) personnellement ; cela constitue la voie rationnelle de l'augmentation. Rien n'est meilleur que de considérer le bien, car son action est transformatrice ; sil y a quelque erreur, elle sera donc corrigée. Voyant le

\footnotetext{
${ }^{1}$ Le texte porte littéralement «le soleil, ou le jour, avance », mais cette expression est
} communément employée avec le sens que lui donnent les commentateurs. 
bien et pouvant changer, il devient donc possible d'accomplir le bien et d'étendre son influence à l'univers entier ; ayant commis des erreurs et les corrigeant, aucune culpabilité ne subsistera, rien ne sera en excès ; ce qui constitue l'augmentation (l'ava ntage) pour les hommes n'est jamais plus grand que ceci.

TSHOU HI. - Les forces inhérentes au vent et à la foudre s'unissent, s'ajoutent, s'aug mentent. Revenir au bien, corriger les erreurs, c'est ce qu'il y a de plus grand dans l'aug mentation (amélioration), et l'aide mutuelle que se prêtent ces deux actions est aussi telle que l'action mutuelle du vent et de la foudre. 


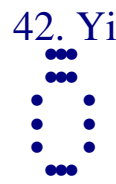

749.

Premier trait nonaire : avantage à agir pour ce qu'il importe de faire ${ }^{1}$; grandeur du présage heureux; pas de culpabilité.

TSHENG TSE. - Le premier trait nonaire est celui de qui dépend le mouvement exprimé par le koua simple tshen ; c'est la perfection de la dureté énergique positive. Existant dans le moment de l'augmentation, ses aptitudes sont suffisantes pour augmenter les êtres et les choses (pour les développer). Bien qu'il occupe le rang le plus inférieur, cependant, en haut, il jouit de la sympathie d'un haut fonctionnaire représenté par le quatrième trait hexaire. Le quatrième, de son côté, est celui de qui dépend la soumission et l'humilité exprimées par le koua simple souen; en haut il est capable d'humilité envers le prince, en bas il est capable de déférence pour les capacités du sage. Celui qui est dans l'infériorité ne peut avoir une action prépondérante ; mais, s'il possède la sympathie de celui qui est dans une haute situation et qui l'écoute, il conviendra qu'il aide et assiste ce supérieur suivant ses moyens ; ce sera un grand avantage pour toutes les affaires concernant l'État : il y a avantage à l'employer pour faire de grandes choses. Puisqu'il occupe un rang inférieur et qu'il est employé par le supérieur pour mettre en pratique ses propres tendances et ses desseins, il faut absolument que ce qu'il fait constitue un grand bien et un présage heureux, et alors il sera sans aucune culpabilité. Si son action ne peut pas constituer un grand présage heureux, non seulement il sera personnellement coupable, mais cette culpabilité rejaillira sur le supérieur ; il sera cause de la culpabilité du supérieur. Pour celui qui est placé dans le rang le plus bas et qui se trouve investi d'une grande autorité, un peu de bien n'est pas suffisant pour répondre à l'importance de sa position ; il faut absolument que son action constitue un présage parfaitement heureux ; c'est alors seulement quill est sans culpabilité.

TSHOU HI. - Bien que le premier trait occupe un rang inférieur, cependant, il est en présence d'un moment d'augmentation et de développement ; c'est c elui qui reçoit l'augmentation du supérieur. Il ne doit pas simplement recevoir cet avantage sans avoir aucun devoir de reconnaissance à remplir; aussi le bien, pour lui, consiste à accomplir de grandes actions ; alors le présage sera parfaitement heureux et ensuite il ne subsistera aucune culpabilité.

\footnotetext{
${ }^{1}$ Sens très incertain.
} 
750.

Grandeur du présage heureux sans culpabilité ; en bas ne pas s'occuper d'affaires importantes.

TSHENG TSE. - Ceux qui sont dans les rangs inférieurs ne doivent essentiellement pas être chargés d'aff aires importantes, c'est-à-dire d'affaires dont les conséquences sont graves. Si on suppose qu'un homme placé dans ces conditions soit investi par le supérieur du soin de telles affaires, ces affaires dont il se trouve chargé doivent évidemment consister à remédier à de graves désordres, de sorte que, si le bonheur qui résulte de son action est complet, il sera sans aucune culpabilité. S’il a pu parvenir à ce résultat parfaitement heureux, il le doit au supérieur qui l'a investi de l'autorité, à cause de sa connaissance des hommes ; lui-même doit se montrer à hauteur de l'autorité dont il se trouve investi ; s’il n'en est pas ainsi, le supérieur et l'inférieur sont tous deux coupables.

TSHOU HI. — Linférieur ne doit essentiellement pas diriger les affaires importantes ; aussi, s'il en est autrement que comme il vient d'être expliqué, la culpabilité ne peut pas être effacée par l'action. 


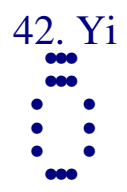

751.

Deuxième trait hexaire : parfois il augmente ; dix paires de tortues ne peuvent aller contre ; présage heureux d'éternelle pureté ; le roi pratique l'offrande à l'être suprême ; présage heureux.

TSHENG TSE. — Le second trait hexaire est placé avec justice et droiture et son essence est la soumission de la douceur; il comporte l'image symbolique du vide intérieur ${ }^{1}$. Lorsqu'un homme se place dans la voie rationnelle de la justice et de la droiture, fait abstraction de ses propres tendances (vide son intérieur) pour rechercher ce qui constitue l'avantage (augmentation), et qu'il peut suivre cette voie avec soumission, qui donc, dans l'univers, ne voudrait le renseigner et lui être avantageux ? Mang Tse dit: «Car s’il aime le bien, entre les quatre mers, chacun méprisant la distance (de milliers de la) viendra lui demander le bonheur. » Mais ce qui est plein ne peut plus recevoir ${ }^{2}$, tandis que ce qui est vide attire les êtres : telle est la raison d'être rationnelle des choses. Aussi, s’il se présente quelque chose qui puisse devenir avantageux, tous ses amis l'aident et l'avantagent. Dix est une expression qui indique l'idée de multitude. Ce que la multitude des hommes accepte comme vérité est ce qui correspond le plus exactement à la raison d'être des choses. La tortue est un être qui présage le bonheur ou le malheur, qui discerne le vrai et le faux ; cela exprime ce qui est absolument certain, et ce qui ne peut être contredit par les oracles de la tortue. Présage heureux parfait et éternel est dit au sujet des aptitudes que comporte le second trait hexaire. Ce second trait possède la justice, la droiture et est évidé au milieu ${ }^{3}$, c'est celui qui peut jouir de l'avantage du concours et de l'action de la foule des hommes. Toutefois, sa matière constitutive, son caractère essentiel est la malléabilité négative, aussi la formule avertit que, avec une fermeté parfaite et durable, le présage sera heureux. La voie rationnelle de la recherche de l'augmentation, ne peut se préserver sans une pureté invariable. Dans le cas du cinquième trait hexaire du koua souen, ce qui fait que, avec dix amis, le présage heureux sera grand, c'est que le trait occupe la situa tion prééminente et se diminue lui-même, qu'il sympathise à la dure té énergique de l'inférieur, qu'il emploie la douceur dans une situation qui comporte la dureté éner gique. La douceur malléable indique ici le vide qui reçoit, c'est-à-dire l'absence de prévention qui écoute; la dureté énergique, dans le même cas, exprime la fermeté inébranlable, et c'est à ces conditions qu'est due l'extrême excellence

\footnotetext{
1 Absence de préventions.

2 Celui qui conserve ses préventions ne peut pas écouter la voie de la raison.

${ }^{3}$ Et est dépourvu de préventions.
} 
dans la recherche de l'aug mentation, par conséquent la grandeur du présage heureux. Lorsqu'il s'agit du second trait hexaire dépourvu de préventions et recherchant l'au gmentation, il a, lui aussi les sympathies de la dureté énergique positive, et il occupe avec douceur un rang qui comporte la douceur; il y a donc lieu de craindre que l'augmentation (amélioration) manque de fermeté, aussi la formule avertit que, s’il pe ut y avoir une fermeté parfaite, permanente et durable, le présage sera heureux. Le roi pratique l'offrande à l'être suprême ; présage heureux: employer l'absence de préventions du second trait et une pureté inaltérable dans l'action de présenter une offrande à l'être suprême doit aussi assurer le bonheur et constitue un présage heureux ; à plus forte raison, cette idée n'est -elle pas évidente quand il s'agit des relations entre les hommes et les êtres ? Chercher à recevoir une augmentation d'autrui, se rait-ce possible sans sympathie ? Le culte rendu au ciel est un des attributs de la puissance de l'empereur, c'est pour cela que le texte dit : le roi emploie.

TSHOU HI. - Le second trait hexaire se trouve en présence d'un moment où il convient d'augmenter ce qui est inférieur ; vide à l'intérieur, il est placé dans un rang inférieur, aussi son image symbolique et son sens divinatoire sont les mêmes que dans le cas du cinquième trait hexaire du koua souen. Toutefois, le trait et la situation sont tous deux négatifs ${ }^{1}$ c'est pourquoi la formule mentionne la pureté éternelle comme avertissement. Puisqu'il occupe un rang inférieur et reçoit un avantage du supérieur, il est donc encore considéré comme ayant un sens augural favorable dans la divination au sujet des sacrifices au ciel.

752.

Parfois il augmente; il vient de l'extérieur.

TSHENG TSE. - Puisqu'il est juste et droit, vide à l'intérieur, qu’il peut recevoir le bien de l'univers et le conserver avec une fermeté inébranlable, il rencontre des occasions d'augmentation, et la foule des hommes vient de l'extérieur pour son avantage. On a dit

Le texte porte «il vient de l'extérieur»; comment cela ne se rapporterait-il pas au cinquième trait ? Réponse : lorsqu'il s'agit de justice, droiture et vide intérieur comme cela est le cas pour le second trait, qui donc, dans l'univers, ne voudrait l'augmenter, c'est -à-dire, agir pour son avantage ? Le cinquième trait lui correspond directement avec sympathie, donc il est certainement compris dans ceux qui en retirent l'avantage.

\footnotetext{
${ }^{1}$ Trait brisé, rang pair.
} 
TSHOU HI. - Parfois, expression qui indique l'idée de multiplicité sans détermination spéciale. 


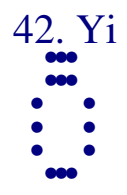

753.

Troisième trait hexaire : augmenter en employant des choses mauvaises; pas de culpabilité ; avoir, confiance, agir avec justice ; avertir le dignitaire revêtu du titre de kong; employer l'insigne secret.

TSHENG TSE. - Le troisième trait occupe le rang supérieur dans la substance du koua simple inférieur ; c'est celui qui occupe un rang au-dessus du peuple, par exemple celui qui fait observer les prescriptions civiles. Il occupe un rang positif et sympathise avec la dureté énergique ; il est placé au sommet du koua qui indique le mouvement ; c'est celui qui, placé au-dessus du peuple, décide avec énergie ce qui doit être fait pour en retirer avantage. Décidant ce qui constitue l'avantage et l'augmentation, même en em ployant des choses néfastes et mauvaises, il sera sans culpabilité. L'expression chose mauvaise désigne des calamités, des difficultés, des choses peu ordinaires ${ }^{1}$. Le troisième trait occupe la position immédiatement inférieure au koua supérieur; étant dans un rang inférieur, il doit en référer au supérieur en employant les formes respectueuses ; comment s'arrogerait-il de lui-même l'autorité de faire ce qui doit constituer l'avantage ? Ce n'est que dans le cas de choses calamiteuses et extraordinaires qu'il pourra apprécier ce qui convient pour répondre à des nécessités imprévues et urgentes; alors, audacieux au mépris de sa propre personne, fort pour protéger le peuple, il sera donc sans culpabilité. Si l'inférieur s'attribue lui -même l'autorité, le supérieur doit en concevoir de la méfiance et des soupçons; bien qu'en présence de périls et de malheurs, ce n'est jamais que par le devoir qu'il doit se laisser guider dans son action, mais il doit être sincère et de bonne foi, et ce qu'il fait doit être conforme à la voie rationnelle de la justice; dans ces conditions sa bonne foi pénétrera le supérieur qui, à son tour, aura confiance en lui. Il ne doit absolument pas agir de sa propre autorité sans que ce ne soit avec le plus sincère amour du peuple et pour servir le supérieur. Bien que mû par la sincérité, si ses actions n'étaient pas conformes à la justice, il ne devrait encore pas se permettre d'agir. Linsigne secret est un objet qui sert d'emblème de sincérité et de signe visible de foi ${ }^{2}$. Le Li king dit : Les hauts dignitaires sont munis d'un insigne de commandement qui leur sert à faire reconnaître la légitimité de leur autorité. Toutes les fois qu'il s'agit de sacrifices, de réceptions et d'audiences à la cour, on emploie des insignes en

\footnotetext{
${ }^{1}$ Troubles, révolutions.

${ }^{2}$ Un objet tel qu'une petite plaque d'ivoire coupée en deux parties qui consti tuent un signe de reconnaissance. L’idée remarquable est celle-ci : on considère les deux moitiés d'un même tout divisé en deux parties égales et par l'une on reconnaît l'autre.
} 
pierres fines (jade) qui servent de signes de reconnaissance. Animé de sincérité et guidé par la bonne foi, possédant d'ailleurs la voie rationnelle de la justice, il pourra faire en sorte d'inspirer de la confiance au supérieur, c'est donc encore «avertir le dignitaire revêtu du titre de kong et employer l'insigne secret »; la confiance peut pénétrer le supérieur. Étant dans l'infériorité et suivant une voie qui comporte la nécessité d'agir, il faut nécessairement avoir de la bonne foi et agir avec justice. D’un autre côté, le troisième trait est négatif et il est dénué de justice ${ }^{1}$, c'est à cause de cela que ce sens est mis en lumière. On a dit: Le troisième trait est malléable et négatif ; comment peut-on donc, au contraire, le considérer comme décidant avec autorité et énergie et s'attribuant la direction des affaires ? Réponse : bien que le caractère naturel du troisième trait soit essentiellement la négativité, cependant la considération qu’il occupe un rang positif montre qu'il se place lui -même avec énergie ; il correspond aussi à un trait énergique, donc ses tendances le portent à la dureté énergique ; il occupe le rang extrême dans le koua simple qui exprime le mouvement, ce qui marque la décision dans l'action. Dans ces conditions, pratiquant la voie de l'augmentation, com ment pourrait-il être sans énergie et décision ? Dans le Yi king, c'est ce qui prédomine qui constitue le sens, aussi il n'est pas question de son propre caractère de négativité.

TSHOU HI. - Le troisième trait hexaire est malléable et négatif, sans justice ni droiture; c'est celui qui ne doit pas recevoir d'augmentation (d'avantages). Toutefois, il est en présence d'un moment d'augmentation de l'inférieur ; il occupe le rang supérieur parmi les inférieurs, aussi il représente quelqu'un qui avantage l'inférieur par le moyen de choses malheureuses. En effet, il avertit, prévient, ébranle et met en mouvement; c'est ainsi quil l'avantage. Si celui qui consulte le sort est dans ces conditions, il pourra ensuite être sans culpabilité. De plus, la formule avertit encore qu'il doit avoir de la bonne foi, agir avec justice et avertir le dignitaire revêtu du titre de kong en employant l'insigne secret ; c'est par l'emploi de l'insigne secret qu'il montre sa bonne foi et inspire la confiance.

754.

Augmenter en employant des choses mauvaises ${ }^{2}$; il y en a certainement.

TSHENG TSE. — Le troisième trait est le seul qui dans le koua yi puisse employer des choses mauvaises ; c'est parce qu'il y en a certainement. Cela signifie qu'il est absolu et ferme et qu'il s'attribue l'autorité dans la direction

\footnotetext{
${ }^{1}$ Il n'est pas au rang du milieu d'un koua simple.

2 On pourrait lire : augmenter l'emploi des choses mauvaises.
} 
des affaires. Occupant un rang inférieur, il doit rendre compte au supérieur, recevoir ses ordres et les exécuter en lui témoignant son respect; ici, s’il s'attribue la direction des affaires, c'e st uniquement pour remédier aux maux dont le peuple souffre, et pour répondre aux nécessités imprévues d'un moment de péril soudain et urgent; dans de telles conditions, son action est licite. C'est là répondre aux nécessités d'une circonstance qui ne pe ut être évitée, de sorte qu'il est sans culpabilité. Si c'était dans un temps ordinaire, il ne devrait pas agir ainsi.

TSHOU HI. - Augmenter en employant des choses mauvaises; il faut que son coar souffre, qu'il apprécie et mesure le mal et qu'il montre d e la fermeté. 


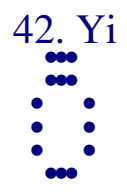

755.

Quatrième trait hexaire : agir avec justice ; avertir le dignitaire revêtu du titre de kong, suivre ; avantage à employer l'action conforme et changer le royaume ${ }^{1}$.

TSHENG TSE. - Le quatrième trait se trouve en face d'un moment d'augmentation ; il est placé près de la situation du prince et il occupe son rang avec droiture. Employant la douceur et se plaisant à aider le supérieur, d'ailleurs correspondant sympathiquement en bas avec la dureté énergique positive du premier trait, il peut ainsi être utile (augmenter) au supérieur. Seulement, il est placé sans se préoccuper de la justice et celui avec qui il sympathise est aussi sans justice; ces conditions montrent qu'il manque de cette qualité, aussi le texte exprime que si, en agissant, il suit la voie rationnelle de la justice, il pourra être utile au prince placé au-dessus de lui : il avertit le supérieur, lui inspire confiance et en est suivi. Faisant partie de la substance de la satisfaction et de la douceur ${ }^{2}$, il manque de décision et de précision, aussi il a avantage à employer l'action conforme et à changer de royaume. Employer l'action conforme, c'est s'appuyer sur le supérieur ; changer de royaume, c'est suiv re passivement l'inférieur et se mettre en mouvement sous son impulsion. En haut il s'appuie sur le prince énergique et juste et il en résulte l'augmentation (l'avantage) ; en bas, il suit passivement les aptitudes de l'énergie positive pour accomplir ses actions ; donc l'avantage consiste à agir ainsi. Depuis l'antiquité, qu'il s'agisse de royaumes ou de districts, quand le peuple n'a pas pu jouir de la paix là où il habitait, il a changé de royaume : cela exprime qu'il imite ce qui est inférieur et se met en mouvement.

TSHOU HI. - Le troisième et le quatrième traits sont tous deux dépourvus de justice, aussi tous deux sont le sujet d'avertissements sur la nécessité d'agir avec justice. Ici, il s'agit de la volonté d'avantager l’inférieur et de se conformer à la justice en agissant; de cette façon, avertissant le dignitaire revêtu du titre de kong, il s'en verra suivi. Le commentaire traditionnel dit: «Le changement de Tsheou vers l'est était motivé par les États de Tsin et de Tshing »; c'est qu'en effe t, dans l'antiquité, plusieurs princes ont changé d'États pour le bien de leurs peuples. Pour exister, il faut qu'il y ait les moyens d'existen ce. Ce trait comporte aussi un présage heureux lorsque l'on consulte le sort au sujet d'un changement de pays.

\footnotetext{
${ }^{1}$ Sens incompréhensible sans les commentaires.

${ }^{2}$ Koua simple souen.
} 
756.

Avertir le dignitaire revêtu du titre de kong, suivre; afin d'augmenter ses tendances.

TSHENG TSE. - La formule du trait dit seulement qu'en agissant conformément à la justice, il avertira le dignitaire revêtu du titre de kong et réussira à en être suivi. Le commentaire symbolique éclaire de nouveau cette même idée : avertir le dignitaire revêtu du titre de kong et réussir à s'en faire écouter, c'est-à-dire l'avertir pour le bien des tendances de l'univers. Du moment où ses tendances le portent à agir pour l'avantage de l'univers, le supérieur doit avoir confiance en lui et écouter ses avis. Celui qui sert le prince ne déplore pas de ne pas être écouté par le supérieur, il déplore le manque de sincérité de ses propres tendances, c'est-à-dire son incapacité à convaincre le prince. 


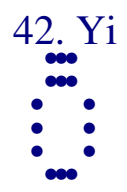

757.

Cinquième trait nonaire : avoir confiance, caur bienveillant; ne pas demander ; grandeur du présage heureux; avoir foi et bienveillance au sujet de mes vertus.

TSHENG TSE. - Le cinquième trait, doué de la dureté énergique positive, de la justice et de la droiture, occupe le rang prééminent. De plus, il jouit de la sympathie, de la justice et de la droiture du second trait hexaire qui agit pour son bien; dans ces conditions, qu'est-ce qui pourrait être sans avantage? Représentant la positivité réelle, et placé au milieu d'un koua simple, il a l'image symbolique de posséder la bonne foi et d'inspirer la confiance. Possédant les vertus du cinquième trait nonaire, ses aptitudes, sa situation, et le sentiment de la justice étant au plus haut point sincère dans la bienveillance qu'il déploie pour le bien des êtres, il est inutile de demander s’il exprime l'extrême excellen ce et la grandeur du présage heureux, car cela est évident. Voilà pourquoi la formule dit : Ne pas demander la grandeur du présage heureux. Le prince occupe la situation qui lui permet de mettre ses desseins à exécution, il possède la puissance qui le met à même de les réaliser; s'il pous se la sincérité à son extrême limite dans son désir de faire le bien (d'augmenter) de l'univers, l'univers reçoit de lui un bonheur complet ; il est donc inutile de mentionner la grandeur du présage heureux. Avoir foi et bienveillance au sujet de mes vertus; si le prince est extrêmement sincère dans son désir de faire le bien de l'uni vers, il n'est personne dans l'univers qui n'éprouve pour lui l'amour le plus sincère, de sorte que l'influence des vertus du prince s'étend comme un bienfait.

TSHOU HI. - Le supérieur est de bonne foi dans sa bienveillance envers les inférieurs, de sorte que les inférieurs, à leur tour, sont de bonne foi dans leur affection bienveillante pour le supérieur. Il n'est pas nécessaire d'interroger au sujet de la grandeur du présa ge heureux, elle est évidente.

758.

Avoir foi, caur bienveillant; ne pas le demander. Affectionner mes vertus ; réussir largement dans ses tendances. 
TSHENG TSE. — Le prince possède une parfaite sincérité dans son désir bienveillant de faire le bonheur de l'univers ; il est inutile de mentionner la parfaite grandeur du présage heureux, aussi la formule dit: « $\mathrm{Ne}$ pas le demander. » L'univers étant extrêmement sin cère dans la reconnaissance de mes vertus dont il reconnaît l'action bienveillante, c'est donc que leur voie rationnelle prévaut largement : les tendances du prince prévalent. 


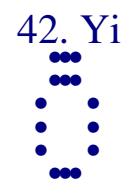

759.

Trait supérieur nonaire : ne pas augmenter; parfois frapper ; affermir le caur sans permanence ; présage malheureux.

TSHENG TSE. - Le trait supérieur occupe un rang qui ne comporte aucune situation définie; c'est celui qui n'agit pas pour l'avantage de l'homme. Employant la dureté éner gique et se plaçant au rang le plus élevé du koua $y i$, c'est celui qui réclame un avantage excessif pour lui-même et sympathise avec la négativité et ce n'est pas par le bien qu'il ch erche à obtenir son propre avantage. L'avantage, c'est ce que tous les hommes recher chent également, mais le désir exclusif de l'avantage personnel constitue un grand mal. Les désirs étant extrêmes, il en est aveuglé et il oublie le devoir et la raison ; recherchant cet avantage avec une ardeur excessive, sa passion le porte à la violence et à l'usurpation et il en résultera de l'inimitié et des ressentiments. Aussi Fou Tse dit: «La passion du luxe, non contenue, engendre les ressentiments »; c'est ce que Mang Tse exprime en disant que si on place l'avantage au-dessus de tout sentiment on ne pourra pas se satisfaire sans violence; ce sont de profonds avertissements donnés par le saint et le sage. Le trait est nonaire, il emploie la dureté énergique et recherche son propre avantage avec une passion extrême; c'est là ce que la foule des hommes déteste et repousse, de sorte qu'il repré sente celui que l'on n'avantage pas et que parfois on combat en le repoussant. Affermir le cour sans permanence, présage malheureux; l'homme saint avertit les hommes qu'ils ne doivent pas se laisser aller à apprécier exclusivement ce qui constitue un avantage ; il dit : Manquer de mesure et de retenue permanente à ce point, c'est une voie rationnelle de malheur, et c'est ce dont on doit s'empresser de se corriger.

TSHOU HI. - Puisque ce trait occupe le rang extrême dans le koua $y i$ c'est qu'il recherche sans cesse son propre avantage, aussi, on ne l'avantage pas et parfois on le frappe. Les expressions «affermir le cour, pas de permanence », sont des avertissements.

760.

Ne pas l'avantager, s'excuser avec partialité ; parfois le frapper, venir de l'extérieur. 
TSHENG TSE. - La raison d'être des choses est ce quill y a de plus immuable dans l'uni vers; le bien-être, ou avantage, est ce que tous les hommes désirent également. Si le coar est dégagé de tout sentiment d'égoïsme et si la droite raison est respectée, l'avantage sera commun à la foule des hommes. Lorsque quelqu'un n'usurpe pas sur les droits d'autrui, chacun veut l'aider et s'allier à lui ; mais l'acharnement dans la recherche de l'avantage conduit à l'aveuglement et à l'oblitération des sentiments d'équité ; on en vient alors à rechercher son propre avantage au détriment des hommes, de sorte que ceux-ci repoussent le mal par la force; ils ne veulent plus avantager et frappent. Ce passage du texte, «ne

pas l'avantager », est une expression qui nie qu’il y ait partialité de sentiment en faveur du trait considéré. S'il n'y a point de partialité et d'égoïsme dans s a conduite, c'est quỉl sui vra la voie rationnelle du désintéressement et de la générosité et alors les hommes agiront aussi pour son propre avantage ; pourquoi le frapperait-on ? Du moment où ce trait représente celui qui demande aux hommes de contribuer à son propre avantage et où il pousse à l'excès cette recherche de l'avantage personnel, il en résulte que tous les hommes le détestent et veulent le combattre et le repousser, de sorte que ceux qui le frappent Viennent de l'extérieur. Lorsqu'un homme $\mathrm{f}$ ait le bien, de mille lis à la ronde chacun sympathise avec lui ; le second trait hexaire, doué de la justice et de la droiture, se dégage de toute prévention égoïste, aussi ceux qui agissent pour son avantage surviennent de l'exté rieur ; sil ne faisait pas le bien, à mille lis à la ronde chacun lui résisterait. Le trait supérieur nonaire recherche l'excès dans l'avantage ; ceux qui le frappent surviennent de l'extérieur. Les formules annexées disent : "Lhomme doué repose son corps et ensuite il se meut; il calme son cour et parle ensuite ; il choisit ses alliances et ensuite il demande assistance. » L'homme doué qui s'applique à ces trois préceptes conserve sa dignité intac te. Mais si quelqu'un s'agite au milieu du péril, le peuple ne s'allie pas à lui ; sîl parle sous l'impulsion de la peur, le peuple ne lui répond pas sympathiquement ; s'il demande avant de s'être créé des alliances, personne ne lui donne et alors ceux qui lui veulent du mal se montrent et l'attaquent. Le Yi king dit: ne pas augmenter; parfois frapper; affermir le coar sans permanence, présage malheureux ; l'homme saint dit que l'action de se mou voir ou de demander doit toujours avoir lieu en suivant la voie rationnelle et que c'est en cela que consiste l'extrême excellence ; autrement on ne recueillera que des blessures et le présage sera malheureux.

TSHOU HI. - C'est celui qu'on n'avantage pas ; cela est encore dit au sujet de ceux qui arguent de prétextes pour rechercher leur propre bien au détriment d'autrui ; en examinant on reconnaît encore que quelqu'un les frappera. 
[] 


\section{Kouae}

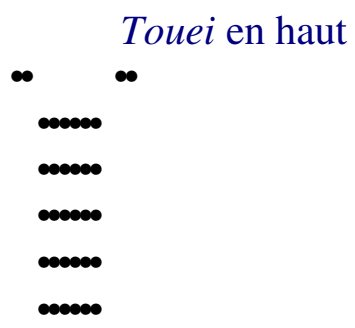

Khien en bas

761. Kouae ; agir ouvertement à la cour du roi ; avec bonne foi, avertir qu'il y a lieu de craindre ; avertir son propre district; pas d'avantage à poursuivre les ennemis ; avantage dans ce qu'il y a à entre prendre.

765. Premier trait nonaire : vigueur dans l'action d'avancer l'orteil ; avancer sans dominer ; cela constitue la culpabilité.

$*$

767. Deuxième trait nonaire : s'inquiéter et avertir ; le soir et la nuit il y a des soldats ; ne pas s'alarmer.

$*$

769. Troisième trait nonaire : énergie sur le front; il y a présage malheureux. L’homme doué est plein de détermination; il agit seul et rencontre la pluie; sil est mouillé il est mécontent; pas de culpabilité.

$*$

771. Quatrième trait nonaire : les fesses sans peau; les actions sont indécises ; conduire des moutons, dissipation des regrets ; entendre parler et ne pas avoir foi.

$*$

773. Cinquième trait nonaire : l'herbe hien lou est très cassante ; agir avec justice, pas de culpabilité.

$*$

775. Trait supérieur hexaire : pas d'avertissement ; finalement il y a présage malheureux.

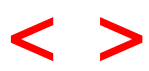




\section{Kouae.}

TSHENG TSE. - Kouae. «L'Ordre des koua » dit : «L'augmentation qui ne cesse pas doit nécessairement s'arrêter brusquement, c'est pourquoi le koua yi est suivi du koua kouae ; kouae, interrompre, séparer brusquement, décider, déterminer. » Lorsque l'augmentation est poussée à son extrême limite, elle doit nécessairement s'arrêter brusque ment, puis rester stationnaire. La raison d'être naturelle des choses ne comporte pas une augmentation constante et indéfinie; augmenter sans cesse, cesser c'est s'arrêter ; c'est pourquoi le koua kouae suit immédiatement le koua yi. Il est constitué par le koua simple touei en haut, et par le koua simple khien, en bas. En en parlant d'après les substances de ces deux koua simples, le marais (touei) est une accumulation d'eau ; ici le marais monte jusque dans le lieu le plus élevé, ce qui donne l'image symbolique du flot qui déborde et envahit. En en parlant d'après les traits, cinq positivités se trouvent en bas ; la positivité monte et grandit, elle est sur le point d'atteindre au maximum ; une seule négativité, occupe le rang supérieur, elle diminue et est sur le point d'être complètement dissoute. La foule des positivités avance en montant et chasse définitivement l'unique négativité, ce qui constitue l'extinction définitive exprimée par le koua kouae. Ce mot comporte le sens de déterminer avec énergie ; la foule des positivités avance et chasse définitivement l'unique négativité. La voie de l'homme doué grandit, l'homme inférieur disparaît et s'efface, il est au moment de disparaître complètement. 


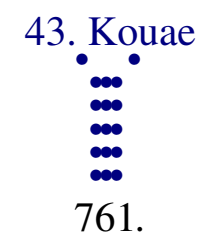

Kouae ; agir ouvertement à la cour du roi ; avec bonne foi, avertir qu' il y a lieu de craindre ; avertir son propre district ; pas d'avantage à poursuivre les ennemis ; avantage dans ce qu'il y a à entre prendre.

TSHENG TSE. - Dans le moment où l'homme inférieur commence à être achevé ${ }^{1}$, la voie rationnelle de l'homme doué ne l'emporte pas encore ; il est impossible qu'il puisse ouvertement chasser l'homme inférieur et mettre fin à son action par la voie rationnelle de la droiture, aussi il se contient et se cache en attendant le moment et médite lentement sur la voie à suivre pour le supprimer. Actuellement, du moment où l'homme inférieur décline et est amoindri, la voie de l'homme doué s'accomplit ; il doit agir ouvertement à la cour afin que les hommes sachent clairement ce qui est le bien et ce qui est le mal ; c'est pour cela que la formule dit : agir ouvertement à la cour du roi. La bonne foi, c'est la sincé rité dans l'intention, la foi intérieure. Le septième caractère du texte, crier, proclamer, est une expression qui comporte aussi le sens de commander, donner des ordres à la multitude. Bien que la voie rationnelle de l'homme doué grandisse et se complète, cependant, il n'ose pas cesser de se tenir en garde et prêt à tout ; c'est pour cela qu'il est extrêmement sincère dans les instructions qu'il donne à la foule, afin qu'elle sache qu'il y a encore des motifs plausibles de crainte et de périls. Même avec cette extrême perfection de sa propre voie, avec l'extrême décadence de l'autre ${ }^{2}$, s’il agissait avec légèreté et sans précaution, il éprouverait le regret de n'avoir point aperçu le danger. Il y a donc encore une raison d'être du péril, son cœur doit être en garde, et alors il ne surviendra aucun malheur. L’idée de l'homme saint en faisant cet avertissement est très profonde; en dirigeant et en gouvernant l'homme inférieur, l'homme doué doit se servir de sa propre voie rationnelle qui le porte au bien pour l'emporter sur ce qui n'est pas le bien et y remédier en supprimant le mal. Aussi, pour supprimer et exterminer le désordre, l'homme saint doit d'abord se réformer lui -même ; tel est le cas de Souen promulguant les «instructions sur la vertu ${ }^{3}$. Le district, son propre district ; avertir son propre district, c'est se réformer d'abord soi -même, avant de réformer les autres. Puisque la totalité des positivités détruit l'unique négativité, la force de l'action est certainement plus que suffisante, mais cependant la dureté énergique ne doit pas être portée à sa plus extrême limite, ce qui serait un excès; l'excès serait par exemple ce qui constitue le

\footnotetext{
1 À être au pouvoir.

2 De l'homme inférieur.

${ }^{3}$ Shou king.
} 
brigandage dans le cas du trait supérieur du koua mong ${ }^{1}$. L'emploi de la guerre et de la force des armes est une action violente; pas d'avantage à poursuivre les ennemis signifie qu'il ne convient pas d'employer continuelle ment la violence. Le quinzième caractère du texte signifie suivre, suivre l'ennemi, c'est -à-dire affectionner les procédés violents. Avantage dans ce qu'il y a à entreprendre ; bien que la positivité tende à se compléter, elle n'est pas encore arrivée à son maximum, dans le trait supérieur. Bien que la négativité soit amoindrie, elle n'est pas encore totalement sup primée ; c'est donc que l'homme inférieur subsiste encore et que la voie rationnelle de l'homme doué n'est pas arrivée à la perfection. C'est à cause de cela qu'il convient d'avancer et d'entreprendre : ne pas attacher un prix exclusif à l'énergie des procédés violents et faire prévaloir sa voie rationnelle, c'est ce qui constitue le bien dans la détermination ${ }^{2}$.

TSHOU HI. - Kouae, trancher, décider, mettre un terme. La positivité met un terme à la négativité. C'est le koua du troisième mois. Suppression d'une négativité par cinq positivités; elles la suppriment. Toutefois en l'exterminan $t$, en prononçant sa sentence, il faut énoncer exactement le nom de la faute qu'elle a commise ${ }^{3}$ et épuiser la sincérité dans la proclamation qui en est faite à la foule. Même dans une action commune, en s'alliant et en réunissant toutes les forces, il peut encore y avoir danger et appréhension d'un péril éventuel; il ne faut pas s'endormir dans la sécurité. De plus, il faut encore commencer par se réformer et se corriger et ne pas attribuer une valeur prépondérante à l'emploi des moyens violents, alors il y aura avantage à entreprendre. Ce sont autant de formules d'avertissement.

762.

Le commentaire traditionnel de la formule déterminative dit: Kouae, trancher, décider, mettre fin à quelque chose; la dureté énergique coupe court à la douceur malléable et la supprime; activité et satisfaction; détermination et harmonie.

TSHENG TSE. - Le koua kouae comporte le sens de détermination nettement tranchée. Cinq positivités suppriment la négativité unique, représentée par la positivité qui occupe le rang supérieur. Activité et satisfaction, détermination et harmonie ; il s'agit des apti tudes exprimées par le koua, déduites de la substance des deux koua simples. En bas activité et en haut satisfaction, c'est posséder l'activité et savoir se contenter. Mettre fin à

\footnotetext{
$1 \S \mathrm{n}^{\circ} 122$.

2 Dans le koua kouae.

${ }^{3}$ Allusion aux prescriptions du code en matière pénale.
} 
quelque chose et pouvoir jouir de l'harmonie, c'est l'extrême excellence dans la détermination. La satisfaction exprimée par le koua simple touei constitue l'harmonie.

TSHOU HI. - Explication du sens du nom du koua et exclamation admirative sur les vertus qu'il comporte.

763.

Manifester à la cour du roi ; la négativité est montée sur cinq traits énergiques; avec foi avertir qu'il y a lieu d'appréhender, le péril est éclatant ${ }^{1}$. Avertir son propre district; pas d'avantage à poursuivre les ennemis; ce qu’il estime est à sa fin ; avantage dans ce qu’il y a à entreprendre ; l'énergie grandit, c'est la fin.

TSHENG TSE. - Bien que la malléabilité négative soit sur le point de s'évanouir, cepen dant, elle occupe une position supérieure au-dessus des cinq traits énergiques, ce qui comporte encore l'image symbolique de fouler aux pieds et de mépriser. La négativité foulant la positivité sous ses pieds, c'est ce qu'il y a de plus contraire à la raison d'être des choses ; du moment où la force naturelle inhérente à l'homme doué est suffisante pour la refouler, celui ci doit montrer clairement devant l'assemblée de la cour la faute qui résul te de cette négativité, afin que la foule sache distinguer le bien et le mal. Épuiser la sincérité et la conviction pour instruire la foule et savoir ce qui est à craindre et constitue un péril, telle est la voie rationnelle de l'homme doué ; en ceci il n'y a aucun doute et la clar té est grande. Mais il convient de se modérer soimême et il ne faut pas apprécier exclusivement les moyens énergiques et violents. Poursuivre l'ennemi, exprime que ce qu'il pré fère, c'est la destruction finale. Dans un moment qui comporte la détermination, ce qui est préférable est l'énergie de l'audace ; bien que la dureté énergique de la positivité soit sur le point d'être complète et prédominante, cependant, sa croissance n'est pas encore arrivée à sa fin ; il existe encore une négativité qu'il faut aussi détruire et chasser, et alors seulement la voie de l'homme doué sera absolument et uniquement prédominante, sans aucun inconvénient ; telle sera la fin de la croissance de l'énergie active.

TSHOU HI. - Ceci est une explication de la formule attachée au koua. La malléabilité négative foule aux pieds cinq traits qui expriment l'énergie ; en en parlant au point de vue de la substance constitutive du koua, cela veut dire qu'un homme inférieur est placé au-dessus. de la foule des hommes doués ; c'est en cela que consiste la faute. Les mots

\footnotetext{
${ }^{1}$ C'est la traduction littérale et elle montre bien l'incohérence du sens admis.
} 
«L'énergie grandit, c'est la fin » expriment que, après une seule transformation (d'un trait), le koua arrive à la pureté absolue de l'énergie active ${ }^{1}$.

764.

Le commentaire traditionnel de la formule symbolique dit: Le marais monte au ciel, détermination; l'homme doué, pour étendre le bonheur jusqu'au bas ${ }^{2}$, s'en tient à la vertu et sera circonspect.

TSHENG TSE. - Marais, amas d'eaux ; cependant, il monte se placer jusqu'au point le plus élevé du ciel, de sorte que l'image symbolique est l'extinction définitive exprimée par le mot kouae. L'homme doué con temple l'image symbolique du marais qui limite ce qui est élevé et submerge ce qui est en bas et il en conclut qu'il a le devoir d'étendre son action bienfaisante jusqu'à ce qu'elle atteigne les inférieurs. Cela exprime que la rosée bienfaisante de ses bienfaits s'étend jusqu'aux inférieurs. Contemplant l'image symbolique du flot qui atteint, limite et submerge, il appliquera sa circonspection à se maintenir dans la vertu. Se maintenir dans la vertu, c'est se contenter de sa propre vertu. Être circonspect, veut dire se limiter à la circonspection, c'est encore comme si on disait qu'il éta blit des limites protectrices et préservatrices; s’il existe des digues préservatrices, le débordement de l'inondation sera évité. Wang Pi explique ces mots par l'expres sion « circonspection intelligente », ce qui est encore intelligible. La formule ne dit pas que le marais est placé au-dessus du ciel, mais «qu'il monte vers le ciel »; avec les mots «monter au ciel», l'idée exprimée comporte linquiétude causée par la présence du flot montant ; lorsqu'on dit que le marais est au-dessus du ciel, c'est une expression qui marque l'idée du repos qui suit un fait accompli.

TSHOU HI. - Le marais monte vers le ciel ; puissance de l'inondation envahissante ; bienfaits atteignant les inférieurs, idée exprimant l'envahissement définitif qui atteint partout. Les mots « se maintenir dans la vertu, alors circonspection », ne sont pas encore bien expliqués.

${ }^{1}$ C'est-à-dire au koua.

2 Jusqu'à 1 'inférieur, ou jusqu'au -dessous, etc. 


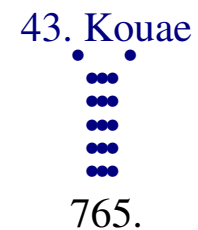

Premier trait nonaire : vigueur dans l'action d'avancer l'orteil ; avancer sans dominer ; cela constitue la culpabilité.

TSHENG TSE. - Le trait est nonaire et positif, il fait partie de la substance de l'activité ${ }^{1}$. Il représente quelque chose énergique et active faite pour la supériorité et qui se trouve en bas pendant un moment qui comporte la détermination ; c'est celui qui met de l'éner gie dans l'action de se porter en avant. Avancer l'orteil, c'est -à-dire progresser en avant; cela exprime l'énergie et la décision de l'homme dans ses actions. S'il agit d'une façon convenable, sa décision sera juste et exacte ; s'il avance sans opportunité, ce sera un excès dans la décision. Aussi, s'il avance sans dominer, cela constituera sa culpabilité. Il est question d'agir dans un moment qui comporte la décision ; agir, c'est montrer de la décision, aussi il est question de dominer et de porter la responsabilité de sa décision. Ce trait est nonaire, il occupe le premier rang et il est énergique dans son mouvement en avant; il met de la précipitation à avancer hâtivement, et c'est à cause de cela que la formule contient un avertissement au sujet de la nécessité de savoir se dominer. Bien que la négativité soit sur le point d'être épuisée, à cause de la précipitation de son propre mouvement, il doit naturellement être coupable de ne pas savoir se dominer et de ne pas tenir compte d'autre chose que de lui -même.

TSHOU HI. - Le cinquième caractère, « devant », a la même valeur que le caractère tsin, «avancer». En présence d'un moment de décision et de détermination, il occupe le rang inférieur, son autorité est considérable, ce qui donne ce sens symbolique et ce sens divinatoire.

766.

Ne pas dormir et agir ; culpabilité.

TSHENG TSE. — L'action de l'homme doit être m esurée ; si le fait doit avoir lieu, il faut s'y décider, et alors il n'y a point de culpabilité. Si la raison d'être des choses fait qu'il est impossible de dominer les circonstances et si

\footnotetext{
${ }^{1}$ Koua simple Khien.
} 
cependant l'homme agit, la culpabilité, l'erreur, est évidente. To us ceux qui sont coupables pour avoir agi, le sont toujours par excès dans leur décision et leur détermination. 


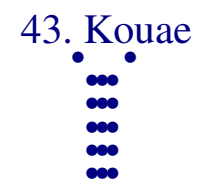

767.

Deuxième trait nonaire : s'inquiéter et avertir ; le soir et la nuit il y a des soldats ; ne pas s'alarmer.

TSHENG TSE. — Le koua kouae marque le moment où la positivité met fin à la négativité, où l'homme doué supprime l'homme inférieur ; dans de telles circonstances, il ne faut pas manquer de se tenir en garde et d'être prêt à toute éventualité. Au moment où l'accroissement de la positivité est sur le point d'atteindre son maximum, le second trait se place avec justice et occupe un rang qui comporte la douceur malléable ; cela ne constitue pas un excès de dureté énergique, et s’il peut se tenir sur ses gardes et prêt à toute éventualité, il se tiendra dans l'extrême excellence de la décision (kouae). Au-dedans défiance et inquiétude, et au-dehors avertissement grave et sincère ; bien que le soir et la nuit il y ait des soldats ennemis, il ne doit cependant pas s'alarmer.

TSHOU HI. - Le second trait nonaire se trouve en présence d'un moment de détermination et d'extinction ; il est dur et énergique, mais il occupe une situation qui comporte la douceur; enfin il possède la voie rationnelle de la justice. C'est pour cela qu'il peut s'inquiéter et prévoir, avertir et conseiller et se tenir lui-même prêt à tout, de sorte que bien que, dans l'obscurité de la nuit, il y ait des ennemis, il est encore possible qu'il n'en résulte aucu n mal.

768.

Il y a des ennemis, ne pas s'alarmer; posséder la voie rationnelle de la justice.

TSHENG TSE. - Dans l'obscurité de la nuit il y a des ennemis ; c'est ce qu'il y a de plus à craindre. Cependant s'il ne doit pas s'alarmer, c'est à cause de l'excellence de la position où il se place. Du moment où il possède la voie rationnelle de la justice et où, de plus, il est capable de circonspection et de prévoyance, où d'ailleurs il se tient sur ses gardes et est prêt à toute éventualité, qu'est-ce qui serait susceptible de l'alarmer ? Un trait nonaire occupant le second rang, bien quil possède la justice, ne se conforme cependant pas à la droiture : Comment cela pourrait-il constituer l'extrême excellence ? Réponse : la positivité met un terme à la négativité ; l'homme 
doué supprime l'homme inférieur et possède la justice ; comment serait-ce là manquer de droiture? Reconnaître le moment et en comprendre la conséquence, telle est la grande règle de l'étude du Yi king. 


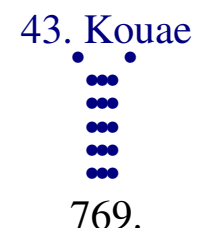

Troisième trait nonaire : énergie sur le front; il y a présage malheureux. L'homme doué est plein de détermination ; il agit seul et rencontre la pluie ; sil est mouillé il est mécontent ; pas de culpabilité.

TSHENG TSE. — La formule du trait est erronée. Hou Kong de Ngan ting transpose le texte et dit: "L'énergie au front est un présage de malheur ; il agit seul et rencontre la pluie, s'il est mouillé il est mécontent ; l'homme doué plein de détermination est sans culpabilité, mais cependant il n'est pas encore tranquille. » On doit lire : Énergie sur le front; il agit seul et rencontre la pluie; l'homme doué est plein de détermination, sîl est mouillé il est mécontent; pas de culpabilité. Le koua kouae marque le moment de la détermination prise en s'appuyant sur la dureté énergique et l'activité. Le troisième trait occupe le rang supérieur dans le koua simple inférieur ; de plus, il se place à l'extrême limite de la substance de l'activité : c'est celui qui porte l'énergie et la dureté dans la déci sion. Le cinquième caractère désigne l'os du front; c'est ce qui est en haut et pas encore au sommet. Le troisième trait occupe la partie supérieure de la substance du koua simple inférieur; bien qu'élevé, cependant il n'est pas encore parvenu à une situation très élevée ; plus haut, il y a le prince et cependant il assume de lui-même l'autorité et décide avec dureté et énergie : c'est là l'énergie dans le front, et c'est une voie rationnelle de malheur. Il agit seul et rencontre la pluie ; le troisième trait avec le sixième trait hexaire sont ceux qui se correspondent par la droiture; au moment où la foule des traits positifs se réunit pour exterminer l'unique négativité, en lui-même il éprouve une sympathie secrète et égoïste pour cette négativité, de sorte qu'il ne se réunit pas avec la foule et qu’il agit seul. Il en résulte que lui et le trait supérieur hexaire, l'un positif, l'autre négatif, s'accor dent ensemble, et c'est à cause de cela que le texte porte que la pluie survient. Dans le Yi king, toutes les fois qu'il est question de la pluie, cela veut toujours dire que la négativité et la positivité s'unissent. La voie rationnelle de l'homme doué se développe ; c'est le moment de chasser et de supprimer définitivement l'homme inférieur et, cependant, lui seul s'accorde avec cet homme inférieur: l'inconvenance de cette manière d'agir est évi dente, l'homme doué seul, dans un tel moment, pourra agir avec une extrême détermination, c'est -à-dire être énergique dans sa décision, mettre de l'énergie dans le parti qu'il adopte. Malgré sa sympathie privée et égoïste, il doit éloigner la négativité et la supprimer; s’il voit l'eau et la boue, il est mécontent, c'est-à-dire qu’il a l'apparence de détester ; étant dans ces conditions il ne commet pas de faute et il est sans culpabilité. Le troisième trait fait partie de la substance de l'activité et se place avec droiture ; il n'est 
pas absolument certain quil commet cette faute, mais ce sens est mis en avant pour servir à l'ensei gnement. Ce qui constitue l'erreur dans la formule du trait, c'est qu'elle contient les mots « rencontrer la pluie », avec les mots «s'il est mouillé », de sorte qu'on croit, par leur position, que le sens des uns se rapporte au sens des autres ${ }^{1}$.

TSHOU HI. - Front, os frontal. Le troisième trait est en présence d'un moment de décision, il emploie la dureté énergique et dépasse la justice ; c'est donc qu'il veut détrui re l'homme inférieur et que son énergie et sa dureté sont visibles sur les traits de son visage. Étant dans ces conditions, sa voie est naturellement marquée de présages malheureux. Mais, cependant, il est au milieu de la foule des traits positifs ; seul entre eux tous il est sympathique au trait supérieur hexaire; s'il peut me ttre une détermination absolue dans sa décision, ne pas se laisser retenir par son affection privée pour la négativité, alors, bien que d'accord avec le trait supérieur hexaire, il sera comme agissant seul, il rencontrera la pluie et en sera comme imprégné et mouillé, ce qui causera le mécontentement de l'homme doué. Toutefois, finalement il pourra nécessairement supprimer et chasser l'homme inférieur et ne sera pas coupable. Le cas de Van Khieou agissant envers Wang Tuen est analogue à ce qu'exprime le te xte.

770.

L'homme doué est plein de détermination ; finalement pas de culpabilité.

TSHENG TSE. — Retenu et entravé par des sentiments d'amitié égoïste à cause desquels il manque de décision, l'homme doué ne se rapproche et ne s'allie que d'après le dev oir ; il rompt lorsquil convient de rompre, de sorte que, finalement, il n'en viendra pas à être coupable.

\footnotetext{
${ }^{1}$ Le plus clair de tout ceci est que le commentateur avoue qu'il n'y comprend rien lui -même.
} 


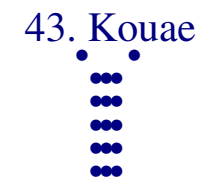

771.

Quatrième trait nonaire : les fesses sans peau ; les actions sont indécises; conduire des moutons, dissipation des regrets ; entendre parler et ne pas avoir foi.

TSHENG TSE. - Les fesses sans peau : ne pas être tranquille en repos. Agir avec indécision : avancer sans marcher droit devant soi ; les huitième et neuvième caractères du texte expriment la condition d'avancer avec difficulté. Le quatrième trait nonaire, avec sa positivité, occupe un rang négatif; il manque de décision et d'énergie ; il voudrait s'arrêter et la foule des traits positifs avance en rangs serrés et monte ; la force des choses fait qu'il ne peut rester en repos. C'est comme s'il avait les fesses blessées et ne pouvait rester tranquille, en repos. Il veut agir, mais comme il occupe un rang qui comporte la douceur malléable et la faiblesse, il manque de décision et d'énergie ; il est incapable d'avancer de force, aussi ses actions manquent de décision. Conduire des moutons, dissipation des regrets : les moutons sont des animaux qui vivent en troupeau; le mot conduire, du texte, a le sens de tirer et de pousser ; cela veut dire que, s'il peut se faire violence, tirer et pous ser afin de suivre l'action de la foule, il lui sera possible de dissiper ses regrets. Mais, puisqu'il occupe une position qui comporte la malléabilité, cela lui sera certainement impossible et bien quil entende de tels conseils (paroles), il ne pourra certainement pas les croire et en faire son profit. Or, commettre des fautes et pouvoir se corriger, entendre le bien et pouvoir l'imiter, se faire violence pour obéir au devoir, tout cela n'est possible qu'à celui qui est doué d'intelligence et d'énergie. Dans les autres koua, lorsqu'un trait nonaire occupe le quatrième rang, l'inconvénient de cette condition n'est jamais poussé à un tel point, mais lorsqu'il s'agit du koua kouae, occuper un rang négatif constitue un très grand mal.

TSHOU HI. - Employant la positivité et occupant un rang négatif, placé sans justice et sans droiture, il ne sera pas satisfait de la position qu'il occupe et, s’il agit, il n'avancera pas. S’il n'avance pas précipitamment avec la foule des traits positifs, sil se contente de la suivre par derrière, alors il lui sera possible de dissiper ses regrets. Toutefois, dans un moment qui comporte la décision, ses tendances le portent à avancer en montant et il ne le pourra certainement pas. Si celui qui consulte le sort entend parler et ajoute foi ${ }^{1}$, le présage malheureux sera écarté et remplacé par un présage heureux. Si celui

\footnotetext{
${ }^{1}$ Entend l'oracle et y ajoute foi.
} 
qui conduit des moutons se place devant eux, ils n'avanceront pas ; s’il les suit et les fait avancer, le troupeau pourra marcher.

772.

L'action est indécise, situation imméritée; entendre des paroles sans y ajouter foi, intelligence peu claire.

TSHENG TSE. - Un trait nonaire occupe un rang hexaire :situation imméritée, inconvenante. Puisqu'un trait positif occupe un rang qui comporte la malléabilité négative, il manque de décision et de détermination, de sorte qu'il ne peut avancer par force et que son action est indécise et incertaine. L'énergie entraîne l'intelligence (clarté brillante), mais placé dans un rang qui comporte la malléabilité négative, il change et s'altère ; il perd sa véritable nature : comment posséderait-il encore l'intelligence ? Aussi, il entend les paroles, mais il ne peut y ajouter foi, et cela précisément parce que son entendement manque de clarté. 


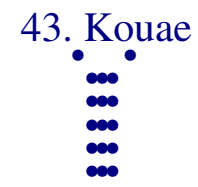

773.

Cinquième trait nonaire : l' herbe hien lou est très cassante ${ }^{1}$; agir avec justice, pas de culpabilité.

TSHENG TSE. - Bien que le cinquième trait possède l'énergie positive, la justice, la droiture, et qu'il occupe la situation prééminente, cependant il est extrêmement rapproché du trait supérieur hexaire. Celui-ci fait partie de la substance du koua simple touei, qui exprime la satisfaction, et c'est la seule négativité de tout le koua kouae; il exprime ce qui est l'objet du rapprochement intime de la positivité. Le cinquième trait est celui de qui dépend exclusivement la suppression de la négativité ; or, au contraire, il se rapproche et se serre contre elle; la culpabilité est grande. Aussi, il faut absolument qu'il décide l'extinction de cette négativité et que cette détermination rappelle l'herbe hien lou ; alors sa vertu de justice dans l'action sera telle qu'il sera sans culpabilité. La justice dans l'action, c'est la voie rationnelle de la justice. L'herbe hien lou est ce qu'on appelle aujourd'hui hien dent de cheval ; c'est une herbe qu'il est extrêmement difficile de sécher et très sensible à l'humidité de l'air, molle et facile à rompre. Si le cinquième trait est comme l'herbe hien lou, bien que très sensible à l'effet de la négativité, à cause de la facilité qu'il aura à rompre cette influence et à prendre une décision, il sera sans aucune culpabilité au sujet de la pratique de la justice ; autrement, il perdra la justice et la droiture. Il y a un très grand nombre de choses sensibles à l'effet de la négativité, mais l'herbe hien lou est considérée comme facile à rompre et c'est à cause de cela que la formule emprunte son image symbolique.

TSHOU HI. - L'herbe hien lou est ce qu'on appelle aujourd'hui hien dent de cheval; c'est une plante très sensible à l'humidité. Le cinquième trai $t$ nonaire est en présence d'un moment de décision et d'extinction ; c'est de lui que cette décision et cette extinction dépendent et, cependant, il est très rapproché du trait supérieur hexaire : il est comme l'herbe hien lou. S'il se détermine et supprime la négativité et si, de plus, il ne commet pas d'excès de violence, il s'accorde à l'action de la justice et sera sans culpabili té. C'est un avertissement à celui qui consulte le sort qu'il doit se maintenir dans ces mêmes conditions.

${ }^{1}$ C'est la même expression traduite au $\mathrm{n}^{\circ} 769$, comme exprimant l'idée d'une très grande détermination. 
774.

Action de la justice, sans culpabilité ; justice pas encore éclatante.

TSHENG TSE. - La formule du koua dit: détermination dans la décision ${ }^{1}$; alors cela sera considéré comme exprimant l'absence de faute dans la pratique de la justice. La formule symbolique revient sur ce sens et le complète par les mots « justice pas encore éclatante $»^{2}$. Or, lorsque le cour de l'homme est droit et que son intention est sincère, c'est là le moment où il peut suivre jusqu'à son extrême limite la voie rationnelle de la justice et de la droiture et la rendre réellement brillante et éclatante. Le cour du cinquième trait est attiré par quelque chose et il tend à s'en rapprocher, comme le devoir exige qu'il rompe cette union, bien qu'allant vers l'extérieur ${ }^{3}$, il ne manque pas au devoir qui résulte de la justice et de la droiture, et il est possible quil soit sans culpabilité. Mais cependant, cela n'est pas encore considéré comme suffisant pour que la voie rationnelle de la justice soit grande et éclatante. En effet, le cour de chaque homme ressent quelque désir qui le porte à s'écarter de la voie rationnelle; dans ce passage Fou Tse montre une profonde connaissance des sentiments de l'homme.

TSHOU HI. - Le commentaire de Tsheng est insuffisant.

\footnotetext{
${ }^{1}$ Le redoublement d'un mot exprime, soit la fréquence, soit l'énergie de l'action.

${ }^{2}$ Littéralement : milieu pas encore éclatant.

${ }^{3}$ Bien qu'il se rapproche du trait supérieur.
} 


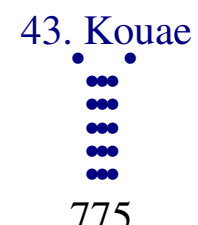

Trait supérieur hexaire : pas d'avertissement ; finalement il y a présage malheureux.

TSHENG TSE. — La croissance de la positivité est sur le point d'atteindre son maximum ; la décroissance de la négativité est sur le point d'être absolue ; une seule négativité occupe encore le rang final. C'est donc le moment où la foule des hommes doués l'emporte en supprimant et en écartant le péril extrême résultant du fait des hommes inférieurs. La force naturelle inhérente à chaque chose fait que cette extinction doit être complète et absolue, aussi la formule dit qu'il serait superflu d'avertir et d'inspirer la crainte, parce que, finalement, le malheur survient nécessairement.

TSHOU HI. - C'est le moment où un homme inférieur, d'un caractère malléable et négatif, occupe une position sans issue ; ceux de même genre que lui ont complètement disparu; il n'a personne qu'il puisse appeler à son secours : finalement, il doit tomber dans le malheur. Si celui qui consulte le sort possède les vertus de l'homme doué, le sens qui lui sera applicable sera exactement le contraire de celui-ci.

776.

Présage malheureux sans avertissement ; finalement cela ne peut durer.

TSHENG TSE. - La voie rationnelle de l'homme doué énergique et positif avance et grandit en se développant parfaitement; la voie rationnelle de l'homme inférieur étant à son extrême limite finale, elle s'évanouit et disparaît naturellement : comment pourrait-elle de nouveau grandir et durer ? Bien qu'il appelle et crie, il n'y a rien qui puisse remédier à sa situation, aussi la formule dit: finalement cela ne peut durer. Les anciens philosophes se basant sur ce fait que, dans certains koua, on trouve les expressions « avertir avec sincérité », «avertir ouvertement», ont voulu considérer les deux caractères du texte ${ }^{1}$ en lisant hao, commander, donner des avertissements, au lieu de lire haò, pleurer, se lamenter, crier, comme si le texte signifiait qu'il

\footnotetext{
${ }^{1} \mathrm{~N}^{\circ} 775$, troisième et quatrième caractères.
} 
est inutile d'avertir de nouveau ${ }^{1}$; cela n'est pas. Où serait l'impossibi lité que, dans un même texte, ce caractère fût employé deux fois avec le son hao et une fois avec le son haò ? Cependant tous ceux qui lisent le Yi king éprouvent la même incertitude ${ }^{2}$. On a dit : Dans son action sur l'univers, bien que l'homme saint éprouve une grande aversion, il ne doit jamais exterminer (le coupable) ; ici, comment serait-il possible qu'il commandât précisément de ne point avertir et qu'il dise que le mal est inévitable ? Réponse : Le koua kouae exprime le moment où la voie de l'homme inférieur disparaît et s'évanouit. Pour supprimer et éloigner la voie rationnelle des hommes inférieurs, comment serait-il indispensable de les exterminer tous ? En les amenant à se corriger et à se transformer, on obtient précisément la disparition totale de leur voie rationnelle ${ }^{3}$. Leur voie rationnelle ne subsiste plus, tel est leur seul malheur.

${ }^{1}$ C'est précisément le sens adopté dans la traduction, et c'est le plus naturel.

2 La traduction de tels commentaires prouve, mieux que l'affirmation d'un traducteur étranger, que depuis longtemps les Chinois eux-mêmes ont perdu le sens du Yi king.

${ }^{3}$ De leur action. 


\section{Keou}

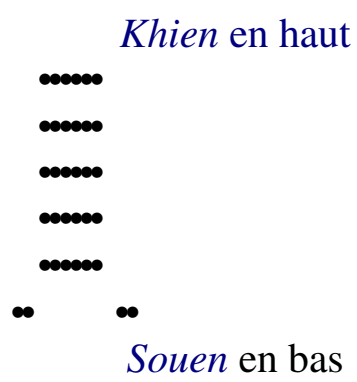

777. Keou, la jeune fille adulte; ne pas employer ; épouser une jeune fille.

784. Premier trait hexaire : retenu et lié à une barre de métal ; présage heureux de pureté ; avoir à entreprendre ; voir le présage malheureux; le porc maigre est porté à sautiller.

$*$

786. Deuxième trait nonaire : le sac contient du poisson ; pas de culpabilité ; pas davantage pour l'hôte.

$*$

788. Troisième trait nonaire : les fesses sans peau ; action indécise ; appréhension ; pas de grande culpabilité.

$*$

790. Quatrième trait nonaire : sac sans poissons ; le présage malheureux débute.

$*$

792. Cinquième trait nonaire : envelopper des courges avec les feuilles de l'arbre ki ; renfermer l'éclat ; il y a quelque chose tombant du ciel.

*

794. Trait supérieur nonaire : rencontrer les cornes ; appréhension, pas de culpabilité.

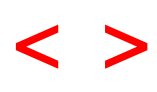




\section{Keou.}

TSHENG TSE. - «Le koua keou suit le koua kouae, qui indique la décision, l'extinction; pour mettre fin à quelque chose il faut qu'il y ait rencontre; c'est pour cela que le koua keou suit le koua kouae; keou, rencontrer. » Kouae, partager en deux moitiés : les moitiés séparées de toute chose coupée en deux doivent se rencontrer et s'unir ; si elles restaient comme à leur origine unies en un seul tout, comment pourraient-elles se rencontrer ? C'est à cause de cela que le koua keou suit le koua kouae. Il est constitué par le koua simple Khien en haut et le koua simple souen en dessous. En en parlant d'après les deux substances de ces koua simples, le vent parcourt l'univers ; au-dessous du ciel il y a la foule des êtres. Le vent, en parcourant l'univers (le dessous du ciel), ne peut pas faire autrement que de rencontrer et de choquer les êtres, ce qui donne l’image symbolique de la rencontre. De plus, une négativité commence à naître en bas du koua : rencontre de la négativité avec la positivité, ce qui fait que le koua exprime la rencontre et est appelé keou ${ }^{1}$.

\footnotetext{
${ }^{1} \mathrm{Keou}$, rencontre et union du principe mâle et du principe femelle.
} 


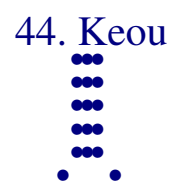

777.

Keou, la jeune fille adulte; ne pas employer ; épouser une jeune fille.

TSHENG TSE. - Une négativité commence à naître; à partir de ce moment elle grandit, peu à peu elle augmente et achève de grandir ; c'est la jeune fille grandissant et sur le point de devenir adulte. La négativité grandissant, la positivité décline ; si la jeune fille est forte, le jeune garçon est faible, aussi la formule avertit qu'il ne faut pas épouser une telle jeune fille. En épousant une jeune fille, on désire qu'elle soit douce et obéissante, afin qu'elle pratique la voie rationnelle de son sexe dans la famille. Le koua keou représente la négativité qui commence à avancer, qui grandit peu à peu, prend de la force et s'oppose à la positivité ; c'est à cause de cela qu'il ne convie nt pas de la choisir pour l'épouser. Si la jeune fille prend peu à peu de l'énergie et de la force, elle manquera aux règles de la droiture entre le principe mâle et le principe femelle et la voie rationnelle de la famille périclitera. Dans le koua keou, bien qu'il n'y ait qu'une seule négativité encore extrême ment petite, cependant celle-ci comporte une voie rationnelle d'augmentation graduelle de sa force, ce qui fait le motif de l'avertissement.

TSHOU HI. - Keou, rencontre ; la suppression absolue de la négativité (kouae) constituerait le koua parfait khien avec son unité de substance, c'est-à-dire le koua du quatrième mois ; survient la rencontre (keou) et alors une négativité devient visible et constitue le koua du cinquième mois. Lorsqu'une chose n'e st pas attendue et que subitement on l'aperçoit devant soi, c'est comme une rencontre imprévue ; c'est pour cela que ce koua exprime la rencontre. La rencontre est déjà dépourvue de régularité ; de plus, il s'agit d'une seule négativité qui rencontre cinq positivités, il en résulte donc que les sept vertus féminines manquent de pureté et que l'énergie et l'ardeur sont extrêmes. La prendre pour en faire sa compagne sera nécessairement nuisible à la positivité, et c'est pour cela que tels sont l'image symboli que et le sens divinatoire.

778.

Le commentaire de la formule déterminative dit: Keou, rencontrer; la douceur malléable rencontre la dureté énergique. 
TSHENG TSE. - Le sens du mot keou est rencontrer. Ce qui fait que ce koua est considéré comme exprimant l'idée de rencontre fortuite, c'est que la douceur malléable rencontre la dureté énergique ; une seule négativité qui vient de naître commence à se rencontrer avec la positivité.

TSHOU HI. - Explication du sens du nom du koua.

779.

Ne pas l'employer pour prendre une épouse ; ne pas s'allier longtemps.

TSHENG TSE. - Une négativité étant née, elle grandit peu à peu et se développe ; la négativité se développant, la positivité doit décliner. Celui qui épouse une jeune fille désire que son union dure longtemps et que sa famille s'achève ; or, ici, cette négativité augmente et se développe graduellement, elle va bientôt effacer la positivité et l'emporter sur elle, il est donc impossible de durer longtemps en état d'alliance avec elle. Toutes les foi s qu'il s'agit de filles, d'hommes inférieurs, de barbares, si leur force inhérente aug mente graduellement, comment serait-il possible qu'une alliance avec eux pût durer longtemps ? Aussi la formule avertit de ne pas prendre une telle jeune fille comme épouse.

TSHOU HI. - Explication de la formule du koua.

780.

Le ciel et la terre se rencontrent; toutes les classes d'êtres en ressentent l'éclat.

TSHENG TSE. - La négativité commence à naître en bas; elle se rencontre avec la positivité : le ciel et la terre se rencontrent. Si la négativité et la positivité ne s'alliaient ensemble en se rencontrant, tous les êtres ne naîtraient point. Le ciel et la terre se rencontrant, ils engendrent tous les genres, et les diverses classes d'êtres en reçoivent l'écl at c'est-à-dire tous les êtres sont brillants et éclairés.

TSHOU HI. - Cela est dit au sujet de la substance du koua. 
781.

La dureté énergique rencontre la justice et la droiture; grande action de l'univers ${ }^{1}$.

TSHENG TSE. - Il est question des aptitudes du koua. Le cinquième et le second emploient tous deux la dureté énergique positive et occupent leur situation avec justice et avec droiture; ils s'accueillent l'un l'autre avec justice et droiture. Le prince possède le serviteur juste et énergique ; le sujet rencontre un prince juste et droit: le prince et le sujet emploient la dureté énergique pour accueillir la justice et la droiture. La voie rationnelle de cette circonstance est qu'il leur est possible d'exercer une grande action sur l'univers.

TSHOU HI. - Ceci désigne le cinquième trait nonaire.

782.

Que le sens et le moment exprimés par le koua keou sont donc grands!

TSHENG TSE. - Exclamation admirative sur la grandeur du moment et du sens du koua keou. Si le ciel et la terre ne se rencontraient pas, les êtres ne naîtraient point; si le prince et le sujet ne se rencontraient pas, les lois de la société et son gouvernement n'exis teraient point; si le saint et le sage ne se rencontraient pas, les vertus de la voie rationnelle ne jouiraient pas de leur libre expansion ; si les choses et les êtres ne se rencontraient pas, leur ouvre et leur effet ne se produiraient point. Le sens et le moment marqués par le koua heou, qui désigne la rencontre, sont extrêmement grands !

TSHOU HI. - C'est à la cause, considérée dans la première apparence de ses effets et si minime qu'elle apparaisse, que l'homme saint attache le plus d'importance.

783.

\footnotetext{
${ }^{1}$ Le mot univers est toujours employé comme traduction des mots « sous le ciel ».
} 
Le commentaire de la formule symbolique dit: Sous le ciel il y a le vent; rencontre; le roi étend ses ordres et ses avertissements aux quatre régions.

TSHENG TSE. - Le vent court sous le ciel; il n'est rien qu'il n'enveloppe. Cela constitue limage symbolique du prince, ou du roi contemplant ce tourbillon enveloppant ${ }^{1}$ et s'appliquant à étendre ses ordres et à faire circuler ses avertissements aux quatre points cardinaux. «Le vent court sur la terre» et «sous le ciel il y a le vent»sont des expressions qui comportent également l'image symbolique d'un mouvement circulaire enveloppant les êtres. Mais sil «agit sur la terre» heurtant, en les enveloppant, tous les êtres, cela constitue le koua kouan ${ }^{2}$, avec l'image symbolique de traverser en regardant et en examinant; si, au contraire, il « agit sous le ciel » enveloppant les quatre régions, cela constitue le koua keou (rencontre), avec l'image symbolique d'étendre et de donner des ordres et des instructions. Les diverses formules symboliques emploient tantôt l'expression « les premiers rois », tantôt l'expression « les rois», tantôt l'expression «homme doué » ou «grand homme». Lorsque c'est l'expression « les premiers rois» qui est employée, il s'agit toujours des premiers rois établissant les règles et les institutions, établissant des états, inventant la musique, découvrant les diverses régions, précisant les règles, fixant les frontières, nourrissant les êtres, offrant des offrandes à l'être suprême ${ }^{3}$. Lorsque c'est l'expression « les rois » qui est employée, il s'agit des actions des princes et des rois complétant la voie rationnelle du ciel et de la terre, étendant leurs ordres et leurs avertissements aux quatre régions. L'expression «homme doué » est une appellation d'un sens plus général ${ }^{4}$; " grand homme » appellation générale des rois et des grands dignitaires.

\footnotetext{
${ }^{1}$ Ce mouvement de révolution circulaire.

${ }^{2}$ Koua $\mathrm{n}^{\circ} 20$.

$3 \mathrm{Ti}$, dieu.

4 Littéralement «appellation générale en haut ou en bas », c'est-à-dire «à peu près », s'appliquant à des personnes d'un rang plus ou moins élevé ; dans le cas actuel on peut lire s'appliquant indistinctement aux deux classes de rois qui viennent d'être indiquées.
} 


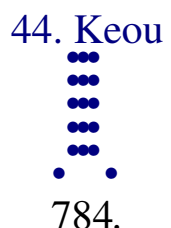

Premier trait hexaire : retenu et lié à une barre de métal; présage heureux de pureté ; avoir à entreprendre ; voir le présage malheureux; le porc maigre est porté à sautiller ${ }^{1}$.

TSHENG TSE. - Le koua keou, c'est le kou a de la négativité qui vient de naître et qui est sur le point de grandir. Une négativité naissant, elle grandira et peu à peu elle se développera complètement. Si la négativité grandit, la positivité s'évanouit : la voie de l'homme inférieur. grandit. Pour la contenir et la limiter, il faut s'y prendre dès le début et au moment où elle n'est pas encore complètement développée. Le sixième caractère du texte désigne une pièce de bois destinée à arrêter un char ; si une borne pour arrêter le char est en métal, elle est au plus haut point solide et résistante. Arrêter avec une barre de métal et de plus lier à cette barre, c'est ce qu'il y a de plus inébranlable. Arrêter solidement la négativité pour l'empêcher d'avancer, de sorte que le présage est heureux par la voie rationnelle de la droiture parfaite de la dureté énergique ; elle grandira progressivement et se complétera de façon à nuire à la positivité : c'est là un présage malheureux qui est manifeste. « Le porc maigre est porté à sautiller »; l'homme s aint en fait l'objet d'un avertissement important; il exprime que, bien que la négativité soit encore extrêmement minime, on ne doit cependant pas la négliger. Le porc est un être négatif et brutal, c'est pour cela qu’il est cité en exemple. Bien qu'un po rc maigre ne soit pas encore fort et puissant, cependant son naturel intérieur le porte à sautiller, c'est-à-dire à bondir de place en place. La négativité encore très minime et à peine manifestée, placée de plus au rang inférieur, est bien quelque chose qui peut être exprimé par l'idée de maigreur ; mais toutefois son naturel la porte constamment à submerger la positivité. Les voies rationnelles de l'homme doué et de l'homme inférieur sont différentes ; bien que l'homme inférieur soit dans un moment de faiblesse et de début, il ne laisse jamais que de blesser les sentiments de l'homme doué : en s'en gardant alors que son développement est encore rudimentaire on l'empêchera d'agir.

TSHOU HI. - Ce qui sert à arrêter un char ; si cet instrument est en métal, sa solidité est évidente. Une négativité vient de naître ; rester en repos avec droiture sera un présage heureux, agir et avancer sera un présage de malheur. Aussi, la formule emploie ces deux idées pour avertir l'homme inférieur, afin qu'il ne soit pas nuisible à l'homme doué, et alors le présage sera heureux, sans aucune apparence funeste. Toutefois, la force naturelle

\footnotetext{
${ }^{1}$ Le texte porte « avait confiance » et sautiller?
} 
inhérente à chaque chose fait qu'il est impossible d'arrêter le développement de la négativité, aussi la formule avertit l'homme doué en lui montrant l'exemple du porc maigre qui bondit, afin quil se prépare à tout et se tienne sur ses gardes.

785.

Attacher à une cheville de métal ; la voie de la malléabilité s'étend ${ }^{1}$.

TSHENG TSE. - «Étendre », conduire et avancer; la négativité commence à naître et avance progressivement; la voie rationnelle de la douceur malléable commence à s'étendre. L'attacher à une cheville de métal, c'est le moyen de l'empêcher d'avancer. Ne pas lui permettre d'avancer, c'est pour qu'elle ne puisse détruire la voie rationnelle de la droiture ; c'est là le présage heureux de la pureté.

TSHOU HI. — «S'étendre », avancer ; c'est parce qu'elle avance qu’il faut l'arrêter.

\footnotetext{
${ }^{1}$ Le caractère traduit par «s'étendre » a essentiellement le sens de «conduire en marchant
} devant». 


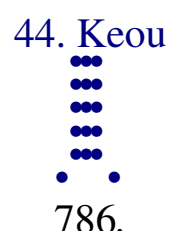

Deuxième trait nonaire : le sac contient du poisson ; pas de culpabilité ; pas davantage pour l'hôte.

TSHENG TSE. - Keou, rencontrer; le second et le premier s'associent en secret : ce sont ceux qui se rencontrent. Dans d'autres koua, le premier trait correspondrait sympathiquement et avec droiture au quatrième, mais dans le koua keou, c'est l'idée de rencontre qui est considérée comme étant la plus importante. La voie rationnelle de la rencontre mutuelle dépend essentiellement de l'unité absolue et exclusive ${ }^{1}$; la justice et l'énergie du second trait supposent la fermeté de la réunion (rencontre) à cause de sa sincérité. Toutefois, la malléabilité négative du premier trait, la foule des positivités qui sont au-dessus de lui, et, de plus, cette circonstance qu'il a la sympathie d'un des traits supérieurs, font que ces tendances le font rechercher. Le caractère spécial de la malléabilité négative lui permet rarement la fermeté et la pureté ; entre le second trait et le premier, il est impossible qu'il y ait sincérité de sentiment. L'absence de sincérité de sentiment dans l'objet rencontré conduit à la destruction de la voie rationnelle de la rencontre (attachement). Sac, sac en chanvre; poisson, la beauté dans ce qui est négatif ${ }^{2}$. De la positivité vis-à-vis de la négativité, c'est le sentiment de l'appréciation de la beauté qui détermine l'affection ; c'est pour cela que la formule choisit l'image symbolique du poisson. De la part du second envers le premier trait, s’il peut y avoir fermeté dans l'attachement, fer meté analogue à celle du lien qui enveloppe le poisson dans le sac du filet, il sera sans culpabilité dans son attachement (sa rencontre). Hôte, celui qui vient de l'extérieur ; pas d'avantage pour l'hôte : comment le poisson enveloppé dans un sac (serré) pourrait-il constituer un avantage pour l'hôte ? Cela exprime qu'il ne faut pas étendre encore cet attachement à des étrangers. La voie de l'attachement (rencontre) doit être l'unité exclu sive ; s'il y a deux objets à cet attachement, il y aura séparation.

TSHOU HI. - Le poisson est un être négatif. Le second et le premier trait se rencontrent; ce qui constitue l'image symbolique du poisson contenu dans un sac. Toutefois comme il ne dépend que de lui-même, il peut encore ne pas y avoir de culpabilité ; s'il ne sait se contenir et est porté à s'unir à la foule (des positivités), le mal qui en résultera sera considérable. C'est pour cela que tels sont l’image symbolique et le sens divinatoire.

\footnotetext{
${ }^{1}$ C'est de l'unité absolue que dépend la rencontre mutuelle.
}

${ }^{2}$ Le plus beau des êtres négatifs. 
787.

Le sac contient le poisson ; le devoir ne s'étend pas à l'hôte.

TSHENG TSE. - La rencontre du premier par le second trait ne doit pas dégénérer en un double attachement du premier trait avec deux traits extérieurs. Il doit en être comme du poisson renfermé dans un sac; le devoir n'en étend pas le bénéfice aux hôtes ${ }^{1}$.

\footnotetext{
${ }^{1}$ Sens absolument arbitraire et à peine intelligible ; un poisson qui est serré et qui n'est pas
} offert aux hôtes ; ceux-ci ne doivent pas en réclamer le bénéfice. 


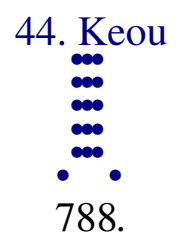

Troisième trait nonaire : les fesses sans peau ; action indécise ; appréhension ; pas de grande culpabilité.

TSHENG TSE. - Du moment où le second et le premier traits se rencontrent ensemble, comme le troisième trait se plaît avec le premier, il se rapproche et se resserre contre le second ${ }^{1}$, ce qui ne peut le satisfaire. De plus il est l'objet de la haine et de la jalousie du second : il reste en place sans être en repos comme celui dont les fesses n'ont plus de peau. Du moment où il n'est pas satisfait de sa position, il doit s'en aller, mais se trouvant dans le moment exprimé par le koua keou, ses tendances le portent à chercher une rencontre avec l'unique négativité placée au rang inférieur, et c'est là ce qu'il désire. Aussi, bien que placé sans être en repos, son action est hésitante. L'expression traduite par action hésitante ou indécise indique la difficulté de progresser en avant; cela veut dire quil ne peut y renoncer rapidement. Toutefois le troisième trait possède la dureté énergique et la droiture et il est placé avec satisfaction ${ }^{2}$; il comporte le sens d'un aveuglement qui n'est pas définitif. S’il vient à reconnaître son manque de droiture et s’il est ému par la crainte du péril, s'il n'ose pas se mouvoir ( agir) hors de propos, il pourra finir par éviter toute grave culpabilité. $\mathrm{Si}$, au contraire, il cherche à provoquer une rencontre contraire au devoir, il est certain qu'il sera coupable. Quand on connaît le péril, si l'on s'arrête, il n'arrive pas à un poin t considérable.

TSHOU HI. - Le troisième trait nonaire est trop durement énergique et il est sans justice ; au-dessous de lui il ne rencontre pas le premier ${ }^{3}$, au-dessus il ne sympathise pas avec le trait supérieur; s’il reste en place il n'est pas tranquille, s'il agit il n'avance pas ; voilà pourquoi tels sont l'image symbolique et le sens divinatoire. Toutefois, du moment où il ne doit rencontrer personne, il ne recevra aucune atteinte du mal causé par la négativité, aussi bien qu’il craigne le péril et éprouve de l'appréhension, il sera sans grande culpabilité.

789.

\footnotetext{
${ }^{1}$ Il veut aller vers le premier et se butte contre le second, sans pouvoir aller plus loin.

${ }^{2}$ Dans le koua simple souen.

${ }^{3}$ Puisquill en est séparé par le second.
} 
L'action est indécise ; l'action ne s'étend pas encore.

TSHENG TSE. - Au début, ses tendances le portent à rechercher la rencontre du premier trait, aussi son action et sa démarche sont lentes et hésitantes. Ne pas s'étendre encore, c'est ne pas encore presser le mouvement. Du moment où il a reconnu le péril et où il se corrige, il n'en vient donc pas à une grande culpabilité. 


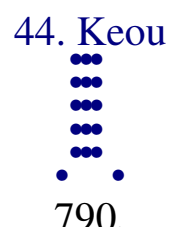

Quatrième trait nonaire : sac sans poissons ; le présage malheureux débute ${ }^{1 .}$

TSHENG TSE. - Sac, ce qui sert à contenir et à réunir ensemble ; poisson, ce qui est joli et agréable à l'œil. Le quatrième trait et le premier sont ceux qui se correspondent sympathiquement et avec droiture ; ce sont ceux qui doivent naturellement se rencontrer, mais le premier trait a déjà rencontré le second. Manquer la rencontre désirée, c'est comme lorsqu'un sac ne contient pas de poisson ; c'est perdre ce que l' on a. Le quatrième trait est en présence du moment de rencontre exprimé par le koua heou; il occupe une situation élevée et perd le concours de l'inférieur. L'abandon par l'inférieur provient de ce que lui-même a déjà perdu sa vertu; linnconvénient présenté par le quatrième trait, c'est qu'il est sans justice et sans droiture. Puisqu'il est sans justice ni droiture et qu'il perd le concours du peuple, c'est par là que survient le présage malheureux. On a dit : Le premier trait suit le second parce quil en est très rapproché : comment serait-ce la faute du quatrième? Réponse : En en parlant au sujet du quatrième, le sens est qu'il doit avoir quelque culpabilité ; il ne peut pas protéger l'inférieur, puisqu'il a perdu la voie ration nelle. Comment pourrait-il se faire que, le supérieur n'ayant point manqué à la voie rationnelle, l'inférieur l'ait cependant abandonné ? La voie rationnelle de la rencontre comporte tous les cas ; du prince et du sujet, de l'homme du peuple et de son maître, de l'époux et de l'épouse, des amis et des camarades. Le quatrième trait et le trait inférieur sont séparés, aussi en en parlant principalement au point de vue de l'homme du peuple, cela exprime le supérieur et l'inférieur séparés l'un de l'autre, et il doit certainement y a voir un changement funeste. Le sixième caractère du texte, élever, s'élever, veut dire «sur le point de naître ». Du moment où le cour du peuple s'est détourné, la difficulté est sur le point de se faire sentir.

TSHOU HI. - Le premier trait hexaire, qui lui correspond suivant la droiture a déjà rencontré le second et n'arrive pas jusqu'à le joindre, aussi tels sont l'image symbolique et le sens divinatoire.

791.

\footnotetext{
${ }^{1}$ Pour obtenir ce sens il faut faire une inversion inadmissible.
} 
Présage malheureux de l'absence de poisson ; il éloigne le peuple.

TSHENG TSE. - L'éloignem ent de l'inférieur provient de son propre fait; ce qui éloigne le peuple c'est que lui-même s'en éloigne. Cela est considéré comme indiquant que celui qui est en bas a des motifs qui le portent à s'éloigner.

TSHOU HI. - Ce qui fait que le peuple s'éloigne de lui, c'est que lui même s'éloigne du peuple. 


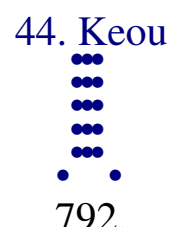

Cinquième trait nonaire : envelopper des courges avec les feuilles de l'arbre $\mathrm{ki}$; renfermer l'éclat; il y a quelque chose tombant du ciel.

TSHENG TSE. - Le cinquième trait nonaire est, lui aussi, sans aucune sympathie de la part de l'inférieur ; il ne rencontre personne. Cependant il se conforme à la voie rationnelle de la rencontre, de sorte que finalement il doit nécessairement arriver à une rencontre. Or, la rencontre du supérieur et de l'inférieur, résulte de ce qu ỉls s'appellent et se désirent mutuellement. L'arbre $k i$ est élevé et ses feuilles sont grandes. Ce qui est placé haut, dont la substance est grande, et qui peut servir à contenir toutes choses, c'est évi demment l'arbre appelé ki. Ce qui est beau, massif et placé bas, c'est la courge. La beauté demeurant dans l'infériorité, donne l'image symbolique du sage humble et effacé. Le cinquième trait nonaire est éminent et il occupe la situation du prince ; de plus, il s'abais se et appelle à lui les capacités du sage. Être très élevé et appeler à soi ce qui est très inférieur, c'est une action analogue à l'emploi des feuilles de l'arbre $k i$ pour envelopper des courges. S'il peut se courber et s’incliner à ce point, et si de plus ses vertus intérieures de justice et de droiture sont complètes, réelles, brillantes et belles, qu'il soit prince, ou homme quelconque, il ne pourra pas manquer de rencontrer ce qu'il désire et ce qu'il recherche. Bien qu'il se courbe et se plie pour appeler le sage, si ses vertus manquent de droiture, le sage ne répondra pas. C'est pour cela qu'il faut absolument qu'il renferme ses qualités belles et brillantes, qu'il les renferme en lui-même et les y développe jusqu'à la plus parfaite sincérité, et alors quelque chose descendra du ciel. C'est comme si la formule disait que quelque chose tombera du ciel et cela veut dire quil obtiendra certaine ment quelque chose. Depuis l'antiquité, les princes et les hommes qui ont su se courber et se plier avec la plus parfaite sincérité, et qui ont suivi la voie rationnelle de la justice et de la droiture pour appeler à eux les sages de l'univers, ne sont jamais restés sans rencon trer ce qu'ils cherchaient. Kao Tsong, ému par ses rêves, Wen Wang, dans ses rencontres en pêchant à la ligne, sont tous deux des exemples de cette même raison d'être.

TSHOU HI. - Les courges sont des choses négatives et qui sont placées très bas, elles sont douces, agréables et salutaires. L'arbre ki est grand et élevé, son bois est dur et résistant. Le cinquième trait, avec sa dureté énergique positive, sa justice et sa droiture, est le maître du koua dans sa partie supérieure, tandis qu'en bas il se tient en garde contre la négativité qui vient de naître et qui tend à provoquer la dissolution de la positivité. Telle est l'im age symbolique. Mais, la négativité et la positivité l'emportent tour à tour, 
comme cela se voit dans le retour successif des saisons; si le cinquième trait peut renfermer et cacher sa beauté et l'éclat de ses qualités, rester immobile pour se dominer, alors il pourra revenir de nouveau, agir et transformer. Il y a quelque chose descendant du ciel: c'est limage symbolique de ce qui, n'existant primitivement pas, finit par apparaître subitement.

793.

Le cinquième trait nonaire renferme ce qui brille; justice et droiture. Il y a quelque chose qui descend du ciel; les tendances ne sont pas contre la destinée.

TSHENG TSE. — L'expression « renfermer, ou contenir ce qui brille » désigne les vertus de justice et de droiture qu'il renferme. Lorsque la vertu est réelle et complète, son apparence devient visible, elle sillumine et éclate. La destinée, c'est la raison d'être indiquée et déterminée par le ciel. Aller contre, abandonner, enfreindre. L'extrême sincérité de la justice et de la droiture se courbe et appelle le secours du sage ; elle maintient ses tendances d'accord avec la raison d'être du ciel, et c'est à cause de cela qu'il y a quelque chose qui descend du ciel et dont il jouira. 


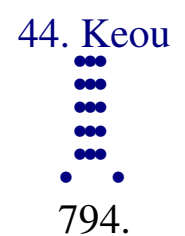

Trait supérieur nonaire : rencontrer les cornes ; appréhension, pas de culpabilité.

TSHENG TSE. - Ce qui est extrêmement dur, énergique et très élevé, ce sont les cornes. Ce trait nonaire avec sa dureté énergique occupe le rang supérieur, de sorte que les cornes sont prises comme symbole. La rencontre (l'accueil, l'alliance) entre les hommes, résulte de ce que chacun se courbe, se contient, pour déférer aux désirs d'autrui, et de la déférence mutuelle avec laquelle ils s'accueillent, ce qui leur permet d'être d'accord entre eux. Le trait supérieur nonaire est excessivement élevé, dur, énergique et altier : qui s'allierait à lui ? Dans ces conditions, rechercher la rencontre doit certainement être un motif d'appréhension. En agissant ainsi soi -même, si les hommes s'écartent, ce n'est pas la faute d'autrui, c'est le résultat de sa propre action, de sorte qu'il n'en faut attribuer la culpabilité à personne.

TSHOU HI. - Les cornes, ce qui est dur et placé haut. Le trait supérieur nonaire emploie la dureté énergique pour se maintenir en haut, mais il est sans situation ; il ne peut obtenir ce qu’il cherche à rencontrer, de sorte que l’image symbolique et le sens divinatoire sont analogues à ce qui a été dit au sujet du troisième trait.

795.

Rencontrer les cornes; en haut la fin, appréhension.

TSHENG TSE. — Étant placé au dernier degré de l'élévation, et la dureté énergique étant aussi à son extrême limite, c'est donc que le (trait) supérieur est à sa fin et qu'il arri ve à l'appréhension. Puisque la dureté én ergique atteint son extrême limite, quill occupe un rang élevé et quill cherche une rencontre (une alliance), n'est -ce pas là une véritable difficulté ? 


\section{Tsouei}

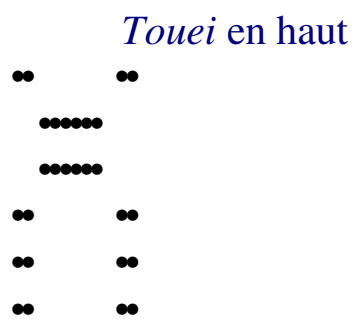

Khouen en bas

796. Tsouei, liberté; le roi parvient à avoir un temple en l'honneur de ses ancêtres ; avantage à voir un grand homme ; liberté ; avantage de la pureté ; présage heureux de l'emploi des grandes pièces de viandes desséchées ; avantage dans ce qu'il y a à entreprendre.

801. Premier trait hexaire : avoir confiance, pas de fin ; c'est le désordre, c'est le rassemblement ; s'il crie, une poignée le considère en riant; ne pas s'alarmer, en entreprenant, pas de culpabilité.

803. Deuxième trait hexaire : présage heureux d'entraînement ; pas de culpabilité ; la confiance sera un avantage en employant les sacrifices appelés tshieou.

$*$

805. Troisième trait hexaire : comme rassemblés, comme pleurant; rien d'avantageux ; en entreprenant, pas de culpabilité ; petite appréhension.

*

807. Quatrième trait nonaire : grand présage heureux ; pas de culpabilité.

$*$

809. Cinquième trait nonaire : le rassemblement a lieu dans une situation, sans culpabilité ; manquer de confiance ; pureté grande et durable ; dissipation des regrets.

$*$

811. Trait supérieur hexaire : gémir, pleurer : pas de culpabilité.

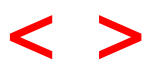




\section{Tsouei.}

TSHENG TSE. - Tsouei. «L'Ordre des koua » dit: «Le koua keou exprime la rencontre; les êtres se rencontrent et ensuite ils se rassemblent et se réunissent; c'est pour cela qu'il est suivi du koua tsouei. Tsouei, rassemblement, réunion. » Les êtres se rencontrent et forment des groupes, ou troupeaux ; c'est ce qui fait que le koua tsouei suit immédiatement le koua keou. Il est constitué par le koua simple touei, en haut, et le koua simple khouen, en bas. Le marais monte sur la terre ; rassemblement, amas des eaux ; c'e st pour cela qu'il est appelé tsouei. La formule ne dit pas que le marais est sur la terre; elle dit qu'il monte sur la terre, cela donne donc le sens de « moment où le rassemblement s'effectue ». 


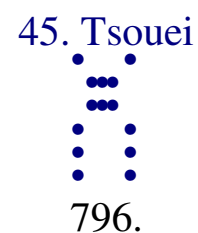

Tsouei, liberté ; le roi parvient à avoir un temple en l' honneur de ses ancêtres ; avantage à voir un grand homme ; liberté ; avantage de la pureté ; présage heureux de l'emploi des grandes pièces de viandes desséchées; avantage dans ce qu'il y a à entreprendre.

TSHENG TSE. - Le « roi » exprime la voie du rassemblement et du groupement de l'univers ; le plus haut degré dans cette voie est d'arriver à avoir un temple élevé à la mémoire des ancêtres. La masse des êtres vivants est extrêmement nombreuse et le roi peut réaliser l'unique idéal vers lequel tous tendent leur vues. Le caur de l'homme ne sait vers quoi se tourner et il peut provoquer la sincérité de son respect. Les esprits et les génies ne sont pas des êtres perceptibles et dont la nature soit appréciable, et cependant il peut parvenir à la déterminer. La voie rationnelle pour rassembler et réunir le coar des hommes et pour donner une seule et même impulsion à toutes leurs tendances, dans tout l'univers, n 'est point unique. La plus grande n'est jamais plus importante que le temple des ancêtres ${ }^{1}$, aussi, lorsque celui qui est roi parvient, dans la voie du groupement et de la réunion de l'univers, jusquà avoir un temple à la mémoire de ses aïeux, c'est qu'il est parvenu à la perfection de la voie du groupement ${ }^{2}$. L'hommage de sacrifices aux mânes des défunts, pour leur rendre grâce de leurs actions, a son origine dans le cour de l'homme et les hommes saints en ont fixé les rites pour compléter et achever les sentiments vertueux. C'est à ce même sentiment qu'il faut encore avoir recours pour comprendre comment les loutres sont capables d'accomplir des sacrifices ; c'est l'effet spontané de la natu re ${ }^{3}$. Après le nom du koua, tsouei, il y a le caractère heng, liberté d'expansion ; c'est une superfétation dans le texte. Ce même caractère se retrouve d'ailleurs plus bas ; mais il n'en est plus comme dans le cas du koua hoan. Dans le cas du koua hoan, il est d'abord question des aptitudes symbolisées par le koua ; ici, au sujet du koua tsouei, il est d'abord question du sens du koua. La formule déterminative est très claire : la foule des êtres sous le ciel (dans l'univers) doit posséder le concours d'un grand homme pour en recevoir le bienfait d'un gouvernement régulier. Lorsque les hommes s'assemblent il y a nécessaire ment confusion et désordre ; lorsque les êtres s'assemblent il y a nécessairement des que relles et

\footnotetext{
${ }^{1}$ C'est la traduction exacte et il est bien difficile d'en préciser le sens.

2 Le commentateur suppose le cas d'un fondateur de dynastie qui achè ve d'asseoir sa domination et couronne l'édifice de sa puissance en élevant ses aïeux à des dignités posthumes.

${ }^{3}$ Les loutres prennent du poisson en grande abondance et en déposent une partie sur la terre, comme offrande, sans les manger. Traditions et légendes populaires.
} 
des luttes; lorsque les choses sont amoncelées en grande quantité il y a désordre. S'il n'y avai t pas un grand homme pour faire régner l'ordre, le rassemblement serait une cause de désordres et de luttes. Si le rassemblement n'était pas conforme à la droiture, les hommes rassemblés seraient unis au hasard de la rencontre, les richesses rassemblées seraient le fruit de l'immortalité ; comment pourrait-il y avoir liberté ? Aussi, il y a avantage dans la pureté. Le koua tsouei indique un moment d'abondance florissante ; l'emploi des biens doit correspondre au but proposé, aussi l'emploi des grandes pièces de viandes séchées comporte un présage heureux. Rien n'est plus important que les sacrifices, aussi la formule parle des offrandes dans les sacrifices ; mais qu'il s'agisse de s'élever jusqu'à s'unir aux esprits et aux génies, ou de condescendre à accueillir le peuple et les êtres, qu'il s'agisse même de tout autre objet quelconque, il n'en est jamais autrement : les moyens doivent être proportionnés au but à atteindre. Dans un moment de rassemblement, et lorsqu'il s'agit de s'unir aux êtres par la gran deur et la générosité, ce sera un présage heureux de liberté de développement de la richesse florissante, l'univers entier jouissant également des bienfaits de ces richesses. S'il s'agit d'un moment de grandeur et d'ampleur et si l'on s'unit aux êtres avec parcimonie, ce n'est plus ce qui indique la liber té d'expansion de ce qui est beau et florissant ; l'univers n'est plus porté à se joindre à cette action mesquine, aussi les regrets et l'appréhension naissent bientôt. C'est qu'en effet il faut toujours se conformer à l'opportunité du moment, se plier à la raison d'être des choses, et agir ensuite. C'est pour cela que, dans le commentaire traditionnel de la formule déterminative $\left(\mathrm{n}^{\circ} 797\right)$, il est parlé de la soumission à la destinée imposée par le ciel, car dans ce qui ne doit pas être fait, la force est insuffisante ${ }^{1}$. C'est parce qu'il s'agit d'un moment de rassemblement qu'il y a avantage dans ce qui est à entreprendre ; dans la majorité des cas, pour commencer un travail, pour fonder quelque chose, ce qui est précieux, c'est de savoir profiter du moment où l'œuvre peut être accomplie. Rassembler et ensuite employer, c'est mettre en mouvement et voir grandir : c'est là précisément la rai son d'être du ciel.

TSHOU HI. - Tsouei, rassembler; le koua simple khouen exprime la soumission et le koua simple touei la satisfaction. Le cinquième trait nonaire est énergique, juste, et le second sympathise avec lui. Ou bien encore, il exprime le marais montant sur la terre image symbolique de tous les êtres se rassemblant, et c'est pourquoi il est appelé tsouei. Le caractère heng est en trop dans le texte. "Le roi parvient à avoir un temple en l'honneur de ses ancêtres » ; cela exprime que le roi peut à cause de cela, parvenir jusque dans l'inté rieur du temple de sa souche ${ }^{2}$ : ceci est un sens augural heureux lorsque le roi consulte le sort au sujet des sacrifices à faire. Le chapitre « des devoirs

\footnotetext{
${ }^{1}$ L'obscurité de tout le commentaire vient de ce qu'il est composé d'une suite de citations de divers textes et, dans ce passage, elle tient encore à ce qu'il s'agit d'une discussion sur les devoirs d'un prince qui fonde une dynastie, c'e st toujours un sujet dangereux sur lequel l'écrivain chinois n'ose guère appuyer autrement que par ses sous -entendus.

${ }^{2}$ Ce mot est expliqué dans le code chinois.
} 
dans les sacrifices » ${ }^{1}$ dit : «Le dignitaire revêtu du titre de kong arrive au grand temple des ancêtres », c'est préci sément le même sens. Le temple des ancêtres sert à réunir les mânes des ancêtres et ascendants; si, en outre, un homme peut rassembler son propre esprit incorporel ${ }^{2}$, lui aussi pourra parvenir au temple des ancêtres et continuer la succession de ses aïeux. Les êtres étant rassemblés, il faudra nécessairement qu’ils voient un grand homme et ensuite ils pourront jouir de la liberté d'expansion. Mais, de plus, il faut encore qu'il y ait avantage pour la droiture ; si ce qui est rassemblé n'est pas conforme à la droiture, il ne pourra pas encore y avoir liberté d'expansion et de développement. «Grandes pièces de viandes séchées »; il faut d'abord les réunir (rassembler), pour les avoir ; une fois qu'elles seront rassemblées, alors il pourra y avoir lieu d'e ntreprendre les sacrifices. Ce sont toujours des expressions augurales comportant un présage heureux et contenant en même temps un avertissement.

797.

Le commentaire de la formule déterminative dit : Tsouei, rassemblement; soumission par ta satisfaction; dureté énergique, justice et correspondance sympathique ; donc rassemblement.

TSHENG TSE. - Le sens du caractère tsouei est « rassemblement ». «Soumission par la satisfaction » est dit au sujet des aptitudes indiquées par les koua simples; en haut satisfaction, en bas soumission, cela constitue bien l'idée de l'emploi de la voie rationnelle du plaisir, ou satisfaction, pour conduire le peuple, en se pliant au désir des hommes. Les inférieurs se réjouissent des institutions qui leur sont données par le supérieur; et ils le suivent (lui obéissant) avec soumission. Du moment où le supérieur et les inférieurs sont satisfaits et soumis, si, de plus, la dureté énergique se place dans une situation qui lui revient suivant la justice et la droiture, et si en bas, au-dessous d'elle, elle rencontre aide et sympathie, c'est à cause de toutes ces conditions réunies, qu'il peut $\mathrm{y}$ avoir rassemblement ${ }^{3}$. Aussi, le rassemblement de l'univers est impossible sans la réunion de toutes ces aptitudes ou qualités.

TSHOU HI. - Explication du sens du nom du koua au moyen des vertus des koua simples et de leur substance.

\footnotetext{
${ }^{1}$ Li king.

2 Probablement une expression à peu près analogue à l'expression « sauver son âme ».

${ }^{3}$ Des hommes se groupent autour du prince.
} 
798.

Le roi parvient à avoir un temple en l'honneur de ses ancêtres; il en résulte l'offrande de la piété filiale. Avantage de la vue de la liberté d'un grand homme: rassemblement par l'emploi de la droiture. Présage heureux de l'emploi de grandes pièces de viandes séchées. Avantage dans ce qu'il y a à entreprendre : soumission aux ordres ${ }^{1}$ du ciel.

TSHENG TSE. - Le roi personnifie la voie rationnelle du rassemblement des hommes en réunissant leur coar dans un même désir, et il pousse l'application de cette voie jusqu'à l'édification et l'érection d'un temple dédié aux mânes de ses ancêtres ; c'est par là que la sincérité de ses sentiments de piété filiale et de dévotion sont manifestés. Les sacrifices sont les actions dans lesquelles le coaur doit s'absorber entièrement, aussi, pour celui qui cherche à renouveler le cour de tous les hommes sous le ciel (dans l'univers), rien ne vaut la piété filiale et la dévotion au culte des ancêtres. Dans la voie rationnelle pour rassembler l'univers, si le roi parvient à avoir un temple en l'honneur de ses ancêtres, ce sera le comble du succès. Dans un moment de rassemblement, voir un grand homme sera pouvoir jouir de liberté. En effet, le rassemblement doit avoir lieu en suivant la voie rationnelle de la droiture; la vue d'un grand homme montrera que le rassemble ment a lieu dans la voie de la droiture, et se conformer à la droiture c'est jouir de la liberté ; si le rassemblement n'avait pas lieu en se conformant à la droiture serait-il possible qu'il y eût liberté d'action et d'expansion 2 ? Les mots «employer de grandes pièces de viandes séchées » se rapportent à la partie précédente du texte où il est question d'avoir un temple en l'honneur des ancêtres ; il s'agit d'offrandes et de sacrifices. Mais il en est de même en toutes choses; dans un moment de rassemblement et d'abondance, celui qui s'allie aux êtres doit faire preuve d'une générosité à la hauteur des né cessités et de la convenance de chaque cas. Lorsque les êtres se rassemblent, leurs forces s'entraident et c'est ainsi qu'il peut y avoir possibilité de faire quelque chose ; aussi il y a avantage dans ce qu'il y a à entreprendre : tout cela est la conséquence naturelle de la raison d'être du ciel, aussi le texte dit : «Soumission à la destinée imposée par le ciel. »

TSHOU HI. - Explication de la formule du koua.

799.

\footnotetext{
${ }^{1}$ Au mandat du ciel, à la destinée.

2 C'est encore une suite d'aphorismes inintelligibles si l'on se borne à chercher le sens apparent.
} 
En regardant ce qui se rassemble, il est possible de voir la nature ${ }^{1} d u$ ciel, de la terre et de tous les êtres.

TSHENG TSE. - En considérant la raison d'être du rassemblement, il devient possible de voir les sentiments du ciel, de la terre et de tous les êtres. Le ciel et la terre dans leur action transformatrice et génératrice, tous les êtres en naissant et en croissant, tout ce qui existe, est toujours le « rassemblement» (la rencontre ou la réunion). La raison d'être de l'être ou du non-être, du mouvement et du repos, de la fin et du commencement n'est jamais autre chose que le rassemblement de la dispersion. Aussi en regardant la cause du rassemblement, on pourra voir le sentiment (la nature) du ciel, de la terre et de tous les êtres.

TSHOU HI. - Dernière et suprême définition de la raison d'être et exclamation admirative.

800.

Le commentaire traditionnel de la formule symbolique dit: Le marais monte sur la terre, rassemblement. L'homme doué pour ${ }^{2}$ régler (déterminer) les armes de guerre ; mettre en garde contre l'imprévu.

TSHENG TSE. - Le marais qui monte sur la terre constitue l'image symbolique du rassemblement exprimé par le koua tsouei, l'homme doué regarde l'image symbolique du koua tsouei (du rassemblement), pour régler et définir ${ }^{3}$ (mettre en ordre) les armes de guerre, à l'effet d'être en garde et prêt pour ce qui est imprévu. Dans tout rassemblement des êtres, il doit nécessairement y avoir des choses imprévues et inattendues, aussi, dans les rassemblements de la foule, il y a des contestations et dans les rassemblements des êtres il doit y avoir abus de la force et usurpation. En général, le rassemblement tient à un grand nombre de motifs divers, aussi, il faut regarder l'image symbolique du rassemble ment et avertir (au sujet de ce qu'il faut en attendre). Tshou veut dire surveiller et mettre en ordre, écarter de qui est

\footnotetext{
1 Plus littéralement «le sentiment». Les anciens, en fixant les caractères de l'écriture, déterminèrent d'abord les caractères relatifs au cour. Les caractères qui représentent les mots « naturel » et « sentiment » sont également classés sous le radical du « cour ». Le «naturel », c'est la raison d'être du cour ; le sentiment c'est précisément l'effet de ce cour. (Tshou Tse).

2 On lit traditionnellement « agit pour».

${ }^{3}$ Le terme tshou a aussi le sens de « rejeter, écarter».
} 
mauvais ; préparer et rassembler, c'est le moyen de mettre en garde contre l'imprévu.

TSHOU HI. - Tshou, expression qui indique l’idée de mettre en ordre et rassembler. 


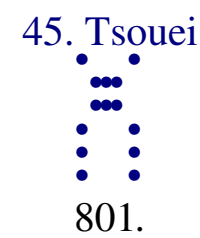

Premier trait hexaire : avoir confiance, pas de fin ; c'est le désordre, c'est le rassemblement ; s'il crie, une poignée le considère en riant ; ne pas s'alarmer, en entreprenant, pas de culpabilité.

TSHENG TSE. - Le premier et le quatrième traits sont ceux qui se correspondent sympathiquement et avec droiture et il en est ainsi parce qu'ils ont confiance en se suivant l'un et l'autre. Toutefois, dans le moment du rassemblement, trois négativités sont réunies en un même groupe, ce qui est un indice de douceur malléable ne conservant pas la droiture. Si le premier trait abandonne celui auquel il correspond sympathiquement et avec droiture, et suit ceux qui sont de son propre genre, c'est là avoir confiance sans résul tat (pas de fin). Là est le désordre ; trouble du cour. Là est le rassemblement, rassemblement avec ceux du même genre que lui. Si le premier trait conserve la droiture et ne la suit pas, s'il crie et appelle pour obtenir le concours de la droiture sympathique, alors «une poignée » s'en rira. Un poignée, c'est ce qu'on entend en disant un groupe, un ensemble ; cela veut dire que la foule le considérera comme un objet de risée. S'il peut ne pas s'en émouvoir et agir en suivant la correspondance sympathique conforme à la droiture de la dureté énergique positive, il sera sans faute et sans culpabilité ; autrement il rentrera dans la foule des hommes inférieurs.

TSHOU HI. — Le premier trait hexaire s'élève sympathiquement vers le quatrième trait nonaire, et il en est séparé par deux négativités. Dans un moment de rassemblement, il ne peut se garder lui-même, c'est là avoir confiance et ne pas réussir. Ses tendances sont désordonnées et le rassemblement est coupable. S'il crie et appelle celui qui lui corres pond sympathiquement avec droiture, la foule le considérera comme un objet de risée. Mais sill ne s'en alarme pas et agit en se conformant à sa sympathie et à sa droiture, il sera sans culpabilité. C'est un avertissement à celui qui consulte le sort, afin qu'il soit dans ces mêmes conditions.

802.

C'est le désordre, c'est le rassemblement, les tendances sont désordonnées. 
TSHENG TSE. - Les tendances de son cour sont troublées par ceux qui sont du même genre que lui, et c'est pour cela quill se réunit (rassemble) à la troupe des négativités. Il ne peut être ferme dans l'observation de ses principes, aussi il sera troublé par l'homme inférieur et il manquera de droiture. 


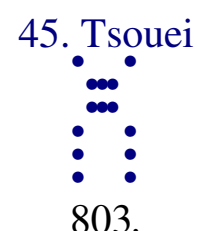

Deuxième trait hexaire : présage heureux d'entraînement; pas de culpabilité ; la confiance sera un avantage en employant les sacrifices appelés tshieou ${ }^{1}$.

TSHENG TSE. - Le premier trait est malléable et négatif, de plus il est sans justice ni droiture; il est à craindre quil ne puisse avoir confiance jusqu'à la fin, aussi ses aptitudes sont citées comme sujet d'avertissement. Bien que le second trait soit malléable et négatif, il possède cependant la justice et la droiture, aussi, bien qu'il comporte encore un avertis sement, cet avertissement est donné avec ménagements. Toutes les fois que la formule d'un trait a rapport à l'un de ces deux sujets : obtenir, ou perdre, elle exprime une règle, ou un avertissement, dans tous les cas elle est établie suivant les qualités ou les aptitudes indiquées par le koua. "Présage heureux d'entraînement sans culpabilité »; entraînement mutuel. Lorsqu’il s'agit de l'union des hommes, sỉls se recherchent mutuellement, ils se réunissent; s'ils s'attendent réciproquement, ils restent séparés. Le second et le cinquième traits sont ceux qui se correspondent sympathiquement avec droiture ; ce sont ceux qui devraient se rassembler et qui s'éloignent mutuellement. D'ailleurs, le second trait se trouve placé au milieu d'une troupe de négativités, qui s'entraînent certainement l'une l'autre et leur rassemblement aura lieu. Le cinquième trait occupe la situation prééminente, il possède les vertus de la justice et de la droiture; le second, lui aussi, suit la voie rationnelle de la justice et de la droiture et entreprend de se joindre à lui ; ceci exprime donc l'accord et la concorde entre le prince et le sujet; comment pourrait-on mesurer la portée de leur action mutuelle ? C'est à cause de cela que le présage est heureux et qu'il n'y a pas de culpabilité. Pas de culpabilité, exprime que le bien compense le mal; si le second et le cinquième ne s'entraînaient pas mutuellement, cela constituerait le mal et la faute. « La confiance sera un avantage dans l'emploi des sacrifices du printemps »; confiance, foi intérieure; expression qui indique la sincérité ; il s'agit des sacrifices les moins importants. Employer des choses communes, ne pas s'attacher à compléter l'appa rat des cérémonies et s'unir directement à l'intelligence de l'esprit par la sincérité de l'idée. Le texte porte « la confiance sera... »; cela signifie que, ayant confiance, agissant de bonne foi, il sera possible de ne pas employer l'ornementation et la recherche des formes et

1 Les moins importants entre les sacrifices des quatre saisons (Tse tien, sacrifices du printemps). 
d'arriver par la plus extrême sincérité à l'union absolue avec le supérieur. Ce qui est dit au sujet des sacrifices du printemps exprime tout simplement l'offrande unique de la sincérité. Lorsque le supérieur et l'inférieur se réunissent ensemble, et qu'ils s'attachent à l'observation des apparences, c'est qu'il n'y a pas encore de sincérité dans leur union. En effet, ce qui est réel en soi n'a pas besoin d'emprunter l'ornementation de l'apparence extérieure ; tel est le sens de l'expression « employer les sacrifices du printemps ». La bonne foi et la confiance sont la base de tout rassemblement (union); et non pas seulement de l'union entre le prince et le sujet ; toute union dans l'univers, est basée uniquement sur la sincérité.

TSHOU HI. - Le second trait correspond sympathiquement au cinquième, mais il est confondu au milieu de deux négativités ; il faut qu'ils s'entraînent et s'attirent mutuellement pour se réunir, c'est ainsi que le présage sera heureux et qu'il n'y aura pas de culpa bilité. De plus, le second est animé par la justice et la droiture, il est malléable et soumis, sans prévention (vide à l'intérieur) et porté à monter vers celui auquel il correspond. Le cinquième trait nonaire est énergique, actif, juste et plein de droiture; sa sincérité est complète et il descend pour s'allier. Aussi, celui qui consulte le sort et possède cette bonne foi sincère pourra sacrifier, même en n'employant que des choses de peu de valeur et très simples.

804.

Présage heureux d'entraînement sans culpabilité; la justice n'est pas encore altérée ${ }^{1}$.

TSHENG TSE. - Dans le moment indiqué par le koua tsouei, obtenir une alliance (rassemblement) est considéré comme constituant un présage heureux, aussi, le quatrième trait nonaire est considéré comme obtenant l'union du supérieur et de l'inférieur. Le second et le cinquième, bien que se correspondant sympathiquement suivant la droiture, sont cependant dans des endroits différents et il y a un intervalle entre eux : ce sont ceux qui devraient s'allier (se rassembler) et qui ne sont pas encore réunis. Aussi, sills peuvent s'attirer l'un l'autre et s'unir, le présage sera heureux et il n'y aura pas de culpabilité. Puisquils ont les vertus de justice et de droiture, ils ne sont pas encore portés à se modifier hâtivement; sills se modifient, ils ne s'attireront plus mutuellement. On a dit : du moment où le second possède les vertus de la justice et de la droiture, et où l'image symbolique exprime qu'il n'y a pas encore transformation, la formule semble être incomplète; comment cela a-t-il lieu ? Réponse : La troupe des négativités est réunie en groupe ; c'est là

\footnotetext{
${ }^{1}$ Ou bien : « le dedans n'est pas encore transformé ».
} 
le genre se réunissant. Au moment où la réunion vient d'avoir lieu, le second trait se trouve placé au milieu du groupe ; s'il peut se préserver i ntact, sans modification, considérer et appeler de loin celui avec lequel il sympathise avec droiture; enfin se maintenir avec énergie en place, comme les deux autres négativités n'ont que les aptitudes qui résultent de la mollesse et de la passivité et que lui-même possède les vertus de justice et de droiture, il est aisé de prévoir qu'il n'ira pas jusqu’à se transformer. Aussi, le commen taire symbolique contient l'idée d'avertissement au sujet de la préservation. 


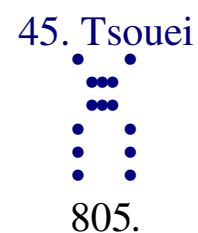

Troisième trait hexaire : comme rassemblés, comme pleurant; rien d'avantageux ; en entreprenant, pas de culpabilité ; petite appréhension.

TSHENG TSE. — Le troisième trait est un homme malléable et négatif dépourvu de justice et de droiture ; il demande à se lier avec quelqu'un et personne ne s'allie à lui. S'il recherche le quatrième, ce ne sera pas celui qui lui correspond sympathiquement avec droiture; de plus, ils ne sont pas du même genre ; c'est donc que le quatrième trait l'aban donne à cause de son manque de droiture. S'il recherche le second, comme celui -ci sympathise de son côté avec le cinquième qui lui correspond avec droiture, c'est donc que le second refuse de s'allier à lui parce qu'il manque de droiture. Il en résulte que sil cherche à s'allier, il est rebuté par chacun et il pleure et se lamente ; il ne peut réussir à former une union, il s'en fâche et crie, au-dessus comme au-dessous personne ne s'alliant à lui, rien ne lui est avantageux ; ce n'est qu'en entreprenant de suivre le trait supérieur hexaire qu’il pourra réussir à obtenir une alliance, ce qui est considéré comme marquant l'absence de culpabilité. Bien qu'entre le troisième trait et le trait supérieur il n'existe pas la correspondance sympathique et correcte de la négativité à la positivité, cependant, dans un moment de rassemblement, ils se suivent mutuellement à cause de leur communauté de genre, puisque tous deux occupent le rang supérieur dans une des deux substances (des koua simples) avec la même malléabilité négative. Enfin, tous deux sont sans alliance, et ils occupent des rangs qui se correspondent. Le trait supérieur est, en outre, placé au comble de la satisfaction passive, de sorte qu'il possède son alliance sans culpabilité. Dans la voie rationnelle des changements ${ }^{1}$, la transformation et le mouvement (ébranlement cause d'action) n'ont pas lieu d'une façon toujours identique et uniforme ; c'est à l'homme de le com prendre. Cependant, comment se fait-il qu'il y ait une petite cause d'appréhension ? C'est que le troisième commence par rechercher l'alliance du quatrième, puis ensuite du second trait ; il n'y réussit pas, et c'est ensuite qu'il entreprend de suivre le trait supérieur hexaire. Si l'action d'un homme est analo gue à celle du troisième trait, bien qu'il obtienne ce qu'il recherche, il y a cependant un motif d'une légère honte et d'appréhension.

TSHOU HI. - Le troisième trait hexaire est malléable et négatif, sans justice ni droiture ; en haut personne ne lui correspond sympathiquement ; il veut rechercher l'alliance de celui qui est proche et ne l'obtient pas, aussi il est

1 «Dans la philosophie du Yi king. » 
comme couvert de confusion et rien ne lui est avantageux. Ce n'est qu'en entreprenant de suivre le trait supérieur qu'il pourra être sans culpabilité. Cependant, n'obtenant pas l'alliance qu'il a recherchée, n'entre prenant qu'après qu'il a essuyé ce malheur, et, enfin, n'obtenant que l'alliance d'un trait sans situation définie et exprimant le dernier degré de la négativité, il y a bien encore là un motif de honte. C'est un avertissement à celui qui consulte le sort, quill convient qu’il écarte les séductions violentes contraires à la droiture et qu'il contracte de loin l'alliance définitive à ce qui lui correspond sympathiquement et avec droiture; dans ces conditions, il sera sans culpabilité.

806.

En entreprenant, pas de culpabilité, en haut satisfaction.

TSHENG TSE. — Le trait supérieur occupe le rang extrême dans le koua simple qui indique la satisfaction de la mollesse ; le troisième entreprend et il est sans culpabilité. Le trait supérieur hexaire s'y prête avec soumission et satisfaction, et l'accueille. 


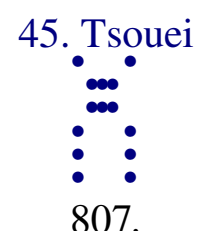

Quatrième trait nonaire : grand présage heureux; pas de culpabilité.

TSHENG TSE. - Le quatrième trait se trouve en présence d'un moment de rassemblement, en haut il se serre contre le prince représenté par le cinquième trait nonaire : c'est la possibilité de l'union du prince et du sujet. En bas, il se rapproche de la foule des négativités qui forme la substance du koua simple inférieur: c'est la possibilité de l'union du peuple et des inférieurs. Posséder le concours du supérieur et des inférieurs, c'est ce qu'on peut appeler le bien. Cependant, le quatrième emploie la positivité pour occuper un rang négatif ; il est sans droiture ; lorsqu’il possède le concours des supérieurs et des inférieurs, il faut quil rencontre un grand présage heureux, et ce n'est qu'ensuite qu'il sera sans culpabilité. Le troisième caractère, grand, a le sens d'entourer et d'atteindre partout par un mouvement circulaire ; une chose qui atteint partout peut être appelée grande et si elle est en tout conforme à la droiture elle constituera un grand présage heureux ; avec un grand présage heureux, le quatrième trait sera donc sans culpabilité. Or, le concours du supérieur et des inférieurs peut certainement être obtenu, dans certains cas, autrement qu'en suivant la voie rationnelle de la droiture. Depuis l'antiquité, nombreux ont été ceux qui ont su plaire au prince tout en agissant contre la raison et en suivant une voie criminelle; d'autres aussi ont quelquefois captivé le peuple par les mêmes moyens illégitimes : tels sont les cas de Tshen Hang de la dynastie de Tsi et de Li Shi du royaume de Lou. Mais ceux-là jouirent-ils de la grandeur du présage heureux ? Furent-ils sans culpabilité ? Il faut donc d'abord que le quatrième trait jou isse de la grandeur du présage heureux, et ensuite il pourra être sans culpabilité.

TSHOU HI. - En haut il s'associe au cinquième trait nonaire ; en bas il se presse contre la foule des négativités ; il jouit du concours. Mais, avec sa nature positive, il occupe un rang négatif et il est sans droiture, aussi la formule avertit celui qui consulte le sort qu'il faut un grand présage heureux et que ce n'est qu'ensuite qu'il pourra ne pas y avoir de culpabilité.

808.

Grand présage heureux sans culpabilité ; situation imméritée. 
TSHENG TSE. - Puisque sa situation est imméritée, il y a lieu de suspecter que son action ne peut pas encore être absolument bonne, aussi la formule mentionne qu'il faut d'abord qu'il jouisse d'un grand présage heureux et que ce n'est qu'ensuite qu'il pourra être sans culpabilité. Si son action n'est pas absolument bonne, comment pourrait-elle constituer un grand présage heureux? 


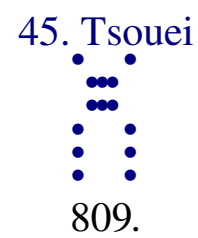

Cinquième trait nonaire : le rassemblement a lieu dans une situation, sans culpabilité ; manquer de confiance ; pureté grande et durable ; dissipation des regrets.

TSHENG TSE. - Le cinquième trait nonaire occupe le rang suprême dans l'univers ; il réunit et groupe autour de lui la foule dans l'univers entier, et la régit en la dirigeant. Il doit répondre exactement aux conditions de cette situation et améliorer ses vertus; puisqu’il emploie la dureté énergique de la positivité et occupe le rang suprême, il est digne de sa situation et il est considéré comme possédant une situation. Comme il suit la voie de la justice et de la droiture, il ne commet pas de faute et il est sans culpabilité. Étant dans de telles conditions, ceux qui n'ont point confiance en lui et qui ne sont pas encore venus se joindre à lui doivent faire retour sur eux-mêmes, réformer la vertu primitive de pureté éternelle et primordiale, de sorte qu'aucun bienfait ne leur manquera et que leurs regrets se dissiperont. La pureté durable et primordiale est la vertu du prince, c'est ce qui attire le peuple, aussi, dans la voie rationnelle du groupement de l'univers 1, comme dans la voie du rassemblement de l'univers ${ }^{2}$ la condition essentielle réside toujours dans ces trois termes: pureté, durée, grandeur. Du moment où le roi possède la situation, et où, de plus, il possède la vertu, alors qu'il est sans faute et sans culpabilité et qu'il possède la jus tice et la droiture, si dans l'univers il existe encore quelqu'un qui n'ait point confiance en lui et ne vienne pas se soumettre, et se grouper autour de lui, c'est que sa voie n'est pas encore grande et éclatante et que la voie rationnelle de la pureté, de la grandeur et de la permanence durable n'est pas encore parvenue à sa limite extrême. C'est par la réforme de la vertu qu'elle y arrive. Ainsi le peuple des Miao se révolta contre la destinée ; le ciel $^{3}$ fit éclater les vertus de la forme ${ }^{4}$; les vertus de Shouen ne manquaient pas d'atteindre à l'extrê me excellence. Or, il y a des différences de proximité ou d'éloignement, d'obscuri té et de clarté, aussi le mouvement qui porte au groupement a lieu plus tôt ou plus tard; si quelque chose n'est pas encore venu se grouper, il convient de réformer les ver tus : ce qu'on entend par le mot vertu, c'est la voie rationnelle de la pureté, grande et éternelle. Le dixième caractère, yuan, exprime la tête, le chef, la grandeur ; il exprime que la vertu du prince apparaît comme dépassant celle des autres

\footnotetext{
${ }^{1}$ Koua $\mathrm{n}^{\circ} 8$, pi.

${ }^{2}$ Koua tsouei.

${ }^{3} \mathrm{Ti}$, Dieu, et aussi l'empereur.

${ }^{4}$ Wen ti : l'éclat manifesté des vertus dans les actions.
} 
êtres, et que la grandeur du prince surpasse la foule de ce qui est vivant : cela donne le sens de grandeur absolue, le sens de domination exclusive. Si à ces conditions on joint encore la fermeté de la pureté et la constance éternelle, ces vertus pénétreront librement et influenceront jusqu'aux génies et aux esprits. Leur éclat illuminant les quatre mers (les quatre points cardinaux), personne ne songera à ne point se soumettre : c'est là ne pas manquer de confiance, et aussi la dissipation des regrets. Ce qu'il faut entendre par regrets, ce sont les tendances pas encore éclatantes, le cour manquant encore de satisfaction ${ }^{1}$.

TSHOU HI. - Le cinquième trait est nonaire, énergique, positif, juste et doué de droiture; il est en présence d'un moment de rassemblement et il occupe le rang prééminent; il est certainement sans culpabilité. S'il ne jouit pas encore de la confiance de tous, il devra, lui aussi, réformer sa vertu de pureté grande et éternelle et ses regrets se dissiperont. C'est un avertissement à celui qui consulte le sort, qu'il convient de se mettre dans ces mêmes conditions.

810.

Le rassemblement possède la situation ; tendances pas encore éclatantes.

TSHENG TSE. - Le commentaire de la formule symbolique cite le premier membre de phrase de la formule de trait ${ }^{2}$. Les tendances du prince doivent nécessairement le porter à désirer manifester une parfaite sincérité envers l'univers ; si elles émeuvent, elles pénétreront librement, et tout ce qui contient la vie ne manquera pas d'en sentir l'influence et de revenir (vers lui). Sil voit encore quelque manque de confiance, c'est que ses ten dances ne sont pas encore grandement éclatantes.

\footnotetext{
${ }^{1}$ C'est encore un de ces échafaudages de lieux communs pris dans des ouvrages anciens et rapprochés avec plus ou moins de discernement. Le sens n'existe que parce qu'il est traditionnellement transmis et appliqué.

2 \$ 809. «Le rassemblement a lieu dans une situation. » Les trois caractères sont les mêmes, mais le sens paraît légèrement modifié par le reste de la sentence.
} 


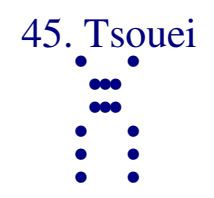

811.

Trait supérieur hexaire : gémir, pleurer : pas de culpabilité.

TSHENG TSE. - Hexaire et maître de la satisfaction ${ }^{1}$; homme inférieur malléable et négatif, se plaisant dans une situation élevée et s'y plaçant : qui donc, dans l'univers, vou drait se joindre à lui ? Il cherche à rassembler et personne ne s'allie à lui ; son malheur est tel qu'il en gémit et pleure. Gémir, lamentations répétées. Si les hommes s'écartent de lui, lui-même en est la cause ; à qui donc encore en attribuer la culpabilité ? Il est abandonné et détesté des hommes, il ne sait que faire, de sorte qu'il en gémit et en pleure. C'est précisément le fait et le caractère de l'homme inférieur.

TSHOU HI. - Il est placé à la fin du moment du rassemblement; il est malléable, négatif, et sans situation; il cherche à rassembler et ne réussit point, aussi la formule avertit celui qui consulte le sort qu'il doit d'abord être dans ces conditions et qu'ensuite il sera sans culpabilité.

812.

Gémir et pleurer; pas encore satisfait de sa supériorité.

TSHENG TSE. - L'homme inférieur, en se plaçant, manque généralement aux convenances; avide et écoutant ses passions, il est incapable de se choisir lui-même une position dont il soit satisfait. Cela va jusqu'à un tel point qu'il est malheureux et qu'il souffre, alors il est bouleversé et ne sait plus que faire. Ce qui fait que ce trait hexaire pleure et se lamente, c'est en effet qu'il n'est pas satisfait en se plaçant au rang supérieur. L'homme doué est attentif dans le choix de la position où il se place; si la position est contraire au devoir, il n'y demeure point ; s'il éprouve des malheurs, s'il se trouve exposé à des périls et souffre, il sait s'en contenter comme de la prospérité, sans que cela influe sur son cour. L'homme inférieur demeure en place sans se mettre en repos, il marche là où il ne devrait pas se tenir. Vienne ensuite le malheur, il s'agite considérablement et sans réflexion et va jusqu'à pleurer, ce qui le conduit fatalement à la hont e. Le terme du texte

\footnotetext{
${ }^{1}$ Trait hexaire placé au dernier rang du koua simple touei.
} 
traduit par les mots «pas encore» est une expression qui marque un mouvement sans rapidité ; comme on dit vulgairement «ce qui ne convient pas encore ». Il ne peut pas encore se contenter de son élévation. Il est négatif et occupe le rang supérieur; il est isolé et personne ne s'allie avec lui ; puisque ce n'est pas la position qui lui convient, comment y serait-il en repos? 


\section{Sheng}

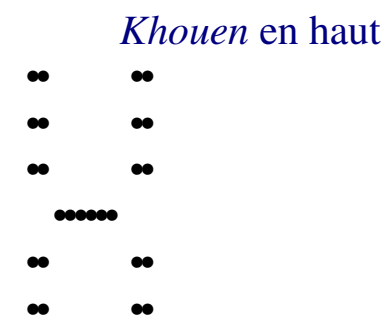

Souen en bas

813. Sheng ; grandeur, liberté ; sert à voir le grand homme; ne pas se chagriner; présage heureux en allant vers le midi.

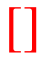

818. Premier trait hexaire : monter avec confiance, grand présage heureux.

$*$

820. Deuxième trait nonaire : confiance, alors avantage dans l'emploi des sacrifices du printemps; pas de culpabilité.

822. Troisième trait nonaire : s'élever dans un district vide.

$*$

824. Quatrième trait hexaire : le roi emploie la liberté sur la montagne de Khi ; présage heureux; pas de culpabilité.

*

826. Cinquième trait hexaire : présage heureux de pureté ; monter des degrés.

$*$

828. Trait supérieur hexaire : s'élever dans l'obscurité ; avantage dans la pureté qui ne cesse point.

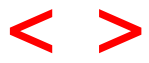


46. Sheng.

TSHENG TSE. - Sheng. «L'Ordre des koua » dit: «Le koua tsouei exprime la réunion des hommes; l'action d'hommes qui se rassemblent et montent s'exprime par le mot Sheng; c'est pour cela que le koua tsouei est suivi du koua sheng. » Les êtres ou les choses s'assemblent, s'accumulent et augmentent en grandeur et en élévation; ils s'assemblent et montent, aussi le koua est appelé sheng et c'est pour cela qu'il suit immédiat ement le koua tsouei. Il est composé du koua simple khouen en haut et du koua simple souen en bas le bois est sous la terre. Cela constitue l'image symbolique de l'arbre qui naît dans la terre. L'arbre naissant dans la terre, grandissant et devenant haut constitue le symbole du koua sheng. 


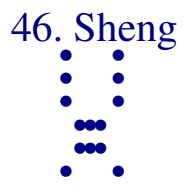

813.

Sheng ; grandeur, liberté; sert à voir le grand homme ; ne pas se chagriner ; présage heureux en allant vers le midi.

TSHENG TSE. - Sheng, avancer et monter. Avancer en montant comporte le sens de liberté d'expansion et, à cause du bien exprimé par les aptitudes des koua simples, il y a symbole de grande liberté. En employant cette voie pour voir un grand homme, il sera inutile de s'inquiéter et de se chagriner. En avançant devant soi le présage est heureux. Aller vers le midi ; avancer devant soi.

TSHOU HI. - Sheng, avancer et monter; provient du koua kiae dans lequel la malléabilité monte occuper le quatrième rang. Au-dehors soumission et au-dedans humilité. Le second trait nonaire est énergique et juste et le cinquième lui correspond sympathiquement. C'est pour cela que le sens divinatoire est tel. Aller vers le midi, avancer devant soi.

814.

Le commentaire de la formule déterminative dit: La malléabilité monte avec le temps.

TSHOU HI. - Explication du nom du koua par la transformation.

815.

Humilité et soumission; la dureté énergique est au-dedans et correspond sympathiquement ; c'est à cause de la grande liberté.

TSHENG TSE. - En en parlant d'après les deux substances, la malléabilité monte; c'est-à-dire que le koua khouen agit en montant. La substance du koua simple souen est humble et s'abaisse, alors le koua khouen 
se conforme au moment et monte : il monte avec le temps. Cela exprime que le moment comporte l'opportunité de l'action de s'élever. Du moment où la malléabilité monte et produit le koua sheng, il en résulte que le bas est humble et le haut soumis : monter en suivant la voie rationnelle de l'humilité et de la soumission, c'est bien ce qu'on peut appeler le temps (le moment ${ }^{1}$ ). Le second trait suit la voie rationnelle de la dureté énergique et de la justice et il sympathise avec le cinquième ; le cinquième avec les vertus de justice et de déférence, sympathise avec le second possibilité de l'humilité et soumission, l'élévation est conforme au moment et c'est à cause de cela quil y a « grandeur et liberté ». Le texte du commentaire de la formule déterminative porte par erreur les mots «grand, liberté », on en verra l'e xplication au sujet du koua tae yeou.

TSHOU HI. - Explication de la formule du koua au moyen des vertus des koua simples et de la substance du koua lui-même.

816.

Sert à voir un grand homme sans s'alarmer; il y a des louanges; présage heureux en allant vers le Midi; les tendances agissent.

TSHENG TSE. - Toute voie rationnelle d'élévation doit provenir du fait d'un grand homme ; s'il s'agit de s'élever à une situation définie dans l'État, c'est par le roi ou les princes ; s’il s'agit d'élévation dans la voie rationnelle, ce sera par le fait du saint ou des sages. Employer la voie rationnelle de l'humilité et de la soumission, de l'énergie active et de la justice pour voir un grand homme doit nécessairement faciliter le mouvement d'élé vation. Ne pas s'alarmer, ne pas se chagriner de l'insuccès. Profiter de l'élévation et la faciliter constituera une cause de bonheur et de louanges pour soi-même, et ce bonheur et ces louanges s'étendront aux êtres. Le Midi, c'est la direction vers laquelle les hommes regardent; aller vers le Midi, c'est avancer droit devant soi. Avançant droit devant soi, on facilite le mouvement d'élévation et on en profite de sorte que les tendances peuvent prévaloir, et c'est à cause de cela qu'il y a un présage heureux.

817.

\footnotetext{
${ }^{1}$ Le moment convenable.
} 
Le commentaire de la formule symbolique dit : Dans l'intérieur de la terre naît l'arbre; mouvement ascensionnel. L'homme doué emploie la vertu de la soumission, il accumule peu à peu, pour s'élever et grandir.

TSHENG TSE. - L'arbre naît dans la terre, il grandit et monte en s'élevant, ce qui constitue l'image symbolique de l'élévation ; l'homme doué regarde l'image symbolique de l'élévation et il l'emploie à la réforme de sa vertu par la soumission; il accumule (procède) peu à peu jusqu'à devenir grand et élevé ${ }^{1}$; en s'y conformant, il lui est possible d'avancer ; s'il y résiste, il recule. Le mouvement en avant de tous les êtres a toujours lieu par la voie rationnelle de la soumission. S'il n'accumulait peu à peu en lui -même le bien, ce serait insuffisant pour achever sa réputation; la certitude et la vérité dans les études et dans les arts, l'illustration brillante dans les vertus de la voie rationnelle, ne se produisent jamais que peu à peu et c'est par l'accumulation lente de petits résultats qu'on parv ient à l'élévation et à la grandeur : tel est le sens du koua sheng.

TSHOU HI. - Dans l'édition de Wang Sou, à la place du mot soumission, se trouve le mot circonspection ; actuellement d'après l'examen de ce passage dans les autres éditions, on voit que beaucoup aussi suivent le présent texte. L'idée qui motive l'emploi du mot circonspection est extrêmement simple, car, en effet, dans l'antiquité les deux carac tères qui expriment ces mots s'employaient l'un pour l'autre. Pour l'explication, voir dans la première partie ${ }^{2}$ le koua mong.

\footnotetext{
${ }^{1} \mathrm{Si}$ on se reporte au commentaire de Tsheng sur le koua $\mathrm{n}^{\circ} 20$ et à ce qui a été expliqué au sujet du sens du caractère prononcé kouan $H$ regarder », ou kouan, être le point de mire des regards, peut-être vaudrait-il mieux, dans ce passage et les sentences analogues, lire: l'homme doué présente aux yeux (ou : est regardé comme) le symbole de l'élé vation.

${ }^{2}$ Koua $n^{\circ} 4$.
} 


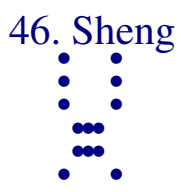

818.

Premier trait hexaire : monter avec confiance, grand présage heureux ${ }^{1}$.

TSHENG TSE. - Le premier trait se sert de la douceur et occupe le rang inférieur dans la substance du koua simple qui exprime l'humilité ; de plus c'est de lui que dépend cette humilité 2 ; il monte servir la dureté énergique du second trait nonaire ; il représente l'extrême humilité. Le second trait emploie les vertus de l'activité énergique et de la justice et il monte sympathiquement vers le prince ; c'est celui qui est investi de l'autorité nécessaire pour s'élever. Yun, troisième caractère du texte, suivre avec confiance. Sa douceur et l'humilité du premier trait le portent à avoir confiance dans le second, et à le suivre ; il suit le second avec confiance et ils s'élèvent en même temps : c'est là un grand présage heureux. Si l'on parle du second trait au sujet de ses vertus, elles seront la dureté énergique et la justice ; si l'on en parle au sujet de sa force, il est investi de la puissance. Le premier trait étant malléable et négatif et de plus ne recevant aucun accueil sympathique, il ne peut s'élever de lui-même, il suit le sage énergique et juste pour avancer ; c'est employer la voie rationnelle de la dureté énergique et de la justice, et le présage heureux est très considérable et grand.

TSHENG TSE. - Le premier trait emploie la douceur et la soumission et il demeure dans l'infériorité ; c'est de lui que dépend l'humilité. En présence d'un moment d'éléva tion, il est humble envers le second trait positif. Si celui qui consulte le sort est dans ces conditions ; la confiance pourra se développer (s'élever, sheng) et ce sera un grand présage heureux.

819.

Grand présage heureux de la confiance dans l'élévation; le supérieur unit ses tendances.

\footnotetext{
${ }^{1}$ Yun, confiance. Mais ce mot a aussi le sens de «tomber ». On pourrait donc lire, et peut-être avec plus de raison, « descendre et se lever».

${ }^{2}$ C'est la transformation d'un trait plein en trait double qui change le koua khien en koua souen.
} 
TSHENG TSE. - Il unit ses tendances à celles de celui qui est au-dessus de lui et ils s'élè vent ensemble. Le supérieur, c'est le second trait. Il suit le second et s'élève, c'est avoir les mêmes tendances que le second. C'est parce qu'il peut avoir confiance en suivant le sage juste et énergique que le présage heureux est très grand. 


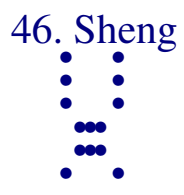

820.

Deuxième trait nonaire : confiance, alors avantage dans l' emploi des sacrifices du printemps ; pas de culpabilité ${ }^{1}$.

TSHENG TSE. - Le second trait représente la dureté énergique positive placée dans l'infériorité ; le cinquième, c'est la douceur négative qui occupe le rang supérieur. Or, employer la dureté énergique et servir la douceur malléable, être positivité et obéir à une négativité, encore qu'il y ait des moments où il en est ainsi, ce n'est cependant pas conforme à la voie rationnelle. Être obscur (manquer d'intelligence) et surveiller ce qui est brillant (intelligent), être fort et servir ce qui est faible, c'est comme être obligé de se plier à la force des choses et ce ne peut pas être une soumission sincère. L'union du supérieur et de l'inférieur, si elle n'est pas basée sur la sincérité, pourrait-elle donc durer longtemps? Serait-il possible qu'elle produisit un effet durable ? Bien que le cinquième trait soit malléable et négatif, cependant, il occupe la situation prééminente ; bien que le second trait soit dur et énergique, il sert le trait supérieur; il doit donc intérieurement conserver la plus extrême sincérité, ne pas emprunter l'élégance de l'ornementation à l'extérieur. La sincérité augmentant à l'intérieur, il ne se servira pas de l'ornementation extérieure de l'apparence, et c'est pour cela que le texte dit : «avantage dans l'emploi des sacrifices du printemps », ce qui signifie ne songer qu'à la sincérité du respect. Depuis l'antiquité, par mi les sujets énergiques et violents qui ont servi des princes faibles et mous on n'en a jamais vu aucun agir sans affecter un appareil orgueilleux. Les sacrifices dont il est question sont les moins importants et ceux pour lesquels on use de la plus grande simplicité. Le texte dit : confiance, alors..., cela signifie que, si la confiance existe, alors il convient de ne pas employer l'ornementation dans les apparences de façon que c'est uni quement par la sincérité qu'on influe sur le supérieur. Dans ces conditions on pourra être sans culpabilité. Puisqu'un sujet énergique et violent sert un prince doux et faible, que de plus c'est dans un moment d'élévation, si tous deux ne s'alliaient point par l'idée de sincérité, pourraient-ils éviter d'être coupables.

821.

\footnotetext{
1 Voir $\$ 803$.
} 
Confiance du deuxième trait nonaire ; il y a de la joie.

TSHENG TSE. — Le second trait peut servir le supérieur par sa confiance et sa sincérité, de sorte que non seulement il achève la voie rationnelle du sujet, sans culpabilité, mais encore il peut parcourir la voie rationnelle de la justice et de l'énergie ; son influence s'étend à tout l'univers ${ }^{1}$, c'est là avoir de la joie. Toutes les fois que le commentaire de la formule symbolique dit qu'il y a des louanges, du bonheur, c'est qu'alors le bon heur atteint les êtres ; toutes les fois qu'il emploie l'expression : il y a de la joie, c'est que les choses dont il s'agit constituent le bien et qu'il y a possibilité de se réjouir. Par exemple, dans le koua tae tshou, lorsqu'il s'agit de la grandeur du présage heureux de la planche placée sur les yeux du jeune buffle, le commentaire de la formule symbolique dit qu'il y a de la joie ; en effet, en plaçant la planche pendant qu'il est jeune l'opération sera facile et on évitera la difficulté de le maintenir par la force, c'est donc qu'il y a possibilité de s'en réjouir ${ }^{2}$.

TSHOU HI. - Pour le sens, voir le koua tsouei ${ }^{3}$.

\footnotetext{
1 «Atteint le ciel et le dessous » ou encore « atteint sous le ciel ».

2 § 486 et 487.

${ }^{3}$ Koua ${ }^{\circ} 45$.
} 


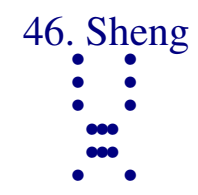

822.

Troisième trait nonaire : s'élever dans un district vide.

TSHENG TSE. - Le troisième trait, avec les aptitudes de la dureté énergique positive, possède la droiture et de plus il est humble; en haut, tous se soumettent, et de plus il est accueilli avec sympathie. Se trouvant dans ces conditions et s'élevant, c' est comme s'il entrait dans un district dépourvu d'hommes ; qui donc le repousserait?

TSHOU HI. - Ce qui est positif est plein, réel ; ce qui est négatif est vide, nul, fictif. De plus, le koua simple khouen comporte l'image symbolique de royaume ou district. Puisque le troisième trait nonaire emploie la dureté énergique de la positivité et se trouve en présence d'un moment d'élévation, puisqu'il monte diriger le koua simple khouen, l'image symbolique et le sens divinatoire sont donc tels.

823.

Monter dans un district vide; rien à redouter.

TSHENG TSE. - Entrer dans un district sans hommes; la marche en avant n'est entra vée par aucune crainte. 


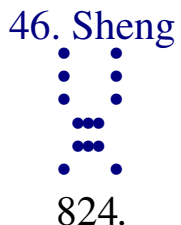

Quatrième trait hexaire : le roi emploie la liberté sur la montagne de Khi; présage heureux; pas de culpabilité.

TSHENG TSE. - Le quatrième trait possède les aptitudes de la malléabilité passive ; au-dessus, il tolère passivement l'élévation du prince ; au-dessous, il tolère passivement le mouvement en avant du trait inférieur ; lui-même reste en place dans le lieu qu'il occupe. C'est parce qu'il est négatif, qu'il occupe un rang qui comporte la douceur malléab le ; c'est parce qu'il est négatif qu'il est dans l’infériorité et qu'il s'arrête dans le lieu où il est placé. Autrefois, lorsque Wen Wang habita au pied de la montagne de Khi, en haut il restait soumis à l'empereur (fils du Ciel) et il voulait simplement l'amener à suivre la voie rationnelle; en bas il était humble et soumis aux sages de l'univers et les poussait à s'éle ver en avançant; quant à lui-même, doux, soumis, modeste et respectueux, il ne sortait pas de son rang. Possédant des vertus aussi éminentes, le roi de Tsheou, dans sa conduite, suivait ces mêmes principes et jouissait de la liberté : si le quatrième trait peut se mettre dans ces mêmes conditions, il jouira de la liberté, le présage sera heureux et, d'ailleurs, il sera sans culpabilité. Les aptitudes du quatrième trait sont certainement bonnes en elles-mêmes; pourquoi donc la formule contient-elle encore les mots pas de culpabilité ? Réponse : bien que les aptitudes du quatrième trait soient bonnes, cependant, sa situation motive un avertissement. Il occupe une situation près du prince; il se trouve dans un moment d'élévation et ne peut pas s'élever d'avantage ${ }^{1}$; s'il s'élevait, le malheur et la cul pabilité seraient évidents. C'est pour cela que la formule avance que, s'il est comme Wen Wang, il sera sans culpabilité et le présage sera heureux. Toutefois, établi dans la situation d'un haut serviteur du roi, il ne peut pas se faire qu'il soit sans embarras au sujet de l'élé vation ${ }^{2}$, au-dessus de lui-même il convient qu'il élève et développe la voie rationnelle du prince ; au-dessous, il doit élever les sages de l'univers, et, quant à lui -même, il restera dans sa propre condition, cependant sa vertu devra s'élever et se développer, sa voie rationnelle devra être libre et pénétrante. Wen Wang seul sut pousser à ses dernières conséquences cette voie rationnelle.

TSHOU HI. - Pour le sens, voir le koua souei ${ }^{3}$.

\footnotetext{
${ }^{1}$ Puisque, au-dessus de lui, il n'y a que le rang du prince.

${ }^{2}$ Qu'il n'ait pas à s'occuper de l'élévation de quelque chose.

${ }^{3}$ Koua $\mathrm{n}^{\circ} 17$.
} 
825.

Le roi use de sa liberté sur la montagne de phi; se conformer aux circonstances.

TSHENG TSE. - Le quatrième trait occupe une situation près du prince et il est en présence d'un moment d'élévation ; c'est celui qui jouit d'un présage heureux et sera sans culpabilité, et cela, parce qu’il possède la vertu de la soumission. Puisqu'il est doux et mal léable et qu'il fait partie du koua khouen, il indique le comble de la passivité. Le cas de Wen Wang sur la montagne de Khi n'est encore rien autre chose qu'un exemple de sou mission aux nécessités du moment: au-dessus, soumission au supérieur; en bas, déférence aux inférieurs ; lui-même se soumettant aux obligations de tous ses devoirs. C'est pour cela que la formule porte les mots : se conformer aux circonstances.

TSHOU HI. - Employer la soumission pour s'élever ; image symbolique de l'action de monter pour accomplir un sacrific e sur une montagne. 


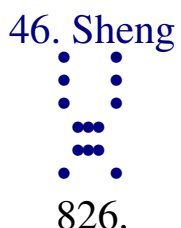

Cinquième trait hexaire : présage heureux de pureté ; monter des degrés.

TSHENG TSE. - Le cinquième trait en s'abaissant rencontre la correspondance sympathique de la dureté énergique et de la justice ; aussi, il peut occuper la situation prééminente et jouir d'un présage heureux. Toutefois sa substance matérielle est essentiellement la douceur malléable de la négativité, de sorte qu'il faut absolument qu' il conserve une pureté et une fermeté parfaite ; c'est ainsi que le présage sera heureux. S'il est inca pable de fermeté et de pureté, il n'aura pas une confiance absolue dans les sages ; il ne les investira pas d'une autorité définitive et entière : comment le présage serait-il heureux ? Degrés, ce qui sert à monter. Investir de l'autorité le sage juste et énergique, le protéger et l'élever, c'est comme s'il le faisait avancer en lui faisant gravir des degrés ; c'est-à-dire en lui en fournissant les moyens et en lui facilitant son mouvement d'ascension. Ceci se rap porte au second trait nonaire, mais tous les sages placés au-dessous du cinquième se servent également de ces degrés pour s'élever ; puisqu'il peut employer les sages, tous s'élè vent également.

TSHOU HI. - Il emploie la négativité et occupe un rang qui comporte la positivité ; il est digne de s'élever et il occupe la situation prééminente. Il doit absolument être capable de droiture et de fermeté et alors il en pourra retirer un présage heureux et s'élever par degrés. Degrés ; ce qui facilite l'ascension.

827.

Le présage heureux de la pureté s'élève par degrés ; la grandeur réussit dans ses tendances.

TSHENG TSE. - Il s'appuie sur les capacités du sage qu'il investit de l'autorité et il est c apable de fermeté dans sa pureté. Étant dans ces conditions et s'élevant, il lui sera pos sible d'arriver à obtenir un ordre parfait dans l'univers ; ses tendances peuvent donc grandement prévaloir. Le malheur pour la voie rationnelle de l'élévation du pri nce, c'est de ne point avoir l'assistance des capacités des sages. Avec cette assistance, il peut s'élever comme en montant des degrés ! 


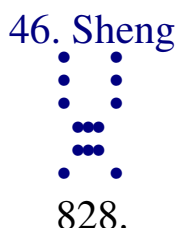

Trait supérieur hexaire : s'élever dans l'obscurité ; avantage dans la pureté qui ne cesse point.

TSHENG TSE. - Ce trait est hexaire et il emploie la négativité pour se maintenir au dernier degré de l'élévation; il est aveuglé et manque de discernement dans son élévation

c'est celui qui sait avancer et qui ne sait s'arrêter. Son action manque absolument d'intel ligence (clarté). Toutefois une volonté qui cherche sans cesse à s'élever peut parfois être employée à des choses pures et correctes qui ne souffrent pas d'interruption, et, dans de tels cas, elle sera opportune et convenable. L'homme doué apporte une activité journalière et une activité incessante à perfectionner sa vertu de droiture parfaite ; il s'effo rce sans cesse, avec une volonté persistante analogue à celle du trait supérieur hexaire. En employant une telle volonté à un tel but, ce sera un avantage. S'il s'agit de changer la volonté insatiable de l'homme inférieur, qui ne sait se limiter, pour l'am ener à avancer dans la voie de la vertu, qu'est -ce qui pourrait être aussi bien?

TSHOU HI. - Avec sa négativité il occupe le dernier degré de l'élévation ; c'est celui qui est aveuglé, sans discernement, et qui ne sait s'arrêter. Si celui qui consulte le sort rencontre ce trait ${ }^{1}$, rien ne lui sera avantageux. Mais il pourra renverser l'effet de la ténacité de sa propre volonté en l'appliquant à un but extérieur ${ }^{2}$ et en l'étendant uniquement et sans cesse à ce qui est légitime et correct.

829.

L'obscurité s'élève et est en haut ; diminution sans richesse.

TSHENG TSE. - Obscurité et aveuglement au dernier degré de l'élévation ; monter sans savoir s'arrêter ; il ne peut y avoir que dissolution et destruction. Comment serait-il encore possible qu'il y eût de nouveau

\footnotetext{
${ }^{1}$ En cherchant une sentence au hasard.

${ }^{2}$ En ne recherchant pas son avantage personnel.
} 
augmentation $(y i)^{1}$. Sans richesse, ne plus augmenter davantage. Du moment où l'élévation est à son extrême limite, il faut rétrograder et ne plus avancer ${ }^{2}$.

\footnotetext{
${ }^{1}$ Koua ${ }^{\circ} 42$.

${ }^{2}$ II est bien évident par la lecture du commentaire que la traduction du texte de Khong Tse n'est pas conforme au sens admis et à la lecture traditionnelle. Un Chinois instruit lira "monter aveuglément et se trouver dans une position supérieure; dissolution, pas de richesse ». Pour justifier ma traduction je me borne à rappeler que c'est en quelque sorte le mot à mot et à donner du caractère ming, qui est évidemment le plus important pour le sens de la sentence, les explications suivantes, tirées du Tse tien. "Obscurité », formé des caractères jour, soleil ; six ; recouvrir d'un voile. Après seize jours la lune commence à diminuer ; exprime encore la nuit. (Shuo Wen). jeunesse ; la jeunesse c'est l'obscurité, le manque de lumière (Yi ya): telles sont les valeurs attribuées à ce caractère ming par les deux plus antiques dictionnaires chinois. Le premier membre de phrase de la sentence est absolument net et précis; les trois derniers caractères, au contraire, ne présentent qu'un sens forcé. On pourrait dire qu'ils ne sont là que pour masquer le sens trop clair de ceux qui les précèdent, et pour entretenir l'équivoque.
} 


\section{Khouen}

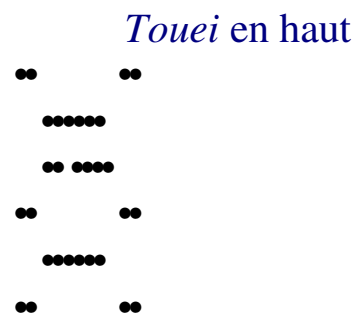

Khan en bas

830. Khouen; liberté ; pureté ; présage heureux du grand homme; pas de culpabilité ; on parle, pas de confiance.

[]

834. Premier trait hexaire : le fond repose misérablement sur un tronc d'arbre dépouillé ; entrer dans une vallée sombre; trois années sans voir.

$*$

836. Deuxième trait nonaire : misère dans le vin et la nourriture, le tablier rouge (vermillon) commence à venir; avantage dans l'emploi de la liberté et des sacrifices ; en entreprenant, présage malheureux; pas de culpabilité.

838. Troisième trait hexaire : misère sur la pierre; appuyer sur des chardons ; entrer dans la chambre intérieure ; ne pas voir l'épouse, présage malheureux.

840. Quatrième trait nonaire : venir tout doucement; misère dans le char d'or ; appréhension; il y a une fin.

*

842. Cinquième trait nonaire: amputer le nez et les pieds; misère dans le tablier rouge cramoisi ; alors doucement il y a satisfaction ; avantage dans l'emploi des sacrifices.

$*$

844. Trait supérieur hexaire; misère du réseau de lianes et de l'équilibre sur une pointe élevée ; il dit : mouvement, regrets ; il y a des regrets ; en entreprenant, présage heureux.

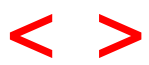




\section{Khouen.}

TSHENG TSE. - Khouen. «L'Ordre des koua » dit : «En montant sans cesse le malheur doit nécessairement survenir, aussi le koua sheng est suivi immédiatement du koua khouen. » Sheng exprime l'action de s'élever en montant de bas en haut; d'en dessous s'élever au -dessus; avancer en employant sa force; si cette action ne s'arrête point, il y a nécessairement malheur. C'est pour cela que le koua khouen est placé immédiatement à la suite du koua sheng. Le caractère khouen exprime le sens de tristesse et de misère ; il est formé du koua simple touei en haut et du koua simple khan en bas. Lorsque l'eau s'étend par -dessus le marais, il y a de l'eau dans le marais ; ici elle est sous le marais, il y a de l'eau dans le marais ; ici elle est sous le marais, c'est donc l'image symbolique de l'arbre sec et altéré, sans eau, et c'est considéré comme exprimant le sens de misère et de malheur. De plus, puisque dans le koua touei la négativité est au rang supérieur, puisque dans le koua khan la positivité occupe un rang inférieur, puisque le trait supérieur hexaire est au-dessus de leur positivité et que le second trait nonaire tombe entre deux négativités, c'est toujours la douceur malléable de la négativité recouvrant et étouffant la dureté énergique positive, et c'est à cause de cela que c'est considéré comme exprimant la misère et le mal heur. Lorsque l'homme doué est recouvert et caché par l'homme inférieur, c'est un moment de misère et de malheur. 


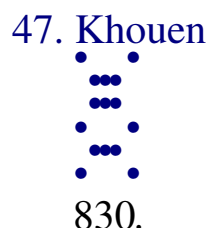

Khouen ; liberté ; pureté ; présage heureux du grand homme ; pas de culpabilité ; on parle, pas de confiance.

TSHENG TSE. - Les aptitudes exprimées par ce koua comportent la misère et la faculté de liberté ; si d'ailleurs on possède encore la droiture et la pureté, cela exprimera la voie rationnelle suivant laquelle le grand homme se place pendant le malheur et la misère, aussi le présage peut être heureux, et il n'y a point de culpabilité. Lorsque le grand homme choisit sa place pendant une période de misère, ce n'est pas seulement sa propre voie qui présente un présage heureux ; se réjouir (des lois) du ciel, se contenter de la destinée, c'est là ne pas perdre le présage heureux ; à plus forte raison, se placer suivant ce qui est le bien, en profitant de l'opportunité du moment, doit de nouveau comporter la grandeur et l'éclat. «Il y a des paroles et pas de confiance »: lorsqu'on parle pendant les moments de misère, qui donc aurait confiance ?

TSHOU HI. - Khouen a le sens d'être dans la détresse sans pouvoir s'en relever soi-même. La dureté énergique du koua simple khan est recouverte et cachée par la douceur malléable dans le koua simple touei; le second trait nonaire est recouvert et caché par les deux négativités; le quatrième et le cinquième traits sont recouverts et cachés par le trait supérieur hexaire, ce qui fait qu'il est considéré comme exprimant le malheur. Khan, péril ; touei, satisfaction : être placé dans le péril et en être satisfait; cela exprime donc que, bien que le corps soit dans la misère, la voie rationnelle ${ }^{1}$ est libre. Le second et le cinquième traits sont énergiques et justes, de plus ils présentent l'image symbolique du grand homme ${ }^{2}$. Si celui qui consulte le sort est placé dans la misère et est capable de liberté, il se conforme à la droiture : Qui pourrait être dans ces conditions, sinon un grand homme ? Aussi la formule dit: pureté, (perfection). Elle ajoute encore que, si le grand homme est intelligent (brillant, éclairé), l'homme inférieur dénué de droiture est incapable de prévaloir. Il parle et on ne le croit pas ; c'est encore un avertissement qu'il doit surtout rester dans l'obscurité et se taire ; il ne doit point toujours parler, ce qui lui attirerait un surcroît de misères.

\footnotetext{
${ }^{1}$ La pensée.

${ }^{2}$ Le prince et le sage.
} 
831.

Le commentaire de la formule déterminative dit:

Khouen ; la dureté énergique recouvre ${ }^{1}$.

TSHENG TSE. - Ce qui fait que le koua est considéré comme exprimant la misère, c'est que la dureté énergique y est recouverte et cachée (yien) par la douceur malléable ; elle tombe en bas ${ }^{2}$ et elle est recouverte (yien) en haut, ce qui constitue la pauvreté. Tomber dans l'abîme c'est encore être recouvert, caché. L’homme doué, énergique et positif est caché et effacé par l'homme inférieur malléable et négatif : c'e st la voie rationnelle de l'homme doué dans un moment de misère et d'obstacles.

TSHOU HI. - Explication du nom du koua au moyen de la substance de ce koua.

\section{2.}

Péril par la satisfaction; misère, mais sans perte de ce qui est liberté; n'est-ce pas l'homme doué seul ? Présage heureux du grand homme doué de Pureté, par la dureté énergique et la justice. Il y a des paroles, pas de confiance : compter sur la bouche, c'est le danger.

TSHENG TSE. - Définition de la voie rationnelle du malheur au moyen des aptitudes des koua simples. En bas péril et en haut satisfaction ; c'est être placé dans le péril et pouvoir être satisfait. Bien que placé au milieu des périls et des difficultés de la misère et du malheur, en cherchant le bonheur dans le ciel ${ }^{3}$ et en se contentant de l'exécution du devoir, il obtient par lui -même la satisfaction et le contentement. Bien que le moment soit malheureux, il se place sans manquer au devoir, de sorte que sa voie rationnelle se fraye elle-même sa liberté : celui qui peut être dans ces conditions, que serait-il sinon un homme doué ? Si le moment comporte la misère et si, au contraire, la liberté subsiste, bien que le corps soit libre, c'est alors la voie rationnelle qui souffre et est entraînée par cette misère. Les appellations homme doué ou

\footnotetext{
${ }^{1}$ On trouve bien dans le Tse tien que le texte signifie : la dureté énergique «est comprimée », mais cette lecture est précisément tirée du commentaire du Yi king. Le caractère yien, du texte, est expliqué comme suit dans le Shuo wen: "de la limite (horizon) en venant par l'Orient, c'est-à-dire croissant, est exprimé par yien ». Si l'on adopte cette valeur on lira : la dureté énergique croit.

${ }^{2}$ Khan, chute, abîme.

${ }^{3}$ Les lois du ciel.
} 
grand homme sont équivalentes; être capable de pureté au milieu de la misère, c'est ce qui fait le présage heureux du grand homme, et c'est l'effet de la voie rationnelle de son énergie active et de sa justice : tel est le cas pour le second et le cinquième traits. Sans la dureté énergique et la justice, ces deux traits perdraient leur droiture. Si quelqu'un parle pendant qu'il est dans le malheur, personne n'a confiance en ses paroles ; aussi vouloir éviter la misère par le secours de la bouche, c'est ce qui conduit à la perte. C'est parce qu'il s'agit de satisfaction dans la misè re que la formule contient l'avertissement au sujet de ceux qui comptent sur leur bouche.

TSHOU HI. - Explication de la formule du koua au moyen de la substance et des vertus des koua simples.

833.

Le commentaire de la formule symbolique dit: Marais sans eau : misère : l' homme doué pour suivre la destinée donne libre cours à ses tendances.

TSHENG TSE. - Marais sans eau, image symbolique de la misère et de la détresse. L'homme doué en présence d'un moment de misère, et après qu'il a épuisé toutes les voies de la prévision et de la circonspection, n'a cependant pas pu l'éviter : c'est donc que telle est la destinée. Il doit suivre sa destinée en donnant libre cours à ses tendances. Sachant que c'est là une conséquence naturelle de sa destinée, il obviera aux malheurs, il n'en fera pas une cause d'agitation pour son cour et se contentera de pratiquer le devoir. S'il ne reconnaissait pas que c'est le résultat de sa de stinée, il craindrait le péril et tomberait dans le péril et le malheur ; ce qu'il conserve serait perdu, comment donc pourrait-il donner cours à ses tendances vers le bien ?

TSHOU HI. - L'eau coule en descendant de sorte que la sécheresse envahit le marais, et c'est pourquoi le texte dit : marais sans eau. Suivre la destinée, c'est comme si l'on disait « imposer la destinée » ${ }^{1}$; cela exprime l'idée d'attendre quelque chose d'autrui et ne point l'avoir. Pouvant être ainsi, bien que la misère survienne, il y aura néanmoins liberté.

\footnotetext{
${ }^{1}$ Le caractère traduit par le mot suivre a le sens de « survenir ; jusqu’à ».
} 


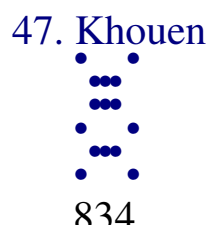

\section{Premier trait hexaire : le fond repose misérablement sur un tronc d'arbre} dépouillé ; entrer dans une vallée sombre ; trois années sans voir.

TSHENG TSE. - Ce trait hexaire, avec sa douceur malléable négative, se place dans la position la plus humble, de plus il se trouve au bas du koua simple khan qui exprime le péril : c'est celui qui est dans la misère sans pouvoir s'en tirer lui-même. Il faut absolument qu'il obtienne l'aide et l'assistance de l'homme énergique et intelligent placé au-dessus de lui, et alors, il pourra remédier aux maux dont il souffre. Le premier trait et le quatrième sont considérés comme se correspondant sympathiquement; le quatrième trait nonaire employant la positivité et demeurant dans une position négative est considéré comme sans droiture; il perd son énergie et il est sans justice. De plus, il est en présence de la misère, sous l'oppression de la négativité ; comment pourrait-il porter remède aux misères d'autrui ? Il en est de lui comme d'un arbre dont le tronc est dépouillé et sous lequel les êtres et les choses ne peuvent trouver d'ombrage et d'abri. Un arbre dépouillé, c'est un tronc dépourvu de branches et de feuilles, le quatrième trait est voisin de la situation du prince; dans d'autres koua, cela n'est pas considéré comme exprimant le manque d'assistance ; c'est parce qu'il se trouve dans le koua khouen, qui exprime la misère, qu'il

ne peut protéger les êtres et c'est pourquoi il est considéré comme un tronc dépouillé. Le fond, c'est ce sur quoi un objet repose, le derrière. Le fond repose misérablement sur un tronc dépouillé; cela exprime qu’il n'a aucune protection et qu'il ne peut être en repos dans la situation qu’il occupe. S’il l'occupait en repos, il ne serait pas misérable. Entrer dans une vallée ténébreuse ; un homme mou et négatif, incapable de se contenter de ce qui lui arrive ; outre qu'il ne peut éviter la misère, il est de plus en plus aveuglé et il s'agite mal à propos : il entre dans une profonde misère. Vallée ténébreuse, un lieu de profonde obscurité. Au moment où il entre de plus en plus dans la misère, il n'a pas la force néces saire pour s'en tirer lui -même, de sorte qu'il reste jusqu'à trois ans sans voir ; c'est la misère définitive. Ne point voir, c'est ne pas rencontrer ce qui constitue la liberté.

TSHOU HI. - Le fond, littéralement le derrière (fesses), le fond d'un objet. Misère sous un tronc dépouillé ; souffrir et ne pas pouvoir jouir du repos. Le premier trait hexaire, avec sa malléabilité négative est placé au bas du koua khouen. Il est dans l'obscurité la plus profonde, aussi tels sont le sens divinatoire et l'image symbolique. 
835.

Entrer dans une vallée sombre; sombre, pas clair.

TSHENG TSE. - Sombre, pas brillant ; cela exprime quil entre de plus en plus dans l'obscurité ; il tombe de lui-même dans une profonde misère ; s'il était éclairé, il n'en arri verait pas jusqu’à y tomber. 


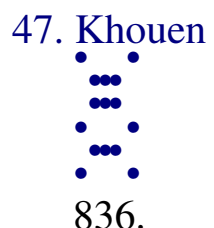

Deuxième trait nonaire : misère dans le vin et la nourriture, le tablier rouge (vermillon) commence à venir ; avantage dans l'emploi de la liberté et des sacrifices ; en entreprenant, présage malheureux; pas de culpabilité.

TSHENG TSE. - Le vin et la nourriture, ce que les hommes désirent et ce qui leur permet d'étendre leur bienveillance. Le second trait emploie les aptitudes résultant de la dureté énergique et de la justice et il se place pendant un moment de misère; c'est un homme doué qui se contente de ce qui lui arrive. La pauvreté, la gêne, les dangers, rien n'émeut et n'agite son cour, il ne se lamente point parce qu'il est misérable ; ce qu'il considère comme misérable, c'est seulement d'être malheureux à cause de ses désirs. Ce que l'homme doué désire, c'est d'étendre l'influe nce de ses vertus aux peuples de l'uni vers et de remédier aux misères de l'univers : le second trait ne peut pas encore satisfaire ce désir d'étendre sa bienveillance, de sorte qu'il est considéré comme malheureux à

cause du vin et de la nourriture. Si le grand homme, l'homme doué, méditent sur la voie rationnelle et sont en bas dans la misère, il faudra qu'il survienne un prince possédant la voie rationnelle, qui les appelle et les emploie, et alors seulement ils pourront manifester les sentiments qui restent cachés en eux-mêmes. Le second trait emploie les vertus de l'énergie et de la justice, il est misérable et dans l'infériorité ; en haut il y a le prince énergique et juste représenté par le cinquième trait nonaire : leur voie est la même, leurs vertus s'accordent; il arrivera nécessairement qu'ils s'appelleront mutuellement, c'est pourquoi la formule dit que le tablier rouge commence à venir. Commencer à venir, commencer à exister et venir ; tablier rouge ; pièce du costume du roi, qui sert à cacher les cuisses, ce qui constitue le sens d'aller et venir. Avantage dans l'emploi des offrandes ${ }^{1}$ et des sacrifices ; offrandes et sacrifices, expriment l'idée de liberté de communication par la plus extrême sincérité, comme dans les offrandes et les sacrifices. Du moment où la vertu est sincère, elle peut, par elle-même et sans efforts, émouvoir le supérieur. Depuis l'antiquité, lorsque le sage intelligent, misérablement placé, s'est trouvé dans l'éloignement et la misère et qu'à la fin sa vertu lui a fait un e renommée, puis que finalement on a eu recours à ses lumières, ça a toujours été uniquement parce qu'il a conservé intacte la plus extrême sincérité. En entreprenant, présage malheureux sans culpabilité; dans un moment de misère, si l'on ne sait se conte nter de sa situation avec la plus entière sincérité

\footnotetext{
${ }^{1}$ Le commentateur lit « offrande » au lieu de « liberté ».
} 
d'intentions, en attendant la destinée, si l'on entreprend et si l'on cherche à obtenir, on brave le danger et on ne rencontre que le présage de malheurs : ce malheur on se l'attire à soi -même, à qui pourrait-on l'imputer ? Entreprendre sans tenir compte du moment, c'est ne pas se contenter de ce qu'on a et être agité par la misère. En perdant les vertus de l'énergie et de la justice, on attire soi-même le présage de malheurs et les regrets; comment et sur qui en rejetterait-on la culpabilité ? Dans tous les koua, lorsque le second trait et le cinquième se correspondent sympathiquement par la négativité et la positivité, c'est un présage heureux ; ce n'est que dans les koua siao tshou et khouen que cette circonstance est considérée comme un inconvénient de la négativité, de sorte que, ayant la même voie rationnelle, ils s'appellent mutuellement. Dans le koua siao tshou c'est la positivité qui est arrêtée par la négativité, tandis que dans le koua khouen la positivité est recouverte et cachée par la négativité.

TSHOU HI. - Misère dans le vin et la nourriture, cela exprime l’idée de l'oppression pénible causée par l'absorption trop considérable d'aliments. Le vin et la nourriture, c'est ce que l'homme désire, cependant l'ivresse et l'excès de nourriture au-delà de ce qui est nécessaire sont, au contraire, ce qui lui cause de la souffrance; le tablier rouge commence à venir : le supérieur lui répond sympathiquement. Le second trait nonaire possède les vertus de la dureté énergique et de la justice; puisqu'il se trouve dans un moment de misère, bien qu'il n'y ait aucun présage malheureux et nuisible, qu'au contraire la misère consiste en ce qu'il possède en trop grande quantité ce dont il a besoin, il présente donc cette image symbolique, et le sens divinatoire exprime l'avantage de l'emploi des offrandes et des sacrifices. S'il entreprend d'agir, ce ne sera point le moment, de sorte que le présage sera malheureux, mais que cependant il n'y aura pas de culpabil ité contre le devoir.

837.

Misère du vin et de la nourriture ; au-dedans il y a bonheur ${ }^{1}$.

TSHENG TSE. - Bien qu'il soit misérable dans ce qu'il désire, il ne peut pas encore étendre sa bienveillance aux hommes; toutefois il conserve ses vertus de dureté énergique et de justice. Il faut qu'il puisse arriver à jouir de la liberté et quỉl obtienne le bon heur. Bien qu'on suppose que le moment ne comporte pas encore la liberté d'expansion et de communication, comme il conserve sa vertu de justice, la voie de l'homme doué est encore libre, c'est en cela que consiste le bonheur.

\footnotetext{
${ }^{1}$ Ou bien, peut-être, « la justice a des louanges ».
} 


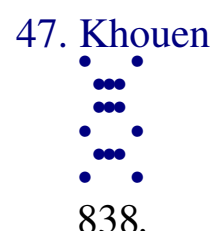

Troisième trait hexaire : misère sur la pierre; appuyer sur des chardons ; entrer dans la chambre intérieure; ne pas voir l'épouse, présage malheureux.

TSHENG TSE. - Le troisième trait hexaire, avec ses caractères de malléabilité négative sans justice ni droiture est placé au plus fort du péril et il emploie la dureté énergique : il occupe un rang positif et emploie la dureté énergique, c'est ce qui est absolument mal se placer dans un moment de malheur. La pierre, c'est quelque chose de dur et de lourd, difficile à supporter. Le chardon est quelque chose d'épineux sur quoi l'on ne peut s'appuyer. Le trois ième trait emploie l'énergie dans le péril et monte en avançant, de sorte que, comme deux traits positifs sont au-dessus, ses forces sont incapables de l'emporter sur eux, sa dureté ne peut les entamer, et il aggrave sa propre misère ; c'est la misère de la pierre. À cause de ses qualités qui ne sont pas bonnes, et demeurant au-dessus du second trait nonaire énergique et juste, il est mal à son aise comme s’il marchait sur des épines ; il appuie sur des chardons. Puisqu'en avançant ou en reculant il aggrave sa misère, il voudrait se contenter de sa situation et il le peut de moins en moins. Chambre intérieure, le lieu de repos ; l'épouse, la personne de qui dépend le repos. Reconnaissant l'impossibilité d'avancer ou de reculer, et voulant se contenter de sa situation, il perd encore ce qui constitue son repos. Il lui est également impossible d'avancer, de reculer, et de demeurer en place : il n'a plus qu'à mourir, donc le présage malheureux est évident. Les formules annexées disent : "Il n'a pas lieu d'être misérable et il est misérable, son nom doit être diffamé ; il ne doit pas appuyer et il appuie, sa personne doit nécessairement être en péril ; le moment de sa mort est sur le point d'arriver ; l'épouse, ce qui est visible, est vicieuse. » Les deux positivités ne doivent pas être bravées, il les brave et il en recueille la misère pour lui-même ; c'est ne pas avoir lieu d'être misérable et se rendre misérable. Son nom est diffamé, ce qu'il fait est mal. Le troisième trait est au-dessus du second, c'est certainement s'appuyer dessus. Cependant, s'il pouvait être modeste et doux pour s’incliner au-dessous du second trait, il n'en éprouverait pas de mal ; au lieu de cela il emploie la dureté et la violence pour le fouler aux pieds, de sorte qu'il n'est pas en repos et qu'il en recueille encore de la misère, comme sil appuyait sur des chardons ou des ronces. Dans ces conditions, l'instant de sa mort est sur le point de survenir ; comment pourrait-il voir celle de qui dépend son repos.

TSHOU HI. - Malléabilité négative et manque de justice et de droiture, c'est pourquoi telle est l'image symbolique, tandis que le sens divinatoire sera 
un présage malheureux. La pierre désigne le quatrième trait ; les chardons, le second trait; la chambre intérieure, c'est-à-dire le troisième rang; enfin l'épouse, c'est le sixième. Le sens est complet dans les formules annexées.

839.

Appuyer sur des chardons, fouler la dureté énergique sous les pieds ; entrer dans la chambre intérieure; ne pas voir l'épouse ; chance défavorable.

TSHENG TSE. - Appuyer sur des chardons, c'est -à-dire marcher sur la dureté énergique du second trait nonaire ; ne pas être en repos, être couché sur des épines. Chance défavorable, début infiniment petit de ce qui n'est pas le bien. Perdre ce qui donne le repos, c'est le résultat de ce qui n'est pas le bien ; c'est pour cela que la formule dit : ne pas voir l'épouse, chance défavorable. 


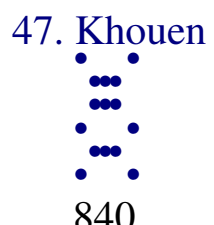

Quatrième trait nonaire : venir tout doucement ; misère dans le char d'or ; appréhension; il y a une fin.

TSHENG TSE. - La force seule est insuffisante, de là résulte la misère. La voie de la liberté, dans un état misérable, consiste nécessairement dans l'aide et l'assistance. Dans un moment de misère, le supérieur et l'inférieur s'appellent mutuellement ; c'est la conséquence naturelle de la raison d'être des choses. Le quatrième trait et le premier sont ceux qui se correspondent sympathiquement, mais le quatrième trait est dans la misère à cause de son manque de justice et de droiture; ses aptitudes sont insuffisantes pour remédier à la misère d'autrui. Le quatrième trait se presse contre le second, qui luimême possède les aptitudes de la dureté énergique et de la justice, ce qui est suffisant pour porter secours à la misère, de sorte qu'il convient quill soit suivi par le premier. Or, ou métal, dureté ; char, ce qui sert à transporter. Le second trait à cause de son énergie, et se mouvant en bas, se supporte luimême, c'est pour cela que la formule emploie les mots char d'or. Le quatrième trait veut suivre le premier et il en est empêché par le second, de sorte qu'il vient en retard, lentement et tout doucement : c'est la misère dans le char d'or. Celui avec qui il sympathise le suspecte de mépris et il en suit un autre ; sur le point de suivre, il est pris d'incertitude et il n'ose se hâter d'aller en avant: comment pourrait-il ne pas en éprouver de la confusion et de l'appréhension ? Avoir une fin, cela exprime que le but est correct et légitime. Le premier et le quatrième se correspondent directement ; finalement ils se suivront certainement l'un l'autre. L'épouse du pauvre érudit, le ministre d'un royaume faible, se contentent chacun de ce qui est droit et légitime; sils profitaient de la force inhérente à quelque autre objet, pour en profiter, ce serait ce quil y aurait de plus odieux ${ }^{1}$ et ce que personne ne pourrait supporter. Le second et le quatrième traits emploient tous deux la positivité et se maintiennent dans un rang qui comporte la négativité ; de plus, le second déploie les aptitudes de la dureté énergique et de la justice, ce qui lui permet de remédier à la misère. Celui qui occupe un rang négatif préfère la douceur ; celui qui possède la justice ne manque pas aux convenances imposées par la dureté ou la douceur.

TSHOU HI. - Le premier trait hexaire est celui qui correspond sympathiquement au quatrième trait nonaire par la droiture ; le quatrième trait nonaire est placé dans une situation quill ne mérite pas, il ne peut pas secourir

\footnotetext{
${ }^{1}$ Trahir le faible et suivre le fort.
} 
les êtres. De plus, le premier trait hexaire commence à être dans la misère au-dessous de lui, et il en est séparé par le second trait nonaire, de sorte que telle est l'image symbolique. Toutefois, le vice ne l'emporte pas sur le droit, aussi, bien que le sens divinatoire exprime qu'il y a des motifs d'appréhen sion, il y aura cependant certainement une fin. Le char d'or, c'est le second trait nonaire. L'image symbolique n'est pas encore clairement expliquée ; peut-être, le koua simple khan a-t-il l'image symbolique de la roue d'un char.

841.

Venir tout doucement, les tendances sont vers le bas; bien que ne méritant pas la situation, il y a alliance.

TSHENG TSE. - Le quatrième trait correspond au premier et en est séparé par le second; ses tendances le portent à descendre et à l'appeler, aussi peu à peu, lentement, il vient. Bien qu’il ne mérite pas la situation quill occupe et qu'il soit considéré comme n'exprimant pas le bien, cependant celui qui lui correspond directement s'allie avec lui, de sorte qu'il y a une fin ${ }^{1}$.

\footnotetext{
${ }^{1}$ Le résultat final n'est pas mauvais.
} 


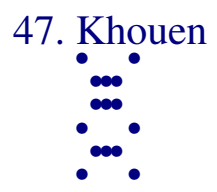

842.

Cinquième trait nonaire : amputer le nez et les pieds ; misère dans le tablier rouge cramoisi ; alors doucement il y a satisfaction; avantage dans l'emploi des sacrifices.

TSHENG TSE. - Couper le nez; blessure dans une partie supérieure (du corps) ; enlever les pieds, les amputer, blessure dans une partie inférieure. Le haut et le bas sont également envahis et recouverts par la négativité, ce qui constitue la blessure et le mal, image symbolique de l'amputation $\mathrm{d} u$ nez et des pieds. Le cinquième rang est la droiture du prince; la misère du prince provient de ce que le supérieur et les inférieurs ne s'allient point entre eux. Tablier rouge (cramoisi), vêtement inférieur du sujet, comporte le sens de quelque chose qui va et vient. La misère du prince provient de ce que l'univers ne vient pas à lui ; si l'univers entier venait vers lui, ce ne serait plus la misère. Bien que le cinquième trait soit dans la misère, cependant, il possède les vertus de la dureté énergique et de la justice ; en bas il y a le sage énergique et juste représenté par le second trait nonaire; leurs vertus sont identiques, leur voie est la même, peu à peu ils doivent nécessairement se répondre l'un à l'autre et venir pour remédier également aux misères de l'univers : au début il y a donc misère, mais peu à peu viennent la joie et la satisfaction. Avantage à employer les sacrifices, tout ce qui concerne les divers genres de sacrifices doit être réglé par le plus sincère respect et alors ces mêmes sacrifices pourront devenir une source de bonheur. Le prince, se trouvant en présence d'un moment de misère, doit méditer sur les misères dont souffre l'univers et appeler les sages de l'univers ; s'il sacrifie comme il vient d'être dit, en développant la sincérité de son respect, il pourra influencer les sages de l'univers entier et, ensuite, remédier à la misère de l'univers. Le cinquième trait et le second ont les mêmes vertus et cependant ce commentaire dit que le supérieur et l'infé rieur sont sans alliance ; comment cela peut-il être ? Réponse : La correspondance mutuelle entre la négativité et la positivité est une correspondance naturelle entre l'époux et l'épouse, entre les os et la chair. Or, le cinquième et le second sont tous deux des traits positifs ; puisque leur vertu d'énergie et de justice est la même et qu'ils se correspondent mutuellement, ils représentent ceux qui s'appellent et qui, ensuite, sont réunis. C'est, par exemple, la réunion par le devoir du prince et du sujet, ou des amis entre eux. Au moment où la misère commence, comment y aurait-il alliance entre le supérieur et l'inférieur ? S’il y avait alliance, il n’y aurait pas de misère, aussi la réunion ne se fait que lentement et ensuite vient la satisfaction. Au sujet du second trait la formule parle d'offrandes et de sacrifices, au sujet du cinquième elle parle de sacrifices ; l'idée fondamentale 
est qu'il convient d'employer la plus parfaite sincérité, et que c'est ainsi qu'on obtiendra le bonheur; d'une façon générale les trois termes employés pour désigner les sacrifices peuvent être pris l'un pour l'autre ; si au contraire on veut distinguer [a] exprime le sacrifice à l'esprit du ciel ; [b] le sacrifice à l'esprit de la terre ; [c] le sacrifice aux mânes de l'homme ${ }^{1}$. Le cinquième rang marquant la situation du prince, il est question de sacrifices à l'esprit du ciel ; le second trait marque le rang inférieur de sorte qu'il n'est question que des sacrifices que la loi religieuse permet à chacun.

TSHOU HI. - Ablation du nez et amputation du pied, blessures en haut et en bas ; le bas étant déjà blessé, il n'y a plus lieu d'employer le tablier rouge cramoisi et, au contraire, cela exprime la misère. Le cinquième trait nonaire est en présence d'un moment de misère ; en haut il est recouvert par la négativité, en dessous, il marche sur la dureté énergique, de sorte qu'il présente cette image symbolique. Toutefois, il est énergique et juste et de plus il fait partie de la substance du koua simple touei qui exprime la satisfaction, de sorte quill pourra attendre et arriver à être satisfait. Le sens divinatoire est complet dans limage symbolique. De plus comme il y a avantage dans l'emploi des sacrifices, après un long espace de temps on obtiendra le bonheur.

843.

Ablation du nez et amputation du pied: les tendances ne prévalent pas encore; alors lentement il y a satisfaction, par la justice et la rectitude; avantage à employer les sacrifices aux esprits du ciel et de la terre; recevoir le bonheur.

TSHENG TSE. - Il commence à être recouvert et caché par la négativité, il n'a d'allian ce ni en haut ni en bas ; c'est le moment où il commence à être dans la misère et où ses tendances ne peuvent pas encore prévaloir. Lentement il arrivera à la satisfaction, en employant la voie rationnelle de la justice et de la rectitude. Ayant le concours du sage qui se trouve en bas, ils portent ensemble remède à la misère. Le texte n'emploie pas les mots « justice et droiture » et « réunion au second trait »; il dit : « rectitude », c'est-à-dire ce qui est convenable et opportun; entre rectitude et droiture il y a une légère différence. En épuisant la sincérité de l'idée, comme en offrant des sacrifices, pour appeler à lui les sages de l'univers, il pourra délivrer l'univers des maux dont il souffre et jouir de la félicité et du bonheur.

\footnotetext{
${ }^{1}$ Le texte porte «liberté » et non pas « offrande»; voir $\S 836$. Le mot traduit par mânes signifie littéralement l'âme négative.
} 


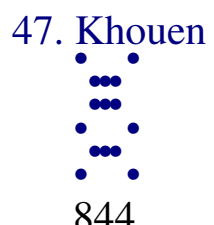

Trait supérieur hexaire; misère du réseau de lianes ${ }^{1}$ et de l'équilibre sur une pointe élevée ${ }^{2}$; il dit ${ }^{3}$ : mouvement, regrets; il y a des regrets; en entreprenant, présage heureux.

TSHENG TSE. - Quand les êtres sont parvenus à leur développement extrême, ils doivent revenir en sens opposé ; quand les choses sont arrivées à leur extrême limite, il faut qu'elles se modifient ; donc, du moment où la misère est à son comble, la raison d'être naturelle exige qu'elle se modifie. Les deux caractères traduits par « réseau de liane » expriment des ligaments entrelacés. Les huitième et neuvième caractères expriment une circonstance dans laquelle le mouvement est périlleux. Ce trait hexaire est placé à l'extrê me limite du koua khouen, il exprime ce qui est enlacé et lié par la misère et ce qui occupe le lieu le plus élevé et le plus périlleux : c'est la misère par le réseau de liane et l'équilibre instable ${ }^{4}$. Mouvement, regrets : aussitôt qu'il y a mouvement, il y a les regrets ; rien qui ne soit misérable. Avoir des regrets, c'est-à-dire au sujet des erreurs déjà commises et avant toute action coupable. Le caractère yue signifie se dire à soi-même ${ }^{5}$. S'il peut ainsi se dire que tout mouvement entraîne des regrets, qu'il doit modifier préalablement les causes par lesquelles il y a des regrets, s'il peut le regretter et ensuite entreprendre, il obtiendra un présage heureux; la misère étant à son maximum, en entreprenant quelque chose, il sortira de la misère, de sorte que le présage sera heureux. Le troisième trait, à cause de sa négativité et se mouvant au rang supérieur dans le koua simple inférieur, comporte un présage malheureux; le trait supérieur occupe le rang le plus élevé dans un koua parfait et ne comporte pas de présage malheureux. Comment cela peut-il être ? Réponse: Le troisième trait occupe un rang qui comporte la dureté énergique et il se place dans le péril ; il est dans le koua qui exprime la misère et il emploie l'énergie en bravant le péril, de sorte que le présage est malheureux. Le trait supérieur emploie la douceur et il se trouve dans le koua simple qui exprime la satisfaction, de sorte qu'il est simplement considéré comme exp rimant le comble de la misère. La misère étant parvenue à son comble, il y a

\footnotetext{
${ }^{1}$ Dans le texte il s'agit d'une liane particulière dont je ne puis traduire le nom.

${ }^{2}$ Les termes du texte ne peuvent être traduits que d'après les commentaires. Le sens est à peu près inconnu.

${ }^{3}$ Traduit d'après le com mentaire. Cela n'a aucun sens ; il faudrait peut-être lire «le soleil se meut, regrets ».

${ }^{4}$ Cette figure serait assez exactement rendue en disant : « danger de marcher sur la corde raide ».

${ }^{5}$ Il signifie « dire ». Le caractère $y i$ signifie « soleil », « jour ».
} 
nécessairement une voie rationnelle de modification de cette misère même. Le trait supérieur, dans le koua khouen comme dans le koua tshouen, occupent tous deux le dernier rang du koua et sont en correspondance sympathique; dans le koua tshouen ${ }^{1}$ les « pleurs de sang » sont « comme ruisselants »; dans le koua khouen il y a des regrets et, en entreprenant, présage heureux. Dans le koua tshouen il s'agit du comble du péril, dans le koua khouen il s'agit de la substance de la satisfaction; là en est la raison. Avançant passivement avec satisfaction, il deviendra possible de sortir de la misère.

TSHOU HI. - C'est parce que, avec la douceur négative, il se trouve placé au comble de la misère qu'il a l'image symbolique d'être misérable par le réseau de lianes et l'équilibre périlleux et de dire qu'en se mouvant il aura des regrets. Toutefois, lorsque les êtres sont arrivés à l'extrême limite de leur développement ils doivent se modifier, aussi le sens divinatoire dit que, s’il peut avoir des regrets, il pourra avoir le moyen d'entreprendre et le présage sera heureux.

845.

Misère du réseau de lianes, ne pas encore être digne. En se mouvant regrets, il y a le présage heureux des regrets ; agir.

TSHENG TSE. - Il est considéré comme étant enlacé par la misère et comme ne pouvant se modifier; il ne s'accorde pas encore à la voie rationnelle : c'est être placé dans une situation dont il n'est pas encore digne. Sachant qu'en se mouvant il aura des regrets, il donne libre cours à ses regrets, et les écarte : il lui devient possible de sortir de la misère, c'est donc qu'il agit et que le présage est heureux.

\footnotetext{
${ }^{1}$ Page 80 et $\S 106$.
} 


\section{Tsing}

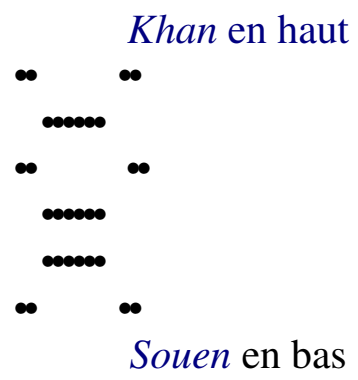

846. Tsing, le puits; déplacer le hameau sans déplacer le puits ; ne rien perdre, ne rien gagner; les allants et venants puisent au puits; la soif survient ; cependant ne pas encore puiser au puits ; casser le vase ; présage malheureux.

850. Premier trait hexaire : puits vaseux, non potable; ancien puits sans oiseaux.

852. Deuxième trait nonaire : puits, torrent qui coule, lancer des flèches à des petits poissons ; le tuyau abîmé laisse fuir l'eau.

$*$

854. Troisième trait nonaire : eau limpide du puits dont on ne boit pas; ce qui rend notre cour triste; on peut s'en servir et y puiser; le roi est intelligent (brillant) ; ils reçoivent également le bonheur.

*

856. Quatrième trait hexaire : le puits est revêtu de briques; pas de culpabilité.

$*$

858. Cinquième trait nonaire : Puits, source fraîche et pure, boire.

$*$

860. Trait supérieur hexaire : le puits recueille sans recouvrir; avoir confiance ; grandeur du présage heureux.

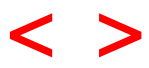


48. Tsing.

TSHENG TSE. - Tsing. «L'Ordre des koua » dit : « La misère en haut doit retomber sur ce qui est bas, c'est pourquoi le koua khouen est suivi du koua tsing. » Cela se rapporte à ce qui a été dit de l'élévation qui ne cesse point et qui doit nécessairement être suivie de misère, celle-ci doit retomber sur ce qui est au-dessous. De toutes les choses qui sont basses, aucune ne l'est autant que le puits ( $t \sin$ ) et c'est ce qui fait que le koua tsing succède immédiatement au koua khouen. Il est formé par le koua simple khan, en haut, et le koua simple souen en bas. Khan, eau ; l'image symbolique du koua souen est l'arbre, le bois ; le sens exprime l'idée d'entrer ; le bois est l'image symbolique de tout ce qui est ustensile, objet, instrument. Le bois pénètre au fond de l'eau et remonte sur l'eau : c'est l'image symbolique de l'action de puiser de l'eau. 


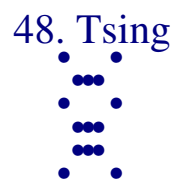

846.

Tsing, le puits ; déplacer le hameau sans déplacer le puits ; ne rien perdre, ne rien gagner; les allants et venants puisent au puits; la soif survient ; cependant ne pas encore puiser au puits; casser le vase ; présage malheureux.

TSHENG TSE. - Entre toutes choses, le puits (tsing) est de celles qui sont permanentes et ne peuvent être déplacées ; le hameau peut être déplacé tandis que le puits ne le peut pas, aussi le texte dit déplacer le hameau sans déplacer le puits. On y puise sans l'assécher, on l'abandonne sans qu'il se remplisse : il ne perd ni ne gagne rien. Ceux qui y parviennent en jouissent également, en allant ou en venant chacun y puise. Ne rien perdre, ne rien gagner, c'est conserver une vertu const ante ; ceux qui vont ou viennent y puisent tour à tour, son effet embrasse tout dans son cercle et est permanent. Embrasser tout dans un même cercle, c'est la voie rationnelle du koua tsing. Le quinzième caractère ${ }^{1}$ signifie le point initial, le début d'un e action; le dix-neuvième caractère veut dire corde. Le puits est une chose utile ; être sur le point d'y arriver et ne pas encore s'en être servi, c'est comme si la corde n'est pas encore descendue au fond du puits. La voie rationnelle de l'homme doué est précieuse par ce qu'elle produit ; les cinq céréales pas mûres ne valent pas mieux que de l'herbe ; un puits creusé jusqu'à la profondeur de neuf coudées sans atteindre la source est comme un puits abandonné ; son but est d'être utile aux êtres et n'est pas atteint, c'est comme s'il n'y avait pas de but. En brisant et détruisant le vase qui sert à puiser et en le perdant, son effet est détruit et c'est par là que le présage est malheu reux. Le caractère du texte a le sens de détériorer et détruire.

TSHOU HI. - Le puits est un endroit creusé dans la terre et d'où sort l'eau. Puisque le bois représenté par le koua simple souen pénètre au-dessous de l'eau représentée par le koua simple khan et remonte l'eau pour la sortir, le koua est considéré comme représentant un puits. Déplacer le hameau sans déplacer le puits, de sorte qu'on ne perd ni ne gagne rien, et que ceux qui vont ou qui viennent puisent tous au puits. Début initial. L'action de puiser l'eau est sur le point d'avoir lieu, la corde n'est pas encore totalement plongée et l'on détruit le vase en le brisant ; le présage est malheureux. Le sens divinatoire exprime que, si les choses restent dans leur ancien état, il n'y aura ni perte, ni gain, et que de plus il convient d'être circonspect et actif. On ne doit pas détruire l'ouvre lors qu'on est arrivé sur le point de la terminer.

\footnotetext{
${ }^{1}$ La soif.
} 
847.

Le commentaire de la formule déterminative dit: entrer dans l'eau et remonter l'eau: puits; le puits désaltère sans fin.

TSHOU HI. - Explication du nom du koua au moyen des images symboliques des koua simples.

848.

Déplacer le hameau sans déplacer le puits; par l'énergie et la justice. La soif survient et cependant ne pas encore puiser au puits; il n'y a pas encore d'effet utile. Briser le vase; c'est par là que le présage est malheureux.

TSHENG TSE. — Ce qui pénètre et entre dans l'eau et remonte l'eau ; le puits. Le puits en désaltérant les êtres a un effet sans fin et illimité ; on y puise sans l'épuiser, ses proprié tés (vertus) sont permanentes et constantes. Le hameau peut changer ; le puits ne peut être transporté : cela exprime encore la persistance constante des propriétés, ou vertus. Les traits qui occupent le second et le cinquième rang comportent les vertus de l'énergie et de la justice et leur persistance est comme celle du puits. Les aptitudes du koua ainsi que le sens sont d'accord. Bien qu'on suppose que le fait soit sur le point d'arriver, du moment où il ne produit pas encore d'effet, il en est cependant comme lorsqu'on ne puise pas encore dans le puits. L'utilité du puits consiste dans son effet réparateur ; cet effet se produit lorsque l'eau en sort : tant qu'elle n'en sort pas, où est l'effet ? Le vase dont il s'agit est le vase qui sert à élever l'eau et à produire l'effet ; si l'on détruit e t si l'on brise le vase, il ne produira plus d'effet, et c'est par là que le présage est malheureux.

TSHOU HI. - Explication de la formule du koua, au moyen de la substance des koua simples. Les deux phrases : ne rien perdre, ne rien gagner ; les allants et venants puisent tour à tour, expriment la même idée que la phrase : ne pas déplacer le puits, aussi il n'en est plus fait mention. Les mots dureté énergique et justice sont employés au sujet du second et du cinquième traits. Briser le vase avant qu'il n' ait produit son effet, c'est la cause du présage malheureux. 
849.

Le commentaire de la formule symbolique dit: Au-dessus du bois il y a de l'eau, puits; l'homme doué pour assurer le repos du peuple exhorte à l'assistance mutuelle.

TSHENG TSE. — Le. bois soulève l'eau et la monte : c'est là l'image symbolique de l'instrument servant à puiser l'eau et à la sortir du puits. L'homme doué contemple l'image symbolique du puits, il se règle sur les propriétés du puits pour affermir et assurer le repos du peuple, et il exhorte à l'assistance mutuelle. Affermir et assurer le repos du peuple, c'est se régler sur l'effet du puits. Exhorter les gens du peuple à s'assister mutuellement, c'est se régler sur les effets produits par le puits.

TSHOU HI. - Au-dessus du bois il y a de l'eau, l'humidité imprègne et mouille en montant : image symbolique du puits. S'efforcer pour assurer le repos du peuple, comme le prince qui veille à l'existence de son peuple. Exhorter à l'assistance mutuelle, afin que les gens du peuple se secourent mutuellement: dans tous les cas c'est toujours le sens des mots : le puits désaltère. 


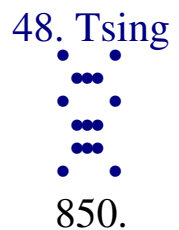

Premier trait hexaire : puits vaseux, non potable ; ancien puits sans oiseaux.

TSHENG TSE. - Les koua tsing (puits) et ting (trépied) représentent tous deux des objets matériels et on se reporte à ces objets pour trouver le sens. Le trait est hexaire, il emploie la douceur malléable négative et reste au rang inférieur ; sil monte, il ne ren contre aucun accueil sympathique : il a l’image symbolique de l'eau qui ne monte point. Ne pouvant pas être utile aux êtres, c'est donc le puits qui ne peut servir à désaltérer ${ }^{1}$, dont l'eau n'est pas potable. Ce qui fait que l'eau des puits n'est pas potable, c'est la vase et la boue, l'impureté de l'eau : l'image symbolique est donc qu'au fond du puits il y a de la vase. L'usage du puits, c'est que son eau désaltère les hommes ; si un puits ne contient pas d'eau, on l'abandonne et on ne s'en sert plus. Lorsque l'eau du puits monte, les hommes profitent de son effet, les animaux et les oiseaux aussi s'y portent et cherchent à en profiter. S'il s'agit d'un puits anciennement abandonné, du moment où les hommes ne s'en servent plus, l'eau ne se répand plus au-dehors, de sorte que les oiseaux et les volatiles n'y retournent plus: c'est qu'en effet il n'a plus d'utilité pour les êtres. Essentiellement, le puits est quelque chose qui sert à l'homme ; un trait hexaire employant la négativité et demeurant au rang inférieur présente l'image symbolique de l'eau qui ne monte plus, aussi il est considéré comme exprimant ce qui ne peut servir à nourrir. Ce qui rend l'eau du puits non potable, c'est la vase. C'est encore comme dans le moment où l'homme doit porter assistance aux êtres, et où ses aptitudes sont faibles, où il ne rencontre point d'aide ; il ne peut rien pour les êtres et alors tout le monde l'abandonne.

TSHOU HI. - Dans le koua tsing, c'est la dureté énergique positive qui est la source, l'origine ; monter et en sortir, c'est l'effet ou usage. Le premier trait hexaire, avec sa négativité, occupe le rang inférieur, de sorte qu'il est considéré comme présentant cette image symbolique. En effet, lorsqu'un puits est sans source et vaseux, les hommes ne peuvent en boire l'eau et les volatiles eux-mêmes n'ont plus de raison d'y venir.

851.

\footnotetext{
${ }^{1}$ Littéralement à « nourrir ».
} 
Puits vaseux, impotable; le fond. Ancien puits sans oiseaux, le temps où il est abandonné.

TSHENG TSE. - Puisqu'il est négatif et occupe le bas du koua tsing (du puits), il présente l'image symbolique de la vase. Pas d'eau, mais de la vase, ce que l'homme ne boit point. Si l'homme n'en boit plu s, l'eau ne monte plus, et il ne peut plus servir aux volatiles et aux oiseaux, de sorte que les oiseaux eux-mêmes n'y vont plus. On considère le fait qu'il ne peut plus être utile aux hommes comme exprimant que c'est le moment où il est abandonné et hors d'usage. S'il pouvait encore étendre son effet aux volatiles et aux oiseaux, il serait encore utile aux êtres. Le mot «abandonner» doit être lu avec le ton montant (sheang) ; et n'a plus le même son que dans l'expression « le moment de l'activité s'étend ».

TSHOU HI. - Cela veut dire qu’il est abandonné dans le temps présent. 


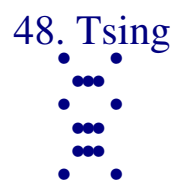

852.

Deuxième trait nonaire : puits, torrent qui coule, lancer des flèches à des petits poissons ; le tuyau abîmé laisse fuir l'eau.

TSHENG TSE. - Bien que le second trait présente les aptitudes de la dureté énergique positive et quil occupe le rang inférieur, il est sans correspondance au-dessus de lui et il se presse contre le second; il présente l'image symbolique de ne pas monter et de descendre. La voie rationnelle du puits, c'est le mouvement d'élévation. L'eau d'un torrent de montagne sort de côté et coule vers le bas ; le second trait fait partie du koua tsing (puits) et se porte vers le trait inférieur ; il manque à la voie rationnelle de ce koua ${ }^{1}$; c'est un puits qui est comme un torrent de montagne. Dans le cas du puits, l'eau monte et sort, de sorte qu'elle désaltère les hommes et est utile aux êtres ; actuellement, l'eau descend vers la vase impure, elle ne coule que pour les têtards et les vers; le sixième caractère est quelquefois considéré comme désignant certains vers, ou aussi des têtards, de petits animaux qui vivent dans la vase des puits. Le cinquième caractère, lancer des flèches, veut dire « couler» : comme le courant d'un torrent d'une gorge de montagne coulant vers les vers et les têtards. Le tuyau abîmé laisse fuir l'eau, comme un tuyau crevé qui fuit. Les aptitudes de la dureté énergique positive rendent ce trait essentiellement capable d'aider au développement physique de l'homme et d'être utile aux êtres ; cependant s'il monte il ne renco ntre aucun accueil sympathique, aussi il ne peut monter et il se porte vers ce qui est bas, de sorte qu'il n'a le mérite d'aucune utilité, ou d'aucun effet. Il en est de lui comme de l'eau dans un tuyau, essentiellement elle peut produire un effet utile, $\mathrm{m}$ ais si le tuyau vient à être abîmé ou brisé, l'eau fuit, et ne peut plus servir à rien. Dans le koua tsing, le premier et le second traits sont sans effet utile et cependant le texte ne parle ni de regrets, ni d'appréhension ; comment cela est-il ? Réponse : lorsquil y a perte, ou manquement, il doit y avoir des regrets, lorsqu'il y a excès, ou erreur, cela doit produire de l'appréhen sion ; mais si, faute d'accueil sympathique, l'effet attendu ne peut être réalisé et achevé, ce n'est pas un motif de regre $t$, ou d'appréhension. Le trait occupe le second rang et se serre contre le premier; n'est-ce donc pas là une faute ? Réponse : être placé dans la justice (au milieu) n'est jamais une faute ; il ne peut monter, faute d'accueil et non pas parce qu'il se ser re contre le premier.

\footnotetext{
${ }^{1}$ Qui est d'agir par l'élévation (puits).
} 
TSHOU HI. - Le second trait nonaire est dur, énergique et juste; il a l'image symbolique de la source ; toutefois, en haut, il ne rencontre aucune correspondance sympathique selon la droiture; en bas, il se serre contre le premier. Son effet utile ne peut se produire par son élévation, de sorte que telle est son image symbolique.

853.

Puits, torrent dans une vallée; lancer des flèches à de petits poissons; pas d'alliance.

TSHENG TSE. - C'est par le mouvement qui fait sortir l' eau en la montant que le puits est utile. Le second trait a les aptitudes de la dureté énergique positive ; essentiellement il peut avoir un effet réparateur et utile ; c'est parce qu'il est dans un rang inférieur et qu'en haut il ne rencontre aucun accueil sympathique quil descend rechercher une alliance et « coule vers les têtards ». Si en haut quelqu'un s'alliait à lui, il serait comme puisé et remonté et il accomplirait l'ouvre utile du puits. 


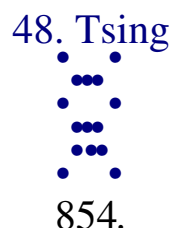

Troisième trait nonaire : eau limpide du puits dont on ne boit pas; ce qui rend notre caur triste ; on peut s'en servir et y puiser ; le roi est intelligent (brillant); ils reçoivent également le bonheur.

TSHENG TSE. — Le troisième trait emploie la dureté énergique positive et il se place conformément à la droiture ; c'est celui qui a les aptitudes nécessaires pour produire un effet utile et réparateur. Il est au rang supérieur dans le koua simple inférieur du koua tsing, qui représente le puits : c'est la partie pure et limpide de l'eau qu'il est possible de boire. L'effet du puits se produit en montant ; ce qui est encore en bas ne produit pas d'effet. La nature de la positivité la porte à monter ; de plus, ses tendances le font correspondre sympathiquement au trait supérieur hexaire; il est placé dans un rang qui comporte la dureté énergique et dépasse la justice ; il est préoccupé d'avancer en montant, c'est là posséder les aptitudes propres à l'usage requis et être pressé d'en déployer et d'en étendre les effets. Ne pouvant pas encore produire d'effet, il est comme l'eau limpide et pure d'un puits en bon état que l'on ne boit cependant pas, ce qui est considéré comme exprimant la tristesse et l'inquiétude du cour. Le troisième trait se trouve dans un moment dont l'emblème est le puits ; il est énergique et dépourvu de justice, aussi il est pressé d'agir ; il est différent de ceux qui, agissant lorsqu'on les emploie, se retirent et se cachent sitôt qu'on les néglige. Cependant, comment un roi intelligent qui emploie les hommes pourrait-il les avoir déjà appelés tous ? Aussi si le roi est intelligent, il en recevra le bonheur. Les aptitudes du troisième trait sont suffisantes pour produire un effet réparateur, comme l'eau claire d'un puits limpide que l'on peut puiser et boire ; si en haut il y a un roi intelligent, il devra l'employer et utiliser ses efforts. Les aptitudes du sage étant manifestées et employées, lui-même pourra suivre cette même voie; le prince profitera de son œuvre et les inférieurs en seront comme imprégnés; le haut et le bas en recevront le bonheur.

TSHOU HI. - Ce qui n'est ni stagnant, ni vaseux, eau limpide du puits dont on ne boit pas, ce qui amène la tristesse du cour de l'homme. Il y a lieu d'y puiser. Si le roi est intelligent il puisera au puits pour servir les êtres et celui qui étend son action, comme celui qui la reçoit, en éprouveront également le bonheur. Le troisième trait nonaire emploie la positivité, occupe un rang positif et se trouve au rang supérieur dans ce qui est inférieur; d'ailleurs le moment n'est pas encore venu de l'employer, aussi tels sont l'image symbo lique et le sens divinatoire. 
855.

L'eau limpide du puits dont on ne boit pas ; en passant, chagrin ; implorer le roi éclairé, recevoir le bonheur.

TSHENG TSE. - Le puits est limpide, réparé, et on ne voit personne y boire : c'est un homme doué de capacité et de savoir, mais que l'on ne voit point employer; ne pouvoir agir est considéré comme constituant un motif d'inquiétude et de chagrin. Du moment où le fait de ne pouvoir agir cause son chagrin, comment se retiendrait-il d'implorer et de demander ? Aussi il implore le roi éclairé et en reçoit le bonheur: ses tendances le rendent impatient d'agir.

TSHOU HI. - En passant, chagrin : les hommes qui passent sur le chemin en éprouvent tous du chagrin ${ }^{1}$.

\footnotetext{
${ }^{1}$ Le texte porte le caractère « agir, marcher, passer, etc. ». J'ai traduit : « en passant. Tsheng Tse lit : « agir, chagrin ». Tshou Hi lit : « les passants ».
} 


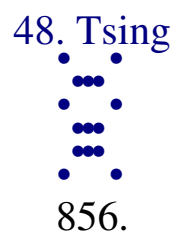

Quatrième trait hexaire : le puits est revêtu de briques ; pas de culpabilité.

TSHENG TSE. - Bien que le quatrième trait soit malléable et négatif et qu'il soit placé sans droiture, au-dessus de lui, il obéit au prince représenté par le cinquième trait nonaire ; ses capacités sont insuffisantes pour être largement avantageuses aux êtres; c'est encore celui qui doit s'observer lui-même. Aussi, s'il peut se corriger et se réformer, il arri vera à être sans culpabilité. Le quatrième caractère exprime l'idée de revêtir avec de la poterie cuite de bas en haut ; c'est-à-dire réparer et mettre en bon état. Bien que chez le quatrième trait les capacités soient faibles, et quill ne puisse pas largement produire un effet utile pour les êtres, cependant lorsqu'on entretient un objet, il n'arrive pas jusqu'à ne pouvoir plus servir du tout ; si l'on ne peut pas le réparer et l'entretenir, il perd toute aptitude à servir au développement des hommes, de sorte qu'il perd la voie rationnelle exprimée par le koua tsing (puits) et que la culpabilité est grande. Occupant une situation élevée, et le prince étant doué de la dureté énergique positive, de la justice et de la droiture, il ne peut que se placer avec droiture pour obéir au supérieur; il n'abandonne pas le soin des affaires et il peut encore éviter d'être coupable.

TSHOU HI. - Hexaire, il occupe le quatrième rang; bien qu'il possède la droiture, il est cependant malléable et négatif, sans initiative ; il ne peut donc que se corriger et s'amender sans produire aucune action bienfaisante pour les êtres, et c'est pour cela que l'image symbolique est un puits revêtu de brique s, tandis que le sens divinatoire exprime qu'il n'y a pas de culpabilité. Si celui qui consulte le sort peut se corriger et s'amender, bien que son avre ne s'étende pas jusqu'aux êtres, il peut cependant être sans culpabi lité.

857.

Le puits est revêtu de briques ; réparation du puits.

TSHENG TSE. - Le quatrième caractère signifie réparer le puits, bien qu'il ne puisse pas grandement étendre son action réparatrice aux êtres, cependant, il peut réparer sans laisser dépérir; aussi il est sans culpabilité. C'est à peine s'il peut éviter la culpabilité ; s'il était à un rang qui comportât la dureté énergique positive, il n'en arriverait naturelle ment pas jusqu'à ce point et alors il serait coupable. 


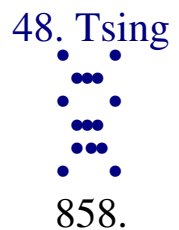

Cinquième trait nonaire : Puits, source fraîche et pure, boire.

TSHENG TSE. - Le cinquième trait emploie la dureté énergique positive, la justice, la droiture, et il occupe la situation prééminente; ses capacités et ses vertus sont absolument bonnes et belles : «puits, source pure et fraîche, buvable », le quatrième caractère signifie pure et agréable au goût ; c'est par sa fraîcheur que la source qui alimente un puits est précieuse ; la source pure et fraîche est buvable pour l'homme, et, dans la voie rationnelle symbolisée par le puits, ces conditions constituent l'extrême excellence. Cependant, la formule ne parle pas de présage heureux ; le puits ne produit son effet que par le mouvement ascensionnel de sortie; tant que ce mouvement ne parvient pas encore jusqu'en haut, l'effet produit n'est pas encore utilisable, aussi ce n'est que lorsqu'il parviendra au rang supérieur que le trait comporte la grandeur du présage heureux.

TSHOU HI. - Pure, claire. Dureté énergique positive, justice et droiture ; son effet atteint les êtres, de sorte qu'il est considéré comme comportant cette image symbolique et ce sens divinatoire. Ayant ces vertus, l'image symbolique sera applicable.

859.

Consommation de la source fraîche; justice et droiture.

TSHENG TSE. - Source fraîche et buvable: c'est ce qu'il y a de meilleur dans la voie rationnelle symbolisée par le puits. La vertu de justice et de droiture du cinquième trait nonaire exprime le sens d'extrême excellence. 


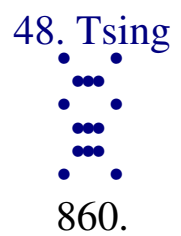

Trait supérieur hexaire : le puits recueille sans recouvrir ; avoir confiance ; grandeur du présage heureux.

TSHENG TSE. - Le puits produit son effet par l'action de sortir en montant; ce trait occupe le rang supérieur du koua tsing; il exprime l'achèvement de la voie rationnelle du puits. Le quatrième caractère, « recueillir, ramasser», veut dire «prendre en puisant», recouvrir, cacher. Prendre et ne pas cacher ; avantage sans fin : l'effet du puits s'étend lar gement et grandement. Avoir confiance, il $\mathrm{y}$ a une règle permanente sans modification ; étendre largement l'effet, avec permanence ; présage heureux d'un grand bien. Or qui donc serait capable de réaliser l'effet du koua tsing (puits), de l'étendre avec permanence, si ce n'est un grand homme ? Le dernier trait d'autres koua exprime le maximum, le plus haut degré, la modification; il n'y a que dans les koua tsing et ting, dans lesquels le trait final est considéré comme exprimant l'achèvement de l'effet utile, et c'est à cause de cela que le présage est heureux.

TSHOU HI. - Recueillir, prendre en puisant ; Tshiao Shi dit que ce mot exprime l'action d'enrouler une corde sur un treuil, cela est encore admissible. Recouvrir, cacher en recouvrant. Avoir confiance, veut dire que ce qui sort est pur et ne cesse point. Le puits produit son action par le mouvement de sortie en montant et l'orifice du trou n'est pas recouvert ; aussi, bien que le trait supérieur hexaire soit dépourvu de la dureté énergique positive, l’image symbolique est ainsi. Mais si celui qui consulte le sort répond à ces conditions, il doit avoir confiance et c'est en cela qu'est la grandeur du présage heureux.[][]

861.

La grandeur du présage heureux est en haut ; grande perfection.

TSHENG TSE. - À cause du présage heureux du grand bien placé au rang supérieur du koua, c'est le plus grand achèvement de la voie rationnelle du puits. C'est en montant que le puits produit son effet. 


\section{Ko}

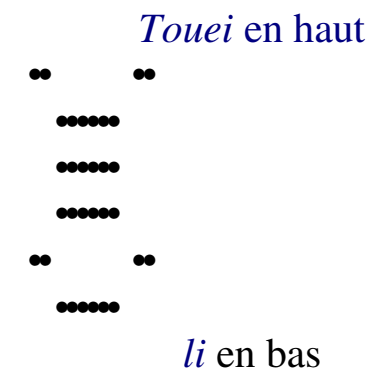

862. Ko, au moment appelé Se ji, alors confiance ; grandeur, liberté ; avantage de la pureté ; regrets dissipés.

[]

867. Premier trait nonaire : lier solidement en employant le cuir du bauf jaune.

$*$

869. Deuxième trait hexaire : le jour fini alors il se renouvelle; entreprendre, présage heureux; pas de culpabilité.

$*$

871. Troisième trait nonaire : en avançant présage malheureux; péril de la pureté ; paroles de renouvellement trois fois ; avoir foi.

$*$

873. Quatrième trait nonaire : regrets dissipés ; avoir foi dans le changement de la destinée; présage heureux.

$*$

875. Cinquième trait nonaire : grand homme, tigre se modifiant; avant de consulter le sort il y a déjà confiance.

$*$

877. Trait supérieur hexaire : homme doué, panthère se modifiant; l'homme inférieur renouvelle son visage; en avançant, présage malheureux; en se maintenant dans la pureté, présage heureux.

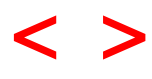


49. Ko.

TSHENG TSE. - Ko. «L'Ordre des koua » dit : «Il est impossible que la voie rationnelle du puits (tsing) ne change pas, aussi le koua tsing est suivi du koua ko.» Le puits (tsing), considéré comme chose (être), doit se corrompre et s'altérer par la durée, se clarifier et se purifier par le changement : il ne peut pas ne pas se modifier, aussi à la suite du koua tsing est placé le koua ko. Il est composé du koua simple touei en haut et du koua simple $l i$ en bas : au milieu du marais il y a du feu. Ko, modifier et changer. L'eau et le feu sont des choses qui s'anéantissent mutuellement ; l'eau éteint le feu, le feu sèche l'eau : ils se modifient et se changent mutuellement. La nature du feu est de monter; celle de l'ea u est de descendre ; si elles marchent en sens contraire ces deux choses se sépareront simplement. Mais ici, le feu est en bas, l'eau est en haut; elles se portent l'une vers l'autre et lut tent ensemble: elles s'anéantissent l'une l'autre, ce qui fait que le koua est considéré comme exprimant le changement. Ou bien encore : deux jeunes filles sont ensemble, mais chacune se porte dans une direction différente; leurs tendances sont différentes, ce qui exprime qu'elles ne s'accordent point, de sorte que le koua exprime la séparation. 


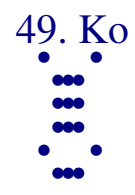

862.

Ko, au moment appelé Se ji, alors confiance ; grandeur, liberté ; avantage de la pureté ; regrets dissipés ${ }^{1}$.

TSHENG TSE. - Ko, renouveler; modifier ce qui est ancien. En modifiant ce qui est ancien, il en résulte que les hommes ne peuvent pas avoir immédiatement confiance (dans le nouvel ordre des choses), aussi il faut absolument achever le jour ${ }^{2}$ et ensuite le coar de l'homme suit avec confiance : grandeur, liberté, avantage de la pureté, regrets dissipés. Lorsque quelque chose est détériorée et abîmée on la renouvelle; c'est en la renouvelant qu'on parvient à en assurer le libre usage, de sorte que c'est par son renouvellement qu'il peut y avoir une grande liberté d'action. En renouvelant il y a avantage pour la voie de la droiture, de sorte qu'il y a le sens de possibilité d'une longue durée et de suppression de ce qui est ancien, sans regrets au sujet de la modification et du mouvement: c'est là la dissipation des regrets. Renouveler sans grand avantage peut encore être une cause de regrets, à plus forte raison encore si le renouvellement est, au contraire, nuisible; c'est à cause de cela que les hommes de l'antiquité attachaient une grande importance à l'action de faire des changements.

TSHOU HI. - Ko, modification et changement. Le koua simple touei, marais, est en haut ; le koua simple $l i$, feu, est en bas : le feu s'allume, donc l'eau sèche ; l'eau coule, le feu s'éteint. Deux jeunes filles, la moyenne et la petite, sont réunies et leur réunion constitue un koua dans lequel la petite est au-dessus et la moyenne au-dessous; leurs tendances ne s'accordent point ensemble, aussi ce koua est considéré comme exprimant le renouvellement. Au début du renouvellement, les hommes n'ont pas encore confiance, aussi il faut que le jour soit fini ${ }^{3}$, et ensuite il y a confiance. De plus, puisqu’à l'intérieur il y a les qualités de la beauté de la forme et de l'éclat, et qu'à l'extérieur il y a l'éther de l'harmonie et de la satisfaction ${ }^{4}$, le sens divinatoire est quill y a quelque chose à changer et à renouveler. De toute manière il y a

\footnotetext{
${ }^{1}$ Le texte porte le terme $s e$ : c'est le nom de l'un des douze signes, ou « moments qui désignent les conjonctions et qui entrent aussi dans la formation des noms des années du cycle de soixante ans. Se ji, le soleil ou le jour du moment se. Or, se désigne le $4^{\mathrm{e}}$ mois, lorsque l'éther positif est entièrement «sorti » et l'éther néga tif entièrement caché. Cela semble bien désigner le soleil levé après avoir passé au point vernal.

${ }^{2}$ Les commentateurs chinois lisent $y i$, fini, achevé. D’après le Tse tien les caractères se et $y i$ auraient la même origine et la distinction serait mal établie. Nous y reviendrons plus loin.

${ }^{3}$ Le commentateur lit yi. Cette lecture est loin de donner un sens satisfaisant.

${ }^{4}$ Cela est basé sur les valeurs symboliques attribuées aux deux koua simples.
} 
toujours grande liberté et accord avec la droiture; ce qui est supprimé et remplacé devait légitimement être supprimé, et les regrets de cette suppression se dissipent. Si même une seule chose était renouvelée contrairement à la droiture, le renouvellement n'inspirerait plus confiance et ne produirait plus librement son effet; au contraire, il y aurait des regrets.

863.

Le commentaire de la formule déterminative dit: Ko, l'eau et le feu se suppriment mutuellement; deux jeunes filles demeurent ensemble; leurs tendances ne s'accordent pas : on l'appelle ho.

TSHENG TSE. - L'humidité et le feu se détruisent mutuellement ; de plus les tendances des deux jeunes filles ne s'accordent pas ens emble, de sorte que le koua exprime le changement. Le caractère traduit par «détruire » signifie éteindre, ou encore arrêter la respiration; mais il exprime aussi la naissance à la vie, le premier souffle de la respiration. Les êtres cessent de respirer et ensuite ils recommencent à respirer, ce qui donne le sens de vivre ; dans le cas du koua ko ce mot a le sens d'arrêter la respiration, « d'éteindre ».

TSHOU HI. - Explication du sens du nom du koua au moyen des images symboliques des koua simples. En général il y a de l'analogie entre ce koua et le koua khouei : toutefois, c'est l'opposition mutuelle qui constitue le koua khouei, tandis que c'est l'extinction mutuelle qui constitue le koua ko. Éteindre, c'est détruire en faisant évanouir et dispa raître ; ce mot a encore le sens de respirer, naître à la vie : extinction du souffle, puis renaissance du souffle ; respiration.

864.

Le jour fini ${ }^{1}$, alors confiance; renouvellement inspirant confiance; apparence brillante par la satisfaction; grande liberté par la droiture; renouvellement et convenance; les regrets sont alors dissipés.

\footnotetext{
${ }^{1}$ Ici le texte porte $y i$, c'est un des dix signes qui, avec ceux de la série des douze signes ou «moments», mentionnés dans le renvoi du $\S 862$, servent à former les noms des soixante années du cycle. Il semble que ces dix nouveaux signes se rapportent aux mouvements des cinq planètes connues dès l'antiquité.
} 
TSHENG TSE. - Lorsqu'il s'agit de suppression et de renouvellement, comment le cour de l'homme pourrait -il avoir aisément confiance ? Il faut que le jour soit fini et alors vient la foi. Pour celui qui occupe le rang supérieur, et lorsqu'il se présente une circons tance où il y a lieu de changer et de faire, il convient d'avertir clairement, de donner des instructions précises jusqu’à la fin du jour, afin que les hommes aient confiance. Si le cœur de l'homme manque de confiance, bien qu'on le contraigne à agir, rien ne peut produire d'effet définitif ${ }^{1}$. Lorsque les premiers rois établirent leurs institutions, le coaur de certains hommes commença par en concevoir de la méfiance. Cependant, à la longue, la confiance survient nécessairement ; on n'a jamais vu le cas d'un gouvernement bien établi n'imposant pas finalement la confiance. L'apparence brille par la satisfaction ; cela exprime la voie rationnelle du changement et du renouvellement au moyen des aptitudes des koua simples. Le koua simple $l i$ exprime l'élégance de la forme ${ }^{2}$; le koua simple touei exprime la satisfaction. Lorsque l'apparence brille (lorsque le texte est clair) la raison d'être ne peut être qu'absolument évidente, aucune chose ne peut rester incomprise. Par la satisfaction, le coar de l'homme est conciliant. Renouveler et pouvoir élucider la raison d'être des choses, faire naître la concorde et la soumission dans le coar des hommes, peut amener une grande liberté et l'accord à la perfection dans la droiture. Dans de telles

conditions, la modification et le renouvellement atteignent au plus haut point de l'opportunité et de la convenance, de sorte que les regrets se dissipent. Lorsqu'il s'agit des affaires de l'univers (de l'empire), si leur renouvellement (changement, innovation) n'est pas conforme à la voie rationnelle, il produira tout au contraire des effets destructifs et nuisibles, aussi le renouvellement comporte une voie rationnelle de regrets ; ce n'est que lorsque le renouvellement est absolument opportun que les regrets relatifs à ce qui est nouveau et à ce qui est ancien seront tous dissipés :

TSHOU HI. - Explication de la formule du koua au moyen des vertus (propriétés) des koua simples.

865.

Le ciel et la terre changent ${ }^{3}$ et les quatre saisons s'achèvent; Theang et Wou ${ }^{4}$ renouvellent (ho) le mandat du ciel ; ils obéissent au ciel et répondent aux besoins des hommes : que le moment du renouvellement est grand!

\footnotetext{
${ }^{1}$ Ici comme dans un grand nombre de cas analogues, le commentateur sous-entend toujours qu'il s'agit de procédés de gou vernement.

2 Nous avons déjà vu souvent ce terme, dont le sens est si douteux. Littéralement « l'apparence brille », mais on peut aussi lire le « texte est clair».

${ }^{3}$ Ko : changent et renouvellent.

${ }^{4}$ Les fondateurs des premières dynasties.
} 
TSHENG TSE. - Si l'on examine la voie rationnelle du renouvellement, elle atteint son maximum d'importance dans les modifications et les changements (yi) du ciel et de la terre, dans le commencement et le fin du cours des saisons. Le ciel et la terre, c'est la négativité et la positivité s'alliant et entraînant la modification et le changement, puis produisant les quatre saisons; c'est par là que tous les êtres naissent et produisent en se développant, s'achèvent et prennent fin, chacun suivant sa voie naturelle. C'est ce renou vellement (ko) qui produit les quatre saisons ; lorsque le cours des saisons est terminé, il doit se renouveler et recommencer de nouveau. L'élévation des rois dépend d'un man dat (destinée) reçu du ciel, aussi un changement de dynastie est appelé « renouvellement du mandat». Lorsque Theang et Wou arrivèrent à régner, c'est qu'en haut ils se confor mèrent avec soumission au mandat céleste, tandis qu'en bas ils répondaient aux aspirations des hommes, telle est la voie. La voie rationnelle du ciel se modifie et change, les choses de ce monde se transforment et se modifient : c'est là l'extrême grandeur du renouvellement. Aussi, Khong Tse s'exclame admirativement et dit : Que le moment du renouvellement est grand !

TSHOU HI. - Dernière expression et exclamation admirative sur la grandeur.

866.

Le commentaire traditionnel de la formule symbolique dit: Dans le marais il y a du feu ; l'homme doué en conclut la détermination des époques ${ }^{1}$.

TSHENG TSE. — L'eau et le feu s'excluant mutuellement constituent le renouvellement; ko, modification. L'homme doué regarde l'image symbolique de la modification et du renouvellement, il observe les changements et la translation du soleil, de la lune, des étoiles et des astres, pour en déduire le calendrier et indiquer clairement l'ordre des saisons. Car la voie rationnelle de la modification et du 'changement est ce qu'il y a de plus grand entre toutes choses ; c'est la plus claire entre les raisons d'être ${ }^{2}$, c'est la plus évidente entre celles dont on peut suivre les traces, et rien n'est comparable aux quatre saisons. Aussi, se conformer à leurs modifications et à leur renouvellement sera se conformer à l'ordre du ciel et de la terre.

TSHOU HI. - Les modifications des quatre saisons, sont ce qu'il y a de plus grand dans le renouvellement symbolisé par le koua $k o$.

1 Calendrier. La traduction la plus naturelle serait: «l'homme doué sert à déterminer le calendrier $\gg$.

${ }^{2}$ Causes explicables. 


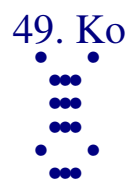

867.

Premier trait nonaire : lier solidement en employant le cuir du bauf jaune.

TSHENG TSE. - La modification et le renouvellement sont ce quil y a de plus grand entre toutes choses. Il faut avoir le moment, avoir la situation, avoir les aptitudes, juger et prévoir et se mouvoir, avec circonspection, alors seulement il sera possible de ne pas avoir de regrets ${ }^{1}$. Ce trait nonaire occupe le premier rang, il marque le commencement dans le temps; se mouvoir au début de quelque chose, c'est manquer de circonspection et de réflexion et se hâter. Dans les symboles du Yi king, ce trait, à cause de la situation qu'il occupe, marque l'infériorité : ce n'est pas le moment, il ne rencontre aucun accueil et il se meut dans un rang inférieur, il sera coupable d'avoir dépassé mal à propos l'initiative qui lui convient et de manquer de puissance dans la force naturelle inhérente à sa nature. Au point de vue des aptitudes, ce trait fait partie de la substance du koua simple li et il est positif; la nature du koua simple $l i$ (feu) le porte à monter et la substance de la dureté énergique est active; toutes ces conditions indiquent également la précipitation dans le mouvement. Ses aptitudes étant telles, s'il a à agir, le présage est malheureu $\mathrm{x}$ et indique la culpabilité au plus haut point ; en effet, il est dur et énergique, dépourvu de justice et d'un caractère trop vif. Ce qui lui manque, c'est la justice et la soumission; il convient donc quil se contienne lui -même par la justice et la soumission, sans agir mal à propos et alors tout lui sera possible. Attacher, lier ; le cuir sert à entourer et à lier. Le jaune est la couleur de la justice ${ }^{2}$. Le boaf est un animal passif et soumis. Lier solidement en employant le cuir du bœuf jaune, c'est -à-dire employer la voie rationnelle de la justice et de la soumission et se contenir avec force pour ne pas s'agiter mal à propos. Comment n'est-il pas parlé de présage heureux ou malheureux ? Réponse: En se mouvant mal à propos il y a présage malheureux de culpabilité ; donc, en se contenant fermement par la justice et la soumission, il n'y aura pas innovation (renouvellement) ; dès lors quelle serait l'utilité de la mention d'un présage heureux ou malheureux ?

TSHOU HI. - Bien qu'en présence d'un moment de renouvellement, ce trait occupant le premier rang, étant sans correspondance sympathique, ne doit pas encore avoir à agir, aussi il donne cette image symbolique. Le troisième caractère signifie «solide, ferme ». Le jaune est la couleur de la justice; le bouf est un animal patient ; le cuir sert à attacher solidement pour affermir

\footnotetext{
${ }^{1}$ Au sujet d'une innovation.

${ }^{2} \mathrm{La}$ « couleur moyenne», ou encore, la couleur qui correspond au rang du milieu dans un koua simple.
} 
quelque chose. Le cuir (ho) a le même nom que le koua du renouvellement (ko), mais le sens n'est pas le même. Le sens divinatoire est qu'il convient de se contenir et de s'observer avec une grande fermeté et qu'il ne faut pas agir. Telle est la prudence de l'homme saint au sujet de la modification et du renouvellement.

868.

Maintenir fermement en employant le bauf jaune ; il ne faut pas employer les motifs d'action.

TSHENG TSE. - Puisque, dans le cas de ce premier trait nonaire, le moment, la situation, les aptitudes ne comportent pas l'emploi des motifs d'action, il faut donc se contenir avec fermeté, par la justice et la soumission. 


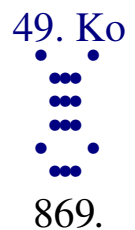

Deuxième trait hexaire : le jour fini alors il se renouvelle ; entreprendre, présage heureux; pas de culpabilité ${ }^{1}$.

TSHENG TSE. - Puisqu'un trait hexaire occupe le second rang, il est doux et soumis et possède la justice et la droiture. De plus c'est de lui ${ }^{2}$ que dépend (le koua simple qui exprime) la clarté de l'apparence (l'élégance de la forme). En haut se trouve le prince doué de dureté énergique positive; ils possèdent les mêmes vertus et ils correspondent sympathiquement. À cause de la justice et de la droiture, il n'y a ni partialité, ni abus.

L'apparence étant brillante ${ }^{3}$, la raison d'être des choses est complètement élucidée. Comme le second trait correspond sympathiquement au trait supérieur, il possède la force inhérente au pouvoir ; comme sa substance est la positivité, il ne se révolte pas et ne fait aucune opposition. Le moment est convenable; la situation s'y accorde ; les aptitudes sont suffisantes : c'est donc celui qui se place dans le koua ho (renouvellement) suivant l'extrême excellence. Toutefois la voie rationnelle du sujet ne comporte pas l'initiative dans les renouvellements ${ }^{4}$; de plus il faut attendre la confiance du supérieur et des inférieurs, et c'est pour cela que le jour étant achevé, al ors, il renouvelle ${ }^{5}$. Avec les vertus et les aptitudes du second trait, étant donné le terrain sur lequel il se trouve, le moment qui se présente, tout est suffisant pour faire disparaître les embarras de l'État et rénover l'ordre dans l'univers ; il doit avancer et monter pour aider le prince à parcourir sa voie rationnelle, de sorte que le présage sera heureux et quil n'y aura pas de culpabilité. Sil n'avan çait pas, il perdrait l'occasion d'agir, ce qui constituerait sa culpabilité. Puisque la substance du second trait est la douceur malléable, et puisqu'il occupe une situation dont il est digne, son mouvement en avant sera lent et mesuré et sa position sera toujours ferme. La modification et le renouvellement sont des choses d'une grande importance et c'est à cause de cette importance que la formule comporte cet avertissement. Le second trait possède la justice et sympathise avec la dureté énergique, de sorte qu’il n'y a pas d'incon vénient résultant de la douceur malléable; l'homme saint profite

\footnotetext{
${ }^{1}$ Les commentateurs chinois ne font pas de différence entre le second caractère de la sentence du $\S 862$ et le troisième caractère de celle-ci. je crois, au contraire, que cette différence existe. ${ }^{2}$ Transformation du koua simple Khien par le changement du second trait qui devient négatif.

${ }^{3}$ On pourrait lire « le texte étant clair ».

${ }^{4}$ Innovations.

${ }^{5}$ Il innove.
} 
de ce qu'il y a un motif de doute et d'avertissement et il éclaire le sens, afin que les sages et les hommes capables ne manquent point le moment d'agir.

TSHOU HI. - Le second trait hexaire est doux et soumis, juste et droit, et c'est de lui que dépend l'élég ance et la clarté de l'apparence. Il rencontre des sympathies au-dessus de lui, de sorte qu'étant dans de telles conditions, il est capable de faire des changements (innovations, renouvellements). Toutefois, il faut absolument finir le jour et ensuite faire ces changements, de sorte qu'en entreprenant le présage est heureux et il n'y a point de culpabilité. C'est un avertissement à celui qui consulte le sort quill ne doit pas encore se hâter de modifier prématurément.

870.

Le jour fini il se renouvelle; en agissant il y a félicité.

TSHENG TSE. - Finir le jour et alors renouveler; en entreprenant le présage sera heureux et il n'y aura pas de culpabilité ; en agissant il y aura de la félicité et des louanges. Cela exprime qu'il est impossible de changer ce qu'il y a de mauvais et de renouveler les choses de l'univers ${ }^{1}$. Si on restait en place sans entreprendre, c'est que le cour ne serait pas porté à remédier aux maux dont souffre l'univers, on manquerait le moment, il y aurait culpabilité.

${ }^{1}$ Affaires de l'État. 


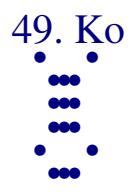

871.

Troisième trait nonaire : en avançant présage malheureux; péril de la pureté ; paroles de renouvellement trois fois ${ }^{1}$; avoir foi.

TSHENG TSE. - Le troisième trait nonaire emploie la dureté énergique et représente l'ascension de ce qui est inférieur. De plus il occupe le rang supérieur du koua simple $l i$ et il ne se conforme point à la justice : c'est celui qui est pressé de se mettre en mouvement pour supprimer et renouveler. Étant dans l'infériorité et pressé de modi fier et de renouveler, et agissant dans ces conditions, il y aura un présage malheureux. Toutefois, il occupe le rang supérieur dans le koua simple inférieur, si quelque chose doit être supprimée, comment pourrait-il ne pas agir ? C'est en observant la p ureté et la droiture et en conservant la crainte du péril, en suivant docilement les conseils désintéressés, quill pourra agir sans provoquer le doute et les soupçons. « Paroles de renouvellement », c'est-à-dire délibération sur la convenance du renouvellement, achever, produire, réunion ${ }^{2}$; examiner et juger jusqu’à trois fois les conseils de changements et de renouvellement et tous seront d'accord, de sorte qu'il sera possible d'avoir confiance. Cela veut dire que, si l'importan ce de la circonspection est poussée à un tel point, il sera possible d'atteindre au plus haut degré de l'opportunité (dans les changements), et alors il y aura foi et confiance. Luimême pourra avoir confiance et la foule aura confiance dans ses actions, dans de telles conditions il devient possible de renouveler. Dans un moment de renouvellement, il occupe le rang supérieur du koua simple inférieur ; s’il est dominé par la crainte et n'ose pas faire les changements qu'il convient de faire, il perdra le moment favorable, ce qui constituera un dommage. Il doit seulement y apporter la plus extrême circonspection et la plus extrême prudence, ne pas s'autoriser de son énergie et de sa propre intelligence, examiner et peser les délibérations désintéressées, jusqu'à y revenir trois fois, et ensuite renouveler, de sorte qu'il ne commettra aucune faute.

TSHOU HI. - Excès d'énergie sans justice ; il occupe le dernier rang du koua simple $l i$; c'est celui qui est pressé de se mouvoir pour effectuer des changements, aussi le sens divinatoire comporte un avertissement au sujet du présage malheureux de l'entreprise et du danger de la pureté. Toutefois le

\footnotetext{
${ }^{1}$ C'est le mot traduit par «fois » dans l'expression trois fois. Essentielle ment, le caractère signifie «parvenir haut». Un commentateur dit : Du premier rang au troisième rang, il y a trois changements de traits; le renouvellement a donc lieu peu à peu et sa voie s'exhausse ; c'est pour cela que le texte dit : pour renouveler; parler (avertir) trois fois et achever.

${ }^{2}$ Le sens est tout à fait incertain.
} 
moment est convenable pour le renouvellement, aussi c'est à ce point qu'après les «paroles de renouvellement trois fois », il y aura encore confiance et le renouvellement sera possible.

872.

Paroles de renouvellement ; trois fois accord ; quoi de plus encore ${ }^{1}$ ?

TSHENG TSE. - Toutes les délibérations de l'enquête, jusqu'au nombre de trois sont finies et arrivent aux mêmes conclusions; le fait est au plus haut point opportun : «Quoi donc de plus encore ?» C'est l'équivalent de l'expression usuelle «Que faire encore ?» Agir quand on est dans ces conditions, c'est suivre la raison d'être des choses et agir selon le moment ; ce n'est plus le désir de faire dans un but d'intérêt privé, de sorte que l'action est convenable.

TSHOU HI. - Le huitième caractère, paroles, veut dire que la chose est déjà délibérée et jugée.

\footnotetext{
${ }^{1}$ Le texte est le même qu'au $§ 870$; la traduction suit le commentaire.
} 


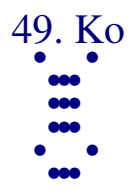

873.

Quatrième trait nonaire : regrets dissipés; avoir foi dans le changement de la destinée ; présage heureux.

TSHENG TSE. - Le quatrième trait nonaire exprime le complet achèvement $\mathrm{du}$ renouvellement. L'énergie positive est l'aptitude au renouvellement; le quatrième trait quitte la substance inférieure et s'avance dans la substance du koua simple supérieur; il marque le moment du renouvellement. Ce trait se trouve aussi au point de contact de l'eau et du feu, ce qui exprime la force inhérente à la suppression et au renouvellement. Il est près de la situation du prince, ce qui indique l'autorité nécessaire au renouvellement. En bas il n'a ni attache ni correspondance sympathique, c'est l'indice de tendances qui le porten tà supprimer et à renouveler. Puisque, étant nonaire, il occupe le quatrième rang, la dureté énergique et la douceur malléable se font mutuellement équilibre, ce qui indique l'effet du renouvellement. Le quatrième trait réunissant toutes ces conditions, on peut dire qu'il marque le moment où le renouvellement est opportun ; les choses susceptibles d'occasionner des regrets étant enfin supprimées et renouvelées, leur renouvellement est donc opportun, et ces regrets se dissipent. Du moment où le renouvellement est opportun, l'unique souci consiste à se placer avec la plus parfaite sincérité, de sorte qu'il y ait foi et alors le changement de la destinée sera un présage heureux. Changement de la destinée, changement de ce qui existe, c'est-à-dire le renouvellement. Dès lors que les choses sont en état convenable et que le mal est supprimé, en agissant avec sincérité, le supérieur inspire la confiance et les inférieurs se soumettent: le présage heureux est donc évident. Le quatrième trait est sans justice ni droiture ; comment se fait-il qu'il marque l'extrême excellence ? Réponse : uniquement parce qu'il est placé à un rang qui marque la douceur malléable, de sorte quỉl est énergique, mais sans excès; quil est proche, mais sans exercer de pression; il obéit avec soumission au prince doué de justice et de droiture, de sorte que c'est lui -même un homme juste et droit. Dans le Yi king, le choix du sens ne dépend pas de règles constantes ; il suit simplement l'occasion.

TSHOU HI. - Puisque la positivité occupe un rang négatif, il en résulte donc des regrets ; toutefois le milieu du koua est dépassé, la rencontre de l'eau et du feu exprime le temps de l'extinction et du renouvellement ; l'énergie et la douceur se balançant sans inclination partiale expriment encore l'effet du renouvellement. C'est à cause de cela que les regrets sont dissipés. Toutefois, il faut encore qu'il y ait d'abord confiance et ensuite renouvellement, et c'est seulement ensuite que le présage heureux peut se réaliser. Ceci indique 
clairement à celui qui consulte le sort que, possédant ces vertus et dans un tel moment, il faut encore avoir confiance, et qu'alors les regrets seront dissipés et le présage heureux se réalisera.

874.

Présage heureux du changement de la destinée ; tendances confiantes.

TSHENG TSE. - Changement de destinée et présage heureux parce que le supérieur et les inférieurs ont confiance dans ses tendances. Du moment où la sincérité est extrême, le supérieur et les inférieurs ont confiance. La voie rationnelle du renouvellement est basée sur la confiance du supérieur et des inférieurs. Si le renouvellement n'est pas opportun, s'il n'y a pas foi, il n'y aura point confiance ; s'il est opportun et s'il n'y a pas confiance, on ne doit pas encore agir: combien donc encore à plus forte raison lorsque le renouvellement n'est pas opportun? 


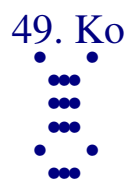

875.

Cinquième trait nonaire : grand homme, tigre se modifiant; avant de consulter le sort il y a déjà confiance.

TSHENG TSE. — Le cinquième trait nonaire emploie les aptitudes de la dureté énergique, les vertus de la justice et de la droiture, et il occupe la situation prééminente : c'est le grand homme. Il emploie la voie rationnelle du grand homme pour renouveler les affaires de l'univers ; il n'est indigne sous aucun rapport ; aucun moment ne lui est inopportun ; dans ce qui est déjà modifié et transformé, la raison d'être des choses est déjà manifeste et claire, brillante comme les dessins de la robe du tigre, de sorte que le texte dit : «tigre se modifiant». Le dragon et le tigre sont des symboles du grand homme. La modification, c'est la modification des choses et des êtres ; alors pourquoi dire «le tigre »? Réponse : Ce que le grand homme modifie, est une modification du grand homme. Modifiant et renouvelant au moyen de la voie rationnelle de justice et de droiture du grand homme, la lumière est brillante, elle illumine et rend manifeste et visible; il n'est pas nécessaire d'attendre que le sens divinatoire soit fixé, et l'on sait immédiate ment que tout est on ne peut plus convenable et opportun et que, nécessairement, l'uni vers entier aura confiance. L'univers, éclairé par les renouvellements du grand homme, n'a pas besoin d'attendre que le sens divinatoire soit déterminé, il reconnaît que tout est on ne peut plus opportun et régulier et en éprouve de la confiance.

TSHOU HI. - Tigre, symbole du grand homme ; « se modifiant », veut dire muant, changeant de peau ; le poil ancien et le nouveau poil déjà poussé, mais pas encore long. Chez le grand homme, cela indique la rénovation personnelle conduisant au plus haut degré de la rénovation du peuple : c'est le moment où il obéit au ciel et répond au désir des hommes. Le cinquième trait nonaire, par sa dureté énergique, sa justice et sa droiture, est celui de qui dépend le renouvellement, de sorte qu'il présente cet te image symbolique. Consultant le sort, et réunissant ces conditions, on pourra s'appliquer cette réponse, mais toutefois il faut encore que, dès avant le moment de la consultation du sort, la personne qui le consulte sache déjà avec confiance que les choses sont ainsi ; alors tout sera suffisant pour mériter l'application de cette réponse. 
Grand homme, tigre se modifiant; son apparence est brillante.

TSHENG TSE. - La raison d'être des choses est claire et brillante comme les dessins de la robe du tigre sont éclatants et complètement brillants ; l'univers peut-il être sans confiance? 


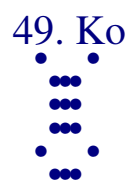

877.

Trait supérieur hexaire : homme doué, panthère se modifiant; l'homme inférieur renouvelle son visage ; en avançant, présage malheureux; en se maintenant dans la pureté, présage heureux.

TSHENG TSE. - Fin du koua ho ; achèvement de la voie rationnelle du renouvellement. Homme doué, c'est-à-dire homme de bien ; bon et vertueux, il a donc suivi l'action du renouvellement (de la rénovation) et s'est modifié. L'évidence est manifeste et visible comme les dessins (fleurs) d e la robe de la panthère. L'homme inférieur, c'est celui qui est stupide, grossier et difficile à changer, bien qu'il ne puisse pas encore modifier son cœur, cependant il change déjà son visage pour suivre les enseignements du supérieur. Le dragon et le tigre sont des symboles du grand homme, aussi au sujet du grand homme, le texte parle du tigre, et au sujet de l'homme doué, il parle de la panthère. La nature des hommes est originellement bonne ; tous peuvent se modifier et se transformer, mais cependant il y en a qui sont inférieurs et grossiers, que les hommes saints eux-mêmes sont incapables de transformer. Lorsque les princes sont des hommes comme Yao et Shouen, lorsque les saints succèdent aux saints et que pendant plus de cent ans l'univers se trans forme sous leur influence, c'est ce qu'on peut appeler une action de longue durée ; cependant il y eut le peuple des Miao, il y eut un homme tel que Seang. Ils approchèrent de la transformation, ils avancèrent dans la voie de l'ordre (de la civilisation), car, en effet, ils ne firent que renouveler leur visage. Quand l'homme inférieur a renouvelé l'apparence extérieure, la voie du renouvellement peut être considérée comme épuisée. Si l'on veut continuer à l'appliquer en poussant plus profondément l'action di rectrice, cela deviendra excessif, or ce qui excessif n'est plus la voie rationnelle. Aussi, parvenu à la fin du renouvellement, si l'on avance encore, le présage est malheureux ; il convient de s'attacher fermement à la pureté pour s'observer et se conten ir soi-même. Le renouvellement arrivé à sa limite, si l'on ne se contient pas par la pureté, ce qui a été renouvelé se modifiera encore de nouveau. Les choses qui concernent l'univers sont telles que, d'abord on se plaint de la difficulté qu'elles présentent à toute tentative de renouvellement, mais une fois renouvelées, on se plaint de l'impossibilité que l'on rencontre pour les conserver et les maintenir. Aussi, à la fin du renouvellement, le texte avertit que le maintien dans la pureté sera un présage heureux. Le maintien dans la pureté n'est-il pas un avertissement motivé par la nature du trait qui est hexaire ? Réponse : cela est dit parce qu’il s'agit de la fin du renouvellement et que rien n'est en dehors de la loi exprimée. La nature de l'homme est originellement bonne : comment se peut-il donc faire qu'il y en ait qui ne sont 
pas susceptibles de rénovation ? Réponse : si l'on discute leur nature, elle est bonne chez tous ; mais si l'on discute leurs aptitudes, alors il y en a qui sont inférieurs et grossiers et qui ne changent point. Ce qu'on appelle infériorité et grossièreté présente deux variétés : se violenter, et s'abandonner. Si l'homme se gouverne par le bien, il n'est rien qu'il ne puisse changer ; bien qu'extrêmement stupide et grossier, il peut tout aiguiser peu à peu et par suite il est susceptible d'avancer. Mais celui qui se violente et se contraint résiste à la transformation parce qu'il n'y a pas confiance ; celui qui s'abandon ne arrête cette transformation parce qu'il n'agit point ; bien qu'initié par la présence d'un homme saint, l'action transformatrice ne peut pas pénétrer. C'est là ce que Khong Tse désigne par les mots « inférieur et grossier». Toutefois, dans l'univers, ceux qui se violentent et s'abandonnent ne sont pas tous nécessairement stupides et grossiers; de temps à autre, il y en a qui sont violents et malfaisants et dont les aptitudes et la force sont supérieures à celles des autres hommes. Tel fut le cas de Sheang Sin. L'homme saint les appelle grossiers parce qu'ils rompent eux-mêmes avec le bien, et d'ailleurs si l'on examine vers quelle fin ils tendent, on sera convaincu de leur stupidité. Du moment où il est dit qu'ils sont inférieurs et grossiers, comment sont-ils capables de changer leur visage ? Réponse : bien que leur cour ait rompu avec la voie du bien, ils craignent l'autorité et fuient les peines et en cela ils sont comme les autres hommes; mais ce quils ont de com mun avec les hommes c'est ce qui fait comprendre que leur faute n'est pas le résultat de 1 eur nature.

TSHOU HI. - La voie de la suppression et du renouvellement est achevée ; la modification de l'homme doué est comme celle de la panthère, et l'homme inférieur, lui aussi, change son visage par obéissance ; on ne doit rien attendre en entreprenant et c'est de demeurer dans la droiture que résultera le présage heureux. Les choses modifiées ou renouvelées ne le sont que par suite de l'impossibilité où l'on se trouve de ne pas effec tuer ces changements; on ne doit point agir avec excès, et les aptitudes du trait supérieur hexaire elles-mêmes ne doivent pas l'autoriser à agir. C'est pour cela que tel est le sens divinatoire.

878.

Homme doué, panthère se modifiant; son apparence est distincte. L'homme inférieur renouvelle son visage ; il est soumis pour suivre le prince.

TSHENG TSE. — L'homme doué obéit à la transformation et s'améliore ; son apparence s'achève et est semblable à l'élégance des fleurs, manifeste et brillante au-dehors. Depuis l'homme ordinaire et au-dessus, personne ne reste sans modification et renouvellement ; même l'homme inférieur, qui ne change pas, n'ose donner cours à ses sentiments odieux ; il supprime et change son 
extérieur pour obéir avec soumission aux enseignements du prince ou du supérieur : c'est là son « renouvellement de figure ». Parvenu à ce point, la voie du renouvellement est achevée ; l'homme inférieur s'efforce et simule le bien, l'homme doué le tolère : s'il entreprenait de le réformer davantage, le présage serait malheureux. 


\section{Ting}

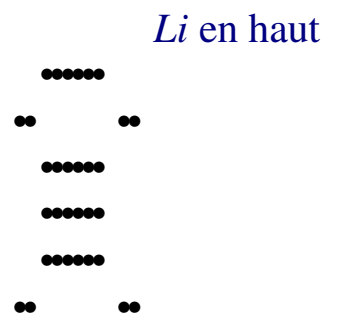

Souen en bas

879. Ting : grandeur, présage heureux, liberté.

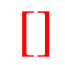

883. Premier trait hexaire : marmite renversée les pieds en haut; avantage à en sortir ce qui est mauvais; posséder une concubine à cause des enfants ; pas de culpabilité.

*

885. Deuxième trait nonaire : la marmite est pleine; nos ennemis sont dangereux, ce n'est pas nous qui devon s approcher; présage heureux.

$*$

887. Troisième trait nonaire : les oreilles de la marmite changent; son action est empêchée; ne pas manger la graisse de la poule sauvage; au moment où

la pluie survient, défaut de regrets ; finalement le présage est heureux.

$*$

889. Quatrième trait nonaire : marmite dont les pieds sont cassés, renverser la nourriture du dignitaire revêtu du titre de kong; la forme est mutilée ; présage de malheur.

$*$

891. Cinquième trait hexaire : marmite à oreilles jaunes ; anse de métal ; avantage de la pureté.

$*$

893. Trait supérieur nonaire : marmite à anse de pierre fine; grand présage heureux; rien sans avantage.

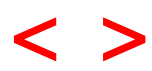


50. Ting.

TSHENG TSE. - Ting. «L'Ordre des koua » dit : «Rien ne change les choses comme la marmite, aussi le koua ko est suivi du koua ting. » L'effet produit par la marmite est le changement ou altération $(\mathrm{ko})$, des choses ; elle transforme ce qui est cru en chose cuite ; elle amollit ce qui est dur. L'eau et le feu ne peuvent subsister ensemble dans le même lieu; pouvoir les amener à produire ensemble un effet utile, sans quils se nuisent mutuel lement, c'est bien là changer $(\mathrm{ko})$ les choses, et c'est ce qui fait que le koua ting suit immédiatement le koua $k o$. Il est constitué par le koua simple $l i$ en haut et le koua simple souen en bas, ce qui est considéré comme représentant la marmite, de sorte que l'on choisit cette image symbolique et ce sens. Il y a deux manières de reconnaître le symbole; si l'on en parle au sujet de la substance complète du koua, le trait inférieur placé sur le sol forme les pieds ; les traits pleins du milieu forment le ventre et présentent l’image symbolique de quelque chose dans l'intérieur ; les deux fragments du trait brisé qui se trouve au-dessus et sont placés en face l'un de l'autre forment les oreilles; le trait qui les dépasse au-dessus, c'est l'anse : c'est bien l'image symbolique d'une marmite ${ }^{1}$. Si on en parle au point de vue des deux substances des koua simples, la partie creuse à l'intérieur est au-dessus, tandis qu'au-dessous il y a des pieds pour soutenir, ce qui constitue encore l’image symbo lique de la marmite. S'il s'agit de choisir le sens, le bois (souen) suit le feu (li); le koua simple souen exprime l’idée d'entrer, de pénétrer, le sens de suivre passivement. Puisque le bois suit le feu et est soumis à son action, cela constitue l'image symbolique du bois brûlant. L'effet utile du feu ne peut être que de brûler et de cuire ; pour brûler il n'a besoin d'aucun instrument, de sorte qu'on choisit l'image symbolique de la cuisson et que le koua est considéré comme représentant une marmite : puisque le bois entre dans le feu, c'est l’image symbolique de la cuisson. L'objet fut-il inventé d'après le symbole du koua, ou bien symbolisa-t-on l'objet pour faire le koua ? Réponse : l'invention de l'objet fut empruntée au symbole ; l'image symbolique existe dans le koua et le koua ne doit pas être antérieur à l'objet. L'homme saint en déterminant l'objet (en l'inventant) n'a pas eu besoin d'attendre la vue du koua pour avoir ensuite la connaissance du symbole ; c'est parce que la foule des hommes ne pouvait pas avoir la connaissance du symbole, quil établit les koua, pour le leur faire comprendre. L'ordre de priorité entre le koua et l'objet n’importe pas au sens. On peut douter si la marmite est un symbole spontané et naturel, ou si elle n'est pas plutôt l'œuvre de l'homme ? Réponse : elle est certainement l'œuvre de l'homme ; mais, la cuisson peut achever et compléter les choses et la forme réglée telle qu'elle existe, de façon à pouvoir être employée, n'est certainement pas l'œuvre de l'homme et est spontanée. Il en est encore de

\footnotetext{
1 Voici la représentation graphique de cette explication bizarre :
} 
même dans le cas du koua tsing ; bien que l'objet existât avant le koua, cependant ce qui a été emprunté pour son invention, c'est précisément l'image symbolique du koua; à son tour, le koua emprunte à l'objet pour définir le sens qu'il comporte. 


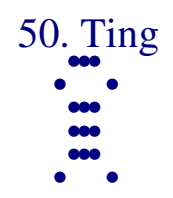

879.

Ting : grandeur, présage heureux, liberté.

TSHENG TSE. - Il s'agit des aptitudes du koua ; avec les aptitudes indiquées par le koua, il devient possible d'arriver à une grande liberté. Il convient de mentionner seulement la grandeur de la liberté, et le présage heureux est une surcharge du texte. Les aptitudes indiquées par le koua sont capables de conduire à une grande liberté, mais il n'y a encore aucune raison pour mentionner la grandeur du présage heureux. Le commentaire de la formule déterminative, de son côté, mentionne seulement la grandeur de la liberté, ce qui prouve clairement la superfétation.

TSHOU HI. - Ting, objet servant à la cuisson des aliments ; on considère le trait inférieur négatif du koua comme représentant les pieds, les second, troisième et quatrième traits positifs comme représentant le ventre, le cinquième trait négatif comme représentant les oreilles et le trait supérieur positif comme représentant l'anse. Ce koua présente le symbole d'une marmite. De plus, puisque le bois représenté par le koua simple souen entre dans le feu symbolisé par le koua simple $l i$, et que la cuisson des aliments en résulte, cela exprime l'usage, ou effet utile de la marmite. C'est pour cela que le koua est appelé ting: En bas, le koua simple souen, humilité ; en haut le koua simple $l i$, qui symbolise les yeux (clarté) et d'ailleurs le cinquième trait représente les oreilles: cela donne donc l'image symbolique de l'humilité intérieure et de l'intelligence extérieure. Ce koua provient du koua souen ${ }^{1}$, dans lequel la négativité avance et vient occuper le cinquième rang en correspondant sympathiquement à la positivité du second trait nonaire placé au-dessous ; c'est pour cela, que la formule divinatoire dit : grande liberté. Le terme «présage heureux » est de trop.

880.

Le commentaire traditionnel de la formule déterminative dit: Ting, marmite; c'est un symbole. Au moyen du bois soumis à l'action du feu on

\footnotetext{
${ }^{1}$ Koua $\mathrm{n}^{\circ} 57$.
} 
opère la cuisson des aliments ${ }^{1}$. L'homme saint les fait cuire ${ }^{2}$ pour les présenter en offrande au souverain suprême ${ }^{3}$, et il jouit dune grande liberté pour nourrir les saints et les sages.

TSHENG TSE. - Ce qui fait que ce koua est appelé ting, c'est qu'il prend l'image symbo lique de la marmite ; ce qui constitue la marmite, comme objet usuel, c'est la conformité de ses usages à l'image symbolique du koua. L'objet n'existe qu'après le symbole, ensuite le koua emploie l'objet comme déterminant le sens. La marmite est un grand objet, lourd et solide, aussi, pour la construire, on fait un moule, en se réglant très exactement sur l'image symbolique ; le nom de ce koua lui convient donc exactement ; les hommes de l'antiquité, en posant les règles, se guidaient sur ce qui est réel et droit. Si on en parle au point de vue de la forme, deux oreilles se dressent en face l'une de l'autre à la partie supé rieure, et des pieds sont posés alternant régulièrement à la partie inférieure. Elle est ronde à l'intérieur et à l'extérieur ; haute ou basse, large ou étroite, chaque détail est réglé et extrêmement régulier. Étant extrêmement régulière, elle constitue et présente l'image symbolique du calme sérieux et grave ; c'est pour cela que la marmite est un ins trument qui imite et reproduit les règles indiquées par les symboles ${ }^{4}$. Si le koua est considéré comme représentant la marmite, c'est à cause de son image symbolique : puisque le bois (souen), est soumis à l'action du feu (h), les deux substances des koua simples expriment l'usage et l'effet utile de la marmite. C'est par le bois introduit dans le feu, par le bois soumis à l'action du feu, que les aliments sont cuits et qu'on peut s'en servir libre ment. Comme objet usuel, la marmite est celui qui est le plus important au point de vue de la vie des hommes; les cas les plus importants entre tous ses usages, sont lorsque l'homme saint s'en sert pour cuire les choses qu'il présente en offrande à l'être suprême, ou pour nourrir librement les saints et les sages. L'expression «l'homme saint » désigne ici les saints rois de l'anti quité. Le mot grand indique la grandeur du but.

TSHOU HI. - Explication du nom et du sens du koua au moyen de la substance et des images symboliques des deux koua simples. La formule cite ce qu'il y a de plus important, comme exemple. Dans l'offrande à Die u, c'est la sincérité d'intention qui est précieuse ; on ne peut employer que de jeunes taureaux. Quand il s'agit de nourrir les sages, c'est une allusion à la cérémonie du plateau assorti de riz et d'aliments cuits. Il convient d'ailleurs de pousser à

\footnotetext{
${ }^{1}$ Le texte porte le caractère heng, "liberté », les commentateurs lisent comme s'il y avait «cuire, cuisson». De là, on conclut que, primitivement, les trois caractères «liberté », «offrandes », et «cuire, cuisson » s'écri vaient de la même manière ? C'est d'autant moins probable que précisément dans la même sentence et quelques mots plus bas, on trouve les deux caractères heng, et heang, chacun avec un sens différent. Je crois que, dans le cas actuel, il vaudrait mieux lire : « libre usage des aliments ».

${ }^{2}$ Heng, en dispose librement, jouit de leur usage pour...

${ }^{3}$ L'Être suprême ; cette expression désigne la divinité, et par extension, l'empereur son repré sentant sur la terre.

${ }^{4}$ Les symboles célestes.
} 
l'e xtrême limite l'accomplissement parfait de chaque rite, ainsi le texte porte « grande liberté ».

881.

Avec l'attention soumise, les yeux et les oreilles voient et entendent clairement; on avance doucement et on monte en progressant; on possède la justice et on sympathise à l'énergie ; c'est par là qu'il y a grande liberté.

TSHENG TSE. - Après avoir parlé plus haut des effets ou usages de la marmite, il est encore question des aptitudes des koua simples; si l'homme peut avoir ces mêmes aptitudes, il lui est possible d'arriver à la grandeur de la liberté. La substance inférieure est l'humilité (souen); elle exprime l'humble soumission à la raison d'être. En haut, c'est la clarté du koua simple $l i$, avec le vide intérieur ${ }^{1}$ ce qui constitue l'image symb olique des oreilles et des yeux entendant et voyant clairement. Toutes les fois que le koua $l i$ est au-dessus, les formules mentionnent également que la douceur malléable avance et monte pour agir. Or la douceur malléable est quelque chose d'inférieur, tand is qu'ici elle occupe le rang prééminent; elle avance et monte pour agir. Puisque éclairée (intelligente), elle occupe la situation prééminente et qu'elle se conforme à la voie rationnelle de la justice et correspond sympathiquement à la dureté énergique, elle peut employer la voie rationnelle de la dureté énergique positive. Le cinquième trait se maintient dans la justice et, de plus, il emploie la douceur malléable et sympathise avec la dureté énergique, il est considéré comme se conformant à la voie rationnelle de la justice ; ses aptitudes étant telles, il en résulte que la grande liberté est possible.

TSHOU HI. - Explication de la formule du koua au moyen des images symboliques des koua simples, de la modification et de la substance du koua lui-même.

882.

Le commentaire traditionnel de la formule symbolique dit : Au-dessus du bois il y a du feu: marmite. L'homme doué par la rectitude de la situation détermine la destinée.

\footnotetext{
${ }^{1}$ Le trait brisé.
} 
TSHENG TSE. - Au-dessus du bois il y a du feu, image symbolique de la cuisson des aliments, ce qui fait que le koua est appelé ting, marmite. L'homme doué contemple ${ }^{1}$ l'image symbolique de la marmite, et par la rectitude de sa situation il fixe la destinée. La marmite est un objet qui imite le symbole ${ }^{2}$. Sa forme est massive et correcte, sa substance la rend immobile et lourde. Si on relève la solidité et la régularité qu'elle symbolise, cela exprime le fait de rectifier la situation c'est-à-dire de rectifier la situation que l'on occupe. L'homme doué, en se plaçant, doit absolume nt suivre la droiture, et cela, même dans les plus petites chose; par exemple si une natte n'est pas posée droit, il ne s'y assied pas ; il n'appuie pas un pied sur l'autre, ni son corps contre quelque chose. Si on relève l'image symbolique d'immobilité e $\mathrm{t}$ de poids qu'elle présente, elle exprime la détermination dans les ordres et les prescriptions, c'est-à-dire le calme et la fixité dans les ordres et les prescriptions. Le caractère traduit par «déterminer» signifie littéralement solidifier, congeler, il exprime le sens de fixité absolue, comme dans la locution usuelle et moderne «immobile comme figé »; ce mot se rapporte aux ordres et prescriptions. Chaque mouvement doit toujours être calme et grave.

TSHOU HI. - La marmite est un objet pesant; c'est pour cela que le koua comporte l'idée de rectitude de situation et de détermination dans les ordres. Le mot traduit par «déterminer» a la même valeur que dans l'expression « la voie rationnelle elle-même n'est pas déterminée ». C'est ce que le grand commentaire exprime en disant: " Il aide au-dessus et au-dessous pour procurer l'assistance du ciel. »

${ }^{1}$ On pourrait lire « montre aux yeux ».

2 Tel que les règles de la construction sont prises dans les symboles représentés par les astres. 


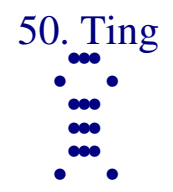

883.

Premier trait hexaire : marmite renversée les pieds en haut; avantage à en sortir ce qui est mauvais; posséder une concubine à cause des enfants; pas de culpabilité.

TSHENG TSE. - Trait hexaire placé en bas du koua, image symbolique des pieds; il monte sympathiquement vers le quatrième; les pieds sont tournés en haut, donc, image symbolique de renversement. La marmite étant renversée, les pieds sont retournés en l'air, et les pieds étant retournés en l'air, le contenu se trouve renversé, ce qui n'est pas conforme à la voie rationnelle. Toutefois, il y a des moments où ce renversement est convenable, par exemple lorsqu'on l'incline pour en sortir la saleté et l'impureté afin de la nettoyer et de la mettre à neuf. C'e st pour cela que le renversement de la marmite est avantageux pour en sortir ce qui est mauvais. Le huitième caractère du texte signifie «mauvais ». Le quatrième rang est la situation du haut fonctionnaire placé près du prince, le premier trait représente un homme placé dans l'infériorité, et il y a correspondance sympathique entre eux; c'est le supérieur implorant l’inférieur et l'inférieur suivant le supérieur. Le supérieur peut utiliser le bien qui existe dans l'inférieur, l'inférieur peut aider à l'a ction du supérieur, ce qui rend possible l'achèvement de l'œavre et de toutes choses et constitue la voie rationnelle du bien. De même que dans le cas du renversement de la marmite, il y a des cas où le renversement est opportun, sans qu'il constitue un ac te contraire à la raison d'être des choses. Posséder une concubine à cause des enfants, pas de culpabilité ; le trait hexaire est négatif et humble, c'est pour cela qu’il est considéré comme représentant une concubine. Posséder une concubine, c'est-à-dire posséder la personne ; si on possède une concubine vertueuse, celle-ci peut aider et assister son maître, de façon que celui-ci ne commette aucune faute et soit sans culpabilité. Le treizième caractère, tse « enfant », désigne le maître ; les mots «à cause de l'enfant » signifient «afin que le maître soit sans faute ni culpabilité ». Hexaire et négatif, placé au rang inférieur, humble et soumis en suivant la positivité, ce trait est l'image symbolique d'une concubine. Puisque ce trait, hexaire, monte et sympathise avec le quatrième, il est considéré comme exprimant le renversement de la marmite et c'est ce sens qui est développé ; le premier trait hexaire est, essentiellement, sans aptitude ni vertus particulières qui puissent être signalées, aussi la formule parle de possession de concubine, ce qui signifie que, suivant la personne, il en sera ainsi.

TSHOU HI. - Ce trait est placé au bas du koua ting, il présente l'image symbolique des pieds de la marmite; en haut il sympathise avec le quatrième 
trait nonaire, donc il y a renversement. Cependant, au début du koua, la marmite n'est pas encore pleine, et elle contient les impuretés accumulées depuis longtemps, c'est en la renversant qu'on les en extrait, ce qui constitue un avantage. C'est par la posses sion des concubines qu'on arrive à la possession des enfants, ce qui présente un sens analogue. L'image symbolique de ce trait est ainsi, et le sens divinatoire est l'absence de culpabilité ; c'est qu'en effet, c'est par la destruction que se produit l'œuv re ; c'est de ce qui est vil que provient la noblesse.

884.

Marmite renversée les pieds en haut, ce n'est pas encore un fait anormal; avantage à en sortir ce qui est mauvais, poursuivre ce qui est précieux.

TSHENG TSE. - La marmite renversée les pieds retournés en l'air ; contradiction anomalique à la voie rationnelle. Cependant, ce n'est pas nécessairement un fait anormal, et, en effet, il y a des moments où il faut l'incliner pour en sortir ce qui est sale et mauvais, jeter ce qui est vieux et y mettre quelque chose de nouveau, laver ce qui est sale et y placer ce qui est bon, ce qui donne le sens de suivre ce qui est précieux, c'est-à-dire s'attacher à ce qui est noble. Correspondre sympathiquement au quatrième trait, c'est s'élever vers ce qui est noble.

TSHOU HI. - Marmite les pieds renversés en l'air ; anomalie de la voie rationnelle. Cependant, par là, il devient possible d'en sortir ce qui est mauvais, pour s'attacher à suivre ce qui est précieux, de sorte que cela ne constitue pas encore un fait contradictoire et contraire à la voie rationnelle. Suivre ce qui est noble, c'est-à-dire sympathiser avec le quatrième trait, cela exprime encore l’idée de renouvellement. 


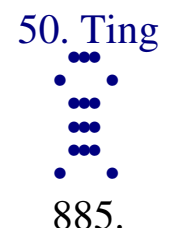

Deuxième trait nonaire : la marmite est pleine ${ }^{1}$; nos ennemis sont dangereux, ce n'est pas nous qui devons approcher; présage heureux.

TSHENG TSE. - Le second trait emploie la dureté énergique, il est massif ${ }^{2}$ et occupe le rang du milieu ; c'est l'image symboliqu e que dans la marmite il y a quelque chose de réel. Ce qu'il y a de solide (matériel) dans la marmite produit son effet en sortant par le haut; le second trait, doué de l'énergie active positive, a les aptitudes nécessaires pour aider à produire l'effet utile. Lui et le cinquième trait se correspondent sympathiquement; il monte pour suivre le prince représenté par le cinquième trait hexaire, de sorte qu'il se conforme à la droiture et que sa voie est susceptible de liberté. Cependant, il est intimement rapproché du premier ; c'est la négativité suivant la positivité. Le second trait nonaire se tient dans la justice et il sympathise avec la justice, il n'arrive pas à manquer à la droiture. Bien que se gardant et s'observant lui -même, l'autre (le premier) l'appelle et l'invoque, aussi la formule comporte l'avertissement qu'il faut pouvoir l'éloigner, afin qu'il ne vienne pas à nous ${ }^{3}$, et qu'alors le présage est heureux. Le septième caractère, « ennemi », désigne ce qui est opposé, ce qui fait contraste ; la négativité et la positivité sont des choses qui contrastent ensemble ; cela désigne le premier trait. S'ils (le premier et le second) se suivent mutuellement, ce sera contraire à la droiture et attentatoire au devoir, et c'est ce qui est exprimé par les m ots « être dangereux » (huitième et neuvième caractères du texte). Le second trait doit employer la droiture pour se préserver, afin que le premier ne puisse venir jusqu’à lui. Si l'homme peut se préserver par la droiture, ceux qui manquent de droiture ne peuvent l'approcher et c'est par là que le présage est heureux.

TSHOU HI. - Puisque la dureté énergique occupe le rang du milieu, c'est l'image sym bolique de la marmite pleine. « Nos ennemis » désigne le premier trait ; négativité et positivité s'appelan t mutuellement et manquant de droiture, de sorte qu'ils tomberont ensemble dans le mal et deviendront ennemis. Le second peut se préserver par son énergie et sa justice, de sorte que le premier, bien que très près, ne peut cependant pas l'atteindre. C'est parce que telle est l'image symbolique et tel est le sens divinatoire que le présage est heureux.

\footnotetext{
${ }^{1}$ Littéralement le texte porte « la marmite à la richesse » ou « la marmite à l'éclat ».

2 Plein.

3 Je, ou nous.
} 
886.

$\ddot{I}$

La marmite est pleine; circonspection en allant ${ }^{1}$, nos ennemis sont dangereux; finalement pas de faute.

TSHENG TSE. — Le plein, pour la marmite, c'est la capacité et le talent pour l'homme ; il convient d'être circonspect dans ce qu'on approche et dans la direction choisie; sans la circonspection dans l'entreprise on tomberait encore dans l'oubli des devoirs. Le second trait peut ne pas conserver de sentiment secret pour le premier, et il monte en obéissant à sa sympathie correcte pour le cinquième trait hexaire; c'est là la circonspection au sujet de la direction. Nos ennemis sont dangereux; citation du texte qui précède. «Nos ennemis» est employé par opposition à «nous-mêmes »; cette expression désigne le premier trait. Le premier trait se presse contre le second, mais il est sans droiture, c'est là « être dangereux ». Du moment où le second trait l'observe et se préserve par la droiture, l'autre ne peut pas l'approcher, de sorte que, finalement, il n'y a pas de faute ni d'erreurs.

TSHOU HI. - Si on est riche et si on n'est pas circonspect en entreprenant quelque chose, ce sera un motif pour que les ennemis surviennent et pour tomber dans le mal.

\footnotetext{
${ }^{1}$ Faire attention où elle va.
} 


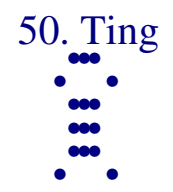

887.

Troisième trait nonaire : les oreilles de la marmite changent ${ }^{1}$; son action est empêchée; ne pas manger la graisse de la poule sauvage; au moment où la pluie survient, défaut de regrets ; finalement le présage est heureux.

TSHENG TSE. - Les oreilles de la marmite, le cinquième trait hexaire ; c'est de lui que dépend le koua ting ${ }^{2}$. Le troisième trait emploie la positivité, il est placé dans l'humilité (souen) et y occupe le rang supérieur. Étant énergique et capable d'humilité, ses aptitudes sont suffisantes pour venir à bout de sa tâche ; cependant, il ne sympathise pas avec le cinquième trait et il n'a pas les mêmes vertus. Le cinquième trait possède la justice et est sans droiture ; le troisième possède la droiture, mais sans la justice ; ce n'est pas la même chose, et c'est celui qui ne s'entend pas encore avec le prince. Ne s'accordant pas encore avec le prince, comment sa voie rationnelle pourrait-elle se produire et agir. Ko, changer, modifier et renouveler et faire quelque chose de différent. Le troisième et le cinquième traits sont différents et ne sont pas d'accord ; l'action est empêchée et ne peut s'opérer librement. Ne s'unissant pas au prince, il ne peut posséder l'autorité ; il n'a pas de moyens d'étendre son influence. La « graisse»; quelque chose qui est agréable au goût, c'est le symbole des émoluments d'une situation officielle. La «poule sauvage » désigne le cinquième trait ; il a les vertus de l'élégance et de l'éclat de l'apparence aussi est -il appelé «poule sauvage » ${ }^{3}$. Le troisième trait a des capacités et des aptitudes, mais il n'obtient de ce cinquième trait hexaire ni émoluments, ni situation; c'est là ne pas réussir à manger la graisse de la poule sauvage. L’homme doué voile ses vertus, à la longue elles doivent briller; sil conserve sa voie rationnelle, à la fin il jouira nécessairement de la liberté. Le cinquième trait présente l'image symbolique de la clarté d'entendement et le troisième est quelque chose qui, finalement, monte en avançant. La négativité et la positivité produisent leur effet en s'unissant et alors il pleut ; au moment où la pluie survient, c'est-à-dire au moment où il va pleuvoir : cela exprime que le cinquième et le troisième traits sont sur le point de s'accorder et de s'unir. « Défauts de regrets ; finalement, présage heureux », veut dire qu'il n'y a pas assez de regrets et que finalement on arrivera à un présage heureux. Le troisième trait cache ses aptitudes et ne voit encore personne venir à lui, aussi il n'a pas assez de regrets ; mais ayant les vertus de la dureté énergique positive, le supérieur étant doué de la clarté

\footnotetext{
${ }^{1}$ Se renouvellent; $k o$, koua $n^{\circ} 49$.

2 C'est par la modification du cinquième trait que le koua se produit.

${ }^{3}$ Peut-être faisan.
} 
d'entendement et l'inférieur de la droiture et de l'humi lité, finalement, ils doivent s'accorder ensemble, de sorte que le présage sera heureux. Bien que le troisième trait soit dépourvu de justice, il fait partie de la substance de l'humi lité de sorte qu'il n'y a pas excès de dureté énergique ; s’il y avait excès de la dureté énergique, comment pourrait-il finalement y avoir présage heureux ?

TSHOU HI. - Puisque, positif, il se trouve placé dans le ventre de la marmite, il représente celui qui, essentiellement, possède des qualités belles et réelles. Cependant, par son excès dans l'énergie, il manque de justice; il saute par-dessus le cinquième trait pour correspondre sympathiquement au trait supérieur; de plus il occupe le rang le plus élevé dans le koua simple inférieur, et comme c'est un moment de modification et de renou vellement, il est considéré comme exprimant que les oreilles de la marmite viennent d'être remplacées et qu'on ne peut la soulever pour la changer de place. Bien quil reçoive l'influence de la clarté de la forme du koua simple supérieur, il a l'excellence de la graisse de la poule sauvage, et ne peut cependant pas servir de nourriture à l'homme. Toutefois, puisque positif il occupe un rang positif, il est considéré comme se conformant à la droiture, et, s'il peut s'observer et se maintenir, la négativité et la positivité seront sur le point de s'accorder harmoniquement et les regrets sur le point de disparaître. Si celui qui consulte le sort est dans de telles conditions, bien que d'abord il soit sans avantage, finalement il jouira d'un présage heureux.

888.

Les oreilles de la marmite se renouvellent; manquer au devoir.

TSHENG TSE. - C'est celui qui commence par renouveler et changer les oreilles de la marmite; il manque au devoir qui existe entre ceux qui s'appellent mutuellement, il ne sympathise pas avec le cinquième trait et manque à la voie rationnelle qui lui prescrit de le rechercher; il est dépourvu de justice, de sorte qu'il ne présente pas limage symbolique de la communauté dans les tendances. C'est à cause de cela que sa marche est entravée et pas libre. Mais en haut se trouve l'intelligence et en bas les capacités ; finalement il doit y avoir entente et accord; aussi la pluie est imminente et le présage est heureux. 


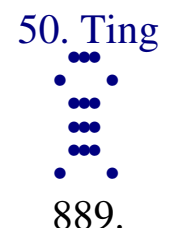

Quatrième trait nonaire : marmite dont les pieds sont cassés ; renverser la nourriture du dignitaire revêtu du titre de kong ; la forme est mutilée ; présage de malheur.

TSHENG TSE. - Le quatrième rang représente la situation des sujets d'un rang très élevé ; il s'agit donc de celui qui a la charge des affaires de l'État. Comment un homme pourrait-il, à lui seul, supporter le fardeau des affaires de l'État ? Il doit nécessairement appeler à lui les gens sages et intelligents dans tout l'univers, pour qu'ils unissent leurs eff orts aux siens, et, sil rencontre de tels hommes, le gouvernement de l'univers peut être parfait, sans nécessiter aucun effort. Au contraire, s'il emploie quelqu'un qui ne répond pas à cette situation, celui-là amènera la décadence des affaires de l'Etat, jusqu'à causer le malheur de l'empire. Le quatrième trait s'abaisse pour correspondre sympathiquement au premier; le premier est un homme inférieur, mou et négatif, qui représente celui qu'on ne doit pas employer. Cependant le quatrième l'emploie, mais comme il n'est pas capable de supporter le poids de sa mission, il compromet les affaires dont il est chargé : il est comme une marmite dont on casse un pied. Une marmite dont un pied est cassé verse sur le côté et renverse la nourriture du noble ou du supérieur. La nourriture, les aliments, ce qui remplit la marmite. Occupant la situation d'un sujet de rang élevé, investi de l'autorité dans l'empire, et employant quelqu'un qui ne doit pas être employé, les choses en arrivent jusquà ce point de renversement et de ruine; c'est là, au plus haut point, n'être pas à la hauteur de l'autorité dont il est investi, et être exposé à la honte et au mépris. Les mots traduits par «forme mutilée », indiquent la rougeur de la honte. Le présage malheureux est évident. Les «formules annexées » disent : «petites vertus et situation prééminente; petit savoir et grandes ambitions ; petites forces et graves responsabilités sont souvent réunies ensemble »; cela exprime le fait de ne pas être à la hauteur de ses devoirs et de son autorité, aveuglé par l'égoïsme, avec des vertus minimes et des connais sances insuffisantes.

TSHOU HI. - Tshiao Shi dit: «Forme mutilée; toutes les éditions remplacent ces mots par ceux-ci : peines et supplices ; c'est-à-dire peines très graves. Actuellement, on suit cette leçon. » Le quatrième trait représente celui qui occupe un rang très élevé et dont l'autori té est considérable ; cependant, il se baisse pour répondre sympathiquement à la négativité du premier trait hexaire : il n'est d onc pas capable de supporter le poids de sa propre autorité. 
C'est à cause de cela que telle est l'image symbolique, et que le sens divinatoire est que le présage est malheureux.

890.

Renverser les aliments du dignitaire ; comment y aurait-il confiance?

TSHENG TSE. — Le sujet d'un rang très élevé, investi de l'autorité sur l'univers, doit être capable de faire régner l'ordre et la paix dans l'univers, et alors il ne décevra pas le prince qui lui délègue cette autorité et qui compte sur lui, ni les espérances du peuple. Avec ces tendances qui le portent à mettre sa personne en avant et à rechercher le pouvoir, il ne faillira pas à sa tâche et c'est là ce qu'on peut appeler être capable d'inspirer la confiance. Autrement, il perdra son rang, il trompera le supérieur qui l’investit de l'auto rité ; cela peut-il amener la confiance ? C'est pour cela que le texte dit : Comment avoir confiance?

TSHOU HI. - Cela exprime que la confiance est perdue. 


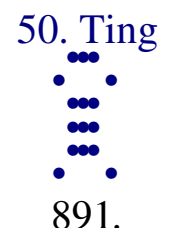

Cinquième trait hexaire : marmite à oreilles jaunes; anse de métal ; avantage de la pureté.

TSHENG TSE. - Le cinquième trait est dans la partie supérieure du koua ting, c'est le haut de la marmite ; image symbolique des oreilles. Ce qui sert à soulever et à transporter la marmite, ce sont ses oreilles ; c'est de l'existence de ce trait que dépend l'existence du koua ting. Le cinquième trait possède la vertu de la justice, aussi la formule parle des oreilles jaunes. L'a nse c'est ce qui est attaché aux oreilles. Le second trait sympathise avec le cinquième ; c'est ce qui vient s'attacher aux oreilles, c'est -à-dire l'anse. Le second trait possède les vertus de la dureté énergique et de la justice. La substance de la positivité est la dureté énergique ; la couleur de la justice est le jaune, aussi est-il considéré comme représentant une anse de métal. Le cinquième trait, à l'élégance de la forme, réunit la justice, et sympathise avec la dureté énergique; le second, énergique et juste, fait partie de la substance de l'humilité et s'élève sympathiquement vers le cinquième. Leurs aptitudes sont en tout point suffisantes, ils sympathisent mutuellement et réalisent l'extrême excellence. Ce qui est avantageux, c'est uniquement la parfaite fermeté ; le cinquième trait hexaire se tient dans la justice et sympathise avec la justice ; il n'en vient pas à manquer à la droiture et ses caractères physiques sont naturellement ceux de la malléabilité négative ; c'est pour cela que la for mule avertit au sujet d'une parfaite fermeté.

TSHOU HI. - Le cinquième trait, dans l’image symbolique, représente les oreilles et il possède la vertu de la justice. C'est pour cela que la formule emploie l'expression : oreilles jaunes. Le métal est une chose dure et solide ; l'anse est ce qui traverse les trous des oreilles pour servir à soulever la marmite. Le cinquième trait, évidé au milieu, c'est-à-dire dépourvu de prétentions, sympathise avec la dureté énergique et la fermeté du second trait nonaire, de sorte que telle est l'image symbolique et, quant au sens divinatoi re, il indique que l'avantage consiste seulement dans la fermeté. On a avancé que l'expression « anse de métal » se rapporte au trait supérieur nonaire. C'est à éclaircir. 
Marmite à oreilles jaunes; la justice considérée comme réalité.

TSHENG TSE. - Dans le cinquième trait hexaire, c'est la possession de la justice ${ }^{1}$ qui est considérée comme constituant le bien ; c'est donc que la justice est considérée comme la vertu réelle. Ce qui fait que le cinquième trait est doué dintelligence et d'entendement, quill est considéré comme celui de qui dépend le koua ling, qu'il se conforme à la voie rationnelle de la marmite, c'est toujours parce qu'il possède la justice.

\footnotetext{
${ }^{1}$ Rang intermédiaire, ou du milieu, dans un koua simple.
} 


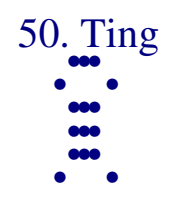

893.

Trait supérieur nonaire : marmite à anse de pierre fine; grand présage heureux; rien sans avantage.

TSHENG TSE. - Dans le koua ting, comme dans le koua tsing, c'est toujours par l'ex traction en montant que se produit l'effet utile. Ce trait est placé au dernier rang; il représente l'achèvement de l'ouvre exprimée par le koua ting (marmite). Étant au-dessus, il a l'image symbolique de l'anse. La pierre précieuse symbolise ce qui est dur et transparent. Bien que ce trait nonaire soit doué de l'énergie active positive, cependant, il occu pe un rang négatif, il marche sur la malléabilité négative, c'est celui qui sans pousser la dureté énergique à sa limite extrême est capable de calme et de dignité. Étant placé dans la voie rationnelle de l'achèvement de l'ouvre, il est simplement placé dans le bien, mais il n’indique rien de plus. La dureté énergique et la douceur malléable sont dans un juste équilibre ; qu'il se meuve ou qu'il reste en repos, il ne commet aucun excès, il est donc considéré comme indiquant un grand présage heureux, et que rien n'est sans être avan tageux. Étant placé au-dessus, il représente l'anse et, bien quil occupe un terrain qui nindique aucune situation spéciale, en réalité, il est précisément en train de produire un effet utile. Ceci est tout à fait différent de ce qui a lieu dans les autres koua, et il en est encore de même dans le cas du koua tsing.

TSHOU HI. - Comme image symbolique, le trait supérieur représente l'anse et, avec sa positivité il occupe un rang négatif : il exprime l'énergie capable de calme et de dignité ${ }^{1}$, de sorte qu'il a l'image symbolique d'une anse en pierre fine et que le sens divinatoire est un grand présage heureux, avantageux en toutes choses. En effet, avec de telles vertus le sens divinatoire doit être applicable.

894.

L'anse de pierre fine est au-dessus; l'énergie et la douceur dans les proportions convenables.

\footnotetext{
${ }^{1}$ Transparent et inaltérable comme une pierre fine.
} 
TSHENG TSE. - Être énergique et calme c'est observer la mesure convenable. Le rang supérieur est le terrain où l'effet utile se produit et où l'œuvre s'achève, aussi, comme l'énergie et la douceur sont dans les proportions convenables, il en résulte un grand présage heureux, et que rien n'est sans avantage. Dans les koua tsing et ting, le puits et la marmite, c'est toujours par l'extraction effectuée par en haut que se produit l'effet utile, mais dans le koua ting la formule n'emploie pas l'expression « grandeur » ${ }^{1}$ et présage heureux ; pourquoi cela ? Réponse : l'œure et l'effet utile du puits (tsing) consistent toujours dans l'extraction par le haut, et, de plus, ce koua indique les vertus, ou propriétés, de vaste étendue de son action et d'effet permanent; c'est à cause de cela qu’il y a gran deur et présage heureux. L'effet de la marmite consiste dans la cuisson des aliments ; être à la partie supérieure indique l'achèvement de cet effet ; ses vertus ou propriétés sont différentes de celles du puits, et comme l'énergie et la douceur sont dans de justes proportions, il y a un grand présage heureux.

${ }^{1}$ C'est mentionné au sujet de la discussion relative aux quatre vertus attributives du koua Khien. Voir koua ${ }^{\circ} 1$. 


\section{Tshen}

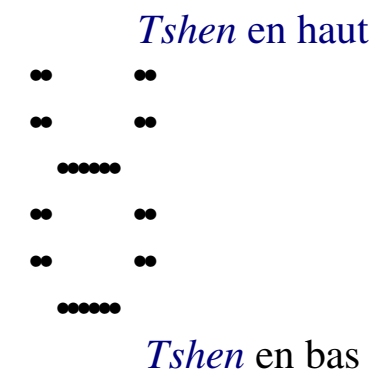

895. Premier trait nonaire : l'ébranlement vient, tremblement causé par la crainte ; ensuite, parler en riant, apparence de gaieté ; présage heureux.

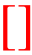

900. Premier trait nonaire : l'ébranlement vient, tremblement causé par la crainte ; ensuite, parler en riant, apparence de gaieté ; présage heureux.

$*$

902. Deuxième trait hexaire : l'ébranlement survient ; péril ; présumer la perte des richesses ; gravir sur neuf collines ; sans poursuivre, atteindre le septième jour.

904. Troisième trait héxaire : ébranlement, être mis hors de soi ; l'ébranleme nt agit sans causer de calamité.

$*$

906. Quatrième trait nonaire : l'ébranlement atteint jusqu'à la base.

$*$

908. Cinquième trait hexaire : ébranlement, en allant et en venant péril ; présumer ne pas perdre, avoir quelque chose.

*

910. Trait supérieur hexaire : ébranlement, tremblement causé par la peur ; regarder avec effarement de tous côtés; en entreprenant le présage sera malheureux. L'ébranlement ne va pas jusqu'à la per sonne, jusqu'à son voisinage; pas de culpabilité ; il y a des représentations au sujet d'un mariage. 
51. Tshen.

TSHENG TSE. - Tshen. «L'Ordre des koua » dit : «Personne n'est le maître des objets autant que le fils aîné ; c'est pour cela que le koua ting est suivi du koua tshen »; ting, l'objet, l'ustensile. Tshen représente le garçon adulte, ainsi le koua prend le sens de domination sur les ustensiles et il est placé à la suite du koua ting. Le fils aîné hérite de l'état, succède dans la situation du chef de la famille et dans ses titres, et c'est pour cela qu'il est considéré comme le maître des ustensiles ou objets mobiliers. L'ordre des koua cite le côté le plus important dans ce sens, c'est-à-dire le sens de succession et d'hérédité. Comme koua simple, le koua tshen est constitué par une positivité naissant au-dessous de deux négativités ; c'est ce qui s'agit e et monte ; ainsi il est appelé tshen, mot qui signifie mouvement, ébranlement initial. Le koua n'est pas appelé tong (mouvement); le mot tshen a plus particulièrement le sens de mouvement ébranlant brusquement, ébranlement effrayant. Dans l'union de l'activité énergique et de la passivité ${ }^{1}$, une première étreinte produit l'ébranlement (tshen) ; c'est le développement de la naissance des êtres, aussi ce koua simple est considéré comme exprimant le principe mâle adulte. Son image symbolique est la foudre; son sens, le mouvement; la foudre est l'image symbolique de l'ébranlement brusque, tandis que le mouvement exprimé par le caractère tong indique le sens de crainte et d'effroi.

\footnotetext{
${ }^{1}$ Khien et khouen.
} 


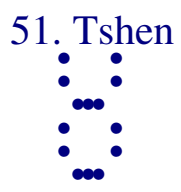

895.

Tshen : liberté ; l'ébranlement vient, tremblement causé par la crainte ; parler en riant, apparence de gaieté ; l'ébranlement effraye à cent lis ${ }^{1}$; ne pas perdre la cuiller et le vin épicé.

TSHENG TSE. - La positivité naît en bas et monte en avançant ; cela comporte le sens de liberté; de plus, le koua simple tshen symbolise le mouvement (tong); il exprime l'effroi et la crainte ; la possession de la direction supérieure. Ébranlement et explosion violente et subite; se mouvoir et avancer, craindre et réformer ; avoir la direction et protéger avec grandeur : toutes ces circonstances sont susceptibles d'amener la liberté d'action et d'expansion, de sorte que le koua tshen comporte la liberté. Au moment où survient l'ébranlement du mouvement, il y a un sentiment de crainte et d'effroi, on n'ose jouir du repos, on regarde autour de soi, on prévoit toutes les conséquences possibles, on est comme tremblant de peur. L'expression constituée par les cinquième et sixième caractères du texte exprime l'apparence de veiller et de se préoccuper sans se laisser aller au repos; le caractère du texte exprime encore la manière de guetter du tigre et du serpent, parce que ces êtres regardent tout autour d'eux et épient sans se laisser aller au repos. Étant ainsi placé au moment de l'ébranlement, on pourra assurer et garantir son repos et son bien-être, ce qui donne l'assurance de parler gaiement et une apparence de gaieté. Cette dernière expression indique que l'apparence extérieure comporte le rire et la gaie té. L'éb ranlement effraye à cent lis ; ne pas perdre la cuiller et le vin épicé ; cela exprime la grandeur de l'ébranlement et en même temps la voie rationnelle de se placer dans une telle circonstance. La grandeur du mouvement n'est jamais telle que dans le cas o ù il s'agit de la foudre. Le koua simple tshen symbolise la foudre, et c'est pour cela qu'il est parlé de la foudre ; l'ébranlement et le mouvement de la foudre s'étendent à cent lis d'éloignement, personne n'échappe à la crainte et tout homme perd la possession de soi-même. Le bruit de la foudre atteint à cent lis, mais, celui qui, sacrifiant dans le temple des ancêtres, tient la cuiller et le vin épicé, ne doit pas cesser de se posséder. La sincérité du respect de l'homme n'est jamais aussi grande que dans les sacrifices et les offrandes. La cuiller sert à retenir ce qui est contenu dans la marmite et à le poser sur le plat; le vin épicé sert à répandre les libations sur la terre pour évoquer et faire descendre l'esprit ${ }^{2}$. Au moment où l'officiant répand les libations pour appeler l'esprit, au moment où il présente les viandes en offrande propitiatoire, il exalte dans

\footnotetext{
${ }^{1}$ De distance.

${ }^{2}$ Cette expression descendre est intéressante à mentionner.
} 
son âme le sentiment du respect et de la vénération, de telle sorte que la menace de l'ébranlement de la foudre elle-même ne peut le distraire et le conduire, par l'effet de la crainte, à cesser d'être maître de lui même. Aussi, en présence de l'effroi causé par la grandeur de l'ébranlement, il peut res ter calme et maître de soi-même, sans éprouver d'autres sentiments que ceux de la sincérité et du respect. C'est là la voie à suivre pour se placer pendant l'ébranlement. La formule ne mentionne pas les aptitudes ou qualités exprimées par le koua; elle ne traite que de la voie pour se placer pendant l'ébranlement.

TSHOU HI. - Tshen équivaut à tong, mouvement ou mettre en mouvement. Une positivité commence à naître au-dessous de deux négativités : ébranlement et mouvement. L’image symbolique est la foudre ; le genre est représenté par le fils aîné ${ }^{1}$. Le koua tshen comporte une voie de liberté. «L'ébranlement vient », il surgit, c'est le moment de l'arri vée de cette secousse. «Tremblement causé par la crainte. » Apparence d'être terrifié par la crainte et de regarder avec effroi. "L'ébranlement effraye à cent lis », ceci est dit au sujet de la foudre. La «cuiller», c'est ce qui sert à enlever le contenu de la marmite. Le "vin épicé », vin extrait d'un certain grain mélangé à du curcuma, qui sert à répandre des libations sur la terre pour évoquer et faire descendre l'esprit. « Ne pas perdre la cuiller et le vin épicé » est dit au sujet du fils aîné ${ }^{2}$. Le sens divinatoire de ce koua est que si on est capable de crainte, il en résultera un bonheur et on ne manquera pas à l'observation de ce qui est important dans les soins dont on a la responsabilité.

896.

Le commentaire de la formule déterminative dit: Tshen, liberté.

TSHOU HI. - Le koua tshen comporte une voie rationnelle de liberté ; il est inutile de le mentionner.

897.

L'ébranlement survient ; tremblement causé par la crainte : de la crainte résulte le bonheur. Parler en riant, apparence de gaieté ; ensuite il y a des règles.

${ }^{1}$ C'est-à-dire le genre des êtres représentés est caractérisé par le jeune homme adulte.

2 Officiant devant l'autel des ancêtres. 
TSHENG TSE. — Le koua tshen comporte par lui-même le sens de liberté d'expansion ; cela ne résulte pas des aptitudes indiquées par le koua simple. Lorsque l'é branlement survient, si on est capable de crainte, on s'amendera, on sera circonspect, de sorte que le bonheur peut en résulter et que le présage peut devenir heureux. Parler en riant, apparence de gaieté ; cela exprime qu'on est naturellement dans ces conditions; c'est parce qu'on est susceptible de crainte et de frayeur que l'on est ensuite capable de se placer sui vant les préceptes et les règles des convenances. Du moment où y a des règles, on se calmera et l'on sera sans crainte ; telle est la voie rationnelle pour se placer pendant l'ébran lement.

TSHOU HI. - La crainte amène le bonheur ; craindre et être effrayé pour que le bonheur en résulte. Règles, préceptes.

898.

L'ébranlement effraye à cent lis; effrayer au loin et craindre de près. En sortant, il peut garder le temple des ancêtres et les autels du ciel et de la terre ; il est considéré comme celui qui préside aux sacrifices.

TSHENG TSE. — L'ébranlement de la foudre atteint à cent lis ; ce qui est loin est effrayé, ce qui est près éprouve de la crainte ; tout cela exprime que la puissance de l'action est grande et s'étend au loin. Dans le texte de la formule déterminative, il manque les mots « ne pas perdre la cuiller et le vin épicé ». La formule du koua ${ }^{1}$ contient cette phrase qui, essentiellement, exprime le comble de la sincérité du respect et de la vénération. La crainte d'une puissance quelconque ne peut l'amener à perdre la possession de lui même. Le commentaire de la formule déterminative, considérant que le fils aîné doit être dans ces mêmes conditions, cite le texte qui précède et emploie indifféremment le sens relatif au fils aîné. Ce commentaire exprime donc que si la sincérité du respect et de la vénération est telle, qu’il soit possible d'éviter de perdre la cuiller et le vin épicé, le prince sortant, il peut cependant veiller sur le temple des ancêtres et sur les autels du ciel et de la terre, comme étant celui qui préside aux sacrifices. Si le fils aîné est dans les mêmes conditions, il sera capable de veiller à l'accompliss ement des sacrifices rituels ordinaires et de s'occuper des affaires de l'état.

TSHOU HI. - Tsheng Tse considère le terme «près » comme désignant le dessous, ce qui est inférieur, et estime quil manque les quatre caractères traduits par les mots «ne pas perdre la cuiller et le vin épicé »; actuellement, on suit cette leçon. «Sortir», en sortant, doit être considéré comme exprimant

\footnotetext{
${ }^{1}$ Texte $n^{\circ} 895$.
} 
l'héritier présomptif, celui qui préside aux sacrifices. On a aussi dit que le caractère sortir, était placé là par erreur à la place du caractère vin épicé.

899.

Le commentaire traditionnel de la formule symbolique dit: Foudre répétée, ébranlement; l'homme doué utilise la crainte et la frayeur en réformant et en examinant.

TSHENG TSE. - Répétition, succession. En haut et en bas, tout est ébranlé. C'est pour cela que le koua exprime la répétition de la foudre ${ }^{1}$ : l'éclat de la foudre étant continu, son intensité et sa puissance se développent et vont en augmentant. L'homme doué contemple l'image symbolique de la répétition de la foudre et de la puissance de l'ébran lement et il met à profit sa crainte et sa frayeur en se corrigeant, en examinant avec attention et en décrivant ce qu'il voit. L'homme doué craint la puissance du ciel, de sorte qu'il réforme sa propre pers onne, qu'il médite sur ses erreurs et ses fautes et qu'il les corrige. Et cela, il ne le fait pas seulement dans le cas de l'ébranlement de la foudre; toutes les fois qu'il rencontre quelque chose d'effrayant, il agit toujours comme ceci.

\footnotetext{
${ }^{1}$ Répétition du koua simple tshen.
} 


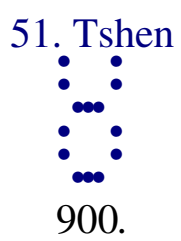

Premier trait nonaire : l'ébranlement vient, tremblement causé par la crainte ; ensuite, parler en riant, apparence de gaieté ; présage heureux.

TSHENG TSE. - Le premier trait nonaire est celui de qui dépend la production de l'ébranlement ${ }^{1}$. Il est au rang inférieur dans le koua, et placé au début de l'ébranlement ; il connaît l'arrivée de cet ébranlement. Au moment où commence l'ébranlement, il est capable de le considérer avec crainte et frayeur, de regarder et de prévoir en embrassant toutes les circonstances possibles, être comme agité d'un tremblement causé par la crain te, sans oser se laisser aller à la sécurité et au repos, alors il assurera son repos final et le présage sera heureux, de sorte qu'ensuite il parlera en souriant et aura l'apparence de la gaieté et du calme.

TSHOU HI. - C'est de lui que dépend la production de l'ébranlement, c'est-à-dire l'achèvement du koua tshen. Il est placé au début de l'ébranlement, de sorte que tel est le sens divinatoire.

901.

L'ébranlement vient, tremblement causé par la crainte; la crainte amène le bonheur. Parler en riant, apparence de gaieté; ensuite il y a des règles établies.

TSHENG TSE. — L'ébranlement survient, il peut éprouver de la crainte et de la frayeur et regarder circulairement tout autour de lui, de sorte quil n'y aura pas de malheur. C'est là savoir utiliser la crainte et la frayeur pour en faire, au contraire, résulter le bonheur. Il utilise la crainte et la frayeur, il se réforme et il s'observe s ans oser contrevenir aux préceptes et aux règles, c'est donc profiter de l'ébranlement pour avoir ensuite des règles fixes et établies, de sorte qu'il peut ainsi assurer son repos et un présage heureux, par conséquent il parle en riant, avec l'apparence du calme et de la gaieté.

\footnotetext{
${ }^{1}$ Sans lui, le koua simple tshen, qui symbolise l'ébranlement, n'existerait pas.
} 


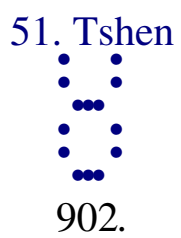

Deuxième trait hexaire : l'ébranlement survient ; péril ; présumer la perte des richesses; gravir sur neuf collines, sans poursuivre, atteindre le septième jour.

TSHENG TSE. - Le second trait hexaire se tient dans la justice et se conforme à la droiture ; c'est celui qui se place bien pendant l'ébranlement. De plus, il foule aux pieds la dureté énergique du premier trait nonaire. $\mathrm{Ce}$ trait nonaire est celui de qui dépend l'ébranlement ; l'ébranlement agite avec énergie et monte soudainement en éclatant; qui donc serait capable de le contenir et de le dominer ? Péril, calamité périlleuse et très intense ; ce qui arrive étant très dangereux, lui-même se trouve en péril. Présumer, compter, évaluer, mesurer. Richesses, les biens ou marchandises qu'il possède. Gravir, monter, faire l'ascension. Les « neuf collines», collines très élevées. Poursuivre, entreprendre d'atteindre. À cause du péril causé par l'ar rivée de l'ébranlement, il calcule qu'il n'est pas capable de résister et qu'il doit nécessairement perdre ce qu'il possède, de sorte qu'il monte dans le lieu le plus élevé, pour fuir. Le mot neuf est employé pour indiquer la répétition ; répétition d'amas de collines; ce qu'il y a de plus élevé. Le nombre neuf indique la multiplicité de la répétition, comme dans les expressions : les neuf cieux, les neuf terres. Sans poursuivre, atteindre le septième jour, ce qui fait la noblesse du second trait, ce qui le rend précieux, c'est la justice et la droiture. Éprouvant la peur et la crainte causées par l'arrivée de l'ébranlement, bien qu'il en mesure la force naturelle, il se retire humblement à l'écart ; il convient qu'il conserve sa justice et sa droiture san s perdre la possession de soi-même. Il présume qu’il doit nécessairement perdre, de sorte quil se retire au loin pour se préserver, et le danger passé, il reprendra ses habitudes ordinaires ; c'est bien là ne pas poursuivre et réussir naturellement à atte indre sans efforts. Poursuivre, c'est suivre quelque chose ; en suivant quelque chose pour l'atteindre, il perdrait ce qu'il veut conserver, aussi la formule avertit de ne pas poursuivre, de se retirer au loin pour se préserver ; c'est là la grande règle dans les cas de l'ébranlement. En agissant comme le second trait, il convient de craindre et de bien se placer. Les situations d'un koua sont au nombre de six ; sept indique que le cycle recommence, que le fait est terminé, que le temps est changé. Il ne perd pas ce qu'il garde, et bien qu'une fois il ne puisse tenir tête à ce qui survient, cependant, le moment passé, le fait fini, il reprend ses habitudes ordinaires, de sorte que la formule dit qu'après sept jours il atteint.

TSHOU HI. - Le second trait hexaire foule aux pieds la dureté énergique du premier trait nonaire, aussi, il fait face au moment où l'ébranlement 
survient et aux périls qui l'accompagnent. Le sixième caractère du texte, présumer, n'est pas encore clairement expliqué. De plus, au moment de perdre ses richesses et ses biens, il monte aussi haut que les sommets des neuf collines ; mais doux et passif, juste et droit, ses qualités sont juste suffisantes pour se préserver, de sorte qu'il n'appelle point à son secours et se protège lui même. Dans la formule de ce trait, le sens divinatoire est complet, l'image symbolique est naturelle, mais les symboles des « neuf collines » ou des « sept jours » ne sont pas encore bien justifiés et éclaircis.

903.

L'ébranlement vient, péril ; fouler l'énergie aux pieds.

TSHENG TSE. - Être en présence de l'ébranlement et fouler l'énergie aux pieds, indique que l'autre est dangereux et que lui-même est en péril ; quand l'énergie de l'ébranlement arrive est -il donc possible de la dominer ? 


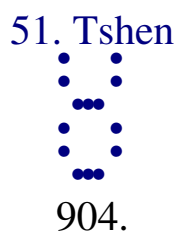

Troisième trait héxaire : ébranlement, être mis hors de soi ; l'ébranlement agit sans causer de calamité.

TSHENG TSE. - L'expression traduite par « être hors de soi » exprime une circonstance dans laquelle le moral et le physique sont totalement déprimés, et où on perd la possession de soi-même. Le troisième trait emploie la négativité et il occupe un rang positif et manque de droiture. En se plaçant sans droiture, même dans un moment ordinaire et tranquille, il est déjà impossible d'être en repos, à plus forte raison lorsqu'il s'agit d'un moment d'ébranlement. Aussi l'ébranlement l'effraye et il est comme hors de lui même ; s'il peut profiter de l'ébranlement et est capable d'agir, quitter ce q ui manque de droiture pour revenir à ce qui est correct, il lui sera possible de ne commettre aucune faute. Le dernier caractère du texte, calamité, signifie ici une faute. Le troisième se mettant à marcher atteindra le quatrième rang, ce qui sera correct. Se mouvoir pour revenir à la droiture est considéré comme constituant le bien, aussi, le second trait atteint naturellement sans poursuivre, et si le troisième entreprend, il ne commet pas d'erreurs. Le manque de droiture coïncidant avec la condition d'être placé pendant l'ébranlement, cause la crainte et il est évident qu'il peut y avoir des fautes commises.

TSHOU HI. - Être mis hors de soi ; être dans un état de prostration et d'annihilation absolue. Puisque, négatif, il occupe un rang positif, qu'il est en présence d'un moment d'ébranlement et quil demeure dans une position contraire aux sentiments de la droiture, cela exprime que si celui qui consulte le sort met à profit la crainte inspirée et agit, pour quitter ce qui est contre la droiture, alors il pourra ne point commettre de fautes et éviter le malheur.

905.

Ébranlement, être mis hors de soi ; la situation est imméritée.

TSHENG TSE. - L'évanouissement des sens est le résultat de la crainte et de la terreur, et la cause en est qu'il est pl acé dans une situation imméritée. Sans justice, sans droiture, est-il donc possible qu'il soit en repos ? 


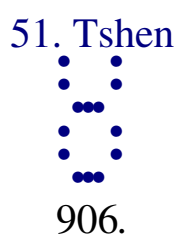

Quatrième trait nonaire : l'ébranlement atteint jusqu'à la base.

TSHENG TSE. - Le quatrième trait nonaire se trouve dans un moment d'ébranlement et d'agitation ; il est sans justice et sans droiture, c'est là une voie rationnelle qui conduit à se placer avec mollesse et à manquer d'énergie et d'activité ; occupant le quatrième rang, il ne possède pas la vertu de la justice, il est embarrassé et comme noyé au milieu de négativités répétées, et c'est celui qui ne peut se secouer et faire effort avec courage. C'est pour cela que la formule parle d'arriver jusqu'à se noyer. Le dernier caractère du texte, boue, vase, désigne ici un flot qui déborde et engloutit. Puisquỉl s'agit d'un trait positif dépourvu de droiture, et qu'au-dessus comme au-dessous de lui se trouvent des négativités accumulées, comment éviterait-il d’être submergé. L'avant-dernier caractère du texte, exprime qu'il n'y a aucune possibilité qu'il en soit autrement. Placé en face de la crainte de l'ébranlement, il ne peut se préserver ; il voudrait se mettre en mouvement et s'ébranler, mais il n'en a pas l'énergie. La voie rationnelle de l'ébranlement est perdue, comment la liberté et l'éclat subsisteraient-ils encore?

TSHOU HI. - Avec son énergie, il se place dans une position qui comporte la mollesse ; il est sans justice comme sans droiture ; il tombe dans l'intervalle entre deux négativités et ne peut se mettre en mouvement. L'avant-dernier caractère indique que l'ídée ne comporte aucune possibilité du contraire. Le dernier caractère signifie boue; se noyer, être submergé par les flots.

907.

L'ébranlement atteint la boue; pas encore d'éclat.

TSHENG TSE. - La positivité est quelque chose d'énergique ; le koua tshen comporte le sens de mouvement. Employer la dureté énergique et se placer dans le mouvement, comporte essentiellement une voie rationnelle d'éclat et de liberté ; mais dans le cas actuel, il y a perte de l'énergie et de la droiture et chute au milieu de négativités répétées, de sorte que cela conduit à atteindre la submersion. Comment pourrait-il encore y avoir de l'éclat? La 
formule mentionne qu'il n'y a pas encore d'éclat pour manifester que l'éner gie positive ne peut pas encore ébranler. C'est à cause de la perte de la vertu qu'il arrive à la submersion. 


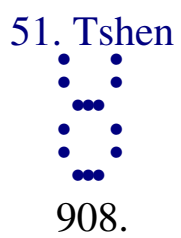

Cinquième trait hexaire : ébranlement, en allant et en venant péril ; présumer ne pas perdre, avoir quelque chose.

TSHENG TSE. - Bien que le cinquième trait hexaire, négatif, demeure dans un rang positif, que la position dans une situation imméritée constitue le manque de droiture, cependant, comme avec sa douceur malléable il occupe un rang qui comporte l'énergie et que, de plus, il possède la justice, il représente donc celui qui possède la vertu de la justice. Ne manquant pas de justice, il ne doit pas aller contre la droiture, et c'est à cause de cela que la justice est considérée comme constituant la noblesse. Dans tous les koua, bien que le second et le cinquième traits ne méritent pas leur situation, le plus souvent c'est la condition de justice qui constitue la beauté dans leurs attributs ; bien que le troisième et le quatrième traits méritent leur situation, il arrive au contraire que le fait qu'ils ne possè dent pas la justice est considéré comme constituant leur erreur ou culpabilité. La condition de possession de la justice est ordinairement plus importante que celle de la possession de la droiture ; en effet, avec la justice on ne fait rien qui soit contre la droiture, tandis que la droiture n'entraîne pas nécessairement la justice. Dans la raison d'être de l'univers, rien n'est plus beau que la justice et cela est visible dans le cas du second et du cinquième traits qui sont hexaires. Quant au mouvement du cinquième, s'il entreprend de monter, sa mollesse le rend incapable de pousser le mouvement à son extrême limite ; s'il cherche à revenir en bas, il brave l'énergie et la provoque, de sorte qu'en allant ou en venant il y a toujours péril. Être en possession de la situation du prince, c'est ce qui est considéré comme exprimant qu'il est celui de qui dépend le mouvement. Suivant l'opportunité, sa sympathie se manifeste, mais elle s'applique uniquement à la justice, de sorte qu'il doit seulem ent apprécier et calculer, de façon à ne point perdre ce qu'il possède. Ce qu'il pos sède, désigne simplement la vertu de justice. S’il ne perd pas la justice, bien qu'il puisse être en péril, il ne lui arrive pas malheur. Apprécier et mesurer, c'est-à-dire méditer et prendre des précautions pour ne pas perdre la justice. Ce qui met le cinquième trait en péril, c'est qu'il n'a point l'énergie de la positivité et qu'il manque d'aide et d'assistance ; s’il employait la dureté énergique positive et sil était aidé, étant le maître de se mouvoir, il jouirait de la liberté. En allant et en venant, il y a également péril : le moment est donc extrêmement difficile, mais en cherchant à ne point perdre la justice, il sera possible qu'il se préserve lui-même. En employant la douceur pour diriger le mouvement, il est très certain qu’il est impossible d'arriver à la liberté d'action et à remédier aux dangers. 
TSHOU HI. - Étant hexaire, il occupe le cinquième rang et il se place dans un moment d'ébranlement ; cela n'est jamais autrement que périlleux. Puisqu'il se conforme à la jus tice, il n'éprouvera aucune perte et peut arriver à un résultat. Si celui qui consulte le sort ne manque point à la justice, bien qu’il soit en péril, il ne subira aucune perte.

909.

Ébranlement, en allant et en venant, péril ; le péril avance. La chose est dans la justice ${ }^{1}$, la grandeur n'est pas perdue.

TSHENG TSE. — En allant et en venant, il y a toujours péril ; en agissant il y aura péril ; quel que soit le mouvement, il sera périlleux. Il s'agit donc exclusivement de ne point perdre ce qu'on possède. La « chose » dont il est question, c'est la justice. Pouvant ne pas perdre la justice, il sera possible de se préserver soi-même. La grandeur n'est pas perdue ; cela veut dire que la grandeur consiste à ne point perdre.

\footnotetext{
${ }^{1}$ Dans le milieu.
} 


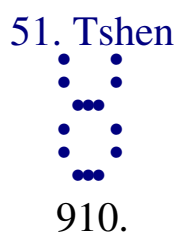

Trait supérieur hexaire : ébranlement, tremblement causé par la peur ; regarder avec effarement de tous côtés; en entreprenant le présage sera malheureux. L'ébranlement ne va pas jusqu'à la personne, jusqu'à son voisinage ; pas de culpabilité ; il y a des représentations au sujet d'un mariage.

TSHENG TSE. - Trembler de peur, effet de la crainte qui cause la perte du sentiment, sans qu'on puisse garder la possession de soi-même ; cela veut dire que la crainte paralyse également la volonté et la force physique. Ce trait hexaire emploie la malléabilité négative et occupe le rang le plus élevé dans le koua qui exprime le mouvement dû à l'ébranle ment. C'est le comble de la frayeur et de la crainte, la volonté et le sentiment sont anéantis. Effarement, apparence du manque de calme et de tranquillité. La volonté et le sentiment sont également anéantis, de sorte que le regard erre de côté et d'autre avec effroi. À cause de son caractère physique, indiquant le manque de justice et de droiture, et étant placé au point le plus extrême dans le mouvement dû à l'ébranlement, sỉl entreprend quelque chose, le présage sera malheureux. L'ébranlement atteint le corps, c'est -à-dire atteint jusqu’à sa propre personne ; s'il n'atteignait pas sa propre personne, cela voudrait dire qu'il n'atteint pas le corps. Le voisinage, c'est tout ce qui est proche de sa personne. Pouvant être ébranlé par la crainte dès avant que le corps ne soit atteint, l'effet ne sera pas poussé à son extrême limite, de sorte qu'il est possible d'éviter toute culpabilité. Tant que l'effet n'est pas poussé à la plus extrême limite, il y a encore une voie rationnelle d'amen dement. L'ébranlement arrivé à sa fin, il doit y avoir transformation; la douceur ne peut pas persister avec fermeté, de sorte que la formule comporte l'avertissement relatif à la crainte du voisinage et le sens de capacité de se modifier. À la fin de l'ébranlement sym bolisé par le koua tshen, l'homme saint avertit les hommes pour quils comprennent le sens de cette idée de la crainte capable d'amender et de corriger ; c'est une exhortation énergique. Le mariage, c'est ce qui crée les relations de parenté par alliance: ici, cette expression désigne ceux qui se meuvent en même temps. Il y a des représentations, c'est-à-dire échange de récriminations verbales. Un trait hexaire occupant le rang supérieur du koua tshen, il commence à être à la tête de ceux qui se mettent en mouvement. Mais actuellement, à cause de la crainte du voisinage, il y a avertissement de ne pas oser avancer, ce qui est différent de ce qui se rapporte à tous les traits du même koua qui représentent ceux qui sont en présence d'un ébranlement. C'est pour cela qu'il y a des représentations au sujet d'une alliance par mariage. 
TSHOU HI. - Puisque la malléabilité négative est placée au moment de l'extrême limi te de l'ébranlement, elle constitue les images symboliques de trembler de peur et d'être effaré, agis sant dans de telles conditions, le présage malheureux est inévitable. Cependant, s’il peut atteindre au moment où l'ébranlement ne gagne pas encore sa propre personne, craindre et être circonspect, réformer et examiner en scrutant attentivement, il sera possible d'éviter toute culpabilité, mais toutefois sans pouvoir éviter les représenta tions au sujet de l'alliance en mariage. Ceci avertit celui qui consulte le sort quil doit se mettre dans ces mêmes conditions.

911.

Ébranlement, trembler de peur ; la justice n' est pas encore atteinte ${ }^{1}$. Bien que le présage soit malheureux, pas de culpabilité ; avertissement de craindre le voisinage.

TSHENG TSE. - Ce qui fait qu'il éprouve une telle crainte de se perdre lui-même, c'est qu'il n'a pas encore atteint la voie rationnelle de la justice. Cela veut dire qu'il a dépassé le rang du milieu dans le koua simple. Si on l'amène à se conformer à la justice, il n'en Vien dra pas jusqu'à trembler de peur. Quand on est parvenu à l'extrême limite d'une condi tion et que l'on continue à avancer, le présage est nécessairement malheureux. S'il peut tenir compte de l'avertissement relatif au voisinage, sil veut craindre, se transformer avant d'arriver à l'extrême limite, il sera sans culpabilité. Le trait supérieur hexaire exprime l'extrême limite du mouvement, et du moment où l'ébranlement est parvenu à son extrême limite, il comporte le sens de modification.

TSHOU HI. - Le mot justice désigne le sentiment de justice du coar.

\footnotetext{
${ }^{1}$ Ou bien : le milieu n'est pas encore atteint.
} 


\section{Ken}

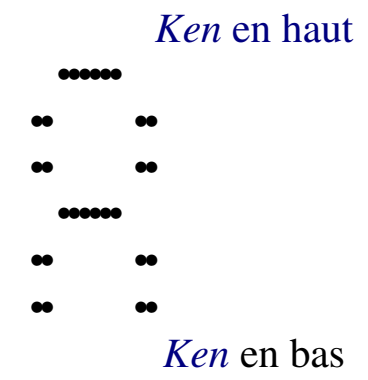

912. Arrêter le dos; ne pas attraper le corps ; parcourir le parois, ne pas voir l'homme; pas de culpabilité.

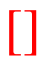

916. Premier trait hexaire : arrêter les orteils, pas de culpabilité ; avantage de la pureté persistante.

*

918. Deuxième trait hexaire :fixer le talon; pour qu'il n'aide pas à suivre le mouvement ; le cour n'e st pas gai.

$*$

920. Troisième trait nonaire : fixer l'articulation de la hanche ; briser les vertèbres ; péril, ardeur du caur.

$*$

922. Quatrième trait hexaire : immobiliser le corps; pas de culpabilité.

$*$

924. Cinquième trait hexaire: immobiliser les angles de la bouche ; parler avec ordre ; dissipation des regrets.

$*$

926. Trait supérieur nonaire : grandeur dans l'immobilité ; présage heureux.

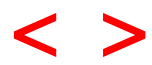




\section{Ken.}

TSHENG TSE. - Ken. «L'Ordre des koua » dit: «Le koua tshen symbolise le mouvement ; les êtres ne peuvent pas se mouvoir indéfiniment ; ils s'arrêtent, aussi le koua tshen est suivi du koua ken : le koua ken symbolise l'arrêt. » Le mouvement et le repos sont la conséquence l'un de l'autre ; s'il y a mouvement, il doit nécessairement y avoir repos, et sil y a repos, il faut qu'il y ait mouvement ${ }^{1}$. La raison d'être des choses ne comporte pas le mouvement perpétuel des êtres, et c'est à cause de cela que le koua ken suit immédiatement le koua tshen. Ken exprime l'arrêt. Le koua n'est pas appelé tshi, arrêt ; le mot ken est l'expression symbolique de la montagne et comporte l'idée d'immobilité, de fixité et de poids, l'idée que le caractère tshi ne rend pas absolument. Dans l'union de l'activité éner gique (khien) et de la passivité (khouen), le troisième trait est échangé et c'est lui qui constitue le koua ken ${ }^{2}$; une positivité se place au-dessus de deux négativités. La positivité est quelque chose qui tend à se mouvoir et à avancer en montant, quand elle est parvenue au troisième rang d'un koua simple, elle doit s'arrêter. La négativité, au contraire, symbolise le repos ; le trait supérieur s'arrête et les traits inférieurs sont en repos, cela constitue donc l'idée d'arrêt exprimée par ce koua ken. Toutefois, quelle différence $\mathrm{Y}$ a-t-il avec l'arrêt exprimé par le caractère tshou? Réponse : l'arrêt exprimé par le mot tshou comporte le sens d'arrêter en contenant le mouvement par force ou par autorité ; c'est arrêter par la force. L'arrêt exprimé par le koua ken comporte le sens d'arrêt pour se reposer, arrêter dans le lieu convenable.

\footnotetext{
${ }^{1}$ Ce passage devient sensé, si on admet qu'il s'agit d'énoncer une loi de l'esprit humain, caractérisé comme incapable de distinguer l'absolu et comme ne pouvant connaître que par comparaison.

${ }^{2}$ Un trait nonaire du koua khien remplace le troisième trait du koua khouen et le transforme en koua ken.
} 


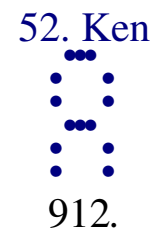

Arrêter le dos ; ne pas attraper le corps ; parcourir le parois, ne pas voir l'homme; pas de culpabilité.

TSHENG TSE. - Ce qui empêche l'homme de jou ir du repos là où il s'arrête, c'est l'agi tation qui résulte de ses désirs; les désirs l'entraînent en avant, et il cherche un lieu pour s'arrêter, sans pouvoir le trouver. Aussi, dans la voie rationnelle de l'arrêt, il convient d'immobiliser le dos. Ce qu'on regarde est en avant et le dos en arrière, c'est ce qu'on ne voit pas. Étant arrêté par ce qu'on ne voit pas, aucun désir ne viendra troubler le cour, et l'arrêt constituera le repos. Ne pas attraper le corps, c'est-à-dire ne pas voir le corps ; c'est s'oublier, faire abstraction de soi -même. Sans égoïsme, on s'arrête ; si l'on est capable d'être sans égoïsme, il n'y a aucune voie rationnelle qui puisse conduire à l'arrêt. Parcou rir le parvis sans voir l'homme ; l'espace compris dans l'enceinte qui porte le parvis de la cour est ce qu'il y a de plus proche; si quelque chose se trouve du côté du dos, bien qu'extrêmement rapprochée, on ne peut la voir ; cela veut dire qu'il n'y a aucune relation avec les êtres ; les êtres extérieurs n'attirant point l' attention, les désirs intérieurs ne naissent point; s'arrêter dans de telles conditions, c'est posséder la voie rationnelle de l'arrêt et s'y accorder ; dans l'arrêt, cela constitue l'absence de culpabilité.

TSHOU HI. - Ken, arrêt, station. Une positivité est arrêtée au-dessus de deux négativités. La positivité monte d'en bas ; parvenue à l'extrême limite de l'ascension, elle s'arrête. L’image symbolique est la montagne. En saisissant cette condition que le trait supérieur du koua khouen, symbole de la terre, se transforme sous une influence florissante, on voit encore l'idée de l'arrêt à l'extrême limite, sans mouvement possible de progression en avant. Celui qui consulte le sort devra être capable de s'arrêter à ce qui est situé derrière son dos sans s'occuper de sa propre personne, parcourir le parvis de la cour sans considérer les hommes, et ainsi, il sera sans culpabilité. En effet, le corps, la personne, est un sujet d'agitation ; le dos seul est fixe et immobile. Fixer le dos, c'est fixer ce qui do it être fixe. Étant arrêté à ce qui doit être arrêté, on ne se laissera pas guider par le corps et on ne sera pas agité ; c'est là ne pas s'occuper du corps, c'est -à-dire de soi-même. Dans ces conditions, bien ,qu'on s'agite dans un espace resserré comme un parvis et encombré d'hommes, on ne fera cependant point attention à ceux -ci. C'est là, en effet, fixer le dos sans s'occuper du corps, s'arrêter et être fixe. Parcourir le parvis et ne point y voir les hommes, c'est marcher et être fixe ; le mouvement et le 
repos sont chacun en leur lieu, et c'est toujours de cette attention que résulte le calme, ce qui rend possible l'absence de toute culpabilité.

913.

Le commentaire traditionnel de la formule déterminative dit : Ken, arrêt ; lorsque le moment est venu de s'arrêter, il s'arrête; lorsque le moment est venu d'agir, il agit ; qu'il s'agisse de se mouvoir ou de s'arrêter, il ne manque jamais le moment opportun. Sa voix est éclatante et claire.

TSHENG TSE. - Ken exprime l'immobilité, l'arrêt ; la voie rationnelle de l'arrêt dépend exclusivement du moment. Qu'on agisse ou qu'on s'arrête, qu'on se meuve ou qu'on reste en repos, si ce n'est pas dans le moment convenable, ce sera toujours mal à propos. Ne pas manquer l'opportunité sera se conformer à la raison d'être des choses et s'accor der au devoir. Chez les êtres, cela constitue le raisonnement ; dans le classement entre les êtres, cela constitue le devoir. Si le mouvement et le repos sont conformes à la raison d'être et au devoir, le moment opportun n'est jamais négligé, et c'est là ce qui constitue l'éclat et la clarté de la voie rationnelle. C'est là ce que l'homme doué apprécie dans le temps ; c'est là ce qui décidait de la lenteur ou de la précipitation du mouvement ou de l'arrêt chez Tshong $\mathrm{Ni}{ }^{2}$. La substance du koua ken est massive et solide, ce qui comporte le sens de clarté et d'éclat.

TSHOU HI. - Ceci explique le nom du koua. Le sens du mot ken exprime l'idée d'arrêt ; toutefois, la progression et l'arrêt ont chacun leur temps, aussi si le temps est venu de s'arrêter, il s'arrête ; si le temps est venu de marcher, il marche de même. La substance du koua ken est massive et solide, de sorte que, de ce côté, on trouve encore le sens de clarté et d'éclat. Dans le koua tae tshou, au sujet du koua simple ken il est aussi question de la splendeur de l'éclat.

914.

Immobiliser lorsqu'il y a lieu d'arrêter. Arrêter dans le lieu convenable ; en haut et en bas équivalence et correspondance, sans alliance entre eux. C'est ainsi que sans posséder le corps, il parcourt le parois sans voir l'homme ; pas de culpabilité.

\footnotetext{
${ }^{1}$ Koua $n^{\circ} 9$ et 26 « petit arrêt » et « grand arrêt».

${ }^{2}$ Khong Tse.
} 
TSHENG TSE. - Immobiliser lorsqu'il y a lieu d'arrêter : cela exprime l'idée de celui qui s'arrête lorsqu'on s'oppose à son mouvement de progression. Celui qui s'arrête lors qu'on l'arrête, s 'arrête parce qu'il a atteint le lieu où il convient de s'arrêter ; s'il s'arrêtait dans un lieu qui ne convienne pas, aucune raison ne pourrait faire qu'il lui fût possible de s'arrêter. Fou Tse ${ }^{1}$ dit : dans l'arrêt, savoir où il convient de s'arrêter, c' est-à-dire le lieu où on doit s'arrêter. Or, lorsqu’il existe des êtres, il doit nécessairement y avoir des lois : le père est arrêté par la tendresse ; le fils est arrêté par la piété ; le prince est arrêté par l'humanité ; et le sujet par le respect. Tous les êtres, toutes choses, ont chacun leur place ; chacune dans son lieu jouit du repos ; placée hors du lieu qui lui convient, elle devient un élément de trouble. Ce que peut l'homme saint, c'est diriger l'univers pour qu'il se soumette à son gouvernement ; il ne peut ni créer les États, ni faire leurs lois ; il ne peut que les contenir (arrêter) chacun dans la place quil doit occuper, et rien de plus. En haut, en bas, équivalence et correspondance ; cela est dit par rapport aux aptitudes exprimées par les koua simples. Les deux substances des koua simples inférieur et supérieur se correspondent mutuellement sans comporter le sens d'alliance; lorsque la négativité et la positivité se correspondent mutuellement, leurs sentiments sont libres et s'accorde nt ensemble ; ici c'est le résultat de leur équivalence, aussi il ne s'allient point. S'ils ne s'allient point, ils se tournent mutuellement le dos, ce qui constitue l'idée d'immobiliser le dos, et le sens d'arrêter. Ils se tournent mutuellement le dos, donc aucun d'eux ne s'empare de la personne et ne voit l'homme. Il en est ainsi à cause de la capacité de s'arrê ter: pouvant s'arrêter, il n'y a aucune culpabilité.

TSHOU HI. - Ceci explique la formule du koua. Tourner le dos, c'est arrêter; puisqu'il éc laire le dos, donc il s'arrête. Le lieu est la partie qui est dans l'immobilité. En en parlant d'après la substance des koua simples, dans le koua simple extérieur et dans le koua simple intérieur, la négativité et la positivité se correspondent avec équivalence, mais sans s'allier ensemble. Puisqu'elles ne s'allient point ensemble, celle qui est à l'inté rieur ne se voit point elle-même, celle qui est à l'extérieur ne voit point l'homme, et il n'y a aucune culpabilité. Tshieu Shi dit: Dans les mots «immobiliser lorsquil y a lieu d'arrêter », on doit, selon la formule du koua, lire « immobiliser le dos ».

915.

\footnotetext{
${ }^{1}$ Khong Tse.
} 
Le commentaire de la formule symbolique dit: accumulation des montagnes du koua ken ${ }^{1}$; l' homme doué en conclut la pensée de ne pas sortir de sa situation.

TSHENG TSE. - En haut et en bas, montagnes sur montagnes, de sorte que ce koua est considéré comme une accumulation de montagnes, ou «montagnes ensemble». Ceci réuni à cela produisent deux choses « ensemble »; c'est-à-dire répétition ${ }^{2}$; c'est l'image symbolique de la répétition du koua simple ken. L’homme doué contemple l'image sym bolique de l'arrêt exprimé par le koua ken, et songe à jouir en repos du lieu où il est arrêté, sans quitter sa situation. Le mot situation exprime la condition dans laquelle il se trouve placé. Chaque chose a un lieu particulier qui lui convient ; lorsqu'une chose est dans le lieu où il convient qu'elle soit, elle s'y arrête et reste en repos. S'il convient de marcher et que l'on s'arrête, sill convient de s e hâter et qu'on s'attarde, que l'on dépasse ce qui doit être ou que l'on reste en deçà, c'est toujours sortir de sa « situation »; à plus forte raison encore, outrepasser les prérogatives de sa condition, n'est -ce pas de la présomption?

\footnotetext{
${ }^{1}$ Répétition du koua simple ken qui représente la montagne.

${ }^{2}$ Le commentateur altère le sens des mots.
} 


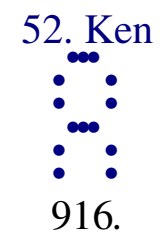

Premier trait hexaire : arrêter ${ }^{1}$ les orteils, pas de culpabilité ; avantage de la pureté persistante.

TSHENG TSE. - Ce trait hexaire occupe le rang le plus inférieur; il présente l'image symbolique des orteils. Le mouvement des orteils précède tous les autres; immobiliser les orteils, c'est arrêter le mouvement à son début. Lorsqu'une chose est arrêtée à son début, elle n'en arrive pas à causer la perte de la droiture, de sorte qu'il n'en résulte aucune culpabi lité. Puisque la douceur malléable est placée dans un rang inférieur, comme c'est dans un moment qui indique les orteils, en agissant elle perdra la droiture, de sorte que l'arrêt exprime l'absence de culpabilité. L’inconvénient de la malléabilité négative réside dans le manque de persistance et de continuité ; elle est incapable de fermeté ; aussi, dès le début de ce qui concerne l'arrêt, la formule avertit que l'avantage consiste dans une pureté persistante et ferme, ce qui préviendra la perte de la voie rationnelle de l'arrêt.

TSHOU HI. - Occuper le premier rang du. koua simple hen, qui exprime l'arrêt, avec la malléabilité négative, est considéré comme l'expression de l'image symbolique de l'arrêt des orteils. Si celui qui consulte le sort est dans ces mêmes conditions, il sera sans culpabilité. De plus, à cause de la malléabilité négative, la formule avertit encore au sujet de la persistance de la perfection.

917.

Fixer les orteils; ne pas encore perdre la droiture.

TSHENG TSE. - Lorsqu'il convie nt de s'arrêter et que l'on marche, ce n'est plus l'observation correcte de la droiture ; en s'arrêtant dès le début, on n'en vient pas jusqu'à manquer à la droiture. Il est aisé d'arrêter quelque chose lorsqu'elle n'est encore qu'à son début, et qu'elle n 'est pas encore arrivée à constituer un vice.

\footnotetext{
${ }^{1}$ Fixer.
} 


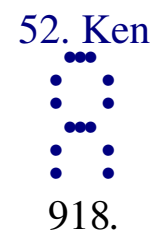

Deuxième trait hexaire :fixer le talon; pour qu'il n'aide pas à suivre le mouvement ; le caur n' est pas gai.

TSHENG TSE. - Le second trait hexaire se maintient dans la justice et possède la droiture ; c'est celui qui possède la voie rationnelle de l'arrêt. En haut, il ne rencontre aucun accueil sympathique ; il ne séduit pas le prince. Le troisième trait occupe le rang supérieur ; c'est de lui que dépend l'achèvement parfait de l'arrêt et c'est lui qui en est le maître. Mais il possède la dureté énergique et perd la justice ; il ne sait saisir l'opportunité de l'arrêt. L'énergie s'arrêtant en haut, elle ne peut descendre et s'abaisser pour réclamer assistance ; bien que le second trait possède les vertus de la justice et de la droiture, il ne peut la suivre. Le mouvement ou l'arrêt du second trait dépendent de celui qui est le maître de sa volonté ; il ne peut pas décider de lui-même, de sorte qu'il présente l'image symbolique du talon. Lorsque la cuisse se meut, le talon suit le mouvement ; l'initiative du mouvement ou de la station résident dans la cuisse et non dans le talon. Du moment où le second trait ne peut agir d'après la voie de la justice et de la droiture pour aider et suppléer au manque de justice du troisième, il doit nécessairement s'efforcer de le suivre ; il est incapable de l'aider, mais il le suit. Outre que la faute ne lui en incombe pas, comment cela serait-il d'ai lleurs sont désir? Ses paroles ne sont point écoutées, sa voie morale n'est point suivie, aussi, son cour n'est pas gai ; il n'est pas à même de suivre l'impulsion de ses propres tendances. Le docteur ${ }^{1}$ qui est placé dans une situation élevée doit aider, assister, et non pas suivre; mais dans une situation inférieure, il y a des cas où il doit consulter et aider, d'autres cas où il doit obéir et suivre ; il y a des cas où il ne peut aider et où, plus tard, il est obligé de suivre.

TSHOU HI. - Le second trait hexaire se maintient dans la justice, il possède la droiture ; il a déjà immobilisé le talon. Le troisième trait représente l'articulation du bassin ${ }^{2}$, c'est là l'origine du mouvement qui suit le talon. De plus, il présente un excès de dureté énergique et manque de justice, puisqu'il est arrêté et maintenu par ce qui lui est supérieur ${ }^{3}$. Bien que le second trait possède la justice et la droiture, et que sa substance soit la faiblesse malléable

\footnotetext{
${ }^{1}$ Lhomme complètement versé dans la doctrine morale et philosophique continuée par Khong Tse.

${ }^{2}$ De la cuisse avec le bassin.

3 Tous ces raisonnements, peu intéressants d'ailleurs, sont à double sens ; la figure anatomique n'est qu'un sy mbole et le sens se rapporte à l'homme qui occupe certains rangs près d'un prince.
} 
et négative, il ne peut entreprendre de remédier aux défauts et aux incapacités du troisième, c'est ce qui fait que son cour n'est pas gai. Le sens divinatoire de la formule de ce trait se trouve dans son image symbolique, et il en est encore de même dans le cas du trait suivant.

919.

Il ne secourt pas ce qu'il suit ; il ne cesse pas encore d'écouter et d'obéir.

TSHENG TSE. - Ce qui fait qu'il ne porte pas aide et remède, c'est uniquement parce qu'il cède à l'impulsion et la suit. Celui qui est au -dessus de lui n'est pas encore capable de s'abaisser à écouter ses avis, il refuse de les écouter et l'inférieur lui obéit.

TSHOU HI. — Le troisième trait est arrêté au-dessus ; il ne veut pas céder et écouter le second. 


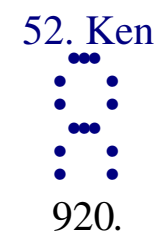

Troisième trait nonaire : fixer l'articulation de la hanche; briser les vertèbres ; péril, ardeur du caur.

TSHENG TSE. - Le cinquième caractère du texte, limite, traduit par articulation de la hanche ${ }^{1}$, indique la séparation ; il exprime la jonction du haut et du bas. Le troisième trait emploie la dureté énergique et occupe un rang qui comporte l'énergie, mais sans la justice ; il est considéré comme celui de qui dépend l'achèvement du koua ken ${ }^{2}$; il indique le comble de l'énergie dans l'arrêt. Étant déjà au rang supérieur de la substance du koua simple inférieur, et formant la limite séparative entre le haut et le bas, toutes ces circonstances contribuent à donner le sens d'arrêt, de sorte qu'il est considéré comme exprimant l'arrêt de la hanche, ce qui constitue la fermeté de l'arrêt et l'impossibilité de pouvoir encore avancer ou reculer. Comme si, dans le corps de l'homme, on désarticulait les vertèbres lombaires qui unissent la partie supérieure à la partie inférieure du corps. Si on désarticule la colonne vertébrale, le haut et le bas du corps ne s'entraînent plus mutuellement, et cela exprime la fermeté inébranlable de l'arrêt de ce qui est inférieur. Ce qui rend la voie rationnelle de l'arrêt précieuse, c'e st la conformité à l'opportunité des circonstances. La marche et l'arrêt ne peuvent résulter d'une détermination unique, prise à l'avance pour toujours. Si l'on y apporte une telle fermeté inébranlable, ce sera une cause de périls extrêmes et de séparation entre tous les êtres. Si un homme reste isolé, à part, se maintenant avec une fermeté absolue dans son arrêt, sans relations avec les autres hommes, il sera plongé dans les peines et les difficultés, agité par la colère et la crainte, à l'intérieur il se consumera par sa propre tristesse : comment pourrait-il être calme et en repos ? «Péril, ardeur du cour », expriment la force naturelle de l'inquiétude qui l'agite et qui brûle en lui.

TSHOU HI. - L'articulation du bassin ; la liaison de la partie supérieure avec la partie inférieure du corps ; précisément ce qu'on appelle le bassin et les cuisses. Vertèbres, la colonne vertébrale. Lorsque le talon est immobilisé, tout mouvement de progression en avant est arrêté. Le troisième trait nonaire, avec son excès de dureté énergique et son manque de justice, se trouve dans une position limite, et il immobilise le bassin, de sorte qu'il ne peut plus se plier, ni se redresser, et que le haut et le bas ${ }^{3}$ sont séparés comme si on avait

\footnotetext{
${ }^{1}$ Ou, peut-être, du bassin.

${ }^{2}$ Qu'il réalise, par la transformation du koua khouen.

${ }^{3}$ Le haut et le bas du corps, ou bien le koua simple inférieur et le koua simple supérieur.
} 
désarticulé la colonne vertébrale. Péril et danger, ardeur du coar, c'est -à-dire manque absolu de calme et de repos.

921.

Fixer la limite ${ }^{1}$; péril, ardeur du caur.

TSHENG TSE. - Cela exprime l'immobilité ferme et absolue sans possibilité d'avancer et de reculer. Préoccupation causée par le péril et la crainte ; entretenir dans le coar une ardeur brûlante.

1 Telle est la traduction la plus littérale et la plus naturelle, en mettant de côté toute interprétation traditionnelle. 


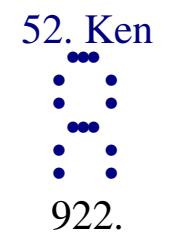

Quatrième trait hexaire : immobiliser le corps; pas de culpabilité.

TSHENG TSE. - Le quatrième rang, c'est la situation du sujet revêtu de dignités élevées. Le quatrième trait représente celui qui arrête ce qui doit être arrêté dans l'univers. Comme il est malléable et négatif et qu'il ne rencontre pas un prince doué de la dureté énergique positive, il n'est pas capable d'arrêter les êtres et il ne peut qu'immobiliser son propre corps, de sorte qu'il peut être exempt de toute culpabilité. Ce qui fait quil peut être exempt de culpabilité, c'est qu'il s'arrête à la droiture. La formule exprime que, arrê tant son propre corps et étant exempt de culpabilité, il est visible qu'il ne peut arrêter les autres êtres et que s’il étendait son action sur les lois et les institutions publiques, il deviendrait coupable. Etre dans une situation élevée et être à peine capable de s'amender soi-même, n'est pas un bien grand motif de louange.

TSHOU HI. - Puisque négatif, il occupe un rang négatif ; puisque le moment comporte l'arrêt et quill s'arrête, il a donc l'image symbolique d'immobiliser sa propre personne, et le sens divinatoire comporte l'absence de culpabilité.

923.

Immobiliser le corps, arrêter toute sa personne.

TSHENG TSE. - Il n'est pas capable de contribuer à contenir (arrêter) l'univers ; tout ce qu'il peut faire est d'arrêter et de contenir sa propre personne : comment cela serait-il suffisant pour mériter le titre de sujet d'un rang élevé ? 


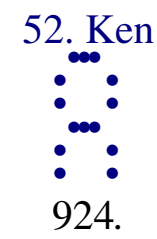

Cinquième trait hexaire : immobiliser les angles de la bouche; parler avec ordre ; dissipation des regrets.

TSHENG TSE. - Le cinquième rang indique la situation du prince ; c'est de lui que dépend l'arrêt et c'e st lui qui est le maître d'arrêter (de contenir) l'univers. Cependant, le cinquième trait possède les aptitudes de la malléabilité négative et cela n'est pas suffisant pour faire face aux devoirs qui lui incombent, aussi c'est seulement par rapport à l'élé vation de son rang que la formule indique le sens d'arrêter ou immobiliser les angles de la bouche. Ce à quoi l'homme doit apporter la plus grande circonspection, lorsqu'il s'agit d'arrêt, c'est surtout à ses paroles et à ses actions. Le cinquième trait e st dans un rang élevé, aussi le trait parle des commissures des lèvres. Les lèvres servent à articuler les paroles; en immobilisant les lèvres, la parole ne s'échappera pas mal à propos et ne viendra jamais qu'avec ordre et opportunité. Si les paroles s'échappent inconsidérément, mal à propos, il en résultera des regrets ; mais en les arrêtant sur les lèvres, les regrets se dissiperont. L'expression « avec ordre » signifie méthodiquement, en suivant un ordre déterminé. Les commissures, les lèvres et la langue sont également les agents de la parole ; les commissures sont dans la partie médiane, de sorte que l'expression « immobiliser les commissures » veut dire immobilité dans ce qui est moyen, entre les extrêmes, ou dans la justice.

TSHOU HI. - Le cinquième trait hexaire représente, par sa position, les commissures des lèvres, aussi telle est son image symbolique, tandis que le sens divinatoire indique que les regrets se dissipent. Le mot « regrets» rappelle la négativité occupant un rang positif.

925.

Immobiliser les coins de la bouche; par la justice et la droiture.

TSHENG TSE. - Le bien, dans le cinquième trait, consiste dans la justice. Immobiliser les coins de la bouche veut dire s'arrêter dans la justice ; cela exprime que la possession de la justice est considérée comme constituant la droiture. Immobiliser les coins de la bouche, afin de ne pas manquer à la justice ; c'est là posséder la droiture et s'y conformer.

TSHOU HI. - Le caractère qui exprime la droiture est une superfétation dans le texte. Cela peut se voir par la consonance de la rime. 


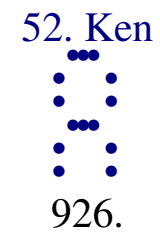

Trait supérieur nonaire : grandeur dans l'immobilité ; présage heureux.

TSHENG TSE. - Nonaire, employant la dureté énergique et la réalité 1 pour occuper le rang supérieur; et, de plus encore, étant celui de qui dépend l'achèvement du koua ${ }^{2}$ et placé à la fin de l'arrêt, c'est celui qui commande l'arrêt avec la fermeté la plus parfaite. "Grandeur», réalité absolue. Il occupe le rang extrême à la limite de l'arrêt, aussi il n’indique pas un excès et est considéré comme indiquant la grandeur. L'arrêt de l'homme est rarement durable et définitif, aussi les règles changent quelquefois avant la fin du jour qui les a vu promulguer et leur observation tombe souvent dans l'oubli avec le temps. Cette versatilité est également à déplorer chez tous les hommes. Le trait supérieur nonaire peut être grand et magnanime jusqu'à la fin ; c'est là l'extrême excellence dans la voie de l'arrêt, et ce qui rend le présage heureux. Entre les vertus des six traits, celle-ci seule est considérée comme constituant un présage heureux.

TSHOU HI. - Avec la dureté énergique de la positivité, il occupe le dernier rang dans l'arrêt ; c'est celui qui porte la g randeur dans l'immobilité.

927.

Présage heureux de la grandeur dans l'arrêt, à cause de la grandeur finale.

TSHENG TSE. - Dans les choses relatives à l'univers, c'est la conservation indéfinie qui est la plus difficile; celui-ci est capable de grandeur dans l'arrêt et peut conserver indéfi niment cette qualité. Le présage heureux du trait supérieur tient à ce qu'il est capable d'une grandeur qui n’a pas de fin.

\footnotetext{
${ }^{1}$ Le trait est plein

2 Par transformation.
} 


\section{Tsien}

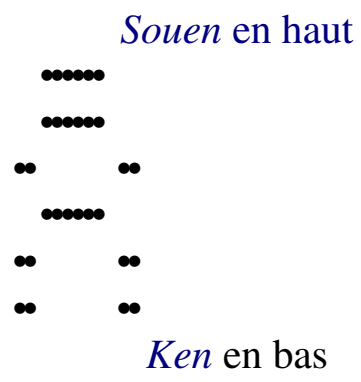

928. Tsien : présage heureux du retour de la fille; avantage de la pureté.

934. Premier trait hexaire: l'oiseau hong avance peu à peu sur le rivage ; péril du petit enfant ; il y a des observations, pas de culpabilité.

*

936. Deuxième trait hexaire : l'oiseau hong avance peu à peu sur le rocher ; il boit, il mange, il est à son aise ; présage heureux.

*

938. Troisième trait nonaire : l'oiseau hong avance peu à peu sur la hauteur ;

l'homme marche sans revenir ; la femme conçoit sans enfanter; présage malheureux ; avantage de la répression du brigandage.

*

940. Quatrième trait hexaire : l'oiseau hong avance peu à peu sur l'arbre ; parfois il rencontre la branche horizontale; pas de culpabilité.

$*$

942. Cinquième trait nonaire : l'oiseau hong avance pe u à peu sur la hauteur ; la femme reste trois ans sans concevoir ; à la fin rien ne le domine ; présage heureux.

*

944. Trait supérieur nonaire : l'oiseau hong avance peu à peu sur la hauteur ; ses plumes peuvent être employées comme ornements somptuaires ; présage heureux.

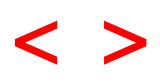




\section{Tsien.}

TSHENG TSE. - Tsien. «L'Ordre des koua » dit : «Ken exprime l'arrêt, les choses ne peuvent pas rester indéfiniment dans l'immobilité, aussi le koua ken est immédiatement suivi du koua tsien; le koua tsien exprime la progression en avant. » L'arrêt doit nécessai rement entraîner le mouvement de progression en avant ; c'est la raison d'être naturelle qui fait que la contraction est suivie de l'extension, et l'inspiration de l'expiration ; il en est de même de l'arrêt qui engendre la progression en avant et qui est l'opposé de la pro gression, et c'est pour cela que le koua tsien suit le koua ken. Avancer par degrés est exprimé par le mot tsien; actuellement encore, on exprime liidée d'avancer lentement à l'aide de ce même mot tsien. Avancer par degrés, méthodiquement, sans sauter brusquement aucun degré, ce qui entraîne la lenteur du mouvement. Comme koua, il est formé par le koua simple souen en haut et le koua simple ken en dessous. Au-dessus de la montagne il y a des arbres; élévation des arbres résultant de la montagne; leur élévation a une cause. Si l'élévation a une cause, c'est que le mouvement de progression suit un ordre établi, et c'est pour cela que le koua est appelé tsien. 


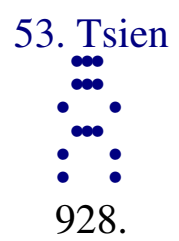

Tsien : présage heureux du retour de la fille; avantage de la pureté.

TSHENG TSE. - Cela est dit au sujet des aptitudes indiquées par les koua simples et aussi à cause du sens du mot tsien. Les koua simples khien et khouen, en se modifiant par transformation, produisent les koua souen et ken ; la superposition des koua simples souen et hen constitue le koua tsien. En en parlant d'après la substance du koua tsien, les deux traits du milieu s'unissent et s'allient ; de l'alliance de ces deux traits résulte que le mâle et la femelle, le garçon et la fille se conforment chacun à la droiture et occupent aussi, chacun, la situation qui lui convient. Bien que le premier et le dernier traits ne soient, ni l'un ni l'autre, di gnes de la situation qu'ils occupent, cependant la positivité est au-dessus et la négativité au-dessous, ce qui est conforme à la droiture, au point de vue de la préséance. Le garçon et la fille se conformant chacun à la droiture et occupant aussi la situation qui lui convient, ce koua et le koua kouei mei forment exactement contraste et opposition. Si l'arrivée de la fille 1 peut avoir lieu dans de telles conditions de droiture, le présage sera heureux. Parmi toutes les choses qui, dans l'univers, doivent absolument se produire avec ordre et mesure, il n'en existe pas qui exigent plus impérieusement cette condition que l'entrée d'une fille dans la famille d'un époux. Les mouvements du sujet qui s'avance à la cour, ceux de l'homme qui s'engage dans quelq ue affaire, doivent très certainement être réglés et méthodiques ; s’ils ont lieu en dépit des règles tracées, il en résulte un manque de décorum et des atteintes au devoir. Le malheur et les fautes doivent en être les suites inévitables. Cependant, au point de vue du plus ou moins de gravité des devoirs, d'après les lois de la décence et de la pudeur, le fait de la jeune fille qui suit un homme est ce qui est considéré comme ce qu'il y a de plus important, aussi le sens est développé d'après les devoirs à observer dans le mariage d'une jeune fille. D'ailleurs, entre toutes les distinctions qui existent entre les choses de l'univers, aucune n'est aussi importante que celle des principes mâle et femelle. On rencontre très fréquemment dans d'autres koua l'ex pression « avantage de la pureté, ou perfection », mais elle peut être appliquée à des choses différentes. Quelquefois, elle est considérée comme un avertissement au sujet de la crainte qu'inspire la propension au manque de droiture ; quelquefois, le sujet de la discussion comportant nécessairement la pureté, il se conforme à cette pureté ; quelquefois, il s'agit de ce qui peut être avantageux et elle indique que l'avantage résulte de la pureté. On trouve un exemple du

\footnotetext{
${ }^{1}$ L'arrivée de la fille dans la famille de son époux.
} 
premier cas dans la formule du second trait nonaire du koua souen ${ }^{1}$. Ce trait est placé à un rang négatif et il se trouve dans le koua simple qui exprime la satisfaction, de sorte que la formule avertit qu'il convient qu'il observe la pureté. Un exemple du second cas se trouve dans le koua tae tshou; il y est dit que ce qui est réuni sera avantageux par la pureté. Enfin, la troisième interprétation se trouve précisément ici dans le koua tsien, où cette expression indique que ce qui fait le présage heureux dans l'alliance de la jeune fille, c'est que ce qui la rend avantageuse est cette pureté et cette droiture. En effet, lorsqu'elle existe avec fermeté, l'expression ne constitue plus un avertissement. Le sens du koua tsien doit pouvoir comporter la liberté d'action, et cependant la formule n e mentionne pas cette liberté ; c'est que, en effet, le mot liberté a aussi le sens de liberté de pénétration et de circulation, ce qui ne répond plus au sens exprimé par le mot tsien qui signifie avancer progressivement et lentement.

TSHOU HI. - Tsien, avancer peu à peu. Ce koua est formé par le koua simple qui exprime l'arrêt ou immobilité en bas, et par le koua simple souen en haut; cela constitue le sens d'avancer sans précipitation. Il représente l'image symbolique de la jeune fille qui s'allie (se $\mathrm{m}$ arie) et entre dans une nouvelle famille. De plus, depuis le second jusqu'au cinquième, chaque trait occupe sa situation avec droiture ${ }^{2}$, aussi le sens divinatoire est que le présage du mariage de la jeune fille est heureux, et d'ailleurs la formule avert it au sujet de l'avantage de la pureté.

929.

Le commentaire traditionnel de la formule déterminative dit: Progression en avant $d u^{3}$ koua tsien ; présage heureux de l'entrée d'une jeune fille dans la famille.

TSHENG TSE. - Avancer dans les conditions exprimées par le sens du mot tsien; là est le présage heureux de l'entrée d'une jeune fille dans la famille : cela veut dire avec droiture et sans précipitation. Le mariage d'une jeune fille est une chose grave. Dans les autres passages, le sens du mot avancer est analogue.

TSHOU HI. - Le caractère tshi du texte, qui indique le génitif « du » paraît être une superfétation dans le texte ; peut-être est-il mis à la place du caractère tsien $^{1}$.

\footnotetext{
${ }^{1}$ Koua $\mathrm{n}^{\circ} 41$.

2 Trait négatif rang impair, trait positif rang pair.

${ }^{3}$ Voir le commentaire de TSHOU HI.
} 
930.

En avançant il obtient une situation; en entreprenant il a du mérite; avançant d'après la droiture, il peut servir à redresser l'État.

TSHENG TSE. - C'est le moment de la progression en avant, lente et mesurée, et la positivité comme la négativité occupent chacune la situation qui leur revient d'après la droiture; il avance et il en acquiert du mérite. Le quatrième trait, à son tour, parvient à une situation qui lui revient d'après la droiture par son mouvement de progression en avant et en montant; le troisième se sépare de ce qui est au-dessous de lui et aide ce qui est au-dessus ; il réussit à obtenir la situation qui lui revient en se conformant à la droiture, ce qui donne toujours le sens d'obtenir une situation par un mouvement de progression en avant. Lorsque le mouvement de progression en avant a lieu d'après la voie rationnelle de la droiture, il devient possible de redresser les états et d'étendre cette action jusqu'à l'uni vers entier. Toutes les fois qu'il s'agit d'un mouvement de progression en avant dans une affaire, dans la voie de la vertu, dans l'obtention d'une situation, ce ne doit jamais être autrement que d'après la droiture.

TSHOU HI. - Explication de l'idée exprimée par les mots « avantage de la pureté », au moyen de la modification des koua simples. En effet, quelle est la transformation qui produit ce koua ? Il provient de la modification du koua hoan $\left(\mathrm{n}^{\circ} 59\right)$ dans lequel le trait nonaire avance pour occuper le troisième rang; il provient de la modification du koua $\operatorname{lin}\left(\mathrm{n}^{\circ} 19\right)$ dans lequel le trait nonaire avance pour occuper le cinquième rang; mais, dans tous les cas, il exprime la droiture dans l'obtention d'une situation.

931.

La situation, c'est l'énergie possédant la justice.

TSHENG TSE. - Le texte qui précède mentionne qu'en avançant il atteint à une situation et qu'en entreprenant il a du mérite; d'une façon générale, cela exprime que la positivité et la négativité occupent chacune la situation qui leur convient et que c'est en avançant que leur ouvre

${ }^{1}$ Dans le premier cas il faudrait lire: «Tsien, progression en avant»; dans le second: « Tsien, avancer lentement et pas à pas. » 
s'accomplit. Actuellement, il s'agit encore de la situation et de l'éner gie possédant la justice. Ce qui est entendu par situation, c'est que le cinquième trait occupe la situation prééminente à cause de sa dureté énergique, de sa justice et de sa droiture. Dans les divers traits, la possession de la droiture peut aussi être appelée possession de la situation, mais cela n'est jamais aussi vrai que dans le cas du cinquième possédant la situation prééminente, et c'est pourquoi il en est spécialement question.

TSHOU HI. - Cela est dit au sujet de la substance entière du koua, mais cela désigne particulièrement le cinquième trait.

932.

Immobilité et humilité ; mouvement sans fin ${ }^{1}$.

TSHENG TSE. - Au-dedans ${ }^{2}$, le koua simple ken : arrêt ; au-dehors, le koua simple souen, soumission. L'arrêt constitue l'image symbolique du repos ; l'humilité donne le sens de concorde et de soumission. Lorsque l'homme avance, s'il est poussé par ses désirs et ses passions, il agit avec précipitation et ne peut procéder graduellement et avec ordre, aussi il est misérable et malheureux ${ }^{3}$. Dans le sens du mot tsien, on trouve le sens de «arrêt et calme intérieurs » et de «soumission et humilité extérieures». Aussi, le mouvement de progression en avant ne peut comporter l'alternative de misère et de pauvreté.

TSHOU HI. - Il s'agit du sens de la progression en avant réglée et graduelle, et il en est parlé au point de vue des vertus exprimées par les koua simples.

933.

Le commentaire de la formule symbolique dit: Au-dessus de la montagne il y a des arbres: Tsien, progression lente et mesurée. L'homme doué s'en inspire pour demeurer dans la sagesse et la vertu et pour améliorer les moaurs.

\footnotetext{
${ }^{1}$ Les koua simples ken et souen.

2 En bas.

${ }^{3}$ Ce caractère a, entre autres, les deux sens « fin » et «pauvreté ». Le commentateur adopte le dernier ;j'ai traduit par le premier.
} 
TSHENG TSE. - Au-dessus de la montagne il y a des arbres; leur élévation a une cause tel est le sens du mot tsien. L'homme doué contemple l'image symbolique exprimée par le koua tsien et il s'en inspire pour se maintenir dans la vertu de la sagesse et du bien, pour améliorer et transformer les mours. La progression de lhomme dans la vertu de la sages se doit nécessairement avoir lieu avec lenteur et par degrés ; il s'y exerce et ensuite il peut y trouver le calme et le repos ; il ne doit pas y parvenir hâtivement en violant les règles et les préceptes. S'il en est ainsi en lui-même, lorsquil agit sur les hommes pour les transformer et les améliorer, s'il ne procè de pas par degrés et avec mesure pourra-t-il donc y réussir? Changer les mours, modifier les coutumes, n'est pas l'affaire d'un jour ; aussi, l'amé lioration des maurs doit se produire par degrés insensibles.

TSHOU HI. - Ces deux choses doivent également progresser avec mesure et lenteur. Il y a lieu de douter si le caractère qui exprime la sagesse n'est point une superfétation. Il est aussi possible qu'il manque un caractère. 


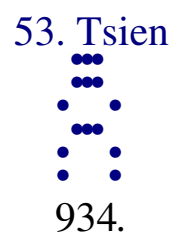

Premier trait hexaire : l'oiseau hong avance peu à peu sur le rivage; péril du petit enfant ; il y a des observations, pas de culpabilité.

TSHENG TSE. - Dans le koua tsien, tous les traits prennent l'image symbolique de l'oiseau hong. Cet animal est un être qui émigre, survient à des époques déterminées et observe un ordre invariable lorsqu'il est en troupe. $\mathrm{Ne}$ jamais manquer le moment opportun, ni intervertir l'ordre de succession, c'est précisément ce qui est exprimé par le caractère tsien. Le sixième caractère du texte représente le bord de l'eau ; l'oiseau aquatique s'arrête sur le bord des eaux, tout à fait à la limite du liquide ; son mouvement de progression en avant peut être caractérisé par le mot tsien, c'est-à-dire qu'il est progressif et lentement mesuré, sans jamais manquer à l'opportunité du moment. Ce trait, hexaire et occupant le premier rang, est dans la position la plus inférieure ; les aptitudes de la négativité sont de la plus extrême faiblesse ; de plus, en haut, il ne rencontre ni sympathie, ni accueil ; avançant dans de telles conditions, c'est une cause ordinaire de tristesse et de chagrin. L'homme doué reconnaît de loin et il élucide la question avec pénétration ; il connaît sur quoi il faut s'appuyer avec calme d'après le devoir et la raison d'être des choses, et ce quill convient de faire selon le moment et la question qui se présente : il se place toujours sans éprouver ni doute, ni incertitude. L'homme inférieur, le jeune enfant, peuvent seulement considérer l'existence du fait, s'en rapporter au savoir du commun des hommes, sans être capables d'en éclaircir la raison d'être. Delà résultent la crainte du péril et, ensuite, les remontrances. En effet, ils ne savent pas que c'est par suite de l'infériorité de leur position qu'ils ont à avancer, que c'est parce qu'ils sont faibles et mous quils ne doivent pas se hâter avec précipitation, que c'est parce qu'ils ne rencon trent aucun accueil sympathique qu'ils peuvent avancer petit à petit et progressivement, sans qu'au point de vue du devoir il en résulte aucune culpabilité. Si, dès le début de la progression graduelle (Khien), on employait la précipitation énergique pour avancer rapidement, ce serait méconnaître le sens du koua Khien, on se trouverait incapable d'avancer, et on se rendrait très certainement coupable.

TSHOU HI. - Les migrations de l'oiseau hong sont réglées et son mouvement de progression est régulier et mesuré. La grève, le bord de l'eau. Commençant à avancer par en bas, il n'y a pas encore lieu de jouir du calme et du repos et, de plus, au-dessus, il ne se présente aucune correspondance sympathique, de sorte que telle est l'image symbolique, tandis que le sens divinatoire sera «le péril du jeune enfant»; bien qu'il y ait des remontrances et des récriminations au point de vue du devoir, il n'y a aucune culpabilité. 


\section{5.}

Péril du petit enfant; devoir sans culpabilité.

TSHENG TSE. - Bien que, pour le petit enfant, ce soit considéré comme un péril et un motif d'appréhe nsion, en réalité, au point de vue du devoir et de la raison d'être des choses, il n'y a aucune culpabilité. 


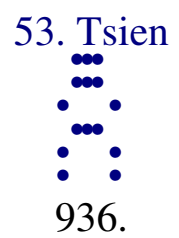

Deuxième trait hexaire : l'oiseau hong avance peu à peu sur le rocher; il boit, il mange, il est à son aise; présage heureux.

TSHENG TSE. - Le second trait se maintient dans la justice, il se conforme à la droiture ; en haut, il correspond sympathiquement au cinquième trait ; c'est celui qui avance dans le repos et la grandeur. Toutefois, il fait partie du koua tsien, de sorte qu'en avançant il ne se hâte pas. Le sixième caractère du texte désigne une pierre plate et lisse, comme il s'en trouve sur le bord des fleuves ; cela symbolise le calme dans la progression en avant. De la grève jusque sur le rocher, il y a encore une progression graduelle. Le second trait et le prince représenté par le cinquième trait nonaire se correspondent sympathiquement par la voie rationnelle de la justice et de la droiture. Le mouvement de progression en avant est calme, certain, facile, égal, sans efforts, aussi il boit, mange, est content, satisfait et à son aise ; le présage heureux est évident.

TSHOU HI. - Rocher, grosse pierre; de plus en plus loin de l'eau ; il avance sur la grève et son bien-être augmente. Bien-être, satisfaction. Le second trait hexaire est malléable et négatif, juste et droit ; il avance, mais progressivement et par degrés, et en haut il jouit de la sympathie du cinquième trait nonaire, de sorte que telle est l'image symbolique et que le sens divinatoire indique un présage heureux.

937.

Boire et manger à l'aise; ne pas se nourrir avec simplicité.

TSHENG TSE. - La formule du trait mentionne la facilité et l'égalité de la progression en avant, aussi elle cite le fait de boire et de manger en repos et à l'aise. Khong Tse, crai gnant que plus tard les hommes ne la comprissent pas clairement, l'explique de nouvea u en disant : l'homme doué, juste et droit, qui rencontre un maître orné des mêmes vertus, avance peu à peu en montant ; il pratique sa voie morale afin d'en faire bénéficier l'uni vers. Ce qui est entendu par les mots manger et boire, être satisfait et à l'aise, c'est réussir selon ses tendances, éprouver de la joie et du bien-être ; cela ne veut pas simplement dire qu'il boit et mange à satiété, « avec simplicité », sans appareil. 


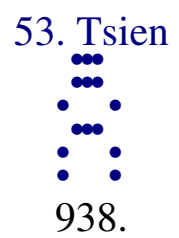

Troisième trait nonaire : l'oiseau hong avance peu à peu sur la hauteur ; l'homme marche sans revenir; la femme conçoit sans enfanter; présage malheureux ; avantage de la répression du brigandage.

TSHENG TSE. - Le sixième caractère du texte désigne une terre unie et élevée, plane et nivelée. Le troisième trait est au rang supérieur du koua simple inférieur; il avance jusque sur la hauteur : c'est la positivité qui monte en avançant. Se trouvant dans le moment exprimé par le koua tsien, ses tendances le portent à avancer peu à peu, et cependant, au-dessus de lui, il ne rencontre ni sympathie, ni accueil. Il doit conserver sa droiture, pour attendre son heure, se placer en paix sur un terrain facile, et alors il sera en conformité avec la voie rationnelle exprimée par le koua tsien. S’il arrive qu’il ne puisse s'observer et se maintenir, si ses désirs l'entraînent, ses tendances sont dirigées vers un but particulier, alors il perd la voie rationnelle de la progression mesurée et lente indiquée par le koua.

Le quatrième trait est négatif, placé au-dessus de lui, et ils sont intimement rapprochés ; c'est ce qui plaît à la positivité. Le troisième trait est positif, il est dans l'infériorité, et ils se rapprochent mutue llement ; c'est l'entraînement ordinaire à la négativité. Ces deux traits se rapprochent et s'associent et d'ailleurs ils n'ont aucune correspondance sympathique. Se rapprochant, ils éprouvent une affection réciproque et se réunissent facilement. Étant sans, correspondance sympathique, chacun se trouve isolé, aussi ils s'appellent mutuellement, et c'est l'objet d'un avertissement. Le septième caractère, l'homme, désigne la positivité ; c'est le troisième trait. Si le troisième trait n'observe pas la droitu re et se joint au quatrième, ce cas répond à ce qu'on entend par l'expression savoir avancer et ne pas savoir revenir. Avancer a, ici, le sens de marcher, et revenir celui d'aller en sens opposé. Ne pas revenir, c'est-à-dire ne pas se retourner vers le devoir et la raison. Le onzième caractère, la femme, désigne le quatrième trait ; si elle s'unit contrairement à la droiture, bien qu'elle puisse concevoir, cependant elle n'enfante pas, et c'est parce qu'elle agit contrairement à la voie rationnelle. S'il en est ainsi, le présage sera malheureux. Pour le troisième trait, ce qui est avantageux c'est de réprimer la perversité et le brigandage. Ce qui survient est contraire à la raison d'être des choses, c'est le brigandage ; maintenir le droit pour entraver le vice, c'est ce qu'on appelle réprimer le brigandage. S'il n'est pas capable de réprimer le brigandage, il se perdra lui-même, et le présage sera malheureux. 
TSHOU HI. - Hong ; c'est un oiseau aquatique ${ }^{1}$; la hauteur, ce qui n'est pas uni. Le troisième trait nonaire est enclin à trop de dureté énergique, il est dépourvu de justice et sans correspondance sympathique; aussi telle est l'image symbolique, et le sens divinatoi re est que, si l'homme avance, il ne reviendra pas, si la femme conçoit, elle n'enfantera point. Le présage malheureux n'est pas absolu, mais toutefois, à cause de l'excès de dure té énergique, l'avantage consiste dans la destruction du brigandage.

939.

L'homme marche sans revenir ; s'il s'écarte de la foule, il en éprouve de la honte. La femme conçoit sans enfanter : elle manque à la voie rationnelle. Avantage de la répression du brigandage: passivement se protéger mutuellement.

TSHENG TSE. - L'homme marche sans revenir en arrière, de sorte qu'il manque à la droiture de la voie du koua tsien (avancer peu à peu); il écoute ses passions et perd la droiture ; il se sépare et s'écarte de la troupe de ceux qui sont de son propre genre, ce qui est considéré comme susceptible d'amener de la honte. Les divers traits du koua ne sont jamais dépourvus de certaines aptitudes pour le bien ; si l'un manque à la droiture, il se sépare de ceux qui sont de son propre genre. La femme conçoit en manquant à la voie rationnelle, ce qui fait qu'elle n'enfante pas. L'avantage consiste dans la répression du brigandage, cela exprime l'assistance mutuelle qui résulte de la soumission à la voie rationnelle. Si l'on compare l'homme doué avec l'homme inférieur, le premier s'observe et se garde par la droiture ; mais comment l'homme doué se bornerait-il à préserver intacte sa propre personnalité ? Il dirige encore l'homme inférieur pour que celui-ci puisse éviter de tomber dans la transgression du devoir, et c'est là ce qui constitue l'assistance mutuelle par la soumission à la voie rationnelle ; enfin il réprime et arrête le mal, et c'est là ce qui est exprimé par les mots réprimer le brigandage.

\footnotetext{
${ }^{1}$ Peut-être l'oie sauvage ?
} 


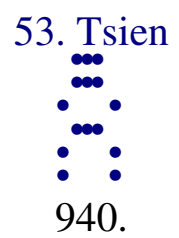

Quatrième trait hexaire : l'oiseau hong avance peu à peu sur l'arbre ; parfois il rencontre la branche horizontale ; pas de culpabilité.

TSHENG TSE. — Dans un moment de progression mesurée, le quatrième trait se sert de la douceur malléable de la négativité et avance pour se mettre au-dessus de la dureté énergique positive. Or, l'énergie positive monte et avance ; comment pourrait-elle se contenter d'être placée au-dessous de la malléabilité négative ? Aussi, le quatrième trait se place sur un terrain qui ne saurait comporter le calme et le repos. Il en est de lui comme de l'oiseau hong qui avance sur un arbre ; peu à peu, l'arbre s'élève et il présente l'image sym bolique d'absence de calme et de repos. L'oiseau hong ales pieds palmés ; il n'est pas capable d'étreindre les rameaux, aussi il ne perche pas sur les branches. Le dixième caractère désigne une branche unie et horizontale ; ce n'est que sur une branche unie et horizontale qu'il peut trouver le repos. Cela exprime que la position du quatrième trait est essentiellement périlleuse. Parfois, il peut de lui-même se conformer à la voie rationnelle du calme et du repos, et alors il sera sans culpabilité. De même que l'oiseau hong placé sur un arbre ne peut essentiellement pas y jouir du repos, mais qu'il arrive quil ren contre une branche horizontale et qu'il s'y place de façon à y être en repos, de même, le quatrième trait se maintient dans la droiture et il est humble et soumis : c'est celui qui doit naturellem ent être sans culpabilité. Tout cela est certainement dit au sujet de, ce quil peut se conformer aux règles ou quil y manque, et c'est par cette conformité ou par ces manquements que le devoir se trouve être mis en lumière.

TSHOU HI. - L'oiseau hong ne perche pas sur les arbres, le terme du texte désigne une branche unie et horizontale. Parfois il rencontre une branche horizontale, de sorte qu'il peut s'y reposer. Le quatrième trait hexaire foule aux pieds l'énergie et il est soumis et humble, de sorte que telle est l'image symbolique. Le sens divinatoire indique que, dans ces conditions, il n'y aura pas de culpabilité.

941.

Parfois il rencontre une branche horizontale; soumission par humilité. 
TSHENG TSE. - Une branche horizontale, un lieu facile et où il peut se reposer. La voie rationnelle de la recherche du repos consiste uniquement dans la soumission et l'humili té. Si on se conforme avec soumission et droiture au devoir, bien que le lieu où l'on se place puisse être humble et inférieur, comment n'y trouverait-on pas le repos? La soumission et l'humilité du quatrième trait, ainsi que sa droiture, sont comme la branche horizontale qui peut servir de lieu de repos. 


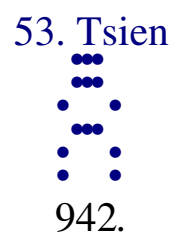

Cinquième trait nonaire: l'oiseau hong avance peu à peu sur la hauteur ; la femme reste trois ans sans concevoir ; à la fin rien ne le domine ; présage heureux.

TSHENG TSE. - Colline, terrain élevé ; l'endroit où s 'arrête l'oiseau hong est le lieu le plus élevé : c'est l'image symbolique de la situation du prince. Bien quil possède la situa tion prééminente dans le moment exprimé par le koua tsien, la voie rationnelle de la progression n'est certainement pas la précipitation. Lui et le second trait se correspondent sympathiquement avec droiture, et la vertu de justice et de droiture est identique chez chacun d'eux. Pourtant, ils sont séparés par le troisième et le quatrième traits ; le troisième se presse contre le second, le quatrième contre le cinquième; tous deux également s'opposent à leur union. Ils ne peuvent pas se réunir immédiatement, de sorte que trois ans s'écoulent avant qu'il y ait conception. Cependant, la voie rationnelle de la justice et de la droiture comporte une raison d'être naturelle de liberté nécessaire ; comment l'absence de droiture pourrait-elle entraver et détruire cette voie ? Aussi, finalement, rien ne peut l'emporter sur elle, mais, toutefois, la réunion n'arrive que graduellement et peu à peu (tsien), aussi le présage finit par être heureux. L'absence de droiture peut, pendant un moment, s'opposer à la droiture et à la justice et les entraver, mais il est impossible qu'elle l'emporte définitivement sur les vertus.

TSHOU HI. - Colline, terrain élevé, butte. Le cinquième trait nonaire occupe le rang préminent; le second trait hexaire lui correspond sympathiquement, au-dessous de luimême, avec droiture, mais ils sont séparés par le troisième et le quatrième traits qui, toutefois, ne peuvent l'emporter définitivement sur la droiture. Aussi telle est l’image symbolique, et si celui qui consulte le sort est dans ces mêmes conditions, le présage sera heureux.

943.

À la fin, rien ne peut l'emporter; présage heureux; obtenir l'objet de ses souhaits.

TSHENG TSE. - Le prince et le sujet s'allient par la justice et la droiture ; leur voie rationnelle doit prévaloir, bien quill y ait quelque obstacle qui les sépare ; finalement, comment cet obstacle pourrait-il l'emporter ?À la longue, ils obtiennent nécessairement ce qu'ils souhaitent, et c'est là le présage heureux du koua tsien. 


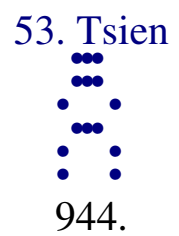

Trait supérieur nonaire : l'oiseau hong avance peu à peu sur la hauteur; ses plumes peuvent être employées comme ornements somptuaires ; présage heureux.

TSHENG TSE. - Hou Kong, de Ngan ting 1, change le sixième caractère en remplaçant le radical [] par le radical [], ce qui lui fait exprimer un lieu auquel plusieurs routes aboutissent; la « route des nuages », c'est-à-dire au milieu de l'espace sans limite. Le $Y i y^{2}$ dit : un carrefour à neuf directions, expression qui comporte le sens de liberté de pénétration sans aucune entrave. Le trait supérieur nonaire est dans la situation la plus élevée et, de plus, il continue encore à monter et à avancer, ce qui est considéré comme exprimant qu'il quitte toute situation établie. Dans d'autres koua, ce serait considéré comme indiquant un excès; dans le moment exprimé par le koua tsien, comme il occupe le rang supérieur dans le koua simple souen (humilité), il doit nécessairement suivre un ordre déterminé, comme l'oiseau hong quittant son lieu de repos et volant au milieu des nuages. Chez l'homme, ce sera celui qui s'élève au-dessus du niveau des choses ordinaires; s'avancer à ce point sans manquer à la régularité mesurée du mouvement est le résultat d'une sagesse et d'une intelligence notoires. Aussi, ces plumes peuvent-elles être employées comme insignes d'un rang ou d'un pouvoir particulier. Les plumes sont l'instrument du mode de progression de l'oiseau hong et, à plus forte raison, elles indiquent la voie rationnelle du mouvement en avant du trait supérieur nonaire.

TSHOU HI. - Hou Shi, Tsheng Shi, disent également que le caractère traduit par « lieu élevé », « colline », doit être remplacé par un autre caractère signifiant « carrefour », « route des nuages », et qu'il exprime la route de l'air. On considère actuellement qu'il convient de lire d'après la consonance du mot elle-même. Les plumes servent d'ornement pour les étendards. Le trait supérieur nonaire est extrêmement élevé et au-delà de toutes les situations auxquelles l'homme peut atteindre, de sorte que les plumes peuvent servir de signe distinctif de son élévation, et, en effet, bien que ce trait soit parvenu à l'extrême limite de l'élévation, il n'est pas considéré comme ayant l’image symbolique de ne comporter aucun sens utile. Aussi, le sens divinatoire étant tel, ce sera un présage heureux.

\footnotetext{
${ }^{1}$ Un commentateur.

2 Dictionnaire.
} 
945.

Les plumes peuvent être employées comme présage heureux d'ornements somptuaires; on ne doit pas commettre de désordre.

TSHENG TSE. - Le mouvement en avant de l'homme doué de vertus part d'en bas et il est ascendant ; de très minime, il finit par devenir éclatant et manifeste ; chaque pas a lieu suivant une gradation fixe et non pas au hasard ; en ne manquant pas à cet ordre, il n'aura aucune raison de ne pas jouir de ce présage heureux. Aussi, bien que ce trait nonaire soit arrivé à la limite extrême de l'élévation, il ne perd pas son présage heureux. C'est ce qui peut être employé comme emblème d'un rang hiérarchique, parce qu’il y a le sens d'ordre fixe, déterminé et qui ne peut être troublé.

TSHOU HI. - Il avance peu à peu et progressivement au-delà de toute hauteur et n'est cepend ant pas considéré comme exprimant l'absence d'usage possible ; ses tendances sont naturellement portées à l'élévation : comment serait-il possible qu'il indiquât le désordre ? 


\section{Khouei mei}

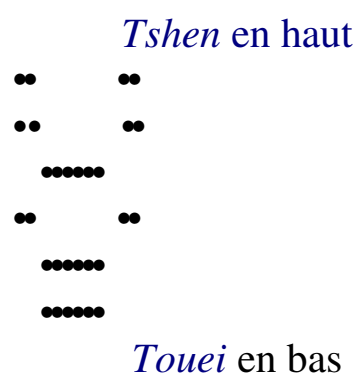

946. Marier la fille; en avançant, présage malheureux; aucune voie avantageuse.

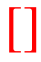

951. Premier trait nonaire : marier la fille comme concubine; boiter et pouvoir marcher; présage heureux de l'entreprise.

$*$

953. Second trait nonaire : yeux affaiblis qui peuvent voir ; avantage de la pureté de l'homme vivant dans la retraite.

$*$

955. Troisième trait hexaire : marier la fille pour attendre; la marier de nouveau comme concubine.

$*$

957. Quatrième trait nonaire : marier la fille, dépasser l'époque fixée ; tarder à la marier jusqu'au moment op portun.

$*$

959. Cinquième trait hexaire : l'empereur Ti Yi marie les jeunes filles ; les parements des manches de la reine ne sont pas aussi beaux que ceux des manches de la concubine; la lune est sur le point d'être pleine, présage heureux.

$*$

961. Trait supérieur hexaire : la fille tient une corbeille pas remplie; le docteur égorge un mouton dépourvu de sang; aucun moyen davantage.

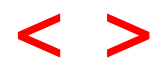


54. Kouei mei.

TSHENG TSE. - Kouei mei. «L'Ordre des koua » dit : «Tsien exprime l'action d'avancer ; pour avancer il faut avoir un but vers lequel tendre, aussi le koua tsien est immédiatement suivi du koua kouei mei. »Pour avancer, il faut nécessairement avoir un but vers lequel on tend ; aussi, le caractère tsien comporte le sens de kouei ${ }^{1}$, et c'est ce qui fait que le koua kouei mei suit immédiatement le koua tsien. Le koua kouei mei se rapporte au mariage de la jeune fille. Mei est une appellation d'une jeune fille. Le koua est formé du koua tshen en haut et du koua touei en bas; puisque la jeune fille suit le garçon adulte, le garçon se meut et la jeune fille est satisfaite ${ }^{2}$. Ou bien encore satisfaction et mouvement, ce qui donne également le sens du garçon satisfait par la fille et de la fille écoutant et suivant le garçon. Les koua qui comportent le sens d'union de la fille et du jeune garçon sont au nombre de quatre qui sont les koua: hien, heng, tsien et kouei mei. Le koua hien exprime l'influence mutuelle que le garçon et la fille exercent réciproquement l'un sur l'autre ; le garçon s'abaisse devant la fille ; les deux éthers s'influencent et se correspondent sympathiquement. Arrêt et satisfaction ${ }^{3}$ : c'est l'image symbolique des sentiments du garçon et de la fille s'influençant réciproquement. Heng, permanence, ordre durable ; le garçon est en haut, la fille est en bas; humilité, soumission et mouvement ${ }^{4}$, la négativité et la positivité se correspondent mutuellement; cela indique le garçon et la jeune fille demeurant ensemble dans une maison, la voie rationnelle persistante et constante de l'époux et de l'épouse, l'un donnant le ton et l'autre s'y accordant ${ }^{5}$. Dans le koua Khien, la jeune fille en entrant dans la famille de son époux se conforme à la droiture ; le garçon s'abaisse devant la jeune fille et chacun occupe la situation qui lui revient suivant la droiture. Arrêt, repos et humilité avec soumission ${ }^{6}$; le mouvement de progression en avant suit un ordre déterminé et mesuré ; c'est l'union entre le garçon et la fille, conforme à la voie rationnelle. Dans le koua kouei mei, il s'agit du mariage de la jeune fille; le garçon est en haut, la jeune fille en bas; la jeune fille suit le jeune garçon et il y a le sens de satisfaction de la jeunesse. Mouvement pour le plaisir, mouvement dirigé par le plaisir, donc il n'y a pas conformité à la droiture. Il en résulte que les situations sont toutes également imméritées ; bien que le premier trait et le trait supérieur occupent les situations qui correspondent à la négativité et à la positivité, cependant, la positivité est au-dessous et la négativité au-dessus, ce qui indique aussi une situation qui n'est pas convenable. Ce koua fait exactement contraste avec le koua tsien;

\footnotetext{
${ }^{1}$ Kouei, revenir à... ; s'unir à... ; marier (en parlant d'une jeune fille).

${ }^{2}$ Le koua simple touei exprime le contentement et il représente aussi la jeune fille. Le koua simple tshen, mouvement, représente le garçon adulte.

${ }^{3}$ Koua simples ken et touei.

${ }^{4}$ Koua simples souen et tshen.

${ }^{5}$ Locution connue.

${ }^{6}$ Koua simples ken et souen.
} 
les koua hien et heng expriment la voie rationnelle de l'époux et de l'épouse ; les koua tsien et kouei mei expriment les devoirs de la jeune fille et de l'époux ; les koua hien et kouei mei expriment les sentiments du jeune garçon et de la jeune fille. Le koua hien indique l'arrêt et la satisfaction ; le koua kouei mei, le mouvement dans la satisfaction : dans les deux cas, à cause du plaisir ou satisfaction. Les koua heng et tsien expriment le devoir entre l'époux et l'épouse ; le koua heng comporte, en effet, l'humilité et le mouvement, l'arrêt graduel avec humilité ; dans tous les cas, soumission à cause de l'humilité. La voie rationnelle du garçon et de la fille, le devoir entre l'époux et l'épous e sont complètement élucidés entre ces quatre koua. Le koua kouei mei est composé du koua simple du marais sur lequel il y a le koua simple de la foudre ${ }^{1}$; la foudre ébranle et l'eau du marais est agitée : image symbolique de l'effet de l'impulsion. Quand il s'agit des choses qui suivent une impulsion, rien n'est comparable à l'eau. Le jeune garçon se meut en haut et la jeune fille le suit : c'est l'image symbolique du mariage qui fait que la fille suit le garçon dans sa famille. Le koua simple tshen représente le jeune garçon; le koua simple touei représente la jeune fille. La jeune fille suit le garçon adulte ; c'est à cause du plaisir qu'elle se meut ; ils se meuvent et en éprouvent un plaisir mutuel. Ce qui fait le plaisir de l'homme, c'est la jeune fille, aussi le texte emploie le caractère $m e i$, jeune fille, et cela constitue l'image symbolique du mariage de la jeune fille entrant dans la famille de son époux. De plus, il y a aussi le sens du grand garçon déjà adulte qui est séduit par la jeune fille, aussi le koua est appelé houei mei, «mariage de la jeune fille $\gg$.

\footnotetext{
${ }^{1}$ Touei et tshen.
} 


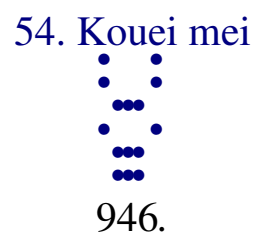

Marier la fille ; en avançant, présage malheureux; aucune voie avantageuse.

TSHENG TSE. - Se laisser aller à la satisfaction et se mouvoir; se mouvoir et être incapable ; aussi, le présage est malheureux. Etre incapable, veut dire être indigne de la situation qu'on occupe. En avançant, présage malheureux : en se mouvant le présage sera malheureux. D'après le sens du koua, il ne s'agit pas seulement du sens de mariage d'une fille, rien de ce qui pourrait être entrepris ne peut être avantageux.

TSHOU HI. - Pour exprimer qu'une femme se marie, on emploie le terme kouei ; mei désigne une jeune fille. La jeune fille personnifiée par le koua simple touei suit le garçon adulte personnifié par le koua simple tshen et leurs sentiments expriment encore le mouvement causé par le plaisir ; c'est toujours l'absence de droiture, aussi le koua est considé ré comme exprimant le mariage de la jeune fille et les divers traits de ce koua, depuis le second jusqu'au cinquième, manquent tous de droiture. Le troisième et le cinquième, avec leur douceur malléable, foulent également aux pieds la dureté énergique, de sorte que le sens divinatoire indique que, en avançant, le présage est malheureux et que rien ne peut être considéré comme avantageux.

947.

Le commentaire de la formule déterminative dit: Kouei mei, le mariage de la fille; c'est le grand devoir du ciel et de la terre ${ }^{1}$. Si le ciel et la terre ne s'unissaient point, les divers êtres ne naîtraient point. Le mariage de la fille, c'est le commencement et la fin de l' homme.

TSHENG TSE. - Une négativité et une positivité constituent ce qu'on appelle la «voie ». La négativité et la positivité s'unissent et s'influencent, le garçon et la fille se joignent en couple : c'est la raison d'être constante du ciel et de la terre ${ }^{2}$. Kouei mei, marier la fille au garçon ; c'est pour cela que la formule parle du grand devoir du ciel et de la terre. Le garçon est au-dessus de

${ }^{1}$ Devoir ; lieu comportant un devoir réciproque.

${ }^{2}$ Qui résulte du ciel et de la terre. 
la fille ; la positivité se meut et la négativité suit l'impulsion, de sorte que ce koua est considéré comme exprimant symboliquement le mariage de la fille. Si le ciel et la terre ne s'unissent poin t, comment tous les êtres pourront-ils en résulter et naître ? Le fait de la fille épousant le garçon exprime la voie rationnelle de l'enchaîne ment des générations. Le garçon et la fille se marient, ils procréent et donnent naissance à de nouveaux êtres ; la génération des êtres assure la continuité sans fin de l'espèce. Ce qui précède aura une suite et ce qui suit a une origine; suite ininterrompue et sans fin; c'est le commencement et la fin de l'homme.

TSHOU HI. - Explication du sens du koua. Le mariage, c'est la fin de la jeune fille; la génération et l'enfantement sont le commencement de l'homme.

948.

La satisfaction entraîne le mouvement, ce qui décide le mariage de la jeune fille.

TSHOU HI. - C'est encore dit au sujet des vertus (qualités) des koua simples.

949.

En entreprenant, présage malheureux; situation imméritée; aucun moyen davantage ; malléabilité marchant sur l'énergie.

TSHENG TSE. - Explication du sens des mots houei mei au moyen des substances des deux koua simples. Le garçon et la fille sinfluencent mutuellement, ils se plaisent et s'agi tent ; c'est le fait d'une petite fille, aussi, lorsque le mouvement résulte de la satisfaction, ce qui est marié, c'est une jeune fille. Ce qui fait que, en entreprenant, le présage doit être malheureux, c'est que tous les divers traits sont également indignes de leur situation, que la position où chacun est placé est également dépourvue de droiture. Dans ces conditions, quel mouvement pourrait donc ne pas être un présage malheureux. Dans la généralité des cas, comment serait-il possible que le mouvement fût le résultat du plaisir sans qu’il en résulte la perte de la droiture ? Non seulement la situation est imméritée, mais encore il y a la faute qui résulte de la positivité foulée aux pieds par la négativité. Le troisième et le cinquième traits foulent 
tous deux l'énergie active sous leurs pieds. Entre le garçon et la jeune fille, il y a un ordre de préséance, entre l'époux et l'épouse, il y a des convenances au point de vue de l'initiative et du concours ; c'est toujours la raison d'être d'un ordre établi et permanent; dans le koua heng il en est encore ainsi. Si ce n'est pas la voie rationnelle de la droiture persistante qui guide les époux, sỉls se laissent aller à leurs sentiments et donnent libre cours à leurs passions, c'est le plaisir seul qui les fait agir, et alors les époux se laissent emporter au courant débordant des passions désordonnées ; le jeune homme entraîné par ses désirs perd son énergie; la femme s'adonne au plaisir et oublie la soumission imposée à son sexe. C'est là le cas de l'énergie foulée aux pieds par la malléabilité dans le koua kouei mei, et ce qui fait que le présage est malheureux et que rien ne peut être avantageux. L'équivalence réciproque de la négativité et de la positivité, le mariage et l'union du jeune homme et de la jeune fille résultent naturellement de la raison d'être immuable et permanente des choses ; s'ils se laissent guider et entraîner par leurs passions au lieu de suivre la voie du devoir et de la raison, la licence et les débordements ne peuvent pas ne pas surgir, blessant le corps, détruisant les vertus et les capacités ; comment serait-ce là la raison d'être de l'homme ? C'est là ce qui fait que le présage du koua kouei mei est malheureux.

TSHOU HI. - Explication de la formule du koua au moyen de la substance même de ce koua. L'union de la jeune fille et du jeune homme est essentiellement conforme à la raison d'être de toutes choses, ce n'est que dans les conditions indiquées par ce koua qu'elle cesse d'être conforme à la droiture.

950.

Le commentaire de la formule symbolique dit: Au-dessus du marais il y a la foudre; mariage de la jeune fille; l'homme doué reconnaît la destruction dans sa fin éternelle.

TSHENG TSE. — La foudre ébranle en haut, le marais reçoit l'impulsion et est agité ; la positivité agite en haut, la négativité en éprouve de la satisfaction et suit l'impulsion. C'est l'image symbolique de la jeune fille suivant le jeune homme, et c'est pour cela que le koua est appelé kouei mei. L’homme doué regarde ${ }^{1}$ l'image symbolique du jeune homme et de la jeune fille s'unissant en couple et perpétuant la génération et par « la fin se renouvelant éternellement ${ }^{2}$, il reconnaît qu’il y a destruction. « Fin éternelle»

\footnotetext{
${ }^{1}$ On pourrait lire : «présente aux yeux ».

${ }^{2}$ C'est une autre lecture des mots traduits dans la formule par fin éternelle, le sens est très vague.
} 
exprime la succession indéfinie et la transmission éternelle du souffle et de la respiration (de la vie animale), de l'extinction et du renouvellement. «Connaître la destruction » veut dire reconnaître que les êtres sont soumis à une voie d'extinction et de destruction, de succession et de renouvellement. La fille se marie, il y aura des naissances, de sorte qu'il y a le sens de " fin éternelle ». De plus, la voie rationnelle des époux est que leur union doit être permanente, définitive et finale, de sorte qu'on doit nécessairement en déduire la raison d'être qui implique la destruction et en faire le sujet d'un avertissement préalable. «Destruction» signifie: «séparation et éloignement»; le koua kouei mei exprime la satisfaction causée par le mouvement ; c'est essentiellement différent de l'humilité dans le mouvement dont il s'agit dans le koua heng ${ }^{1}$ et de l'arrêt, avec humilité, du koua tsien ${ }^{2}$. Le plaisir de la jeune fille consiste dans l'émotion des sentiments; par l'émotion ou mouvement, elle perd la droiture; ce n'est pas une voie rationnelle de persistance de la femme dans la droiture. À la longue, il doit y avoir destruction et ruine; sachant qu'il doit y avoir des truction et ruine, il convient donc de songer éternellement à la fin inévitable. Toute animosité en ce monde provient toujours de l'incapacité de comprendre la « fin éternelle». Cela n'est pas seulement vrai dans le cas de la raison d'être des époux, dans toutes les affaires de ce monde, aucune n'est sans fin et sans destruction, aucune n'est exempte d'une voie comportant la succession et la perpétuité. En regardant le koua kouei mei, il convient de penser à l'avertissement de la « fin éternelle ».

TSHOU HI. - La foudre agite, le marais subit l'impulsion : c'est l'image symbolique du mariage de la jeune fille. L'homme doué contemple le manque de droiture dans l'union et il sait y reconnaître la nécessité finale de destruction qu'elle comporte. Cela s'étend aux choses et aux êtres, et il n'est rien qui ne soit soumis à la même loi.

\footnotetext{
1 Voir koua $\mathrm{n}^{\circ} 32$.

2 Voir koua $n^{\circ} 53$.
} 


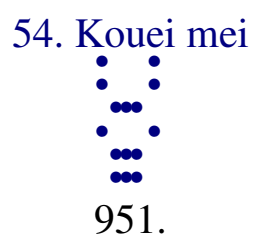

Premier trait nonaire: marier la fille comme concubine; boiter et pouvoir marcher; présage heureux de l'entreprise.

TSHENG TSE. - Mariage de la fille ; ce trait occupe le rang inférieur et est dépourvu de correspondance sympathique conforme à la droiture : image symbolique d'une concubi ne. La dureté énergique positive, chez la femme, constitue la vertu de la parfaite sagesse, de sorte que le fait d'être placée avec soumission dans un rang humble constitue la droiture et la sagesse de la concubine. Etre placée au milieu des plaisirs, occuper le rang inférieur, constitue le sens de soumission. L'infériorité et l'humilité de la concubine la rend incapable, quelle que soit d'ailleurs sa sagesse, de faire quoi que ce soit ; elle n'est pas capable d'autre chose que de soigner sa propre personne, de s'améliorer elle-même, pour aider et assister son seigneur. Il en est d'elle comme du boiteux qui est encore capable de marcher. Sa parole ne peut être entendue et porter au loin; cependant, dans sa condition, c'est considéré constituant le bien, de sorte que, en agissant ainsi, le présage sera heureux.

TSHOU HI. - Le premier trait est nonaire, il occupe le rang inférieur et il est dépourvu de correspondance sympathique, de sorte quil est considéré comme présentant l'image symbolique de la concubine. Toutefois, la dureté énergique positive, chez la fille, constitue les vertus de la sagesse et de la droiture. C'est uniquement à cause de la vilité du rang de concubine qu'elle est à peine capable d'aider et d'assister le prince, de sorte que ce trait comporte encore l'image symbolique du boiteux capable de marcher et que le sens divinatoire est que, en entreprenant, le présage sera heureux.

952.

Marier la fille comme concubine, à cause de la permanence. Boiter et pouvoir marcher, présage heureux; s'entraider mutuellement.

TSHENG TSE. - Le sens du koua kouei mei est : se mouvoir à cause du plaisir; ce n'est pas une voie rationnelle qui puisse être durable chez la femme. Etre nonaire, c'est comporter la dureté énergique et avoir les vertus de la sagesse et de la pureté. Bien que dans un rang aussi infime que celui d'une 
concubine, ce trait est capable de constance. Bien qu'il soit dans l’infériorité, il ne peut avoir aucune action à exercer ; il en est de lui comme d'un boiteux capable de marcher. Cependant, en entreprenant, le présage sera heureux, parce qu'il est capable de prêter ou de recevoir une assistance qui sera mutuelle. Pouvoir aider le prince, c'est le plus heureux présage pour une concubine.

TSHOU HI. - Le mot heng désigne la vertu de la constance et de la persistance. 


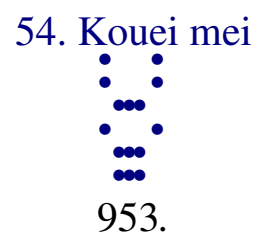

Second trait nonaire : yeux affaiblis qui peuvent voir ; avantage de la pureté de l'homme vivant dans la retraite.

TSHENG TSE. - Le second trait nonaire est positif et énergique et, de plus, il possède la justice ; il représente la droiture et la sagesse de la jeune fille. En haut, il rencontre un trait qui lui correspond sympathiquement suivant la droiture, mais dont la substance est au contraire la malléabilité négative et qui représente le mouvement pour le plaisir. Il s'agit donc d'une jeune fille sage mise en parallèle avec une autre dépourvue de bonnes qualités, de sorte que, bien que le second trait possède la sagesse, il ne peut donner naturellement cours à ses tendances pour achever l'ouvre de l'assistance intérieure ${ }^{1}$. Il est juste, capable d'employer ses qualités à améliorer sa propre personne et à étendre quelque peu son influence. Il en est de lui comme de celui dont les yeux sont affaiblis, mais qui peut simplement distinguer les objets extérieurs, c'es t-à-dire sans percer au loin. La réunion du jeune homme et de la jeune fille doit avoir lieu conformément à la droiture et en se conformant aux règles rituelles et morales. Bien que le cinquième trait manque de droiture, le second, de son côté, observe la droiture et la pureté en gardant le repos dans la retraite ; c'est en cela que consiste son avantage. Le second trait possède les vertus de la droiture et de l'énergie, c'est l'homme qui vit calme dans la retraite et l'obscurité. Telles sont les aptitudes du second trait et la formule mentionne l'avantage de la perfection, ou pureté ; avantage signifie qu'il convient d'avoir une telle pureté ; cela est bien suffisant pour être pris pour motif d'avertissement.

TSHOU HI. - Yeux affaiblis capables de voir ; cela est dit par rapport à la formule du trait précédent. Le second trait nonaire est énergique et positif, il possède la droiture; il exprime la sagesse de la jeune fille; en haut il rencontre une correspondance sympathique d'une trait qui, au contraire, exprime la douceur malléable négative et l'absence de droiture. C'est donc une jeune fille sage, mal alliée, qui ne peut pas accomplir largement l'œuvre de l'assistance intérieure, de sorte que le trait est considéré comme repré sentant l'image symbolique des yeux affaiblis, quoique capables de voir, et que le sens divinatoire indique quil y aura avantage dans la perfection de l'homme qui vit caché dans le calme de la retraite. L'homme caché dans la retraite, c'est encore celui qui s'attache à la voie $r$ ationnelle et observe la droiture, mais qui ne rencontre pas celui qui répondrait à ses aptitudes.

\footnotetext{
1 À l'intérieur de son foyer.
} 
954.

Avantage de la pureté de l'homme vivant dans la retraite ${ }^{1}$; il ne modifie point ce qui est ordinaire.

TSHENG TSE. - Conserver la perfection dans la retraite; ne pas encore perdre la voie rationnelle de la droiture persistante entre l'époux et l'épouse. Les modernes considèrent comme règle naturelle la familiarité et le laisser-aller entre ceux qui sont unis, de sorte que la pureté et le calme sont considérés comme modifiant ce qui est ordinaire ; c'est là ne pas connaître la voie rationnelle de la permanence durable.

${ }^{1}$ De l'homme caché. 


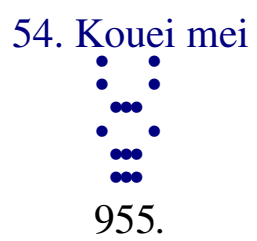

Troisième trait hexaire : marier la fille pour attendre; la marier de nouveau comme concubine.

TSHENG TSE. - Le troisième trait occupe le rang supérieur du koua simple inférieur; essentiellement il ne représente pas ce qui est vil, et c'est parce qu'il a perdu sa vertu et qu'il est sans correspondance sympathique quil est considéré comme voulant se marier et ne pouvant pas encore y réussir, le sixième caractère du texte signifie attendre, ne pas encore posséder ce qui lui convient. Un trait hexaire occupant le troisième rang ne mérite pas sa situation; sa vertu n'est pas la droiture. Il est malléable et doux, mais il affectionne l'emploi de la dureté énergique ; il agit sans soumission. C'est de lui que dépend la satisfaction ${ }^{1}$; il cherche à se marier parce qu'il recherche le plaisir, et se meut sans observer les règles de la bienséance. Au-dessus de lui, il ne rencontre aucune correspondance sympathique ; c'est celui que personne ne peut supporter. Il ne rencontre personne qui lui convienne, aussi, il attend. Quel est l'homme qui épouserait une fille dans de telles conditions ? Elle est indigne de devenir la compagne d'un homme ; elle doit au contraire revenir et chercher à être acceptée comme concubine dans un rang inférieur, ce dont elle peut être capable, parce qu'elle manque de droiture et qu'elle a manqué son sort.

TSHOU HI. - Le troisième trait hexaire est malléable, négatif, et sans justice ni droiture. De plus, c'est de lui que dépendent le plaisir et la satisfaction. La fille qui manque de droiture ne sera point choisie par un homme, de sorte qu'elle ne peut pas encore ren contrer celui qui lui convient et que le trait présente limage symbolique de la fille qui, au contraire, est mariée comme concubine. On a dit aussi que le sixième caractère du texte exprime une jeune fille d'un rang humble et vil ?

956.

Marier la jeune fille cadette pour attendre, elle n' en est pas encore digne.

TSHENG TSE. - «Ne pas encore être digne » indique que le bien, la vertu, la voie suivie pour la recherche du mariage ne sont également pas convenables, de sorte qu'on ne l'épouse point, ce qui fait qu'elle attend.

\footnotetext{
${ }^{1}$ C'est ce trait qui par transformation produit le koua simple touei.
} 


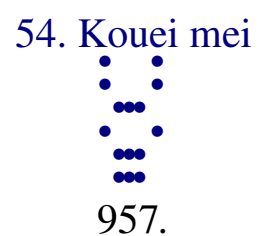

Quatrième trait nonaire : marier la fille, dépasser l'époque fixée ; tarder à la marier jusqu'au moment opportun.

TSHENG TSE. - Ce trait, nonaire, emploie les qualités de la positivité et occupe le quatrième rang; ce rang fait partie du koua simple supérieur et indique un terrain élevé. La dureté énergique, chez la fille, constitue la vertu de la droiture et l'intelligence de la sagesse. Absence de correspondance sympathique conforme à la droiture : Ne pas encore savoir avec qui se marier ; elle dépasse l'âge convenable sans se marier, aussi la formule dit : "Au-delà de l'époque fixée. » Une jeune fille qui occupe une position sociale élevée et noble, qui est douée de la sagesse et de l'intelligence, est l'objet de s recherches des sentiments de l'homme, aussi le retard apporté à l'époque fixée, ou à l'âge convenable, résul te seulement des conditions du temps présent. En effet, c'est qu'elle -même ajourne, et cela ne vient pas de ce qu'elle ne trouve pas à se placer; elle attend le moment où elle trouvera un parti avantageux et alors elle se décidera. Ce trait nonaire occupe le quatrième rang; bien quill ne réponde pas exactement à la situation qu’il occupe, cependant, il est dans une position qui comporte la malléabilité, ce qui constitue la voie rationnelle de la femme. Puisqu'il ne possède aucune correspondance sympathique, cette circonstance indique le sens de dépasser l'époque convenable, et l'homme saint en montre la raison naturelle : puisque la fille est sage et ajourne l'époque convenable, c'est qu'elle a des motifs pour attendre.

TSHOU HI. - Le quatrième trait nonaire étant positif et faisant partie de la substance du koua simple supérieur, dépourvu de toute correspondance sympathique, présente l'image sym bolique d'une fille sage, qui ne se donne pas légèrement à un homme, qui dépasse l'époque ordinaire et attend pour se marier une occasion qui lui plaise. Ce trait exprime exactement le contraire du sens du troisième trait hexaire.

958.

Tendances à dépasser le délai ; avoir d'attendre, puis agir. 
TSHENG TSE. - Ce qui fait qu'elle dépasse le moment ordinaire, dépend de sa propre initiative, et non de celle d'autrui. Une fille sage est l'objet des souhaits de l'homme, qui voudrait l'épouser ; ce qui fait qu'elle dépasse le moment ordinaire, c'est que ses ten dances et ses goûts la portent à attendre. Elle attend qu'elle rencontre un parti avantageux et elle se décide ensuite. 


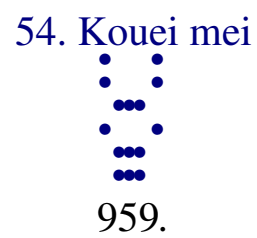

Cinquième trait hexaire : l'empereur Ti Yi marie les jeunes filles; les parements des manches de la reine ne sont pas aussi beaux que ceux des manches de la concubine ; la lune est sur le point d'être pleine, présage heureux.

TSHENG TSE. - Le cinquième trait hexaire occupe la situation prééminente; c'est la noblesse et l'élévation de la jeune fille. En bas, elle correspond sympathiquement avec le second trait, ce qui constitue l'image symbolique de se marier en s'abaissant. Les princesses s'abaissent en se mariant, et cela fut ainsi depuis l'antiquité, jusqu'à ce que l'empe reur Ti Yi et ses successeurs eussent réformé les cérémonies et les règles rituelles relatives aux mariages en précisant la condition du jeune homme et de la jeune fille. Bien qu'il s' agisse de la jeune fille la plus noble, elle ne doit pas manquer à la voie rationnelle de l'humilité et de la douceur et entretenir des pensées orgueilleuses au sujet de sa noblesse. Aussi, dans le Yi king, lorsque la douceur malléable est dans un rang éminent et condescend à faire preuve de modestie, on dit «l'empereur Ti Yi marie les jeunes filles »; c'est le cas du cinquième trait hexaire du koua thae. En se mariant, les jeunes filles nobles doivent simplement shumilier modestement pour se conformer aux lois rituelles, et c'est en cela que consiste la vertu de la noblesse et de l'élévation. Elles ne doivent pas se servir de la parure du visage pour plaire aux hommes ; la parure du visage est le fait des concubines et des filles de joie. Les parements des manches constituent un ornement et une parure. Le cinquième trait hexaire représente une jeune fille d'une condition émi nente et élevée, se préoccupant des règles rituelles et non pas de parure, aussi la richesse de ses parements n'égale pas la riches se des parements d'une concubine. Richesse, beauté, élégance. La pleine lune correspond au plein développement de la négativité ; lorsqu'elle est pleine, elle ne le cède point à la positivité. «Sur le point de » veut dire qu'elle n'est pas encore complè tement pleine. L'élévation et la noblesse du cinquième trait, ne sont pas encore arrivées à la limite extrême de leur développement total, de sorte que la femme, représentée par le trait, ne l'emporte pas encore sur son époux, ce qui constitue un présage heureux. Cela exprime la voie rationnelle de la jeune fille de rang noble et éminent.

TSHOU HI. - Le cinquième trait hexaire, doux et malléable, doué de justice, occupe un rang prééminent ; au-dessous de lui, il sympathise avec le second trait nonaire ; il estime la vertu et n'apprécie pas la parure, de sorte qu'il est considéré comme présentant l’image symbolique de la fille de l'empereur qui s'abaisse en se mariant et dont la parure n'est pas extrême. 
Toutefois, la perfection de la vertu chez la jeune fille ne peut pas dépasser ce niveau, de sorte que le trait est aussi considéré comme l'image symbolique de la lune sur le point d'être pleine, et que si celui qui consulte le sort est dans ces conditions, le présage sera heureux.

960.

L'empereur Ti Yi marie sa fille cadette; son luxe n'est pas comparable à celui des parements élégants d'une concubine; la situation est moyenne ; elle agit avec noblesse.

TSHENG TSE. - Il est question de la voie rationnelle tracée par l'empereur Ti Yi au sujet du mariage des filles cadettes. Les parements de celle-ci ne sont pas comparables, par leur élégance, à ceux d'une concubine ; elle estime la décence et non pas le luxe. Le cinquième trait emploie la douceur malléable et la justice dans une situation élevée et prééminente ; il pratique une voie moyenne malgré son élévation et l'éminence de son rang. Doux, malléable et passif, il s'abaisse et se courbe ; il estime les règles rituelles et n'apprécie pas l'ornementation, c'est là une voie rationnelle de justice.

TSHOU HI. - C'est parce quill possède la noblesse des vertus de la justice et qu'il les pratique, qu'il n'estime pas l'ornementation. 


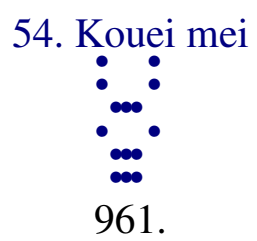

Trait supérieur hexaire : la fille tient une corbeille pas remplie ; le docteur ${ }^{1}$ égorge ${ }^{2}$ un mouton dépourvu de sang ; aucun moyen davantage.

TSHENG TSE. - Le trait supérieur hexaire est à la fin du koua relatif au mariage de la fille et il est dépourvu de correspondance sympathique : il représente le mariage de la jeune fille qui ne s'achève pas. L'épouse est le moyen de continuation de la lignée des ancêtres; elle a un office dans les cérémonies des sacrifices du culte établi en leur honneur ; si elle est incapable de remplir un office dans ces sacrifices, elle ne doit pas être considérée comme épouse ${ }^{3}$. Il est dans les attributions de l'épouse de préparer le contenu des corbeilles et de les présenter ${ }^{4}$. Dans l'antiquité, les provisions de l'intérieur du palais, telles que les conserves de végétaux crus ou cuits et autres choses de ce genre étaient surveillées sous l'autorité de l'épouse du roi ; dans leurs sacrifices, les divers princes feudataires découpaient eux-mêmes les viandes des animaux et il en était de même pour tous ceux qui étaient revêtus des titres de Meng, shi, ta fou et autres du même genre. Égorger, c'est recueillir le sang pour les sacrifices; le Li king dit : "Le sang est l'essence parfaite des sacrifices. » La femme doit présenter les corbeilles, et celles-ci ne sont pas pleines ; n'étant pas pleines, il n'y a rien à offrir en sacrifice, ce qui exprime qu'elle est incapable de remplir son office dans les cérémonies des sacrifices. L'époux et l'épouse accomplissent ensemble les cérémonies dans les temples des mânes des ancêtres; si la femme est incapable de contribuer à préparer les sacrifices, l'époux ne pourra pas les offrir et les accomplir, de sorte qu'égorgeant le mouton, celui -ci est dépourvu de sang, ce qui indique encore qu'il n'y a rien à présenter en offrande et ce qui veut dire qu'il ne doit pas effectuer le sacrifice. La femme étant incapable de remplir son office dans les sacrifices, il convient de la répudier, ce qui indique que le lien entre l'époux et l'épouse n'est pas indissoluble : que pourrait-on donc entreprendre qui fût avantageux ?

TSHOU HI. - Le trait supérieur hexaire, avec la douceur malléable, occupe le rang final dans le koua qui symbolise le mariage de la fille et il est sans correspondance sympathique : il fixe l'époque du mariage et ne l'achève pas. C'est pour cela que tel est son image symbolique et que, pour celui qui consulte le sort, il indique que rien ne peut être avantageux.

\footnotetext{
${ }^{1}$ Lettré ; homme complètement versé dans la doctrine dite confucéenne.

2 Dans un sacrifice.

${ }^{3}$ Elle doit être répudiée.

${ }^{4}$ Pendant les sacrifices.
} 
962.

Le trait supérieur n'est pas plein : présenter des corbeilles vides.

TSHENG TSE. - Des corbeilles qui ne sont pas remplies sont des corbeilles vides; des corbeilles vides sont-elles propres à servir dans les sacrifices ? Cela exprime que ce trait ne peut pas servir lorsqu'il s'agi t de préparer des sacrifices. Si la femme n'est pas capable de remplir son office dans la préparation des sacrifices, il n'y a qu'à la répudier, et c'est là «l'absence de fin » de la femme ou de l'époux. 


\section{Fong}

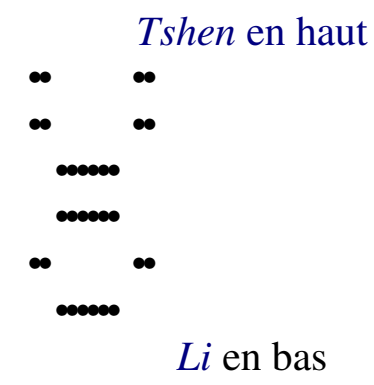

963. Fong, liberté : le roi s'en autorise; ne pas se chagriner; justement le soleil au milieu de sa course.

968. Premier trait nonaire : rencontrer le maître équivalent; bien qu'une décade, pas de culpabilité ; en entreprenant il y a des louanges.

$*$

970. Deuxième trait hexaire : l'écran se développe ; le soleil étant au milieu de sa course voir l'étoile polaire ; en entreprenant il y aura l'inconvénient du doute; il y a confiance et elle se manifeste ; présage heureux.

$*$

972. Troisième trait nonaire : agrandir le rideau; le soleil au milieu de sa course, voir les petites étoiles; briser le bras droit ; pas de culpabilité.

974. Quatrième trait nonaire : épaissir l'écran ; au milieu du jour vair l'étoile polaire ; rencontrer le maître égal ; présage heureux.

*

976. Cinquième trait hexaire : la beauté vient; il y a des félicitations et des louanges; présage heureux.

$*$

978. Trait supérieur hexaire : agrandir la splendeur de l'habitation ; protéger la famille par un écran; chercher à espionner par la porte ; regarder les yeux grands ouverts sans voir l'homme; trois ans sans voir ; présage malheureux.

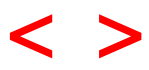




\section{Fong.}

TSHENG TSE. — Fong. «L'Ordre des koua » dit : « La possession de ce qui vient s'adjoindre ${ }^{1}$ doit entraîner la grandeur, de sorte que le koua kouei mei est suivi du koua fong; fong, grandeur. » Les êtres en se réunissant et en se rassemblant consomment et achèvent la grandeur, aussi à la suite du koua kouei mei est placé le koua fong : Le caractère fong comporte le sens de grandeur achevée, complète ; le koua est formé par le koua simple tshen en haut, et le koua simple li en bas. Le koua simple tshen exprime le mouvement; le koua simple $l i$ exprime la clarté, la lumière. Profiter de la lumière et se mouvoir; se mouvoir et pouvoir le faire avec intelligence : c'est toujours également une voie rationnelle conduisant à la grandeur. La lumière est propre à éclairer, le mouvement est capable d'amener la liberté, et après peut arriver la grandeur parfaite et florissante.

\footnotetext{
${ }^{1}$ Kouei, marier.
} 


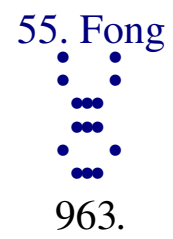

Fong, liberté : le roi s'en autorise ${ }^{1}$; ne pas se chagriner ; justement le soleil au milieu de sa course.

TSHENG TSE. - Fong exprime la perfection de la grandeur; le sens indique la liberté même d'expansion ; pousser à l'extrême limite l'éclat et la grandeur de l'univers ${ }^{2}$. Seul le roi peut y atteindre. Le quatrième caractère veut dire parvenir à, atteindre à. La prééminence de la situation du ciel, la richesse des quatre mers, la foule de tout ce qui vit et existe, la grandeur de la voie rationnelle du roi, la voie rationnelle de la grandeur extrême, tout cela n'est -il pas résumé dans le roi ? Dans un moment de grandeur florissante, la foule du peuple est innombrable, les affaires et toutes choses sont compliquées et importantes : comment serait-il facile d'embrasser le tout pour gouverner ? Il convient de posséder l'intelligence (clarté) parfaite du soleil illuminant largement de tous côtés et dont les rayons atteignent partout; ensuite il n'y aura aucune inquiétude ni chagrins.

TSHOU HI. - Fong, grandeur; se mouvoir avec intelligence; force inhérente au parfait développement de la grandeur, aussi, le sens divinatoire comporte la voie rationnelle de liberté. Toutefois, si le roi atteint ce point, comme lorsque la perfection est arrivée à son extrême limite elle doit être suivie de décadence, il observe cette voie rationnelle d'inquiétudes et de chagrins. L'homme saint considère ces chagrins et ces inquiétudes comme vains et sans utilité ; il faut exclusivement maintenir les règles permanentes et immuables, pour éviter l'excès da ns la perfection. Aussi, la formule avertit au sujet de l'abandon des inquiétudes et de l'effet convenable du soleil au milieu de sa course.

964.

Le commentaire traditionnel de la formule déterminative dit: Fong, grandeur ; la lumière se meut ${ }^{3}$, donc splendeur.

\footnotetext{
${ }^{1}$ Les commentateurs donnent un sens étranger à ce caractère ; voir la note suivante.

2 «Dessous du ciel » ou encore « ciel et dessous ».

3 «Se meut » ou « sert à mettre en mouvement ».
} 
TSHENG TSE. - Fong a le sens de perfection de la grandeur; le koua simple $l i$ exprime la lumière, ou l'intelligence, et le koua simple tshen exprime le mouvement. La lumière et le mouvement s'entraident mutuellement et produisent la perfection de la grandeur.

TSHOU HI. - Explication du sens du nom du koua au moyen des vertus des koua simples.

965.

Le roi y parvient ${ }^{1}$, il estime la grandeur; ne pas se chagriner, justement le soleil au milieu de sa course ; il convient d'éclairer l'univers.

TSHENG TSE. - Le roi possède l'immensité des quatre mers, la foule des millions d'âmes, il épuise ce qui peut être la grandeur dans l'univers. Aussi la voie rationnelle de la grandeur, ou splendeur exprimée par le koua fong est telle que le roi seul peut y atteindre. Ce qu'il possède constituant déjà la grandeur, la voie rationnelle quil doit suivre pour protéger et régir ses possessions doit également comporter la grandeur, et c'est là ce qui fait que ce que le roi estime est l'extrême grandeur. Ses possessi ons étant immenses, son gouvernement s'étendant sur la foule innombrable, il doit se préoccuper et s’inquiéter en craignant de ne pouvoir tout embrasser dans la portée de ses capacités ; il convient qu'il soit comme la parfaite clarté du soleil parvenu au milieu de sa course, qu'il illumine l'univers sans omettre aucun point, et alors il ne devra plus s’inquiéter et se chagriner. Étant dans ces conditions il pourra ensuite assurer et maintenir la grandeur florissante de l'univers. Garantir le maintien de la grandeur et de la splendeur, est-ce là une action qui soit à la portée d'une habileté médiocre et d'un faible savoir ?

TSHOU HI. - Explication de la formule du koua.

966.

Le soleil parvenu au milieu de sa course doit décliner; la lune pleine doit s'échancrer $^{2}$; ciel et terre, plein et effacement, avec le temps succession de l'apparence et de la disparition; combien à plus forte raison pour l'homme! Combien à plus forte raison pour les génies et esprits.

\footnotetext{
${ }^{1}$ Version admise.

${ }^{2}$ Littéralement « se manger ».
} 
TSHENG TSE. - Après avoir parlé de l'extrême développement de la splendeur, le texte parle encore de l'impossibilité de la permanence invariable comme constituant le sujet d'un avertissement. Le soleil étant parvenu à l'extrême limite de son éclat, au milieu de sa course, il doit décliner et s'abaiss er; la lune étant arrivée à son plein parfait, elle doit décroître ; le plein, ou l'effacement (vide) du ciel ou de la terre se produisent et expirent encore avec le temps : combien à plus forte raison en est-il de même pour l'homme, l'esprit et les génie s ? Le plein et l'effacement désignent la prospérité et la décadence; se produire et expirer désigne le mouvement en avant ou en arrière; le mouvement de révolution du ciel et de la terre se produit aussi en avançant ou en reculant, avec le temps et les saisons. Les « esprits et génies », c'est-à-dire les traces visibles de l'action créatrice et transformatrice dont on peut voir les alternatives de progression et de régression dans le développement et la décrépitude de tous les êtres. Donner un tel avertissement au moment du parfait développement de la splendeur, c'est vouloir amener les hommes à se maintenir dans un juste milieu sans dépasser la perfection; comment serait-il facile de se placer dans la voie exprimée par le koua fong.

TSHOU HI. - Ceci développe de nouveau et éclaire la formule du koua au point de vue des idées qui n'y sont point énoncées, en disant qu'il ne faut pas dépasser le juste milieu.

967.

Le commentaire traditionnel de la formule symbolique dit: La, foudre et l'éclair éclatent en même temps: splendeur; l'homme doué applique ce principe en prononçant les jugements et en appliquant les peines.

TSHENG TSE. - La foudre et l'éclair éclatent en même temps, la lumière et le mouvement agissent à la fois. Les deux substances s'uni ssent ensemble et c'est pour cela que la formule dit « éclatent en même temps » la lumière et le mouvement se complètent mutuellement: c'est l'image symbolique du développement complet de la splendeur. Le koua simple $l i$ exprime la lumière ; c'est l'image symbolique de l'examen et du jugement qui porte la clarté dans les détails de l'investigation. Le koua simple Men représente le mouvement et donne l'image symbolique de l'autorité dans la décision. Pour prononcer une sentence, il faut élucider l'intention et le fait et la clarté seule peut inspirer la confiance; pour infliger une peine, afin d'imposer la crainte au vice et aux déporte ments, c'est surtout la précision qui rend l'effet exemplaire. Aussi, l'homme doué voit l'image symbolique de la foudre et de l'éclair éclairant et agitant et il applique cet exemple en rendant 
les jugements et en prononçant les peines. Le koua she ho parle des premiers rois qui formulèrent les règles; le koua fong parle de l'homme doué prononçant les jugements. Puisque la clarté est au-dessus, qu'elle s'écarte de l'autorité et du mouvement, il s'agit d'une affaire qui dépend du roi, de sorte qu'il s'agit d'édicter des peines et d'établir des règlements ; puisque la clarté est au-dessus et qu'elle s'écarte de l'autorité et du mouvement, il s'agit de l'effet de l'action de l'homme doué, de sorte qu'il est question de rendre des jugements et de prononcer des peines. Dans le koua she ho la clarté est au-dessus et la formule parle de l'homme doué ; mais dans le koua fong il est question de la circonspection dans l'emploi des peines pour ne pas prolonger indéfiniment la durée de l'emprisonnement. Quand il s'agit de l'homme doué il en est toujours ainsi.

TSHOU HI. - La formule relève l'image symbolique de l'autorité et de l’intellig ence qui agissent en même temps. 


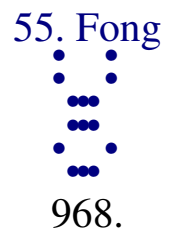

Premier trait nonaire : rencontrer le maître équivalent ${ }^{1}$; bien qu'une décade, pas de culpabilité ; en entreprenant il y a des louanges.

TSHENG TSE. - La foudre et l'éclair surviennent également : image symbolique du parfait développement de la splendeur. La clarté et le mouvement s'entraident mutuelle ment; c'est la voie rationnelle d'où résulte la grandeur. Sans la lumière, rien ne peut briller; sans le mouvement rien ne peut se déplacer; ces deux termes s'appe llent mutuellement comme la forme et l'ombre. S'entraider mutuellement, comme les deux faces d'un même objet se complètent l'une l'autre. Le premier trait nonaire, c'est le début de la lumière ; le quatrième trait nonaire est l'origine du mouvement ; il convient qu'ils s'attendent réciproquement pour produire leur effet, aussi, bien qu'égaux, ils se corres pondent sympathiquement. Leur situation fait qu'ils se correspondent sympathiquement, leur effet les conduit à s'entraider mutuellement, aussi le premier trait désigne (nomme) le quatrième trait comme étant le maître de l'équivalence, c'est -à-dire celui qui, avec lui-même, constitue une paire. Le caractère traduit par «équivalent» ou «le second d'une paire », exprime au moins ce qui approche de la condition d'égalité avec la chose énoncée. Ainsi dans l'expression « équivalent au ciel pour se mettre au rang de l'homme doué », le premier par rapport au quatrième est appelé équivalent ; le quatrième par rapport au premier est appelé « barbare », bien qu'égaux, pas de culpabilité. Le huitième caractère du texte qui signifie décade, ou semaine de dix jours, a ici le sens de «égal à ». Ceux qui dans l'univers se correspondent sympathiquement ne sont généralement pas rigoureusement égaux et équivalents ; cela est visible, par exemple, dans la correspondance sympathique entre la négativité et la positivité, dans la mollesse se plaisant à suivre l'énergie, dans la propension de l'inférieur à s'adjoindre au supérieur. Si les deux objets étaient rigoureusement égaux, pourquoi voudraient-ils se suivre l'un l'autre ? Il n'y a que dans le cas du premier et du quatrième traits du koua fong, que leurs effets conduisent à s'entraider mutuellement, que leur correspondance sympathique complète l'un l'autre, de sorte que, bien qu'égaux par leur énergie positive, ils se suivent mutuellement, mais sans commettre aucune faute coupable. En effet, sans la clarté, le mouvement ne saurait où se porter; sans le mouvement, la lumière serait sans effet. Ils s'entraident mutuellem ent et produisent chacun son effet ; «dans un même bateau on traverse un lac avec le même cour »; dans le danger commun les ennemis eux-mêmes réunissent leurs forces : la force des

\footnotetext{
${ }^{1}$ Le maître de l'équivalence.
} 
choses l'exige ainsi. Ils tentent une entreprise et se suivent mutuelle ment, de sorte qu'ils peuvent achever la grandeur de leur auvre, et c'est pour cela que la formule mentionne qu'il y a des louanges ; il y a lieu de leur donner des louanges. Dans d'autres koua, ils ne se feraient point de concessions mutuelles et ils se sépareraient.

TSHOU HI. - «Maître équivalent» désigne le quatrième trait; le caractère dont le sens est décade est pris ici avec le sens d'égal ; cela exprime qu'ils sont tous deux positifs. Dans un moment de splendeur florissante, la lumière et le mouvement s'entraident mutuelle ment, aussi, le premier trait nonaire, rencontrant le quatrième également nonaire ; ils conduisent, bien que tous deux énergiques et positifs, à ce sens divinatoire.

969.

Bien que dix jours, pas de culpabilité ; au-delà de dix jours, calamité.

TSHENG TSE. - L'homme saint suit le moment et se place selon l'opportunité ; il se conforme à la raison suivant la nature de chaque affaire. Or, lorsque la force naturelle et inhérente est égale chez deux êtres, ceux-ci ne s'abaissent pas mutuellement l'un devant l'autre ; telle est la raison d'être générale. Mais il y en a qui, bien qu'opposés par leurs qua lités, se complètent et s'entraident mutuellement, de sorte qu'ils s'appellent ; tel est le cas du premier et du quatrième trait, et c'est ce qui fait que, bien qu'après dix jours, il n'y a cependant pas de culpabilité. Lorsque l'on est de même force que quelqu'un et que les énergies sont égales des deux côtés, qu'on est porté à s'abaisser pour s'appeler mutuelle ment, à réunir ses forces pour répondre aux circonstances, comme en détruisant d'abord le sentiment de sa propre personnalité pour renforcer les idées d'un supérieur, le mal doit nécessairement bientôt en résulter, aussi, la formule dit: au-delà de «l'égalité » calamité. Être égal à quelqu'un et se mettre soi -même en avant, c'est enfreindre l'égalité ; si l'un des deux veut l'emporter sur l'autre, tous deux ne peuvent pas être égaux.

TSHOU HI. - Avertissement à celui qui consulte le sort quill ne doit pas chercher à l'emporter sur celui qui est son égal. C'est encore une idée en dehors de la formule du trait. 


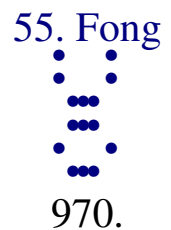

Deuxième trait hexaire : l'écran se développe ${ }^{1}$; le soleil étant au milieu de sa course voir l'étoile polaire; en entreprenant il y aura l'inconvénient du doute; il y a confiance et elle se manifeste ; présage heureux.

TSHENG TSE. - La lumière et le mouvement s'entraident mutuellement ${ }^{2}$, ainsi peut se produire la grandeur de la splendeur. Le second trait est le maître de la lumière ${ }^{3}$; de plus il possède la justice et la droiture et c'est celui qui peut être désigné par l'appellation de « la lumière ». D'ailleurs te cinquième se trouve sur le terrain de la correspondance sympathique il est malléable, négatif, et sans droiture ; c'est celui qui est incapable de mouve ment. Sans que le second et le cinquième traits soient tous deux négatifs, cependant, se trouvant dans le moment où la lumière et le mouvement s'entraident et se complètent mutuellement, occupant un terrain de correspondance sympathique mutuelle, si les aptitudes du cinquième sont insuffisantes, et si celles du trait qui lui correspond sympathiquement sont aussi insuffisantes pour y suppléer, il en résulte que la lumière seule est incapable d'achever la grandeur du développement. Du moment où elle ne peut achever la grandeur du développement, elle perd le mérite qui résulte de sa clarté (ou intelligence) de sorte que ce trait est considéré comme ayant l'image symbolique du développe ment de l'écran et de voir l'étoile polaire lorsque le soleil est dans sa position moyenne. Le second trait a les qualités et les aptitudes de l'extrême lumière; comme celui qui lui correspond sympathiquement est incapable de l'aider, et qu’il ne peut ache ver le développement de la grandeur, il détruit l'œavre de sa clarté et, ne produisant plus de clarté, il est considéré comme représentant les ténèbres, aussi la formule dit : «voir l'étoile polai re », l'étoile polaire ne se voit que pendant l'obscurité de la nuit; le caractère traduit par le mot écran indique le sens d'un écran ou voile circulaire; c'est-à-dire de l'emploi de quelque chose d'obscur et d'opaque pour intercepter la lumière. L'étoile polaire appar tient au genre négatif, et elle précise le mouvement de révolution uniforme ${ }^{4}$; c'est le sym bole du cinquième trait employant la malléabilité négative et occupant la situation du prince. Lorsque le soleil est au milieu ${ }^{5}$, c'est le moment où la

\footnotetext{
${ }^{1}$ Sens très vague ; quelque chose propre à masquer la lumière, et qui grandit.

2 Ou se complètent mutuellement.

${ }^{3}$ Celui qui entraîne la transformation du koua simple $l i$.

${ }^{4}$ Egal, uni.

${ }^{5}$ Cette expression, déjà traduite par « soleil au milieu de sa course » peut aussi exprimer le soleil décrivant l'horizon de l'observa teur.
} 
lumière est complète alors, apercevoir l'étoile pol aire, c'est comme rencontrer celui de qui dépend la malléabilité négative dans le moment de la grandeur du développement et de l'éclat. L'étoile polaire n'est rendue visible que par l'obscurité ; la formule parle de voir l'étoile polaire, cela veut donc di re que la clarté disparaît et qu'il fait sombre. Bien que le second trait possède les aptitudes de l'extrême clarté (intelligence), de la justice et de la droiture, ce qu'il ren contre c'est la malléabilité dépourvue de lumière, représentant un prince dénué de droiture. Du moment où ce prince ne peut s'abaisser pour venir implorer son aide, s'il entre prenait d'aller lui -même implorer ce prince il ne rencontrerait, au contraire, que le doute, l'aversion et le soupçon. Ainsi prévaut l'obscurité. Mais alors s'il en est ainsi, qu'est -ce qui sera possible ? l'homme doué qui sert son supérieur, s’il ne possède point son caur, ne pourra qu'épuiser le comble de la sincérité afin d'émouvoir et d’influencer son jugement et ses idées. Si la sincérité de ses intentions peut l'émouvoir, alors, bien que dépourvu de lumière et aveuglé, il est encore possible de lui ouvrir les yeux. Bien que faible et malléable, il peut encore aider ; bien que dépourvu de droiture, il peut encore continuer à redresser. Les hommes de l'anti quité qui servirent des princes médiocres, ou des maîtres de valeur ordinaire, et qui d'ailleurs furent capables de réunir et de suivre la voie rationnelle n'y réussirent qu'en faisant apprécier et comprendre au -dessus d'eux la sincérité de leurs intentions, en la rendant visible aux princes et en leur inspirant la confiance. Tels sont par exemple, les exemples de Kouan Tshong servant Heng Kong et de Khong Ming aidant son dernier maître. S’il est possible par la bonne foi et la sincérité de révéler ses tendances et ses intentions, il sera possible de faire prévaloir sa voie rationnelle, et c'est cela qui constitue un présage heureux.

TSHOU HI. - Le second trait hexaire se trouve dans un moment de développement de la grandeur; c'est de lui que dépend l'exis tence du koua simple $l i^{1}$, c'est celui qui est le plus brillant par l'intelligence. D'ailleurs, en haut, il correspond sympathiquement à la douceur et à l'obscurité du cinquième trait hexaire, de sorte qu'il est considéré comme comportant l'image symbolique de l'agrandissement de l'écran et de la vue de l'étoile polaire. Écran, voile qui masque la vue d'un objet, qui cache et obscurcit ; agrandir le voile qui obscurcit, de sorte que, au milieu du jour ${ }^{2}$, il fait sombre. S'il entreprend et poursuit son entreprise, étant celui de qui dépend l'obscurité, il doit nécessairement regarder en arrière avec suspicion. Ce n'est qu'en développant la sincérité dans les idées, afin d'émouvoir et d'influencer, que le présage pourra être heureux. C'est un avertisse ment à celui qui consulte le sort, qu'il convient qu'il se mette dans ces mêmes conditions, qu'il se débarrasse de toute prévention, ce qui est l’image symbolique d'avoir la foi et la confiance.

\footnotetext{
${ }^{1}$ Au point de vue de la transformation.

${ }^{2}$ Ou pendant le jour.
} 
971.

Avoir foi et le montrer; confiance en manifestant ses tendances.

TSHENG TSE. - Avoir foi et la faire éclater, cela exprime que, avec sa propre confiance ou bonne foi, il influence et fait éclore les tendances du cour du supérieur. S’il peut émouvoir, le présage heureux est évident; bien qu'obscur et malléable, il possède une voie qui peut influencer. 


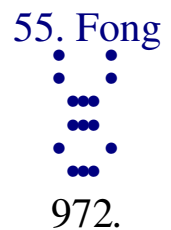

Troisième trait nonaire : agrandir le rideau; le soleil au milieu de sa course, voir les petites étoiles; briser le bras droit ; pas de culpabilité.

TSHENG TSE. - Le cinquième caractère est quelquefois remplacé dans certaines éditions par [] et désigne un rideau ou écran en tissu. Wang Po considère ce caractère comme exprimant des rideaux de lit, ce qui justifie bien l'équivalence de ces de ux caractères. Les rideaux de lit, ou moustiquaires, et les draperies servent à s'abriter à l'intérieur des appartements ; en augmentant les tentures, l'obscurité devient plus grande qu'avec un écran ${ }^{1}$. Le troisième trait fait partie de la substance de la lumière $(l i)$, et cependant, il est plus obscur que le quatrième: c'est parce que ce qui lui est sympathique est l'ombre et la négativité. Ce troisième trait occupe le rang supérieur dans la substance de la lumière (li $)$; il est positif et énergique; c'est celui qui, essentiellement est capable d'éclairer. La voie rationnelle de la splendeur doit se produire par l'aide mutuelle de la lumière et du mouvement ; le troisième trait sympathise avec le trait supérieur; le trait supérieur est malléable et négatif, de plus, il est sans situation et il est placé à la fin du koua simple tshen qui exprime le mouvement : puisqu'il est à la fin, il y a arrêt ; c'est celui qui ne peut se mouvoir. Dans les autres koua parvenir au dernier rang exprime la limite extrême ; lorsqu'il s'agit du koua tshen, parvenir à la fin indique l'arrêt. Le troisième trait n'est pas celui avec qui le trait supérieur sympathise, de sorte qu’il ne peut achever la grandeur du développement. Le neuvième caractère du texte exprime les plus petites étoiles, sans nom, comme innombrables ; pour voir les plus petites étoiles, il faut une obscurité absolue. Dans un moment de grandeur, rencontrer le trait supérieur hexaire, c'est comme voir les plus petites étoiles au milieu du jour. L'épaule droite ou le bras droit, ce qui sert le plus à l'homme ; ici, le texte dit qu'il est brisé ; il est donc évident qu'il ne peut plus servir à rien. Les aptitudes du sage instruit rencontrent un prince intelligent, de sorte qu'elles peuvent agir pour le bien de l'univers ; si, au-dessus, elles ne rencontrent aucun maître sur qui elles puissent s'appuyer, elles ne pourront produire aucun effet, comme lorsque le bras droit de quelqu'un est cassé. Si ce que l'homme fait présente un défaut ou inconvénient, c'est à lui que la faute en incombera ; on dit alors : « à cause de cela, il résulte ceci ». Sîl veut se mouvoir et s’il est privé de l'usage de son bras droit, il désire agir, mais au-dessus de lui, il n'a personne sur qui

\footnotetext{
${ }^{1}$ Ou pylône, masquant la vue de l'intérie ur, ou bien encore un écran en bambou ou store à l'entrée de la maison.
} 
s'appuyer, de sorte que cela lui sera fina lement impossible ; que pourrait-on lui dire de plus ? Aucune faute ne peut lui être imputable.

TSHOU HI. - Le caractère [] équivaut à [] et désigne une moustiquaire ou une tenture de lit; l'obscurcissement qui en résulte est plus épais encore que celui d'un store ou écran. [] les petites étoiles. Le troisième trait est placé au rang extrême dans le koua li qui exprime la clarté, et il sympathise avec le trait supérieur hexaire. Bien qu'il ne puis se produire aucun effet, cependant il n'en est pas coupable, de sorte que tels sont le sens divinatoire et l'image symbolique.

973.

Épaissir les tentures ; impossibilité de faire de grandes choses; briser le bras droit ; finalement, ne pouvoir produire aucun effet.

TSHENG TSE. — Le troisième trait correspond sympathiquement au trait supérieur ; le trait supérieur qui lui correspond est sans situation déterminée ; malléabilité négative sans force naturelle et déjà placée au dernier rang : est-ce là une condition qui puisse permettre de remédier à des périls graves ? Du moment où il ne peut s'appuyer sur personne, il est comme celui dont le bras droit est cassé et qui est définitivement incapable d'agir. 


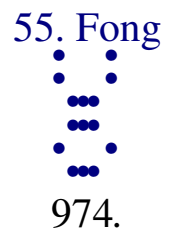

Quatrième trait nonaire : épaissir l'écran; au milieu du jour vair l'étoile polaire ; rencontrer le maître égal ${ }^{1}$; présage heureux.

TSHENG TSE. - Bien que le quatrième trait soit énergique et positif et qu'il soit celui de qui dépend la transformation du koua simple tshen, du mouvement, que, de plus, il possède la situation du sujet de rang élevé, cependant, à cause de son manque de justice et de droiture rencontrant celui de qui dépend l'obscurité négative et la faiblesse de la malléa bilité, comment pourrait-il arriver à la grandeur du développement de la splendeur ? Aussi, il est considéré comme exprimant l'épaississement des stores ou écrans ; le store ou écran est quelque chose qui cache en entourant de tous côtés ; ce qui est entouré de tous côtés ne peut être grand; ce qui est masqué et voilé ne peut être clair et brillant. Voir l'étoile polaire au milieu du jour ; cela exprime que, au milieu d'un moment de clarté parfaite, il se rencontre un moment d'obscurité et de ténèbres. « Maître égal »; de rang égal. Ils se correspondent mutuellement avec sympathie, et c'est pour cela que la formule emploie l'expression de «maître ». Le premier et le quatrième traits sont également positifs et occupent tous deux le premier rang dans un koua simple : ce sont ceux qui possèdent les mêmes vertus. De plus, ils occupent des positions qui comportent la sympathie mutuelle, de sorte quils sont considérés comme maîtres de même rang. Occupant la situation d'un sujet de rang élevé, et possédant le concours du sage placé au-dessous de lui dans l'in fériorité, doués des mêmes vertus et s'entraidant mutuellement, comment le résultat de cette assistance pourrait-il être minime ? C'est en cela que consiste le présage heureux. Avec les aptitudes du quatrième trait, possédant comme auxiliaire le sage placé au rang inférieur, ne pourra-t-il pas atteindre à une grandeur florissante ? Réponse : si celui qui est dans un rang supérieur est digne de la situation qu'il occupe, celui qui est au rang inférieur sera un auxiliaire pour lui, et, inversement, si celui qui est au rang inférieur possède les aptitudes que dénote la sagesse, celui qui est dans une position supérieure deviendra pour lui un protecteur ; comment cela pourrait-il exister sans quil en résultât un effet avantageux ? Donc le présage est heureux. Mais sil s'agit de la splendeur et de la grandeur de l'univers, il faut être le prince pour être à même de la produire; or le cinquième trait est malléable et négatif, il occupe le rang prééminent et fait partie de la substance du koua simple tshen qui exprime le mouvement; il est dépourvu de l'image symbolique de l'absence de préventions et de la soumission humble aux conseils de la sagesse ; quant au

\footnotetext{
${ }^{1}$ Très douteux.
} 
trait inférieur, de son côté, bien que présentant de nombreux caractères de sagesse, cependant rien n'indique qu'il soit sur le point de devoir exercer une action importante. C'est que sans la dureté énergique positive, sans la justice et la droiture, il est impossible de conduire l'univers vers la grandeur et la splendeur florissante.

TSHOU HI. - L'imag e symbolique est la même que celle du second trait hexaire. L'antépénultième caractère du texte signifie égal, de même rang, et se rapporte au premier trait. Le sens divinatoire exprime qu'il doit grandir et qu'il rencontre celui de qui dépend l'obscurité ; s'il descend pour se porter vers celui qui est doué des mêmes vertus, le présage sera heureux.

975.

Épaissir le store (écran); situation imméritée; au milieu du jour voir l'étoile polaire; ombre sans clarté ; rencontrer le maître de même rang est un présage heureux; agir.

TSHENG TSE. - «Situation imméritée > exprime qu’il occupe une situation élevée sans posséder ni justice ni droiture, et qu'il en résulte qu'il est obscur et ne peut parvenir à la splendeur. " Au milieu jour (ou de la course du soleil) voir l'étoile polaire, ombre sans clarté » exprime que l'obscurité empêche l'éclat de la lumière ; cela est ainsi parce que le prince est mou et négatif et le sujet dépourvu de justice et de droiture. Le dernier membre de phrase signifie que si la positivité et l'énergie active se rencontrent ensemble, ce sera l'effet du présage heureux. C'est parce que ce trait descend et se porte vers le premier, que le texte porte le mot agir (marcher). Le fait de s'abaisser pour réclamer assistance sera considéré comme un présage heureux. 


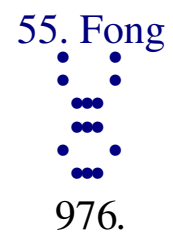

Cinquième trait hexaire : la beauté vient; il y a des félicitations et des louanges; présage heureux.

TSHENG TSE. - Le cinquième trait emploie les aptitudes de la malléabilité négative et c'est lui de qui dépend la splendeur; il ne peut certainement pas achever la grandeur de cette splendeur. S'il peut simplement parvenir aux aptitudes qui résultent de la beauté du trait inférieur et les employer, il recevra des félicitations et sera heureux et, de plus, il recevra des louanges sur sa beauté, ce qui est indiqué par l'expression «présage heureux ». Le second trait hexaire possède la beauté et l'élégance de la forme, la justice et la droiture, ce qui constitue les «aptitudes de la beauté »; c'est lui qui est investi de l'auto rité par le cinquième trait et dont la parfaite sincérité peut atteindre jusqu'à la plus haute situation. Il lui est possible d'atteindre à l'éclat de la grandeur et de la splendeur, d'être salué par l'appellation de beauté, de sorte que le présage est heureux. L'expression « aptitudes de la beauté » est principalement employée au sujet du second trait, cependant, le premier, le troisième et le quatrième traits possèdent, tous également les aptitudes de l'énergie positive ; si le cinquième trait est capable d'employer les sages, ceux-ci s'avan cent de concert. Bien que le second trait soit négatif, il possède les vertus de l'élégance de la forme, de la justice et de la droiture ; c'est le grand sage placé dans l'infériorité. Bien que le cinquiè me et le second traits ne soient pas en correspondance sympathique correcte par la négativité et la positivité, cependant ils sont dans un moment où la lumière (li) et le mouvement (tshen) s'entraident mutuellement, ce qui comporte le sens de pro duire un même effet en agissant de concert. Bien que le cinquième trait puisse arriver à la beauté, ce qui lui vaudra des félicitations et des louanges, et ce qui constituera un présage heureux, cependant, ce cinquième trait est hexaire et il ne comporte pas le sens d'absence de préventions personnelles et d'abaissement volontaire devant la sagesse. L’homme saint ${ }^{1}$ précise le sens pour en faire un enseignement.

TSHOU HI. - Bien que son caractère physique indique la douceur et l'obscurité, s’il peut cependant arriv er à la clarté de l'inférieur ${ }^{2}$, il recevra des félicitations et des louanges, et le présage sera heureux. C'est, en effet, à cause de sa malléabilité et de son obscurité que cette explication est posée, et cela

\footnotetext{
${ }^{1}$ Khong Tse.

2 On pourrait lire : « à la clarté qui est au-dessous ».
} 
précisément pour y remédier. Si celui qui consulte le sort peut se mettre dans ces conditions, le sens divinatoire lui sera applicable.

977.

Présage heureux du cinquième trait hexaire; il y a des félicitations.

TSHENG TSE. - Ce qui est appelé présage heureux, c'est cette condition qu'il est capable d'étendre à l'univers la félicité et l'éclat dont il jouit lui même. Le prince, bien que malléable et obscur, pourra, sîl sait employer les aptitudes de la sagesse, faire le bonheur de l'univers ; il ne déplore que son incapacité personnelle. 


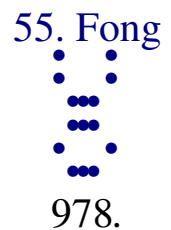

Trait supérieur hexaire : agrandir la splendeur de l' habitation; protéger la famille par un écran ${ }^{1}$; chercher à espionner par la porte ; regarder les yeux grands ouverts sans voir l'homme; trois ans sans voir ; présage malheureux.

TSHENG TSE. - Ce trait hexaire comporte les caractères physiques de la malléabilité négative et il est placé au dernier rang du koua fong. Placé à la fin du koua simple tshen, sa présomption et sa précipitation à se mouvoir sont extrêmes. Étant placé dans un moment de grandeur et de splendeur, il convient qu'il soit modeste et sache se courber, mais comme il se place au rang le plus élevé, il en résulte que l'effet de la grandeur et de la splendeur est attaché à l'énergie active ; or, il est, par lui-même, d'une substance molle et négative. Investi de la puissance de produire la grandeur et la splendeur, se trouvant dans le moment où cette grandeur devrait se produire, il est indigne de la situation qu'il occupe. Dans les conditions où se trouve le trait supérieur hexaire, ne répondant en rien aux nécessités requises, le présage malheureux est évident. « Agrandir la splendeur de l'habi tation », c'est se placer très haut, «protéger la famille par un écran », c'est se placer dans un lieu dépourvu de clarté. Puisque malléable et négatif, il est placé dans la splendeur, mais dans un rang qui ne comporte aucune situation reconnue, c'est quil dépasse les limites convenables de l'élévation et qu'il est dans les ténèbres et l'obscurité ; il rompt de lui-même avec les hommes : quel est donc l'homme qui s'allierait à lui ? Aussi, il épie par la porte et il regarde, les yeux grands ouverts, l'absence d'hommes. Cela dure jusqu'à trois années, sans qu'il soit capable de se modifier, et le présage est naturellement malheureux. « Ne pas voir » veut dire qu'il continue à ne pas voir d'homme ; c'est que, en effet, il ne se modifie point. Un trait hexaire qui occupe le dernier rang à la fin du koua comporte le sens de modification, tandis que celui-ci est incapable de changer ; c'est donc que ses aptitudes sont insuffisantes.

TSHOU HI. - Puisqu'il est négatif, malléable et placé au comble de l'éclat de la splen deur, qu'il occupe le dernier rang dans le koua simple tshen qui marque le mouvement, et que, à l'encontre de ces conditions, il est obscur et manque de clarté, il est considéré comme exprimant l'action « d'agrandir la splendeur de l'habitation » et comme comportant l'image symbolique de se cacher lui-même. Les expressions «absence d'homme » et «ne pas voir » indiquent encore la profondeur de l'obscurité dans laquelle il se cache : le présage est extrêmement malheureux.

\footnotetext{
${ }^{1}$ Stores ( ?)
} 
979.

Agrandir l'habitation; voler dans les espaces du ciel, épier par la porte, regarder, les yeux grands ouverts, l'absence d'hommes; se cacher dans la retraite.

TSHENG TSE. - Hexaire et placé au comble de la grandeur, étant en haut et s'élevant encore, il est comme planant dans les espaces du ciel, ce qui exprime l'extrême grandeur de l'élévation. Épier par la porte et absence d'hommes ; bien qu'il occupe l'extrême degré de la grandeur, il est cependant placé sur un terrain qui ne comporte aucune situation définie. Lui-même se cachant dans l'obscurité, les hommes l'abandonnent tous ; il se retire à l'écart et personne ne l'app roche.

TSHOU HI. - Se cacher dans la retraite ; c'est-à-dire se masquer derrière un écran pour ne pas être vu. 


\section{Lou}

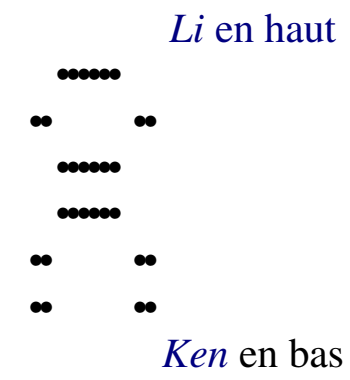

980. Lou ; petite liberté ; pureté du voyageur, présage heureux.

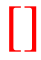

984. Premier trait hexaire : voyageur infime ; s'attendre à le voir éprouver des calamités.

$*$

986. Deuxième trait hexaire : le voyageur approche du lieu de repos ; il veille sur son bien; il jouit de la pureté du jeune serviteur.

988. Troisième trait nonaire : le voyageur brûle le lieu de repos; il perd le jeune garçon et le serviteur ; pureté et péril.

$*$

990. Quatrième trait nonaire : le voyageur arrive à son but; il jouit de ses biens et de ses instruments; son cour n'est pas satisfait.

$*$

992. Cinquième trait hexaire : lancer une flèche à la poule sauvage et la perdre ; à la fin recevoir des louanges et un ordre du prince.

*

994. Trait supérieur nonaire : l'oiseau brûle son nid ; le voyageur commence par rire et se lamente ensuite; il perd son bouf par négligence ; présage malheureux.

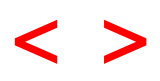




\section{Lou.}

TSHENG TSE. - Lou. «L'Ordre des koua » dit : «Fong, grandeur ; en épuisant la grandeur il doit nécessairement y avoir perte de la position occupée, de sorte que le koua fong est suivi du koua lou. » La perfection absolue de la grandeur parvenue à son comble, elle doit perdre ce qui fait son repos, et c'est cette raison qui fait que le koua lou suit immédia tement le koua fong. Il est composé avec le koua simple $l i$ au-dessus et le koua simple hen au-dessous ; la montagne arrête et ne change pas ; le feu marche et ne reste pas en place c'est l'image symbolique de s'éloigner dans des directions opposées, sans rester en place, de sorte que le koua est appelé lou. De plus, le péril est au-dehors, ce qui constitue encore l'image symbolique du voyageur ${ }^{1}$.

\footnotetext{
${ }^{1}$ Lou, voyageur.
} 


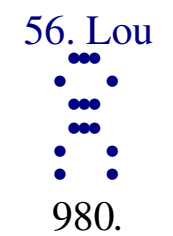

Lou ; petite liberté ; pureté du voyageur, présage heureux.

TSHENG TSE. - Cela est dit d'après les aptitudes exprimées par les koua simples. Avec les aptitudes de ces koua, il est possible de jouir d'une petite liberté, de posséder la droiture parfaite du voyageur, et de présenter un présage heureux.

TSHOU HI. - Lou, étranger voyageur. La montagne arrête en bas, le feu brûle et flambe en haut, ce qui constitue l'image symbolique de s'écarter de ce qui reste en place, sans jamais demeurer en un même lieu, de sorte que le koua est appelé lou. Comme le cinquième trait hexaire possède la justice à l'extérieur ${ }^{1}$ et qu'il s'incline avec soumission devant les deux positivités placées au-dessus et au-dessous de lui, comme le koua simple ken exprime l'arrêt, comme le koua simple li est inséparable de liidée de lumière, le sens divinatoire est qu'il y a possibilité d'une petite liberté et que s'il peut observer la droiture convenable dans le voyageur, le présage sera heureux. Le voyageur ne demeure pas perpétuellement en un même lieu; comme celui qui peut demeurer dans l'incertitude ; cependant, la voie rationnelle subsiste toujours et en tous lieux, de sorte qu'il a sa propre droiture dont il ne doit pas s'écarter, même pour un seul instant.

981.

Le commentaire de la formule déterminative dit: Lou, petite liberté; la douceur malléable se conforme à la justice du dehors et elle se soumet à la dureté énergique. Arrêt attaché à la clarté : c'est à cause de la petite liberté que la pureté énergique du voyageur présente un présage heureux.

TSHENG TSE. - Un trait hexaire monte occuper le cinquième rang; la douceur malléable possède la justice dans le koua simple extérieur ; elle est attachée aux deux traits énergiques placés au-dessus et au-dessous d'elle, ce qui exprime la soumission à la dureté énergique. En bas, le koua simple ken arrête ; en haut le koua simple li grossit et réunit arrêt et jonction avec la

\footnotetext{
${ }^{1}$ Dans le koua simple extérieur.
} 
lumière. La douceur malléable comportant la soumission et réussissant à se conformer à la justice dans le koua simple extérieur, ce qui arrête peut joindre et réunir à la lumière, et c'est à cause de cela qu'il y a une petite liberté, conformité à la droiture parfaite du voyageur, et enfin, présage heureux. Dans le moment de misère indiqué par le koua lou, si on ne possède l'aide de la positivité énergique, de la justice et de la droiture placées en bas, au-dessous de soi, il est impossible d'atteindre à une grande liberté ; c'est ce qu'on appelle atteindre à la justice à l'extérieur ${ }^{1}$. La justice n'affecte pas une seule et même forme; dans le koua lou, il s'agit de la justice convenable au voyageur. Arrêt joint à la clarté, ce qui fait que l'opportunité du moment n'est pas perdue et que, ensuite, on peut atteindre à la voie rationnelle de l'action de se placer pendant un voyage.

TSHOU HI. - Explication de la formule du koua, d'après les vertus et la substance des deux koua simples.

982.

Que le moment et le sens exprimés par le koua lou sont grands!

TSHENG TSE. - Dans les choses de l'univers, il convient de suivre, en chaque cas, la convenance et l'opportunité du moment. Cependant, le koua lou exprime la difficulté de se placer, de sorte que cette formule mentionne la grandeur du sens et du moment indiqué.

TSHOU HI. — Le temps ou moment exprimé par le koua lou exprime la difficulté de se placer.

983.

Le commentaire traditionnel de la formule symbolique dit: Sur la montagne il y a du feu; lou, l'homme doué, en conclut la circonspection dans l'emploi des peines et il ne laisse pas prolonger la détention.

TSHENG TSE. — Le feu étant placé très haut, il n'existe rien qui ne soit illuminé par sa clarté. L'homme doué contemple l'image symbolique de la clarté projetant sa lumière et illuminant, et il en conclut la circonspection dans

\footnotetext{
${ }^{1}$ Etre placé au milieu du koua simple extérieur.
} 
l'emploi des peines. L'intelligence ne doit pas se fier à elle -même, aussi la formule avertit de la nécessité de la circonspection. Clarté et arrêt : c'est encore une image symbolique de circonspection. Regardant l'image symbolique du feu qui agit sans rester en place, il ne laisse pas les procès criminels traîner en longueur. Les jugements criminels n'on été institués que pour les cas où il est absolument impossible de s'en passer; mais si quelqu'un, dans la nation, a commis une faute et tombe sous le coup de l'application d'une peine, comment serait -il licite de laisser la question traîner en longueur?

TSHOU HI. - Circonspection dans l'application des peines, comme l'exprime le koua qui représente la montagne ; absence de retards et de lenteurs, comme dans l'action du feu. 


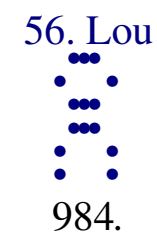

Premier trait hexaire : voyageur infime; s'attendre à le voir éprouver des calamités.

TSHENG TSE. - Ce trait est hexaire, il emploie les qualités de la malléabilité négative et se trouve dans un moment indiquant le voyage. Il se place dans un rang inférieur et humble ; c'est un homme faible et mou, placé au milieu des misères d'un voyage, qui se trouve dans un rang humble et vil et qui se maintient en cet état sordide et infime. Un homme dont les tendances sont humbles et qui, se trouvant placé dans les circonstances pénibles d'un voyage, est encore rustique, vil et sans valeur ; il est exposé à tout, et c'est à cause de cela qu'il éprouve de la honte, des calamités et qu’il est coupable. Être infime, c'est une des c onditions qui s'attachent à la grossièreté rustique. Dans le moment des misères qui résultent de la situation d'être en voyage, ses aptitudes et ses caractères physiques étant tels, bien que au-dessus de lui il rencontre accueil et assistance, il est incapable de rien faire. Le quatrième trait possède la nature de la positivité et fait partie de la substance du koua simple $l i$; de plus, ce n'est pas quelqu'un qui soit porté à descendre ; enfin, dans le koua lou, cela exprime une condition différente de celle du sujet de rang élevé dont il s'agit dans les autres koua.

TSHOU HI. - En présence d'un moment qui indique le voyage, il occupe la situation inférieure avec ses qualités de mollesse négative; aussi, tels sont l'image symbolique et le sens divinatoire.

985.

Voyageur infime ; ses tendances Le conduisent finalement à des calamités.

TSHENG TSE. - Ses tendances et ses idées le poussent aux excès, ce qui fait qu'il s'expo se de plus en plus aux calamités. Lorsque les calamités éventuelles sont désignées par deux mots à peu près synonymes, cela indique qu'il y a une distinction à faire entre les genres de ces calamités. Lorsqu'un seul terme est employé, comme dans le cas actuel, il s'agit simplement de calamités et de malheurs. 


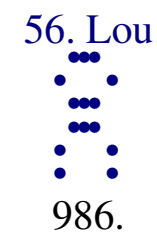

Deuxième trait hexaire : le voyageur approche du lieu de repos; il veille sur son bien ; il jouit de la pureté du jeune serviteur.

TSHENG TSE. - Le second trait possède les vertus de la soumission et de la douceur, de la justice et de la droiture ; puisqu'il est soumis et doux, la foule s'allie à lui, puisqu'il est juste et droit, en se plaçant il ne manque pas aux convenances. Il résulte de là qu'il est capable de garantir ce quill possède ; le jeune serviteur, à son tour, épuise la fidélité et la bonne foi. Bien qu'il ne possède pas comme le cinquième trait les vertus qui résultent de l'élégance de la forme et l'assistance de ceux qui sont au -dessus et au-dessous de lui, cependant, il est encore placé dans des conditions avantageuses, étant donné qu'il s'agit du koua lou, qui exprime l’idée de voyages et de voyageurs. Lieux de repos, lieux ou les voyageurs se reposent; richesses et marchandises; les biens d'un voyageur ; jeune serviteur, qui accompagne le voyageur et l'assiste. Il réussit à atteindre le lieu de repos ; il réunit ses biens et veille à leur conservation et enfin il jouit de la pureté du jeune serviteur honnête : c'est la meilleure condition pour un voyageur. Celui qui est doux et faible, et placé dans l'infériorité, c'est le jeune garçon ; celui qui est fort et vigoureux et qui est placé au-dehors, c'est le serviteur. Le second trait, doux et soumis, doué de justice et de droiture, possède le concours et le cour de l'extérieur et de l'intérieur ; étant en voyage, ceux avec lesquels il vit en intimité sont le jeune garçon et le serviteur. La formule ne parle pas de présage heureux : Dans les circonstances et les hasards d'un voyage, pouvoir éviter les maux et les calamités, constitue déjà un bien.

TSHOU HI. - Approchant d'un relais ${ }^{1}$ il se reposera; veillant sur son bien, il sera prospère; possédant la confiance et la pureté d’intentions du jeune garçon et du serviteur, il ne sera point déçu et saura sur qui compter : c'est ce qui constitue le plus grand présage heureux du voyageur. Le second trait possède les vertus de la justice et de la droiture de la malléabilité douce et passive, aussi tels sont le sens divinatoire et l'image symbolique.

987.

\footnotetext{
${ }^{1}$ Halte, station de voyageurs.
} 
Possédant la pureté du jeune serviteur, finalement pas de regrets.

TSHENG TSE. - Lhomme qui voyage compte surtout sur ses serviteurs ; du moment où il jouit de la fidélité et de la pureté d’intention du jeune serviteur, finalement, il n'aura aucun regret. 


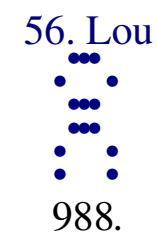

Troisième trait nonaire : le voyageur brûle le lieu de repos ; il perd le jeune garçon et le serviteur; pureté et péril.

TSHENG TSE. - La voie rationnelle pour se placer pendant un voyage, consiste d'abord dans la douceur modeste, la déférence et l'humilité ; le troisième trait est énergique et sans justice ; il occupe le rang supérieur dans la substance du koua simple inférieur, lui et le trait supérieur du koua simple hen ont l'image symbolique de s'exalter eux -mêmes. Étant en voyage et doués de trop de dureté énergique, s'exaltant eux -mêmes, c'est là une voie qui doit nécessairement conduire aux calamités. S'exaltant et s'élevant, il manque de soumission au supérieur, aussi, celui-ci ne s'allie pas avec lui et brûle son lieu de halte et de repos ; il perd ce qui assurait son repos. Le koua simple $l i$, placé au-dessus, exprime liimage symbolique de lincendie et des calamités qui en résultent. Ayant trop de dureté énergique, il est cruel avec ses inférieurs, aussi, ceux-là l'aband onnent et il perd la confiance et la pureté du jeune garçon et de l'esclave, c'est -à-dire qu'il perd leur coar, dans ces conditions, c'est une voie rationnelle de périls et de dangers.

TSHOU HI. - Excès d'énergie sans justice occupant le rang inférieur dans le koua simple inférieur, aussi tels sont l'image symbolique et le sens divinatoire. Perdant le jeune garçon esclave, il ne perd pas seulement son coaur, aussi le caractère traduit par le mot pureté suit immédiatement dans la phrase suivante et indique le sens.

989.

Le voyageur brûle le lieu de repos ${ }^{1}$; c'est encore pour en souffrir; puisqu'il voyage vers le bas, le sens est «perdre».

TSHENG TSE. - Le voyageur brûle et perd sa station de repos, c'est encore un moyen de s'attirer de la souffrance. A vec le moment du voyage, et suivant la voie de l'alliance avec l'inférieur comme cela est exprimé dans le texte, le sens comporte une perte. Étant en voyage et se mettant très haut dans

\footnotetext{
${ }^{1}$ Le relais.
} 
ses relations avec les inférieurs à cause de son excès d'énergie, il doit naturellement perdre la pureté de la fidélité, c'est-à-dire perdre le cour de ses inférieurs. Être en voyage et perdre le cour de ses serviteurs, constitue un motif de péril et de crainte.

TSHOU HI. - Puisqu'il s'agit du moment d'un voyage, et qu'il pr atique ainsi la voie de l'alliance avec les inférieurs, le devoir doit être violé et oublié. 


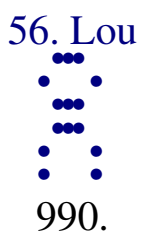

Quatrième trait nonaire : le voyageur arrive à son but; il jouit de ses biens et de ses instruments; son cour n' est pas satisfait.

TSHENG TSE. - Le quatrième trait, énergique et positif, bien que n'étant pas dans une position qui comporte la justice, se place cependant au rang inférieur dans le koua simple supérieur, ce qui comporte la douceur ; et présente l'image symbolique de l'emploi de la douceur et de la capacité de s'abaisser, ce qui est conforme aux conditions de l'état de voyageur. À cause de ses aptitudes d'énergie et de clarté (intelligence), c'est à lui que le cinquième trait s'allie, c'est à lui que le premier répond sympathiquement, et c'est lui qui constitue le bien dans le koua lou. Toutefois, le quatrième rang n'est pas la situation qui lui revient suivant la droiture, aussi, bien qu'il y trouve un lieu de repos, cette condition n'est pas comparable à celle du second trait se portant vers un lieu de repos et d'habitation. Ayant les aptitudes de l'énergie et de l'intelligence, il représente celui avec qui le supérieur et l'inférieur s'allient, c'est donc un voyageur et il j ouit de ses biens et du bénéfice de l'emploi de ses outils et de ses instruments. Bien que dans le koua lou cela constitue le bien, cependant au-dessus de lui, il ne rencontre pas l'alliance et le concours de la dureté énergique positive et, au-dessous, il ne rencontre que les sympathies de la malléabilité négative, de sorte quill ne peut étendre l'effet de ses aptitudes, faire prévaloir ses tendances, et que son cour n'est pas joyeux. Dans le texte le dixième caractère, moi, je, est employé pour désigner le quatrième trait.

TSHOU HI. - Puisque positif, il occupe un rang négatif, puisqu’il se place au rang inférieur du koua simple supérieur, qu'il emploie la douceur et est capable de s'abaisser, tels sont donc limage symbolique et le sens divinatoire. Mais ce rang n'est pas la situation qui lui revient conformément aux règles de la droiture ; de plus, au-dessus de lui, il ne jouit pas du concours de la dureté énergique positive ; au-dessous, il ne rencontre que la sympathie de la malléabilité négative, de sorte qu'il y a des motifs pour que son cour ne soit pas joyeux. 
Le voyageur au repos; il ne possède pas encore de situation; il possède ses biens et ses armes; son caur n'est pas encore satisfait.

TSHENG TSE. - Le quatrième trait étant près du prince, est considéré comme digne de sa situation. Dans le koua lou, le cinquième trait ne comporte pas le sens de prince, aussi le quatrième est considéré comme ne possédant pas encore de situation. On a dit: Mais alors, puisque nonaire, il occupe le quatrième rang, l'absence de droiture doit être consi dérée compte un fait de culpabilité ? Réponse : Puisque énergique, il occupe un rang qui comporte la douceur, il possède les qualités nécessaires au voyageur. Nonaire, il emploie les aptitudes de l'éne rgie et de l'intelligence, il veut profiter du moment et faire prévaloir ses tendances ; aussi, bien qu'il possède ses biens et ses armes, ce qui, pour un voyageur, constitue le bien, son cœar n'est cependant pas encore satisfait. 


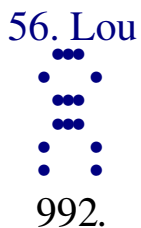

Cinquième trait hexaire : lancer une flèche à la poule sauvage et la perdre ; à la fin recevoir des louanges et un ordre du prince ${ }^{\text {. }}$

TSHENG TSE. - Le cinquième trait hexaire possède les vertus de l'élégance de la forme ${ }^{2}$ et de la soumission passive ; il est placé dans la voie de la justice, et le supérieur comme l'inférieur s'allient avec lui ; c'est celui qui, dans le koua lou, se place dans les conditions d'extrême excellence. Lorsque l'homme se trouve en voyage et qu'il peut se conformer à la voie de l'élégance de la forme, cela peut certainement être qualifié du mot «bien ». Un voyageur étranger qui se met en mouvement, peut parfois éprouver des pertes, et alors la misère et la honte s'ensuivent pour lui ; s'il se met en mouvement et ne fait pas de pertes, cela constitue le bien. Le koua simple $l i$ représente la poule sauvage, c'est-à-dire un être élégant et brillant; lancer une flèche veut dire choisir les règles de l'élégance de la forme et s'y conforme $r$. Si on lance une flèche à la poule sauvage et si on la perd, cela ne signifie pas que la flèche n'a pas été bien dirigée, de sorte que, finale ment, il y a des louanges et une mission. Louanges, compliments flatteurs; mission, appointements d'une fonction. Le cinquième trait occupe la situation qui comporte l'élé gance de la forme et l'éclat brillant ; il possède les vertus qui en résultent, de sorte que s'il se meut, il atteindra à la voie rationnelle de cette élégance et de cette clarté. Le cinquième rang est la situation du prince, mais un prince n'est pas un voyageur ni un marchand ; s'il voyageait en pays étranger, comme un marchand, il perdrait sa situation, de sorte que la formule de ce trait ne relève pas le sens de prince.

TSHOU HI. - La poule sauvage est un animal élégant et brillant ; c'est l'image symbo lique exprimée par le koua simple $l i$. Le cinquième trait hexaire est soumis et doux, beau et brillant ; de plus, il possède la voie de la justice et c'est lui qui est le maître du koua simple $l i{ }^{3}$, c'est à cause de cela qu'il est accompagné de cette formule et qu'il est considé ré comme exprimant l'image symbolique de l'action de lancer une flèche à la poule sau vage. Bien qu'il ne puisse éviter de subir une perte, ce qu’il perd n'est pas considé rable et, finalement, il reçoit des louanges et une mission.

\footnotetext{
${ }^{1}$ Nomination à une fonction.

2 Ou clarté du style.

${ }^{3}$ Celui qui donne naissance au koua $l i$ en remplaçant un trait plein dans le koua simple khien.
} 
993.

À la fin, par des louanges et une mission royale, il atteint au rang supérieur.

TSHENG TSE. - Il a les vertus de l'élégance de la forme et de la soumission passive, de sorte que, en haut et en bas, on s'allie à lui. Le mot atteindre veut dire aussi « allier ${ }^{1}$.

Étant capable d'obéissance et de soumission envers le supérieur, et le supérieur s'alliant avec lui, il est donc atteint par le supérieur. Être au-dessus et atteindre à ce qui est au-dessous, c'est être soi-même l'objet auquel l'inférieur atteint en montant. Étant en voyage, et le supérieur comme l'inférieur s'alliant à lui, il en résulte nécessairement des louanges et une mission. Le koua lou exprime le moment de misère avant de pouvoir atteindre au repos ; "finalement, à cause des louanges et d'une mission royale », cette phrase du texte exprime quil mérite d'arriver à recevoir des louanges et une mission ; quand il sera arrivé à ce résultat, il ne sera plus un voyageur (lou). La misère et l'isolement constituent le sens du mot lou ; il n'est pas nécessaire qu'il y ait voyage à l'extérieur.

TSHOU HI. — « Le supérieur atteint » est une expression qui indique les louanges et la mission conférée par le supérieur.

\footnotetext{
${ }^{1}$ Le commentateur lit : « alliance avec le supérieur ».
} 


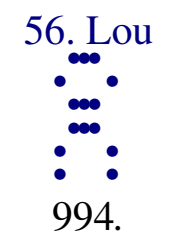

Trait supérieur nonaire : l'oiseau brûle son nid; le voyageur commence par rire et se lamente ensuite; il perd son bauf par négligence ; présage malheureux.

TSHENG TSE. — L'oiseau, ce qui vole et se place haut. Le trait supérieur nonaire et énergique, est dépourvu de justice et se place extrêmement haut ; de plus, il fait partie de la substance du koua simple $l i$, et l'excès est évident, ce qui fait qu'il prend l'image symbo lique de l'oiseau. Se trouvant dans le moment exprimé par le koua lou, la modestie qui s'abaisse, la douceur qui porte à la concorde sont des garanties de la possibilité de se protéger soimême ; or, ce trait comporte l'excès d'énergie et lui -même s'élève et s'exalte ; il perd ce qui fait son repos, c'est infaillible. Le nid, c'est le lieu de repos où l'oiseau s'arrête et se retire ; brûlant son nid, il perd ce qui assurait son repos et n'a plus de lieu où il puisse s'arrêter. Être au rang supérieur du koua simple $l i$, est considéré comme présentant l'image de l'action de brûler (incendier). Positif et énergique, se plaçant de lui-même au point le plus élevé, il commence par donner satisfaction à ses idées, de sorte que, d'abord il rit ; plus tard, quand il a perdu son repos et qu'il ne sait vers quoi se porter, il se lamente. La légèreté et la négligence font qu'il perd ses vertus de soumission, et de là résulte le présage malheureux. Le bouf est un animal soumis et passif; perdre le bouf par négligence veut dire que sa précipitation et sa négligence lui font perdre sa soumission première. Le koua simple $l i$ désigne le feu dont la nature est de monter, et il donne l'image symbolique de l'emportement et de la légèreté. La première phrase se rapporte à l'oiseau qui brûle son nid, de sorte que, à la suite, le texte mentionne la qualité de «voyageur»; słil ne portait pas les mots «l'homme voyageur », ce serait l'oiseau qui rirait ou pleurerait.

TSHOU HI. - Le trait supérieur hexaire indique un excès d'énergie ; il se place au rang supérieur du koua lou qui représente le voyage. Le comble de l'éclat (la), l'orgueil sans soumission, sont également la voie rationnelle d'où résulte un présage malheureux, de sorte que tels sont limage symbolique et le sens divinatoire. 
Puisque le voyageur est au rang supérieur, le sens indique l'incendie; perdre le bouf par légèreté ; à la fin, rien ne peut être entendu.

TSHENG TSE. - Puisqu'il s'agit du koua lou et du trait supérieur, que, de plus, il se place lui-même avec hauteur dans un lieu très élevé, comment pourrait-il y assurer son séjour? Le sens indique quil doit y avoir quelque chose d'analogue à l'incendie du nid. Au moment où, par l'extrême limite de l'énergie, il s'exalte et s'élève, il est considéré comme réussissant à faire prévaloir ses tendances et comme riant de plaisir ; il ne sait pas que la perte de la vertu de soumission, résultant de sa légèreté et de sa précipitation, fera que finalement, il n'écoutera plus rien, ce qui signifie que, finalement, il n'aura plus aucun entendement, ni savoir. Pour lui rendre conscience de ses actions, avant qu'il ne soit parvenu au faite de l'élévation, il est réduit à se lamenter. Énergique et positif, dépourvu de justice et se plaçant au faîte de l'élévation, il présente certainement l’image symbolique de l'excès de précipitation dans les mouvements, et comme, de plus, le feu brûle et monte en flambant, cela indique encore quelque chose d'excessif. 


\section{Souen}

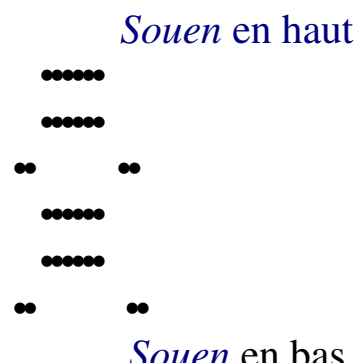

996. Souen, petite liberté ; avantage dans ce qu'il y a à entreprendre ; avantage à voir un grand homme.

[]

1000. Premier trait hexaire : avancer et reculer; avantage de la pureté de l'homme hardi.

1002. Deuxième trait nonaire : l'humilité est au-dessous du lit; employer les devins et les charmeurs nombreux ; présage heureux, sans culpabilité.

1004. Troisième trait nonaire : précipitation dans l'humilité ; appréhension. $*$

1006. Quatrième trait hexaire : dissipation des regrets. Chasser et capturer les trois catégories; il y a du mérite.

1008. Cinquième trait hexaire : pureté, présage heureux, dissipation des regrets ; rien sans avantage; pas de commencement et fin; trois jours avant le jour kang ; trois jours après le jour hang ; présage heureux.

*

1010. Trait supérieur nonaire : entrer sous le lit; perdre lesbiens et les armes ; présage malheureux de la pureté.

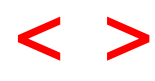




\section{Souen.}

TSHENG TSE. - Souen. «L'Ordre des koua » dit: «Les voyages conduisent à l'absence d'abri, de sorte que le koua lou est suivi du koua souen; souen signifie : entrer. » Si l'étran ger, voyageur, isolé, est dépourvu de soumission et d'humilité, qui le supportera ? S'il est capable de soumission et d'humilité, bien que placé au milieu des misères qui résultent des voyages, que pourrait-il entreprendre sans réussir ? C'est là ce qui fait que le koua souen suit le koua lou. Comme koua simple, il est constitué par une négativité placée au-dessous de deux positivités: humilité et soumission envers la positivité, et c'est ce qui fait que ce koua est appelé souen. 


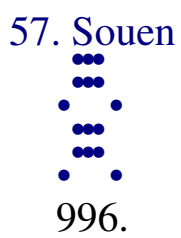

Souen, petite liberté ; avantage dans ce qu'il y a à entreprendre; avantage à voir un grand homme.

TSHENG TSE. - Étant données les aptitudes que comporte ce koua, il est possible, avec une petite liberté, d'avoir avantage dans ce quil y a à entreprendre et avantage à voir un grand homme. Les koua souen et touei comportent également l'énergie, la justice et la droiture, et enfin, le sens des mots souen et touei sont analogues. Mais dans le koua touei il y a «liberté », tandis que dans le koua souen, il ne s'agit que d'une « petite liberté ». Touei exprime ce qui est produit par ia positivité, tandis que souen exprime ce qui est produit par la négativité. Dans le koua touei, la malléabilité est à l'extérieur ; c'est l'effet de la mal léabilité ; dans le koua souen, la malléabilité se trouve à l'intérieur, c'est la nature de la malléabilité, et c'est ce qui fait le peu de liberté du koua souen.

TSHOU HI. - Souen, entrer. Une négativité courbée au-dessous de deux positivités: sa nature lui permet d'être humble pour entrer. L'im age symbolique est considérée comme exprimant le vent, ce qui provient encore du sens du mot «entrer ». La négativité est maîtresse de la situation, aussi le sens divinatoire est considéré comme exprimant une petite liberté. Puisque la négativité suit la positivité, il y a aussi avantage dans ce quill y a à entre prendre. Toutefois, il faut être certain que ce qui est suivi est conforme à la droiture, aussi, la formule ajoute : avantage à voir un grand homme.

997.

Le commentaire de la formule déterminative dit: Répétition d'humilité pour redresser le sort.

TSHENG TSE. - Répétition du koua simple souen, répétition de l'humilité. Le supérieur se conforme à la voie rationnelle en donnant ses ordres; l'inférieur reçoit les ordres et s'y conforme avec so umission: le supérieur et l'inférieur font tous deux preuve de soumission, ce qui donne l'image symbolique de la répétition de la soumission. D'ailleurs, le caractère traduit par le mot répétition comporte le sens de redoublement. L'expression 
homme doué comporte également le sens de répétition d'humilité, afin de redresser et de répéter les ordres du destin. Ici, le mot redresser prend le sens de renouvellement ; c'est une expression qui indique la paix et le repos.

998.

Énergie et soumission à la justice et à la droiture, action des tendances; la malléabilité est toujours soumise à la dureté énergique, c'est par là qu'il y a une petite liberté. Le bien a un moyen de se produire; avantage à voir un grand homme.

TSHENG TSE. - Cela est dit au sujet des aptitudes du koua. L'énergie active de la positivité fait partie de la substance du koua souen et elle possède la justice et la droiture; c'est la voie rationnelle de la soumission et de l'humilité dans la justice et la droiture. La nature de la positivité est de monter ; ses tendances la portent à agir en s'élevant dans la voie de la justice et de la droiture. De plus, la malléabilité, qu'elle soit placée au -dessus ou audessous, est toujours soumise à la dureté énergique ; les aptitudes indiquées étant telles, bien que la qualité intérieure soit la malléabilité, il est cependant possible qu'il existe une petite liberté. Dans la voie de la soumission et de l'humilité, il n'est aucune entreprise qui ne puisse réussir, aussi il y a avantage dans ce qu'il y a à entreprendre. Bien que la soumission et l'humilité constituent une voie de bien, cependant il faut encore savoir à qui on obéit ; pouvoir être humble et soumis à la dureté énergique du grand homme doué -de justice et de droiture sera considéré comme constituant un avantage, aussi il y a avantage à voir un grand homme. Par exemple, la justice et la droiture de la dureté énergique du cinquième ou du second trait indiquent un grand homme. L'humilité et la soumission envers tout autre que le grand homme ne doivent pas, nécessairement, ne pas être considérées comme constituant un excès.

TSHOU HI. - Explication de la formule du koua au moyen de la substance du koua luimême. L'énergie soumise à la justice et à la droiture et dont les tendances peuvent prévaloir, cela désigne le cinquième trait nonaire ; la malléabilité désigne le premier et le quatrième.

999. 
Le commentaire traditionnel de la formule symbolique dit : Suivre le vent ${ }^{1}$, humilité ; l'homme doué s'applique à répéter les ordres et à pratiquer les affaires.

TSHENG TSE. - Deux vents répétés l'un sur l'autre : les vents se suivent; le mot « suivre » du texte comporte le sens de soumission. L'homme doué considère l'image symbolique de la répétition du koua simple souen se succédant à lui-même avec humilité, et il s'applique à renouveler ses ordres et ses instructions ; il agit pour redresser et réformer les affaires. Le mot suivre comporte le sens de répétition, ce qui indique que, au-dessus et au-dessous, il $\mathrm{y}$ a toujours soumission. Le supérieur se soumet à l'inférieur et se met en avant ; linférieur se soumet au supérieur et le suit : le supérieur et l’inférieur font tous deux preuve de soumission, ce qui comporte le sens de répétition de l'humilité ${ }^{2}$. Dans les questions relatives aux décrets, aux ordonnances et aux affaires du gouvernement, si le prince se conforme à la raison d'être des choses, il est d'accord avec le cour du peuple et le peuple se soumet et obéit.

TSHOU HI. — Suivre ; c'est le sens de suivre mutuellement.

${ }^{1}$ Les commentateurs lisent : répétition du vent ; cela n'est pas dans le texte.

${ }^{2}$ Répétition du koua simple souen. 


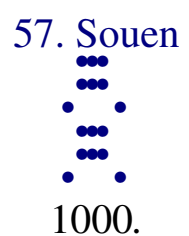

Premier trait hexaire : avancer et reculer; avantage de la pureté de l'homme hardi.

TSHENG TSE. - Ce trait est hexaire ; avec sa malléabilité négative, il occupe une position humble et inférieure, mais il est sans justice. Il est placé au rang le plus bas et il est soumis à l'énergie : il exprime l'excès d'humilité. Un homme malléable et négatif qui pousse l'abaissement et l'humilité jusqu'à l'excès, de sorte que ses tendances et ses idées sont toujours sous l'impres sion de la crainte et quil ne peut jouir du repos et de la tranquillité. Tantôt il avance, tantôt il recule ; il ne sait quel parti prendre, et ce qui peut lui être avantageux, c'est la perfection ou pureté de l'homme hardi. S'il peut employer les tendances de la pureté et de l'énergie de l'homme hardi, c'est ce qui conviendra. En s'efforçant d'être énergique, il ne tombera point dans la faute qui consiste dans l'excès de l'humilité et de la crainte.

TSHOU HI. - Le premier trait, négatif, occupe le rang inférieur ; il est le maître du koua simple souen ${ }^{1}$; il représente l'excès dans l'abaissement et l'humilité, aussi il est consi déré comme exprimant l'image symbolique de l'action d'avancer et de se retirer, sans décision. Si l'on se place dans cette position avec la perfection de l'homme hardi, il y aura moyen de remédier à ce qui fait défaut et d'atteindre au degré convenable.

1001.

Avancer et reculer; tendances hésitantes; perfection de l'homme hardi, jugement raffermi.

TSHENG TSE. - C'est celui qui avance, puis recule, sans savoir où trouver le repos; son jugement est indécis et craintif. L'avantage consistera dans l'emploi de la pureté et de l'énergie de l'homme hardi, afin de déterminer son jugement, de sorte qu'il soit calmé et affermi. Le cara ctère employé a le sens de réparer et consolider.

\footnotetext{
${ }^{1}$ Puisque en se transformant de l'état de trait plein à celui de trait brisé, il donne naissance au koua simple souen qui remplace le koua simple khien.
} 


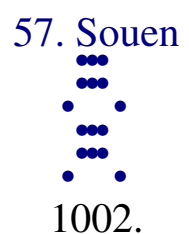

Deuxième trait nonaire : l'humilité est au-dessous du lit ; employer les devins et les charmeurs nombreux; présage heureux, sans culpabilité.

TSHENG TSE. — Le second trait se trouve dans le moment exprimé par le koua souen, qui indique l'humilité et l'abaissement ; nonaire, il est placé à un rang qui comporte la négativité et il est au rang inférieur; il exprime l'excès dans l'humili té. Le lit est ce qui sert à l'homme pour se reposer : « humble et placé plus bas que le lit » est un excès d'humilité et c'est dépasser le niveau qui assure le repos. Si l'excès d'humilité et d'abaissement chez l'homme ne résulte pas de la crainte et de la pusillanimité, il sera causé par l'esprit de flat terie et d'adulation; c'est toujours l'effet du manque de droiture. Le second trait est plein, énergique et juste ; bien qu'il fasse partie de la substance du koua souen (humilité) et qu'il occupe un rang négatif, il est considéré comme exprimant un excès dans l'humi lité, sans avoir un cour vicieux et méchant. L'excès dans l'humilité respectueuse, bien que ne constituant pas la droiture dans la bienséance, peut cependant prévenir et éviter la honte et les affronts et supprimer les causes de reproches et toute culpabilité, c'est donc encore une voie rationnelle dont le présage est heureux. Les devins et les charmeurs, ce sont tous ceux qui sont en communication d'idées avec l'intelligence des génies. Les dixième et onzième caractères du texte ont le sens de "grande multiplicité ». S’il y a extrême sincérité et calme dans la modestie et l'humilité, ceux qui pourront comprendre cette sincérité d'idée seront nombreux ; le présage sera heureux, et il n'y aura aucune culpabilité, ce qui veut dire que la sincérité sera suffisante pour émouvoir les hommes. Si l'homme n'examine et ne recherche pas la sincérité de l'idée, alors il considérera l'excès d'humilité comme de l'adulation.

TSHOU HI. - Le second trait emploie les qualités de la positivité et il se place à un rang négatif dans une position inférieure ; cela comporte l’idée d'absence de tranquillité. Tou tefois, en présence d'un moment d'abaissement (souen), il n'opprime pas les humbles et, de plus, il possè de la justice ; il ne pousse pas les choses à l'extrême, de sorte que le sens divinatoire est considéré comme exprimant la possibilité de l'excès dans l'humilité et comme comportant la répétition continuelle et fastidieuse des mêmes paroles pour faire prévaloir sa propre doctrine. Delà résulte la possibilité du présage heureux et de l'absen ce de culpabilité et aussi le sens divinatoire indiquant le présage heureux du fait d'épui ser la sincérité de l'idée dans l'accomplissement des sacrifices. 
1003.

Présage heureux de la multiplicité ; possession de la justice.

TSHENG TSE. - Puisque le second trait occupe un rang qui indique la douceur et quil est dans une situation inférieure, il constitue l'image symbolique de l'excès dans l'humili té et c'est celui qui peut faire apprécier et connaître la sincérité de son idée. La foule nombreuse s'agite et réussit ainsi à se conformer à la justice. Lorsqu'un trait positif occu pe le rang du milieu (possède la justice), il constitue l’image symbolique de la plénitude intérieure ${ }^{1}$; du moment où la justice est complète, les hommes doivent naturellement avoir confiance et, à cause de la sincérité de l'idée, il ne s'agit ni du résultat de la crainte, ni de flatterie et d'adulation ; c'est par là que le présage est heureux et qu'il n'y a pas de culpabilité.

${ }^{1}$ De la justice absolue. 


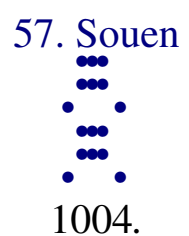

Troisième trait nonaire : précipitation dans l'humilité ; appréhension.

TSHENG TSE. - Le troisième trait avec sa positivité se place dans un rang qui comporte l'énergie ; il ne possède point la justice et de plus il est au rang supérieur dans le koua simple inférieur. Les caractères physiques indiquent l'excès dans l'énergie, et se trouvant dans le moment de la soumission et de l'humilité, c'est celui qui est incapable de s'humi lier. Il s'efforce pour être humble et il échoue continuellement dans ses tentatives. Il se trouve dans un moment d'humiliation, il se place dans l'infériorité, et le supérieur le regarde avec humilité. Enfin le quatrième trait se sert de la douceur et de la soumission et ils se serrent l'un contre l'autre en se rapprochant; ce quill foule aux pieds est énergique et au-dessus de lui se trouvent encore deux positivités répétées. Quand même il voudrait ne pas être humble, le pourrait-il ? Aussi, il échoue continuellement dans ses tentatives et il essaye de nouveau d'être humble, et c'est bien là un motif d'appréhension.

TSHOU HI. - Excès de dureté énergique sans justice, occupant le rang supérieur dans le koua simple inférieur: c'est celui qui est incapable d'humilité. Il s'efforce et il échoue pe rpétuellement dans ses tentatives : c'est une voie rationnelle d'appréhension, et aussi, tels sont le sens divinatoire et l’image symbolique.

1005 .

Appréhension de l'humilité continuelle; tendances épuisées.

TSHENG TSE. - Les caractères physiques et les aptitudes du troisième trait le rendent essentiellement incapable d'humilité ; d'ailleurs le supérieur le surveille ${ }^{1}$ avec humilité ; il est soumis à une répétition d'énergie et il foule des énergies aux pieds. La force inhérente aux choses montre qu'il ne peut pas faire prévaloir ses tendances, aussi il échoue continuellement, et continuellement il recommence et essaye d'être humble. Donc, ses tendances sont contrariées et il en souffre, ce qui est, au plus haut point, un motif d'appréhension.

\footnotetext{
${ }^{1}$ Le regarde d'en haut.
} 


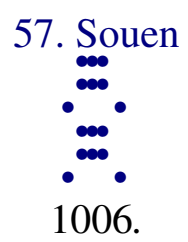

Quatrième trait hexaire : dissipation des regrets. Chasser et capturer les trois catégories; il y a du mérite.

TSHENG TSE. - Malléabilité négative qui ne reçoit aucun accueil, pliée sous l'énergie et rencontrant l'én ergie sous ses pieds; il doit naturellement y avoir des regrets. Mais le quatrième trait avec sa qualité de trait négatif occupe encore un rang négatif ; il se conforme à la droiture dans l'humilité. Placé au rang inférieur de la substance du koua simple supérieur, il occupe un rang élevé et il est capable de s'abaisser ; demeurant au rang inférieur du koua simple supérieur, il est humble envers les supérieurs ; surveillant les inférieurs avec humilité, il est humble envers ses inférieurs. Étant bien placé comme il vient d'être dit, il est possible que ses regrets se dissipent. Ce qui fait que ses regrets peuvent se dissiper, c'est qu'il est comme celui qui chasse et qui obtient trois parts. Chasser et capturer trois parts ; c'est atteindre en haut et en bas ; le gibier capturé à la chasse est partagé en trois catégories, l'une pour sécher, une autre pour offrir aux hôtes et invités et pour les besoins de la cuisine, une autre pour les rabatteurs et les aides de la chasse. Le quatrième trait est capable d'humilité envers les traits positifs placés au-dessus et au-dessous de lui ; il est comme celui qui obtient les trois parts à la chasse, c'est-à-dire qu'il atteint partout, en haut et en bas. Le terrain, ou rang, occupé par le quatrième trait comporte essentiellement l'existence de regrets ; comme il s'y place pour le mieux, ses regrets se dissipent, et en outre, il a du mérite. Dans les affaires de ce monde, si on se place bien il est parfois possible que les regrets se dissipent et qu'il en résulte du mérite.

TSHOU HI. - Malléabilité négative sans correspondance sympathique ; ce qui est au-dessus et au-dessous est toujours énergique, de sorte qu'elle doit naturellement éprouver des regrets. De plus, négatif, ce trait occupe un rang négatif (pair) ; il se place au rang inférieur du koua simple supérieur, de sorte qu'il réussit à oublier ses regrets, et qu'en outre il a un sens divinatoire heureux lorsqu'on consulte le sort au sujet du résultat futur d'une chasse à entreprendre. Les trois catégories sont les pièces à faire sécher, la part des hôtes et des invités, la part destinée aux cuisines. 
Chasser et capturer les trois catégories; il y a du mérite.

TSHENG TSE. - Humble envers les supérieurs et les inférieurs, comme celui qui, à la chasse, capture les trois catégories et atteint en haut et en bas. Achèvement du mérite de l'humilité. 


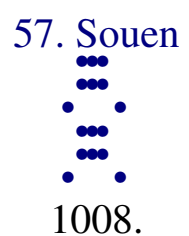

Cinquième trait hexaire : pureté, présage heureux, dissipation des regrets ; rien sans avantage ; pas de commencement et fin ; trois jours avant le jour kang ; trois jours après le jour hang ; présage heureux.

TSHENG TSE. - Le cinquième trait occupe la situation prééminente ; c'est de lui que dépend la formation du koua souen, c'est de lui que proviennent les décrets, les ordonnances et les instructions. En se plaçant il se conforme à la justice et à la droiture, et il épuise le bien dans la voie de l'humilité. Toutefois, l'humilité est une voie de douceur et de soumission ; ce qui la rend avantageuse, c'est sa pureté ; ce n'est pas que le cinquième trait ne le possède suffisamment, mais dans le cas du koua souen, il convient d'en faire l'objet d'un avertissement. Du moment que la pureté est atteinte, le présage est heureux et les regrets se dissipent. Il n'est rien qui n'en retire un avantage ; la pureté, c'est la droi ture et la justice. Pour quiconque se place avec humilité en donnant des instructions, c'est toujours à cause de la justice et de la droiture que le présage est heureux. La douceur et l'humilité sans la pureté doivent donner lieu à des regrets ; comment pourrait-il se faire que rien restât sans en retirer un avantage ? Dans l'action de lancer des décrets ou des ordonnances, il y a lieu de procéder à des changements et à des renouvellements. Ne pas avoir de commencement ; c'est-à-dire que les débuts sont mauvais. Il y a une fin, commencement du bien aussi par les changements et les renouvellements. Si le bien était déjà réalisé, à quoi servirait l'emploi des ordonnances ? pourquoi ferait-on des changements et renouvellerait-on les lois? Trois jours avant le jour hang, trois jours après le jour kang, présage heureux ${ }^{1}$; la voie rationnelle quand on promulgue des lois ou qu'on prescrit des réformes doit être ainsi, le caractère [] indique le début d'une chose 2 ; le caractère kang, désigne le commencement du changement. Dans les dix caractères du cycle décimal, les signes [] et [] sont au milieu : le milieu dépassé il y a transformation, aussi ce signe est appelé kang (changement). Dans le changement et le renouvellement des choses, tout doit dépendre du commencement et tendre vers la fin; , c'est le sens de l'expression : «avant le jour kia, après le jour hia » et, comme cela, le présage est heureux, l'explication de cette expression se trouve dans les commentaires du koua kou $\left(\mathrm{n}^{\circ} 18\right)$.

TSHOU HI. - Le cinquième trait nonaire est énergique, actif, juste et droit, et il fait partie de la substance du koua souen, aussi, il y a des regrets ;

\footnotetext{
${ }^{1}$ Le caractère kang est un des dix caractères d'un cycle qui sert à nommer les jours.

${ }^{2}$ L'un des caractères du même cycle (cycle décimal).
} 
c'est parce qu'il possède la pureté que le présage est heureux. Aussi, il peut oublier ses regrets et rien n'est sans avan tage. Avoir des regrets, c'est ne pas avoir de commencement ; les oublier, c'est avoir une fin. Kang, exprime le changement; la transformation des choses. Trois jours avant le jour kang, c'est le jour ting; trois jours après le jour kang, c'est le jour kouei. Le mot ting exprime ce qui cause le trouble avant la transformation; le mot kouei exprime l'appréciation et la mesure après la transformation. Celui qui a quelque changement à effectuer, et qui obtient ce sens divinatoire en consultant le sort, saura quill comporte un présage heureux.

1009.

Présage heureux du cinquième trait nonaire; situation juste et droite.

TSHENG TSE. - Le présage heureux du cinquième trait nonaire provient de ce quill se place avec justice et droiture ; posséder la voie rationnelle de droiture et de la justice, c'est un présage heureux, aussi ses regrets se dissipent. Justice et droiture signifient qu'il n'y a ni excès, ni défaut et que le juste milieu est exactement atteint. Quand il s'agit de se pla cer avec humilité, et en même temps de promulguer des règlements, c'est uniquement la possession de la juste mesure qui constitue le bien; si l'on manque à la justice, on en éprouve des regrets. 


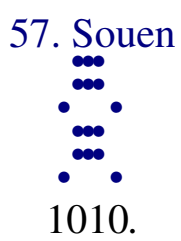

Trait supérieur nonaire : entrer sous le lit ${ }^{1}$; perdre lesbiens et les armes; présage malheureux de la pureté.

TSHENG TSE. — Le lit, c'est le lieu de repos de l'homme ; être dessous le lit, comporte un sens qui indique l'excès dans le repos. Un trait nonaire occupant le rang extrême dans le koua souen, exprime l'excès dans l'humilité. Le neuvième caractère du texte signifie les biens, ce qu'on possède; le dixième, hache, instrument pour couper. L'énergie positive comporte essentiellement la décision (couper); puisque le trait indique un excès d'humilité et qu' il perd l'énergie de sa décision ${ }^{2}$, il perd ce qu'il avait, donc « perte des biens et de la hache ». Il occupe le rang supérieur et son humilité est excessive, à ce point qu'elle cause sa propre perte ; dans la voie rationnelle de la droiture, cela est considéré comme constituant un présage malheureux.

TSHOU HI. — L'humilité est sous le lit ; c'est l'excès dans l'humilité. Il perd ses biens et sa hache ; il perd ce qui lui servait à trancher et à décider. Dans ces conditions, bien qu'avec la pureté, le prés age est encore malheureux. Il occupe l'extrême limite du koua souen, il perd ses vertus d'énergie et de décision, et c'est pourquoi tels sont le sens divina toire et l'image symbolique.

1011.

Entrer sous le lit; l'élévation est épuisée; perdre les biens et la hache; la droiture dans le présage malheureux.

TSHENG TSE. - Entrer sous le lit : excès d'humilité ; il est placé au rang supérieur du koua: l'humilité atteint à l'extrême limite. Il occupe le rang supérieur et son humilité atteint la limite de l'excès possible, à ce point qu'il se perd lui-même: Cela peut-il être considéré comme constituant la possession de la droiture ? C'est évidemment une loi logique d'un présage malheureux. L'humilité est essentiellement bonne à pratiquer ; aussi on peut

${ }^{1}$ Ou bien, comme au n 1002 « humilité au-dessous du lit ».

${ }^{2} \mathrm{Ce}$ qui lui servait à trancher, à décider. 
douter si ce trait peut être considéré comme se conformant à la droiture, aussi la formule prononce de nouveau et affirme le présage malheureux.

TSHOU HI. - Précisément un présage malheureux ${ }^{1}$; cela est une affirmation précise de l'existence du présage mal heureux.

\footnotetext{
${ }^{1}$ La droiture consiste dans l'existence du présage malheureux.
} 


\section{Touei}

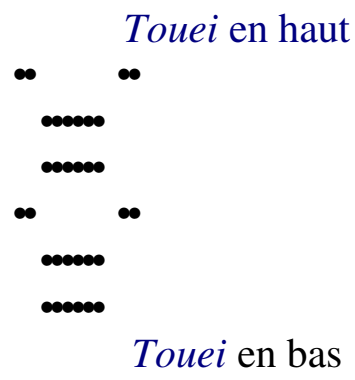

1012. Touei ; liberté ; jouir de la pureté.

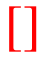

1016. Premier trait nonaire : satisfaction et harmonie ; présage heureux.

$*$

1018. Deuxième trait nonaire : foi et satisfaction; les regrets se dissipent.

$*$

1020. Troisième trait hexaire : il vient se réjouir ; présage malheureux.

$*$

1022. Quatrième trait nonaire : apprécier la satisfaction sans être déjà en repos ; limiter l'inconvénient et avoir du plaisir.

$*$

1024. Cinquième trait nonaire : foi dans l'usure ; il y a péril.

*

1026. Trait supérieur hexaire : amener le plaisir.

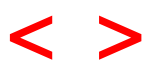


58. Touei.

TSHENG TSE. - Touei. «L'Ordre des koua » dit: «Le koua souen exprime l'idée d'entrer. Entrer et ensuite s'en réjouir, aussi le koua souen est suivi du koua touei; touei, plaire. » Lorsque les êtres s'accordent (entrer) ensemble, ils se plaisent mutuellement; lorsquils se plaisent mutuellement, ils s'accordent ensemble; c'est là ce qui fait que le koua touei suit immédiatement le koua souen. 


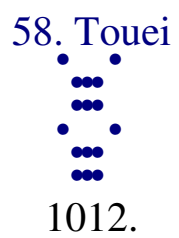

Touei ; liberté ; jouir de la pureté.

TSHENG TSE. - Touei, plaire, satisfaction ; la satisfaction conduit à une voie rationnelle de liberté; si quelque chose peut plaire aux êtres, tous les êtres sans exception en éprouvent du plaisir et s'y attachent, ce qui est suffisant pour produire la liberté. Toutefois, ce qui est considéré comme la voie rationnelle de la satisfaction, est avantageux par la perfection et la droiture. Chercher à plaire autrement que par la voie rationnelle sera de l'adulation vicieuse et il en rés ultera des regrets et des fautes coupables, aussi la formule avertit de l'avantage de la pureté.

TSHOU HI. - Touei, plaire, satisfaction, une négativité avance au-dessus de deux positivités; manifestation extérieure du contentement. L'image symbolique est le marais, à cause du sens de satisfaction produite chez tous les êtres. Ce koua prend encore l'image symbolique d'entraver le cours de l'eau, et de l'empêcher de couler vers le bas. Dans la substance du koua, l'énergie est à l'intérieur et la douceur ma lléable est à l'extérieur. L'énergie est à l'intérieur, aussi elle plaît et cause la liberté ; la douceur est à l'extérieur, de sorte que l'avantage consiste dans la pureté. En effet, la satisfaction comporte la voie rationnelle de liberté, et le plaisir illicite ne peut pas ne point être le sujet d'un avertisse ment, de sorte que tel est le sens divinatoire. De plus, la malléabilité est à l'extérieur, de sorte qu'elle indique la liberté de satisfaction ; l'énergie est à l'intérieur, de sorte qu'elle est av antageuse pour la pureté ; c'est encore le même sens.

1013.

Le commentaire de la formule déterminative dit : Touei, plaire.

TSHOU HI. - Sens du nom du koua.

1014.

Énergie au-dedans et douceur malléable au-dehors; plaire par l'avantage de la pureté; c'est par là qu'il obéit au ciel et qu'il est sympathique aux hommes. Se plaire à précéder le peuple, le peuple oublie ses fatigues; se 
plaire à braver le danger, le peuple oublie la mort; combien la grandeur de la satisfaction stimule le peuple!

TSHENG TSE. — Le sens du mot touei est «plaire »; une négativité est placée au-dessus de deux positivités. La négativité se plaît avec la positivité et c'est elle qui cause la satisfac tion de la positivité. La dureté énergique positive occupe l'intérieur (le milieu); le milieu, ou intérieur, est l'image symbolique de la sincérité réelle du cœur. Le trait mal léable est à l'extérieur, ce qui donne l'image symbolique d'accueillir les êtres avec dou ceur et bienveillance, aussi il exprime la satisfaction et l'ap titude à la pureté. « Avantage de la pureté »; la voie rationnelle de la satisfaction comporte la droiture ; le koua indique les vertus de la dureté énergique : c'est l'aptitude à la pureté. La satisfaction et l'aptitude à la pureté, c'est s'élever avec s oumission vers la raison d'être du ciel et s'abaisser pour répondre aux sentiments du cour de l'homme : c'est l'extrême droiture et l'extrême excellence dans la voie rationnelle de la satisfaction. Mais, résister à la voie rationnelle pour rechercher les louanges de la foule, c'est une voie rationnelle de satisfaction malsai ne ; résister à la voie rationnelle et ne pas se soumettre à la raison d'être du ciel, recher cher les louanges sans répondre aux aspirations des hommes, c'est chercher inconsidérément la satisfaction exclusive d'un moment. Tout cela n'est point la voie rationnelle de la droiture de l'homme doué ; la voie rationnelle de l'homme doué consiste en ceci : la satisfaction qu'il donne au peuple est comme l'effet produit par le ciel et la ter re ; il influence et émeut leur cour, et chacun sans distinction se soumet avec satisfaction. Aussi, si on emploie cette voie pour précéder le peuple, le cour du peuple est satisfait de suivre et oublie la fatigue et la peine du moment; mais si on veut guider le peuple en le mettant en lutte avec la difficulté, le cour du peuple, tout en aimant à se soumettre au devoir, ne peut cependant accepter l'idée de la mort. Le peuple n'est jamais indifférent à l'entraî nement causé par la grandeur de la voie rationnelle de la satisfaction. Ne pas être indifférent, veut dire que cette grandeur inspire la confiance et que chacun développe ses efforts pour suivre docilement limpulsion. Dans la voie rationnelle du prince souverain, ce qui est essentiel, c'est que le caur des hommes les porte à se soumettre volontairement, aussi l'homme saint s'exclame sur cette grandeur.

1015.

Le commentaire traditionnel de la formule symbolique dit : Marais contigus, satisfaction; l'homme doué instruit et exerce au moyen de ses amis.

TSHENG TSE. - Marais contigus : deux marais qui se suivent et communiquent ensemble; deux marais contigus produisant simultanément 
leur effet en réunissant l'influence de leur humidité ; cela comporte l'image symbolique de réciprocité dans l'avantag e de l'effet de l'un à l'autre. Aussi, l'homme doué, considérant cette image symbolique, emploie ses amis pour instruire et exercer; les amis instruisant et exerçant, il y a avantage mutuel. Les anciens philosophes ${ }^{1}$ disaient que, dans ce qui peut réjouir l'uni vers, rien n'égalait l'instruction et l'exercice par les amis, et en réalité, c'est certainement ce qu'il y a de plus grand dans ce qui peut causer de la satisfaction. Cependant, il est indispensable d'éclairer cette idée de mutualité de l'avantag e.

TSHOU HI. - Deux marais qui se suivent et qui s'accroissent mutuellement l'un l'autre. Dans l'enseignement mutuel entre les amis, telle est l’image symbolique.

${ }^{1}$ De la doctrine dite confucéenne. 


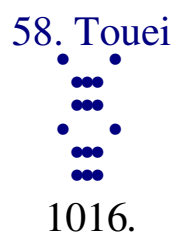

Premier trait nonaire : satisfaction et harmonie ; présage heureux.

TSHENG TSE. - Bien que le premier trait soit positif, il est rangé dans la substance du koua qui exprime la satisfaction et il occupe le rang le plus bas, sans avoir aucun concours sympathique sur quoi s'appuyer ; cela indique qu'il est capable de s'abaisser et de shumi lier, de se soumettre avec douceur pour trouver sa satisfaction et que, de plus, il n'a aucun lien d'intérêt privé et de partialité qui le dirige et le retienne. Lorsque la satisfaction résulte de l'esprit de concorde et qu'elle n'est pas entraînée par aucun sentiment d'égoïsme, c'est ce qui constitue la droiture de cette satisfaction. L'énergie active positive n'est pas humble ; l'acceptation d'un rang inférieur comporte au contraire l'humilité ; être placé dans la satisfaction indique la possibilité de la douceur et de la concorde ; l'absence de correspondance sympathique exprime quill n'y a aucune partialité. Lorsqu'on se place ainsi dans la satisfaction, c'est ce qui entraîne comme conséquence un présage heureux.

TSHOU HI. - Puisqu'il s'agit d'un trait positif faisant partie du koua qui exprime la satisfaction, et puisqu'il est placé au rang le plus bas, que d'ailleurs il n'est reten u par aucune sympathie, tels sont donc l'image symbolique et le sens divinatoire.

1017.

Présage heureux de la satisfaction et de l'harmonie; agir sans éprouver déjà des doutes.

TSHENG TSE. - Il y a appel, recherche et concorde, de sorte que cela conduira à la flatterie et à l'affectation. Le premier trait se place avec soumission et selon le moment ; son cour n'est retenu par aucun lien, il n'a rien à faire ; il emploie simplement la concorde et l'harmonie, et c'est par là que le présage est heureux. Dans le commentaire de la formule symbolique, comme il se place avec satisfaction dans l'infériorité et qu'il est dépourvu de justice et de droiture, il est dit qu'il agit et qu’il n'éprouve pas encore de doute. Son action n'a encore rien qui puisse inspir er le doute et faire naître des soupçons; cela veut dire qu'on n'y voit encore rien qui constitue un 
inconvénient. S’il possédait la justice et la droiture, il n’y aurait pas lieu d'ajouter ces mots. Dans la satisfaction, c'est la justice et la droiture q ui constituent la condition essentielle ; la formule du trait indique exactement ce sens, le commentaire symbolique l'étend et le complète.

TSHOU HI. - Il occupe le premier rang dans le koua ; ce qui plaît, c'est la droiture ; il n'y a encore rien qui puis se inspirer le doute. 


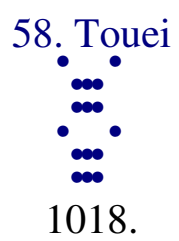

Deuxième trait nonaire : foi et satisfaction; les regrets se dissipent.

TSHENG TSE. - Le second trait supporte la malléabilité négative et se presse contre elle; la malléabilité négative indique l'homme inférieur; puisqu'il se plaît avec lui il en aura des regrets. Le second trait possède les vertus, de l'énergie et de la justice ; il est rempli de bonne foi et de confiance ; bien qu'il se rapproche de l'homme inférieur, il s'observe lui -même et ne commet point de manquements. L’homme doué s'accorde mais il n’imite pas ; il éprouve de la satisfaction mais sans perdre son énergie et sa justice, aussi le présage est heureux et les regrets se dissipent. Sans son énergie et sa justice, le second trait aurait des regrets, et c'est parce qu'il s'observe lui -même que les regrets se dissipent.

TSHOU HI. - L'énergie et la justice sont considérées comme comportant la bonne foi ; occuper un rang négatif constitue une cause de regrets. Si celui qui consulte le sort emploie la bonne foi et veut en être satisfait, le présage sera heureux, et les regrets se dissiperont.

1019.

Présage heureux de la satisfaction causée parla bonne foi ; tendances à la confiance.

TSHENG TSE. - Ce qui subsiste et persiste dans le coar ${ }^{1}$ constitue les tendances. Le second trait est énergique, réel ${ }^{2}$ et il se maintient dans la justice ${ }^{3}$. La confiance et la bonne foi se maintiennent et existent dans la justice; le jugement conserve la sincérité et la confiance: Comment en viendrait-il à se plaire avec l'homme inférieur et à se perdre lui -même ? C'est à cause de cela que le présage est heureux.

\footnotetext{
${ }^{1}$ L'esprit, la volonté.

2 Trait plein, sincérité.

${ }^{3}$ Trait du milieu.
} 


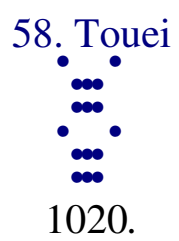

Troisième trait hexaire : il vient se réjouir ; présage malheureux.

TSHENG TSE. — Le troisième trait hexaire est malléable et négatif, c'est un homme sans justice ni droiture ; s'il est satisfait, c'est autrement que par la voie rationnelle. Il vient se réjouir ; c'est -à-dire qu’il survient pour chercher sa propre satisfaction. Il se presse contre les positivités qui sont au-dessous de lui, se courbe sans souci de la voie morale. C'est parce qu'il arrive pour chercher sa propre satisfaction que le présage est malheureux. Arriver en dedans venant de l'extérieur, c'est venir ; au-dessus et au-dessous de lui, les traits sont positifs et lui seul se dirige vers l'intérieur. Il fait partie de la même substance ${ }^{1}$, mais sa nature, qui est la malléabilité, le porte à descendre et à s'abaisser ; il perd la voie rationnelle et agit en descendant.

TSHOU HI. - Malléabilité négative, sans justice ni droiture ; c'est de lui que dépend la satisfaction exprimée par le koua simple touei ${ }^{2}$. Au-dessus de lui il ne rencontre aucune sympathie et il revient vers les deux positivités placées au-dessous pour rechercher sa propre satisfaction: c'est une voie rationnelle de présage malheureux.

1021.

Présage malheureux de venir pour se réjouir ; situation imméritée.

TSHENG TSE. - Se plaçant sans justice ni droiture, sans alliances et cherchant à tort sa satisfaction, c'est ce qui produit le présage malheureux.

\footnotetext{
${ }^{1}$ Du même koua simple.

${ }^{2}$ Il donne naissance à ce koua par transformation du koua simple khien.
} 


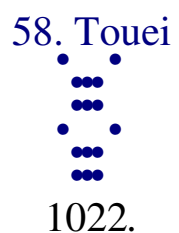

Quatrième trait nonaire : apprécier la satisfaction sans être déjà en repos ; limiter l'inconvénient et avoir du plaisir.

TSHENG TSE. - Le quatrième trait s'élève pour servir la justice et la droiture du cinquième et il descend pour s'associer à la faiblesse vicieu se du troisième. Bien qu'éner gique et positif, il se place sans se conformer à la droiture. Le troisième trait malléable et négatif plaît à la positivité, aussi, le quatrième ne peut se décider et il calcule et apprécie sans être déjà fixé et en repos. Cela exprime qu'il discute et délibère sur ce qu'il doit suivre, sans être déjà décidé, sans pouvoir avoir déjà pris un parti définitif. Ce qui est placé entre deux choses différentes s'appelle une limite ; c'est ce qui divise et sépare (septième caractère du texte). Dans le caractère qui représente les limites entre les pièces de terre, on a ajouté le caractère qui représente un champ ${ }^{1}$, mais le sens est le même. Aussi, lorsqu'il s'agit de préceptes ou de règles que l'homme doit observer on emploie ce mot de «limite » avec le sens de restriction, restreindre, limiter. Sil se restreint et se limite ainsi, en observant la droiture, et s'il coupe court au mal et au vice, il aura du plaisir. Suivre le cinquième, c'est la droiture ; se placer avec le troisième, c'est le vice et le mal. Le quatrième trait est placé près de la situation du prince. Sil se restreint énergiquement et observe la droiture, sil coupe court au vice et au mal, il est sur le point de s'accorder avec le prince pour pratiquer la voie rationnelle de celui-ci, le bonheur se répand sur les êtres, ce qui constitue une cause de plaisir. Dans le cas du quatrième trait, le bien et le mal sont encore incertains ; cela dépendra de ce quill suivra.

TSHOU HI. - Le quatrième trait monte se soumettre à la justice et à la droiture du cinquième et il descend s'associer à la malléabilité vicieuse du troisième, de sorte que rien ne peut être décidé et qu'il est en train d'apprécier ce qui le satisfera; il ne peut pas encore prendre de décision. Toutefois, ses caractères physiques comportent essentiellement la dureté énergique positive, aussi il peut se contenir et observer la droiture et restreindre le mal et le vice ; de cette façon il éprouvera du plaisir. Le sens divinatoire et l'image symbolique étant ainsi, ils constituent un avertissement profond.

\footnotetext{
${ }^{1}$ Champ cultivé, principalement les rizières.
} 
1023.

Joie du quatrième trait; il y a des louanges.

TSHENG TSE. - Ce qu'on entend par joie, plaisir, c'est par exemple se maintenir dans la droiture et causer la satisfaction du prince, de sorte quil devient possible de pratiquer la voie rationnelle de la dureté énergique et que le bonheur atteint tous les êtres. 


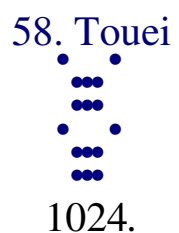

Cinquième trait nonaire : foi dans l'usure ; il y a péril.

TSHENG TSE. - Le cinquième trait nonaire, possède la situation prééminente et il se place avec justice et droiture; il accomplit le bien dans la voie rationnelle de la satisfaction ${ }^{1}$, cependant, l'homme saint pose encore l'avertissement relatif à l'existence du péril. C'est qu'en effet, la perfection accomplie de Shouen et de Yeou eux-mêmes n'existe jamais sans comporter quelque avertissement. Un avertissement ne se rapporte qu'à l'objet qui le rend nécessaire ; bien que des hommes saints ou des sages occupent les rangs supérieurs, cela n'empêche pas que l'univers renferme toujours quelques hommes inférieurs, mais ceux-ci n'osent pas donner libre cours à leurs sentiments pervers. L'homme saint de son côté se réjouit de les voir capables de concentrer leurs efforts pour modifier leur apparence, tandis que ceux-là, hommes inférieurs, savent toujours reconnaître la satisfaction qu'ils causent à l'homme saint. Par exemple, c'est le cas des « quatre méchants » placés à la cour de Yeou, qui, d'une nature vicieuse et négative, se conformaient cependant avec soumission à ses ordres ${ }^{2}$; l'homme saint ${ }^{3}$ n'ignorait pas qu'ils étaient incorrigibles, mais il exploitait leur crainte des châtiments pour les forcer à se conduire vertueusement. Si le cinquième trait croit sincèrement que la fausse apparence de vertu de l'homme inférieur tient à une vertu réelle, et s'il ne sait reconnaître ce que cache réellement cette apparence simulée, ce sera une voie rationnelle de péril. Quant à l'homme inférieur n'étant pas suffisamment tenu en défiance, il ira contre le bien: liidée qui conduit l'homme saint ${ }^{4}$ à cet avertissement est profonde. Le cinquième caractère du texte, user par le frottement, exprime la diminution graduelle de la positivité ; c'est la négativité usant peu à peu la positivité, et en effet ce passage désigne le sixième trait hexaire, de sorte que la confiance dans l'usure sera périlleuse. Puisque le cinquième trait se trouve dans un moment qui comporte la satisfaction, et qu'il se rapproche secrètement du trait supéri eur hexaire, il y a donc lieu à un avertissement : bien qu'avec la sainteté de Shouen et même en se défiant de la flatterie et de la dissimulation, il est impossible de ne pas être sur ses gardes. La satisfaction émeut et influence le cour de l'homme, elle l'enva hit facilement et il y a lieu de la craindre; tel est le sens.

\footnotetext{
${ }^{1}$ Il atteint à la perfection dans cette voie.

${ }^{2}$ Citation historique tirée du Shou king

${ }^{3}$ Yeou.

${ }^{4}$ L'auteur du texte.
} 
TSHOU HI. - Usure se rapporte à la capacité que possède la négativité d'user et de diminuer la positivité. Le cinquième trait nonaire est énergique et positif, juste et droit ; cependant, en présence d'un moment de satisfaction et occupant la situation prééminente, il est très voisin du trait supérieur hexaire ; celui-ci est malléable et négatif ; c'est de lui que dépend l'existence du koua touei ${ }^{1}$. Placé au comble de la satisfaction, il pourrait se plaire autrement qu'au bien et user la positivité, c'est pourquoi la formule borne l'avertis sement à prévenir que la confiance dans le trait supérieur hexaire serait périlleuse.

1025.

Confiance dans l'usure ; situation directement méritée.

TSHENG TSE. - L'avertissement au sujet de la confiance dans l'usure résulte de ce que la situation où se place le cinquième trait comporte exactement cet avertissement. Se rapprochant intimement de la malléabilité négative, il y a une voie rationnelle de satisfaction mutuelle entre eux deux, aussi l'avertissement est relatif à la confiance qu'il peut avoir en lui.

TSHOU HI. - Identiquement comme dans le cas du cinquième trait nonaire du koua $l i^{2}$.

${ }^{1}$ Il est le maître de la satisfaction.

${ }^{2}$ Koua n $^{\circ} 10$. 


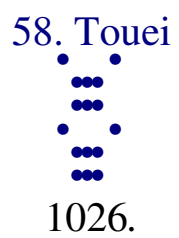

Trait supérieur hexaire : amener le plaisir.

TSHENG TSE. - Dans les autres koua, lorsque le dernier degré est atteint, il y a modification et transformation; dans le koua touei, qui exprime la satisfaction, le degré extrême atteint, il y a encore une satisfaction plus grande. Il occupe le rang extrême dans ce koua, et il représente celui qui est incapable de s'arrêter et de se modérer. Aussi, alors que la satisfaction est déjà arrivée à son extrême limite, il l'étend encore et l'augmente et cepen dant il n'en résulte ni regrets, ni culpabilité ; comment cela peut-il être ? Réponse : en disant qu'il ne sait pas s'arrêter, on ne voit pas encore si ce qui fait l'objet de sa satisfaction est le bien ou le mal. De plus, au-dessous de lui, il foule aux pieds la justice et la droiture du cinquième trait nonaire, il ne peut donc produire chez qui que ce soit une satisfaction malsaine ${ }^{1}$. Le troisième trait hexaire, lui, est entre deux traits qui dénotent l'absence de droiture, et c'est à cause de cela qu'il comporte un présage malheureux.

TSHOU HI. - Le trait supérieur hexaire est celui de qui dépend l'achèvement du koua qui exprime la satisfaction ; puisqu'il est négatif et qu'il occupe le rang extrême dans ce koua, il entraîne les deux traits positifs placés au-dessous de lui à s'allier avec lui pour leur satisfaction, mais il ne peut pas affirmer que ceux-ci le suivront. Aussi, au sujet du cinquième trait nonaire il y avait lieu de formuler un avertissement, tandis que, dans le cas de ce trait supérieur, la formule ne parle ni de présage heureux, ni de présage malheureux.

1027.

Le trait supérieur hexaire entraîne la satisfaction ; pas encore d'éclat.

TSHENG TSE. - La satisfaction est déjà arrivée à son comble; il continue encore à la produire et l'augmente. Bien que sa passion pour la recherche de la satisfaction soit insatiable, cependant la raison d'être de cette satisfaction a déjà passé ; en réalité, il n'y a plus de motif de satisfaction. Quand une chose est achevée et complète elle doit être brillante et éclatante.

\footnotetext{
${ }^{1}$ Le cinquième trait étant incapable de se laisser induire au mal.
} 
Puisque la satisfaction est déjà arrivée à son extrême limite et qu'il cherche encore à l'augmenter, son manque de jugement et de goût est considérable : en quoi serait-il brillant? Pas encore, est une expression qui comporte l'indécision et l'incertitu de; dans les commentaires symboliques, elle est fréquemment employée ; ne pas devoir nécessairement briller et être éclatant, veut dire ne pas pouvoir avoir d'éclat. 


\section{Hoan}

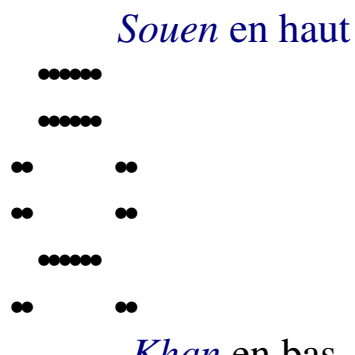

1028. Hoan; liberté, le roi parvient à avoir un temple pour les mânes de ses ancêtres ; avantage à traverser un grand cours d'eau ; avantage de la pureté.

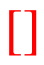

1033. Premier trait hexaire : servir à aider la vigueur du cheval, présage heureux.

$*$

1035. Deuxième trait nonaire : le flot court rapide et recouvre le point le plus élevé ; dissipation des regrets.

*

1037. Troisième trait hexaire : submerger la personne ; pas de regrets.

1039. Quatrième trait hexaire : disperser la troupe ; grandeur du présage heureux; ce qui était dispersé est rassemblé ; ce n'est pas ce que la médiocrité pense.

$*$

1041. Cinquième trait nonaire : disperser les grandes ordonnances comme le cors disperse la sueur; disperser les biens du roi, pas de culpabilité.

$*$

1043. Trait supérieur nonaire : disperser (répandre) le sang et le répandre rapidement; pas de culpabilité.

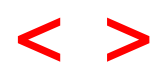




\section{Hoan.}

TSHENG TSE. — Hoan. «L'Ordre des koua » dit : «Touei, satisfaction ; la satisfaction se dissipe, aussi le koua touei est suivi du koua hoan; hoan, séparation. » La satisfaction se dissipe et passe; l'humeur de l'homme s'ép aissit lorsqu'il est triste et elle se dilate au contrai re lorsqu'il est satisfait ; aussi le mot qui exprime la satisfaction comporte le sens de dilatation et c'est encore ce qui fait que le koua hoan est placé à la suite du koua touei. Il est constitué par le koua simple souen en haut et le koua simple khan au-dessous. Le vent agit à la surface de l'eau ; lorsque le vent survient, l'eau se répand et se disperse ; c'est pour cela que ce koua est appelé hoan. 


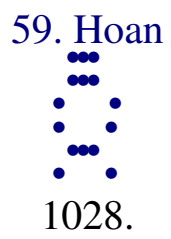

Hoan ; liberté, le roi parvient à avoir un temple pour les mânes de ses ancêtres; avantage à traverser un grand cours d'eau; avantage de la pureté.

TSHENG TSE. - Hoan, séparation et dispersion; l'effusion, chez l'homme, provient de l'intérieur ; lorsque le cour de l'homme se sépare (de l'objet de son affection), ses senti ments le portent vers des objets divers et le remède à cette dispersion est essentiellement basé sur la justice. Si on peut réussir et rassembler le cour des hommes, ces affections dispersées au hasard pourront se réunir en faisceau. Aussi le sens, dans le koua, est toujours basé sur la question de justice. «Avantage de la pureté » exprime que la voie de réunion de ce qui est dispersé consiste dans la fermeté de la droiture.

TSHOU HI. - Hoan, dispersion ${ }^{1}$; ce koua est formé du koua simple khan en bas, et souen en haut; le vent courant et agissant à la surface des eaux ; c'est l'image symbolique de la séparation et de la dispersion, et c'est à cause de cela qu'il est appelé hoan. Au point de vue de la transformation, il provient essentiellement du koua tsien ${ }^{2}$ dans lequel le trait nonaire vient occuper le second rang et se conforme à la justice, tandis que le trait hexaire va occuper le troisième rang, prend la situation du trait nonaire et monte avec le quatrième, de sorte que le sens divinatoire est quill y a possibilité de liberté. De plus, les esprits de ses ancêtres étant dispersés, le roi doit venir jusqu’à l'autel consacré au culte de leurs mânes pour les réunir. Enfin, le koua simple souen correspond à l'arbre, au bois, et le koua simple khan à l'eau ; cela donne limage symbolique du gouvernail d'une barque, de sorte qu'il y a avantage à traverser un grand cours d'eau. La formule ajoute « avantage de la pureté »; c'est un avertissement profond adressé à celui qui consulte le sort.

1029.

Le commentaire de la formule déterminative dit: Hoan, liberté ; la dureté énergique vient et ne finit point; la douceur malléable possède la situation à l'extérieur et monte de même.

\footnotetext{
${ }^{1}$ Le sens le plus archaïque est « changement $»$.

${ }^{2}$ Koua ${ }^{\circ} 53$.
} 
TSHENG TSE. — La possibilité de la liberté du koua hoan provient de ce que telles sont les aptitudes exprimées par ce koua. L'achèvement de la dispersion indique que le koua hoan provient de ce que le trait nonaire vient occuper le second rang, tandis que le trait hexaire monte occuper le quatrième. L'arrivée de la dureté énergique positive n’ira pas jusqu'à atteindre le dernier rang vers le bas, et en se plaçant, elle se maintiendra dans la justice ${ }^{1}$. Le mouvement de départ de la douceur malléable lui fera occuper la situation qui lui revient suivant la droiture, à l'extérieur, et monter comme la justice du cinquième trait. Se conformer avec satisfaction et douceur, c'est « monter de même ». Le quatrième et le cinquième rang indiquent les situations du sujet et du prince ; ils sont en présence d'un moment de dispersion et ils se serrent l'un contre l'autre et se rapprochent, leur sentiment du devoir fait qu'ils se comprennent. «De même que le cinquième » veut dire suivre la justice. Étant en présence d'un moment de dispersion et observant la justice, ils n'en viendront pas à la séparation et à la dispersion, de sorte que la liberté de communication est possible.

1030.

Le roi parvient à avoir un temple pour les mânes de ses ancêtres ; le roi, alors, est dans la justice.

TSHENG TSE. - Le sens de la phrase : «Le roi parvient à avoir un temple pour les mânes de ses ancêtres » est clairement expliqué dans le koua khouen ${ }^{2}$. Dans le temps où l'univers est désuni et séparé, le roi rass emble et réunit les coaurs, et il parvient à avoir un temple pour les mânes de ses ancêtres ${ }^{3}$; cela est ainsi parce qu'il se maintient dans la jus tice. «Dans la justice » veut dire qu'il s'efforce de posséder la justice ; c'est une expression qui indique qu'il domine ses passions et ses sentiments. La justice, ou milieu, est l'image symbolique du cour. La dureté énergique vient, mais pas jusqu'au bout, la douceur malléable possède la situation et monte de même ; le sens indiqué par les aptitudes du koua dépend de la possession de la justice. La voie rationnelle du roi pour porter remède à la dispersion et à la désunion, consiste uniquement dans la possession de la justice. Mang Tse dit : il y a une voie rationnelle pour posséder la puissance sur le peuple; si on possède son coaur, on le domine. L'adoration de l'être suprême, l'édification de temples en l'honneur des mânes des ancêtres sont des actions qui entraînent toujours le coaur du peuple et le portent à se soumettre ; la voie rationnelle pour ramener

\footnotetext{
${ }^{1}$ Trait intermédiaire.

2 Koua $n^{\circ} 47$.

${ }^{3}$ Après une révolution, un prétendant s'empare du pouvoir et s'attribue les honneurs souverains.
} 
le cour des hommes n'est jamais plus grande qu'en ceci, aussi le texte dit qu'il va jusqu'à posséder un temple pour les mânes de ses aïeux. C'est là le dernier ressort pour rassembler ce qui a été dispersé.

TSHOU HI. - Justice, au milieu, intérieur; cela veut dire : dans l’intérieur du temple des ancêtres ${ }^{1}$.

1031.

Avantage à traverser un grand cours d'eau; se servir du bois et en retirer du mérite.

TSHENG TSE. - La voie rationnelle pour remédier à la dispersion est qu'il convient de traverser les périls et les difficultés, et le koua présente l'image symbolique de l'action de se placer sur du bois pour traverser un cours d'eau. En haut, le koua simple souen représente le bois ; en bas le koua simple khan représente l'eau, c'est un cours d'eau. L'avant age consiste à traverser le péril pour remédier aux inconvénients de la désunion. Le bois est sur l'eau, ce qui constitue l'image symbolique de se placer sur le bois, ou du bois surnageant ; le bois surnageant est ce qui sert à traverser un cours d'eau. En traversant on aura le mérite de l'œuvre qui consiste à réunir ce qui est séparé et désuni. Le koua présente ce sens et cette image symbolique.

1032.

Le commentaire de la formule déterminative dit : Le vent court sur l'eau; dispersion; les premiers rois employaient les offrandes à l'être suprême et l'établissement des temples aux mânes de leurs ancêtres.

TSHENG TSE. - Le vent court sur l'eau, ce qui comporte l'image symbolique de dispersion et de désunion, agitation troublée. Les premiers rois, considérant cette image symbolique, remédiaient à la désunion et à la dispersion de l'univers, et ils allaient jusqu'à offrir des sacrifices à l'être suprême et à établir des temples aux mânes de leurs ancêtres. Pour réunir le coaur des hommes, rien n'est co mparable à la pratique religieuse des sacrifices aux mânes des ancêtres et aux actions de grâces envers les génies. Ces pratiques sont la manifestation d'un sentiment du cour, aussi les deux actions

\footnotetext{
${ }^{1}$ Le commentateur lit : «Le roi, alors, est dans l'intérieur. »
} 
mentionnées sont le meilleur moyen de ramener le coar de s hommes. Attirer le cour des hommes, c'est la voie rationnelle pour réunir ce qui est séparé et dispersé, et, dans cette voie, rien n'est plus important que ceci.

TSHOU HI. - C'est toujours le moyen de réunir et de rassembler ce qui est dispersé et séparé. 


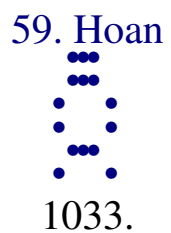

Premier trait hexaire : servir à aider la vigueur du cheval ${ }^{1}$, présage heureux.

TSHENG TSE. - Ce trait hexaire occupe le premier rang dans le koua; il indique le commencement de la dispersion. La dispersion commence et il y porte remède. De plus, il possède l'énergie du cheval vigoureux, ce qui constitue le présage heureux. Entre les six traits, il n'y a que dans le cas du premier où la formule ne parle pas de dispersion. La force naturelle est inhérente à la dispersion et à la séparation, exige que l'on s'y prenne de bonne heure pour y porter remède ; si, dès le moment de son début, on y remédie, les choses n'en viendront pas jusqu'à la séparation et à la dispersion ; ceci constitue donc un enseignement profond. Le cheval est l'auxiliaire que l'homme emploie ; ici, il se sert d'un cheval vigoureux, et c'est pour cela qu'il peut remédier à la dispersion. Le « cheval » désigne le second trait. Le second trait possède les aptitudes de la dureté énergique et de la justice ; le premier est malléable, négatif et soumis; tous deux sont également sans correspondance sympathique. Étant sans correspondance sympathique ils se serrent l'un contre l'autre et s'appellent mutuellement. La mal léabilité passive du premier, s'appuyant sur les aptitudes de l'énergie et la justice pour remédier à la dispersion, c'est comme la possession d'un cheval vigoureux lorsqu'il s'agit d'aller au loin : l'action est nécessairement efficace, donc, le présage est heureux. Lorsque l'on porte remède dès le début à la dispersion, avec de la force, la tâche est facile : c'est là se conformer au temps.

TSHOU HI. - Il occupe le premier rang dans le koua, au début de la dispersion et de la séparation, si on porte remède à la dispersion dès son début, c'est une tâche qui est facile à la force ; si de plus on possède un cheval vigoureux, le présage heureux devient évident. Le premier trait hexaire ne possède pas les aptitudes nécessaires pour traverser sans danger cette période de séparation et de dispersion; il n'est capable que d'obéir au second trait nonaire, c'est pour cela que tels sont l'image symbolique et le sens divinatoire.

1034.

\footnotetext{
${ }^{1}$ Les commentateurs chinois lisent « employer l'aide d'un cheval vigoureux ».
} 
Présage heureux du premier trait hexaire ; soumission.

TSHENG TSE. - Ce qui fait que le premier trait présente un présage heureux, c'est qu'il peut suivre avec soumission les aptitudes de l'énergie et la justice ${ }^{1}$. La dispersion commence et il agit pour y remédier; il est donc capable de se soumettre aux nécessités du moment.

${ }^{1}$ Du second trait. 


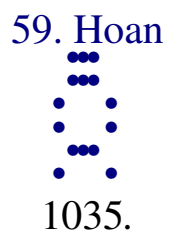

Deuxième trait nonaire : le flot court rapide et recouvre le point le plus
élevé $^{1}$; dissipation des regrets.

TSHENG TSE. - Dans chaque formule des traits le troisième caractère du texte est toujours employé ; hoan, dispersion ; cela exprime le moment de la dispersion, ou séparation. Dans un moment de séparation et de dispersion, et placé au milieu du péril ${ }^{2}$, il est visible qu’il doit y avoir des regrets ; s’il peut courir et se porter rapidement vers un lieu de repos, ses regrets pourront se dissiper. Le sixième caractère du texte exprime l'idée d'un appui sur lequel on s'affermit en se baissant. Courir rapidement, aller en se pressant. Bien que le premier trait et le second ne soient pas en correspondance sympathique selon la droiture, cependant, en présence d'un moment de séparation et de dispersion, tous deux étant également sans alliance, s'appellent mutuellement l'un l'autre à cause de leurs caractères de négativité et de positivité ; donc, ce sont ceux qui comptent et s'appuient l'un sur l'autre. C'est à cause de cela que, au sujet du second, le premier est considéré comme un point fixe ${ }^{3}$, tandis que dans le cas du premier, le second est considéré comme représentant un cheval. Le second trait se hâte de se porter vers le premier pour trouver le repos, et alors ses regrets peuvent se dissiper. Le premier, bien que faisant partie de la substance du koua simple khan, ne se trouve cependant pas au milieu du danger. Mais on a douté si la minime malléabilité du premier trait pouvait servir de point d'appui à quoi que ce soit En effet, dans un moment de dispersion et de séparation, c'est la réunion des efforts qui permet le succès. Tous les anciens philosophes ${ }^{4}$ ont considéré le cinquième trait comme représentant le «point d'appui »; cela n'est pas exact. Au moment où la séparation et la dispersion commencent, comment les deux positivités pourraient-elles suivre des voies identiques ? Si elles le pouvaient, l'œuvre de remédier à la séparation serait arrivée au maximum de sa grandeur ; comment $\mathrm{y}$ aurait-il simplement dissipation des regrets ? Ce sixième caractère du texte traduit par «point d'appui » signifie «s'appuyer sur quelque chose en se baissant ».

\footnotetext{
${ }^{1}$ Le troisième caractère du texte désigne aussi et essentiellement l'eau, le flot qui monte et submerge. Dans le cas actuel, on lit généralement : « la dispersion atteint rapidement le point d'appui ».

${ }^{2}$ Au milieu du koua simple khan.

${ }^{3}$ Appui, pieu planté en terre ; barre de bois.

${ }^{4}$ Commentateurs du Yi king.
} 
TSHOU HI. - Nonaire et occupant le second rang, il doit avoir des regrets ; mais en présence d'un moment de séparation, il vient sans aller jusqu'au bout : c'est donc celui dont les regrets peuvent se dissiper, aussi tels sont l'image symbolique et le sens divinatoi re. En effet, la qualité de trait nonaire masque la rapidité de mouvement, tandis que le second rang est le point le plus élevé ${ }^{1}$.

1036.

La dispersion court rapidement vers le point d'appui ${ }^{2}$; satisfaction des désirs.

TSHENG TSE. — Dans le moment de la dispersion indiquée par le koua hoan, c'est l'unio n qui assure le repos. Le second trait est placé au milieu du koua simple qui exprime le péril ; il se hâte de se porter vers le premier pour chercher le repos. Il s'appuie sur lui comme sur un banc et ses regrets se dissipent ; c'est là la satisfaction de ses désirs.

\footnotetext{
${ }^{1}$ Ainsi que je le montrerai plus tard, les trois rangs des traits d'un koua simple désignent le début ou « lever », le maximum d'élévation et le déclin ou « coucher».

${ }^{2} \mathrm{Ce}$ sont les mêmes caractères traduits au $\mathrm{n}^{\circ} 1035$ par les mots : «le flot court rapide et recouvre le point le plus élevé ».
} 


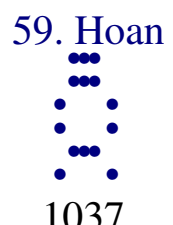

Troisième trait hexaire : submerger la personne ${ }^{1}$; pas de regrets.

TSHENG TSE. - Le troisième trait se trouve dans un moment de dispersion et, seul, il possède une correspondance sympathique et des alliances; il n'éprouve donc pas les regrets causés par la séparation et la dispersion. Cependant, à cause de ses caractères physiques de malléabilité négative et des aptitudes qui résultent de l'absence de justice et de droiture, enfin de l'élévation du lieu où il se trouve et qui ne comporte cependant aucune situation définie, comment pourrait-il être capable de remédier à la dispersion (désunion) des hommes et de leur faire ressentir son influence ? C'est seulement pour sa propre personnalité qu'il est possible qu'i 1 n'y ait point de regrets. En tête de la formule on a ajouté le caractère hoan; cela exprime que dans un moment de dispersion, lui-même n'éprouve point le regret de la séparation.

TSHOU HI. - Malléable, négatif et dépourvu de justice et de droiture, il comporte l'image symbolique d'égoïsme et de partialité pour ses propres intérêts. Toutefois, il occupe une situation positive ${ }^{2}$; ses tendances le portent à remédier aux inconvénients du moment, il est capable de secouer ${ }^{3}$ son égoïsme afin de n'éprouver aucun regret, de sorte que tel est le sens divinatoire. En général, dans le cas de chacun de ces quatre traits supérieurs l'idée de dispersion exprimée par le mot hoan est appliquée à l'action de remédier à la dispersion ${ }^{4}$.

1038.

Dispersion du corps ${ }^{5}$, tendances vers l'extérieur.

TSHENG TSE. - Ses tendances sont sympathiques au trait supérieur : c'est-à-dire, à l'extérieur. Avec le trait supérieur, ils s'allient

\footnotetext{
${ }^{1} \mathrm{Si}$ on lit comme les commentateurs chinois, le sens sera : « la dispersion de la personne ».

${ }^{2}$ Un rang impair.

${ }^{3}$ Disperser.

${ }^{4}$ Dispersion de la dispersion.

${ }^{5}$ Même remarque qu'au sujet du no 1036.
} 
sympathiquement, aussi sa personne peut éviter la dispersion ${ }^{1}$ et il n'éprouve point de regrets. La dissipation des regrets indique essentiellement qu'il y a eu des regrets et qu'ils s'effacent ; l'absence de regrets indique essentiellement que ces regrets n'ont pas existé.

${ }^{1}$ Ou la submersion. 


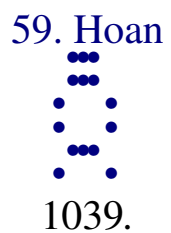

Quatrième trait hexaire : disperser la troupe ${ }^{1}$; grandeur du présage heureux ; ce qui était dispersé est rassemblé ; ce n'est pas ce que la médiocrité pense.

TSHENG TSE. - Dans le koua hoan, le sens de chacune des formules des quatrième et cinquième traits est réciproque et l'un entraîne l'autre, aussi il en est parlé de la même manière et c'est pour cela que le commentaire de la formule déterminative dit: "monte de même » ${ }^{2}$. Le quatrième trait est humble, soumis et plein de droiture ; il occupe la situation du sujet de rang élevé. Le cinquième possède l'énergie, la justice et la droiture, il occupe la situation du prince. Le prince et le sujet réunissent leur force, la dureté énergique et la douceur malléable s'entraident et se tempèrent mutuellem ent afin de remédier à la dispersion dont souffre l'univers. Au moment où commencent la séparation et la dispersion, en employant la dureté énergique, il serait impossible de diriger les hommes de façon à les rallier et à les réunir : en employant la douceur malléable, ce serait insuffisant pour les ramener et les soumettre. Le quatrième, en employant la voie de la droiture dans l'humilité et la soumission, aide le prince doué d'énergie, de justice et de droiture ; le prince et le sujet ont un même mérite, et c'est ainsi qu'ils peuvent remédier à la dispersion. Lorsque l'univers se trouve dans un moment de dispersion ${ }^{3}$, et que l'on est capable de le grouper en réunissant les hommes en un seul troupeau, c'est ce que l'on peut appe ler le présage heureux d'u n grand bien. Ce qui était dispersé est rassemblé, ce n'est pas ce que la médiocrité pense : c'est là une expression admirative. Le dixième caractère du texte signifie grandeur du rassemblement. Au moment où la dispersion commence, il est capable d'arriver à la grandeur du rassemblement, donc le mérite de l'œuvre est extrêmement grand et l'œuvre elle -même est très difficile, tandis que l'effet est merveilleux. Le douzième caractère du texte signifie plain, uni, égal, ordinaire ; ce n'est pas ce que quel qu'un d'une intelligence médiocre est capable de penser ou de méditer; sans l'intelli gence de la sagesse, qui donc serait capable d'atteindre ce but ?

TSHOU HI. - Il occupe un rang négatif et possède la droiture ; il monte pour se mettre au service du cinquième trait nonaire : c'est celui qui est actuellement investi de l'autorité nécessaire pour remédier à la dispersion du moment. Au-dessous de lui, il ne rencontre ni sympathie, ni alliance, ce qui constitue l’image symbolique d'être capable de se séparer de ses propres

\footnotetext{
1 Ou submerger le troupeau.

2 Voir $\$ 1029$.

${ }^{3}$ Morcellement de l'empire.
} 
attaches particulières. Si celui qui consulte le sort est dans de telles conditions, ce sera un grand bien et un présage heureux. Cela exprime encore la capacité de disperser une petite réunion pour constituer une grande société, de telle sorte que ceux qui sont dispersés se rassemblent et forment une multitude ; ce n'est donc pas un résultat vers lequel les pensées et les préoccupations d'un homme ordinaire puissent tendre.

1040.

Dispersion de la troupe ${ }^{1}$, grandeur du présage heureux; grandeur et éclat.

TSHENG TSE. - Lorsque l'expression « grandeur du présage heureux » est employée, elle indique que le mérite et la vertu sont pleins d'éclat et de grandeur. Ces mots : «grandeur du présage heureux, grandeur et éclat », ne sont pas employés à propos du cinquième trait et sont appliqués au quatrième. Le sens des formules de ces deux traits est analogue ; dans le cas du quatrième trait il s'agit de l'effet produit ; dans le cas du cinquième trait il s'agit de l'œuvre accomplie, ce qui répo nd à la distinction entre le prince et le sujet.

${ }^{1} \mathrm{Ou} \ll$ submerger la troupe $»$. 


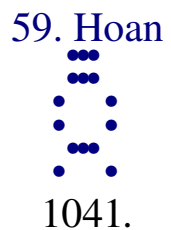

Cinquième trait nonaire : disperser les grandes ordonnances comme le corps disperse la sueur ; disperser les biens du roi, pas de culpabilité ${ }^{1 .}$

TSHENG TSE. - Le cinquième et le quatrième traits sont le prince et le sujet qui unissent leurs vertus ; ils emploient la voie rationnelle de la dureté énergique, de la justice, de la droiture et de l'humilité dans la soumission pour remédier aux maux de la dispersion. Pour se conformer à cette voie, il faut uniquement influencer le cour des hommes comme en l'imprégnant, et alors ceux-là se soumettent et obéissent. Il convient pour cela de publier des instructions et des ordonnances pour adoucir le cour des hommes et qui agissent comme les humeurs du corps qui se répandent dans les quatre membres ; alors les hommes ont confiance, se soumettent et obéissent. Dans ces conditions, il devient possible de remédier à la désunion et à la dispersion dans l'univers, et celui qui pratique cette voie est digne de la situation du prince et il est sans culpabilité. «Grandes ordonnances », ordonnances sur les grandes institutions civiles dans l'État, c'est-à-dire les grands édits de rénovation du peuple ${ }^{2}$ et les grandes institutions politiques pour remédier à la dispersion ${ }^{3}$. La formule reparle une seconde fois de la dispersion ; d'abord, il est question d'un moment de dispersion, ensuite il est dit qu'en se plaçant ainsi pendant la dispersion on sera sans culpabilité. Au sujet du quatrième trait, il a déjà été question de la grandeur du présage heureux; au sujet du cinquième, il est seulement question d'être digne de la situation indiquée. Le cinquième et le quatrième traits du koua hoan expriment la même idée ; dans le changement, c'est la séparation et la dispersion qui constituent le mal ; on y porte remède pour arriver à la réunion : serait-il possible au prince et au sujet d'y porter aucun remède s'ils n'étaient animés d'une même volonté et s'ils ne réunissaient pas leurs efforts? Le sens de chacun des deux traits entraîne celui du second et est une conséquence naturelle du moment exprimé.

TSHENG TSE. - Positif, énergique, juste et droit, il occupe la situation prééminente. Dans le moment de la dispersion, pouvoir répandre les ordonnances et les mandements ainsi que les biens déjà accumulés, c'est ce qui donne la possibilité de remédier à cette dispersion et d'éviter toute culpabilité. C'est pour cela que tels sont l'image symbolique et le sens divinatoire. Le cinquième trait nonaire fait partie de la substance du koua

\footnotetext{
${ }^{1}$ Sens tout conventionnel impossible à lire dans le texte, sans les commentaires.

${ }^{2}$ Les lois fondamentales que promulgue le premier prince de toute nouvelle dynastie.

${ }^{3}$ Dans tout ce qui précède ce mot « dispersion »est pris allégoriquement pour exprimer les dissensions civiles dans les temps de révolutions.
} 
simple qui symbolise l'humilité et il indique limage symbolique d'ordonnances et d'édits. Le qua trième caractère du texte, sueur, a le même sens que dans l'expression « la sueur sort et ne rentre pas ». " Disperser les biens du prince », c'est ce que Lou Tshi exprime en disant « disperser un peu pour réunir beaucoup ».

1042.

Le roi reste sans culpabilité ; c'est la situation correcte ${ }^{1}$.

TSHENG TSE. - Les neuvième et dixième caractères du texte veulent dire situation correcte conforme à la droiture; la situation prééminente du prince. Celui qui est capable d'agir comme le cinquième trait occupera la situation prééminente, il en sera digne, et sera sans culpabilité.

${ }^{1}$ Ce passage prouve au moins que Khong Tse ne lisait pas le texte comme les commentateurs postérieurs. Il coupe en effet la phrase après le troisième caractère du texte qui, dès lors, fait partie du premier membre de la phrase. 


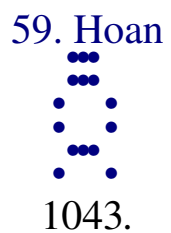

Trait supérieur nonaire : disperser (répandre) le sang et le répandre rapidement; pas de culpabilité.

TSHENG TSE. - Tous les traits du koua hoan sont également sans attache ni correspondance sympathique et comportent aussi l'image symbolique de la dispersion et de la séparation. Le trait supérieur seul correspond sympathiquement au troisième, et le troisième est placé à l'extrême degré de péril et de l'empêchement ${ }^{1}$; si le trait supérieur ne s'abais sait pour suivre le troisième, il serait incapable de sortir de cette situation caractérisée par le koua hoan ${ }^{2}$. Le péril comporte l'image symbolique de tout ce qui blesse ou nuit, de tout ce qui inspire la crainte, aussi la formule parle de la crainte causée par l'écoulement du sang. Toutefois, ce trait est nonaire, il emploie l'énergie de la positivité et se place à l'exté rieur du koua qui indique le changement, de sorte quill a l'image symbolique de sortir de l'état indiqué par ce koua. De plus, il occupe le rang extrême du koua simple souen, ce qui est considéré comme exprimant la capacité de pouvoir se conformer avec soumission à la raison d'être des choses, aussi la formule dit que, s'il peut faire que le sang coule, les inquiétudes disparaîtront et il n'y aura aucune culpabilité. Le quatrième caractère du texte indique l'objet dont il s'agit et qui existe. Dans le moment marqué par le koua hoan, c'est la capacité de réunir qui constitue le mérite de l'action. Le trait nonaire qui occupe le rang extrême seul capable d'avoir une relation et il surveille de haut le péril, de sorte que, pour lui, c'est de pouvoir sortir de cet état de changement et éloigner le mal qui constitue le bien.

TSHOU HI. - Le trait supérieur nonaire emploie les qualités de la positivité pour se maintenir au rang extrême du koua hoan; il est capable de sortir de cet état de changement, et c'est pour cela que tels sont l'image symbolique et le sens divinatoire. Le «sang » indique ce qui blesse et nuit; le septième caractère du texte doit être remplacé par le caractère [ $]^{3}$; comme dans le cas du quatrième trait hexaire du koua siao tshou, cela veut dire que, par la dispersion, le sang coule, et que par le changement les inquiétudes se dissiperont ${ }^{4}$.

\footnotetext{
${ }^{1}$ Représenté par le koua simple khan.

2 Changement brusque ; dispersion, séparation.

${ }^{3}$ Le premier signifie « s'éloigner rapidement », le second signifie « préoccupation ».

4 Voir $\$ 200$ : «Le sang coule, la préoccupation déborde. »
} 
1044.

Répandre le sang ; éloigner le mal.

TSHENG TSE. - Selon le texte du commentaire symbolique, il s'agit de répandre le sang; c'est une expression analogue à celle de la formule « obstacle à l'influence » ${ }^{1}$, mais le sens n'est pas le même. En effet, après le cinquième caractère du texte, il manque le caractère [] du texte ${ }^{2}$. Le sang coule, l’inquiétude s'éloigne ${ }^{3}$; cela veut dire que si l'on peut écarter le mal, il n'y aura aucune culpabilité.

$1 \S 104$, et commentaire de TSHOU HI.

${ }^{2} \mathrm{~N}^{\circ} 1043$.

${ }^{3}$ C'est la lecture du commentateur chinois, tandis que le texte porte : «le sang coule et se répand rapidement ». 


\section{Tsie}

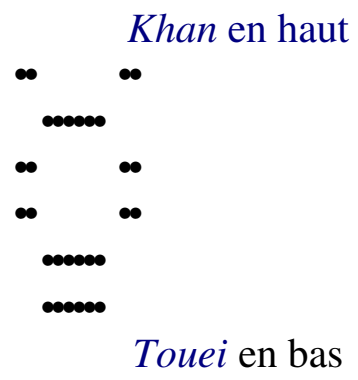

1045. Tsie ; liberté, les préceptes cruels ne peuvent pas conduire à la perfection.

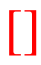

1051. Premier trait nonaire : ne pas sortir de la cour de la porte intérieure; pas de culpabilité.

$$
\text { * }
$$

1053. Deuxième trait nonaire : ne pas sortir de la cour de la porte extérieure; présage malheureux.

1055. Troisième trait hexaire : comme sans principes; comme devant se lamenter; pas de culpabilité.

$*$

1057. Quatrième trait hexaire : jouir en paix des préceptes, liberté.

$*$

1059. Cinquième trait nonaire : préceptes agréables ; présage heureux; en entreprenant il y aura des félicitations.

$*$

1061. Trait supérieur hexaire : préceptes cruels; présage malheureux de la perfection ; dissipation des regrets.

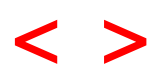


60. Tsie.

TSHENG TSE. - Tsie. «L'Ordre des koua » dit : «Hoan, séparation; les êtres ne peuvent pas résulter d'une séparation indéfinie, aussi le koua hoan est suivi du koua tsie. » Quand les êtres sont séparés et dispersés, il convient de les contenir et de les arrêter et c'est là ce qui fait que le koua tsie suit immédiatement le koua hoan. Comme koua, il est constitué par le marais sur lequel s'étend l'eau. La capacité du marais est limitée ; si l'on y verse de l'eau, lorsqu'il sera plein, il débordera, ce qui constitue l'image symbolique de limitation et ce qui fait que le koua est appelé tsie ${ }^{1}$.

${ }^{1}$ Tsie, arrêter, contenir ; définir et réglementer par des préceptes. 


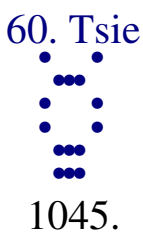

Tsie ; liberté, les préceptes cruels ne peuvent pas conduire à la perfection.

TSHENG TSE. - Du moment où les choses sont réglées par des préceptes, elles peuvent parvenir à se développer librement et c'est pour cel a que le koua tsie comporte le sens de liberté. La noblesse des principes réside dans leur conformité à la justice, et si cette justice est dépassée, ils deviennent cruels et abusifs. Lorsque les préceptes arrivent à être cruels, comment pourraient-ils constituer des règles permanentes? Ils ne peuvent pas être observés et maintenus avec fermeté pour devenir permanents, de sorte qu'ils ne peuvent atteindre à la perfection.

TSHOU HI. - Tsie ; avoir des limites et être arrêté. Ce koua est composé du koua simple touei au-dessous et du koua simple khan en dessus. Il y a de l'eau au-dessus du marais ; sa contenance est limitée, de sorte qu'il constitue une limitation ou réglementation. Les principes comportent certainement une voie de liberté. De plus, dans la substance de ce koua, les traits positifs et négatifs sont en nombre égal et, d'ailleurs, le cin quième et le second traits sont tous les deux positifs, de sorte que le sens divinatoire comporte la liberté de développement. Toutefois poussés à l'excès, les préceptes de toute réglementation deviennent gênants et cruels, de sorte que la formule avertit encore qu'il ne faut pas les observer comme sils constituaient la perfection.

1046.

Le commentaire de la formule déterminative dit: Tsie, liberté; participation de la dureté énergique et de la douceur malléable, la dureté énergique possédant la justice.

TSHENG TSE. - La voie rationnelle du koua tsie comporte essentiellement le sens de liberté d'expansion et de pénétration ; lorsque les choses sont réglées par des principes fixes ( $t$ sie) elles peuvent se développer librement. De plus, dans les aptitudes du koua, la dureté énergique et la douceur malléable se partagent les positions ; la dureté énergique possède la justice et n'est pas excessive et c'est en core la raison pour laquelle le koua est 
considéré comme exprimant la réglementation et pour laquelle il est susceptible de liberté.

TSHOU HI. - Explication de la formule du koua au moyen de la substance même de ce koua.

1047.

Les préceptes cruels ne peuvent pas conduire à la perfection; la voie rationnelle est limitée.

TSHENG TSE. - Les préceptes poussés à l'extrême limite et devenant cruels et pénibles à supporter, il devient impossible de les maintenir avec une fermeté persistante et de les observer d'une façon permanente ; la voie rationnelle est alors parvenue à son extrême limite.

TSHOU HI. - Il en est encore parlé au point de vue de la raison d'être du fait.

1048.

Se plaire à s'exposer au danger; mériter sa situation d'après les préceptes ; justice et droiture par la liberté.

TSHENG TSE. - En en parlant d'après les aptitudes indiquées par les koua simples, au-dedans satisfaction, au-dehors danger : se plaire à marcher dans le danger. Dans ce qui lui plaît, l'homme ne sait pas se borner ; s'il rencontre inopinément le danger, il pense seulement à s'arrêter. Or, s'arrêter au moment où il est satisfait constitue le sens du précepte et l'idée de principe qui limite et retient ; il mérite sa situation à cause des préceptes. Le cinquième trait occupe le rang prééminent et mérite sa situation; il est au-dessus du marais, il a des principes ; il mérite sa situation et c'est par ses principes, c'est lui de qui dépendent les préceptes et les principes. En se plaçant, il se conforme à la justice et à la droiture, c'est avoir des principes et pouvoir les communiquer librement. La justice et la droiture constituent la liberté, l'excès les rendrait odieux et cruels.

TSHOU HI. - Il en est encore question au point de vue de la substance et des vertus des koua simples; K mériter la situation, justice et droiture », se 
rapporte au cinquième trait. De plus le koua simple khan est considéré comme indiquant la libre transmission.

1049.

Le ciel et la terre marquent les règles (tsie) et les quatre saisons s'achèvent; les préceptes (tsie) servent à déterminer et à régler; ils ne détruisent pas la richesse, ils ne nuisent pas au peuple.

TSHENG TSE. - Il est question de déduire la voie rationnelle des préceptes et de toute réglementation. Le ciel et la terre sont soumis à des règles (tsie), aussi, ils peuvent engendrer les quatre saisons ; sans ces règles, ou préceptes (tsie), l'ordre de ces saisons serait troublé et interverti. L'homme saint institue des règles et des principes qui constituent des préceptes et il peut le faire sans nuire à la richesse publique et sans nuire au peuple. Sans les préceptes qui les réglementent, les passions de l'homme n'auraient pas dé limite ; elles le conduiraient aux déportements de la licence effrénée et jusqu’à la destruction de sa richesse, au détriment du peuple.

TSHOU HI. - Dernière analyse de la voie rationnelle de la réglementation par les préceptes.

1050.

Le commentaire traditionnel de la formule symbolique dit: Au-dessus du marais il y a de l'eau; retour par des préceptes (tsie). L'homme doué emploie les règlements et les prescriptions sur les mesures et sur les nombres; il délibère sur la vertu et l'action.

TSHENG TSE. - L'aptitude du marais à contenir de l'eau, ou sa capacité, a des limites ; s'il y en a trop il se ra rempli et débordera. C'est là un exemple de limitation (tsie), aussi le koua est considéré comme exprimant la réglementation restrictive. L'homme doué considère l'image symbolique présentée par le koua tsie : et il l'applique à l'institution et à l'étab lissement de règles sur les nombres. Toute chose, importante ou futile, légère ou grave, élevée ou minime, virtuelle ou réelle, correspond toujours à un nombre et à une mesure déterminée, et qui est considéré comme un principe (tsie). Le nombre exprime la multiplicité plus ou moins grande ; la mesure exprime la règle instituée qui limite et définit. Délibérer sur la vertu et les actions 
c'est-à-dire maintenir intactes les vertus intérieures et innées, tandis que leur manifestation extérieure constitue leur action. Les vertus et les actions de l'homme doivent être pondérées, après délibération, et alors elles sont conformes aux préceptes. Délibérer veut dire discuter et apprécier, afin de chercher à atteindre au degré (tsie) juste et convenable. 


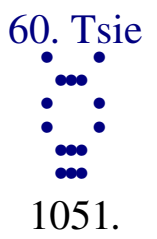

Premier trait nonaire : ne pas sortir de la cour de la porte intérieure; pas de culpabilité.

TSHENG TSE. - Cour de la porte intérieure, la cour en dehors de la porte intérieure ; cour de la porte d'entrée, la cour à l'intérieur de la porte principale d'entrée. Le premier trait, avec sa positivité, est au rang inférieur ; en haut, il rencontre en outre une correspondance sympathique, c'est celui qui est incapable de retenir et de limiter par des préceptes. De plus, il est en présence du moment qui exprime le début de la réglementation, aussi la formule avertit-elle à son sujet qu'il faut observer et contenir avec circonspection, jusqu'à ce point qui consiste à ne point sortir de la cour de la porte intérieure, et qu'alors on sera sans culpabilité. Lorsqu'au début on peut s'observer avec fermeté, il arrive quel quefois que finalement on se laisse aller à dépasser le milieu convenable ; mais sans circonspection, dès le début, comment serait-il possible d'atteindre jusqu'au bout sans défaillance ? Aussi, dès le début du koua tsie; ceci constitue un avertissement très grave.

TSHOU HI. - Cour de la porte intérieure, cour hors de la porte des appartements intérieurs. Positivité énergique se conformant à la droiture et occupant le premier rang du koua tsie ; elle n'est pas encore capable d'agir : c'est celui qui peut se conformer aux pré ceptes et se retenir, aussi, tels sont le sens divinatoire et l'image symbolique.

1052.

Ne pas sortir de la cour de la porte intérieure; savoir reconnaître ce qui est libre ou obstrué ${ }^{1}$.

TSHENG TSE. - Au début du koua mie, la formule du trait avertit d'observer avec pru dence, aussi elle dit qu'en ne sortant point de la cour de la porte intérieure, on sera sans culpabilité. Le commentaire de la formule symbolique craint que les hommes ne se laissent entraîner par la lettre, en négligeant l'esprit, aussi, il revient sur le même sujet en expliquant que, bien

\footnotetext{
1 Praticable ou impraticable.
} 
qu'il convienne d'observer avec prudence sans sortir hor s de la cour de la porte intérieure, il faut encore savoir juger de ce qui est praticable ou impraticable, suivant le moment considéré. Si quelque chose est praticable, on agira ; si c'est imprati cable, on s'arrêtera ; si le devoir exige qu'on sorte, on sortira. La crédulité de Vi Sheng restant dans l'eau sans en sortir, c'est précisément ne pas savoir reconnaître ce qui est praticable ou impraticable. C'est pour cela que l'homme doué est pur et point crédule. Ce que les « formules annexées » expliquent spécialement est que, ce qu'il faut contenir et limiter, chez l'homme, c'est exclusivement la parole et les actions. En restreignant les paroles il est évident qu'on restreindra par là même les actions, mais il convient de commencer par les paroles. 


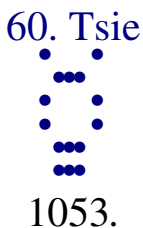

Deuxième trait nonaire:
pe pas sortir de la cour de la porte extérieure;
preureux.

TSHENG TSE. - Bien que le second trait réunisse les caractères physiques qui dénotent l'énergie et la justice, cependant il occupe un rang négatif et est placé dans le koua simple qui exprime la satisfaction, il obéit à la mollesse négative. Se plaçant dans un rang négatif, il est sans droiture ; faisant partie du koua simple qui exprime la satisfaction, il perd son énergie; obéissant à la mollesse, il est bien près de commettre le mal. La voie rationnelle de la retenue (tsie) comporte l'emploi de l'énergie, de la justice et de la droiture; le second trait perd ses vertus d'énergie et de jus tice et il diffère du cinquième trait nonaire énergique, juste, et doué de droiture. Ne pas sortir de la cour de la porte extérieure ; ne pas aller au-dehors ; cela veut dire qu'il ne suit pas le cinquième trait. Bien que le second et le cinquième traits ne se correspondent pas sympathiquement suivant la droiture, ils se correspondent par la condition de négativité et de positivité, ce qui fait qu'ils ne se suivent pas l'un l'autre ; sills s'accordent ensemble par la voie rationnelle de la justice et de l'énergie, ils seront encore capables de produire l'œuvre de la régularisation et de la rete nue par les préceptes (tsie). C'est simplement parce que, perdant ses vertus, il manque l'occasion, quill y a un présage malheureux. Ne pas s'accorder avec le cinqui ème, c'est un fait (tsie) de manquer de droiture. La justice, l'énergie et la droiture sont considérées comme constituant des principes régulateurs (tsie), comme propres à réprimer la colère, à limiter les passions, à diminuer les excès, et à augmenter le reste. Comme exemple de principes sans droiture, on peut citer la parcimonie dans les choses nécessaires et la timidité excessive dans les actions.

TSHOU HI. - Cour de la porte intérieure, cour en dedans de la porte d'entrée princi pale. Le second trait nonaire est en présence d'un moment où il doit agir et il manque d'énergie et de droiture ; il est dépourvu d'alliances sympathiques au-dessus de lui; il sait retenir et restreindre (tsie) et il ne comprend pas la liberté d'action et de communication, auss i tels sont le sens divinatoire et l'image symbolique. 
Présage malheureux de ne pas sortir de la cour de la porte extérieure; manquer absolument l'occasion.

TSHENG TSE. - Il est incapable de monter suivre la voie rationnelle de justice et de droiture du cinquième trait nonaire et d'accomplir l'œuvre de restriction par les principes (tsie); c'est qu'il est attaché et retenu par la malléabilité négative dans des liens égoïstes, et c'est là manquer absolument l'occasion et le moment favorable, d'o ù résulte le présage malheureux. Manquer l'occasion, c'est manquer le moment opportun. 


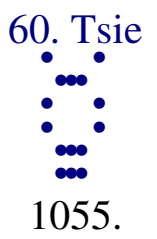

Troisième trait hexaire : comme sans principes; comme devant se lamenter; pas de culpabilité.

TSHENG TSE. — Le troisième trait hexaire est sans justice ni droiture ; il s'appuie sur l'énergie et surveille le péril, il sera très certainement coupable. Cependant, malléable, soumis, doué d'aménité, s’il peut se contenir lui -même par des principes et se conformer au devoir, il pourra ne pas commettre de fautes. Autrement, le présage malheureux de la culpabilité surgira nécessairement, et il y aura de quoi se lamenter et souffrir. Aussi, sîl est comme sans préceptes et livré à ses passions, il sera comme souffrant et se lamentant, et ce sera le résultat de sa propre conduite, sans quill en puisse rejeter sa culpabilité sur qui que ce soit.

TSHOU HI. - Malléable et négatif, sans justice ni droiture, c'est parce que dans un moment où il conviendrait de se retenir et de se modérer par des préceptes, il est incapable d'obéir à aucune règle restrictive. Aussi, tels sont le sens divinatoire et l'image symbolique.

1056.

Lamentations causées par le manque de principes; sur qui en rejeter la culpabilité ?

TSHENG TSE. - En suivant les préceptes, il deviendra possible d'éviter les fautes, tandis que l’incapacité de se régler sur les préceptes deviendra le sujet de lamentations ; à qui en attribuerait-on la faute ?

TSHOU HI. - Dans cette formule ${ }^{1}$ l'expression «absence de culpabilité » a un sens différent de celui qu'elle comporte dans tous les autres koua ; elle signifie que la faute ne peut être rejetée sur personne.

\footnotetext{
$1 \S 1055$.
} 


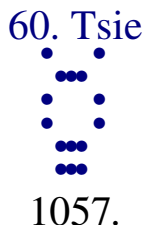

Quatrième trait hexaire : jouir en paix des préceptes, liberté.

TSHENG TSE. — Le quatrième trait obéit avec soumission au cinquième trait nonaire et se soumet à sa voie rationnelle d'énergie, de justice et de droiture : c'est prendre la justice et la droiture pour préceptes, ou principes (tsie). Avec les qualités de la négativité il occupe un rang négatif : il assure son repos par la droiture. Être digne de la situation occupée, est considéré comme constituant l'im age symbolique d'avoir des principes fixes auxquels on se conforme. En bas, il sympathise avec le premier trait. Le quatrième trait fait partie de la substance du koua simple khan, qui représente l'eau ; lorsque l'eau monte et débor de elle représente l'ab sence de préceptes, c'est-à-dire de limitation ; lorsqu'elle descend, elle représente la conformité aux préceptes et aux règles naturelles. D'après le sens du quatrième trait, il ne s'agit pas de se violenter pour se conformer aux préceptes ; il s'agit de ceux qui trouvent leur calme et leur repos dans l'observation naturelle des règles et des préceptes. Aussi il peut en résulter une liberté complète d'action et de communication. Ce qui constitue le bien dans les préceptes, c'est lorsqu'ils peuvent conduir e au repos et à la paix ; si on s'y conforme par force et sans y trouver la paix, ils ne peuvent pas constituer des règles permanentes et durables; comment pourraient-ils assurer la liberté ?

TSHOU HI. - Malléabilité négative et obéissante possédant la droiture ; au-dessus de lui, il obéit au cinquième trait nonaire : c'est celui qui se conforme naturellement et spontanément aux préceptes, et c'est pourquoi tels sont l’image symbolique et le sens divinatoire.

1058.

Liberté résultant du calme dans l'observation des préceptes; suivre la voie rationnelle d'en haut.

TSHENG TSE. - Le sens qui résulte de l'aptitude du quatrième trait à trouver le repos dans l'observation des préceptes n'est pas unique; le commentaire symbolique ne relève que le plus important. Le quatrième monte pour obéir à la voie rationnelle d'énergie, de justice et de droiture du 
cinquième trait nonaire, qu'il considère comme constituant une règle et un ensemble de préceptes; cela est suffisant pour assurer la liberté. Les autres biens qui résultent de cette voie ne sont pas étrangers à la justice et à la droiture. 


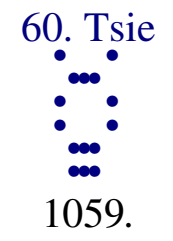

Cinquième trait nonaire : préceptes agréables ; présage heureux ; en entreprenant il y aura des félicitations.

TSHENG TSE. - Le cinquième trait nonaire est énergique, juste et droit, il occupe la situation prééminente ; c'est de lui que dépendent les préceptes. Ce qu'on appelle être digne de sa situation par les préceptes, c'est la liberté résultant de la justice et de la droiture. Pour lui-même cela constitue l'action calme et sereine ; pour l'univers il en résulte la satisfaction dans l'obéissance et la soumission; c'est ce qui fait la douceur des préceptes. Le présage heureux est évident. Agir dans ces mêmes conditions doit nécessairement contribuer à la grandeur de l'ouvre accomplie, aussi, en entreprenant, il sera possible de recueillir des louanges et des félicitations.

TSHOU HI. - Ce qu'on appelle « mériter la situation par les préceptes » c'est la liberté résultant de la justice et de la droiture. Aussi, tels sont le sens divinatoire et limage sym bolique.

1060.

Présage heureux de la douceur des préceptes; occuper justement sa situation.

TSHENG TSE. - Occupant déjà la situation prééminente, il se conforme encore à la voie rationnelle de la justice, ce qui fait que le présage est heureux et qu'il a du mérite. C'est la justice qui constitue la noblesse des préceptes ; en se conformant à la justice, on se conforme à la droiture. La droiture ne peut pas à elle seule équivaloir absolument à la justice. 


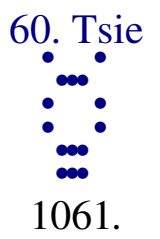

Trait supérieur hexaire : préceptes cruels ; présage malheureux de la perfection; dissipation des regrets.

TSHENG TSE. - Le trait supérieur hexaire occupe le rang extrême dans le koua tsie (préceptes); il exprime ce qu'il y a de pénible et de dur dans les préceptes. Il occupe le rang extrême dans le koua simple khan, qui exprime le péril, ce qui donne encore le sens de rigueur pénible. En observant les préceptes et en s'y maintenant avec une fermeté inébranlable, le présage sera malheureux ; mais sîl a des regrets, le présage malheureux disparaîtra. Le mot « regret » est une expression qui indique la diminution de ce qui est en excès pour revenir à ce qui est juste. L'expression « dissipation des regrets », dans le koua tsie et cette même expression dans les autres koua, identiques dans la forme, différent cependant par le sens.

TSHOU HI. - Il occupe le rang extrême dans le koua tsie (qui symbolise l'idée de pré ceptes), aussi il est considéré comme exprimant la rigueur pénible des préceptes. Étant déjà placé trop au sommet, bien qu'il possède la droiture, il ne peut se soustraire au présage malheureux. Cependant, si les règles rituelles sont trop surchargées, les entraînements de la dissolution n'en sont que plus rares, de sorte que bien qu'éprouvant des regrets, à la fin ceux-ci pourront se dissiper.

1062.

Présage malheureux de la perfection dans la rigueur des préceptes; la voie rationnelle est à sa fin.

TSHENG TSE. - Du moment où les préceptes sont rigoureux et où on les observe avec une rigidité parfaite, le présage devient malheureux. En effet, la voie rationnelle des préceptes est parvenue à la fin de son extrême limite. 


\section{Tshong fou}

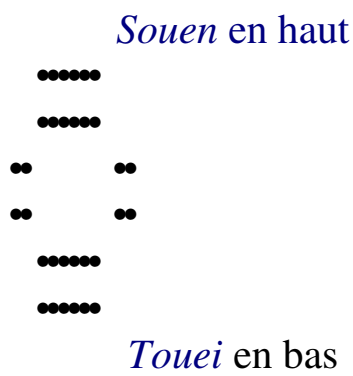

1063. Tshong fou, présage heureux du fretin; avantage à traverser un grand cours d'eau; avantage de la perfection.

1068. Premier trait nonaire : présage heureux de l'appréciation ; s'il y a autre chose, pas de calme.

1070. Deuxième trait nonaire : l'oiseau ho qui chante est dans l'obscurité ; son petit l'accompagne ; j'ai un beau titre; moi et toi nous le désirons.

1072. Troisième trait hexaire : il possède un équivalent; parfois battre la mesure, parfois cesser; parfois pleurer, parfois chanter.

$*$

1074. Quatrième trait hexaire : la lune est sur le point d'être pleine ; chevaux dépareillés; pas de culpabilité.

$*$

1076. Cinquième trait nonaire : avoir bonne foi et comme attaché ; pas de culpabilité.

$*$

1078. Trait supérieur nonaire : le bruit des ailes monte au ciel ; pureté, présage malheureux.

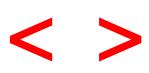


61. Tshong fou.

TSHENG TSE. - Tshong fou. «L'Ordre des koua » dit: «De l'observation des préceptes résulte la confiance, aussi le koua tsie est suivi du koua tshong fou. » Le koua tsie est considéré comme exprimant l'action de formuler des préceptes, afin qu'on ne puisse les enf reindre; il faut qu'ils inspirent la confiance et ensuite ils peuvent produire leur effet. Les supérieurs pouvant les maintenir avec confiance, les inférieurs s'y conforment avec une confiance égale ; ce sont des préceptes d'où résulte la confiance et c'e st pour cela que le koua tshong fou (foi intérieure) suit immédiatement le koua tsie. Il est constitué par un marais sur lequel y a du vent; le vent agissant sur le marais et son action étant ressentie jusqu'à l'intérieur de l'eau, donne l'image symbolique de la «foi intérieure ». Le caractère traduit par «faire ressentir son action » signifie littéralement émouvoir, influencer et mettre en mouvement. Les traits intérieurs et extérieurs du koua sont également des traits pleins, tandis que les traits du milieu sont vides, ce qui constitue l'image symbolique de la foi intérieure. Enfin, le second et le cinquième traits sont également positifs et pleins au milieu, ce qui donne encore le sens de bonne foi. Dans les deux substances des koua simples le trait du milieu est plein; dans la substance totale du koua parfait le milieu est évidé. L’intérieur vide ${ }^{1}$ est la base essentielle de la confiance; l'intérieur plein ${ }^{2}$ est le caractère physique qui indique la confiance.

${ }^{1}$ L'absence de préventions.

${ }^{2}$ La sincérité. 


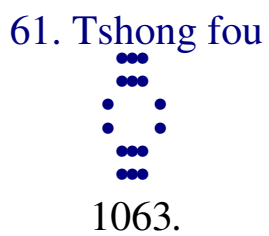

Tshong fou, présage heureux du fretin ${ }^{1}$; avantage à traverser un grand cours d'eau; avantage de la perfection.

TSHENG TSE. - Le troisième caractère du texte signifie ce qui est vif et sautillant ; le poisson est un être dénué d'intelligence et difficile à influencer ${ }^{2}$. Si la bonne foi et la confiance peuvent influencer le poisson frétillant, il est clair que rien ne pourra rester en dehors de cette influence d'où résulte le présage heureux. La bonne foi et la confiance rendent possible le fait de marcher sur l'eau ou au travers du feu, à plus forte raison enco re, elles permettent de traverser un grand cours d'eau. L'observation permanente et durable de la bonne foi consiste dans une ferme droiture, aussi il y a avantage dans la perfection.

TSHOU HI. - Le deuxième caractère du texte équivaut à [], confiance, sincérité, bonne foi. Dans le koua, les deux négativités sont à l'intérieur et les quatre positivités sont à l'extérieur. De plus les deux positivités qui occu pent le second et le cinquième rangs occupent chacune le milieu d'un koua simple, c'est-à-dire possèdent la justice. En en parlant d'après le koua lui -même, il indique le vide intérieur ${ }^{3}$; en en parlant au point de vue des substances des deux koua simples, ils expriment la plénitude intérieure ${ }^{4}$, c'est donc toujours une image symbolique de sincérité et de bonne foi. De plus, l'inférieur se plaît dans la correspondance sympathique avec le supérieur; le supérieur est modeste et écoute les conseils de l'in férieur, ce qui constitue encore le sens de bonne foi. Le fretin c'est-à-dire des animaux sans intelligence ni entendement. De plus, le bois se trouve au-dessus du marais ; au-dehors réalité solide et au-dedans vacuité, ce qui constitue également l'image symbolique du gouvernail d'une embarcation. La plus extrême sincérité est capable d'influencer et d'émouvoir les petits poissons eux -mêmes; elle brave les périls et les difficultés et est incapable de perdre sa pureté, aussi, si celui qui consulte le sort peut atteindre jusqu'à la sympathie du fretin, le présage sera heureux et il y aura avantage à traverser un grand cours d'eau ; de plus, il y aura certainement avantage dans la pureté parfaite.

\footnotetext{
${ }^{1}$ Petits poissons frétillant.

${ }^{2}$ Il est difficile d'agir sur son intelligence.

3 Absence de parti pris ou de préventions.

${ }^{4}$ La sincérité intérieure.
} 
1064.

Le commentaire traditionnel de la formule déterminative dit: Tshong fou, sincérité intérieure; la malléabilité est au-dedans et la dureté énergique possède la justice; satisfaction et humilité ; la bonne foi alors transforme les États.

TSHENG TSE. - Les deux malléabilités sont à l'intérieur du koua : image symbolique du vide intérieur qui constitue la bonne foi et la parfaite sincérité. Deux duretés énergiques occupent le rang du milieu dans la substance de chacun des deux koua simples inférieur et supérieur. Le « milieu réel ${ }^{1}$ constitue l'image symbol ique de la bonne foi ; c'est à cause de cela que le koua est appelé tshong fou, sincérité intérieure. Satisfaction et humilité, cela exprime les effets du koua en en parlant d'après les substances des deux koua simples. En haut modestie et humilité, en bas satisfaction, ce qui constitue chez le supérieur la plus extrême sincérité en se soumettant humblement aux conseils de l'infé rieur, tandis que l'inférieur est de bonne foi en obéissant avec satisfaction au supérieur. Lorsqu'il en est ainsi la bonne foi et la sincérité sont capables de transformer les États et les royaumes. Si un homme ne se complaît pas dans l'obéissance, ou s'il viole et transgres se la raison d'être des choses, comment serait -il capable de transformer l'univers ?

TSHOU HI. - Explication du sens du nom du koua au moyen de sa substance et des propriétés des koua simples qui le composent.

1065.

Présage heureux du menu fretin; la bonne foi atteint jusqu'au menu poisson. Avantage à traverser un grand cours d'eau; monter dans le canot de bois évidé.

TSHENG TSE. - La sincérité peut atteindre jusqu'au menu poisson frétillant ; la voie de la sincérité est poussée à l'extrême et c'est par là que le présage est heureux. Employer la sincérité et la bonne foi pour braver les périls et les dangers est une voie aussi avantageuse que le moyen qui consiste à se servir du bois pour traverser un cours d'eau et à creuser un canot. Avec

\footnotetext{
${ }^{1} \mathrm{Ou}$ « plénitude intérieure ».
} 
un canot évidé, il n'y a plus de danger de chavirer ${ }^{1}$. Le koua est évidé au milieu ; c'est une image symbolique d'un ca not creusé.

TSHOU HI. - Il est question de l'image symbolique du koua.

1066.

La sincérité intérieure emploie l'avantage de la pureté; c'est là correspondre au ciel.

TSHENG TSE. - Sincérité intérieure et pureté ; alors il y a correspondance sympathique avec le ciel ; la voie rationnelle du ciel n'est rien autre chose que la parfaite sincérité.

TSHOU HI. - Sincérité et droiture, alors il y a correspondance sympathique aux principes du ciel.

1067.

Le commentaire traditionnel de la formule symbolique dit: Au-dessus du marais il y a du vent; sincérité intérieure ${ }^{2}$; l'homme doué l'applique à la délibération dans les jugements criminels et il ajourne la peine de mort ${ }^{3}$.

TSHENG TSE. - Sur le marais il y a du vent, il influence l'intérieur de l'eau. La substance de l'eau est fluide (vide), aussi le vent peut la pénétrer ; le cour de l'homme est vide, aussi les êtres peuvent l'émouvoir et l'influencer. Le mouvement communiqué au marais par le vent est comme l'influence pénétrante des êtres, ce qui constitue l’image symbolique de la bonne foi intérieure (tshong fou). L'homme doué contemple cette image symbolique et il applique sa sincérité à la discussion des jugements criminels et à l'ajourne ment de la peine de mort. L'action de l'homme doué dans la dé libération au sujet des affaires criminelles consiste uniquement dans le fait d'épuiser les limites de la sincérité ; dans l'application de la peine de mort, elle consiste uniquement à pousser la mansuétude à son extrême limite, aussi la sincérité de son sentiment de justice le porte continuellement à rechercher les motifs

\footnotetext{
${ }^{1}$ L'idée primitive me paraît être celle -ci. Un tronc d'arbre peut servir à traverser un cours d'eau, mais il est sujet à rouler sur lui -même ; creusé en forme de canot il devient stable.

${ }^{2}$ Tshong fou.

${ }^{3} \mathrm{Il}$ accorde un sursis. Voir le code chinois et annamite.
} 
d'ajournement et de sursis, c'est -à-dire les motifs d’indulgence. Dans les affaires de l'univers, il ne manque jamais d'épuiser la sincérité et la justi ce, mais la délibération ou jugement des affaires criminelles et la clémence dans les sentences capitales sont certainement les plus graves entre toutes.

TSHOU HI. - Le vent influence et émeut, l'eau subit cette influence ; c'est l'image sym bolique de la bonne foi intérieure. La délibération dans les jugements criminels, les sursis dans les cas de condamnation capitale sont toujours le résultat de l'idée de la bonne foi intérieure. 


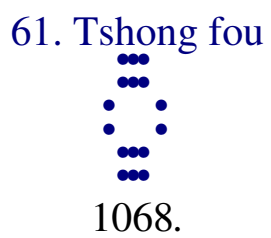

\section{Premier trait nonaire : présage heureux de l'appréciation; s'il y a autre chose, pas de calme.}

TSHENG TSE. - Ce trait nonaire se trouve au début de la confiance intérieure, aussi la formule avertit au sujet du jugement relatif à ce qu'il croit. Le troisième caractère du texte signifie mesurer, apprécier, évaluer ; mesurer ce qu'il est possible de croire avec confiance, et ensuite s'y conformer. Bien qu'animé d'une confiance extrême, si cette confiance est appliquée à un fait qui n'en est pas digne, il en résultera des regrets et de la culpabilité, aussi c'est en mesurant et en appréciant d'abord et en ajoutant foi ensuite que le présage sera heureux. Du moment où l'objet de cette confiance est vérifié, la confiance doit être entière et parfaite, si elle est appliquée à d'autres objets, le calme et la sérénité de l'assurance deviennent impossibles. Calme, sérénité majestueuse. «S’il y a autre chose » indique que le jugement est indécis ; lorsque le jugement de l'homme manque de fixité et de certitude, il est troublé et manque de calme et d'assurance. Le pre mier et le quatrième traits sont en correspondance sympathique conforme à la droiture. Le quatrième fait partie de la substance qui indique l'humilité et occupe son rang avec droiture, rien en lui n'est autrement que bien. Dans les sentences des traits, ce qui est important est surtout le sens du début ou commencement, aussi ce n'est pas le sens de correspondance sympathique mutuelle qui est relevé ; si on s'occupait de cette correspondance sympathique, il n'y aurait plus discer nement et jugement.

TSHOU HI. - Au moment du début de la confiance intérieure ${ }^{1}$, en haut, il correspond sympathiquement avec le quatrième trait hexaire; s'il est capable d'apprécier ce qu'il doit croire, et ensuite de le croire, le présage sera heureux. S'il y a d'autres sujets de confiance, il perd la droiture qui résulte de son premier jugement, et il ne pourra pas jouir de la certitude qui assurerait son repos et sa tranquillité : c'est une expression qui indique l'avertissement donné à celui qui consulte le sort.

1069.

\footnotetext{
${ }^{1}$ On pourrait peut-être encore lire « juste confiance » ou « confiance justifiée ».
} 


\section{Premier trait nonaire, présage heureux de l'appréciation; le jugement (les tendances) pas encore modifié.}

TSHENG TSE. - Au moment où la confiance commence à naître, les tendances n'ont encore aucun objet par lequel elles peuvent être fixées et maintenues; aussi, si l'on mesu re et si l'on apprécie ce qu'il convient de croire, on atteindra à la droiture dans le jugement porté ; c'est par là que le présage est heureux. En effet, les tendances n'ont pas encore été modifiées et mises en éveil ; lorsque le jugement est formé et qu'il y a une croyance à observer, alors les tendances sont, au contraire, altérées et modifiées, et si l'on veut juger, on ne juge plus avec la droiture primordiale. Dès le début du koua, cette sentence exprime qu'il faut chercher la voie rationnelle dans laquelle il faut avoir confiance. 


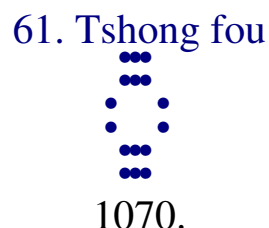

Deuxième trait nonaire : l'oiseau ho ${ }^{1}$ qui chante est dans l'obscurité ${ }^{2}$; son petit l'accompagne ; j'ai un beau titre ; moi et toi nous le désirons.

TSHENG TSE. - Le second trait est celui qui est énergique et absolu dans l'extrême confiance intérieure. La bonne foi, étant poussée à un degré extrême, peut émouvoir et influencer librement. L'oiseau ho chante dans un lieu solitaire et sombre ; on ne l'entend pas, mais son petit et lui se répondent mutuellement et s'acco rdent; les désirs intimes de leurs cours sont librement en communication par leurs accents. Le beau titre c'est moi qui le possède et l'autre aussi y est attaché par un désir ardent ; l'idée d'estimer et de dési rer le beau titre est la même chez tous les deux. Lorsqu'il y a bonne foi à l'intérieur, les êtres répondent tous sans exception avec sympathie, parce que la sincérité est la même. Pour l'extrême sincérité, il n'y a pas d'intervalle, ou de distinction, entre ce qui est près ou éloigné, profond ou superficiel, aussi les «formules annexées » disent que, lorsqu’il s'agit du bien, on y répond sympathiquement de mille lis à la ronde, tandis que lorsqu'il s'agit de ce qui n'est pas le bien, on s'en détourne à mille lis de distance ; cela exprime que la sincérité des sentiments se communique librement. L'extrême sincérité est la raison d'être de la libre communication des influences ; celui qui connaît la voie rationnelle est à même de le comprendre.

TSHOU HI. - Le second trait nonaire exprime la réalité de la confiance intérieure et le cinquième trait nonaire lui correspond sympathiquement, aussi par la réalité de la confiance intérieure, de sorte qu’il présente l'image symbolique de l'oiseau ho qui chante et de son petit qui s'accorde à ses accents et de l'idée exprimée par les mots : «mon titre et ton désir ». L'oiseau ho est dans la négativité, c'est-à-dire que, nonaire, il occupe le second rang. Un «beau titre », c'est-à-dire se conformer à la justice. L'avant-dernier caractère du texte signifie désirer, avoir envie de; cela exprime que les grandes vertus sont ce que l'homme estime et apprécie. Aussi, bien que je sois moi-même seul à posséder ce beau titre, cependant, chacun y est aussi fortement attaché et le désire ${ }^{3}$.

\footnotetext{
${ }^{1}$ Échassier (cigogne ?), corps blanc, pennes noires, sommet du crâne rouge ; chante au milieu de la nuit et son chant est entendu à huit ou neuf lis de distance. Tie tien.

${ }^{2}$ Littéralement dans la «négativité ».

${ }^{3}$ Le texte ne présente aucun sens saisissable et les commentaires sont loin de l'éclairer.
} 
1071.

Son petit s'accorde avec lui ; désir intérieur du cour.

TSHENG TSE. — Désirer dans le coar ; cela veut dire « ce que désire la sincérité de l’idée »; c'est pour cela qu’ils se comprennent librement et se répondent mutuellement avec sympathie. 


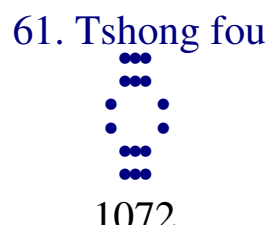

Troisième trait hexaire : il possède un équivalent; parfois battre la mesure, parfois cesser; parfois pleurer, parfois chanter.

TSHENG TSE. - Le quatrième caractère du texte signifie équivaloir ; contracter, former parallèle ou opposition avec...; cela veut dire que ce qui forme le lien de la confiance, c'est uniquement la correspondance sympathique avec le trait supérieur nonaire. Le troisième et le quatrième trait sont tous les deux, à cause de leur évidement intérieur ${ }^{1}$ ceux de qui dépend l'achèvement de la bonne foi. Toutefois leur manière de se placer est dif férente. Le quatrième possède une situation définie à laquelle il s'accorde ${ }^{2}$ et il se maintient dans la droiture ${ }^{3}$, aussi il oublie celui qui fait paire avec lui, afin de suivre le trait supérieur. Le troisième trait est dépourvu de justice ${ }^{4}$ et perd la droiture, aussi il s'accorde avec (possède) quelque chose d'équivalent à lui-même, qui influe sur ses propres tendances. À cause de ses caractères physiques de malléabilité portée au plaisir, du moment où il y a quelque chose qui le retient et l'attire, il ne suit que ce qui lui inspire de la confiance. Parfois il se met en évidence ${ }^{5}$, parfois il cesse et abandonne ; parfois il est triste et pleure, parfois il chante et fait de la musique. Agité ou en repos, triste ou gai, c'est tou jours parce qu'il est retenu et attiré par ce qui lui inspire confiance, de sorte qu'on ne sait encore si le présage est heureux ou malheureux. Toutefois, ce n'est pas la manièr e d'agir de l'homme doué, intelligent.

TSHOU HI. — «Équivalent », désigne la confiance indéfinie dans le trait supérieur nonaire. Le troisième trait hexaire, malléable et négatif, sans justice ni droiture, se laisse aller à se maintenir définitivement dans le plaisir et s'allie avec le trait supérieur qu'il considère comme lui correspondant sympathiquement. Il en résulte qu'il n'est pas maître de lui -même et que l’image symbolique est telle.

1073.

\footnotetext{
${ }^{1}$ Absence de préventions.

${ }^{2}$ Rang pair, trait négatif.

3 Il correspond au premier trait positif.

${ }^{4}$ Il n'est pas au milieu d'un koua simple.

${ }^{5}$ Battre la mesure.
} 
Parfois il bat la mesure, parfois il cesse ; situation imméritée.

TSHENG TSE. - Il occupe une situation imméritée ; parfois rien ne le domine et il ne suit que ce qui lui inspire confiance. La position qu'il occupe est conforme à la droiture de sorte que ce qui lui inspire confiance sera correct. 


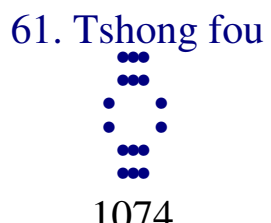

Quatrième trait hexaire : la lune est sur le point d'être pleine ; chevaux dépareillés; pas de culpabilité.

TSHENG TSE. — Le quatrième trait est celui de qui dépend l'achèvement de la bonne foi ; il occupe une situation voisine de celle du prince ; sa position est conforme à la droiture et le trait supérieur a confiance en lui au plus haut point: c'est celui qu'il convient d'investir de l'autorité avec une entière confiance. C'est comme lorsque la lune est tout à fait sur le point d'être pleine. Quand elle est pleine elle est en opposition. Si un sujet est en opposition avec le prince, les malheurs et la décadence doivent bientôt survenir, aussi le moment où il s'agit d'être sur le point d' être entièrement éclatant (pleine lune) constitue l'extrême limite de la perfection. Chevaux dépareillés : le quatrième et le premier traits sont ceux qui se correspondent sympathiquement suivant la droiture ; ce sont ceux qui sont appareillés. Dans l'ant iquité, on employait quatre chevaux pour traîner un char; lorsqu'on ne pouvait les réunir tous de la même couleur, alors les deux de dehors et les deux de dedans ( ?) étaient accouplés par couleur. De plus, il fallait encore qu'ils fussent appareillés comme taille, de sorte que deux chevaux constituaient une paire et étaient appelés «équivalents» (couple). Les chevaux sont des êtres qui marchent et circulent, le premier trait monte et sympathise avec le quatrième, tandis que celui-ci avance également et suit le cinquième; ils agissent tous deux en montant, aussi l'image symbolique est empruntée aux chevaux. La voie de la bonne foi consiste dans l'unité absolue des tendances; du moment où le quatrième trait suit le cinquième, s'il était encore retenu en bas par le premier, l'unité n'existerait plus et cela nuirait à la bonne foi et constituerait l'exis tence de la culpabilité. Aussi, si les chevaux appareillés s'oublient mutuellement, il n'y aura point de culpabilité. Le trait supérieur ${ }^{1}$ suit le cinquième et n'est pas retenu par le trait inférieur, c'est donc que les chevaux appareillés s'oublient et se quittent. S’il était retenu par le premier, il n'avancerait pas, et il ne pourrait achever l'œuvre de la bonne foi.

TSHOU HI. - Le quatrième trait hexaire occupe un rang négatif et se conforme à la droiture ; sa situation est voisine de celle du prince, ce qui constitue l'image symbolique de la lune sur le point d'être pleine. Chevaux appareillés, ou couple de chevaux, c'est-à-dire que le premier et le quatrième forment une paire. Mais le quatrième rompt avec le premier et monte à cause

\footnotetext{
${ }^{1}$ C'est probablement une faute et il faut lire le « quatrième trait suit... ».
} 
de la confiance qu'il place dans le cinquième, de sorte que cela constitue l'image symbolique de chevaux dépareillés, ou qui s'abandonnent mutuellement. Si celui qui consulte le sort est dans ces conditions, il n'y aura pas de culpabilité.

1075.

Chevaux dépareillés, rompre avec ceux de son genre et monter.

TSHENG TSE. - Il abandonne ceux de son genre et monte suivre le cinquième. « Genre » est employé pour « correspondance sympathique ». 


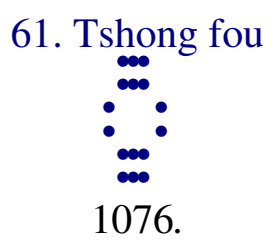

Cinquième trait nonaire : avoir bonne foi et comme attaché ; pas de culpabilité.

TSHENG TSE. - Le cinquième trait occupe la situation du prince. La voie rationnelle du prince doit être basée sur la plus extrême sincérité et pénétrer tout l'univers en l'influençant librement, afin d'inspirer la confiance et de toucher le cour de l'univers entier en l'attachant par des liens indissolubles; alors le prince sera sans culpabilité. Si la bonne foi du prince est incapable de produire cet attachement inébranlable, alors, parmi des millions et des millions de cours, comment garantir qu'il ne se produira pas des cas d'abandon et d'éloignement ?

TSHOU HI. - Le cinquième trait nonaire est énergique et actif, juste et droit; il exprime la réalité dans la bonne foi, et il occupe la situation prééminente : c'est celui de qui dépendent la confiance et la bonne foi. En bas, il correspond sympathiquement au second trait nonaire; ils possèdent les mêmes vertus, aussi, tels sont l'image symbolique et le sens divinatoire.

1077.

Avoir confiance et comme attaché ; situation directement méritée.

TSHENG TSE. - Le cinquième trait occupe la situation prééminente du prince; par la voie rationnelle de droiture et de justice il peut inspirer confiance à l'univers entier, comme en se l'attachant d'une manière inébranlable. C'est là être digne de la situation qu'il occupe ; la voie rationnelle du prince doit être ainsi. 


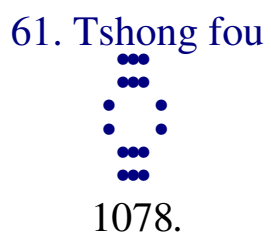

Trait supérieur nonaire : le bruit des ailes monte au ciel ; pureté, présage malheureux.

TSHENG TSE. — Le bruit des ailes, le bruit vole, mais la réalité ne suit point. Ce trait est placé à la fin du koua qui symbolise la confiance ; lorsque la confiance cesse, tout se corrompt ; la fidélité intérieure se perd, la beauté et l'élégance extérieures s'évanouissent, aussi la formule parle du bruit des ailes montant au ciel. La droiture à son tour est détruite : la nature de la positivité est d'avancer en montant; le caractère physique du vent est de voler en tourbillonnant. Ce trait nonaire se trouve dans le moment exprimé par le koua tshong fou ; il est placé au rang le plus élevé : c'est celui qui a confiance dans son mouvement ascensionnel et qui ne sait point s'arrêter. L'extrême degré dans l'action du bruit du battement d'ailes dans le vol des oiseaux, c'est de monter jusqu'au ciel d'où il peut être entendu ; en poussant à tel point la fermeté absolue d'une action, sans être capable de la modifier, le présage malheureux est évident. Fou Tse ${ }^{1}$ dit : «aimer à croire et ne pas aimer à étudier : c'est l'aveuglement et il est destructif »; c'est une expression pour désigner l'opiniâtreté sans intelligence.

TSHOU HI. - Il occupe le rang extrême dans le koua qui exprime la confiance ; il exprime l'extrême crédulité et l'incapacité de se corriger ; bien qu'il possède la pureté, c'est cependant une voie rationnelle de présage malheureux, et c'est pour cela que tels sont l'image symbolique et le sens divinatoire. La poule est désignée par l'expression « bruissement des ailes »; elle est l'image symbolique de l'humilité. Être placé au rang extrême du koua simple qui exprime l'humilité, c'est ce qui est considéré comme le fait de parvenir jusqu'au ciel. La poule n'est pas un animal capable de s'élever jusqu'aux cieux et cependant elle voudrait y atteindre ; elle croit ce qu'elle ne devrait pas croire et est incapable de modifier ses aspirations. C'est encore le même sens.

1078 bis.

Le bruit des ailes monte jusqu' au ciel : comment cela pourrait-il durer?

\footnotetext{
${ }^{1}$ Khong Tse.
} 
TSHENG TSE. - Conserver la confiance jusquà sa plus extrême limite et ne pas savoir changer : comment un tel état pourrait-il durer longtemps ? Opiniâtreté sans intelligence; dans de telles conditions, le présage est malheureux. 


\section{Siao kuo}

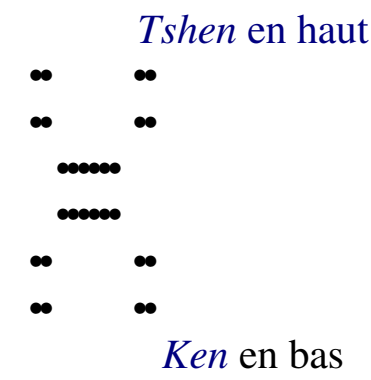

1079. Petit excès; liberté ; avantage de la pureté ; capacité dans les petites choses, incapacité dans les grandes choses ; bruit qui suit le vol de l'oiseau ;

il ne convient pas de monter, il convient de descendre; grand présage heureux.

1086. Deuxième trait hexaire : dépasser l'aïeul ; rencontrer l'aïeule ; ne pas atteindre le prince ; rencontrer le sujet ; pas de culpabilité.

$*$

1088. Deuxième trait hexaire : dépasser l'aïeul ; rencontrer l'aïeule ; ne pas atteindre le prince ; rencontrer le sujet; pas de culpabilité.

$*$

1090. Troisième trait nonaire : ne s'en gardant point jusqu'à l'excès ; suivre et peut-être le tuer; présage malheureux.

$*$

1092. Quatrième trait nonaire : point de culpabilité ; le rencontrer sans le dépasser ; en entreprenant péril, avertissement nécessaire ; ne pas employer une pureté constante et éternelle.

1094. Cinquième trait hexaire : nuages épais sans pluie; de nos plaines de l'ouest; le kong lance une flèche et capture l'autre dans l'antre.

*

1096. Trait supérieur hexaire: ne pas le rencontrer et le dépasser; l'oiseau qui vole s'en sépare ; présage malheureux; c'est-à-dire calamités et maux.

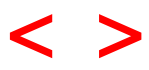




\section{Siao kuo.}

TSHENG TSE. - Siao kuo. «L'Ordre des koua » dit : «Celui qui a confiance en quelque chose agit nécessairement dans ce sens, aussi le koua tshong fou est suivi du koua siao kuo. » Ce que lhomme croit, il le fait certainement; en agissant, il en arrive à l'excès, et c'est ce qui fait que le koua siao kuo suit immédiatement le koua tshong fou. Il est composé des koua simples de la montagne au-dessus duquel il y a le koua simple de la foudre. La foudre ébranle dans un lieu élevé ; son bruit dépasse les limites ordinaires, et c'est pour quoi le koua est considéré comme exprimant un «petit excès » (siao kuo). De plus, la négativité occupe la situation prééminente ; la positivité perd cette situation et est dépourvue de justice ${ }^{1}$; sa petitesse dépasse le niveau ordinaire, et en effet, c'est un excès de ce qui est petit. Enfin, le koua exprime encore l'excès dans les petites choses, et un petit excès dans quelque chose.

\footnotetext{
${ }^{1}$ Le cinquième trait, négatif, est au milieu, et le trait positif passe au rang supérieur.
} 


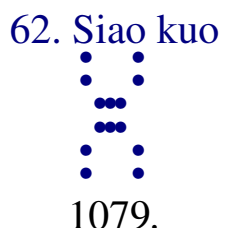

Petit excès ${ }^{1}$; liberté ; avantage de la pureté ; capacité dans les petites choses, incapacité dans les grandes choses ; bruit qui suit le vol de l'oiseau; il ne convient pas de monter, il convient de descendre ; grand présage heureux.

TSHENG TSE. - Kuo, outrepasser les règles normales, comme, par exemple, l'orgueil et la licence entreprenant sur le droit. L'excès est ce qui ramène au droit. Dans les affaires, quelles qu'elles soient, il y a des moments où tout répond à la convenance naturelle au sujet; il y a des moments où il faut outrepasser les règles ordinaires et où la liberté résulte ensuite de cet excès. C'est pour cela que le koua siao kuo comporte par lui-même le sens de liberté. Avantage de la pureté, c'est-à-dire que la voie rationnelle de l'excès est avantageuse par la pureté. Ne pas manquer à l'opportunité du moment, c'est ce qu'on appelle la correction ou droiture ; c'est ce qui fait que l'excès ramène à la justice. L'excès a lieu dans les petites choses; comment pourrait-il y avoir excès dans les choses importantes ? Cela est d'ailleurs clairement discuté au sujet du koua tae kuo, grand excès ${ }^{2}$. Le son laissé derrière lui par l'oiseau qui vole, désigne ce qui ne peut pas dépasser une limite très rapprochée. Il ne convient pas de monter, il convient de descendre ; c'est-à-dire que la soumission convient. Avec la soumission ce sera un grand présage heureux; dépasser pour se rapprocher ensuite, c'est en effet se conformer à la raison d'être des c hoses. Dépasser et se conformer ensuite à la raison d'être est nécessairement un grand présage heureux.

TSHOU HI. - Siao, ce qui est petit ; cela signifie la négativité ${ }^{3}$; le koua est constitué par quatre traits négatifs placés au-dehors et deux traits positifs placés à l'intérieur ; les traits négatifs sont plus nombreux que les traits positifs : ce qui est petit dépasse et l'emporte. Du moment où la négativité surpasse la positivité, elle peut jouir de la liberté. Mais toutefois, il faut nécessairement qu'elle soit avantageuse par l'observation de la pureté, de sorte que la formule ne peut pas ne point comporter d'avertissement. Le second et le cinquième traits du koua emploient tous les deux la douceur et possèdent la justice, de sorte qu'ils sont capables de petites choses; le troisième et le quatrième perdent tous les deux leur situation à cause de leur dureté énergique et tous deux manquent de justice, de sorte qu'ils sont incapables de grandes choses. La constitution physique du koua est la solidité

\footnotetext{
${ }^{1}$ Ou bien « dépasser un peu ».

2 Koua $\mathrm{n}^{\circ} 28$, ce qui est grand traverse.

${ }^{3}$ Il faudrait alors lire « ce qui est petit surpasse ».
} 
réelle au-dedans et le vide à l'extérieur ; comme dans le vol de l'oiseau, le son descend et ne monte pas, sil peut y avoir la concordance du son laissé par l'oiseau en volant, il conviendra de descendre et le présage sera grandement heureux ${ }^{1}$. Cela comporte encore le sens d'incapacité pour les grandes choses.

1080.

Le commentaire de la formule déterminative dit: Siao kuo; ce qui est petit dépasse et jouit de liberté.

TSHENG TSE. — La positivité, c'est la grandeur ; la négativité c'est la petitesse. La négativité possède la situation, la positivité perd cette situation et manque de justice : c'est la petitesse qui surpasse ${ }^{2}$. Aussi est-ce considéré comme exprimant que les petites choses l'emportent, ou comme la petitesse dans l'excès. Ce qui est petit, comme aussi les petites choses, doivent nécessairement à certains moments l'emporter et surpasser ; mais l'excès lui même est petit, aussi est-ce considéré comme exprimant le petit excès. En toute chose il y a nécessairement besoin d'un excès dépassant la limite convenable pour amener ensuite la possibilité de liberté d'action ; c'est par là que l'excès peut entraîner la liberté d'action à sa suite.

TSHOU HI. - Explication du sens du nom du koua et de la formule au moyen de la substance du koua lui-même.

1081.

Surpasser par l'avantage de la pureté, agir suivant le moment.

TSHENG TSE. - Excès et avantage dans la pureté ; cela veut dire agir conformément au moment. Le moment nécessite un excès et il y a excès, alors ce n'est plus un excès, c'est la co nséquence nécessaire du moment, et c'est cela qui est appelé la droiture.

\footnotetext{
${ }^{1}$ C'est une règle d'interprétation d'un présage.

${ }^{2}$ Le cinquième trait du koua tshong fou, qui était positif a été remplacé, dans le koua siao kuo par un trait négatif.
} 
1082.

La malléabilité possède la justice, c'est ce qui fait que dans les petites choses le présage est heureux.

TSHOU HI. — Cela est dit au sujet du second et du cinquième traits.

1083.

La dureté énergique perd sa situation et manque de justice, c'est ce qui fait son incapacité dans les grandes choses.

TSHENG TSE. - La voie rationnelle du petit excès, c'est que l'excès dans les petites choses sera un présage heureux. Aussi, le commentaire de la formule déterminative parle du sens du présage heureux d'après les aptitudes exprimées par le koua. La malléabilité possède la justice : le second et le cinquième traits occupent le rang du milieu dans chaque koua simple. La douceur malléable négative possède la situation ${ }^{1}$, il peut en résulter un présage heureux dans les petites choses, mais cela ne peut être suffisant pour les choses importantes. La dureté énergique perd la situation et manque de justice, c'est ce qui la rend incapable de grandes choses. Sans les aptitudes de la dureté énergique il est impossible d'arriver aux affaires graves et importantes. Le troisième trait est sans justice, le quatrième perd sa situation ; c'est par là qu’ils sont incapables de grandes choses. Dans le moment indiqué par le petit excès, il y a nécessairement incapacité d'accomplir de grandes choses et, de plus, les aptitudes du koua sont également au-dessous d'une tâche importante, ce qui concorde avec le moment.

TSHOU HI. - Cela est dit au sujet du troisième et du quatrième traits.

1084.

Il y a l'image symbolique de l'oiseau qui vole. Le son laissé par l'oiseau qui vole ne doit pas monter, il doit descendre; c'est un grand présage heureux. Monter c'est la rébellion et descendre c'est la soumission.

\footnotetext{
${ }^{1}$ Le cinquième rang.
} 
TSHENG TSE. - Il y a l'image symbolique de l'oiseau qui vole ; cette phrase n'a pas de rapport avec la substance du commentaire de la formule déterminative et c'est, en effet, une phrase tirée d'un commentateur et introduite par erreur dans le commentaire de la formule déterminative. Au-dedans énergie, au-dehors douceur ; c'est l'image symbolique de l'oiseau volant. Le koua a cette image symbolique, de sorte que, visant cette circonstance, on considère que l'oiseau qui vole constitue le sens exprimé par le koua. Dans toutes choses, il y a des moments où il convient de dépasser les limites ordinaires et c'est par là qu'il est possible de profiter de l'opportunité ; mais comment l'excès pourrait -il être considérable ! Par exemple l'excès dans les marques de respect extérieur, l'excès de douleur dans le deuil, l'excès dans l'économie ne sont pas supportables s'ils sont très considérables, et c'est à cause de cela qu’il s'agit de petit excès, un excès dans les petites choses. Ce qui est en excès doit être comme le son laissé derrière lui par l'oiseau qui vole. Lorsque l'oiseau vole rapidement, son corps a déjà quitté la place où le bruit de ses ailes se produit, cependant il serait impossible que le corps et le bruit fussent très éloignés l'un de l'autre. Il en est encore de même dans toutes les choses où il convient de dépasser le niveau ordinaire ; le corps ne peut être très éloigné du bruit qu'il produit, les choses ne peuvent pas dépasser de beaucoup la proportion ordinaire ; c'est en cela que consiste la convenance. Ce qui ne convient pas en haut convient en bas ; cela se rapporte encore plus directement au bruit de l'oiseau et précise le sens de l'opportunité de l'obéissance. La voie rationnelle dans l'excès doit être comme le bruit que l'oiseau qui vole laisse derrière lui. Or, si le bruit, contrairement à la loi naturelle, monte et s'élève, cela indique une difficulté ; si au contraire, il se conforme passivement à cette loi et descend, ce sera l'indica tion d'une chose aisée et facile. Aussi dans ce qui est élevé, il y aura grandeur. Sur la montagne il y a la foudre ; c'est ce qui constitue l'excès. Dans l'excès, la voie rationnelle est que l'obéissance et la soumission comportent un présage heureux, comme le son laissé derrière lui par l'oiseau en vol ant, qui doit être la suite naturelle du vol. Ce qui constitue l'excès, c'est alors la soumission aux convenances du moment. S'il peut y avoir soumis sion à l'opportunité, c'est ce qui constitue la grandeur du présage heureux.

TSHOU HI. - Cela est dit au sujet de la substance du koua.

1085.

Le commentaire de la formule symbolique dit: Sur la montagne il y a la foudre; petit excès. L'homme doué emploie l'excès dans la dignité de sa contenance extérieure ; l'excès de douleur dans le deuil ; l'excès d'économie dans la satisfaction de ses besoins. 
TSHENG TSE. - La foudre ébranle sur la montagne, son bruit est extraordinaire, c'est pour cela que le koua est considéré comme exprimant un petit excès. Dans les affaires de l'univers, il y a des moments où l'exc ès convient, mais cet excès ne doit pas être très considérable, ce qui constitue la petitesse de l'excès. L'homme doué considère l'image symbo lique du petit excès : dans les choses où il convient de dépasser le niveau moyen, il s'effor ce de le dépasser; dans sa conduite et ses actions il pousse à l'excès les marques de respect dans son attitude extérieure ; dans le deuil, il pousse à l'excès la douleur ; dans la satisfaction de ses besoins, il pousse l'économie à l'excès. Tel est le sens. Lorsque l'exc ès est convenable, il dépasse le niveau ordinaire, c'est là ce qui convient ; mettre de l'excès là où l'excès ne convient pas, c'est une faute.

TSHOU HI. - Sur la montagne il y a la foudre ; son bruit est légèrement excessif. Dans les trois cas d'excès cités dans le texte, il s'agit toujours d'excès dans ce qui est petit. L'excès est supportable dans les petites choses et il ne le serait pas dans les grandes; on peut commettre un léger excès et on ne doit pas pousser cet excès à un point considérable : c'est là ce qu'exprime le commentaire de la formule déterminative en disant qu'il convient dans les petites choses et qu'il est opportun chez les inférieurs. 


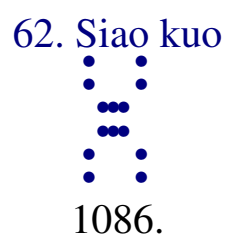

Premier trait hexaire : l'oiseau qui vole est généralement un présage malheureux.

TSHENG TSE. — Le premier trait hexaire est malléable et négatif et il se trouve au rang inférieur: c'est l'image symbolique de l'homme inférieur. De plus, il s'élève sympathi quement vers le quatrième trait. Le quatrième trait fait en outre partie de la substance du mouvement ${ }^{1}$. Lhomme inférieur est brusque et léger et il rencontre de l'aide et du concours au-dessus de lui ; dans les choses où il conviendrait de dépasser le niveau ordinaire, il arrivera nécessairement à commettre un excès considérable ; combien encore à plus forte raison, lorsqu'il n'y a pas lieu de dépasser ce niveau et que néanmoins il le dépasse ! L'excès dans ses actions est comme la rapidité et la précipitation dans le vol de l'oiseau, et c'est par là que le présage est malheureux. Une telle brusquerie unie à tant de précipitation constitue la rapidité dans l'excès et son extension lointaine : on n'est jamais à temps pour y remédier et le contenir.

TSHOU HI. - Le premier trait hexaire est malléable et négatif ; il s'élève sympathiquement vers le quatrième trait positif ; de plus, il se trouve dans un moment qui comporte l'excès : c'est celui qui, étant dans une position élevée, ne sait pas s'abaisser. Le bruit lais sé derrière lui par l'oiseau en volant ne doit pas monter et il doit descendre; c'est pour cela que tels sont limage symbolique et le sens divinatoire. Un commentateur dit que, lorsqu'on rencontre ce trait en consultant le sort, il peut indiquer un désastre qui surviendra par le fait d'un insecte ailé.

1087.

L'oiseau qui vole est généralement un présage de malheur. On ne peut y remédier d'aucune manière.

TSHENG TSE. — Linconvénient de l'excès est rapide comme le vol de l'oiseau : comment y remédier et l'arrêter ? Le présage malheureux en est la conséquence naturelle, et on ne peut y remédier d'aucune manière ; la force est impuissante.

\footnotetext{
${ }^{1}$ Koua simple tshen.
} 


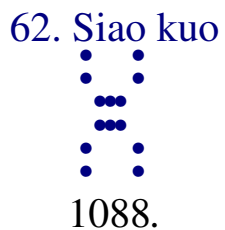

Deuxième trait hexaire : dépasser l'aïeul ; rencontrer l'ä̈eule ; ne pas atteindre le prince ; rencontrer le sujet ; pas de culpabilité.

TSHENG TSE. - La position de la positivité dans un rang supérieur donne l'image symbolique du père. Ce qui est prééminent par rapport au père, donne l'image symbolique de l'aïeul. Le quatrième trait est au -dessus du troisième, de sorte qu’il est considéré comme représentant l'aïeul. Le second et le cinquième traits occupent des rangs et des situations qui se correspondent et concordent; ils ont de même les vertus de la justice et de la malléabilité. Les tendances du second trait ne le portent ni vers le troisième, ni vers le quatrième trait, aussi il dépasse le quatrième et se porte vers le cinquième, c'est là dépasser l'aïeul. Le cinquième trait est malléable et prééminent, c'est l'image symbolique de l'aïeule ; il a les mêmes vertus que le second trait et ils se correspondent sympathiquement. Dans d'autres koua, cela indiquer ait que la négativité et la positivité s'appellent réciproquement, mais dans le moment de l'excès, les conséquences doivent dépasser les limites ordinaires, aussi le sens est-il différent. Rien n'est sans excès, aussi le second suit le cinquième et c'est e ncore un avertissement au sujet de l'excès. Ne pas atteindre le prince et rencontrer le sujet, c'est-à-dire, avancer en montant mais sans empiéter sur les droits du prince et en suivant exactement la voie rationnelle du sujet ; dans ces conditions, il n'y aura aucune culpabilité. Le terme rencontrer, du texte, veut dire ici « conforme à... »; conforme à la condition de sujet ; s'il outrepassait les droits qui correspondent à la condition du sujet, la culpabilité serait évidente.

TSHOU HI. - Le second trait hexaire est donc obéissant, juste et droit. S’il avance il dépasse le troisième et le quatrième rangs et rencontre le cinquième trait hexaire ; c'est là dépasser la positivité et atteindre la négativité qu'il rencontre. Dans ces circonstances, il atteind ra naturellement à sa propre condition sans atteindre le cinquième trait hexaire ; c'est là ne pas atteindre le prince et se conformer exactement à la condition de sujet. C'est toujours dépasser, mais sans commettre d'excès et cela comporte l'idée de se ma intenir dans la droiture en observant la justice ; c'est une voie rationnelle qui indique l'absence de toute culpabilité et c'est pour cela que tels sont l'image symbolique et le sens divinatoire. 
Ne pas atteindre le prince; le sujet ne doit pas le dépasser.

TSHENG TSE. - Dans le moment de l'excès, il n'est rien qui ne dépasse les limites ordinaires; aussi, s'il s'agit d'avancer en montant, il y aura l'avertissement relatif au fait d'atteindre le prince. Le sujet ne doit pas dépasser la condition de sujet.

TSHOU HI. - Ce qui fait qu'il n'atteint pas le prince et quil revient vers le rang de sujet, c'est que le sujet ne doit pas outrepasser ses droits. 


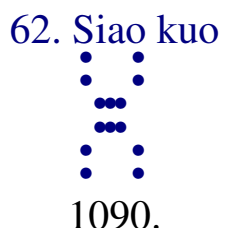

Troisième trait nonaire : ne s'en gardant point jusqu'à l' excès; suivre et peut-être le tuer; présage malheureux.

TSHENG TSE. - Le koua siao kuo marque le moment où la négativité dépasse la positivité qui perd la situation. Le troisième trait, seul, observe la droiture, mais il est dans une position inférieure, il n'est pas capable de rien faire et il est détesté et haï par la négativité, de sorte que la chose dans laquelle il est convenable qu’il pousse jusqu'au -delà des limites ordinaires, c'est l'excès de préc aution contre les hommes inférieurs. S’il ne s'en garde pas jusqu'à l'excès, peut -être qu'ils le suivront et le tueront, de sorte qu'ainsi le présage est malheureux. Le troisième trait, pendant le moment marqué par l'excès de la négativi té, emploie la positivité et occupe un rang qui comporte la dureté énergique : c'est un excès dans l'énergie. Après l'avertissement au sujet de l'excès dans la précaution pour se garder, l'excès dans l'énergie donne encore lieu à un nouvel avertissement. La voie rationnelle pour se garder contre les hommes inférieurs, consiste d'abord dans le redres sement de sa propre personne ; le troisième trait ne manque pas à la droiture, aussi le trait ne comporte pas nécessairement le sens de présage malheureux; s'il peut prendre un excès de précaution en se gardant, il évitera le malheur. Le troisième trait occupe le rang supérieur dans le koua simple inférieur ; tout ce qui est supérieur entre les inférieurs, est dans des conditions analogues.

TSHOU HI. - Dans le moment du petit excès, l'excès est opportun dans chaque chose et c'est après cet excès que la juste limite se trouve atteinte. Le troisième trait nonaire emploie la dureté énergique et se maintient dans la droiture : c'est lui que la foule des négativités veut détruire. Mais il s'appuie et se fie sur sa seule énergie et il ne veut pas s'appuyer sur un excès de sa propre action, de sorte que tels sont l'image symbolique et le sens divinatoire. Si celui qui consulte le sort est capable d'un excès de précaution en se gardant, il pourra éviter le danger qui le menace.

1091.

Suivre et peut-être le tuer; comment le présage ne serait-il pas malheureux? 
TSHENG TSE. - Dans le moment de l'excès de la négativité, celle-ci doit infailliblement nuire à la positivité. La voie de l'homme inférieur florissant, il doit nuire à l'homme doué ; il convient de pousser à l'excès les mesures de précautions. Si ces mesures de précautions ne sont pas poussées à leur plus extrême limite, cela sera cause qu'il sera tué ${ }^{1}$, aussi la formule dit : comment le présage ne serait-il pas malheureux ? Cela veut dire «combien le présage est malheureux !»

\footnotetext{
${ }^{1}$ On ne sait trop à qui cela se rapporte ; à l'homme doué, sans doute.
} 


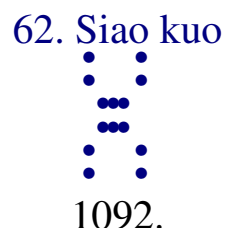

Quatrième trait nonaire : point de culpabilité ; le rencontrer sans le dépasser ; en entreprenant péril, avertissement nécessaire ; ne pas employer une pureté constante et éternelle.

TSHENG TSE. - Le quatrième trait se trouve en présence d'un moment de petit excès ; il emploie la dureté énergique et se place dans un rang qui comporte la douceur malléable : la dureté énergique n'est pas un excès, et c'est ainsi qu'il n'y a pas de culpabilité ; du moment où elle n'est pas en excès, c'est qu'elle est dans les limites convenables. C'est pour cela que la formule parle de rencontre, c'est-à-dire ce qui est conforme à la voie rationnelle. En entreprenant, il y aura péril, donc il est nécessaire d'avertir et de mettre en garde. Entreprendre, ce serait abandonner la douceur et se porter en avant en employant la dureté énergique. Ne pas employer une pureté éternelle ; la nature de la positivité est la dureté énergique et la fermeté, aussi la formule avertit au sujet de la nécessité de se conformer aux nécessités et aux convenances du moment, sans s'opiniâtrer dans une même manière d'agir. $\mathrm{Au}$ moment où commence l'excès de la négativité, où la dureté énergique positive perd sa situation, alors, l'homme doué doit se conformer au moment et se plier aux circonstances sans s'en tenir avec opiniâtreté à sa manière ordi naire d'agir. Le quatrième trait occupe une haute situation, et il est sans relation avec les traits supérieurs et inférieurs; bien quill soit proche du cinquième et qu'il sympathise avec le premier, dans le moment où commence l'excès de la négativité, comment ceux -là voudraient-ils consentir à suivre un trait positif ? Aussi, s'il entreprend, il sera en péril.

TSHOU HI. - En présence d'un moment où il convient de dépasser le niveau ordinaire, employer l'énergie et se placer dans un rang qui comporte la douceur, c'est ce qui constitue le fait de dépasser les limites ordinaires de la dignité manifestée dans les actions extérieures et la contenance. C'est une voie qui ne comporte aucune culpabilité. Le rencontrer sans le dépasser veut dire ne pas mettre d'excès dans la dureté é nergique et en déployer juste autant qu'il en faut dans les circonstances actuelles ; en allant au-delà, il y aurait excès, aussi ce serait périlleux et il convient d'avertir. La nature de la positivité est la dureté énergique et la fermeté, aussi la formule avertit encore de ne pas employer une perfection invariable, ce qui signifie qu'il faut suivre les nécessités du moment, sans s'atta cher avec une opiniâtreté invincible à une manière unique de voir et de faire. On a dit: le rencontrer sans le dépasser peut être interprété de deux façons : si c'est d'après les règles applicables dans le cas du second trait hexaire, l'interprétation sera conforme à ce qui vient 
d'être dit, mais si on suit les règles posées au sujet du troisième trait nonaire, alors les mots « excès » et « rencontrer » doivent comporter le même sens que l'expression « excès dans les mesures de précaution en se gardant». Ce point n'a pas encore été claire ment élucidé et il convient de réserver tout jugement à cet égard.

1093.

Le rencontrer sans le dépasser; situation imméritée; en entreprenant péril, il faut absolument avertir ; finalement cela ne peut durer longtemps.

TSHENG TSE. - La situation n'est pas méritée ; cela veut dire quill se place dans un rang qui comporte la douceur malléable. Le quatrième trait nonaire se trouve en présence d'un moment d'excès ; or non seulement il ne pousse pas la dureté énergique à l'excès, mais au contraire il se tient dans la douceur, ce qui est se conformer aux nécessités du moment. C'est pour ce la que la formule dit : «le rencontrer», rencontrer ce qui convient et s'y conformer. Puisque, nonaire, il occupe le quatrième rang, la situation ne convient pas ; se maintenir dans la douceur, c'est s'adapter aux nécessités du moment. En présence d'un moment d'excès de la négativité, la positivité se recule, et il lui suffit de se garer et de se garantir; comment, finalement, pourrait-elle se compléter et se développer? Aussi, en entreprenant il y aurait péril, et il faut absolument avertir et prévenir. Le caractère du texte (durer) doit être lu avec le ton sheang; si on lui donnait le ton p'ing (avec le sens de haut, grand, élevé), on s'écarterait grandement de l'idée du livre des change ments (Yi king). Cela peut se voir dans les koua kouae ${ }^{1}$ et $p^{2}{ }^{2}$. Le texte est le même que dans le commentaire symbolique du koua po, mais l'idée est différente.

TSHOU HI. — Le sens de la formule du trait n'est pas encore éclairci ; il faut aussi s'abs tenir sur ce passage.

${ }^{1} \mathrm{~N}^{\circ} 43$.

${ }^{2} \mathrm{~N}^{\circ} 23$. 


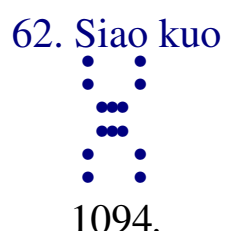

Cinquième trait hexaire : nuages épais sans pluie ; de nos plaines de l'ouest ; le kong ${ }^{1}$ lance une flèche et capture l'autre dans l'antre.

TSHENG TSE. - Le cinquième trait emploie la douceur malléable négative et occupe la situation prééminente ; bien qu'il désire faire quelque chose de plus ${ }^{2}$, comment serait-il capable d'achever son auvre ? Il en est encore comme des nuages épais qui ne peuvent produire de pluie ; ce qui les empêche de produire la pluie, c'est qu'ils proviennent des plaines de l'ouest. La négativité ne peut produire la pluie ; cela a déjà été expliqué dans le koua siao tshou ${ }^{3}$. Le kong lance une flèche et capture celui-là dans l'antre ; le douzième caractère du texte a le sens de lancer une flèche contre un but et de saisir ce but; le caractère ordinairement employé pour exprimer le tir au moyen de l'arc, n'exprime que l'action de lancer le trait, tandis que le douzième caractère a le sens de saisir, prendre. Antre, caverne dans une montagne, un creux évidé ; «situé dans l'antre » désigne le second trait. Le second et le cinquième ne sont pas essentiellement et naturellement en concordance sympathique; ici, l'un lance un trait et capture l'autre. Le cinquième trait est en possession de la situation, aussi il est désigné par le terme honorifique kong, c'est-à-dire celui qui est au-dessus des autres dignitaires revêtus de ce titre. Ils sont de même genre et se prennent mutuellement, mais bien que d'accord ensemble, comment deux négativités pourraient-elles accomplir de grandes choses ! Il en est encore d'elles comme de nuages épais qui ne peuvent pas produire de pluie.

TSHOU HI. - Employant la négativité et occupant un rang prééminent, de plus en présence d'un moment où la négativité doit l'empor ter et dépasser le niveau ordinaire, il ne peut rien y avoir à faire, il lance une flèche et capture le second trait pour s'en faire aider, ce qui produit cette image symbolique. «Dans l'antre », quelque chose de négatif; les deux négativités s'accordent ensemble, leur incapacité à produire de grandes choses est évidente.

1095.

\footnotetext{
1 Titre d'un dignitaire.

${ }^{2}$ Se distinguer par ses actions.

${ }^{3}$ Koua $n^{\circ} 9$.
} 
Nuages épais sans pluie ; déjà élevé.

TSHENG TSE. - La positivité descend, la négativité monte; en s'accordant elles produi raient l'harmonie et causeraient la pluie. Du momen $t$ où la négativité est déjà en haut, bien que les nuages soient épais, comment pourraient-ils produire la pluie ? Le sens est que lorsque la négativité l'emporte (dépasse) elle ne peut rien accomplir de grand.

TSHOU HI. — Déjà haut ; très élevé. 


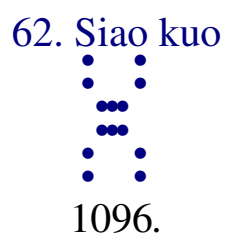

Trait supérieur hexaire : ne pas le rencontrer et le dépasser; l'oiseau qui vole s'en sépare ; présage malheureux ; c'est-à-dire calamités et maux.

TSHENG TSE. - Ce trait hexaire est négatif et il fait partie de la substance du koua simple tshen qui exprime le mouvement. Il est placé à l'extrême limite de l'excès, et il ne s'accorde pas avec la raison. Sill se meut, c'est toujours au -delà de ce qui conviendrait; ses dérogations à la juste raison d'être en toutes choses dépassent les limites ordinaires. C'est une agitation inquiète et précipitée comme le battement des ailes de l'oiseau qui vole, et c'est par là que le présage est malheureux. Se séparer, dépasser de loin. Calamités et maux ; c'est-à-dire que les calamités et les maux en sont la conséquence naturelle. Il s'agit des calamités et maux ou fléaux qui viennent soit du ciel, soit de l'action des hommes. Du moment où l'extrême limite de l'excès est dépassée, comment les conséquences pour raient-elles être limitées à des maux résultant du fait des hommes ? Il est évident que les fléaux célestes doivent également survenir de sorte que la raison d'être du ciel et les actions humaines concourent au même but.

TSHOU HI. - Le sixième trait emploie la négativité et occupe le rang supérieur dans la substance du mouvement; il se place au comble de l'excès de la négativité. Cet excès est déjà élevé et dépasse de loin les bornes ordinaires. C'est pour cela que tels sont le sens divinatoire et l'image symbolique. On a dit au sujet des quatrième et cinquième caractères du texte ${ }^{1}$, « rencontrer et dépasser», que peut-être il y a lieu de supposer qu’il fau drait lire «dépasser et rencontrer » et que le sens est le même que dans le cas du quatrième trait nonaire. On ne sait encore s’il en est ainsi ou non.

1097.

Ne pas le rencontrer, le dépasser; déjà dépassé.

\footnotetext{
${ }^{1}$ Le premier caractère signifie rencontrer, et le second dépasser.
} 
TSHENG TSE. - Il occupe le dernier rang dans l'excès ; il ne s'accorde pas à la raison d'être des choses ${ }^{1}$ et la dépasse ; l'excès est déjà arrivé à sa dernière limite, le présage malheureux en est la conséquence naturelle.

${ }^{1}$ Littéralement « il ne rencontre pas ». 


\section{Ki tsi}

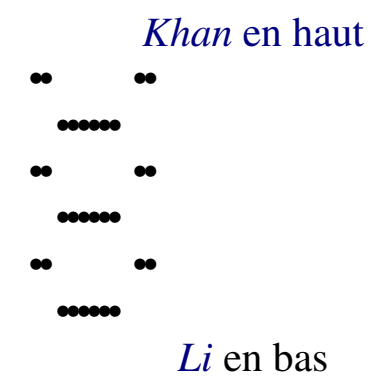

1098. Ki tsi ; liberté dans ce qui est petit ; avantage de la perfection ; au début présage heureux, à la fin désordre.

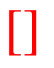

1104. Premier trait nonaire : tirer la roue en arrière ; mouiller la queиe; pas de culpabilité.

$*$

1106. Deuxième trait hexaire : l'épouse oublie les stores ; ne pas poursuivre; après sept jours réussir.

*

1108. Troisième trait nonaire : Kao Tsong détruit le peuple des Kouei fang ; en trois ans il le dompte; l'homme inférieur est sans effet.

$*$

1110. Quatrième trait hexaire : de couleur sombre avoir un vêtement déchiré ; à la fin du jour, avertissement.

$*$

1112. Cinquième trait nonaire : le hameau oriental tue un bauf; cela ne vaut pas le petit sacrifice du hameau occidental ; il reçoit certainement le bonheur.

$*$

1114. Trait supérieur hexaire : mouiller la tête ; péril.

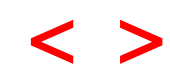




\section{Ki tsi.}

TSHENG TSE. - Ki tsi. «L'O rdre des koua » dit : «Celui qui a plus qu'autrui doit prêter assistance, aussi le koua siao kuo est suivi du koua $k i$ tsi. » Ses facultés dépassent celle des êtres, il doit les employer pour prêter son aide, et c'est pour cela que, à la suite du koua siao kuo se trouve placé le koua $k i$ tsi. Il est constitué par l'eau placée au-dessus du feu; l'eau et le feu se joignant produisent leur effet. Chacun de ces deux éléments suffit à son usage, de sorte que le koua est considéré comme exprimant que chaque chose est déjà mise en ordre ; c'est le moment où toutes les choses de l'univers sont déjà régularisées. 


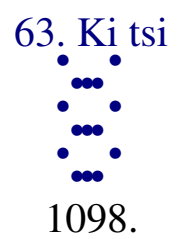

Ki tsi ; liberté dans ce qui est petit ; avantage de la perfection ; I au début présage heureux, à la fin désordre.

TSHENG TSE. - Dans le moment exprimé par le koua ki tsi, ce qui est grand jouit déjà de liberté ; ce qui est petit est encore dénué de liberté. Bien que dans le moment exprimé par ce koua ${ }^{1}$ il n'est pas possible qu’il n'existe pas encore quelque manque de liberté au sujet de petites choses. Dans le texte le quatrième caractère siao, petit, est placé après le troisième caractère heng, liberté ; c'est l'ordre naturel dans le langage. Si le texte portait siao heng; siao qualifierait le mot heng et on devrait lire «petite liberté ». Avantage de la perfection; placé dans le moment de l'ordre déjà rétabli, l'avantage consiste dans l'emploi d'une parfaite fermeté pour le maintenir et le conserver. $\mathrm{Au}$ début, le présage est heureux ; c'est-à-dire au moment où l'ordre commence à être établi. Finalement désordre ; quand le rétablissement de l'ordre est arrivé à sa dernière limite, il y a renversement dans la voie opposée.

TSHOU HI. - Ki tsi, « déjà rétabli », c'est-à-dire quand une chose est terminée. Le koua est composé par l'eau et le feu se joignant ensemble, chacun pouvant produire son effet ${ }^{2}$. La situation de chacun des six traits correspond dans chaque cas à la nature positive ou négative de ces traits, selon la droiture, aussi le koua exprime que l'ordre est établi. Au lieu de "heng siao » on doit dire «siao heng». Dans la plupart des cas, la formule de ce koua ainsi que les formules divinatoires des six traits comportent presque toujours une idée d'avertissement et de prudence: c'est la conséquence naturelle du moment dont il s'agit.

1099.

Le commentaire traditionnel de la formule déterminative dit: $\mathrm{Ki}$ tsi; liberté ; ce qui est petit jouit de la liberté.

\footnotetext{
${ }^{1}$ Dont le nom peut se traduire par « ordre déjà rétabli ».

${ }^{2}$ Par leur ordre de superposition.
} 
TSHOU HI. - Au-dessous du caractère $t s i$, il semble qu'il manque le caractère siao, petit.

1100.

Avantage de la perfection; énergie et douceur placées suivant la droiture et situation méritées.

TSHENG TSE. — Dans le moment où l'ordre est rétabli ( $k i$ tsi) ce qui est grand jouit certainement déjà de la liberté ; il n'y a que ce qui est petit qui n'en jouit pas encore. Du moment où le temps comporte déjà la régularité, il convient certainement de s'attacher avec fermeté à conserver l'ordre déjà obtenu. Les aptitudes du koua, énergie, ou malléabilité, correspondent dans chaque cas à la situation occupée. La règle constante est que chaque trait mérite la situation qu'il occupe et correspond aux nécessités de cette situa tion, ce qui donne le sens de droiture et de fermeté. L'avantage consiste dans une telle perfection ; la négativité et la positivité, possèdent chacune la situation qui leur revient selon les règles de la droiture, et c'est par là que le koua exprime l'ordre déjà établi.

TSHOU HI. - Cela est dit au sujet de la substance du koua.

1101.

Au début présage heureux; la douceur possède la justice.

TSHENG TSE. - Le second trait emploie la douceur et la soumission, l'élégance de la forme, et il possède la justice, aussi il peut accomplir l'œuvre du rétablissement de l'ordre en toutes choses. Le second trait fait partie de la substance du koua simple inférieur ; c'est lui qui marque le commencement du moment où l'ordre est nouvellement établi. De plus encore, il se place suivant la loi du bien, c'est ce qui rend le présage heureux.

TSHOU HI. - Cela désigne le second trait hexaire. 
Finalement il s'arrête, alors il y a désordre; la voie rationnelle du koua est arrivée à sa limite finale ${ }^{1}$.

TSHENG TSE. - Pour toutes les choses de l'univers, lorsqu'il n'y a plus progrès en avant il y a régression en arrière ; aucun ne comporte une raison d'être de permanence dans un niveau déterminé. À la fin du rétablissement de l'ordre, il n'y a plus progrès en avant et il y a arrêt ; mais l'arrêt ne peut avoir de permanence et le désordre et les troubles surviennent. C'est qu'en effet la voie rationnelle du koua, parvenue à sa limite extrême, est épuisée. Les aptitudes du cinquième trait nonaire sont loin de ne point êtres bonnes, mais le moment est passé, la voie rationnelle est épuisée, la raison d'être des chos es exige nécessairement une modification. Que fera l'homme saint dans de telles circonstances ? Réponse : L'homme saint seul est en état de comprendre la modification avant même que la voie rationnelle ne soit complètement épuisée ; il n'est pas dans la nécessité d'attendre jusqu'à cette dernière limite. Tels furent Yao et Shouen; aussi, il peut arriver que la fin du moment considéré arrive sans qu'il se produise de désordre.

1103.

Le commentaire traditionnel de la formule symbolique dit: L'eau est au-dessus du feu; ki tsi, l'ordre est rétabli. L'homme doué en conclut qu'il doit songer aux malheurs et les prévenir à l'avance.

TSHENG TSE. - Du moment où l'eau et le feu se joignent déjà, et où chacun de ces deux éléments produit son effet, cela constitue la régularité déjà rétablie. Le moment étant celui où la régularité vient d'être rétablie, il n'y a d'autres préoccupations que la prévision des maux ou des dangers qui peuvent surgir, aussi en y pensant on les prévient, et on les empêche d'arriver jusqu'au point où ils produiraient le mal. Depuis l'antiquité, quand l'ordre est rétabli dans l'univers, s'il survient des calamités, c'est que l'on a été incapable de les prévoir et d'y aviser à l'avance pour s'en garantir.

\footnotetext{
${ }^{1}$ Et elle change.
} 


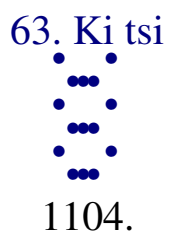

Premier trait nonaire : tirer la roue en arrière ; mouiller la queue; pas de culpabilité.

TSHENG TSE. - Le premier trait, avec sa positivité, occupe le rang inférieur ; en haut, il sympathise avec le quatrième. De plus, il fait partie de la substance du feu ; ses tendances à se porter en avant sont vives et décidées. Mais, du moment où l'instant dont il s'agit com porte la régularité déjà rétablie, le mouvement de progression en avant qui ne cesse point doit conduire à des regrets et à la culpabilité. Aussi, «tirer la roue en arrière », « mouiller la queue » expriment le moyen d'éviter la culpabilité. La roue, est ce qui fait avancer (le char?), elle est tirée à l'envers pour arrêter le mouvement en avant. Lorsque les quadrupèdes traversent un cours d'eau à la nage, ils sont obligés de relever la queue hors de l'eau ; s'ils mouillent leur queue ils ne peuvent plus nager ${ }^{1}$. Au début du rétablissement de l'ordre, pouvoir arrêter le mouvement en avant, c'est pouvoir éviter toute culpabilité ; ceux qui sont incapables de s'arrêter en viendront forcément à être coupables.

TSHOU HI. - La roue est au-dessous (du char ?), la queue est par derrière: c'est l'image symbolique du premier trait. En tirant la roue en arrière, le char n'avance plus, en mouillant sa queue, le second ne peut plus traverser les cours d'eau. Au début du rétablissement de l'ordre, si l'on peut être ainsi circonspect et en éveil, ce sera une voie rationnelle d'absence de culpabilité. Si celui qui consulte le sort est dans ces mêmes conditions, il sera sans culpabilité.

1105 .

Tirer la roue en arrière; le sens est l'absence de culpabilité.

TSHENG TSE. - Au début, du moment où la régularité est établie, et étant capable d'arrêter son propre mouvement en avant, il ne poussera pas les choses à l'extrême. Le sens comporte naturellement l'absence de culpabilité.

\footnotetext{
${ }^{1}$ Ceux qui expliquent ce passage citent l'écureuil, le renard, etc., comme exemples.
} 


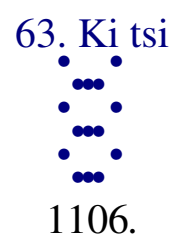

Deuxième trait hexaire : l'épouse oublie les stores ; ne pas poursuivre ; après sept jours réussir ${ }^{1}$.

TSHENG TSE. — Le second trait emploie les vertus de l'élégance de la forme, de la justice et de la droiture ; en haut, il sympathise avec le prince doué de l'énergie positive, de la justice et de la droiture, représenté par le cinquième trait nonaire. Il est naturel quîl parvienne à faire prévaloir ses tendances. Cependant, du moment où le cinquième trait occupe la situation prééminente, le moment comportant déjà la régularité établie, même sans continuer à avancer davantage, son action peut néanmoins se produire et s'exercer ; comment donc, alors, aurait-il l'idée de s'adresser au sage capable placé dans l'infériori té, pour l'employer ? C'est pour cela que le second ne doit pas réussir à donner libre cours à ses propres actions. Depuis l'antiquité, les princes qui, l'ordre une fois rétabli, ont été capables d'employer les hommes, ont toujours été rares. Si le premier fondateur de la dynastie des Theang fut capable d'écouter les avis, il fut toujours long à les suivre ; cela ne fit que s'aggraver avec le temps et les générations. Dans un tel moment, l'énergie et la jus tice se dénaturent et deviennent de la suffisance. Les koua simples khan et li sont considérés ici comme constituant ensemble une antithèse. L'homme capable de reconnaître le moment et de savoir la modification qui s'accomplit peut être considéré comme capable de parler des changements (Yi king). Le second trait est négatif, aussi il en est question comme représentant une femme. Le sixième caractère du texte traduit par « stores » désigne un écran que les femmes emportent, lorsqu'elles sortent, pour se dérober aux regards, « oublier l'écran », cela indique limpossibilité où elle se trouve de sortir. Le second trait n'étant ni appelé, ni employé par le cinquième, ne peut agir ; il est comme une femme qui a oublié son écran. Toutefois, la voie rationnelle de la justice et de la droiture ne peut jamais être oubliée et abandonnée : ce moment passé, il agira. Poursuivre, courir après quelque être. En poursuivant les êtres, il négligerait l'observation de soimême, aussi la formule avertit de ne pas poursuivre, et de s'observer soi même sans commettre aucune négligence, et alors, après sept jours, il pourra de nouveau réussir. Les koua comportent six situations, donc le nombre sept indique qu'il survient une modifica tion et que le koua se transforme, " après sept jours, réussir » est une expression qui indique que le moment se modifie. Bien qu'il ne soit pas employé par le supérieur, la voie rationnelle de la jus tice et de la droiture ne peut jamais être définitivement abandonnée et oubliée ; il

\footnotetext{
${ }^{1}$ Il faut sous-entendre le mot « après » qui n'est pas dans le texte.
} 
lui est impossible d'agir présentement, donc il agira dans un autre temps les exhortations de l'homme saint sont profondes ?

TSHOU HI. - Le second possède les vertus de l'élégance de la forme, de la justice et de la droiture; en haut, il sympathise avec le prince doué d'énergie positive, de justice et de droiture, représenté par le cinquième trait nonaire ; il est naturel qu'il réussisse à agir et à faire prévaloir ses ten dances. Mais, du moment où le cinquième trait nonaire se trouve dans un moment où l'ordre est déjà rétabli, il est incapable de s’incliner devant les sages pour pratiquer et suivre leur voie rationnelle. Aussi le second comporte l'image symbolique d'une fe mme qui oublie son écran. Le terme «écran » désigne les stores de la voiture d'une femme ; cela exprime qu'il perd ce qui lui permet de circuler et de sortir. Cependant la voie rationnelle de la justice et de la droiture, ne peut être définitivement abandonnée, le moment passé, elle prévaudra de nouveau. Aussi, la formule comporte encore l'avertissement de ne pas poursuivre et que le succès viendra naturellement.

1107.

Après sept jours réussir ${ }^{1}$; par la voie rationnelle de la justice.

TSHENG TSE. - Bien que la voie rationnelle de la justice et de la droiture ne soit pas utilisable dans le moment considéré, cependant, aucune raison ne peut faire qu'elle soit définitivement impraticable. Aussi après avoir oublié l'écran pendant sept jours, on doit le retrouver; cela veut dire que, observant soi-même la justice, dans un autre temps elle prévaudra nécessairement ; ne pas perdre la justice constitue la droiture.

\footnotetext{
${ }^{1}$ Littéralement « sept jours, réussir ».
} 


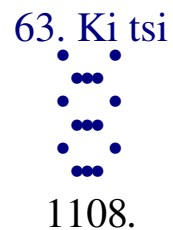

Troisième trait nonaire : Kao Tsong détruit le peuple des Kouei fang ; en trois ans il le dompte ; l'homme inférieur est sans effet ${ }^{1}$.

TSHENG TSE. - Le troisième trait nonaire se trouve en face d'un moment où l'ordre est déjà rétabli ; il emploie la dureté énergique et il occupe un rang qui comporte la dureté énergique ; c'est l'extrême degré de l'emploi de la dureté énergique. L'ordre étant déjà établi, et employant une telle dureté énergique, cela rappelle le fait de Kao Tsong détruisant le peuple des Kouei fang. Kao Tsong ${ }^{2}$, désigne certainement Kao Tsong de la dynastie des Sheang. Les affaires de l'empire étant déjà en ordre, il poursuivit au loin et détruisit les rebelles à son autorité. L'autorité et la puissance des armes, capables d'atteindre, et guidées par le désir d'assurer le bonheur du peuple, tel est le fait du prince; il n'y a que les princes sages et saints qui en soient capables. Mais la violence irréfrénée dans la répression, l'emportement contre ceux qui ne sont pas encore soumis, l'ambition de domi ner sur de vastes territoires conduisent à la destruction des peuples et à la satisfaction injuste des passions. C'est à cause de cela que la formule contient l'avertis sement de ne pas employer les hommes inférieurs ${ }^{3}$; si c'est un homme inférieur qui agit, il ne se laissera conduire que par ses ressentiments personnels et son intérêt privé ; s'il n'est pas poussé par son ambition et par sa colère il ne voudra rien faire. «En trois ans il le dompte»; ceci indique l'extrême difficulté que présente la tâche à accomplir. L'homme saint montre que le troisième trait nonaire est en présence d'un moment où l'ordre est déjà rétabli et emploie néanmoins la dureté énergique; il développe cette idée afin d'enseigner aux hommes ce qui constitue les règles et les av ertissements; comment pourrait-il considérer avec légèreté l'extension possible d'une telle action et sa portée lointaine ?

TSHOU HI. - Dans un moment où l'ordre est déjà établi, employer la dureté énergique et occuper un rang qui comporte la dureté énergique ; cela exprime l'image symbolique de Kao Tsong détruisant le peuple des Kouei fang. «En trois ans il le dompte »; cela exprime la durée de l'action et ensuite le succès. C'est un avertissement à celui qui consulte le sort qu'il ne doit pas songer à se mouvoir à la légère. «Ne pas employer l'homme inférieur »; la règle de divination est la même que dans le cas du trait supérieur hexaire du koua $s h i^{4}$.

\footnotetext{
${ }^{1}$ Littéralement « l'homme inférieur ne l'emploie pas ».

${ }^{2}$ Kao Tsong, souche élevée, ou illustre, appellation du premier fondateur d'une dynastie.

${ }^{3}$ Ou de ne pas appliquer ce sens, lorsqu'il s'agit d'hommes infé rieurs.

${ }^{4}$ Koua $n^{\circ} 7, \S 170$.
} 
1109.

En trois ans il vient à bout; fatigue pénible.

TSHENG TSE. — La formule dit : «fatigue pénible », afin de montrer l'extrême difficulté du fait en question. Lorsque c'est Kao Tsong qui agit, le fait devient possible; sans le génie et le cœur de Kao Tsong ce ne serait que passions et colères pour détruire le peuple. 


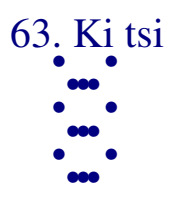

1110.

Quatrième trait hexaire : de couleur sombre avoir un vêtement déchiré ${ }^{1}$; à la fin du jour, avertissement.

TSHENG TSE. - Le quatrième trait fait partie du koua $t s i^{2}$ et du koua simple qui exprime l'eau, aussi c'es t le sens de barque, ou bateau, qui est relevé et mis en lumière. Le quatrième trait est près de la situation du prince ; il représente celui qui est dépositaire de l'autorité ${ }^{3}$. En présence du moment où l'ordre est déjà établi, ce qui est urgent, c'est de se mettre en garde contre les revers de la fortune et de prévoir les troubles et les révolutions, afin de les prévenir. Le troisième caractère du texte, « soie foncée ${ }^{4}$ », doit être remplacé par le caractère, «être mouillé »; cela exprime une fuite ou voie d'eau qui entraîne la submersion. La barque est percée d'un trou par lequel elle fait de l'eau, alors on le bouche avec des habits déchirés. Il y a un habit déchiré pour boucher la voie d'eau. De plus, jusqu'à la fin du jour la circonspection craintive ne se relâche point : telle doit être la prévoyance contre les dangers et les maux. Il n'est pas question de présage heureux ; c'est quill s'agit d'un moment où l'on est à peine soustrait au danger. Dans le moment où l'ordre vient d'être rétabli, c'est déjà assez d'être soustrait au danger ; que pourrait-on encore ajouter de plus?

TSHOU HI. - Dans le moment où l'ordre vient d'être rétabli, ce trait emploie la douceur malléable et occupe un rang qui comporte cette même douceur; il représente celui qui peut se tenir sur ses gardes et prévenir les maux en les redoutant; c'est pour cela que telle est l'image symbolique. Tsheng Tse dit: Le troisième caractère du texte doit être remplacé par un caractère analogue qui veut dire être mouillé ; l'habit déchiré, c'est ce qui sert à aveugler la voie d'eau pour empêcher la barque de couler.

1111.

\footnotetext{
1 Aucun sens apparent ; voir l'interprétation des commentateurs chinois.

${ }^{2}$ Littéralement « traverser à la nage ».

${ }^{3}$ Déléguée par le prince.

${ }^{4} \mathrm{Ou}$ bien fil à coudre.
} 
Le jour fini avertir; il y a lieu de craindre ${ }^{1 .}$

TSHENG TSE. - Jusqu'à la fin du jour être en éveil et craindre ; suspecter continuellement l'imminence du danger. Étant placé dans un moment où l'ordre est déjà rétabli, il faut être ainsi craintif et circonspect.

${ }^{1}$ Ou de $\ll$ douter $»$. 


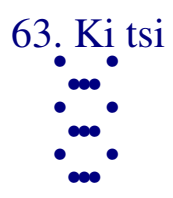

1112.

Cinquième trait nonaire : le hameau oriental tue un bauf; cela ne vaut pas le petit sacrifice du hameau occidental; il reçoit certainement le bonheur.

TSHENG TSE. — Lintérieur du cinquième trait est plein : bonne foi ; le second est vide au milieu: sincérité. Aussi, tous deux prennent un sens se rapportant aux sacrifices des divers ordres. Hameau oriental, positivité ; cela désigne le cinquième trait. Hameau occidental, négativité ${ }^{1}$; cela désigne le second trait. Tuer un bouf ; cela désigne un sacrifice complet et parfait; au contraire, les mots traduits par petits sacrifices désignent plusieurs genres de sacrifices plus modestes. Ce qui est complet ne vaut pas ce qui est superficiel, cela tient à la différence qui existe entre les moments considérés. Le second et le cinquième traits ont, tous deux également, les vertus de la bonne foi, de la sincérité, de la justice et de la droiture. Le second est en bas du koua tsi (traverser à la nage), il a encore à avancer, aussi il obtient le bonheur; le cinquième est placé au faîte du même koua (ordre déjà établi), il n’a plus aucun motif d'avancer. C'est à cause de la plus extrême sincérité qu'il apporte à se maintenir dans la droiture et la justice, qu'il n'en arriv e pas encore à un renversement de l'ordre établi ; la raison d'être des choses ne comporte pas l'extrême déve loppement d'une action sans un renversement final dans l'ordre inverse ; étant déjà parvenu à l'extrême limite, et bien que placé pour le mieux, pourquoi échapperait-il à cette règle ? Aussi la formule du trait et le commentaire symbolique ne parlent que du moment.

. TSHOU HI. - Orient, positivité ; occident, négativité ; il s'agit du cinquième trait nonaire qui occupe le rang prééminent alors que le moment est déjà passé ${ }^{2}$. Ces conditions ne valent pas celles où se trouve le second trait hexaire, placé dans l'infériorité, qui commence seulement à jouir des avantages du moment exprimé. De plus, ceci correspond aux événements relatifs à Wen Wang et à Tshieou, aussi le sens symbolique et le sens divinatoire sont tels. Dans la formule déterminative les mots « d'abord présage heureux, ensuite désordre et troubles » comportent la même idée.

1113.

\footnotetext{
${ }^{1}$ Au lieu de hameau on peut lire « région».

${ }^{2}$ Le moment exprimé par le koua.
} 
Le hameau oriental tue le bauf; cela ne vaut pas le moment du hameau occidental; il reçoit certainement le bonheur; les présages heureux arrivent et grandissent.

TSHENG TSE. - Les aptitudes et les vertus du cinquième trait sont loin de ne pas être bonnes, mais le moment n'est pas comparable à celui qui est exprimé par le second trait. Le second trait est en bas et dans le moment où il lui faut avancer, aussi il est animé de bonne foi, de justice et de droiture, de sorte que le présage heureux vient et grandit ; c'est ce qui est exprimé par le mot «recevoir le bonheur». Ce qui fait que le présage heureux vient et grandit, c'est que le moment de l'ordre déjà établi est considéré comme expri mant la grandeur qui vient et approche ; c'est par exemple la liberté dans les petites choses et le présage heureux du premier trait. 


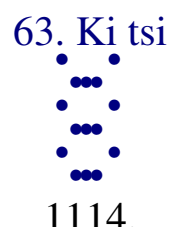

Trait supérieur hexaire : mouiller la tête ; péril.

TSHENG TSE. - Au dernier degré de la régularité établie, le calme est certainement loin de régner et le péril est proche. De plus la malléabilité négative occupe ce rang et elle est placée au rang supérieur de la substance (du koua simple) qui exprime le péril. Le koua simple khan représente l'eau ; le caractère tsi, du texte, comporte aussi un sens relatif à l'eau ${ }^{1}$, aussi le trait exprime l'acuité extrême du péril qui ne va pas à moins qu'à submerger la tête. Le péril est évident ; c'est la fin de l'ordre établi, et un homme inférieur occupe la situation: la décadence et la ruine doivent être attendues instantanément.

TSHOU HI. - Dernier degré de l'ordre établi ; rang supérieur dans la substance du péril, et, de plus, le rang est occupé par la malléabilité négative. Cela est considéré comme exprimant l'image symbolique du renard traversant un cours d'eau à la nage et mouillant sa tête. Si celui qui consulte le sort manque de circonspection, c'est une voie rationnelle de péril.

1115.

Mouiller la tête, péril ; comment cet état pourrait-il durer?

TSHENG TSE. — À la fin de l'ordre établi, le péril atteint des proportions telles que le fait de mouiller (submerger) la tête ; est-il donc possible que cet état puisse durer?

\footnotetext{
${ }^{1}$ Tsi, traverser un cours d'eau à la nage.
} 


\section{Vi tsi}

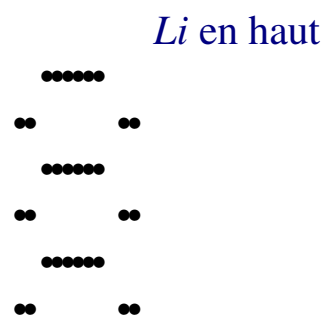

Khan en bas

1116. Vi tsi ; liberté ; le petit renard est sur le point de traverser le cours d'eau, il mouille sa queue ; aucun moyen davantage.

[]

1120. Premier trait hexaire : mouiller la queue ; appréhension.

$*$

1122. Deuxième trait nonaire : tirer la roue en arrière ; pureté, présage heureux.

$*$

1124. Troisième trait hexaire : pas encore régularisé ; en avançant présage malheureux; avantage à traverser un grand cours d'eau.

$*$

1126. Quatrième trait nonaire : présage heureux de la perfection; regrets dissipés; l'ébranlement de la foudre a pour effet la destruction du peuple des Kouei fang ; après trois ans il y a récompense d'un grand royaume.

$*$

1128. Cinquième trait hexaire : présage heureux de la perfection; pas de regrets ; éclat de l'homme doué ; avoir foi ; présage heureux.

$*$

1130. Trait supérieur nonaire : avoir foi pour boire le vin; pas de culpabilité ; mouiller la tête ; avoir foi, perdre ce qui est.

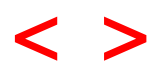




\section{Vi tsi.}

TSHENG TSE. — Vi tsi. «L'Ordre des koua » dit : «Les êtres ne peuvent pas être éternels, aussi le koua hi tsi est suivi du koua $v i$ tsi, qui est le dernier. » La régularité établie, c'est la fin des êtres ; si les êtres, parvenus à leur fin ne se modifiaient pas, il n'y aurait aucune rai son qui puisse les faire cesser d'exister. Le mot $y i$ de $Y i$ king exprime la modification et le changement sans fin ; aussi, après l'ordre établi vient immédiatement le koua $v i$ tsi, qui est le dernier du livre. Vi tsi signifie pas encore traversé, pas encore fini, pas encore régularisé ; ce koua doit donc comporter le sens de continuité infinie dans la succession des êtres et dans la transmission de la vie. Le koua est constitué par le koua simple $l i$ en haut, et le koua simple khan en bas : le feu est au-dessus de l'eau ; ils ne peuvent produire aucun effet utile l'un par l'autre, aussi le koua est considéré comme exprimant l'ordre non encore établi. 


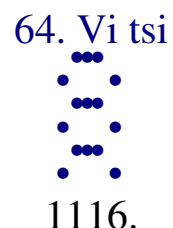

Vi tsi ; liberté ; le petit renard est sur le point de traverser le cours d'eau, il mouille sa queue; aucun moyen davantage.

TSHENG TSE. - Dans le moment où la régularité n'est pas encore établie, il existe une raison d'être naturelle de liberté et, en outre, les aptitudes indiquées par le koua comportent une voie rationnelle d'où résulte la liberté, qui réside uniquement dans la circonspection ou dans l'action de se placer. Le renard peut traverser l'eau à la nage, mais s'il mouille sa queue, il ne sera plus capable de nager. Quand le renard est déjà vieux, il est très méfiant et craintif, c'est pour cela qu'en marchant sur la glace il écoute, craignant qu'elle ne vienne à s'effondrer. Mais lorsqu'il est jeune, il est encore incapable de crainte et de circonspection, aussi il est assez hardi pour s'aventurer à traverser l'eau à la nage. Le sixième caractère du texte dont le sens est « sur le point de », doit être lu [] expression qui indique la vigueur et le courage violent et hardi. Le Shou king dit : «Hardi ! hardi ! l'homme courageux. » Le petit renard montre sa décision pour traverser l'eau à la nage ; il mouillera sa queue et ne pourra nager. Dans le moment où la régularité n'est pas enco re établie, et en cherchant la voie rationnelle pour traverser ( $t s i)$ cette période, il convient d'avoir la plus grande circonspection, et alors il pourra y avoir liberté. Mais en agissant avec la décision imprudente du jeune renard, on sera incapable de remédier ( $t s i$ ) aux dangers du moment. Puisque l'on est incapable d'y remédier, rien ne peut être avanta geux.

TSHOU HI. - Vi tsi : c'est le moment où les choses ne sont pas complètement achevées. L'eau et le feu ne s'allient point, et ne produisent aucun effet ensemble. Les six traits de ce koua manquent tous à leur situation ${ }^{1}$, aussi il est considéré comme exprimant que la régularité n'est pas encore rétablie ; sur le point de, au moment du début de l'action ; au moment où il commence à nager, il mouille sa queue ; cela exprime encore le sens des mots vi tsi, «pas encore traversé ». Si celui qui consulte le sort est dans ces conditions, qu'est -ce qui pourrait être avantageux ?

1117.

\footnotetext{
${ }^{1}$ Les rangs impairs sont occupés par trois traits négatifs.
} 
Le commentaire traditionnel de la formule déterminative dit: Vi tsi; liberté ; la douceur malléable possède la justice.

TSHENG TSE. - Il s'agit des aptitudes qui résultent de la structure du koua. Ce qui fait qu'il y a possibilité de liberté, c'est que la douceur malléable possède la justice. Le cinquième trait occupe le rang prééminent avec sa douceur malléable ; il occupe un rang qui comporte la dureté énergique et il sympathise avec la dureté énergique; il possède ce qui constitue la justice dans la douceur malléable. L'énergie et la douceur sont dans de justes proportions, aussi placé dans un moment où l'ordre n'est pas encore établi, il est possible de jouir de la liberté.

TSHOU HI. - Ceci désigne le cinquième trait hexaire.

1118.

Le petit renard est sur le point de traverser l'eau à la nage; il n'est pas encore sorti dedans ${ }^{1}$; il mouille sa queue, rien ne peut être avantageux; il ne peut arriver à la fin; bien que la situation ne soit pas méritée, la dureté et la douceur sympathisent.

TSHENG TSE. - Cela est dit au sujet du second trait. Le second trait emploie la dureté énergique positive et demeure au milieu du péril ${ }^{2}$, c'est celui qui est sur le point de se mettre à la nage. De plus, en haut, il sympathise avec le cinquième. Un lieu périlleux ne peut être un lieu de calme et de repos ; le cinquième comporte une raison d'être qui oblige à le suivre, aussi, le second est aussi décidé à traverser qu'un jeune renard pe ut être entêté à se mettre à la nage. Du moment où il est absolument décidé à traverser à la nage, il doit lui arriver le malheur qui consiste à mouiller sa queue, de sorte quill ne peut pas encore sortir du milieu du péril ${ }^{3}$. Comme son mouvement en avant est très vif, son mouvement de recul est très précipité. Bien qu'au commencement il soit assez courageux et hardi pour se mettre à nager, il est incapable de continuer jusqu'au bout et d'achever la tâche qu'il a entreprise, aussi il n'y a rien à entrepren dre et qui puisse lui être avantageux. Bien que la négativité et la positivité ne méritent également pas la situation qu'elles occu pent, cependant, partout, la douceur et la dureté sympathisent ensemble. En présence d'un moment où l'ordre n'est pas encore établi, il y a cependant alliance, relation entre elles deux, aussi, si le second trait est capable de

\footnotetext{
${ }^{1} \mathrm{Ou}$ « sorti au milieu »; on pourrait encore lire «il n'est pas encore allé dedans », mais je ne crois pas que tel soit le sens.

2 2. - Second trait du koua simple khan, péril.

3 3. -Le commentateur donne un sens au texte qui n'en comporte pas.
} 
redoubler de circonspection, il y aura nécessairement une raison d'être naturelle qui lui donnera la possibilité de traverser les dangers du moment et d'y remédier (tsi). Le second trait est sur le point de traverser et il emploie la hardiesse pour traverser, aussi il mouille sa queue. Tous les traits du koua sont également sans rapport avec la situation qu'ils occupent, aussi le trait est considéré comme exprimant que l'ordre n'est pas encore rétabli. Le commentaire intitulé «Mélanges sur les koua» dit que le koua vi tsi marque la fin du principe mâle, cela veut dire que les trois traits positifs sont également sans relation avec la situation qu'ils occ upent ; c'est bien là le sens, et si on l'entend, on pénètre tout ce qui y est caché.

1119.

Le commentaire traditionnel de la formule symbolique: Le feu est au-dessus de l'eau; l'ordre n'est pas encore rétabli; l'homme doué, par son attention circonspecte, distingue les êtres et pose les règles.

TSHENG TSE. — L'eau et le feu ne s'unissent point, ils ne se complètent pas ${ }^{1}$ mutuellement pour produire un effet utile, aussi, le koua est considéré comme exprimant que l'ordre n'est pas encore établi. Le feu est au-dessus de l'eau ; ce n'est pas la position qui lui convient. L'homme doué regarde l'image symbolique de la situation imméritée (qui ne convient pas), et il l'applique avec circonspection en plaçant les choses et les êtres ; il discerne ce qui convient à chacun d'eux, il les place chacun dans le lieu qui lui convient, c'est-à-dire qu'il les fixe chacun en sa place.

TSHOU HI. - L'eau et le feu sont des choses absolument dissemblables et chacune occupe sa propre place, aussi l'homme doué considère les symboles et il discerne et distingue.

${ }^{1}$ Littéralement, «ils ne remédient pas mutuellement aux mouvements propres à chacun d'eux ». 


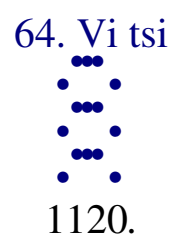

Premier trait hexaire : mouiller la queue ; appréhension.

TSHENG TSE. - Ce trait hexaire emploie la douceur négative et se trouve au rang inférieur; il est placé dans le péril et il sympathise avec le quatrième. Étant placé dans le péril, il en résulte qu'il n'est pas satisfait de la position quil occupe ; ayant une correspondance sympathique, ses tendances le portent à s'élever. Mais outre qu'il est lui-même négatif et malléable, le quatrième trait étant dépourvu des qualités de justice et de droiture est incapable de l'accueillir et de l'aider à traverser le péril. Lorsque les quadrupèdes traversent l'eau en nageant, il faut qu'il s relèvent leur queue ; si leur queue est mouillée, ils ne peuvent plus nager. «Mouiller la queue» veut dire «ne pouvoir traverser à la nage ». Il ne mesure ni ses capacités ni ses forces et il avance ; à la fin il ne peut traverser le péril (tsa); c'est un motif plausible de honte et d'appréhension.

TSHOU HI. - Employant la négativité et occupant le rang inférieur, en présence du début de l'ordre non rétabli, il ne peut pas avancer de lui-même, aussi tels sont l'image symbolique et le sens divinatoire.

1121.

Mouiller la queue, c'est encore ne point connaître l' extrême limite.

TSHENG TSE. - Il ne mesure ni ses aptitudes, ni ses forces, et il se porte en avant ; cela est poussé au point que la queue est mouillée : c'est le comble de l'ignorance.

TSHOU HI. - Le mot traduit par limite extrême, n'est pas encore clairement interprété ; si on se reporte aux rimes précédentes, il ne s'accorde pas non plus. Il est peut-être possible de soupçonner quill est mis là pour le caractère qui signifie respect. Actuellement, d'ailleurs, on en fait abstraction dans la lecture. 


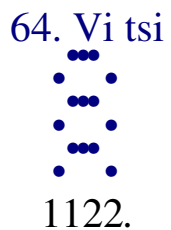

Deuxième trait nonaire : tirer la roue en arrière ; pureté, présage heureux.

TSHENG TSE. - Dans les autres koua, lorsqu'un trait nonaire occupe le second rang, il est considéré comme se maintenant dans la douceur et précédant la justice; cela exprime le sens qu’il n'y a pas abus de dureté énergique. Dans le koua vi tsi, l'homme saint relève attentivement le sens symbolique du koua pour en faire l'objet d'un avertissement et montre clairement la voie rationnelle pour servir le supérieur avec respect et obéissance. Le koua vi tsi exprime le moment où la voie rationnelle de l'homme doué est pleine de difficultés. Le cinquième trait, avec sa malléabilité, est placé dans la situation du prince, tandis que le second, avec ses capacités d'énergie entière positive, et occupant un lieu qui indique leur mutuelle sympathie, est celui quill convient d'employer. Le mot dureté énergique comporte encore le sens d'opprimer la négativité ; l'eau comporte encore l'image symbolique de l'aptitude à éteindre le feu. Au moment où commence la difficulté, celui sur qui il compte et s'appuie, c'est le sujet capable ; celui-ci doit absolument pousser l'accomplissement de la voie rationnelle du respect et de l'obéissance, aussi la formule contient l'avertissement de tirer la roue en arrière, alors la droiture sera respectée et le présage sera heureux. Tirer la roue à l'envers, c'est atténuer la force naturelle de l'action et ralentir le mouvement de progression en avant. C'est un avertissement au sujet de l'excès dans l'emploi de la dureté énergique. Lorsquil y a excès de dureté énergique, on en arrive facilement à braver et mépriser les supérieurs et l'obéissance est incomplète. Tels furent les cas relatifs à plusieurs personnages historiques de la dynastie des Theang. Au moment où le péril n'est pas encore conjuré, pouvoir. pousser le respect et l'obéissance à leur plus extrême limite, c'est le moyen qui assure la conformité à la droiture et à la possibilité de garantir et d'assurer la fin heureuse des événements qui s'accomplissent. Au sujet du cinquième trait hexaire, il sera question du pureté, de présage heureux et d'éclat illustre pour épuiser le bien dans la voie rationnelle du prince ; mais dans le cas du second trait nonaire, il s'agit seulement d'un avertissement relatif au respect et à l'obéis sance, pour épuiser la droiture dans la voie rationnelle du sujet; ensemble, ces deux formules épuisent la voie rationnelle du supérieur et de l'inférieur.

TSHOU HI. - Comme le second trait nonaire correspond sympathiquement avec le cinquième trait hexaire, et comme il occupe un rang qui comporte la douceur malléable et possède la justice, il est considéré 
comme exprimant la capacité de se contenir soi-même sans avancer; il possède ce qui constitue la droiture dans l'infériorité et, c'est pour cela, que tels sont l'image symbolique et le sens divinatoire.

1123.

Deuxième trait nonaire ; pureté, présage heureux ; justice pour pratiquer la droiture.

TSHENG TSE. — Le second trait nonaire représente celui qui possède la droiture et dont le présage est heureux ; c'est en tirant la roue en arrière et en se conformant à la voie rationnelle de la justice que l'on atteint à la droiture.

TSHOU HI. - Un trait nonaire occupe le second rang; essentiellement cela ne constitue pas la droiture ; c'est à cause de la justice qu'il est conforme à la droiture. 


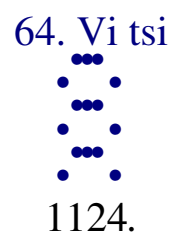

Troisième trait hexaire : pas encore régularisé ; en avançant présage malheureux; avantage à traverser un grand cours d'eau.

TSHENG TSE. — La régularité n'étant pas encore rétablie, en avançant le présage est malheureux ; cela veut dire qu'il demeure dans le péril et qu'il ne produit aucun résultat propre à l'en tirer, de sorte que s'il agit et entreprend quelque chose, le présage est malheureux. Il faut d'abord qu'il sorte du péril, et ensuite, il lui sera possible d'avancer et d'entreprendre. À cause de ses aptitudes de malléabilité négative sans justice ni droiture, et parce quỉl est placé dans le péril, le troisième trait n'est pas capable de suffire aux nécessités du moment pour rétablir l'ordre. Il n'a encore aucune voie $r$ ationnelle de possibilité de remédier à l'état actuel des choses et il ne produit aucun effet capable de le faire sortir du péril, de sorte que, s'il avance et entreprend quelque chose, c'est ce qui conduit à un présage malheureux. Cependant, lorsque l'ordre n'est pas encore rétabli, il y a naturellement une voie rationnelle qui permet d'y remédier ; lorsque le péril est à sa fin, il y a une raison d'être qui conduit à sortir du péril. En haut, il jouit de la sympathie de la dureté énergique positive ; sil l peut s'aventurer à traverser le péril pour aller suivre le trait supérieur, tout sera réparé et mis en ordre. C'est pour cela quill y a «avantage à traverser un grand cours d'eau ». Toutefois, comment la régularité malléable du troisième trait serait-elle capable de sortir du péril et d'avancer ensuite ? Tant que le moment n'en sera pas venu, elle en sera incapable ; ses aptitudes ne le lui permettent pas.

TSHOU HI. - Malléabilité négative sans justice et sans droiture, placée dans le moment où l'ordre n'est pas encore rétabli ; c'est en entreprenant quelque chose qu'elle entraînera le présage malheureux. Cependant, foulant la dureté énergique aux pieds par sa douceur malléable, ce trait est sur le point de sortir du péril exprimé par le koua simple khan; il comporte l'image symbolique de traverser un cours d'eau à la nage, de sorte que tel est le sens divinatoire. En effet, sỉl agit il peut être entraîné par le courant des eaux, tandis que d'un autre côté, il ne lui est pas possible de suivre la voie de la terre ferme. On doute si au-dessus du septième caractère du texte il ne devrait pas y avoir la négation. 
Régularité pas encore établie, en avançant, présage malheureux; situation imméritée.

TSHENG TSE. - Le mouvement en avant du troisième trait conduit au présage malheureux ; c'est parce que sa situation est imméritée. Cela veut dire qu'il est malléable et négatif, sans justice ni droiture, et dépourvu des aptitudes qui le rendraient capable de remédier aux périls. S'il peut traverser le péril et s'y exposer pour suivre celui avec qui il correspond sympathiquement, ce sera un avantage. 


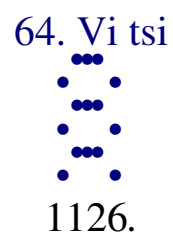

Quatrième trait nonaire : présage heureux de la perfection ; regrets dissipés ; l'ébranlement de la foudre a pour effet la destruction du peuple des Kouei fang ; après trois ans il y a récompense d'un grand royaume.

TSHENG TSE. - Le quatrième trait, doué de la dureté énergique positive, occupe la situation du sujet de rang élevé ; au-dessus de lui se trouve un maître juste et sans préventions ${ }^{1}$, intelligent et docile aux conseils. De plus, lui-même sort du péril ${ }^{2}$; l'ordre non régularisé a déjà dépassé la moitié de sa période de durée, et il y a une voie rationnelle de possibilité d'y remédier. Sans les aptitudes de l'activité et de la dureté énergique positive, il n'est pas possible de remédier aux périls et aux difficultés de l'univers. Ce trait nonaire, bien que positif, occupe cependant le quatrième rang, aussi la formule avertit au sujet du présage heureux de la perfection et de la dissipation des regrets. Sans perfection on ne pourra rétablir l'ordre, et cela constituera l'existence de regrets. L'ébranlement dû à la foudre, c'est le maximum de l'ébranlement. Ce qui exigea le maximum de l'emploi des forces, chez les hommes de l'antiquité, ce fut la destruction des peuples appelés Kouei fang ${ }^{3}$, aussi cette expression est employée comme comportant un sens allégorique ${ }^{4}$. Les forces mises en activité détruisent au loin ; cela dure jusqu'à trois ans, et ensuite l'ouvre est accomplie et il y a jouissance d'un grand royaume en récompense ; c'est ainsi quil faut absolument être pour pouvoir rétablir l'ordre et remédier aux périls. La voie rationnelle pour régulariser l'univers est qu'i 1 convient d'employer une parfait fermeté, comme il vient d'être expliqué ; c'est parce que le quatrième trait occupe un rang qui comporte la douceur malléable que cet avertissement est mentionné.

TSHOU HI. - Étant nonaire, il occupe le quatrième rang; c'est un manque de droiture et il en éprouve des regrets; il peut exercer ses forces et ses capacités, et le faire avec perfection, ses regrets se dissipent. Toutefois, comme il n'est pas doué de la perfection, il a la volonté d'exercer ses efforts, de chercher à atteindre à la perfection dans ce genre; sil ne pousse pas à l'extrême son énergie positive, s'il n'y consacre pas ses efforts pendant une longue durée, il n'y parviendra pas. C'est pour cela qu'il est considéré comme comportant l'image symbolique de détruire les Kouei fang et d'avoir une récompense au bout de trois ans.

\footnotetext{
1 «Vide à l'intérieur ».

2 Quitte le koua simple khan.

${ }^{3}$ Kouei fang, région des démons.

4 Proverbe.
} 
1127.

Présage heureux de la perfection et dissipation des regrets ; tendances qui prévalent.

TSHENG TSE. - Avec les capacités du quatrième trait et le moment étant opportun, ajoutant à cela la perfection dans la fermeté, il peut donc faire prévaloir ses tendances. Le présage est heureux et les regrets se dissipent. La destruction des Kouei fang, c'est l'excel lence dans la perfection. 


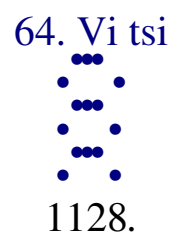

Cinquième trait hexaire : présage heureux de la perfection; pas de regrets ; éclat de l'homme doué ; avoir foi ; présage heureux.

TSHENG TSE. — Le cinquième trait est le maître de l'élégance de la forme ${ }^{1}$; il occupe un rang qui comporte la dureté énergique et il correspond sympathiquement à la dureté énergique ${ }^{2}$. La place quill occupe est conforme à la justice ; il dégage son coaur de toute prévention ${ }^{3}$ et la positivité lui vient en aide. Bien qu'il emploie la douceur malléable en occupant la situation prééminente, il s'y place cependant avec l'extrême droiture et l'extrême excellence et il n'est insuffisant sous aucun rapport, du moment où il se conforme déjà à la parfaite droiture, il en résulte que le présage est heureux et qu'il est sans regrets. Il possède certainement cette perfection ; il ne s'agit pas ici d'un avertissement à ce sujet. Employant ces qualités pour remédier au désordre, il n'est rien qui ne soit régu larisé par son action. Le cinquième trait est celui de qui dépend l'intelligence de la forme, aussi il est question de son éclat. C'est la perfection de la splendeur des vertus de l'homme doué et son œuvre y répond et en est digne. Il y a foi. Au commencement de la formule, le texte parle de présage heureux, au sujet de la perfection ; être malléable et doux, et en même temps capable de la perfection dans une autre, c'est ce qui constitue le présage heureux de cette vertu; à la fin de la formule, le texte parle encore de présage heureux, au sujet de l'oavre accomplie, du moment où il est éclatant et où il $\mathrm{y}$ a bonne foi, c'est que le moment comporte la régularisation.

TSHOU HI. - Puisque c'est un trait hexaire qui occupe le cinquième rang, il y a encore manque de droiture ; mais, cependant, c'est de lui que dépend l'intelligence (clarté) de la forme ; il conserve la justice et sympathise avec la dureté énergique, il maintient son cour vide de préventions pour réclamer l'aide de ceux qui sont au-dessous de lui, aussi il possède la perfection, son présage est heureux ; et d'ailleurs il est sans regret. De plus encore, il a la perfection de l'éclat et de la splendeur, il est sincère et sans fausseté : tout cela comporte une suite de présages heureux.

\footnotetext{
${ }^{1}$ Le trait du milieu du koua $l i$; celui de qui dépend l’intelligence de l'image, ou de la figure ou forme.

${ }^{2}$ Du second trait.

${ }^{3}$ Il s'évide au milieu.
} 
1129.

Éclat de l'homme doué ; son reflet est un présage heureux.

TSHENG TSE. — Lorsque l'éclat est parfait il a des reflets : le reflet est la dispersion de l'éclat. L'homme doué croit jusqu'à un état complet ${ }^{1}$ et son éclat est parfait, jusqu'à ce point qu'il a des reflets ${ }^{2}$; c'est l'extrême excellence, aussi la formule répète les mots présage heureux.

TSHOU HI. — Le reflet, c'est la dispersion de l'éclat.

${ }^{1}$ Telle est bien la traduction littérale; le sens traditionnel est quill accumule la vertu jusqu’à la posséder complètement. Le sens littéral s'adapte exactement à la croissance de la lune.

${ }^{2}$ L'effet de son éclat réagit sur les autres hommes. 


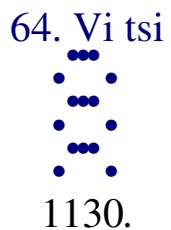

Trait supérieur nonaire : avoir foi pour boire le vin ; pas de culpabilité ; mouiller la tête; avoir foi, perdre ce qui est.

TSHENG TSE. - Ce trait nonaire emploie la dureté énergique et il est au rang supérieur : c'est l'extrême degré de la dureté énergique. Il occupe le rang supérieur du koua simple ${ }^{1}$ qui représente la clarté : c'est l'extrême degré de la clarté ${ }^{2}$. La dureté énergique atteignant l'extrême degré mais étant capable d’intelligence, cela n'est plus considéré comme exprimant la précipitation, mais comme exprimant la netteté de la décision. Lintelligence peut éclairer la raison d'être, la dureté énergique peut déterminer le devoir. Il occupe le rang extrême dans le koua vi tsi (pas encore régularisé) ce n'est pas une situation qui lui permette de régulariser; il ne comporte aucune raison d'être de possibilité de régularisation, de sorte qu'il doit se contenter de jouir des bienfaits du ciel, et de suivre sa destinée. Si l'époque de décadence est à sa fin il devra y avoir réaction, ce sera la modification du moment. La régularité n'étant pas encore rétablie, il n'y a aucune raison pour que les choses soient déjà arrivées à leur limite extrême et qu'elles se régula risent d'elles -mêmes, aussi este seulement considéré comme exprimant l'extrême degré de l'ordre non établi. Avec une parfaite sincérité, en se contentant de la destinée et de l'accomplissement des devoirs et en en faisant sa joie, il sera possible d'être sans culpabilité. «Boire le vin », c'est se réjouir, «faire sa joie ». Si l'on ne se contente pas de sa situation, on sera livré à la colère et à l'emportement et on tombera dans les embarras, c'est -à-dire qu'on sera plongé dans la culpabilité et que les présages seront funestes. Si au contraire, on s'adonne exclusivement au bien-être et aux plaisirs et si l'on se livre à la volupté avec excès et en dépassant toutes les lois morales, jusqu'à s'y « plonger (mouiller) la tête », il sera encore impossible de vivre en paix en se contentant de sa situation. «Avoir foi », c'est-à-dire avoir la confiance dans le cour. «Perdre ce qui est », perdre la notion des convenances; dans ces conditions, ce sera considéré comme constituant l'inconvénient du fait « d'avoir foi ». L’homme étant placé dans le malheur, lorsqu'il reconnaît qu'il n'a rien à faire où à tenter et qu'il se laisse aller sans réagir, comment pourrait-on dire qu'il se contente de sa destinée et de l'accomplissement de ses devoirs ${ }^{3}$.

\footnotetext{
$1 \mathrm{Li}$.

${ }^{2}$ Le sens admis est « intelligence ».

3 Comme en beaucoup d'autres cas, d'ailleurs, il n'y a aucun rapport entre le texte et les développements brodés par le commentateur.
} 
TSHOU HI. - Avec son énergie et son intelligence (clarté) il occupe le rang extrême dans le koua vi $t s i$; le moment où il y aura quelque chose à faire est sur le point d'arriver, et il a confiance en lui-même, il se fortifie en attendant le destin : c'est une voie rationnel le qui exclut la culpabilité. Si au contraire il se laissait aller sans réagir, comme le renard qui traverse l'eau à la nage et qui laisse submerger (mouiller) sa tête, cela serait un excès de confiance en soi-même et cela constituerait un manquement au devoir.

1131.

Boire du vin et s'en mouiller la tête; c'est encore ne pas connaître les préceptes.

TSHENG TSE. - Boire du vin jusqu'à s'en mouiller la tête, c'est la dernière limite de l'ignorance des préceptes de la morale. Ce qui conduit à un tel état, c'est l'incapacité de savoir se co ntenter du devoir et de la destinée ; si l'on pouvait s'en contenter, on ne man querait pas aux lois immuables et éternelles. 


\title{
FORMULES ANNEXÉES
}

\author{
PREMIÈRE PARTIE
}

\section{DU COMMENTAIRE TRADITIONNEL}

TSHOU HI. - La rubrique «formules annexées » désignait originellement les formules ou sentences écrites par Wen Wang et Tsheou Kong et inscrites (annexées) au-dessous des koua et des traits; c'est précisément ce qu'on appelle aujourd'hui le texte : king. Le présent chapitre est composé du commentaire traditionnel c'est-à-dire de l'ensemble des formules écrites et annexées par Khong Tse, en continuation de l'œuvre primitive. Comme ces formules traitent indistinctement de la matière essentielle et des diverses règles de tout le texte ( king), il était impossible de les rattacher spécialement à aucune partie de ce texte, de sorte qu'on les a recueillies à part, et divisées (comme le texte) en première et seconde parties ${ }^{1}$.

1132.

Le ciel est élevé, la terre est basse ${ }^{2}$ : par là sont définis les koua khien et khouen ; l'infériorité et l'élévation servent à déterminer ce qui est noble ou humble; le mouvement et le repos sont soumis à des lois constantes qui déterminent l'énergie et la douceur ; les principes sont classés par genre, les êtres sont divisés par catégories, le faste et le néfaste naissent ${ }^{3}$. Dans le ciel s'achève le symbole, dans la terre s'achève la forme: les changements et les transformations sont visibles.

TSHOU HI. - Le ciel et la terre sont la substance réelle et matérielle de la négativité et de la positivité, de l'apparence et de l'essence ${ }^{4}$; khien et khouen sont, dans le Yi king, les noms des koua qui expriment l'absolue négativité et l'absolue positivité. Infériorité et élévation, marquent les

\footnotetext{
${ }^{1}$ Les formules annexées sont aussi désignées sous le nom de « grand commentaire ».

2 Ou bien « le ciel est en haut, la terre est au-dessous».

${ }^{3}$ On peut lire aussi « le bien et le mal naissent», mais ki a aussi le sens de «nouvelle lune».

${ }^{4}$ De la forme et de l'éther constitutif.
} 
situations relatives du ciel et de la terre et de tous les êtres. Noblesse et humilité marquent l'infériorité ou la supériorité des situations des traits des koua, dans le $Y i$ king. Le mouvement, c'est l'état permanent de la positivité ; le repos, c'est l'état permanent de la négativité. Énergie et douceur, appellation, dans le $Y i$ king, de la positivité et de la négativité des traits des koua. Le caractère fang ${ }^{1}$, principe, désigne le but vers lequel tendent les choses, c'est-à-dire ce qui inspire aux choses ou aux êtres l'attraction du bien ou la répulsion du mal ; chacun est classé d'après son genre. Enfin le faste et le néfaste, c'est, dans le Yi king, la formule augurale qui détermine le sens divinatoire relatif aux traits du koua. Symbole, les divers phénomènes ${ }^{2}$ relatifs au soleil, à la lune, aux étoiles, aux phases. Forme, les divers accidents ou caractères tels que montagnes, cours d'eau, êtres qui se meuvent (comme les animaux) ou qui existent sur place (comme les végétaux). Changements et transformations; dans le Yi king, cela désigne les brins dherbe sèche qui servent à représenter les traits du koua en consultant le sort; la négativité se transforme et devient positivité, la positivité se change en négativité. Ceci exprime que les hommes saints qui ont fait le Yi king ont déduit les règles relatives aux traits des koua, de la substance matérielle et réelle de la négativité et de la réalité. C'est précisément ce qu'exprime Tseang Tsheou en disant : «Le Yi king a pour objet de définir la négativité et la positivité. »

1133.

C'est pourquoi l'énergie active et la douceur malléable s' enlaçant ${ }^{3}$, les huit koua simples se déduisent mutuellement l'un de l'autre.

TSHOU HI. - Ceci parle des modifications et des transformations des koua dans le $Y i$ king. L'origine première des soixante-quatre koua consiste uniquement dans les deux traits qui expriment la dureté énergique et la douceur malléable. Ces deux traits s'enla cent et forment quatre groupes ; ces quatre groupes se rapprochent et forment huit trigrammes; ces huit trigrammes se modifient mutuellement et constituent les soixante-quatre koua.

1134.

Elles éveillent ${ }^{4}$ par la foudre et le tonnerre; elles imprègnent par le vent et la pluie. Le soleil et la lune se suivent tour à tour ${ }^{5}$ : l'un est le froid, l'autre la chaleur ${ }^{1}$.

\footnotetext{
${ }^{1}$ Droit, correct, perpendiculaire, loi, règle ; région.

${ }^{2}$ Littéralement « ce qui tient du soleil, de la lune, des étoiles, etc. »

${ }^{3}$ Littéralement «se frottent », « se pressent l'une contre l'autre ».

${ }^{4}$ Le terme du texte exprime l'action du printemps qui fait fendre et éclater l'enveloppe du germe dans les semences. C'est aussi le nom d'un gros tambour d'appel.

${ }^{5}$ Circulent à la suite l'un de l'autre.
} 
TSHOU HI. - C'est l'achèvement du symbole par la modification et la transformation.

1135.

La voie de l'activité énergique (khien) produit ${ }^{2}$ le genre masculin; la voie de la passivité (khouen) produit le genre féminin.

TSHOU HI. - Ceci est l'achèvement de la forme par la modification et la transformation. Ces deux paragraphes éclairent encore ce que le Yi king rend manifeste dans sa substance matérielle ${ }^{3}$; ils s'éclairent réc iproquement avec le texte qui précède.

1136.

L'activité énergique (khien) préside ${ }^{4}$ au commencement de la grandeur; la passivité (khouen) fait et achève les êtres.

TSHOU HI. - Le mot connaître, du texte, a encore le sens de diriger, présider à, être le maître dont dépend quelque chose. L'activité énergique (khien) crée les êtres (présider à leur commencement), et la passivité (khouen) les produit et les achève. Ceci se rapporte au texte qui précède au sujet du principe mâle et du principe femelle, et exprime la raison d'être du ciel et de la terre. En effet, toute chose dépend nécessairement de l'un de ces deux principes : négativité ou positivité ; rien n'échappe à cette loi, mais rien n'est non plus aussi important que la distinction des genres (ou sexes). En général, la positivité précède ; la négativité suit ; la positivité provoque par son action, la négativité subit et reçoit l'impression. La pure essence de la positivité n'a pas encore de forme, tandis que la matière plus lourde de la négativité laisse des traces visibles.

1137.

\footnotetext{
${ }^{1}$ Ou encore « une alternative de chaud et une alternative de froid ».

2 Achève le garçon, achève la fille.

3 Ses traits.

4 « Sait, connaît ».
} 
L'activité énergique (khien) sert à faciliter la connaissance ${ }^{1}$; la passivité (khouen) sert à distinguer la possibilité.

TSHOU HI. - Khien exprime l'activité et le mouvement ; précisément ce qui crée. Cette activité peut naturellement commencer (créer) les êtres et rien ne lui est impossible; c'est pourquoi la formule dit : " sert à faciliter et à diriger le grand commencement $\gg^{2}$. Khouen exprime la passivité et le repos; tout ce que peut ce principe, c'est toujours de suivre l'impulsion de la positivité sans rien faire de lui-même, de sorte qu'il est considéré comme servant à séparer et distinguer et comme pouvant achever les êtres.

1138.

Facilitant ${ }^{3}$, on connaîtra facilement ; distinguant, on suivra facilement. Connaissant facilement, il y aura rapprochement ; suivant facilement, il y aura mérite. Quand il y aura rapprochement, il pourra y avoir durée; quand il y aura mérite, il pourra y avoir grandeur. Quand il pourra y avoir durée, ce sera la vertu du sage ; quand il pourra y avoir grandeur, ce sera l'auvre du sage.

TSHOU HI. - Si l'action de l'homme est comme la «facilité » de l'activité (khien) son intelligence est brillante et claire et l'homme sait facilement ; si elle est comme la «distinction» de la passivité (khouen), les choses seront déterminées et limitées et l'homme les suivra facilement (s'y pliera facilement). Connaissant facilement, ceux qui auront la même volonté que lui seront nombreux, de sorte quil y aura rapprochement ${ }^{4}$; suivant facilement, ceux qui lui prêteront leur concours et l'aideront de leurs forces seront en multitude, de sorte qu'il aura du mérite (de la gloire). Puisqu'il y aura rapprochement il y aura unité de sentiment au-dedans, de sorte qu'il sera possible qu’il y ait durée ; puisqu’il aura du mérite, il embrassera indifféremment l'extérieur, de sorte qu'il sera suscep tible de grandeur. Vertu se rapporte à ce qu'il possède en lui-même ; œure se rapporte à son action sur les choses. En commençant, le texte exprime que les vertus de l'activité (Khien) et de la passivité (khouen) ne sont pas les mêmes ; maintenant il s'agit de l'homme se réglant sur la voie de l'activité et de la passivité, et arrivant à ce résultat : alors il pourra être considéré comme sage.

\footnotetext{
${ }^{1} \mathrm{Ou}$ « sert à diriger facilement », mais il faut déjà interpréter.

${ }^{2}$ Le texte est dénaturé.

3 Ce passage est très obscur; je crois quil faudrait lire : le premier caractère $y i$ « changement » et le second caractère $y i$ avec la valeur «facilement». On lirait donc «par le changement il est facile de savoir, par la distinction il est facile de suivre »?

${ }^{4}$ On se rapprochera de lui.
} 
1139.

Facilité et distinction : la raison d'être du ciel et de la terre existe ${ }^{1}$. La raison d'être du ciel et de la terre existant, il en résulte qu' elle détermine une situation dans le milieu.

TSHOU HI. - Achever une situation, veut dire déterminer la situation de l'homm e ; le milieu, c'est-à-dire le milieu du ciel et de la terre. Parvenue à ce point, la réalisation de la voie rationnelle a produit son chef-d'œuvre : la capacité de l'homme saint, dans les affaires, peut être égalée à celle du ciel et de la terre.

Ceci forme le premier paragraphe ; il éclaire la raison d'être de la doctrine du king (texte) par la substance réelle de la création et de la transformation. Il traite aussi de la raison d'être, de l'activité et de la positivité, manifestées séparément dans le ciel et la terre, et dont l'homme participe également.

1140.

L'homme saint établit les koua montrant ${ }^{2}$ les symboles, il y annexe des formules, et éclaire le faste et le néfaste.

TSHOU HI. - Symbole, ce qui est ressemblant aux choses. Ceci exprime que les hommes saints en faisant le Yi king regardèrent les symboles des traits et des koua et les expliquèrent dans des formules.

1141.

La dureté énergique et la douceur malléable se combinent ensemble et engendrent la modification et la transformation.

TSHOU HI. - Cela exprime que la négativité et la positivité des traits du koua se heurtent, se pressent et se combinent mutuellement et que tantôt la négativité modifie la positivité, tantôt la positivité transforme la négativité. C'est dans cette action combi née que les hommes saints contemplèrent les

\footnotetext{
${ }^{1}$ Est comprise, ou bien encore « agit, prévaut, dirige ». Le texte porte thien hia « dessous le ciel », ce qu'on lit généralement « l'univers ».

${ }^{2}$ On peut lire « regarde », le même mot signifiant « regarder » et « être l'objet des regards ».
} 
symboles et qu'ils en déduisirent les formules. C'est par cette auvre que la foule des hommes a pu consulter le sort par le moyen de la recherche des koua au moyen des brins d'herbe ${ }^{1}$.

1142.

C'est pourquoi le faste et le néfaste sont les symboles de la perte et de l'obtention ${ }^{2}$; le regret et l'appréhension sont le symbole du chagrin imprévu.

TSHOU HI. - Présage heureux, présage malheureux, regrets, appréhensions, sont également des expressions du $Y i$ king; la perte et l'obtention, le chagrin imprévu résultent des modifications des choses. Obtenir, ou posséder, sera le présage heureux ; perdre, manquer, sera le présage malheureux. Le chagrin imprévu, bien que ne constituant pas un présage malheureux est déjà suffisant pour motiver des regrets et attirer la honte. En effet, les présages, heureux ou malheureux, se font opposition, tandis que les regrets et l'appréhension constituent des degrés intermédiaires ; le regret provient du malheur et tend vers le présage heureux ; l'appréhension provient du présage heureux et vise le présage malheureux. Aussi, l'homme saint contemple les traits des koua, qui parfois présentent ces symboles, et il le marque par l'emploi de l'un de ces termes.

1143.

La modification et la transformation sont les symboles du mouvement en avant ou en arrière; la dureté et la douceur sont les symboles du jour et de la nuit. Le mouvement des six traits marque la voie rationnelle des trois termes extrêmes.

TSHOU HI. - La douceur malléable se modifie et tend vers la dureté énergique; le mouvement en arrière porté à son extrême limite devient un mouvement en avant. La dureté énergique transforme et porte à la douceur malléable: le mouvement en avant parvenu à son maximum devient mouvement en arrière. Du moment où il y a modification et par suite dureté énergique, le jour survient avec la positivité; du moment où il $\mathrm{y}$ a

\footnotetext{
${ }^{1}$ Allusion au procédé ordinaire de la divination par les koua.

${ }^{2}$ C'est l'ordre observé dans le texte et il est à remarquer, le mot perte semblant correspondre à faste et le mot gain à néfaste.
} 
transformation et par suite douceur malléable, c'est la nuit et la négativité ${ }^{1}$. Dans les six traits, le premier et le second représentent la terre; le troisième et le quatrième représentent l'homme; le cinquième et le trait supérieur représentent le ciel. Le mouvement, c'est exactement la modification et la transformation. Le terme $k i$, du texte, exprime l'idée de comble ; extrême limite, maximum, résultat. Les trois $(k i)$ extrêmes, sont l'extrê me raison d'être du ciel, de la terre et de l'homme ; les trois capacités (puissances) ont chacune une limite extrême ${ }^{2}$. Ceci éclaire comment la dureté énergique et la douceur malléable s'influencent mutuellement pour engendrer la modification et la transformation, et comment la modification et la transformation parvenues à leur extrême limite redeviennent dureté énergique et douceur malléable. Le courant d'action circule entre les six traits de chaque koua et celui qui consulte le sort peut déduire des traits quỉl obtient, pour décider ce qui est faste et néfaste.

1144.

C'est pourquoi, ce à quoi l'homme doit se tenir et ce dont il se contente ${ }^{3}$, c'est l'ordre du $\mathrm{Yi}^{4}$; ce dont il se réjouit et dont il se pénètre, ce sont les formules des traits.

TSHOU HI. — L'ordre du Yi king ; c'est-à-dire la raison d'être des choses telle qu'elle est exposée par les traits des koua ; l'ordre logique et naturel. Se pénétrer, comprendre par la contemplation.

1145.

C'est pourquoi, l' homme doué, étant placé, contemplera le symbole ${ }^{5}$ et il se pénétrera de la formule; étant en mouvement, il contemplera la modification et il se pénétrera du sens divinatoire. C'est parce que le ciel l'aidant, le présage heureux n'est jamais sans avantage.

\footnotetext{
${ }^{1}$ Dans l'esprit du commentateur chinois, le jour et la nuit ne sont très certainement pris que comme des cas particuliers, cités en exemples. Dans le texte de Khong Tse, selon moi, ils semblent être et sont, en réalité, à double sens.

${ }^{2}$ Tae ki, voir tome I, Exégèse chinoise. Tout ce passage est fortement teinté des doctrines taoïstes.

${ }^{3}$ Le terrain sur lequel il se tient.

${ }^{4} \mathrm{Ou}$ : l'ordre des changements.

${ }^{5}$ Ou bien « offrira aux yeux le symbole »; il en est de même plus bas.
} 
TSHOU HI. - Le symbolisme, les termes, la modification ont déjà été exposés plus haut. Toutes les fois que le terme modification est employé seul, il comporte aussi le sens du terme transformation. Sens divinatoire, c'est-à-dire les diverses alternatives de présages heureux ou malheureux que l'on rencontre successivement.

Ceci constitue le second paragraphe; il traite de la méthode des hommes saints, auteurs du Yi king, et de l'étude du Yi king par l'homme doué.

1146.

La formule déterminative traite $d u$ symbole; les formules des traits parlent de la modification.

TSHOU HI. - Le terme formule déterminative désigne la formule relative au koua; c'est l'ouvre de Wen Wang. Les formules des traits sont celles qui suivent chaque trait et sont l'œuvre de Tsheou Kong. Pour désigner toute la substance on emploie l'expression formule déterminative; pour désigner un point en particulier on emploie l'expression modification.

1147.

Présage heureux ou présage malheureux se rapportent à la perte ou à l'obtention. Regrets et appréhension se rapportent à de légers inconvénients. Pas de culpabilité, exprime que le bien compense le mal.

TSHOU HI. - Règles générales applicables aussi bien aux koua eux-mêmes qu'aux traits.

1148.

C'est pourquoi la distinction entre ce qui est noble et vil réside dans la situation; le classement entre ce qui est petit ou grand existe dans les koua; la distinction entre le faste et le néfaste se trouve dans l'expression ${ }^{1}$.

\footnotetext{
${ }^{1}$ La sentence ou formule.
} 
TSHOU HI. - Situation, c'est-à-dire la situation de chacun des six traits, classer, déterminer. Petit désigne la négativité ; grand désigne la positivité.

1149.

Le chagrin, les regrets, l'appréhension existent dans l'intervalle; l'ébranlement, la non-culpabilité existent dans le regret.

TSHOU HI. - Intervalle, démarcation dans la séparation et la distinction ; par exemple le moment où le bien et le mal sont déjà en action (mouvement) mais sans avoir déjà revêtu une forme précise ; si à ce moment on en éprouve de l'inquiétude, on n'arrivera pas jusqu'aux regrets et à l'appréhension. Ébranlement, mouvement.. Si on est susceptible d'éprouver des regrets, il y aura à se mouvoir pour compenser la volonté de mal faire, et alors il sera possible d'être sans culpabilité.

1150.

C'est pourquoi dans les koua, lorsqu'il y a les termes petitesse ou grandeur, lorsque dans les formules il y a les expressions difficulté ou facilité, dans chaque cas, elles désignent le résultat.

TSHOU HI. - Petitesse et difficulté, grandeur et facilité ; dans chaque cas suivant ce à quoi elles se rapportent.

Ce troisième paragraphe explique les règles générales relatives aux termes employés dans les formules des koua et des traits.

1151.

Le Yi king prend ses règles dans le ciel et la terre ${ }^{1}$, de sorte qu'il peut définir totalement la voie rationnelle du ciel et de la terre.

TSHOU HI. - Les formules des koua et des traits dans le livre des changements contiennent et embrassent en entier la raison d'être du ciel et de la terre ; elle sont réglées et basées sur elle. Le caractère du texte exprime le sens de relier deux choses l'une à l'autre comme en les cousant ensemble, et il contient liidée des extrémités rapprochées ensemble, comme se faisant suite ;

\footnotetext{
${ }^{1}$ On lirait mot à mot : «yi (le changement) équivaut à ciel et terre ».
} 
un autre caractère exprime l’idée de suivre de point en po int les délinéaments d'une figure pour en comprendre tout l'ensemble.

1152.

Lever les yeux pour le contempler dans les apparences du ciel ; les baisser pour l'apprécier dans la raison d'être de la terre ${ }^{1}$, c'est pourquoi on connaîtra les causes de l'obscurité et de la clarté. Origine, commencement, retour et fin: c'est pourquoi on connaît les expressions de mort et de naissance. L'éther pur (incorporel) constitue l'être; l'âme positive qui en résulte constitue la modification; c'est pourquoi on connaîtra les circonstances et la nature des esprits et des génies.

TSHOU HI. - Ceci épuise la raison d'être des choses et leur cause et la manière dont l'homme saint emploie le livre des changements. Le changement (yi), n'est rien autre chose que la négativité et la positivité. L'obscurité, la lumière, la mort, la vie, les esprits et génies ne sont rien autre chose que des modifications de la négativité et de la positivité c'est la voie rationnelle du ciel et de la terre. Dans les apparences du ciel il y aura le jour et la nuit, le haut et le bas; dans la raison d'être de la terre il y aura le sud et le nord, l'élévation et la profondeur. Origine, ce qu'on recherche et qui précède ; retour, ce qui est nécessaire et postérieur. L’absolue pureté de la négativité et l'éther de la positivité se réunissant et produisant les êtres, c'est -à-dire le redressement, ou expansion de l'esprit ${ }^{2}$; l'âme positive en dérive, l'âme négative descend, elles se séparent et constituent la modification : c'est la contraction du génie ${ }^{3}$.

1153.

Avec le ciel et la terre ils sont semblables entre eux ${ }^{4}$, c' est pourquoi ils ne s'éloignent pas; la science (le savoir) embrasse tous les êtres et la voie rationnelle traverse l'univers (le ciel et le bas), de sorte qu'il n'y a point

\footnotetext{
${ }^{1}$ Cette phrase semble bien donner raison à la lecture mot à mot que j’ai indiquée dans le précédent renvoi ; «le contempler », c'est-à-dire «contempler le changement», il ne peut s'agir du livre lui -même.

${ }^{2}$ Principe immatériel.

${ }^{3}$ Autre nom du même principe immatériel. Le premier désigne l'expansion favorable, le bon esprit; le second la contraction en sens inverse, le mauvais esprit. Ce n'est pas le lieu de développer le sens que les commentateurs chinois attachent à ces divers mots; il nous suffit $d e$ bien mettre en lumière le peu de rapport qui existe entre l'interprétation et le texte.

${ }^{4}$ Lire « les changements avec le ciel et la terre sont équivalents .
} 
d'erreur. Ils agissent parallèlement et sans flotter (être entraîné). Se réjouir du ciel et connaître la destinée, de sorte qu'il n'y aura pas d'inquiétude; se contenter du sol et être grand par l'humanité, de sorte qu'il est possible d'aimer ${ }^{1}$.

TSHOU HI. - Ici, l'homme saint épuise ce qui est relatif à la nature des choses. La voie rationnelle du ciel et de la terre consiste exclusivement dans la connaissance de l'huma nité (amour de l'homme). Ce dont la science embrasse tous les êtres, c'est le ciel ; la voie rationnelle qui traverse l'univers, c'est la terre. Connaissant (instruit) et d'ailleurs humain, on saura et on ne commettra pas d'erreur. Agir parallèlement ${ }^{2}$ c'est la science de l'action pondérée. Ne pas flotter (se laisser entraîner), c'est l'humanité, résultant de la conservation de la droiture ; du moment où on se complaît dans la raison d'être du ciel, et où, en outre, on connaît la destinée imposée par le ciel, il en résulte qu’il est possible d'éviter le chagrin et l'inquiétude, et que la science a ugmente et s'approfondit. Il devient possible d'être satisfait en quelque position qu'on soit placé et d'éviter tout germe de manque d'humanité, de sorte qu'on ne peut oublier la pensée de secourir les êtres et que la vertu d'humanité augmente en intensité . C'est qu'en effet, l'humanité c'est la raison d'être de l'amour et que l'amour est l'effet de l'humanité, de sorte que l'un et l'autre s'entraînent et s'enchaînent mutuellement comme il vient d'être dit.

1154.

Il trace le moule et les limites de la transformation du ciel et de la terre et ne les dépasse pas; il suit dans toutes leurs sinuosités les délinéaments ${ }^{3}$ de tous les êtres, sans aucune omission; il pénètre dans la voie rationnelle du jour et de la nuit et connaît; aussi l'esprit est sans région particulière et le changement n'a point de substance.

TSHOU HI. - Ici, il s'agit de l'homme saint ${ }^{4}$ qui suit jusqu'à la fin la destinée. Moule, comme le moule qui sert à couler le métal en fusion ; limite, comme l'enceinte d'une for teresse. Les transformations du ciel et de la terre n'ont point de fin et l'homme saint les considère comme un moule et une limite, qui ne permettent pas de dépasser la voie rationnelle de la justice : c'est

\footnotetext{
${ }^{1} \mathrm{Si}$ je traduisais toutes les annotations recueillies dans tous les commentaires on verrait facilement que la traduction est absolument fidèle et que le texte n'est compris quà la condition d'être étendu et dénaturé.

2 Ou latéralement.

3 Les détails circonstanciés d'une chose quelconque ; littéralement «le redressement et l'achèvement ».

${ }^{4}$ Le texte est absolument obscur et il est impossible de préciser qui est le sujet. je considère que c'est le $y i$ «le changement». Les commentateurs chinois prennent pour sujet l'homme saint; je cherche à rendre un sens résultant exclusivement du texte, les commentateurs s'efforcent de lire, à côté du texte lui -même, un sens qui concorde avec leurs préjugés.
} 
ce qu'on entend par l'expression « définir et achever». «Pénétrer», c'est-à-dire «comprendre indifféremment». Il trace indifféremment la voie du jour et de la nuit ${ }^{1}$. Le jour et la nuit, c'est simplement une expression pour désigner ce qui est obscur ou brillant, la mort ou la vie, les génies et les esprits. De cette façon, et après cette étude, il lui devient possible de voir jusqu'à la cause mystérieuse de l'esprit. Il n'y a pas de région ni de lieu : cela veut dire que les transformations et les modifications du Yi king n'ont pas une forme propre et une substance réelle.

Quatrième paragraphe; traite de la grandeur de la voie rationnelle du Yi king et montre comment l'homme saint l'utilise.

1155.

Une négativité et une positivité sont ce qu'on appelle la voie rationnelle.

TSHOU HI. - Le retour alternatif de la négativité et de la positivité, c'est l'éther. La raison d'être de ce fait est ce qu'on appelle la voie rationnelle.

1156.

Ce qui succède immédiatement, c'est le bien; ce qui en résulte immédiatement, c'est la nature.

TSHOU HI. - La voie rationnelle est contenue dans la négativité et elle agit dans la positivité. Succéder, exprime ce qui surgit; le bien, exprime l'œuvre de la transformation et de la génération : c'est le fait de la positivité. Résulter (littéralement, achever), veut dire embrasser complètement, ou être contenu dans. Nature, c'est ce que reçoivent les êtres ; cela exprime que les êtres, en naissant, ont une nature propre, et que chacun contient et renferme en entier cette voie rationnelle : c'est le fait de la négativité. Tsheou Tse, dans son livre, traite complètement cette question ${ }^{2}$.

1157.

${ }^{1}$ Le caractère du texte veut dire «pénétrer, comprendre, passer librement de la cause à l'effet » et par extension, il exprime l'idée de faire une chose librement, sans entrave.

2 Voir tome I, exégèse chinoise. 
L'humanité, il l'y voit et elle est appelée humanité; la science, il l'y voit et elle est appelée la science. La foule des hommes l'emploie journellement et ne le sait pas. Aussi, la voie rationnelle de l'homme doué, c'est la clarté ${ }^{1}$.

TSHOU HI. - L'humilité, est une vertu de la positivité, la science est une chose négative (un résultat et non pas une cause). Chacune exprime une face de cette voie rationnelle, aussi l'œil de celui qui regarde con sidère ce qu'il voit comme constituant le tout. Employer journellement sans savoir : ainsi, tout le monde boit et mange, mais « peu » (sien) sont capables d'apprécier le goût. Il en est encore de même dans tout ce qui suit, rien n'est étranger à cette voie rationnelle. On a dit : Dans une proposition précédente, on a parlé de la science comme d'une chose dépendant du ciel ${ }^{2}$ et de l'humanité comme dépendant de la terre ; cela n'est pas d'accord avec ce qui est dit ici ; comment cela peut-il être ? Réponse dans l'autre cas, il est question de ce qui est pur et limpide, ou de ce qui est trouble et épais ; ici il est question du mouvement et du repos.

1158.

Elle est manifeste dans l'humanité ${ }^{3}$, elle est cachée dans tous les effets. Elle ébranle et se fait voir dans tous les êtres et n'a pas les mêmes chagrins que l'homme saint. Que sa vertu est achevée et que son auvre est parfaite!

TSHOU HI. - Manifester (éclairer, rendre éclatant) c'est-à-dire aller du dedans au dehors. Humanité, auvre de l'action transform atrice, effet de la vertu. Cacher, de l'exté rieur aller vers l'intérieur. Effet, ou usage, désigne le secret mystérieux du mécanisme, la base de l'ouvre. Tsheng Tse dit : Le ciel et la terre n'ont point de cour et ils achèvent et transforment ; l'homme saint a un cour et il ne produit point.

1159.

La richesse se désigne par l'expression "grande auvre », la rénovation journalière ${ }^{4}$ est désignée par l'expression «vertu parfaite».

\footnotetext{
${ }^{1}$ Sien, signifie «nouveau, brillant, le bien », dans quelques cas, et suivant Tshou Hi, il prend le sens de «peu de, un petit nombre». Sous une autre forme graphique, le même mot sien désigne « le soleil éclatant qui avance».

2 Positivité.

${ }^{3}$ On peut aussi bien lire « elle éclaire chaque, ou toutes les humanités».

${ }^{4}$ C'est bien le sens admis de cette expression composée de deux mots : soleil et nouveau, ou rapprochement.
} 
TSHOU HI. - Tsheang Tse dit: La richesse, c'est la grandeur qui contient tout ; le renouvellement journalier, c'est la durée sans fin.

1160.

La vie succédant à la vie ${ }^{1}$ est exprimée par le mot changement (yi).

TSHOU HI. — La négativité engendre la positivité ; la positivité engendre la négativité ; cette modification n'a pas de fin : la raison d'être et le livre ${ }^{2}$ sont tous deux ainsi.

1161.

L'achèvement du symbole est désigné par le mot khien; la manifestation conforme à la loi est désignée par le mot khouen.

TSHOU HI. - Manifestation, ce qui fait voir et montre ; loi, règle, ce qui est visible dans le compréhensible ou le mystérieux de l'action transformatrice.

1162.

Épuiser le nombre et connaître l'avenir est désigné par l'expression « sens divinatoire »; la libre modification est appelée le fait, la chose.

TSHOU HI. - Sens divinatoire, consulter le sort ; la « chose », le « fait », qui n'est pas encore déterminé, arrivé. Ceci dépend de la positivité. « Chose», résultat de l'action, la chose qui se poursuit. Lorsque le sens divinatoire est déjà fixé, il appartient au principe négatif. Épuiser le nombre et connaître l'avenir, c'est ce qui permet de pénétrer les modi fications des « choses». Tsheong Tshong Ting Kong dit que les affaires publiques dépendent de la négativité et de la positivité ; l'idée est bien eff ectivement celle-ci.

${ }^{1}$ Shang « vie, engendrer, naître ».

2 -Les traits du Yi king. 
1163.

L'insondable dans la négativité et la positivité est appelé l' esprit ${ }^{1}$.

TSHOU HI. - Tsheang Tse dit : Les deux principes sont certainement insondables (ne peuvent être mesurés).

Cinquième paragraphe; traite de la substance et des effets de la voie rationnelle, qui ne sont jamais autre chose que la négativité et la positivité, tandis que la cause qui fait qu'il en est ainsi ne s'appuie jamais spécialement sur la négativité ou la positivité.

1164.

Or, le changement (yi) est vaste et grand; si on en parle lorsqu'il est lointain, alors il ne cesse point; si on en parle lorsqu' il est proche, alors il est calme et régulier; si on en parle lorsqu'il est entre le ciel et la terre, alors il est complet.

TSHOU HI. — «Ne cesse point », c'est-à-dire « pas épuisé »; « calme et régulier» veut dire qu'il atteint les êtres et que la raison d'être en est persistante. «Complet » exprime que rien n'y manque.

1165.

Dans (le koua) khien, le repos est absolu, le mouvement est régulier, et c'est par là que la grandeur naît. Dans (le koua) khouen, le repos est caché, le mouvement est libre : c'est par là que l'immensité naît.

TSHOU HI. - Les koua khien et khouen comportent chacun le mouvement et le repos et on le voit par les quatre vertus attributives ${ }^{2}$; le repos, c'est la substance, tandis que le mou vement en est l'effet ; le repos sépare et le mouvement relie. Khien, l'activité, c'est l'unité et la certitude matérielle, de sorte quîl en est parlé d'après ce caractère physique et que le

\footnotetext{
${ }^{1}$ Le phénomène de développement et de redressement.

2 Voir koua $n^{\circ} 1$.
} 
terme employé est la «grandeur»; khouen, la passivité, c'est la dualité et le vide, de sorte quiil en est parlé par appréciation, et que le terme employé est le mot «immensité ». En effet, bien que la forme visible du ciel ${ }^{1}$ entoure à l'extérieur de la terre, cependant l'éther agit (marche) constamment à l'intérieur de la terre. C'est à cause de ceci que le ( yi) changement est grand et immense.

«Immensité » et « grandeur» équivalent à «ciel»et «terre ${ }^{2}$; la modification et la libre pénétration correspondent (équivalent) aux quatre saisons ; le sens de «négativité »et «positivité» équivaut à «soleil»et «lune ». Le bien de la «facilité » équivaut à «l' extrême vertu».

TSHOU HI. - La libre pénétration de la modification, la grandeur et l’immensité du changement (yi) ainsi que les définitions données de la négativité et de la positivité, la vertu de la facilité, la voie rationnelle du ciel et les choses relatives à l'homme qui résul tent de cette équivalence, tout est comme il vient d'être dit.

Fin du sixième paragraphe.

\footnotetext{
${ }^{1} \mathrm{Ou}$ « les formes visibles dans le ciel »; si on voulait dire « la forme du ciel», il n'y aurait pas lieu d'employer le caractère qui marque le génitif possessif. Toutefois le sens prête au doute. Si au lieu d'un commentaire il s'agissait d'un texte, je n'hésiterais pas à traduire « bien que les signes visibles dans le ciel décrivent leur cercle au-dehors de la terre...

2 Équivalent à : « faire paire avec », «faire contraste à... ».
} 
FORMULES ANNEXÉES

PREMIERE PARTIE DU COMMENTAIRE TRADITIONNEL

S U I T E

1167.

Khong Tse dit: Que le changement est extrême ${ }^{1} ! C^{\prime}$ est par le changement (yi) que l'homme saint honore la vertu et élargit les talents; le savoir donne l'illustration, les rites donnent l'humilité ; L'illustration prend ses règles dans le ciel, l'humilité prend les siennes dans la terre.

TSHOU HI. - Les commentaires désignés par l'expression « les dix coups d'aile » sont tous également l'auvre de Khong Tse et il n'est pas nécessaire de commencer par les mots «Tse dit»; il y a lieu de penser que ces mots ont été ajoutés au texte par des commentateurs des époques postérieures. En scrutant jusqu'à ses dernières limites la raison d'être des choses, la connaissance devient brillante (illustre) comme le ciel, et la vertu sillustre. En se conformant à la raison d'être, les rites conduiront à l'humilité analogue à celle de la terre et les talents s'élargiront. Ceci est cité comme analogie et, de plus, distingue entre ce qui est plus ou moins pur ou mélangé.

1168.

Le ciel et la terre établissent les situations et le changement (yi) agit (marche) entre eux, il produit et engendre avec persistance, c'est la porte du sens de la voie rationnelle ${ }^{2}$.

TSHOU HI. - Le ciel et la terre déterminent les situations et la modification et la transformation agissent ${ }^{3}$, c'est comme si on disait que la connaissance des règles rituelles et la préservation de la nature (sentiments naturels) rend visible le sens de la voix rationnelle. Les caractères [] [] du texte signifient «la nature produite », c'est-à-dire la nature originellement produite. [] [] conservation et conservation, idée de persistance sans fin ${ }^{4}$.

\footnotetext{
${ }^{1}$ Extrême a le sens de superlatif en importance et en toutes ses conséquences.

2 Porte d'accès au sens de...

3 Dans le commentaire le sens devient étroit et il est évidemment question des koua du $Y i$ king. Le texte, au contraire, est vague et parle plutôt du «changement » dont il est question dans le Yi king.

${ }^{4}$ Ce mot désigne bien la nature, dans le sens de «naturel d'un être », mais il a aussi le sens de faire naître, engendrer. Je ne crois pas que la lecture du commentateur chinois soit bonne; elle résulte d'idées arrêtées plutôt que de l'étude du texte.
} 
Septième paragraphe.

1169.

L'homme saint a le moyen de voir ce qui est obscur dans le monde (sous le ciel) et d'en déterminer toutes les apparences et les circonstances; il symbolise ${ }^{1}$ ce qui est relatif aux êtres, aussi on le désigne ${ }^{2}$ par l'expression image symbolique.

TSHOU HI. - Obscur, c'est-à-dire confus, mélangé. Image symbolique, image symbolique du koua, tel qu'il est énuméré dans la « définition des koua $»^{3}$.

1170.

L'homme saint a le moyen de voir le mouvement de l'univers (de ce qui est sous le ciel) et il montre aux regards la réunion (conjonction) et la libre pénétration, pour pratiquer les règles rituelles. Il annexe les formules pour déterminer le présage heureux ou malheureux, et c'est pourquoi on les désigne par l'expression de «formules des traits» 4 .

TSHOU HI. - Réunion, c'est-à-dire ce qui est rassemblé par la raison d'être et ce qui ne peut pas être dérangé de sa place. Libre pénétration veut dire la possibilité de pratiquer la raison d'être des choses sans aucune erreur de lieu et d'application. Ainsi dans la « désarticulation du bouf », la réunion, c'est « l'articulation » ${ }^{5}$, la « libre pénétration », c'est le vide.

1171.

\footnotetext{
${ }^{1}$ Exprimer une idée par une idée analogue.

2 Obscurité ; il ne peut s'agir de «l'homme saint», donc il s'agit probablement du changement (ya).

3 Titre d'un des commentaires de Khong Tse.

${ }^{4}$ [] Relier, lier; symboliser une règle. Désigne aussi les traits des koua et les formules annexées à ces traits.

${ }^{5}$ Sens très douteux ; peut-être le tendon ? C'est une citation d'un passage mal expliqué d'un ouvrage ancien.
} 
Il explique ce qu'il y a de plus obscur dans l' univers (sous le ciel) et ce qui ne doit pas être considéré comme odieux; il explique l'extrême mouvement dans l'univers (sous le ciel) et ce qui ne peut pas être troublé ${ }^{1}$.

TSHOU HI. - Être odieux, ce qui provoque le dégoût par la satiété.

1172.

Il s'en rend compte et ensuite il parle; il délibère et ensuite il se meut; il décide et délibère pour produire la modification et la transformation ${ }^{2}$.

TSHOU HI. - Il regarde l'image symbolique et se pénètre de la formule ; il regarde la modification et se pénètre du sens divinatoire. Puis il la traduit en règle et la pratique. Cette formule et les sept suivantes posent des règles d'interprétation.

1173.

L'oiseau kou chante dans la négativité ${ }^{3}$; ses petits accordent leur chant au sien. J'ai un beau titre; moi et toi nous le désirons. Tse ${ }^{4}$ dit : L'homme doué occupe l'abri ${ }^{5}$; il prononce de bonnes paroles, et à plus de dix mille lis à la ronde on lui répond sympathiquement; combien à plus forte raison ceux qui sont proches! Occupant labri, s'il prononçait de mauvaises paroles, à plus de mille lis à la ronde on s'écarterait de lui, et à plus forte raison ceux de son voisinage. La parole sort du corps, elle agit sur le peuple; l'action se révèle dans ce qui est proche, elle se manifeste dans ce qui est lointain. La parole et l'action sont le pivot de l'action de l'homme doué. L'effet qui résulte de ce pivot, origine de tout effet, c'est la cause de toute gloire ou de toute honte. La parole et l'action sont les moyens par lesquels l'homme doué remue et ébranle le ciel et la terre. Est-il possible de ne pas y apporter de circonspection?

\footnotetext{
${ }^{1}$ Le texte commence par le caractère [] expliquer verbalement, et aussi, la «parole », le langage. Je crois quill faudrait lire : «la parole est ce qu'il y a de plus mystérieux dans l'univers et elle ne doit pas être déna turée ; la parole est ce qu'il y a de plus mobile et on ne doit pas y introduire la confusion. »

2 [] Décider, se rendre compte d'un fait ; [] discuter, délibérer.

${ }^{3}$ Dans l'ombre. Un certain échassier, peut -être une cigogne « chante au milieu de la nuit et sa voix est entendue à huit ou neuf lis de distance ». Tse tien.

${ }^{4}$ Khong Tse.

${ }^{5}$ Maison, toit
} 
TSHOU HI. - Explication du sens du second trait nonaire du koua tshong fou ${ }^{1}$.

1174.

Dans le koua thong jen d'abord pleurs et cris, ensuite rire. Tse dit: La voie rationnelle de l'homme doué lui prescrit tantôt de sortir, tantôt de rester; tantôt d'être silencieux, tantôt de causer. Lorsque deux hommes ont le même caur ${ }^{2}$, leur force brise le métal. Les paroles qui résultent de caurs identiques sont odorantes comme les parfums.

TSHOU HI. - Explication du sens du cinquième trait nonaire du koua thong jen. Il s'agit de la voie rationnelle de l'homme doué. Au début elle ne semble pas unique, mais ensuite elle ne présente aucune différence. Les expressions «briser le métal », « odorant comme les parfums », expriment que les êtres ne peuvent pas être divisés et que les paroles ont une saveur.

1175.

Premier trait hexaire: tapisser en employant du chaume blanc; pas de culpabilité ${ }^{3}$. Tse dit: S'il est possible de placer directement sur le sol, et si cependant on le tapisse avec du chaume, quelle faute commettrait-on? C'est la plus extrême circonspection. Or, le chaume est une matière commune, mais son effet peut devenir précieux. En portant la circonspection dans les intentions, avant d'agir, c'est un moyen d'éviter tout inconvénient.

TSHOU HI. - Explication du premier trait hexaire du koua tae kuo.

1176.

L'homme doué méritant et modeste; il a une fin heureuse. Tse dit: Il se donne du mal et il ne s'en vante pas; il a du mérite et il ne le montre pas; c'est la plus extrême grandeur d'âme. Celui qui proclame son propre mérite est un homme inférieur. Au sujet de la vertu, on emploie les mots «perfection

\footnotetext{
${ }^{1} \mathrm{~N}^{\circ} 1070$.

${ }^{2}$ La même volonté.

${ }^{3}$ [signet p514]
} 
achevée.>, au sujet des convenances (rites), on emploie l'expression « respect dans la contenance ». C'est là la modestie ; c'est pousser le respect aussi loin que possible, pour conserver la situation acquise.

TSHOU HI. - Explication du sens du troisième trait nonaire du koua Khien ${ }^{1}$. «Au sujet de la vertu parler avec perfection, au sujet des rites parler avec respect »; cela veut dire en parlant de la vertu vouloir qu'elle soit complète, et, au sujet des rites, vouloir le respect ${ }^{2}$.

1177.

Dragon élevé : il y a regret ${ }^{3}$. Tse dit: Noble et sans situation, élevé et sans peuple; l'homme sage est dans une situation inférieure et ne l'aide pas ; c'est là se mouvoir et avoir des regrets.

TSHOU HI. - Explication du trait supérieur nonaire du koua Khien. Ceci devrait faire partie du «commentaire sur l'expression des représentations »; c'est effectivement une répétition.

1178.

Ne pas sortir de la porte de la cour intérieure; pas de culpabilité ${ }^{4}$. Tse dit : La cause d'où naît le désordre, c'est la parole et la conversation ${ }^{5}$; c'est là la suite des degrés qui y conduisent. Si l'homme doué n'est pas discret, il perdra le sujet; si le sujet n' est pas discret, il se perdra lui-même. Être indiscret dans les préliminaires dune affaire quelconque entravera son achèvement. C'est à cause de cela que l'homme doué est discret, circonspect, et qu'il ne sort pas au-dehors.

TSHOU HI. - Explication du sens du premier trait nonaire du koua tsie.

1179.

\footnotetext{
${ }^{1}$ [signet p295]

${ }^{2}$ Le commentaire n'est pas plus clair que le texte.

3 [signet p018]

${ }^{4}$ [signet p060]

${ }^{5}$ Les bavardages.
} 
Tse dit: Ceux qui ont fait le Yi king connaissaient-ils le vol ? Le Yi dit: porter sur les épaules et d'ailleurs en voiture; il en résulte que les brigands surviennent ${ }^{1}$. Porter sur les épaules, c'est le fait de l'homme inférieur ; la voiture est un objet de l'homme doué. Si un homme inférieur se sert d'un objet réservé à un homme doué, les brigands méditent de le dépouiller : Lorsque les supérieurs sont négligents et les inférieurs trop hardis, les brigands songent à les détruire tous. Serrer négligemment ${ }^{2}$, c'est appeler les voleurs; se farder le visage, c'est appeler la luxure. Le Yi dit: porter sur les épaules et d'ailleurs en voiture, il en résulte que les brigands surviennent ; c'est appeler les brigands.

TSHOU HI. - Explication du sens du troisième trait hexaire du koua kiae.

Huitième paragraphe. Traite de l'usage des formules des traits.

1180.

Le ciel est un, la terre est deux; le ciel est trois, la terre est quatre; le ciel est cinq, la terre est six; le ciel est sept, la terre est huit; le ciel est neuf, la terre est dix.

TSHOU HI. - Cette tablette ${ }^{3}$, était primitivement en tête du dixième paragraphe. Tsheng Tse dit qu'elle doit être placée ici, et actuellement on suit cette leçon. - Il s'agit des nombres du ciel et de la terre. La positivité c'est l'unité ; la négativité c'est la dualité ; c'est précisément ce qu'on appelle le «plan du fleuve » ${ }^{4}$. Les situations caractérisées par les nombres un et six sont en bas; deux et sept sont en haut; trois et huit sont à gauche; quatre et neuf à droite; cinq et dix au milieu. En en parlant au point de vue de cette proposition, le nombre cinq du milieu représente l'extension de ce qui engendre et produit ; le nombre dix qui vient à côté représente l'extension de ce qui est engendré. Les nombres un, deux, trois et quatre représentent les situations des quatre symboles; les nombres six, sept, huit et neuf sont les nombres qui correspondent à ces quatre symboles. Les deux nombres vieux ont leur situation au nord-ouest, les deux nombres jeunes ont leur situation au sud-est. Ces nombres, chacun d'après leur genre, sont rangés à l'intérieur.

\footnotetext{
1 [signet p719]

${ }^{2}$ Les objets précieux.

${ }^{3}$ Feuille de palmier servant à écrire.

${ }^{4}$ Un certain tableau représentant probablement des constellations.
} 
1181.

Il y a cinq nombres célestes et cinq nombres terrestres. Dans chacune des cinq situations, deux nombres concordent ensemble. La somme des nombres célestes est vingt-cinq et la somme des nombres terrestres est trente; ensemble les nombres célestes et terrestres donnent pour somme cinquante-cinq. C'est ceci qui accomplit la modification et la transformation et qui agit dans les démons et les génies ${ }^{1}$.

TSHOU HI. - Ce feuillet se trouvait primitivement après le paragraphe suivant, depuis on a reconnu qu'il doit être placé ici. Les cinq nombres célestes, un, trois, cinq, sept, neuf, sont tous impairs; les cinq nombres terrestres. deux, quatre, six, huit, dix, sont tous pairs. Ils concordent ensemble ; c'est-à-dire qu'un et deux, trois et quatre, cinq et six, sept et huit, neuf et dix, l'un impair et l'autre pair, forment un groupe de même genre et concordent ensemble. Dans chacune des cinq situations deux nombres correspondent ensemble ; cela veut dire un et dix, deux et sept, trois et huit, quatre et neuf, cinq et dix, chaque groupe comprend toujours deux nombres qui s'accordent. Vingt-cinq est la somme totale des cinq nombres impairs ; trente est la somme totale des cinq nombres pairs. Modification et transformation ; cela veut dire que l'unité se modifie et engendre l'eau, tandis que le nombre six la transforme et la produit; le nombre deux transforme et engendre le feu et le nombre sept le modifie et l'achève. Trois modifie et engendre le bois et huit le transforme et l'achève; quatre transforme et engendre l'or, et neuf le modi fie et l'achève ; cinq modifie et engendre la terre et dix la transforme et l'achève. Génies et esprits, sont des termes qui expriment tous les phénomènes de contraction et de redressement, d'aller et de retour, engendrés et produits par l'unité et par la dualité ${ }^{2}$.

1182.

Le total de la "grande somme» est cinquante; on n'en emploie que quarante-neuf. On les partage et on en fait deux parts pour symboliser les deux principes. On en place un entre le petit doigt de la main gauche pour symboliser le troisième agent (l'homme). On les compte par quatre, pour symboliser les quatre moments (saisons). On ramasse le reste entre l'index et le médius de la main gauche pour symboliser les mois complémentaires. Dans cinq années, il y a deux mois complémentaires, aussi on refait l'opération du reste en éliminant par quatre et ensuite on en replace un entre le petit doigt.

\footnotetext{
${ }^{1}$ Phénomènes d'expansion et de contraction.

${ }^{2}$ Les cinq agents, eau, feu, terre, bois et métal ; ce sont aussi les noms des cinq planètes.
} 
TSHOU HI. - Le nombre total de la « grande somme » est cinquante. En effet, d'après le « centre secret» du «tableau du fleuve », le nombre céleste cinq multiplie le nombre terrestre quatre et on obtient le nombre cinquante. Mais lorsqu'on veut emp loyer ce nombre pour consulter le sort au moyen des brins de paille, on borne l'emploi à quaran te-neuf, de sorte que chacune de ces pratiques est une conséquence naturelle de la raison d'être des choses et que la force ou la science des hommes ne peut rien y ajouter ou y retrancher. Les « deux principes », expression qui désigne le ciel et la terre. Le caractère [] du texte, koua, signifie « en suspendre un entre le petit doigt de la main gauche et le doigt suivant». Le caractère trois du texte désigne les trois causes efficientes, ou les trois agents ${ }^{1}$. Éliminer, séparer et emporter à part. Le « reste ${ }^{2}$, ce qui reste après qu'on a compté, quatre par quatre. Ramasser dans les deux intervalles du médius de la main gauche. Mois complémentaire l'ensemble des jours en plus de l'année lunaire et formant un mois pour remettre l'année lunaire en concordance avec l'année solaire. Dans l'intervalle de cinq années on est obligé de réunir deux fois ces jours et d'en former deux fois un mois complémentaire, aussi, en cinq ans il y a toujours deux mois complémentaires. Aussitôt l'opération terminée, on relève le tout, on réunit et l'on sépare comme après la pre mière fois, de façon à faire le même groupement dans les deux mains et l'on recommence ainsi la même opération ${ }^{3}$.

1183.

Les brins relatifs au koua khien sont au nombre de deux cent seize; les brins relatifs au koua khouen sont au nombre de cent quarante-quatre, en tout trois cent soixante, équivalent au nombre de jours d'une révolution.

TSHOU HI. - Ces divers nombres de brins résultent des quatre symboles. En effet, le «tableau du fleuve» a quatre faces ; la parfaite positivité réside dans l'unité et est suivie du nombre neuf ; la jeune négatitivé réside dans le nombre deux et est suivie du nombre huit. La jeune positivité réside dans le nombre trois et est suivie du nombre sept. L'extrê me négativité réside dans le nombre quatre et est suivie du nombre six. La règle pour compter et éliminer les brins de l'herbe $s h{ }^{4}$ consiste à compter ensemble ce qui reste après les trois modifications, à écarter l'unité dès le commencement ; à compter chaque groupe de quatre comme une nouvelle unité et chaque groupe de huit comme une paire ou dualité. L'unité est entourée circulairement par trois ; la dualité

\footnotetext{
${ }^{1}$ Le ciel, la terre et l'homme.

${ }^{2}$ Le caractère du texte désigne seulement l'unité.

${ }^{3}$ C'est la description de la manière d'opérer pour pratiquer la divination avec des brins de paille.

4 « L'herbe spirituelle».
} 
est entourée en carré par quatre ; trois emploie la totalité ; quatre emploie la moitié. En réunissant le tout et en le comptant, cela donne les nombres six, sept, huit, neuf, et après trois éliminations, tout se trouve encore réuni. En effet, il reste trois unités en excès (ce qui répété trois fois donne donc neuf; les éliminations sont aussi au nombre de neuf, les brins sont donc au nombre de 4 x $9=36$. C'est ce qui est considéré comme constituant l'extrême positivité qui réside dans l'unité. S'il reste deux unités et une dualité, cela fait huit et les éliminations sont aussi au nombre de huit, ce qui fait que les brins sont au nombre de 4 × $8=32$, c'est ce qui est considéré comme constituant la jeune négativité résidant dans le nombre deux. Deux dualités et une unité font le nombre sept et les éliminations sont aussi au nombre de sept, de sorte que les brins sont au nombre de $4 \times 7=28$, ce qui est considéré comme constituant la jeune positivité résidant dans le nombre trois. Trois dualités feront six et le nombre des éliminations étant de six, les brins seront au nombre de 4 × $6=24$, ce qui est considéré comme constituant la négativité vieille résidant dans le nombre quatre. Tel est le mystère merveilleux de la transformation, du mouvement d'aller et de retour, de la séparation et de la réunion. C'est toujours une conséquence spontanée et naturelle de la nature même des choses et quelque chose que l'homme ne pourrait faire de lui même. La jeune négativité se retire avant d'être à son extrême limite dans le vide (o u néant) ; la jeune positivité avance et n'est pas encore à son extrême limite comme plénitude. C'est pour cela que l'on tient seulement compte de la vieille positivité et de la vieille négativité et que l'on ne compte que les brins des traits du koua Khien et du koua khouen. Dans tous les autres cas on ne peut déduire la règle d'après ce qui précède. Le terme révolution, veut dire une révolution d'une année, en tout trois cent soixante-cinq jours et un quart. Ceci a exclusivement pour but de montrer la formation des nombres ${ }^{1}$.

1184.

Les brins qui correspondent aux deux parties du Yi king sont au nombre de onze mille cinq cent vingt, ce qui correspond au nombre de toutes choses ${ }^{2}$.

TSHOU HI. - Les deux parties du Yi king, la première et la seconde. Les koua contiennent en tout cent quatre-vingt-douze traits positifs, et le même nombre de traits négatifs. Or $192 \times 36=6.912$ et $192 \times 24=4.608$, en tout 11.520 brins correspondant à autant de formules de divination.

\footnotetext{
${ }^{1}$ Le commentaire ne se rapporte pas directement au $Y i$ king. Il vise exclusivement les pratiques de la divination au moyen des brins d'herbe.

${ }^{2}$ Il s'agit, enfin de compte, du nombre d'oracles que l'on peut tirer du Yi king en pratiquant une certaine méthode de divination. Le mot traduit par brin (d'herbe) a plusieurs significations. Dans le Shou king il est dit que Hoang Ti se servait de ces brins d'herbe pour en déduire les périodes du calendrier.
} 
1185.

C'est pour cela que quatre opérations achèvent un changement ; dix-huit modifications complètent un koua.

TSHOU HI. - Quatre opérations ; cela veut dire, division des brins en deux groupes, suspension d'un brin, élimination par quatre, recueil du reste ${ }^{1}$. Changement, modification; une modification. Trois modifications déterminent une formule de trait; dix-huit modifications correspondent aux six traits d'un koua.

1186.

Huit koua et petit achèvement.

TSHOU HI. - Cela veut dire que neuf transformations achèvent de déterminer trois traits, ce qui donne un koua intérieur ${ }^{2}$.

1187.

Les étendre et en faire des déductions; les appliquer à chaque genre et les développer, et les choses possibles dans l'univers sont épuisées.

TSHOU HI. - Cela veut dire que les six traits étant complets, et en regardant ceux de ces traits qui se modifient, comme aussi ceux qui ne se modifient pas et en les considérant comme représentant, les uns le mouvement, les autres le repos, il en résultera qu'un même koua peut, rien que par ses modifications, devenir successivement l'un quelconque des soixante-quatre traits et servir à déterminer les présages heureux ou malheureux. Ces modifications, appliquées à chaque koua, engendrent ces mêmes koua de quatre mille quatre-vingt-seize façons différentes ${ }^{3}$.

\footnotetext{
$1 \S n^{\circ} 1181$.

2 Intérieur, quand les koua de six traits sont rangés en cercle, c'est-à-dire le koua inférieur d'un koua de six traits.

${ }^{3} 64 \times 64=4096$.
} 
1188.

La voie rationnelle, rendue brillante et claire, les vertus de l'esprit

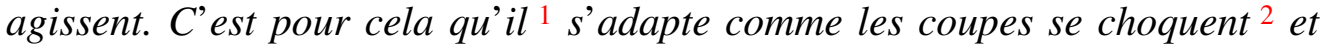
qu'il peut concourir à la transformation.

TSHOU HI. - La voie rationnelle est rendue manifeste et évidente par les formules. L'action se produit par les nombres et sur les esprits ( ?). Les termes du texte expriment l’idée de répondre à une libation par une libation et d'aider à l'œuvre de la transforma tion de l'esprit (génie).

1189.

Tse dit : Celui qui connaît la voie rationnelle de la modification et de la transformation, celui-là connaît ce qui est produit par l' esprit ${ }^{3}$.

TSHOU HI. - La voie rationnelle de la modification et de la transformation; c'est préci sément les règles relatives aux nombres, telles qu'elles sont exposées dans le texte qui pré cède. C'est toujours quelque chose hors de la portée des hommes, aussi, Fou Tse s'écrie admirativement de sorte que les disciples du philosophe ont ajouté les mots : «Tse dit» afin de les distinguer du reste du texte.

Neuvième paragraphe. Il traite des règles de la grande somme des nombres, de la méthode de trouver les brins d'herbe shi pour rechercher dans les koua un oracle répondant à une question. Toutefois, ces questions ne sont traitées que dans leurs traits essentiels et généraux; il est probable que les explications complètes se trouvaient développées au sujet des fonctionnaires chargés de la divination ${ }^{4}$, mais il est actuellement impossible de le contrôler. Ce qui peut en être déduit est complètement copié dans le commentaire intitulé « dissipation des ténèbres » 5 .

\footnotetext{
${ }^{1}$ Le Yi king.

2 2. - Cette expression rend le sens sans être littérale.

3 «L'action de l'esprit », expression qui distingue d'une façon vague et générale un fait, ou phénomène quelconque dont la cause est inconnue ou mal comprise.

${ }^{4}$ Une partie perdue du Tcheou li.

${ }^{5}$ De TSHOU HI.
} 
1190.

Le Yi king contient les voies rationnelles de l'homme saint, au nombre de quatre: par la parole, dans les formules; par le mouvement, dans les modifications ; par la détermination des objets usuels, dans les symboles ; par la divination, dans les formules divinatoires.

TSHOU HI. - Ces quatre distinctions constituent toute la voie rationnelle de la modification et de la transformation et sont l'auvre des esprits.

1191.

C'est à cause de cela que l'homme doué est sur le point d'avoir à faire, sur le point d'avoir à agir. Il demande, et c'est par la parole qu'il reçoit la réponse. Il reçoit l'ordre qui prescrit sa destinée comme répondu par un écho. Il n’y a (pour lui) rien d'éloigné, rien de près ; rien d'obscur, rien de caché ; il a spontanément connaissance et conscience des êtres qui arrivent. Sans la plus extrême pureté d'essence, dans l'univers, qui donc pourrait prévoir à l'avance, à ce point?

TSHOU HI. - Il s'agit ici de ce qui a rapport à ce qui se trouve dans les formules et dans le sens divinatoire. Il s'agit de l'homme consultant le Yi king à l'aide des brins d'herbe shi, cherchant à obtenir la formule du trait ou du koua, et s'en servant pour expliquer et pour pren dre un parti au sujet de quelque chose. C'est donc que le Yi king donne une réponse aux hommes au sujet de la destinée, et qu'il contient ce qui est nécessaire pour l'avertir et le renseigner. Le Yi king lui répond comme un écho renvoie le son, afin de décider du présage heureux ou malheureux au sujet des événements qui ne sont pas encore arrivés. Par la parole ; comme en parlant, c'est considérer les paroles employées dans les formules avec le sens qu'elles comportent. Destinée ; c'est une expression relative aux invocations adressées au moment où on est sur le point de consulter le sort. Cela a de l'analogie avec le passage du Tcheou li où il est dit que, le jour de la consultation du sort, « le maître proclame la destinée ». 
Par trois, par cinq ${ }^{1}$, par les modifications; mélanger et confondre les nombres. Pénétrer les modifications, achever naturellement et sans effort les signes du ciel et de la terre. Épuiser les nombres, et en déterminer naturellement les symboles du ciel et de la terre. Sans l'extrême modification de l'univers, qui donc serait capable de ceci?

TSHOU HI. - Ceci est relatif à la considération des symboles. La modification, c'est le symbole avant qu’il ne soit déterminé. Par trois, compter par trois ; par cinq, compter par cinq ${ }^{2}$. Après avoir compté trois par trois pour la modification ${ }^{3}$, on compte encore cinq par cinq pour la modification, une fois avant et une fois après. On recherche encore une fois de plus pour s'assurer de la réalité du plus ou du moins. Mélanger veut dire réunir, mettre ensemble une partie à droite, une partie à gauche. Confondre, c'est mettre tout ensemble et réunir dans une même main ; c'est une expression qui indique qu'une partie est en dessous et l'autre en dessus. Tout cela se rapporte encore à ce qui est relatif aux opérations relatives à la recherche d'un koua qui doit donner une réponse à une question. En effet, on compte par trois en relevant les brins contenus dans les deux mains, afin d'arriver à déterminer complètement s'il s'agit d'une négativ ité ou d'une positivité vieille ou jeune. On recherche les nombres sept et huit, neuf et dix, pour déterminer le symbole de mouvement ou de repos présenté par le koua ou par le trait. Ces expressions «par trois, par cinq, mélanger, confondre », sont toutes déjà anciennes, mais les deux premiers termes «par trois, par cinq », sont particulièrement difficiles à comprendre. Si on observe que Suien Tse dit : «En surveillant ce qui est opposé pour maîtriser les modifications, on veut par cinq pour tripler »; que Wei Fei dit : «Scruter les analogues et les différences dans les paroles, afin de connaître les distinctions entre membres d'une même association ${ }^{4}$; survient l'épreuve par « trois et par cinq » pour s'assurer de la réalité des mots; qu'il dit encore : «tripler», afin de comparer les êtres, « quintupler» pour corroborer l'épreuve « par trois »; qu'enfin le Shou king dit : « il faut absolument tripler et quintupler », et aussi «par trois et par cinq sans erreur »; que le livre des Han dit : «tripler et quintupler le prix », en réunissant ces diverses citations et en comparant leur sens à chacune, cela paraît encore suffisant pour éclairer le sens du passage qui nous occupe.

1193.

\footnotetext{
${ }^{1}$ Tripler, quintupler.

${ }^{2}$ Ou peut-être : compter trois, compter cinq fois?

3 Probablement pour connaître la modification.

${ }^{4}$ Sens très douteux. La plupart de ces citations, prises isolément, hors du texte dont elles font partie, n'ont plus de sens précis. Il semble cependant qu'il est question de reconnaître des signes d'affiliation à certaines associations.
} 
Le yi ${ }^{1}$ n'a pas de pensée et n'a pas d'action ${ }^{2}$; il est naturellement le repos sans mouvement. Il influence ${ }^{3}$ et aussitôt on comprend et on pénètre sans effort les causes de l'univers ${ }^{4}$. Sans l'extrême génie de l'univers, qui donc pourrait prévoir à l'avance comme ceci?

TSHOU HI. - C'est là ce qui fait que la subs tance de ces quatre choses 5 existe et ce qui fait que leur effet se produit. Le mot yi du texte désigne les brins de l'herbe shi et les koua. Absence de pensée et d'action ; cela exprime l'absence d'individualité (cour). « Repos naturel », c'est la substance de ce qui cause l'impression ressentie ; influencer, pénétrer. L'effet du repos, c'est le mystère du cour humain ${ }^{6}$. Tels sont bien le repos et le mouvement.

1194.

Or, le yi, c'est le moyen par lequel l'homme saint scrute la limite extrême de la profondeur et juge le début.

TSHOU HI. - Juger, examiner. Début, ce qui est infiniment petit. Ce qui lui sert à pousser la profondeur à ses dernières limites, c'est sa parfaite pureté d'essence ; ce qui lui permet de juger le début initial, c'est l'extrême modification.

1195.

Il pense à la profondeur, aussi il peut pénétrer les desseins de l'univers; il pense au début initial, aussi il peut achever et terminer les affaires de l'univers ; il pense à l'esprit, aussi il peut aller vite sans précipitation, et parvenir sans agir.

TSHOU HI. - Ce qui fait quîl pénètre les desseins et quill achève les œuvres, c'est l'action de l'esprit.

\footnotetext{
${ }^{1}$ Le « Yi king », ou bien le changement.

2 Il ne fait pas.

${ }^{3}$ Il fait ressentir son impression.

${ }^{4}$ Du ciel, et du dessous.

5 Voir $\$ 1189$.

${ }^{6}$ Le mystère de l'intelligence humaine.
} 
1196.

Tse dit: C'est là ce qu'on entend par les quatre voies rationnelles de l'homme saint contenues dans le yi.

TSHOU HI. - Dixième paragraphe. - Continuation du sujet du paragraphe précédent. Il explique que l'effet du Yi king comprend ces quatre conséquences.

1197.

Tse dit : Or, comment le yi agit-il ? Le yi développe les êtres et achève le fait ; il recouvre la voie rationnelle de l'univers. Il est ainsi, et rien de plus. C'est pour cela que l'homme saint l'emploie pour pénétrer les desseins de l'univers, pour déterminer les auvres et les actions de l'univers, pour trancher les doutes de l'univers.

TSHOU HI. - Développer, ou expliquer les êtres et achever les choses ; cela veut dire engager les hommes à consulter le sort afin de connaître les présages heureux ou malheureux et de terminer ensuite les affaires et les entreprises. Recouvrer la voie rationnelle de l'univers veut dire que, lorsque les koua et les traits sont déterminés, la voie rationnelle de l'univers s'y trouve toujours renfermée.

1198.

$C$ est pour cela que la vertu des brins de l'herbe shi est ronde et spirituelle; que la vertu des koua sert de règle pour le savoir ${ }^{1}$; que le sens des six traits change pour avertir. L'homme saint s'en sert pour purifier son caur, il se retire dans le secret. Il subit, comme les hommes, les présages heureux ou malheureux. L'esprit sert à connaître l'avenir; le savoir sert à cacher ce qui est passé. Qui donc pourrait égaler ceci? Ceux qui furent intelligents et lucides dans leur entendement, dans l'antiquité, doués d'un grand savoir, ils étaient redoutables par l' esprit et ne causaient point de mal.

TSHOU HI. - «Ronde et spirituelle»; cela veut dire que la transformation et la modification ont lieu sans règle ${ }^{2}$. " Règle du savoir »; cela veut dire que les choses ont toutes une raison d'être déterminée et établie.

\footnotetext{
${ }^{1}$ Littéralement on lirait « est carrée à cause du savoir ».

${ }^{2}$ Or, le mot fang, règle, veut aussi dire « carré », de sorte qu'on peut lire « sans carré ».
} 
« Change pour avertir»; cela veut dire changer pour avertir et prévenir les hommes ${ }^{1}$. L'homme saint met en pratique les vertus de ces trois conditions et il n'est pas retenu par une seule attache ${ }^{2}$. Quand il n'y a rien à faire, son cour est naturellement en repos et l'homme ne peut pas le percevoir ; quand il y a à faire, alors l'effet de l'esprit (phénomène) et le savoir se suivent et influencent en se correspondant: c'est ce qui est exprimé par les mots « connaître le présage heureux ou malheureux sans consulter le sort ». «L'esprit redoutable ne cause point de mal »; expression indiquant la conformité à la raison d'être sans dénaturer les choses.

1199.

C'est ainsi qu'il ${ }^{3}$ sert à éclairer la voie ${ }^{4}$ rationnelle $d u$ ciel et à rechercher et examiner les causes qui influent sur les peuples. C'est là relever l'esprit et les êtres pour précéder les effets des hommes. L'homme saint s'en sert pour régulariser et avertir, afin de redresser et d'éclairer la vertu ${ }^{5}$.

TSHOU HI. — «L'esprit et les êtres »; cela désigne les brins d'herbe et la tortue ${ }^{6}$. "Redresser» est une expression qui indique l'absolue pureté naturelle ; «avertir» est une expression qui indique le respect naturel et spontané avec une attention craintive. Il éclaire la voie rationnelle du ciel, de sorte que l'on connaît la possibilité de manif estation (action de s'élever, de surgir) des phénomènes et des êtres. Examiner les causes qui influent sur les hommes, de sorte qu'on peut connaître l'impossibilité qu'il y a à ce que l'effet attendu ne se produise pas, et cela afin de la manifester (l'annoncer) à l'avance. C'est par là qu'il 7 établit la divination, pour enseigner aux hommes, et dans cette action il régularise et avertit, afin de rechercher le sens divinatoire, afin que le cour et l'esprit soient éclairés sans limite, comme l'aptitude des démons et des génies à connaître l'avenir.

1200.

C'est pour cela que fermer une porte est appelé khouen ; ouvrir une porte est appelé khien; une occlusion et une séparation sont désignées par l'expression « une modification». L'aller et le retour sans fin constituent ce

\footnotetext{
${ }^{1}$ L'acception « avertir » donnée au caractère du texte, vient précisément de l'explication de ce passage, donc elle ne prouve rien.

${ }^{2}$ Qui empêcherait la liberté de son jugement.

${ }^{3}$ Il s'agit du yi ce qui peut désigner le Yi king, ou le «changement ».

${ }^{4}$ À jeter la lumière dans la voie...

${ }^{5}$ Se rapporte probablement aux « hommes ».

${ }^{6}$ Les moyens de divination.

${ }^{7}$ Probablement l'homme saint ?
} 
qu'on appelle «libre pénétration». Ce qui est manifesté et visible est appelé un «symbole»; ce qui a une forme est appelé «objet»; en déterminer les dimensions et l' emploi est appelé « créer une règle »; l'effet utile, la sortie ou l'entrée que les hommes emploient tous, est appelé le phénomène ${ }^{1}$.

TSHOU HI. - Fermer et ouvrir, cause initiale du repos et du mouvement. Il est d'abord question du koua khouen : c'est que c'est par le repos que se produit le mouvement, l'action. Les koua khien et khouen circulent librement : c'est l'auvre de la transformation et de la production. Symbole de la manifestation, objet et forme, tel est l'ordre dans la génération des êtres. La règle, ce que l'homme saint établit pour reconstituer la voie rationnelle. Enfin le phénomène, ou esprit, ce que le peuple emploie journellement et spontanément.

1201.

C'est pour cela que le yi comporte l'extrême origine; c'est là ce qui engendre les deux équivalents, ou règles; les deux règles engendrent les quatre symboles, et les quatre symboles engendrent les huit koua simples.

TSHOU HI. - L'unité engendre la dualité : c'est la raison d'être naturelle. Le $y i$, c'est la modification de la négativité et de la positivité. L'extrême origine : c'est la raison d'êtr e. Les deux équivalents ${ }^{2}$ : c'est d'abord la création d'un trait pour distinguer la négativité de la positivité. Les quatre symboles ; c'est, ensuite, créer deux traits afin de distinguer ce qui est à sa limite et ce qui débute. Les huit koua simples ; c'est, encore plus tard, la création de trois traits, pour compléter les symboles des trois causes efficientes. Ces quelques paroles indiquent bien effectivement l'ordre de succession naturel dans lequel l'homme saint a fait le Yi king. L'ouvre s'est accomplie sans qu'il ait eu à emprunter une parcelle, si minime qu'elle soit, de son savoir ou de sa force. Qu'il s'agisse de tracer les koua, ou de manipuler les brins d'herbe pour la divination, l'ordre des opérations est toujours ainsi et clairement expliqué et classé en règles dans le commentaire intitulé Khi mong ${ }^{3}$.

1202.

\footnotetext{
${ }^{1}$ L'esprit ?

2 Règles, lois.

${ }^{3}$ Traité de la divination par le Yi king de TSHOU HI.
} 
Les huit koua simples déterminent le présage heureux ou malheureux; les présages heureux ou malheureux donnent naissance à la «grande science ».

TSHOU HI. - Il y a des présages heureux, il y a des présages malheureux : c'est ce qui engendre la grande science.

1203.

C est pour cela que les règles et les symboles ne sont jamais aussi grands que dans le cas du ciel et de la terre; que la modification et la libre circulation ne sont jamais plus grandes qu'au sujet des quatre saisons; que la mise en évidence des symboles ${ }^{1}$ et la manifestation de la clarté n'est jamais plus grande que pour le soleil et la lune; que l'illustration et l'élévation ne sont jamais plus grandes que dans la richesse et la noblesse des êtres. Le complément des séries des êtres et les effets qu'ils produisent, l'établissement ${ }^{2}$ et l'achèvement des objets usuels pour le bien de l'univers n'est jamais plus grand que dans l'auvre de l'homme saint. La recherche dans la confusion de l'obscurité, l'action de sonder dans les profondeurs les plus éloignées, afin de déterminer les présages heureux ou malheureux pour l'univers, et l'achèvement de l'effort ${ }^{3}$ constant de l'univers, n'est jamais plus grand que dans les brins d' herbe shi et la tortue ${ }^{4}$.

TSHOU HI. - Richesse et noblesse, veut dire possession de la situation du souverain de l'univers. Après le caractère [] traduit par «établissement » dans la phrase : «l'établisse ment et l'achèvement des objets usuels... », il y a lieu de croire qu'il manque quelques mots du texte. Achèvement de l'effort ; s'efforcer sans relâche ; le doute engendrerait la paresse, mais comme il y a décision et certitude, donc il y a effort constant.

1204.

C'est pour cela que le ciel engendre les esprits (phénomènes) et les êtres, et que l'homme saint en formule les règles; le ciel et la terre se modifient et se transforment, et l'homme saint en explique les lois. Le ciel montre les symboles, manifeste le présage heureux ou malheureux, et l'homme saint les

\footnotetext{
${ }^{1}$ Littéralement : suspendre un symbole en l'exposant en évidence.

2 Voir le commentaire de TSHOU HI.

${ }^{3}$ Le sens du terme du texte est à peu près inconnu.

${ }^{4}$ Instruments de la divination par le Yi king.
} 
représente ${ }^{1}$. Du fleuve sort le tableau, du lac sort le livre ${ }^{2}$ et l'homme saint en formule les règles ${ }^{3}$.

TSHOU HI. - Ces quatre choses sont les moyens que l'homme saint utilise pour faire le $Y i$ king. Pour le «tableau du fleuve » et le «livre du lac », voir le Khi mong ${ }^{4}$.

1205

Le yi (changement) a quatre symboles, qui lui servent pour avertir et enseigner. Les formules qui y sont annexées servent à expliquer l'avertissement. La détermination des présages heureux ou malheureux sert à décider.

TSHOU HI. - Les quatre symboles, c'est-à-dire la négativité, la positivité, vieilles ou jeunes. Avertir et enseigner, c'est-à-dire montrer aux hommes par les traits des koua qu'ils rencontrent dans la divination.

Onzième paragraphe ; traite spécialement de la divination par les koua.

1206.

Le Yi king dit : "Par le présage heureux de l'assistance du ciel, rien n'est sans avantage ${ }^{5}$. Tse dit : aider, donner assistance. Ce que le ciel aide, c'est la soumission; ce que l'homme aide, c'est la confiance. Marcher avec confiance et penser à la soumission ; de plus s'appliquer à estimer la sagesse. C'est à cause de cela que le ciel laide et que le présage heureux n' est jamais sans avantage.

TSHOU HI. - Explication du sens de la formule du trait supérieur nonaire du koua tae yeou. Toutefois, placée ici, cette explication ne se rapporte à rien; il y a lieu de craindre qu'il n'y ait eu une transposition erronée du texte et que celui-ci devrait être à la fin du huitième paragraphe ?

1207.

\footnotetext{
${ }^{1}$ Les symbolise.

2 Ou plutôt « l'écriture ».

${ }^{3}$ Le « tableau du fleuve » et le « livre du lac », deux dessins mystérieux, ou signes, tracés sur le dos d'une tortue ou d'un dr agon. Les dessins me paraissent représenter des constellations.

4 Voir renvoi du $\S 1201$.

${ }^{5}$ Koua tae yeou; $\S 285$.
} 
Tse dit : L'écriture ${ }^{1}$ ne peut épuiser la parole ${ }^{2}$; la parole ne peut épuiser l'idée; mais, cependant, les idées de l'homme saint ne pourront-elles pas être manifestées? Tse dit: L'homme saint établit des symboles pour épuiser l'idée ; il institue des koua pour épuiser le vrai et le faux; il annexe des formules pour épuiser la parole; il transforme et pénètre librement pour épuiser ce qui peut être avantageux; il manifeste et expose pour épuiser l'esprit ${ }^{3}$.

TSHOU HI. - Ce qui est transmis par la parole est superficiel, tandis que ce qui est enseigné par les symboles est profond. La seule considération des deux traits, l'un repré sentant l'unité, l'autre la dualité, renferme complètement la modification et la transformation et il est visible que celles-ci n'ont ni fi n ni épuisement total. Les mots transformer et pénétrer librement, manifester et exposer se rapportent aux choses ou circonstances quelconques. Les mots « Tse dit » sont répétés deux fois; dans les deux cas ce sont des mots ajoutés plus tard par des hommes des temps postérieurs au temps où le commentaire fut écrit, ce qui a été cause de cette erreur, et c'est une superfétation. C'est un cas analogue à la mention «Tsheou Tse dit» ajoutée en tête de chaque proposition par des commentateurs postérieurs dans le livre, plus moderne cependant, intitulé Thong shou et écrit par Tsheou Tse. Il en est exactement de même dans les passages mis sous forme de demandes et de réponses.

1208.

Les mots khien et khouen désignent précisément l'essence mystérieuse du $y i$. Khien et khouen déterminent l'ordre des situations, et le changement (yi) a lieu entre les deux. Le khien et le khouen détruits, il n'y aurait plus de moyen de voir le changement; si le changement est impossible à voir, le khien et le khouen ont peut-être leur cause initiale dans un renouvellement.

TSHOU HI. - Mystérieux, caché, ce qui est enveloppé et caché, comme un vêtement habille et cache. Ce qui produit le changement, c'est uniquement la négativité et la positivité. Tout ce qui est positif est du genre khien, tout ce qui est négatif est du genre khouen. En traçant les koua et en déterminant les situations, ces deux antithèses déterminent la classification, et la substance du Yi king existe dès lors. Le khien et le khouen détruits, c'est-à-dire si les koua n'existaient pas, l'arrêt, ou renouvellement du khien et du khouen, c'est-à-dire la transformation, n'agirait pas encore.

\footnotetext{
${ }^{1}$ Littéralement shou, livre.

2 Rendre complètement la parole.

${ }^{3}$ Les caractères du texte traduits par manifester et exposer signifient frapper sur un tambour pour marquer la mesure et danser en mimant, épuiser l'esprit, c'est -à-dire analyser complètement la cause des phénomènes.
} 
1209.

C'est pour cela que tout ce qui est antérieur à la forme est appelé voie rationnelle, tout ce qui est postérieur à la forme est appelé «objet». Transformer et délimiter est exprimé par le mot modifier; déduire et pratiquer est exprimé par les mots pénétrer librement, ou comprendre. Choisir et placer est ce que les hommes de l'univers expriment par les mots propriétés des choses.

TSHOU HI. - Les koua et leurs traits, la négativité et la positivité sont toujours également des exemples de ce qui est postérieur à la forme ; la raison d'être de ces choses est dans la voie rationnelle. Montrer la spontanéité de la transformation et en tracer les lois, c'est le sens du mot modifier. Les deux mots modifier et pénétrer sont employés au sujet du ciel dans le texte de la proposition précédente; dans cette proposition, les deux mêmes mots se rapportent à l'homme.

1210.

C'est pour cela que, dans les symboles, l'homme saint a le moyen de voir le mystère de l'univers ${ }^{1}$ et de décider quant à la forme et à l'apparence, de symboliser les circonstances relatives aux êtres, et c'est pour cela qu'ils sont appelés symboles. L'homme saint a le moyen de voir le mouvement de l'univers et de considérer la réunion et la libre circulation, pour pratiquer les règles rituelles, et d'annexer des formules, pour décider des présages fastes et néfastes. C'est à cause de cela que les traits et les formules y annexées sont appelés hiao ${ }^{2}$.

TSHOU HI. - Ceci est une transition pour amener le texte qui suit.

1211.

Ce qui est l' extrême limite du mystère dans l'univers subsiste dans les koua; ce qui est produit par l'ébranlement causé par le mouvement de l'univers subsiste dans les formules.

${ }^{1} \mathrm{Du}$ « ciel et du dessous ».

2 Règle, loi, relier, imiter un exemple. 
TSHOU HI. — Les koua sont précisément les symboles ; les formules sont précisément ce qu'on appelle hiao, ou imitations.

\section{2.}

La transformation et ce qui la détermine subsistent dans la modification (des koua); la déduction et sa mise en pratique subsistent dans la libre pénétration (ou adaptation?). Ce qui en est la cause mystérieuse et ce qui l'éclaire subsiste dans l'homme. Le mutisme qui détermine la confiance sans discours subsiste dans la pratique des vertus.

TSHOU HI. - Ce qui cause la modification et la circulation des koua et de leurs traits réside dans l'homme ; ce qui fait que l'homme peut en tracer la cause mystérieuse et l'éclaircir, c'est la vertu.

Douzième paragraphe. 


\section{DEUXIÈME PARTIE}

\section{DU COMMENTAIRE TRADITIONNEL}

1213.

Les huit koua déterminent l'ordre et la classification et les symboles s'y trouvent compris; en les répétant par superposition, limitation des exemples (hiao) s'y trouve comprise.

TSHOU HI. - Déterminer, ou activer l'ordre et la classification, c'est-à-dire : le koua simple khien représente un, le koua simple touei, deux ; $l i$, trois ; tshen, quatre ; souen, cinq; khan, six; ken, sept; khouen, huit, et ainsi de suite. Symbole, désigne la substance et la forme du koua. En les répétant par superposition, c'est-à-dire que sur chacun de ces koua on place successivement chacun des huit koua, ce qui constitue les soixante-quatre koua parfaits, ou de six traits. Le mot hiao, imitation d'un exemple, désigne les six traits de chaque koua. Du moment où les koua simples sont superposés, les koua nouveaux ont chacun six traits (hiao).

1214.

La dureté énergique et la douceur malléable se succèdent en se remplaçant; la modification s'y trouve inclue. Les formules annexées y attachent une destinée, et le mouvement s'y trouve compris.

TSHOU HI. - La dureté énergique et la douceur malléable se succèdent mutuellement, et la modification du koua et des traits a lieu, l'échange et le mouvement alternatif sont toujours également visibles. L'homme saint imite cet ordre et à chacun il annexe une formule pour prononcer sur le présage heureux ou malheureux de la destinée, de sorte que, pour celui qui consulte le sort, le symbole et l'exemple du mouvement qu'il convient de f aire d'après le trait qu'il rencontre pour la divination, n'est jamais en dehors de ceci. 
Les présages heureux ou malheureux, les regrets et l'appréhension naissent également du mouvement.

TSHOU HI. - Les présages heureux ou malheureux, les regrets et l'appréhension sont toujours le résultat de la destinée tracée par les formules. Mais, toutefois, ce n'est que par le mouvement des koua et des traits ${ }^{1}$ que ces présages deviennent évidents.

1216.

L'énergie et la douceur sont la base fondamentale ; la modification et la libre pénétration sont la résultante des moments.

TSHOU HI. - Une dureté et une douceur malléable ayant chacune sa situation; de ces deux choses résulte le mouvement afin de suivre les instants.

1217.

Les présages heureux et malheureux; c'est la perfection (ou pureté) qui l'emporte.

TSHOU HI. - Perfection, droiture; ce qui est la loi constante et ordinaire. C'est par leur rectitude exacte que les choses sont considérées comme étant constantes. Dans les choses relatives à l'unive rs, si le présage n'est pas heureux, il est néfaste ; sill n'est pas néfaste, il est heureux. Les choses qui sont constantes et continues l'emportent tour à tour et se succèdent sans cesse.

1218

La voie rationnelle du ciel et de la terre, c'est l'enseignement pur; la voie rationnelle du soleil et de la lune, c' est la parfaite lumière; le mouvement de l'univers, c'est l'absolu dans l'unité.

TSHOU HI. - Enseignement, ce qui montre et commande la loi à suivre. Le mouvement de l'univers est sans fin dan s ses modifications ; toutefois, par

\footnotetext{
${ }^{1}$ Dans la modification du koua.
} 
sa conformité à la raison d'être, il comportera un présage heureux ; par son opposition à la raison d'être, il comportera un présage malheureux. Il en résulte que ce qui est régulier et constant n'est jamais autre chose que la seule raison d'être.

1219.

Or la fixité ${ }^{1}$ de l'activité (khien) enseigne aux hommes le changement, tandis que l'abaissement de la passivité (khouen) enseigne aux hommes l'observation.

TSHOU HI. - Fixité, apparence de fermeté et de rectitude ; abaissement, apparence de soumission. C'est précisément ce qui est entendu par l'expression « enseignement pur $»^{2}$.

1220.

Les hiao (traits), sont l'imitation de ceci; les symboles sont ce qui est semblable à ceci.

TSHOU HI. - Ceci traite de la raison d'ê tre de ce qui, d'après le texte précédent, est enseigné par l'activité (Khien) et la passivité (khouen). L'état d'unité ou de dualité des traits ${ }^{3}$; l'extinction et la réapparition du koua ${ }^{4}$ sont ce qui sert à imiter et à représenter.

1221.

Les hiao (traits) et les symboles se meuvent et s'agitent à l'intérieur, les présages fastes ou néfastes sont manifestés à l'extérieur. l'auvre et le mécanisme sont manifestés dans les modifications, le sentiment de l'homme saint est manifesté dans les formules.

\footnotetext{
${ }^{1}$ La traduction suit le commentaire, mais si on se reporte au second membre de la phrase, qui est évidemment destiné à faire opposition au premier, il sera plus naturel de lire : l'élévation représentée par le mot khien.

$2 \S 1117$.

${ }^{3}$ Simples ou brisés.

${ }^{4}$ Dans leurs modifications.
} 
TSHOU HI. - Au-dedans veut dire dans les brins d'herbe ${ }^{1}$ et dans les koua. À l'extérieur veut dire en dehors des brins d'herbe et des koua. Modification, précisément la modification qui s'agite en dedans. Formules, c'est-à-dire manifesté dans les formules extérieures.

1222.

La grande vertu du ciel et de la terre est appelée la vie ${ }^{2}$; la grande chose précieuse chez l' homme saint est appelée la situation. Ce qui sert à conserver la situation est appelé humanité ; ce qui sert à rassembler les hommes est appelé richesse. Déterminer la raison d'être de la richesse, redresser les expressions, défendre aux hommes de faire ce qui est injuste est appelé le devoir.

TSHOU HI. - Le caractère jen, homme, de l'expression « soleil et homme », est actuellement remplacé par le caractère [], humanité ${ }^{3}$. Lu Shi suit l'ancienne forme ; en effet, c'est ce qui est exprimé dans les mots : « sans la foule comment conserver l'état »? - Premier paragraphe. Traite du présage heureux ou malheureux des traits et des koua et de l'ordre et du mécanisme de la création.

1223.

Dans l'antiquité, Pao Hi Shi ${ }^{4}$, gouvernant le monde, leva les yeux et aperçut les symboles dans le ciel, il baissa les yeux et aperçut les règles sur terre. Il remarqua les formes des oiseaux et des animaux ainsi que les productions de la terre. Auprès, il prit les divers ${ }^{5}$ corps ; au loin, il prit les divers êtres. C'est dans cela qu'il commença à faire les huit koua, afin de pénétrer la vertu de l'intelligence et du génie, afin de classer les sentiments et le genre de tous les êtres.

TSHOU HI. - Wang Tchieou So dit: Entre les mots «sur terre » la plupart des éditions portent le caractère ciel. Baisser et lever les yeux ; au loin et auprès ; donc ce qu'il prit ou recueillit ${ }^{6}$ est loin d'être unique ; toutefois cela ne dépasse en aucune façon l'examen attentif de ces deux principes,

\footnotetext{
${ }^{1}$ Herbe shi, servant à la divination.

2 Ou la naissance.

${ }^{3}$ La vertu, le sentiment d'humanité.

${ }^{4}$ Fou Hi.

${ }^{5}$ Ou sur le corps.

${ }^{6}$ Releva, remarqua.
} 
l'expiration et la croissance de la négativité et de la positivi té. Vertu de l'intelligence et du génie ; par exemple la nature de l'activité et de la passivité, du mouvement et de l'ar rêt. Le naturel ou sentiment de tous les êtres, par exemple les symboles de la foudre et du vent, de la montagne et du marais.

1224.

Il fit des nauds de cordes et constitua les filets et les rets pour les usages de la chasse et de la pêche. Et il tira (prit) cela du koua li ${ }^{1}$.

TSHOU HI. - «Deux yeux échangeant leurs regards et les êtres existent $»^{2}$.

1225.

Pao Hi Shi mort, Shen Nong Shi gouverna; l'équarrit le bois pour faire une herse ; il ploya le bois pour faire une charrue. Les bienfaits de la charrue et de la herse servirent à instruire l'univers. Et en effet il tira (prit) cela du koua yi ${ }^{3}$.

TSHOU HI. - Les deux substances (koua simples du koua yi) sont également le bois ; en haut, entrer; en bas, mouvement. L'avantage ( yi) de l'univers n'es t jamais plus grand qu'en ceci.

1226.

Le soleil au-dedans ${ }^{4}$ constitue le marché, afin que les hommes de tout l'univers y réunissent les produits de l'univers, y fassent leurs échanges et se retirent, chacun ayant acquis ce qui lui convient. Et en effet il tira (prit) cela dans le koua she ho ${ }^{5}$.

\footnotetext{
${ }^{1}$ Lecture traditionnelle, maison peut lire, sans prévention, des diverses séparations. Voir koua $\mathrm{n}^{\circ} 30$.

2 Citation

${ }^{3} \mathrm{Yi}$ : croître, avancer.

${ }^{4}$ Le soleil au milieu.

${ }^{5}$ Koua ${ }^{\circ} 21$.
} 
TSHOU HI. - Le soleil au-dedans constitue le marché ; en haut clarté et en bas mouvement. De plus, c'est emprunter au mot she le sens de marché ; ho veut dire réunir.

1227.

Shen Nong Shi mort, Hoang Ti, Yao et Shouen Shi gouvernèrent. Ils pénétrèrent les modifications et dirigèrent le peuple pour qu'il ne fût pas oisif; ils l'éclairèrent et le transformèrent afin que le peuple s'y conformât. Lorsque le changement est fini, il y a modification; par la modification, il y a pénétration; pénétration, donc durée. C'est à cause de cela que le ciel l'assiste et que rien n'est sans avantage. Hoang Ti, Yao et Shouen secouant le pan de leur robe, l'univers fut gouverné et en ordre. En effet, ils prirent ceci dans les koua khien et khouen.

TSHOU HI. - Les koua khien et khouen se modifient et se transforment sans agir directement.

1228.

Ils fendirent un arbre en deux parties pour faire une pirogue; ils coupèrent le bois pour faire un aviron; l'utilité de la pirogue et de l'aviron, c'est de permettre de traverser un cours d'eau qui empêche la libre circulation, et de parvenir au loin pour le bien de l'univers. Or ils tirèrent (prirent) cela du koua hoan ${ }^{1}$.

TSHOU HI. - Le bois est au-dessus de l'eau ; parvenir au loin pour le bien de l'univers ; ces mots paraissent en trop dans le texte.

1229.

Ils lièrent les baufs et montèrent les chevaux et transportèrent au loin ce qui est lourd, pour l'avantage de l'univers; or ils prirent cela dans le koua souei ${ }^{2}$.

TSHOU HI. - En bas mouvement, en haut satisfaction.

\footnotetext{
${ }^{1}$ Koua n ${ }^{\circ} 59$.

${ }^{2}$ Koua ${ }^{\circ} 17$.
} 
1230.

Ils doublèrent les portes ${ }^{1}$ et frappèrent les bâtonnets ${ }^{2}$ pour accueillir les hôtes dangereux; or ils prirent cela dans le koua yu ${ }^{3}$.

TSHOU HI. - Idée de circonspection ; prévenir à l'avance.

1231.

Ils coupèrent un arbre pour faire un pilon et creusèrent la terre pour faire un mortier. L'avantage du mortier et du pilon est de subvenir aux besoins de tous les peuples. Or ils tirèrent ceci du koua siao kuo ${ }^{4}$.

TSHOU HI. — En bas arrêt, immobilité, en haut mouvement.

1232.

Ils ployèrent le bois pour faire un arc et taillèrent le bois pour faire des flèches; l'usage des flèches et de l'arc est d'intimider et d'imposer le respect à l'univers. Or ils prirent cela dans le koua khouei ${ }^{5}$.

TSHOU HI. - L'opposition (Moues) est suivie de l'intimidation qui conduit à la soumission.

1233.

Dans la plus haute antiquité les hommes habitaient les cavernes et vivaient dans les déserts; plus tard, les hommes saints changèrent ces lieux

\footnotetext{
${ }^{1}$ Portes répétées les unes en avant des autres.

${ }^{2}$ Deux petits morceaux de bois dur et résonnant que l'on frappe pour montrer que l'on veille.

${ }^{3}$ Koua $n^{\circ} 16$.

${ }^{4}$ Koua ${ }^{\circ} 62$.

${ }^{5}$ Koua ${ }^{\circ} 38$.
} 
en maisons et en habitations. Ils dressèrent des colonnes et inclinèrent les formes, afin de prévenir le vent et la pluie. Or ils prirent cela dans le koua ta tshang ${ }^{1}$.

TSHOU HI. — Idée de solidité inébranlable.

1234.

Dans l'antiquité, les funérailles consistaient à recouvrir le corps sous une couche épaisse de menu bois; on plaçait les sépultures au milieu des bois (déserts), sans faire de tumulus, sans planter d'arbres. La durée des deuils n'était pas fixée. Plus tard, pendant les générations postérieures, les hommes saints changèrent cela par l'usage des cercueils intérieurs et extérieurs; or ils tirèrent cela du koua tae kuo ${ }^{2}$.

TSHOU HI. — Les funérailles sont des choses des plus importantes.

1235.

Dans la plus haute antiquité, on faisait des nouds sur des cordelettes pour transmettre les règles ${ }^{3}$; plus tard, les hommes saints les changèrent en caractères d'écriture et en tablettes. Les divers magistrats s'en servirent pour instruire ${ }^{4}$. Tous les hommes s'en servirent pour examiner ${ }^{5}$. Or ils prirent cela dans le koua kouae ${ }^{6}$.

TSHOU HI. - Explication au sujet de l'idée de décision. - Deuxième paragraphe. - Traite des choses relatives aux hommes saints déterminant les objets usuels en imitant les symboles.

1236.

\footnotetext{
${ }^{1}$ Koua $\mathrm{n}^{\circ} 34$.

2 Koua $n^{\circ} 28$.

3 Pour servir de caractères d'écriture.

${ }^{4}$ Gouverner.

5 S'instruire ?

${ }^{6}$ Koua $n^{\circ} 43$.
} 
C'est pour cela que le changement (yi), c'est le symbole; symbole, imitation ressemblante.

TSHOU HI. — Similitude à la figure et à la raison d'être des koua du $Y i$ king.

1237.

Thouan, «formule déterminative », la substance essentielle, élémentaire.

TSHOU HI. - La formule déterminative traite de la substance d'un ko ua.

1238.

Hiao (formule d'un trait, ou le trait lui-même) ; c'est ce qui enseigne ${ }^{1}$ le mouvement de l'univers.

TSHOU HI. - Hiao, étendre, disperser, imiter.

1239.

C'est pour cela que les présages fastes ou néfastes naissent et que les regrets et l'appréhension se manifestent.

TSHOU HI. - Le regret et l'appréhension sont essentiellement insensibles dans leur origine, c'est par ceci qu'ils deviennent évidents.

Troisième paragraphe.

1240.

Les koua positifs renferment beaucoup de négativités; les koua négatifs renferment beaucoup de positivités.

${ }^{1}$ Ou ce qui imite. 
TSHOU HI. - Les koua simples, tshen, khan, ken sont considérés comme koua positifs ; tous sont formés d'un trait positif et de deux traits négatifs. Les koua simples souen, li, touei, sont considérés comme koua négatifs et sont tous formés d'une négativité et de deux positivités.

1241.

Quelle en est la cause? C'est que les koua positifs représentent l'unité et les koua négatifs la dualité.

TSHOU HI. - Chaque koua positif contient toujours cinq traits ; chaque koua négatif contient toujours quatre traits ${ }^{1}$.

1242.

Quelle en est la vertu et l'action? Dans les koua positifs il y a un prince et deux sujets : c'est la voie rationnelle de l'homme doué. Dans les koua négatifs il y a deux princes et un sujet, c'est la voie rationnelle de l'homme inférieur.

TSHOU HI. - Prince, c'est-à-dire une positivité ; sujet, c'est-à-dire négativité.

Quatrième paragraphe.

1243.

Le yi dit: «aller et venir avec incertitude; les amis suivent la pensée » ${ }^{2}$. Tse dit: Dans l'univers pourquoi penser? pourquoi s'inquiéter ?Dans l'univers tout arrive au même point par des voies diverses. Pour un résultat, cent prévisions ; dans l'univers, pourquoi penser, pourquoi s'inquiéter?

TSHOU HI. - Citation et explication de la formule du quatrième trait nonaire du koua hien. Cela exprime que, essentiellement, la raison d'être des choses n'est pas double, et que les routes diverses et les cent préoccupations ne sont jamais autre chose que la conséquence naturelle de cette raison d'être.

\footnotetext{
${ }^{1}$ Un trait plein et deux traits brisés, cinq traits. Un trait brisé et deux traits pleins, quatre traits.
} $2 \S 571$, koua $n^{\circ} 31$. 
Dès lors à quoi bon penser, prévoir et agir? Il faut y réfléchir et s'y conformer, et alors ce à quoi on se conformera sera aussi d'accord ${ }^{1}$.

1244.

Le soleil sen allant, alors la lune vient; la lune s'en allant, le soleil revient. Le soleil et la lune s'entraînent mutuellement et la lumière naît. Le froid s'en va, alors la chaleur vient; la chaleur s'en va, alors le froid revient ; le froid et le chaud s'entraînent mutuellement et l'année se complète. Le départ (s'en aller), c'est la contraction; le retour (venir), c'est le redressement. La contraction et le redressement ${ }^{2}{ }^{\prime}$ ' influencent mutuellement et le bien-être naît.

TSHOU HI. - Il s'agit de l'aller et du retour, de la contraction et de l'extension et c'est toujours le résultat d'une influence $\mathrm{r}$ éciproque, conséquence spontanée de la raison d'être constante et universelle. Si on y ajoute les mots indécision ou incertitude ${ }^{3}$, il s'agit alors du résultat de l'intérêt privé. C'est à cause de cela ${ }^{4}$ qu'il devient nécessaire de pen ser et ensuite de suivre la raison d'être.

1245.

La contraction du ver (?) provient de la nécessité de se détendre; l'enroulement du dragon et du serpent résulte du besoin de préserver leur corps. La pureté du devoir pénètre dans la cause spirituelle, afin de produire son effet; il est avantageux de reposer le corps, afin d'illustrer la vertu.

TSHOU HI. - Cela est dit au sujet de la raison d'être des phénomènes de contraction et d'extension, d'aller et de retour ; mais, de plus, le commentaire en tire des déductions afin de montrer que l'étude a aussi une cause essentielle et spontanée. Examiner et scruter la pureté du devoir, jusqu'à pénétrer la cause spirituelle, c'est le comble de la contrac tion ${ }^{5}$. Mais, c'est par là que l'effet est ensuite produit à l'extérieur. Am éliorer l'effet pro duit, sans prendre de repos jusqu'à ce que ce but soit atteint, c'est le comble du redresse ment (réaction), mais c'est précisément ce qui est considéré comme le don de l'illustration de

\footnotetext{
1 Doctrine taoïste.

${ }^{2}$ Littéralement le texte porte «confiance ». C'est précisément d'après le passage que l'on donne, dans le Tse tien, le sens de « redressement et tension » à ce caractère.

3 Voir $\$ 1142$.

${ }^{4}$ De cette dérogation à ce qui est conséquence spontanée de la raison d'être.

${ }^{5}$ Retour sur soi-même et méditation.
} 
la vertu. L’intérieur et l'extérieur s'unissent e t se développent mutuellement ; ils s'entraînent et se manifestent l'un par l'autre.

1246.

Dépasser ceci afin de s'en aller; pas encore de quai peut-être savoir ${ }^{1}$; épuiser la cause spirituelle et connaître la transformation, c'est la perfection de la vertu.

TSHOU HI. - Il s'agit des études des choses ci-dessus citées ; d'épuiser ses forces au sujet de la pureté absolue du devoir et de l'amélioration des effets produits, de la cause invisible de l'union et du développement pour la manifestation mutuelle. Cette étude ne peut naturellement jamais finir ; en exceptant celui-là, il n'y aura point d'objet auquel il convienne d'appliquer les efforts. Quant à ce qui est dit de pousser jusqu'à la limite extrê me l'étude de la cause spirituelle et la science de la transformation, c'est le résultat de l'éclat de la vertu et de l'ardeur du sentiment d'humanité. Toutefois, ceux qui ne savent point entreprennent et se contractent et ce qui résulte naturellement de leurs efforts, c'est qu'ils reviennent et se détenden $\mathrm{t}$; or, ce n'est encore jamais rien autre chose que la raison d'être naturelle de l'influence mutuelle et correspondante. Tsheang Tse dit: L'éther ${ }^{2}$ comporte la négativité et la positivité, l'action qui résulté de leur concours produit graduellement la transformation ; réuni en une seule unité insondable, cela constitue le génie (cause des phénomènes). Les quatre propositions qui précèdent ont toutes pour but d'expliquer la formule du quatrième trait nonaire du koua hien.

1247.

Le yi dit: Misère sur la pierre; appuyer sur les chardons; entrer dans la chambre intérieure; ne pas voir l'épouse; présage malheureux ${ }^{3}$. Tse dit : Ce n'est point un sujet de misère et on le considère comme misère ; la réputation (le nom) sera diffamée; il n'y a pas lieu de se baser là-dessus et on s'en sert comme de base. Le corps doit nécessairement être en péril ; du moment où il y a honte et de plus péril, le moment de la mort est sur le point d'arriver ; est-il donc possible de voir l'épouse?

\footnotetext{
${ }^{1}$ Construction inintelligible : on pourrait lire : « au-delà de ceci ».

${ }^{2}$ Substratum de toutes choses.

${ }^{3} \S 838$.
} 
TSHOU HI. - Explication du sens du troisième trait hexaire du koua khouen ${ }^{1}$.

1248.

Le yi dit: Le dignitaire revêtu du titre de Kong emploie la flèche; l'oiseau sin est posé sur une muraille élevée; il le capture; rien n'est sans avantage ${ }^{2}$. Tse dit; Sin, c'est un oiseau ; arc et flèche, objets, outils; celui qui tue, c'est l'homme. L'homme doué porte les objets sur son corps; il attend le moment et se meut; que pourrait-il y avoir qui ne fût avantageux. Il se meut et n'attache point ${ }^{3}$, cela est ainsi parce qu'il sort et capture : cela est dit au sujet de l'achèvement des objets (outils) et du mouvement.

TSHOU HI. - Attacher, faire obstacle. Ce passage explique le sens du trait supérieur hexaire du koua kiae.

1249.

Tse dit : L'homme inférieur n'a pas de honte et manque d'humanité ; il ne craint rien et ne connaît pas le devoir; sil n'y voit point un avantage, il n'exerce point son effort; il ne respecte aucune autorité s'il n'est pas réprimandé ; une petite réprimande est un grand avertissement : là est le bonheur de l'homme inférieur. Le Yi dit: Chausser des entraves de bois qui détruisent les orteils; pas de culpabilité : c'est précisément ce que signifie ce passage ${ }^{4}$.

TSHOU HI. - Explication du sens du premier trait nonaire du koua she ho.

1250.

Si le bien n'est pas accumulé, c'est insuffisant pour illustrer une réputation; si le mal n'est pas accumulé, ce n'est pas suffisant pour causer la

\footnotetext{
${ }^{1}$ Koua $\mathrm{n}^{\circ} 47$.

${ }^{2}$ Koua $n^{\circ} 40, \S 725$.

${ }^{3}$ Les commentateurs lisent : n'entrave point, ne fait point obstacle.

${ }^{4}$ Koua n $^{\circ} 21, \S 392$.
} 
destruction du corps. L'homme inférieur considère un peu de bien comme sans valeur et il ne de fait point; l'homme inférieur considère un peu de mal comme n'étant pas nuisible et il ne l'évite pas. Aussi, le mal s'accumule et ne peut plus être caché, la faute devient considérable et ne peut plus être absoute. Le Yi king dit: Porter sur les épaules une entrave qui détruit les oreilles ; présage malheureux ${ }^{1}$. ho.

TSHOU HI. - Explication du sens du trait supérieur nonaire du koua she

1251.

Tse dit: Le péril, c'est ce qui assure le repos dans la situation; la destruction est ce qui garantit la conservation; le désordre, c'est ce qui fait l'ordre. C'est pour cela que l'homme doué est tranquille et il n'oublie pas le péril ; il se maintient et n'oublie pas la destruction; il gouverne et n'oublie pas le désordre. C'est par là que sa personne est en repos et que l'État est préservé. Le yi dit: La perte! la perte! être attaché à un bosquet de mûriers ${ }^{2}$.

TSHOU HI. - Explication du sens de la formule du cinquième trait nonaire du koua pi (décadence).

1252.

Tse dit: Vertu minime et situation éminente; petit savoir et grande ambition; peu de force et grave responsabilité : il est rare qu'il n'en soit pas ainsi. Le yi dit : Marmite dont les pieds sont cassés; renverser la nourriture du dignitaire revêtu du titre de Kong; la forme est mutilée : présage de malheur.

TSHOU HI. - Explication du sens du quatrième trait positif du koua ting (marmite).

1253.

$1 \S 402$.

${ }^{2}$ Koua $^{\circ}{ }^{12}$, § 249. 
Tse dit: Connaître la cause, n'est-ce pas le génie ? L'homme doué monte et s'allie sans employer la flatterie ; il descend et s'allie sans mépris : n'est-ce pas connaître la cause initiale? Cette cause initiale, c'est le mouvement à peine manifesté ; c'est avant la manifestation du présage heureux. L'homme doué regarde la cause initiale et il agit; il n'attend pas la fin du jour. Le yi dit: Solidité de la pierre; ne pas attendre au dernier jour ; présage heureux de la perfection ${ }^{1}$. Étant ferme comme la pierre, à quoi bon attendre la fin du jour? Il est capable de décider et de connaître. L' homme doué connaît ce qui est encore infiniment petit, il connaît ce qui est brillant; il connaît ce qui est encore infiniment petit, il connaît ce qui est brillant; il connaît la douceur malléable et la dureté énergique: c'est l'objet de l'attente de tous les hommes.

TSHOU HI. - Explication du sens du second trait hexaire du koua $y u$ (satisfaction). Dans le livre des Han, entre le mot présage heureux et celui qui suit, il y a aussi le caractère dont le sens est présage malheureux.

1254.

Tse dit: Le péril du fils de Ngan Shi était presque absolu; s'il y avait quelque chose qui ne fût pas bien, il n'était jamais sans le reconnaître, et quand il l'avait reconnu, il ne manquait jamais de le réparer. Le yi dit: Retour pas éloigné ; sans arriver aux regrets ; bonheur absolu ${ }^{2}$.

TSHOU HI. - Péril, misère, pauvreté presque absolue, idée de proximité ; près de la voie rationnelle. Ceci est une explication du premier trait nonaire du koua fou (retour).

1255.

Le ciel et la terre en union intime; tous les êtres se transforment absolument. Les deux sexes, masculin et féminin, en réunissant leurs essences transforment et engendrent tous les êtres. Le yi dit : Trois hommes marchent; donc diminuer un homme; un homme marche, donc il possède des amis ${ }^{3}$. Cela exprime la tendance vers l'unité.

TSHOU HI. - Les deux caractères yin yuan expriment une circonstance de jonction et union mystérieuse. Absolument, c'est-à-dire pleinement et

\footnotetext{
${ }^{1}$ Koua $^{\circ}{ }^{16}$, $\$ 310$.

${ }^{2}$ Koua n $^{\circ} 24, \S 445$.

${ }^{3}$ Koua n ${ }^{\circ} 41, \S 736$.
} 
solidement. Il s'agit de la transformation de l'éther, transformer et engendrer, donner naissance ; c'est la transfor mation de la forme. C'est une explication du sens du troisième trait hexaire du koua souen (décroissance, diminution).

1256.

Tse dit: L'homme doué assure le calme de sa personne et ensuite il se meut ; il adoucit son cour, et ensuite il parle ; il établit ses liaisons et ensuite il cherche et appelle. L'homme doué soigne ces trois choses, de sorte qu'il les maintient entières. S'il se mouvait quand il est en péril, le peuple ne se joindrait pas à lui ; s'il parlait pendant qu'il a des motifs de crainte, le peuple ne lui répondrait pas sympathiquement; s'il demandait avant d'avoir des alliances, le peuple ne lui accorderait rien. Personne ne s'alliant à lui, le mal qui en résulterait serait extrême. Le yi dit: Ne pas augmenter; parfois frapper ; affermir le caur sans permanence ; présage malheureux ${ }^{1}$.

TSHOU HI. - Explication du sens du trait supérieur nonaire du koua yi (avantage).

Cinquième paragraphe.

1257.

Tse dit: Khien et khouen, activité et passivité ; c'est la porte d'accès du Yi king. Khien désigne les choses positives; khouen désigne les choses négatives. La négativité et la positivité unissent leurs vertus et la dureté énergique comme la douceur malléable ont une substance. Ces deux principes servent à matérialiser l'action du ciel et de la terre et à pénétrer les vertus de la cause mystérieuse et brillante (intelligente).

TSHOU HI. - Dans les divers koua, la substance de la dureté énergique et de la douceur malléable se produit toujours par l'union des vertus du koua Khien et du koua khouen. Aussi la formule dit que Khien et khouen sont la porte du Yi. Action; ce qui est fait; choses.

1258.

${ }^{1}$ Koua $\mathrm{n}^{\circ} 42, \S 759$. 
Les noms donnés sont variés et ne s'écartent jamais de l'examen du genre de l'objet nommé. N'est-ce pas là une idée particulière aux siècles de décadence?

TSHOU HI. - Quelque nombreux que soient les divers êtres, ils ne résultent jamais d'autre chose que des modifications de la négativité et de la positivité. Aussi le sens des traits des koua, bien qu'il s'adapte à des sujets variés, ne comporte cependant point d'erreurs. Mais toutefois ce n'étai t pas là quelque chose à quoi peuvent atteindre les pensées et les préoccupations du temps de la pure simplicité de la haute antiquité. Aussi c'est considéré comme le résultat des idées des âges de la décadence, et, en effet, cela désigne les temps de Wen Wang et de Tshieou ${ }^{1}$.

1259.

Or, le Yi (changement) illumine ce qui s'en va et scrute ce qui vient et il rend manifeste et découvre ce qui est infiniment ténu et caché. Il ouvre (sépare) et distingue les êtres en leur donnant un nom approprié; il exprime exactement et détermine la formule, et tout est alors complet.

TSHOU HI. - Il y a lieu de croire que ce passage a été altéré.

1260.

Les noms donnés sont humbles, le genre embrassé par ce nom est grand; le sujet est éloigné, la formule est ornée ; les mots sont appropriés au détail de chaque objet et atteignent juste ; les choses sont étendues et cachées ( ?). Par le doute, il sert à remédier aux maux du peuple, il sert de récompense en montrant ce qui est perdu ou obtenu ${ }^{2}$.

TSHOU HI. - Étendu ; développé. Le caractère du texte qui signifie deux doit être considéré comme indiquant le doute. - Sixième paragraphe. Il présente beaucoup de lacunes et de mots très douteux; on ne peut pas le pénétrer complètement ; il en est de même dans les paragraphes suivants.

\footnotetext{
1 Ce passage donne lieu à plusieurs hypothèses. Les commentateurs varient dans leurs appréciations, l'un dit : «les noms, c'est-à-dire les noms des koua ». Il peut aussi être question des noms donnés au koua khien et au koua khouen : activité et passivité ; dureté et douceur, et ainsi de suite, c'est là, semble -t-il, le vrai sens à adopter.

${ }^{2}$ Ce qui est d'accord ou contraire au devoir, sens très obscur.
} 
1261.

Le développement (la rédaction) du Yi n'eut-il pas lieu dans la moyenne antiquité ? Ceux qui firent le Yi king n'étaient-ils pas dans le chagrin et le malheur?

TSHOU HI. - À la fin des dynasties des Hia et des Sheang, la voie rationnelle (la doctrine) du Yi était dans la décadence. Wen Wang était prisonnier à Yeou li et y annexait les formules déterminatives ${ }^{1}$; c'est là une renaissance de la doctrine.

1262.

C est pour cela que le koua li ( $\left.n^{\circ} 10\right)$ exprime la base de la vertu; le koua khien ( $\left.n^{\circ} 15\right)$ la poignée de la vertu ${ }^{2}$; le koua fou ( $\left.n^{\circ} 24\right)$ son origine; le koua heng $\left(n^{\circ} 32\right)$ sa fermeté ; le koua souen $\left(n^{\circ} 41\right)$ la réforme de la vertu; le koua yi ( $\left.n^{\circ} 42\right)$, son extension; le koua khouen ( $\left.n^{\circ} 47\right)$ les distinctions entre les vertus ; le koua tsing ( $\left.n^{\circ} 48\right)$ le lien de la vertu et le koua souen $\left(n^{\circ} 57\right)$ les lois de cette même vertu.

TSHOU HI. - $L i\left(\mathrm{n}^{\circ} 10\right)$, rites, bienséance; en haut le ciel, en bas le marais, détermination et destruction immuable ; il faut être circonspect en ceci et ensuite la vertu a une base assurée sur laquelle elle peut se maintenir debout. Khien $\left(\mathrm{n}^{\circ} 15\right)$, la modestie, c'est shumilier et exalter les hommes (autrui). De plus, c'est ce que les préceptes rituels doivent maintenir et conserver et ce qui ne peut être négligé. Les neuf koua cités expriment tous quelque chose relatif au retour sur soi-même et à l'amélioration de la vertu, pour faire face aux causes de chagrin et au malheur; enfin, ils expriment encore un ordre fixe et déterminé. La base est ce qui sert à supporter; la poignée c'est ce qui sert à tenir l'outil dans la main pour en faire usage. Le koua fou exprime que le cour n'est point altéré et que le principe du sentiment du bon y est intact. Heng, permanence, conserver sans modification et faire durer d'une façon immuable; se mettre en garde contre la colère, barrer la voie des passions et amender l'individu, ramener au bien, corriger les défauts et les excès pour développer les bons sentiments. Khouen (misère), mesurer ses propres forces ; tsing (puits) exprime l'idée de ne point quitter le terrain choisi. Après cela, l'humilité devient possible, on peut sincliner devant la raison d'être des choses pour diriger et contenir les modifications de toutes choses.

\footnotetext{
${ }^{1}$ La première formule après le koua.

${ }^{2}$ Le moyen d'action.
} 
1263.

La bienséance (li) entraîne la concorde et l'harmonie qui se développe; la modestie (khien) rend illustre et éclatant; le retour à la petitesse (fou) sert à distinguer les êtres; la permanence (heng) montre la diversité sans confusion $^{1}$; la diminution (souen) montre d'abord la difficulté et ensuite la facilité $^{2}$; l'augmentation (yi), ou avantage, c'est la croissance sans grandissement; la misère (khouen), la fin, épuisement suivi de compréhension; la fixité (tsing), demeurer dans le même lieu et s'y transformer ; l'humilité (souen), c'es t-à-dire mériter et se tenir caché ${ }^{3}$.

TSHOU HI. - Ceci est quelque chose d'analogue aux neuf vertus du Shou long: La bienséance consiste à ne point violenter les choses du moment; mais, toutefois, les choses, quelles qu'elles soient, parviennent toutes à leur plus extrême développement. La modestie consiste à s'humilier soi-même et à exalter autrui. Dans le koua fou, la positivité est encore infiniment minime et ne se mêle point à la foule des négativités. La persistance montre que, placées d'une façon quelconque, les vertus essentielles ne se confondent point. La diminution montre que les désirs rencontrent d'abord des difficultés, mais que par l'exercice répété tout devient aisé et facile. L'augmentation avantageuse, c'est seule ment l'accroissement de ce qui existe sans qu'il y ait action de faire ou de créer. Le mot misère indique la misère où se trouve la personne tandis que la voie rationnelle est libre et pénétrable. Fixité sans mouvement qui n'empêche point d'atteindre partout. Humilité, être apte à répondre aux besoins et aux nécessités des êtres et se retirer à l'ombre, sans se mettre en évidence.

1264.

La bienséance (li) sert à établir la concorde dans les actions; la modestie (khien) sert à tracer les règles de la bienséance; le retour (fou) sert à se connaître soi-même; la permanence (heng) sert à l'unité de la vertu; la diminution (souen) permet d'éloigner le mal ; l'augmentation (yi) sert à faire naître l'avantage; la misère (khouen) sert à diminuer le ressentiment; la fixité (tsing) sert à tracer le devoir ; l'humilité sert à agir avec puissance.

TSHOU HI. - Diminuer le ressentiment veut dire diminuer le sentiment de mauvais vouloir qu'on peut éprouver. Tracer le devoir signifie que, étant au

\footnotetext{
${ }^{1}$ Peut-être « la diversité dans ce qui est unique ».

$2 \mathrm{Ou} \ll$ le changement $»$.

${ }^{3}$ Plus littéralement « nommer et cacher ».
} 
repos, il est possible de méditer. - Septième paragraphe. Troisième exposition de la valeur de ces neuf koua, pour éclairer la voie rationnelle suivant laquelle il convient de se placer pendant le chagrin et le malheur.

1265.

Ce qui constitue le livre des changements (Yi), c'est, on ne doit pas l'oublier, la voie rationnelle. Elle change continuellement, se transforme et se meut sans rester en place; elle circule et parcourt les six vides ${ }^{1}$; elle monte ou descend sans suivre une règle continue. La dureté énergique et la douceur malléable se transforment mutuellement sans qu'on puisse assigner une règle immuable ; il n'y a que la modification qui y soit équivalente.

TSHOU HI. - Le caractère yuan du texte (éloigner) veut dire oublier. «Circuler et parcourir les six vides », cela veut dire que la négativité et la malléabilité circulent et agissent dans les six situations du koua.

1266.

Sa sortie ou son entrée a lieu par des règles graduées; au-dehors ou au-dedans pour qu'on connaisse et qu'on craigne.

TSHOU HI. - Cette phrase n'est pas encor e clairement expliquée ; il y a lieu de craindre une lacune ou une interpolation.

1267.

De plus elle porte la clarté dans le chagrin et le malheur comme cause; rien et avoir dirigent et assurent ${ }^{2}$ comme considérant le père et la mère.

TSHOU HI. - Bien qu'il n'y ait pas un maître qui gouverne, et comme si un père et une mère surveillaient ; c'est le plus extrême degré de la crainte et du respect.

\footnotetext{
${ }^{1}$ Six espaces vides.

2 Rien et avoir; c'est-à-dire, l'être et le non-être; l'avoir et le non-avoir. On traduit généralement autrement. Voir le commentaire de TSHOU HI.
} 
Tshou Shi Tshen dit: De plus elle éclaire ce qui, chez une personne, doit être une cause de chagrin et de malheur et les motifs qui amènent cette cause ; il n'y a pas un maître qui assure, enseigne et montre et cependant on la craint ; c'est comme lorsqu'un père et une mère surveillent personnellement et que leurs enfants les respectent et les aiment : cela montre quel est le sentiment de l'homme saint ${ }^{1}$.

1268.

D'abord elle motive la formule et trace la règle; lorsqu'il y a des préceptes permanents, si ce n'est la personne, la voie rationnelle n'agit pas en vain.

TSHOU HI. - Règle ${ }^{2}$, désigne la voie rationnelle. Le livre commence par la formule pour tracer la raison d'être des choses, ce qui sert à rendre manifestes les préceptes permanents. Cependant la pénétration de la cause et l'intelligence de cette voie rationnelle résident entièrement dans l'homme. Huitième paragraphe.

1269.

Ce qui constitue le livre des changements (Yi), c'est qu'il a son origine dans le commencement et son but dans la fin, et cela pour constituer sa matière (son caractère particulier). Les six traits se mêlent ensemble: ils expriment simplement le moment et l'être (la chose).

TSHOU HI. - Matière, ou caractère particulier, cela désigne la substance des koua. Dans les koua il faut absolument relever le commencement et la fin et ensuite la substance en est déterminée. Les traits ne sont rien autre chose que l'indication du moment et de l'être.

1270.

\footnotetext{
${ }^{1}$ Cela est beaucoup plus clair, mais le texte ne le dit pas.
}

${ }^{2}$ Fang, carré, régulier, règle. 
Le début (commencement) est difficile à connaître; le dessus ${ }^{1}$ est facile à connaître: Origine et fin. D'abord, la formule détermine; après, fin de l'achèvement.

TSHOU HI. - Ceci traite du premier trait et du trait supérieur seulement.

1271.

S'il s'agit de choses diverses, de vertus particulières, il détermine ce qui est et ce qui n'est pas ${ }^{2}$; de sorte que, sans les traits du milieu, ce serait incomplet.

TSHOU HI. - Ceci parle des quatre traits intermédiaires des koua.

1272.

Ah! il en résulte encore nécessairement la conservation ou la disparition, le présage faste ou néfaste, de sorte qu'il devient possible de connaître sans changer de place. Celui qui sait considère la formule déterminative, et sa pensée atteint et dépasse déjà plus de la moitié ${ }^{3}$.

TSHOU HI. - Formule déterminative, celle qui embrasse à la fois la substance des six traits d'un koua ${ }^{4}$.

1273.

Le second et le quatrième produisent le même effet, mais dans des situations différentes; le bien qu'ils expriment n'est pas le même; le second comporte beaucoup de louanges; le quatrième inspire beaucoup de crainte : proximité. Ce qui constitue la voie rationnelle de la douceur malléable, c'est quelle n'est pas avantageuse pour ce qui est au loin. La nécessité est sans culpabilité ; l' effet est compris dans la douceur malléable ${ }^{5}$.

\footnotetext{
${ }^{1}$ Peut désigner le trait supérieur.

${ }^{2}$ Le vrai et le faux, le pour et le contre.

${ }^{3}$ De ce qui y est contenu?

${ }^{4}$ La première formule du texte chinois.

5 Intelligible.
} 
TSHOU HI. - À partir de ce passage il est question des traits intermédiaires. "Même effet», ou même ouvre, c'est -à-dire qu'il s'agit également de situations négatives. "Situations différentes », c'est-à-dire loin ou près, pas de même. Le quatrième rang est proche du prince, de sorte qu'il y a beaucoup à craindre. La douceur malléable n'est pas avanta geuse à ce qui est éloigné et le second trait comporte beaucoup de louanges : c'est parce que ce trait comporte la douceur malléable et la justice.

1274.

Le troisième et le cinquième ont le même effet et des situations différentes. Le troisième est très néfaste, le cinquième a beaucoup de mérite : degrés de noblesse et de virilité. La douceur négative y est périlleuse, la dureté énergique l'emporte sur le mal.

TSHOU HI. - Le troisième et le cinquième rangs ont également des situations positives, mais la noblesse ou l'humilité de ces situations n'est pas la même. Toutefois, si elles sont occupées par un trait malléable et négatif, ce sera un péril, et ce n'est que la positivi té qui peut surmonter ce péril.

Neuvième paragraphe.

1275.

Ce qui constitue le livre des changements (Yi), ce qui le rend immense et absolument complet, c'est qu'il contient la voie rationnelle du ciel, c'est qu'il contient la voie rationnelle de l'homme, c'est qu'il contient la voie rationnelle de la terre. Il réunit à la fois les trois causes efficientes et double chacune d'elles, ce qui fait six. Ces six choses ne sont rien autre chose : c'est la voie rationnelle des trois causes efficientes.

TSHOU HI. - Trois traits suffisent à compléter les trois causes efficientes; on les répète, c'est pourquoi il y en a six ; les deux traits supérieurs ${ }^{1}$ sont considérés comme représentant le ciel, les deux traits du milieu (de chaque koua simple) sont considérés comme représentant l'homme, et les deux traits inférieurs représentent la terre.

1276.

\footnotetext{
${ }^{1}$ Le trait supérieur de chaque koua simple.
} 
La voie rationnelle comporte la modification et le mouvement : c'est pour cela qu'on emploie l' expression hiao ${ }^{1}$ ou trait ; les hiao, ou traits, comportent des degrés, aussi on dit «êtres » ${ }^{2}$; les êtres se confondent ensemble, aussi on dit wen (figure, image, caractère d'écriture); la forme ou mirage wen ne répond pas exactement, c'est pourquoi les présages fastes ou néfastes naissent.

TSHOU HI. - La voie rationnelle comporte la modification et le mouvement, cela désigne la substance d'un koua entier. Degrés, degrés d'éloignement ou de proximité, de noblesse ou de virilité ; se confondre ensemble, se mélanger; cela veut dire que les situations qui comportent la dureté énergique et la douceur malléable sont intercalées. Ne pas répondre exactement, c'est-à-dire que les traits ne correspondent pas toujours exactement à la situation qu'ils occupent.

Dixième paragraphe.

1277.

Le développement du Yi (Yi king) eut lieu vers la fin de la dynastie des Yin, et alors que les Tsheou achevaient le développement de leurs vertus, au moment des événements auxquels participèrent Wen Wang et Tshieou. C'est à cause de cela que les formules parlent du péril. Le péril conduit au calme, l'absence de difficultés conduit aux catastrophes. La voie rationnelle est extrêmement grande, aucun être ne peut s'y soustraire. Si la circonspection dure du début jusqu'à la fin, les conséquences seront sans culpabilité ; ceci est ce qu'on appelle la voie rationnelle du Yi (changement).

TSHOU HI. - C'est par le péril et par la crainte qu'il inspire qu'on peut atteindre au calme et au repos ; l'absence de difficultés conduit au laisser-aller qui amène à sa suite les catastrophes : telle est la voie rationnelle du Yi king.

Onzième paragraphe.

1278.

\footnotetext{
${ }^{1}$ Sens très douteux. Je traduis généralement par trait, mais il s'agit tantôt du trait, tantôt de quelque chose qui s'y rapporte, imitation, peut -être aussi modèle ?

${ }^{2}$ Wou, être, chose.
} 
Or, le koua khien exprime l'extrême activité de l'univers ; la pratique des vertus qu'il comporte, constante et facile, fait connaître le danger. Le koua khouen exprime l'extrême passivité de l'univers ; la pratique des vertus qu'il comporte, constante et aisée, fait connaître les obstacles.

TSHOU HI. - Avec l'extrême activité, toutes les actions seront sans difficulté, donc elles seront faciles ; avec l'extrême patience, toutes les actions seront simples et sans complication, de sorte qu'elles seront aisées. Mais, quelles que soient les affaires dont il s'agit, il faut toujours en connaître le degré de difficulté et on ne doit pas se permettre de les négliger pendant qu'on s'en occupe. C'est ainsi que lorsqu'elles présentent des ennuis et des difficultés, avec l'activité on les considérera comme d'un point élevé d'où on peut embrasser d'un coup d'œil ce qui est au -dessous de soi, et on reconnaîtra leurs dangers ; avec la patience, on les regardera comme on regarde d'en bas un endroit escarpé et on connaîtra l'obstacle qu'elles présentent. En effet, bien qu'elles soien t faciles, si on peut connaître les difficultés qu'elles présentent, on ne tombera point dans les dangers qui peuvent en résulter et les accompagner; lorsqu'elles sont aisées et qu'on sait mesurer les obstacles qui se dressent pour en prévenir l'exécution, on ne souffrira point de ces obstacles. C'est par là que la capacité de connaissance du péril et de circonspection ne conduira pas aux catastrophes qui accompagnent ce qui est aisé et en apparence agréable.

1279.

Ils peuvent réjouir tous les caurs, ils peuvent déterminer les préoccupations des divers feudataires ${ }^{1}$, fixer les présages fastes ou néfastes de l'univers, et achever les efforts constants dans l'univers entier.

TSHOU HI. - Les deux caractères [] [], sont en trop et on doit lire «déterminer toutes les préoccupations », réjouir tous les coars : lorsque le cour embrasse la raison d'être d'une chose, c'est là le fait de l'idée exprimée par le mot khien. Déterminer toutes les préoccupations; la raison d'être entraîne comme conséquence l'analyse d es préoccupations, c'est là le fait de l’idée exprimée par le mot khouen. Ils réjouissent tous les cours, de sorte qu'il y a de quoi servir à déterminer les présages fastes et néfastes. Ils déterminent toutes les préoccupations, de sorte qu'il y a de quoi servir à achever et à compléter les efforts constants.

1280.

\footnotetext{
${ }^{1}$ Voir le commentaire de TSHOU HI.
} 
C'est pour cela que la modification et la transformation décrivent ${ }^{1}$ le fait; les choses dont le présage est heureux ont un sens favorable; le symbolisme de la chose fait connaître l'objet ${ }^{2}$; le sens divinatoire au sujet d'une chose fait connaître l'avenir.

TSHOU HI. - La modification et la transformation décrivent le fait, c'est pourquoi le symbolisme des choses peut servir à faire connaître l'objet ; le présage heureux de quelque chose a un sens favorable, c'est pourquoi le sens divinatoire d'une chose peut servir à faire connaître l'avenir.

1281.

Le ciel et la terre établissent et marquent les situations; l'homme saint complète ses capacités; les desseins de l'homme, les combinaisons des esprits, chaque homme y égale ses capacités.

TSHOU HI. - Le ciel et la terre déterminent les situations et l'homme saint fait le $Y i$ king afin d'y compléter leur auvre. C'est là que les desseins des hommes, les combinaisons des génies, et même les préoccupations des plus grossiers parmi les hommes, trouvent toujours l'adaptation de leurs capacités.

1282.

Les huit koua simples servent à symboliser les avertissements, les hiao (formules des traits) et les formules déterminatives servent à l' expression du sentiment $^{3}$. La dureté énergique et la douceur malléable occupent des positions alternées et il est possible d'y voir les présages heureux ou malheureux.

TSHOU HI. - Symboles, c'est-à-dire les traits des koua; hiao et formules déterminatives, désignent les formules relatives aux koua et à chaque trait.

1283.

\footnotetext{
${ }^{1}$ Littéralement : « disent » le fait.

${ }^{2}$ Imité de ce symbole.

3 Plus littéralement on peut lire : servent à exprimer la réalité.
} 
La modification et le mouvement servent à exprimer l'avantage; le présage heureux ou malheureux change d'après le sentiment. C'est pourquoi l'amour et la haine combattent ensemble et les présages fastes ou néfastes naissent; l'éloignement et la proximité se déduisent mutuellement l'un de l'autre, et les regrets ou le chagrin naissent. La vérité et le mensonge s'influencent réciproquement, le bien et le mal naissent. Toutes les fois que les vérités exprimées par le Yi king sont rapprochées sans concorder ensemble, c'est que le présage est malheureux; parfois il en résulte du mal, des regrets, et, d'ailleurs, de l'appréhension.

TSHOU HI. - Sans concorder ensemble, c'est-à-dire en opposition, en contradiction. Mal, regrets, appréhension, ce qui en résulte toujours.

1284.

Ce qui est sur le point d'aller contre, la formule le flétrit ; ce qui est relatif au doute dans le caur, la formule le soutient. Les formules relatives aux hommes heureux sont en petit nombre; les formules relatives aux hommes trop hâtés sont nombreuses; les formules relatives à l'homme qui calomnie le bien sont superficielles; les formules relatives aux manquements à une règle qui devrait être observée sont restreintes.

TSHOU HI. - Les formules des koua et des traits sont bien ainsi.

Douzième paragraphe. 


\section{DÉFINITIONS des KOUA}

«Recueil varié de dissertations » ${ }^{1}$. - Khong Shi King Ta dit : Fou Hi, après avoir tracé les huit koua simples, les combina pour faire les soixante-quatre koua parfaits. Khong Tse, suivant pas à pas l'œuvre de Fou $\mathrm{Hi}$, explique d'une façon générale et à grands traits, dans ses « formules annexées », le petit appareil des koua simples; il part de là et développe ensuite ce sens. Il dit encore: L'ordre des huit koua étant déterminé, les symboles s'y trouvent compris. En les combinant, le sens des traits s'y trouve inclus. Il dit encore : Il regarda les symboles dans le ciel et les règles sur la terre ; il regarda les formes et l'appa rence des oiseaux et des animaux, ainsi que les productions naturelles de la terre. Près, il prit ses exemples ( ?) sur le corps, au loin il les prit dans les êtres. Il fit d'abord les huit koua, afin de pénétrer et d'interpréter les vertus de la cause mystérieuse de la clarté et afin de classer en genres le naturel de tous les êtres. Ensuite, par déduction et en continuant, il redoubla les koua simples de trois traits pour achever les koua complets de six traits. Là encore, pendant qu'il n'était pas encore éclairé (avant qu'il n'eût compris), il leva les yeux pour contempler et les baissa pour examiner les symboles, près de lui sur son propre corps, au loin sur les êtres également considérés comme n'étant pas encore mani festes. Aussi, dans ce commentaire, il est de nouveau question de compléter l'étude de l'ordre de succession et de l'origine des koua, ainsi que des symboles représentés par les huit koua simples ; il est appelé définition des koua.

1285.

Autrefois, l' homme saint, en faisant le Yi king, aperçut secrètement les causes mystérieuses de la lumière ${ }^{2}$ et il créa les brins de paille ${ }^{3}$.

TSHOU HI. - «Aider mystérieusement au développement de l'intelligence », c'est comme si on disait « aider à l'action créatrice ». Le commentaire traditionnel de la «Divination par la tortue» dit: L'univers étant en paix et en repos, la voie rationnelle des rois prévalut et sur les grandes tiges se développèrent mille rameaux.

\footnotetext{
${ }^{1}$ Titre d'un commentaire du Yi king.

2 Je traduis le plus naturellement et sans tenir compte des préjugés des commentateurs. L'on peut lire, en suivant le sens traditionnel, les causes de l'intelligence. Voyez le commentaire de TSHOU HI.

${ }^{3}$ Pour servir à la divination.
} 
1286.

Il tripla le ciel, doubla la terre, et s'appuya sur les nombres ${ }^{1}$.

TSHOU HI. — Le ciel est rond et la terre est carrée ; ce qui est rond est un et ce qui entoure est trois. Dans trois, chaque partie est un, aussi en triplant le ciel cela constitue trois. Ce qui est carré est un et ce qui entoure est quatre ; quatre réunit deux paires, aussi en doublant la terre cela constitue deux. C'est de cette considération que naissent tous les nombres, aussi à la fin de trois opérations consistant à mettre un brin à part et à relever les autres, le restant étant composé de trois unités, trois fois trois font neuf ; si le reste est composé de trois dualités, trois fois deux font six. Deux dualités et un nombre trois font sept ; deux nombres trois et une dualité font huit ${ }^{2}$.

1287.

Il considéra les modifications dans la négativité et la positivité et établit les koua ; il porta la clarté dans l'énergie et dans la douceur et il créa les traits, se conformant passivement aux vertus de la voie rationnelle et suivant la raison d'être dans le devoir. Il épuisa la raison d'être et embrassa complètement la nature de l'homme, afin de parvenir à la destinée.

TSHOU HI. - Suivre en s'accordant et en se conformant sans aller contre en quoi que ce soit; cela est dit d'une façon générale, au sujet de tout l'ouvrage. Suivre la ra ison d'être dans le devoir, c'est -à-dire suivre la raison d'être en la scrutant dans chaque détail et en s'y conformant de point en point ; cela est dit d'une façon particulière et au sujet d'un détail. Épuiser la raison d'être de l'univers, scruter complè tement les sentiments de l'homme et se conformer à la voie rationnelle du ciel : là est la grande aure accomplie par les hommes saints qui firent le Yi king.

Première proposition.

1288.

\footnotetext{
${ }^{1}$ Peut-être devrait-on lire : il apprécia le ciel et mesura la terre.

2 Toute cette explication est basée sur des raisonnements très peu intelligibles sur la méthode de divination qu'on prétend exposée dans les formules annexées.
} 
Autrefois l'homme saint en faisant le Yi king procéda en se conformant passivement à la raison d'être des sentiments et de la destinée. C'est à cause de cela qu'en établissant la voie rationnelle du ciel, le livre parle de la négativité et de la positivité ; qu'en établissant la voie rationnelle de la terre, il parle de la douceur malléable et de la dureté énergique ; qu'en établissant la voie rationnelle de l'homme, il parle de l'humanité et du devoir. Il embrassa indifféremment les trois causes efficientes et il les doubla ${ }^{1}$, c'est pour cela que dans le Yi il y a six traits pour constituer un koua parfait. Il distingua la positivité et la négativité et les employa tour à tour; il employa de même la douceur et la dureté énergique ; c'est pour cela que dans chaque changement (Yi, ou koua), il y a six situations, ce qui achève la distinction.

TSHOU HI. - Embrasser indifféremment les trois causes efficientes et les doubler: cela désigne les six traits d'une façon générale. De plus il distingua minutieusement, ce qui établit les situations négatives et positives et ce qui complète l'exposition complète du texte.

Deuxième proposition.

1289.

Le ciel et la terre déterminent les situations; la montagne et le marais mélangent librement leurs éthers; la foudre et le vent se heurtent et entrent en contact; l'eau et le feu ne se détruisent pas mutuellement: les huit koua simples se combinent ensemble.

TSHOU HI. - Un commentateur dit : Ceci est la disposition des huit koua telle qu'elle fut adoptée par Fou Hi ; le koua khien au midi, le koua khouen au nord, le koua li à l'es t, le koua khan à l'ouest, le koua touei occupant le sud-est, le koua tshen le nord-est, le koua souen le sud-ouest et le koua ken le nord-ouest. C'est de là que résultent les combinaisons des huit koua simples qui constituent les soixante-quatre koua parfaits de six traits, ou ce qu'on appelle l'étude de « ce qui précède le ciel ».

1290.

Connaître et compter ce qui est passé, c'est ce qui est conforme (à la voie ordinaire ?) ; connaître ce qui arrivera, c'est ce qui est contraire (à cette voie ordinaire?). C'est pour cela que le Yi king va contre les nombres.

\footnotetext{
${ }^{1}$ En répétant les koua simples de trois traits l'un sur l'autre.
} 
TSHOU HI. - En commençant au koua tshen et en suivant par les koua $l i$ et touei jusqu'au koua khien, cela donne les koua dans lesquels le nombre est déjà né. Du koua souen en passant au koua khan et au koua ken, jusqu'au koua khouen, cela donne les koua dans lesquels le nombre n'est pas encore né. Dans le Yi king, les koua naissent dans l'ordre suivant, partant du koua khien et en suivant par les koua touei, li, tshen, souen, khan, ken et khouen. De sorte que tous sont rangés dans un ordre contraire aux nombres.

Troisième proposition.

1291.

La foudre a pour effet d'ébranler; le vent disperse, la pluie imbibe, le soleil réduit en vapeur. L'obstacle sert à arrêter, le koua touei réjouit, le koua khien régit et le koua khouen embrasse et contient.

TSHOU HI. - Ceci met en parallèle les situations des divers koua comme dans les propositions précédentes.

Quatrième proposition.

1292.

L'être suprême résulte du mouvement (tshen), il s'égale dans le koua souen, se voit dans le koua li, jusqu'à ce qu'enfin il agisse dans le koua khouen. Il parle dans le koua touei, combat dans le koua khien, s'efforce dans le koua khan et achève la parole dans le koua ken.

TSHOU HI. - Ti, celui qui régit le ciel. Shiao Tse dit : Ces situations des koua ont été fixées par Wen Wang; ce qu'en dit ce passage se rapporte à l'étude de ce qui vient « après le ciel ${ }^{1}$ ».

1293.

Tous les êtres sortent (résultent) du koua tshen (mouvement); ce koua tshen représente la région de l'est. Ils s'égalent (ou se régularisent) dans le

${ }^{1}$ Ces passages sont, pour le moment, absolument impossibles à traduire. Les commentateurs chinois n'y comprennent évidemment rien. Pour les expressions «avant le ciel », «après le ciel », voir t.1, Exégèse chinoise où j’en ai dit quelques mots. 
koua souen, c'est le koua de la région du sud-est. Le caractère tsi, égaler, régulariser, désigne le renouvellement de tous les êtres ${ }^{1}$. Le koua li désigne la clarté ; tous les êtres se voient mutuellement à cause de cette clarté ; c'est le koua de la région du sud. L'homme saint se tourne vers le sud et il écoute l'univers ; il fait face à la lumière et il gouverne, c'est qu'en effet il se base sur les règles ci-dessus. Le koua khouen, c'est la terre; tous les êtres se réunissent pour se développer, c'est pourquoi le texte dit agir dans le koua khouen. Le koua simple touei représente directement l'automne, c'est ce qui cause le bien-être et la satisfaction des êtres, c'est pour cela que le texte dit : Parler dans le koua touei. Combattre dans le koua khien; khien, koua de la région du nord-ouest; cela veut dire que la négativité et la positivité se heurtent et se froissent mutuellement. Le koua simple khan désigne l'eau, c'est le koua de la région du vrai nord, le koua qui exprime les efforts, c' est à lui que tous les êtres font retour, et c'est pour cela que le texte dit : S'efforcer dans le koua khan. Ken, koua du nord-est; le lieu où tous les êtres se complètent et finissent et où ils recommencent, c'est pourquoi le texte dit : Achever la parole dans le koua ken.

TSHOU HI. - Plus haut, le sujet est Ti, l'être suprême ; ici il est question de tous les êtres qui suivent cet être Ti pour sortir ou entrer.

Cinquième proposition. - Ce qui y est dit au sujet des situations des koua est pour la plus grande partie incompris ${ }^{2}$.

1294.

Le caractère ( $\mathrm{du} \S 1184)$ shen ${ }^{3}$ exprime les causes mystérieuses de tous les êtres. Parmi tout ce qui remue les êtres ou les choses, rien n'est plus rapide que la foudre; parmi tout ce qui les entraîne, rien n'est plus rapide que le vent; parmi tout ce qui les échauffe, rien n'est plus brûlant que le feu; parmi tout ce qui plaît aux êtres, rien ne leur est plus agréable que le marais; parmi tout ce qui imprègne les êtres, rien ne les imbibe autant que l'eau. Pour finir ou pour commencer les êtres, rien n'est plus parfait que le koua ken. C'est pour cela que l'eau et le feu s'atteignent mutuelle ment, que la foudre et le vent sont simultanés, que la montagne et le marais ont le même éther. Il en résulte qu' ensuite la transformation peut s'accomplir et achever les êtres.

\footnotetext{
${ }^{1}$ D'après un commentaire chinois.

${ }^{2}$ Ce qui excuse le traducteur. Il me paraît évident qu'il existe une interprétation cachée et absolument inconnue des commentateurs chinois, dont les énormes compilations n'apportent aucun éclaircissement à la question.

3 Traduit par « cause secrète ».
} 
TSHOU HI. - Ceci traite exclusivement des six koua qui sont engendrés par les koua khien et khouen en laissant ces deux-là de côté. Il s'agit de distinguer la cause naturelle de ce qui existe et de l'ordre des situations de ces koua. Ce passage emploie encore les mêmes expressions que le précédent et on n'en connaît pas encore le sens.

Sixième proposition.

1295.

Khien, activité ; khouen, passivité ; tshen, mouvement; souen, entrer; khan, tomber; li, rapprochement ou séparation; ken, arrêt; touei, satisfaction.

TSHOU HI. - Il s'agit de la nature des huit koua.

Septième proposition.

1296.

Khien, c'est le cheval ; khouen est le bauf ; tshen, c'est le dragon ; souen est la poule; khan est le porc; li est le faisan; ken est le renard et touei le bélier.

TSHOU HI. - Le Yi king prend ses exemples partout, au loin comme auprès.

Huitième proposition.

1297.

Khien, c'est la tête; khouen, le ventre; tshen, les pieds; souen, la cuisse ; khan, l'oreille ; li, l'ail ; ken, la main ; touei, la bouche.

TSHOU HI. - Le Yi king prend ses exemples partout, même sur le corps.

Neuvième proposition. 
1298.

Khien, le ciel, aussi on l'appelle le père; khouen, la terre, aussi on l'appelle la mère; tshen exprime une secousse ${ }^{1}$, une agitation, et il représente le principe mâle, aussi est-il appelé le fils adulte. Souen exprime aussi une agitation et représente une fille, aussi il est appelé la fille adulte. Khan, une nouvelle secousse d'où résulte le sexe mâle, de sorte qu'il est appelé le garçon moyen. $\mathrm{Li}$, une autre secousse d'où résulte une fille, il est donc appelé la fille moyenne. Ken, troisième secousse d'où résulte le principe mâle, de sorte qu'il est appelé le jeune garçon. Touei, troisième secousse et principe femelle, de sorte qu'il est appelé la jeune fille encore petite.

TSHOU HI. - Le terme traduit par secousse veut dire demander, chercher à obtenir ; c'est-à-dire lancer les brins d'herbe shi et les relever pour obtenir une formule de divination. Les mots garçon et fille désignent un trait négatif, ou un trait positif dans un koua.

Dixième proposition.

1299.

Khien, c'est le ciel ; c'est ce qui est rond, c'est le prince, le père, la pierre précieuse, le métal (ou or), le froid, la glace, le rouge foncé ; c'est le cheval rapide, le vieux cheval, le cheval maigre, le cheval blanc, c'est l'arbre sec.

TSHOU HI. - Dans une ancienne copie, à la suite de ces mots, il y avait : c'est le dragon, ce qui est droit et correct, c'est le vêtement, c'est la parole.

1300.

Khouen, c'est la terre, la mère, l'étoffe, la hache, c'est l'économie, légalité ; c'est l'enfant, la mère du bauf ; c'est le char, c'est l'apparence, la foule, la poignée de l'outil ou le manche de l'objet. Sur la terre, c'est le noir.

TSHOU HI. - Dans la copie citée plus haut, il y avait encore les mots : c'est la vache, l'obscurité, ce qui est carré ; c'est le sac, la jupe, le jaune, l'étoffe teinte, la mouche.

\footnotetext{
${ }^{1}$ Le caractère en question n'a pas de sens bien défini. II signifie aussi un « lien », ou bien « dispersion».
} 
1301.

Tshen représente la foudre, le dragon ${ }^{1}$, le jaune sombre, l'influence causative, le grand chemin, le fils aîné ; la précipitation, la tige de bambou développée, certain végétal. Par rapport au cheval, c'est le chant harmonieux, la balzane blanche du pied du cheval; le pied, la crinière. Par rapport à la graminée, c'est le retour à la vie. Dans la recherche des règles, c'est la répétition ${ }^{2}$.

TSHOU HI. - Dans une copie, on lit: c'est la pierre précieuse, le corbeau, le tambour.

1302.

Souen, c'est le bois, le vent, la jeune fille adulte, le fil raidi ${ }^{3}$; c'est le travail, le blanc, la longueur, l'élévation, c'est avancer et se reculer, c'est la branche de l'arbre, c'est l'odorat. Dans l'homme, c'est la rareté des cheveux, le front large, c'est l'ail blanc. C'est un bénéfice prochain, résultat de trois échanges : dans la recherche, c'est la précipitation.

TSHOU HI. - Une copie porte : c'est l'arbre yang, c'est un certain oiseau.

1303.

Khan représente l'eau, c'est le fossé plein d'eau; c'est ce qui est secret et caché ; c'est la couverture du char, la corde de l'arc. Dans l'homme, c'est ce qui cause le chagrin, c'est le malaise du caur, la maladie de l'oreille, la révolution du sang: C'est le rouge. Dans le cheval, c'est une belle échine, l'ardeur, l'abaissement de la tête, le pied fin; la housse, la couverture du char. C'est la multitude des calamités, c'est la libre circulation, la lune, le voleur. Dans le bois (l'arbre), c'est la dureté du cour.

TSHOU HI. - Une copie porte encore : c'est l'antre, ou appartement intérieur ; la règle, ou note de musique ; c'est la possibilité, c'est la colonne en bois, c'est le buisson d'épines, c'est le renard, certaine plante, l'entrave de bois.

\footnotetext{
${ }^{1}$ Voir $§ 1198$, commentaire de TSHOU HI.

${ }^{2}$ La plupart de ces termes sont traduits par à peu près.

${ }^{3}$ Peut-être la trame?
} 
1304.

Li représente le feu, le soleil, l'éclair, la jeune fille d'âge moyen, c'est la suite, la postérité, l'arme $d u$ soldat. Dans l'homme, c'est un ventre proéminent, c'est le koua khien, certains insectes ou reptiles, la tortue. Dans l'ordre, c'est le fruit placé en haut de la tige.

TSHOU HI. — Une copie porte : c'est la vache.

1305.

Ken représente la montagne, le sentier étroit, la petite pierre; c'est la fermeture de la porte, certain fruit; le religieux; le doigt. C'est le renard, la souris. Dans l'arbre, c'est la solidité de nombreux rameaux.

TSHOU HI. - Une copie porte : c'est le nez, c'est 1 e tigre, c'est le loup.

1306.

Touei, c'est le marais, c'est la petite fille, c'est la divination ou le devin, la bouche ou la langue; c'est la rupture, c'est la décision dans l'union ou la séparation. Dans la terre, c'est la dureté ; c'est une concubine, c'est le bélier.

TSHOU HI. - Une copie porte : c'est la permanence ordinaire, c'est l'assistance.

Onzième proposition. Extension du symbolisme des huit koua simples ; la plus grande portée est incompréhensible et si on se reporte au texte il est encore impossible d'y rap porter le tout. 


\section{O R D R E des K O U A}

Aussitôt que le ciel et la terre existent, tous les êtres naissent; ce qui comble l'interval le entre le ciel et la terre n'est rien autre que tous les êtres et toutes les choses ; aussi le koua tshouen $\left(\mathrm{n}^{\circ} 3\right)$ suit immédiatement les koua Khien $\left(\mathrm{n}^{\circ} 1\right)$ et khouen $\left(\mathrm{n}^{\circ} 2\right)$, qui représentent le ciel et la terre. Tshouen signifie remplir. Tshouen exprime le commencement de la naissance des êtres $\left(n^{\circ} 3\right)^{2}$.

Lorsque les êtres naissent, ils sont nécessairement incapables de discernement, c'est pourquoi ce koua est suivi du koua mong. Le koua mong exprime l'aveuglement et le manque de discernement ; c'est le jeune âge des êtres $\left(n^{\circ} 4\right)$.

Pendant le jeune âge des êtres, il est indispensable de les nourrir, c'est pourquoi le koua mong est suivi du koua su. Le koua su exprime la voie rationnelle de l'acte de boire et de manger $\left(\mathrm{n}^{\circ} 5\right)$.

L'obligation de boire et de manger doit nécessairement donner lieu aux contestations, aussi le koua su est-il immédiatement suivi du koua song $\left(\mathrm{n}^{\circ} 6\right)$.

$\mathrm{Du}$ moment où il $\mathrm{y}$ a contestation, il doit nécessairement $\mathrm{y}$ avoir soulèvement de la foule, aussi le koua song est suivi du koua shi ; shi exprime la foule $\left(\mathrm{n}^{\circ} 7\right)$.

La foule doit nécessairement voir quelqu'un autour de qui elle se groupe ; c'est pour quoi le koua shi est suivi du koua $p i$; $p i$, se rapprocher et s'assister $\left(n^{\circ} 8\right)$.

Du moment où il y a association, il doit y avoir quelque chose à réunir, aussi le koua pi est suivi du koua siao tshou: rassemblement, petit arrêt $\left(n^{\circ} 9\right)$.

Aussitôt que les êtres sont arrêtés, il y a des règles rituelles, aussi le koua siao tshou est suivi du koua li. Règles rituelles ( $\left.\mathrm{n}^{\circ} 10\right)$.

Lorsque les hommes sont policés, ils prospèrent; il en résulte quils sont en paix. C'est pour cela que le koua $l i$ est suivi du koua thae; prospérité $\left(n^{\circ} 11\right)$.

Thae prospérité: liberté; les choses ne peuvent pas se développer indéfiniment en liberté, aussi le koua thae est immédiatement suivi du koua $p i$, qui exprime la décadence $\left(\mathrm{n}^{\circ} 12\right)$.

\footnotetext{
${ }^{1}$ C'est un des commentaires attribués à Khong Tse. Comme je n'ai traduit aucune annotation, j'ai pensé inutile de continuer à marquer les par agraphes.

${ }^{2}$ On trouvera les commentaires relatifs à ces propositions dans le commentaire de Tsheng Tse de chaque koua. Nous ne les répéterons pas ici.
} 
Les êtres et les choses ne peuvent pas être indéfiniment dans la décadence et l'adversi té ; c'est pour cela que le koua $p i$ est suivi du koua thong jen; être les mêmes que les hommes $\left(\mathrm{n}^{\circ} 13\right)$.

Les choses ou êtres doivent nécessairement faire retour à quelqu'un, c'est pourquoi le koua thong jen est suivi du koua tae yeou : grand avoir ( $\left.\mathrm{n}^{\circ} 14\right)$.

Lorsque l'avoir est grand, on ne doit pas s'en enorgueillir, aussi le koua tae yeou est suivi du koua Khien, modestie $\left(\mathrm{n}^{\circ} 15\right)$.

L'avoir étant grand et pouvant être modeste, il y a nécessairement satisfaction, aussi le koua khien est suivi du koua $y u$, satisfaction $\left(\mathrm{n}^{\circ} 16\right)$.

La satisfaction fait nécessairement suivre, c'est pourquoi le koua yu est suivi du koua souei $\left(\mathrm{n}^{\circ} 17\right)$.

Lorsqu'on suit quelqu'un par plaisir, il doit en résul ter quelque chose (suites fâcheuses), aussi le koua souei est suivi du koua kou. Kou signifie affaires $\left(\mathrm{n}^{\circ} 18\right)$.

Ce n'est qu'après que les difficultés sont révélées que la grandeur est possible ; aussi le koua kou est suivi du koua lin ; lin, grandeur ( $\left.\mathrm{n}^{\circ} 19\right)$.

Aussitôt que les choses sont devenues grandes, on peut les voir, aussi le koua lin est suivi du koua kouan; kouan, regarder ou être en vue $\left(\mathrm{n}^{\circ} 20\right)$.

Lorsqu'il est possible de regarder, il y a moyen d'unir, aussi le koua kouan est suivi du koua she ho, s'accorder à, s'unir à. Ho veut dire réunir $\left(\mathrm{n}^{\circ} 21\right)$.

Les êtres ne peuvent pas n'être que réunis temporairement, aussi le koua she ho est suivi du koua $p i ; p i$ veut dire orner $\left(\mathrm{n}^{\circ} 22\right)$.

En poussant l'arrangement à l'extrême, il en résulte qu'à la fin la facu lté de liberté se trouve épuisée ; c'est pour cela que le koua $p i$ est suivi du koua po ; po signifie user $\left(\mathrm{n}^{\circ} 23\right)$.

Les êtres et les choses ne peuvent pas finir d'une façon absolue ; lorsque l'usure, ou déclin, épuise le dessus, le dessous reprend en sens c ontraire, c'est pour cela que le koua po est suivi du koua fou ; retour $\left(\mathrm{n}^{\circ} 24\right)$.

Puisqu'il y a retour, il n'y a donc point de désordre ; c'est pourquoi le koua fou est suivi du koua wou wang, pas de désordre $\left(\mathrm{n}^{\circ} 25\right)$.

Aussitôt qu’il y a absence de désordre, il peut y avoir groupement, c'est pourquoi le koua wou wang est suivi du koua tae tshou, grand arrêt (et aussi troupeaux) $\left(n^{\circ} 26\right)$.

Aussitôt que les êtres sont arrêtés (réunis en troupeaux), il faut les nourrir, aussi le koua tae tshou est suivi du koua yi, nourrir $\left(\mathrm{n}^{\circ} 27\right)$.

Sans la nutrition, le mouvement est impossible, aussi le koua yi est suivi du koua tae kuo, excès de grandeur $\left(\mathrm{n}^{\circ} 28\right)$.

Les êtres ne peuvent pas indéfiniment pousser les choses à l'excès, c'est pourquoi le koua tae kuo est suivi du koua khan; khan, tomber, chute $\left(\mathrm{n}^{\circ} 29\right)$. 
Dans la chute, il y a nécessairement un point d'arrivée, aussi le koua khan est immédiatement suivi du koua $l i ; l i$, venir se joindre à $\left(n^{\circ} 30\right)$.

Le ciel $^{1}$ et la terre existant, l'existence de tous les êtres en résulte immédiatement; de là résulte la distinction des sexes, et de celle-ci la relation d'époux à épouse. De la relation d'époux et d'épouse résulte nécessairement, à son tour, celle de père et d'enfant, et de même, de celle-ci, celle de prince et de sujet. De cette dernière résulte encore la relation de supériorité et d’infériorité, d'où découle, comme conséquence, que les règles rituelles et les devoirs réciproques peuvent être définis. La voie rationnelle de l'époux et de l'épouse ne peut pas ne pas avoir de durée, c'est pourquoi le koua hien (influence) ( $\left.\mathrm{n}^{\circ} 31\right)$ est suivi du koua heng; heng signifie longue durée $\left(\mathrm{n}^{\circ} 32\right)$.

Les êtres ne peuvent pas occuper leur situation avec une persistance durable, aussi le koua heng est suivi du koua thouen; thouen, se retirer en arrière, se garer $\left(\mathrm{n}^{\circ} 33\right)$.

Les êtres ne peuvent pas rester indéfiniment dans la retraite, aussi ce koua est suivi du koua ta tshang; grande force $\left(\mathrm{n}^{\circ} 34\right)$.

Les êtres ne peuvent pas jouir indéfiniment de l'épanouissement de la vigueur et c'est pourquoi le koua ta tshang est suivi du koua tsin; tsin, avancer $\left(n^{\circ} 35\right)$.

En avançant, il y a nécessairement des causes de blessures, et c'est pour cela que ce dernier koua est suivi du koua ming yi, blessures, accidents nuisibles $\left(\mathrm{n}^{\circ} 36\right)$.

$Y i$, c'est blesser ; la blessure faite à autrui fera souffrir son auteur ; aussi le koua ming yi est suivi du koua kia jen. Kia jen, hommes de la famille ( $\left.\mathrm{n}^{\circ} 37\right)$.

La voie rationnelle de la famille épuisée, il y a nécessairement opposition ; c'est pour cela que le koua kia jen est suivi du koua khouei ; khouei signifie opposition $\left(\mathrm{n}^{\circ} 38\right)$.

L'opposition comporte nécessairement le danger, de sorte que le koua khouei est immédiatement suivi du koua kien; kien exprime la difficulté $\left(n^{\circ} 39\right)$.

Les êtres ne peuvent pas être définitivement plongés dans les difficultés, aussi le koua kien est suivi du koua kiae ; kiae, ajourner, amnistier ( $\left.\mathrm{n}^{\circ} 40\right)$.

En ajournant, il doit en résulter des inconvénients, c'est pourquoi le koua kiae est suivi du koua souen, diminuer $\left(\mathrm{n}^{\circ} 41\right)$.

La diminution ne cessant pas, il doit nécessairement y avoir augmentation; c'est pourquoi le koua souen est suivi du koua $y i$ (augmentation) ( $\left.\mathrm{n}^{\circ} 42\right)$.

\footnotetext{
${ }^{1}$ C'est ici que commence la seconde partie du Yi king.
} 
L'augmentation ne cessant pas doit nécessairement s'arrêter brusquement, c'est pourquoi le koua yi est suivi du koua kouae, interrompre brusquement, décider $\left(\mathrm{n}^{\circ} 43\right)$.

Pour mettre fin à quelque chose, il faut qu'il y ait rencontre, c'est pour cela que le koua keou suit le koua kouae; keou, rencontrer $\left(\mathrm{n}^{\circ} 44\right)$.

Les êtres se rencontrent et ensuite ils se rassemblent et se réunissent, c'est pour cela que le koua keou est suivi du koua tsouei; tsouei, rassembler $\left(n^{\circ} 45\right)$.

L'action de monter en se rassemblant s'exprime par le mot sheng; c'est pour cela que le koua tsouei est suivi du koua sheng $\left(\mathrm{n}^{\circ} 46\right)$.

En montant sans cesse, le malheur doit nécessairement survenir, aussi le koua sheng est suivi immédiatement par le koua khouen. Khouen, tristesse, misère $\left(n^{\circ} 47\right)$.

La misère en haut doit retomber sur ce qui est en bas ; c'est pour cela que le koua khouen est suivi du koua tsing ( $\left.\mathrm{n}^{\circ} 48\right)$.

Il est impossible que la voie rationnelle du puits ne change pas, aussi le koua $t$ sing est suivi du koua $k o$, changer, modifier $\left(\mathrm{n}^{\circ} 49\right)$.

Parmi tout ce qui change les choses, rien n'est comparable à la marmite, aussi le koua $k o$ est suivi du koua ting ; ting, marmite $\left(\mathrm{n}^{\circ} 50\right)$.

Personne n'est le maître des objets autant que le fils aîné ; aussi le koua ting est suivi du koua tshen; tshen, le mouvement ( $\left.\mathrm{n}^{\circ} 51\right)$.

Les êtres ne peuvent pas se mouvoir indéfiniment; ils s'arrêtent, aussi le koua tshen est suivi du koua ken. Ken, arrêt ( $\left.\mathrm{n}^{\circ} 52\right)$.

Les êtres ne peuvent pas rester indéfiniment dans l'immobilité, aussi le koua ken est suivi du koua tsien; Khien, progression en avant $\left(\mathrm{n}^{\circ} 53\right)$.

Pour avancer, il faut avoir un but vers lequel on tend ; aussi le koua tsien est suivi du koua kouei mei $\left(\mathrm{n}^{\circ} 54\right)$.

La concision de ce qui vient s'adjoindre doit entraîner la grandeur, aussi le koua kouei mei est suivi du koua fong; fong, grandeur $\left(\mathrm{n}^{\circ} 55\right)$.

L'épuisement de la grandeur doit nécessairement conduire à la perte de la situation occupée, aussi le koua fong est suivi du koua lou $\left(\mathrm{n}^{\circ} 56\right)$.

Les voyages conduisent à l'absence d'abri, de sorte que le koua lou est suivi du koua souen; souen, entrer ( $\left.{ }^{\circ} 57\right)$.

Entrer et ensuite s'en réjouir, aussi le koua souen est suivi du koua touei ; touei, plaire $\left(\mathrm{n}^{\circ} 58\right)$.

La satisfaction se dissipe, aussi le koua touei est suivi du koua hoan; hoan, séparation ( $\left.\mathrm{n}^{\circ} 59\right)$. 
Les êtres ne peuvent pas demeurer toujours séparés, aussi le koua hoan est suivi du koua tsie $\left(\mathrm{n}^{\circ} 60\right)$.

De l'observation des préceptes résu lte la confiance, aussi le koua Mie est suivi du koua tshong fou $\left(\mathrm{n}^{\circ} 61\right)$.

Celui qui a confiance en quelque chose agit nécessairement d'après sa conviction, aussi le koua tshong fou est suivi du koua siao kuo $\left(\mathrm{n}^{\circ} 62\right)$.

Celui qui a plus qu'autrui doit prêter assistance, aussi le koua siao kuo est suivi du koua ki tsi ( $\left.{ }^{\circ} 63\right)$.

Les êtres ne peuvent pas être éternels, aussi le koua ki tsi est suivi du koua vi tsi, qui est le dernier ( $\left.\mathrm{n}^{\circ} 64\right)$. 


\section{O P P O I T I O N des KOUA}

Khong Shi Ying Te dit : «L'Ordre des koua » donne l'ordre dans lequel ces koua sont rangés depuis les temps de Wen Wang; dans ce commentaire sur les «Oppositions des koua», Khong Tse change cet ordre et établit une autre classification par leur opposition, classification tout à fait différente de celle de «L'Ordre des koua ».

Khien (1), activité ; khouen (2), passivité.

$P i(8)$, joie ; shi (7), chagrin.

Lin (19) et houan (20), le sens est tantôt donner, tantôt demander.

Tshouen (3), manifeste et qui ne perd point sa situation; mong (4), confusion et distinction.

Tshen (51), s'élever ; ken (52), arrêter; souen (41) et yi (42) marquent le commencement de la plénitude et de la décroissance.

Tae tshou (26), le moment (temps) ; wou wang (25), calamité.

Tsouei (45), réunion, et sheng (46), ne pas venir ; khien, mépris, et $y u$, vanité.

She ho (21), manger ; Siao tshou ${ }^{1}$ (22), absence de couleur.

Touei (58), manifeste et visible ; souen (57), caché.

Souei (17), absence de cause ; kou (18), alors commandement.

Po (23), user ; fou (24), retour en sens opposé.

$T \sin$ (35), le lever du soleil ; ming yi (36), détruire ${ }^{2}$.

Tsing (48) et khouen (47), se rencontrer ensemble.

Hien (31), subit, rapide ; heng (32), de longue durée.

Hoan (59), séparation ; tsie (60), arrêt, séjour.

Kiae (40), délivrance ; kien (39), empêcher, retenir.

Khouei (38), dehors ; kia jen (37), dedans.

$P ’ i$ (12), thae (11), distinguent deux genres opposés ${ }^{3}$.

Ta tshang (34), alors s'arrêtera ; thouen (33), se retirer en arrière.

\footnotetext{
${ }^{1}$ Koua $\mathrm{n}^{\circ} 22$, représente l'ornementation. Dans ce commentaire, c'est souvent un sens tout opposé à celui qui est développé dans le King qui est relevé.

${ }^{2}$ Yu Shi Hien dit : Le rapprochement du soleil a lieu au-dessus, donc le lever du soleil ; la clarté rentre en dedans de la terre, donc destruction. - Un autre commentateur dit : Ces deux koua sont les symboles de l'aube et du crépuscule.

${ }^{3}$ Thae, prospérité, premier mois, printemps ; $P$, décadence, septième mois, automne.
} 
Tae yeou (14), la foule; thong jen (13), parents, proches.

Ko (49), écarter les causes ; ting(50), prendre auprès.

Siao kuo (62), dépasser ; tshong fou (61), confiance.

Fong (55), beaucoup de causes ; peu de parents, lou (56).

Li (30), au-dessus, monter ; khan (29), descendre.

Siao tshou (9), peu ; li (10), ne pas rester en place.

Su (5), ne pas avancer; song (6), ne pas s'approcher.

Tae kuo (28), sommet, culmination; keou (44), rencontrer. La dureté énergique rencontre la douceur malléable.

Tsien (53), la fille qui se marie attend que le jeune homme agisse (la demande). Yi (27), nourrir la droiture, la développer. Ki tsi (63), établir ; kouei mei (54), la fin de la jeune fille. Vi tsi (64), la fin du jeune homme. Kouae (43), décider; la dureté énergique limite la douceur malléable. La voie de l'homme doué s'élève, la voie de l'homme infé rieur décline ${ }^{1}$.

1 À partir du koua tae kuo (28), l'opposition entre deux koua consécutifs n'est plus r elevée. 


\section{T A B L E A N A L Y T I Q U E}

de quelques expressions remarquables dans les formules des koua.

Le titre : $Y i$, changement; Tsheou, mouvement circulaire qui entoure.

Koua $\mathrm{n}^{\circ}$ 1. - Khien : La force, ou cause active, activité, dureté, l'unité, le ciel. - Koua simples intérieurs et extérieurs ; formation des koua : Le ciel, le dragon. Koua du quatrième mois, correspond au solstice d'été.

Koua $\mathrm{n}^{\circ} 2 .-\S 58$. Khouen : passivité, douceur, dualité, jument. Koua du dixième mois, correspond au solstice d'hiver. — $\$ 59$. Voie rationnelle de la terre. $-\S 61$. Le cheval. — $§ 65$. Définition du terme hexaire : givre, glace, le froid, refroidissement progressif. — $\$ 65$. Régularité de la règle. $-\S 68$. La voie de la terre est éclatante. - $\$ 73$. Jupe jaune : présage heureux. - Tshou $\mathrm{Hi}$, définition de la jupe. - $-\$ 74$. La chose dont il est question a lieu à l'intérieur, elle est invisible, mais elle se manifeste au-dehors par ses effets. $\S 75$. Encore le dragon, sang noir et jaune, ombres et lumière. — $§ 76$. La voie du koua khouen, de la terre, est épuisée et la voie du ciel (khien) recommence. — 90 . Khi. souffle, éther, air.

Koua $\mathrm{n}^{\circ}$ 3. - Tshouen : remplir, ce qui remplit. Le commencement de la naissance des êtres. C'est le résultat de l'union de la négativité et de la positivité. Analogie et différence avec le koua khan: foudre. — §91. Avantage à instituer des princes feudataires: ce qui permet d'établir des divisions entre les régions. — $\$ 96$. Borne, pieu, limite d'états, maria ge, enlèvement de la fiancée par des hommes à cheval. Remarquer l'expression « demeurer au rang ». Le sens du mot pan, mettre en rang. Ce radical avec le même sens dans les langues aryennes. Le même mot autrement accentué, pan : moitié, demi. — § 100. Le daim. — § 106. Pleurs de sang. — § 107. Tsheng Tse, une hypothèse.

Koua $\mathrm{n}^{\circ}$ 4. - Mong : privation de lumière qui suit la naissance des êtres. Clarté imparfaite au moment du lever du soleil. — $§ 112$. Dissipation des ténèbres, châtiment, entraves, supplices, appréhension d'un mal futur. $\S 114$. Tolérer et contenir ; l'enfant. — $§ 116$. Épousailles. — $§ 118$. Misère. — $\$ 120$. Jeune garçon. — $§ 122$. Brigandage, violence. 
Koua $\mathrm{n}^{\circ} 5$. $-S u$ : idée de boire et de manger, attente. $-\S 124$. Grand cours d'eau. $-\S 126$. «Milieu » et «justice». - $§ 128$. Attendre dans la plaine. Persévérance, permanence. $-\S 130$. Attendre sur la grève, sable. $\S 132$. Attendre dans la boue. - $§ 134$. Attendre dans le sang, gouffre. $\S 136$. Vin et nourriture. - $\S 138$. Caverne.

Koua $n^{\circ}$ 6. - Song : origine des contestations, grand homme. $-\S 141$. En haut, activité ; en bas, péril ; contestation. _ $§ 144$. Ne pas éterniser. $\S 146$. Revenir et se mettre à l'abri, district, ou hameau, famille, calamités. $\S 148$. Nourrir la vertu. — $§ 150$. Retour à la destinée. — $§ 154$. Présent, don, grande ceinture ; pan.

Koua $\mathrm{n}^{\circ}$ 7. - Shi: expression de l'idée de multitude, armée, troupe. $\S 155$. L'hom me au bâton. — $§ 160$. Tshou Hi, sens des caractères dénaturés par les commentateurs. - $\$ 164-166$. Sens dénaturé. — $§ 168$. Rizières, gibier (ailé), fils aîné, cadet. — $§ 170$. Homme inférieur, grand prince, fonder des royaumes.

Koua $\mathrm{n}^{\circ}$ 8. $-P i$ : rapprochement. $-\S 172$. Divination, consulter le sort. — $\S 174$. Valeur du nom de ce koua. Analogie avec un préfixe des langues agglutinantes. — $§ 177$. Foi ou bonne foi, confiance. $-\S 179$. L'association se produit du dedans vers le dehors. $-\S 181$. Hommes indignes. $-\S 183$. L'e xtérieur s'y associe. - $§ 185$. Association illustre, manifeste, visible. Le roi. Détails sur les chasses. L'homme du hameau. Le sens de plusieurs mots dénaturé. - § 187. S'y associer sans se mettre à la tête, ou absence de tête.

Koua $\mathrm{n}^{\circ}$ 9. - Siao tshou: petit rassemblement, ou petit arrêt, groupement. — $§ 189$. Épais, mystérieux, nuages, pluie, plaines de l'ouest. — Tsheng Tse, éther, air, vapeur, effluves. — $§ 194$. Revenir par la voie. $\S$ 196. Entraîner par son retour. — § 196. Char privé d'essieu. — § 198. Char, essieu, époux et épouse. $\_\S 200$. Le sang s'écoule. — $\$ 202$. Foi comme inébranlable. — § 204. La lune près de son plein, homme doué. Quel rapport y a-t-il entre ces deux expressions ?

Koua $\mathrm{n}^{\circ} 10 .-L i$ : règles rituelles ? $-\S 206$. Fouler aux pieds, queue du tigre, mordre. - $\$ 211$. Démarche. - $\$ 213$. Marcher dans la voie, homme retiré, ou obscur (renvoi). — $\$ 215$. CEil qui voit mal, pied blessé, homme violent, grand prince. — $\$ 217$. Cruauté et inquiétude, fin. — $\$ 219$. Décision. — \$221. Regarder la démarche pour scruter un présage, mouvement circulaire embrassant tout l'horizon. 
Koua $\mathrm{n}^{\circ}$ 11. - Thae: prospérité, croissance. Koua du premier mois, équinoxe du printemps. - $§ 225$. Éther, air, solidifier, rendre corporel. $\S 226$. Arracher des herbes, genre, catégorie, choses qui s'entraînent l'une l'autre. - $§ 228$. Amis qui s'oublient. - $§ 230$. Pas d'action sans réaction, pas de départ sans retour, difficulté. - $\$ 232$. Voltiger, voisinage, avertissement. - Ti, empereur, et aussi dieu, marier une princesse. $-\S 236$. Le rempart retombe dans le fossé, district, hameau, Tshou $\mathrm{Hi}$, fin de la prospérité, apogée suivie de décadence.

Koua $\mathrm{n}^{\circ} 12$. - $P^{\prime} i$ : décadence, absence d'homme, pas d'avantage pour l'homme doué, ce qui est grand s'en va, ce qui est petit vien t. Koua du septième mois, équinoxe d'automne. Le mot ling, esprit, essence spirituelle, correspond au mot $\cdots$ cos, raison et parole. $-\S 241$. Analogies entre ce $\S$ et le $\S 226$. - $§ 243$. Petit homme et grand homme. - $\$ 247$, catégories, entraînement mutuel des choses d'un même genre. Séparation. — $\$ 249$. Arrêter la décadence, grand homme, perte. Être attaché. — $\$ 251$. La décadence est suivie de joie. L'apogée atteinte, réaction en sens inverse.

Koua $\mathrm{n}^{\circ}$ 13. - Thong jen : mêmes hommes. - $\$ 253$. Désert, sens de cette expression, grand cours d'eau, homme doué. — $\$ 258$. Mêmes hommes à la porte, (men) porte à deux battants. — § 260. Souche, origine commune, association, groupe. - $\$ 262$. Armes, herbes épaisses, colline élevée, armée, élever, commencer, édifier. - $\$ 264$. Monter sur le mur, vaincre, l'emporter sur quelque chose. - $\$ 266$. Cris, lamentations et ensuite rires, rencontre mutuelle. — $§ 268$. Plaine, regrets.

Koua $\mathrm{n}^{\circ}$ 14. - Tae yeou : grand avoir. — $\$ 274$. Joindre le malheur. \$ 276. Char, porter sur un char. Kong: titre, dignité, le fils du ciel, petit homme. Sens arbitraire donné à un caractère. - $\$ 280$. Ce n'est pas l'ampleur. Sens très douteux, idée de distinguer clai rement quelque chose. $\S 282$. Autorité imposante. - $§ 285$. Assistance provenant du ciel. Intelligence ou clarté sont synonymes.

Koua $\mathrm{n}^{\circ}$ 15. - Khien : modestie, homme doué, avoir une fin. — $§ 288$. Voie du ciel et voie de la terre, Tsheng Tse. - § 290. La terre, infériorité, abaissement. — $§ 293$. Renommée. — $\$ 295$. Mérite et modestie, homme doué. - \$299. Envahir, réduire par les armes. - $\$ 301$. Districts et royaumes.

Koua $n^{\circ} 16$. - Yu : calme, repos, satisfaction. - $§ 303$. Institution de princes feudataires, idée d'une division d'un territoire, ou d'un espace. $\S 306$. Soleil et lune, jour et mois, moment. — $§ 310$. Solidité, pierre. - 
$\S 312$. Retards et regret, regarder en haut. — $\$ 314$. Exemple d'un passage incompréhensible. Nouvel exemple d'une interprétation forcée d'un caractère. — §316. Inconvénient, infirmité, continuité. — §318. Aveugles, obscurité. Achèvement suivi de modification.

Koua $\mathrm{n}^{\circ}$ 17. - Souei : suivre. — $\$ 322$. L'univers suit le temps (shi). $\S 325$. Kouan : magistrat, fonctionnaire, sortir de la porte (men) pour se joindre. - $\$ 327$. S'attacher au petit enfant, perdre l'homme au bâton, analogie avec le mot dandino — $§ 329$. Hypothèse inverse, suivre et chercher à atteindre, demeurer. — $§ 331$. Suivre et attraper, avoir foi dans la voie, à cause de la clarté. — § 332. À cause de la clarté. — § 333. Foi dans le bien, hia. — $§ 335$. Montagnes de l'ouest, analogie avec le $\S 278$.

Koua $n^{\circ}$ 18. - Kou : affaires, troubles, difficultés. - § 341, 343, 345. Support, tuteur, tige. $-\S 351$. Servir un roi, un prince, estimer haut.

Koua $\mathrm{n}^{\circ}$ 19. - Lin : grandeur, et aussi surveiller. - $\S 353$. Parvenu au huitième mois il y a présage malheureux (voir koua $\mathrm{n}^{\circ} 20$, kouan). — $§ 356$. Croissance. - $-\S 359,361$. Surveiller et émouvoir. - $§ 362$. Pas encore et déjà. — § 363. Surveillance agréable, en avoir du chagrin. — § 365. Extrême grandeur, ou extrême surveillance. - $\_$367. Savoir surveiller, convenance d'un grand prince. — $\$ 369$. Grandeur, ampleur dans la surveillance.

Koua $\mathrm{n}^{\circ} 20$. - Kouan : point de mire, être exposé aux regards. Effet de l'accent tonique. - $§ 371$. Rites dans certains sacrifices; nouveau sens du mot houan. - $\$ 374$. Absence d'irrégularité dans les quatre saisons, danses mimées représentant le mouvement des astres. — $\$ 376$. Le jeune garçon est exposé aux regards, petit homme, homme doué. — \$ 378. Épier, regarder à la dérobée, jeune fille. - $§ 380$. Mouvement en avant et en arrière. - $\$ 382$. Éclat du royaume, hôtes. - $§ 384$. Regarder ma naissance, le pronom je ou moi. — §386. Regarder sa naissance, ou sa vie.

Koua $n^{\circ} 21$. - She ho: s'accorder, s'unir à. - $§ 388$. Couper avec les dents, peines, châtiments, supplices. - $§ 392$. Chausser des entraves de bois, détruire les orteils. — $\$ 394$. Peau, nez. — $\$ 396,398$. Viande séchée pointe de flèche en métal. — $\$ 400$. Viande séchée, métal jaune ou or jaune. $\S 402$. Entraves pour le cou, oreilles.

Koua $\mathrm{n}^{\circ} 22$. $-P i$ : orner, ornementation extérieure, farder. $-\S 404$. Petit avantage. - $\$ 408$. Regarder l'apparence du ciel afin d'examiner les modifications des moments, ou des saisons. - $§ 410$. Position des pieds, quitter le char et aller à pied. — $§ 412$. La barbe. — $\$ 416$. Blanc écru, cheval 
blanc, brigands, enlèvement d’une épousée. — $§ 418$. Jardin, enclos, colline, pièce d'étoffe de soie. Sens très douteux. — $\$ 420$. Blanc.

Koua $\mathrm{n}^{\circ}$ 23. - Po: user, usure, tomber. Koua du neuvième mois. $\S$ 422. Pas d'avan tage. — $§ 424$. Mouvement du ciel. $— \S 426$. Usure du lit par les pieds, destruction. - $§ 428$. Traverses du lit, destruction. — $§ 430$. L'user. — § 432. User le lit par la peau. — $\$ 434$. Enfiler des poissons en chapelet, palais, faveur. - $§ 436$. Gros fruit, homme doué, petit homme, user la maison.

Koua $\mathrm{n}^{\circ}$ 24. - Fou: retour, recommencer. Koua du onzième mois. Pourquoi il représente le onzième mois. — $\$ 438$. Sortir ou entrer sans inconvénient, amis qui viennent, aller et revenir en suivant la voie. Au bout de sept jours revenir de nouveau. Tshou Hi, explication du mot fou. — $\$ 441$. Parcourir la voie en divers sens, Tshou HI. Le mouvement de translation du ciel. — §443. Évanouissement et croissance. Solstice d'hiver. — $§ 445$. Retour peu éloigné. - $\$ 447$. Retour du bien. - $\$ 448$. Jen, homme et humanité. — § 449. Retours consécutifs. — $\$ 451$. Revenir seul. — $\$ 452$. En suivant la voie. — $§ 453$. Grandeur dans le retour. — $\$ 455$. Aveuglement dans le retour, ou retour obscurci, calamités, grande déroute, destruction ; dix ans, dix est la limite des nombres.

Koua $\mathrm{n}^{\circ} 25$. - Wou wang : pas de désordre, retour à la voie rationnelle (tao) donc absence de désordre, ou d'irrégularité. — $\$ 457$. Voie du ciel pas de désordre. Tshou Hi, autre forme de la même expression. — $§ 459$. Parfaite concordance aux moments, saisons. - § 460. Pas de désordre. — $\$ 462$. Récolter sans labourer, rizières. - $§ 464$. Pas de désordre, bouf attaché, homme du district. - $\$$ 468. Inconvénient de l'absence de désordre, joie, guérison sans remède. $-\$ 470$. Absence de désordre, calamités.

Koua $\mathrm{n}^{\circ}$ 26. - Tae tshou : grand arrêt. — § 472. Grand arrêt, traverser un grand cours d'eau. — $\$ 476$. Répondre au ciel, ou correspondre au ciel. $\S 480$. Avantage à cesser. — $§ 482$. Le char manque d'essieu. — $§ 484$. Un bon cheval poursuit, chars, enceintes. Caractère douteux, Tshou Hi. — $§ 486$. Jeune taureau, encore dépourvu de cornes. — $\$ 488$. Défenses du porc. $\S$ 490. Liberté d’influence du ciel.

Koua $\mathrm{n}^{\circ}$ 27. - Yi : nourrir. — $\$$ 492. Regarder, ou montrer la bouche, bouche pleine. - $§ 494$. Le ciel et la terre nourrissent (développent, engendrent) tous les êtres. — $§ 495$. Les termes relatifs à la boisson et à la nourriture déduits d'un symbole, par «l'homme doué ». — $\$ 496$. Tortue spirituelle, mouvement des mâchoires. — $§ 500,502$. Transgression dans la 
nourriture, subversion dans l'acte de se nourrir, atteindre brusque ment par bonds. — $-\S 503$. À cause de la nourriture.

Koua $\mathrm{n}^{\circ}$ 28. - Tae kuo : excès de grandeur, sens du mot kuo. - $§ 508$. L'arête du toit. — $\$ 510$. L'origine et l'extrémité sont faibles. — $\$ 514$. Chaume blanc, nattes. — $\$ 516$. Le saule desséché pousse des bourgeons, éther positif. — $\$ 518$. Arête recourbée d'un toit. — $\$ 520$. Éclat du faîte. $\S 522$. Saule desséché qui donne des fleurs, vieille épouse et jeune époux. $\S 524$. Traverser en nageant.

Koua $\mathrm{n}^{\circ} 29$. - Khan: tomber, chute. Répétition d'un koua simple : obstacles, accumulation de périls, débordement. — $\$ 532$. Fissure, gouffre précipice. - $\$ 534$. Chute périlleuse. $-\$ 536$. Périls nombreux, entrer dans le gouffre de l'abîme. — $§ 538$. Urne, vin, offrandes, fenêtre, ouverture. $\S 540$. L'abîme ne se remplit pas. — § 542, 543. Corde, attacher, buisson d'épines, trois ans.

Koua $\mathrm{n}^{\circ} 30$. - Li : se joindre à, se séparer de, jour, clarté. — $\$ 544$. Réunir des vaches en troupeau. - $\$ 545$. $L i$, contact, le soleil et la lune sont en contact avec le ciel. Répétition de clartés. — $\$ 547$. Clarté qui jaillit deux fois. — $\$ 548$. Démarche hésitante. — $\$ 540$. Clarté jaune. — $\$ 552$. Clarté du soleil qui décline. Tsheng Tse, in fine. Vieillesse et soleil qui décline. $\S 554$. Courant rapide, brûlant, mort abandonné. — § 556. Larmes, pleurs, tristesse, lamentations. $-\S 558$. Le roi est sur le point de s'en aller.

Koua $n^{\circ} 31$. - Hien: être influencé, ressentir l'influence. - $\$ 560$. Épouser une jeune fille. — $\$ 563$ (suite). Le ciel et la terre s’influencent. $\S 565$. L'orteil. — $§ 567$. Le mollet. — $\$ 569$. La jambe. — 5571. Mouvement incertain, aller et venir. Amis. Tsheng Tse, exemples et explications. — $§ 573$. La colonne vertébrale. — $§ 575$. Maxillaires, langue.

Koua $\mathrm{n}^{\circ}$ 32. - Heng : longueur de la durée. — $\$ 583$. Persistance. $\S 584$. Manque de discernement, ou obscurité. - $§ 589$. Rizières sans oiseaux. — $\$ 593$. Ébranler la persévérance.

Koua $\mathrm{n}^{\circ} 33$. - Thouen: se retirer en arrière, se garer. Koua du sixième mois. - § 598. Commencement de la croissance de la négativité. — $\$ 600$. Retirer la queue. — $\$ 602$. Cuir du bouf jaune. — $\$ 604$. Être retenu par des attaches, des liens. — $\$ 606$. Aimer la retraite, le petit homme se réduit. $\S 608$. Se plaire dans la retraite. — $§ 610$. Profiter de la retraite; augmentation, croissance. 
Koua $\mathrm{n}^{\circ}$ 34. - Ta tshang: grande force. Koua du deuxième mois. $\S 614$ (suite). Conformité de la grandeur à la droiture. — § 616. Vigueur dans le gros orteil. - $\$ 620$. Le petit homme emploie la vigueur, homme doué, jeune bélier qui heurte avec la tête, user les cornes. Analogie avec un passage de Lucrèce. - $§ 624$. Disparition du bélier dans le changement. — $§ 625$. Disparition. - $\S 626$. jeune bélier qui heurte avec la tête, incapable de se retirer en arrière ou de poursuivre.

Koua $\mathrm{n}^{\circ} 35$. - Tsin : avancer. Le soleil sort sur la terre. — $§ 628$. Feudataires, chevaux, trois rencontres, ou entrevues. Le soleil émerge au-dessus de l'horizon. — $§ 632$. Avancer et se retirer. — $§ 634$. La mère du roi. - $§ 636$. Confiance universelle. — $§ 638$. Avancer comme le rat des champs. - $\$ 640$. Ne pas s’inquiéter de perdre ou d'obtenir. — $\$ 642$. Avancer la corne, détruire le district.

Koua $\mathrm{n}^{\circ}$ 36. - Ming yi: blessures nuisibles. La clarté entre dans l'intérieur de la terre, sens diamétralement opposé à celui du koua tsin; le soleil rentre dans la terre, la lumière s'altère. — $\$ 642$. Difficulté. — $\$ 648$. Vol, ailes, lumière qui change, homme doué, trois jours. - $§ 650$. Blessure dans la cuisse gauche, cheval vigoureux. $-\$ 652$. La lumière s'obscurcit vers la limite méridionale, grande tête. — $\$ 654$. Viscères de gauche, au sortir de la porte du parvis. — $§ 656$. Obscurcissement de la clarté, Ki Tse. — $§ 658$. Il n'éclaire pas l'obscurité, s'élever au ciel et rentrer dans la terre.

Koua $\mathrm{n}^{\circ}$ 37. - Kia jen: personne de la famille. — §665. Régler la famille : difficultés d'interprétation. - $\$ 667$. Pas de moyen de réussir. $\S 669$. Répétitions de caractères très douteux. — $\$ 673$. Le roi a une famille.

Koua $\mathrm{n}^{\circ}$ 38. - Khouei : opposition. Loucher, regarder des deux côtés à la fois. — $§ 677$. Petites choses. — $§ 682$. Regrets dissipés : perte du cheval, il revient de lui-même ; les hommes méchants. — $§ 684$. Rencontrer le maître dans la ruelle. $-\S 686$. Expressions douteuses et sens arbitraires. — $§ 688$. Séparé et isolé. - §690. Regrets dissipés, morsure. — \$ 692. Séparé et isolé, brigands ou mariage, rencontrer la pluie : la raison d'être des choses.

Koua $n^{\circ} 39 .-$ Ken : difficulté, danger, empêchement. — § 694, 696. Avantage du côté du sud-ouest, région opposée au nord-est, grand homme. $\S 700$. Roi et sujet, difficultés. — $\$ 702$. En allant péril, en venant, c'est le contraire. — § 704. En allant péril, en venant, entraînement. — § 706. Grand empêchement, amis qui viennent. — $§ 708$. En venant : grandeur ; avantage à voir un grand homme. 
Koua $\mathrm{n}^{\circ}$ 40. - Kiae: délivrance, séparation. - $\$ 710$. Avantage du sud-ouest, agir de bonne heure. — $§ 713$. Le ciel et la terre séparés, les germes se développent, le moment exprimé est grand, solennel, important. — $\$ 717$. Rizière ou chasser, trois renards, flèche jaune, expressions mal comprises. $\S 719$. Porter sur les épaules, les brigands surviennent. — $\$ 721$. Délivrance du gros orteil, amis. — $§ 723$. L’homme doué pense à la déli vrance, avoir foi dans le petit homme. $-\$ 725$. Employer la flèche, l'oiseau sin.

Koua $\mathrm{n}^{\circ}$ 41. - Souen : diminuer, décroître. Éther. — $\$ 729$. Diminuer en bas, augmenter en haut; la voie monte et agit. - $\$ 730$. Diminution et augmentation, plein et vide, ils se succèdent toujours avec le temps, remplir et vider. — $§ 732$. Apprécier la diminution. — $§ 734$. Ne diminuer ni augmenter. — 736. Trois hommes. — $\$ 738$. Diminuer l'inconvénient. — $§ 740$. Tortue des dix amis, contrevenir. — Ne diminuer ni augmenter, avoir des serviteurs.

Koua $\mathrm{n}^{\circ}$ 42. - Yi : augmentation, avantage, bénéfice. $-\S 745$. Diminuer en haut et augmenter en bas, de haut, descendre en bas, voie brillante. $\S 747$. journellement avancer sans limite, ou le soleil avance sans cesse ? $\S 751$. Dix paires de tortues, aller contre, être suprême, ou empereur, roi. $\S 753$. Augmenter en employant ce qui est mauvais, insigne secret, signe d'affiliation. - $\$ 755$. Agir avec justice ou suivre le milieu, changer le royaume. - $§ 757$. Bonne foi, bienveillance, mes vertus. — $\_759$. Ne pas l'augmenter, frapper, affermir le cour.

Koua $\mathrm{n}^{\circ}$ 43. - Kouae: décider, déterminer, interrompre, séparer brusquement. - $\$ 761$. Avertir son propre district. — $\$ 765$. Vigueur dans l'action d'avancer l'orteil. — $§ 767$. Le soir et la nuit il y a des ennemis, ne pas s'alarmer. — $\$ 769$. Vigueur dans le front, homme doué. — $\$ 771$. Fesses sans peau, action indécise, conduire des moutons. - $\$ 774$. Reduplication, sens fréquentatif. Comment les commentateurs chinois entendent le Yi king.

Koua $\mathrm{n}^{\circ}$ 44. - Keou : rencontrer. $-\$ 777$. Jeune fille adulte, épouser une jeune fille. - $\$ 780$. Le ciel et la terre se rencontrent, éclat qui en résulte. $\S 781$. Univers. — $§ 782$. Le moment exprimé est important. — $§ 783$. Tsheng Tse, tourbillon enveloppant, « homme doué » et « grand homme ». — $\$ 784$. Attacher à un pieu en métal : porc maigre sautillant. — § 786. Sac contenant du poisson. — § 788. Action indécise. — § 790. Sac sans poisson. — $\$ 792$. Envelopper des courges, renfermer l'éclat, quelque chose tombe du ciel. $\S 794$. Rencontrer les cornes.

Koua $\mathrm{n}^{\circ} 45$. - Tsouei : rassemblement, réunion. - $§ 796$. Temple en l'honneur des ancêtres, grand homme, viande desséchée, religiosité de la 
loutre. - $\S 801$. Foi, pas de fin, désordre, rassemblement, une poignée de... — $\S 803$. Entraînement, sacrifices du printemps. — $§ 809$. Le rassemblement a lieu dans une situation sans culpabilité, dissipation des regrets. $— \S 811$. Gémir, pleurer, lamentations.

Koua $\mathrm{n}^{\circ}$ 46. - Sheng : avancer, monter. — $\$ 813$. Voir un grand homme, midi ou sud, présage heureux. — $§ 817$. Tsheng Tse. $\_\S 818$. Monter avec confiance, remarque au sujet du caractère yun. - $\S 820$. Sacrifices du printemps. - $-\S 821$. S'élever dans un dis trict vide. $-\$ 823$. Encore la montagne de $k h i$. — $§ 826$. Monter des degrés. — $\$ 828$ et 829. S'élever dans l'obscurité, Tsheng Tse.

Koua $\mathrm{n}^{\circ}$ 47. - Khouen : tristesse et misère. - $\$ 830$. Grand homme, présage heureux. - $\$ 831$. La dureté énergique recouvre. $-\S 834$. Le fond repose sur un tronc d'arbre dépouillé, vallée sombre, trois années sans voir. $\S 836$. Manger et boire, tablier rouge qui commence à venir, sacrifices. $\S 838$. Misère sur la pierre, appuyer sur des chardons, chambre intérieure, ne pas voir l'épouse. - $\$ 840$. Char d'or. $-\$ 842$. Amputer le nez et les pieds, tablier rouge cramoisi. $\_\S 844$. Réseau de lianes, au sujet du caractère traduit par « dire », renvois.

Koua $\mathrm{n}^{\circ}$ 48. - Tsing : le puits. $-\S 846$. Changer le hameau sans changer le puits, la soif survient, casser le seau. $-\S 850$. Puits vaseux, non potable, puits sans oiseaux. $-\S 852$. Torrent qui coule, lancer des flèches. $-\S 854$. Puits limpide, roi intelligent (brillant). — $§ 856$. Puits revêtu de briques. $\S 858$. Puits frais et pur. $-\S 860$. Le puits recueille sans recouvrir.

Koua $\mathrm{n}^{\circ}$ 49. - Ko : modifier, changer, renouveler ce qui est ancien. - Le moment appelé $S e j i$. $-\$ 865$. Renouvellement des quatre saisons, changement du ciel et de la terre. $-\$ 866$. Détermination des époques, calendrier. - $\$ 867$. Couleur jaune et justice. $-\$ 869$ et 870 . Renouvellement du jour. - $\$ 875$. Grand homme, tigre. Le dragon et le tigre, symboles du grand homme, c'est-à-dire, probablement, de la pleine lune. $\S 877$. Panthère se modifiant. La panthère, symbole de l'homme doué.

Koua $\mathrm{n}^{\circ}$ 50. - Ting: trépied, marmite. - Discussion au sujet de l'antériorité du symbole ou de l'objet. — $§ 883$. Marmite renversée les pieds en l'air. — $§ 891$. Marmite à oreilles jaunes. — $\$ 893$. Marmite à anse en pierres fines.

Koua $\mathrm{n}^{\circ}$ 51. - Tshen: ébranlement initial, cause première de toute création ou génération, foudre. — $\$ 902$. Gravir neuf collines, atteindre le 
septième jour. Sens du mot neuf, sens du mot sept. Ces mots ne sont pas compris, Tshou HI. — $\$ 911$. Justice ou milieu ?

Koua $\mathrm{n}^{\circ}$ 52. - Ken: arrêt. — §912. Arrêter le dos. - $\$ 915$. Accumulation de montagnes. — $§ 916$. Arrêter les orteils, les fixer. — $§ 918$. Fixer le talon. - $\$ 920$. Fixer l'arti culation de la hanche, briser les vertèbres. — § 922. Immobiliser le corps. — § 924. Immobiliser les coins de la bouche.

Koua $\mathrm{n}^{\circ}$ 53. - Tsien : avancer méthodiquement par degrés. - $\$ 928$. Retour de la jeune fille, arrivée dans la famille de l'époux. — § 934. L'oiseau hong avance peu à peu sur le rivage, péril du petit enfant.

Koua $\mathrm{n}^{\circ}$ 54. - Kouei mei : but vers lequel on tend. $-\S 946$. Marier une fille. - $\S 950$. Fin éternelle, destruction signifie séparation et éloignement. $\S 953$. Yeux affaiblis qui peuvent cependant voir, l'homme caché dans la retraite. - $\$ 954$. L'homme caché dans la retraite, il ne modifie pas la règle ordinaire. — § 959. La lune sur le point d'être pleine.

Koua $n^{\circ}$ 55. - Fong: grandeur, éclat achevé. — $\$ 963$. Le soleil au milieu de sa course. — §964. La lumière se meut. — $\$ 966$. Le soleil et la lune : déclin, décroît, apparence et disparition. — \$ 970. Écran, étoile visible au milieu de la course du soleil. - Tsheng Tse, le mouvement diurne uniforme. — $\$ 972$. Le soleil au milieu de sa course, voir les petites étoiles. $\S 974$. Étoile polaire visible au milieu de la course du soleil. — §975. Situation imméritée. — $\$ 978$. Absence de l'homme, se cacher dans la retraite.

Koua n 56. — Lou : voyageur. — §994. L'oiseau brûle son nid.

Koua $\mathrm{n}^{\circ}$ 57. - Souen : entrer. — $§ 999$. Suivre le vent, soumission. $\S 1000$. Avancer et reculer, hésitation, humilité. — § 1010. La hache.

Koua $\mathrm{n}^{\circ}$ 58. - Touei : plaire. — $§ 1016$. Satisfaction et harmonie. $\S 1024$. Usure, diminution graduelle de la positivité.

Koua $\mathrm{n}^{\circ}$ 59. Hoan : séparation, submersion. 
Koua $\mathrm{n}^{\circ} 60$. - Tsie : préceptes. - $§ 1051$. Ne pas sortir de la porte intérieure. - $§ 1053$. Ne pas sortir de la porte extérieure. — $\$ 1059$ et 1061. Préceptes agréables ou cruels.

Koua $\mathrm{n}^{\circ}$ 61. - Tshong fou : foi intérieure, confiance. — $\$ 1070$. L'oiseau qui chante dans l'obscurité. - — $\$ 1072$. Avoir un équivalent, faire paire avec ou opposition à quelque chose. — $§ 1074$. La lune est sur le point d'être pleine.

Koua $\mathrm{n}^{\circ}$ 62. - Siao kuo : petit excès. — $\$ 1086$. L'oiseau qui vole. $\S 1088$. Dépasser l'aïeul, rencontrer l'aïeule. — § 1092. Rencontrer sans dépasser. — $§ 1094$. Nuages épais sans pluie. — $§ 1096$. L'oiseau qui vole s'en sépare, calamités.

Koua $\mathrm{n}^{\circ}$ 63. - Ki tsi: ordre établi. - $\$ 1104$. Tirer la roue en arrière, mouiller la queue. — $§ 1106$. Après sept jours, réussir. — $\$ 1108$. Intervalle de trois ans. — $\$ 1110$. Avertissement de la fin du jour. — $\$ 1114$. Mouiller la tête.

Koua $\mathrm{n}^{\circ}$ 64. - Vi tsi: pas complètement achevé. — $\$ 1116$. Le petit renard. — $§ 1120$. Mouiller la queue. $\_\S 1122$. Tirer le char en arrière. $\S 1124$. Ordre pas encore établi. 


\section{O N C L U S I O N}

J'ai déjà dit ${ }^{1}$ les motifs qui me font considérer Fou Hi comme un mythe, personnification masculine des phases de la lune, mythe moins gracieux que Diane, dont le croissant est remplacé par des cornes.

D'après la tradition, Fou $\mathrm{Hi}^{2}$ aurait tiré la première idée des koua de la contemplation des phénomènes astronomiques; il aurait pris la première notion de chaque idée dans le ciel et l'origine de cette notion est appelée le symbole ; puis, par déduction ou simple analyse, il aurait appliqué le nom de ce symbole à une chose terrestre.

Le premier résultat de cette contemplation aurait été la distinction entre l'unité et la dualité et cette distinction aurait été immédiatement fixée et graphiquement représentée par un trait plein —, appelé $k i$ et un trait double — - appelé ngeou.

Comme les huit koua simples de trois traits, qui sont attribués à Fou Hi, sont composés par la répétition de ces deux traits, il en résulterait déjà, d'après la tradition, l'unité d'ori gine des soixante-quatre koua de six traits qui, tout au moins, dériveraient ainsi d'obser vations faites sur les astres.

Voyons d'abord le sens des deux mots qui servent à désigner ces traits.

Le premier, $k i$, est donné comme exprimant l'unité de tous les êtres ; unité, un seul. Mais, si l'on fait abstraction de la forme graphique pour ne tenir compte que du son donné à ce caractère, en consultant un dictionnaire phonétique, on remarquera de suite que le même son exprime le passage du néant à l'être ; la première manifestation sensible d'une cause dans ses effets ; - examiner, reconnaître la cause d'une chose ; - le support de toutes choses ; la révolution annuelle de 365 jours ; - l'action de compter ; le nom de la troisième étoile de la grande ourse.

Le mot ngeou, sous diverses formes, exprime la dualité ; - deux bœufs labourant ensemble sous le même joug; — une paire ; - contraste ; rencontre ; - opposition ; faire la paire ; former contraste avec quelque chose.

La lecture et l'étude approfondie du Yi king m'ont depu is longtemps amené à l'inter prétation suivante : L'unité désigne le cas où le soleil et la lune sont ensemble dans le même hémisphère, visibles au-dessus de l'horizon. La dualité désigne le cas où le soleil est dans un hémisphère et la lune dans l'autre, le soleil sous l'horizon, la lune visible au-dessus. De là une distinction fondamentale entre une région et une seconde région. La réflection sur les divers aspects de la lune, dans les deux circonstances, amenant la constatation

\footnotetext{
1 Tome VIII, page 6, Genèse du langage, Paris, Ernest Leroux, 1879, p. 168.

${ }^{2}$ Genèse du langage, p. 99 à 103. -Fou : caché, masqué, disparu. Hi, brillant, éclairé.
} 
de sa présence tantôt au-dessus, tantôt au-dessous de l'horizon aurait ensuite conduit à exprimer le rapport de position d'un objet placé « au-dessus » à un autre objet placé «au-dessous ». Par exemple, la présence de l'astre élevé dans le ciel devenant un terme de comparaison pour tout ce qui est élevé, «au-dessus », et son mouvement descendant suivi de disparition devenant le symbole de « bas », « dessous ».

Dans cet ordre d'idées, ce que dit Khong Tse, ou plutôt ce qu'il rapporte, peut prendre un sens et cesser d'être absurde ; on peut au moins entrevoir une interprétation rationnelle possible des paragraphes qui suivent immédiatement de 1223 à 1234 et 1244, 1257. Il n'y serait très probablement pas seulement question des inventions dont il y est parlé, et il s'agirait surtout de l'adaptation de sons, ou mots, exprimant des aspects, des circonstances de positions relatives des deux astres, soleil et lune, pour nommer des parties du corps, des animaux, des objets ou des instruments. Le paragraphe $n^{\circ} 1244$ me semble, au milieu de beaucoup d'autres presque inintelligibles, venir très nettement à l'appui de ma thèse. On ne manquera pas d'objecter à cela que Khong Tse cite ces faits comme de simples exemples d'une théorie tout à fait en dehors de la simple question astronomique ; c'e st bien là, en effet, l'apparence qui résulte de la rédaction que nous pouvons à peine lire, mais n'est-ce pas plutôt une lueur de vérité, un jalon lumineux conservé à dessein (ou échappé par inadvertance) et qui permet de retrouver la vérité dissimulée sous un fatras d'idées qui paraissent absurdes ?

Remarquons encore l'idée fondamentale, qu'on retrouve dans toutes les parties du Yi king, de la suite des phénomènes qui s'entraînent mutuellement comme conséquences inévitables les uns des autres : «contraction » suivie de «dilatation», «action» suivie de «réaction contraire» et encore : «augmentation», suivie de «diminution». Par la similitude des sons employés pour exprimer des idées souvent très éloignées en apparence, je suis encore ramené au même ordre d'idées : " phases de la lune », "croissance et déclin» comme source invariable de ces aphorismes si souvent répétés : « aucun être ne peut croître, se développer et persister définitivement »; « tout développement parvenu à son extrême limite est suivi de décadence et de destruction », destruction qui engendre et reproduit une nouvelle série de phénomènes analogues, «mouvement de circulation » sans fin qui « entoure » l'univers, c'est -à-dire le «ciel» et le «dessous» et qui est la «voie rationnelle » et la « raison d'être » de toutes choses.

Mais cette idée si confuse, si difficile à saisir, et que j’ai déjà essayé d'analyser et d'exposer dans le tome $1^{\text {er }}$ des Annales ${ }^{1}$ est-elle un simple accident ? est-elle exclusive à l'antiquité chinoi se ? À défaut de textes précis, tels que les souhaiterait M. Zeller ${ }^{2}$, pour établir une communication, une transmission de doctrine de l'Orient aux Grecs, il me semble qu'il suffit de considérer les fondements de leurs doctrines. Ces trois idées, que « tout résulte

\footnotetext{
${ }^{1}$ Exégèse chinoise.

${ }^{2}$ La Philosophie des Grecs, trad. par M. Boutroux. $1^{\mathrm{e}}$ partie, chap. II, p. 24 - p. 31.
} 
de l'air ${ }^{1}$ par raréfaction et condensation »; que le principe du mouvement, ou cause motrice, réside dans la chaleur et entraîne le monde dans un mouvement de circulation; enfin, les spéculations inintelligibles sur les propriétés des nombres, tout cela ne me semble pas susceptible d'avoir été inventé deux fois. Il y a peut-être eu communication, dans des temps relativement récents, mais il y a essentiellement transmission, suivant la filiation des races, et l'idée mère du «mouvement de circulation », de beaucoup la plus antique, a dû laisser une trace qui ne s'est pas effacée. Les mêmes erreurs d’interprétation donnant lieu, avec le temps, à des spéculations de plus en plus distinctes, chaque race y imprimant son cachet propre, le principe restant toujours le même.

Ce qui précède suffit à montrer, sans aucune hypothèse étrangère, et en ne tenant compte que de la tradition chinoise, de rapprochements entre les sens différents attribués à des mots homophones, et du sens qui semble souvent résulter de la traduction des textes lorsqu'on cherche à les lire sans se conformer aux leçons arbitraires des commentateurs, comment il est possible de découvrir le véritable sujet des idées exprimées par les koua.

Nous allons voir si l'étude particulière de ces signes et des lambeaux de texte qui y sont attachés depuis une très haute antiquité est susceptible de confirmer notre premier jugement, et même de le fortifier, en l'éclairant par des exemples dont quelques-uns, au moins, puissent paraître indiscutables. Pour cela, je reviens aux deux premiers traits de Fou Hi, traits qu'on désigne ensemble par l'expression : «Les deux $\mathrm{Yi}$ », ou règles.

Au-dessus de chacun de ces deux traits, Fou Hi, dit la tradition, les répéta tous les deux, ce qui donna quatre groupes de deux traits.

$\overline{\text { Le premier groupe est donné comme représentant }} \overline{\overline{-}} \overline{-}$ l'extrême positivité », le second «la petite négativité », le troisième «la petite positivité » et le dernier «l'extrême négativi té ».

Sil me fallait nécessairement chercher un sens à ces quatre signes, en les considérant comme des symboles, j’inclinerais à penser, en tenant compte du sens que je crois reconnaître à «l'unité » et à «la dualité », que le premier pourrait symboliser cette idée : que les deux astres sont alternativement tous deux ensemble au-dessus de l'horizon et au-dessous ; le second, que, quand les deux astres sont visibles ensemble sous l'horizon, on ne voit que l'un d'eux au-dessus de l'horizon ; le troisième, l'i dée réciproque, que quand on voit les deux astres ensemble au-dessus de l'horizon, on n'en voit qu'un seul à la fois sous l'horizon ; enfin le quatrième, analogue au premier, que dans les deux hémisphères alternativement il n'y a qu'un astre visible à la fois. Mais je doute qu'il faille leur chercher une valeur particulière et, en dépit de ce que la tradition rapporte au sujet de la matière dont furent formés les koua de trois traits, il me paraît possible que ceux-ci aient été imaginés sans avoir d'abord employé les quatre combinaisons ci-dessus, et je vais dire plus loin pourquoi.

\footnotetext{
${ }^{1}$ Khi. - On croirait lire la traduction d'un texte chinois ancien !
} 
Selon la tradition, en répétant encore chacun des deux traits au-dessus de ces quatre groupes, Fou Hi obtint enfin les huit koua simples :

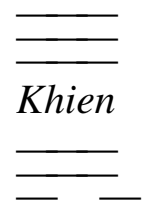

Souen

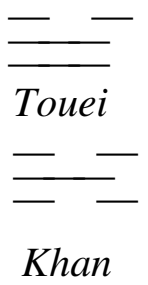

Khan

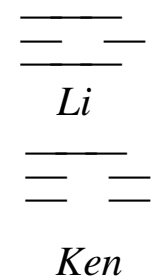

Ken

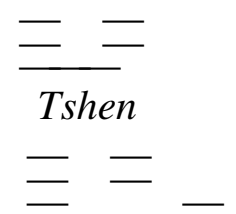

Khouen

Khong Tse dit $\left(n^{\circ} 1257\right)$ que le koua khien et le koua khouen, qui expriment, l'un la cause active, l'autre l'effet de cette cause, sont la « porte d'entrée ou d'accès » du livre, c'est-à-dire la clef de son intelligence. Si nous pouvons établir que ces deux signes symbolisent deux faits astronomiques, il sera bien prouvé que, pour Khong Tse la matière du Yi king est bien relative à l'astronomie.

Il résulte de la lecture de l'ouvrage entier que les trois rangs des traits d'un koua simple comportent chacun et invariablement un sens particulier. Le premier rang marque le début, l'infériorité, le mouvement ascendant; le second celui de milieu, et en même temps celui de supériorité ; le troisième marque la fin, le mouvement descendant, le commencement d'un fait inverse de celui dont il est question. Voilà pour les koua simples.

Les koua complets, de six traits, sont indubitablement, par la tradition et généralement par le sens attribué à quelques-uns d'entre eux, formés par la combinaison de deux koua simples superposés. De plus, il résulte encore de tout l'ouvrage quils ne se fondent pas en un seul tout. Chaque koua simple subsiste, les rangs marquent les mêmes idées dans chacun des deux koua simples superposés et il existe une «correspondance » entre la valeur d'un trait de l'un d'eux et du trait de même rang dans l'autre. Il faut donc admettre que le koua complet marque un parallèle entre deux faits correspondants et analogues.

Derrière l'idée vague de «droiture » dans l'expression « correspondance sympathique conforme, ou contraire à la droiture », il y a un sens, peut-être assez simple à saisir, et qui serait que deux traits, de même rang, pour se «correspondre avec droiture » doivent représenter deux phénomènes concordants tandis que, lorsque cette droiture fait défaut, les deux phénomènes, par leur nature ne peuvent pas naturellement coexister.

Enfin, le koua complet marque un «moment », littéralement, un temps, ou instant.

Ceci posé, il me semble résulter de la lecture du Yi king que le premier trait indique généralement, sinon toujours, une conjonction qui a lieu à l'horizon, à l'équinoxe du printemps. Le second trait indique ou une conjonction ou une opposition au moment où le soleil est au solstice d'été au solstice d'hiver ; dans le premier cas la conjonction est visible, dans le second 
la lune est pleine quand elle atteint sa position culminante dans le ciel. Enfin le troisième trait marque encore une conjonction au moment du coucher du soleil, à l'équinoxe d'automne.

Cette interprétation rend compte de la valeur attribuée à chaque rang d'un koua simple. De la nature de chacun des trois traits dépend le sens, ou l’idée exprimée par le koua simple de trois traits.

Deux koua simples superposés établissent une corrélation entre les phénomènes observés de «jour» ou de «nuit» et ils expriment une conclusion sur le mouvement combiné des deux astres. Le nom du koua désigne le jugement porté sur l'observation.

Là encore, l'interprétation rend compte des termes que j'ai rappelés ci dessus. Par exemple, il est clair que le koua marque un «moment»; de plus, si les traits de même rang indiquent deux phénomènes coexistant qui ont lieu chacun dans un des deux hémisphères, la correspondance est correcte, c'est-à-dire « conforme à la droiture », cela semble résulter par exemple de la lecture des paragraphes 1265 à 1275.

Je vais maintenant essayer de donner quelques preuves à l'appui de mon interprétation.

Il est facile de remarquer ${ }^{1}$ que les soixante-quatre koua parfaits sont rangés en trente-deux groupes dans lesquels le premier est linverse du second, ou représente une formation parallèle à celle du second.
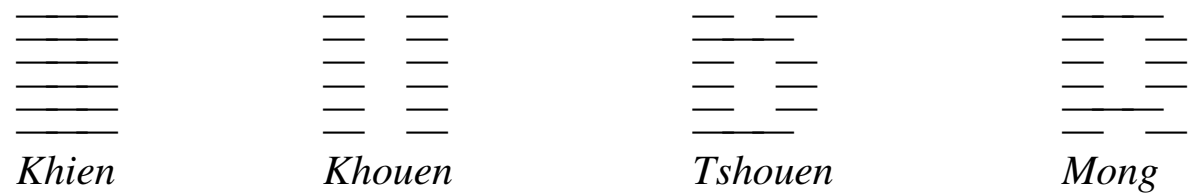

Aussi khien et khouen présentent deux formes parallèles, il en sera de même dans un grand nombre d'autres koua.

tshouen et mong présentent une inversion, puisque le koua supérieur dans le premier est le koua inférieur dans le second, et ensuite un parallélisme, puisque le koua inférieur de tshouen et le koua supérieur de mong ont à la fois une forme parallèle et présentent en plus une inversion ${ }^{2}$.
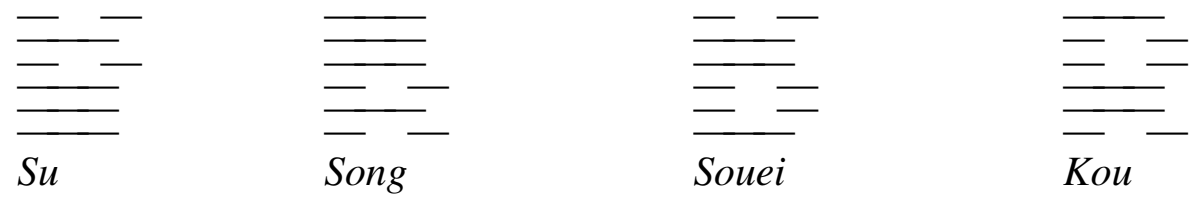

\footnotetext{
${ }^{1}$ Et cela a été de tout temps connu des Chinois, comme cela a été dernièrement remarqué par M. L. Feer. Voir, jaumal asiatique, année 1891. Tome XVIII, p. 373 et suivantes.

2 J'entends par parallélisme une symétrie dans l'arrangement des mêmes koua simples, ou dans celui des traits des deux koua simples différents.
} 
Les deux koua su et song, présentent seulement une inversion ; il en est de même dans les koua 7 et 8 par exemple. Les deux koua 17 et 18, Souei et kou, ne présentent qu'un simple paralléli sme de traits et ce cas est également fréquent Ces rapprochements de traits ou de koua simples concordent encore avec le sens que j'attribue aux koua et pour essayer de le prouver j'analyserai d'abord les deux premiers koua khien et khouen.

Khong Tse dit $\left(\mathrm{n}^{\circ} 1275\right)$ que ce qui constitue le $Y i$ king (livre des changements), ce qui le rend immense et complet, c'est qu'il contient la voie rationnelle du ciel, celle de l'homme et enfin celle de la terre, c'est -à-dire qu'il montre à la fois la nature des trois causes actives, ou efficientes, et enfin, c'est quil double chacune d'elles.

Si Khien, symbole du ciel, de ce qui est élevé, du jour, exprime la conjonction visible de la lune et du soleil au-dessus de l'horizon ; si khouen, symbole de la terre, de ce qui est bas, au-dessous, de la nuit, exprime que cette conjonction a eu lieu sous l'horizon ; enfin si jen, l'homme, symbolise le changement, le renouvellement de la lune; si le koua simple inférieur représente l'étude faite, par comparaison déduction et analogie, du mouvement de

la lune et du soleil, dans l'hémisphère inférieur, tandis que le koua simple supérieur représente cette étude faite directement sur les mêmes phénomènes astronomiques quand on observe les deux astres ou l'un d'entre eux dans l'hémis phère supérieur, ce passage de Khong Tse devient très simple et très naturel.

Le mot tao, que dans le passage ci-dessus j'ai traduit par «voie rationnelle », acception scolastique opposée à $l i$, « raison d'être » dont cette voie est la conséquence, désigne, en astronomie, au moyen de divers qualificatifs, l'équateur, l'écliptique, l'orbite de la lune.

Les six traits du koua Khien sont considérés comme symbolisant le dragon, long.

Long, le plus grand des reptiles; capable de se cacher et de devenir brillant ; capable de devenir très petit ou très grand, très long ou très court. À l'équinoxe du printemps il monte au ciel ; à l'équinoxe d'automne il plonge dans l'abîme (Shuo wen). — nom d'un ministre de Hoang Ti qui gouvernait la région de l'est ; nom d'un min istre de Shouen.

On peut encore rapprocher de ces diverses valeurs le mythe du dragon qui dévore la lune pendant les éclipses et il semble possible de supposer que le dragon représente le renouvellement de la lune, disparaissant pour reparaître ensuite.

Premier trait : la première conjonction a lieu à l'horizon dans l'hémisphère inférieur ; pour l'observateur placé dans l'hémisphère supérieur le soleil et la 
lune disparaissent à l'horizon (équateur), la conjonction va avoir lieu, mais il ne la verra pas : «le dragon est caché. » La lune va rester environ quinze jours sous l'horizon, elle réapparaîtra que plei ne et sur son déclin.

Le second trait marque la plus haute situation dans l'hémisphère inférieur, le « milieu » de la course de l'astre au solstice d' hiver, la conjonction a lieu sous l'horizon ; la lune apparaît dans l'hémisphère supérieur à son premier quartier, croissante, à l'hori zon, à la «surface de la terre »; elle monte, est pleine au bout de sept jours environ au moment de sa plus grande hauteur : "Voir le dragon au niveau de la terre, ce qui permet de voir le grand homme. »

Quel est ce grand homme ?

Jen, homme ; ce qu'il y a de plus précieux dans la nature du ciel et de la terre (Shuo wen). - Jen, homme ; la vertu du ciel et de la terre ; la liaison entre la négativité et la positivité ; la réunion du démon et de l'esprit (du mal et du bien, de ce qui se contracte et s'obscurcit et de ce qui se dilate en s'éclaircissant) ; l'éther subtil des cinq éléments (ou cinq agents : eau, feu, terre, bois, métal ; ou les cinq planètes) (Tse tien).

Le sens primitif, la filiation de ces diverses acceptions, la valeur cachée du mot homme sont bien difficiles à préciser, mais ces citations, que je ne cherche pas à multiplier, suffisent à montrer que l'int erprétation proposée n'est ni gratuite, ni arbitraire.

Le troisième trait occupe le rang qui marque la fin du phénomène exprimé par un koua simple, donc la fin de la course du soleil dans l'hémisphère inférieur, puisqu'il s'agit du koua inférieur. La conjo nction a lieu sous l'horizon et près de l'horizon ; la pleine lune aura lieu près de l'horizon, dans l'hémisphère supérieur, ce qui annonce le retour prochain du jour, tandis que c'est le « soir », la « fin du jour » dans l'hémisphère inférieur.

Le koua inférieur, marquant la période qui sera la nuit dans l'hémisphère de l'observateur, est fini et le koua extérieur va marquer les trois «moments correspondants » dans la période qui sera le jour pour l'observateur.

Quatrième trait, correspond au premier, c'est-à-dire qu'il marque l'origine du jour, mais dans l'hémisphère supérieur ; la correspondance n'est pas « conforme à la droiture », parce que ce trait exprime le même fait que le premier, savoir les deux astres visibles ensemble, et que cette circonstance ne peut coexister dans les deux hémisphères. La formule parle encore du dragon (conjonction), mais le sens est trop incertain pour rien conclure. Il est question d'un fait qui « peut » avoir lieu sous l'horizon ou au-dessus de l'horizon, et il est à remarquer que le commentaire traditionnel reporte encore à des observations astronomiques en parlant du temps où Shouen faisait des observations pour établir le calendrier.

Le cinquième trait qui marque le « milieu », la « situation prééminente » le « maître du koua » parle du «Dragon qui vole dans le ciel », ce qui, je crois, signifie simplement que les conjonctions ont lieu au-dessus de l'horizon et ce 
qui permet de voir le «grand homme ». Cette dernière partie de la phrase semble contraire à mon interprétation, le «grand homme » désigne la pleine lune, toutefois, il est à remarquer que, d'après la tra dition, il s'agit du « grand homme » représenté par le second trait ; ce passage pourrait donc simplement indiquer que le phénomène qui a lieu sous les yeux de l'observateur lui permet de se rendre compte du phénomène analogue, lorsqu'il a lieu sous l'horizon.

Le trait supérieur correspond au troisième trait, il marque le dernier moment de la période du jour dans l'hémisphère supérieur, période caractérisée par le mot «Ciel». Le «Dragon redescend», la dernière conjonction visible pour l'observateur ; elle annonce le retour de la nuit, de l'hiver ; il «y a des regrets ».

Dans ce koua, et par une exception qui n'a lieu que pour les deux koua Khien et khouen nous trouvons' une formule de plus que dans les autres koua : «Dans la troupe des dragons visibles, aucun ne précède les autres, présage heureux ». Cette formule a certainement pour objet d'expliquer l'usage exclusif des traits pleins, mais le sens est trop vague pour en tirer aucun argument probant.

Avant d'aller plus loin, il faut en passant, relever une des expressions les plus remarquables du Yi king, que nous rencontrons pour la première fois dans la formule du troisième trait : «l'homme doué ». Le premier caractère de cette expression désigne d'une façon générale tout roi ou prince souverain possédant des États ; par extension tout homme éminent, titre honorifique, appellation ou qualification très honorable réservée aux parents, aux supérieurs ; avec le second caractère il forme une expression composée qui désigne un mérite accompli. Il est impossible de rien tirer de là au point de vue de l'étymologie du mot, mais en se reportant à la formule du trait supérieur du koua siao tshou il semble que ce symbole représente particulièrement la pleine lune observée pendant la nuit. On peut voir à ce sujet $\S 783$, Tsheng Tse et $\S 784$, Tsheng Tse; mais c'est bien plus le rapprochement d'un nombre considérable de citations, qu'aucune citation particulière, qui peut amener à une conviction sur la valeur du sens du Yi king.

Voyons actuellement si l'analyse succincte du koua khouen nous conduit à des conclusions identiques.

1.- $\$ 20$.

2. - $§ 204$, koua $n^{\circ} 9$.

La formule attachée au koua parle de la faculté que possède «l'homme doué » de circuler : pendant la nuit la pleine lune est visible.

Il y est encore question de deux régions opposées sud-ouest d'une part, nord-est de l'autre ; le sud est le « dessous », le nord le « dessus ». Quand une des deux régions jouit de la vue d'un certain phénomène, l'autre région cesse d'en jouir. Ici le phénomène en question est exprimé par un caractère qui représente (dans sa forme actuelle) deux lunes côte à côte, et qui a un sens 
vague comportant l'idée de réunion, groupe, marcher ensemble, choses de même genre. Si j'avance qu'il s'agit des phases de la lune on pourra m'objecter que j'attribue une valeur bien restreinte à un mot dont le sens est très général, mais je demande pourquoi ce signe graphique de la lune répété à côté de lui-même ? pourquoi cette distinction de deux régions opposées ?

Premier trait ; commencement de la nuit dans l'hémisphère austral, les deux astres sont dans les deux régions opposées; il s'agit donc, pour l'observateur, du commence. ment de l'hiver. Or la formule parle de froid, de glace qui durcit, de refroidissement progressif

Deuxième trait ; correspond au solstice d'été dans l'hémisphère supérieur, la pleine lune éclaire l'hémisphère inférieur.

Troisième trait ; fin de la nuit dans l'hémisphère inf érieur.

Quatrième trait; commencement de la nuit dans l'hémisphère de l'observateur.

Dans les formules relatives à ces deux derniers traits le sens est trop vague pour y trouver aucun rapprochement.

Cinquième trait; le milieu de la nuit dans l'hémisphère supérieur; la conjonction a eu lieu sous l'horizon au moment du solstice d'hiver ; la lune apparaît à l'horizon au premier quartier, le décrit et monte, c'est le présage du retour du jour. Qu'indique l'expression jupe jaune ? Je remarque simplement que la route parcourue par le soleil, la lune et les cinq planètes est appelée « la route jaune », c'est une zone contenant l'écliptique, et que si, en dernière analyse, toute recherche sur le sens d'un mot ou l'origine d'une expres sion ou d'une forme graphique nous ramène invariablement à l'étude du mouvement des astres, il faudra bien arriver à reconnaître un lien entre les origines du langage et ces mêmes phénomènes.

Sixième trait; fin de la nuit dans l'hémisphère nord ; "Dragons combattant dans le désert »; le désert c'est ce qui est au -delà des bornes de la région occupée ; les conjonctions ont lieu près de l'horizon, mais en dessous. «Le sang répandu est jaune sombre »; serait-ce une allusion à la lumière de l'aurore ${ }^{1}$ ?

Dans ce koua, comme dans le précédent, une septième formule mentionne l'emploi exclusif de traits doubles.

D'après moi, ces deux koua «positivité absolue» et «négativité absolue », unité de chaque substance, expriment simplement un premier fait : le «jour» comme la «nuit» existent de la même façon dans chaque hémisphère et ce jugement résulte de la comparaison des phénomènes observés successivement, le jour et la nuit, dans le même hémisphère. Dans «L'Ordre des koua » nous lisons : «Après que le ciel et la terre existent... »; khien est donc le ciel et khouen la terre ; consultons ensuite les «oppositions de koua » commentaire également attribué à Khong Tse et nous y lisons que

\footnotetext{
1 «Le noir et le jaune, c'est le rapprochement du ciel et de la terre. »
} 
khien c'est l'énergie et khouen la soumission; les diverses explications commentées traditionnellement nous montrent que khien est une cause active dont les effets se manifestent et s'expliquent par le koua khonen : tout cela est très vague et inexplicable pris à la lettre, mais devient très simple si on admet que le mot khouen exprime simplement cette pensée que tous les phénomènes observés la nuit, sur la lune, sont les effets du soleil qui se trouve alors dans l'autre hémisphère : il y a bien alors cause active, d'une part, et effet reçu passivement de l'autre.

Ajoutons que le sens vrai des formules est moins probant que dans le koua précédent, ce qui est assez naturel, puisque les idées se compliquent et que les phénomènes sont moins simples à analyser. Mais si on m'objectait que les allégations que j'avance sont bien vagues, je répondrais, une fois pour toutes que je ne prétends pas donner une version définitive mais offrir seulement une voie à suivre pour arriver au résultat et qu'enfin l'évidence de ma théorie résulte non d'un seul passage, mais, au contraire, du groupement d'un nombre presque indéfini d'allusions et de sous -entendus qui font transsuder la vérité à travers les voiles dont elle est enveloppée.

Il me paraît naturel, dans la recherche du sens des koua simples, de procéder par l'étude des koua complets formés par la répétition du m ême koua simple, comme nous l'avons fait pour les deux premiers koua khien et khouen. Par conséquent, je suis naturellement amené à étudier s'il est possible de poursuivre cette interprétation dans le koua $n^{\circ} 57$. Souen «entrer », qui, dans l'« ordre des koua» fait paire, ou contraste, avec le koua touei.

Idée de répétition d'un même fait ; ( 9997$)$. Provient du koua simple khien dans lequel le trait inférieur s'est transformé en trait brisé (Tshou Hi, § 1000).

D'autre part, Khong Tse, dans les « oppositions des koua»dit : «Touei, manifester, souen, cacher ». Entrer a le sens de «s'abriter, se mettre hors de vue ».

Premier trait ; la formule parle d'avancer et reculer. Le caractère souen est considéré comme ayant été couramment employé dans l'antiquité avec le sens de « se retirer en arrière ».

Je ne vois rien de probant dans les formules des traits suivants jusqu'au cinquième, où on lit : «pas de commencement et fin »; trois jours avant le jour kang ; trois jours après le jour kang ». (§ 1008 Tsheng Tse et Tshou Hi.)

Kang est le nom d'un des dix caractères qui servent à former les noms des jours, et aussi, les noms des années. D'après le Shuo wen, «la position du signe kang (dans le cycle des dix signes) correspond à la région de l'ouest et symbolise le moment de l'automne; les êtres ont tous atteint leur développement parfait ».

Dans le Tse tien, parmi les explications données au sujet du caractère fou (du nom de Fou Hi) dont le sens général est " cacher, recourir, voiler », on retrouve cette expression : «trois kang », ou «troisième kang », et kang est 
pris avec le sens de changement: "Après le solstice d'été, le troisième changement constitue (ou est appelé) première disparition, ou commencement de la disparition; le quatrième changement (kang) est le milieu de la disparition ; le premier changement après le milieu de l'automne est appelé la fin de la disparition. » Bien que le sens exact me paraisse très douteux, il est certain qu'il s'agit encore ici d'un moment particulier des phases de la lu ne et de la révolution apparente du soleil autour de la terre.

Dans le koua $\mathrm{n}^{\circ} 58$ touei gloire, satisfaction. La formule $(\S 1024) \mathrm{du}$ cinquième trait parle de «l'usure », diminution par frottement, terme que nous verrons plus loin comme synonyme de décroissance de la lune.

Ici, le trait brisé (dualité, les deux astres dans deux hémisphères différents) est au dernier rang du koua simple touei, c'est-à-dire au rang qui marque la fin du jour, ou la fin de la nuit.

En rapprochant ces deux koua, qui marquent certainement une opposition ou contraste entre deux faits analogues ou contraires, je serais porté à leur attribuer les significations suivantes. Souen : au commencement du jour dans chaque hémisphère, les deux astres se trouvent dans les hémisphères opposés et la lune se cache, entre sous l'horizon.

Touei : à la fin du jour dans chaque hémisphère les deux astres sont dans les hémisphères opposés et la lune apparaît sur l'horizon.

Après souen et touei, viennent les deux koua li (30) khan (29), et Khong Tse dans les «oppositions des koua » dit: «Li monter, khan descendre » 1 . «L'Ordre des koua » dit : «khan, tomber ; li séparation ».

Dans le koua khan, un trait plein est au milieu de deux traits brisés ; c'est le contraire dans le koua $l i$ :

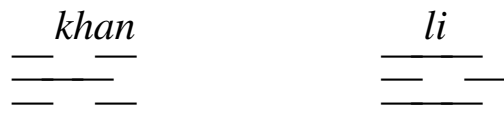

Si nous acceptons les quatre lectures précédentes comme bonnes, sans recourir au texte ou à la traduction, nous serons naturellement amenés à dire :

Khan ; au milieu de la nuit, dans chaque hémisphère, les deux astres sont ensemble dans un même hémisphère.

$L i$; au milieu du jour, dans chaque hémisphère, les deux astres sont dans les hémisphères opposés.

Mais le sens ne serait pas bien précis.

\footnotetext{
${ }^{1}$ Dans l'ordre que l'on appel le ordre des koua selon Fou Hi, li, désigne l'est et khan désigne l'ouest.
} 
$L i$, exprime le rapprochement, la lumière, la clarté, le feu ; deux choses se rapprochant mutuellement; être en contact (\$ 545) «s'attacher, se joindre à quelque chose, comme par exemple, le soleil et la lune sont attachés au ciel». Dans la formule du second trait on lit «clarté jaune ».

« Clarté du soleil qui décline » $(\$ 542)$.

Khan exprime le danger, la chute, le péril, répétition de chute ou de péril ; deux périls superposés ${ }^{1}$.

En tenant compte de ces diverses acceptions des deux mots $l i$ et khan, je suis porté à interpréter comme suit les deux sens que je viens d'attribue r à ces deux koua.

Khan ; au milieu de la nuit, c'est-à-dire au solstice d'hiver, la conjonction a lieu au moment où les deux astres sont au point le plus bas de leur course et les deux astres sont ensemble sous l'horizon, c'est -à-dire que le moment où les deux astres sont en conjonction sous l'horizon marque le milieu de la nuit, le moment le plus pénible à cause du froid et de l'absence de lumière.

$L i$; au milieu du jour quand la lune est pleine, les deux astres sont dans les deux hémisphères opposés, c'est-à-dire que le moment où les deux astres sont en opposition marque le milieu du jour.

Les répétitions de chacun des deux koua simples indiquent que le fait a lieu de la même manière successivement dans les deux hémisphères.

Ces deux koua terminent la première partie du Yi king.

${ }^{1} \mathrm{Li}$ symbolise le feu; khan symbolise l'eau. Or l'une des anciennes formes du caractère paraît précisément représenter le koua simple khan. Voir Shuo wen. 
Il nous reste encore à examiner deux koua complets formés par la superposition d'un koua simple sur lui-même : ce sont les koua

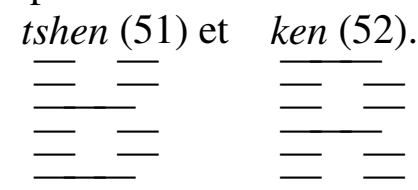

L'Ordre des koua » dit seulement «tshen, mouvement ; ken, arrêt. » Dans les « oppositions des koua» on lit «tshen, s'élever ; ken, s'arrêter ». Mais Khong Tse rapproche de cette opposition celle qui existe entre les koua souen (41) et $y i$ (42) «qui marquent le commencement de la décadence (décroissance) et du parfait développement (croissance).

Tshen, région de l'est ; la foudre, ce qui cause la crainte, le respect; s'élever.

Deuxième trait $(\$ 902)$ « atteindre le septième jour ».

Ken, arrêt.

Sur ces deux koua qui forment évidemment opposition, les formules ne nous donnent aucun indice relativement au fait quils symbolisent et nous sommes réduits, pour le moment, à chercher à les interpréter par analogie. Tshen semble indiquer un phénomène qui a lieu au commencement de la nuit, ken un phénomène analogue qui a lieu à la fin de la nuit.

Par exemple: tshen, au commencement de la nuit, dans chaque hémisphère la lune nouvelle s'élève au-dessus de l'horizon.

Et ken, à la fin de la nuit, dans chaque hémisphère la lune disparaît.

On pourra tout d'abord objecter à cette lecture, qui n'est qu'une hypothèse, que le nom du koua, ken, signifie arrêt. Mais là encore il y a lieu de craindre un cercle vicieux. Le sens d'arrêt, ou de limite, vient très probablement, sinon des commentaires traditionnels, du moins d'une nouvelle idée éveillée par le phénomène primitivement considéré. D’après le Shuo wen, ken signifie désobéir, aller contre, aller en sens contraire, yeux qui se lèvent ensemble et qui ne s'abaissent pas en semble.

D’après les commentaires, ken est aussi le symbole de la montagne, de l'obstacle, de ce qui par conséquent arrête la vue, à l'horizon.

Il ne peut entrer dans mon plan d'entreprendre ici une analyse complète de chaque koua et d'essayer d'expliquer, au moyen du sens que je crois voir représenté par chaque koua simple, le sens plus complexe de l’idée représentée par les koua complets de ses traits. J'ai voulu donner une traduction complète de l'ouvrage entier, textes et commentaires les plus intéressants, et par là mettre un plus grand nombre de personnes à même de se former une opinion sur la question. Mon travail est surtout un guide pour les sinologues qui voudront étudier le $Y i$ king et, au besoin, plus tard, en recommencer l'analyse. Dans mon esprit, cette analyse ne sera jamais 
complètement fructueuse que si elle est faite en suivant mot à mot le texte chinois lui-même.

Aussi, je vais terminer en relevant simplement et très rapidement le sens astronomique de quelques koua plus particulièrement faciles à interpréter.

Le koua khouen est le koua du dixième mois ; le moment qu'il marque correspond par conséquent au solstice d'hiver ; c'est le moment de la nuit la plus épaisse dans l'hémjsphè re boréal et la pleine lune qui suit brille de tout son éclat.

Le koua fou $\left(\mathrm{n}^{\circ} 24\right)$ est le koua du onzième mois, il exprime le retour et les «oppositions des koua» contiennent ce passage «Po, maturité, clarté ; fou, retour ${ }^{1} »$. Si le dixième mois correspond au solstice d'hiver, le onzième marque bien le commencement du retour du soleil vers l'hémisphère boréal. Po $\left(\mathrm{n}^{\circ} 23\right)$ est le koua du neuvième mois et son nom est considéré comme exprimant l'usure, la destruction par l'usage répété.

$\mathrm{Si}$ on se reporte au sens que nous avons proposé pour les huit koua simples,

est formé du koua khouen surmonté du koua ken et devrait se lire :

Nuit dans l'hémisphère austral ; à la fin de la nuit la lune se couche décroissante (par suite elle se lève décroissante dans l'hémisphère boréal).

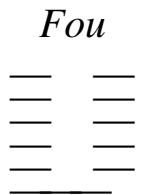

formé du koua supérieur khouen et du koua inférieur tshen, ce qui pourrait se lire :

Nuit dans l'hémisphère boréal, au commencement de la nuit la lune se lève croissante.

Mais il est bien douteux que chaque koua simple puisse ainsi conserver sa signification propre ; au contraire, il y a tout lieu de croire que le sens du koua complet ne tient à celui de chacun des koua simples que par une association d’idées plus ou moins impossible à retrouver au premier abord et $\mathrm{p}$ ar suite de combinaisons d'acceptions ultérieurement données à chacun de ces koua simples.

\footnotetext{
${ }^{1}$ Retour, « renaissance ».
} 
Les « oppositions des koua » portent encore « $P$ ' $i$, thae.. expriment des choses contraires. »

Thae $\left(\mathrm{n}^{\circ} 11\right)$, prospérité, koua du premier mois.

$P ’ i\left(n^{\circ} 12\right)$, décadence, koua au septième mois.

Ces deux koua désignaient dans les deux équinoxes :
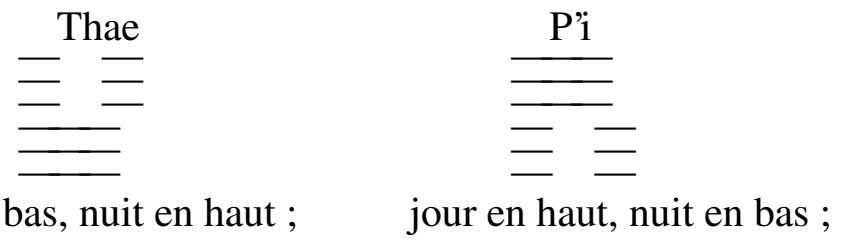

J'arrête ici toute citation ou tout complément d'analyse. Mon travail est une traduction discutée, rien de plus. Malgré ses imperfections, les erreurs inévitables qu’il peut renfer mer, je crois pouvoir dire que mon essai est le plus indépendant et en même temps le plus fidèle de ceux qui ont été entrepris jusqu'à ce jour et dont j'ai pu avoir connaissance. Je serais heureux de le voir discuter; le problème que j'indique est assez grandiose pour appeler l'attention des sinologues. Si mes données sont fausses, on peut en démontre $r$ la fausseté, mais elles méritent mieux qu'un silence dédaigneux. 


\section{P O S T F A C E}

Le premier volume de cette traduction a paru en 1885 ; des circonstances absolument indépendantes de moi, ont fait durer l’impression $\mathrm{du}$ second volume jusqu'à ce jour, après plusieurs interruptions, dont l'une a duré près de trois ans.

Dans cet intervalle d'autres sinologues ont à leur tour abordé la recherche de la solution du problème étrange que présente le Yi king. Je veux parler d'a bord de M. C. de Harlez et ensuite de MM. les Drs A. de Lacouperie et Douglas ${ }^{1}$, mais je ne m'occuperai que de la version de M. de Harlez, la seule dont j'ai quelques notions.

Tout en remerciant M. de Harlez de la bienveillance avec laquelle il parle de mon travail, je suis obligé de relever quelques-unes de ses appréciations. D’abord, j’ai vécu plus de vingt -deux ans dans un milieu presque absolument chinois et on me croira, je pense, facilement si j'affirme que jamais je n'ai pu me dire «c'est du chinoi s et l'on ne doit point juger cela comme les produits des terres occidentales ».

Quant aux «bizarreries des explications des lettrés de l'empire des fleurs », il n'y a aucun doute à avoir qu'elles ne m'ont pas échappé, surtout après ce que j'en ai écrit, en 1879, dans la Genèse du langage, p. 161 et suivantes.

J'ai donc quelque droit d'être étonné de ne pas me voir rangé dans la classe des sinologues qui «ne se sont pas contentés de ces traditions des fils de han ». Je me reporte encore, à ce sujet, à ce que j'ai écrit dans la préface du premier volume du Yi king, en citant le P. de Prémare et en rappelant que les écrivains chinois admettent très généralement que la véritable interprétation du Yi king est perdue. J'y ai expliqué, en même temps, la néces sité de lire et de traduire les principaux commentaires chinois.

Enfin, il ressort de toute ma traduction que je ne me sers de ces commentaires que pour y rechercher des indices, laissés par la tradition, sur le véritable sens du Yi king et pour prouver que les lectures admises faussent le sens naturel des mots et le dénaturent.

\footnotetext{
1 Je ne connais ces travaux que par les articles de M. de Harlez, insérés dans le Journal asiatique. Le Texte originaire du Yih-king, sa nature et son interprétation, par M. C. de Harlez, 1887, Sér. 8, T. 9, pp. 424-456. Le texte originaire du Yih king, sa nature et son interprétation, par M. C. de Harlez, addition 1891, Sér. 8, T. 17, pp. 164-170, et un article de M. L. Feer, paru dans les Nouvelles et mélanges: le Yi-king, texte primitif rétabli, traduit et commenté par Ch. de Harlez, t. XVIII, p. 373,1891.
} 
Il résulte de ceci que, si les critiques chinois m'ont devancé, ce qui est assez naturel, je puis néanmoins revendiquer l'honneur, si c'en est un, d'être ${ }^{1}$ le premier critique européen qui ait reconnu l'existence d'un sens caché et inconnu des commentateurs et qui ait proclamé la nécessité de rejeter toutes les interprétations traditionnelles pour chercher dans le texte seul un sens moins dénaturé.

Je ne puis que me féliciter de voir que mes idées ont attiré l'attention de savants aussi distingués que M. Ch. de Harlez sur la recherche du sens propre du Yi king, mais ma méthode diffère de la sienne.

Je prétends qu'on ne peut étudier le Yi king qu'en s'aidant de la tradition ; non pas en l'acceptant sans critique, mais en y cherchant des indices sur la nature du livre.

M. Ch. de Harlez, de son côté, fait table rase, ou du moins croit faire table rase de tous les commentaires. M. de Harlez, que je ne cite que d'après les articles du Journal asiatique, considère le koua comme un hiéroglyphe et le $Y i$ king comme un dictionnaire desdits hiéroglyphes.

Ainsi $^{2}$, M. de Harlez admet que le koua khouen ci-dessous est un hiéro-

$$
\vdots \vdots \vdots
$$

glyphe qui représente la terre ; que le mot khouen, jusqu'ici considéré comme servant à désigner le koua en rappelant ce qu'il représente, a simplement le sens de « terre, en tant que recevant l'action de la force universelle ».

Jusqu'ici, le traducteur a bien réellement renoncé à tous les commentaires. Mais alors, il eût dû s'en tenir là. Au lieu de cela, il accepte encore « certains mots, certaines phrases où l'on voit l'exposé des pronostics divinatoires qu'il $\mathrm{y}$ a à tirer de la figure expliquée » et il dit «qu'ayant admis les deux premiers points, cette troisième partie du Yi king ne sera pas moins claire si l'on sait y reconnaître des explications, des exemples, des développements, tels que les lexiques chinois sont habitués à en donner ».

Or, ces phrases, cette troisième partie, constituent, d'après toutes les traditions, le plus ancien commentaire. M. de Harlez fait donc un choix ; il ne rejette pas tout en bloc, ou bien il nie simplement, et sans aucune preuve à l'appui de sa négation, la tradition qui attribue cette partie du texte à Wen Wang, quel que soit d'ailleurs ce personnage. De plus, et sans discuter la traduction que $\mathrm{M}$. de Harlez donne de cette troisième partie, je remarque encore que ce traducteur déclare telle partie «ajoutée tardivement», telle autre «phrase stéréotypée » ${ }^{3}$, et que, parfois, il ne craint pas de se servir des commentaires plus modernes pour trouver un sens concordant avec sa manière de voir ${ }^{4}$. Pour être absolument conséquent avec la méthode qu'il prétend

\footnotetext{
${ }^{1}$ Après le P. Prémare qui y cherchait autre chose.

2 Journal asiatique, $8^{\mathrm{e}}$ série, t. IX, p. 431.

${ }^{3}$ Loc. cit., p. 437, renvoi 2 et renvoi 3.

${ }^{4}$ Loc. cit., p. 442, renvois 1 et 2 .
} 
suivre, il aurait peut-être été meilleur de renoncer absolument à se servir de toutes les lectures admises et il eût été intéressant de savoir si, mis en face du texte qu'il a traduit et rien que de ce texte, il eût trouvé exactement le sens qu'il indique ?

M. de Harlez sent fort bien que son interprétation, qui heurte toutes les traditions et prétend n'en pas tenir compte, est faite pour soulever quelques doutes ; il va au-devant de certaines de ces objections, et, pour y répondre, il avance que «le Yih King n'était pas fort répandu »; et il ajoute : «il était même probablement peu connu». Sur quelles données appuie-t-il cette assertion contraire à toutes les traditions ?

Je remarque avec intérêt que $\mathrm{M}$. de Harlez a comme moi ${ }^{1}$, mais avec un jugement différent, songé à rapprocher les signes des koua et les traits des caractères cunéiformes ${ }^{2}$; puis il arrive à cette conclusion qu'il est presque impossible de ne pas voir dans le Yi king «quelque chose comme des notes écrites sur le carnet d'un homme politique chinois », une « sorte de matière de méditation, de memento quotidien ».

Enfin il parle de l'origine probable des lignes pleines ou coupées qui servent à composer les koua et se demande si on n'y pourrait voir une « sorte de représentation des cordes droites et simples ou nouées qui, selon la tradition, servirent de premier instrument graphique pour l'expression des idées et des mots. »C'est là un fait affirmé par la tradition écrite, en Chine, et je l'ai également déjà relevé ${ }^{3}$.

Je regrette de n'avoir pu lire la traduction de $\mathrm{M}$. de Harlez pour pouvoir juger de ce qu'il avance que « des commentaires plus modernes expliquent le texte comme lui ». Mais, après la lecture de ces deux articles, j'avoue que je ne suis pas ébranlé dans mes convictions à l'égard du Yi kinget du sens caché qu'il peut renfermer. Je diffère surtout et absolument d'opinion avec lui sur un point; M. de Harlez considère que la question est «si simple qu'on se demande en vain comment on n'y a jamais p ensé ». Après de longues années d'études, je crois que le problème est soluble, mais que sa difficulté est extrême et que, pour arriver au résulat, il faudra du temps et le concours de nombreux ouvriers, ce qui fait que je me réjouis de voir des hommes tels que M. de Harlez, et de Lacouperie s'en occuper à leur tour.

Cette discussion des opinions de M. de Harlez m'était en quelque sorte imposée. Après le long intervalle écoulé entre l’impression du premier volume de ma traduction et celle du second, et comme la traduction a été achevée dès 1879, alors que je me trouvais à Hué, on eût pu se demander si l'opinion de M. de Harlez n'aurait pas modifié mes idées sur ce sujet et si, par conséquent la suite de ma traduction conservait son utilité et sa valeur, ce que j'ai tenu à affirmer en rétablissant en même temps la véritable idée que j’ai du Yi king, de

\footnotetext{
${ }^{1}$ Genèse du langage, p. 185, Paris, Ernest Leroux, 1879.

2 Journal asiatique, 1887, p. 454.

${ }^{3}$ Genèse du langage, p. 168 et 175.
} 
la manière de le traduire, et l'antériorité, sinon la priorité de mes vues sur cette question. 
1. Khien - 2. Khouen - 3. Tshouen - 4. Mong - 5. Su - 6. Song - 7. Shi - 8. $\underline{\text { Pi }-9 . ~ S i a o ~ t s h o u ~-~ 10 . ~ L i ~-~ 11 . ~ T h a e ~-~ 12 . ~ P ’ ~}$

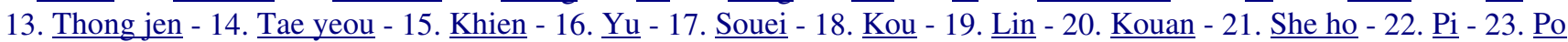
24. Fou - 25. Wou wang - 26. Tae tsou - 27. Yi - 28. Tae kuo - 29. Khan - 30. Li - 31. Hien - 32. Heng - 33. Thouen 34. Ta tshang - 35. Tsin - 36. Ming yi - 37. Kia jen - 38. Khouei - 39. Kien - 40. Kiae - 41. Souen - 42 . Yi - 43 . Kouae 44. Keou - 45. Tsouei - 46. Sheng - 47. Khouen - 48. Tsing - 49. Ko - 50. Ting - 51. Tshen - 52. Ken - 53. Tsien - 54. Kouei

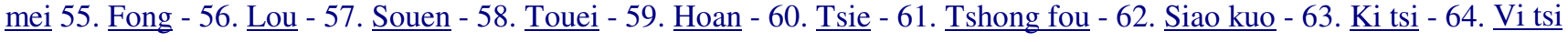

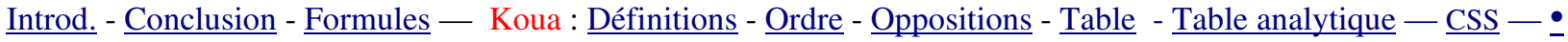


Nom du document : Yi_king.rtf

Dossier :

Modèle :

C:\CSS\Envoi021204\classiques

DatalMicrosoft $\backslash$ Modèles $\backslash$ Normal.dot

Titre :

Sujet :

Auteur :

C:IWINDOWS $\backslash$ Application

Mots clés :

Commentaires :

iences_sociales/index.html

Yi king (Yi Jing, I Ching)

série Chine

divers

Date de création : $\quad$ 05/12/04 10:44

$\mathrm{N}^{\circ}$ de révision : 2

Dernier enregistr. le : 05/12/04 10:44

Dernier enregistrement par : Pierre Palpant

Temps total d' édition1 Minute

Dernière impression sur : $\quad$ 05/12/04 10:45

Tel qu' à la dernière impression

Nombre de pages : $\quad 1271$

Nombre de mots : 317285 (approx.)

Nombre de caractères : 1808528 (approx.) 